Agnieszka Hunstiger und Uwe Koreik (Hg.)

\title{
Chance Deutsch
}

Schule - Studium - Arbeitswelt

34. Jahrestagung des Fachverbands Deutsch als Fremdsprache an der Leibniz Universität Hannover 2006

\section{Band 78}

Materialien

Deutsch als Fremdsprache

\section{fadaf}



Agnieszka Hunstiger und Uwe Koreik (Hg.)

Chance Deutsch

This work is licensed under the Creative Commons License 2.0 "by-nd", allowing you to download, distribute and print the document in a few copies for private or educational use, given that the document stays unchanged and the creator is mentioned.

SORE RIIGHIS RESERVED 
erschienen als Band 78 in der Reihe „Materialien Deutsch als Fremdsprache” in den Universitätsdrucken im Universitätsverlag Göttingen 2009 
Agnieszka Hunstiger und

Uwe Koreik (Hg.)

\section{Chance Deutsch}

Schule - Studium - Arbeitswelt

34. Jahrestagung des Fachverbandes

Deutsch als Fremdsprache 2006

an der Leibniz Universität

Hannover

Materialien

Deutsch als Fremdsprache

Band 78

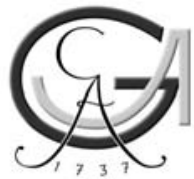

Universitätsverlag Göttingen

2009 


\section{Bibliographische Information der Deutschen Nationalbibliothek}

Die Deutsche Nationalbibliothek verzeichnet diese Publikation in der Deutschen Nationalbibliographie; detaillierte bibliographische Daten sind im Internet über $<$ http://dnb.ddb.de $>$ abrufbar.

Die „Materialien Deutsch als Fremdsprache“ sind eine Reihe des Fachverbands Deutsch als Fremdsprache e.V. (FaDaF), in der Tagungsergebnisse, Dissertationen und andere wichtige Einzeldarstellungen aus dem Bereich Deutsch als Fremdsprache veröffentlicht werden.

http://www.fadaf.de/de/Publikationen/mat_daf/

\section{fadaf}

Dieses Buch ist nach einer Schutzfrist auch als freie Onlineversion über die Homepage des Verlags sowie über den OPAC der Niedersächsischen Staats- und Universitätsbibliothek (http:/ / www.sub.uni-goettingen.de) erreichbar. Es gelten die Lizenzbestimmungen der Onlineversion. Es ist nicht gestattet, Kopien oder gedruckte Fassungen der freien Onlineversion zu veräußern.

Titelabbildung: http://www.flickr.com/photos/iik-duesseldorf/ Satz und Layout: Uwe Koreik

(C) 2009 Universitätsverlag Göttingen http://univerlag.uni-goettingen.de ISBN: 978-3-941875-04-3

ISSN: $1866-8283$ 


\section{Inhalt}

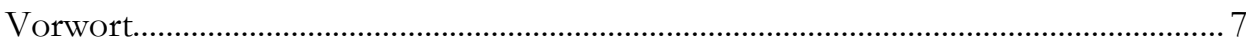

\section{Themenschwerpunkt 1: \\ Integrationskurse $\mathrm{DaZ}$}

\section{Jörg Matthias Roche}

Wie Deutsche mit Ausländern sprechen und was man daraus für den

Spracherwerb und den Sprachunterricht lernen kann...

\section{Christoph Hausschild / Erika Hoffmann}

Die Integrationskurse des Bundes - Bilanz und Weiterentwicklung. 35

\section{Rosemarie Buhlmann}

Die Konzeption des Bundesamtes für Migration und Flüchtlinge für die Zusatzqualifizierung von Lehrkräften im Bereich Deutsch als Zweitsprache

\section{Redaktionsteam der ZQDaZ (Landesverband Niedersachsen)}

Lehrkräftequalifizierung für den Unterricht in Integrationskursen. Konzept des Landesverbandes der Volkshochschulen Niedersachsens e.V.

\section{Sabine Jentges}

Zur Rolle von Alphabetisierung und Nullanfängerunterricht in der Ausbildung von DaF-Lehrpersonen. 65

\section{Beate Blüggel / Amadeus Hempel / Simone Henke}

Die organisatorischen und finanziellen Rahmenbedingungen der Integrationskurse des BAMF und die daraus resultierenden pädagogischen Konsequenzen. 


\section{Themenschwerpunkt 2: \\ Testen und Prüfen in DaZ / DaF}

\section{Christian Krekeler}

Auswahltests für alle ausländischen Studienbewerber statt Feststellungsprüfung für wenige?

\section{Dorrie Goossens}

Eine Prüfung für $\mathrm{DaF}$ in den Niederlanden am Beispiel Hörverständnis 109

\section{Sylwia Adamczak-Krysztofowicz}

Zur Überprüfung des Hörverstehens in der neuen Fassung der DaF-

Abiturprüfung in Polen.

\section{Tibor Vígh}

Der Prüfungsteil Schreiben im neuen ungarischen Abitur für Deutsch als Fremdsprache

\section{Katalin Petneki}

Problematik der Niveaustufen im neuen Abitur für Deutsch als Fremdsprache in Ungarn

\section{Ulrike Arras}

Wie es zu einer Beurteilung kommt. Ein Forschungsbericht zu Strategien bei der Beurteilung schriftlicher Leistungen im Kontext der Prüfung TestDaF.

\section{Beate Zeidler}

Niveaustufenbeschreibungen vergleichbar machen: "Is my B1 your B1?"

\section{Roland Forster}

Mündliche Noten - wofür? und wie?. 


\section{Christina Eberle / Matthias Perner}

DSH-Qualitätssicherung am Fachsprachenzentrum der Leibniz Universität Hannover

Themenschwerpunkt 3:

Schreiben und Sprechen in den Wissenschaften

\section{Henning Hermann-Trentepohl / Martin Lange}

Dimensionen wissenschaftlichen Schreibens -Problemaufriss und Vorschläge

für den Unterricht in studienbegleitenden DaF-Kursen

\section{Gabriele Graefen}

Der lästige „Kleinkram“ beim (wissenschaftlichen) Schreiben in der

Fremdsprache.

\section{Jin Zhao / Antje Stork}

Die wissenschaftliche Seminararbeit: Stolperstein für chinesische

Studierende?

\section{Michaela Reinhardt}

Sprechen in den Wissenschaften - zunächst mit Humor.

\section{Anja Centeno García}

Das mündliche Seminarreferat zwischen Theorie und Praxis 323

\section{Ingrid Mummert}

Subjektivität beim Schreiben in der Fremdsprache 


\section{Themenschwerpunkt 4: \\ DaZ und Mehrsprachigkeit}

\section{Ernst Apeltauer}

Sprachliche Frühförderung von Kindern mit Migrationshintergrund.... 355

\section{Gunde Kurtz}

Förderunterricht Deutsch als Sprache.

\section{Andrea Wagner}

$\mathrm{DaF} / \mathrm{DaZ}$, Mehrsprachigkeit und gelebte Internationalität - Beispiele aus dem

Bildungsangebot und Alltag an einer International School in Deutschland....

\section{Martina Rost-Roth}

Korrekturen und Ausdruckshilfen im Deutsch-als Zweitsprache-Unterricht.

Fallstudien und Vergleiche mit anderen Kontexten der Sprachförderung.....

\section{Katja Francesca Cantone}

Sprachmischungen im simultanen Erwerb zweier Muttersprachen: Ein Fall von Sprachdominanz?.

\section{Sigrid Luchtenberg}

Deutsch als Zweitsprache und Mehrsprachigkeit - Widerspruch oder Möglichkeit? 461

\section{Christoph Chlosta}

Deutsch als Zweitsprache im Rahmen der Lehrerausbildung an der Universität Duisburg-Essen

\section{Andrea Esgen}

Förderung zweisprachiger Kinder. 


\section{Forum DaF}

\section{Dagmar Schimmel / Linda Meier / Florian tom Wörden}

Anfängerintensivkurse als Vorbereitung auf das Fachstudium

\section{Roya Moghaddam}

Interkulturelle Kommunikation und Geschlecht?

\section{Anke Stöver-Blahak}

Besser sprechen durch „Loreley“ und „Mondnacht“

\section{Wolfgang Rug}

Aktive Mitarbeit deutschsprachiger Hospitant/innen im DaF-Unterricht, eine Methode der sprachlichen Förderung und Integration internationaler Studierender - ein Praxisbericht aus Tübingen

\section{Julia Richter}

„Das klingt irgendwie komisch“. Ausspracheschulung für DaF-Studierende 555

\section{Susanne Duxa}

Präsentationstraining auf der Mittelstufe

\section{Christiane Beinke / Stefan Thürmer / Melanie Brinkschulte / Lothar Bunn}

Akademisches Schreiben für ausländische Studierende: Ein Lehr- und Übungsbuch für Fortgeschrittene

\section{Manfred Kaluza / Karin Schmidt / Sabine Schmidt}

Erinnerungsorte im Unterricht Deutsch als Fremdsprache

\section{Sabine Jentges}





\section{Vorwort}

In dem hier vorgelegten Band 78 der Reihe Materialien Deutsch als Fremdsprache (MatDaF) publiziert der Fachverband Deutsch als Fremdsprache die Vorträge, Referate und Ergebnisse der 34. Jahrestagung Deutsch als Fremdsprache, die vom 08.06.2006 bis 10.06.2006 an der Leibniz Universität Hannover stattfand.

Bei der Veröffentlichung des Bandes gab es Probleme, die vorher nicht absehbar waren: Die Druckerei, bei der die Reihe Materialien Deutsch als Fremdsprache in den letzten Jahren erstellt wurde, war plötzlich nicht mehr erreichbar. Lange Zeit waren damit verbundene finanzielle und rechtliche Fragen ungeklärt, und dies in einer Phase, in der der Übergang der Reihe in den Universitätsverlag Göttingen vorbereitet wurde.

Die erste Version war für den Druck von Armin Wolff und seinem Mitarbeiter Reinhold Zollner vorbereitet worden, die aber nur die Grundlage für die nun erheblich veränderte textliche Gestaltung, eine redigierte und im Hinblick auf die Diagramme und Abbildungen völlig neu erstellte Fassung ist.

Unter dem Thema „Chance Deutsch: Schule - Studium - Arbeitswelt" waren vom Vorstand des $\mathrm{FaDaF}$ für die Hannoveraner Tagung die folgenden Themenschwerpunkte vorbereitet worden:

TSP 1:

Integrationskurs DaF

Koordination: Prof. Dr. Hiltraud Casper-Hehne, Dr. Marijana Kresic, Dr. Matthias Jung

TSP 2:

Testen und Prïfen, Korrektur und Bewertungsverfahren

Koordination: Dr. Silvia Demmig, Christina Eberle

TSP 3:

Sprechen und Scbreiben in den Wissenschaften

Koordination: Martin Lange, Dr. Maria Muallem, Dr. Sigrun Schroth-Wiechert

TSP 4:

Deutsch als Zweitsprache und Mehrsprachigkeit

Koordination: Prof. Dr. Christian Krekeler, Prof. Dr. Hans Bickes, Christoph Chlosta

Forum Deutsch als Fremdsprache

Erfabrungen und Problemstellungen aus der Praxis des Unterrichtsfaches Deutsch als Fremdsprache

Koordination: Dr. Susanne Duxa, Anke Stöver-Blahak 

Themenschwerpunkt 1:

Integrationskurse $\mathrm{DaZ}$ 



\section{Wie Deutsche mit Ausländern sprechen und was man daraus für den Spracherwerb und den Sprach- unterricht lernen kann ${ }^{1}$}

Jörg Matthias Roche, München

\section{$1 \quad$ Einleitung}

Darüber, wie Sprecher einer Zielsprache mit all denen kommunizieren, die diese Sprache nicht oder nur rudimentär beherrschen, wird sehr viel spekuliert. Obwohl die Sprachumgebung oder Eingabe im Spracherwerb anerkanntermaßen eine bedeutende Rolle spielt, gibt es erstaunlich wenige solide Untersuchungen des kom-

\footnotetext{
${ }^{1}$ Bei diesem Beitrag handelt es sich um den Plenarvortrag (gehalten am 9. Juni 2006) im Themenschwerpunkt 1 »Integrationskurse DaZ« im Rahmen der 34. Jahrestagung Deutsch als Fremdsprache des Fachverbandes Deutsch als Fremdsprache $(\mathrm{FaDaF})$ vom 8.-10. Juni 2006 an der Universität Hannover zum Rahmenthema »Chance Deutsch: Schule - Studium - Arbeitswelt«. Der Beitrag orientiert sich sehr stark am mündlichen Stil des Plenarvortrages und wurde nur leicht modifiziert. In einem solchen Vortrag ist es auch nur bedingt möglich, die relevante Forschungsliteratur angemessen darzustellen. So enthält dieser Beitrag naturgemäß einige Verkürzungen. Über die im Literaturverzeichnis angegebenen Referenzen kann der interessierte Leser dazu leicht Zugang finden.
} 
munikativen Verhaltens der Zielsprachensprecher in diesen ungleichen Kommunikationssituationen. Die Äußerungen der Lerner sind dagegen wesentlich besser untersucht. So kommt es, dass ein in Wirklichkeit recht komplexes, reiches, differenziertes und adaptives sprachliches Verhalten der Zielsprachensprecher hinter den vorwiegend indirekten, sporadischen und anekdotischen Beobachtungen versteckt bleibt. In diesem Beitrag möchte ich zunächst die authentische Sprache von Deutschen in der Kommunikation mit Lernern der Zweit- und Fremdsprache Deutsch präsentieren und analysieren. Sie wird dann im Kontext von Sprachentwicklungsprozessen mit anderen Formen und Registern reduzierter und grammatikalisierter Sprache verglichen. Abschließend wird ein Verfahren skizziert, das die systematische Integration reduzierter Sprachformen in den DaZ- und DaFUnterricht vorsieht. Dies erfordert jedoch eine Neudefinition des Progressionskonzeptes im Unterricht.

\section{Hypothesen über die Kommunikation mit Ausländern}

Wenn man sich ansieht, welche Hypothesen es zur Kommunikation mit Ausländern gibt, dann fallen am deutlichsten negative Einschätzungen auf. Es scheint wenig förderlich, wenn man mit Ausländern nicht korrekt redet, so die landläufige Vermutung. Der Begriff ,Stigmatisierung' erscheint häufig in solchen Kontexten. Eine andere Hypothese geht davon aus, dass eine mögliche gegenseitige Imitation von Lernern und Sprechern der Zielsprache den Spracherwerb verhindert. Wie soll ein Lerner schließlich auch die Sprache lernen, wenn beide rudimentäres Deutsch sprechen? Die nativistische Position zu dem Thema ist noch einfacher zusammengefasst: Der Input spielt dort naturgemäß eine sehr geringe Rolle. Schließlich wird davon ausgegangen, dass Lerner trotz unvollständigen Inputs die korrekte Grammatik entwickeln. Der Input ist also weder für den Spracherwerb noch für den Sprachunterricht relevant. Zu guter Letzt gibt es auch eine nicht sehr umfangreiche amerikanische Forschung zu diesem Bereich, die davon ausgeht, dass es keine besonderen Anpassungen der Zielsprachensprecher gibt, weil sie sie nicht beobachten konnte.

Vielleicht sagen Sie jetzt: Genau so ist es eigentlich, diese Stigmatisierung, dieses vermeintlich schlechte Deutsch, das wir mit Ausländern häufig reden, das kann ja nichts bringen. Ich möchte Ihnen dazu gleich eine kleine Begebenheit erzählen, die diese Annahme vielleicht bestätigt. Aber die Auflösung gebe ich Ihnen dann doch nicht gleich, denn die Geschichte nimmt noch eine überraschende Wendung. Es war also auf einer wichtigen großen internationalen Tagung, die interessanterweise noch auf Deutsch gehalten wurde, und in der Mittagspause saß ein kenianischer Konferenzteilnehmer beim Essen und ein deutscher Kollege setzte sich zu ihm, klopfte ihm dabei auf die Schulter und fragte: »Hamm hamm gut?« und man unterhielt sich ansonsten nicht, weil man sich mit 
vollem Mund bekanntlich nicht gut unterhalten kann. Schließlich prostete der Deutsche seinem Kollegen mit den Worten zu: »Gluck gluck gut?« .

\section{Struktur und Variation in der Kommunikation mit Ausländern}

So ungefähr stellen wir uns also die Kommunikation mit Ausländern vor. Aber in Wirklichkeit ist diese Sache viel differenzierter, und das möchte ich Ihnen nun anhand eines Transskripts zeigen. Dabei müssen Sie sich etwas an die Transkriptionsart gewöhnen. Die hat zwar phonetische Züge, kommt aber ohne phonetische Sonderzeichen aus und nutzt das graphemische System des Deutschen. Die Aufnahme stammt aus einem Korpus, das ich bereits vor einiger Zeit erstellt habe, und zwar mit Beobachtungen von authentischen Gesprächen zwischen Deutschen und Ausländern am Arbeitsplatz und in Geschäften und auch in privater Kommunikation. Wir haben also hier einen deutschen Sprecher D, nennen wir ihn mal Dietmar, und seinen türkischen Kollegen T, nennen wir ihn Tekin, die beide Mitte 20 sind, sich sehr gut kennen und befreundet sind. Und der Deutsche erzählt in dieser längeren Sequenz, was er am Vorabend gemacht hat ${ }^{2}$ :

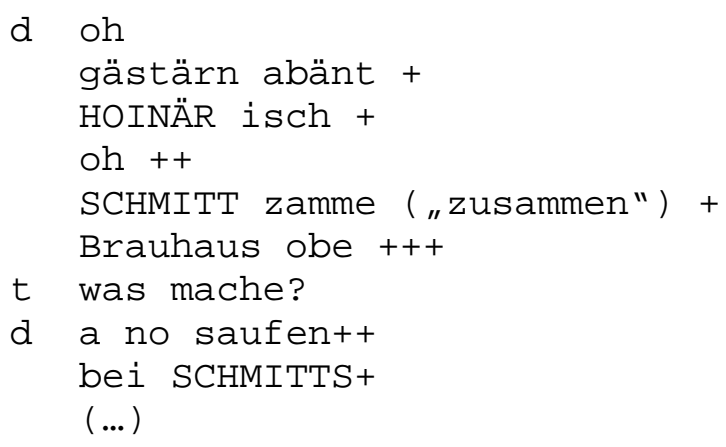

Natürlich kann man sagen, dass hier einige Funktionswörter, Endungen und andere Elemente fehlen. Es fehlt hier sicher mehr, als da ist. Man könnte dieses Gespräch als ungrammatisch beschreiben. Aber ich gehe davon aus, dass $90 \%$ von Ihnen schon verstanden haben, worum es hier geht, trotz der rudimentären Grammatik. Das ist auch mein Vorschlag, dass wir uns bei solchen Gesprächen nicht so sehr davon leiten lassen, was unserer Meinung nach falsch ist oder fehlt, sondern zunächst einmal sehen, was eigentlich da ist. So machen wir das im Übrigen ja mittlerweile auch in der Spracherwerbsforschung.

Die Struktur dieser Äußerungen, wie zum Beispiel »Gestern Abend Heiner isch«, lässt sich als fortlaufender Fokus beschreiben, d. h. als eine systematische Progres-

\footnotetext{
2 Zur Erläuterung der wichtigsten Zeichen: $\mathrm{c}=$ unbetontes $\mathrm{e}, \mathrm{x}=$ ach-Laut, $\mathrm{C}=$ ich-Laut, $B=$ schLaut, $\mathrm{z}=$ stimmhaftes $\mathrm{s},+=$ Pausenlänge (je circa 1 Sekunde), alle Namen sind geändert.
} 
sion von einem thematischen Element, in diesem Fall einem Temporaladverbial, zum Fokus. Und so geht das systematisch weiter. Der Fokus, die zusätzliche Information, die neue oder hervorgehobene Information, wird im Grunde einfach addiert. Hier ist ein weiteres Beispiel aus einer anderen Geschichte, das uns nachher noch etwas intensiver beschäftigen wird.

\begin{tabular}{|l|}
\hline där \\
\hline der
\end{tabular}$\rightarrow$\begin{tabular}{|c|}
\hline naxtßißt \\
\hline Nacht -
\end{tabular}$\rightarrow$\begin{tabular}{|l|}
\hline mäsär unt schärc \\
\hline Messer und Sche- \\
\hline
\end{tabular}

Thema

Fokus

Fokus

Sie können aus diesen wenigen Begriffen bereits ahnen, wie sich diese Geschichte weiterentwickelt. »Messer und Schere« ... und dann kommen die einzelnen Ereignisse. Hier liegt also die gleiche Struktur vor: Thema - Fokus und diese Struktur wird ergänzt durch den fortlaufenden Fokus. In der Literatur spricht man bei dieser Art Kommunikation von ,Foreigner Talk', ,Telegrammdeutsch' oder ,Ausländerdeutsch‘. Man könnte Neudeutsch auch ,SMS-Deutsch` sagen. Wegen der größeren Variation, die zu beobachten ist, und der Nähe zu anderen »-lekten« habe ich den Begriff ,Xenolekt' vorgeschlagen.

Sehen wir uns hier nun eine etwas komplexere Erzählung an und betrachten etwas genauer die Variation in dem Gespräch.

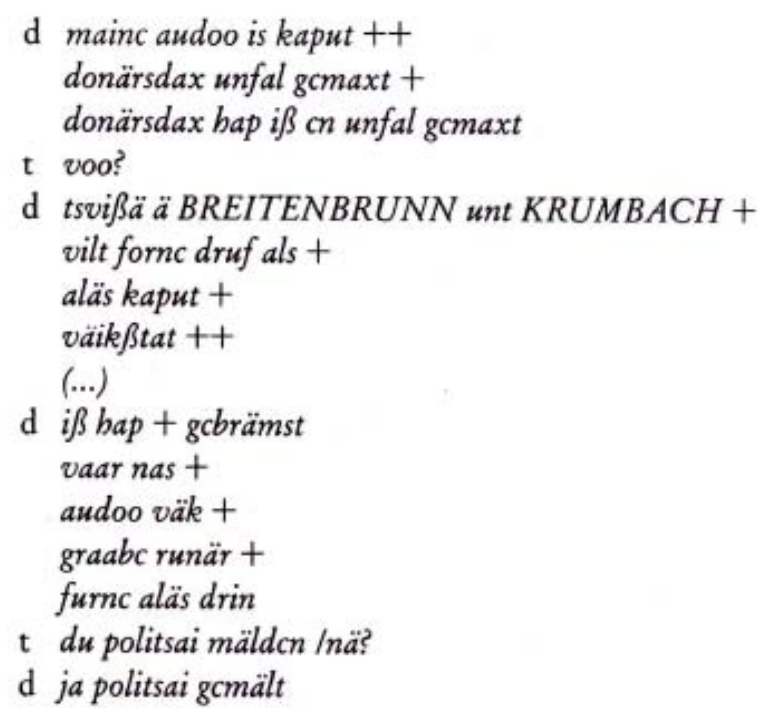

$\mathrm{d} i ß$ hap + gcbrämst vaarnas +

d ja politsai gcmält 


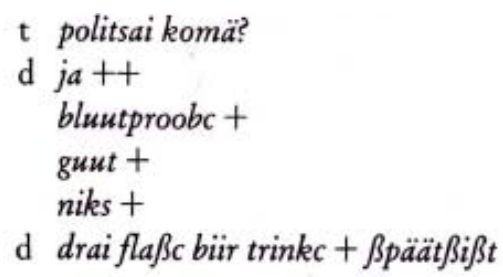

Sie erkennen natürlich auch den leichten dialektalen (hessischen) Einschlag. Den müssen wir dem Sprecher natürlich nachsehen. Hochdeutsch heißt es also:

»Mein Auto ist kaputt«.

»Donnerstag hab ich einen Unfall gemacht«.

$»$ Wo $\ll$ ? -

"Zwischen Breitenbrunn und Krumbach«.

»Wild vorne drauf. Alles kaputt - Werkstatt«.

»Ich hab’ gebremst, war nass. Auto weg. Graben runter. Vorne alles drin«.

$»$ Du Polizei melden«?

»a, Polizei gemeldet. Polizei gekommen. Ja. Blutprobe. Gut, nix. Drei Flaschen Bier trinken, Spätschicht«.

Das Interessante an diesem Gespräch ist außer dem Inhalt, seiner Darstellung und der überraschenden Pointe die Mischung der verschiedenen Äußerungstypen. Wenn man sich das Gespräch nämlich genauer ansieht, stellt man fest, dass einige der Äußerungen sehr rudimentär, infinitivartig und reduziert sind, d. h. dass Äußerungen nur mit Inhaltselementen auskommen. Es enthält aber auch Aussagen, die weniger verändert oder reduziert sind, wie zum Beispiel »Meine Auto ist kaputt", "Donnerstag hab' ich ein Unfall gemacht«. Einige sind sogar umgangssprachlich und enthalten eigentlich keinen Fehler. Und das ist eine ganz typische Sache, die wir noch ein bisschen weiter verfolgen sollten. Wir sehen gar keine registerartige Veränderung im Sinne eines Ausländerregisters, sondern einen enormen Variantenreichtum. Interessanterweise ist dieser Variantenreichtum bei vielen Sprechern zu beobachten, zumindest allen Sprechern, die ich untersucht habe, und die kamen aus ganz unterschiedlichen Gegenden und sozialen Schichten. Wir können also davon ausgehen, dass die hier beobachtete Variation kein Einzelfall ist, sondern systematisch auftritt. Wenn man sich jetzt den Textaufbau von solchen Erzählungen ansieht, dann stellt man fest, dass diese meistens nach dem folgenden Schema strukturiert sind:

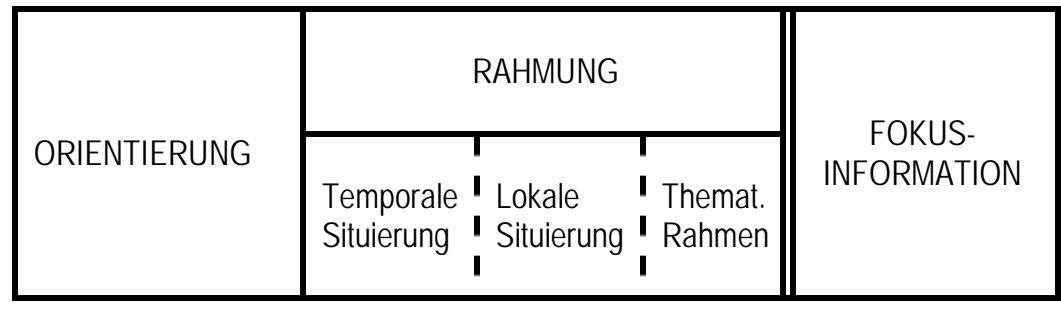


Es kann natürlich sein, wie in allen anderen Erzählungen auch, dass die eine oder andere Position nicht realisiert ist, aber im Grunde finden wir dieses Strukturmuster immer wieder: Eine Orientierung, wie in diesem Fall »Meine Auto ist kaputt«, die den Rahmen der Erzählung angibt, dann die temporale und thematische Situierung "Donnerstag hab ich ein Unfall gemacht«, in der normalerweise auch die handelnden Personen genannt werden. Im Grunde entspricht dieses Muster dem, was man in der Literaturwissenschaft eine ,Exposition' nennt, in der die wichtigsten Parameter am Anfang eingeführt werden.

Sehen wir uns nun noch einen weiteren Bereich an, nämlich den der semantischen Simplifizierungen. Zu beobachten ist in Xenolekten allgemein, dass häufiger eine Substitution durch Synonyme oder vom Sprecher als Synonym erachtete Begriffe - ich nenne sie ,Pseudosynonyme - und Paraphrasen erfolgt. Also statt »Leben« wird »Wohnen« gesagt, statt »ärgern« »aufregen«, wahrscheinlich wegen der Ausdifferenzierung, die durch das Präfix erfolgen kann. Statt »überprüfen« wird »Kontrolle« verwendet, möglicherweise, weil »Kontrolle« als Internationalismus vermutet oder erkannt wird. 1500 wird aufgeteilt in »1000 - 500«, »jedes Mal« wird zu »immer«. Zudem gibt es auch einige Neologismen, die spontan geschaffen werden, wie zum Beispiel anstatt »einen Kredit aufnehmen« »über Kredit gehen« oder anstatt »Schleudertouren« »Schleuderzahl«, weil der Sprecher »Zahk offensichtlich als prägnanter erachtet. Zusätzlich scheinen metonymische Kürzungen ein beliebtes Mittel zu sein: »Möbelabteilung« erscheint als »Möbel«, »Teppichabteilung« als »Teppiche«. Auf der anderen Seite werden semantische Elemente analytisch präsentiert wie in »große Baum mit Blätter« statt »Eiche«, »viel Reparatur« statt »reparaturanfällig« (Quantifizierung), »meist gängige« statt »das gängigste« (Komparation) und »Monat, Monat« statt »monatlich" (Iteration). Solche analytischen Wiederholungsformen sind übrigens auch ein beliebtes Mittel von Lernern im Zweitsprachenerwerb, um wiederholte oder anhaltende Handlungen auszudrücken.

\section{Sprecher- und adressatenspezifische Variation}

Kommen wir nun zu dem Thema ,Variation unter den Sprechern und adressatenspezifische Variation'. Ich habe bisher versucht, das System der Xenolekte darzustellen, aber man könnte sich ja im Anschluss daran fragen, wie einheitlich diese Kommunikation eigentlich ist. Reden wir relativ pauschal mit Ausländern, spielt der Ausländerstatus, die Erscheinungsweise des Adressaten eine Rolle, passen wir uns an das Sprachniveau an und gibt es individuelle, sprechertypische Präferenzen? Dazu möchte ich Ihnen aus meinem Korpus exemplarisch nur einen Sprecher vorstellen und kurz erklären, was diese Abbildung bedeutet (aus: Roche 1989: 148). 


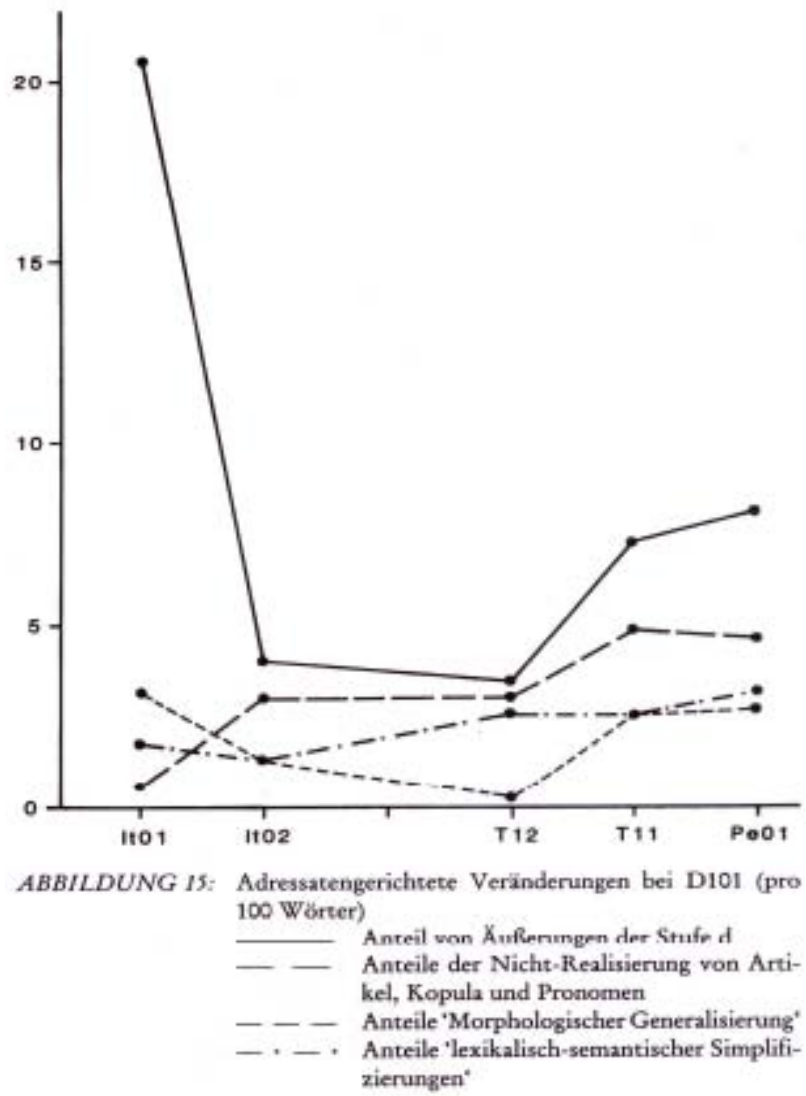

Auf der vertikalen Achse ist die Vorkommenshäufigkeit und auf der horizontalen Achse sind die verschiedenen Adressaten abgetragen. IT01 ist ein italienischer Gastarbeiter, der wirklich sehr schlecht Deutsch spricht, obwohl er zum Zeitpunkt der Aufnahme 15-20 Jahre in Deutschland gelebt hat. IT02 ist auch ein italienischer Gastarbeiter, der aber über etwas bessere Kenntnisse verfügt. T12 ist ein türkischer Gastarbeiter, T11 ein türkischer Krankenpfleger und PE01 ein peruanischer Student mit sehr guten Deutschkenntnissen. Die Sprachkenntnisse dieser Versuchspersonen, die ich hier nur grob beschrieben habe, habe ich natürlich in einem Blindversuch von einer Kontrollgruppe bewerten lassen. Dazu habe ich Sprachausschnitte aus den Aufnahmen genommen, habe sie der Kontrollgruppe vorgestellt und habe sie in Bezug auf die Verständlichkeit bewerten lassen. Daraus ergibt sich diese Reihung, wobei die Abstände zwischen den Sprechern nicht maßstabsgetreu sind. Aus der Bewertung ergibt sich immerhin eine signifikante Zunahme der Verständlichkeit nach rechts. Der Peruaner spricht fast muttersprachlich Deutsch. IT01 kann tatsächlich nur ein paar ganz rudimentäre Brocken Deutsch. 
Anhand von verschiedenen Merkmalen kann damit gemessen werden, wie sich ein deutscher Verkäufer in einem großen Kaufhaus, der täglich mit unzähligen Kunden zu tun hat, in einer Standardsituation verhält. Die Standardsituation bestand hier aus dem Waschmaschinenverkauf, Garantieanfragen, Reparaturfragen und Ähnlichem. Alle meine Versuchspersonen hatten dabei die Aufgabe, die gleichen Fragen an diese Person zu stellen. Allerdings geschah dies mit einem Abstand von mehreren Tagen oder Wochen, so dass der Verkäufer keinen Verdacht schöpfen konnte.

Die durchgezogene Linie bildet die Infinitiväußerungen $\mathrm{ab}$, das sind diese rudimentären Äußerungen, die wir anfangs gesehen haben. Es zeigt sich dabei, dass der Verkäufer gegenüber dem sehr schlecht Deutsch sprechenden und verstehenden Italiener eine ganz hohe Anzahl der rudimentären Äußerungen benutzt. Circa die Hälfte aller Äußerungen des deutschen Verkäufers sind solche Infinitiväußerungen. Das fällt dann rapide ab. Die anderen Strategien, wie die Nicht-Realisierung von Artikel, Kopula und Pronomen, verwendet er viel seltener. Morphologische Generalisierungen, damit sind vor allem Flexionsausfälle und -reduktionen gemeint, verwendet er bei allen Adressaten, aber relativ wenig, und lexikalischsemantische Simplifizierungen benutzt er nur gelegentlich. Aber das Auffällige hieran ist, dass er seine Sprache sehr stark an den jeweiligen Adressaten anpasst. Gegenüber dem Italiener IT01 erwarten wir das. Dass die starken Anpassungen so rapide abfallen, ist aber nicht unmittelbar verständlich. Dass die starken Veränderungen bei den besser sprechenden Adressaten wieder ansteigen, scheint auf den ersten Blick vielleicht sogar paradox. Diese Adressaten sprechen eigentlich ganz gut Deutsch, also müsste man annehmen, dass der Sprecher mit ihnen auch ganz normales Deutsch spricht. Woran liegt das? Sehen wir uns dazu noch eine weitere Folie an. Sie illustriert, wie verschiedene Sprecher sich gegenüber den gleichen Versuchspersonen verhalten. Hier habe ich mittels eines Verfahrens Typen eines Wortes und Tokens, also ihre Häufigkeit, gemessen. Es geht um die Inhaltsdichte des Gesprächs anhand des Vorkommens von Wörtern und ihrer Häufigkeit (vgl. Roche 1989: 119). 


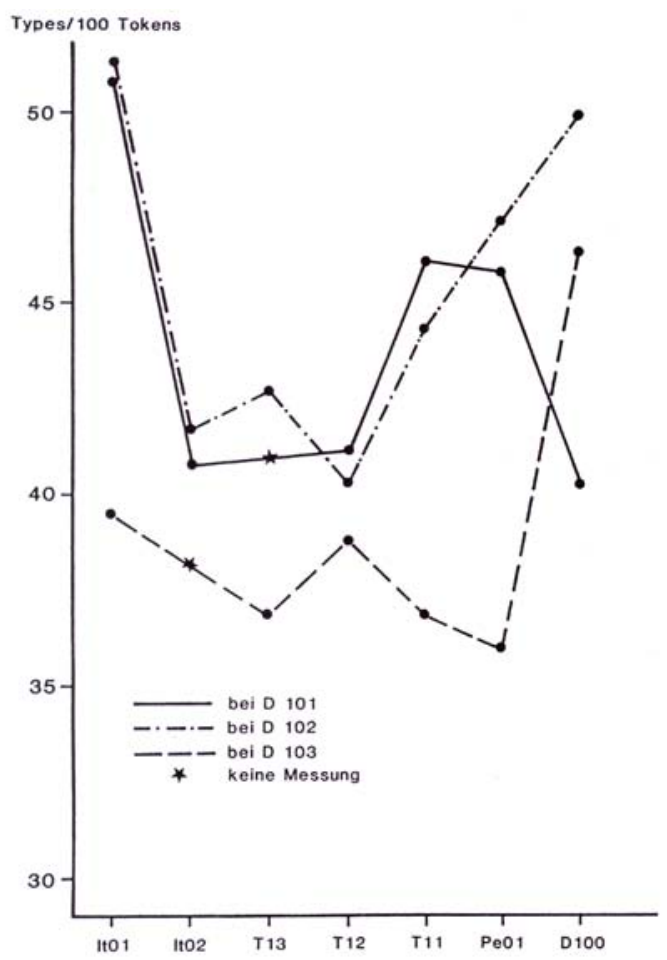

ABBILDUNG 13: Type-Token-Verhältnis in den Auskunftsdaten

Hier sehen Sie die Werte von drei Versuchspersonen: D101, einem anderen Verkäufer D102, der Fernsehgeräte verkauft, und D103, der in einer Lotterieannahmestelle arbeitet. Und Sie sehen schon, ohne dass ich auf die Einzelheiten eingehe, dass es große Unterschiede zwischen den Sprechern gibt. Beachten Sie bitte, die Informanten reden immer mit den gleichen Adressaten, die Thematik ist die gleiche und dennoch haben sie andere Präferenzen. Bei D101 und D102 sehen wir eine gewisse Übereinstimmung, aber gegenüber dem Peruaner und einer muttersprachlichen Vergleichsperson (D100) zeigen sich doch ganz andere Strategien. Hier ist das Verhältnis von Wörtern und Vorkommen relativ hoch, d. h., es liegt eine sehr hohe Inhaltsdichte vor. Fast jedes Wort kommt maximal zweimal vor, während gegenüber den weiteren Adressaten das Type-Token-Verhältnis niedriger ist. Das bedeutet, die Sprecher verwenden gegenüber IT01 sehr viele Inhaltswörter und lassen die Funktionselemente weg, die ja im Deutschen hochfrequent sind. Der erneute Anstieg bei den Adressaten rechts lässt sich auf qualitative Unterschiede zurückführen. Gegenüber den schlecht sprechenden Adressaten beschränken sich die Sprecher auf einfachste Erklärungen, z. B. zum Funktionieren der 
Maschine, des Fernsehers oder des Lottosystems, und auf ein paar kurze Informationen. Schluss. Die fortgeschrittenen Adressaten sind dagegen viel flexibler, die können nachfragen, die können Zusatzinformationen verlangen. Dementsprechend geben die deutschen Sprecher nicht nur kurze Erklärungen. Sie reagieren auf die Nachfragen und geben entsprechende Nachlieferungen, und die sind teilweise sehr komprimiert, aber dann auch wieder ausgedehnt. Das erklärt, warum sich das Type-Token-Verhältnis auch bei besseren Adressaten erhöht. Das Gespräch von D101 und der deutschen Versuchsperson, der letzte Punkt in der Abbildung, findet ganz im Dialekt, ganz umgangssprachlich, statt. Es handelt sich um ein sehr facettenreiches Gespräch, aber es verläuft in den Routinen der Umgangssprache, und die sind häufig unterspezifiziert. Da heißt es anstatt »Waschmaschine« zum Beispiel einfach »Ding«, weil man sich auf eine gemeinsame Basis beziehen kann, also können die Wörter und Strukturen auch unterspezifiziert und elliptisch sein. Wir sehen hier, dass es sowohl eine sprecherspezifische Varianz für verschiedene Strategien gibt und eine gewisse adressatenspezifische Anpassung. Als Sprecher der Zielsprache scheren wir also nicht alle ausländischen Adressaten über einen Kamm, wie das häufig vermutet wird. Xenolekte sind kein einheitliches Register.

Aber es geht noch weiter. Sehen wir uns nun das, was wir gerade quantifiziert betrachtet haben, noch einmal unter qualitativen Gesichtspunkten genau an. Dieses Gesprächssegment stammt aus einem etwa 30-40minütigen Gespräch zwischen einem deutschen und einem türkischen Kollegen. Dabei erzählt der Deutsche von verschiedenen Sachen, von einem Unfall, von einer Messerattacke und Ähnlichem. Eine Beispieläußerung haben wir vorhin schon gesehen. Sehen wir mal, wie seine Sprache variiert:

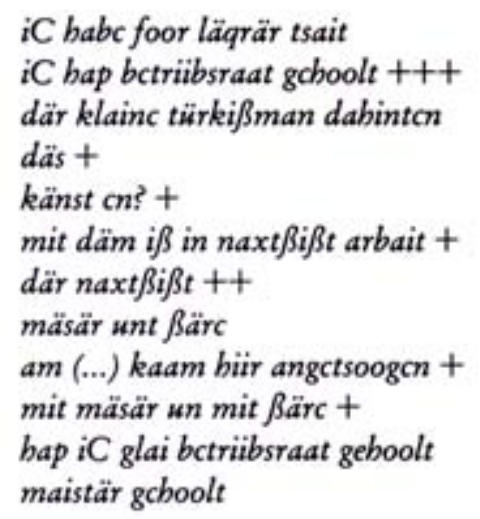

»Ich habe vor einer längeren Zeit, der kleine Türkischmann da hinten«, diese ÄuBerung entspricht im Grunde einer umgangssprachlichen Äußerung mit der Ausnahme von »Türkischmann« als semantischer Simplifizierung. Grammatisch ist diese Äußerung korrekt. Dann gibt es aber auch eine Reihe ganz stark veränderter 
Äußerungen, also infinitivartige, rudimentäre Äußerungen. Es finden sich aber weitere rein umgangssprachliche Äußerungen wie diese Nachfrage »kennst ihn« und diese Äußerung »kam hier angezogen«. Zudem gibt es Äußerungen, die in einem Element verändert sind, wie zum Beispiel »ich hab' Betriebsrat geholt« statt wich hab' den... « oder »mit dem ich in Nachtschicht Arbeit» anstatt »mit dem ich in der ...«. Es fehlt hier also der Artikel. Es ist typisch für diese Art von Äußerungen, und auch über Sprechergrenzen hinweg, dass immer nur ein Element von Auslassungen betroffen ist. Es fehlt entweder eine Präposition oder ein Artikel, aber es fehlt in diesem Äußerungstypus in ganz seltenen Fällen beides. Das Inventar der Sprecher besteht demnach aus vier Äußerungstypen:

- umgangssprachlichen Äußerungen,

- semantisch vereinfachten oder verlangsamten Äußerungen,

- in einem Element betroffenen Äußerungen oder

- infinitivartigen Äußerungen.

\section{$5 \quad$ Pragmatische Variation}

Der deutsche Sprecher erzählt von der Messerattacke und rekurriert dabei auf einen türkischen Kollegen. Den realisiert er in seinen Erzählvarianten unter anderem als »Türkischmann«, »des«, »««, »er«, »den«, »der«, mit seinem Vornamen und auch als Ellipse (»ich kann dir zeigen«). Diese Variantenvielfalt gilt für alle der beobachteten Sprecher und betrifft nicht nur die Lexik, sondern, wie wir schon gesehen haben, auch die Grammatik. Wenn man sich den Codewechsel genauer ansieht, dann kann man feststellen, dass es mindestens sechs verschiedene Veränderungsniveaus gibt, die jeweils spezifische Funktionen markieren: Ein

- Ausgangsniveau

- Erklärungs- und Erzählniveau

- Komprimierungsniveau

- Zitatniveau

- Nachlieferungs- und Nebenthemenniveau

- Kommentarniveau

Das Ausgangsniveau bildet eine erste augenscheinliche Einschätzung ab. Darunter fallen auch Kriterien wie Hautfarbe und weitere sprachliche und außersprachliche Merkmale der »Ausländerhaftigkeit«, die wir rational gar nicht immer erklären können. Ich nenne es ,Ausgangsniveau', weil es sehr instabil ist. Nach meiner Beobachtung versuchen die Sprecher, schnell von diesem Niveau wegzukommen. Sie versuchen eigentlich nur zu reduzieren und einfacher zu sprechen, wo es unbedingt nötig ist. Dabei sollte man berücksichtigen, dass ein reduziertes Sprechen eigentlich sehr viele Kapazitäten verlangt, weil es von unserer automatisierten Gesprächsroutine massiv abweicht. Als zweites Niveau lässt sich das Erklä- 
rungs- und Erzählniveau bestimmen. Es betrifft die zentralen, inhaltlichen Elemente, also die zentrale Bedeutungsvermittlung (Proposition). Die Erklärungsund Erzählsequenzen sind in der Regel am stärksten verändert oder reduziert. Wo die inhaltliche Vermittlung, der propositionale Gehalt, uns Sprechern ganz besonders wichtig ist, da reduzieren wir, da konzentrieren wir uns, da verwenden wir die 1-Wort-Äußerungen und die infinitivartigen Sätze. Ganz ähnlich betrifft das Komprimierungsniveau kurzfristige Erklärungen als Antwort auf Nachfragen, etwa nach den Schleudertouren einer Waschmaschine. Dann wird temporär mit einzelnen Begriffen komprimiert und vertieft, aber sofort wieder in ein anderes Niveau gewechselt.

Das Zitatniveau ist ebenfalls ganz interessant, weil es eine Strategie repräsentiert, die wir automatisch beherrschen, um uns zu helfen. Es lässt sich beobachten, dass Sprecher, die irgendetwas erzählen oder erklären, gerne auf originale Referenzen Bezug nehmen und original zitieren. Sie übernehmen Zitate von Dritten eins zu eins und betten diese auch in komprimierte Kontexte ein.

Zudem gibt es so etwas wie ein Nachlieferungs-/Nebenthemenniveau, das in der Regel viel weniger reduziert oder angepasst wird, als die anderen Äußerungen. Wenn ein Sachverhalt oder Begriff erklärt wurde und den Sprechern auffällt, dass das eine oder andere Element noch erklärt werden muss, dann wird diese Information im Sinne von »ich meine .... nachgeliefert. Äußerungen werden dann viel weniger reduziert als bei dem Erzähl- und Erklärungsniveau. Schließlich gibt es noch das Kommentarniveau, das an die Codewechsel in Luthers Tischreden erinnert. Sprecher nutzen es ohne erkennbaren Zeitverlust, um Textpassagen oder Sachverhalte zu kommentieren, und wechseln dabei zum Beispiel von einer stark komprimierten Äußerung in ihren Dialekt und wieder zurück, so wie Luther vom Lateinischen ins Deutsche wechselte, wenn er seine Reden kommentierte oder schimpfte. Wenn zum Beispiel D101 das Funktionieren einer Waschmaschine erklärt und illustriert und die Tür geht in diesem Moment gerade nicht zu oder auf, dann fängt er an zu fluchen. Das tut er aber nicht in den xenolektalen Äußerungen, sondern da wechselt er sofort in die Begrifflichkeit seines Dialektes, wie gesagt nahtlos. Der Kunde ist in solchen Fällen nicht der primäre Adressat.

Kann man nun sagen, wie es ja häufig gemacht wird, diese Kommunikation drückt vor allem die Abwertung der Ausländer, eine Stigmatisierung, aus? Fragen wir hier stellvertretend Dietmar, von dem die vorhin besprochene Erzählung stammt, wie er das sieht, trotz der Messerattacke. Er sagt:

ich hab' nix gegen türkischmann, türkischfrau

hab ich nimmer

nur ihr anders sprechen wie wir ne?

anders sprechen

andre sprache

ich hab nix dagegen 
Es geht bei Xenolekten also mit Sicherheit primär nicht um die Stigmatisierung oder Abwertung der Ausländer/Lerner, sondern es geht vor allem um die Aufrechterhaltung der Kommunikation. Die Art und Weise mit Ausländern zu reden ist ein produktives und nicht ein abwertendes Mittel. Die meisten meiner Informanten empfinden diese Anpassungen als große Hilfe. Einige haben dies jedoch eingeschränkt, nämlich diejenigen, die schon besser Deutsch konnten. Sie wollen verständlicherweise nicht mehr so angesprochen werden, wenn sie schon Deutsch können. Aber auch sie haben bestätigt, dass Komprimierungen eine groBe Hilfe für sie darstellten, als sie noch nicht so gut Deutsch sprachen, denn so konnten sie überhaupt etwas verstehen. Das ist ein interessanter Befund, der so gar nicht in die Landschaft vorgefertigter Meinungen passt.

\title{
6 Englischsprachige Xenolekte
}

Noch ein interessanter Befund: in der englischsprachigen Literatur wird oft behauptet, dass es solche Xenolekte gar nicht gebe, und wenn, dann sei das eine deutsche Eigenart. Zu dieser Annahme habe ich vor einigen Jahren mit Unterstützung der Alexander-von-Humboldt-Stiftung in Kanada eine kontrastive Studie durchgeführt.

\author{
Explaining 'a simpler washing machine' \\ Salesman: well this + \\ here is a simpler ++ \\ this is a very simple + \\ okay? ++ \\ this very basic \\ is just all normal + \\ no delicate \\ no permanent press + \\ okay? \\ is just very basic + \\ you go from here + \\ and then when you go to this one here + \\ see \\ this one has a three speeds + \\ and a washer \\ its watertemperature + \\ but that one you can't do much with is \\ ES: okay is much simpler \\ Salesman: yeah +++
}


In diesen wenigen Äußerungen des Verkäufers (in einem großen Warenhaus in Toronto) finden sich einige Parallelen zu den deutschen Gesprächen. Typisch für Sprecher in beiden Sprachen ist dabei, welche Probleme sie haben, das richtige Niveau zu finden. Diese Schwierigkeiten manifestieren sich in den vielen Abbrüchen am Anfang des Gesprächs. Der Sprecher entwickelt hier hörbar einen Plan und während er den Plan entwickelt, bemerkt er, dass sein Gegenüber das vermutlich gar nicht verstehen wird, weil er es auf Grund der Hautfarbe vermutet, weil die Kundin aus Ecuador eine ungewöhnliche oder spezifische Gestik oder Mimik an den Tag legt oder weil irgendwelche anderen Anzeichen vorhanden sind, die Nicht-Verstehen oder Verstehensprobleme signalisieren. Daraus resultieren die vielen Ansätze, bis dann das passende Niveau ausgetestet ist.

Ich habe in Kanada auch Zugang zu einem bedeutenden Archiv des Ontario Folklife Centres gehabt, für das Kolleginnen und Kollegen Interviews mit Immigranten durchgeführt hatten, die in den 50er Jahren nach Kanada gekommen waren. Aus diesem Korpus zeige ich Ihnen hier einen Ausschnitt aus einem langen Gespräch zwischen dem englischsprachigen Interviewer Marc (MF) und einem älteren Informanten aus Litauen (GZ). Der litauische Informant fragt nun auch den Interviewer ein paar Sachen:

\section{Mennonite}

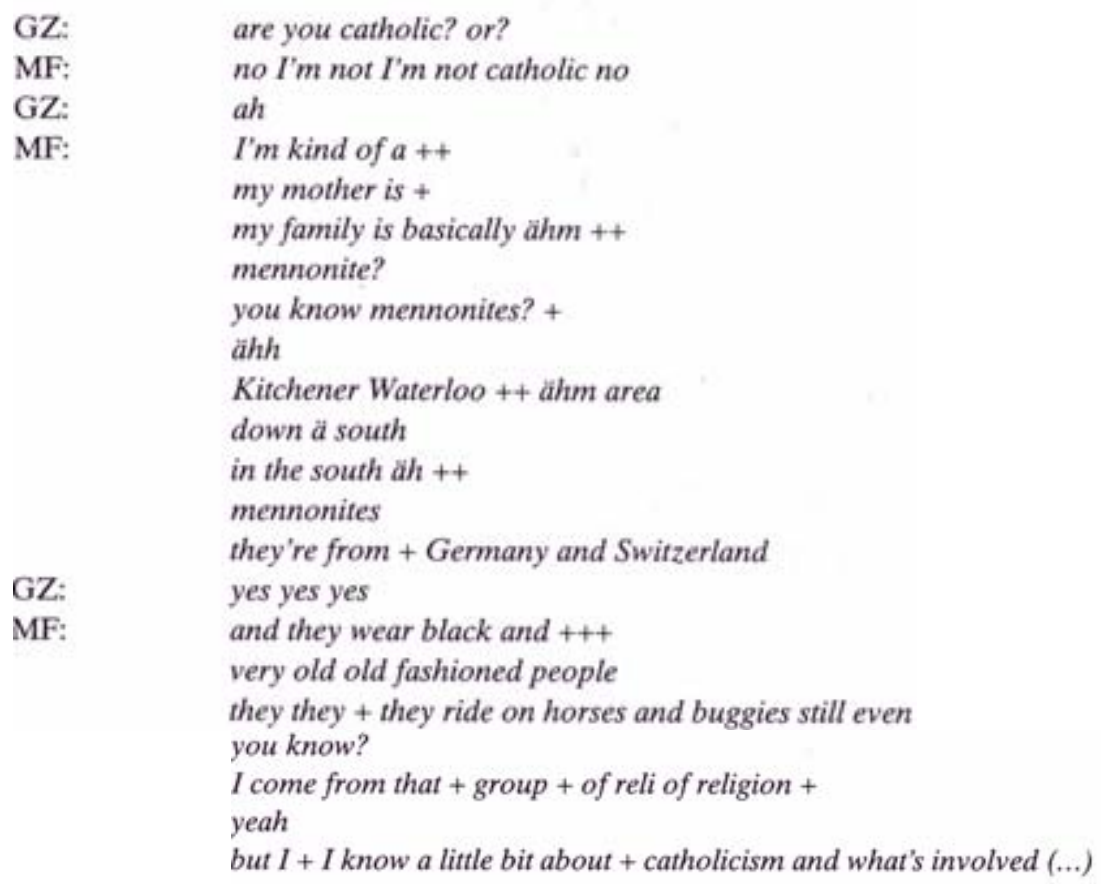


Dazu müssen Sie wissen, dass die genannten Orte ,Kitchener ${ }^{6}$ und ,Waterloo“ Ansiedlungen südlich von Toronto sind, in denen viele Menonniten leben. Der Interviewer versucht hier mit einer metonymischen Ortsangabe, ein kulturelles und religiöses Konzept zu erklären, und all das in sehr rudimentären Äußerungen. Also auch dieses Stereotyp, dass Xenolekte in dieser Ausprägung typisch für das Deutsche sind, können wir beiseite legen. Xenolekte gibt es auch im Englischen. Für das Türkische hatte sie bekanntlich Volker Hinnenkamp (vgl. Hinnenkamp 1982) bereits belegt. Die Akzeptanznormen sind aber in verschiedenen Kulturen unterschiedlich.

Wenn man etwa einen Kanadier mit einer solchen Aufnahme konfrontiert, was ich bei MF gemacht habe, dann läuft er rot an und es ist ihm sehr peinlich. Das erklärt, dass bei amerikanischen Untersuchungen bisher wenige verlässliche Daten produziert wurden. Hier wurden nämlich zum Großteil Informanten aufgenommen, die im Geschäftsbereich öffentlich tätig sind, und da redet man natürlich nicht so rudimentär wie am Arbeitsplatz, und schon gar nicht, wenn ein Mikrofon eingeschaltet ist.

\section{$7 \quad$ Pragmatischer und syntaktischer Modus}

Zum Abschluss möchte ich das skizzierte System in einen sprachgenetischen Kontext einbetten, wie ihn Givòn schon 1979 vorgestellt und später weiter entwickelt hat. Sein Modell beschreibt sowohl die phylogenetische als auch die ontogenetische Sprachentwicklung als ein Wechselspiel von einem pragmatischen und einem syntaktischen Modus. Alle die Elemente, die wir in den Äußerungen der Xenolektsprecher gesehen haben, eine klare syntaktische Struktur, langsamere Sprechgeschwindigkeit, pragmatische Prinzipien der Informationsverteilung, ein insgesamt hohes Verhältnis von Verben und Nomen - also den wichtigsten Inhaltswörtern -, wenig grammatische Morphologie und ganz bestimmte Intonationsmuster, sind typische Merkmale des pragmatischen Modus. Der syntaktische Modus dagegen beschreibt den Zustand der Grammatikalisierung, wie sie etwa in der deutschen Hochsprache ausgeprägt ist. Allerdings zeigen uns die Tendenzen der deutschen Gegenwartssprache genau das Wechselspiel zwischen pragmatischem und syntaktischem Modus: Ist der syntaktische Modus voll entwickelt, setzen Verschleifungserscheinungen ein. Man sieht das im Gegenwartsdeutsch unter anderem

- an der Reduktion des Kasusinventars;

- an der Tendenz zu analytischen statt synthetischen Formen, etwa beim Konjunktiv mit ,würde', dem Präteritumschwund oder dem Verschwinden starker Verbformen (,backte' statt ,buk');

- an der Hauptsatzstellung bei ,weil'; 
- an der Reduktion der Flexionsmorphologie in der gesprochenen Sprache; an der Verkürzung der Klammerstrukturen zugunsten von Ausklammerungen, Nachträgen und Ähnlichem.

Das Englische ist uns hier bekanntlich schon etwas weiter voraus. Das, was Givòn als Modi beschreibt, lässt sich aus Lernersicht etwa folgendermaßen darstellen (Roche 2005: 101):

\section{Prinzipien des Spracherwerbs}

- Bekannte und gegebene Information steht vor neuer Information.

- Thematisierende Elemente stehen vor fokussierenden Elementen.

- Bedeutungsmäßig zusammengehörige Elemente stehen möglichst nahe beieinander.

- In einer Reihung von Nomen hat das erste Element den größten Einfluss.

- Funktionale Elemente wie kein, viel, alle werden einheitlich vor (oder einheitich hinter) die von ihnen bestimmten Elemente gestelt.

- Orientierende Elemente wie Orts- oder Zeitangaben stehen am Anfang einer Außerung.

- Ereignisse werden nach inrer tatsächlichen (chronologischen) Reihenfolge berichtet.

- Die Betonung bestimmt, ob es sich um eine Aussage, eine Frage oder eine Anweisung handelt.

- Die Betonung markiert auch die fokussierten Elemente.

Die Prinzipien des Lerners zeigen dabei eine frappierende Übereinstimmung mit den Vereinfachungsstrategien der Xenolektsprecher. Daraus könnte man schließen, dass sich Lerner und Zielsprachensprecher gegenseitig so imitieren, dass am Ende ein gemeinsames Pidgin herauskommt. Wir haben aber bereits in der Variation der Xenolekte gesehen, dass dies nicht der Fall ist. Eingabe ist nicht gleich Ausgabe. Vielmehr treffen die Lerner bekanntlich auf der Basis ihrer Vorkenntnisse und Annahmen eine bestimmte Auswahl (Aufnahme) und verarbeiten diese nach sequenztypischen, sprachtypischen und individuellen Möglichkeiten. Daraus entsteht dann die Lernerproduktion (Ausgabe).

\section{Konsequenzen für den Sprachunterricht}

Fassen wir also kurz den Zwischenstand zusammen:

1. Die Eingabe bietet ein reichhaltiges Inventar.

2. Sie wird individuell variiert und enthält viele Paraphrasen.

3. Die Variation ergibt sich oft aus expliziten Aushandlungsprozessen. 
4. Die Variation ist keine registerartige pauschale (auch wenn pauschalierende Einschätzungen der Adressatenkompetenz besonders am Anfang von Kontakten vorkommen), sie ist pragmatisch bestimmt, nach der Art der Illokution und nach dem Gewicht des propositionalen Gehalts.

5. Der Anpassungsaufwand für Sprecher ist hoch. Sie versuchen daher so schnell wie möglich zu ihrer »normalen« Sprache zurückzukehren.

6. Xenolekte gibt es nicht nur im Deutschen.

Aus der Sicht des Lerners ergibt sich daraus:

1. ein höchst vielfältiger Input;

2. die interaktive Aushandlung verstehbaren Inputs und die Abstimmung von Erwerbsstand und Eingabe (allerdings nicht im naiven Sinne von Krashens Inputtheorie) (vgl. Krashen 1985);

3. eine Hilfsfunktion der Xenolekte statt einer Stigmatisierungsintention.

Aus diesen Beobachtungen lassen sich meines Erachtens daher einige brauchbare Folgerungen für die Erprobung im Unterricht ableiten:

1. Die Art und Weise, wie die Eingabe ausgehandelt wird. Es sollte grundsätzlich viel direkter an das Niveau der Lerner angeknüpft als einer vorgeschriebenen, von der Forschung nicht abgesicherten grammatischen Progression gefolgt werden. Dazu muss man sich auch undogmatischer Mittel bedienen, und zwar nicht nur im lexikalischen Bereich (etwa durch die Verwendung von Internationalismen), sondern auch im grammatischen.

2. Die Progression bei produktiven und rezeptiven Fertigkeiten kann und sollte konsequent anders gestaltet werden. Nicht alles, was ein Lerner verstehen kann, kann er auch sofort umsetzen.

3. In den Progressionen ist eine angemessene Nutzung von Textsorten im Sinne der Abstimmung auf die Verarbeitungsmöglichkeiten der Lerner und die natürlichen Anpassungsstrategien der Zielsprachensprecher angeraten.

Welche Textsorten dafür in Frage kommen, will ich an folgenden Beispielen illustrieren. 


\section{rasen}

\section{tötet}

freunde kinder tante bekannte

kumpel opa schwester oma mutter

arbeitskollegin partner bruder

ehefrau vater opa neffe oma

freundin onkel lebensgefährtin

kind mutter opa bekannte oma

ehemann neffe $\mathbf{b e k a n n t e}$ kind

onkel mutter tante vater oma

freund bekannte nichte freunde

kind tante bekannte kumpel

schwester mutter arbeitskollegin

partner bruder ehefrau vater opa oma

freunde freundin onkel bruder

freund bekannte nichte freunde

kind tante onkel neffe vater mutter

ehefrau freun

lebensgefährt

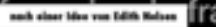

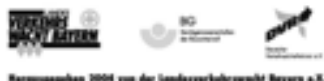

Dieser Text der Verkehrswacht, der lange an vielen Autobahnen zu lesen war, enthält außer der 2-Wort-Äußerung des Titels nur einzelne Wörter und eignet sich damit sehr gut für den Aufbau eines Wortfeldes in den ersten lexikalischen Phasen des Spracherwerbs. Lehrwerke, wie Schritte international (2005), setzen diesen Ansatz bereits folgendermaßen um, wobei durchaus auch einzelne Wortketten (Phrasen, »Chunks«) vorkommen können, die allerdings nicht grammatisch analysiert werden brauchen.

2 B3 Ratsel: Wer bin ich? Sprechen Sie mit Ihrer Partnerin / Ihrem Partner.

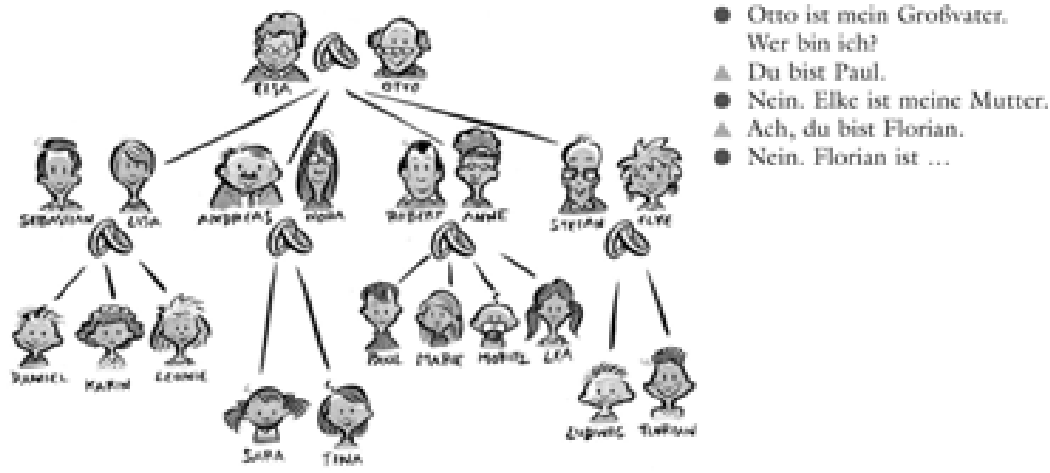


In den folgenden beiden kombinierten Textsorten finden Sie zudem weitere völlig authentische Äußerungsstrukturen (Sätze, Texte), die allerdings nach pragmatischen Prinzipien gebildet werden, also einfach gesagt, ohne Grammatik auskommen und dennoch völlig korrektes Deutsch darstellen.

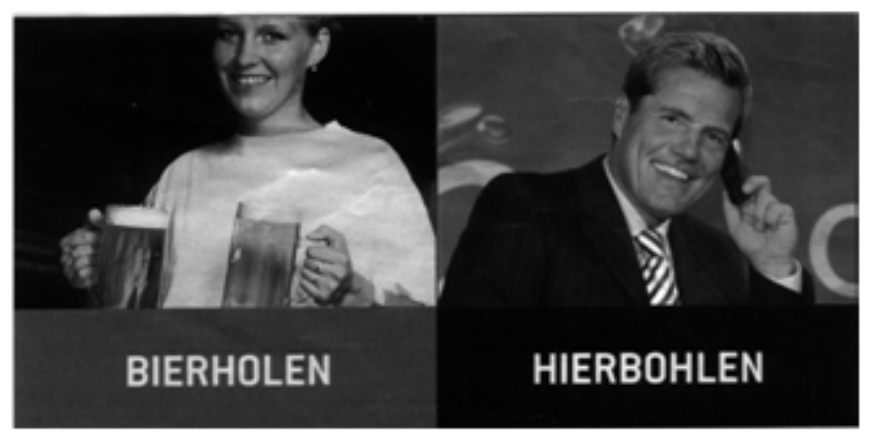

In den beiden folgenden Beispielen aus Tangram (2001) und Schritte international (Band 1, 2005) sehen Sie, wie diese pragmatischen Strukturen produktiv (und motivierend) für das Sprachverstehen und die Sprachproduktion bei Anfängern eingesetzt werden können. Die Lerner können so viel schneller als durch zu frühe und zu intensive Grammatikalisierung kommunikative Flexibilität erlangen.

Beispiel: Verwendung einfacher Strukturen im Unterricht (aus Tangram und Schritte International 1):

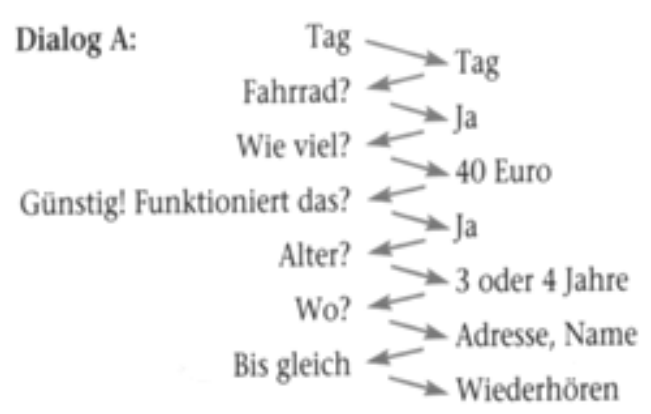


D4 Hören Sie das Telefongespräch und sprechen Sie dann mit Ihrem Namen.

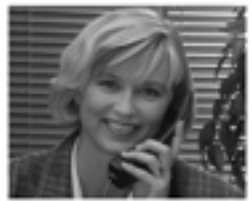

Firma Teletec, Iris Pfeil, guten Tag.

Guten Tag, Herr K...

Entschuldigung, wie ist thr Name?

Ah ja, Herr Khosa. Tut mir Leid, Frau Söll ist nicht da.

Auf Wiederhoren, Herr Khosa.

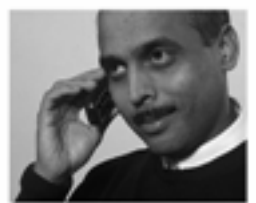

Guten Tag. Mein Name ist Khosa. Ist Frau Soll da, bitte?

Khosa.

Khosa. Ich buchstabiere: $\mathrm{K}-\mathrm{H}-\mathrm{O}-\mathrm{S}-\mathrm{A}$.

Ja, gut. Danke. Auf Wiederhören.

An dem folgenden bekannten authentischen Text kann man leicht erkennen, wie facettenreich Wortschatz mit einfachen grammatischen Strukturen genutzt werden und damit sukzessive zum Aufbau einer grammatischen Kompetenz beitragen kann. Nach der lexikalischen Phase geht es also um die Nutzung einfacher grammatischer Strukturen wie hier der prädikativen Nutzung der Adjektive statt der der flexionsreicheren attributiven.

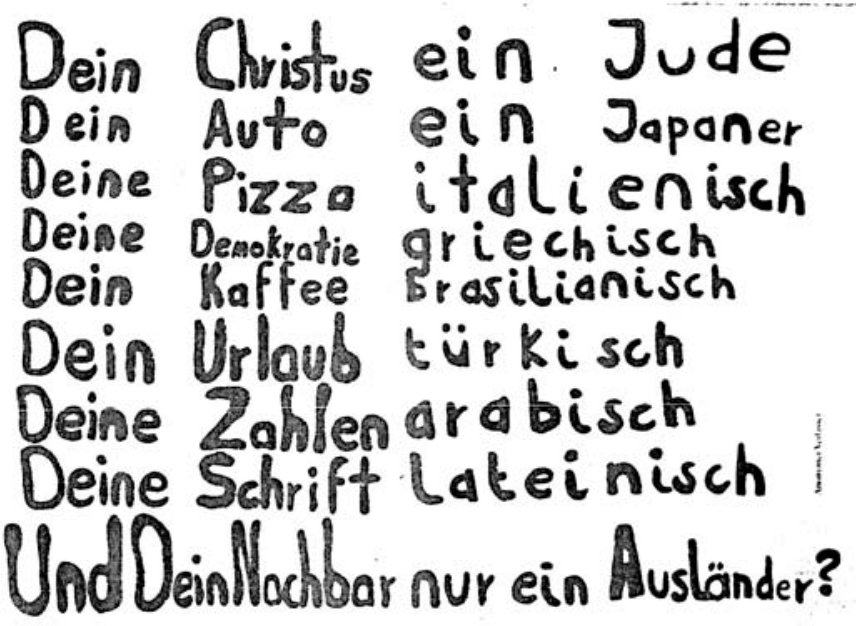

్ㅟ 
Regeln lassen sich im Sinne der Lernerprinzipien oft erstaunlich einfach darstellen und erklären (hier das Serialisierungsprinzip in Schritte international 1, 2005). Hieraus entsteht übrigens die Grundmotivation für didaktisierte Grammatiken.

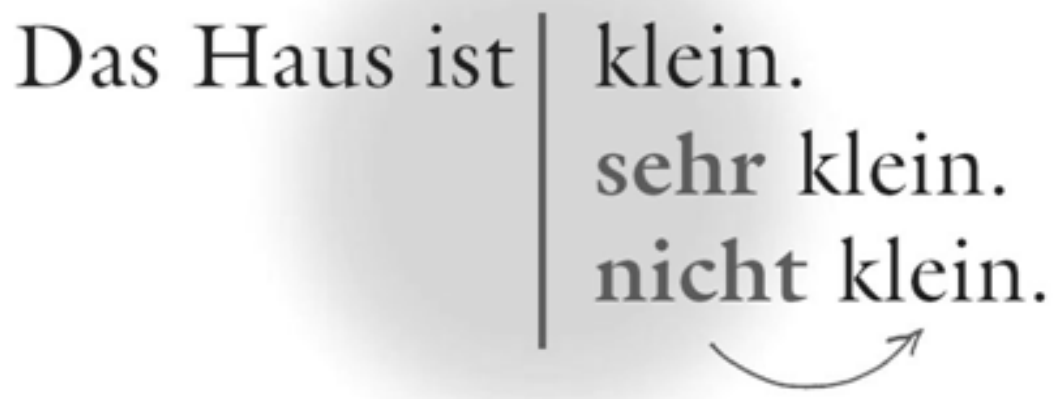

Animationen, wie dieses Beispiel aus uni-deutsch.de (www.deutsch-uni.com), fungieren als Einstieg in die Regelerklärung und die Vertiefung der grammatischen Kenntnisse.

Aussage

Position 1 Position 2

Mein Name ist Iris Pfeil.

Ich bin Timo.

Ich komme aus Deutschland.

Sie sprechen gut Deutsch.

\section{W-Frage}

Position 1 Position 2

Wer ist das?

Wie heißen Sie?

Woher kommen Sie?

Was sprechen Sie?

\section{wiederholen}

Chunks können zu jeder Zeit vermittelt und genutzt werden, ohne dass die Grammatik erklärt werden muss. Lerner sind erstaunlich aufnahmebereit für sol- 
che Ketten, die ähnlich verarbeitet werden wie einzelne Wörter (Beispiel aus Schritte international 1, 2005).

\author{
Etwas bewerten: Das ist _. \\ Das ist aber schön. \\ Das Bad ist doch nicht kein. \\ Meine Wohnung ist nicht so schorn. \\ Aber sie ist billig. \\ Das Haus gefallt mir. \\ Die Frau ist verruckt.
}

\title{
9 Schluss
}

Es wäre sinnvoll im Sprachunterricht, das nachzuempfinden, was in natürlicher Kommunikation passiert. Das bedeutet eine bessere Abstimmung von Eingabe und Aufnahmemöglichkeit der Lerner. Wir benötigen damit eine andere Vorstellung von grammatischer Progression in unseren Lehrwerken, auch eine andere, als sie der Referenzrahmen vorschlägt. Spracherwerb ist nun mal ein Prozess, den man nicht beliebig steuern kann. Im Mittelpunkt stehen immer die Begriffe, die Inhalte. Die Grammatik kommt zu den Inhalten, zu den Begriffen, und nicht umgekehrt.

Sinnvolle Konsequenzen aus diesen Beobachtungen können daher sein:

1. Eine bessere Abstimmung von Strukturen der Eingabe und Aufnahmeund Verarbeitungsmöglichkeiten der Lerner.

2. Eine Änderung und Differenzierung im Verständnis von Progression. Diese müsste ausgerichtet sein am L2-Erwerb und den Verarbeitungsmöglichkeiten der Lerner, d. h., pragmatische Elemente sind in den Vordergrund zu rücken und es ist auf einem lexikalischen Ansatz aufzubauen (vgl. hierzu die CD-ROM grenzenlos, www.grenzenlos-life.de).

3. Die Progression müsste nach rezeptiven und produktiven Kriterien konsequent unterschieden werden. Der Input sollte demnach Varianten mit verschiedener Komplexität (reiche Eingabe) enthalten. Sprachmischungen könnten dabei durchaus auch eine Brückenrolle spielen. In der Produktion sollten von den Lernern zunächst nur solche authentischen Textsorten genutzt werden, die einfache Strukturen und Formeln enthalten, jedoch keine grammatisch falschen Äußerungen und Texte. Auf dieser Basis kann dann die zunehmende Grammatikalisierung erfolgen.

4. In didaktisierten Grammatiken müssten die auf linguistischer und erwerbslinguistischer Forschung basierenden Bausteine systematisch zur 
Verfügung gestellt, in Lehrwerken konsequent umgesetzt und mit diesen empirisch erprobt werden. Anders wird sich die verbreitete Frustration beim Erwerb und der Vermittlung sprachlicher Strukturen nicht nachhaltig beseitigen lassen.

\section{Literatur}

Handwerker, Brigitte: Chunks, Raster und Regeln: Vom Lexikon zur Grammatik in der Fremdsprachenvermittlung. In: Börner, Wolfgang; Vogel, Klaus (Hrsg.): Kognitive Linguistik und Fremdsprachenerwerb. Tübingen: Gunter Narr, 2002, 207-230.

Givòn, Talmy: »Fro Discourse to Syntax: Grammar as a Processing Stategy«. Discours and Syntax. In: Givòn, Talmy (Hrsg.): Syntax and Semantics. Volume 12, New York: Academic Press, 1979, 81-112.

Hinnenkamp, Volker: Foreigner Talk und Tarzanisch. Eine vergleichende Studie über die Sprechweise gegenüber Ausländern am Beispiel des Deutschen und des Türkischen. Hamburg: Buske, 1982.

Krashen, Stephan: The Input Hypothesis: Issues and Imlications. London, New York: Longman, 1985.

Roche, Jörg: Xenolekte. Struktur und Variation im Deutsch gegenüber Ausländern. Berlin, New York, de Gruyter, 1987.

Roche, Jörg: Variation in Xenolects. In: Ammon, Ulrich et al. (Hrsg.): Variationslinguistik. Tübingen, Niemeyer, 1998. 117-139.

Roche, Jörg: Interkulturelle Sprachdidaktik. Eine Einführung. Tübingen, Gunter Narr, 2001 (= narr studienbücher).

Roche, Jörg: Fremdsprachenerwerb - Fremdsprachendidaktik. Tübingen: Franke, 2005. 



\section{Die Integrationskurse des Bundes - Bilanz und Weiterentwicklung}

Christoph Hausschild / Erika Hoffmann, Berlin

\section{Integrationspolitische Weichenstellungen}

„Voneinander lernen - gemeinsam leben“: Unter dieses Motto hat das Bundesamt für Migration und Flüchtlinge seine Arbeit im Bereich der Integration gestellt und verweist damit auf die Umorientierung und neue Schwerpunktsetzung in der Ausländer- und Integrationspolitik, die seit einigen Jahren in Deutschland stattfindet und die durch einen - manchmal auch beschwerlich verlaufenden - Diskussionsprozess weiter vorangetrieben wird. Zunehmend wird die Tatsache anerkannt, dass bereits seit Jahrzehnten eine Einwanderung nach Deutschland erfolgt gleichzeitig jedoch in steigender Zahl auch eine Abwanderung aus Deutschland; kurzum: Wissenschaft und Politik wenden ihre Aufmerksamkeit Migrationsprozessen zu, die in einer globalisierten Welt zunehmend an Bedeutung und Einfluss auf die einzelnen Nationalstaaten gewinnen. Nicht nur die Steuerung der Zuwanderung durch verschiedene Instrumente, sondern auch das Bemühen um nachholende Integration für bereits seit längerem hier lebende Migrantinnen und Migran- 
ten stehen im Mittelpunkt dieser Debatte, die auf allen politischen Ebenen und im öffentlichen Raum ausgetragen wird. Unter der Maßgabe des „Forderns und Förderns" von staatlicher Seite her zur Verbesserung des Zusammenlebens der Migrantengruppen und der Aufnahmegesellschaft beizutragen, haben sich das Bundesministerium des Innern und insbesondere seine untergeordnete Behörde, das Bundesamt für Migration und Flüchtlinge, auf die Fahnen ihrer integrationspolitischen Arbeit geschrieben. Es obliegt dem Bundesamt, die Maßnahmen und Neuerungen umzusetzen, die in dem am 1. Januar 2005 in Kraft getretenen Zuwanderungsgesetz vorgesehen sind. Mit jenem Gesetz wurden erstmals Integrationsmaßnahmen für Migrantinnen und Migranten auf eine gesetzliche Grundlage gestellt. Trotz öffentlich angemahntem Nachbesserungsbedarf in den gesetzlichen Ausformulierungen und in deren Folge in der Umsetzung ist deshalb darin ein Meilenstein für die Integrationspolitik in Deutschland zu sehen.

\section{Die Integrationskurse des Bundes}

Einen Schwerpunkt der Maßnahmen bilden die Integrationskurse des Bundes, deren Struktur und Durchführungsmodalitäten in der Intergrationskursverordnung (IntV) geregelt sind. Die Kurse setzen sich aus einem Sprachkursteil mit 600 Unterrichtseinheiten (UE) á 45 Minuten und einem anschließenden Orientierungskurs mit $30 \mathrm{UE}$ zusammen. Ein erfolgreicher Abschluss des Integrationskurses setzt das Bestehen einer Abschlussprüfung voraus, die aus einer Sprachprüfung auf dem Sprachniveau B1 nach dem Gemeinsamen europäischen Referenzrahmen (GER) und einem Test zum Orientierungskurs besteht. Das modular aufgebaute Kurssystem bildet erstmals ein bundesweit einheitliches Sprachfördersystem als Grundangebot der Integration, das grundsätzlich allen erwachsenen Migranten offen steht und ein gemeinsames Erlernen der deutschen Sprache einen ersten Schritt gelebter Integration - ermöglicht. Obgleich die Einheitlichkeit bezüglich Kursaufbau, methodisch-didaktischer Ausrichtung und Lernzielsetzung als grundlegend erachtet wird, versucht ein System aus gezielter Einstufung in Module und Progressionstypen dennoch, dem einzelnen Lerner ein ihm angemessenes Lernumfeld anzubieten. Teilnehmerorientierung und erwachsenengerechte Vermittlung der Lerninhalte stehen dabei im Mittelpunkt des methodischen Konzeptes, das auf den Prinzipien der kommunikativen Didaktik basiert. Teilnehmerorientierung kann als eine fortgesetzte Zielgruppenorientierung auf didaktischer Ebene verstanden werden, zu der auch die IntV beiträgt, indem sie in $\int 13$ spezielle Zielgruppen ausweist und für diese die Bereitstellung spezifischer Kurskonzeptionen vorsieht. Es handelt sich dabei um drei Gruppen: Jugendliche und junge Erwachsene bis zur Vollendung des 27. Lebensjahres, die eine schulische oder berufliche Ausbildung anstreben; Eltern und Frauen, die aus familiären oder kulturellen Gründen nicht an dem allgemeinen Integrationskurs teilnehmen können 
sowie Personen, die das Lesen und Schreiben noch nicht oder nicht hinreichend beherrschen.

Die bundesweite erfolgreiche Implementierung der Integrationskurse durch Bereitstellung pädagogischer Konzeptionen und Qualifizierungsangebote für Lehrkräfte, eines Zulassungsverfahrens für Kursträger und Teilnehmer sowie eines funktionierenden Verwaltungs- und Abrechnungssystems der Kurse in einem äußerst kurzem Zeitraum brachte zwangsläufig Kompromisse auf allen Seiten mit sich. An verschiedenen Stellen werden nun Defizite sichtbar und lassen Nachsteuerungsbedarf erkennen. Die Kritik an diesen oder jenen Punkten darf jedoch nicht überdecken, dass es mit Einführung der Integrationskurse gelungen ist, Sprachförderung nicht nur für Neuzuwanderer, sondern gerade auch im Sinne der nachholenden Integration für länger hier lebende Migrantinnen und Migranten attraktiv und qualitativ hochwertig zu gestalten. Allein die Förderdauer von 630 $\mathrm{UE}$, die vielfach als unzureichend kritisiert wird, insbesondere für spezielle Zielgruppen, darf hier nicht als ausschließliches Bewertungskriterium gelten. Entscheidend ist, dass eine objektive Zielsetzung, nämlich das Erreichen des Sprachniveaus B1 nach dem GER, und ein verbindlicher Kriterienkatalog für Unterricht und Organisation der Kurse erstmals umfassende Maßnahmen zur Qualitätssicherung und eine Evaluation der Sprachförderung und ihrer Ergebnisse ermöglichen und somit eine Vergleichbarkeit von Angeboten herstellen.

\section{Bilanz des Jahres 2005}

Vom Bundesamt wurde zu Beginn des Jahres 2006 eine erste Bilanz der Integrationskurse für das Jahr 2005 veröffentlicht, in der die wichtigsten Zahlen und Fakten zusammengefasst sind. Demzufolge begannen im Jahr 2005 insgesamt 8.196 Kurse, in denen sich 115.158 Teilnehmende eingefunden haben. Die Zahl der Berechtigungen zur Teilnahme lag mit 215.651 Personen jedoch fast doppelt so hoch. Von der Möglichkeit der Verpflichtung von Teilnehmenden machten die zuständigen Ausländerbehörden zu Beginn nur zögerlich Gebrauch. Immerhin einem Anteil von 28,2\% aller Berechtigten gegenüber wurde eine Verpflichtung ausgesprochen, entweder aufgrund ihres Status als Neuzuwanderer oder aufgrund noch mangelnder Sprachkenntnisse trotz bereits längeren Aufenthaltes in der Bundesrepublik. Die Betrachtung der Zusammensetzung der Kurse zeigte, dass die bereits vor 2005 in Deutschland lebenden Personen den größten Anteil bildeten. Allein bei den vom Bundesamt auf Antrag ausgesprochenen Zulassungen - das waren in Zahl 103.146 Personen - erreichte dieser Personenkreis einen Anteil von 47,8\%.

Frauen stellten 63\% der Kursteilnehmenden. Dies spiegelt einen erfreulichen Trend wider, denn die Bedeutung von Frauen als Multiplikatorinnen für die Integration weiterer Familienmitglieder und insbesondere der Kinder steht außer Frage. Gleichwohl ist dieser Trend aber auch deshalb so positiv zu bewerten, weil damit die gesellschaftliche und eine spätere berufliche Integration der Frauen selbst vo- 
rangetrieben wird. Dieser Trend eines höheren Anteils von Frauen setzt sich nach Erkenntnissen des Bundesamtes auch im Jahr 2006 fort.

An der bislang freiwilligen Abschlussprüfung nahmen im Jahr 200517.482 Personen teil. 12.151 Teilnehmende, also 69,5\%, beendeten die Prüfung mit Erfolg und wiesen damit nach, dass sie nunmehr über das Sprachniveau B1 verfügten. Dieses anspruchsvolle Ziel erreicht zu haben, hilft den Betroffenen nicht nur bei einer selbstständigen und sicheren Bewältigung des Alltags in Deutschland, sondern gilt auch als Nachweis einzelner Voraussetzungen zur Erlangung einer Niederlassungserlaubnis ( 9 Abs. 2 S.1 Ziff. 7 u. 8 AufenthG) und führt darüber hinaus zu einer Verkürzung der Einbürgerungsfrist von acht auf sieben Jahre bei der Anspruchseinbürgerung (\$ 11 Abs. 3 StAG). Im Jahr 2006 hat sich der Anteil derjenigen, die bislang erfolgreich an der Prüfung teilgenommen haben, leicht erhöht auf vorläufig ca. 71\% (Stand 3. Quartal 2006).

\section{$4 \quad$ Evaluation der Integrationskurse im Jahr 2006}

Nichtsdestotrotz bleibt zu konstatieren, dass ein großer Prozentsatz der Teilnehmenden den Integrationskurs nicht erfolgreich abschließt. Hier und an anderen Stellen zeigt sich, dass dieses Integrationsangebot noch optimiert werden kann. Zur Weiterentwicklung des Kurssystems findet daher seit Anfang dieses Jahres eine umfassende Evaluation der Integrationskurse statt, die noch bis zum 31. Dezember 2006 andauert und von der Firma Rambøll Management durchgeführt wird. Das europäische Beratungsunternehmen mit Standorten in Dänemark, Schweden, Norwegen, Deutschland und Belgien kann eine dreißigjährige Erfahrung in der Beratung des öffentlichen Sektors vorweisen.

Im Fokus der Analyse stehen dabei die Bereiche „Verfahrenseffizienz“, „Finanzierung“ und „Methodik/Didaktik“. Untersucht werden beispielsweise die Regelungen und Verfahren zur Kursdurchführung, das Finanzierungsmodell sowie das Verfahren der Zulassung von Kursträgern. Geprüft wird auch, inwieweit die Kurse das Ziel, den Teilnehmern Deutschkenntnisse bis zum Sprachniveau B1 zu vermitteln, tatsächlich erreichen und worin die Gründe für ein Nichterreichen in erster Linie zu suchen sind.

Nach dem Projektstart am 1. Januar 2006 begann das Unternehmen bereits Anfang Februar mit der Bestandsaufnahme. Dazu wurden verschiedenste Daten und Dokumente untersucht, Interviews mit Mitarbeiterinnen und Mitarbeitern des Bundesamtes und seiner Regionalstellen sowie mit Mitarbeitern zahlreicher Ausländerbehörden und Kursträger geführt. Es fanden auch zwei Vor-Ort-Analysen statt. Als nächster Schritt wurde eine Breitenanalyse durchgeführt, zu der eine umfangreiche Onlinebefragung aller Kursträger zählte. Daran schloss sich eine Tiefenanalyse an, bei der wiederum rund 80 weitere Vor-Ort-Analysen bei Kursträgern, etwa 30 bei Ausländerbehörden und etwa zehn bei Arbeitsgemeinschaften der Jobagenturen und Kommunen erfolgten. Die weiteren Phasen des Projektes 
umfassen eine internationale Vergleichsanalyse, Gutachtenerstellung und Berichtslegung.

Bereits im Juni 2006 hat das Bundesministerium des Innern einen Zwischenbericht dieser „Evaluation der Integrationskurse nach dem Zuwanderungsgesetz“ veröffentlicht. Darin ist als ein Hauptkritikpunkt der Träger an dem administrativen Verfahren der für sie entstehende Verwaltungsaufwand aufgenommen; gleichzeitig wurde von Trägerseite die überaus positive Rolle der Regionalkoordinatoren in den Regionalstellen des Bundesamtes betont und einer allgemeinen Zufriedenheit mit deren Arbeit Ausdruck verliehen. Die Finanzierungsgrundlage von 2,05 Euro pro Teilnehmer und UE stand ebenfalls in der Kritik: Etwa zwei Drittel der befragten Kursträger gaben an, die Kurse kostendeckend durchführen zu können. Die Dozentenhonorare sind nach Trägerangaben aber seit Einführung des Integrationskurssystems im Durchschnitt um zwei Euro pro Lehrkraft und UE gesunken. Hinsichtlich der Zielerreichung des Sprachniveaus B1 gaben die Kursträger die Einschätzung ab, dass etwa die Hälfte der Teilnehmenden das Ziel erreichen könne, gut ein Drittel hingegen den Integrationskurs mit dem Sprachniveau A2 beenden würde. Rambøll Management wird diese und weitere Daten einer genauen Analyse unterziehen und Verbesserungsvorschläge unterbreiten, die auch in den Erfahrungsbericht der Bundesregierung an den Bundestag einfließen werden.

\section{Optimistischer Blick in die Zukunft}

Das Bundesministerium des Inneren zieht eine insgesamt positive Bilanz der ersten eineinhalb Jahre Integrationskurse. In der Praxis haben sich die Neuerungen weitgehend bewährt, auch wenn an vielen Stellen Nachbesserungsmöglichkeiten offenbar werden. Ein Überdenken einzelner zugrunde gelegter Förderprinzipien kann sich im Verlauf der weiteren politischen Auseinandersetzung mit aktuellen Fragen der Zuwanderung und Integration einstellen. Das Bundesamt seinerseits setzt seine Arbeit im Bereich Konzeptionierung und Verfahrensoptimierung fort und trägt somit kontinuierlich zur Weiterentwicklung der Integrationskurse bei. Grundsätze der Ökonomie lassen sich zwar nicht ohne Weiteres auf die Unterrichts- und Lernsituation übertragen, aber die Effizienz dieses Systems der Integrationskurse wirkt sich auch hier positiv auf die Motivation und die Bereitschaft des Einzelnen aus, seine Möglichkeiten voll auszuschöpfen, um somit den Integrationsprozess zum Erfolg zu führen. 



\section{Die Konzeption des Bundesamtes für Migration und Flüchtlinge für die Zusatzqualifizierung von Lehrkräften im Bereich Deutsch als Zweitsprache}

Rosemarie Bublmann, München

In den letzten beiden Jahren hat der Bereich „Deutsch als Zweitsprache“ des Goethe-Instituts im Auftrag des Bundesamtes für Migration und Flüchtlinge eine Konzeption für die Zusatzqualifizierung von Lehrkräften für die Integrationskurse erarbeitet, dazu ein Qualitätssicherungskonzept entwickelt und zusammen mit dem Bundesamt erprobt. Die Konzeption dient interessierten Institutionen, die Lehrkräfte für die Integrationskurse qualifizieren und sich dazu beim Bundesamt akkreditieren lassen möchten, als Referenz.

Die Konzeption des Bundesamts steht seit 13. Februar 2006 im Netz. Ich möchte deshalb in diesem Beitrag nicht ihren Inhalt referieren, sondern

- auf die Philosophie abheben, die dahinter steht

- die Überlegungen ansprechen, die zu der Form geführt haben, in der die Konzeption jetzt vorliegt 
- auf die Merkmale der Ziele und Methoden der Zusatzqualifizierung abheben

und

- Hinweise auf die Arbeit mit der Konzeption geben.

\section{Das Vorgehen bei der Entwicklung der Konzeption des Bundesamtes für Migration und Flüchtlinge für die $\mathrm{Zu}-$ satzqualifizierung von Lehrkräften im Bereich Deutsch als Zweitsprache}

\subsection{Festlegung der Ziele des DaZ-Unterricht für Zugewanderte}

Die Qualifizierungsziele dieser Konzeption sind keine willkürliche Setzung. Sie wurden aus den Anforderungen an den DaZ-Unterricht abgeleitet. Diese wiederum resultieren aus den Zielsetzungen, Einflussfaktoren und Merkmalen des DaZUnterrichts. Bezüglich der Ziele des Sprachunterrichts in den Integrationskursen stellt das Bundesamt fest:

„Ziel der Sprachkurse ist die Förderung der sozialen und beruflichen Integration von ausländischen Arbeitnehmern und ihren Familienangehörigen. Dieses Ziel ... soll durch einen handlungsorientierten Sprachunterricht“" erreicht werden, „der auf die Verbesserung der Kommunikationsfähigkeit in der deutschen Sprache ausgerichtet ist und sich an der realen Situation orientiert, in der sich die ausländischen Arbeitnehmer und ihre Familienangehörigen befinden" $" 1$

Der Erwerb von deutschen Sprachkenntnissen wird also verstanden als die wesentliche Grundlage der Integration. Es wird ein Unterricht gefordert, der primär auf Kommunikationsfähigkeit abzielt und sich dabei an der Lebenswirklichkeit der Zugewanderten orientiert.

So verstanden muss der Sprachunterricht zu folgenden, vom sprachlichen Aspekt her formulierten Zielen beitragen:

a) die Verbesserung der Voraussetzungen für die Teilnahme der Zugewanderten am sozialen Leben in der Bundesrepublik Deutschland,

z. B.

- Erwerb von sprachlichen Mitteln zur Bewältigung des eigenen Alltags

- Erwerb von Kommunikationsmitteln zur Bewältigung von Behördengängen, zur Regelung der Probleme der Kinder in Schule

\footnotetext{
${ }^{1} \mathrm{http} /$ /www.bafl.de/template/integration/content_integration_sprachkurs-foerderung_20... (09. 07. 2003)
} 
und Kindergarten, zur Kommunikation beim Arzt, auf der Bank, mit Vermietern etc.

- Erwerb von sprachlichen Mitteln zur Kommunikation mit der Nachbarschaft, Kollegen und deutschsprachigen Bekannten bzw. Freunden in der Freizeit

b) die Verbesserung der Voraussetzungen für die Teilnahme der Zugewanderten am Erwerbsleben in Deutschland, z. B.:

- Erwerb von Kommunikationsmitteln zur Bewältigung der beruflichen Qualifizierungsmaßnahmen

- Erwerb von Kommunikationsmitteln zur Bewältigung der Arbeitssituation

c) die Verbesserung der Voraussetzungen für die Teilnahme der Zugewanderten am gesellschaftlichen Leben in Deutschland

- Erwerb der sprachlichen Mittel zur Kommunikation in Versammlungen, Treffen, Gruppierungen, Vereinen etc.

- Erwerb der sprachlichen Mittel zur Information über politische, wirtschaftliche und gesellschaftliche Ereignisse

- Erwerb von sprachlichen Mitteln zur Kommunikation im kommunalen Kontext

d) die Verbesserung der Voraussetzungen für die Teilnahme der Zugewanderten am kulturellen Leben in Deutschland

- Erwerb von sprachlichen Mitteln zur Information über kulturelle Ereignisse

- und Teilnahme an entsprechenden Veranstaltungen wie Stadtteilfesten, Informationsveranstaltungen etc.

Um diese Ziele in die Wirklichkeit umsetzen zu können, sind jedoch noch andere Ziele zu erreichen, die im DaZ-Unterricht integriert sein müssen wie z. B.:

- Förderung der Fähigkeit der Zugewanderten, sich mit den eigenen Fähigkeiten, Stärken, Schwächen und Interessen aktiv in die deutsche Gesellschaft einzubringen

- Steigerung des Selbsthilfepotentials der Zugewanderten durch Förderung von Selbsttätigkeit und Eigenverantwortung

- Förderung der Identitätsfindung und Handlungsfähigkeit in den Kulturen

- Förderung der Fähigkeit zum interkulturellen Lernen

- Förderung der Fähigkeit des reflektierenden Lernens

- Förderung der Fähigkeit zum lebenslangen Lernen

Diese Liste ließe sich fortsetzen. Aus der Auflistung einiger der Hauptziele, die nicht rein sprachlicher Natur sind, geht aber bereits hervor, dass der DaZ-Unterricht 
auch nicht-sprachliche Kenntnisse aus den verschiedensten Bereich transportieren bzw. die Teilnehmer in die Lage versetzen muss, sich diese selbst zu erschließen.

Aus den Zielen des DaZ-Unterrichts, von denen hier lediglich Rahmenziele aufgeführt werden können, und aus seinen Merkmalen und Bedingungsfaktoren, die allgemein bekannt sind und die ich hier ausspare, ergeben sich die Anforderungen an diesen Unterricht.

\subsection{Anforderungen an den DaZ-Unterricht für Zugewanderte}

Die wichtigsten Anforderungen sollen im Folgenden aufgeführt werden. Sie werden mit Hilfe der folgenden Kriterien zusammengefasst - die Aufzählung bedeutet keine Hierarchisierung. Der DaZ-Unterricht muss ermöglichen bzw. gewährleisten:

- Interkulturelles Lernen

- Lernerorientierung

- Differenzierung im Unterricht

- Selbstgesteuertes Lernen

- Lebensnahes Lernen

- Ganzheitliches Lernen

- Handlungsorientiertes Lernen

- Entdeckendes Lernen

- Nutzung von Fehlern als Lernanstoß

- Reflektierendes Lernen

- Methodische, mediale und soziale Vielfalt

- Offene Unterrichtsgestaltung

Eine ausführliche Darstellung der Inhalte dieser Anforderungen und der Wirkung ihrer Erfüllung findet sich in der Broschüre der Konzeption auf S. 7 - 112).

\subsection{Anforderungen an die Lehrkräfte für den DaZ-Unterricht für Zugewanderte}

Die Anforderungen an die Person der Lehrkraft in Bezug auf ihre Kompetenzen ergeben sich aus den o. g. Anforderungen an den DaZ-Unterricht. Sie wurden u. a. als Ergebnis einer Recherche in der einschlägigen Literatur und von über 25 Jahren DaZ-Lehrkräftequalifizierungserfahrung zu einem Anforderungsprofil zusammengestellt. Dieses ist Ergebnis einer Auswahl von über 150 Einzelanforderungen und der Anordnung der Anforderungen nach bestimmten Kriterien. Da eine Arbeit mit dem Kompetenzbegriff als Ordnungskriterium keine eindeutigen

\footnotetext{
2http://www.bamf.de/cln_042/SharedDocs/Anlagen/DE/Integration/Downloads/Integrationskurse/Leh rkraefte/konzept-zusatzqualifizierung-

lehrkr_C3_A4fte,templateId=raw,property=publicationFile.pdf/konzept-zusatzqualifizierung-lehrkräfte.pdf
} 
Ergebnisse, d. h. keine zwingenden Zuordnungen brachte, wurden als Ordnungskriterien die Tätigkeiten gewählt, die die DaZ-Lehrkraft für Zugewanderte in Ausübung ihres Berufes ausführen muss. Diese lassen sich in Handlungsfelder zusammenfassen, die ihrerseits das entsprechende Berufsbild ergeben.

Die Minimalanforderungen, die DaZ-Lehrkraft erfüllen muss, wenn sie pädagogisch handlungsfähig sein soll, wurden (auf S. 11 - 19 der Broschüre) in die folgenden 12 Handlungsfelder eingeordnet:

\begin{tabular}{|ll|}
\hline \multicolumn{2}{|l|}{ Handlungsfelder } \\
\hline I. & $\begin{array}{l}\text { Bedürnisse, Ziele, Beweggründe, Einstellungen und Voraussetzungen der Zuge wan } \\
\text { derten für den Kurs ermitteln }\end{array}$ \\
\hline II. & $\begin{array}{l}\text { adressatenspezifischen DaZ-Unterricht innerhalb eines Standardangebots im Bereich } \\
\text { A1 - B1 planen }\end{array}$ \\
\hline III. & $\begin{array}{l}\text { adressatenspezifischen DaZ-Unterricht innerhalb eines Standardangebots im Bereich } \\
\text { A1 - B1 vorbereiten }\end{array}$ \\
\hline IV. & $\begin{array}{l}\text { adressatenspezifischen DaZ-Unterricht innerhalb eines Standardangebots im Bereich } \\
\text { A1 - B1 durchführen }\end{array}$ \\
\hline V. & $\begin{array}{l}\text { adressatenspezifischen DaZ-Unterricht innerhalb eines Standardangebots im Bereich } \\
\text { A1 - B1 auswerten }\end{array}$ \\
\hline VI. & Kursteilnehmer einstufen, testen und prüfen \\
\hline VII. & Kursteilnehmer bezüglich ihres Spracherwerbs beraten \\
\hline VIII. & das Migrationsumfeld und die Migrationssituation in den DaZ-Unterricht einbeziehen \\
\hline IX. & Teilnehmer über öffentliche Beratungsangebote informieren \\
\hline X. & mit Kollegen zusammenarbeiten \\
\hline XI. & die eigene Institution nach außen vertreten \\
\hline XII. & die eigene Persönlichkeit und Professionalität weiter entwickeln \\
\hline
\end{tabular}

Bei der Zuordnung wurde auch deutlich, dass es neben den fachlichen auch persönliche Anforderungen und Voraussetzungen gibt, die unabdingbar für die Ausübung dieses Berufs sind und ohne die eine Qualifizierung für die Lehrtätigkeit nicht sinnvoll erscheint. Sie müssen für den Besuch einer Qualifizierungsmaßnahme vorausgesetzt werden. Das sind z. B. Anforderungen wie z. B. Kenntnis von Hintergründen, die zur Migration führen (Handlungsfeld I), Beherrschung der deutschen Standardsprache schriftlich und mündlich auf dem Niveau von C1, Verfügen über eine deutliche Aussprache... (Handlungsfeld IV), Fähigkeit, Kritik und kollegiale Beratung anzunehmen, zu reflektieren und ggf. Ergebnisse bzw. Lösungsvorschläge zu verarbeiten und umzusetzen... (Handlungsfeld X), Bewusstsein bzw. -werden der eigenen Stärken und Schwächen sowie der eigenen Entwicklungspotentiale als Lehrende und Lernende sowie Fähigkeit, Stärken weiter zu entwickeln und Schwachstellen zu verbessern bzw. zu den eigenen Grenzen zu stehen... (Handlungsfeld XII). Diese Voraussetzungen schlagen sich deshalb 
nicht in Qualifizierungszielen nieder und führen dementsprechend auch nicht zu einer Formulierung von Inhalten.

\subsection{Das Anforderungsprofil als Grundlage des Soll-Zustandes der DaZ-Lehrkräfte}

Die Erfüllung der Minimalanforderungen an die DaZ-Lehrkräfte spiegelt einen Soll-Zustand wieder. Aus den Minimalanforderungen lässt sich nun ein Minimalzielkanon für die Qualifizierungsveranstaltungen ableiten und präzise formulieren, der den Soll-Zustand der DaZ-Lehrkräfte umreißt. Vergleicht man den SollZustand mit dem Ist-Zustand, der durch ihre Eingangsqualifikationen bzw. -voraussetzungen charakterisiert wird, so ergeben sich als Differenz die Lerninhalte, die in der Qualifizierungsmaßnahme vermittelt werden müssen. Das Anforderungsprofil bildet also die Grundlage für die Formulierung des Soll-Zustandes und die Ableitung von Lernzielen für die Qualifizierung.

\subsection{Ableitung von Zielen aus Anforderungen}

Für alle Anforderungen lassen sich also Qualifizierungsziele formulieren. Ich möchte dies anhand eines Beispiels durchspielen, um auf diese Weise die Vorteile und die Problematik der expliziten Dokumentation eines solchen Verfahrens aufzuzeigen.

Ich wähle die Anforderung Nr. 2 „Kenntnis der einschlägigen Unterrichtsmaterialien“ aus Handlungsfeld III: ,adressatenspezifischen Unterricht innerhalb eines Standardangebots im Bereich A 1 - B 1 vorbereiten“ (S. 21 der Broschüre). Diese Anforderung führt nicht nur zu dem Qualifizierungsleitziel: „Die Lerner können DaF- und DaZ-Lehrwerke analysieren und zielgruppengerecht auswählen.", sondern steht auch mit den übrigen vier auf Seite 46 genannten Anforderungen in enger Verbindung.

Die o. g. Anforderung muss zielmäßig formuliert und in Grobziele herunter gebrochen werden. Im obigen Fall erhält man mindestens drei Grobziele, die in der Grafik auf S. 46) aufgeführt sind. Die Grobziele müssen ihrerseits wieder herunter gebrochen werden in Feinziele, denen die entsprechenden Inhalte zuzuordnen sind, wenn die Leitziele in Qualifizierungsveranstaltungen in überprüfbarer Weise erreichbar sein sollen. 
Anforderung: Leitziele

Qualifizierungsgrobziele Feinziele

Die Daz-Lehrkrätte können Das beinhaltet:

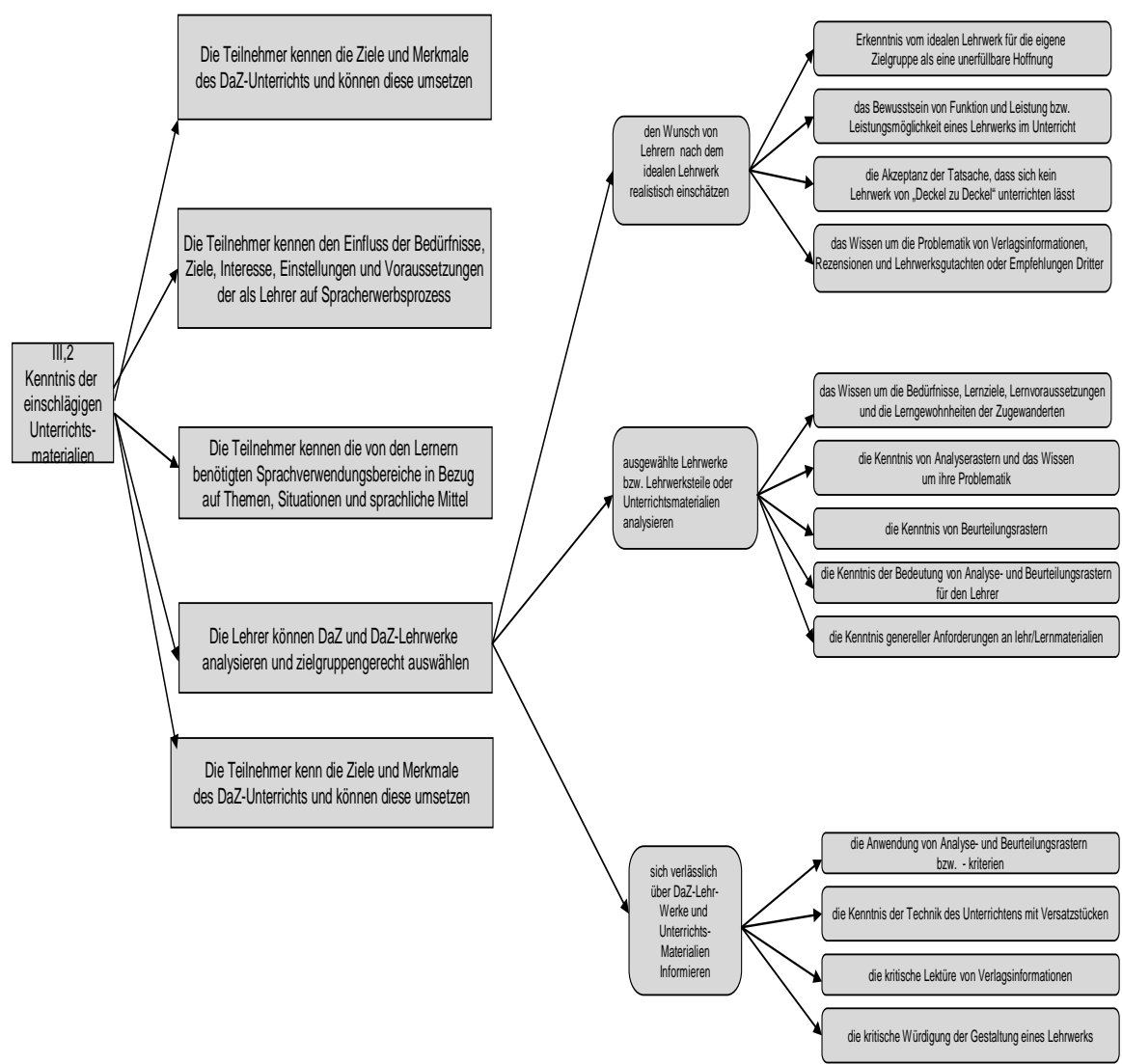

In gleicher Weise wäre nun mit den anderen Anforderungen zu verfahren, die in der Anforderung „Kenntnis der einschlägigen Unterrichtsmaterialien“ enthalten sind. Die konsequente Ausführung dieser Operation, d. h. die Ableitung von Qualifizierungszielen aus den entsprechenden Anforderungen, ergibt neben zahlreichen Verästelungen, Verflechtungen und Überschneidungen auch Doppelnennungen von Zielen, die - bildlich gesprochen - einem mehrschichtigen Teppich von Zielen und Inhalten vergleichbar sind. Dieser ist einerseits nicht besonders übersichtlich, andererseits ist das entsprechende Zielgeflecht nicht ohne weiteres 
in transparente Qualifizierungsveranstaltungen umsetzbar, weil immer wieder Rückgriffe auf grundlegende Ziele nötig wären, die den thematischen Zusammenhang im besten Fall überdehnen würden.

Dazu kommt noch, dass die aus der Erreichung des Lernziels: „Die Lerner können DaF- und DaZ-Lehrwerke analysieren und zielgruppengerecht auswählen." resultierende Fähigkeit aber auch die Voraussetzung ist, um einen Teil der Anforderung Nr. 5 im Handlungsfeld III zu erfüllen, nämlich die „Fähigkeit, Unterrichtsmaterialien zu beurteilen, gezielt auszuwählen und für die Zielgruppen zu adaptieren.“ (S. 21 der Broschüre). Dementsprechend müsste auch hier aufgefächert werden, der Teppich würde sich vergrößern und verdichten.

Deshalb wurde aus Gründen der Übersichtlichkeit und Umsetzbarkeit der Weg beschritten, die verschiedenen Schichten eines solchen Teppichs nicht in ihrer naturgegebenen Überlagerung zu präsentieren, sondern sie gewissermaßen in einer Ebene auszulegen. Das erlaubt anschließend eine thematische Bündelung zu Gruppen von Zielen und eine progrediente Anordnung dieser Gruppen dergestalt, dass z. B. grundlegende Gruppen von Zielen mit einer Benennung „Merkmale des DaZ-Unterrichts“ oder „Analyse von DaZ-Unterricht“ am Anfang einer Qualifizierungsveranstaltung die erforderlichen Grundlagen gewährleisten, auf die immer wieder zurückgegriffen werden kann.

Bei der Bündelung und Ordnung von Qualifikationszielen wurde deshalb so vorgegangen, dass - thematisch geordnet - mit den aus den Anforderungen abgeleiteten Grobzielen begonnen und die zu Erreichung der Grobziele zu erreichenden Feinziele samt den zugehörigen Inhalten, die ja alle Ergebnis der Bündelung von Anforderungen sind, anzuschließen. Parallel dazu wurden der Übersichtlichkeit halber Leitziele formuliert. Die Korrelation zwischen Anforderungen und Zielen wurde zur Kontrolle mit einem gesonderten Nachweis belegt.

1.6 Hierarchisierung der Ziele und Inhalte der Qualifizierung: Form der Darstellung

\section{a) Leitziele, Grobziele, Feinziele und Inhalte der Zusatzqualifizierung}

Insgesamt wurden 14 Leitziele aus den Anforderungen an die DaZ-Lehrkräfte abgeleitet (aufgeführt auf S. 19 der Broschüre). Diese Leitziele wurden jeweils in Grobziele herunter gebrochen. Die zu den Leitzielen gehörigen Grobziele wurden, wie schon erwähnt, nach inhaltlichen Gesichtspunkten gebündelt und mit Feinzielen und Inhalten versehen.

Die unter inhaltlichen Gesichtspunkten zusammengestellten Grobziele mit den Feinzielen und Inhalten haben wir als curriculare Bausteine bezeichnet. Insgesamt wurden für die unverkürzte Zusatzqualifizierung nach thematischen Gesichtspunkten 34 Bausteine gebildet, 28 davon sind als obligatorisch zu betrachten, 6 Themen stehen zur Wahl. Die folgende Übersicht enthält eine Zeitempfehlung für die Umsetzung der einzelnen Bausteine: 
obligatorische curriculare Bausteine in Präsenzphasen

1. Migration und Migranten $4 \mathrm{UE}$

2. Merkmale des DaZ-Unterrichts $4 \mathrm{UE}$

3. Selbsterfahrung der Seminarteilnehmer $4 \mathrm{UE}$ durch Fremdsprachenanfängerunterricht

4. Unterrichtsbeobachtung und -beurteilung $2 \mathrm{UE}$ im Rahmen der angebotenen Hospitation

5. Analyse von DaZ-Unterricht $4 \mathrm{UE}$

6. Methodische Ansätze im DaF- und DaZ-Unterricht $2 \mathrm{UE}$

7. Unterrichtsmaterialanalyse und -beurteilung im DaZ-Unterricht $4 \mathrm{UE}$

8. Wortschatzvermittlung im DaZ-Unterricht $6 \mathrm{UE}$

9. Lesen im DaZ-Unterricht $6 \mathrm{UE}$

10. Hören im DaZ-Unterricht $6 \mathrm{UE}$

11. Phonetik im DaZ-Unterricht 4 UE

12. Sprechen im DaZ-Unterricht $6 \mathrm{UE}$

13. Schreiben im DaZ-Unterricht $6 \mathrm{UE}$

14. Kombinierte Fertigkeiten im DaZ-Unterricht

15. Grammatik im DaZ-Unterricht $6 \mathrm{UE}$

16. Übungstypologien, Sozialformen 4 UE und Arbeitsanweisungen im DaZ-Unterricht

17. Spielerische Übungen, Sprachlernspiele 4 UE und Spiele im DaZ-Unterricht

18. Projektarbeit im DaZ-Unterricht $4 \mathrm{UE}$

19. Visualisierung im DaZ-Unterricht $2 \mathrm{UE}$

20. Fehler und Fehlerkorrektur im DaZ-Unterricht 2 UE

21. Kommunikationsmittel im DaZ-Unterricht $2 \mathrm{UE}$

22. Heterogenität und Binnendifferenzierung im DaZ-Unterricht $6 \mathrm{UE}$

23. Interkulturelles Lernen im DaZ-Unterricht $4 \mathrm{UE}$

24. Lernen Lernen im DaZ-Unterricht 2 UE

25. Testen und Prüfungen im DaZ-Unterricht $6 \mathrm{UE}$

26. Planen, Vorbereiten und Erteilen von DaZ-Unterricht $16 \mathrm{UE}$

27. Evaluation im DaZ-Unterricht $2 \mathrm{UE}$

28. Kursteilnehmerberatung im DaZ-Unterricht $\underline{2 \mathrm{UE}}$

insgesamt $120 \mathrm{UE}$

Themen zur Wabl in Präsenzphasen

1. Konfliktmanagement im DaZ-Unterricht

2. Kollegiale Beratung

3. Berufsorientierender DaZ-Unterricht

4. Alphabetisierung im DaZ-Unterricht

5. Schule mal anders - Eltern lernen Deutsch

6. Neue Medien im DaZ-Unterricht 


\section{b) Die Darstellung eines curricularen Bausteins}

Die Einzeldarstellung der curricularen Bausteine erfolgt in tabellarischer Form. Der curriculare Baustein „Lesen im DaZ-Unterricht“, dessen Lernziele für die Erfüllung von Anforderungen aus Handlungsfeld II - VIII erreicht sein müssen, ist als Beispiel auf S. 49-50 abgedruckt.

In der linken Spalte der Tabelle sind jeweils die Grobziele in „can do"-Formulierungen angegeben, in der rechten Spalte werden die Feinziele und Inhalte aufgelistet, die die Erreichung dieser Ziele beinhalten. Sowohl die Grobziele als auch die Feinziele und Inhalte sind progredient angeordnet - beginnend mit den grundlegenden Zielen und Inhalten fortschreitend zu den komplexeren und abschließenden Zielen, die die Erreichung der grundlegenden voraussetzen.

\section{Lesen im DaZ-Unterricht}

Die Teilnehmer können:

\begin{tabular}{|c|c|}
\hline Die leslnehmer konnen: & Das belnnalt \\
\hline $\begin{array}{l}\text { den Stellenwert des Lesens authentischer } \\
\text { Texte für die Zielgruppe der Zugewanderten } \\
\text { richtig einschätzen }\end{array}$ & 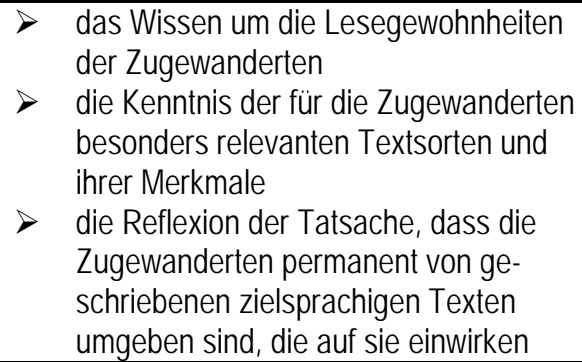 \\
\hline $\begin{array}{l}\text { Entschlüsselungsstrategien beim Lesen von } \\
\text { Texten identifizieren }\end{array}$ & $\begin{array}{l}\text { die Kenntnis der gängigsten Entschlüs- } \\
\text { selungsstrategien }\end{array}$ \\
\hline $\begin{array}{l}\text { die Einsetzbarkeit eines Textes im Unterricht } \\
\text { beurteilen }\end{array}$ & 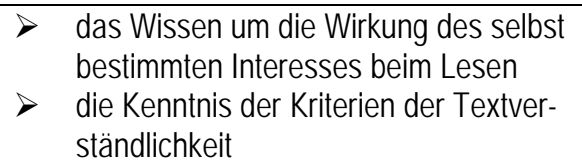 \\
\hline die Funktionalität einer Leseübung beurteilen & $\begin{array}{l}\text { die Kenntnis der Abhängigkeit des Le- } \\
\text { sestils von Leseabsicht und Textsorte } \\
\text { das Wissen um die Abhängigkeit des } \\
\text { Lesestils von der Aufgabenstellung } \\
\text { die Kenntnis von Leseübungstypolo- } \\
\text { gien, ihren Voraussetzungen und Leis- } \\
\text { tungen }\end{array}$ \\
\hline $\begin{array}{l}\text { einen angemessenen authentischen Text } \\
\text { auswählen und didaktisieren }\end{array}$ & $\begin{array}{ll}>\quad \text { das Wissen um die Formen der Vor- } \\
\text { entlastung } \\
>\quad \text { die Auswahl einer funktionalen } \\
\text { Übungsform und die Ausarbeitung } \\
\text { einer funktionalen Leseübung } \\
>\quad \text { die Formulierung einer funktionalen }\end{array}$ \\
\hline
\end{tabular}




\begin{tabular}{|l|l|}
\hline & $\begin{array}{l}\text { Arbeitsanweisung zu der für den } \\
\text { Nachweis des Textverständnisses } \\
\text { am besten geeigneten Übungsform } \\
\end{array}$ \\
$>\begin{array}{l}\text { die Auswahl der optimalen Sozial- } \\
\text { form } \\
\text { die Kenntnis der Formen möglicher } \\
\text { Nachbereitung des Textes }\end{array}$ \\
\hline
\end{tabular}

Alle curricularen Bausteine sind gleich aufgebaut. Sie repräsentieren Ziele und Inhalte, die grundsätzlich zu erreichen bzw. umzusetzen sind. Ihr Umfang, d. h. die Menge und Kleinteiligkeit ihrer Feinziele und Inhalte, ergibt sich in der Praxis der jeweiligen Seminarveranstaltung aus den Bedürfnissen und Lernvoraussetzungen der Teilnehmer, die wiederum durch die Anforderungen bestimmt werden, die an ihre Lehrtätigkeit im Bereich DaZ gestellt werden.

\section{Merkmale der Konzeption}

\subsection{Merkmale der Ziele}

Aus dem bisher Gesagten wurde deutlich, dass die Ziele, mit deren Erreichung die DaZ-Lehrkraft sich für den DaZ-Unterricht qualifiziert, organisch aus den Zielen des DaZ-Unterrichts, den Anforderungen an den DaZ-Unterricht und an die DaZ-Lehrkraft abgeleitet sind. Zum anderen wurde klar, dass es sich dabei nicht um Lehrziele handelt, sondern um Lernziele, die so konkret definiert sind, dass ganz deutlich wird, was die Lehrkraft am Ende der Qualifizierung können soll. Aus diesem Grunde braucht eigentlich nicht gesagt zu werden, dass es sich bei Seminaren zur Qualifizierung nicht um die Vermittlung deklaratorischen Wissens handelt, sondern um den Aufbau bzw. der Förderung von Kompetenzen. Dazu dienen nicht nur die Umsetzung der didaktischen Aspekte, sondern auch die exemplarische Anwendung der im Folgenden aufgeführten Methoden, die auch im DaZ-Unterricht wichtig sind bzw. in Unterrichtsmethoden ihre Äquivalente haben und den Teilnehmern im Sinne einer Selbsterfahrung nahe zu bringen sind.

\subsection{Merkmale der methodischen Empfehlungen}

Der Unterricht in „Deutsch als Zweitsprache“ stellt hohe Anforderungen an die methodische Kompetenz der Lehrkräfte. Die Steigerung der methodischen Kompetenz der Lehrkräfte ist also ein wichtiges durchgängiges Ziel der Qualifizierungsmaßnahmen. Bei der Arbeit an diesem Ziel sollte in Qualifizierungsveranstaltungen nicht so vorgegangen werden, dass methodische Verfahren nur vorgestellt und beschrieben bzw. im Rahmen eines kleinen abgeschlossenen Seminarteils 
kurz behandelt werden - sie sollten vielmehr bei der Vermittlung der Inhalte durchgängig praktiziert werden. Die Teilnehmer sollten also die methodischen Verfahren mit ihren Wirkungen und Ergebnissen selbst erleben können. Diese Erfahrung wird ergänzt durch die Reflexion der dabei erlebten Prozesse, Ergebnisse, Emotionen und Erfolge.

Die folgenden Methoden sollten also in Seminaren durchgängig integriert und angewendet werden:

- Teilnehmerzentrierung

- Erfahrungsaustausch

- Praxisorientierung

- Handlungsorientierung

- Induktives Vorgehen

- Exemplifizierung

- Medienunterstützung

- Visualisierung

- Spielen

- Methodische, mediale und soziale Vielfalt

- Offene Unterrichtsgestaltung

- Team-Teaching

- Reflektivität

Einzelheiten dazu finden sich in der Broschüre auf S. 20 - 24.

\section{Arbeiten mit der Konzeption}

\subsection{Regelungen}

Für die Durchführung von Seminarveranstaltungen bestehen nur wenige Regelungen, nämlich

- die Definition der Eingangsvoraussetzungen (s. Matrix des Bundesamtes unter „Zulassungskriterien für die Zusatzqualifizierung von Lehrkräften“)

- die Dauer der Maßnahme, abhängig von den Eingangsvoraussetzungen, nämlich

$>$ die unverkürzte Zusatzqualifizierung mit 140 UE Präsenzphase + mindestens 140 UE Eigentätigkeit

$>$ die verkürzte Zusatzqualifizierung mit 70 UE Präsenzphase + mindestens 70 UE Eigentätigkeit

(Echtes) Team-Teaching ist erwünscht, aber nicht Bedingung. Die Anzahl der Teamer bzw. Seminarleiter ist nicht festgelegt. Die Erfahrung zeigt jedoch, dass es aufgrund der Notwendigkeit intensiver Koordination nicht günstig ist, wenn zu viele Teamer im Einsatz sind. Da die Seminarteile aufeinander bezogen und inei- 
nander verzahnt sind, muss die jeweilige Seminarleitung wissen, was vorher „gelaufen ist", um ein Optimum an Teilnehmerzentriertheit zu erreichen. Grundsätzlich gilt: Je höher die Anzahl der Teamer bzw. Seminarleiter, desto aufwändiger wird das Koordinationsverfahren.

\subsection{Empfehlungen für die Vorbereitung von Qualifizierungsveranstaltungen}

Im Vorfeld von Seminarveranstaltungen auf Basis der Konzeption sind im Sinne der Qualitätssicherung etliche Aufgaben vorzunehmen, die nicht direkt aus der Konzeption zu entnehmen, die aber im Akkreditierungsleitfaden enthalten sind, der zusammen mit dem Auditleitfaden die Akkreditierung einer Institution für die DaZ-Lehrkräftequalifizierung regelt. Dabei hat der/die Verantwortliche für die DaZ-Lehrkräftequalifizierung folgende Aufgaben: Er/sie

- schafft geeignete Voraussetzungen für die Umsetzung der Konzeption

- trifft eine geeignete Auswahl der Qualifizierenden

- gewährleistet die Einführung der Qualifizierenden in die gesamte Konzeption und deren Umsetzung

- gewährleistet kontinuierliche Fortbildung der Qualifizierenden

- gewährleistet Supervision der Qualifizierenden

- stellt Beratung und Betreuung der TN sicher

- veranlasst eine differenzierte Ermittlung der Lernvoraussetzungen der TN sowie Auswertung und Umsetzung der Ergebnisse

- koordiniert die Qualifizierenden bzw. veranlasst die Koordinierung

- garantiert Informationsfluss und Rückmeldung zwischen den Qualifizierenden

- veranlasst eine regelmäßige Auswertung der einzelnen Seminarteile und die Umsetzung der Ergebnisse, soweit möglich

- kümmert sich um Rückmeldungen seitens der TN und veranlasst ggf. Konsequenzen

- sorgt für angemessene Seminarräume

- stellt geeignete Medienausstattung sicher

- stellt einen ausreichenden und für die TN zugänglichen Handapparat zur Verfügung.

\subsection{Vorgehen bei der Planung}

Die Planung sollte als Ganzes vorgenommen werden. Auf diese Weise lassen sich insbesondere die Lernziele von Bausteinen optimal platzieren, die mit anderen kombinierbar sind, wie z. B. Visualisierung o. ä. D. h., es lässt sich entscheiden, ob Lernziele integrierbarer Bausteine in andere Bausteine integriert oder als selbständiger Block umgesetzt werden. Bei der Planung eines einzelnen Bausteins sollte anhand einer differenzierten Ermittlung der Lernvoraussetzungen der TN ent- 
schieden werden, bei welchen Lernzielen eines Baustein begonnen werden muss, d. h. welche Lernziele ggf. bereits als erreicht vorausgesetzt werden können etc. Während des Seminars kontinuierlich vorgenommene Auswertungen müssen umgesetzt werden, d. h. die Ergebnisse müssen ggf. die Planung noch ausstehender Bausteine modifizieren.

\subsection{Vorgehen bei der Durchführung}

Genau so wichtig wie eine gemeinsame Planung ist eine möglichst weitgehende Abstimmung bei der Durchführung. Dazu gehört nicht nur die Umsetzung von Ergebnissen von Zwischenevaluationen in noch ausstehenden Seminarteilen, sondern auch Maßnahmen wie gemeinsame Durchführung oder Hospitationen, ggf. Supervision in den Seminarveranstaltungen von Kollegen, um methodisch und didaktisch ein möglichst hohes Niveau bei der Durchführung zu erreichen.

3.5 Das Portfolio als Nachweis einer erfolgreichen Qualifizierung seitens des Seminarteilnehmers

\section{a) Ziele des Portfolios}

Der zu Qualifizierende soll durch die Erstellung eines Portfolios nachweisen, inwieweit er mit dem Besuch einer Qualifizierungsveranstaltung das angestrebte Ziel, nämlich eine den Qualitätskriterien des BAMF entsprechende Qualifizierung, erreicht hat, also die notwendigen Kompetenzen erworben hat, um in vom BAMF geförderten Integrationskursen erfolgreich und effizient unterrichten zu können.

Das Portfolio weist die Form eines Präsentationsportfolios auf. Zur Erstellung eines solchen Qualifizierungsnachweises führen sowohl prozessorientierte Aktivitäten als auch ergebnisorientierte Leistungen. Der Aufbau des Portfolios soll Multidimensionalität verdeutlichen: Die Teilnehmer an der Qualifizierungsmaßnahme haben die Möglichkeit, ihre Erfahrungen und Kenntnisse auf verschiedenen Ebenen aufzuzeigen. Sie sollen die Fähigkeit zum Ausdruck bringen, die eigenen Entwicklungspotentiale als Lehrende und Lernende zu reflektieren und weiterzuentwickeln. Voraussetzung dafür ist, dass die eigenen Verhaltensmuster als Lehrkraft erkannt und das eigene Rollenverhalten sowie die eigenen methodischdidaktischen Fähigkeiten und Ressourcen reflektiert und ggf. erweitert werden.

Die Aktualisierung der eigenen Fachkompetenz in Bezug auf die spezifischen Anforderungen durch die heterogenen Zielgruppen der Zuwanderer soll in Teilen des Portfolios verdeutlicht werden: Dies schließt u. a. ein, dass in der ausschließlich schriftlichen Arbeit am Portfolio die Fähigkeit nachgewiesen wird, Lernziele und Lerninhalte unter Berücksichtigung der spezifischen Bedürfnisse der Sprachkursteilnehmer lebensnah und realistisch auszuwählen, zu definieren und progredient anzuordnen. Dies beinhaltet, dass die DaZ-Lehrkraft nach der Qualifizie- 
rung in der Lage ist, ihren Lernern in ihrem Unterricht durch methodische, mediale und soziale Vielfalt und eine soweit wie möglich offene Unterrichtsgestaltung:

- adressatengerechten und effizienten Zielsprachenerwerb

- interkulturelles Lernen

- selbstgesteuertes und differenziertes Lernen

- lebensnahes, lernerorientiertes Lernen

- ganzheitliches und handlungsorientiertes Lernen

- entdeckendes und reflektierendes Lernen

- die Nutzung von Fehlern als Lernanstoß

zu ermöglichen.

Darüber hinaus macht das Portfolio qualifizierenden Institutionen deutlich, inwieweit in der Qualifizierung das Verständnis von Unterricht der lernenden Lehrenden tatsächlich beeinflusst wurde. Damit dient es auch der Evaluation der Veranstaltungen zur Zusatzqualifizierung.

\section{b) Inhalte des Portfolios}

Der Qualifizierungsnachweis greift wesentliche Teile der Einzelanforderungen und die daraus resultierenden Ziele, Inhalte und Methoden der Zusatzqualifizierung auf und verlangt deren konkrete, praxisbezogene Umsetzung. Die Lernerträge aus den behandelten curricularen Bausteinen der „Konzeption..." (Broschüre S. 25 - 61) werden für die Erstellung des Portfolios vorausgesetzt. Es werden jedoch keine beschreibenden Texte im Sinne der Reproduktion von Wissen gefordert. Vielmehr machen die Lehrkräfte ihre in der Qualifizierung erworbenen Kompetenzen durch die Bearbeitung DaZ-spezifischer und transferbezogener Aufgaben transparent.

Das verlangte Portfolio besteht aus einer Sammlung verschiedener Arbeiten und Leistungen der Teilnehmer in der Qualifizierung. Dazu gehören:

- Reflexion des durch die Qualifizierung erworbenen Lernzuwachses, der Lernprozesse, der damit gemachten Lernerfahrungen, zukünftiger Professionalisierung usw.

- Transfer auf Unterrichtsebene: Unterrichtsentwurf, Lehrwerkanalyse

Konkret weist das Portfolio die folgenden Teile auf:

- Im einleitenden Teil geht es zunächst darum, Auskunft über einige persönliche Daten und den beruflichen Werdegang zu geben,

- der Teil „Ausgewählte Leistungen“ enthält die Bearbeitung von Aufgaben, die in direktem Zusammenhang stehen mit der Praxis des eigenen unterrichtlichen Handelns,

- der abschließende Teil bezieht sich auf die in der Zusatzqualifizierung gemachten Lernerfahrungen, die reflektiert werden.

Eine genaue Übersicht über das Portfolio und seine Teile würde den hier gesetzten Rahmen sprengen. 


\section{Die Konzeption und die Qualitätssicherung bei ihrer Durchführung}

Um zu gewährleisten, dass die Konzeption der DaZ-Lehrkräftequalifizierung zielführend umgesetzt wird, müssen Institutionen, die die DaZ-Lehrkräftequalifizierung anbieten wollen, ein Akkreditierungsverfahren durchlaufen. Dieses bezieht sich auf die Qualität der Institution, auf die Qualität ihres Qualifizierungsangebots und auf die Qualität der Durchführung des Angebots. Die Überprüfung Qualität der Durchführung des Angebots wird im Auditleitfaden geregelt. Der Auditleitfaden enthält:

- Ziel und Funktion des Audits

- Prinzipien bei der Auditierung

- die Zuständigkeit für die Auditierung

- die Qualifikationen der AuditorInnen

- Planung, Vorbereitung und Durchführung des Akkreditierungsaudits

- Auditberichterstattung

- Themen/curriculare Bausteine als Grundlage einer Hospitation

- Kriterien für die Bewertung der Institution

- Kriterien für die methodisch-didaktische Umsetzung des Qualifizierungsangebots

Letztere schließen den Kreis zu den didaktischen und methodischen Merkmalen von Qualifizierungsveranstaltungen auf der Basis der DaZ-Lehrkräftequalifizierungskonzeption des Bundesamtes.

\section{Literatur}

Konzept für einen bundesweiten Integrationskurs (08. 11. 05)

Konzeption für die Zusatzqualifizierung von Lehrkräften im Bereich Deutsch als Zweitsprache (13.02.06)

Zulassungskriterien für die Zusatzqualifizierung von Lehrkräften (16.05. 06)

Alle unter:

http://www.bamf.de/cln_043/nn_565180/DE/Integration/_Function/Do

wnload/Lehrkraefte/download-lehrkraefte-mehr-node.html_nnn=true 


\section{Lehrkräftequalifizierung für den Unterricht in In- tegrationskursen. Konzept des Landesverbandes der Volkshochschulen Niedersachsens e.V.}

Redaktionsteam der ZQDaZ

(Landesverband der Volkshochschulen Niedersachsens)

\section{Adressaten, Ziele, Lehrgangsstruktur}

Der im Folgenden beschriebene Lehrgang richtet sich an Lehrkräfte in Integrationskursen, die eine Zusatzqualifizierung nachweisen müssen.

Dabei sind zwei Lehrgangstypen zu unterscheiden: Die unverkürzte und die verkürzte Form. Die Zugangsvoraussetzungen zur Qualifizierung sind über die entsprechende Matrix des BAMF „Zulassungskriterien für die Zusatzqualifikation von Lehrkräften in Integrationskursen" geregelt (www.bamf.de).

Der im Folgenden beschriebene Lehrgang richtet sich an Kursleiter, die eine unverkürzte Zusatzqualifizierung nachweisen müssen. Zielsetzung und Inhalte entsprechen den Anforderungen der „Konzeption für die Zusatzqualifizierung von Lehrkräften im Bereich Deutsch als Zweitsprache“, die das Goethe-Institut 
im Auftrag des Bundesamtes für Migration und Flüchtlinge (BAMF) entwickelt hat. Der Deutschunterricht, den die teilnehmenden Kursleiter planen und durchführen sollen, zielt auf Kommunikationsfähigkeit ab und orientiert sich an der Lebenswirklichkeit der Zugewanderten, wobei der Erwerb von deutschen Sprachkenntnissen als wesentliche Grundlage der Integration verstanden wird.

Das Curriculum der Zusatzqualifikation besteht aus einer bestimmten Anzahl an Bausteinen, deren Ziele und Inhalte für die Qualifizierung unerlässlich sind (obligatorische curriculare Bausteine) und solchen, deren Ziele und Inhalte in Auswahl (Themen zur Wahl) - je nach Bedarf der Zielgruppe - in Qualifizierungsveranstaltungen umgesetzt werden können. Die obligatorischen Bausteine sollen die methodisch-didaktische Kernkompetenz für den Unterricht in unspezifischen Integrationskursen entwickeln, mit den fakultativen Bausteinen werden weitere Themenbereiche aufbereitet, die für den DaZ-Unterricht allgemein relevant sind (Konfliktmanagement im DaZ-Unterricht, Neue Medien im Unterricht) oder für den Unterricht mit bestimmten Adressatengruppen qualifizieren sollen (Alphabetisierung im DaZ-Unterricht, DaZ-Unterricht mit Jugendlichen, Berufsorientierender DaZ-Unterricht).

Der Lehrgang „Zusatzqualifikation DaZ (ZQDaZ)“ setzt die curricularen Vorgaben in ein didaktisches Konzept um, das den Erwerb von Lehrkompetenz nicht primär als Wissensvermittlung, sondern als subjektive (Re)konstruktion von Handlungswissen begreift: Die Lehrkräfte werden Schritt für Schritt an qualifiziertes Unterrichten herangeführt und erarbeiten sich dabei ihr Wissen im Grundsatz selbst. Der Lehrgang verbindet daher die theoretische Reflexion in Seminarform prozessorientiert mit praktischem Unterricht, Selbststudium, angeleitetem Erfahrungsaustausch im Kollegium und der Betreuung durch Tutoren. Die Ausbildung erstreckt sich über ungefähr sechs Monate. Lehrkräfte, die den Lehrgang erfolgreich durchlaufen, erhalten am Ende ein Zertifikat, das zur Erteilung von DaZ-Unterricht in vom BAMF geförderten Integrationskursen berechtigt. Nach fünf Jahren ist jährlich der Besuch geeigneter weiterer Fortbildungsmaßnahmen nachzuweisen.

Das Lehrgangsformat weist einen hohen Grad an Verbindlichkeit in der praktischen Durchführung aus: Lehrkräfte, die an dem ZQDaZ-Lehrgang teilnehmen möchten, verpflichten sich zu kontinuierlicher Teilnahme sowie zu den Leistungen und Beiträgen, die sich aus dem inhaltlichen Aufbau des Lehrgangs ergeben; der Kursträger verpflichtet sich parallel zu einem qualifizierten Tutoring.

\section{Lehrgangskonzept}

Anders als Einführungsseminare der üblichen Art, die meist als geschlossener Block am Anfang einer Lehrtätigkeit stehen, folgt der ZQDaZ-Lehrgang einem prozessorientierten Ansatz, der nicht auf Beispielen, Instruktion und Vermittlung, sondern primär auf erfahrungsgeleitetem Lernen beruht: Theorie und Praxis fallen nicht in separate Blöcke auseinander, sondern Aufgabenverständnis und Hand- 
lungskompetenz entwickeln sich schrittweise in der Wechselwirkung von praktischem Handeln, angeleiteter Reflexion und kontinuierlicher Begleitung und Supervision.

Theoretische Grundlage ist ein handlungstheoretisch-konstruktivistisches Modell des Lehrens. Die Aufgabe des Lehrenden in der Weiterbildung liegt darin, von einem bestimmten Ausgangszustand (Modul 2) unter kontrollierten, nicht-zufälligen Bedingungen mit seiner Lerngruppe zu bestimmten Zielen zu gelangen (Modul 3), indem er unter Berücksichtigung der vorgegebenen Lehrpläne und Materialien (Modul 4) und der Lernvoraussetzungen seiner Zielgruppe (Modul 5) geeignete, erwachsenengemäße Lernanordnungen auswählt und unterrichtspraktisch umsetzt (Modul 6, 7 und 8). Dabei überzeugt er sich kontinuierlich davon, dass die ausgewählten Lernanordnungen erfolgreich im Sinne der Lehrziele sind und den Bedürfnissen seiner Lerngruppe entsprechen (Modul 9). Die Lehrgangsteilnehmer sollen verstehen, dass man Sprachkenntnisse nicht nach einem festen, prozessunabhängigen Schema „vermitteln“ kann, sondern dass es immer neu die Aufgabe des Lehrenden ist, flexibel und phantasievoll die unter den jeweils gegebenen Voraussetzungen passenden Lernanordnungen (aktiv-konstruktiv) zu finden und unterrichtspraktisch umzusetzen (Vielau 2001, 10).

Fünf Bestandteile kennzeichnen den Lehrgang:

\section{$2.1 \quad$ Unterrichtspraxis}

Spätestens mit Lehrgangsbeginn übernimmt der Kursleiter eigenverantwortlichen DaZ-Unterricht, möglichst im Bereich der Grundstufe (Europäischer Referenzrahmen, Niveaustufe A1 oder A2). Dazu gehört die Planung, Vorbereitung, Durchführung und Evaluation des Unterrichts unter Anleitung des Tutors und in kollegialer Kooperation.

\subsection{Seminarbesuch}

Das Konzept sieht für den unverkürzten Lehrgang die Beteiligung an 9 Pflichtmodulen mit insgesamt 120 Unterrichtsstunden vor. Die Module greifen jeweils bestimmte handlungspraktisch wichtige Aspekte aus der laufenden Lehrtätigkeit heraus und bringen sie in einen theoretischen Bezugsrahmen. Dieser gemeinsam erarbeitete Bezugsrahmen soll als „Expertentheorie“ und reflektiertes Aufgabenverständnis nach und nach die subjektiven (häufig nur erfahrungs- und traditionsgeprägten) Unterrichtstheorien, auf deren Basis die Lehrkraft zunächst ihre Aufgaben wahrnimmt, überlagern und nötigenfalls ersetzen. Die Module decken alle Inhalte der in den Standards aufgeführten obligatorischen curricularen Bausteine ab. 


\subsection{Die Pflichtmodule:}

Modul 1: Orientierungsseminar

- Eine erste Kursstunde in einer langue inconnue. Selbsterfahrung und methodische Analyse

- Dynamik von Lernprozessen

- Innerer und äußerer Lehrplan

- Auf dem Weg zum autonomen Lerner

- Prinzip des entdeckenden Lernens

Modul 2: Migration und Integration - Ausgangsbedingungen von DaZ-Unterricht

- Migration und Migranten

- Merkmale des DaZ-Unterrichts

- Kursteilnehmerberatung

- Interkulturelles Lernen

- Unterrichtsbeobachtung und -beurteilung im Rahmen der angebotenen Hospitation

Modul 3: Lernziele

- Leitziel Kommunikationsfähigkeit

- Interkulturelle Kommunikation

- Exkurs zur Kommunikationstheorie

- Erweiterter Begriff von Kommunikationsfähigkeit: Diskursanalyse und Szenarienansatz

- Lernzielbeschreibung des GERR

- Umsetzung in neueren Lehrwerken

- Methodische Ansätze im DaZ-Unterricht

Modul 4: Lernwege und Kursplanung

- Heterogenität und Binnendifferenzierung

- Planen, Vorbereiten und Erteilen von DaZ-Unterricht

- Exkurs: Übungstypologien, Sozialformen und Arbeitsanweisungen

- Exkurs: Projektarbeit im DaZ-Unterricht

Modul 5: Spracherwerb, Unterrichtskonzept, Lehrplanung

- Faktoren des Spracherwerbs

- Zweitspracherwerb im Land der Zielsprache

- Bausteine für ein Unterrichtskonzept DaZ

- Unterrichtsplanung: Ein praktisches Beispiel 
Modul 6: Vorkommunikative Fertigkeiten

- Phonetik

- Wortschatz

- Grammatik

Modul 7: Rezeptive Fertigkeiten

- Hörverstehen

- Leseverstehen

Modul 8: Produktive Fertigkeiten

- Mündliche Kommunikation im DaZ-Unterricht

- Schriftliche Kommunikation im DaZ-Unterricht

Anm.: Die Methodenseminare 6, 7 und 8 werden ergänzt durch zwei Exkurse, die aus organisatorischen Gründen den Anfang von Modul 9 bilden.

Modul 9: Febler, Testen und Prïfen, Evaluation

- Exkurs: Spiele

- Exkurs: Visualisierung

- Fehler und Fehlerkorrektur

- Testen und Prüfen im DaZ-Unterricht

- Unterrichtsmaterialanalyse und -beurteilung (prüfungsbezogen)

- Evaluation von DaZ-Unterricht

Inhalt und Reihenfolge der Pflichtmodule sind verbindlich, da sich die Themen inhaltlich ergänzen und oft auch wechselseitig voraussetzen.

Zusätzlich zu den Pflichtmodulen belegt der Kursleiter nach eigenem Ermessen, aber in einem festgesetzten Stundenumfang (wenigstens 20 Unterrichtsstunden) die in der Konzeption des BAMF aufgeführten Wahlmodule zu den Themenbereichen Konfliktmanagement im DaZ-Unterricht, Berufsorientierender DaZ-Unterricht, Alphabetisierung im DaZ-Unterricht, DaZ-Unterricht mit Jugendlichen, Neue Medien im DaZ-Unterricht.

\subsection{Selbststudium}

Die einzelnen Lehrgangsmodule sind mit jeweils acht Unterrichtsstunden (Tagesseminar) bzw. sechzehn Unterrichtsstunden (Wochenendseminar) sehr kompakt gehalten. Im Allgemeinen wird es daher kaum möglich sein, die umfangreichen Wissensbestände, die in den einzelnen Modulen thematisiert werden, schon während der Seminarveranstaltungen zu erarbeiten. Der Seminarbesuch muss daher durch ein vertiefendes, Zusammenhang stiftendes Selbststudium ergänzt werden. Entsprechend sieht jedes der Module bestimmte Literaturverweise und Aufgaben- 
stellungen für das Selbststudium vor. Einheitliche Bezugsliteratur und jedem Seminarteilnehmer zur Verfügung stehend ist Axel Vielaus „Handbuch des Fremdsprachenunterrichts an Volkshochschulen“, das vom Landesverband der Volkshochschulen Niedersachsens herausgegeben wurde. Jedes Kapitel enthält Angaben zu weiterführender und vertiefender Literatur.

\subsection{Hospitation und Konferenzen}

Das Konzept sieht die kontinuierliche Betreuung des Lehrgangsteilnehmers durch einen örtlichen Tutor vor, der ihm als Ansprechpartner und kollegialer Berater zur Seite steht. Verpflichtende Bestandteile der Ausbildung sind die Beratungshospitation (d. h. Teilnahme an einer Lernberatung), mindestens eine gegenseitige kollegiale Hospitation und eine Fremd-Hospitation durch den Tutor im eigenen Kurs, alle mit entsprechender Dokumentation und anschließenden Auswertungsgesprächen.

\subsection{Dokumentation und Zertifizierung}

Eindrücke und Erfahrungen sind flüchtig, werden rasch durch Neues überlagert oder verdrängt. Man kann sie oft schon nach kurzer Zeit nicht mehr sinnvoll besprechen oder auswerten, wenn sie nicht in bestimmter Weise schriftlich fixiert werden. Jeder Lehrgangsteilnehmer ist daher verpflichtet, einen kurs- und seminarbezogenenen Lehrgangsordner zu führen. Neben Angaben zu seinem Kurskonzept, dem Stoffverteilungsplan, seiner Unterrichtsplanung, -durchführung und -auswertung enthält der Lehrgangsordner die Seminarunterlagen und -nachweise und das Lernprotokoll, in dem der Lehrgangsteilnehmer seine eigenen Lernergebnisse dokumentiert und analysiert.

Dazu erhält er vom Kursträger jeweils nach örtlichem Muster (örtliches QMSystem) die entsprechenden Unterlagen und Formblätter, die er zur laufenden Dokumentation seines Unterrichts benötigt.

Diese Dokumentation im Lehrgangsordner, die vom Tutor auf Vollständigkeit und Angemessenheit überprüft, mit einem fachlichen Kommentar versehen und abgezeichnet wird, ist also die Basis der Zertifizierung.

Nach Abschluss des Lehrgangs muss darüber hinaus ein Portfolio mit schriftlichen Ausarbeitungen nach Vorgaben des Bundesamtes vorgelegt werden. Gefordert ist dafür die Feinplanung einer Unterrichtseinheit, eine Analyse von Unterrichtsmaterial, wahlweise unter dem Aspekt Heterogenität und Binnendifferenzierung, Interkulturelles Lernen oder Handlungsorientiertes Lernen, sowie eine Selbsteinschätzung bezüglich der Lernerfahrungen während der Qualifizierungsmaßnahme, die durch ein Formblatt vorstrukturiert ist.

Das Portfolio wird vom Maßnahmeträger nach vorgegebenen Kriterien des BAMF bewertet. Der Träger meldet dem BAMF die Teilnehmer, für die das Zerti- 
fikat ausgestellt werden soll. Die Portfolios werden beim Maßnahmeträger archiviert und können jederzeit vom BAMF eingesehen werden.

\section{Seminarleitung und Arbeitsformen}

Die einzelnen Lehrgangsmodule sind so weit inhaltlich vorstrukturiert und materialmäßig aufbereitet, dass sie nach entsprechender Schulung von den Seminarleitern in die Praxis umgesetzt werden können. Jedes Modul beginnt mit einer Inhaltsübersicht. Dann folgt der detaillierte Seminarplan mit Zeitverteilung, Lernzielen, wichtigen Arbeitsschritten und didaktisch-methodischem Kommentar. Außerdem enthält es im Anhang alle erforderlichen Arbeitsblätter, Folien und Texte sowie Hinweise zum Selbststudium nebst Literaturangaben.

Natürlich können die Seminarleiter bei der praktischen Umsetzung, d. h. bei Arbeitsformen oder einzelnen Übungen, auch eigene Wege gehen. Verbindlich für jedes Modul bleibt aber auf jeden Fall der Lernzielkatalog, damit aus Sicht der Teilnehmer die inhaltliche Kohärenz, die innere Logik im Ablauf der Lehrgangsmodule gewahrt bleibt.

Die Zusatzqualifikation DaZ versteht sich als ein flexibel lernendes System, in dem die vorgeschlagenen Arbeitsformen immer wieder getestet und kontinuierlich verbessert werden müssen. Eine Arbeitsgruppe (Redaktionsteam) evaluiert fortlaufend die Erfahrungen, und auch für die Seminarleiter und Tutoren sind spezielle Schulungen und Möglichkeiten zum Erfahrungsaustausch vorgesehen. Die Materialien werden in der Form einer Sammlung loser Blätter zur Verfügung gestellt; praktische Erfahrungen aus der Durchführung können auf diese Weise jederzeit in das Material einfließen.

\section{Literatur:}

Vielau, Axel: Handbuch des Fremdsprachenunterrichts an Volkshochschulen, Hannover: Landesverband der Volkshochschulen Niedersachsens 2001

ders.: Methodik des kommunikativen Fremdsprachenunterrichts, Berlin:

Cornelsen 1997 



\section{Zur Rolle von Alphabetisierung und Nullanfängerun- terricht in der Ausbildung von DaF-Lehrpersonen}

Sabine Jentges, Marburg

\section{$1 \quad$ Einleitung}

Die im vergangenen Jahr in Verantwortung des Bundesamtes für Migration und Flüchtlinge (BAMF) bundesweit angelaufenen Integrationskurse, die neben der Vermittlung sprachpraktischen Wissens auch landeskundlich ausgerichtete Orientierungskurse einbeziehen, finden inzwischen in der universitären und außeruniversitären Aus- und Weiterbildung von Lehrpersonen für Deutsch als Fremdsprache (DaF) bzw. Deutsch als Zweitsprache (DaZ) Berücksichtigung. Auch Lehrbuchverlage bringen entsprechende Kursbücher und Materialien auf den Markt.

Nullanfängerunterricht und vor allem Alphabetisierung ${ }^{1}$, die ebenfalls Bestandteil der Integrationskurse sind, werden hingegen weiterhin als „Stiefkinder“ behandelt. In kaum einer Fortbildung oder universitären DaF-/DaZ-Lehreraus-

\footnotetext{
1 Wenn hier von Alphabetisierung die Rede ist, ist immer die Alphabetisierung in DaZ gemeint, geht es um die Alphabetisierung in der Muttersprache Deutsch, ist dies ausdrücklich vermerkt.
} 
bildung werden diese Aspekte berücksichtigt. Insbesondere für den Bereich der Alphabetisierung existieren nur wenig kommerzielle Materialien. Dieser Beitrag möchte sich daher auf den Bereich der Alphabetisierung in DaZ konzentrieren und eine erste Annäherung an deren Rolle in der Ausbildung von DaF-/DaZLehrpersonen versuchen.

\section{Nullanfängerunterricht im Bereich DaF/DaZ}

Im Bereich des Nullanfängerunterrichts $\mathrm{DaF} / \mathrm{DaZ}$ gibt es Dutzende unterschiedliche Lernsituationen, -motivationen, -voraussetzungen usw., die zum Besuch eines solchen Kurses führen können.

Um nur ein paar Beispiele zu nennen: $\mathrm{Da}$ ist der amerikanische Doktorand der Philosophie, der Deutsch lernen will, um auch nicht ins Englische übersetzte Texte, z. B. von Nietzsche und Schopenhauer, verstehen und ggf. auch bereits übersetzte im Original lesen zu können.

$\mathrm{Da}$ ist die japanische Opernsängerin, die endlich einmal den Sinn dessen, was sie beispielsweise bei der Zauberflöte zu singen hat, verstehen möchte. Und da ist z. B. die in Deutschland lebende, aus der Türkei stammende Hausfrau und Mutter, die wenig Deutsch spricht, etwas versteht und nicht lesen und schreiben kann und jetzt, z. B. weil sie nach dem Zuwanderungsgesetz dazu verpflichtet ist, beginnt (bzw. beginnen muss) in einer gesteuerten Situation Deutsch zu lernen².

Diese drei Beispiele zeigen Personen, die zum ersten Mal in einer gesteuerten Sprachlernsituation mit Deutsch konfrontiert werden, wobei sowohl die Japanerin als auch die Türkin bereits (zwar unterschiedliche) Vorerfahrungen mit der deutschen Sprache gemacht haben. Wahrscheinlich verfügt die Japanerin darüber hinaus bereits über Erfahrungen im gesteuerten Fremdsprachenerwerb und somit auch über sprachliche Strukturen und systematisches Lernen, nämlich zumindest mit Englisch, vielleicht auch der Amerikaner. In lateinischer Schrift alphabetisiert ist der amerikanische Doktorand und aller Erwartung nach auch die Japanerin, beide über Englisch.

\footnotetext{
2 Das Beispiel der türkischen Hausfrau wurde insofern als exemplarisches gewählt, da in Deutschland stattfindende Alphabetisierungskurse für Nicht-Muttersprachler, soweit überhaupt Angaben über die Teilnehmer existieren, von deutlich mehr Frauen als Männern besucht werden: In den Jahren 1986 bis 1994 wurden die Alphabetisierungskurse des Sprachverbandes zu mindestens 79,9\% bis maximal 90,7 \% von Frauen besucht (vgl. Schramm 1996: 14). Für diese Kurse und diesen Zeitraum liegt darüber hinaus die Angabe vor, dass etwa $70 \%$ der Kursteilnehmer Hausfrauen waren (vgl. ebd.). Die aktuellen Zahlen des BAMF für Teilnehmer an Integrationskursen mit Alphabetisierung im Jahre 2005 gehen ebenfalls - wenn auch weniger deutlich - in diese Richtung. Hier gab es 68,4 \% Teilnehmerinnen (und somit auch hier in den Alphabetisierungskursen deutlich mehr Frauen als allgemein in Integrationskursen, dort gab es 59,7\% Teilnehmerinnen, vgl. www.bamf.de). Ob diese Zahlen aber wirklich bestätigen, dass die nicht-muttersprachlichen Analphabeten in Deutschland überwiegend weiblich sind, kann hieraus nicht geschlussfolgert werden.
} 
Diese drei gemeinsam in ein- und demselben Kurs zu unterrichten, darüber dürfte Einigkeit bestehen, ist wenig sinnvoll.

Der hier nur angerissene teilweise problematische Bereich von sehr unterschiedlichen Lernmotivationen, -vorerfahrungen und bereits vorhandenem Wissen, das sich nicht nur konkret auf (minimale) Kenntnisse der zu lernenden Sprache in dem einen oder anderen Bereich bezieht, ist sicherlich jeder DaF-/DaZLehrkraft bewusst und besonders in Anfängerkursen werden wir häufig damit konfrontiert. „Den“ Anfängerunterricht gibt es also nicht und im Idealfall bräuchte jeder der drei genannten Lernenden einen völlig anders konzipierten Kurs, der i.d.R. auch gefunden werden kann.

Allerdings wird die Türkin in dem Alphabetisierungskurs, den man ihr empfehlen würde, im Vergleich zu den beiden anderen (fast überall in Deutschland) auf die heterogenste Teilnehmergruppe treffen.

Was aber bedeutet Analphabetismus? Ist dies im heutigen Deutschland überhaupt noch ein Thema? Und wer sind die Betroffenen?

\section{Analphabetismus}

Zu Beginn seines Mercury, or the Secret and Swift Messenger (1641) erzählt John Wilkins:

Es gibt [...] eine schöne Geschichte über einen indischen Sklaven, den sein Herr mit einem Korb Feigen und einem Brief losschickte und der auf dem Weg einen großen Teil dieser Feigen verspeiste und den Rest der Person übergab, für die sie bestimmt waren; diese beschuldigte, als sie den Brief empfing und nicht die darin angegebene Menge Feigen fand, den Sklaven, sie gegessen zu haben, und teilte ihm mit, was der Brief gegen ihn ausgesagt hatte. Aber der Inder leugnete (trotz des Beweises) rundheraus die Tatsache und beschimpfte den Brief als falschen und lügenhaften Zeugen. Als er später wieder mit einer gleichartigen Last losgeschickt wurde und mit einem Brief, in dem stand, wie viele Feigen er abliefern sollte, aß er wiederum, wie beim erstenmal, auf dem Weg einen großen Teil der Feigen. Doch bevor er sie anrührte, nahm er (um jede mögliche Anklage zu verhindern) den Brief, versteckte ihn unter einem Stein und beruhigte sich mit dem Gedanken, dass dieser, wenn er ihn beim Essen der Feigen nicht gesehen habe, auch nichts davon erzählen könne; als er sich aber dieses Mal noch härter als zuvor angeklagt sah, gestand er seine Schuld und versprach für die Zukunft die größte Zuverlässigkeit bei jedem Auftrag. (Wilkins 1717: 3f, zitiert bei Eco 1992: 11).

Diese aus dem 17. Jahrhundert stammende Anekdote dürfte den meisten von uns heute unvorstellbar sein, Schrift ist fester Bestandteil unseres täglichen Le- 
bens; dass das Konzept Schrift einem Menschen in unserer heutigen modernen Gesellschaft nicht bekannt sein könnte, wirkt auf uns nahezu unbegreiflich. Aber auch die Vorstellung, dass eine in unserer Gesellschaft lebende Person zwar das Konzept Schrift kennt, aber selbst nicht oder nur den Ansprüchen der Umgebung unzureichend lesen und schreiben kann, erstaunt die meisten, die der Schrift produktiv und rezeptiv mächtig sind, wie folgende Teilnehmerin der Fachtagung des Bundesverbandes Alphabetisierung e.V. 2003 berichtet. Sie beschreibt zunächst ihre Gedanken, bevor sie mit dem Thema Analphabetismus konfrontiert wurde:

Analphabetismus? Hier bei uns im ,zivilisierten und ach so fortschrittlichen“ Deutschland? „Niemals ...“, dachte ich. Bis ich eines besseren belehrt wurde und das oft so totgeschwiegene Thema Einzug hielt in meine kleine heile Azubi-Welt. Wenn man selbst des Lesens und Schreibens mächtig ist und noch dazu in einem Unternehmen ausgebildet wird, das mehr als jedes andere von Lesekundigen lebt, einem Verlag nämlich, geht man meist mit der irrigen und arroganten Annahme durch die Welt, der Rest der Menschheit müsse über dieselben Fähigkeiten verfügen wie man selbst. (Bühler 2004: 206)

Dass aber die Fähigkeit, Lesen und Schreiben zu können, auch bzw. selbst in unserem ,zivilisierten und ach so fortschrittlichen“ Deutschland keineswegs eine Fähigkeit ist, die von allen in Deutschland lebenden Personen beherrscht wird, zeigen die folgenden Zahlen.

Allein in der Bundesrepublik Deutschland nehmen jährlich 20.000 Menschen an den Alphabetisierungskursen der Volkshochschulen teil, und das ist nur die Spitze des Eisbergs, denn es fällt nach wie vor vielen Menschen sehr schwer, sich zu ihrer Lese- und Schreibschwäche zu bekennen und von sich aus den ersten und wichtigsten Schritt zu tun, nämlich sich helfen zu lassen.

Neueste Zahlen gehen von mehr als vier Millionen funktionalen Analphabeten in Deutschland aus [...] und von 10 Millionen [ $=12,5 \%$ (sj)] Menschen in unserem Land wird angenommen, dass sie nur auf niedrigstem Niveau lesen und schreiben können - trotz einer allgemeinen Schulpflicht von neun Jahren! (Spotka 2004: 10)

Die hier genannten, nur geschätzten Zahlen beziehen sich ganz allgemein auf Analphabetismus in Deutschland, unabhängig davon, ob es sich um Muttersprachler handelt, die trotz einer deutschen Schullaufbahn betroffen sind, oder um in Deutschland lebende Migranten. Inwiefern im Bereich DaF/DaZ Alphabetisierung eine Rolle spielt und was in diesem Bereich unter Alphabetisierung fällt bzw. verstanden werden kann und wie unterschiedlich die Erscheinungsformen sind, wird im Folgenden dargestellt. 


\subsection{Erscheinungsformen von Analphabetismus}

Man unterscheidet generell, unabhängig von Alphabetisierung in Mutter-, Fremdoder Zweitsprache zwischen primären Analphabeten, funktionalen sowie sekundären Analphabeten.

Unter primären Analphabeten (auch als natürliche Analphabeten bezeichnet) versteht man Menschen, die nie eine Schule besucht haben und daher keinerlei Lese- und Schreibkenntnisse haben, also in überhaupt keiner Form jemals lesen oder schreiben gelernt haben. Dieses Phänomen findet man in Deutschland fast ausschließlich im DaZ-Bereich, also bei Migranten, die keinerlei schulische Bildung in ihren Heimatländern genießen konnten.

Von funktionalem Analphabetismus spricht man, wenn Menschen zwar eine Schule besucht haben, aber dennoch nicht in der Lage sind, sich schriftlich so mitzuteilen bzw. Schriftliches so zu rezipieren, wie es für das Leben in der sie umgebenden Gesellschaft erforderlich wäre. Dies kann aus unterschiedlichsten Gründen eintreten. Für den DaZ-Bereich könnte dies z. B. bei Personen der Fall sein, die in ihren Heimatländern nur eine sehr rudimentäre Schulausbildung durchlaufen haben und dadurch nicht ausreichend in lateinischer Schrift alphabetisiert wurden, oder bei Kindern von Migranten, die trotz Schulbesuch nicht über ausreichende Schriftsprachkenntnisse verfügen (vgl. auch Schramm 1996: 8).

Menschen, die nicht ausreichend alphabetisiert wurden und z. B. durch Unsicherheiten oder Ängste Lese- und Schreibsituationen gemieden haben und dadurch schließlich ihre schriftsprachlichen Kenntnisse verlernen, werden hingegen als sekundäre Analphabeten bezeichnet (vgl. Schramm 1996: 7). Auch dies kann bei Personen mit DaZ-Hintergrund der Fall sein, z. B. in dem Sinne, dass sie Lese- und Schreibkompetenz in ihrer Muttersprache verlieren, diese also sozusagen verlernen, da sie keine Gelegenheit haben oder diese nicht suchen, mit der Schrift der Muttersprache konfrontiert zu werden.

Alle drei dieser genannten Formen von Analphabetismus können folglich in einem Alphabetisierungskurs in DaZ vorkommen, darüber hinaus gibt es noch eine vierte Gruppe, die ausschließlich im DaZ-Bereich existiert. Sie wird häufig als „Umalphabetisierung" bezeichnet; gemeint sind nämlich Menschen, die in einem nicht-lateinischen Schriftsystem alphabetisiert wurden und ein solches auch nicht über Fremdsprachenerwerb gelernt haben (vgl. Feldmeier 2004: 108). Hier kann man nicht von Analphabeten sprechen, dennoch muss in das lateinische Schriftsystem eingeführt werden, genau wie in lateinischer Schrift Alphabetisierte, wollen sie z. B. Arabisch, Kyrillisch usw. lernen, eine neue Schrift erwerben müssen oder Zeichen für die Schriftsprachen des Chinesischen oder Japanischen, also Kanjis, z. B.

\subsection{Aspekte in der Alphabetisierung in DaZ}

Die Realität für Lehrende und Lernende in einem solchen Alphabetisierungskurs ist in aller Regel durch Heterogenität, nicht nur basierend auf unterschiedlichen 
Erscheinungsformen von Analphabetismus der Teilnehmer ${ }^{3}$, geprägt. Auf weitere Aspekte wird im Folgenden eingegangen.

\subsubsection{Herkunftsländer und Muttersprache}

Zunächst gibt es meist keine Kurse speziell für Teilnehmer einer Nationalität bzw. einer bestimmten Ausgangs- also Muttersprache. Es ist üblich, dass Teilnehmer mit unterschiedlichsten Muttersprachen in einem Kurs sitzen. Die Möglichkeit im Unterricht also auf die Muttersprache der Teilnehmer oder eine Mittlersprache zurückzugreifen, ist nicht gegeben. Aus den unterschiedlichen Muttersprachen resultiert, ebenfalls auch für Kurse ohne Alphabetisierung, aber im Falle der Alphabetisierung noch viel stärker, das Problem des Einbezugs von typischen Ausspracheschwierigkeiten, die auf die Muttersprache der Teilnehmer zurückzuführen sind, da bei der Alphabetisierung zunächst eine Laut-Buchstaben-Zuordnung (und NICHT Buchstaben und Name des Buchstaben) geschehen muss ${ }^{4}$. Teilnehmer, deren Muttersprachen bestimmte im Deutschen existierende Laute nicht realisieren, müssen hier - im Gegensatz zu einer Alphabetisierung für deutsche Muttersprachler - besonders trainiert werden ${ }^{5}$. Nur dazu müsste sich der Kursleiter ein irgendwann nach längerer Tätigkeit sich vielleicht aus Erfahrung entwickelndes aber zunächst doch sehr vielfältiges Wissen über eine ganze Reihe von Sprachen aneignen. Um hier in der Alphabetisierung Lehrende zu entlasten bzw. besser vorbereiten zu können, wäre es daher von größter Wichtigkeit, gesicherte Angaben über die Herkunftsländer bzw. Muttersprachen der Teilnehmer solcher Kurse, also der Betroffenen zu haben, so dass über Sprachvergleiche gezielt mögliche Schwierigkeiten der Teilnehmer in diesem Bereich prognostiziert werden könnten. Gesicherte Zahlen über die Herkunftsländer der Betroffenen bzw. Kursteilnehmer liegen allerdings nicht vor. Schramm legt basierend auf die Analphabetenrate der jeweiligen Herkunftsländer der in Deutschland lebenden Migranten für das Jahr 1990 eine Schätzung vor, nach der die mit Abstand größte Gruppe der Analphabeten mit DaZ-Hintergrund aus der Türkei käme (327.067), gefolgt von Personen aus dem ehemaligen Jugoslawien (48.376), dem Iran (42.397), Marokko (35.146), Griechenland (21.772) und Afghanistan (21.594) (vgl. Schramm 1996: 4f). Schramm selbst gibt jedoch zu bedenken, dass diese Zahlen eine nur sehr unge-

\footnotetext{
${ }^{3}$ Anne Roder beschreibt die Teilnehmer ihrer Alphabetisierungskurse in DaZ in Frankfurt a.M. z. B. wie folgt: „Es gibt TN, die in ihrer Heimat die Schule besucht haben und in ihrer Sprache zu schreiben gelernt haben. Sie nutzen diese Kenntnisse teilweise, um sich neu gelernte deutsche Wörter zu übersetzen. Manche sind in der Lage, ein Wörterbuch zu benutzen. Andere haben noch nie eine Schule besucht und sind primäre Analphabeten" (Roder 2006: 6).

${ }^{4}$ Zumindest wenn man davon ausgeht, bei der Alphabetisierung von Migranten auf unterster Lernstufe anzusetzen, also vom Leichten zum Schweren zu gelangen, folglich mit Buchstaben als kleinste Einheit der Schrift und Lauten als kleinste des Gesprochenen.

${ }^{5} \mathrm{Je}$ nach Muttersprache und vor allem abhängig von der Vorbildung kann das sogar bedeuten, dass erst einmal gelernt werden muss, was ein einzelner Laut ist, wie klein ein solcher Einzellaut ist und wie dieser nun aus einer Menge anderer Laute heraus erkannt werden kann.
} 
fähre Annäherung an die Realität sein können ${ }^{6}$. Feldmeier hält die besagten Daten vom Sprachverband ebenfalls nicht für repräsentativ, da bei den Kursen „ausschließlich Teilnehmer aus EU-Mitgliedsländern, aus den ehemaligen Anwerbeländern Türkei, dem früheren Jugoslawien, Marokko und Tunesien sowie ehemalige DDR-Vertragsarbeitnehmer aus Angola, Mosambik und Vietnam gefördert wurden“ (Feldmeier 2004: 104). Feldmeier selbst - sich auf die eigene Unterrichtserfahrung beziehend - gibt für die letzten zwei bis drei Jahre (als persönliche Einschätzung) an, dass „die Kurden aus dem Irak zusammen mit den Kurden aus der Türkei die größte Analphabetengruppe dar[stellt]; thailändische Frauen folgen an dritter Stelle. Auch Marokkaner fanden oft den Weg in den Alphabetisierungskurs. Vereinzelt nahmen Griechen, Chinesen, Litauer, Roma und Menschen aus weiteren Ländern am Unterricht teil"“ (Feldmeier 2004: 104). Anne Roder nennt als Herkunftsländer ihrer Teilnehmer vor allem Afghanistan, Marokko, Eritrea, Äthiopien und die Türkei, als weitere Herkunftsländer u.a. auch Thailand, Vietnam und Indien (vgl. Roder 2006: 5).

Für die ,neuen“ Integrationskurse mit Alphabetisierung liegen bisher keine gesonderten Zahlen nach Herkunftsländern bzw. Muttersprachen vor ${ }^{7}$.

\subsubsection{Mündliche und schriftliche Vorkenntnisse}

Auch die Kenntnisse der Teilnehmer im Bereich der deutschen Sprache sind sowohl im mündlichen als auch schriftlichen Gebrauch sehr unterschiedlich, was eine extreme Herausforderung für Kursleiter bedeutet. Für den mündlichen Bereich reicht das Spektrum von absoluten Nullanfängern bis zu Teilnehmern, die seit Jahren oder Jahrzehnten in Deutschland sind und - natürlich mit strukturellen Schwächen - fließend sprechen können, also kaum oder nur geringe Schwierigkeiten in kommunikativen Situationen haben ${ }^{8}$. Anne Roder schreibt beispielsweise über ihre Kursteilnehmer:

\footnotetext{
${ }^{6}$ Zum einen wurden nur alphabetisierte Ausländer als Gastarbeiter angeworben, zum anderen sind durch Familienzusammenführungen auch nicht alphabetisierte Familienangehörige eingereist. Bei politischen Flüchtlingen ist „auf Grund der erhöhten Fluchtbereitschaft für Angehörige gehobener Bildungsschichten“ (Schramm 1996: 7) ein niedrigerer Prozentsatz von Analphabeten als in den jeweiligen Herkunftsländern zu erwarten (vgl. Schramm 1996: 7 sowie Feldmeier 2004: 104).

${ }_{7}$ Die Angaben der Kursteilnehmer aller Integrationskurse (ohne Spätaussiedler) werden vom BAMF für das Jahr 2005 wie folgt angegeben: 20,8 \% Türkei, 17,7\% Russische Föderation, 8,3\% Ukraine, 7,3 \% Kasachstan, 2,9 \% Polen, 2,7 \% Irak, je 2,0 \% Thailand und Serben (Serbien und Montenegro) sowie 4,1 \% Unbekannte und 32,6 \% Sonstige (vgl. www.bamf.de ).

${ }^{8}$ Diese Teilnehmer werden häufig an Alphabetisierungskurse für Muttersprachler verwiesen. Dies ist jedoch aus mehreren Gründen problematisch. „Die meisten von ihnen unterscheiden sich [...] nicht nur hinsichtlich ihrer Muttersprache von deutschen funktionalen Analphabeten, sondern auch aufgrund ihrer anderen Lernbiografie. Im Gegensatz zu den meisten deutschen Analphabeten hatten viele Ausländer aus ganz unterschiedlichen Gründen noch keine Gelegenheit an Lese- und Schreibunterricht teilzunehmen und empfinden ihr Analphabetentum daher nicht als selbstverschuldet" (Schramm 1995: 102). Darüber hinaus gibt es auch Teilnehmer, die die Schrift ihrer Muttersprache gelernt haben, aber eben nicht das lateinische Alphabet. Und schließlich finden die bereits genannten, aus der jeweiligen Muttersprache resultierenden Ausspracheschwierigkeiten, die bei NichtMuttersprachlern häufig zu Problemen bei der Graphem-Phonem-Zuordnung führen, in Kursen für
} 
Ein Großteil der TN lebt schon seit vielen Jahren in Deutschland, oftmals sind ihre Kinder schon hier eingeschult worden und sprechen Deutsch als oder wie eine zweite Muttersprache. Diese TN haben ungesteuert Deutsch gelernt, und verfügen über ausreichende mündliche Deutschkenntnisse, so dass sie die meisten Alltagssituationen kommunikativ bewältigen können.

Andere TN haben keine oder geringe Deutschkenntnisse, weil sie neu nach Deutschland eingereist sind oder - im Falle vieler Frauen - sehr isoliert in ihrer Familie gelebt haben. (Roder 2006: 6)

Auch die Vorkenntnisse im lateinischen Schriftsystem können als ähnlich heterogen beschrieben werden.

Buchstaben- und Wortkenntnisse können auf verschiedene Weise erworben worden sein, so z. B. im Zusammenhang mit dem Erlernen einer Sprache, die in der lateinischen Schrift verschriftet wird, meist der französischen oder englischen Sprache, oder über Verwandte und Bekannte, die lesen und schreiben können. Diese Kenntnisse können sich auf wenige Buchstaben beschränken oder schon auf einer Ebene sein, die Wortbildung zulässt, aber eine unzureichende oder auf Interferenz zurückzuführende Phonem-Graphem-Zuordnung zeigt. (Roder 2006: 6)

Feldmeier berichtet darüber hinaus auch von funktionalen Analphabeten ${ }^{9}$, die an diesen Kursen teilnehmen. Diese Personen haben in der Regel „lediglich Schwierigkeiten, seltene Buchstaben und Buchstabengruppen [...] zu erkennen und zu benennen. Doch auch innerhalb dieser Gruppe gibt es deutliche Unterschiede im Kenntnisstand. Das Spektrum reicht von Teilnehmern, die auch beim Lesen noch größte Schwierigkeiten haben bis zu solchen, die bereits über eine gewisse Lesefertigkeit verfügen, aber kaum schreiben können" (vgl. Feldmeier 2004: 105).

Diese Teilnehmer zählen auch zu denjenigen, die bereits Konzepte über Schriftsprache haben, also z. B. ein Verständnis davon haben, was ein Wort ist, ggf. sogar einzelne Wortklassen bzw. deren Funktionen kennen, auch wenn ihnen die Fachausdrücke (Substantiv, Verb, Adjektiv usw.) unbekannt sind (vgl. Feldmeier 2004: 106).

Im Gegensatz dazu kann im Extremfall, wie im einleitenden Zitat von John Wilkins beschrieben, ein Konzept von Schriftsprache überhaupt nicht vorhanden sein. Feldmeier berichtet z. B. von Teilnehmern, ,die überhaupt keine Vorstellung darüber haben, was ein Wort ist (weder ein gesprochenes noch ein geschriebenes Wort in der Mutter- oder in der Zweitsprache Deutsch). Solche Teilnehmer

deutsche Muttersprachler keine Berücksichtigung. Eine Teilnahme an Alphabetisierungskursen für deutsche Muttersprachler kann also für diese Zielgruppe nicht als ideale Alternative gesehen werden. ${ }^{9}$ Hier sind Personen mit Migrationshintergrund gemeint, die eine deutsche Schulausbildung durchlaufen haben. 
schreiben oft ganze Sätze als ein Wort; manchmal werden die Wortgrenzen nur leicht durch Punkte oder Striche gekennzeichnet“ (Feldmeier 2004: 106).

Aus den schriftsprachlichen Vorkenntnissen resultiert ein weiterer Aspekt, bei dem üblicherweise bei den Kursteilnehmern mit unterschiedlichstem Fertigkeitenstand gerechnet werden muss, nämlich im Bereich von nicht-sprachlichen Fertigkeiten wie Motorik, Akustik und visuelle Wahrnehmung z. B.:

Es gibt beispielsweise Teilnehmer, denen allein der Umgang mit dem Bleistift größte Schwierigkeiten bereitet [...]. Striche können nicht gerade gezogen werden, Bleistiftspitzen brechen ständig ab, Unter- und Oberarm verkrampfen sich. [...] Andere Teilnehmer haben wiederum große Schwierigkeiten, ähnlich lautende oder ähnlich aussehende Buchstaben zu unter-

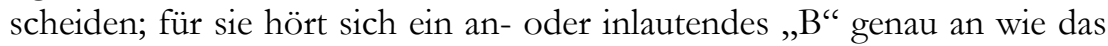
an- oder inlautende „P“ und die Buchstaben „n“ und „h“ unterscheiden sich optisch nicht voneinander. Die Größenverhältnisse innerhalb der Buchstaben werden trotz mehrmaliger Behandlung im Unterricht nicht wahrgenommen. Die Beachtung der Raumlage stellt sie ebenfalls vor Schwierigkeiten: Die Buchstaben „, $\mathrm{b}^{\prime}$, , $\mathrm{q}$ “, „d“ und „,p“ werden in deren Raumlage nicht unterschieden. Den genauen Gegensatz dazu bilden Teilnehmer, die bereits in einem nicht lateinischen Schriftsystem alphabetisiert wurden. Sie haben kaum Probleme im Umgang mit Schreibwerkzeug und haben gelernt, auf all die Kleinigkeiten zu achten, die Buchstaben optisch, akustisch und in ihrer Raumlage voneinander unterscheiden. (Feldmeier 2004: 105f)

Für den Bereich der mündlichen und schriftlichen Vorkenntnisse in DaZ wäre es sowohl für Lehrende als auch Teilnehmer von höchster Wichtigkeit, ein vor allem nach Vorkenntnissen und ggf. auch nach Muttersprachen differenzierteres Kursangebot zu entwickeln. Realistisch betrachtet ist dies jedoch nur in größeren Städten bzw. Ballungsgebieten zu leisten, wie dies in Ansätzen zumindest in einigen größeren Städten bereits zu realisieren versucht wird. Z. B. wurde mit der Einführung der Integrationskurse an der Volkshochschule Frankfurt, „das Angebot von 4 auf 6 Stufen für die Alphabetisierung erhöht. [...] Nach Möglichkeit werden die Interessenten nach ihrem schriftsprachlichen Kenntnisstand, ihrer bisherigen Schulerfahrung und ihren mündlichen Kenntnissen entsprechend in die Kurse verteilt" (Roder 2006: 6).

In vielen anderen Orten, insbesondere in ländlicheren Gebieten, ist aber weiterhin die beschriebene Heterogenität in Kursen Realität. Von den Lehrenden ist hier also eine starke Binnendifferenzierung während des Unterrichts gefordert und zwar in viel stärkerem Maße als das in „,normalen“ Sprachkursen erforderlich ist. 


\section{$4 \quad$ Alphabetisierung in den Integrationskursen}

Im Jahr 2005 hatten insgesamt 215.651 Personen eine Berechtigung zur Teilnahme an einem Integrationskurs, tatsächlich besucht wurden Integrationskurse von etwas mehr als der Hälfte der Berechtigten, nämlich von 115.158 Personen, die an insgesamt 8.196 Integrationskursen teilgenommen haben. Von diesen 8.196 Kursen waren 227 Alphabetisierungskurse mit insgesamt 2.294 Teilnehmern in der Alphabetisierung. Diese Zahlen würden hochgerechnet auf alle Personen, die 2005 an Integrationskursen teilgenommen haben, bedeuten, dass hier „nur“ knapp zwei Prozent eine Alphabetisierung benötigten. Im Verhältnis zu den geschätzten Zahlen (funktionaler) Analphabeten in Deutschland insgesamt und insbesondere zu Erhebungen über die Analphabetenrate unter Migranten in Deutschland aus den letzten Jahrzehnten ${ }^{10}$, wären diese Zahlen überraschend niedrig. Dass diese Zahlen aber nicht ganz den Bedarf an Alphabetisierung im Integrationskursbereich widerspiegeln, wird deutlich, da laut BAMF der Schwerpunkt im Jahr 2005 auf den allgemeinen Integrationskursen lag. „Kurse für spezielle Zielgruppen wurden daher nur in geringer Zahl angeboten, werden aber ein Schwerpunkt der Weiterentwicklung im Jahr 2006 sein", so das BAMF"1.

Dennoch schließt das Bundesamt aufgrund einer Mitte letzten Jahres durchgeführten Umfrage unter 1.304 Teilnehmern in 100 zufällig ausgewählten Integrationskursen, „dass der Bedarf an Kursen für spezielle Zielgruppen wohl deutlich niedriger einzuschätzen ist, als bisher angenommen“ (ebd.). Inwiefern diese doch recht optimistische Prognose aufrechterhalten werden kann, bleibt vorerst offen. Zu bedenken bleibt aber, dass im Jahr 2005 Kurse für spezielle Zielgruppen nur in geringer Zahl angeboten wurden und eine Umfrage unter Teilnehmern allgemeiner Integrationskurse kaum den Bedarf an Alphabetisierungskursen aufdecken kann. Berücksichtigt werden sollte in diesem Kontext auch, dass 2005 die „Teilnehmerzahl der Ausländer mit längerem Aufenthalt in Deutschland, die noch von den Ausländerbehörden zur Teilnahme an einem Integrationskurs verpflichtet wurden, [noch sehr gering ist]. Sie belegen mit 7.945 [von insg. 18.330] Teilnehmern [nur] 6,9\% der Kursplätze“ (ebd.). Insbesondere bei dieser Zielgruppe muss vermutet werden, dass der Anteil zumindest funktionaler Analphabeten deutlich höher ist, als bisher angenommen.

Die aktuellsten vom BAMF vorgelegten Zahlen ${ }^{12}$ über die Beteiligung an Integrationskursen im ersten Quartal 2006 weisen dann auch auf eine gestiegene

\footnotetext{
${ }^{10}$ Dierks und Zander gingen 1975 davon aus, dass mehr als ein Drittel aller Migranten nicht oder nur rudimentär lesen und schreiben konnten (Dierks / Zander 1975: 105); eine Untersuchung aus dem Jahre 1985 ergab, dass 55,9 \% der Migranten nach eigenen Angaben Deutsch nicht schreiben konnten (Sargut u.a. 1988: 38, vgl. auch Schramm 1996: 4).

11 BAMF (2006): Integrationskurse. Jahresbilanz 2005: 4. Dieses Dokument sowie alle weiteren hier vom BAMF genannten, können auf der Homepage des Amtes, www.bamf.de kostenfrei eingesehen bzw. heruntergeladen werden (Stand: 31.08.2006), sie sind deshalb nicht einzeln in der Literaturliste aufgeführt.

12 Das BAMF weist darauf hin, dass die Werte vorläufig sind und unter Vorbehalt nachträglicher Ergänzungen stehen.
} 
Zahl an Kursen für spezielle Zielgruppen und somit auch an Alphabetisierungskursen hin: So machten die 3.966 Teilnehmer an Alphabetisierungskursen in diesem Zeitraum knapp 3,4 \% aller Teilnehmenden an Integrationskursen aus (insgesamt 117.859). Betrachtet man nur die Zahlen der im ersten Quartal 2006 begonnenen Kurse, so haben die 153 Alphabetisierungskurse einen Anteil von über sieben Prozent an den insgesamt 2.123 Integrationskursen ${ }^{13}$. Diese Zahlen belegen also, dass durchaus Bedarf an Alphabetisierungskursen auch im Bereich der Integrationskurse besteht und somit auch Bedarf an qualifizierten Lehrkräften für diesen Bereich.

\section{Qualifizierung von Lehrkräften für Alphabetisierungskurse}

Die ab 2009 bindenden Zulassungskriterien ${ }^{14}$ für die Zusatzqualifikation von Lehrkräften in Integrationskursen definiert die Zulassungsvoraussetzungen für Lehrkräfte wie folgt:

Lehrkräfte, die im Integrationskurs Deutsch als Zweitsprache unterrichten, müssen ein erfolgreich abgeschlossenes Studium Deutsch als Fremdsprache oder Deutsch als Zweitsprache vorweisen.

Soweit diese fachlichen Qualifikationen nicht vorliegen, ist eine Zulassung zur Lehrtätigkeit nur möglich, wenn die Lehrkraft an einer vom Bundesamt vorgegebenen Qualifizierung teilgenommen hat. (ebd.)

Genauere Angaben über zusätzliche Auflagen für Lehrkräfte in Kursen für spezielle Zielgruppen, also auch für Alphabetisierung, liegen (zumindest bisher noch) nicht vor ${ }^{15}$.

\subsection{Zusatzqualifizierung für Lehrkräfte in Integrationskursen}

Bei der Zusatzqualifizierung für Lehrkräfte in Integrationskursen, davon sind Personen mit einem Hochschulabschluss in DaF/DaZ auch ohne Praxiserfahrung, wie oben erwähnt, nicht betroffen, ist bisher für den Aspekt Alphabetisierung Folgendes vorgesehen:

In der unverkürzten Zusatzausbildung sind 28 obligatorische, curriculare Bausteine enthalten, darüber hinaus ist „Alphabetisierung“ eines von insg. fünf Themen ${ }^{16}$ zur Wahl, wobei die unverkürzte Zusatzqualifikation insg. 160 UE á 45

\footnotetext{
13 BAMF (2006): Quartalsstatistik Integrationskurse 1/06.

${ }^{14}$ Bis zum 31. Dezember 2009 kann das Bundesamt auf Antrag der Kursträger Lehrkräfte zulassen, die die im Folgenden genannten Voraussetzungen nicht erfüllen (vgl. BAMF (2006): Lehrkräftequalifizierung für Integrationskurse).

15 In der BAMF-Broschüre „Konzept für einen bundesweiten Integrationskurs“ heißt es „Sowohl für den Integrationskurs als auch für seine zielgruppenspezifischen Varianten werden Rahmencurricula entwickelt" (ebd.: 5).

${ }^{16}$ Die weiteren sind: Konfliktmanagement im DaZ-Unterricht, Berufsorientierter DaZ-Unterricht, DaZ-Unterricht für Jugendliche, Neue Medien im DaZ-Unterricht.
} 
Minuten Präsenzveranstaltung ausmacht, davon 140 UE im obligatorischen Bereich und 20 UE im Wahlbereich, wobei hier vier der fünf Themen gewählt werden, so dass fünf UE pro Thema zur Verfügung stehen, also auch für die Alphabetisierung (= in Zeit 225 Minuten) ${ }^{17}$. Das Wahlthema „Alphabetisierung“ wird wie folgt beschrieben:

\begin{tabular}{|c|c|}
\hline Die Teilnehmer können: & Das beinhaltet: \\
\hline $\begin{array}{l}\text { den Stellenwert schriftlicher } \\
\text { Kompetenz in der Zielsprache } \\
\text { für die Zugewanderten richtig } \\
\text { einschätzen }\end{array}$ & $\begin{array}{l}\text { - } \text { das Wissen um die Bedeutung der Schriftsprache } \\
\text { in der deutschen Gesellschaft, insbesondere im } \\
\text { Berufs- und Alltagsleben } \\
\text { - } \quad \text { die Kenntnis der für die Zugewanderten besonders } \\
\text { relevanten kommunikativen Anforderungen }\end{array}$ \\
\hline $\begin{array}{l}\text { die Auswirkungen der For- } \\
\text { men des Analphabetismus } \\
\text { auf den Spracherwerb der } \\
\text { Zugewanderten richtig ein- } \\
\text { schätzen }\end{array}$ & $\begin{array}{ll}\text { - } & \text { die Kenntnisse der Formen des Analphabetismus } \\
\text { - } & \text { die Kenntnisse der Lernziele und Lerninhalte für } \\
& \text { Lerner mit unterschiedlichen Formen von Analpha- } \\
& \text { betismus }\end{array}$ \\
\hline $\begin{array}{l}\text { Methoden und Ansätze bei } \\
\text { der Alphabetisierung bezüg- } \\
\text { lich ihrer Eignung für eine } \\
\text { definierte Zielgruppe }\end{array}$ & $\begin{array}{ll}\text { - } & \text { das Wissen um die Lernvoraussetzungen und } \\
& \text { Lerngewohnheiten der Zielgruppe } \\
\text { - } & \text { die Kenntnis der Methoden der Alphabetisierung, } \\
\text { ihrer Voraussetzungen und Progressionen } \\
\text { - } & \text { das Wissen um die Eignung von Alphabetisie- } \\
\text { rungsmethoden für Zielgruppen bestimmter Mutter- } \\
\text { sprachen }\end{array}$ \\
\hline $\begin{array}{l}\text { Angemessenheit von } \\
\text { Übungsformen in Unter- } \\
\text { richtsmaterialien zur Alphabe- } \\
\text { tisierung beurteilen }\end{array}$ & $\begin{array}{l}\text { - } \\
\text { die Kenntnis der Aufgaben- und Übungstypologien } \\
\text { zur Alphabetisierung } \\
\text { - } \quad \text { die Beurteilung von Aufgaben und Übungen hin- } \\
\text { sichtlich ihrer Funktionalität und ihrer Ziele }\end{array}$ \\
\hline $\begin{array}{l}\text { Lehrbücher und Übungsmate- } \\
\text { rialien zur Alphabetisierung } \\
\text { analysieren und bezüglich } \\
\text { ihrer Eignung für die Ziel- } \\
\text { gruppe beurteilen und aus- } \\
\text { wählen }\end{array}$ & $\begin{array}{ll}\text { - } & \text { die Kenntnis von Lehrwerken und Zusatzmateria- } \\
& \text { lien zur Alphabetisierung im DaZ-Unterricht } \\
\text { - } & \text { die Kenntnis der Analysekriterien für Unterrichtsma- } \\
& \text { terialien zur Alphabetisierung } \\
\text { - } & \text { die Fähigkeit, zielgruppenspezifisches Material } \\
& \text { auszuwählen, anzupassen oder selbst zu erstellen } \\
& \text { und in eine zielgruppengerechte Progression zu } \\
\text { bringen }\end{array}$ \\
\hline
\end{tabular}

Thema zur Wahl in der Zusatzqualifikation: Alphabetisierung im DaZ-Unterricht ${ }^{18}$

\footnotetext{
17 Vgl. BAMF/GI (2005): Konzeption für die Zusatzqualifizierung von Lehrkräften im Bereich Deutsch als Fremdsprache: 25. Die verkürzte Zusatzqualifikation besteht aus insg. 80 UE, davon 70 im obligatorischen und $10 \mathrm{UE}$ im Wahlbereich, wobei hier formuliert wird „um die Wünsche und Bedürfnisse der Teilnehmer möglichst weitgehend berücksichtigen zu können und eine möglichst große Flexibilität zu erreichen, sollte mind. 2 UE pro Einheit angesetzt werden“ (vlg. ebd.: 65). ${ }_{18}$ BAMF (2006): Konzeption für die Zusatzqualifizierung von Lehrkräften im Bereich Deutsch als Fremdsprache: 59 .
} 
Sicherlich handelt es sich hier um berechtigte und sinnvolle Inhalte. Zu berücksichtigen bleibt allerdings, dass in der Alphabetisierung DaZ völlig andere Ansätze und Methoden ${ }^{19}$ eingesetzt werden (müssen), als in einem Fremdsprachenunterricht ohne Alphabetisierung, dass Übungsformen und Unterrichtsmaterialien nicht einfach aus dem „herkömmlichen“ Fremdsprachenunterricht übertragen werden können, dass sowohl Lehrbücher als auch Übungsmaterialien für die Alphabetisierung in $\mathrm{DaZ}^{20}$ einen völlig anderen - wenn auch bedauerlicherweise recht überschaubaren - Bereich als diejenigen für den $\mathrm{DaF}$-/DaZ-Unterricht ohne Alphabetisierung darstellen.

Punkte wie „Methodische Ansätze“, „Übungstypologien, Sozialformen und Arbeitsanweisungen“ sowie „Unterrichtsmaterialanalyse und -beurteilung“", die in der Zusatzqualifikation als eigene, obligatorische curriculare Bausteine behandelt werden und kaum vom DaF-/DaZ-Unterricht auf die Alphabetisierung übertragen werden können, werden hier für den Bereich der Alphabetisierung gemeinsam mit allgemeinen Grundlagen und weiteren Aspekten in einem einzelnen Wahlbaustein behandelt. Dass dies eine kaum zu realisierende Forderung für fünf Unterrichtseinheiten, also 225 Minuten, ist, dürfte außer Frage stehen.

Darüber hinaus muss bedacht werden, dass Vieles, was für den Baustein Alphabetisierung als Inhalt formuliert ist, lediglich aus langjähriger Berufserfahrung weitergegeben werden kann, da zum einen in der kurz bemessenen Zeit für diesen Baustein kein Raum für eigene Praxiserfahrung, und seien es nur Hospitationen, bleiben dürfte, und da es darüber hinaus an wissenschaftlich gesicherten Erkenntnissen für diesen Bereich mangelt. Was beispielsweise sind die für die Zugewanderten besonders relevanten kommunikativen Anforderungen und welche Lernziele und Lerninhalte resultieren aus unterschiedlichen Formen von Analphabetismus? Auch über die Lernvoraussetzungen und Lerngewohnheiten der Zielgruppe ist bisher kaum wissenschaftlich gesichertes Wissen zusammengetragen worden. Ebenso fehlen begleitende Studien zur Überprüfung der Eignung von Alphabetisierungsmethoden, z. B. für Zielgruppen bestimmter Muttersprachen, wenn es denn überhaupt solche Kurse gibt, für den DaZ-Bereich gänzlich (vgl. Schulte-Bunert 2000: 51).

Und schließlich stellt sich die Frage, ob nicht über das Wissen um die Bedeutung der Schriftsprache in der deutschen Gesellschaft hinaus, angehenden Lehr-

\footnotetext{
${ }^{19}$ Bei der Alphabetisierung in DaZ wird üblicherweise sowohl auf Methoden für den Erstspracherwerb als auch auf solche für die muttersprachliche Alphabetisierung im Erwachsenenalter zurückgegriffen wie z. B. auf den Spracherfahrungsansatz (Language Experience Approach), die MorphemMethode, den Fähigkeiten-Ansatz, das Konzept der „conscientização“ von Freire (vgl. u. a. SchulteBunert 2000: 51ff, Schramm 1995: 105ff).

${ }^{20}$ Kommerzielle, für den Bereich der Alphabetisierung in den Integrationskursen zugelassene Lehrwerke sind: „Das Alpha-Buch“ (Brandt / Brandt / Frohn 1992), „Hamburger ABC“ (Wäbs 2000), „Lesen und Schreiben“ (Lonnecker / Schröder 2001), „Mosaik“ (Knechtel 2004), „Projekt Alphabet neu“ (Volkmar-Clark 2004) sowie „Der Vorkurs“ (Bastani / Ragg 2005), der sich an eine Zielgruppe mit mündlichen Vorkenntnissen im Deutschen richtet, die sich zwischen Alphabetisierung und „normalem“ Anfängerunterricht befindet, und der Online-Kurs bzw. die interaktive CD „Alphabetisierung und deutsche Sprache" (Proson GmbH 2004).
} 
kräften in der Alphabetisierung vor allem Kenntnis über das Gegenteil, also ein Leben ohne Schrift in einer verschriftlichten Welt, vermittelt werden müsste.

Zweifelsohne ist aber festzuhalten, dass die für den Baustein „Alphabetisierung im DaZ-Unterricht" formulierten Inhalte und Ziele als wünschenswerte Bestandteile einer entsprechenden Aus- bzw. Fortbildung gesehen werden können. Anzuzweifeln bleibt aber, ob die Inhalte und Ziele tatsächlich in einer solchen Kürze erreicht werden können. Sicherlich macht ein entsprechender Wahlbaustein in der Zusatzqualifizierung in diesem Rahmen insofern Sinn, als dass er Lehrkräfte in Integrationskursen allgemein für das Phänomen Alphabetisierung sensibilisieren kann, denn auf niedrigen Kursstufen passiert es durchaus, dass Lehrpersonen mangelnde Lese- und Schreibkenntnisse erkennen, aber nicht angemessen reagieren bzw. helfen können. Als Vorbereitung einer ansonsten für den DaF-/DaZ-Bereich ausgebildeten Lehrkraft auf den Einsatz in einem ,reinen“ Alphabetisierungskurs allerdings, mag der hier vorliegende curriculare Baustein zwar ein erster Schritt sein, aber ausreichend ist er in diesem Umfang sicher nicht. Daraus kann sich nicht die Forderung ableiten, dass dem Punkt Alphabetisierung allgemein in der Zusatzqualifikation ein größerer Stundenumfang eingeräumt werden sollte, schließlich sind die Integrationskurse in der überwiegenden Mehrheit solche ohne Alphabetisierung. Es stellt sich aber die Forderung, solchen Lehrkräften, die in Alphabetisierungskursen arbeiten (wollen), tatsächlich eine zusätzliche Qualifikation oder einen stärkeren Schwerpunkt in der derzeit durchgeführten Zusatzqualifikation für den Bereich der Alphabetisierung anzubieten.

\subsection{Alphabetisierung in der universitären DaF-/DaZ-Ausbildung}

Lehrkräfte mit einem Hochschulabschluss in DaF oder DaZ (auch als Ergänzungs- bzw. Aufbaustudium) benötigen keine Zusatzqualifizierung für den Einsatz in Integrationskursen, dies gilt also auch für den Bereich der Alphabetisierung. Der Bereich der Integrationskurse steht somit universitären DaF-/DaZ-Absolventen offen und kann sicherlich als ein solch großer gesehen werden, dass sich auch der universitären DaF-/DaZ-Lehrerausbildung die Aufgabe stellt, auf diesen Arbeitsmarkt vorzubereiten. Somit sollte auch hier zumindest in einem gewissen Maße auf den Aspekt Alphabetisierung eingegangen werden.

In ersten Ansätzen finden insbesondere in den DaZ-Studiengängen Integrationskurse und sehr vereinzelt auch Alphabetisierung Berücksichtigung in der universitären Lehre. Dies müsste aber noch stärker geschehen. Das bedeutet als Fazit nicht, dass Alphabetisierung ein fester Bestandteil in den DaF-/DaZ-Curricula sein muss, aber die Aspekte Alphabetisierung, Anfängerunterricht und auch das Thema Integrationskurse im Allgemeinen sollten auch in der universitären Ausbildung zumindest im Wahlbereich Berücksichtigung finden. Denkbar wäre sicherlich auch eine stärkere „Spezialisierung“ einzelner universitärer DaF-/DaZ-Abteilungen auf den einen oder den anderen Bereich. Forderungen darüber hinaus 
könnte man nur basierend auf der Erhebung umfangreicherer Zahlen anstellen, also einer Ermittlung, wie viel Bedarf es in welchem Bereich tatsächlich gibt und welche Lehrkräfte (also mit welcher Vorbildung) dort vor allem eingesetzt werden.

\subsection{Konsequenzen für die Aus- und Fortbildung}

Für Aus- und Fortbildungsangebote von Lehrkräften für die Alphabetisierung in DaZ dürfte sicherlich das vom Goethe-Institut erarbeitete und vom BAMF für den curricularen Baustein vorgelegte Profil inhaltlich eine erste Richtlinie darstellen. Um dieses sinnvoll umzusetzen, müsste aber eine größere zeitliche Flexibilität eingeräumt werden.

Darüber hinaus wäre ein stärkerer Praxis-Einbezug, z. B. über Hospitationen in verschiedenen Alphabetisierungskursen oder Praktika in Institutionen mit einem solchen Kursangebot erstrebenswert (vgl. auch Feldmeier 2005: 36).

Auch der Erwerb von zumindest Basiskenntnissen der Muttersprachen der häufigsten Herkunftsländer der Teilnehmer solcher Kurse würde den künftigen Kursleitern sicherlich helfen (vgl. ebd.). Um hier allerdings gesicherte Empfehlungen auszusprechen, wäre es nötig über umfassendere und detaillierte Angaben der Teilnehmer solcher Kurse zu verfügen. Die Einführung der zentral geregelten Integrationskurse könnte hierzu erstmals eine Chance darstellen, tatsächlich repräsentative Angaben zu erhalten. Nach den bisher vorgelegten geschätzten Zahlen und Erfahrungsberichten von Kursleitern wäre der Erwerb von Basiskenntnissen in Türkisch, Arabisch und Kurdisch sowie einer asiatischen Sprache, z. B. Thai, oder zumindest Wissen über die Besonderheiten der jeweiligen Sprache, beispielsweise im phonetischen Bereich, über die Art des Schriftsystems, strukturelle Besonderheiten usw., zu empfehlen. Zweifelsohne können (künftige) Kursleiter dies nur für einzelne der vielen möglichen Herkunftssprachen leisten. Im Rahmen von Aus- und Fortbildung aber sollte hierauf gezielt eingegangen werden.

Das Lernen einer nicht-indogermanischen Sprache bzw. einer mit einem anderen Schriftsystem als dem lateinischen, wie es in den meisten universitären DaF/DaZ-Studiengängen vorgesehen ist, kann künftige Kursleiter auch in einem gewissen Rahmen dafür sensibilisieren, was Teilnehmer in einer Alphabetisierung in DaZ zu leisten haben. Eine Vorstellung von diesem Lernprozess bzw. Verständnis für hierbei auftauchende Schwierigkeiten kann darüber hinaus durch „Simulationen " bzw. in die Fort- bzw. Ausbildung integrierte Szenarien verstärkt werden. Sehr einfach lässt sich dieses z. B. mit einem Unterrichtsexperiment durchführen, wenn Muttersprachler von Sprachen mit nicht-lateinischem Schriftsystem in der Gruppe sind. Diese werden gebeten, in Kleingruppen ihre eigene Muttersprache zu unterrichten und erhalten dazu die folgende Aufgabenstellung: 
Aufgabe für Unterrichtende:

Vor dem Unterrichtsexperiment:

Überlegen Sie sich, was Sie unterrichten wollen und wie Sie das vermitteln könnten.

Was sind Ihre Lernziele?

Wie sollen diese erreicht werden?

Beachten Sie bitte, dass Sie während der Unterrichtszeit ...

$>$... KEIN Wort Deutsch sprechen oder verstehen!!!

> ... nicht auf Englisch als Ausweichsprache/Zweitsprache ausweichen!

Falls Sie Arabisch unterrichten: BITTE nicht auf Französisch ausweichen!

> ... nicht Schriftsprache vermitteln sollen, sondern NUR Kommunikation!

Nach dem Unterrichtsexperiment:

Formulieren Sie bitte spontan Ihre persönlichen Eindrücke (binnen 60 Sekunden ca.).

Bitte notieren Sie später in Ruhe, was Ihr Gesamteindruck von dem Unterrichtsexperiment ist.

(Auf der Rückseite des Blattes.)

Beantworten Sie dabei bitte auch die folgenden Punkte:

$>$ Welche der vorab formulierten Lernziele konnten erreicht werden, welche nicht, warum?

$>$ Inwiefern konnten Sie Ihre vorab überlegte Vermittlungsmethode beibehalten?

$>$ Was war besonders schwierig, was besonders einfach?

Was hat Sie - positiv oder negativ - überrascht?

Der Rest der Gruppe wird in Kleingruppen geteilt und je nach Fremdsprachenkenntnissen einem der jetzt „Lehrenden“ zugeordnet. Wichtig ist dabei, darauf zu achten, dass keine Sprache gelernt wird, zu der oder einer verwandten bereits Vorkenntnisse vorhanden sind. Die Arbeitsanweisungen für diese Gruppe werden, wie folgt, formuliert:

\section{Aufgabe für Lernende:}

Vor dem Unterrichtsexperiment:

Formulieren Sie bitte, welche Erwartungen Sie persönlich an die erste Unterrichtsstunde in einer Ihnen völlig fremden Sprache haben.

Was wären Ihre Lernziele?

Wie sollten bzw. könnten diese Ihrer Meinung nach erreicht werden?

Beachten Sie bitte, dass Sie während der Unterrichtszeit ...

... nicht auf Deutsch oder Englisch als Ausweichsprache/Zweitsprache ausweichen!

Falls Sie Arabisch lernen: BITTE nicht auf Französisch ausweichen!

... keine Kompetenz im Bereich Schriftsprache erwerben sollen, sondern NUR kommunizieren! 
Nach dem Unterrichtsexperiment:

Formulieren Sie bitte spontan Ihre persönlichen Eindrücke (binnen 60 Sekunden ca.).

Bitte notieren Sie später in Ruhe, was Ihr Gesamteindruck von dem Unterrichtsexperiment ist.

Beantworten Sie dabei bitte auch die folgenden Punkte:

Welche Ihrer vorab formulierten Erwartungen wurden erfültt, welche nicht, warum?

Inwiefern entsprach die Vermittlungsmethode Ihren eigenen Vorstellungen/Wünschen?

$>$ Was war besonders schwierig, was besonders einfach?

$>$ Was hat Sie - positiv oder negativ - überrascht?

In analoger Form kann dies für die reine Schriftsprachvermittlung durchgeführt werden, hier würde also formuliert, dass nicht mündliche Kommunikation vermittelt werden soll, sondern Schriftsprache. Insbesondere der Erwerb einer unbekannten Schriftsprache kann noch weiter verstärkt werden, z. B. um auch evt. auftauchende motorische Schwierigkeiten zu simulieren, durch die Anweisung von links nach rechts zu schreiben oder gar nur mit der linken Hand zu schreiben (bzw. für Linkshänder mit der rechten).

\subsection{Weitere Konsequenzen}

Um viele der genannten Aspekte, die in eine Ausbildung einfließen sollten, überhaupt sinnvoll und theoretisch fundiert vermitteln $\mathrm{zu}$ können, wären auch wissenschaftliche Untersuchungen zu dem Bereich Alphabetisierung in DaZ erforderlich. Neben den bereits genannten Punkten, nämlich dass genauere Angaben über Betroffene (Herkunft, Kenntnisse usw.) von Nöten sind, wären begleitende Forschungen zu Kursen, in denen auch eine Analyse von Erfolgen und Misserfolgen stattfindet, die sich mit der Eignung einzelner methodischer Vermittlungsansätze für bestimmte Zielgruppen auseinandersetzen bzw. überhaupt zunächst einmal Lese- und Schreiblernprozesse bei der Alphabetisierung Erwachsener in DaZ dokumentieren, äußerst wünschenswert. Aber auch die Frage, welche Lernvoraussetzungen und Lerngewohnheiten bei der Zielgruppe bestehen, sollte erforscht werden. Dabei stellen sich ebenfalls Fragen, wie z. B., welche Fähigkeiten haben sich entwickelt, wenn Menschen als Analphabeten in einer „verschriftlichten“ Welt leben? Kann man diese Fähigkeiten nutzbar machen? - Dieser Aspekt ist besonders interessant bei Personen, die einen hohen Grad an ungesteuertem DaZ-Erwerb haben, also mehr oder weniger problemlos kommunizieren können. Wie wurde hier, wenn auch nur unbewusst, gelernt; welche dieser Strategien und Fertigkeiten können nutzbar gemacht werden, um Lesen und Schreiben zu lernen?

Aber auch die Publikation von mehr Berichten aus der Praxis wäre insbesondere in diesem Bereich zu wünschen. Dies gilt auch für Materialienproduktionen aus der Praxis, denn das Angebot an kommerziellen Materialien ist doch als recht 
überschaubar zu bezeichnen und aus der Praxis wird nicht selten ein Mangel an geeigneten Lehrmaterialien beklagt (vgl. Rost-Roth o.J.: o.S.).

Für den Bereich der Finanzierung bleibt abschließend nochmals zu betonen, dass vielerorts weiterhin ein breiteres Kursangebot wünschenswert wäre, das mindestens die Aspekte nicht-schriftsprachliche und schriftsprachliche Vorkenntnisse und ggf. Muttersprache berücksichtigt. Dies würde viele der immer wieder auftretenden und vor allem aus der Heterogenität der Kursteilnehmer resultierenden Probleme von Teilnehmern und Lehrkräften entschärfen. Denn Nullanfängerunterricht und - falls erforderlich - Alphabetisierung sind das Fundament für den weiteren gesteuerten Fremdsprachenerwerb und somit - auch für einen Teil der Zielgruppe von Integrationskursen - äußerst wichtig. Frustration auf dieser Lernstufe kann schnell zur Aufgabe des Fremdsprachenlernens führen, wodurch schon hier das Ziel sprachlicher Integration verfehlt würde. Daher ist nicht nur die Qualifizierung von Lehrkräften in diesem Bereich zwingend erforderlich, sondern auch ein differenziertes Kursangebot.

\section{Literatur}

Bastani, Atousa / Ragg, Christina: Der Vorkurs. Einführung ins Deutsch-Lernen, Stuttgart: Klett 2005.

Brandt, Elke / Brandt, Karl-Heinz / Frohn, Bernd: Das Alpha-Buch, Ismaning: Hueber 1992.

Bühler, Kristina: „Wie ich mir vornahm, einmal Analphabetin zu sein ...“. In: Genz, Julia (Hrsg.): 25 Jahre Alphabetisierung in Deutschland, Stuttgart: Klett 2004, 209-212.

Eco, Umberto. Die Gren₹en der Interpretation, München: Hanser 1992.

Dierks, Manfred / Zander, Hartwig: Sprachgebrauch und Erfahrung: Soziolinguistische Grundfragen pädagogischer Praxis, Kronberg: Scriptor 1975.

Feldmeier, Alexis: „Die Alphabetisierung von Erwachsenen nicht deutscher Muttersprache“. In: Genz, Julia (Hrsg.): 25 Jahre Alphabetisierung in Deutschland, Stuttgart: Klett 2004, 101-138.

Feldmeier, Alexis: „Unterrichtsmethoden in der muttersprachlichen Alphabetisierung und ihre Anwendbarkeit im fortgeschrittenen Alphabetisierungsunterricht in der Zweitsprache Deutsch“", Deutsch als Zweitsprache 2 (2004), 8-17.

Feldmeier, Alexis: „Alphabetisierung ausländischer Erwachsener. Empfehlungen für die Beschäftigung von Kursleitern und Durchführung von Alphabetisierungskursen“, Deutsch als Zweitsprache 1 (2005), 33-37. 
Knechtel, Inge: Mosaik. Der Alphabetisierungskurs, Berlin: Cornelsen 2004.

Lonnecker, Georgia / Schröder, Beate: Lesen und Schreiben, Ismaning: Hueber 2001.

Prosodon GmbH: Alphabetisierung und deutsche Sprache, http://www.prosonsprachschule.de/ (Stand: 07.08.2006).

Roder, Anne: Bedeutung und didaktische Möglichkeiten von Ausspracheunterricht in der Alphabetisierung in Deutsch als Fremdsprache (Diplomarbeit), Marburg: PhilippsUniversität 2006.

Rost-Roth, Martina: Deutschkurse für Mütter nicht-deutscher Herkunftssprache, http://www2.hu-berlin.de/ffz/dld/rost.pdf (Stand: 07.08.2006).

Sargut, Sener u.a.: „Überlegungen zu Stand und Entwicklung des DaF- und Weiterbildungsangebots für Ausländer“. In: Vielau, Axel (Hrsg.): Fremdsprachen an der Volkshochschule: Arbeitsschwerpunkte und Entwicklungstendenzen, Bonn: Deutscher Volkshochschul-Verband/Pädagogische Arbeitsstelle 1988, 38-46.

Schramm, Karin: „Alphabetisierung erwachsener Ausländer in der Zweit- oder Zielsprache Deutsch“, Deutsch lernen 20, 2 (1995), 99-124.

Schramm, Karin: Alphabetisierung ausländischer Erwachsener in der Zweitsprache Deutsch, Münster: Waxmann: Münster 1996.

Schulte-Bunert, Ellen: „Alphabetisierung kurdischer Jugendlicher aus dem Irak in der Drittsprache Deutsch“. In: Apeltauer, Ernst / Glumpler, Edith / Luchtenberg, Sigrid (Hrgs.): Erziehung für Babylon. Interkulturelle Erziehung in Praxis und Theorie, Bd. 22, Baltmannsweiler: Schneider Hohengehren 1998: 88-107.

Schulte-Bunert, Ellen: Alles noch einmal von vorn? Zweitschrifterwerb für Seiteneinsteiger in der Sekundarstufe I, Baltmannsweiler: Schneider Hohengehren 2000.

Spotka, Alfred: „Grußworte“. In: Genz, Julia (Hrsg.): 25 Jahre Alphabetisierung in Deutschland, Stuttgart: Klett 2004, 10-11.

Volkmar-Clark, Claudia: Projekt Alphabet neu, Berlin: Langenscheidt 2004.

Wäbs, Herma: Hamburger ABC, Hamburg: Arbeitsgemeinschaft Karolinenviertel e.V. 2000. 



\section{Die organisatorischen und finanziellen Rahmenbe- dingungen der Integrationskurse des BAMF und die daraus resultierenden pädagogischen Konsequenzen}

Beate Blïggel/Amadeus Hempel/Simone Henke, Stuttgart

In diesem Beitrag soll dargestellt werden, wie sich aus Sicht der einzelnen Akteure die Umsetzung der Integrationskurse in die Praxis gestaltet. Zu diesen Akteuren gehören die Kursteilnehmer, die Lehrkräfte und die Mitarbeiter bei den Sprachkursträgern. Außerdem spielen auch die Ausländerbehörden, die Jobcenter und die Außenstellen des Bundesamtes für Migration und Flüchtlinge (BAMF) eine wichtige Rolle.

Die Durchführung der Integrationskurse ist interessanterweise im Gesetz zur „Steuerung und Begrenzung der Zuwanderung“ geregelt. Diese Tatsache wird bei der Umsetzung immer wieder spürbar. 


\section{Vor dem Kurs}

Wenn ein Zuwanderer Deutsch lernen möchte, braucht er eine sogenannten „Bestätigung über die Berechtigung zur Teilnahme am Integrationskurs“ - kurz: Berechtigungsschein.

Wenn er einen solchen Schein erhalten will, muss er genau analysieren, was für ein Teilnehmertyp er ist. Davon hängt ab, wo und wie er einen Berechtigungsschein erhalten kann.

Ist er „Neuzuwanderer“, d. h. nach dem 01.01.2005 eingereist, bekommt er ihn von der Ausländerbehörde, aber nur, wenn er kein EU-Bürger ist. Ist er schon länger in Deutschland, bekommt er ihn entweder auf Antrag bei der Außenstelle des Bundesamtes oder, wenn er Sozialleistungen bezieht, über eine Verpflichtung durch das Jobcenter über die Ausländerbehörde, dies gilt jedoch nicht, wenn der letzte Aufenthaltstitel nach dem 01.01.2005 erteilt wurde, dann ist wieder nur die Ausländerbehörde zuständig. Die EU-Bürger bekommen den Berechtigungsschein auf Antrag bei der Außenstelle des BAMF unter Vorlage einer Freizügigkeitsbescheinigung, die bei der Ausländerbehörde beantragt werden muss, dort aber nur ungern ausgestellt wird, weil „die EU-Bürger eigentlich seit 01.01.2005 sowieso Freizügigkeit in der EU haben“. (Originalton Ausländerbehörde). Ist der Teilnehmer Aussiedler, bekommt er seinen Berechtigungsschein in Friedland bei der Einreise, ist er schon länger da und hat noch keinen Sprachkurs nach SGB III besucht, muss er ihn beim Bundesverwaltungsamt in Köln beantragen, das gleich noch einmal nachfragt, ob die Person schon einen SGB III-Kurs besucht hat. Angehörige von Aussiedlern wiederum bekommen ihren Berechtigungsschein nicht in Friedland, sie dürfen aber trotzdem einen Sprachkurs machen, müssen aber ihren Berechtigungsschein von der Ausländerbehörde bekommen oder... (s.o.).

Wenn man nun den Integrationskurs machen möchte, weil man ja Deutsch erst lernen will, ist es doch sehr schwierig zu durchblicken, wie man an den Berechtigungsschein kommt.

So gehen die meisten potentiellen Integrationskursteilnehmer zunächst einmal zu einer Sprachschule, die andere Zuwanderer kennen und empfehlen. Dort sitzen dann in der Regel Menschen, die das System wenigstens ansatzweise durchblicken und auch in der Lage sind, die entsprechenden Anträge zu verstehen und auszufüllen, selbstverständlich als Service und ohne Vergütung.

Mit dem Antrag auf einen Berechtigungsschein erhält der Teilnehmer ein Merkblatt, in dem er darauf hingewiesen wird, dass er ,als rechtmäßig und dauerhaft in Deutschland lebende(r) Ausländer (in) bzw. als Bürger(in) eines Mitgliedstaates der Europäischen Union nach $₫ 44$ Abs. 4 AufenthG...zur Teilnahme an einem Integrationskurs ...zugelassen werden, sofern Sie einen gesetzlichen Teilnahmeanspruch nach $\int 44$ Abs.1 AufenthaltsG nicht oder nicht mehr besitzen.“ Außerdem soll er den ,vollständig ausgefüllten Antrag .... in einem ausreichend frankierten Umschlag an die zuständige Außenstelle des Bundesamtes senden.“ 
Davon abgesehen, dass selbst viele Muttersprachler diesen Text nicht verstehen, wäre es doch schön, wenn zumindest am Anfang ein „Herzlich Willkommen in Deutschland!“" stehen würde.

Ist der Berechtigungsschein beantragt, stellt sich die Frage der Finanzierung. Normalerweise kostet der Kurs $1 €$ pro Stunde, der Sprachkursträger bekommt noch 1,05 vom Bundesamt dazu. Bekommt der Teilnehmer jedoch Sozialleistungen, kann er einen Antrag auf Befreiung von den Kosten stellen, dieser muss jedoch vor dem 1. Kurstag gestellt sein - unter Vorlage des Bescheids über die Sozialleistungen. Auch wenn der Teilnehmer vom zuständigen Jobcenter zu dem Integrationskurs verpflichtet wurde, was am Berechtigungsschein abzulesen ist, muss er trotzdem diesen Antrag stellen, sonst muss er zahlen, eine Tatsache, die der Teilnehmer nur schwer versteht.

Dann gibt es noch Härtefallanträge, bei denen sämtliche Unterlagen von der Lohnabrechnung über den Mietvertrag bis hin zum Kindergeldbescheid eingereicht werden müssen. Da das Bundesamt meist nicht vor Ort vertreten ist, müssen diese Unterlagen vom Sprachkursträger eingetrieben und verschickt werden. Dieser hat auch ein fundamentales Interesse an der Regelung dieser Fragen, da er sonst auf den Kosten sitzen bleibt. Das Bundesamt zahlt eben einfach nicht, wenn etwas fehlt, und der Teilnehmer, der z. B. Arbeitslosengeld II bekommt, kann nicht $100 €$ für einen Monat Sprachkurs einfach hinlegen.

Mit dem Berechtigungsschein erhält der Teilnehmer eine Liste der Sprachkursträger, die befugt sind, die Integrationskurse durchzuführen. In Berlin sind das 99 Sprachschulen, sortiert nach Postleitzahl. Aus diesen soll sich der Teilnehmer dann einen passenden Kurs aussuchen. Die Zulassung von so vielen Sprachschulen an einem Ort führt natürlich dazu, dass sich bei jeder Sprachschule nur wenige Teilnehmer einfinden, sodass die Wartezeit bis zum Kursbeginn sehr lang ist. Meist findet eine Differenzierung nach Sprachniveau oder nach Alter aus diesem Grunde nicht statt. Die Kurse werden mangels Teilnehmern immer wieder verschoben. Wenn die Sprachschule, die die ganze Bürokratie zum Nulltarif erledigt hat, Pech hat, geht der Teilnehmer jetzt in eine andere Sprachschule, die vielleicht bei ihm um die Ecke liegt.

\section{Während des Kurses}

Nun beginnt endlich der Kurs, dessen Verlauf von vielen guten und schlechten Überraschungen geprägt ist.

Die erste böse Überraschung ist am ersten Tag, wenn der Teilnehmer feststellt, dass er in einem Kurs mit 25 Teilnehmern sitzt, deren Bildungsniveau vom Analphabeten bis zum Professor reicht. Die hohe Teilnehmerzahl ergibt sich aus der mageren Finanzierung von 2,05€ pro Teilnehmer, sodass der Kurs nur finanziert werden kann, wenn viele Personen teilnehmen. Die mangelnde Differenzierung ergibt sich aus der viel zu hohen Anzahl von Sprachkursträgern, die zur Durch- 
führung der Kurse zugelassen wurde. Bei 25 Teilnehmern bleibt im Schnitt pro Teilnehmer eine Sprechzeit von 9 Minuten täglich, aber nur, wenn der Lehrer nichts sagt. So soll er dann schnell und effektiv Deutsch lernen.

Nun betritt ein weitere Akteur die Bühne: Die Lehrkraft. Aufgrund der schlechten Finanzierung der Kurse wird er in der Regel auf Honorarbasis beschäftigt, die Honorare liegen derzeit zwischen 9 und $25 €$ je Unterrichtsstunde, im Durchschnitt bei ca $16 €$. Hat die Lehrkraft eine Familie zu ernähren, muss sie sehr viele Stunden in der Woche unterrichten, um finanziell über die Runden zu kommen. So gibt es Lehrkräfte, die bis zu 50 Stunden in der Woche bei unterschiedlichen Sprachschulen unterrichten. Es ist klar, dass dann die Unterrichtsstunden nicht mehr adäquat vorbereitet werden können und dass eine gewisse Ermüdung eintritt. Viele Lehrkräfte sind zusätzlich frustriert, da sie durch die Einführung der Integrationskurse ihre festen Arbeitsverträge in den Vorgängerprogrammen (Garantiefonds/SGB III) verloren haben. Viele leben seitdem am Rande des Existenzminimums.

Die Anwesenheitslisten müssen von den Lehrern akribisch geführt werden und führen zu sowohl positiven als auch negativen Überraschungen der Teilnehmer: fehlt ein Teilnehmer mit einem ärztlichen Attest, bekommt er das Geld für die gefehlten Stunden zurück, 2,05€ je Stunde. Deshalb werden ärztliche Atteste von einigen Teilnehmern gern in Anspruch genommen, jedenfalls wenn es sich unter Berücksichtigung der Praxisgebühr rechnet.

Die negative Überraschung entsteht bei unentschuldigten Fehlzeiten, wenn das Bundesamt dann dem Sprachkursträger kein Geld zahlt, sodass dieser dem Teilnehmer eine Rechnung stellt, dies macht er allerdings nur, wenn es sich mit dem damit verbundenen bürokratischen Aufwand rechnet, der vom Bundesamt nicht vergütet wird. Nicht entschuldigt ist der Teilnehmer z. B., wenn er ein Bewerbungsgespräch oder einen Termin beim Arbeitsamt hat.

Im Verlauf des Kurses macht der Sprachkursträger insgesamt 592 Kopien für einen Kurs mit 20 Teilnehmern, die wiederum vom Bundesamt verwaltet, beantwortet und abgelegt werden müssen. Für diesen Aufwand wird dem Sprachkursträger 7,- $€$ Verwaltungsgebühr pro Teilnehmer bezahlt, nicht pro Monat, sondern insgesamt.

Am Ende des Kurses steht die Abschlussprüfung zum Zertifikat Deutsch. Im Jahr 2005 bestanden bundesweit nur 42\% der Teilnehmer, die einen Kurs beendet hatten, die Prüfung, Abbrecher nicht eingerechnet.

Für viele Teilnehmer, vor allem die mit weniger Bildungshintergrund, ist das Zertifikat Deutsch nicht in 630 Stunden erreichbar, so machen viele Teilnehmer die Erfahrung, dass sie das gesteckte Ziel nicht erreichen und nicht zur Prüfung angemeldet werden können. Er hat dann die Möglichkeit, auf eigene Kosten weiter zu lernen, was aber viele nicht finanziell bewältigen können.

Für die Anmeldung zur Prüfung sind wieder zahlreiche Anträge zu stellen, an das Bundesamt und an das WBT, das die Prüfung durchführt. Aufgrund des er- 
forderlichen, nicht finanzierten Aufwandes und der geringen Anzahl Teilnehmer an der Prüfung führen viele Sprachschulen die Zertifikatsprüfung gar nicht erst durch. Das vom Bundesamt gesteckte Ziel „Zertifikat Deutsch“ als Ziel für alle wird bei weitem nicht erreicht.

Nach dem Kurs sind die meisten Teilnehmer nicht in der Lage, einen qualifizierten Beruf auszuüben oder gar eine Ausbildung zu machen oder eine Schule zu besuchen. Deshalb kann von Nachhaltigkeit der Integrationskurse nicht die Rede sein. Da die Kenntnisse zu gering sind, kehren vor allem die meisten sozial schwachen Teilnehmer wieder zurück in ihre Peergroup und vergessen schnell wieder, was sie im Kurs gelernt haben. Tests haben ergeben, dass die Teilnehmer, die anschließend an den Kurs wieder zu Hause bleiben, innerhalb von wenigen Monaten wieder auf das Anfängerniveau zurückfallen. Die Chance auf einen Arbeitsplatz oder eine Ausbildung und ein Aufbrechen der Isolation ist gering. Von daher sind die Integrationskurse in ihrer jetzigen Form nur begrenzt dazu geeignet, zur Integration der Zuwanderer beizutragen.

\section{$3 \quad$ Fazit}

Prinzipiell ist es sehr zu begrüßen, dass die Zuwanderer alle die Möglichkeit und die Pflicht haben, einen Deutschkurs zu besuchen.

Das Ziel der Kurse wird bisher nur von ca. einem Drittel der Teilnehmer erreicht. Selbst wenn es erreicht ist, reicht das Niveau noch nicht aus, um eine qualifizierte Berufstätigkeit, Ausbildung oder Schulbesuch aufzunehmen. Um eine Nachhaltigkeit der Kurse zu erreichen, muss die Stundenzahl vor allem für junge Teilnehmer unbedingt erhöht werden.

Mit den Kursen ist ein hohes Ausmaß an Bürokratie verbunden, das von den Teilnehmern selbst nicht erledigt werden kann. Die Sprachschulen leisten diese Erfordernisse bisher ohne Vergütung. Zusammen mit der geringen Finanzierung der Kurse führt dies zur Einrichtung von zu großen Gruppen und zu einer sehr geringen Bezahlung der Lehrkräfte, die wiederum zu Mängeln bei der Durchführung des Unterrichts führen. Die Bearbeitung der vielen Unterlagen beim Bundesamt selbst produziert hohe Kosten. Dieses Geld wäre dringend für eine bessere Finanzierung der Kurse und für eine Erhöhung der Unterrichtsstunden notwendig. 

Themenschwerpunkt 2:

Testen und Prüfen in $\mathrm{DaZ} / \mathrm{DaF}$ 



\section{Auswahltests für alle ausländischen Studienbewer- ber statt Feststellungsprüfung für wenige?}

Christian Krekeler, Konstanz.

\section{Ausgangslage}

An der Fachhochschule Konstanz wird ein Auswahltest für ausländische Studienbewerber an Fachhochschulen entwickelt (und inzwischen auch eingesetzt), der „Test für ausländische Studienbewerber, Konstanz" (TASK). Der Test beruht auf den Erfahrungen mit dem Studienkolleg und der Feststellungsprüfung (FSP). In diesem Beitrag stelle ich den Test und seine Konzeption vor. Ich stelle Überlegungen zur Studienvorbereitung an und gehe der Frage nach, ob der TASK möglicherweise die FSP ersetzen kann. Dann würden sowohl Kandidaten mit Direktzulassung als auch Kandidaten, die sich über das Studienkolleg qualifizieren müssen, an dieser Prüfung teilnehmen. Vor der eigentlichen Vorstellung des TASK möchte ich die Diskussion um Auswahltests und das Zulassungsverfahren skizzieren.

Der geringe Studienerfolg ausländischer Studienbewerber verdeutlicht Probleme mit der Dienstleistung „Studium in Deutschland“. Da sich diese Probleme 
zunächst nicht in einer geringen Nachfrage äußerten - die Zahl ausländischer Studienbewerber war seit mehreren Jahren auf einem hohen Niveau - und da deutsche Hochschulen die Ausbildung von ausländischen Studierenden nicht als ihre Kernaufgabe betrachteten, war der Änderungsdruck nicht allzu groß. Doch für ausländische Studierende ist ein Scheitern eine persönliche Katastrophe, da ein Lebensentwurf zerstört oder zumindest in Frage gestellt wird. Auch für deutsche Hochschulen und für Deutschland als internationalen Wissenschaftsstandort hat die geringe Erfolgsquote ausländischer Studierender langfristig negative Auswirkungen: Es ist nicht ökonomisch und dem Ansehen deutscher Hochschulen abträglich, wenn so viele Studierwillige ohne einen Studienabschluss bleiben. Derzeit zeichnet sich zudem ein Rückgang der Zahl der ausländischen Studierenden ab. Ich vermute, dass dies nicht nur den bevorstehenden Studiengebühren zuzuschreiben ist, sondern möglicherweise auch den schlechten Erfahrungen der Rückkehrer, die neue Bewerber abschrecken.

Will man den Studienerfolg ausländischer Studierender erhöhen, so gibt es zwei mögliche Stoßrichtungen: Man verändert die Studienbedingungen und/oder die Auswahl der Kandidaten. Beide Reaktionen sind derzeit zu beobachten. Eine Internationalisierung des Studienangebots, die Umstellung auf international anerkannte Abschlüsse, die Verbesserung von Betreuungsangeboten - derartige Reformen und Ansätze zielen auch darauf ab, deutsche Hochschulen für ausländische Studierende attraktiver zu machen und den Studienerfolg ausländischer Studierender zu erhöhen. Die zweite Reaktion, die Veränderung des Auswahlprozesses, soll auch eine Veränderung der Zielgruppe bewirken. Möglicherweise haben deutsche Hochschulen bislang nicht die richtige Zielgruppe angesprochen; möglicherweise sind nicht genügend Studieninteressierte aus dem Ausland nach Deutschland gekommen, die auch in der Lage sind, ein wissenschaftliches Hochschulstudium zu absolvieren. Es ist häufig ein völlig studienfremdes Argument, das bei ausländischen Studienbewerbern ein Interesse an einem Studium an einer deutschen Hochschule weckt: Die bislang fehlenden Studiengebühren, das vermeintlich kostenlose Studium. Vor allem bei ausländischen Studienbewerbern für den grundständigen Bereich ist die Motivation, ein Studium an einer deutschen Hochschule aufzunehmen, weiter zu hinterfragen: Deutsche Hochschulen ziehen vor allem Studierende aus Schwellenländern und aus Entwicklungsländern an. In diesen Ländern können die eigenen Hochschulen die stark steigende Nachfrage häufig nicht befriedigen. Bei den Studienbewerbern in Deutschland kann es sich auch um diejenigen handeln, denen es nicht gelungen ist, im Heimatland einen Studienplatz zu erhalten. Ein Studium im Ausland ist im Normalfall jedoch als schwieriger einzustufen als ein Studium im Heimatland.

Die Hoffnung ist also, dass eine verbesserte Auswahl einen Beitrag zur Erhöhung des Studienerfolgs ausländischer Studierender leistet. Bei Studienbewerbern mit deutscher Hochschulzulassung nutzen viele Studiengänge Kriterien, die über die Note des Reifezeugnisses hinausgehen. Häufig werden relevante Fächer (Deutsch, 
Mathematik) besonders stark gewertet. Andere Merkmale wie Berufserfahrung oder - aus Leistungsgesichtspunkten fragwürdig - Wartezeit können ebenfalls berücksichtigt werden, weiter finden studienfachspezifische Auswahlverfahren Anwendung. Die Qualität der Auswahlverfahren für deutsche Studienbewerber allgemein zu beurteilen, ist kaum möglich. Aus den vielfältigen Aktivitäten in diesem Bereich kann man allenfalls schlussfolgern, dass ein gewisser Handlungsdruck besteht.

Das Auswahlverfahren ausländischer Studienbewerber ist nicht zufrieden stellend. Wenn die Studienbewerber sich direkt bewerben können, entscheidet die Hochschule in der Regel allein nach der Heimatnote über die Zulassung. Die Heimatnote ergibt sich aus dem Schulabschlusszeugnis, aus Studienleistungen oder einem Studienabschluss. Sie wird in das deutsche System umgerechnet. Die Verwendung der Heimatnote führt zu Problemen, die vor allem auf unterschiedlichen „Notenkulturen“ beruhen. In manchen Ländern ist es beispielsweise üblich, dass die beste Note von keinem Kandidaten erreicht wird, in anderen kann es durchaus vorkommen, dass ein größerer Teil der Kandidaten die Bestnote erreicht. Wenn es mehr Bewerber als Studienplätze für ausländische Studierende gibt, setzen sich Bewerber aus Ländern durch, in denen es möglich ist, die Bestnote zu erhalten.

Anders und wohl Erfolg versprechender ist das Verfahren bei Bewerbern, die den Hochschulzugang über die Feststellungsprüfung (FSP) erreichen. An der FSP müssen ausländische Studienbewerber teilnehmen, die nicht direkt zum Studium zugelassen werden. Sie besuchen vorher ein Studienkolleg, wo sie in zwei Semestern (manchmal auch in nur einem Semester) auf die FSP und auf das Fachstudium vorbereitet werden. Dies gilt beispielsweise für Bewerber aus Peru, die nach Abschluss der elfjährigen Schulbildung die Hochschule im Heimatland besuchen dürfen, in Deutschland aber nur studieren dürfen, wenn sie die FSP ablegen. Die Bewerbungsnote bildet sich bei dieser Gruppe aus der Note der FSP und der (umgerechneten) Heimatnote.

Der Studienerfolg der ausländischen Studierenden, die sich über die FSP qualifiziert haben, dürfte über dem Studienerfolg der ausländischen Studierenden liegen, die direkt zum Studium zugelassen werden. Dies legt eine Studie des Hochschul-Informations-Systems (HIS) nahe (Heublein, 2005; Heublein/ Sommer/Weitz, 2004). Man verfolgte den Studienverlauf ausländischer Studierender an insgesamt vier Hochschulen. An der LMU München konnte außerdem der Studienverlauf von ausländischen Studierenden mit und ohne FSP verglichen werden. Nach 16 Semestern verfügten 21 Prozent der ausländischen Studierenden, die direkt zum Studium zugelassen wurden, über einen Abschluss. 70 Prozent hatten die Hochschule verlassen, neun Prozent waren noch eingeschrieben. Von den Studierenden, die sich über die FSP qualifiziert haben, verfügten 39 Prozent zu diesem Zeitpunkt über einen Abschluss, 42 Prozent hatten die Hochschule verlassen, 19 Prozent waren noch eingeschrieben (siehe Abbildung 1). 


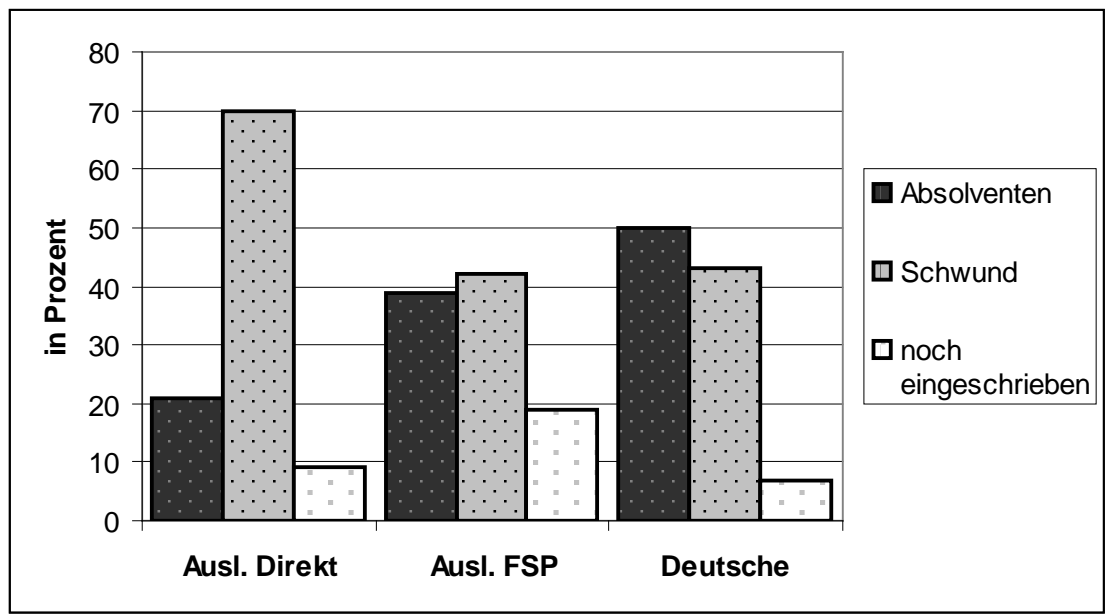

Abbildung 1: Durchschnittlicher Studienverlauf an der LMU München nach 16 Semestern: ausl. Studierende mit Direktzulassung, ausl. Studierende mit Feststellungsprüfung und deutsche Studierende im Vergleich; Quelle: nach Häublein, 2005.

Der Unterschied zwischen dem Studienverlauf von ausländischen Studierenden mit und ohne FSP ist beträchtlich: Der Anteil der Absolventen ist fast doppelt so hoch, der Anteil der Studierenden, die noch eingeschrieben sind, ist ebenfalls doppelt so hoch.

Wie groß der Unterschied zwischen dem Studienverlauf von ausländischen Studierenden mit und ohne FSP ist, wird darüber hinaus deutlich, wenn man den Studienverlauf der deutschen Studierenden als Vergleich hinzuzieht. 50 Prozent der deutschen Studierenden hatten nach 16 Semestern das Abschlusszeugnis in der Tasche, acht Prozent waren noch eingeschrieben und 42 Prozent sind ausgeschieden. Der Unterschied zwischen den Studienverläufen der ausländischen Studierenden mit FSP und den deutschen Studierenden ist gering. Der Anteil der Studierenden, welche die Universität verlassen haben, ist etwa gleich. Allerdings haben etwa zehn Prozent mehr deutsche als ausländische Studierende das Studium bereits abgeschlossen; dementsprechend sind etwa zehn Prozent mehr ausländische Studierende noch eingeschrieben. Bei der Interpretation der Zahlen ist allerdings zu berücksichtigen, dass es sich um die Situation an einer Hochschule mit einer relativ kleinen Stichprobe handelt. Die Daten decken sich aber mit unsystematischen Beobachtungen an anderen Hochschulen.

Der vermutlich höhere Studienerfolg der ausländischen Studierenden, die über die FSP zum Studium zugelassen wurden, ist durchaus überraschend, denn schließlich handelt es sich um die ursprünglich geringer qualifizierte Gruppe. Als Ursachen für den höheren Studienerfolg können vor allem der Auswahlprozess und die Studienvorbereitung im Studienkolleg angeführt werden. Die Auswahl der 
Kandidaten zum Studienkolleg erfolgt normalerweise nach inhaltlichen Kriterien. In der Regel müssen ausländische Studienbewerber an einem Aufnahmetest teilnehmen, wenn sie das Studienkolleg besuchen müssen; es werden also nicht alle ausländischen Studienbewerber ausgebildet, die sich über die FSP für ein Studium qualifizieren können. Die Heranführung an das Studium im Studienkolleg dürfte ein weiterer Faktor für den Erfolg sein. Die Teilnehmer sind dadurch auch mit dem Studienumfeld vertraut, sie können die Erwartungen deutscher Hochschullehrer/Prüfer einschätzen, sie setzen sich mit ihrem Studienwunsch auseinander und haben ihn häufig revidiert. Möglicherweise werden die hohen Schwundquoten der ausländischen Studienbewerber mit Direktzulassung bereits über das Studienkolleg vorweggenommen. Wer das Studienkolleg erfolgreich durchlaufen und die FSP bestanden hat, hat bereits ein gewisses Durchhaltevermögen an den Tag gelegt, die Studienmotivation ist bereits auf den Prüfstand gestellt worden.

Die HIS-Studie offenbart meiner Ansicht nach ein weiteres Problem: Nicht nur der Studienerfolg der ausländischen Studierenden ist gering, auch die Absolventenquote der deutschen Studierenden ist nicht zufrieden stellend. Dies galt für alle untersuchten Hochschulen. Unter Berücksichtigung der Studienwechsler dürfte sich, so mutmaßt man in der Studie, allerdings eine insgesamt deutlich höhere Absolventenzahl ergeben (Heublein/Sommer/Weitz, 2004). Die hohe Schwundquote verdeutlicht jedoch, dass die Auswahl der Studierenden an vielen Hochschulen nicht vor, sondern erst während des Studiums durch eine Reihe von Selektionshürden stattfindet. Eine Auswahl während des Studiums hat möglicherweise zwar den Vorteil, dass vielen Bewerbern eine Studienchance gegeben wird (vgl. Deidesheimer Kreis, 1997: 72-77). Dies geht jedoch nur, wenn die Hochschulen über ihre Kapazität hinaus zulassen. Außerdem verlassen viele Studierende die Hochschule frustriert und ohne Abschluss. Insgesamt ist meiner Ansicht nach eine Auswahl vor der Aufnahme des Studiums vorzuziehen. Auch wenn Hochschulen verstärkt bemüht sind, eine Auswahl der Bewerber nach Eignung vor dem Studium durchzuführen, bezweifele ich, dass sich die Absolventenzahlen rasch verändern werden. Ändern müsste sich auch die Einstellung zum Studienerfolg durch die Lehrkräfte an Hochschulen. Es ist immer noch verbreitet, hohe Schwundquoten als Qualitätsmerkmal und nicht als Problem wahrzunehmen.

\section{Der „Test für ausländische Studienbewerber, Konstanz" (TASK)}

Ausgehend von den guten Erfahrungen mit der Studienvorbereitung im Studienkolleg und der Aussagekraft der FSP wurde an der Hochschule Konstanz ein Test entwickelt, mit dem die einschlägigen Vorkenntnisse der ausländischen Studienbewerber erhoben werden sollen. Die Ergebnisse aus diesem Test können im Zulassungsverfahren der Fachhochschulen in Baden-Württemberg berücksichtigt 
werden. Die Prüfung wird vom Ausländerstudienkolleg an der Hochschule Konstanz durchgeführt, das für die Fachhochschulen in Baden-Württemberg tätig ist. Die Testentwicklung wird vom DAAD im Rahmen der PROFIS-Initiative gefördert.

Die Entwicklung des TASK wurde durch die Erfahrungen mit der FSP beeinflusst. Beim TASK handelt es sich wie bei der FSP um einen studienfeldbezogenen Kenntnistest. Es wurden zwei Versionen entwickelt: Für wirtschaftswissenschaftliche Studiengänge (W-Test) und für Studiengänge aus den Ingenieur- und Naturwissenschaften (T-Test). Der TASK besteht aus jeweils drei Prüfungsteilen: Der W-Test besteht aus Teilprüfungen in Deutsch, Mathematik und Wirtschaft. Der T-Test enthält die Teilprüfungen Deutsch, Mathematik und Physik / Technische Grundlagen. Für jede Teilprüfung wird ein Ergebnis in Form einer Note ausgewiesen (1,0-5,0). Als Endergebnis wird das arithmetische Mittel aus den drei Teilprüfungen gebildet. Ich gehe auf jede Teilprüfung kurz ein.

Wir haben diese beiden Testversionen erstellt, weil es sich um große Fächergruppen handelt, die von den Fachhochschulen in Baden-Württemberg angeboten werden. Aber nicht alle Studienfächer werden durch den Test erfasst: Design und vergleichbare Studiengänge berücksichtigten wir zunächst nicht, weil diese Studiengänge häufig über eigene Auswahlverfahren verfügen, die auch für ausländische Studienbewerber gelten. Diese Auswahlverfahren beinhalten in der Regel praktische Arbeiten. Andere Studiengänge wie Sozialwesen oder Fortwirtschaft wurden nicht berücksichtigt, weil es sich um recht kleine Bewerbergruppen handelt. Es ist allerdings nicht ausgeschlossen, dass Testversionen für weitere Fächergruppen entwickelt werden.

Bei der Deutschprüfung handelt es sich um den Deutschteil der FSP, der sich aus der schriftlichen und der mündlichen Prüfung zusammensetzt. Die schriftliche Prüfung enthält Hörverstehen, Textproduktion und Leseverstehen mit wissenschaftssprachlichen Strukturen. Der Deutschteil der FSP orientiert sich also an der DSH; im Unterschied zur DSH wird das Ergebnis im Deutschteil der FSP (und damit auch beim TASK) nicht mit einer Prozentzahl und DSH-Ergebnisklassen ausgewiesen, sondern als Note. Der TASK enthält eine Deutschprüfung, damit den ausländischen Studienbewerbern die Bedeutung des Deutscherwerbs für den Studienerfolg signalisiert wird. Ein Zusammenhang zwischen den Kenntnissen der Zielsprache und dem Studienerfolg scheint auf dem ersten Blick plausibel, er ist aber empirisch kaum nachzuweisen und in einigen Studien sogar in Frage gestellt worden (vgl. Graham, 1987).

Mathematikkenntnisse werden als eine wichtige Voraussetzung für ein technisches oder wirtschaftswissenschaftliches Studium angesehen. Dies geht beispielsweise aus einer Umfrage hervor: Mathematik wurde von Hochschullehrem als besonders wichtig für die Wirtschaftswissenschaften und die Ingenieurswissenschaften eingestuft (Heldmann, 1984: XXIII). Daher wird auch für deutsche Studienbewerber die Mathematiknote häufig stärker gewichtet als die Noten in anderen Fächern. In wirtschaftswissenschaft- 
lichen Studiengängen ist die Nichtbestehensquote in den quantitativen Fächern besonders hoch. Bei der Mathematikprüfung im TASK werden grundlegende mathematische Fähigkeiten geprüft, wobei sich die Anforderungen im W-Test und im T-Test leicht unterscheiden. Das Leitkriterium bei der Auswahl der Themen war die Frage, ob das Fehlen von Kenntnissen zu diesem Thema zu Schwierigkeiten am Beginn des Studiums führen wird. Es geht also weniger darum, das deutsche Abitur abzubilden, als vielmehr, studienfeldspezifische Themen zu prüfen. Der Anforderungskatalog für den Prüfungsteil Mathematik umfasst folgende Bereiche:

- Beherrschung der Grundlagen der Mengenlehre

- Rechnen im Bereich der reellen Zahlen

- Lösen von Gleichungen und Ungleichungen

- Lösen praktischer Aufgaben unter Verwendung von Proportionen; Prozentrechnung

- Kenntnisse der Elementargeometrie

- Eigenschaften elementarer Funktionen

- Differentialrechnung

- Integralrechnung

Beispielaufgabe für Bewerber technischer Studiengänge:

Beispiel 1

Der Durchmesser $d_{1}$ eines Öltanks (Kreiszylinder) soll um 20\% verkleinert werden. Um wie viel

Prozent verringert sich das Volumen $\mathrm{V}_{1}$ ?

Beispielaufgabe für Bewerber wirtschaftlicher Studiengänge:

Beispiel 2

Die Mehrwertsteuer(= $p$ \% vom Nettopreis) ist eine Umsatzsteuer:

Bruttopreis $=$ Nettopreis + Mehrwertsteuer

Ein Auto kostet mit Mehrwertsteuer 23.200 Euro. Wie viel kostet das Auto, wenn die Mehrwertsteuer von $16 \%$ auf $19 \%$ erhöht wird?

Ähnlich wie bei der Mathematik werden im Prüfungsteil Physik/Technische Grundlagen Themen geprüft, die als inhaltliche Voraussetzung für technische Studienfächer anzusehen sind. Dazu zählen:

- Grundlagen des physikalischen Arbeitens (z. B. physikalische Größen und Einheiten)

- Mechanik (z. B. Energieerhaltungssatz)

- Elektrizitätslehre (z. B. elektromagnetische Induktion)

- Wärmelehre (z. B. Grundgesetz des Wärmeaustauschs) 
- Schwingungen und Wellen (z. B. mechanische Wellen)

Beispielaufgabe für den Prüfungsteil Physik/ Technische Grundlagen:

\section{Beispiel 3}

Bei einer Messung der Siedtemperatur von Wasser in verschiedenen Höhen über dem Meersspiegel wurden folgende Werte gemessen:

\begin{tabular}{|l|l|l||l||ll|l|}
\hline Höhe & $h$ & {$[\mathrm{~m}]$} & 0 & 3.000 & 10.000 & 15.000 \\
\hline \hline Siedetemperatur & $\mathrm{J}$ & {$\left[{ }^{\circ} \mathrm{C}\right]$} & 100 & 90,1 & 66,7 & 50,0 \\
\hline
\end{tabular}

a) Zeichnen Sie ein Temperatur-Höhe-Diagramm!

b) Beschreiben Sie den Zusammenhang von der Höhe $h$ und der Siedetemperatur J des Wassers!

c) Was ist die Ursache für die Veränderung der Siedetemperatur des Wassers?

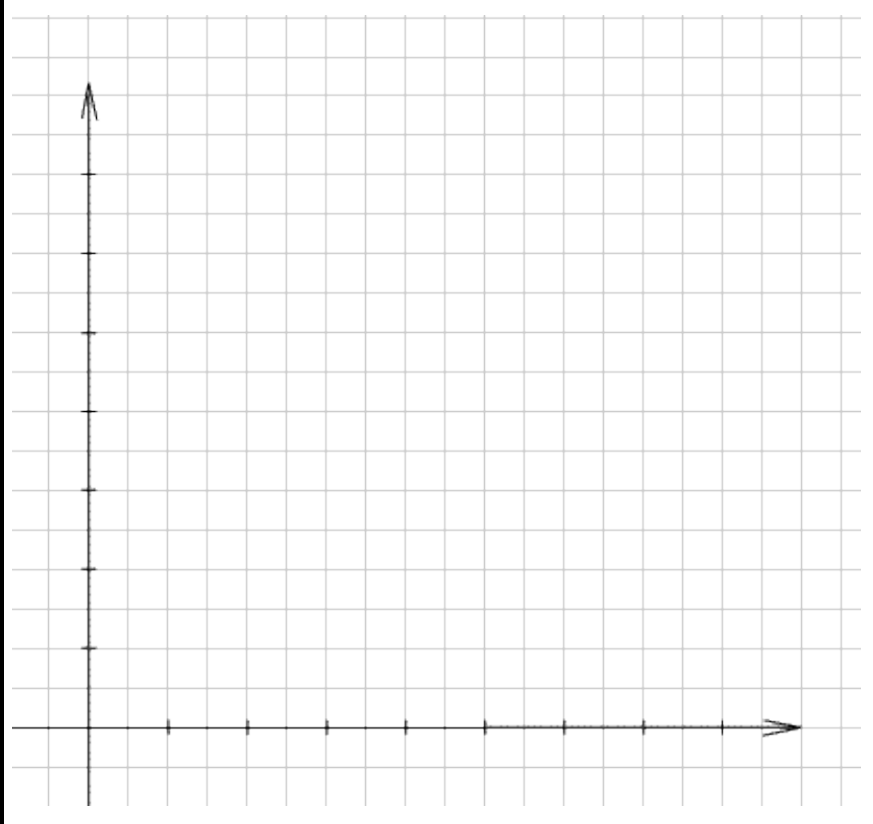


Der Prüfungsteil Wirtschaft folgt nicht dem Muster der übrigen Prüfungsteile. Es handelt sich nicht um einen Kenntnistest, sondern um einen studienfeldspezifischen Eignungstest. Das heißt, es werden nicht volks- oder betriebswirtschaftliche Fachkenntnisse geprüft, sondern die Fähigkeit, typische Aufgaben zu lösen. Von Kandidaten, die ein wirtschaftswissenschaftliches Studium aufnehmen möchten, werden zu Beginn keine einschlägigen Kenntnisse verlangt. Daher können auch keine Vorkenntnisse über die Betriebs- oder Volkswirtschaftslehre geprüft werden. Es wäre beispielsweise nicht sinnvoll, nach konkreten Fakten zu fragen (Wie hoch ist die Einlage, die bei der Gründung einer GmbH oder einer AG zu leisten ist?) oder Begriffe ohne weitere Erläuterung erläutern zu lassen (Was zeigt die Grenzkostenkurve?). Erfragt werden im Prüfungsteil Wirtschaft eher die Auswertung, Interpretation und Anwendung von wirtschaftlichen Zusammenhängen, die in einem Text oder einem Schaubild erläutert werden. Dementsprechend allgemein wurde auch der Anforderungskatalog formuliert:

- Fähigkeit, Informationen sinnvoll und effizient aufzunehmen und zu verarbeiten.

- Fähigkeit zum logischen Denken.

- Fähigkeit, Diagramme und Tabellen richtig zu analysieren und zu interpretieren.

Die Aufgaben enthalten in der Regel einen kurzen Text, in dem ein Thema erläutert wird. Die Aufgaben können also nur von Kandidaten gelöst werden, die über gute Deutschkenntnisse verfügen.

Beispielaufgabe für den Prüfungsteil Wirtschaft:

Beispiel 4

Alternative Produktionsmöglichkeiten

In einer Volkswirtschaft stehen nur eine begrenzte Anzahl der Produktionsfaktoren (z. B. Arbeitskräfte, Kapital, Energie) zur Verfügung. Da ist eine Entscheidung zu treffen, ob diese Faktoren zur Herstellung von Maschinen oder zur Herstellung von Verbrauchsgütern für die Bevölkerung eingesetzt werden. 
Im folgenden Diagramm ist eine Kapazitätslinie dargestellt.

Diese Linie zeigt den Zusammenhang an, wie viele Landmaschinen und wie viel Tonnen Nahrungsmittel maximal mit den vorhandenen Produktionsfaktoren gleichzeitig produziert werden können.

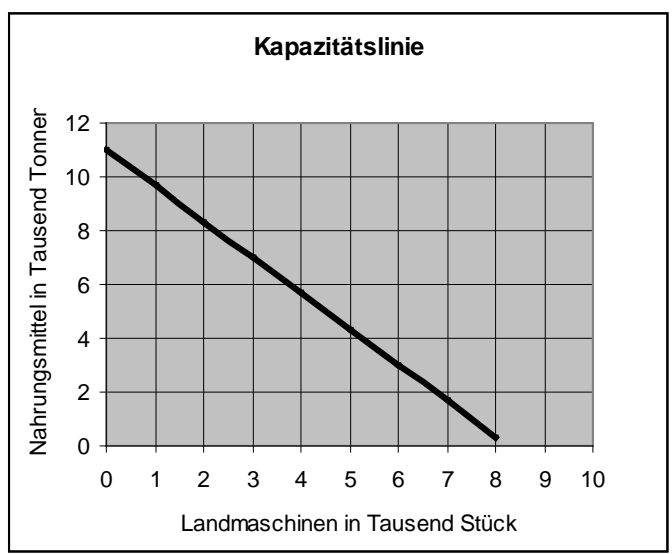

a. In einer Volkswirtschaft ist zwischen zusätzlicher Energiegewinnung und dem Ausbau der elektrischen Schienewege zu entscheiden.

Bei P1 sind 2 Mill. kWh Energie und 5.000 km Schienen möglich. Bei P2 können 10 Mill. kWh Energie produziert und 1.000 km neue Schienenwege gebaut werden. Zeichnen Sie die Kapazitätslinie!

b. Zeichnen Sie in das Diagramm den Punkt P4 $(2 ; 4)$ !

Was besagt der Punkt P4 für die Energiegewinnung und für den Bau von Schienen?

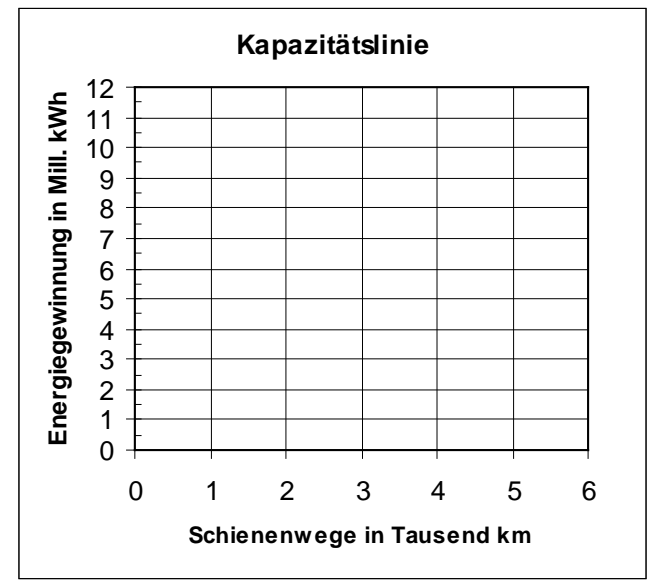


Am TASK können alle ausländischen Studienbewerber teilnehmen, die ein Studium an einer Fachhochschule in Baden-Württemberg anstreben. Das TASKZeugnis gibt Auskunft über die Ergebnisse in den Teilprüfungen, über die TASKGesamtnote und die Heimatnote. Die Hochschulen können entscheiden, wie sie das Ergebnis des TASK bei der Zulassung berücksichtigen.

\section{TASK im Vergleich mit anderen Prüfungen}

Im Vergleich mit der FSP und dem Studierfähigkeitstest von ITB-Consulting und TestDaF-Institut (Test AS) soll die Konzeption des TASK nun weiter verdeutlicht werden. Beim Test AS handelt es sich um einen standardisierten Studierfähigkeitstest für ausländische Studienbewerber, der zur Auswahl der Kandidaten eingesetzt werden kann.

Könnte der TASK die FSP ersetzen? Dies ist nicht vorgesehen, und es gibt aus meiner Sicht wenig Argumente für den Ersatz der FSP durch eine andere Prüfung. Zielgruppe der FSP sind ausländische Studienbewerber ohne Direktzulassung, die sich (normalerweise) über das Studienkolleg qualifizieren. Es handelt sich also um eine Qualifikation, welche von den Studienbewerbern erreicht werden muss, um die Hochschulzugangsberechtigung zu erhalten. Zielgruppe des TASK sind ausländische Studienbewerber, die die Voraussetzungen für eine Zulassung zum Studium bereits erfüllen. Hauptziel des TASK ist es also, den Hochschulen zusätzliche Informationen über den Kenntnisstand der Bewerber bereit zu stellen und damit einen Beitrag für eine verbesserte Auswahl zu leisten.

Die FSP ist eine typische Kursabschlussprüfung. Sie wird von den Lehrkräften erstellt, welche die Kursteilnehmer unterrichten; Sie bezieht sich mehr oder weniger direkt auf die Lehrgebiete, die im Kurs unterrichtet wurden. Wenn Prüfungskandidaten nicht am Kurs teilgenommen haben, sind ihre Erfolgschancen gering, da sie den Stoff nicht im Detail kennen und die spezifischen Anforderungen der Lehrkraft kaum einschätzen können. Der TASK ist weniger abhängig von einem bestimmten Kurs, von bestimmten Lehrkräften. Es gibt nicht nur für die Deutschprüfung, sondern auch für die übrigen Prüfungsteile einen detailliert beschriebenen Anforderungskatalog. Bei den Aufgaben wird auf Fragestellungen verzichtet, die ein ganz spezielles Wissen voraussetzen.

Diese Vorgehensweise beim TASK bedingt einen weiteren Unterschied: das unterschiedliche Anforderungsniveau beider Prüfungen. Das objektive Anforderungsniveau des TASK liegt unter der FSP. Dies ist zu rechtfertigen, da die Funktionen der Prüfungen unterschiedlich sind, die FSP als formale Qualifikation für den Hochschulzugang und der TASK als zusätzliches Auswahlinstrument. Dies ist weiter zu rechtfertigen, da sich die Vorbereitung unterscheidet: Auf die FSP werden fast alle Kandidaten in zwei Semestern vorbereitet. Auch auf den TASK kann man sich vorbereiten; spezielle Kurse werden allerdings allenfalls ein Semester dauern. 
Auch zwischen dem Test AS und dem TASK bestehen grundlegende Unterschiede. Der Test AS st ein Studierfähigkeitstest, während es sich beim TASK um einen Kenntnistest handelt. Ein Studierfähigkeitstest will ermitteln, ob die Kandidaten in der Lage sind, sich bestimmte Kompetenzen anzueignen. Die zentrale Funktion von Studierfähigkeitstests ist die prädiktive Validität. Das Testergebnis soll also Aufschlüsse über den künftigen Studienerfolg ermöglichen, ja den Studienerfolg vorhersagen. Die Argumentation ist: Wenn die Kandidaten im Test relevante intellektuelle Fähigkeiten nachweisen, dann können Sie sich auch im Studium behaupten. Beim TASK ist die Argumentation etwas anders; hier beruht die prädiktive Validität auf den Kenntnissen, welche sich die Kandidaten bereits angeeignet haben. Die Interpretation der Testergebnisse lautet also: Wenn die Kandidaten über Kenntnisse verfügen, die für ein Studium relevant sind, werden sie in den ersten Semestern weniger Schwierigkeiten haben; wenn sie sich diese Kenntnisse bereits erfolgreich angeeignet haben, können sie sich auch weitere Kenntnisse und Fähigkeiten aneignen.

Die unterschiedlichen Vorgehensweisen beruhen auf der Einstellung zur Trainierbarkeit. Beim Test AS legt man Wert darauf, dass sich die Ergebnisse sich nicht durch eine kurzfristige Vorbereitung verbessern lassen. Beim TASK gehört die Trainierbarkeit zum Programm. Häufig wird die Trainierbarkeit von Auswahltests als negativ angesehen. Als „Anforderung an zukunftsfähige Auswahlmodelle“ postulieren Trost und Haase (2005) beispielsweise:

Bei allen eignungsdiagnostischen Verfahren, die zu Auswahlzwecken verwendet werden, ist zu verlangen, dass deren Ergebnisse nicht durch kurzfristiges, massives und gezieltes Training so beeinflusst werden können, dass nicht mehr die eigentliche Qualifikation, sondern lediglich das Ausmaß an Vorbereitung das Ergebnis bestimmt (Trost/Haase, 2005: 18).

Dieser Feststellung ist grundsätzlich nicht zu widersprechen. Der TASK wurde jedoch so konzipiert, dass eine gezielte Vorbereitung auf die Prüfung gleichzeitig auch eine sinnvolle Studienvorbereitung darstellt. Wenn sich Bewerber für technische Studiengänge vor dem Studium mit der Zielsprache, mit mathematischen und technischen Grundlagen beschäftigen, ist das produktiv und sinnvoll. Mit der Vorbereitung auf den TASK soll Kandidaten, die sich schon zu einem Studium an einer Hochschule in Baden-Württemberg entschieden haben, ein Weg aufgezeigt werden, wie sie sich auf das Studium vorbereiten können. Dann ist es legitim, wenn sich die Anstrengungen in einem guten Testergebnis und guten Chancen beim Auswahlverfahren niederschlagen. Der TASK soll also ein Instrument sein, das - ähnlich wie die FSP - zu einer intensiven Studienvorbereitung führt. 
Damit wird ein weiterer Unterschied zwischen Test AS und dem TASK deutlich: Die Zielgruppen. Mit dem TASK sollen nur Bewerber angesprochen werden, die sich bereits für ein bestimmtes Studium an einer bestimmten Hochschule entschieden haben und die sich auf dieses Studium vorbereiten möchten. Normalerweise halten sich diese Bewerber in Deutschland auf. Diesem Bewerberkreis steht mit dem TASK eine Prüfung zur Verfügung, auf die sie sich systematisch vorbereiten können. Mit der Vorbereitung auf die Prüfung geht auch eine Vorbereitung auf die Studieninhalte und eine Auseinandersetzung mit dem Studienziel einher. Bewerber, die sich im Ausland aufhalten und/oder rasch zum Studium zugelassen werden möchten, werden am Test AS teilnehmen.

Die Entwicklung des TASK beruht schließlich auch auf der besonderen Situation der Fachhochschulen. Es handelt sich um vergleichsweise kleine Hochschulen, für die es nicht einfach ist, international bekannt zu werden. Um qualifizierte ausländische Studierende zu gewinnen, ist es sinnvoll, alternative Wege zum Studium aufzuzeigen. Durch die Teilnahme an einem alternativen Auswahltest, der nur von den Fachhochschulen anerkannt wird, werden Bewerber auf das attraktive Studienangebot der Fachhochschulen aufmerksam. Mit dem Test wird also auch beabsichtigt, einen Marketingeffekt zu erzielen und qualifizierte Studienbewerber an eine Gruppe von Hochschulen zu binden.

\section{$4 \quad$ Zulassung ausländischer Studienbewerber}

Wenn das Ergebnis eines Auswahltests im Zulassungsverfahren berücksichtigt werden soll, gibt es zwei Möglichkeiten: Man kann von allen Bewerbern die Teilnahme an einem Auswahltest verlangen oder man kann die Teilnahme freistellen.

Wird die Teilnahme an einem Auswahltest zwingend vorgeschrieben, könnte die Bewerbungsnote aus dem arithmetischen Mittel aus Auswahltest und Heimatnote gebildet werden. Beispiel: Aus der Heimatnote 1,0 und TASK-Note 3,0 ergibt sich die Bewerbungsnote 2,0. Was aber macht man, wenn Bewerber nicht am Auswahltest teilgenommen haben, obwohl es in der Zulassungsordnung vorgesehen ist? In diesem Fall sollten Bewerber nicht vom Verfahren ausgeschlossen werden, sondern es sollte die niedrigste Bestehensnote angenommen werden. Beispiel: Aus der Heimatnote 1,0 und der fiktiven TASK-Note 4,0 ergibt sich die Bewerbungsnote 2,5.

Was geschieht, wenn man die Teilnahme am Auswahltest nicht zur Zulassungsvoraussetzung macht? Dann könnten Bewerber, die an einem Auswahltest teilgenommen haben, ihre Bewerbungsnote verbessern, wenn das Ergebnis im Auswahltest über demjenigen der Heimatnote liegt. Für Kandidaten, die bereits eine gute Heimatnote haben, lohnt sich die Teilnahme am Auswahltest nicht. Wird die Teilnahme an einem Auswahltest nicht vorgeschrieben, bietet die Teilnahme am Auswahltest für die Studienbewerber die Gelegenheit, ihre Heimatnote zu verbessern. Meine Empfehlung ist, dass die Teilnahme an einem Auswahltest 
zur Zulassungsvoraussetzung gemacht wird. Nur auf diese Weise kann die Ungenauigkeit der Heimatnote relativiert werden. Ein freiwilliger Aufnahmetest würde nur Kandidaten mit einer schlechten Heimatnote dienen. Aus Sicht der Hochschule würde die Auswahl kaum verbessert.

\section{Resümee}

Der geringe Studienerfolg ausländischer Studierender ist nicht nur für die betroffenen Personen, sondern auch für den internationalen Studienstandort Deutschland problematisch. Ein Ansatz zur Verbesserung dieser Situation liegt in der gezielteren Auswahl von ausländischen Studienbewerbern. Mit dem TASK wurde ein Auswahlinstrument konzipiert, das sich an der FSP orientiert. Ebenso wie die FSP soll der TASK eine intensive Studienvorbereitung auslösen und damit zur Erhöhung des Studienerfolgs beitragen. Ersetzen kann der TASK die FSP nicht. Es handelt sich um ein Auswahlinstrument, dessen Niveau unter demjenigen der FSP liegt. Bewerber an Fachhochschulen in Baden-Württemberg können in Zukunft entscheiden, ob sie am Studieneignungstest von Test AS teilnehmen oder am TASK. Die Entscheidung dürfte vor allem dann für den TASK fallen, wenn die Bewerber an studienvorbereitenden Kursen teilgenommen haben. Wer sich noch im Heimatland aufhält oder wer rasch mit dem Studium beginnen möchte, wird sich für den Test AS entscheiden. Die FSP bleibt von dieser Entscheidung unberührt: Wer sich nur über die FSP für ein Studium qualifizieren kann, hat auch in Zukunft keine Wahl.

Zu Beginn meines Beitrags habe ich zwei mögliche Reaktionen auf den geringen Studienerfolg ausländischer Studierender angesprochen, mich in den folgenden Ausführungen jedoch nur auf einen Aspekt konzentriert, die Verbesserung der Auswahl. Ich möchte zum Schluss noch einmal darauf hinweisen, dass verbesserte Auswahlverfahren und eine verbesserte Auswahl allein nicht ausreichen, um Deutschland als internationalen Studienstandort zu fördern. Auch wenn man nur noch vermeintlich „geeignete“, besonders „fähige“ Studienbewerber aus dem Ausland zulässt, bleibt als Aufgabe, ein Studien- und ein Betreuungsangebot bereit zu stellen, das auf die Situation ausländischer Studienbewerber abgestimmt ist. Der Studienerfolg hängt nicht allein von Kenntnissen und intellektuellen Fähigkeiten ab. Schwierigkeiten mit der Finanzierung, die Studienmotivation und die Betreuung im Studium dürften eine ebenso große Rolle spielen. Diese Aspekte werden von Auswahltests jedoch nicht erfasst. 


\section{Literatur}

Deidesheimer Kreis. 1997. Hochschulzulassung und Studieneignungstests. Göttingen und Zürich: Vandenhoeck und Ruprecht.

Graham, Janet G. 1987. English language proficiency and the prediction of academic success. TESOL Quarterly 21(3), S. 505-521.

Haase, Klaudia; Trost, Günter. 2005. Hochschulzulassung: Auswahlmodelle für die Zukunft. Essen und Stuttgart: Stifterverband für die Deutsche Wissenschaft und Landesstiftung Baden-Württemberg.

Heldmann, Werner. 1984. Studierfähigkeit, Ergebnisse einer Umfrage (Schriften des Hochschulverbandes, 29). Göttingen: Otto Schwartz.

Heublein, Ulrich. 2005. Studienverlauf im Ausländerstudium. Vortrag auf der Jahrestagung der Direktorinnen und Direktoren von Studienkollegs am 3. Mai 2005 in Darmstadt.

Heublein, Ulrich; Sommer, Dieter; Weitz, Birgitta. 2004. Studienverlauf im Ausländerstudium. Eine Untersuchung an vier ausgewählten Hochschulen (Dokumentationen \& Materialien). Bonn: Deutscher Akademischer Austauschdienst.

Trost, Günter. 2003. Deutsche und internationale Studierfähigkeitstests. Arten, Brauchbarkeit, Handhabung (Dokumentationen \& Materialien, 51). Bonn: Deutscher Akademischer Austauschdienst. 



\section{Eine Prüfung für $\mathrm{DaF}$ in den Niederlanden am Beispiel Hörverständnis}

Dorrie Goossens, Arnheim (Niederlande)

\section{Cito}

Cito ist die Abkürzung für Zentrales Institut für Testentwicklung (auf Niederländisch: Centraal Instituut voor Toetsontwikkeling). Cito wurde 1968 vom niederländischen Kultusministerium gegründet, um Materialien zur Evaluierung von Schülerleistungen zu entwickeln und zu erstellen - damals in erster Linie die zentralen Abschlussprüfungen. Im Laufe der Jahre wurde Cito immer weiter erweitert und ab 1999 zum größten Teil privatisiert.

Im September 2006 hatte Cito etwa 500 Mitarbeiter und Hunderte von „,freien“ Mitarbeitern, meistens Lehrkräfte, die z. B. einige Stunden pro Woche mit einem Cito-Testexperten an den zentralen Abschlussprüfungen arbeiten. 


\section{Abteilungen:}

Cito hat 3 Primärabteilungen:

- $\mathrm{VO}=$ Sekundarunterricht; subventioniert vom Kultusministerium und unter desssen Verantwortung; Entwicklung und Erstellung der zentralen Abschlussprüfungen.

- $\quad$ POVO = Primar- und Sekundarunterricht; Entwicklung und Erstellung von Test- und Unterrichtsmaterialien unter eigener Verantwortung; nur einige „Produkte“ sind subventioniert. U. a. die Hörverständnistests gehören zu dieser Abteilung, aber auch der Abschlusstest für die Grundschule und die Schüler- und Unterrichtsmonitoringsysteme für den Primar- und den Sekundarunterricht.

- $\mathrm{BBE} / \mathrm{HO}=$ Beruf, Betrieb und Ausbildung/Hochschulunterricht; Entwicklung und Erstellung von Test- und Unterrichtsmaterialien für berufsbildende Schulen, für den Tertiarbereich und für Betriebe; dieser Bereich wird nicht subventioniert.

Bei Business Development werden Tests und Examen- und Monitoringsysteme für Betriebe und Dachverbände (z. B. Makler, Arbeitsschutz u.dgl.) entwickelt und erstellt, aber auch implementiert.

Die beiden internationalen Abteilungen beschäftigen sich vor allem mit der Entwicklung, Erstellung oder Anpassung von Unterrichtsmateralien für das Ausland, aber auch mit der Unterstützung von Kultusministerien oder Exameninstituten in anderen Ländern wie z. B. in Deutschland, Frankreich, den Vereinigten Staaten, der Türkei, Montenegro, Azerbeidschan und Malaysia.

In Deutschland (Butzbach), in der Türkei und in den Vereinigten Staaten hat Cito inzwischen eigene Büros.

\section{Das Unterrichtssystem in den Niederlanden}

In den Niederlanden findet die Einschulung der meisten Kinder statt, wenn sie vier Jahre alt sind: Schulpflicht gibt es aber erst ab fünf.

\section{Primarunterricht}

In der Grundschule (auf Niederländisch basisschool) werden die 8 Jahrgangsstufen Gruppen genannt. Die ersten zwei Gruppen, also die Vier- und Fünfjährigen werden noch mehr oder weniger spielerisch „unterrichtet“, ab Gruppe drei (also die Sechsjährigen, in Deutschland erste Klasse) wird Lesen, Schreiben und Rechnen u. dgl. gelehrt und gelernt. Ab Gruppe sieben gibt es Englisch als Pflichtfach, eine andere Fremdsprache als Englisch wird in der Grundschule (mit einigen Ausnahmen) nicht unterrichtet, wohl gibt es eine Möglichkeit, dass Migrantenkinder zusätzlichen Unterricht in ihrer eigenen Muttersprache bekommen, der dann außerhalb der regulären Unterrichtssstunden stattfindet. 


\section{Sekundarunterricht}

Am Ende der Grundschule gibt es einen Test (an $85 \%$ der Schulen den „Cito Eindtoets Basisonderwijs“) und gibt die Lehrkraft von Gruppe 8 eine Empfehlung. Diese Beiden bestimmen, welche Sekundarschule das Kind besuchen wird, obwohl natürlich auch der Wunsch der Eltern und des Kindes eine Rolle spielen. Im Sekundarunterricht gibt es meistens eine ein- oder zweijährige mehr oder weniger heterogene „Brücken“-Periode, worin die allgemeinbildenden Fächer unterrichtet werden. Neben Englisch als Fremdprache gibt es dann in der ersten Klasse (jetzt spricht man von Klassen und fängt man wieder bei „eins“ an, die SchülerInnen sind \pm 12 Jahre alt) auch Französisch und meistens erst in der zweiten Klasse Deutsch. Praktische Fächer gibt es nur im „vmbo“, aber auch da in den ersten zwei Jahren wenig.

Nach einem oder zwei Jahren wird dann, anhand der Noten, bestimmt, welches Unterrichtsniveau am besten geeignet ist für den Schüler/die Schülerin.

Im „Vmbo“ (vorbereitend berufsbildend), wo es 4 sog. Lehrgänge, d. h. Niveaus, von rein theoretisch bis mehr praktisch orientiert gibt (TL-GL-KB-BB), wird dann auch der Unterrichtszweig, der sog. Sektor für die letzten zwei Jahre bestimmt. Es gibt eine Wahl aus Wirtschaft, Landwirtschaft, Technik, Verpflegung und Sozialwesen.

Im „havo“ (allgemeinbildend) und im „vwo“ (vorbereitend wissenschaftlich) findet diese Wahl ein Jahr später statt, da gibt es vier sog. Profile:

1. Natur und Technik, Schwerpunkt Mathematik und Physik;

2. Natur und Gesundheit, Schwerpunkt Biologie und Chemie;

3. Wirtschaft und Gesellschaft, Schwerpunkt Wirtschaftslehre und Erdkunde und

4. Kultur und Gesellschaft, Schwerpunkt Sprachen und Kunst .

Alle Sekundarschulen werden mit einer zur Hälfte zentralen (siehe unten Abschn. 4) Prüfung abgeschlossen. Im „vmbo“ nach insgesamt vier Jahren. Nach dem „vmbo“ besuchen die meisten SchülerInnen noch die Berufsfachschule (mbo), sie sind dann meistens auch noch schulpflichtig, die Ganztagsschulpflicht dauert bis zum vollendeten 16. Lebensjahr, die Teilzeitschulpflicht bis zum 18.

Der „mbo“ (weiterführende berufsbildende Unterricht) dauert vier Jahre, auch wieder eingeteilt in vier Klassen, aber auch vier Unterrichtsniveaus, von Niveau eins bis Niveau vier. Meistens gibt es im dritten Jahr ein Praktikum.

Der „havo“ wird nach insgesamt fünf Jahren abgeschlossen und die meisten SchülerInnen besuchen dann eine Fachhochschule, die vier Jahre dauert und worin es auch ein Praktikum gibt. Die Fachhochschule wird mit dem „Bachelor“Examen abgeschlossen.

Der „Vwo“ wird nach sechs Jahren abgeschlossen, also ein Jahr weniger als in den meisten Bundesländern und dann studieren die AbiturientInnen entweder an 
einer Fachhochschule oder an einer Universität weiter. Meistens dauert ein akademisches Studium vier Jahre, manchmal fünf, drei/vier Jahre für das „Bachelor“Examen, ein/zwei Jahr(e) für das „Master“-Examen.

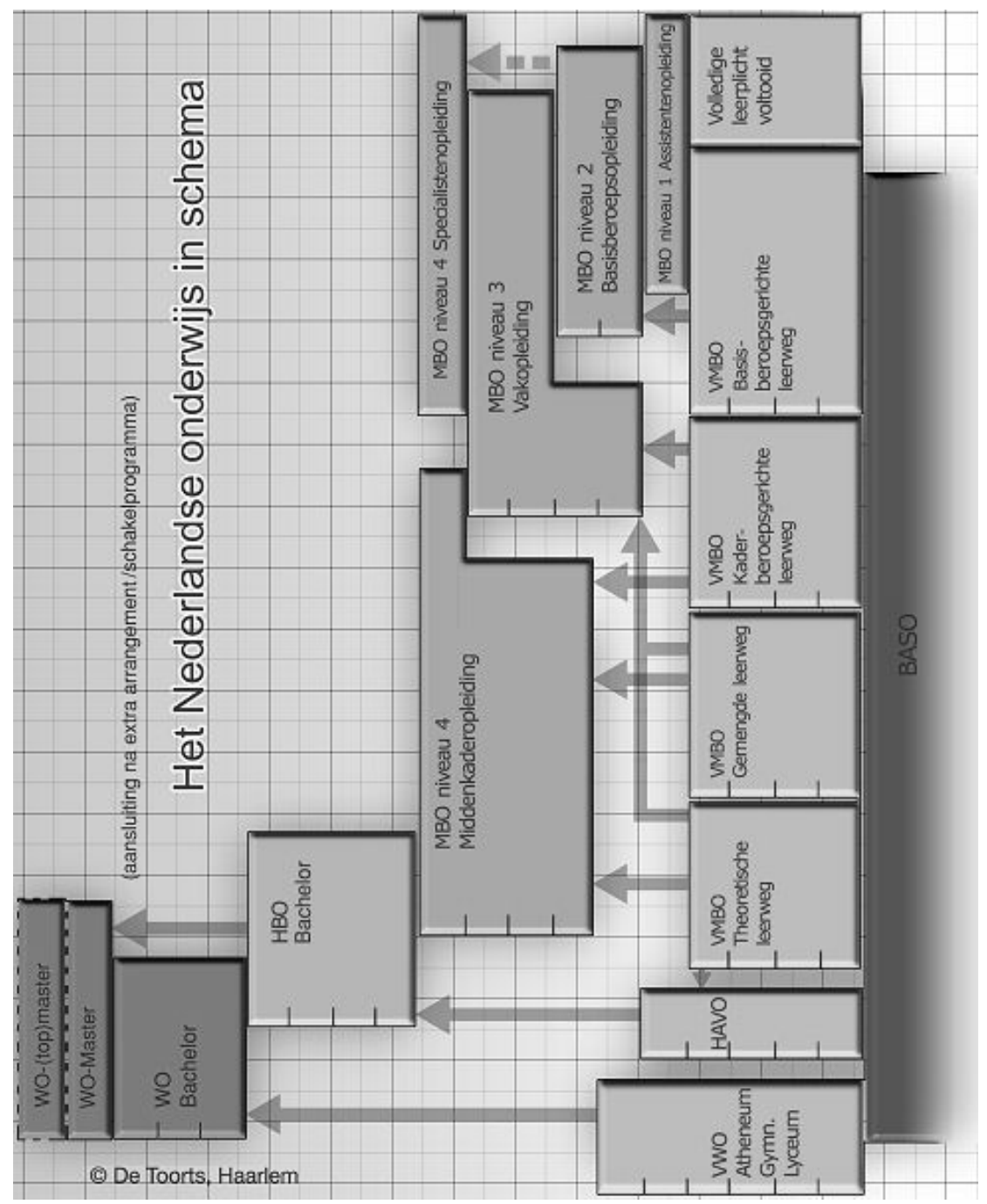

\section{Die Position von DaF in den Niederlanden}

Die Sekundarschulen dürfen schon in der ersten Klasse mit Deutsch anfangen, aber meistens wird das nicht gemacht. Insgesamt gibt es dann im ganzen Sekundarunterricht nämlich nicht mehr Unterrichtsstunden. 
Im „vwo“ ist Deutsch drei Jahre Pflichtfach, (wenn schon in der 1. Klasse angefangen wird vier Jahre) dann Wahlfach, aber die SchülerInnen müssen, neben Englisch, eine zweite Fremdsprache wählen. Diese Fremdsprache kann Deutsch oder Französisch sein, ist es meistens auch, kann aber auch Arabisch, Türkisch, Friesisch, Spanisch oder Russisch sein. Im altsprachlichen Gymnasium können Latein oder Griechisch als zweite Fremdsprache gewählt werden.

Im „havo“ ist Deutsch zwei oder drei Jahre Pflichtfach, danach Wahlfach, es gibt aber keine Verpflichtung eine zweite Fremdsprache zu wählen.

Im „vmbo“ muss in den drei obersten Lehrgängen (KB, GL und TL) eine zweite Fremdsprache angeboten werden, das braucht kein Deutsch zu sein, ist es aber meistens wohl. Nach einem oder zwei Jahren ist Deutsch dann Wahlfach.

Deutsch als Wahlfach verliert immer mehr an Bedeutung, das gilt auch für Französisch. Englisch ist für alle SchülerInnen in allen Schulen verpflichtet und das genügt den meisten. Dass die Niederlande jährlich Milliarden an Einkünften verlieren, nur weil es zu wenige Leute gibt, die Deutsch und Französisch können, ist zwar bei vielen Leuten bekannt, aber anscheinend noch nicht genug.

In einigen Jahren wird wenigstens bei Deutsch sowieso ein großes Problem entstehen, weil es dann wahrscheinlich viel zu wenig DeutschlehrerInnen gibt. Es ist schon viele Jahre so, dass es in Deutschland mehr StudentInnen gibt, die Niederländisch studieren, als in den Niederlanden, die Deutsch studieren. Jetzt schon gibt es in vielen Schulen deutsche DeutschlehrerInnen, was an sich natürlich eine gute Sache ist.

\section{Schulexamen und Abschlussprüfungen}

\section{Schulexamen}

Das Schulexamen wird unter eigener Verantwortung der Schule, aber unter Aufsicht der Schulaufsichtsbehörde in den letzten zwei Jahren des Sekundarunterrichts abgenommen, der größte Teil im letzten Jahr.

Bei den Fremdsprachen werden das Hörverständnis, die Schreibfertigkeit, die Sprechfertigkeit und - bei „,vwo“ - die Literaturkenntnisse getestet. Die Examen oder Tests dürfen von den LehrerInnen selber erstellt werden, aber meistens (auBer für Literatur) wird die Expertise von Cito einberufen. Cito entwickelt und erstellt für die Fremdsprachen Schulexamentests für alle Fähigkeiten und für alle Niveaus.

Die in einer vorher festgelegten Prozedur zustanden gekommene Durchschnittsnote für das Schulexamen bildet die erste Hälfte der Abschlussnote. 


\section{Zentrale Abschlussprüfung/Zentralabitur}

Im Mai des letzten Jahres finden die zentralen Abschlussprüfungen oder das Zentralabitur statt, d. h. dass alle ExamenkandidatInnen oder AbiturientInnen in den Niederlanden an der gleichen Uhrzeit am gleichen Examen arbeiten, das auch in der gleichen Weise mit einer vorher festgelegten Korrekturvorschrift von der eigenen Lehrkraft und von einer Lehrkraft einer anderen Schule nachgesehen wird. Bei den Fremdsprachen wird dann das Leseverständnis getestet.

\section{Abschlussnote}

Die Note für die zentrale Abschlussprüfung oder das Zentralabitur bildet die zweite Hälfte der „Abschlussnote“.

Die beiden nicht ab- oder aufgerundeten Noten werden zusammengezählt, der Mittelwert wird errechnet und dann wird auf- oder abgerundet, so dass eine Ziffer zwischen 1 (die schlechteste Note) und 10 (die beste Note) entsteht. Das ist dann die „Abschlussnote“.

Von einem der 6, 7 oder 8 Zentralfächer darf Ende Juni eine Wiederholungsprüfung (nur vom Zentralteil) gemacht werden. Die beste Note gilt dann.

\section{$5 \quad$ Hörverständnis}

Der Hörverständnistest ist bei den Fremdsprachen für alle Niveaus ein obligatorischer Teil vom Schulexamen. Es ist vom Ministerium festgelegt worden, welche Hörstrategien auf welchem Niveau getestet werden sollen und welchen minimalen Anforderungen die Ausgangsmaterialien genügen müssen.

\section{Geschichte}

Im Schuljahr 1972-1973 wurden nach vielen Untersuchungen und einigen Pilots die ersten Hörverstandnistests von Cito vorgelegt und sie waren vom Anfang an sehr erfolgreich. Es ist den LehrerInnen gestattet, selber Tests zu erstellen oder den Test bei einem anderen Institut zu kaufen, aber das geschieht nur sehr wenig. Im Moment ist es so, dass sogar $95 \%$ der Schulen unsere Hörverständnistests abnehmen.

Am Anfang gab es nur Audiotests, zuerst Tonband, dann Audiokassette, jetzt CD, dann DVD. Ab 2001 wurde ein Teil vom den zwei Teilen des Audiotests von einem Videoteil ersetzt.

\section{Hörstrategie und Aufgabenformats}

Die Hörstrategie bestimmt zum größten Teil das Aufgabenformat. Alle Aufgaben sind Mehrwahlaufgaben. 
Im „vwo“ sind die Aufgaben auf Deutsch, im „havo“ und „vmbo“ auf Niederländisch. Die verschiedenen Hörstrategien mit den dazu gehörenden Aufgabenformaten sind:

1. Globales Hören mit Frage und drei alternativen Antworten.

2. Detailliertes Hören mit Frage und zwei oder drei alternativen Antworten oder zwei alternativen Behauptungen.

3. Selektives Hören mit zwei alternativen Behauptungen (immer Niederländisch).

4. Vorhersagendes Hören mit sog. „cloze“-Aufgaben, wobei ein Wort oder eine Wortgruppe am Ende einer Aussage von einem Pieps ergänzt wird und der Schüler/die Schülerin die Wahl hat zwischen zwei alternativen Ergänzungen.

Die Hörstrategie ihrerseits wird vom Ausgangsmaterial bestimmt, also, wenn eine bestimmte Hörstrategie getestet werden soll, wird bestimmtes Ausgangsmaterial gesucht oder erstellt.

\section{Ausgangsmaterialien}

Die TestexpertInnen im „Cito“ machen Interviews mit deutschsprachigen Personen, die einen für die SchülerInnen interessanten Beruf und/oder ein interessantes Hobby haben und darüber mit einer angenehmen, akzentarmen oder akzentfreien Stimme erzählen können.

Dann gibt es Nachrichten, die auch von den TestexpertInnen aus kleinen Zeitungs- oder Internetmitteilungen zusammengestellt werden. Sie werden dann von einem Muttersprachler vorgelesen.

Weiter werden für den Audioteil aus dem Radio allerhand Sendungen mitgeschnitten, wie z. B. „Hallo Ü-Wagen“ oder „Neugier genügt“ vom WDR oder „Journal am Vormittag“ vom Deutschlandfunk.

Beim Videoteil gibt es Ausschnitte von Sendungen wie „Odysso“ (vorher WiesoWeshalb-Warum), 37 Grad, Menschen hautnah, die große Reportage und SpiegelTV.

\section{Konstruktion eines Seh- und Hörtests}

Die Konstruktion eines Seh-Hörtests dauert insgesamt ungefähr zwei Jahre.

\section{Materialbeurteilung}

Zuerst wird eine große Menge Material beurteilt, und nur ein sehr kleiner Teil wird für brauchbar erklärt. Bei der Auswahl der Materialien achten wir gleich darauf, für welche Hörstrategie, für welches Aufgabenformat und für welches Niveau das Material verwendet werden kann. Das macht auch noch die aus drei DozentInnen bestehende Beratungskommission, die das Material beurteilt. 


\section{Lizenzanfrage}

Dann muss beim Produzenten die Lizenz beantragt werden, was nicht immer sehr leicht ist und oft sehr teuer werden kann.

\section{Auswabl der Passagen, Selektion, Erstellung und Selektion der Aufgaben, 1. Konzept,} Screening und Besprechung, 2. Konzept

Wenn das alles stimmt, kann mit der Bearbeitung angefangen werden. Vom Testexperten werden die geeigneten Passagen (30-60 Sekunden) ausgesucht, dazu wird eine Frage erstellt, dann die richtige Antwort formuliert (oft auch andersherum: Zuerst die Antwort, dann die Frage) und schließlich die „falschen“ Antworten. Nach einigen Revisionen und langem „Feilen“ ist dann das erste Konzept fertig und wird zuerst einem Fachkollegen der gleichen Sprache vorgelegt und dann einem Fachkollegen einer anderen Fremdsprache, bei uns also Französisch oder Englisch. Mit diesem Kollegen wird das 1. Konzept des Testteils dann besprochen und bei der Besprechung wird das Screening des Fachkollegen einbezogen und werden die endgültigen Passagen selektiert und die Fragen und Antworten revisiert. So entsteht das zweite Konzept.

\section{Montage, Kontrollen, Beurteilung Beratungskommission, 3. Konzept, Pretest}

Dann werden die niederländischen Ein- und Anleitungen aufgenommen und findet die erste Montage im Studio statt. Nach den Kontrollarbeiten wird der montierte Test der Beratungskommission vorgelegt und wird von ihr kontrolliert und kommentiert. Mithilfe dieses Kommentars wird vom Testexperten das dritte Konzept der Aufgaben gemacht und dann geht das Testteil als Pretest in die Schulen.

Endgïltige Auswabl der Passagen und Aufgaben, Zusammenstellung des gesamten Tests, Kommentierung Beratungskommission

Nach dem Pretest werden die Ergebnisse sehr ausführlich analysiert und bekommt der Testexperte eine ganze Reihe von psychometrischen Daten: Natürlich der P-Wert, d. h. wieviel Prozent der SchülerInnen haben die richtige Antwort gegeben, der Durchschnitts P-Wert, die Zuverlässigkeit des Tests, die Rir und RarWerte der Aufgaben, d. h. ob die ,guten“ SchülerInnen die Aufgabe richtig gemacht haben und die „schlechten“ SchülerInnen falsch usw. Auch werden die Ergebnisse dieses Tests und dieser KandidatInnen mit den Ergebnissen aller vorherigen Tests und KandidatInnen verglichen und anlässlich dieser Daten kann man sehen, wie gut oder schlecht bestimmte Aufgaben funktionieren und passen. All diese Daten werden dazu benutzt, einen zuverlässiggen und validen Test zusammenzustellen, der auch, was das Niveau betrifft, zu dem Unterrichtsniveau passt, wozu er gedacht worden ist. 
Dieser Vorschlag wird der Beratungskommission wieder vorgelegt und sie kommentiert nur die Auswahl, an den einzelnen Aufgaben darf in dieser Phase nichts mehr geändert werden.

\section{Emeute Montage, Kontrollen, Zusammenstellung der Aufgaben- und Texthefte}

Nach der zweiten Montage (einschließlich einer Fassung mit längeren Pausen für dyslektische SchülerInnen) und den endlosen Kontrollen werden die Aufgabenhefte in drei Fassungen (gegen das Abschreiben während des Tests) zusammengestellt und wird ein Textbuch gemacht, das gebraucht werden kann, wenn der Test nachher mit den SchülerInnen besprochen wird.

\section{Test, Normierung und Evaluierung mithilfe der psychometrischen Daten}

Dann wird alles zu den Schulen geschickt und kann der Test abgenommen werden. Wir empfehlen dafür ein bestimmtes Datum, aber können die Schulen nicht dazu verpflichten.

Die Ergebnisse, die Cito von den Schulen bekommt, werden wieder analysiert und mithilfe dieser Daten wird dann die Normierung bestimmt und den Schulen mitgeteilt. Übrigens sind die Schulen frei, diese Normierung anzuwenden, sie dürfen auch eine andere, strenger oder milder, nehmen.

Schließlich wird der Test mithilfe der psychometrische Daten und der Schülerund Lehrerumfragen evaluiert.

\section{$7 \quad$ Forschung}

Schon vor der Einführung der Hörverständnistests im Jahr 1974 gab es sehr viele Untersuchungen nach dem Testen von Hörverständnis. Wie erstellt man einen zuverlässigen und validen Test, wie soll man die Ergebnisse interpretieren und normieren, welche Aufgabenformats funktionieren gut usw. usw.

Aber auch nach der Einführung wurde weiter geforscht und das ist bis heute so geblieben. Einige Beispiele:

- Validität von Hörverständnistests

- Funktionieren von Falsch-Richtig-Aufgaben

- Aufgabenformats und Zuverlässigkeit

- Niederländisch in den Aufgaben

- Funktionieren von Videotetsts

\section{Untersucbungen der letz̦ten Zeit}

Einige Untersuchungen, die in der letzten Zeit durchgeführt worden sind:

1. Der Gebrauch von offenen Fragen in den Seh- und Hörtests 
Ergebnis: Offene Fragen funktionieren eigentlich besser als geschlossene; sind ein bisschen schwieriger, werden aber als viel schwieriger empfunden; werden sowohl von SchülerInnen als von LehrerInnen abgelehnt.

\section{Niveaurückgang bei Deutsch und Englisch}

Ergebnis: Seit dem Jahr 1994 hat das Niveau, sowohl bei Hörverständnis als auch bei Leseverständnis bei Deutsch und bei Französisch, nicht aber bei Englisch, beträchtlich abgenommen, vor allem bei den „unteren“ Niveaus („vmbo“ und „havo“).

\section{GERR}

Im Moment ist Cito dabei, zu untersuchen, ob und wie die Hörverständnistests mit den „Kann-Beschreibungen“ und den Niveaus des Gemeinsamen Europäischen Referenzrahmens (GERR) verbunden werden können. Die ersten Ergebnisse sind erschienen, aber weitere Forschung ist notwendig. Bis jetzt hat sich erstens herausgestellt, dass das Niveau der drei Fremdsprachen in den Hörverständnistests vergleichbar ist, aber dass das Niveau bei Französch meistens etwas niedriger ist als bei Deutsch und Englisch, und zweitens, dass es einen deutlichen Niveauunterschied gibt zwischen den Tests für die verschiedenen Schultypen. Die „,vwo“-Tests haben \pm B2-Niveau, die „havo“-Tests \pm B1, die „Vmbo“-Tests (zwei Niveaus, KB und TL) zwischen A2 und B1.

\section{8 Übungs-CD-ROM}

Seit einigen Jahren hat Cito auch Übungs-CD-ROMs für die Fremdsprachen Deutsch, Englisch und Französisch für alle Niveaus („,vwo“, „havo“ und „,vmbo“) entwickelt.

\section{Zum selbstständigen Üben}

Mithilfe des CD-ROMs kann ein(e) Schüler(in) selbstständig, zu Hause oder in der Schule oder sonstwo, am Computer Hörverständnis testen und üben. Umfragen haben ergeben, dass das Hörverständnis tatsächlich zunimmt.

Die Tests können natürlich so oft wie erwünscht wiederholt werden, der Schüler/die Schülerin kann so lange oder so kurz über wie er/sie möchte, er/sie bekommt Feedback, d. h. dass nicht nur die richtige Antwort gegeben wird, sondern auch, dass der Text (mit Worterklärungen) abgerufen werden kann und dass im Text die Stellen, welche die Basis für die richtige Antwort bilden, markiert worden sind. Die Ergebnisse werden gespeichert.

\section{Alle Hörstrategien und Aufgabenformats; Extra: Offene Fragen}

Auf den CD-ROMs sind alle oben genannten (Abschn. 5) Hörstrategien und Aufgabenformats abgedeckt, jede CD-ROM hat einen Audio- und einen Videoteil und zusätzlicht 
gibt es auch noch Videofragmente mit offenen Fragen (extensives Hören). Die Aufgaben sind nach ansteigendem Schwierigkeitsgrad eingestuft und gegliedert.

Eine Demo-CD-ROM ist verfügbar. Auf dieser Demo-CD-ROM mit deutscher Bedienungsanleitung und Navigation gibt es fünf Teile von französischen Seh- und Hörtests, nach ansteigendem Schwierigkeitsgrad A2 - C1 (GERR) eingestuft.

\section{Blick in die Zukunft}

Abschließend noch einige Bemerkungen über die Zukunft der Seh- und Hörtests:

- Ab 2007 sowohl Audio als auch Video auf DVD

- Ab 2007 Pilot Computertabschlussexamen havo mit nach Themen zusammengestellten Leseverständnis- und Seh/Höraufgaben

- $A b$ ? verbunden mit Kannbeschreibungen und Niveaus des GERR

- In noch nicht absehbarer Zukunft: Onlinetesting mit adaptiven Aufgaben, vom Computer nachgesehen und normiert

Weitere Informationen:

Dorrie Goossens MA

Testexpertin DaF

Zuständig für: Schulexamen Hörverständnis „vmbo“, „havo“ und „vwo“

Zentralabschlussprüfung „,havo“

Pilot Computerabschlussexamen „havo“

Schulexamen Leseverständnis „,vwo“

Cito Arnheim (Niederlande)

Tel. 0031-263521507 (Direktwahl)

E-Mail: dorrie.goossens@cito.nl

\section{Website:}

www.cito.nl (auf Niederländisch und teilweise auf Englisch)

http://www.cito.nl/de_index.htm (auf Deutsch, nur Cito Deutschland) 



\section{Zur Überprüfung des Hörverstehens in der neuen Fassung der DaF-Abiturprüfung in Polen}

Sylwia Adamczak-Krysztofowicz, Poznań (Polen)

\section{Einleitung: Ziel des Aufsatzes}

Dem Hörverstehen wird ein besonderer Stellenwert bei der Entwicklung der kommunikativen Kompetenz zugeschrieben. Es nimmt nach Werner Kieweg (2003: 23) in der muttersprachlichen Alltagskommunikation im Vergleich zu anderen Fertigkeiten mit 55\% eine führende Position ein, gefolgt vom Sprechen mit 23\%, dem Lesen mit 13\% und schließlich dem Schreiben mit 9\%. Dieses rezeptive Teillernziel zählt somit zu den Basisfertigkeiten, ohne die eine zwischenmenschliche Interaktion nicht möglich wäre. Es wird auch als notwendige Vorstufe und Voraussetzung für das Sprechen angesehen.

Trotz dieser Tatsache steht die graduelle Förderung des Hörverstehens nach wie vor hinter der Ausbildung von anderen Fertigkeiten in fremdsprachlicher Unterrichtspraxis zurück (vgl. die Kritik bei Bernd Voss 1984: 69, Dietrich Eggers 1996: 16, Eynar Leupold 2000: 4 sowie Gert Solmecke 2001: 894). 
Mit den Problemen der auditiven Sprachverarbeitung setzen sich zwar in den letzten Jahren immer mehr fachdidaktische Veröffentlichungen auseinander, wenn man aber das Augenmerk auf die zu diesem Thema erschienene Literatur richtet, kann man ziemlich schnell feststellen, dass es sich bei diesen Publikationen vorwiegend um Seminarberichte und Zeitschriftenaufsätze handelt, die sich durchweg mit muttersprachlichen Verarbeitungsprozessen im Hörverstehen, mit möglichen Schwierigkeiten fremdsprachlichen Hörverstehens sowie optimalen unterrichtlichen Verfahren bei der Schulung des Hörverstehens in fremdsprachlicher Unterrichtspraxis beschäftigen (vgl. u. a. Dietrich Eggers 1999, Claudia Wiemer, 1999 Dieter Wolff 1999, Eynar Leupold 2000 sowie Gert Solmecke 2003).

Darüber hinaus gibt es nur wenige Aufsätze, deren Autoren sich auch um relevante Probleme der Hörverstehensprüfungspraxis im FU kümmern ${ }^{1}$. Das Ziel des vorliegenden Beitrags ist es daher, einen differenzierten Einblick in die unterschiedlichen Hörstile, Hörtexte und Aufgabentypen von Hörverstehenstests anzubieten und sich auf dieser Grundlage mit den Vor- und Nachteilen des Hörverstehensteils der neuen seit 2005 geltenden Fassung der polnischen Abiturprüfung im Fach Deutsch als Fremdsprache auseinander zu setzen.

In einem ersten Schritt werden in diesem Zusammenhang die wichtigsten Aufgaben- und Fragentypen in Hörverstehenstests unter Berücksichtigung verschiedener Hörstile und Hörtexte dargestellt und ihre Vor- und Nachteile diskutiert. Im Mittelpunkt des Interesses wird dabei die Frage stehen, welche Hörfähigkeiten mit welchen Hörtexten und mit welchen Aufgabenstellungen überprüft werden können. In einem zweiten Schritt sollen einige grundsätzliche Überlegungen zur Konzeption der neuen polnischen schriftlichen und mündlichen Reifeprüfung skizziert werden.

Nach diesen einführenden Anmerkungen soll der Hörverstehensteil des im Mai 2005 durchgeführten schriftlichen Abiturexamens vorgestellt und analysiert werden.

\section{Hörstile}

Der Schwierigkeitsgrad eines Hörverstehenstests ist von den gewählten Hörtexten, den jeweilig zu diesen Hörvorlagen gestellten Hörverstehenstestaufgaben und den zu ihrer Lösung erforderlichen Hörstilen abhängig. Unterschiedliche Hörtexte werden mit verschiedenen Verstehensabsichten rezipiert und erfordern in diesem Zusammenhang jeweils andere Hörverstehenstestaufgaben. Die differenzierten Hörverstehensaufgaben legen dagegen die Hörstile fest, welche die Prüfungskandidaten bei Hörverstehenstests zu verfolgen haben. Im Folgenden sollen daher

\footnotetext{
1 Zu den Ausnahmen zählen z. B. die Artikel von Bernd Voss (1984), Dietrich Eggers (1989), Jan van Weeren (1992), Gerhard Bickes (1996) und Sigrid Zehren (1999).
} 
zuerst die wichtigsten Hörstile charakterisiert werden, die bei der Überprüfung des Hörverständnisses in Frage kommen.

In der Fachliteratur ${ }^{2}$ werden beim Hörverstehen mehrere Hörstile unterschieden, die entweder intensives oder extensives Verstehen voraussetzen.

Beim intensiven Hören sind alle der Hörvorlage entnommenen Informationen relevant. Um die Gesamtaussage zu verstehen, müssen auch einzelne inhaltliche Details (z. B. Zahlenangaben, Namen oder Einzelinformationen) herausgehört werden. Bei dieser Hörart sollen sowohl die Makro- als auch die Mikrostruktur des Textes sowie logische Relationen, Modalaussagen und Sprecherintentionen erfasst werden. Um die Einzelheiten richtig zu verstehen, muss man den Text also gleichzeitig auch global hören und verstanden haben. Bei dieser Hörart ist daher gutes Sprachwissen und Sprachkönnen erforderlich, weil die Details nicht nur den Inhalt, sondern auch Grammatik, Lexik, Phraseologie und Intonation umfassen können. Intensives Hören wird in der Fachterminologie daher auch als detailliertes Hörverstehen (auch/inkl. reflektierendes Verstehen) bezeichnet.

Beim extensiven Hören genügen dagegen zentrale Informationen des Textes, um die Gesamtaussage richtig zu verstehen. Von den Fachdidaktikern werden als verschiedene Typen extensiven Hörens solche Hörstile wie; globales Hören (auch kursorisches Hören genannt), selektives Hören und selegierendes Hören unterschieden.

Globales (=kursorisches) Hörverstehen bezeichnet einen Hörstil, bei dem die Hauptaussagen eines Textes verstanden werden sollen. Relevant bei dieser Hörart ist die Konzentration auf die Makrostruktur, das Erkennen des Themas oder der agierenden Personen, das Erfassen der Situation, in der die Hörhandlung abläuft, das Identifizieren der Intention und der Gesamtaussage, Einblick in die Textstruktur sowie Erkennen der Schlüsselbegriffe. Vereinfachend bei diesem Hörverhalten wirkt eine gute Strukturierung des Hörtextes. Da das globale Hören der ersten Orientierung dienen soll, werden bei der Förderung dieser Hörstrategie am häufigsten folgende Fragen gestellt: Um welche Hörtextsorte handelt es sich? Wie ist der Text gegliedert? Wer spricht zu wem? Worüber wird gesprochen? Was ist passiert? Wichtig sind bei der Beantwortung von solchen Fragestellungen keine Einzelinformationen, da es vielmehr darum geht, sich beim Hören einen Überblick zu verschaffen.

Beim selektiven Hörverstehen geht es dagegen in allererster Linie um das rasche Auffinden von bestimmten Informationen. Der Hörer muss dabei die ihn interessierenden Details aus einer Fülle von Informationen auswählen. Das selektive Hören wird durch Vorgaben gesteuert, die bereits vor dem ersten Hören zugänglich sein müssen. Die Konzentration des Hörenden kann dabei auf punktuelle Daten, Eigennamen oder Zahlen gelenkt werden, aber auch auf bestimmte Definitionen, Argumente Pro und Contra sowie konkrete Thesen und Fakten. Bei Übungen zum selektiven Hörverstehen werden häufig solche Textsorten wie Wet-

\footnotetext{
2 Bei der Charakteristik der Hörstile folge ich im wesentlichen Barbara Dahlhaus (1994:79), Dietrich Eggers (1996:20) und Claudia Wiemer (1999:37).
} 
terberichte, Verkehrsdurchsagen, Hörerumfragen, Nachrichten auf dem Anrufbeantworter oder kurze Ansagen (z. B. am Bahnhof oder im Kaufhaus) eingesetzt.

Beim selegierenden Hörverstehen werden auch wie beim selektiven Hören einzelne Informationen entnommen. Die Auswahl erfolgt bei dieser Hörart allerdings nicht durch konkrete Aufgaben, sondern der Hörer entscheidet selbst, gelenkt durch seine subjektiven Erwartungen und Interessen, welche wesentlichen Inhaltsinformationen er dem Hörtext entnehmen möchte, um ihn beispielsweise resümieren zu können. Diese Hörart kommt deshalb authentischen monologischen und dialogischen Hör- und Sprechanlässen im Alltag besonders nahe und ist daher für Prüfungen weniger relevant.

Die gerade genannten Hörstile richten sich nach den Hörtexten, die den Schwierigkeitsgrad eines Hörverstehenstests in besonderem Maße bestimmen. Daher sollen die bei der Überprüfung des Hörverständnisses in Frage kommenden auditiven Textsorten sowie an ihre Auswahl gestellten Forderungen in dem nächsten Teil des Beitrags kurz beschrieben werden.

\section{Hörtexte}

In der gesichteten Fachliteratur zu Möglichkeiten der Klassifizierung von gesprochenen Textsorten werden leider keine konkreten Vorschläge hinsichtlich einer speziellen Typologie im Hinblick auf die Überprüfung des Hörverständnisses auf angemessenem Niveau thematisiert. Aus diesem Grunde wird sich die Autorin in diesem Abschnitt vor allem auf die sehr allgemeinen Typologien von auditiven Texten stützen müssen.

Da Hörtexte sich in einer großen Vielgestaltigkeit präsentieren, werden sie von den Fremdsprachendidaktikern nach unterschiedlichen Kriterien gruppiert.

Werner Hüllen (1977: 35) gliedert beispielsweise gesprochene Textsorten nach ihren Präsentationsformen. Nach diesem Kriterium können dann auditive Texte in drei Gruppen aufgeteilt werden und zwar:

- monologische Hörtexte: Zu dieser Gruppe zählen vor allem Kurzmeldungen im Radio, Werbung, Präsentationen, Berichte, Kommentare, Reportagen, Vorträge;

- dialogische Hörtexte: Dazu gehören Telefonanrufe, Interviews, an denen zwei Gesprächspartner beteiligt sind oder Dialoge aus Filmen oder Theaterstücken und

- multilogische Hörtexte: zu dieser Gruppe zählen Gruppeninterviews, Talkshows, Unterhaltungen, Diskussionen.

Zwischen monologischen und multilogischen Texten stehen nach Werner Hüllen (ebd.) auktoriale Textsorten, also erzählte oder verlesene Texte, zu denen vor allem Hör-Darbietungen der erzählenden Literatur gehören. 
Dietrich Eggers (1996: 34) geht bei der Klassifikation von Hörtexten von gesprochener Rede aus und unterscheidet zwischen spontan gesprochenen Hörtexten, die nur im Gespräch, Dialog oder Multilog vorkommen und vorbereiteten Hörtexten, die bei Nachrichten, Kommentaren oder Vorlesungen anzutreffen sind.

Herbert Rück (1991: 130ff., zitiert nach Gert Solmecke 1996: 89) greift dagegen die funktionale Ebene (Intention des Textproduzenten, potentielle Texterwartung des Rezipienten) für die Systematisierung von auditiven Texttypen auf und schlägt dabei folgende Typologie vor:

- expositorische Texte, d. h. Texte, in denen Sachverhalte beschrieben werden. $\mathrm{Zu}$ dieser Gruppe zählen viele informierende Sach- und Gebrauchstexte sowie landeskundliche Informationstexte;

- instrumentelle Texte, d. h. Texte, die produziert werden, um die Rezipienten zu bestimmten Handlungen aufzufordern. Zu dieser Gruppe von Texten gehören Arbeitsanleitungen, Aufforderungen, Werbeanzeigen, Einkaufsgespräche, Bitten um Auskunft etc.;

- narrative Texte, zu denen alle erzählenden Texte wie z. B. Kurzgeschichten zählen;

- argumentative Texte; dienen dazu, Meinungsunterschiede zu klären und zu überwinden;

- Kontakttexte, d. h. solche, in denen die Kontaktfunktion und Regelung von Beziehungen dominieren, wie es z. B. bei einem small talk oder einer Begrüßung der Fall ist;

- poetische Texte, d. h. Texte, die dem ästhetischen Genuss dienen und schließlich

- dramatische Texte; zu dieser Gruppe zählen Texte, die Mischformen der vorhergehenden Textypen sind.

Andere Kriterien, die bei der Frage der Klassifikation und Auswahl von Hörtexten für die fremdsprachige Prüfungspraxis erwähnt werden sollen, sind die verschiedenen Kommunikationssituationen, also die Hörverstehensüberprüfung in einer direkten face-to-face-Hörsituation während einer mündlichen Prüfung oder in einer indirekten d.h. medienvermittelten Kommunikation, wie es im schriftlichen Teil eines fremdsprachigen Examens der Fall ist. Die genannten Kriterien korrelieren wiederum teilweise mit solchen text(sorten)bezogenen Orientierungsmarken wie der Schwierigkeitsgrad und Authentizität der für den Einsatz in Hörverstehenstests zur Verfügung stehenden Hörvorlagen.

Der Schwierigkeitsgrad und damit zusammenhängende Progression von Hörtexten wird in der Fachliteratur ${ }^{3}$ anhand folgender Faktoren bestimmt:

\footnotetext{
3 Vgl. dazu u.a. Eynar Leupold (2000:8) oder Gert Solmecke (2001:896f.).
} 
- Sprecher: Zahl der Sprecher, ihr Sprechtempo, ihre Aussprache, Dialektfärbungen, Sprecherwechsel, Nebengeräusche, die das Hören erschweren.

- Inhalt, d. h. thematisch-inhaltliche Komplexität, die von der Vertrautheit der Hörer mit dem thematischen Gegenstand, Umfang der vorausgesetzten Sachkenntnisse, der Informationsdichte (viele Informationen werden dicht gedrängt angeboten) und der Informationsredundanz (eine neue Information wird ausführlich beschrieben) abhängig ist.

- Sprache, d. h. sprachlich-syntaktische Komplexität, die von dem Anteil neuer Vokabeln und unbekannter syntaktischer Erscheinungen, Abweichungen von der Standardsprache, Zahl der zu entschlüsselnden Metaphern und Anspielungen auf lexikalischer und syntaktischer Ebene (also vom Grad der Verschlüsselung), Zahl der Gliederungssignale und von der sprachlichen Authentizität bestimmt wird.

- Textsorte, d. h. Vertrautheit bzw. fehlende Vertrautheit der Hörer mit der Textsorte und der Textorganisation sowie mit den relevanten soziokulturellen und soziolinguistischen Merkmalen des Textes, Textlänge, Textgliederung, Authentizität der Textsorte.

Die Frage nach dem Schwierigkeitsgrad eines Hörtextes wird immer gestellt, wenn man nach einer geeigneten Hörvorlage für die Hörverstehensüberprüfung sucht. Damit ist auch die Frage nach dem Einsatz von authentischen Hörvorlagen in fremdsprachlicher Unterrichtspraxis eng verbunden. Die Forderung nach Authentizität von Hörtexten wird in der Fachliteratur seit fast 30 Jahren erhoben 4 . Verwirrend ist dabei allerdings, dass der Begriff ,authentisch“ ganz unterschiedlich definiert wird. Es gibt nach wie vor Fremdsprachendidaktiker, die authentische Texte als nicht für den Gebrauch im Unterricht produzierte Texte auffassen. Andere Autoren (z. B. Gert Solmecke 1996:80ff.), denen ich mich auch anschlieBen möchte, setzen sich dagegen für den Begriff der pädagogisch authentischen, didaktisch authentischen bzw. quasi-authentischen Texte ein. Diese Begriffe bezeichnen allerdings alle Textsorten, die sowohl von Muttersprachlern als auch von Nichtmuttersprachlern im Hinblick auf die Lernsituation produziert wurden, die jedoch in einer realen Kommunikationssituation zum Einsatz kommen könnten. Dabei müssen sie aber möglichst „echt“ wirken und eine Reihe von typischen textinternen und wesentlichen textexternen Merkmalen der jeweils beabsichtigten Textsorte erfüllen ${ }^{5}$, d. h. reale situative Einbettung und eine wirkliche Autorenintention aufweisen, einen wirklichen Adressaten haben, themabezogen sein und erkennbare Textstrukturmuster besitzen. Nach der hier vorgestellten Definition sollen die in Hörverstehenstests einzusetzenden Hörtexte einerseits relevante Merkmale einer authentischen Hörvorlage aufweisen, zu denen u. a. umgangssprachliche Wörter,

\footnotetext{
${ }^{4}$ Zur Problematik des textlich-sprachlichen Begriffs von Authentizität siehe z. B. S. AdamczakKrysztofowicz (2003:85ff.).

${ }^{5}$ Siehe dazu genauer die Auflistung bei Irmgard Honnef-Becker (1996:50f.).
} 
Pausen, Satzabbrüche, Reformulierungen, Selbstkorrekturen, Missverständnisse, Rückmeldesignale und störende Geräusche (Lachen, gleichzeitiges Sprechen) und Passagen schneller Wechselrede gehören.

Andererseits darf der Hörtext aber die Prüfungskandidaten nicht überfordern. In diesem Zusammenhang muss jede Hörvorlage einer strengen Kontrolle unterzogen werden. Dabei sollen die Lehrenden und/oder die Prüfenden bei der Textauswahl folgende Forderungen an authentische wie quasi-authentische Hörtexte stellen:

- Der Hörtext muss die Erwartungshaltung der Hörer berücksichtigen, also für sie hörenswert sein.

- Der Hörtext soll mit eindeutigen Überschriften versehen sein, um topdown Prozesse in Gang zu setzen.

- Der Hörtext soll eine textsortenspezifische Makrostruktur aufweisen und deutlich in Teilthemen, Abschnitte, wichtige und unwichtige Nebeninformationen gegliedert sein.

- Die Anzahl der Redundanzen muss gegenüber einem Lesetext in einem Hörtext wesentlich höher sein, die Redundanzen sollen gleichmäßig über den ganzen Text verteilt sein, zusätzliche Redundanzen (wie Beispiele) sollen argumentative Ausführungen, Engstellen wie Definitionen und komplexere Deskriptoren entlasten.

- Nominale Häufungen oder Nominalkomposita sollen in einem Hörtext nicht vorkommen.

- Der Anteil unbekannter Wörter muss begrenzt sein. Den Lernenden unbekannte Wörter sollen eher in inhaltlich redundanten Textteilen geäußert werden.

- Der Hörtext soll keine bzw. sehr wenige Metaphern, Exkurse und Ironisierungen aufweisen und einen gradlinigen Gedankengang besitzen. Intentionale Standpunkte des Sprechers sollen außerdem möglichst klar geäußert werden.

- Informationsschwerpunkte sollen über den ganzen Text verteilt sein, um den fremdsprachigen Prozess des Textverstehens nicht zu behindern.

- Der Hörtext muss an das vorhandene Sprach- und Weltwissen des Hörers anknüpfen, Wissensbestände erweitern und zur Hypothesenbildung sowie zu Stellungnahmen herausfordern.

- Dialogische und multilogische Texte sollen darüber hinaus nicht zu kurz sein, damit sich Gedanken entfalten lassen.

- Die technische Qualität der über Tonträger abgespielten Hörtexte muss schließlich auch garantiert sein ${ }^{6}$.

\footnotetext{
${ }^{6}$ Die Liste von Forderungen an die in Hörverstehenstests einzusetzenden Hörtexte wurde in Anlehnung an Dietrich Eggers (1996: 35f.) und Claudia Wiemer (1999: 49) zusammengestellt.
} 
Die bis jetzt gemachten Erläuterungen zu Hörtexten samt vorangegangenen Ausführungen zu Hörstilen können nun den Hintergrund abgeben zu einer kurzen Darstellung der relevanten Hörverstehenstestaufgaben im Allgemeinen und der Diskussion der Zusammenhänge zwischen allen bis jetzt genannten Variablen und ihrer Vor- und Nachteile bei der Überprüfung des Hörverständnisses ${ }^{7}$ insbesondere.

\section{Hörverstehenstestaufgaben}

Mit den bereits dargestellten Hörtexten und Hörstilen hängen unterschiedliche Hörverstehenstestaufgaben zusammen. Von ihnen hängt, wie bereits in Abschnitt 2 erwähnt, der Schwierigkeitsgrad eines Hörverstehenstests entscheidend ab. Daher soll auf die Typologie von Höraufgabentypen in informellen und standardisierten Tests $^{8}$ in dem vorliegenden Teil des Beitrags detaillierter fokussiert werden.

Die bei Sprachtests in Frage kommenden Testaufgaben und Übungstypen werden in der Fachliteratur (vgl. u. a. Hans-Georg Albers und Sibylle Bolton 1995: 27ff. sowie Sigrid Zehren 1999: 59ff.) nach dem Grad ihrer Offenheit in offene, balboffene und geschlossene Aufgaben gruppiert. Im folgenden Abschnitt werden diese drei Grundtypen von Testaufgaben beschrieben und ihre Vor- und Nachteile in Bezug auf die Überprüfung des Hörverständnisses analysiert. Im Mittelpunkt der Analyse wird dabei die Frage stehen, welche Hörstile mit welchen Hörtexten von diesen Aufgabenstellungen überprüft werden können.

\subsection{Offene Aufgaben}

Offene Aufgaben sind Aufgaben, die von dem Prüfungskandidaten relativ frei und selbständig formuliert werden müssen. Diese Aufgaben eignen sich vor allem zur Überprüfung der produktiven Fertigkeiten. Aber auch das Hörverstehen kann mit offenen Aufgaben überprüft werden. Dieser Aufgabentypus kann nämlich sowohl zur Kontrolle und Bewertung des extensiven Hörens (also des Globalverstehens und des selektiven Hörverstehens) als auch des intensiven Hörens (also des Detailverstehens) verwendet werden. Offene Aufgabenstellungen enthalten vor allem offene Fragen zum Textinhalt und zu Textzusammenhängen, stichwortartige Beantwortung von globalen W-Fragen, Eintragung von einzelnen Informationen in

\footnotetext{
${ }^{7}$ Gerhard Bickes differenziert zwischen „Hörverstehen als einem durch auditiven Input ausgelösten Verarbeitungsprozess und Hörverständnis als dem hieraus resultierenden Bewusstseinsinhalt" (1996: 151). Seiner Ansicht nach ist nur das über andere sprachliche bzw. nichtsprachliche Tätigkeiten vermittelte Hörverständnis nachvollziehbar und dadurch überprüfbar.

8 Auf die Differenzierung zwischen standardisierten und informellen Tests sowie feine Unterschiede zwischen Einstufungstests (Placementtests), Diagnosetests, lernzielorientierten Tests

(Achievementtests), Aptitudetests und Proficiencytests kann im Rahmen des vorliegenden Beitrags nicht näher eingegangen werden. Siehe dazu u. a. Dietrich Eggers (1989: 83f.), Hans-Georg Albers und Sibylle Bolton (1995: 14ff.) oder Sigrid Zehren (1999: 57f.)
} 
Raster oder Zusammenfassungen der relevanten Hauptinformationen von Texten. Offene Aufgaben lassen testmethodisch gesehen jegliche Vielfalt der potentiellen Hörtexte zu. Am häufigsten werden jedoch für die Beantwortung von offenen Fragen Geschichten, Rundfunkberichte, Radiokommentare, Reportagen, Rundfunkmeldungen oder kurze Gesprächsbeiträge eingesetzt. Der Vorteil des offenen Aufgabentyps liegt darin, dass offene Fragen oft viel leichter und schneller formuliert werden können als z. B. Multiple-choice-Aufgaben. Allerdings muss man bei eng mit dem Text verbundenen Fragestellungen beachten, dass die Testkandidaten (vor allem in der Grundstufe) bei ihren Antworten die zu der jeweiligen Frage passende Textstelle aus dem Gedächtnis reproduzieren können, ohne diese Textpassage wirklich verstanden zu haben. Bei offenen freien Aufgabentypen muss man dagegen darauf Rücksicht nehmen, dass die Prüfungteilnehmer bei der Hörverstehensüberprüfung auch produktive Schreibleistungen erbringen müssen, bei denen viele stilistische Unvollkommenheiten sowie verschiedene orthographische und grammatische Fehler entstehen können. Sigrid Zehren (1999: 60) empfiehlt in dieser Situation bei der Beurteilung zu erwägen, ob und in welcher Gewichtung sprachliche Fehler bei der Bewertung offener Übungstypen sanktioniert werden sollen. Jan van Weeren (1992: 58) schlägt dagegen noch eine andere Lösung vor:

Diesem Problem könnte insofern Rechnung getragen werden, als die Schüler die Sprache ihrer Wahl benutzen dürfen, Muttersprache, Fremdsprache oder sogar eine Mischsprache, und etwaige Unzulänglichkeiten in der Formulierung nicht mit bewertet werden. Es wird dann nur auf wesentliche Begriffe, Aussagen und Relationen in der Schülerantwort geachtet.

Das Problem mit einer mangelnden objektiven ${ }^{9}$ Bewertung von offenen Fragestellungen kann man aber auch vermeiden, indem man geschlossene Aufgabentypen zur Überprüfung des Hörverstehens verwendet.

\subsection{Geschlossene Aufgaben}

Geschlossene Aufgaben lassen die Testteilnehmer die richtige Lösung unter den vorgegebenen Antwortalternativen nur herausfinden und markieren. Die Prüfungskandidaten müssen also bei diesem Aufgabentyp ihre Antworten nicht selbständig formulieren. Zu den typischen geschlossenen Aufgabentypen gehören:

- Multiple-choice-Aufgaben (in der Fachliteratur auch als MehrwahlantwortAufgaben bzw. Mehrfachwahl-Aufgaben bezeichnet): Diese Aufgabenformen bestehen aus drei bis vier Alternativen d. h. aus einer richtigen

\footnotetext{
${ }^{9} \mathrm{Zu}$ Gütekriterien für Tests und Prüfungen siehe Dietrich Eggers (1989:83), Hans-Georg Albers und Sibylle Bolton (1995:21ff.) sowie Rüdiger Grotjahn (in diesem Band).
} 
Lösung und zwei bis drei falschen Lösungen (sog. Distraktoren). Dieser Aufgabentyp überprüft vor allem das Detailverstehen, kann aber auch bei der Überprüfung selektiven Hörens eingesetzt werden. Wenn Antwortmöglichkeiten vorgegeben werden, ist das Hörverständnis schnell und einfach auszuwerten. Ein weiterer Vorteil von Mehrfachwahl-Aufgaben liegt darin, dass sie objektiv mit früher für jede richtige Lösung festgelegten Punkwerten bewertet werden können. Allerdings überprüft dieser Aufgabentypus durch Verstehen und Erkennen der richtigen Lösung nicht nur das Hörverstehen, sondern auch das Leseverstehen. In diesem Zusammenhang empfiehlt Jan van Weeren (1992: 58) dem störenden Einfluss des Leseverstehens durch unkomplizierten Satzbau, angemessene Wortwahl und Knappheit vorzubeugen. Die klar formulierten Antwortangaben sollen auch nach Sigrid Zehren (1999: 60) in ihrem Schwierigkeitsgrad dem Hörtext entsprechen. Ein weiteres Problem von Multiplechoice-Aufgaben ist mit der Tatsache verbunden, dass bei ihrer Lösung keine produktive Sprachleistung erforderlich ist und daher die Gefahr besteht, die richtige Antwort durch die Eliminierung von Distraktoren und Raten anzukreuzen. Schwierigkeiten bei diesem Aufgabentypus kann schließlich auch die Konstruktion von akzeptablen und attraktiven Distraktoren bereiten.

- Alternativantwort-Aufgaben (d. h. Ja/Nein ankreuzen oder Richtig/Falsch ankreuzen bzw. Zwei-Thesen-Aufgabe): Bei diesen Aufgabenformen sollen die Prüfungskandidaten entscheiden, ob die zu dem Textinhalt formulierten Aussagen mit dem Text übereinstimmen oder nicht. Dieser Aufgabentyp kann sowohl intensives als auch extensives Hören überprüfen. Ein Vorteil von Alternativantwort-Aufgaben liegt in der sehr leichten und schnellen Auswertung der Tests. Ein Nachteil ist dagegen die zu hohe Ratewahrscheinlichkeit.

- Zuordnungsaufgaben: Diese Aufgabenformen testen in erster Linie das selektive Hörverstehen. Dabei müssen die Testteilnehmer passende Informationen oder Teile einander zuordnen. Bei der Überprüfung des Hörverständnisses können z. B. Aussagen aus einem Hörtext einzelnen Personen oder Stichwörter, Überschriften bzw. Bilder oder Cartoons passenden Texten zugeordnet werden. In diesem Zusammenhang werden die Prüfungskandidaten aufgefordert, die Zuordnungen durch Pfeile bzw. in ein vorgegebenes Raster einzutragen oder entsprechende Rubriken anzukreuzen.

Für alle oben präsentierten geschlossenen Aufgabentypen werden verschiedene Hörtexte eingesetzt u. a. Hörerumfragen, Nachrichten, Rezepte, Berichte, Interviews, oder Gespräche usw. 
Neben offenen und geschlossenen Aufgaben lässt sich das Hörverstehen auch durch halboffene Aufgaben überprüfen.

\subsection{Halboffene Aufgaben}

Bei halboffenen Aufgabenformen müssen die Testteilnehmer ihre Antworten innerhalb eines präzise begrenzten Kontextes nur teilweise selbst formulieren. $\mathrm{Zu}$ den halboffenen Aufgaben zählen:

- Ergänzugsanfgaben: Hierunter werden vor allem Einzelsätze, Listen und Tabellen mit Lücken verstanden, die durch passende Wörter, Stichwörter oder Satzteile ergänzt werden müssen.

- Lückentexte: Bei diesem Aufgabentyp sollen die Prüfungskandidaten die von dem Autor des Tests gezielt vorgegebenen Lücken in einem zusammenhängenden Text ergänzen.

- Cloze-Tests: Hierunter werden die Testteilnehmer aufgefordert, die mechanisch getilgten Wörter (z. B. jedes sechste Wort) zu ergänzen.

Für halboffene Aufgabentypen eigenen sich u. a. solche Hörtextsorten wie Verkehrsdurchsagen, Wetterberichte, Ausschnitte einer Rede, Nachrichten oder Werbetexte.

Halboffene Aufgaben kommen relativ selten bei der Überprüfung des Hörverständnisses (in der Regel nur des Detailverstehens) zum Einsatz, weil diese mit umfangreicheren Schreibarbeiten verbundenen Testaufgaben häufig zu hohe Anforderungen an das Mitschreiben stellen und dadurch Speicher- und Antizipationsprozesse behindern können.

Neben der oben beschriebenen Aufgabentypologie zum Testen des Hörverständnisses wird auch in der Fachliteratur (vgl. dazu Bernd Voss 1984: 74ff. oder Eynar Leupold 2000: 9) eine grundsätzliche Unterscheidung getroffen zwischen Aufgaben, die eine sprachliche Antwort erfordern (sog. verbalen Aufgaben) und solchen, die durch eine nichtsprachliche Antwort erfüllt werden (sog. nonverbalen Aufgaben).Verbale Aufgaben, wie die Beantwortung von Fragen, kommen in allen Sprachtests und Stufen zum Einsatz. Nonverbale Aufgaben eignen sich dagegen vor allem für die Grundstufe und lassen sich gut in informellen Tests einsetzen. Diese Aufgabenformen können beispielsweise durch das Nachzeichnen einer im Hörtext gegebenen Wegbeschreibung oder das Ergänzen eines vorgegebenen Bildes nach Instruktion ausgeführt werden.

Die bis jetzt unter Berücksichtigung verschiedener Hörstile und Hörtexte im Überblick dargestellte Hörverstehenstestaufgabentypen sollen im Abschnitt 6 des vorliegenden Beitrags die Grundlage für die Analyse des Hörverstehensteils der polnischen DaF-Abiturprüfung bilden. 


\section{$5 \quad$ Einige grundsätzliche Anmerkungen zur Konzeption der neuen DaF-Abiturprüfung in Polen}

Bevor in einem nächsten Schritt der Hörverstehensteil der neuen polnischen DaFReifeprüfung vorgestellt und diskutiert wird, ist es notwendig, einige grundsätzliche Überlegungen zur Überprüfung vom Fach Deutsch als Fremdsprache in dem neuen Konzept des polnischen Abiturs zu skizzieren, was im Folgenden nun beabsichtigt wird.

Die neue für neun lebende Fremdsprachen geltende Reifeprüfungsform wird von einer zentralen Prüfungskommission erstellt, organisiert und an allen polnischen Oberschulen nach genau definierten Richtlinien jedes Jahr (im Mai und Januar) durchgeführt und ausgewertet. Die seit 2005 für alle polnischen Abiturklassen geltende Reform ermöglicht den Abiturienten je nach erworbenen Sprachkenntnissen selbst das Prüfungsniveau zu bestimmen, was in der alten Fassung des polnischen Abiturs nicht der Fall war (vgl. Magdalena Ptak 2006: 31). Die Grundstufe der Reifeprüfung soll ca. ein Leistungsniveau der Stufe B1 nachweisen, die erweiterte Version des Abiturs dagegen den Abschluss der Stufe B2.

Das neue Prüfungskonzept, das solche Bereiche wie sprachliches Wissen, Rezeption, Produktion, Interaktion und Mediation berücksichtigt, prüft alle produktiven und rezeptiven Fertigkeiten. In diesem Zusammenhang besteht die dem neuen Modell angepasste Prüfungsform aus einer schriftlichen Gruppenprüfung und einer mündlichen Einzelprüfung. Der schriftliche Teil des Grundniveaus dauert insgesamt 120 Minuten und enthält verschiedene Aufgaben zum Lesen, Hören und Schreiben, die auf dem ersten Prüfungsbogen abgebildet sind (siehe Tabelle 1).

Aufgaben zum Hör- und Leseverstehen für die Grundstufe sind meistens als „richtig/falsch“ oder „multiple choice“-Aufgaben konzipiert und bestehen aus einer breiten Palette von didaktisierten Texten, die folgende Themenbereiche betreffen können: Mensch und seine Umgebung, Wohnen, Schule, Arbeit, Familien- und Gesellschaftsleben, Gesundheit, Ernährung, Einkäufe und Dienstleistungen, Reisen und Touristik, Kultur, Sport, Natur, Wissenschaft und Technik, Staat und Gesellschaft und schließlich landes- und kulturkundliche Grundinformationen über die deutschsprachigen Länder.

Bei der Überprüfung des Schreibens sieht das Curriculum des Grundniveaus auch viele differenzierte Textsorten vor, von der Umfrage, über den informellen bzw. formellen Brief bis hin zur Einladung.

Die Abiturienten, die sich für die erweiterte Stufe des Abiturs entschieden haben, sind verpflichtet, innerhalb von 180 Minuten den zweiten und den dritten Prüfungsbogen auszufüllen. Diese Bögen enthalten sowohl schwierigere Aufgaben zum Hörverstehen, Leseverstehen und Schreiben als auch einige lexikalischgrammatische Aufgabentypen. Bei den Leseaufgaben für die erweiterte Stufe handelt es sich nicht nur um das Leseverstehen selbst, sondern auch um das Leseverstehen und Erkennen sowie Gebrauch von grammatisch-lexikalischen Strukturen. 
In dem Schreibteil des erweiterten Abiturs müssen die Schüler eine Rezension, Erörterung, Beschreibung oder Erzählung verfassen.

Die bis jetzt skizzierten Anmerkungen zur Konzeption des schriftlichen Teils der polnischen Reifeprüfung lassen sich zur summarischen Orientierung mit Hilfe der folgenden Tabelle darstellen und ergänzen:

\begin{tabular}{|c|c|c|c|c|c|}
\hline Niveau & $\begin{array}{l}\text { Prüfungs- } \\
\text { bogen }\end{array}$ & Sprachfertigkeit & $\begin{array}{l}\text { Aufgabenzahl- } \\
\text { und typ }\end{array}$ & \begin{tabular}{|l} 
Zeit \\
in \\
Min. \\
\end{tabular} & $\begin{array}{l}\text { Punkt- und } \\
\text { Prozentzahl }\end{array}$ \\
\hline \multirow{3}{*}{ Grundniveau } & \multirow{3}{*}{1} & Hörverstehen & $\begin{array}{l}\text { 2-3 geschlossene } \\
\text { Aufgaben }\end{array}$ & 20 & $15(30 \%)$ \\
\hline & & Leseverstehen & $\begin{array}{l}\text { 2-3 geschlossene } \\
\text { Aufgaben }\end{array}$ & \multirow{2}{*}{100} & $20(40 \%)$ \\
\hline & & Schreiben & 2 offene Aufgaben & & $15(30 \%)$ \\
\hline \multirow{4}{*}{$\begin{array}{l}\text { das erweiterte } \\
\text { Niveau }\end{array}$} & \multirow[b]{2}{*}{ II } & Hörverstehen & $\begin{array}{l}\text { 2-3 geschlossene } \\
\text { Aufgaben }\end{array}$ & 25 & $15(30 \%)$ \\
\hline & & $\begin{array}{l}\text { Leseverstehen u. } \\
\text { Erkennen von lex.- } \\
\text { gr. Strukturen }\end{array}$ & $\begin{array}{l}2-3 \text { geschlossene } \\
\text { Aufgaben }\end{array}$ & 45 & $12(24 \%)$ \\
\hline & \multirow[t]{2}{*}{ III } & $\begin{array}{l}\text { Gebrauch von } \\
\text { lexikalisch- } \\
\text { grammatischen } \\
\text { Strukturen } \\
\end{array}$ & $\begin{array}{l}\text { 1-2 offene } \\
\text { Aufgaben }\end{array}$ & \multirow[t]{2}{*}{110} & $5(10 \%)$ \\
\hline & & Schreiben & 1 offene Aufgabe & & $18(36 \%)$ \\
\hline
\end{tabular}

Tabelle 1: Struktur des schriftlichen Teils der neuen polnischen Abiturprüfung

Bei der Analyse der in der Tabelle abgebildeten Struktur schriftlichen Gruppenprüfung fällt sofort ins Auge, dass dem Teiltest Leseverstehen größerer Stellenwert als dem Untertest Hörverstehen zukommt. Die prozentuale Verteilung der einzelnen Prüfungsteile wird allerdings von der Prüfungskommission nicht begründet.

Die mündliche Prüfung wird ausschließlich als Einzelprüfung durchgeführt. Dabei bezieht sich der mündliche Prüfungsteil auf die bereits genannten thematischen Schwerpunkte.

Bei dem Grundniveau des mündlichen Teils wird erwartet, dass der Abiturient ein Photo beschreibt und drei kurze gelenkte Gespräche führt, bei denen er eine in der jeweiligen Aufgabe vorgegebene Rolle übernimmt, d. h. verhandelt, berichtet bzw. bestimmte Informationen erteilt oder erfragt.

Bei der erweiterten Stufe ist es im mündlichen Teil dagegen erforderlich, dass der Kandidat in der ersten Aufgabe eine Collage beschreibt und interpretiert und in der zweiten Aufgabe eine vielfältige und längere Aussage zu einem Themenbereich präsentiert. 
Die gerade skizzierte mündliche Prüfung kann vereinfacht in der folgenden Tabelle dargestellt werden:

\begin{tabular}{|c|c|c|c|}
\hline Niveau & Aufgabenzahl- und typ & $\begin{array}{l}\text { Punktzahl: } \\
\text { Inhalt }\end{array}$ & $\begin{array}{l}\text { Punktzahl: } \\
\text { Sprache }\end{array}$ \\
\hline \multirow{2}{*}{ Grundniveau } & $\begin{array}{l}\text { Aufgabe 1: } 3 \text { gelenkte Gespräche: } \\
\text { Informieren, Berichten, Verhan- } \\
\text { deln }\end{array}$ & $3+3+3$ & \multirow[t]{2}{*}{5} \\
\hline & $\begin{array}{l}\text { Aufgabe 2: Bildbeschreibung und } \\
\text { Beantwortung von zwei Fragen }\end{array}$ & 6 & \\
\hline \multicolumn{4}{|c|}{ Vorbereitungszeit: 5 Min./ Prüfungszeit: 10 Min./ Gesamtpunktzahl: 20} \\
\hline \multirow{2}{*}{$\begin{array}{l}\text { das erweiterte } \\
\text { Niveau }\end{array}$} & $\begin{array}{l}\text { Aufgabe 1: Collagebeschreibung- } \\
\text { und besprechung }\end{array}$ & 6 & \multirow{2}{*}{7} \\
\hline & $\begin{array}{l}\text { Aufgabe 2: Präsentation zu einem } \\
\text { Themenbereich und Diskussion }\end{array}$ & 7 & \\
\hline
\end{tabular}

Tabelle 2: Struktur der mündlichen Abiturprüfung

Nach dieser kurzen Schilderung des neuen Prüfungskonzepts werden nun relevante Hörstile, Hörtexte und Aufgabentypen des Hörverstehensteils in der neuen Fassung des polnischen DaF-Abiturs beschrieben.

\section{Analyse des Hörverstehensteils in der neuen polnischen DaF-Abiturprüfung}

Das Hörverstehen wird in dem neuen Prüfungskonzept zweimal getestet und zwar sowohl während des mündlichen Teils in direkter mündlicher Kommunikation mit der Prüfungskommission, in der die Prüfungskandidaten als Hörer und Sprecher zugleich agieren müssen, als auch während des schriftlichen Teils in indirekter Kommunikation, d. h. in Form von medienvermittelten Texten und schriftlich fixierten Einzelaufgaben. Im Rahmen des vorliegenden Beitrags möchte ich auf den schriftlichen Teil des polnischen Abiturs näher eingehen.

Anhand von einem mir zur Verfügung stehenden kompletten Prüfungssatz mit Hörtexten und Aufgabentypen (vgl. Anhang) aus dem Hörverstehensteil der im Mai 2005 durchgeführten Abiturprüfung ${ }^{10}$ werde ich die Überprüfung des

$10 \mathrm{Zu}$ Beispielprüfungssätzen siehe u.a. die Internetseiten der polnischen Prüfungskommission: www.cke.edu.pl/index und www.oke.poznan.pl/pliki/informatory/niemiecki.pdf oder die veröffentlichte Sammlung von Beispielprüfungssätzen von Krystyna Luniewska und Zofia Wassik (2004). 
Hörverstehens sowohl im nicht-erweitertem als auch im erweitertem Niveau analysieren und kritisch bewerten. Dabei möchte ich von folgenden Fragestellungen ausgehen:

1. Was wird genau gefordert und geprüft? (Um welche Hörstile geht es vorwiegend?)

2. Auf welche Weise wird das Hörverstehen getestet? (Um welche Hörtexte und Aufgabentypen handelt es sich hauptsächlich?)

3. Wie wird der Hörverstehensteil bewertet?

\subsection{Geprüfte Hörstile}

Die Autoren der neuen Konzeption der Reifeprüfung bleiben in dem AbiturRatgeber für das Jahr 2005 (vgl. Informator maturalny od 2005 roku z języka niemieckiego: 35, www.oke.poznan.pl/pliki/informatory/niemiecki.pdf) in ihrer Lernzielbeschreibung und Differenzierung der nicht-erweiterten und erweiterten Stufe des Hörtests eher allgemein, indem sie fordern, dass die Prüfungskandidaten des Grundniveaus in der Lage sein sollten, einen inhaltlich einfachen und lexikalisch-grammatisch wenig differenzierten Hörtext zu verstehen. Im Gegensatz dazu sollen ihrer Auffassung nach die Prüfungsteilnehmer der erweiterten Stufe, imstande sein, einem sowohl inhaltlich als auch lexikalisch und grammatisch komplexen Hörtext zu folgen. Diese Zielsetzung impliziert für die Autoren der neuen Konzeption in den beiden Stufen die Fähigkeiten, den Grundgedanken des Textes bzw. der einzelnen Textteile zu bestimmen, relevante Informationen auszusortieren, zwischen einem informellen und formellen Textstil zu unterscheiden sowie die Intention des Autors und den Situationskontext (wie z. B. Raum- und Zeitverhältnisse) dem gehörten Text zu entnehmen.

Im analysierten Hörverstehensteil der Prüfung für das Grundniveau wird aber in der Wirklichkeit vor allem geprüft, ob die Prüfungskandidaten die Hörtexte detailliert verstehen können (vgl. die Aufgaben 1.1 bis 1.6 und 2.1 bis 2.9 im Anhang I).

In dem untersuchten Testteil der erweiterten Stufe zielen insgesamt sechs Aufgaben zur Überprüfung von Hörverstehen auf die anderen in dem AbiturRatgeber genannten verbindlichen Hörstrategien d. h. sie testen auch das selektive (vgl. Aufgaben 7.1 bis $7.4 \mathrm{im}$ Anhang II) und globale (siehe Aufgaben 8.1 und 8.2 im Anhang II) Hörverstehen.

\subsection{Getestete Hörtextsorten}

Dem Abitur-Ratgeber für das Jahr 2005 (vgl. Seite 23) kann man die Informationen entnehmen, dass die Prüfungskandidaten in dem Grundniveau zwei bis max. drei Texte von insgesamt 6 Minuten Dauer hören sollten. Die für die Prüfung ausgewählten Textsorten, zu denen Gespräche, Interviews, Diskussionsbeiträge, Durchsagen, Arbeitsanleitungen und narrative Texte gehören sollten, müssen für 
die Abiturienten geeignet sein, d. h. im Schwierigkeitsgrad angemessen und für sie auch adaptiert sein.

Für die erweiterte Stufe werden in dem Abiturratgeber (vgl. Seite 24) auch zwei bis max. 3 Originaltexte bzw. leicht adaptierte Texte vorgesehen. Der Abiturratgeber listet allerdings dabei die bereits in der Grundstufe genannten Textsorten auf und enthält keinerlei Informationen zur Authentizität und Entstehungsgeschichte der Hörtexte. Die Begriffe „Originaltexte“ oder „(leicht) adaptierte Textsorten" werden auch nicht näher definiert.

Die Hörvorlagen werden während des Hörtests sowohl in dem nichterweiterten Niveau als auch in der erweiterten Stufe gleich bearbeitet. Die Prüfungskandidaten erhalten vor dem Hören den entsprechenden Prüfungsbogen und das Antwortblatt und lesen alle Aufgaben zu dem ersten Hörtext ca. drei Minuten lang. Dieses Verfahren hilft ihnen, ihre Aufmerksamkeit auf diejenigen Textpassagen zu konzentrieren, die für die Auswahl der richtigen Aussage ausschlaggebend sind. Nach dem Lesen der Aufgaben hören die Prüfungsteilnehmer zum ersten Mal den Hörtext als Ganzes von der CD mit langen Pausen zum Lösen aller zu dem ersten Text gehörenden Aufgaben. Dann wird dieser Hörtext noch einmal abgespielt und einige Minuten Zeit für die richtige Lösung und Markierung der entsprechenden Antwort auf dem Antwortbogen zur Verfügung gestellt. Der zweite bzw. dritte Hörtext wird auf dieselbe Art und Weise d. h. jeweils zweimal als Ganzes mit zwei langen Pausen vorgespielt, so dass der gesamte Prüfungsteil in der nicht-erweiterten Stufe ca. 20 und in dem erweiterten Niveau ca. 25 Minuten dauert ${ }^{11}$. In dem von mir analysierten Prüfungssatz besteht der Testteil Hörverstehen für das Grundniveau aus zwei unabhängig voneinander zu bearbeitenden monologischen Hörtexten mit jeweils anderen Aufgabentypen. Die beiden Hörtexte zeichnen sich durch eine langsame Sprechgeschwindigkeit und künstliche Intonation aus. Typisches Merkmal der analysierten Texte ist ein deutlicher Leseton, der wenig lebendig und natürlich wirkt, viel zu wenig Merkmale der Mündlichkeit enthält und kaum dem Gesprächs- bzw. Interviewstil in der Wirklichkeit entspricht. Man hat gleich den Eindruck, dass die beiden Texte zuerst geschrieben und dann bei der Aufnahme mit der gleichmäßigen Stimmführung abgelesen wurden.

In dem Testteil Hörverstehen für die erweiterte Stufe überwiegen auch monologisch konstruierte Beiträge, die trotz geänderter Geschwindigkeit und sprachlich-grammatischer Komplexität nicht authentisch wirken, weil sie zu glatt verlaufen, Charakteristika der schriftlichen Kommunikation aufweisen, keine Spuren von Interaktivität enthalten und typische Merkmale der Mündlichkeit wie Pausen, Reformulierungen, Abbrüche, Kooperation der Gesprächspartner durch Sprecherund Hörersignale ausklammern.

\footnotetext{
11 Um den Lesern einen Eindruck von Schwierigkeitsgrad der Hörtexte zu vermitteln, werden in Anhang III und IV die Transkritptionen von Hörtexten des analysierten Pürfungssatzes abgedruckt.
} 


\subsection{Testaufgaben und ihre Bewertung}

Laut dem Abitur-Ratgeber sollen die Testaufgaben zu den Hörvorlagen sowohl in der nicht-erweiterten als auch in der erweiterten Stufe aus einfachen geschlossenen Aufgabentypen bestehen. Die von den Autoren der neuen Konzeption aufgelisteten Aufgabentypen lassen testmethodisch gesehen jegliche Vielfalt vermissen. In der für beide Stufen geltenden Liste (vgl. Seite 23 und 24 des Abitur-Ratgebers für das Jahr 2005) sind zunächst einfache Ankreuzaufgaben zu nennen: Die FalschRichtig-Antworten und drei- bzw. viergliedrige Mehrfachwahl-Aufgaben. Als die dritte und zugleich letzte Möglichkeit kommen verschiedene Zuordnungen in Frage. Den Unterschied zwischen dem Grundniveau und dem erweiterten Programm des Hörverstehenstests bekommt man leider auch in dem Punkt „Aufgabentypen" des Abitur-Ratgebers nicht transparent aufbereitet.

Zur Bewertung des gesamten Prüfungsteils Hörverstehen in beiden Leistungsstufen ist zu sagen, dass es immer pro Aufgabe einen Punkt gibt. Dabei wird in der Benotung zwischen Haupt- und Nebeninformationen nicht differenziert. Die Prüfungskandidaten erhalten für jede richtige Lösung einen Punkt. Bei 15 möglichen Ankreuzmöglichkeiten können sie also insgesamt 15 Punkte bekommen. Das sind sowohl in dem Grundniveau als auch in dem erweiterten Niveau 30\% der maximal erreichbaren Gesamtpunktzahl. Da die Rate-Menge bei den empfohlenen geschlossenen Ankreuzaufgaben relativ hoch ist und die formulierten Aussagen kurz und einfach sind, können sich die Schüler in diesem Teil der Abiturprüfung nicht überfordert fühlen.

\section{Fazit: Empfehlungen für das Erstellen von Hörtestunterlagen}

Aus der kurzen Schilderung des neuen Prüfungskonzepts ist erkennbar, dass die Überprüfung des Hörverstehens in der polnischen Reifeprüfung noch optimiert werden könnte. In diesem Zusammenhang lassen sich für das optimale Erstellen des Prüfungsteils Hörverstehen folgende Empfehlungen formulieren:

- Durch die gezielte und sorgfältige Auswahl von Hörvorlagen sollen in den für die nächsten Jahre geltenden Prüfungsunterlagen solche Hörmaterialien eingesetzt werden, die den Bedingungen von authentischen bzw. quasi-authentischen Hörtexten entsprechen, keinen Eindruck der Künstlichkeit und Schriftlichkeit erzeugen sowie typische Merkmale der Mündlichkeit aufweisen (vgl. Abschnitt 3 des vorliegenden Beitrags).

- Durch differenzierte und dem jeweiligen Leistungsniveau angemessene Hörverstehenstestaufgaben (vgl. Abschnitt 4 des vorliegenden Aufsatzes), soll der Unterschied zwischen dem Grundniveau und dem erweiterten Niveau transparenter gemacht werden. Darüber hinaus könnten entsprechende Aufgabenformulierungen intensiver der Tatsache Rechnung tra- 
gen, dass ein Hörtext aus Hauptinformationen, relevanten und unwichtigen Nebeninforamtionen und Teilthemen sowie Redundanzen besteht.

- Der Hörverstehensteil des Abiturs soll nicht nur auf die Überprüfung von Detailaussagen angelegt werden, sondern es müssen auch solche Aufgaben konzipiert werden, bei denen die Prüfungskandidaten mehr zum globalen und kursorischen Hörverstehen veranlasst würden (vgl. dazu Teil 2 des vorliegenden Beitrags). Gerade in dem Hörverstehensteil für die Grundstufe sollte mehr Globalverstehen und selektives Hören überprüft werden.

- Man könnte zwischen den einzelnen Testaufgaben in den beiden Stufen noch stärker differenzieren, indem man jeweils eine Höraufgabe zu jedem Hörstil konzipieren und ausgewählte Hörtexte (z. B. für den Teil Globalverstehen) nur einmal vorspielen würde.

- Durch geeignete (d. h. kürzere und häufigere) Pausen zwischen den einzelnen Textpassagen des jeweiligen Textes könnte die Konzentration beim Hörverstehen und dadurch die benutzerfreundliche Lösung des gesamten Hörverstehensteils unterstützt werden.

Die hier kurz genannten Empfehlungen zur Optimierung des Prüfungsteils Hörverstehen der polnischen Reifeprüfung, welche die Prüfungskandidaten mit unterschiedlichen Hörstilen, authentischen Hörtexten und differenzierten Testaufgaben konfrontieren würden, stellen mit Sicherheit eine besondere Herausforderung sowohl für die Konzeption als auch für die Beurteilung des das Hörverstehen überprüfenden Prüfungssatzes. Einerseits erfordern diese Postulate von der Prüfungskommission solide fachdidaktische Kenntnisse, mehr Engagement, eine zeitaufwendigere Vorbereitung dieses Teils des Abiturs und schließlich auch eine größere Verantwortung für die didaktische Gestaltung des Deutschunterrichts an den polnischen Oberschulen. Andererseits sind diese Empfehlungen jedoch unerlässlich, damit sich die Abiturienten in dem auf die Abiturprüfung vorbereitenden Deutschunterricht an authentische fremdsprachige Texte und ihre Eigenschaften sowie Sprecher gewöhnen und Techniken sowie Strategien des globalen, selektiven und detaillierten Verstehens auf allen Ebenen automatisieren können.

\section{Literatur}

Adamczak-Krysztofowicz, Sylwia: Texte als Grundlage der Kommunikation zwischen Kulturen. Eine Studie zur Kultur- und Landeskundevermittlung im DaF-Studium in Polen. Hamburg: Verlag Dr. Kovač 2003.

Albers, Hans-Georg/ Bolton, Sibylle: Testen und Prüfen in der Grundstufe: Einstufungstests und Sprachstandsprüfungen. Fernstudieneinheit 7. Berlin u.a.: Langenscheidt 1995. 
Bickes, Gerhard: „Hörverstehensüberprüfung als methodisches Problem“. In: Kühn, Peter (Hrsg.). Hörverstehen im Unterricht Deutsch als Fremdsprache. Theoretische Fundierung und unterrichtliche Praxis. Frankfurt/Main u.a.: Peter Lang 1996, 149-170.

Dahlhaus, Barbara: Fertigkeit Hören. Fernstudieneinheit 5. Berlin u.a.: Langenscheidt 1994.

Eggers, Dietrich: „Einige Aspekte zur Analyse von Hörverstehenstests“. In: Eggers, Dietrich (Hrsg.). Didaktik Deutsch als Fremdsprache. Hörverstehen Leseverstehen - Grammatik. Materialien Deutsch als Fremdsprache. Heft 28. Regensburg: Becker-Kuns Verlag 1989, 83-90.

Eggers, Dietrich: „Hörverstehen: Bestandsaufnahme und Perspektiven“. In: Kühn, Peter (Hrsg.). Hörverstehen im Unterricht Deutsch als Fremdsprache. Theoretische Fundierung und unterrichtliche Praxis. Frankfurt/Main u.a.: Peter Lang 1996, 13-44.

Eggers, Dietrich: „Hörverstehen aus andragogischer Sicht - Einführung“. In: Eggers, Dietrich (Hrsg.). Hörverstehen aus andragogischer Sicht. Sprachlern- und Spracherwerbsstrategien im Fremdsprachenunterricht mit Erwachsenen. Mainz 1999, 5-16.

Grotjahn, Rüdiger: „Testen und Prüfen: Ein Überblick“ (in diesem Band).

Honnef-Becker, Irmgard: „Hörverstehen in Sprachlehrwerken Deutsch als Fremdsprache“. In: Kühn, Peter (Hrsg.). Hörverstehen im Unterricht Deutsch als Fremdsprache. Theoretische Fundierung und unterrichtliche Praxis. Frankfurt/Main u.a.: Peter Lang 1996, 45-78.

Hüllen, Werner: „Hörverstehen im Fremdsprachenunterricht der Sekundarstufe I“. In: Dirven, René (Hrsg.). Hörverständnis im Fremdsprachenunterricht. Kronberg: Scriptor 1977, 27-39.

Kieweg, Werner: „Möglichkeiten zur Verbesserung der Hörverstehenskompetenz“, Der Fremdsprachliche Unterricht Englisch 37, 64/65 (2003), 23-27.

Leupold, Eynar: „Didaktische Aspekte des Hörverstehens“, Der Fremdsprachliche Unterricht Französisch 48, 6 (2000), 410.

Łuniewska, Krystyna/ Wąsik, Zofia: Język niemiecki. Zakresy podstawowy i rozszerzony: zestawy i arkusze egzaminacyjne, teksty do słuchania, przykłady wypowiedzi pisemnych. Warszawa: Wydawnictwa Szkolne i Pedagogiczne 2004.

Ptak, Magdalena: „, „Trening maturalny« Vorbereitung auf das neue Abitur“. In: Hallo Deutschleher! Zeitschrift des polnischen Deutschlehrerverbands 22 (2006) , 31-33. 
Solmecke, Gert: „,Ohne Hören kein Sprechen. Bedeutung und Entwicklung des Hörverstehens im Deutschunterricht“. In: Fremdsprache Deutsch. Zeitschrift für die Praxis des Deutschunterrichts 7 (1992), 4-11.

Solmecke, Gert: „Authentische Texte - authentisches Hören?“‘. In: Kühn, Peter (Hrsg.). Hörverstehen im Unterricht Deutsch als Fremdsprache. Theoretische Fundierung und unterrichtliche Praxis. Frankfurt/Main u.a.: Peter Lang 1996, 79-92.

Solmecke, Gert: „Hörverstehen“. In: Helbig, G. et al. (Hrsg.). Deutsch als Fremdsprache. Ein internationales Handbuch. 2. Halbband, Berlin/ New York: Walter de Gruyter 2001, 893-900.

Solmecke, Gert: „Das Hörverstehen und seine Schulung im Fremdsprachenunterricht", Der Fremdsprachliche Unterricht Englisch 37, 64/65 (2003), 4-11.

Van Weeren, Jan: „Zum guten Schluss: ein Test. Zur Erstellung und Auswertung informeller Hörverstehenstests“, Fremdsprache Deutsch. Zeitschrift für die Praxis des Deutschunterrichts 7 (1992), 58-60.

Voss, Bernd: „Zur Überprüfung von Hörverstehen im Fremdsprachenunterricht“. In: Schumann, Adelheid/ Vogel, Klaus/ Voss, Bernd (Hrsg.). Hörverstehen. Grundlagen, Modelle, Materialien zur Schulung des Hörverstehens im Fremdsprachenunterricht der Hochschule. Tübingen: Narr 1984, 69-85.

Wiemer, Claudia: „Aspekte des Hörverstehens im fremdsprachlichen Lernprozess“. In: Eggers, Dietrich (Hrsg.). Hörverstehen aus andragogischer Sicht. Sprachlern- und Spracherwerbsstrategien im Fremdsprachenunterricht mit Erwachsenen. Mainz: Zentralstelle für universitäre Fort- und Weiterbildung 1999, 37-55.

Wolff, Dieter: „Hörverstehen in einer Fremdsprache: Ein psycholiguistisches Ratespiel?". In: Eggers, Dietrich (Hrsg.). Hörverstehen aus andragogischer Sicht. Sprachlern- und Spracherwerbsstrategien im Fremdsprachenunterricht mit Erwachsenen. Mainz: Zentralstelle für universitäre Fort- und Weiterbildung 1999, 17-35.

Zehren, Sigrid: „Hörverstehenstests“. In: Eggers, Dietrich (Hrsg.). Hörverstehen aus andragogischer Sicht. Sprachlern- und Spracherwerbsstrategien im Fremdsprachenunterricht mit Erwachsenen. Mainz: Zentralstelle für universitäre Fort- und Weiterbildung 1999, 57-69.

\section{Internetseiten:}

www.cke.edu.pl/index

www.oke.poznan.pl/pliki/informatory/niemiecki.pdf 


\section{Anhang}

Anhang I: Hörverstehensaufgaben für die Grundstufe

Zadanie 1 (6 pkt)

Zapoznaj sie z. treścia zadań 1.1. - 1.6. Po dwukrotnym wystuchaniu wypowiedzi Kathariny wybierz wtaściwe, zoodne zjej trésicia dokończenia zdań, zakesélajac litere $A, B$ lub C. Za każde poprawne rozwiazanie otraymasz 1 punk.t.

1.1. Katharinas Familie

A. besteht aus vier Personen.

B. trifft sich oft mit Tanten und Onkeln.

C. verbringt ihren Urlaub immer zu Hause.

1.2. Ihr Bruder

A. ist zwei Jahre älter als sie.

B. ruft Katharina oft an.

C. besucht seine Familie regelmäßig am Wochenende.

1.3. Katharinas Eltern

A. akzeptieren nicht immer ihre Pläne.

B. erziehen sie sehr streng.

C. besuchen oft mit ihr Kulturveranstaltungen.

1.4. Katharina

A. hat manchmal Streit mit ihren Eltern.

B. bekommt ab und zu Ausgehverbot.

C. spricht selten mit ihren Eltern.

1.5. Sie

A. verbringt viel Zeit in ihrem Zimmer.

B. möchte schon selbstständig sein.

C. braucht ihre Familie noch.

1.6. In zwei Jahren

A. macht sie Abitur.

B. wird sie Kauffrau von Beruf.

C. möchte sie eine eigene Familie haben 
Zadanie 2. (9 pkt)

Zapoznaj sie z treścia zdań 2.1. - 2.9. Po dwukrotnym wystuchaniu wypowiedzi trzech osób zaznacz znakiem $X w$ tabeli, które zpodanych zdan sa prawdziwe (richtig - R), a które fatsaywe (falsch - F). Za każde poprawne rozwiazanie otraymasz 1 punkt.

\begin{tabular}{|l|l|l|l|}
\hline \multicolumn{2}{|l|}{ Gina aus Kanada } & R & F \\
\hline 2.1. & In Frankfurt hat sie Menschen aus vielen Nationen getroffen. & & \\
\hline 2.2. & Das deutsche Bier hat ihr sehr gut geschmeckt. & & \\
\hline 2.3. & $\begin{array}{l}\text { Sie hat am Ende der Reise eine Schifffahrt auf dem Rhein } \\
\text { gemacht. }\end{array}$ & & \\
\hline Frans aus Südafrika & & \\
\hline 2.4. & Er hat eine Dienstreise nach Deutschland gemacht. & & \\
\hline 2.5. & Er meint, in Deutschland macht man viel für die Umwelt. & & \\
\hline 2.6. & Er besucht Deutschland noch einmal. & Ihre Lieblingsstädte sind Berlin und Boppard. & \\
\hline Lucy aus den USA & Sie war zum ersten Mal in Deutschland. & & \\
\hline & & & \\
\hline
\end{tabular}


Anhang II: Hörverstehensaufgaben für die erweiterte Stufe

Zadanie 7. (4 pkt)

Ustyszysz. dwnkerotnie zapowiedzi różnych programów telewizyjnych (A - F). Wybierz. program odpowiadajacy zainteresowaniom każdej z. wymienionych poniżej osób. Wpisz. do tabeli wlaściwa litere. Dwa programy nie pasuja do ich zainteresowań. Za każde poprawne rozwiazanie otraymasz. 1 punkt.

\begin{tabular}{|l|l|l|}
\cline { 2 - 3 } \multicolumn{1}{l|}{} & Person & Programm \\
\hline 7.1. & $\begin{array}{l}\text { In diesem Jahr macht Sandra Abitur. Sie möchte später Ge- } \\
\text { schichte studieren. }\end{array}$ & \\
\hline 7.2. & $\begin{array}{l}\text { Manfred bleibt oft lange im Büro. Er möchte sich abends über } \\
\text { die Tagesthemen informieren. }\end{array}$ & $\begin{array}{l}\text { Gabi spielt kein Instrument. Sie interessiert sich aber für klas- } \\
\text { sische Musik. }\end{array}$ \\
\hline 7.3. & $\begin{array}{l}\text { Marc sieht nicht oft fern. Wenn er aber abends Zeit hat, wählt } \\
\text { er einen Actionfilm oder Thriller. }\end{array}$ & \\
\hline
\end{tabular}

Zadanie 8. (2 pkt)

Zapoznaj sie z treścia zadan 8.1. - 8.2. Po dwnkrotnym wystuchaniu fragmentu tekstu, dotyczacego mtodych ludzi, uybierz. wtaściwe, zgodne z nim dokończenia zdań, zakereślajac litere A, B, C lub D. Za każde poprawne rozwiazanie otraymasz. 1 punkt.

8.1. Der Text beinhaltet:

A. ausschließlich Kritik

B. viele gute Ratschläge

C. höfliche Bitten

D. überwiegend Vergleiche

8.2. Der richtige Titel für den Hörtext lautet:

A. Klare Regeln bei Partys

B. Jugendliche und ihre Körpersprache

C. So verhandelst du richtig mit deinen Eltern

D. Das Jugendschutzgesetz gilt für alle. 
Zadanie 9. (9 pkt)

Po dwukrotnym wystuchaniu wypowiedzi trzech osób zaznacz znakiem X w tabeli, do których osób odnoszq sie informacje umiesz̧zone w kolumnie $A$. Za każde poprawne rozwiazanie otryymasz 1 punk.t.

\begin{tabular}{|c|c|c|c|c|}
\hline & A & Stefan & Yvonne & Ismail \\
\hline 9.1. & ... hatte sowohl gute als auch schlechte Lehrer. & & & \\
\hline 9.2. & ... hat die Schule sehr gern besucht. & & & \\
\hline 9.3. & ... fand die Schule lebensfern. & & & \\
\hline 9.4. & ... hatte Schwierigkeiten bei Klassenarbeiten. & & & \\
\hline 9.5. & ... hat den Mathe- und Physiklehrer bewundert. & & & \\
\hline 9.6. & ... hat selten eine eigene Meinung geäußert. & & & \\
\hline 9.7. & ... hat der Englischlehrerin viel zu verdanken. & & & \\
\hline 9.8 . & $\begin{array}{l}\text {... hat die Schule mit einer sehr guten Note in } \\
\text { Deutsch abgeschlossen. }\end{array}$ & & & \\
\hline 9.9. & ... hat sich fürs Schauspielern interessiert. & & & \\
\hline
\end{tabular}




\section{Anhang III: Transkription der Hörtexte für die Grundstufe}

\section{Aufgabe 1 \\ Katharina}

„Ich wohne mit meinen Eltern in der Nähe von Stuttgart. Wir sind zu viert, meine Eltern, mein Bruder und ich. Ich habe zwar noch viele Tanten und Onkel, die auf drei Kontinenten verteilt sind, aber weil die weiter weg wohnen, haben wir nicht viel mit ihnen zu tun. Eigentlich können wir nur in den Ferien in vielen Ländern günstig Urlaub machen - indem wir einfach unsere Familie besuchen. Mein Bruder ist vor zwei Jahren, gleich nach dem Abitur, ausgezogen, das hat aber nicht viel verändert. Wir verstehen uns immer noch sehr gut, schreiben uns regelmäßig E-Mails und telefonieren. An Familienritualen wie Weihnachtsfeiern halten wir fest, mein Bruder kommt dann zu uns nach Stuttgart. Mein Bruder hatte es sicher schwerer als ich, er hat mir schon einiges freigekämpft. Im Gegensatz zu ihm muss ich nicht lange bitten, wenn ich ausgehen will oder wenn Freunde bei mir über Nacht bleiben möchten. Ich bin wohlbehütet aufgewachsen, meine Eltern haben mich vielleicht ein bisschen öfter gefragt, wo ich hingehe und mit wem, als meine Freunde das von ihren Eltern gefragt wurden. Für mich war das aber immer normal, ich hatte damit nie ein Problem. Manchmal wundern sich meine Freunde, weil ich mit meinen Eltern auch oft etwas unternehme, ins Kino gehe zum Beispiel oder ins Museum. Das heißt nicht, dass es bei uns nie Streit gibt. Aber wir kriegen das dann schon immer wieder hin. Ich hatte zum Beispiel nie Hausarrest, höchstens mal zwei Stunden Fernsehverbot. Das Positive an meiner Familie ist, dass ich ein gutes Verhältnis zu meinen Eltern habe. Ich kann immer $\mathrm{zu}$ ihnen kommen und mit ihnen reden. Ich glaube, das können andere wahrscheinlich nicht so. Meine Zimmertür steht fast immer offen, ich finde es einfach schön, rauszukommen und mit jemandem zu reden. Deswegen kann ich es mir jetzt auch noch nicht vorstellen, alleine zu leben. Weil ich gerade einfach noch jemanden brauche. In zwei Jahren, wenn meine Ausbildung zur Kauffrau abgeschlossen ist, möchte ich dann schon ausziehen, meine Eltern stehen mir da aber nicht im Weg. Zum Schluss: So eine Familie möchte ich irgendwann auch haben." nach: Fluter, Nr. 06 April 2003

\section{Aufgabe 2 \\ Vor dem Abflug \\ Gina aus Kanada}

Auf meiner Europa-Reise machte ich in Frankfurt kurz Aufenthalt. Es hat mir gut gefallen, obwohl ich nur kurz hier war. Zuerst hieß es, in Frankfurt gebe es nichts zu tun - eine glatte Fehlinformation. Zusammen mit meinem Mann war ich bummeln, und dabei haben wir eine Menge nette Leute getroffen. Die größte Überraschung aber war, wie multikulturell es hier ist. Es gab zum Beispiel sehr viele Asiaten. Zu schaffen macht mir aber auch etwas: das deutsche Bier. Es war einfach zu 
gut. Unsere Ziele für die nächste Deutschland-Reise stehen bereits fest: Ski laufen in den Alpen und eine Schifffahrt auf dem Rhein.

\section{Frans aus Südafrika}

Ich bin drei Wochen mit Freunden durch Deutschland gereist. An die Gastfreundschaft der Menschen hier werde ich mich noch lange erinnern. Überall wurden wir sehr, sehr freundlich aufgenommen. Sehr beeindruckt bin ich auch von dem Grün überall. Wir waren am Bodensee, auf der Insel Mainau, im Schloss Neuschwanstein, im Schwarzwald und im Kloster Maulbronn. Und egal, welche Gegend wir besucht haben - überall gab es Wald. Die Deutschen setzen anscheinend alles daran, ihre Natur zu erhalten. Das gefällt mir sehr. Für meine Familie in Südafrika nehme ich ein Geschenk mit: Baumkuchen. Der hat mir während meines Kurzurlaubes hier wirklich gut geschmeckt. Ich werde wiederkommen, ganz bestimmt. Dann werde ich mir Berlin anschauen.

\section{Lucy aus den USA}

Ich mag Deutschland einfach. Es gibt sehr viel Kultur zu sehen, man kann viel unternehmen - und das alles mit einem hervorragenden Nahverkehrssystem. Jedes Mal, wenn ich wieder hier bin, ich habe Deutschland bereits mehrfach besucht, versuche ich, meine beiden Lieblingsorte zu besuchen: Berlin. Das ist eine moderne Stadt, die wirklich lebt. Und als Kontrast das kleine romantische Boppard am Rhein. Das Land ist nicht nur sauber, auch die Menschen sind richtig nett. Die Deutschen sind zwar etwas reserviert, aber nach einer gewissen Zeit tauen sie auf. Inzwischen habe ich auch Freunde in Deutschland gefunden. Und nicht nur darum werde ich wiederkommen.

nach: Deutschland, Nr. 5/2002 Oktober/November

\section{Anhang IV: Transkription der Hörtexte für die erveiterte Stufe}

Aufgabe 7

Fernsehprogramme

A. Zeitgenössische Musik gilt vielen Menschen als schwierig und unzugänglich. Mit seiner sechsteiligen Doku-Serie beweist Paul Crossley das Gegenteil. Der britische Pianist stellt im ersten Teil der Serie allgemein verständlich bedeutende Komponisten des 19. Jahrhunderts und deren wichtigste Werke vor. Fortsetzung folgt.

B. Wie überall in Mitteleuropa zählen die Schwarzstörche auch in Tschechien zu den stark bedrohten Arten. Ein Kamerateam begleitet ein Projekt, bei dem versucht wird, junge Zootiere wieder auszuwildern.

C. Als der Ingenieur Bowman im südamerikanischen Tecala von Rebellen entführt wird, stellt sich heraus, dass seine Firma die Kidnapping-Versicherung gekündigt hat. Trotzdem lässt sich Verhandlungsexperte Terry Thorne von Bowmans Frau Alice überreden, ihr zu helfen. Er versucht routiniert, das Lö- 
segeld herunterzuhandeln. Doch schließlich bleibt nur ein Ausweg: Terry und seine Männer müssen Bowman mit Waffengewalt befreien.

D. Vor 125 Jahren begannen in Pergamon deutsche Archäologen mit ihren Grabungen. Ihre sensationellen Funde gaben dem Haus auf der Berliner Museumsinsel seinen Namen und machten es weltberühmt. Carola Wedel erzählt über das Museum, das nun für 500 Millionen Euro restauriert und modernisiert wird.

E. Deutschland zur Zeit des Wirtschaftswunders. Daniel Finkbeiner, Fabrikant für argbeschläge, spürt, dass sich in seinem Betrieb etwas ändern muss. Nach einem Herzinfarkt ruft er seine drei Söhne Kurt, Erwin und Fritz ans Krankenbett: Wer als Erster eine Braut und einen Stammhalter präsentiert, erbt die Firma.

F. Der Abend mit Christoph Teuner gibt einen Überblick über das Tagesgeschehen. Anschließend: Sportberichte vom Tage.

nach: TV magazin, 28. August 2003 und TV direkt, Nr. 7/2001

\section{Aufgabe 8}

Der Zeitpunkt. Warte einen günstigen Moment ab, in dem du in Ruhe mit deinen Eltern über deine Pläne sprechen kannst.

Die Kontaktaufnahme. Fragst du deine Eltern rechtzeitig um Erlaubnis, bleibt dir bei einem „Nein" immer noch genügend Zeit, um ihre Meinung unter Umständen zu ändern. Die Verhandlung. Eltern interessieren vor allem zwei Fragen: „Wohin?" und „Mit wem?". Die Aussage-Verweigerung ist eine schlechte Taktik, Lügen fliegen meist auf. Ehrlichkeit verschafft dir die beste Chance, dass sie über das „Bis wann?" mit sich reden lassen. Gehe dabei auch mal einen Kompromiss ein. Besser ein wenig erreichen als gar nichts. Frage bei einem „Nein", was du tun kannst oder sich ändern müsste, damit du das „Okay" bekommst.

Die Ausrüstung beim Ausgehen. Nie ohne Ausweis! Außerdem wäre ein Handy empfehlenswert, ebenso wie ein paar Euro eiserne Reserve für Notfälle, etwa wenn du ein Taxi brauchen solltest. Ruf im Notfall zuerst deine Eltern an!

Kläre ab, wie du sicher zum Ort des Geschehens und wieder nach Hause kommst, und informiere deine Eltern darüber.

http.V/www.jungoesterreicher.at/2003-10-08

\section{Aufgabe 9}

Was denken Soap-Stars über ihre Schulzeit?

Die Stars aus "Marienhof" erinnern sich an Mathelehrer, Prüfungsängste und Freundschaften im Klassenzimmer.

Ich habe die prominenten Schul-Impressionen gesammelt.

\section{Stefan}

„Wenn ich an Schule denke, denke ich an ein undemokratisches System. Einer hat das Sagen auf Grund seines Alters und seiner Position. Was er sagt, ist Recht und 
Gesetz, alle anderen haben Unrecht. Sehr beeindruckt hat mich mein Klassenlehrer, bei dem ich Mathe und Physik hatte. Er war ein Außenseiter in der DDR, nicht stromlinienförmig, und er hatte mir beigebracht, wie wichtig es ist, immer offen sein Gesicht zu zeigen.

Mir fällt aber auch meine Theater-AG ein. Meine Englischlehrerin, die diese AG leitete, hat mich stark geprägt. Ohne sie hätte ich wohl nicht meine Liebe fürs Schauspielern entdeckt. Leider wurde die Theater-AG inzwischen eingestellt, weil Lehrerstunden gestrichen wurden und die nachfolgende Schülergeneration sich in der freiwilligen AG nicht engagieren wollte“.

\section{Yvonne}

„Ich hatte leidenschaftliche, teilweise sogar grandiose Lehrer, aber auch total unmotivierte, die nur den Stoff durchzogen und einen grauenhaften Unterricht abhielten. Mit schlimmen Folgen. Denn Schüler, denen nicht vermittelt wird, wie wichtig gute Noten für die spätere Berufswahl sind und die deshalb keinen Kämpfergeist entwickeln, können später oftmals ihren Lebenstraum nicht verwirklichen. Insgesamt bereitet die Schule zu wenig aufs Leben vor. Da gibt es kaum Spielräume. Ich wusste, wie meine Lehrer denken, habe ihnen zum Schluss nur noch nach dem Mund geredet. Dadurch waren meine Noten gut, und ich hatte meine Ruhe“.

\section{Ismail}

„Ich hatte in der Schule viel Spaß, der Zusammenhalt der Klasse war super. Ich glaube, wenn man sich mit den Klassenkameraden versteht, geht man auch gerne zur Schule. Meistens jedenfalls. Ich hatte nur dann Probleme, wenn Klausuren und Prüfungen anstanden. Ich habe übrigens erst im Kindergarten Deutsch gelernt, weil bei uns zu Hause nur Türkisch gesprochen wurde. Trotzdem bestand ich meine Abschlussprüfung in Deutsch über Andorra von Max Frisch mit 1,4 - als Bester meines Jahrgangs".

nach: Fluter, Nr. 5/2002 


\section{Der Prüfungsteil Schreiben im neuen ungarischen Abitur für Deutsch als Fremdsprache ${ }^{1}$}

Tibor Vigh, Szeged (Ungarn)

In dem vorliegenden Beitrag werden einige Ergebnisse meiner empirischen Forschungsarbeit zur Analyse des Prüfungsteils Schreiben im neuen ungarischen Abitur dargestellt.

Das Hauptziel der Forschung war anhand der Analyse von Schülerleistungen, der Testaufgabe und der Bewertung des Probeabiturs im Jahre 2004 ein Bild darüber zu bekommen, wie die ungarischen Abiturienten ein angegebenes kommunikatives Ziel schriftlich erreichen konnten bzw. was ihnen dabei Schwierigkeiten

${ }^{1}$ Der Beitrag ist eine Zusammenfassung der Forschungsergebnisse meiner Diplomarbeit, die 2005 an der Universität Szeged vorgelegt wurde. Im Rahmen des PhD Programms: Pädagogische Leistungsmessung wurde die Analyse weiterentwickelt, indem mehrere Schülerarbeiten auch mit statistischen Verfahren untersucht wurden. Die wichtigsten Ergebnisse daraus werden hier auch dargestellt. 
bereitet. In der Studie wird neben der Zusammenfassung der Forschungsergebnisse auch behandelt, welche Konsequenzen die von den Schülern erbrachten Leistungen für die Bewertung und die Testerstellung haben.

\section{$1 \quad$ Merkmale des Prüfungsteils Schreiben}

Das fertigkeitsorientierte Messen der fremdsprachlichen Kompetenz ist im neuen Abitur eines der grundlegenden Prinzipien (vgl. Einhorn 2000, 2002, 2004; Petneki 2002). So enthält diese Prüfung den Prüfungsteil Schreiben, dessen Ziel ist zu messen, ob der Kandidat fähig ist, sich in der Fremdsprache schriftlich auszudrücken, bzw. schriftliche Aufgaben zur Bewältigung verschiedener Kommunikationsziele auszuführen (Prüfungsregelung und -beschreibung. DaF. 2003: 763). Um die drei Niveaustufen des Gemeinsamen Europäischen Referenzrahmens im zweistufigen Abitur niveaugerecht messen zu können, gibt es Unterschiede in den Themen, und auch in dem Grad der Ausführlichkeit der Themenbehandlung.

Die Testaufgaben sollen den Prüfungsanforderungen entsprechend gestellt werden, deshalb unterscheiden sie sich nach Niveaustufen. Es gibt Unterschiede im angegebenen Input, in der Textsorte und Textlänge, die die Kandidaten schreiben sollen, und auch die Steuerung der Textproduktion ist durch die inhaltlichen Leitpunkte verschieden. Außerdem besteht der Prüfungsteil immer aus zwei Aufgaben, wovon die Kandidaten in der zweiten unter zwei angegebenen Themen wählen können. Die Themen sollten immer unter gleichen Bedingungen gelöst werden, aber die dazu gehörenden kommunikativen Ziele sind unterschiedlich. Die in den Tabellen 1 und 2 angegebene Aufgabe aus dem Probeabitur 2004 (vgl. S. 159, 160) soll als Beispiel für den Aufbau einer Testaufgabe auf der Mittelstufe dienen.

Bei der Bewertung auf den zwei Stufen gibt es Unterschiede in den Bewertungskriterien. Zu diesen gehört je eine analytische Skala, die das Punktesystem für verschiedene Leistungen beschreibt. Bei dem gleichen Bewertungskriterium der verschiedenen Niveaus ist die Beschreibung der gegebenen Punktzahlen anders².

\section{Analyse des Prüfungsteils Schreiben im Probeabitur 2004 auf der Mittelstufe}

\subsection{Problemstellung}

Im Weiteren möchte ich zeigen, zu welchen Ergebnissen das Probeabitur 2004 und das Abitur 2005 geführt hat (Abbildung 1).

\footnotetext{
2 Vgl. Petneki, S. 182.
} 


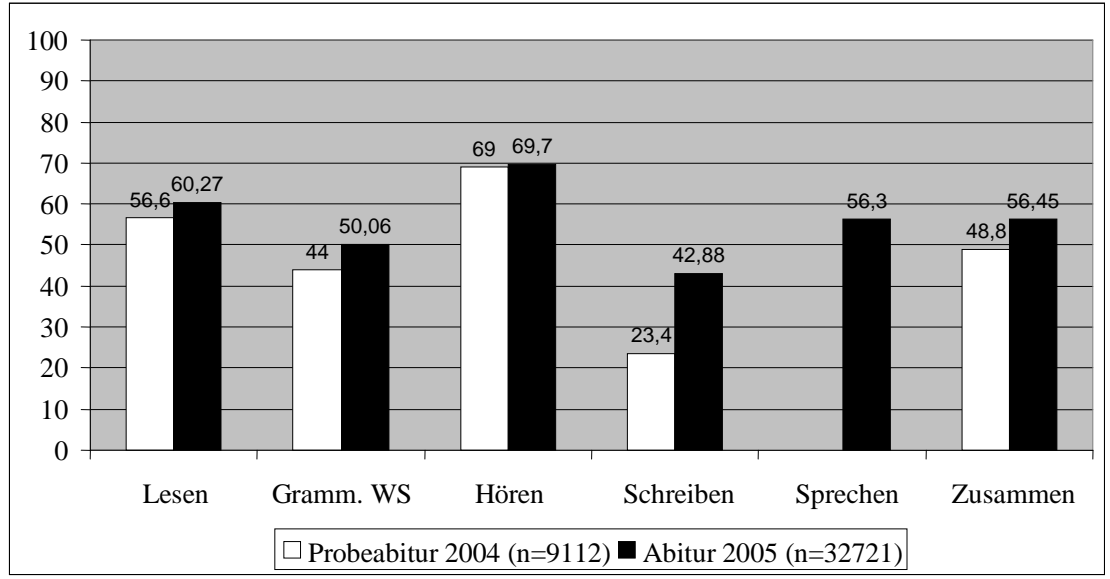

Abbildung 1: Prüfungsergebnisse auf der Mittelstufe im Probeabitur 2004 und im Abitur $2005^{3}$

Das Ziel des Probeabiturs war auszuprobieren, inwieweit die Schüler auf die neue Prüfungsform vorbereitet sind. Die Ergebnisse bei der Schreibfertigkeit ( $A b$ bildung 1) zeigen, dass es den Schülern Schwierigkeiten bereitet, einfache Texte zu schreiben und die kommunikativen Ziele zu bewältigen. Die Abiturprüfung im Mai 2005 auf der Mittelstufe zeigt bessere Ergebnisse, aber die Lösungsrate dieses Prüfungsteils ist immer noch am niedrigsten im Vergleich zu den anderen (etwa $40 \%$ ). So scheint diese Problematik aktuell zu sein.

\subsection{Forschungsfragen}

Um den Prüfungsteil Schreiben analysieren zu können, wurden unter anderem die folgenden Fragen gestellt:

- Inwieweit wurden die in der zweiten Aufgabe angegebenen Themen von den Schülern erfasst und ausgeführt und wie haben sie die kommunikativen Ziele erreicht?

- Wie angemessen sind die Testaufgaben? Wenn darin Probleme auftauchen, wo liegen sie dann und welche Schwierigkeiten verursachten sie sowohl den Schülern bei der Produktion als auch den Lehrern bei der Bewertung?

\footnotetext{
${ }^{3}$ Nach: Einhorn/Major 2005; Einhorn 2006.
} 


\subsection{Forschungsgegenstände}

Die Testaufgabe (Tabelle 1 und 2) und die Schülerarbeiten (Abbildung 2) bilden die Forschungsgegenstände der Analyse. Zuerst wird die Aufgabe vorgestellt.

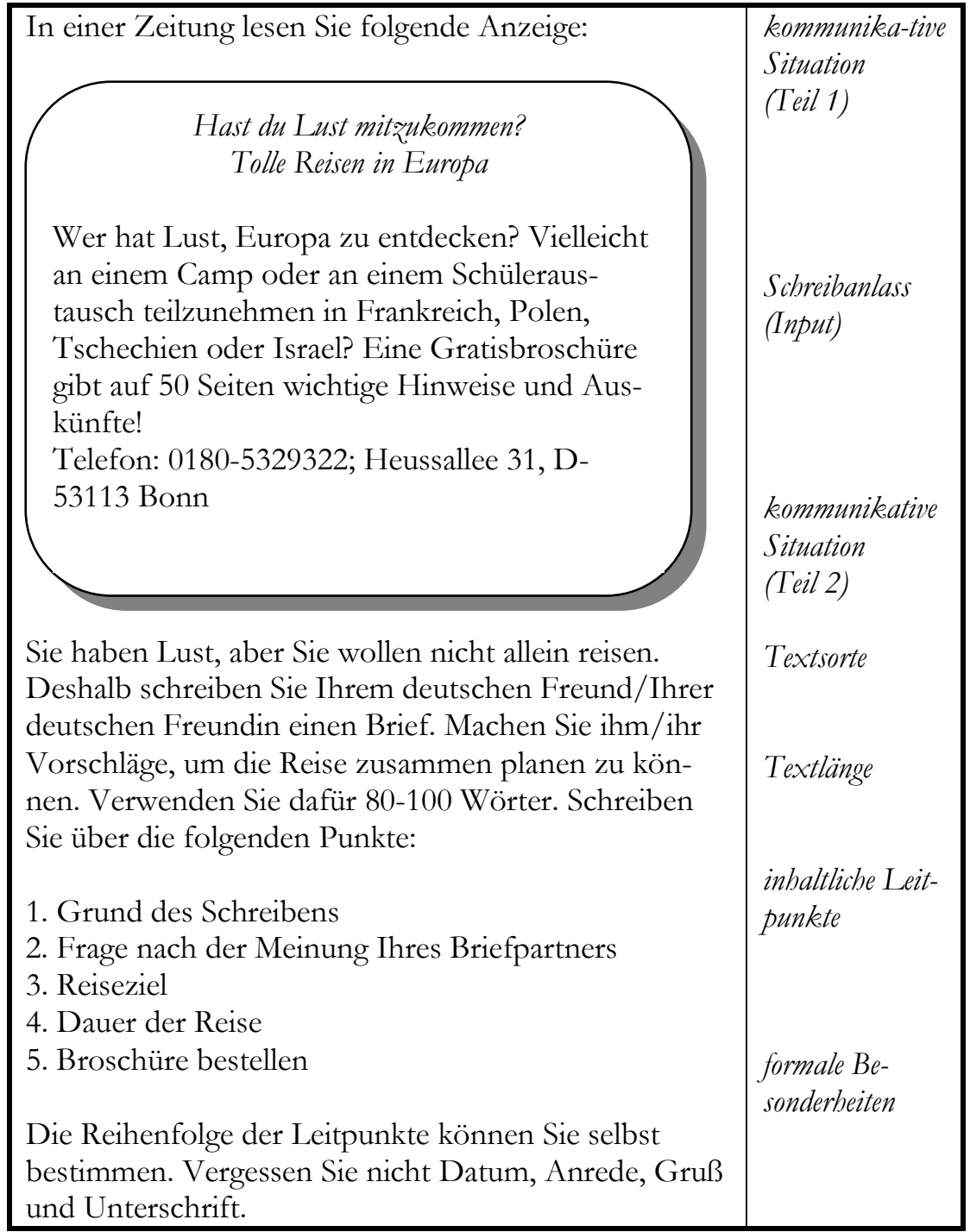

Tabelle 1: Testaufgabe zum Thema Planung einer Reise ${ }^{4}$

${ }^{4}$ Probeabitur 2004. DaF. Mittelstufe. Prüfungsteil Schreiben. 


\begin{tabular}{|c|c|}
\hline $\begin{array}{l}\text { Vorige Woche haben Sie Ihren Geburtstag gefeiert. } \\
\text { Sie haben von Ihrem deutschen Brieffreund den fol- } \\
\text { genden Geburtstagsgruß bekommen: }\end{array}$ & $\begin{array}{l}\text { kommunikative } \\
\text { Situation } \\
\text { (Teil 1) }\end{array}$ \\
\hline $\begin{array}{l}\text { Herzlichen Glückwunsch und alles Gute } \\
\text { zum Geburtstag! } \\
\text { Schreib mir unbedingt, wie du gefeiert } \\
\text { hast. } \\
\text { Markus }\end{array}$ & $\begin{array}{l}\text { Schreibanlass } \\
\text { (Input) }\end{array}$ \\
\hline $\begin{array}{l}\text { Schreiben Sie Ihrem deutschen Freund einen Brief } \\
\text { über Ihre Geburtstagsparty. Verwenden Sie dafür 80- } \\
100 \text { Wörter. Schreiben Sie über die folgenden Punkte: }\end{array}$ & $\begin{array}{l}\text { Textsorte } \\
\text { Textlänge }\end{array}$ \\
\hline $\begin{array}{l}\text { 1. Datum und Dauer der Party } \\
\text { 2. Vorbereitungen für die Party (Getränke, Musik usw.) } \\
\text { 3. Gäste } \\
\text { 4. Programm } \\
\text { 5. Geschenke }\end{array}$ & $\begin{array}{l}\text { inhaltliche Leit- } \\
\text { punkte }\end{array}$ \\
\hline $\begin{array}{l}\text { Die Reihenfolge der Leitpunkte können Sie selbst } \\
\text { bestimmen. Vergessen Sie nicht Datum, Anrede, Gruß } \\
\text { und Unterschrift. }\end{array}$ & $\begin{array}{l}\text { formale Be- } \\
\text { sonderheiten }\end{array}$ \\
\hline
\end{tabular}

Tabelle 2: Testaufgabe zum Thema Geburtstagsparty

Die Analyse erfolgte anhand des Bewertungskriteriums Inhalt (Tabelle 3). Dieses Kriterium ist primär, weil da entschieden wird, ob der Kandidat die kommunikative Zielsetzung erreicht hat oder nicht. Das hat zur Folge, dass - falls der Kandidat nämlich nach diesem Kriterium keinen Punkt bekommt - die ganze Arbeit mit 0 Punkt bewertet wird.

Bei der Analyse des Kriteriums Inhalt spielen die Leitpunkte folgende Rollen: sie steuern einerseits die Textproduktion, andererseits ermöglichen sie die Vergleichbarkeit der Schülerergebnisse (vgl. Bolton 1996: 82 f., Kast 1999: 151 f.). Aus der Sicht der Analyse kann man so sowohl die Schülerleistungen als auch die Testaufgabe und die Bewertung untersuchen. 


\begin{tabular}{|l|l|}
\hline Punkte & Beschreibung \\
\hline 5 & $\begin{array}{l}\text { Der Kandidat arbeitete fünf Leitpunkte ausführlich aus. } \\
\text { Der Kandidat erreichte die kommunikativen Ziele angemessen }\end{array}$ \\
\hline $4-3$ & $\begin{array}{l}\text { Der Kandidat arbeitete vier oder drei Leitpunkte ausführlich, die anderen } \\
\text { nur oberflächlich oder gar nicht aus. } \\
\text { Der Kandidat erreichte die kommunikativen Ziele meistens angemes- } \\
\text { sen. }\end{array}$ \\
\hline \multirow{2}{*}{$2-1$} & $\begin{array}{l}\text { Der Kandidat arbeitete zwei Leitpunkte ausführlich, die anderen nur } \\
\text { oberflächlich oder gar nicht aus. } \\
\text { oder: Der Kandidat arbeitete einen Leitpunkt ausführlich aus und es gibt } \\
\text { einen oder mehrere Leitpunkte, die er oberflächlich behandelt. } \\
\text { oder: Der Kandidat behandelte mindestens vier Leitpunkte oberflächlich. } \\
\text { Der Kandidat erreichte die kommunikativen Ziele teilweise. }\end{array}$ \\
\hline $\begin{array}{l}\text { Der Kandidat arbeitete nur einen Leitpunkt ausführlich aus und er be- } \\
\text { handelte die anderen nicht. } \\
\text { oder: Der Kandidat behandelte nur drei oder weniger Leitpunkte ober- } \\
\text { flächlich. } \\
\text { Das grundsätzliche kommunikative Ziel erreicht er nicht. } \\
\text { oder: Der Kandidat schreibt über ein anderes Thema }\end{array}$ \\
\hline
\end{tabular}

Tabelle 3: Die analytische Skala zur Bewertung von Inhalt ${ }^{5}$

Anhand der Bewertungsskala (Tabelle 3) lässt sich feststellen, dass die einzelnen Leitpunkte drei Werte haben: (1) ausführlich, (2) oberflächlich, (3) nicht angemessen behandelter Leitpunkt. So müssen die Prüfer folgende Entscheidungen treffen: Zuerst eine quantitative Entscheidung, d.h., Ist die Behandlung des jeweiligen Leitpunktes vorhanden oder nicht? und dann eine qualitative Entscheidung, also, Ist der behandelte Leitpunkt ausführlich oder oberflächlich ausgeführt?

Am Ende des Beitrags wird dargestellt, welche Verfahren es in der untersuchten Testaufgabe gibt, die eine objektive Entscheidung ermöglichen sollen.

\section{Ergebnisse der Analyse}

Im Weiteren versuche ich die wichtigsten Ergebnisse der Forschungsarbeit mit Hilfe von Diagrammen und in einigen Fällen mit konkreten Beispielen aus den Schülerarbeiten so zu präsentieren, dass die Antworten auf die am Anfang erwähnten Forschungsfragen thematisiert werden.

5 Probeabitur 2004. DaF. Mittelstufe. Schriftliche Prüfung. Korrektur- und Bewertungsanleitung, S. 16 


\subsection{Analyse der Schülerleistungen}

In Abbildung 2 wird die Anzahl der untersuchten Schülerarbeiten zum ersten (über die Planung einer Reise) und zum zweiten Thema (über die Geburtstagsparty) angegeben.

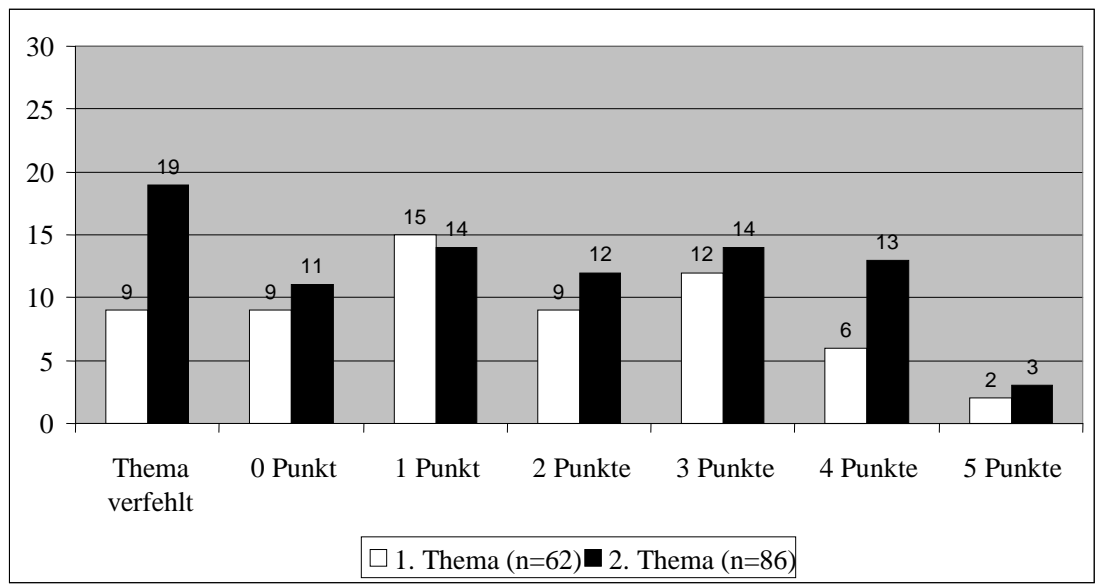

Abbildung 2: Anzahl der untersuchten Schülerarbeiten ( $n=148)$

Bei der Analyse der Schülerarbeiten wurden drei Gruppen gebildet:

1. Die Gruppe von Schülerarbeiten, in der die Schüler vom Thema abgekommen sind, d.h. sie haben das grundsätzliche kommunikative Ziel nicht erreicht (Tabelle 4, 5).

2. Die Gruppe der schwachen Schülerleistungen, in der die kommunikativen Ziele teilweise erreicht wurden (0-2 Punkte) (Abbildung 3, 5).

3. Die Gruppe der guten Schülerleistungen, in der die kommunikativen Ziele meistens angemessen bewältigt wurden (3-5 Punkte) (Abbildung 4, o). 


\begin{tabular}{|l|l|}
\hline Problem & Beispiele aus den Schülerarbeiten \\
\hline $\begin{array}{l}\text { Der Kandidat hat einen } \\
\text { halboffiziellen Brief für die } \\
\text { Zeitung geschrieben. }\end{array}$ & $\begin{array}{l}\text { "Sehr geehrte Damen und Herren! Meine Freunden } \\
\text { und ich würden in Tschechien reisen möchten. [... } \\
\text { Wir haben einige Fragen: Was können Sie die Her- } \\
\text { berge anbieten? Was kostet das? [...] Wir interessie- } \\
\text { ren noch die Weise für die Reise, und die Reiseziel." }\end{array}$ \\
\hline $\begin{array}{l}\text { Der Schüler hat einen } \\
\text { Leitpunkt ausgewählt. }\end{array}$ & $\begin{array}{l}\text { "Reiseziel: Frankreich. Ich möchte hingehen in Frank- } \\
\text { reich. Dort auch in Paris. Interessieren die Schön- } \\
\text { heitspflege. Sich auszeichnen Loreal Name Marke." }\end{array}$ \\
\hline $\begin{array}{l}\text { Das im Input angegebene } \\
\text { Reiseziel hat den Kandida- } \\
\text { ten verwirrt. }\end{array}$ & $\begin{array}{l}\text { "Ich habe eine Anzeige gelesen, und Gestern habe ich } \\
\text { für sie telefoniert. Sie haben gesagt, dass wir nach } \\
\text { Israel auch nicht fahren müssen. (Israel ist zwischen } \\
\text { den Reisezieles auch gewesen.) Wir dürfen nur in }\end{array}$ \\
\hline $\begin{array}{l}\text { Der Kandidat hat den } \\
\text { Partner zu der Reise } \\
\text { eingeladen, aber es gab } \\
\text { keinen Hinweis auf den } \\
\text { Input. }\end{array}$ & $\begin{array}{l}\text { [Dann beschreibt er seine ehemalige Reise] } \\
\text { ein tolle, schöne Land und ich habe einen Reiseplan } \\
\text { geschrieben. [...] Ich habe diese Reise geplant." }\end{array}$ \\
\hline
\end{tabular}

Tabelle 4: Beispiele für verfehlte Themenbehandlung im ersten Thema

\begin{tabular}{|c|c|}
\hline Problem & Beispiele aus den Schülerarbeiten \\
\hline $\begin{array}{l}\text { Viele Kandidaten haben } \\
\text { sowohl den Inputtext als } \\
\text { auch die kommunikative } \\
\text { Situation missverstanden, } \\
\text { weil sie Markus zur Ge- } \\
\text { burtstagsparty eingeladen } \\
\text { haben. }\end{array}$ & $\begin{array}{l}\text { "Liebe Markus, } \\
\text { Ich bekamm diese Geburtstagsgruß. Ich freue mich } \\
\text { sehr darüber. [...] Jetzt schreibe ich, wie wird die Party } \\
\text { organisieren. Sie wird in der Nächste Woche, am } \\
\text { Freitag abwickeln, und dauert bis Mitternacht. [...] Ich } \\
\text { hoffe, dass du kommst." } \\
\text { "Liebe Markus, } \\
\text { ich freute mich, als du mich einen Brief geschrieben } \\
\text { hast. Ich möchte dich für meiner Party einladen. Es } \\
\text { wird im erste Mai." }\end{array}$ \\
\hline $\begin{array}{l}\text { Außerdem wurde nicht } \\
\text { Markus, sondern eine } \\
\text { andere Person angespro- } \\
\text { chen. }\end{array}$ & $\begin{array}{l}\text { "Hallo Peter, } \\
\text { Ich möchte dich meine Party einladen. Die Party be- } \\
\text { ginnt am Samstag um } 6 \text { Uhr. Die Vorbereitungen geht } \\
\text { für die Party prächtig. Für die Party kannst du viele Art } \\
\text { Getränke wählen." }\end{array}$ \\
\hline
\end{tabular}

Tabelle 5: Beispiele für verfehlte Themenbehandlung im zweiten Thema 
Die Beispiele in Tabelle 4, 5 deuten darauf hin, dass die Schüler zur Lösung der Testaufgabe ihre Lesekompetenz intensiv einsetzen sollen, d. h. sie sollen die Testaufgabe detailliert verstehen, sonst können sie die Aufgabe nicht lösen.

Im Folgenden werden die Ergebnisse der Analyse der zwei Themen getrennt vorgestellt. Zuerst wird die Anzahl der Schülerarbeiten nach der Qualität der Behandlung der Leitpunkte in den zwei Leistungsgruppen im ersten Thema angegeben (Abbildung 3, 4).

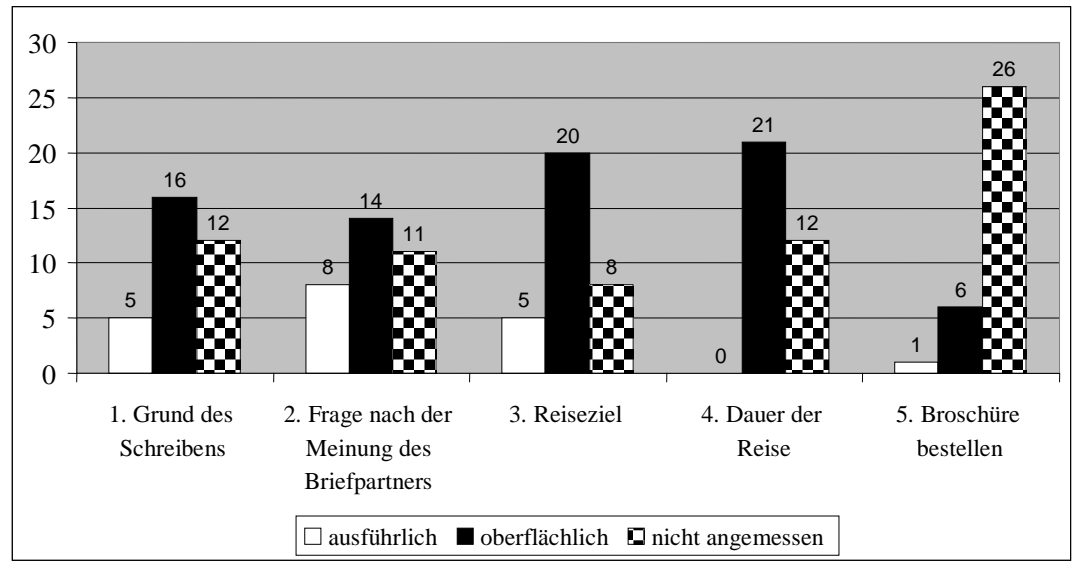

Abbildung 3: Schwache Schülerleistungen (0-2 Punkte) im ersten Thema ( $n=33)$

Die Abbildung 3 zeigt, dass einerseits die Behandlung des dritten Leitpunktes in dieser Gruppe keine großen Schwierigkeiten bereitete, weil die Anzahl der nicht angemessen ausgeführten Leitpunkte am niedrigsten ist. Andererseits konnten die Schüler den fünften Leitpunkt gar nicht ausarbeiten. Die Lösungsrate der anderen drei Leitpunkte ist ähnlich, aber es ist zu bemerken, dass sie den vierten Leitpunkt ausführlich nicht behandeln konnten. 


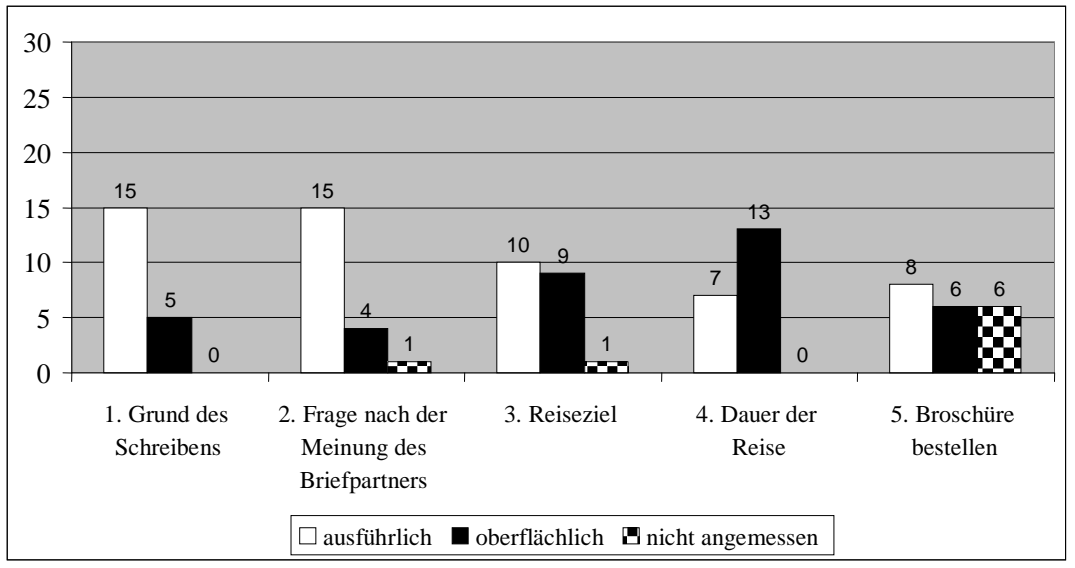

Abbildung 4: Gute Schülerleistungen (3-5 Punkte) im ersten Thema $(n=20)$

In der zweiten Leistungsgruppe (Abbildung 4) konnten die Schüler die zu den ersten zwei Leitpunkten gehörenden kommunikativen Ziele meistens angemessen erreichen.

Die ausführliche Behandlung des dritten und insbesondere des vierten Leitpunktes hat den Schülern Probleme bereitet. Aus den Beispielen in der Tabelle 6 geht hervor, dass die Schüler nicht wie in der Testaufgabe vorgesehen Vorschläge gemacht, sondern konkrete Aussagen geschrieben haben.

\begin{tabular}{|l|l|}
\hline Problem & Beispiele aus den Schülerarbeiten \\
\hline $\begin{array}{l}\text { Viele Schüler haben keine } \\
\text { Vorschläge gemacht, } \\
\text { sondern sie haben das } \\
\text { Reiseziel konkret ange- } \\
\text { ben. }\end{array}$ & $\begin{array}{l}\text { "Ich glaube dass, wir nach Frankreich fahren." } \\
\text { "Dächte in Israel gehen, und du auch." } \\
\text { Stadt sehen!" }\end{array}$ \\
$\begin{array}{l}\text { Die Kandidaten haben das } \\
\text { Datum der Reise konkret } \\
\text { angegeben. }\end{array}$ & $\begin{array}{l}\text { "Die Reise wird in August sein und [...] dauert ein } \\
\text { Woche." }\end{array}$ \\
& $\begin{array}{l}\text { "Wir fahren am Moi Woche." } \\
\text { "Diese Reise werde 11 Tage dauern." } \\
\text { "Der Reise dauert: von 30. Mai bis 13. Juni." }\end{array}$ \\
\hline
\end{tabular}

Tabelle 6: Beispiele für Probleme bei der Behandlung des dritten und vierten Leitpunktes Reiseziel und Dauer der Reise 
Die Behandlung des fünften Leitpunktes bereitete sowohl in den schwachen als auch in den guten Schülerarbeiten Probleme. In Tabelle 7 sind einige Beispiele für die Problematik der Behandlung dieses Leitpunktes angeführt.

\begin{tabular}{|l|l|}
\hline Problem & Beispiele aus den Schülerarbeiten \\
\hline $\begin{array}{l}\text { Einige Schüler haben ver- } \\
\text { sucht, Teile aus dem Input- } \\
\text { text und den Leitpunkt in } \\
\text { den eigenen Text einzu- } \\
\text { bauen. }\end{array}$ & $\begin{array}{l}\text { "[Reiseziele] Die Broschüre gibt auf 50 Seiten wichti- } \\
\text { schreib zurück!" }\end{array}$ \\
\hline $\begin{array}{l}\text { "Kommst du mit mir nach Frankreich?] Die Zeitung } \\
\text { schickt eine Gratisbroschüre. [Ich möchte 1,5 oder 2 } \\
\text { rende kommunikative Ziel } \\
\text { Wurde nicht erreicht. }\end{array}$ & $\begin{array}{l}\text { "Ich habe schon fahren." } \\
\text { bestellt, was ich für dich auch schicken werde." }\end{array}$ \\
\hline
\end{tabular}

Tabelle 7: Beispiele für Probleme bei der Behandlung des fünften Leitpunktes Broschüre bestellen

Hier werden noch zwei Beispiele für angemessene Behandlung angegeben:

$>$ „Wenn du mit mir fahren möchtest (ich möchte nicht allein gehen), dann muss ich eine Broschüre bestellen, und studieren was die Bedienungen sind.“

$>$ „Vor allem müssen wir Broschüre bestellen und es ist gratis! In einer Broschüre können wir die wichtige Hinweise lesen."

Im Folgenden wird das zweite Thema (über eine Geburtstagsparty) behandelt.

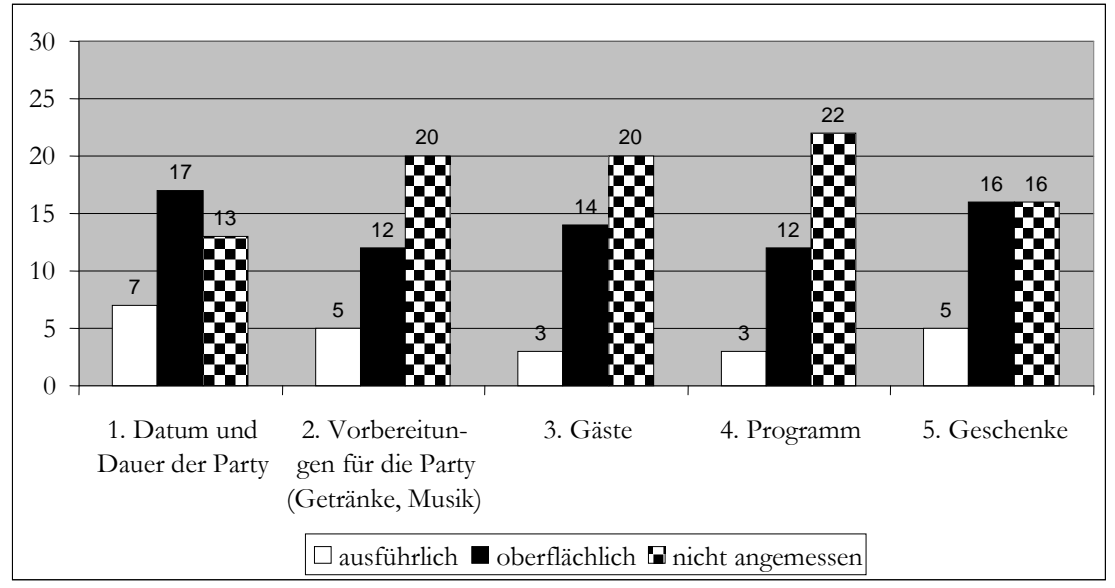

Abbildung 5: Schwache Schülerleistungen (0-2 Punkte) im zweiten Thema ( $n=37)$ 
Die Abbildung 5 der schwachen Schülerleistungen zeigt, dass die meisten Schüler nur den ersten Leitpunkt behandeln konnten. Die Ausführung der anderen Leitpunkte war für die meisten Kandidaten in dieser Leistungsgruppe problematisch.

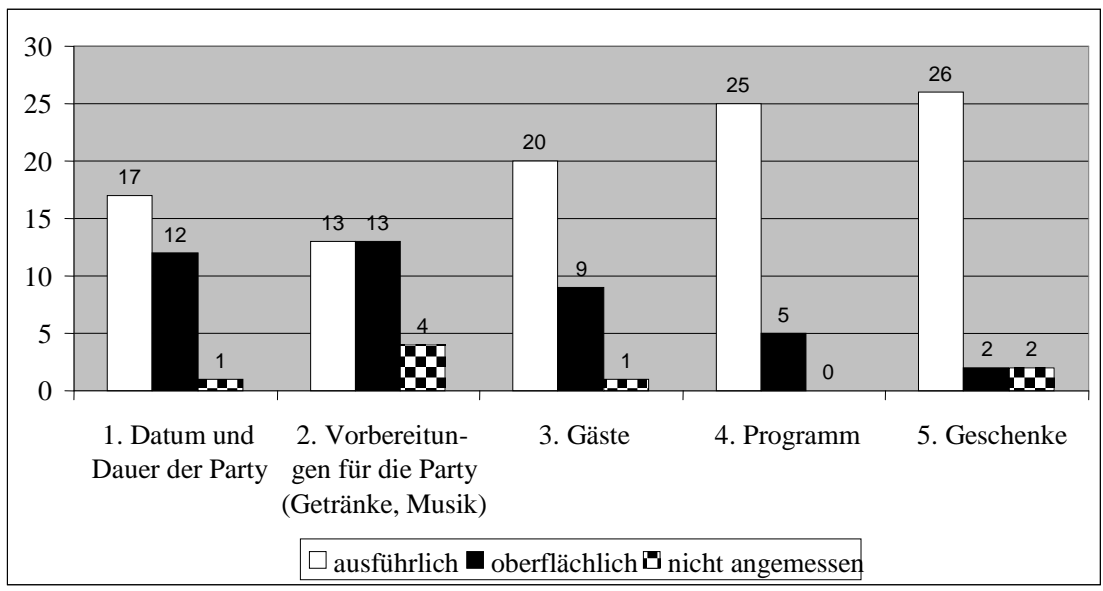

Abbildung 6: Gute Schülerleistungen (3-5 Punkte) im zweiten Thema ( $n=30)$

In dieser Leistungsgruppe (Abbildung O) kann man sehen, dass die Testpersonen den dritten, vierten und fünften Leitpunkt ausführlich behandeln konnten. Außerdem ist festzustellen, dass bei diesen drei Leitpunkten die Lösungsrate der zwei Leistungsgruppen deutlich voneinander unterscheidet (Abbildungen 5 und 6 ).

Bei der Ausführung dieses Themas bereitete es den Schülern Schwierigkeiten, den ersten und zweiten Leitpunkt ausführlich zu behandeln (Tabelle 8).

\begin{tabular}{|l|l|}
\hline Problem & Beispiele aus den Schülerarbeiten \\
\hline Das Datum fehlt. & $\begin{array}{l}\text { "Die Party hat um 14 Uhr begonnen und sie hat bis 21 } \\
\text { Uhr gedauert." }\end{array}$ \\
\hline $\begin{array}{l}\text { Die angemessene Formulierung } \\
\text { der Dauer der Party hat den } \\
\text { Schülern Probleme bereitet. }\end{array}$ & $\begin{array}{l}\text { "Mein Geburtstagsparty war im 15. Mai. Das Party } \\
\text { begann um 8 Uhr." }\end{array}$ \\
\hline $\begin{array}{l}\text { Der Leser bekommt keine } \\
\text { Information. }\end{array}$ & $\begin{array}{l}\text { "Die Vorbereitungen für die Party könnten schwer } \\
\text { zusammenkommen. Einmal war keine Getränke, dann } \\
\text { wird der Musiker krank. Und sie kamen keine Tänzer." }\end{array}$ \\
\hline $\begin{array}{l}\text { Die Schüler haben nur über } \\
\text { eine Angabe geschrieben. }\end{array}$ & $\begin{array}{l}\text { "Ich habe ein Dj eingeladen und er brachte die Musik. } \\
\text { Meine Mutter hat gebackt, sie backt den ganzen Tag." } \\
\text { [Beim Programm: „Wir hat getrunken."] }\end{array}$ \\
\hline
\end{tabular}

Tabelle 8: Beispiele für Probleme bei der Behandlung des ersten und zweiten Leitpunktes Datum und Dauer der Party und Vorbereitungen für die Party (Getränke, Musik usw.) 
Aus den Beispielen in der Tabelle 8 geht hervor, dass das Problem wahrscheinlich darin liegt, dass diese Leitpunkte weitere Angaben enthalten, und der Leitpunkt wird nur dann ausführlich behandelt, wenn die Schüler alle weiteren Angaben behandeln (vgl. Probeabitur 2004. DaF. Mittelstufe. Scbriftliche Prüfung. Korrekturund Bewertungsanleitung, S. 12).

\subsection{Analyse der Testaufgabe}

Im Folgenden wird behandelt, in welchem Zusammenhang die inhaltlichen Leitpunkte zueinander stehen. Dazu habe ich Regressionsanalyse vorgenommen, um analysieren zu können, wie die Lösungsrate der einzelnen Leitpunkte die Gesamtpunktzahl erklärt. Bei den zwei Themen wurden die zwei Leistungsgruppen getrennt analysiert (Abbildung 7, 8).

Schwache Schülerleistungen ( $n=33)$ Gute Schülerleistungen $(n=20)$ abhängige Variable: Gesamtpunktzahl

\begin{tabular}{|c|c|c|c|c|c|}
\hline & Sig. & $\%$ & & Sig. & $\%$ \\
\hline 1. Leitpunkt &, $000^{*}$ & 38,52 & 5. Leitpunkt &, $000^{*}$ & 37,99 \\
\hline 2. Leitpunkt &, $002^{\star}$ & 26,10 & 4. Leitpunkt &, $000^{*}$ & 21,74 \\
\hline 4. Leitpunkt &, $047^{\star}$ & 12,25 & 1. Leitpunkt &, $002^{*}$ & 15,67 \\
\hline 3. Leitpunkt & ,306 & 5,43 & 2. Leitpunkt &, $006^{*}$ & 7,70 \\
\hline 5. Leitpunkt & ,057 & 4,45 & 3. Leitpunkt &, $003^{*}$ & 1,29 \\
\hline \multicolumn{2}{|c|}{ Erklärte Varianz } & 86,75 & \multicolumn{2}{|c|}{ Erklärte Varianz } & 84,39 \\
\hline
\end{tabular}

Abbildung 7: Ergebnisse der Regressionsanalyse im ersten Thema

Der Erklärungswert der ersten zwei Leitpunkte (Grund des Schreibens und Frage nach der Meinung des Briefpartners) in den schwachen Schülerarbeiten ist am höchsten. Diese Leitpunkte haben aber kleineren Wert in der anderen Leistungsgruppe.

Die Lösungsrate des fünften Leitpunktes (Broscbüre bestellen) erklärt die Gesamtpunktzahl in den guten Schülerarbeiten am besten, dieser Leitpunkt hatte aber in der anderen Leistungsgruppe keine signifikante Wirkung. So ist er am schwierigsten zu lösen.

Der dritte Leitpunkt (Reisezię) hat den kleinsten Erklärungswert sowohl in der Gruppe der schwachen als auch in der guten Schülergruppe, so ist dieser Leitpunkt primär. 
Schwache Schülerleistungen ( $n=37)$ Gute Schülerleistungen $(n=30)$ abhängige Variable: Gesamtpunktzahl

\begin{tabular}{|c|c|c|c|c|c|}
\hline & Sig. & $\%$ & & Sig. & $\%$ \\
\hline 1. Leitpunkt &, $002^{*}$ & 29,06 & 4. Leitpunkt &, $000^{\star}$ & 26,73 \\
\hline 2. Leitpunkt &, $001^{*}$ & 20,63 & 5. Leitpunkt &, $001^{*}$ & 18,99 \\
\hline 4. Leitpunkt &, $001^{*}$ & 18,32 & 2. Leitpunkt &, $000^{\star}$ & 13,53 \\
\hline 3. Leitpunkt &, $012^{*}$ & 12,38 & 3. Leitpunkt &, $008^{\star}$ & 11,59 \\
\hline 5. Leitpunkt & 322 & 6,02 & 1. Leitpunkt &, $000^{\star}$ & 5,83 \\
\hline \multicolumn{2}{|c|}{ Erklärte Varianz } & 86,41 & \multicolumn{2}{|c|}{ Erklärte Varianz } & 76,67 \\
\hline
\end{tabular}

Abbildung 8: Ergebnisse der Regressionsanalyse im zweiten Thema

Beim zweiten Thema (über die Geburtstagsparty) möchte ich wegen der niedrigen erklärten Varianz in der zweiten Gruppe (Abbildung 8 ) nur auf zwei Tendenzen hinweisen:

(1) Der erste Leitpunkt (Datum und Dauer der Party) erklärt am besten die Gesamtpunktzahl in den schwachen Schülerarbeiten, hat aber den niedrigsten Wert in der anderen Leistungsgruppe.

(2) Der fünfte Leitpunkt (Geschenkee) hatte keinen signifikanten Wert in der ersten Gruppe, in den guten Schülerarbeiten steht er an der zweiten Stelle. Die anderen drei Leitpunkte haben ähnlichen Erklärungswert in den beiden Leistungsgruppen.

So kann man anhand der Regressionsanalyse bei den verschiedenen Leitpunkten auch Umgestaltungen sehen, und das deutet darauf hin, dass die Leitpunkte zwischen den Leistungsgruppen differenzieren.

\subsection{Analyse der Bewertung der Qualität der Leitpunkte}

Zum Schluss wird die folgende Frage behandelt: Welche Verfahren sichern eine eindeutige Entscheidung von ausführlicher und oberflächlicher Behandlung eines Leitpunktes?

Bei der Analyse konnte ich feststellen, dass die objektive Bewertung:

(1) durch den Input und Mitteilungsabsicht,

(2) durch die Aufgabenstellung,

(3) durch die Definition in der Bewertungsanleitung, und

(4) durch die kommunikative Situation erfolgen kann.

$\mathrm{Zu}$ diesen Verfahren gebe ich die Leitpunkte aus den Testaufgaben an. Beim Leitpunkt Grund des Schreibens sollten die Schüler auf eine Zeitungsanzeige reagieren (Input) und Lust auf eine gemeinsame Reise machen (Mitteilungsabsicht). 
Die objektive Bewertung der Qualität der Leitpunkte Reiseziel, Dauer der Reise wird durch die Aufgabenstellung (Machen Sie ibm/ibr Vorschläge, um die Reise zusammen planen zu können) bestimmt.

Bei der Frage nach der Meinung des Briefpartners bedeutet eine angemessene Fragestellung, dass der Schüler den Leitpunkt ausführlich behandelte. Die Leitpunkte Datum und Dauer der Party und Vorbereitungen für die Party (Getränke, Musik usw.) enthalten weitere Angaben, und die Behandlung kann nur dann für ausführlich gehalten werden, wenn der Kandidat alle weiteren Angaben behandelt (vgl. Probeabitur 2004. DaF. Mittelstufe. Schriftliche Prüfung. Korrektur- und Bewertungsanleitung, S. 12f.). Die Leitpunkte Broscbüre bestellen; Programm werden durch die kommunikative Situation determiniert. Die Bewertung von ausführlicher und oberflächlicher Behandlung von Gäste und Geschenke war in einigen Fällen problematisch. Dazu gibt es Beispiele in der Tabelle 9.

\begin{tabular}{|c|c|}
\hline $\begin{array}{l}\text { Beispiele für oberflächlich gehaltene } \\
\text { Behandlung }\end{array}$ & $\begin{array}{l}\text { Beispiele für ausführlich gehaltene } \\
\text { Behandlung }\end{array}$ \\
\hline $\begin{array}{l}\text { "Dort war } 40 \text { Menschen (nur meine } \\
\text { Freunde)." } \\
\text { „Ich habe auf meine Party zirka } 60 \\
\text { Leuten eingeladen." } \\
\text { „Zusammen ist zehn Leute gekommen.” }\end{array}$ & $\begin{array}{l}\text { "Die Gäste waren zehn." } \\
\text { "In der Party waren zirka } 20 \text { Leuten." } \\
\text { "Ich lud meine Freunde ein, und sie kam } \\
\text { alle bei uns." }\end{array}$ \\
\hline $\begin{array}{l}\text { "Die Geschenke waren sehr schön, und } \\
\text { interessanten." } \\
\text { "Meine Freund habe ich eine schöne } \\
\text { Puli bekommen." }\end{array}$ & $\begin{array}{l}\text { "Ich bekam viele schönen Geschenke, } \\
\text { ich fraute über sie sehr." } \\
\text { „Die Geschänke sind überhaupt ver- } \\
\text { schiede Kleinigkeiten." }\end{array}$ \\
\hline $\begin{array}{l}\text { "Ich habe viele Geschänke bekommen. } \\
\text { Ich habe ein Fußball, und eine sehr } \\
\text { schöne Halskette." } \\
\text { "Ich habe viele Geschenke bekommen. } \\
\text { Ich habe Bücher, Torte bekommen." }\end{array}$ & $\begin{array}{l}\text { "Ich habe CD-s, Bücher, Kleider, Blumen } \\
\text { bekommen." } \\
\text { "Ich bekam die Geschenke: ein Fahrrad, } \\
\text { Kleider, Bücher und ein Radio." }\end{array}$ \\
\hline
\end{tabular}

Tabelle 9: Beispiele für gleiche Behandlung des Leitpunktes aber unterschiedliche qualitative Entscheidung beim dritten und fünften Leitpunkt Gäste und Geschenke

\section{$4 \quad$ Konklusionen}

Anhand der Analyse von Schülerleistungen und der Testaufgabe kann man folgende Schlussfolgerungen ziehen:

(1) Den meisten Schülern ist es ein Problem, auf alle inhaltlichen Leitpunkte einzugehen, und sie angemessen zu behandeln. So wurden einige Leitpunkte sehr ausführlich, die anderen dagegen nur oberflächlich oder gar nicht behandelt. Daraus folgt, dass die Schüler das angemessene Verhält- 
nis zwischen den einzelnen Leitpunkten kaum bzw. nur mit Schwierigkeiten finden können.

(2) Die Bewältigung der kommunikativen Ziele der Themen war unterschiedlich: Bei dem ersten Thema hat die Mehrheit der Schüler die kommunikativen Ziele nur teilweise bei dem zweiten Thema meistens angemessen erreicht.

(3) Außerdem ist festzustellen, dass die Gruppe der schwachen und guten Schülerleistungen voneinander stark abweichen, weil die Leitpunkte zwischen den einzelnen Leistungsgruppen differenzieren.

Aus der Analyse der Bewertung erfolgt, dass die Formulierung der Leitpunkte folgende Voraussetzungen erfüllen sollten. Erstens sollten sie aus dem Input erfolgen, voneinander gut trennbar sein, und auch ermöglichen, dass bei ihnen möglichst keine Überlappungen vorkommen. Zweitens sollten die Leitpunkte die Textproduktion der Schüler eindeutig steuern, aber sie sollten auch so formuliert werden, dass die Schüler sie bei der Behandlung verändern. Drittens ist es wichtig, dass für den Prüfer die Entscheidung hinsichtlich der ausführlichen oder oberflächlichen Behandlung der Leitpunkte eindeutig ist.

Aus der Forschungsarbeit lässt sich feststellen, dass im Fremdsprachenunterricht noch mehr Gewicht auf die Förderung der Schreibfertigkeit gelegt werden soll. Es scheint auch notwendig zu sein, den Deutschlehrern weitere, der Bewertungsanleitung entsprechend korrigierte und bewertete Schülerarbeiten zur Verfügung zu stellen. Außerdem ist es bei diesem Prüfungsteil wichtig, die Testaufgaben vorher zu erproben und die Ergebnisse zu analysieren und wenn es nötig ist, die Testaufgabe zu überarbeiten.

Im Weiteren könnte man auch die Entwicklungstendenzen bei Schülerleistungen, Bewertungsverfahren und Testaufgaben bei der Schreibfertigkeit im Rahmen der Abiturprüfungen untersuchen.

\section{Literatur}

Bolton, Sibylle: Probleme der Leistungsmessung. Lernfortschrittstests in der Grundstufe.

Fernstudieneinheit 10. Berlin etc.: Langenscheidt 1996.

Einhorn, Ágnes: „Die erste Erprobung im Rahmen der Abiturreform für DaF“ Deutschunterricht für Ungarn 2000/1, 35-54.

Einhorn, Ágnes: „Die Revision der ungarischen Abschlussprüfung in DaF.

Prinzipien und Ergebnisse der Forschungsarbeit 1996-2002“. In: Ágel, Vilmos / Herzog, Andreas (Hrsg.): Jahrbuch der ungarischen Germanistik. 2002, 315-329.

Einhorn, Ágnes (Hrsg.): Kézikönyv a német érettségi reformjához: [Handbuch zur

Abiturreform für DaF] 2004. Online im Internet:

http://www.om.hu/letolt/kozokt/erettsegi2005/tanaroknak/nemet/német_nyelv.htm 
Einhorn, Ágnes: A 2005. évi német érettségi vizsga tanulságai. [Die Erfahrungen bei dem Abitur 2005 Deutsch als Fremdsprache] Unveröff. Manuskript. (2006)

Einhorn, Ágnes / Major, Éva: "2004-es próbaérettségi tapasztalatai - idegen nyelvek“" [Die Erfahrungen bei der Probeprüfung 2004 - Fremdsprachen], Új Pedagógiai Szemle, 2005/3, 11-27. Online im Internet: http://www.oki.hu/oldal.php?tipus=cikk\&kod=2005-03-ta-tobbek-2004

Kast, Bernd: Fertigkeit Schreiben. Fernstudieneinheit 12. Berlin etc.: Langenscheidt 1999.

Petneki, Katalin: „Entwicklung des Abiturkonzepts für den Deutschunterricht“, Deutschunterricht für Ungarn, 2002/1-2, 33-42.

Petneki, Katalin: „Schreibfertigkeit im neuen Abitur. Ergebnisse der Probeprüfung 2004 auf der Oberstufe.“ In: Szatmári, Petra (Hrsg.):

Gedenkschrift an Prof. János Kohn. Szombathely. (Erscheint im Jahre 2006)

Próbaérettségi (2004. május): Német nyelv. Középszint. Íráskészség. Probeabitur (Mai 2004): DaF. Mittelstufe. Prüfungsteil Schreiben.] Budapest: OKÉV; OKI. Online im Internet:

http://www.om.hu/letolt/okev/doc/probaerettsegi_2004/nemet_kz_irkesz.pdf

Próbaérettségi (2004. május): Német nyelv. Középszint. Írásbeli vizsga. Javításiértékelési útmutató. [Probeabitur (Mai 2004): DaF. Mittelstufe. Schriftliche Prüfung. Korrektur- und Bewertungsanleitung] Budapest: OKÉV; OKI. 1018. Online im Internet:

http://www.om.hu/letolt/okev/doc/probaerettsegi_2004/nemet_kz_utmut.pdf

Vígh, Tibor (2006): „Der Prüfungsteil Schreiben im neuen Abitur für DaF“. In: Orosz, Magdolna / Albrecht, Terrance (Hrsg.): Jahrbuch der ungarischen Germanistik 2005, 335-351.

„Vizsgakövetelmények és vizsgaleírás. Német nyelv.“ [Prüfungsregelung und beschreibung. DaF] In: Magyar Közlöny: 2003/57/II. 731-773. 



\section{Problematik der Niveaustufen im neuen Abitur für Deutsch als Fremdsprache in Ungarn}

Katalin Petneki, Szeged (Ungarn)

\section{Das neue Abitur für Deutsch als Fremdsprache in Ungarn}

Das neue Abitur ist eine zweistufige, standardisierte und - bei den Fremdsprachen - eine einsprachige Prüfung. Es wurde im Mai 2005 in Ungarn eingeführt. Die zwei Stufen der Prüfung haben eigentlich nichts mit dem Gemeinsamen europäischen Referenzrahmen zu tun. Für alle Schulfächer, in denen das Abitur abgelegt wird, gilt, dass die Prüfung auf einer sog. „Mittelstufe“ oder auf einer sog. „, Oberstufe“ abgelegt werden kann. Die „Mittelstufe“ bedeutet den Abschluss der Schulausbildung, die „Oberstufe“ dient als Einstiegsprüfung für ein Studium. Bei Fremdsprachen orientiert sich das Abitur selbstverständlich an den Niveaustufen des Gemeinsamen europäischen Referenzrahmens. Um die Stufen des ungarischen Abiturs für Deutsch mit den Sprachniveaus vergleichen zu können, habe ich die Prüfungen des Goethe-Instituts als Beispiel genommen. 


\begin{tabular}{|l|l|l|l||}
\hline $\begin{array}{l}\text { Niveaustufen des } \\
\text { Gemeinsamen } \\
\text { Eurpoäischen } \\
\text { Referenzrahmens }\end{array}$ & Prüfungen des \\
Goethe-Instituts & $\begin{array}{l}\text { Abitur } \\
\text { (Abschluss der } \\
\text { Ausbildung) }\end{array}$ & $\begin{array}{l}\text { Abitur } \\
\text { (Aufnahme- } \\
\text { prüfung) }\end{array}$ \\
\hline C2 Mastery & ZOP, KDS & & \\
\hline C1 Effektive Operational Profiency & ZMP & & \\
\hline B2 Vantage & & & ,Oberstufe" \\
\hline B1 Treshold Level & Zertifikat Deutsch & ,Mittelstufe" & \\
\hline A2 Waystage & Fit in Deutsch 2 & Ungarn & \\
\hline A1 Breakthrough & Fit in Deutsch 1 & & \\
\hline
\end{tabular}

Tab. 1: Niveaustufen des neuen Abiturs im Vergleich

Die zweistufige Prüfung erfüllt auch bei Fremdsprachen zwei verschiedene Funktionen: Die Mittelstufe bedeutet den Schulabschluss, die Oberstufe bedeutet den Einstieg ins Studium. Diejenigen Schüler also, die z. B. Germanistik studieren möchten, brauchen keine Aufnahmeprüfung extra abzulegen.

Das Abitur besteht generell aus zwei Teilen: Aus einem schriftlichen Teil, der auf der „Mittelstufe“ 180 Minuten lang, auf der „Oberstufe“ 240 Minuten lang dauert, und aus einem mündlichen Teil, der 15 bzw. 20 Minuten lang dauert. Dies gilt für alle Fächer einheitlich.

Die Prüfung bei Fremdsprachen besteht auf beiden Stufen aus fünf Teilen. Die schriftliche Prüfung umfasst vier Prüfungsteile, die mündliche nur einen Prüfungsteil. Die vier Fertigkeiten werden in der Prüfung gleichmäßig bewertet, der Prüfungsteil „Strukturen und Wortschatz” hat aber auf der „Mittelstufe“ einen geringeren Anteil. Der Grund dafür liegt in den Niveaustufen. Auf dieser Stufe sollen die Prüfungskandidaten ja nur die einfachsten bzw. elementaren Strukturen und nur den grundlegenden Wortschatz verwenden können. Sobald die Mitteilung des Kandidaten verständlich ist, wird die Aussage akzeptiert. Es geht also darum, dass der Kandidat zeigt, dass er auf einer Elementarstufe in der Zielsprache handlungsfähig ist. 


\begin{tabular}{||c|c|c|c||}
\hline \multirow{2}{*}{ Prüfungsteil: } & & $\begin{array}{c}\text { Prüfungszeit } \\
\text { (Minuten) }\end{array}$ & Anteil (\%) \\
\hline \multirow{3}{*}{ Schriftliche } & Leseverstehen & 60 & 22 \\
\cline { 2 - 4 } Prüfung: & Strukturen und Wortschatz & 30 & 12 \\
\cline { 2 - 4 } & \multicolumn{2}{|c|}{ PAUSE } & 22 \\
\cline { 2 - 4 } & Hörverstehen & 30 & 22 \\
\cline { 2 - 4 } & Schreibfertigkeit & 60 & - \\
\hline \multirow{2}{*}{ Mündliche Prüfung: } & Insgesamt: & 180 & 100 \\
\hline & Sprechfertigkeit & 15 & 22 \\
\hline \hline
\end{tabular}

Tab. 2: Struktur des Abiturs auf der sog. Mittelstufe

Die Prüfung auf der Oberstufe besteht aus denselben Teilen wie auf der Mittelstufe. Der Unterschied liegt einerseits im Zeitrahmen: Für diese Prüfung steht mehr Zeit zur Verfügung, andererseits ist die Gewichtung der einzelnen Prüfungsteile hier identisch. Das bedeutet, dass auf der sog. Oberstufe die angemessene Wortwahl und die grammatische Korrektheit genauso wichtig sind wie die einzelnen Fertigkeiten. Diese Prüfung bedeutet schließlich gleichzeitig die Zulassung zum Studium (z. B. Germanistik).

\begin{tabular}{|c|c|c|c|}
\hline \hline \multirow{2}{*}{ Prüfungsteil: } & & $\begin{array}{c}\text { Prüfungszeit } \\
\text { (Minuten) }\end{array}$ & Anteil (\%) \\
\hline \multirow{3}{*}{$\begin{array}{c}\text { Schriftliche } \\
\text { Prüfung: }\end{array}$} & Leseverstehen & 70 & 20 \\
\cline { 2 - 4 } & Strukturen und Wortschatz & 50 & 20 \\
\cline { 2 - 4 } & \multicolumn{2}{|c|}{ PAUSE } \\
\cline { 2 - 4 } & Hörverstehen & 30 & 20 \\
\hline & Schreibfertigkeit & 90 & 20 \\
\hline Mündliche Prüfung: & Insgesamt: & 240 & - \\
\hline & Sprechfertigkeit & 20 & 20 \\
\hline
\end{tabular}

Tab. 3: Struktur des Abiturs auf der sog. Oberstufe 


\section{Kurze Entstehungsgeschichte des neuen Abiturs}

Das neue Prüfungsmodell hat einen ziemlich langen Weg bis zur Einführung hinterlegt. Es hat vor 10 Jahren angefangen, als ein Team im Staatlichen Institut für Unterrichtswesen in Budapest mit der Erarbeitung des Prüfungsmodells (für jedes Schulfach) angefangen hat. Die fachliche Arbeit musste sich an dem neuen Curriculum bzw. an der neuen Prüfungsregelung orientieren. Mit dem Wandel schulpolitischer Auffassungen wurden immer wieder neue Aufgaben an die Entwickler des Modells gestellt. Hier folgt eine kurze Zusammenfassung, wie dieser Weg verlaufen ist.

> 1996: Ein Jahr nach der Einführung eines neuen Curriculums wurde das Bedürfnis formuliert, nicht nur den grundlegenden Lehrplan, sondern auch das Abitur zu verändern. Die erste Aufgabe war, die gegenwärtige Lage zu analysieren, im internationalen Kontext zu vergleichen. Die ersten Hypothesen über das neue Prüfungs modell wurden formuliert und diskutiert - ein Studienband wur de darüber 1998 veröffentlicht.

> 1997: Als gerade der erste Modellentwurf entstanden ist, wurde das neue Gesetz als Prüfungsregelung erlassen, wonach das neue Modell im Jahre 2004 eingeführt werden sollte.

> 1998: Die ersten Modellaufgaben wurden entwickelt und ausprobiert.

$>$ 2000: Nach den ersten Erfahrungen wurden Aufgaben überarbeitet und neue entwickelt, darauf folgte wieder eine Erprobung.

2001: Im Schulwesen wurde ein Rahmenlehrplan eingeführt, so musste das Prüfungsmodell an die neuen Anforderungen angeglichen werden.

2002: Lehrerhandreichungen wurden zur neuen Prüfung erarbeitet und im Internet zugänglich gemacht.

2003: Die Prüfungsanforderungen wurden nochmals überarbeitet und eine landesweite Probeprüfung wurde sowohl schriftlich als auch mündlich durchgeführt.

2004: Die letzten Änderungen im Prüfungsmodell wurden anhand der bisherigen Erfahrungen durchgeführt. Die letzte Probeprüfung wurde nur noch im schriftlichen Prüfungsteil durchgeführt. Mit der Prüferschulung wurde landesweit begonnen.

2005: Die erste Prüfung wurde nach dem neuen Modell durchgeführt.

\section{3 Änderungen im neuen Abitur}

Welche Änderungen hat das neue Abitur mit sich gebracht? Wie war es vorher? Um zu verstehen, welche Bedeutung die neue Prüfung für den Fremdsprachenun- 
terricht hat, wird hier kurz geschildert, worin die Unterschiede zum früheren Abitur bestehen.

Vor 2005:

$>$ Niveaustufe ist unklar

$>$ zweisprachig, wobei die Übersetzung eine zentrale Rolle spielt

$>$ Schwerpunkt: grammatische und lexikalische Kenntnisse
Ab 2005:

$>$ die Prüfung ist standardisiert

$>$ Niveaustufen sind klar definiert

$>$ einsprachig

> Schwerpunkt: kommunikative Sprachverwendung

Tab. 4: Unterschiede in den Grundprinzipien

Vor 2005:

Die Fertigkeiten wurden nicht gleichmäßig geprütt:

$>$ Hörverstehen wurde nicht geprüft

> Schreibfertigkeit war mit Übersetzung identisch

$>$ Sprechfertigkeit wurde unterschiedlich geprüft.
Ab 2005:

Alle Fertigkeiten werden gleichmäßig behandelt:

$>$ Hörverstehen wird auch geprüft

$>$ produktive Fertigkeiten werden durch gut definierte Aufgaben geprütt.

Tab. 5: Unterschiede beim Anteil der kommunikativen Fertigkeiten

Vor 2005:

Die Aufgabentypen:

$>$ waren aus testtypologischer Sicht problematisch

$>$ waren meist schematisch und geschlossen

$>\quad$ waren bei der mündlichen Prüfung nicht festgelegt und so war die Leistung nicht vergleichbar.

\section{Ab 2005:}

Die Aufgaben:

$>$ bauen auf authentische Texte $u$. Situationen, sind abwechslungsreich

$>$ sind nicht nur geschlossen

$>$ verlangen sowohl schriftlich als auch mündlich sprachliche Handlungsfähigkeit

Tab. 6: Unterschiede bei der Aufgabentypologie

\begin{tabular}{|c|c|}
\hline $\begin{array}{l}\text { Vor 2005: } \\
\text { Bewertung: } \\
\quad \text { Bewertungsanleitung nur global } \\
\quad \text { Abweichungen vor allem bei der } \\
\text { Bewertung der produktiven Fertig- } \\
\text { keiten (subjektiv) }\end{array}$ & $\begin{array}{l}\text { Ab 2005: } \\
\text { Bewertung: } \\
\quad \text { detaillierte Bewertungsanleitung mit } \\
\text { analytischen Skalen zur Bewertung } \\
\text { der produktiven Fertigkeiten - neu } \\
\quad \text { für die Lehrer }\end{array}$ \\
\hline$>$ Ergebnisse: Nicht zuverlässig & $>$ Ergebnisse: Zuverlässig \\
\hline
\end{tabular}

Tab. 7: Unterschiede bei der Bewertung 
Die Uneinheitlichkeiten des früheren Abiturs rühren unter anderem daher, dass die Aufgaben des alten Abiturs - vom Schultyp und Lehrplan abhängig - von drei verschiedenen Institutionen erstellt wurden. Es ist vorgekommen, dass gleichzeitig dreizehn verschiedene Prüfungssätze eingesetzt wurden. So konnte das Grundprinzip der Standardisierung nicht verwirklicht werden. Da der Fremdsprachenunterricht in den 90er Jahren eine große didaktisch-methodische Entwicklung durchgemacht hat, war es unerlässlich, eine zuverlässige, standardisierte Prüfung einzuführen.

\section{$4 \quad$ Wirkung der Abiturreform}

Was hat man vom neuen Abitur erwartet? Wenn man die Antwort auf diese Frage sucht, dann findet man den Schlüssel dazu in der Lage des Fremdsprachenunterrichts Mitte der 90er Jahre. Es gab einerseits eine große Entwicklung in didaktisch-methodischer Hinsicht, hinzu kam noch eine große Auswahl an Lehrmaterialien, den Lehrern wurde große Freiheit in der Schule gesichert, wie man unterrichtet, andererseits bestand die Möglichkeit, weiterhin unerschüttert einen grammatikorientierten Frontalunterricht zu machen, mit der Begründung, das werde von der (damaligen) Prüfung verlangt.

1995 wurde das neue Curriculum eingeführt, in denen neue Ziele deklariert wurden, aber die Verwirklichung dieser Ziele konnte nur so erreicht werden, wenn sich auch die Prüfungsanforderungen daran orientieren. Das bedeutet, die Ziele des Nationalen Grundlehrplans können sich nur dann in der Unterrichtspraxis verwirklicht werden, wenn die Anforderungen der Abschlussprüfung überprüfen können, ob die gesetzten Ziele erreicht worden sind oder nicht.

\begin{tabular}{|l|l|}
\hline Neue Ziele im FSU: & Neue Messziele: \\
\hline $\begin{array}{l}\text { Entwicklung der Fertigkeiten und Kompeten- } \\
\text { zen }\end{array}$ & Fertigkeiten / Kompetenzen gezielt messen \\
\hline Handlungs- und schülerorientierter Unterricht & Handlungsorientierte Aufgaben \\
\hline Authentische Texte und Situationen & Prinzip der Authentizität \\
\hline Klare Ziele & Transparente Anforderungen \\
\hline
\end{tabular}

Tab. 8: Zusammenhang zwischen den Zielen im Curriculum und in den Messzielen

Gerade diese Erwartungen werden vom neuen Prüfungsmodell gesichert.

\section{$5 \quad$ Drei Sprachniveaus auf zwei Stufen}

Von den drei Niveaus sind zwei auf der sog. Mittelstufe. Die sog. Oberstufe ist dagegen auf dem Niveau B2. Der Gemeinsame europäische Referenzrahmen differenziert aber innerhalb dieses Niveaus auch noch weiter, so kann man auch in 
diesem Prüfungsteil im Schwierigkeitsgrad unter den Aufgaben kleinere Unterschiede feststellen. Wie funktionieren die drei Sprachniveaus auf zwei Prüfungsstufen?

Mittelstufe: A2-B1

$>$ Beim schriftlichen Teil wird der Schwierigkeitsgrad der Aufgaben innerhalb eines Testsatzes anhand von Hypothesen stufenweise erhöht.

$>$ Bei der Schreibfertigkeit gibt es Unterschiede sowohl bei den Aufgaben, als auch bei der Bewertung durch differenzierte Kriterien.

$>$ Beim mündlichen Teil erfolgt die Differenzierung bei der Bewertung in den Punktzahlen.
Oberstufe: B2

$>$ Es stellt eine einheitliche Niveaustufe dar.

$>$ Schwierigkeit hängt vom Text und von der Aufgabe ab.

$>$ Bewertungskriterien orientieren sich an einer höheren Stufe.

Tab. 9: Realisierung der Niveauunterschiede auf den zwei Prüfungsstufen

Die Schreibfertigkeit wird auf beiden Stufen immer jeweils mit zwei Aufgaben geprüft. Da die Schreibfertigkeit sowohl in den Aufgaben, als auch in der Bewertung die Unterschiede zeigt, folgt hier ein Beispiel aus diesem Prüfungsteil, wie die Sprachniveaus da erscheinen.

\begin{tabular}{|c|c|c|c|}
\hline $\begin{array}{l}\text { Mittelstufe A2 } \\
\text { 1. Aufgabe } \\
\text { (40-50 Wörter) } \\
\text { Eine persönliche } \\
\text { Reaktion auf eine E- } \\
\text { Mail oder Karte }\end{array}$ & $\begin{array}{l}\text { Mittelstufe B1 } \\
\text { 2. Aufgabe } \\
\text { (80-100 Wörter) } \\
\text { Persönlicher Brief } \\
\text { über ein Thema }{ }^{1} \\
\text { anhand von } 5 \\
\text { Leitpunkten }\end{array}$ & $\begin{array}{l}\text { Oberstufe B2 } \\
\text { 1.Aufgabe } \\
\text { (100-120 Wörter) } \\
\text { Halboffizieller Brief } \\
\text { anhand von } 5 \\
\text { Leitpunkten }\end{array}$ & $\begin{array}{l}\text { Oberstufe B2 } \\
\text { 2. Aufgabe } \\
\text { (200-250 Wörter) } \\
\text { Essay (Leserbrief) zu } \\
\text { einem Thema }{ }^{2} \text { nach } \\
4 \text { Leitpunkten }\end{array}$ \\
\hline
\end{tabular}

Tab. 10: Unterschiede in der Aufgabe nach Niveaustufen im Prüfungsteil Schreiben

Man kann aus zwei Themen eins wählen.

2 Man kann aus zwei Themen eins wählen. 


\begin{tabular}{|c|c|c|}
\hline $\begin{array}{l}\text { Mittelstufe A2 } \\
\text { 1. Aufgabe } \\
\text { Bewertung: } \\
>\quad \text { Inhalt } \\
>\quad \text { Sprachliche } \\
\quad \text { Richtigkeit }\end{array}$ & $\begin{array}{l}\text { Mittelstufe B1 } \\
\text { 2. Aufgabe } \\
\text { Oberstufe B2 } \\
\text { 1.Aufgabe } \\
\text { Bewertung: } \\
>\text { Inhalt } \\
>\text { Textmerkmale } \\
>\text { Textgestaltung } \\
>\text { Wortschatz } \\
>\text { Grammatik }\end{array}$ & \begin{tabular}{|l} 
Oberstufe B2 \\
2. Aufgabe \\
Bewertung: \\
$>$ Inhalt \\
$>$ Textgestaltung \\
$>$ Wortschatz \\
$>$ Grammatik
\end{tabular} \\
\hline
\end{tabular}

Tab. 11: Unterschiede in der Bewertung nach Niveaustufen im Prüfungsteil Schreiben

Die zweite Aufgabe der Mittelstufe und die erste Aufgabe der Oberstufe werden scheinbar gleich bewertet. Es geht ja hier in beiden Aufgaben um eine ähnliche Textsorte (Brief). Aber eine weitere Differenzierungsmöglichkeit bieten die analytischen Skalen, in denen die einzelnen Bewertungskriterien beschrieben werden. Da die sprachliche Richtigkeit bzw. Grammatik immer bewertet wird, soll hier anhand dieses Kriteriums ein Beispiel angeführt werden, wie die Differenzierung realisiert wird. Das Beispiel bezieht sich auf die maximale Punktzahl, also wenn das Kriterium gut erfüllt wird.

\begin{tabular}{|c|c|c|}
\hline $\begin{array}{l}\text { Mittelstufe, A2 } \\
\text { 1. Aufgabe: } \\
\text { Der Kandidat verwendet die } \\
\text { elementaren, grundlegenden } \\
\text { Strukturen meistens richtig. }\end{array}$ & $\begin{array}{l}\text { Mittelstufe, B1 } \\
\text { 2. Aufgabe: } \\
\text { Der Kandidat verwendet } \\
\text { die einfachen, häufig verwen- } \\
\text { deten Strukturen meistens } \\
\text { richtig. }\end{array}$ & $\begin{array}{l}\text { Oberstufe, B2 } \\
\text { 1. und 2. Aufgabe: } \\
\text { Der Kandidat verwendet } \\
\text { anspruchsvolle Strukturen } \\
\text { richtig und die Sätze sind } \\
\text { abwechslungsreich gestaltet. }\end{array}$ \\
\hline
\end{tabular}

Tab. 12: Unterschiede bei der Bewertung der grammatischen Richtigkeit im Prüfungsteil Schreiben

Die ersten Erfahrungen bei den Probeprüfungen 2003 und 2004 haben gezeigt, dass die Aufgaben für die Schüler - vor allem im Prüfungsteil Schreiben recht schwer sind. Der Prüfungsteil Hörverstehen, wovon man am meisten Angst hatte, schien leicht gewesen zu sein. Das bedeutet, dass die Fähigkeiten der Schüler in den einzelnen Fertigkeiten noch nicht gleichmäßig entwickelt wurden. Deshalb war es besonders wichtig, zuerst die Lehrer auf das neue Prüfungsmodell vorzubereiten. Nach den anfänglichen Schwierigkeiten konnten die meisten ungarischen DaF-Lehrer/innen durch Fortbildungsmaßnahmen für das neue Prüfungsmodell gewonnen werden. 


\section{Erfahrungen der ersten Prüfung im 2005 Mai}

Im Jahr der Einführung der neuen Prüfung haben sich die meisten Schüler in allen Fächern eher für die Mittelstufe entschieden. Offenbar wollten sie auf Nummer sicher gehen bzw. sie haben eventuell vor der höheren Stufe Angst gehabt. Diese Tendenz war auch für die Fremdsprachen charakteristisch.

In Ungarn muss man das Abitur in Muttersprache, Mathematik, Geschichte und in einer gewählten Fremdsprache ablegen. Zu diesen Pflichtfächern kommt noch ein weiteres Wahlpflichtfach. Die Anzahl der Abiturienten war im Jahr der Einführung 82.652. Davon haben die meisten beim Wahlpflichtfach Fremdsprache das Abitur in Englisch und in Deutsch abgelegt.

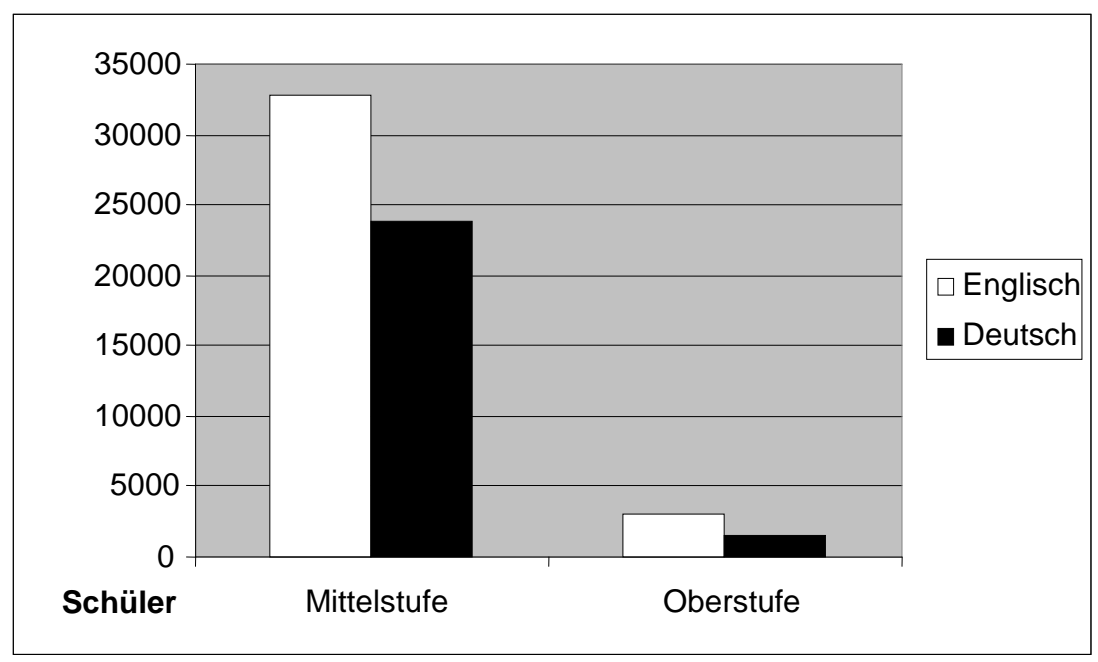

Abb. 1: Anzahl der Schüler, die das neue Abitur in Englisch und Deutsch gemacht haben

Die Prüfungsergebnisse zeigen, dass die Oberstufe für die wenigen Schüler, die sich getraut haben die Oberstufe abzulegen, eigentlich leicht war, während die Mittelstufe nicht mehr so leicht war. 


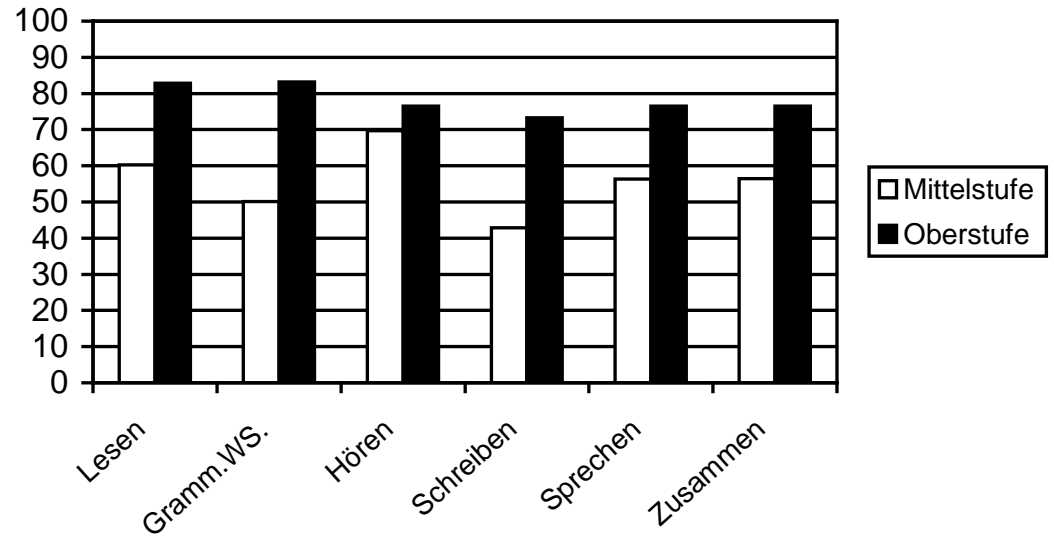

Abb. 2: Ergebnisse der ersten Prüfung in Deutsch nach dem neuen Modell in Prozent

Im Vergleich mit den Ergebnissen der Probeprüfungen ist das neue Abitur besser gelungen als man erwartet hat. Das ist auf mehrere Gründe zurückzuführen.

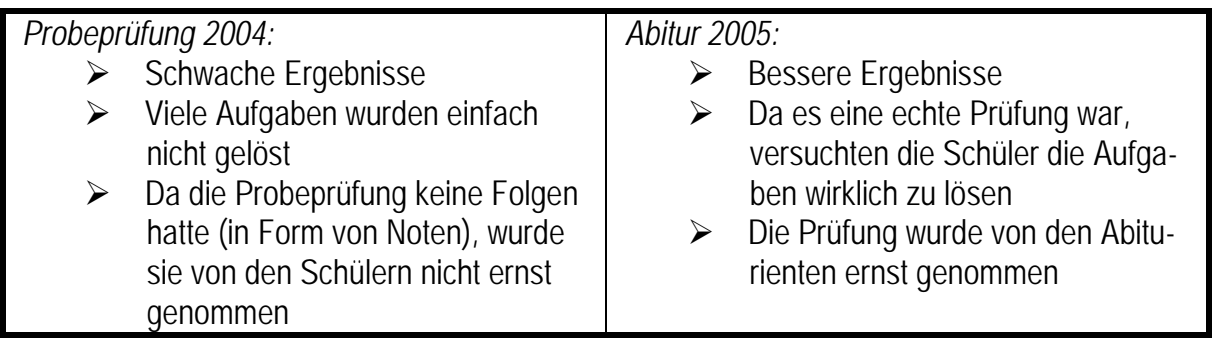

Tab. 13: Vergleich der Ergebnisse zwischen der Probeprüfung 2004 und der ersten Prüfung 2005

\section{Gegenwärtige Aufgaben}

Die Ergebnisse haben gezeigt, dass die Schüler mit einem konsequent kommunikativ durchgeführten Unterricht auf das neue Prüfungsmodell vorbereitet werden können. Die weitere Analyse der Prüfungsergebnisse kann auch dazu beitragen, dass die didaktisch-methodische Vorbereitung verbessert wird. Hier sollte auch die Lehrerausbildung mehr dafür tun, die jungen Lehrkräfte auf diese Arbeit vorzubereiten. Daher wurde an der Universität Szeged damit angefangen, Arbeiten aus der letzten Probeprüfung in die Lehrerausbildung einzubeziehen. So konnten (und können auch jetzt) Studierende in Germanistik als Thema ihrer Abschlussar- 
beit einen Prüfungsteil auswählen, um diesen zu analysieren. Bislang entstanden drei Diplomarbeiten an der Universität Szeged, wo jeweils ein Aspekt untersucht wurde. Eine Arbeit ${ }^{3}$ hat sich mit dem Schwierigkeitsgrad der Aufgaben zum Hörverstehen untersucht. Früher war die Annahme weit verbreitet, dass Hörverstehen eine vernachlässigte Fertigkeit sei, die Aufgaben sollen also nicht zu schwer sein. Die Arbeit hat mit einer Item-Analyse gezeigt, wie schwer bzw. leicht die Aufgaben der Probeprüfung 2004 für die Schüler waren. Eine andere Studentin ${ }^{4}$ hat ein Jahr später typologisch ähnliche Aufgaben auf verschiedenen Stufen im Prüfungsteil „Strukturen und Wortschatz“ untersucht. Auch die Bewältigung der Aufgaben in Schreibfertigkeit war Thema einer Diplomarbeit ${ }^{5}$. Die Studierenden, die noch das frühere Abitur kannten, aber eine solche Untersuchung durchgeführt haben, haben die neue Prüfung recht bald mit anderen Augen gesehen.

Um das Niveau der einzelnen Prüfungsaufgaben besser einschätzen bzw. die Leistung angemessen bewerten zu können, soll der Einklang zwischen Anforderungen und Schwierigkeitsgrad gefunden und gesichert werden. Dazu sind detaillierte Analysen notwendig. Gegenwärtig werden die Aufgaben etappenweise erprobt, analysiert und dann zur Prüfung freigegeben. Das ist eine große Herausforderung sowohl für die Aufgabenersteller, als auch für die Gutachter der Aufgaben. Um das Ziel zu erreichen, muss die Qualitätssicherung kontinuierlich erfolgen.

\section{Literatur:}

Einhorn Ágnes: „Die erste Erprobung im Rahmen der Abiturreform für DaF“. In: DUfU. Deutschunterricht für Ungarn ${ }^{6} .2000 / 1.35-54$.

Einhorn, Ágnes: „Die Revision der ungarischen Abschlussprüfung in DaF.

Prinzipien und Ergebnisse der Forschungsarbeit 1996-2002“. In: Ágel, Vilmos / Herzog, Andreas (Hg.): Jabrbuch der ungarischen Germanistik. 2002, 315-329.

Einhorn, Ágnes (Hrsg.): Kéziikönyv a német érettségi reformjához: [Handbuch zur Abiturreform für DaF] 2004. On-line im Internet:

http://www.om.hu/letolt/kozokt/erettsegi2005/tanaroknak/nemet/német_nyelv.htm

Einhorn, Ágnes: „A 2005. évi német érettségi vizsga tanulságai“. [Die

Erfahrungen bei dem Abitur 2005 Deutsch als Fremdsprache] Unveröff.

Manuskript, 2006.

\footnotetext{
${ }^{3}$ Gonda, Orsolya: Leistungsmessung der Hörfertigkeit. Diplomarbeit. Szeged, 2005.

${ }^{4}$ Fröhling, Edit: Fehleranalyse beim Prüfungsteil 'Wortschatz und Grammatik' auf Grund des Probeabiturs 2004. Eine Fallstudie. Diplomarbeit. Szeged, 2006.

5 Vigh, Tibor: Der Prüfungsteil Schreiben im neuen Abitur für DaF. Diplomarbeit. Szeged. 2005.

Vgl. den Beitrag von Tibor Vigh.

6 Zeitschrift des Ungarischen Deutschlehrerverbands.
} 
Einhorn, Ágnes / Major, Éva: „A 2004-es próbaérettségi tapasztalatai - idegen nyelvek“. [Die Erfahrungen bei der Probeprüfung 2004 - Fremdsprachen] Új Pedagógiai Sžemle. März 2005, 11-27. On-line im Internet:

http://www.oki.hu/oldal.php?tipus=cikk\&kod=2005-03-ta-tobbek-2004

Petneki, Katalin: „Entwicklung des Abiturkonzepts für den Deutschunterricht“. In: DUfU. Deutschunterricht für Ungarn. 2002/1-2. 33-42.

Petneki, Katalin: „Schreibfertigkeit im neuen Abitur. Ergebnisse der Probeprüfung 2004 auf der Oberstufe“. In: Szatmári, Petra (Hrsg.):

Gedenkschrift an Prof. János Kohn. Szombathely. (Erscheint im Jahre 2006)

Trim, John/North, Brian/Coste, Daniel: Gemeinsamer europäischer Referenzrabmen für Sprachen: lernen, lehren, beurteilen. Übersetzt von Jürgen Qetz. Berlin-MünchenWien-Zürich-New York: Langenscheidt 2001.

Vígh, Tibor: „Der Prüfungsteil Schreiben im neuen Abitur für DaF“. In: Orosz, Magdolna / Albrecht, Terrance (Hrsg.): Jahrbuch der ungarischen Germanistik. 2005. Budapest, Gesellschaft der ungarischen Germanisten - Bonn, Deutscher Akademischer Austauschdienst: 2006, 335-351.

Viæsgakövetelmények és vįsgaleírás. Német nyelv. [Prüfungsregelung und -beschreibung. DaF] In: Magyar Közlöny (2003): 2003/57/II. 731-773. 


\title{
Wie es zu einer Beurteilung kommt. Ein Forschungs- bericht zu Strategien bei der Beurteilung schriftlicher Leistungen im Kontext der Prüfung TestDaF.
}

\author{
Ulrike Arras, Hagen
}

\section{$1 \quad$ Problemaufriss}

Die valide und reliable Beurteilung von Prüfungsleistungen stellt neben der Erstellung geeigneter Testaufgaben und der objektiven Prüfungsdurchführung eine weitere Säule der Qualitätssicherung eines Tests dar. Zwar nehmen Schulung und Kalibrierung der BeurteilerInnen von Prüfungsleistungen, die anhand von offenen Items erbracht wurden, gerade bei standardisierten Tests wie dem TestDaF eine zentrale Rolle ein. Jedoch wissen wir trotz kriterienorientierten Vorgehens und der Operationalisierung von Beurteilungsmaßstäben und -verfahren im Grunde wenig darüber, wie die BeurteilerInnen bei ihrer Arbeit vorgehen, mit welchen Strategien sie die Beurteilung einer (schriftlichen oder mündlichen) Leistung bewerkstelligen, worauf sie ihr Augenmerk richten und welche Textfaktoren, aber auch von der konkreten Leistung unabhängige Faktoren die Wahrnehmung und damit das Urteil beeinflussen. Gründe genug also, den Blick auf die BeurteilerInnen selbst zu 
lenken. Die hier vorzustellende Untersuchung möchte zur Erhellung der Frage beitragen, wie es überhaupt zu einer Beurteilung fremdsprachlicher Prüfungsleistungen kommt, indem sie die BeurteilerInnen, ihr (individuelles) Verhalten bei der Beurteilung schriftlicher Prüfungsleistungen zum Forschungsgegenstand macht. Eine explorativ-interpretative Untersuchung kann zum Verständnis dieses Gegenstands beitragen, indem mit Hilfe eines Mehrmethodendesigns und introspektiver Verfahren Einblicke in die Strategien und Prozesse der Beurteilungsarbeit ermöglicht werden.

\section{Der TestDaF}

Der TestDaF (Test Deutsch als Fremdsprache) ist ein seit 2001 vom TestDaFInstitut administriertes Testsystem für ausländische StudienbewerberInnen. Die Prüfung testet Deutschkenntnisse auf fortgeschrittenem Niveau, die für ein Studium an einer deutschsprachigen Hochschule relevant sind: Damit fungiert der TestDaF als Nachweis ausreichender Sprachkenntnisse, um ein Hochschulstudium aufzunehmen. Die vier Fertigkeiten Lesen, Hören, Schreiben und Sprechen werden getrennt in je eigenen Subtests gemessen und die Leistungen entsprechend getrennt auf einem Zeugnis ausgewiesen. Dank seiner Standardisierung und Orientierung an wichtigen Referenzsystemen wie dem Gemeinsamen europäischen Referenzrahmen für Sprachen des Europarats und der Skala der Association of Language Testers in Europe (ALTE) ${ }^{1}$ ist ein TestDaF-Zeugnis jedoch auch für berufliche Zwecke, insbesondere für akademische Berufsfelder, von Nutzen. Die Prüfung wird weltweit durchgeführt, deshalb kann sie bereits im Heimatland abgelegt werden und vereinfacht dadurch den Zugang zu einem Hochschulstudium in Deutschland (Althaus 2004, Arras 2005).

\section{Der Prüfungsteil Schriftlicher Ausdruck: Aufgabenformat und Beurteilungsverfahren}

Die Überprüfung der schriftlichen Ausdrucksfähigkeit erfolgt anhand lediglich einer Texterstellungsaufgabe (Arras/Grotjahn 2003). Es handelt sich um eine direkte Erfassung der Fähigkeit auf der Basis eines offenen Itemformats, wobei jedoch schriftlich bzw. grafisch präsentierte Vorgaben die Aufgabe steuern. Die Prüfungsteilnehmenden sollen zeigen, ob sie in der Lage sind, zu einem bestimmten Thema einen zusammenhängenden und klar aufgebauten, diskursiven Text zu schreiben. Gefordert werden im Wesentlichen zwei Schreibhandlungen, die für den akademischen Kontext von besonderer Bedeutung sind: Das Beschreiben und

\footnotetext{
${ }^{1}$ Die Niveaustufen-Beschreibungen des Gemeinsamen europäischen Referenzrahmens für Sprachen s. www.coe.int sowie Europarat (2001). Die Niveaustufen-Beschreibungen der ALTE

s.: www.alte.org.
} 
Zusammenfassen von statistischen Daten, die in Form einer Grafik oder einer Tabelle präsentiert werden, sowie das Argumentieren, indem beispielsweise zu einer Frage oder einem Problem begründet Stellung genommen werden soll und dabei i.d.R. unterschiedliche Meinungen zu paraphrasieren und zu berücksichtigen sind ${ }^{2}$.

Ein standardisierter Test, der weltweit abgenommen wird, muss sich eines kriterienorientierten Beurteilungsverfahrens bedienen. Das bedeutet: Ausschlaggebend bei der Bewertung von Leistungen ist nicht die (durchschnittliche) Leistung der Gesamtgruppe, i. e. alle Teilnehmenden an einem Prüfungsereignis, sondern die durch die TestDaF-Niveaustufen ausgewiesene Leistung selbst. Aus diesem Grunde werden die Leistungen aus den Prüfungsteilen zur Erfassung der produktiven Fähigkeiten, d. h. die schriftlichen und mündlichen Texte, zentral beurteilt und zwar von BeurteilerInnen, die eigens durch das TestDaF-Institut geschult werden und regelmäßig an Kalibrierungsveranstaltungen teilnehmen. Das wichtigste Instrument der Beurteilung sind die Bewertungskriterien in Form von skalierten Deskriptoren (s. Beurteilungsraster im Anhang). Das standardisierte Bewertungsverfahren sieht vor, dass die individuelle Prüfungsleistung hinsichtlich vorgegebener, das Testkonstrukt widerspiegelnder Aspekte mit den Deskriptoren des Beurteilungsrasters abgeglichen wird. Diese Kriterien erfassen zum einen die Gesamtwirkung bei der Rezeption eines Textes. Es handelt sich um eine eher holistische Erfassung der Leistung. Zum anderen werden die sprachliche und die inhaltliche Umsetzung der jeweiligen Aufgaben beurteilt, was ein eher analytisches Vorgehen bei der Bewertung erfordert. Um die Schwierigkeit der verschiedenen Aufgaben über verschiedene Testsätze bzw. Testereignisse hinweg konstant zu halten, bedarf es darüber hinaus einer testsatzspezifischen Kalibrierung. Die entsprechenden Instrumente bestehen aus zwei Teilen: Zum einen werden die Anforderungen aufgabenspezifisch festgehalten. Das bedeutet, zu jeder Aufgabe wird anhand von Texten aus den Erprobungen eruiert, welche Leistungen bzw. Anforderungen die Aufgabe elizitiert und welche Maßstäbe bei der Umsetzung der jeweiligen Aufgabe anzulegen sind. Hiermit wird also versucht, die Schwierigkeit über verschiedene Testereignisse und Aufgaben konstant zu halten, indem festgelegt wird, inwieweit bei eher komplexen Aufgaben geringere Anforderungen an die Umsetzung zu stellen sind als bei Aufgaben, die z. B. eher einfach zu erfassende Darstellungen statistischer Daten aufweisen. Zum anderen werden ebenfalls anhand von Leistungen aus der Erprobungsphase der jeweiligen Aufgabe Texte auf unterschiedlichen Leistungsniveaus ausgewählt und durch ein ExpertInnenGremium, bestehend aus geschulten BeurteilerInnen und TestentwicklerInnen, bewertet. Die Urteile und entsprechenden begründeten Einstufungen werden schriftlich fixiert und stellen eine weitere Orientierungshilfe für alle BeurteilerInnen dar, die für die Bewertung schriftlicher bzw. mündlicher Leistungen aus einem

${ }^{2}$ Modellaufgaben zum Prüfungsteil Schriftlicher Ausdruck sind auf der Internetseite des TestDaFInstituts unter www.testdaf.de einsehbar. Die im Rahmen der Untersuchung verwendete Aufgabe ist mittlerweile als „Musterprüfung 1“ veröffentlicht worden (TestDaF-Institut 2005). 
Testereignis eingesetzt werden. Diese Kalibrierungsmaßnahmen haben zum Ziel, die Beurteilungsmaßstäbe zu bestimmen. Die konstante Interpretation dieser Maßstäbe ist zum einen wichtig, um die Reliabilität der Beurteilungen zu erhöhen und zum anderen, um die Schwierigkeit der Aufgabe zu justieren. Die Konstanthaltung der Schwierigkeit schließlich ist erforderlich, um das Gütekriterium der Validität zu erfüllen.

Da trotz Schulung, Kalibrierung und anderen Monitoring-Maßnahmen Menschen unterschiedlich strenge Beurteilungsmaßstäbe anlegen, wird ein weiteres Instrument eingesetzt, um zu zuverlässigen und damit fairen Leistungsbeurteilungen zu gelangen, nämlich die Erfassung der individuellen Strenge der einzelnen Beurteilerin bzw. des einzelnen Beurteilers mit Hilfe des Multi-Facetten-RaschModells. Hierbei wird bei der Ermittlung der tatsächlich erreichten Leistungsstufe u. a. auch der Strengekoeffizient der individuellen Beurteilerin bzw. des individuellen Beurteilers einbezogen (Eckes 2003, 2004). Die Erfassung verschiedener Determinanten - also die Leistungseinstufungen hinsichtlich der verschiedenen Aspekte wie sie im Kriterienraster vorgegeben sind, die Aufgabenschwierigkeit sowie die individuelle Strenge und Konsistenz der BeurteilerInnen - ermöglicht schließlich eine faire endgültige Stufenzuweisung. Die mannigfaltigen Faktoren, die bei der Beurteilung einer (schriftlichen) Prüfungsleistung wirksam werden, können in einem Modell zusammengestellt werden. 


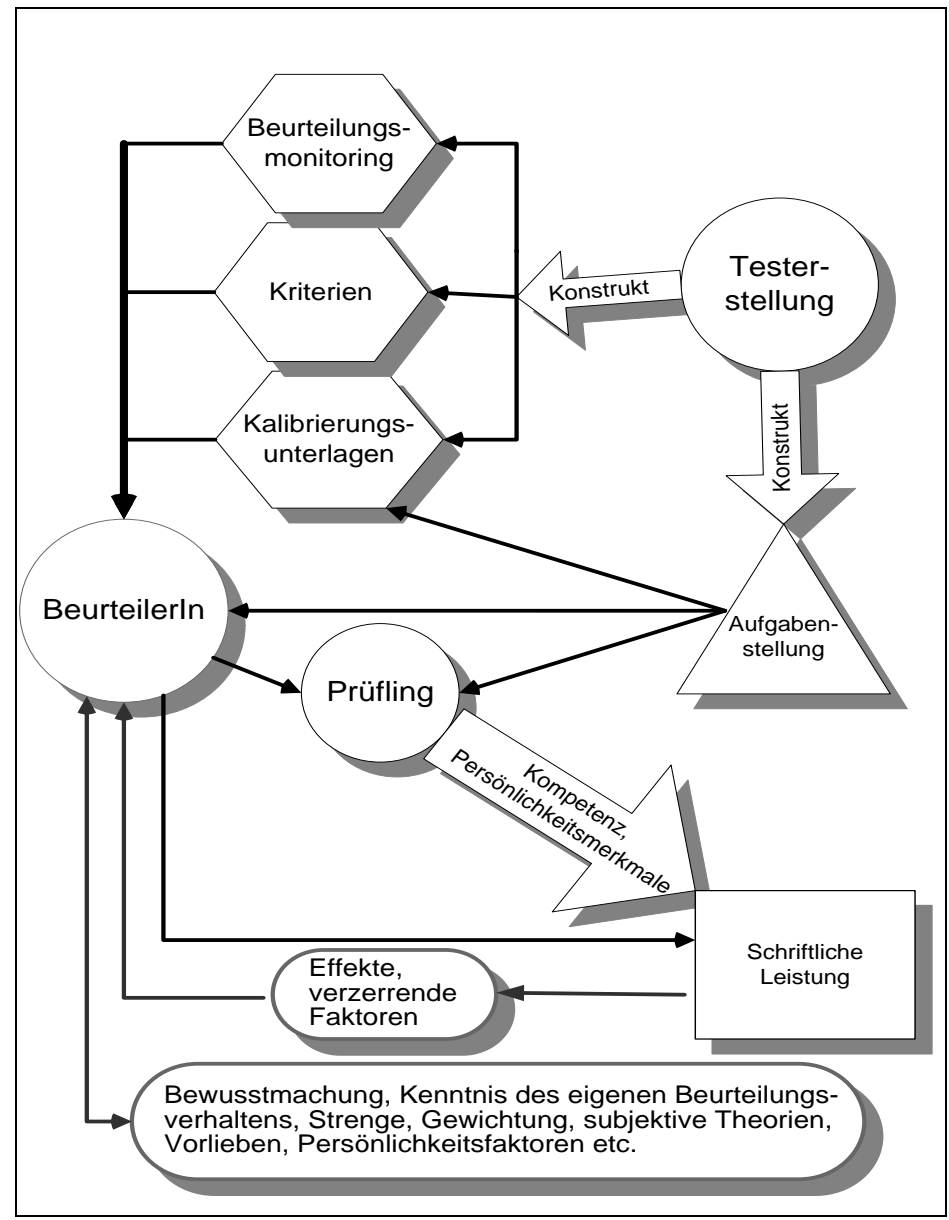

Abbildung: Faktorenmodell Schreibtest

Wie das Modell zeigt, spielen nicht allein die institutionell geprägten Faktoren wie Testkonstrukt, Kalibrierung, Schulung etc. eine Rolle. Vielmehr wird die Beurteilung durch Persönlichkeitsfaktoren sowie subjektive Theorien u. ä. Faktoren gesteuert. Es sind nicht zuletzt diese Faktoren, welche die hier vorzustellende empirische Studie zu eruieren sucht.

\section{$4 \quad$ Fragestellung}

Dass die Bewertung unserer Leistung nicht unbedingt unserer subjektiv empfundenen Selbsteinschätzung entspricht und auch nicht stets einer wie auch immer geregelten objektiven Bewertung standhält, ist eine Erfahrung, die wir spätestens 
in der Schule machen müssen, wo nicht nur das Testen, sondern auch Klagen über ungerechte Noten an der Tagesordnung sind. Es ist daher überraschend, dass im deutschsprachigen Raum das Problem (subjektive) Bewertung (fremdsprachlicher Leistungen) nur sporadisch Gegenstand der Fachliteratur ist. Selbst in Fachzeitschriften, in denen Lehrkräfte selbst zu Wort kommen, finden sich nur gelegentlich Ansätze zur kritischen Auseinandersetzung damit. In der Regel werden dann jedoch lediglich Einzelaspekte wie die „Abmilderung“ von oder Kritik an Fehlerquotienten (Drexel-Andrieu/Kahl 1991, Schnädter 1991) behandelt. Eine Ausnahme stellt die Studie von David (1991) dar: Schriftliche Abiturprüfungen im Fach Englisch wurden von verschiedenen Englischlehrkräften bewertet. Das Ergebnis ist deprimierend, denn die Lehrkräfte kommen zu völlig unterschiedlichen Beurteilungen, so dass erhebliche Zweifel an der Zulässigkeit dieses Prüfungsverfahrens gerechtfertigt erscheinen, zumal es sich bei der Abiturprüfung immerhin um einen High-Stakes-Test handelt, der zudem den Anspruch erhebt, in Ansätzen standardisiert zu sein. Tatsächlich erfüllt zumindest die untersuchte Prüfung wichtige Testgütekriterien nicht.

Es befremdet, dass in Deutschland - auch angesichts solcher, freilich singulärer Studien - der Gegenstand Testen und Prüfen (im Kontext Fremdsprachenerwerb) erst seit kurzem Beachtung findet ${ }^{3}$. In der englischsprachigen Fachliteratur hingegen werden unterschiedliche Aspekte der Testerstellung und -auswertung seit Langem behandelt. Insbesondere im Kontext der großen - standardisierten Testsysteme zur englischen Sprache mit langer Tradition werden seit den 60er Jahren zunehmend die Probleme der Bewertung schriftlicher, später auch mündlicher, Leistungen erforscht. Mehrere empirische Studien vergleichen beispielsweise die Bewertung von Prüfungsleistungen durch verschiedene Gruppen von BeurteilerInnen (,lay" vs. ,expert", „,native speaker“ vs. „, non-native speaker"); sie kommen freilich zu recht unterschiedlichen Ergebnissen (Überblick s. Charney 1984, Lumley 2005). Tendenziell ist jedoch festzuhalten, dass vor allem mangelnde Interrater-Reliabilität (s. die o. g. Studie von David), aber auch mangelnde IntraraterReliabilität Anlass zur Besorgnis geben. Den Untersuchungen gemeinsam ist, dass sie im Wesentlichen das Ergebnis der Bewertung fokussieren. Die gleichen Arbeiten werden von einer Versuchs- sowie von einer Kontrollgruppe bewertet, die Ergebnisse werden sodann miteinander verglichen. Wie jedoch bewertet wird, welche - insbesondere auch individuellen - Strategien oder auch subjektiven Theorien und Erfahrungen die BeurteilerInnen leiten, inwiefern Persönlichkeitsfaktoren, Erwartungen, Vorlieben und Idiosynkrasien, eine Leistungsbewertung mitbestimmen - und vor allem warum - bleibt weitgehend im Dunkeln.

Solche Fragen sind seit Beginn der 90er Jahre mehr und mehr in das Interesse der Forschung gerückt. Es sind wiederum die großen englischsprachigen Testsys-

\footnotetext{
${ }^{3}$ Für den schulischen Bereich exemplarisch Freudenstein 1994; einen historischen Forschungsüberblick zu subjektiven Fehlerquellen bei der Zensurvergabe liefern Ingenkamp 1995 und Kieweg 2001. Zum Rater-Verhalten im Kontext TestDaF s. Eckes (in Vorbereitung).
} 
teme, die in den letzten Jahren zunehmend den Blick auf die mentalen Prozesse der Beurteilungsarbeit gerichtet haben - mit entsprechend methodischen Konsequenzen. Das bedeutet: Nicht mehr allein das Reliabilitätsproblem steht im Mittelpunkt, vielmehr wird nunmehr auch Fragen zur Validität von Bewertungsverfahren und -skalen nachgegangen. Arbeiten wie die von Weigle (1994), Milanovic et al. (1996), Cumming et al. (2002) sowie von Lumley (2005) erheben Daten mittels introspektiver Methoden, um das Beurteilungsverhalten, die spezifischen sowie individuell geprägten Beurteilungsstrategien und Entscheidungsprozesse zu analysieren.

Die vorliegende Untersuchung versteht sich als Teil dieser Entwicklung, um zur Theoriebildung des Gegenstandes Beurteilung von Prüfungsleistungen beizutragen. Sie widmet sich in erster Linie den bei der Beurteilung schriftlicher Leistungen beobachtbaren Strategien und geht der Frage nach, in welchen Schritten die Beurteilungsarbeit verläuft. Die folgenden Aspekte stehen dabei im Vordergrund:

- Wie gestaltet sich der individuelle sowie durch das Bewertungsverfahren vorgegebene Prozess der Rezeption und Urteilsfindung?

- Welche Strategien werden hierbei eingesetzt?

- Auf welche Probleme treffen die BeurteilerInnen und mittels welcher Strategien lösen sie diese?

- Gibt es Unterschiede in der Herangehensweise sowie bei der Interpretation der Leistungen und der Beurteilungsmaßstäbe?

Die Untersuchung erfolgt anhand eines spezifischen Aufgabentyps im Kontext einer High-Stakes-Prüfung mit entsprechend spezifischem Testkonstrukt und klar definiertem Testziel. Auch das Beurteilungsverfahren sowie die Beurteilungsinstrumente sind - wie erwähnt - analog den speziellen Anforderung der Prüfung konzipiert. Insofern scheint zunächst Vorsicht geboten bei der Generalisierung der Ergebnisse und der Übertragbarkeit bestimmter Befunde auf andere Kontexte. Allerdings lassen sowohl die verwendeten Methoden als auch die aufgefundenen Strategien und Beurteilungsprozesse gerade den Schluss zu, dass das Beurteilungsinstrumentarium ebenso wie seine Operationalisierung von so zentraler Bedeutung für das Beurteilungsverhalten ist, dass Aussagen möglich sind, die sich nicht allein auf den Kontext TestDaF beziehen, sondern auch allgemeine, bei der Konzipierung von Testsystemen und der Betreuung von BeurteilerInnen relevante Aspekte benennen.

\section{$5 \quad$ Zum Untersuchungsdesign}

Angesichts der Komplexität des Problems Leistungsbeurteilung liegt ein Mehrmethodendesign nahe, um durch Triangulation zur Verbesserung der Validität beizutragen (Flick 2002). Dies ist insbesondere vor dem Hintergrund erforderlich, dass 
die Studie im Wesentlichen auf Fallstudien gründet. Im Folgenden werden die einzelnen Schritte der Untersuchung kurz skizziert.

Die Untersuchung gliedert sich in zwei Vorstudien und die Hauptuntersuchung:

- Bei Vorstudie I handelt es sich um eine Fragebogenerhebung. TestDaFBeurteilerInnen des Subtests Schriftlicher Ausdruck (39 Frauen, 11 Männer, Rücklauf ca. 50\%) wurden mit Hilfe von offenen Fragen um eine Evaluierung bzw. kritische Rückmeldung zu den Schulungsmaßnahmen, den Beurteilungskriterien sowie zu den testsatzspezifischen Kalibrierungsmaterialien gebeten. Die Daten wurden einer Inhaltsanalyse unterzogen.

- Die Vorstudie II wurde im Anschluss an Vorstudie I mit vier Versuchspersonen durchgeführt und gliederte sich in zwei Teile: ein problemzentriertes, halbstrukturiertes und leitfadenorientiertes Interview und ein Laut-Denken-Protokoll während der Beurteilung einer schriftlichen Prüfungsleistung. Ziel dieses Teils war nicht zuletzt, das Laut-DenkenVerfahren zu erproben und seine Anwendbarkeit im Kontext Beurteilung zu prüfen.

Dank der beiden Vorstudien konnten erste Erkenntnisse sowie methodische Konsequenzen in das Design der Hauptstudie einfließen. Dies betraf die folgenden Entscheidungen:

- Auswahl und Anzahl der Versuchspersonen

- Auswahl der Aufgabenstellung

- Auswahl, Anzahl und Anordnung der zu beurteilenden schriftlichen Leistungen

- Prozedere und Durchführung der introspektiven Verfahren, also der Laut-Denken-Protokolle und der retrospektiven Interviews

Die Hauptuntersuchung erfolgte anhand von vier Fallbeispielen: Vier Versuchspersonen, es handelt sich um geschulte und erfahrene Beurteilerinnen von TestDaF-Prüfungsleistungen, beurteilten zunächst per Laut-Denken-Verfahren acht schriftliche Leistungen. Als Warming-up bzw. zu Trainingszwecken wurde ein Text vorgeschaltet, um das Laut-Denken-Verfahren zu trainieren und Rückmeldungen zu ermöglichen. Denn während der eigentlichen Laut-DenkenSitzungen sollte seitens der Forscherin nach Möglichkeit nicht eingegriffen werden. Die Protokolle wurden unmittelbar nach Abschluss der Beurteilungsarbeit auditiv einer Grobanalyse unterzogen, so dass am Folgetag im Rahmen der introspektiven Interviews auf Basis der Protokolle problemzentriert das semistrukturierte Interview durchgeführt werden konnte. Diese Interviews wurden transkribiert und einer Inhaltsanalyse unterzogen. Die Laut-Denken-Protokolle wurden 
angesichts der Fragestellung der Studie, die nicht allein die Strategien, sondern auch die Prozesshaftigkeit der Beurteilungsarbeit fokussiert, transkribiert, segmentiert und zu Analysezwecken anhand eines eigens entwickelten Kodiersystems kodiert ${ }^{4}$. Die Codes ermöglichen eine detaillierte Analyse der einzelnen Beurteilungsschritte und zeigen, in welchen Kontexten bestimmte Strategien auftreten und in welchen Abfolgen einzelne Strategien angeordnet sind.

\section{Vorläufige Befunde}

Die Verbaldaten aus den Laut-Denken-Protokollen haben eine Fülle an Handlungen und Strategien beobachtbar gemacht, deren Kontext und Motivierung durch die retrospektiven Interviews genauer betrachtet werden können, Erkenntnisse aus den beiden Vorstudien zu spezifischen Beurteilungsproblemen und -strategien validieren zudem die Befunde. Im Folgenden sollen erste Befunde aufgezeigt werden. Dabei können beobachtete Strategien lediglich skizziert werden, auf ausführliche Belege mit Hilfe der Verbaldaten muss weitgehend verzichtet werden. Im Wesentlichen sollen folgende Aspekte dargelegt werden:

- Der Beurteilungsvorgang, also die Phasierung der Beurteilungsarbeit im Kontext des vorgegebenen Beurteilungsverfahrens

- Individuell und institutionell determinierte Beurteilungsstrategien

- Die Funktion und Anwendung der Deskriptoren, auch individuell geprägte Interpretationen und Strategien des Ausgleichens, des Abwägens u. Ä.

Dabei lassen sich Strategien auf der Makroebene von solchen auf der Mikroebene der Beurteilungsarbeit unterscheiden. Zunächst zeigt sich, dass sich der Bewertungsablauf auf Makroebene sehr stark an dem standardisierten Verfahren orientiert, so dass folgende Phasen differenziert werden können:

- Erste Wahrnehmung und Identifizierung der zu beurteilenden Leistung

- Lesedurchgang (zur Beurteilung holistischer Aspekte)

- Lesedurchgang (zur Beurteilung analytischer Aspekte)

- Resümee.

Der Ablauf der Beurteilungsarbeit kann wie folgt dargestellt werden:

\footnotetext{
${ }^{4}$ Zur Aufbereitung und Analyse von Verbalprotokollen s. beispielsweise Green 1998.
} 


\begin{tabular}{|c|c|c|c|}
\hline $\begin{array}{l}\text { Erste Wahrneh- } \\
\text { mung und Identifi- } \\
\text { zierung: }\end{array}$ & 1. Lesedurchgang & 2. Lesedurchgang & Resümée \\
\hline $\begin{array}{l}\text { - Konstituierung } \\
\text { einer ersten } \\
\text { Erwartung } \\
\text { - unter Einbezug } \\
\text { von Faktoren } \\
\text { wie Hand- } \\
\text { schrift, Text } \\
\text { länge etc. }\end{array}$ & $\begin{array}{l}\text { - totales Lesen } \\
\text { - ggf. wiederhol- } \\
\text { tes Lesen } \\
\text { - unter Einbezug } \\
\text { des korrigieren- } \\
\text { den Lesens, } \\
\text { Sinn- } \\
\text { Rekonstruktion } \\
\text { - holistische } \\
\text { Beurteilung } \\
\text { mittels } \\
\text { Deskriptoren } \\
\text { - ... }\end{array}$ & $\begin{array}{l}\text { - scannen } \\
\text { - paraphrasieren } \\
\text { - ggf. erneut } \\
\text { totales } \\
\text { Lesen } \\
\text { - Beurteilung } \\
\text { analytischer } \\
\text { Aspekte } \\
\text { mittels Desk } \\
\text { riptoren } \\
\text { - ... }\end{array}$ & $\begin{array}{l}\text { - Einbezug des } \\
\text { Testkons- } \\
\text { trukts } \\
\text { - Einbezug } \\
\text { der „real } \\
\text { world“ } \\
\text { - ... }\end{array}$ \\
\hline
\end{tabular}

Diese vier Phasen sind unterschiedlich lang. Den größten Raum nehmen die eigentlichen Lesephasen, also der erste sowie der zweite Lesedurchgang ein. Vor allem in diesen Phasen zeigt sich auf der Mikroebene eine breite Palette an unterschiedlichen Strategien, die der Rezeption der Leistung sowie der Entscheidungsfindung in Bezug auf das Urteil dienen. Die Strategien, die in den vier verschiedenen Phasen eingesetzt werden, lassen sich wie folgt gruppieren:

1. Zu den Rezeptionsstrategien, im Wesentlichen also jene des Lesens und der Wahrnehmung des Textes, können folgende Strategien gezählt werden:

- Identifizierung der zu beurteilenden Leistung

- Totales Lesen

- Scannen und Search Reading

- Paraphrasieren

- Korrigierendes Lesen

- Interpretierendes Lesen

2. Strategien, die die Beurteilungsinstrumente sowie das institutionalisierte Testkonstrukt einbeziehen; hier sind insbesondere zu nennen:

- Abgleich zwischen Leistung und Deskriptoren

- Ausgleichen

- Abwägen

- Einbezug der Aufgabenstellung

- Gewichten von Aspekten der Leistung 
3. Strategien, die auf eigene Erfahrungen und auf subjektive Theorien (auch subjektive Testkonstrukte) rekurrieren:

- Konstituierung der Erwartung(en)

- Konstituierung des ersten Eindrucks

- Einbezug externer Faktoren wie Handschrift, Textlänge etc.

- Einbezug des Testkonstrukts (auch vor dem Hintergrund eigener - beruflicher etc. - Erfahrungen)

- Entwicklung einer Vorstellung von der Person des Prüflings

4. Eine weitere, für die Beurteilungsarbeit wichtige Strategiengruppe liegt in Form von Strategien zur Arbeitsorganisation vor. Hierzu zählen im Wesentlichen weitgehend individualisierte Notizformen („Gedächtnisstützen“) sowie die Arbeitseinteilung und das Zeitmanagement.

Eine weitere Kategorisierung bietet sich hinsichtlich der Referenz der eingesetzten Strategien an:

- Strategien, die sich der zur Verfügung stehenden Beurteilungsinstrumente bedienen

- Strategien, die sich auf das übergeordnete Testkonstrukt beziehen

So referieren Strategien wie das Abwägen auf die Deskriptoren des Beurteilungsrasters, während bei Strategien wie dem Resümieren Bezug zum zugrunde gelegten Testkonstrukt genommen wird. Zu der Kategorie der verfahrensgesteuerten Strategien zählen solche, die im Kontext des Leseprozesses eingesetzt werden sowie jene Strategien, die auf der Anwendung der Deskriptoren beruhen. Es handelt sich um Strategien, die durch das (standardisierte) Beurteilungsverfahren geprägt sind. Hierzu zählen Faktoren wie das Lesen, die Anwendung der Kriterien, die Berücksichtigung des Testkonstrukts, die Einbeziehung von Erfahrungen und Erkenntnissen aus Kalibrierungen. Es handelt sich um Strategien, die von allen Versuchspersonen angewendet werden, wenn auch in unterschiedlich starkem Ausmaß. Zu den individuell geprägten Strategien zählen beispielsweise Strategien, die der Arbeitsorganisation dienen, etwa individuelle Notizformen oder Maßnahmen zum Zeitmanagement, aber auch solche Strategien, die von Faktoren der Persönlichkeit geprägt sind wie der Einbezug von Erfahrungen, Vorlieben bei der Beurteilung von Leistungen u. Ä.

Die Strategien interagieren oder korrespondieren miteinander und ergänzen sich, etwa wenn die Strategie des Abwägens die Strategie des wiederholten Lesens einzelner Passagen notwendig macht, um Belege für oder gegen ein vorläufiges Urteil vorzubringen. Damit formieren sich Strategiengruppierungen. Das bedeutet: Strategien ergeben sich aus bereits zuvor verwendeten Strategien. Beispielsweise besteht eine enge Verbindung zwischen der Strategie des Abwägens und der Strategie der Verifizierung oder Revision des Urteils. Zu dieser Gruppe gesellt sich 
auch eine spezifische Lesestrategie, nämlich die des Scannens zur Suche nach Belegen für oder gegen eine (vorläufige) Entscheidung. Solche Strategiencluster liegen auch hinsichtlich des Einbezugs der Beurteilungskriterien vor: So erfolgt die Einstufung eines Einzelaspekts prozesshaft mittels Aussage zur Qualität i. d. R. unter Zuhilfenahme der Deskriptoren in paraphrasierter Form, oder indem der geeignet erscheinende Deskriptor zitiert wird.

Diese meist sehr dichte Abfolge verschiedener Strategien soll anhand eines Auszugs aus einem der Protokolle illustriert werden. In dem zitierten Auszug beurteilt die Versuchsperson die Qualität der geforderten Schreibhandlung Beschreiben statistischer Daten, die in einer Grafik präsentiert werden ${ }^{5}$.

\section{Transkript}

Beschreibung der Grafik. [räuspert sich] [.]

Ist ein bisschen quer mit der Grafik. Ist NICHT GANZ KLAR gedacht.

ALSO KLAR, 4 IST KLAR UND FOLGERICHTIG WIEDERGEGEBEN. [..]

Während die Anteile der Eltern Studierende unter $24 . . .$. noch groß sind betragen sie ziehmlich wenig.

Es ist NICHT KLAR ausgedrückt. Es ist irgendwie

Anteile der Wohngemeinschaft nimmt nicht so auffällig ab. [!]

Was ist das, nimmt nicht so auffällig ab?

Was soll uns das sagen? Das ist irgendwo nonsense.

Und die Schwankung dazwischen ist auch Quatsch.

Dann so ein paar Zahlen aneinander gereiht. [..]

Eh ist nicht so richtig schön in Zusammenhang.

ALSO KLAR UND FOLGERICHTIG, ne 4, würde ich da nicht geben, sondern da tendiere ich eher zu der 3.

Schreibe ich jetzt mal hin.

Die Informationen der Grafik werden überwiegend aufzählend wiedergegeben.

Ja, ÜBERWIEGEND AUFZÄHLEND.

Es ist zwar ein bisschen ein Versuch, so quer zu denken,

aber es ist [...] ja es ist auch nicht natürlich, auch nicht, nicht total AUFZÄHLEND, ABER ÜBERWIEGEND AUFZÄHLEND, kann man, könnte man vertreten. [...]

Die Informationen der Grafik werden folgerichtig, klar und folgerichtig wiedergegeben.

Ne 4, das ist es nicht.

ES IST NICHT KLAR UND FOLGERICHTIG.

ES IST KLAR, NICHT [!] KLAR, was da

der Anteil der Wohngemeinschaft nimmt nicht so auffällig ab wie die obengenannte

Was soll das?

Gut, bleibe ich bei der 3 .

\footnotetext{
${ }^{5}$ Bei den im Transkript in Kapitälchen gesetzten Äußerungen handelt es sich um Äußerungen, die direkt (fett) oder in paraphrasierter Form Bezug zu den Deskriptoren nehmen (s. Beurteilungsraster im Anhang). Wenn die Versuchsperson die zu beurteilende schriftliche Leistung liest, ist diese Äußerung kursiv gesetzt. Aus Gründen der besseren Lesbarkeit sind die Zeitleiste des Protokolls sowie die Codes hier nicht abgebildet.
} 
Der Auszug zeigt zum einen, wie intensiv die Deskriptoren bei der Urteilsfindung herangezogen werden (Kapitälchen). Sie werden meist paraphrasiert, nur manchmal direkt zitiert (Kapitälchen fett), vermutlich dann, wenn ein direkter Vergleich zwischen dem Wortlaut der Deskriptoren und dem Eindruck der Leistung erforderlich ist. Damit erweisen sich die Deskriptoren als zentrales Instrument bei der konkreten Beurteilungsarbeit. Sie sind von den vier Versuchspersonen derart verinnerlicht, dass Formulierungen und Wortmaterial der Deskriptoren verwendet werden. Mit Hilfe des sprachlichen Materials aus den Deskriptoren wird schließlich eine Beurteilung formuliert. Oder aber das sprachliche Material der Deskriptoren wird in Form einer an den zu beurteilenden Text gerichteten Frage bzw. in Form einer Antwort verwendet, eine Entscheidung herbeizuführen. Die zuletzt genannte Vorgehensweise zeigt sich im folgenden Auszug:

\author{
Transkript \\ Und Korrektheit. \\ Ja, da sind also manchmal Fehler. \\ Frage, SIND DIE FEHLER SO, DASS ES MEIN VERSTEHEN BEEINTRÄCHTIGT? \\ $\mathrm{Ja}$, ich glaube fast, DIE FEHLER SIND SO, DASS ES MEIN VERSTEHEN MANCHMAL BEEINTRÄCHTIGT.
}

Zum anderen wird die Verbindung der einzelnen Strategien zu Clusters deutlich. Die Urteilsfindung erfolgt im steten Wechsel zwischen der genannten Referenz auf die Deskriptoren (und zwar jenen der angrenzenden Niveaustufen), der Überprüfung der Leistung, der Suche nach Belegen und dem Abwägen.

Die hier genannten Strategien können auch unter dem Gesichtspunkt der Referenz kategorisiert werden:

- Strategien, die durch das vorgegebene Beurteilungsverfahren bestimmt werden (Lesen, Abgleichen)

- Strategien, die durch die Person bzw. durch individuelle Faktoren der Beurteilerin geprägt sind (z. B. solche, die nur von bestimmten Versuchspersonen verwendet werden, von anderen nicht)

- Strategien, die sowohl auf individuelle Faktoren der Versuchspersonen als auch auf den standardisierten Bewertungsverlauf zurückzuführen sind (z. B. die Erwartungen an die Leistung, die wiederum bestimmt wird zum einen durch die Berufserfahrung, zum anderen aber auch durch Faktoren wie Kalibrierungen)

Die meisten der in der vorliegenden Untersuchung eruierten Strategien weisen sowohl Einflüsse aus dem persönlichen oder beruflichen Erfahrungsschatz als auch aus dem weitgehend standardisierten Beurteilungsverfahren auf. Danach entsteht eine Teilmenge durch das Zusammentreffen von institutionell geprägten 
Strategien, etwa solchen, die auf das Testkonstrukt oder auf das Beurteilungsinstrumentarium referieren, und jenen, die von Persönlichkeitsfaktoren geprägt sind und auf individuellen Erfahrungen, subjektiven Theorien u. Ä. beruhen.

\section{$7 \quad$ Erste Schlussfolgerungen}

Der Forschungsstand, insbesondere auch die auf der Basis von introspektiven Daten entwickelten Modelle und Systematisierungen der Beurteilungsstrategien und -prozesse zeigen, dass die Bewertung (schriftlicher) Leistungen eine komplexe kognitive Handlung, bestehend aus verschiedenen, sich ergänzenden und aufeinander aufbauenden Einzelhandlungen, darstellt. Diese wiederum sind geürägt durch Faktoren wie persönliche Erwartungen und Erfahrungen, aber auch durch institutionelle Faktoren wie das Beurteilungsinstrumentarium und vor allem auch das Training, durch welches Testkonstrukt und Maßstäbe transportiert werden. Beurteilen stellt damit eine kontinuierliche ,problem-solving activity” dar, bei der die BeurteilerInnen die Informationen und Begriffe der Beurteilungsmaßstäbe interpretieren ,and then reconcile this interpretation with the specifics of the text. Thus, evaluating writing when using a scoring rubric is a constructive activity." (DeRemer 1998: 13). Angesichts der Komplexität und der teils individuellen Prägung der Beurteilungsstrategien erscheint eine Auseinandersetzung mit den sie determinierenden Faktoren, ja eine Bewusstmachung des eigenen Handelns während der Beurteilung von besonderer Bedeutung. Diese vorläufige Schlussfolgerung stellt ein starkes Argument dar für die Bedeutung von Schulungen. Die Auseinandersetzung mit dem kriterienorientierten Beurteilen und den eigenen Beurteilungsstrategien, die auf subjektiven Theorien, auf eigenen (persönlichen und beruflichen) Erfahrungen und anderen, auch affektiven und sozialen, Faktoren beruhen, trägt zur Professionalisierung der Beurteilungsarbeit bei, und zwar unabhängig davon, ob es sich um BeurteilerInnen handelt, die Leistungen im Kontext eines speziellen Tests beurteilen, oder ob es um angehende oder fortzubildende (Fremd-)SprachenlehrerInnen geht, die für ein breites Spektrum an Leistungsmessung - verschiedene Formen des classroom assessment bis hin $\mathrm{zu}$ (standardisierten) High-Stakes-Prüfungen wie insbesondere das Abitur - vorbereitet und trainiert werden müssen. Trotz der eingeschränkten Generalisierbarkeit der anhand von vier Fallbeispielen eruierten Befunde können einige praktische Vorschläge vor allem für die Optimierung von Trainings gemacht werden. Eine Möglichkeit für Schulungsmaßnahmen kann in der Verwendung des Laut-Denken-Verfahrens liegen. Das Verfahren macht die eigenen Verhaltensweisen und Strategien bei der Beurteilung schriftlicher Leistungen bewusst und somit zugänglich für Reflexion. Damit kommt dem Verfahren ein Lerneffekt zu, dessen sich Schulungen bedienen können. Dieser Ansatz wird auch in anderen Untersuchungen zu Problemlösestrategien vertreten. So kommt Dominowski (1998: 43) zu dem Schluss, dass das 
Verfahren des Lauten Denkens gezielt für die Effektivierung von Problemlösestrategien eingesetzt werden sollte:

Having people simply think aloud while working on a problem can provide useful information about problem-solving processes; task performance typically is not changed. Asking more specific questions [...] can elicit additional information and might affect problem solving, presumably for the better. Asking people to focus on their own problem solving, to explain what they are trying to do, promotes metacognitive processing and leads to more effective problem solving, even when the questions are no longer asked. (Dominowski 1998: 43).

Diese Überlegungen werden durch Daten der Untersuchung gestützt. Alle vier Beurteilerinnen der Studie, aber auch die vier Versuchspersonen der Vorstudie II geben nach den Laut-Denken-Sitzungen an, dass das Verfahren, während der Bewertung die kognitiven und emotionalen Prozesse zu verbalisieren, ihnen interessante Aufschlüsse über sich selbst und ihr Verhalten gab. Die vier Versuchspersonen der Hauptstudie wurden im retrospektiven Interview am Tag nach den Laut-Denken-Sitzungen einleitend um Kommentierung des Laut-DenkenVerfahrens selbst gebeten. Hier merkten alle Beurteilerinnen an, dass dieses Verfahren zwar anstrengend und zeitintensiv war, dass sie jedoch ganz persönlich davon profitiert hatten, denn das Laute Denken, v. a. im Beisein einer weiteren Person, führe zu intensiver Reflexion des eigenen Verhaltens. Im Gegensatz zu Erkenntnissen aus der Erforschung von Lern- und anderen Problemlösestrategien liegt im Falle der Beurteilungsstrategien jedoch ein gewichtiger Unterschied vor: das Gebot der Standardisierung. Im Falle beispielsweise des Fremdsprachlernens geht es darum,

dem einzelnen Lerner zu helfen, aus dem präsentierten Strategienspektrum die zum jeweiligen Lerntyp und der Aufgabe passenden Strategien bewusst auszuwählen, praktisch zu erproben und selbst zu evaluieren, damit er so schrittweise sein Strategienrepertoire erweitern und modifizieren kann (Kleppin/Tönshoff 2000: 113, Hervorhebung im Original).

Geht es im Falle von Lernstrategien also um Individualisierung bzw. um Diversifikation, so ist es Anliegen eines standardisierter Tests wie dem TestDaF, ein gewisses $\mathrm{Maß}$ an Standardisierung auch in Form von Beurteilungsverfahren zu gewährleisten, um das Testkonstrukt und die Validität zu berücksichtigen. Ein standardisiertes Beurteilungsverfahren schränkt damit die Freiheit in der Auswahl an Beurteilungsstrategien notgedrungen ein. Aus diesem Grunde erscheint die Bewusstmachung eigener, aber auch das Angebot an bislang nicht eingesetzten Strategien sowie ihrer unter Berücksichtigung des Testkonstrukts begründeten Evaluation und Revision besonders naheliegend. 


\section{Literatur}

Althaus, Hans-Joachim: „Der TestDaF“. In: DAAD (ed.): Die internationale Hochschule: Ein Handbuch für Politik und Praxis, Band 8. Bielefeld: Bertelsmann 2004, 80-87.

Arras, Ulrike: „Der TestDaF. Konzept und Prinzipien des standardisierten Tests Deutsch als Fremdsprache". In: Fòrum - Anuari de l'Associació de Germanistes de Catalunya. Akten des sechsten Kongresses des Katalanischen Deutschlehrer-und Germanistenverbandes (A.G.C.), Tarragona, April 2005, Número 12.

Arras, Ulrike / Grotjahn, Rüdiger.: „TestDaF: Einige aktuelle Entwicklungen“. In: Katzorke, Heidrun (ed.): Fremdsprachen an Hochschulen: IntegrationInterdisziplinarität - Internationalität. Dokumentation der 22. Arbeitstagung 2002. Bochum: AKS-Verlag 2003, 40-50.

Charney, Davida: "The validity of using holistic scoring to evaluate writing: a critical overview", Research in the Teaching of English 18 (1984), 65-81.

Cumming; Alister / Kantor, Robert / Powers, Donald E.: „Decision making while rating ESL/EFL writing tasks. A descriptive framework“, Modern Language Journal 86/1 (2002), 67-96.

David, Reinhard: „Einheitliche Prüfungsanforderungen in der Abiturprüfung Englisch? Eine Betrachtung nach einer Vergleichskorrektur", Die Neueren Sprachen 90/6 (1991), 624-635.

DeRemer, Mary L.: „Writing assessment: Raters’ elaboration of the rating task“, Assessing Writing 5/1 (1998), 7-29.

Dominowski, Roger L.: „Verbalization and problem solving“. In: Hacker, Douglas J./ Dunlosky, John / Graesser, Arthur C. (Hrsg.): Metacognition in Educational Theory and Practice. Mahwah, NJ, London: Erlbaum (1998), 25-45.

Drexel-Andrieu, Irène / Kahl, Detlev: „Empfehlungen zur Fehlerbewertung im schriftlichen Abitur“, Die Neueren Sprachen 90/6 (1991), 686-687.

Eckes, Thomas: „Qualitätssicherung beim TestDaF: Konzepte, Methoden, Ergebnisse“, Fremdsprachen und Hochschule, 69 (2003), 43-68.

Eckes, Thomas: „Facetten des Sprachtestens: Strenge und Konsistenz in der Beurteilung sprachlicher Leistungen“. In: Wolff, Armin / Ostermann, Torsten / Chlosta, Christoph (Hrsg): Integration durch Sprache. Regensburg: FaDaF 2004, 485-518.

Eckes, Thomas (in Vorbereitung): „Rater types in writing performance assessments: a classification approach to rater variability“, Language Testing.

Europarat: Gemeinsamer europäischer Referenzrabmen für Sprachen: Lernen, lebren, beurteilen. Berlin et al.: Langenscheidt 2001. 
Flick, Uwe: Qualitative Sozialforschung. Eine Einführung. 6. Auflage. Reinbek bei Hamburg: Rowohlt 2002.

Freudenstein, Reinhold: „Fremdsprachentests für die Schule. Ein Plädoyer für die objektivierte Leistungsmessung“, PRAXIS $41 / 4$ (1994), 339-347.

Green, Alison J. K.: Verbal Protocol Analysis in Language Testing Research. A Handbook. UCLES, Cambridge University Press 1998.

Ingenkamp, Karlheinz (Hrsg.): Die Fragwürdigkeit der Zensurengebung. Texte und Untersuchungsberichte. 9. Auflage. Weinheim, Basel: Beltz 1995.

Kieweg, Werner: Evaluation fremdsprachlicher Leistungen im schulischen Kontext. In: FluL 30 (2001), 65-86.

Kleppin, Karin / Tönshoff, Wolfgang: „Autonomiefördernde Strategievermittlung als Gegenstand und Verfahren in der Ausbildung von Fremdsprachenlehrern". In: Helbig, Beate / Kleppin, Karin / Königs Frank G. (Hrsg.): Sprachlehrforschung im $W$ andel. Beiträge zur Erforschung des Lehrens und Lernens von Fremdsprachen. Festschrift für Karl-Richard Bausch zum 60. Geburtstag. Tübingen: Stauffenburg, 2000, 113-128.

Kvale, Steinar: InterViews. An introduction to qualitative research interviewing. Thousand Oaks et al.: SAGE Publications 1996.

Lumley, Tom: Assessing Second Language Writing. The Rater's Perspective. Frankfurt/Main: Peter Lang 2005.

Milanovic, Michael / Saville, Nick / Pollitt, Alastair / Cook, A. (1996):

„Developing rating scales for CASE: theoretical concerns and analyses”. In: Cumming, Alister / Berwick, Richard (Hrsg.): Validation in Language Testing. Clevedon: Multilingual Matters 1996, 15-38.

Schnädter, Herbert: „Der Fehlerindex - ein zuverlässiger Bewertungsfaktor? Zur Korrekturpraxis im Fach Französisch“, Die Neueren Sprachen 90/6 (1991), 636-652.

Steinke, Ines: Kriterien qualitativer Forschung. Ansätze zur Bewertung qualitativempirischer Sozialforschung. Weinheim, München: Juventa 1999.

Steinke, Ines: „Gütekriterien qualitativer Forschung“. In: Flick, Uwe / Kardorff, Ernst v. / Steinke, Ines (Hrsg.): Qualitative Forschung. Ein Handbuch. 2. Auflage. Reinbek bei Hamburg: Rowohlt 2003, 319-331.

TestDaF-Institut: Musterprüfung 1. Ismaning: Hueber 2005.

Weigle, Sara C.: „Effects of training on raters of ESL compositions”, Language Testing 11/2 (1994), 197-223. 


\section{Internet-Seiten}

www.alte.org

www.coe.int

www.testdaf.de 


\section{Niveaustufenbeschreibungen vergleichbar machen: "Is my B1 your B1?"}

Beate Zeidler, Frankfurt am Main

Seitdem 2001 die Endfassung des Gemeinsamen Europäischen Referenzrahmens für Sprachen (im Weiteren: GERR) vorgelegt wurde, werden die dort definierten sechs Niveaustufen zunehmend zur näheren Bestimmung und Beschreibung von Sprachkursen, Lehrwerken und Sprachprüfungen benutzt. Das ist zunächst einmal in Einklang mit der Intention des GERR, ein gemeinsames Bezugssystem zu schaffen, um so beispielsweise Sprachprüfungen miteinander vergleichbar zu machen. So heißt es in Kapitel 9 des GERR („,Beurteilen und Bewerten“) zu den „,drei wichtigsten Möglichkeiten, den Referenzrahmen [im Kontext des Bewertens von Sprachleistungen] zu verwenden“: „1. Zur genauen Beschreibung des Inhalts von Tests und Prüfungen ... 2. Zur Festlegung der Kriterien, mit deren Hilfe man entscheiden kann, ob ein Lernziel erreicht wurde ... 3. Zur Beschreibung des Kompetenzniveaus bei bereits existierenden Tests und Prüfungen, wodurch deren Vergleich über verschiedene Qualifikationssysteme hinweg ermöglicht wird.“ 
Der GERR ging zu diesem Zeitpunkt davon aus, mit den darin enthaltenen Niveaustufenbeschreibungen sei ein hinreichendes Instrumentarium vorhanden, um eine solche Stufenzuordnung von Sprachenprüfungen vorzunehmen - und eine große Zahl von Prüfungsanbietern folgte dieser impliziten Aufforderung. Jedoch wurde die Methode der mehr oder weniger intuitiven Zuordnung schon bald nicht mehr als ausreichend empfunden, und bereits 2002 veranstaltete der Europarat ein Seminar in Helsinki, welches das Ziel hatte, ein Verfahren zu finden, um diesen Prozeß besser zu spezifizieren und so eine Antwort auf die von J. Charles Alderson aufgeworfene und seither schon sprichwörtlich gewordene Frage „How do I know that my Level B1 is your Level B1?“ zu finden. Eine Arbeitsgruppe wurde gebildet, die 2003 die Pilotfassung eines Handbuches "Relating Language Examinations to the Common European Framework of Reference for Languages" vorlegte. Die in diesem Handbuch (das von den Internetseiten des Europarates, www.coe.int, heruntergeladen werden kann) vorgeschlagenen Methoden werden zurzeit von vielen Sprachprüfungsanbietern erprobt und auf ihre Tauglichkeit geprüft. Die folgenden Überlegungen sind im Verlauf der Mitarbeit und aus der Erfahrung im Rahmen dieses internationalen Projekts entstanden und formulieren Zwischenstände und Desiderate für die weitere Arbeit.

Im Wesentlichen zielt das „Manual“ darauf ab, die Kategorien des GERR im Bewusstsein der Testautoren zu verankern (Kap. 3: Familiarisation), die Prüfung in den Termini des GERR zu beschreiben (Kap. 4: Specification), die Kriterien, nach denen ein/e Testteilnehmer/in besteht oder nicht besteht, auf den GERR bezogen zu beschreiben (Kap. 5: Standardisation of Judgements), und diese Prozesse empirisch zu untermauern (Kap. 6: Empirical Validation). Zentral ist das Kapitel 5, denn hier geht es darum, die Kontaktstelle zwischen den Prüfungsteilnehmenden und dem Messinstrument zu beschreiben. Auf die Problemstellung dieses Kapitels soll deshalb hier näher eingegangen werden. In Anlehnung an Aldersons Zitat ist im Folgenden der Einfachheit halber immer von der Verortung auf dem B1-Niveau die Rede. Das B1-Niveau dient hier natürlich nur als Beispiel; das Gesagte gilt genauso für die anderen Niveaustufen.

Das Erkenntnisziel einer Prüfung ist, welches Sprachniveau die/der Prüfungsteilnehmende, die/der sich ihr unterwirft, erreicht hat (je nach Anlage der Prüfung global oder pro Teilfertigkeit). Es ist also sinnvoll, eine B1-Prüfung als eine Prüfung zu definieren, die die Entscheidung erlaubt, ob TN das B1-Niveau erreicht also die untere Schwelle des B1-Bereichs überschritten - hat oder nicht. Darüber hinaus könnte das Prüfungsergebnis weitere Informationen enthalten, z. B. eine qualitative Aussage darüber, in welchem Ausmaß das der Fall ist (also eine Benotung), oder eine Aussage darüber, ob die/der TN auch eine weitere Niveaustufe, also etwa B2, erreicht hat. Informationen dieser Art sollen hier aber nicht weiter berücksichtigt werden.

Die Entscheidung, ob eine Niveaustufe erreicht wurde, erfolgt auf zwei unterschiedlichen Wegen. Für die produktiven Fertigkeiten ist die TN-Leistung direkt 
zu beobachten und muss mit der Leistung eines/einer „typischen“ TN verglichen werden, um so zu einer Aussage zu kommen, ob der/die zu beurteilende TN schwächer als, genauso wie oder besser als der/die Referenz-TN die Sprache beherrscht. Für die rezeptiven Fertigkeiten muss anhand der Prüfungsaufgaben ein Indizienbeweis geführt werden, denn hier geht es ja um das nicht direkt beobachtbare Verstehen von gelesenen oder gehörten Texten. Unter der Voraussetzung, dass die Aufgaben grundsätzlich geeignet sind, diesen Indizienbeweis zu führen, muss dann festgelegt werden, wieviele Aufgaben mindestens gelöst werden müssen, um das Überschreiten der Niveauschwelle anzuzeigen. Dabei ist die Einschätzung notwendig, welche Aufgaben an der angezielten Niveaustufenschwelle zu verorten sind. Man muss also pro Aufgabe in der Lage sein, zu sagen, ob es sich um eine B1-Aufgabe handelt, d.h. um eine Aufgabe, die ein/e TN, der/die gerade das B1-Niveau erreicht hat, lösen können müsste.

Wir haben somit das Problem der Niveaueinschätzung von der Ebene der Prüfung auf die Ebene der einzelnen Aufgabe verschoben. Das macht es handhabbarer, aber es ist natürlich trotzdem noch nicht gelöst. Denn aufgrund welcher Kriterien kann man eine Aussage darüber machen, welche Niveaustufe eine Aufgabe repräsentiert?

Es bietet sich an, die Schwierigkeit als Kriterium zu benutzen, denn Schwierigkeit ist quantifizierbar als Anteil von TN in einer Stichprobe, die die Aufgabe richtig lösen konnten oder als Wahrscheinlichkeit, mit der ein/e TN einer bestimmten Fähigkeit eine Aufgabe richtig lösen kann. Im Prinzip könnte man also alle zur Verfügung stehenden Aufgaben nach Schwierigkeit ordnen und eine Stelle auswählen, die die Schwierigkeit der unteren B1-Schwelle bezeichnet. Die Auswahl dieser Stelle muss allerdings nach inhaltlichen Kriterien erfolgen, denn es ist der Anspruch der Niveaustufenbeschreibungen des GERR, eine inhaltliche Aussage zu treffen zu den Sprachhandlungen, zu denen jemand in der Lage ist, wenn sie/er die entsprechende Stufe erreicht hat. Die Aussage „hier ist die untere B1Schwelle“ ist also überhaupt nur sinnvoll, wenn der entsprechende Schwierigkeitswert in Bezug gesetzt werden kann zu den Inhalten der Aufgaben, die an dieser Stelle zu finden sind.

Tatsächlich lautet die Frage, die beantwortet werden muss, also: Welche inhaltlichen Faktoren machen eine Aufgabe mehr oder weniger schwierig? Wenn die Frage so gestellt wird, wird im Übrigen auch die dahinterliegende erste Hypothese sichtbar, die lautet: Es gibt einen direkten und eindeutigen Bezug zwischen bestimmten inhaltlichen Faktoren einer Aufgabe und ihrer Schwierigkeit. Aus dem sprachenübergreifenden Ansatz des GERR ergibt sich darüberhinaus die weitergehende zweite Hypothese: Dieser Bezug ist sprachenunabhängig. Die Beantwortung der Ausgangsfrage wird Rückschlüsse auf die Gültigkeit dieser Hypothesen ermöglichen.

Es liegt nahe, von den inhaltlichen Faktoren auszugehen, die in den Niveaustufenbeschreibungen des GERR genannt werden. Da der GERR sich aber nicht explizit der Beantwortung dieser Frage widmet, sondern die erforderlichen Infor- 
mationen nur implizit enthält, sind diese zunächst aus den Deskriptoren herauszupräparieren. Dies war der Ansatz des „Dutch Construct Project“, das zum Ziel hatte, zu dem beschriebenen Zweck ein inhaltliches Beschreibungsraster für Aufgaben zu entwickeln.

A. Unter Annahme der o.g. ersten Hypothese müsste es dann möglich sein, anhand der inhaltlichen Beschreibung/Bewertung der Aufgabe eine Erwartung hinsichtlich der Aufgabenschwierigkeit zu formulieren, die erstens bei mehreren Bewertern konsistent sein müsste und zweitens sich in der teststatistischen Erprobung als zutreffend erweisen müsste. Umgekehrt müsste es auch möglich sein, Aufgaben gezielt unter Beachtung der Beschreibungsparameter so zu entwickeln, dass sie ebenfalls von einer Bewertergruppe w.o. eingestuft werden könnten und diese Einstufung ebenfalls empirisch bestätigt werden könnte. Das Ziel der teststatistischen Bestätigung muss dabei zunächst nur sein, zu derselben Schwierigkeitsreihenfolge zu kommen wie in dem Bewertungsverfahren.

B. Wenn die zweite Hypothese zutrifft, müsste es außerdem möglich sein, aufgrund der inhaltlichen Beschreibung einer Aufgabe eine parallele Aufgabe in einer anderen Fremdsprache zu formulieren, die in einer Rangfolge von Aufgaben den gleichen Platz einnehmen würde wie die ursprüngliche Aufgabe in der Rangfolge der Aufgaben in der ursprünglichen Fremdsprache, und zwar sowohl bei der Bewertung durch Gutachter als auch nach teststatistischer Schwierigkeit.

Zur Überprüfung der Testbedingung A. mussten zunächst Beschreibungskategorien für die Aufgaben ermittelt werden. An beschreibenden Kategorien sind im GERR selbst aufgeführt: Verwendungskontext (Domäne, Orte, Institutionen, Personen, Objekte, Ereignisse, Handlungen, Texte) (Tabelle 5, S. 54), Themen (Kap. 4.2), Kommunikative Ziele (Kap. 4.3), Kommunikative Aktivitäten und Strategien (Kap. 4.4), Textsorte und Textlänge (Kap. 4.6.2/4.6.3), Kommunikative Aufgaben (Kap. 7), erforderliche linguistische, soziolinguistische, pragmatische und strategische Kompetenzen (Kap. 5.2.1, 5.2.2, 5.2.3, 4.4.3). Diese werden in Kapitel 4 des „Manuals“ als hilfreiche Grundlage für die Beschreibung von Testteilen genannt.

Der Projektbericht des „Dutch Construct Project“ (Alderson u.a. (2005)) geht hingegen nicht von den explizit genannten Kategorien, sondern von den in den Deskriptoren des GERR implizit enthaltenen aus und nennt als direkt aus dem GERR abgeleitete Kategorien zur Beschreibung von Aufgaben die folgenden: „,Textsorte“, „Themen“, „Domäne“ sowie die Kategorie „Was“ (,What") als Sammelbegriff für das Objekt derjenigen mentalen Operation, die im Deskriptor beschrieben wird (Alderson u.a. (2005), Appendix 1, S. 24). Diese Kategorien wurden zunächst induktiv ermittelt, dann praktisch ausprobiert und mehrmals modifiziert. 
Im Laufe der Arbeit stellte sich heraus, dass die genannten im GERR aufgefundenen Kategorien nicht ausreichen, um die o. g. Bedingungen zu erfüllen. Es wurden deshalb weitere Kriterien hinzugezogen. Aus Lern- und Testtheorie wurden die Kategorien „Operation“, „Discourse type“ und „Text structure“ abgeleitet. Aus der praktischen Erfahrung als Testautoren wurden die Kategorien „Item types“, „Vocabulary“, „Grammar“, „Text length“, „Number of participants“, „Text speed“, „Accent/Standard Pronunciation“, „Readability“, „How often played“" (Alderson u.a. (2005), Appendix 1, S. 24) bezogen.

Die folgende Tabelle zeigt in der linken Spalte die Parameter, die zur Beschreibung der Aufgaben verwendet werden, und in der rechten Spalte die Beurteilung ihrer Eignung zur Identifikation des Schwierigkeitsgrades der Aufgaben nach einem Anwendungsversuch mit fünf Beurteilern, die 77 Testitems (16 Texte mit teilweise mehreren Aufgaben) beschrieben. In Klammern ist die entsprechende Seite im Projektbericht angegeben. Die Merkmalsausprägungen, die zur Beschreibung jeweils zur Verfügung standen, sind dem Raster (s. Anhang) zu entnehmen. Es ist zu beachten, dass die Ergebnisse auf der Basis des „Grid 3“, der bislang vorletzten Entwicklungsstufe des Rasters, ermittelt wurden, welches deshalb auch im Anhang wiedergegeben wird. Hervorhebungen wurden zur besseren Übersicht vorgenommen.

\begin{tabular}{|l|l|}
\hline TEXT & \\
\hline Textquelle & $\begin{array}{l}\text { no obvious association between text source and CEF level of the task } \\
\text { (117) }\end{array}$ \\
\hline Authentizität & $\begin{array}{l}\text { When the text alone is considered, there is no association between Au- } \\
\text { thenticity and CEF level. (114) }\end{array}$ \\
\hline Diskurstyp & $\begin{array}{l}\text { no clear association between broadly defined discourse type and CEF } \\
\text { level.(117); ... no association between discourse type, narrowly defined, } \\
\text { and CEF level. (118) }\end{array}$ \\
\hline Domäne & $\begin{array}{l}\text { no clear association by Domain although there is a tendency for Occupa- } \\
\text { tional to be associated with the higher levels (115) }\end{array}$ \\
\hline Thema/Inhalt & $\begin{array}{l}\text { no clear association with CEF level, although there was a tendency for } \\
\text { the world of work to predominate at the higher levels. (119) }\end{array}$ \\
\hline $\begin{array}{l}\text { Abstraktions- } \\
\text { niveau }\end{array}$ & $\begin{array}{l}\text { no significant association between whether a text was concrete or ab- } \\
\text { stract and its CEF level. (120) }\end{array}$ \\
\hline Textlänge & $\begin{array}{l}\text { no association between text length and CEF level (121) ... However, if } \\
\text { one casts the frequencies as percentages ..., an interesting tendency } \\
\text { emerges. This suggests that (although we only are dealing with 16 tasks } \\
\text { and there is herefore a substantial sampling effect) we could formulate } \\
\text { the hypothesis that texts tend to be longer when levels raise. The C1 } \\
\text { tasks run against this tendency, but this could easily be a result of the } \\
\text { small sample. (122) }\end{array}$ \\
\hline
\end{tabular}




\begin{tabular}{|c|c|}
\hline Vokabular & $\begin{array}{l}\text { significant association between vocabulary and CEF level }(p<.05) \text {. At } \\
\text { higher levels texts contain more extended vocabulary, which is in line } \\
\text { with the descriptions in the CEF-scales. }(115)\end{array}$ \\
\hline Grammatik & $\begin{array}{l}\text { no significant association with Grammar, although there is some evi- } \\
\text { dence that at higher levels sentence structure is more complex than at } \\
\text { lower levels, in accordance with the CEF scales (116) }\end{array}$ \\
\hline Geschwindigkeit & (nur HV, nicht kommentiert) \\
\hline Anzahl Sprecher & (nur HV, nicht kommentiert) \\
\hline Akzent/ Färbung & (nur HV, nicht kommentiert) \\
\hline $\begin{array}{l}\text { Klarheit der Aus- } \\
\text { sprache }\end{array}$ & (nur HV, nicht kommentiert) \\
\hline Wie oft gehört & (nur HV, nicht kommentiert) \\
\hline \multicolumn{2}{|l|}{ ITEM } \\
\hline Itemtyp & $\begin{array}{l}\text { no significant relationship between item type and CEF level. This is } \\
\text { hardly surprising given the lack of variety of test methods used in this } \\
\text { sample of tasks. In any case, it is unlikely that test methods will vary by } \\
\text { CEF (108) }\end{array}$ \\
\hline Mentale Operation & $\begin{array}{l}\text { In sum, there are clearly substantial differences among analysts as to } \\
\text { which operations they identify in the various items. This is in line with } \\
\text { findings in the literature on reading in a foreign language (see Alderson } \\
\text { 2000) but it presents considerable difficulties for those who wish to claim } \\
\text { that CEFlevels can be distinguished by operations or "skills", and it rein- } \\
\text { forces the finding that it is in fact rather difficult to reach agreement on } \\
\text { what operations are required by any given item, at any CEF level. } \\
\text { However, Tables } 15 \text { and } 16 \text { seem to indicate a tendency that at lower } \\
\text { levels items are more focused on retrieving explicit information from } \\
\text { texts, while at higher levels inferring from and evaluating texts become } \\
\text { more prominent, and items tend to deal more with implicit information. } \\
\text { Thus, some hope is provided by rather coarser-grained analyses at three } \\
\text { CEF levels, reinforcing the desirability of further research using larger } \\
\text { samples of texts and items in order to explore possible relations, espe- } \\
\text { cially if the analysts are trained in advance and discuss their analyses } \\
\text { among themselves before reaching final decisions. (113) }\end{array}$ \\
\hline $\begin{array}{l}\text { Geschätztes } \\
\text { Niveau }\end{array}$ & $\begin{array}{l}\text { When the modal value of the item level estimated by analysts is com- } \\
\text { pared with the claimed CEF level, there is a highly significant association } \\
\text { (Table } 4 \text { above also showed a significant correlation between analysts } \\
\text { and CEF level). However, almost half of the items had more than one } \\
\text { mode - ie analysts disagreed perhaps more here than with the other } \\
\text { analytic dimensions. There was notable agreement at B2, but C2 had } \\
\text { less agreement, and bizarrely, at least two analysts appear to have con- } \\
\text { sidered an A2 item to be C1. (108) }\end{array}$ \\
\hline
\end{tabular}

Im Ergebnis erweisen sich nur zwei Parameter als aussagekräftig, nämlich „Vokabular“ und die holistische Einschätzung der Aufgabe durch die Bewertenden („Geschätztes Niveau“), die ja gerade keinen analytischen Parameter dar- 
stellt. Für die Parameter „Domäne“, „Thema/Inhalt“, „Textlänge“, „Grammatik“ und „Mentale Operationen“"werden Tendenzen festgestellt, die eventuell in weiteren Versuchen Zusammenhänge ergeben könnten. Der Parameter „Itemtyp“ muss aus der Betrachtung ausgeklammert werden, da in dem Versuch keine hinreichende Bandbreite von Itemtypen betrachtet wurde. Die verbleibenden fünf Parameter „Textquelle“, „Authentizität“, „Diskurstyp“ und „Abstraktionsniveau“ lassen keinen Zusammenhang mit der Aufgabenschwierigkeit erkennen.

Die verwendete Parameter fallen in zwei Klassen: qualitative und quantitative. Tatsächlich lässt sich also eine Progression der Schwierigkeit am besten mit einer quantifizierbaren Größe, nämlich der Breite des geforderten Vokabulars, beschreiben. Ein weiterer quantitativer Parameter, die Textlänge, zeigte zwar keine signifikante Unterscheidungskraft, obwohl man dies intuitiv erwarten würde, jedoch kann eine Tendenz festgestellt werden. Ähnlich verhält es sich mit dem dritten und letzten quantifizierbaren Parameter „Grammatik“" (quantifizierbar, weil hier der Umfang der zur Verfügung stehenden Strukturen gemeint ist). Wesentlich schlechter schneiden die nominalskalierten Parameter ab. Was könnten die Gründe sein?

\section{Aussagekraft der Parameter hinsichtlich der Schwierigkeit}

Die Anfangshypothese, dass diese Parameter etwas mit Aufgabenschwierigkeit zu tun haben, könnte falsch sein. Es handelt sich um „Textquelle“, „Authentizität“, „Diskurstyp“ und „Abstraktionsniveau“. Es liegt nahe, die Hypothese anhand einer Versuchsanordnung mit eigens zu konstruierenden Aufgaben zu überprüfen, die sich jeweils nur in der betrachteten Dimension unterscheiden. Es zeigt sich allerdings bei dem Versuch, entsprechende Aufgaben zu entwerfen,

- dass der Bedeutungsgehalt der Parameter einer genaueren Definition bedarf - die momentan durch die Merkmalsausprägungen gegebene Definition ist nicht hinreichend

- dass zwischen den Parametern eine Abhängigkeit besteht, so dass beispielsweise der Diskurstyp nicht unabhängig von der Textquelle geändert werden kann. Insofern wird wahrscheinlich eine andere Auswahl der verwendeten Parameter konsistentere Ergebnisse erbringen. Abhängigkeiten treten dabei zwischen zwei oder auch mehr Parametern auf, z. B. fokussiert „Authentizität“ - wie aus den Merkmalsausprägungen ersichtlich auf vereinfachten im Gegensatz zu nicht vereinfachtem Text. Vereinfachungen werden in der Regel auf der Ebene von Wortschatz und Strukturen vorgenommen, so dass eine Abhängigkeit der Kategorie „Authentizität“ von „Vokabular“ und „Grammatik“ bestehen dürfte. Diese werden jedoch wiederum in gewissem Umfang durch die Thematik des Textes, also „Thema/Inhalt“, in Verbindung mit dem Abstraktionsniveau bedingt.

Im Folgenden einige Überlegungen zu Abhängigkeit in den einzelnen Kategorien: 


\subsection{Textquelle}

Je nach Beschreibungstiefe ist für diese Kategorie eine unendlich große Anzahl von Merkmalsausprägungen denkbar, beispielsweise kann die Ausprägung „Newspapers“ weiter nach „Boulevardpresse“ und „seriösen Tageszeitungen“ differenziert werden usw., auch nach Themenfeldern (Politik / Wirtschaft / Feuilleton / Vermischtes / Kleinanzeigen usw.). Jede dieser Unter-Ausprägungen enthielte sehr verschiedenartige Texte, so dass die Kategorie „Textquelle“ ohne „The$\mathrm{ma} /$ Inhalt" und „Abstraktionsniveau“ keine eigene Aussagekraft besitzen dürfte.

\subsection{Authentizität}

Ein Text, der von vornherein von Vokabular und Strukturen her für Lernanfänger geeignet ist, braucht nicht vereinfacht zu werden und bleibt insofern ,,authentisch“. Es kann jedoch ein anderer Text soweit vereinfacht werden, dass auch er für Anfänger geeignet ist, dieser wäre dann zwar für dieselbe Niveaustufe gedacht, aber ,nicht authentisch“.

\subsection{Diskurstyp}

Weder genereller noch spezieller Diskurstyp zeigte Aussagekraft bezüglich der Niveaustufe. Es liegt nahe, dass auch für den Diskurstyp das unter „Textquelle“ gesagte gilt, da sehr unterschiedliche Texte als Operationalisierungen der Diskurstypen vorstellbar sind.

\subsection{Abstraktionsniveau}

„Abstraktionsniveau“ ist bei dem im Projektbericht veröffentlichten Grid 3 dichotom angelegt (abstrakt/nicht abstrakt), für die Erprobung wurde jedoch offenbar bereits eine erweiterte Skala verwendet, wie sie dann in Grid 4 erscheint (Alderson u.a. (2005), Appendix 11, Table 33, S. 119f.)

Möglicherweise besteht eine direkte Abhängigkeit zwischen wahrgenommenem Abstraktionsniveau und Niveaustufe dergestalt, dass bei Texten einer höheren Niveaustufe ein höheres Abstraktionsniveau als „normal“ empfunden wird und somit das Abstraktionsniveau als „only“ oder „mostly concrete“ beschrieben wird, obwohl es nicht mit dem Abstraktionsniveau einer Aufgabe auf niedrigerer Niveaustufe zu vergleichen ist. Hierzu zwei Lesetexte aus den Dialang-Aufgaben, die auf der Beispiel-CD des Europarates vorgestellt werden. Beide werden als „mostly concrete“ eingestuft, einer davon auf der Niveaustufe B1, der andere auf der Stufe A1/A2 (diese Aufgaben dienen nur als Beispiel, sie gehören nicht zu den Aufgaben, mit denen das Raster erprobt wurde): 
„A spokeswoman for the RSPCA (the Royal Society for the Prevention of Cruelty to Animals), Jo Crozier, said: 'If it hadn't been for DNA testing, we would never have been able to prove that it was these men who killed the animal, even though they were found only 600 yards from the body. All we would have had was circumstantial evidence and that wouldn't have been enough to convict them."

"Bike Doc - A Worker's Co-op

Mountain bikes, bikes, tourers, city bikes, racers, hybrids, folders, tandems, and more.

Spares, accessories, clothing, friendly helpful service, everything you should get from the best all round bike shop in Manchester.

Access, Visa, Switch, 0\% Finance, Xmas Club.

Hotline $01612241303^{\prime c}$

Um die Parameter auf ihre Tauglichkeit zu überprüfen und weitere Parameter zu gewinnen, sollte als nächster Schritt die Entwicklung von Aufgaben entlang der bisher vorliegenden Parameter systematisch versucht werden. Eine Einbeziehung der Parameter, die im DESI-Projekt (vgl. http://www.dipf.de/desi) entwickelt wurden, wäre sicher zu erwägen. Anhang 14 des Projektberichts enthält Anmerkungen zur Anwendung des Beschreibungsrasters auf die englischen Hörverstehens-Aufgaben des DESI-Projektes und weist darauf hin, dass in diesem Projekt mit drei Parametern eine gute Aussagekraft hinsichtlich der Niveaustufe einer Aufgabe erzielt werden konnte. Diese Parameter liegen teilweise quer zu dem im Dutch Construct Project entwickelten: TC1 (Item: Focus on Content) enthält die Dimension "Abstraktionsniveau", jedoch mit anderen Merkmalsausprägungen (Item focuses on concrete aspects in everyday contexts/ Item focuses on abstract aspects in every day contexts (e.g. emotions, attitudes)/ Item focuses on abstract aspects such as text structure); TC2 (Comprehension: Purposes) und TC3 (Comprehension: Information Processing) enthalten jeweils Aspekte der Dimension "Mentale Operation" (TC2: Understanding linguistically and contextually simple detail to construct local understanding/ Integrating two aspects of the content to construct global understanding + Integrating two aspects of the content to construct global understanding / Integrating several aspects of the content which are scattered across the entire text to construct global understanding + Understanding linguistically and contextually very complex detail to construct local understanding; TC3: Recognising and retrieving information which is explicitly stated in the text/ Recognising and retrieving information which is implicitly stated in the text + Understanding paraphrases in the item which refer to explicitly stated information in the text / Making inferences about information which is implicitly stated in the text). Darüberhinaus verwendet DESI Parameter zu Vokabular und Grammatik (Nold/Rossa (2005), S. 133 - 137).

DESI macht also mit gutem Erfolg Gebrauch von Kategorien, die in der Erprobung des Dutch Construct Project sich als nicht (Abstraktionsniveau) bzw. 
nicht signifikant (Mentale Operation) aussagekräftig zeigten. Es liegt nahe, den Grund in der unterschiedlichen Formulierung der Merkmalsausprägungen zu vermuten. Eine vergleichende Untersuchung wäre wünschenswert.

\section{Vollständigkeit der Merkmalsausprägungen}

Die Parameter könnten (teilweise) geeignet zur Beschreibung der Aufgabenschwierigkeit sein, aber die vorgegebenen Merkmalsausprägungen könnten nicht hinreichend differenzierend sein, so dass zuviel Interpretationsspielraum hinsichtlich der Zuordnung von Aufgabenmerkmalen zu den Merkmalsausprägungen besteht.

Diese Annahme wird durch die Tatsache unterstützt, dass unter den fünf Bewertern teilweise nur wenige übereinstimmende Bewertungen abgegeben wurden, wie die folgende Tabelle zeigt (Alderson u.a. (2005), S. 120). „Mean Agreement" bezeichnet die durchschnittlich erreichte Übereinstimmung bei der Bewertung der 16 Aufgaben, wobei zunächst für jede Aufgabe die Übereinstimmung unter den fünf Bewertern ermittelt und dann das arithmetische Mittel dieser Werte gebildet wurde.

Table 35 Mean agreement by dimension

\begin{tabular}{|l|l|l|}
\hline Dimension & Mean agreement & Standard Deviation \\
\hline Authenticity & $82.5 \%$ & 19.15 \\
Domain & $81.25 \%$ & 17.08 \\
Broad Discourse Type & $77.5 \%$ & 19.15 \\
Text Source & $73.75 \%$ & 18.93 \\
Narrow Discouirse Type & $70 \%$ & 21.91 \\
Vocabulary & $68.75 \%$ & 16.28 \\
Concrete/Abstract & $66.25 \%$ & 17.46 \\
Grammar & $62.5 \%$ & 19.15 \\
Topic & $62.5 \%$ & 24.08 \\
Task level estimated & $46.25 \%$ & 12.04 \\
\hline
\end{tabular}

(Alderson u.a. (2005), Appendix 11, S. 120)

Es ist zu erwarten, dass große Abweichungen unter den Bewertern tendenziell zunehmen, wenn die Anzahl der zur Verfügung stehenden Optionen steigt. Dies ist jedoch nicht durchgängig der Fall:

\begin{tabular}{|l|l|}
\hline Dimension & Anzahl Optionen in Grid 3 \\
\hline Authentizität & 3 (wenn "Lesen" zugrundegelegt wird) \\
\hline Domäne & 4 \\
\hline Diskurstyp (generell) & 5 \\
\hline
\end{tabular}




\begin{tabular}{|l|l|}
\hline Textquelle & $37 / 18$ \\
\hline Diskurstyp (speziell) & 12 \\
\hline Vokabular & 4 \\
\hline Abstraktionsniveau & 4 \\
\hline Grammatik & 4 \\
\hline Thema/Inhalt & 14 \\
\hline Geschätzte Niveaustufe & 13 \\
\hline
\end{tabular}

Relativ wenig Übereinstimmung wird bei den Kategorien „Vokabular“, „Grammatik“ und „Abstraktionsniveau“ erzielt, obwohl hier nur vier Optionen zur Verfügung stehen - und das, obwohl sich „Vokabular“ trotzdem als die Kategorie mit der besten Aussagequalität erwiesen hat. Es liegt nahe anzunehmen, dass die Bewerter jeweils sehr unterschiedliche Auffassungen von der Bedeutung der Begriffe „frequent“", „komplex/weniger komplex“, „konkret/abstrakt" usw. hatten. Dies ist auch meine Erfahrung bei der probeweisen Anwendung des Rasters. Insofern repliziert die Erprobung die Ergebnisse einer Analyse der GERRTerminologie, die Teil des Projektberichts ist und die (hier auf den GERR bezogen) feststellt „For example, 'simple' is frequently used in the scales, but it is not clear how one is to decide what is simple and what is less simple, and especially 'very simple“" (Alderson u.a. (2005), S. 12) Um die Schwierigkeit zu lösen, dass die Bewerter keine Bezugsgröße haben, an der „komplex“ etc. gemessen werden kann, schlägt Alderson vor, dass sie ein Training mit Musterbewertungen durchführen. Dieses Verfahren wirft allerdings weitere Fragen auf:

- Wieviele Übungsbewertungen sind notwendig, um die in den Musterbewertungen enthaltenen Maßstäbe als Bezugsgröße zu internalisieren? Um Aussagen hinsichtlich der Vergleichbarkeit der Bezugsgrößen treffen zu können: wie beschreiben die Bewerter die internalisierten Bezugsgrößen?

- Wie stabil ist der Trainingseffekt, d.h. muss das Verfahren wiederholt werden, und ggf. wie oft?

- Genügt die Rezeption der Musterbewertungen, oder ist die Diskussion unter mehreren Bewertern unabdingbar, um eine gemeinsame Bezugsgröße zu konstituieren?

- Wenn letzteres der Fall ist, kann dann trotzdem bei mehreren Bewertergruppen von gleichartigen Bezugsgrößen ausgegangen werden, oder sind diese nur innerhalb einer Gruppe konsistent?

Es sollte deshalb versucht werden, die Merkmalsausprägungen auch explizit weiter zu operationalisieren. Darüberhinaus sollten weitere Erprobungen der Trainingsaufgaben vorgenommen werden, um deren Geeignetheit für das Verfahren zu untersuchen und zu optimieren.

Nicht konsistente Bewertungen könnten einerseits durch inkompatible interne Maßstäbe der Bewerter erklärt werden, andererseits durch Eigenschaften, die der 
Aufgabe inhärent sind. Um diese beiden Effekte auseinanderhalten zu können, sollte die Dimension „Sicherheit bei der Einschätzung“ in die Betrachtung mit einbezogen werden, d. h. für jede getroffene Bewertung sollten die Bewerter angeben, ob sie dabei sehr sicher, sicher oder eher unsicher sind. Aufgaben oder Dimensionen, bei denen die Bewertenden sich mehrheitlich unsicher sind, bedürfen einer gesonderten Analyse.

Aufschlüsse hinsichtlich der Verfahren und Maßstäbe, die die Bewertenden bei der Bewertung verwenden, könnten Lautdenkprotokolle geben.

Alle oben zitierten Ergebnisse wurden aufgrund einer Aufgabenstichprobe aus Englischprüfungen ermittelt. Von den genannten zu testenden Hypothesen konnte somit zunächst nur Hypothese A in Angriff genommen werden. Allerdings kann Hypothese B erst getestet werden, wenn aus den beschreibenden Kategorien mit entsprechender Sicherheit Spezifikationen abgeleitet werden können, die die Konstruktion von anderssprachigen Parallelaufgaben erlauben. Die Aufgaben sollten dann einer Bewertung/Einordnung durch Gutachter unterzogen werden und auch mit einer TNStichprobe erprobt werden, so dass zwei Schwierigkeitsrangfolgen entstehen, die weitgehend übereinstimmen sollten. Schließlich wäre zu überprüfen, ob die Schwierigkeitsrangfolgen auch mit den Rangfolgen der Parallelaufgaben in anderen Fremdsprachen übereinstimmen.

\section{Literatur}

Gemeinsamer Europäischer Referenzrahmen für Sprachen: lernen, lehren, beurteilen. (2001) Europarat, Rat für kulturelle Zusammenarbeit, Modern Languages Division, Strasbourg, deutsch: Berlin etc. (Langenscheidt), 2001

„Relating Language Examinations to the Common European Framework of Reference for Languages: Learning, Teaching, Assessment (CEF). Manual. Preliminary Pilot Version“" (2003), Language Policy Division, Council of Europe DGIV/EDU/LANG (2003) 5 rev. 1, Strasbourg

Relating Language Examinations to the Common European Framework of Reference for Languages: Learning, Teaching, Assessment (CEF).Reading and Listening Items and Tasks", Council of Europe, Language Policy Division 2005 (CD-ROM)

Alderson, J. Charles/Figueras, Neus/Kuijper, Henk/Nold, Günter/Takala, Sauli/Tardieu, Claire: "Final Report of the Dutch CEF Construct Project", in: Relating Language Examinations to the Common European Framework, of Reference for Languages: Learning, Teaching, Assessment (CEF).Reading and Listening Items and Tasks, Council of Europe, Language Policy Division 2005 (CD-ROM)

Nold, Günter/Rossa, Henning: "Applying the Grid to the EFL listening tests in DESI", in: Relating Language Examinations to the Common European Framework of Reference for Languages: Learning, Teaching, Assessment (CEF).Reading and Listening Items and Tasks, Council of Europe, Language Policy Division 2005 (CD-ROM) 


\section{Anhang}

Merkmalsausprägungen in Grid 3 (Grundlage der Erprobung, Alderson u.a. (2005), Appendix) und Grid 4 nach der Fassung im Internet

(http://www.lancs.ac.uk/fss/projects/grid/grid.php), 12.08.2006

\begin{tabular}{|c|c|c|}
\hline $\begin{array}{l}8 \\
8 \\
8\end{array}$ & Grid 3 & Grid 4 \\
\hline 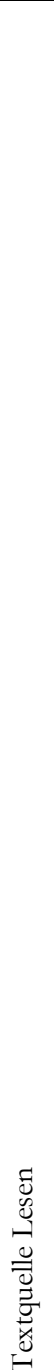 & $\begin{array}{l}\text { 1. Teletext } \\
\text { 2. Guarantees } \\
\text { 3. Recipes } \\
\text { 4. Instructional material } \\
\text { 5. Novels, magazines } \\
\text { 6. Newspapers } \\
\text { 7. Junk mail } \\
\text { 8. Brochures } \\
\text { 9. Personal letters } \\
\text { 10. Broadcast and recorded spoken } \\
\text { texts } \\
\text { 11. Business letter } \\
\text { 12. Report, memorandum } \\
\text { 13. Life and safety notices } \\
\text { 14. Instructional manuals } \\
\text { 15. Regulations } \\
\text { 16. Advertising material } \\
\text { 17. Labelling and packaging } \\
\text { 18. Job description } \\
\text { 19. Sign posting } \\
\text { 20. Public announcements and notices } \\
\text { 21. Labels and packaging } \\
\text { 22. Leaflets, graffiti } \\
\text { 23. Tickets, timetables } \\
\text { 24. Notices, regulations } \\
\text { 25. Programmes } \\
\text { 26. Contracts } \\
\text { 27. Menus } \\
\text { 28. Sacred texts, sermons, hymns } \\
\text { 29. Authentic texts (as above) } \\
\text { 30. Textbooks, readers } \\
\text { 31. Reference books } \\
\text { 32. Blackboard text } \\
\text { 33. Computer screen text } \\
\text { 34. Videotext } \\
\text { 35. Exercise materials } \\
\text { 36. Journal articles } \\
\text { 37. Abstracts }\end{array}$ & $\begin{array}{l}\text { 1. Abstracts } \\
\text { 2. Advertising material } \\
\text { 3. Blackboard text } \\
\text { 4. Broadcast \& recorded spoken text } \\
\text { 5. Brochures } \\
\text { 6. Business letter } \\
\text { 7. Computer screen text } \\
\text { 8. Contracts } \\
\text { 9. Dictionaries } \\
\text { 10. Exercise materials } \\
\text { 11. Guarantees } \\
\text { 12. Instructional manuals } \\
\text { 13. Instructional material } \\
\text { 14. Job description } \\
\text { 15. Journal articled } \\
\text { 16. Junk mail } \\
\text { 17. Labeling and packaging } \\
\text { 18. Leaflets and graffity } \\
\text { 19. Life safety notices } \\
\text { 20. Magazines } \\
\text { 21. Menus } \\
\text { 22. Newspapers } \\
\text { 23. Notices, regulations } \\
\text { 24. Novels } \\
\text { 25. OP text } \\
\text { 26. Personal letters } \\
\text { 27. Programmes } \\
\text { 28. Public announcements and notices } \\
\text { 29. Recipes } \\
\text { 30. Reference books } \\
\text { 31. Regulations } \\
\text { 32. Report, memorandum } \\
\text { 33. Sacred texts, sermons, hymns } \\
\text { 34. Signposting } \\
\text { 35. Teletext } \\
\text { 36. Textbooks, readers } \\
\text { 37. Tickets, timetables } \\
\text { 38. Videotext } \\
\text { 39. Visiting cards }\end{array}$ \\
\hline
\end{tabular}




\begin{tabular}{|c|c|c|}
\hline 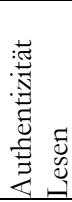 & $\begin{array}{l}\text { authentic } \\
\text { abridged/adapted/simplified } \\
\text { pedagogic }\end{array}$ & $\begin{array}{l}\text { genuine } \\
\text { adapted/simplified } \\
\text { pedagogic }\end{array}$ \\
\hline 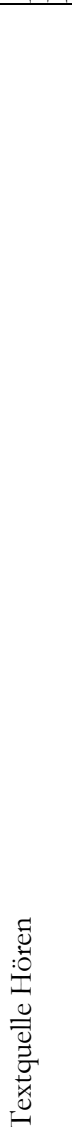 & 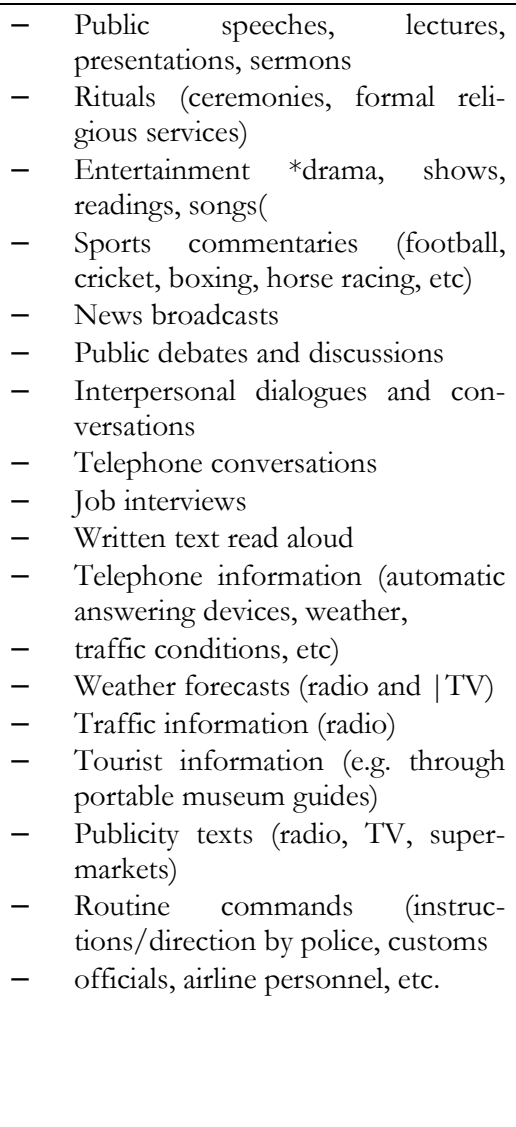 & $\begin{array}{l}\text { 1. Debates and discussions (both live } \\
\text { and on the media) } \\
\text { 2. Entertainment (drame, shows, } \\
\text { readings, songs) } \\
\text { 3. Interpersonal dialogues and conver- } \\
\text { sations } \\
\text { 4. Interview (both live and on the } \\
\text { media) } \\
\text { 5. News broadcasts } \\
\text { 6. Public announcements and instruc- } \\
\text { tions } \\
\text { 7. Public speeches, lectures, presenta- } \\
\text { tions, sermons } \\
\text { 8. Publicity texts (e.g. radio, TV, su- } \\
\text { permarkets) } \\
\text { 9. Radio phone-in } \\
\text { 10. Recorded Tourist information } \\
\text { 11. Rituals (ceremonies, formal religious } \\
\text { services) } \\
\text { 12. Routine commands (instructions/ } \\
\text { direction by police, customs officials, } \\
\text { airline personnel, etc) } \\
\text { 13. Sports commentaries (football, } \\
\text { cricket, boxing, horse racing, etc) } \\
\text { 14. Songs and poems } \\
\text { 15. Telephone conversations } \\
\text { 16. Telephone information (automatic } \\
\text { answering devices, weather, traffic } \\
\text { conditions etc) } \\
\text { 17. Traffic information } \\
\text { 18. TV/radio documentaries } \\
\text { 19. Weather forecasts }\end{array}$ \\
\hline
\end{tabular}




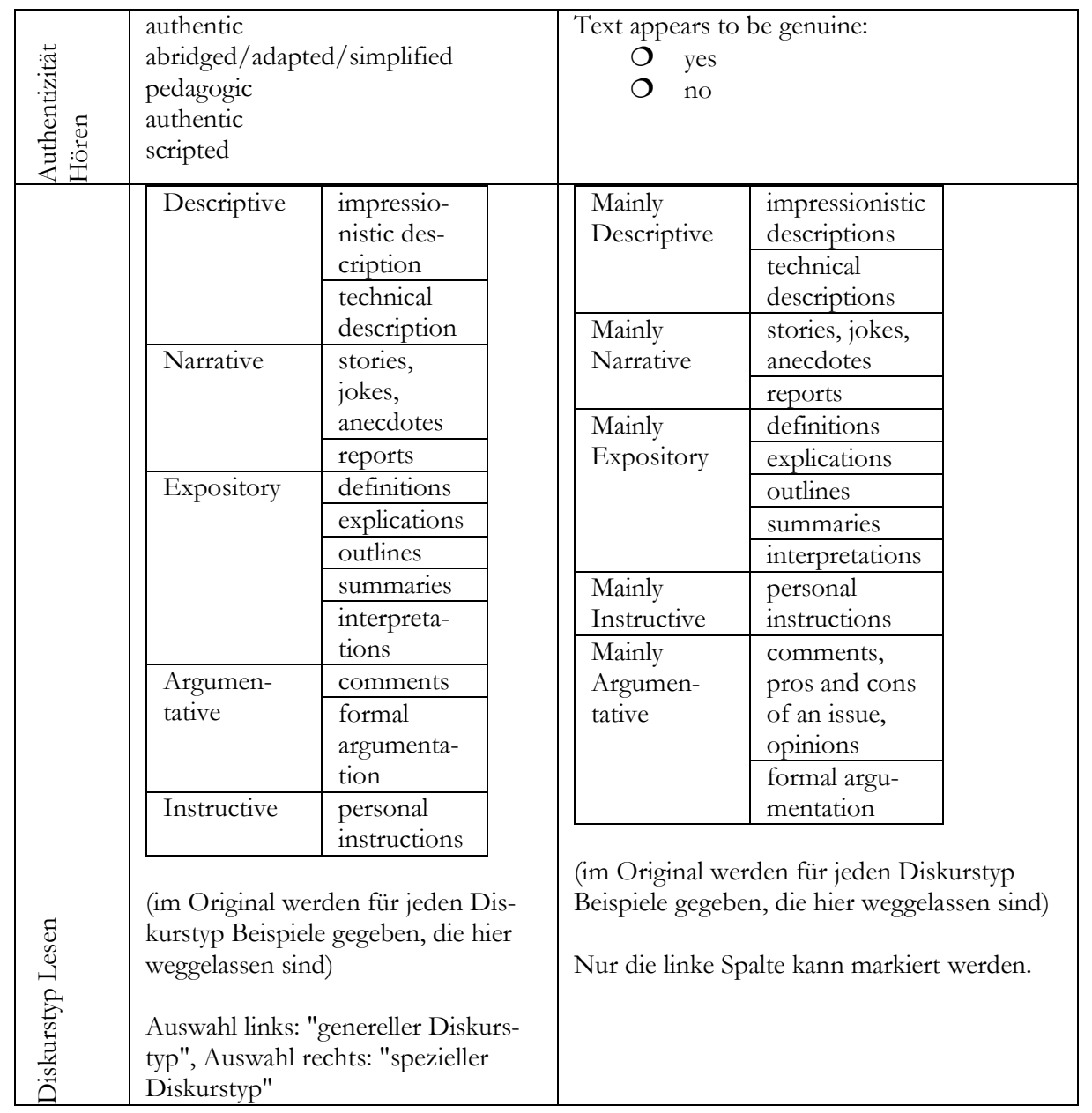




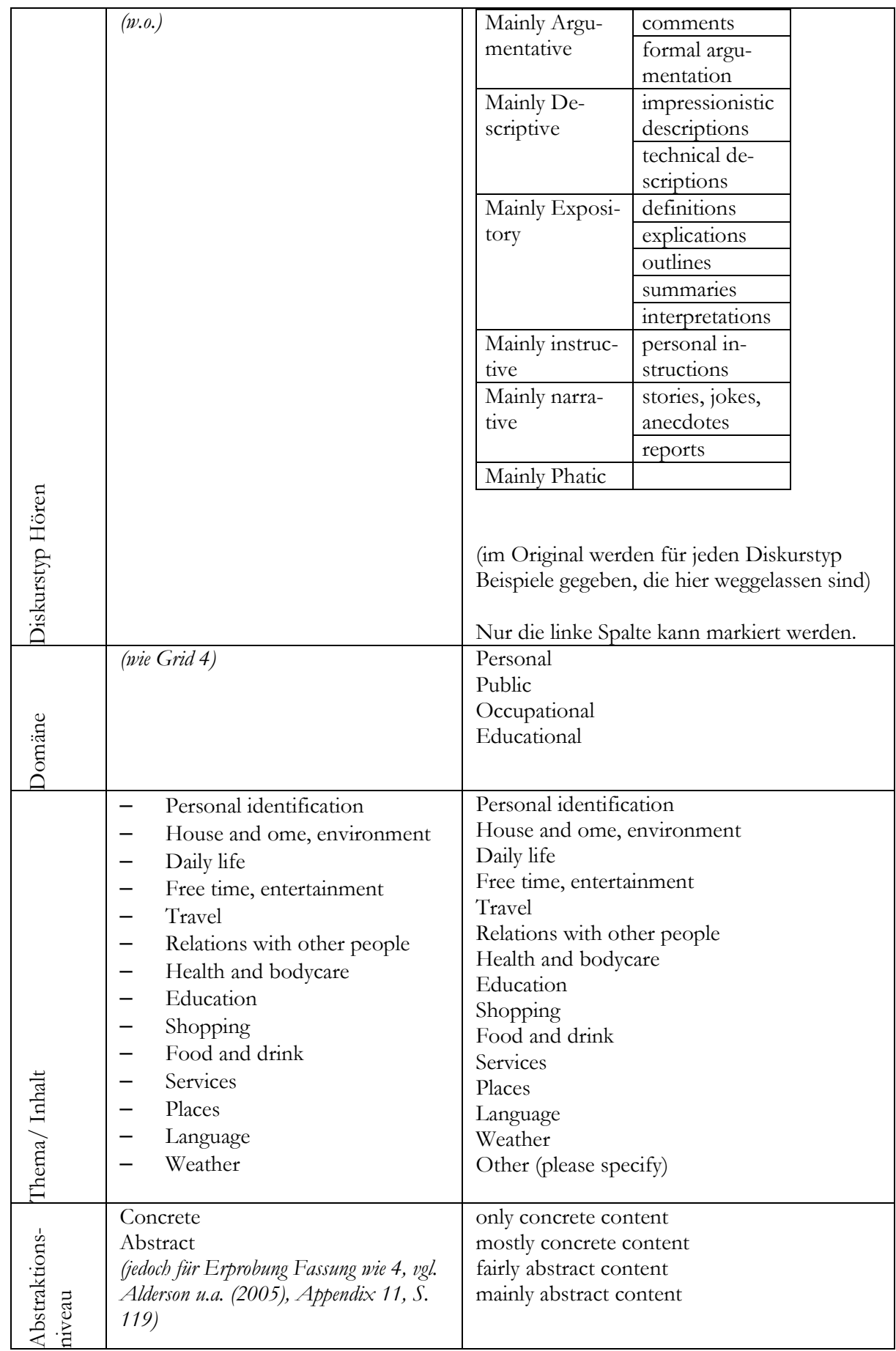




\begin{tabular}{|c|c|c|}
\hline 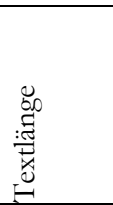 & (wie Grid 4) & words/minutes,seconds \\
\hline 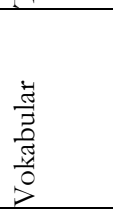 & (wie Grid 4) & $\begin{array}{l}\text { only frequent vocabulary } \\
\text { mostly frequent vocabulary } \\
\text { rather extended } \\
\text { extended }\end{array}$ \\
\hline 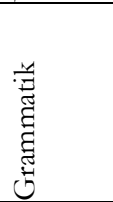 & $\begin{array}{l}\text { only simple sentences } \\
\text { mostly simple sentences } \\
\text { frequent compound sentences } \\
\text { many complex sentences }\end{array}$ & $\begin{array}{l}\text { only simple structures } \\
\text { mainly simple structures } \\
\text { limited range of complex structures } \\
\text { wide range of complex structures }\end{array}$ \\
\hline 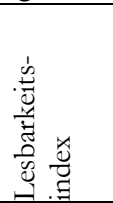 & Readability & (nicht mehr gefragt) \\
\hline 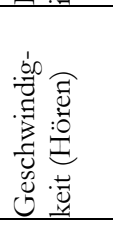 & $\begin{array}{l}\text { artificially slow } \\
\text { very slow } \\
\text { slow } \\
\text { normal } \\
\text { rapid }\end{array}$ & $\begin{array}{l}\text { artificially slow } \\
\text { slow } \\
\text { normal } \\
\text { fast }\end{array}$ \\
\hline 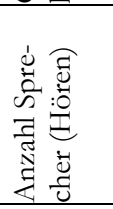 & (wie Grid 4) & $\begin{array}{l}\text { one } \\
\text { two } \\
\text { more than two }\end{array}$ \\
\hline 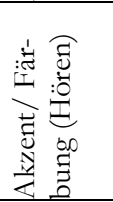 & $\begin{array}{l}\text { Dialect } \\
\text { Accent } \\
\text { Standard }\end{array}$ & $\begin{array}{l}\text { standard accent } \\
\text { slight regional accent } \\
\text { strong regional accent } \\
\text { non-native accent }\end{array}$ \\
\hline 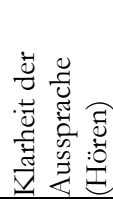 & $\begin{array}{l}\text { Artificially articulated } \\
\text { Clearly articulated } \\
\text { Normally articulated } \\
\text { Unclearly articulated }\end{array}$ & $\begin{array}{l}\text { artificially articulated } \\
\text { clearly articulated } \\
\text { normally articulated } \\
\text { sometimes unclearly articulated }\end{array}$ \\
\hline 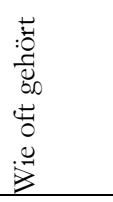 & $\begin{array}{l}\text { played once } \\
\text { played twice } \\
\text { played three times }\end{array}$ & $\begin{array}{l}\text { played once } \\
\text { played twice } \\
\text { played three times } \\
\text { played more than three times }\end{array}$ \\
\hline
\end{tabular}




\begin{tabular}{|c|c|c|}
\hline 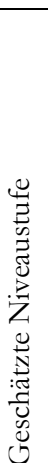 & $\begin{array}{l}\text { (Task und Item zusammen beurteilt, } \\
\text { Ausprägungen wie 4) }\end{array}$ & $\begin{array}{l}\text { below A1 } \\
\text { A1 } \\
\text { A1/A2 } \\
\text { A2 } \\
\text { A2/B1 } \\
\text { B1 } \\
\text { B1/B2 } \\
\text { B2 } \\
\text { B2/C1 } \\
\text { C1 } \\
\text { C1/C2 } \\
\text { C2 } \\
\text { beyond C2 }\end{array}$ \\
\hline & (wie Grid 4) & $\begin{array}{l}\text { Auswahlantwort: } \\
\quad \text { A. Multiple choice } \\
\text { B. Banked multiple choice } \\
\text { C. True/false } \\
\text { D. Multiple matching } \\
\text { E. Sequencing/ordering jumbled text } \\
\quad \text { F. Citing } \\
\text { Kurzantwort: } \\
\text { 1. Short answer } \\
\text { 2. Cloze (every nth) } \\
\text { 3. Gap-filling (one word) } \\
\text { 4. C-Test } \\
\text { 5. Summary completion } \\
\text { 6. Information transfer } \\
\text { 7. Sentential response } \\
\text { 8. Justify by citing } \\
\text { Freie Antwort: } \\
\text { 1. Essay } \\
\text { 2. Summary } \\
\text { 3. Report in own words } \\
\text { 4. Justify in own words } \\
\text { Andere Typen: }\end{array}$ \\
\hline 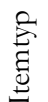 & & \\
\hline
\end{tabular}




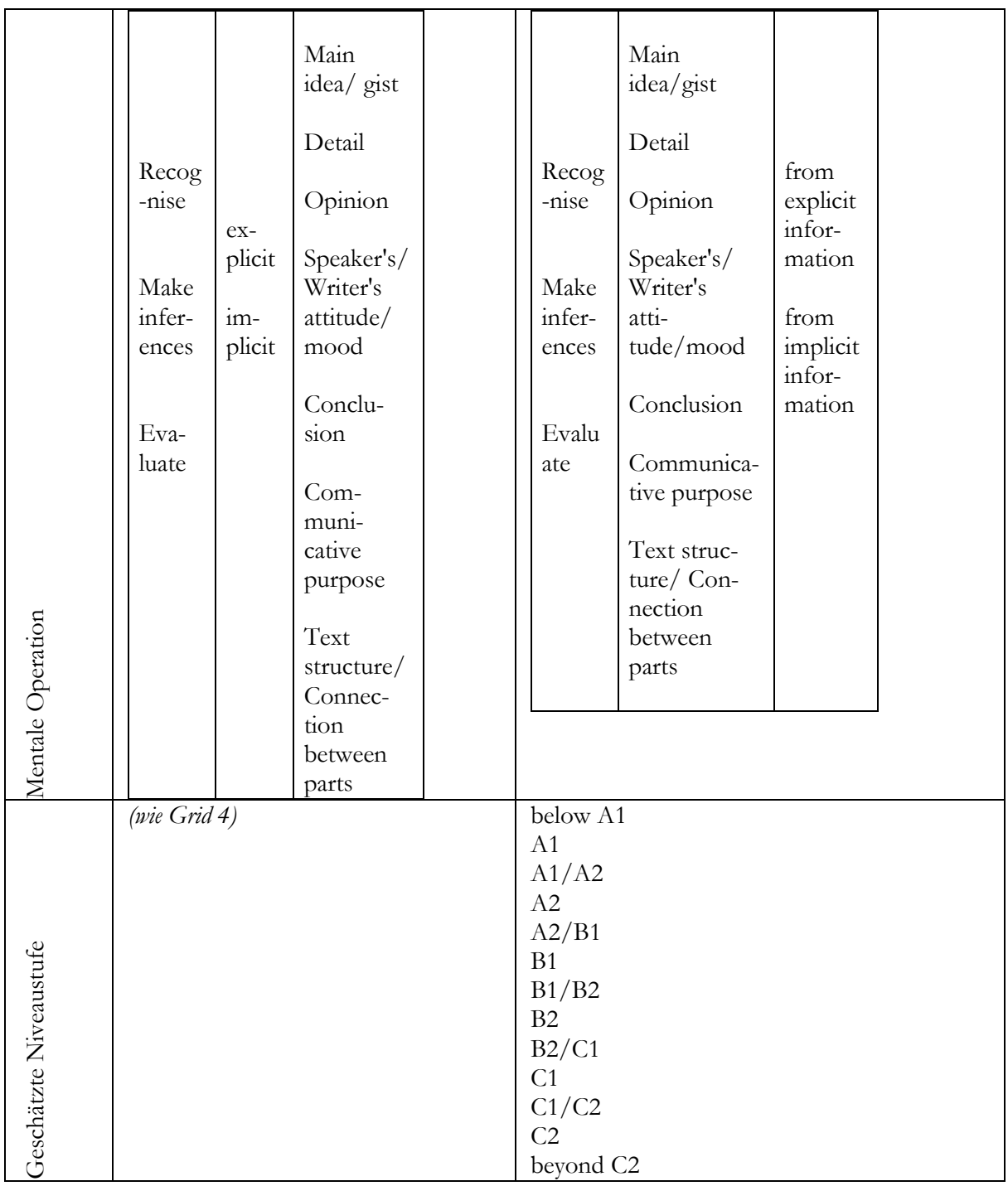





\title{
Mündliche Noten - wofür? und wie?
}

\author{
Roland Forster, Saarbrücken
}

\section{1}

Die Notwendigkeit, dieses Thema anzupacken, soll mit drei Beobachtungen gestützt werden.

In vielen Ländern Mittelosteuropas werden seit geraumer Zeit die Systeme, nach denen Teile des Abiturs auf Deutsch abgelegt werden können, neu gestaltet. In allen mir bekannten Fällen betrifft dies in besonderer Weise den Bereich der Mündlichkeit, insbesondere natürlich die mündliche Abschlussprüfung. Entsprechend groß ist bei den betroffenen Kolleginnen und Kollegen der Bedarf an Beratung und Weiterbildung.

In den „Bildungsstandards“ (KMK 2004), die für immer mehr Fächer entstehen und vorerst für den mittleren Bildungsabschluss konzipiert werden, spielt die mündliche Kompetenz eine große Rolle. Das Thema wird häufig nachgefragt für Weiterbildungsveranstaltungen oder für Pädagogische Tage kompletter Kollegien und wird zunehmend in die Ausbildung von jungen Lehrerinnen und Referendaren integriert. 
Der dritte Punkt: auf der FaDaF-Tagung 2006 war die ,DSH neu' mit ihren Fortschritten, aber auch Defiziten und Unklarheiten ein Dauerthema. Eine nicht hoch genug einzuschätzende Entwicklung der neuen Prüfungsordnung besteht darin, dass die mündliche Kompetenz (endlich?) ein notenrelevanter Teilbereich geworden ist. Verwaltungstechnisch argumentierend bedeutet dies, dass die Stellen, die die DSH abnehmen, durch Einbeziehen von entsprechenden Leistungen die Anzahl der mündlichen Prüfungen verringern können. Die Studierenden müssen dazu nachweisen, dass sie eine sprachliche Studierfähigkeit auch in der mündlichen Kompetenz erreichen.

Dies alles bedeutet in der Summe eine Aufwertung der mündlichen Kompetenz, in der Praxis - eine Erfahrung aus der Lehrer- und Dozentenweiterbildung im In- und Ausland - entstanden Irritationen und auch Unklarheiten, was genau und vor allem wie zu bewerten sei.

\section{2}

Zu klären ist demnach im nächsten Schritt die Frage, was wir als DaF-Lehrende zu lehren/unterrichten/vermitteln haben, um es später vielleicht auch zu beurteilen, zu bewerten oder sogar zu benoten. Konkret bedeutet dies: Welche Formen der mündlichen Kommunikation ziehen wir heran, um begründet und nachvollziehbar zu Beurteilungen, Bewertungen und Noten zu kommen? Im Kontext dieser Problematik hört man öfter als erste Ideen, dass sicher Aussprache ein gewichtiges Kriterium darstelle, Akzent wohl auch, und welche Körperhaltung ein Lernender beim Vortrag einnehme, dürfe ebenfalls nicht außer Acht gelassen werden. Hier soll es aber nicht um diese instinktive, eher reflexhafte Bewertung des Muttersprachlers gehen. Stattdessen soll versucht werden, vom Ziel her zu denken - im Übrigen eine Vorgehensweise, die die Autoren der Bildungsstandards demonstrieren. Typisch für diese Art von Denken ist ein lernerorientiertes, kompetenzorientiertes Arbeiten, bei dem die so genannte „exit competence“ eine maßgebliche Rolle spielt.

Für unsere Zusammenhänge bedeutet dies: Wir haben uns mit Gesprächsfähigkeit und Redefäbigkeit zu beschäftigen, also mit den beiden Großformen mündlicher Kommunikation. Damit ist immer auch Gesprächsverstehensfähigkeit gemeint und Redeverstehensfähigkeit; diese analytische Ebene ist von der produktiven Kompetenz nicht abzukoppeln. An dieser Stelle sollen für diese Phänomene keine neuen Definitionen geliefert werden. Es geht darum, mit anderen zu sprechen, also Gespräche zu führen, und es geht darum, zu anderen zu reden, also Reden zu halten. Für DaF-Lehrende erwächst hieraus die Frage: wie kann man diese Kompetenzen feststellen?

Von diesen komplexen Prozessen „Gespräch“ und „Rede“ sind die Elementarprozesse nicht ablösbar, die oft fatalerweise noch längere Zeit vorrangig bewertet werden - was allerdings nachvollziehbar ist. Ein Akzent ist leichter feststellbar 
und auch benennbar als etwa die Argumentationsstruktur eines Gesprächs zu analysieren. Zu diesen Elementarprozessen - die Sprechwissenschaft / Sprecherziehung gebraucht daneben den Terminus „Sprechbildung“ - zählen:

\section{Artikulation}

normiert, überprüfbar; Normverstoß $=$ Fehler, nachweisbar, bewertbar

\section{Intonation}

nicht normiert, „klingt deutsch“ vs. „klingt nicht deutsch“; abhängig von Situation, Intention, ähnlich: Korrekturen

\section{Tempo}

a. Sprechgeschwindigkeit: zu langsam - zu schnell - angemessen

b. Pausen: keine - zu viele - zu wenige - sinnvolle, Spannungs- und Lösungspausen; auch interkulturell kritisch: Pausendauer;

beides nicht normiert, abhängig von Sprecherpersönlichkeit, Kommunikationssituation, Kommunikationspartnern, vermutetem oder bekanntem Vorwissen

\section{Dynamik}

a. Betonung: normiert, also bewertbar (Ausnahme: Korrekturbetonung)

b. Lautstärke: nicht normiert, wird höchst subjektiv empfunden

Dies alles wahrzunehmen und zu beurteilen ist sicherlich nicht einfach und bereitet vor allem Berufsanfängern Schwierigkeiten. Trotzdem soll an dieser Stelle erneut der Hinweis erfolgen: wenn mündliche Kompetenz entwickelt und optimiert werden soll, und wenn vielleicht sogar Noten dafür gegeben werden müssen, geraten sämtliche beschriebenen Phänomene in den Blickpunkt. Es geht letztendlich immer um Gesprächskompetenz und Redekompetenz, und darin integriert um Elementarprozesse, die in der Summe den Sprechausdruck bedeuten. An dieser Stelle kann auch bereits die Antwort auf die Frage „Wie?“ aus dem Titel des Vortrages beantwortet werden. Erforderlich bei dieser Art von Sprachlehrarbeit - zu der Arbeit mit Muttersprachlern gibt es hier übrigens keinen Unterschied - ist immer wieder der Dreiklang beobachten, dokumentieren, bewerten.

\section{3}

Wenn über das vorliegende Themenfeld diskutiert wird, muss immer auch über Probleme gesprochen werden.

Die erste Schwierigkeit lautet: Wie kommen wir an Material, das wir beurteilen und bewerten können? Wenn Gesprächsfähigkeit festgestellt werden soll, gibt es 
nur eine Möglichkeit: Die Lernenden führen ein Gespräch und stellen damit ihr eigenes Material her, mit dem anschließend weitergearbeitet werden kann. Was dabei im Einzelnen beurteilt werden wird, ist abhängig von verschiedenen Faktoren, zum Beispiel: Was konnte (vorher) geübt werden? Wie wird mündliche Kompetenz verstanden? Was wird - wie - beobachtet? In vielen Teams und Kollegien ist sogar die entscheidende Frage: Wer kann mündliche Kommunikation lehren, unterrichten, trainieren?

Wer sich als Muttersprachler fragt: Wann sind für mich Gespräche gut?? Wann haben sie mir, was gebracht?? Wann hätte ich mir ein Gespräch ,sparen können'?, stößt schnell auf das Problem der höchst subjektiv vorhandenen Vorstellungen über Wertigkeit. Für den Teil „Rede“ ist Einigkeit, vor allem im universitären Kontext, sicher schnell herstellbar: Vorträge und Referate sollten nicht auswendig gelernt sein, nicht abgelesen werden, so frei wie möglich geredet und im unmittelbaren Hörerkontakt realisiert werden. Für „Gespräch“ ist dies bereits erheblich schwieriger: Sicher sollten sie zielorientiert sein, auch sachbezogen, wohl auch partnerorientiert, dann aber auch argumentierend (nicht: behauptend) und metakommunikativ. Die Ausschreibungen in den einschlägigen Weiterbildungseinrichtungen, vor allem auch im Unternehmensbereich, geben hier interessante Hinweise, was Gespräche noch alles sein können bzw. sollten.

Problematischer ist aber die Frage, wie Gesprächsfähigkeit und Redefähigkeit bewertet werden kann, wie nahe also jemand an das Erreichen von Zielvorstellungen herankommt. Als Beispiel sei angeführt für den Unterricht in einer heterogenen Lerngruppe von ausländischen Studierenden: Jemand vertritt eine andere Meinung, widerspricht also - ist das ein Zeichen für Gesprächsfähigkeit? Bekommt er oder sie also eine gute Note? - Und: In derselben Lerngruppe widerspricht jemand nicht, ist also nicht gesprächsfähig, erhält also eine schlechte Note? Diese Überlegungen führen immer wieder zur selben Frage zurück: Wo finden wir eine Bewertungsgrundlage und einen Bewertungsmaßstab für die Beurteilung der mündlichen Leistungen von Lernenden? Hinzu kommt: Was bewerten wir bewusst? was unbewusst? Was sind persönliche Vorlieben und Abneigungen, auf die - reflektiert oder eben nicht - reagiert wird? Wie bewerten Kollegen derselben Institution? Was für den einen eine „,perfekte Gesprächsführung“ ist, erscheint anderen als „schon ganz gut, aber nicht direkt perfekt, irgendwie“.

Ein typisches Unterrichts- bzw. Trainingsproblem soll hier ebenfalls erwähnt werden: das so genannte Beobachterparadoxon, was bedeutet: Eine Gruppe von Lernenden muss beobachtet werden können, um mit ihnen zu arbeiten. Wenn sie beobachtet werden, tun sie verschiedene Dinge nicht, die sie normalerweise tun würden - weil sie eben beobachtet werden. Andere Dinge hingegen kommen dazu, die üblicherweise nicht vorkämen. Wenn sie allerdings nicht beobachtet werden, um diese Effekte auszuschalten, kann nicht mit ihnen gearbeitet werden, weil eben nichts beobachtet werden konnte. Aus dieser Falle kommt kein Lehrender heraus, der mündliche Kommunikation weiterentwickeln will. 
Interkulturelle Probleme schließen sich an, es sind im Wesentlichen Schwierigkeiten, die die Zielgruppe direkt betreffen. Vor allem bei heterogenen Gruppen müssen methodische Schritte wohl überlegt werden. Eine bekannte Erfahrung ist, dass Teilnehmende rasch verstummen, wenn isolierte Kommunikationsleistungen beurteilt werden, wie längere Gesprächsbeiträge oder Reden. Viele geraten unter erhöhten Stress, sollten sie dazu gezwungen werden, weil diese Leistungen eine notwendige Bedingung für Noten sind, die unter Umständen von der mündlichen Abschlussprüfung befreien. In diesem Zusammenhang ist auch nicht von der Frage der kulturellen Gebundenheit abzusehen: Darf und kann etwas bewertet werden, das in der eigenen Kultur sozialisiert wurde und von der Zielkultur abweicht? Es handelt sich vor allem um Formen der Beteiligung am Unterricht, um Initiativ-Sein in Gesprächen, um widersprechen, leise oder laut reden und ähnliches. Das dahinter liegende Kernproblem, das hier allerdings nicht erörtert werden kann, ist die Frage: Wann schlägt eine Lernsituation (in der Fehler zum Beispiel einen notwendigen Erkenntnisschritt darstellen) in eine Leistungssituation um (in der Fehler eine Normabweichung darstellen und sanktioniert werden)?

Ein sehr spezielles Problem, das die Beurteilung mündlicher Leistungen von Lernenden erschweren kann, soll nicht ausgespart bleiben. Es handelt sich um die Eigensprechleistung der Lehrenden. Die Unterrichtenden sind in der typischen Lebenssituation deutscher Studienkollegiaten häufig das einzige Sprechvorbild der Lernenden. Folglich wird ihre Ausprägung von Standardsprache aufgenommen, eine eventuell vorhandene regionale Färbung, der Artikulationsort in seiner individuellen Variante wird nachgeahmt, gleiches gilt für den Kieferöffnungswinkel. Wenn von der Eigensprechleistung der Lehrenden die Rede ist, muss auch ihre Rezeptionsfähigkeit, konkreter: die Hörfähigkeit, betrachtet werden. Was hören Bewertende, was davon können sie benennen, was beurteilen sie - und was wird überhaupt nicht zur Kenntnis genommen, zum Beispiel als Hinweis auf eventuell vorhandene Lernschwierigkeiten?

\section{4}

Hier sollen nun Lösungsmöglichkeiten für diese Schwierigkeiten und Hürden betrachtet werden. Die zentrale Frage, von der wir ausgehen, lautet: Wie kann man etwas so Komplexes wie die Gesprächsfähigkeit und die Redefähigkeit von DaFLernenden bewerten, vielleicht sogar benoten? Welche Form der Beurteilung ist sinnvoll, der Mehrzahl der Unterrichtenden möglich und im Zusammenhang stehend mit dem vorangegangenen Unterricht?

Als erstes sollte das Kommunikationsverhalten im ,normalen' Unterricht unterschieden werden von den Ausprägungen der mündlichen Kompetenz, wie man sie in besonderen Veranstaltungen zum Schwerpunkt „Gespräch“ (=Gruppenleistungen) und zu „Rede“ (=Einzelleistung) zu sehen und zu hören bekommt. Im Folgenden sollen dazu in Kurzform die wichtigsten Punkte aufgeführt werden. 
Möglichkeit A: Bewerten/Beurteilen/Benoten während des Unterrichts

zentral: Wird ein Lernender verstanden als Gesprächsteilnehmer?

Versteht er/sie andere Teilnehmer in Gesprächen?

in Betracht kommen: Häufige kommunikative Handlungen in den bekannten Formen von Unterricht, wie: Dialoge Lehrende - Lernende, Gespräche zwischen Lernenden, kürzere Vorträge, eventuell Sprechen von Texten darin eingehend: Beurteilung der Elementarprozesse (=Sprechausdruck)

Noten geben in möglichst hoher Frequenz, resultierend aus permanenter Beobachtung am Ende der Unterrichtszeit (z. B. Semesterende): Note in mündlicher Kommunikation, oder: Einschätzung okay, nicht okay

notwendig (ebenso bei anderen Varianten): Transparenz - das heißt: Klarheit bei Lehrenden und Lernenden darüber, was und wie bewertet wird dazu erforderlich: Beobachtungsinstrumentarien verabreden für Teilkompetenz ,Reden halten' (im Hochschulkontext: Referate, Vorträge): wird ein Lernender verstanden, wenn er einen Vortrag hält oder ein (längeres) Statement abgibt?

(Ob ein Lernender seinerseits Vorträgen folgen kann, wird im schriftlichen Prüfungsteil ,Hörverstehen' bereits geprüft.)

\section{Möglichkeit B: Gesprächstraining}

als Möglichkeit, eine Beurteilung ,mündliche Kompetenz' zu erstellen angeleitet, systematisch, was bedeutet: erwünschte kommunikative Aktivitäten in Gesprächen ansteuern

Beispiele: Zuhören können, eigene Gedanken verständlich darstellen, Gesprächsbeiträge auf ein Ziel hin orientieren, Fragen stellen und sich fragen lassen usw.

als Hilfestellung zur Systematisierung der Auswertung, zur fundierten Rückmeldung: Diagnosebogen oder Auswertungskatalog, auch als Grundlage einer Bewertung (vgl. Anlage 1 und 2).

weitere Hilfestellung zum Thema:

GER, C 1; DSH-Prüfungsordnung neu, Kap. 9; S. 37 ff. („Beurteilungsraster zur mündlichen Kommunikation")

\section{Möglichkeit C: Redetraining / Reden und Präsentieren}

systematisches Heranführen an möglichst freies Reden, weitergehend Medien einsetzen zur Unterstützung der Darstellung, somit Präsentieren (vgl. Anlage 3).

\section{Möglichkeit D: „3-Kisten-Modell“ (für DSH neu)}

Es ist kaum begründbar, ob ein Lernender 61,5\% für seine mündlichen Leistungen erhält oder nur 58,9\%. Hier bietet die neue Prüfungsordnung an, gewissermaBen pauschalisiert eine Note festzusetzen in drei Stufen: $62 \%$, $75 \%$ oder $90 \%$. 
Folgt man dieser Möglichkeit des Ablegens in drei „Kisten“ (nicht die Menschen, sondern die Leistungsnachweise und Notenvorschläge!), sind Detailnoten nicht mehr länger erforderlich, sondern pauschale Beurteilungen sind legitim.

Abschließend soll deutlich gemacht werden, wozu wir raten:

1. Wir raten dazu, Lösungsmöglichkeiten zu suchen in Abhängigkeit von der eigenen Arbeitssituation. Das bedeutet konkret: Eine Gruppe von Lehrenden / eine Institution wird sich klar und einigt sich über:

- Inhalte

- Methoden

- Umfang der Leistungen, die zur Bewertung herangezogen werden

- Bewertungsverfahren (Basis?) Vorher muss geklärt worden sein: Was verstehen wir unter mündlicher Kommunikation? Wann ist jemand für uns mündlich kompetent? Hilfestellung geben hier neben verschiedenen Veröffentlichungen zu den Themen ,Mündliche Prüfung ${ }^{6}$ und „Bildungsstandards` der Europäische Referenzrahmen (GER) und das DSH-Handbuch.

2. Sobald und so lange Noten gegeben werden müssen, plädieren wir für eine Kurslehrerkonferenz beziehungsweise eine Besprechung aller mit bestimmten Kandidaten arbeitenden Lehrenden. So kann die Objektivität der Notenfindung erhöht werden, außerdem lässt sich damit eine Forderung der Prüfungsordnung für den Fall einer Befreiung erfüllen $(\$ 4, \mathrm{Ab}$ satz 3).

3. Wenn möglich, sollten für mündliche Leistungen keine Noten gegeben werden, sondern Wortbeurteilungen werden erstellt und kommuniziert. Diese beschreiben den Lernenden den erreichten Stand in einem höchst komplizierten Prozess. Eine solche Beurteilung lässt sich fundierter erstellen und wird von den Betreffenden besser verstanden und verarbeitet.

4. Zumindest die Inhalte und die Bewertungsverfahren müssen den Lernenden gegenüber kommuniziert werden.

In einem Punkt möchten wir nicht missverstanden werden: Die Komplexprozesse „Gespräch“ und „Rede“, überhaupt Formen der mündlichen Kommunikation, zu bewerten durch das Erteilen von Noten, halten wir für überaus problematisch - auch dann, wenn klar abgrenzbare Leistungen vorliegen. Der aktuelle Stand innerhalb des Prozesses „Sprachenlernen“ ist dagegen jederzeit zu beschreiben und liefert nachvollziehbare Rückmeldungen für den Lernenden. 


\section{5}

Im letzten Teil sollen Konsequenzen aus dem bisher dargelegten formuliert werden. Diese Konsequenzen in der appellativen Form von Fragen spiegeln pädagogische, didaktische und methodische Entscheidungen und empfehlen eine Vorgehensweise, um mündliche Kommunikation zu lehren, zu beschreiben, zu beurteilen, zu bewerten - und vielleicht sogar zu benoten.

a. Was soll bewertungsrelevant sein für eine bestimmte Lerngruppe an einer bestimmten Institution?

Diese Überlegung führt zur Beschreibung der sogenannten exit competence.

b. Was soll gelehrt, unterrichtet (und später auch bewertet!) werden? Erforderlich ist die Orientierung an den Komplexprozessen ,Gespräch ${ }^{6}$ und ,Rede'.

c. Wie soll unterrichtet werden?

Es werden methodische Fragestellungen geklärt.

d. Wie kommen wir zu einer Bewertung/zu Noten?

Das beschlossene Vorgehen muss innerhalb der Institution verbindlich sein.

e. Welche Bewertung/Note bekommen die Kandidaten wofür?

Erwartungshorizont und Bewertungsraster werden beschlossen.

Zentral bei allen diesen Schritten sind die Reflexion der Lehrenden, die Transparenz für alle Beteiligten und verbindliche Vereinbarungen innerhalb der Institution.

\section{Literatur}

Forster, Roland: Mündliche Kommunikation in Deutsch als Fremdsprache:

Gespräch und Rede. St. Ingbert: Röhrig 1997

ders.: Deutsch als Fremdsprache und Sprechwissenschaft / Sprecherziehung. In:

Altmayer, Claus / Forster, Roland (Hrsg.): Deutsch als Fremdsprache:

Wissenschaftsanspruch - Teilbereiche - Bezugsdisziplinen. Frankfurt/M: Lang 2003, 225-254

ders.: Sprecherziehung im Unterricht "Deutsch als Fremdsprache". - in: Pabst-

Weinschenk, Marita (Hrsg.): Grundlagen der Sprechwissenschaft und

Sprecherziehung. München: Reinhardt 2004, 324-326 
Meier, D.: Accelerated Learning. Handbuch zum schnellen und effektiven Lernen in Gruppen. Bonn: managerSeminare 2004

Pabst-Weinschenk; Marita et al. (Hrsg.): Sprecherziehung im Unterricht. München: Reinhardt 1997

Schneider, Günther / North, Brian: Fremdsprachen können - was heißt das? Skalen zur Beschreibung, Beurteilung und Selbsteinschätzung der fremdsprachlichen Kommunikationsfähigkeit. Chur/Zürich: Ruegger 2000

Slembek, Edith: Mündliche Kommunikation - interkulturell. St. Ingbert: Röhrig 1997 


\section{Anlagen}

FaDaF-Jahrestagung 2006, Hannover, 08. - 10.06.06

Dr. Roland Forster: Mündliche Noten - wofür? und wie?

I. Auswertung Rede / Präsentation

- Inbalt

- Gliederung / Aufbau / Struktur

- Sachlogik: der Sache, dem Gegenstand angemessen?

- Psychologik: den Hörern in dieser Form in dieser Zeit angemessen?

- Zeitlogik: angemessen und durchführbar in der zur Verfügung stehenden Zeit?

- Sprache

- Wortwahl: kompliziert - einfach / konkret - abstrakt / Fachsprache / Reizwörter

- Satzbau: klar, überschaubar - verschachtelt / assoziativ - gezielt entwickelnd

- muttersprachliche Muster? Grammatikverstöße?

- Sprechen

- Artikulation: präzise - undeutlich - korrekt - unsicher - Interferenzfehler?

- Intonation: monoton, wenig lebendig - mehrere Tonhöhen, ausdrucksstark

- Dynamik: Lautstärke (laut, leise, angemessen, variabel); korrekte Betonung

- Tempo: schnell, langsam, angemessen, variabel; viele, wenige, spannende, lösende Pausen - bewusst und gezielt eingesetzt?

- Hörerbezug

für sich gesprochen - zu den Zuhörern geredet? frei in der Kontaktgestaltung - eng an Vorlage und Material geklammert?

- Schauform

1) Mimik: authentisch - verkrampft - künstlich, aufgesetzt

2) Gestik: authentisch - verkrampft, antrainiert - sparsam - großräumig

3) Kinesik: Körperarbeit allgemein

4) Proxemik: Verhalten im Raum, in Bezug zu Hörern, Raum ausgenutzt?

- Redevorlage / Stichwortkonzept 
übersichtlich - Mängel? - zu viel/zu wenig Text - Anordnung der Informationen - graphische Gestaltung

- Medien / Präsentationsmaterial

zweckmäßig - ausreichend - ansprechend

\section{Elementarprozesse}

\section{Artikulation}

2. Intonation

normiert, überprüfbar; Normverstoß = Fehler, damit bewertbar

nicht normiert, ,klingt deutsch'vs. ,klingt nicht deutsch“

abhängig von Situation, Intention, Korrekturabsicht usw.

3. Tempo

a. Sprechgeschwindigkeit: zu schnell - zu langsam - angemessen im Deutschen: 250 Silben pro Minute $=$ mittelrasch (?)

b. Pausen: keine - viele - zu viele - wenige - zu wenige - sinnvolle ,richtige' oder ,falsche', Spannungspausen, Lösungspausen

4. Dynamik interkulturell kritisch: Pausendauer

a. Betonung: normiert, also bewertbar (Ausnahme: Korrekturbetonung)

b. Lautstärke: nicht normiert, wird höchst subjektiv empfunden

\section{Hintergrundinformationen in}

- Gemeinsamer Europäischer Referenzrahmen

- Bildungsstandards der Länder zu verschiedenen Fächern, insbesondere Deutsch

- DSH-Prüfungsordnung

- Handbuch DSH 



\section{DSH-Qualitätssicherung am Fachsprachenzentrum der Leibniz Universität Hannover}

„Prüfen ist der Versuch, durch entsprechende schriftliche und/ oder mündliche Aufgabenstellungen oder Fragen Kenntnisse, Fähigkeiten und Leistungen einer Person [...] auf einem bestimmten Gebiet so objektiv wie möglich festzustellen". (Jung 2005: 180).

\section{Christina Eberle/Matthias Perner, Hannover}

In der kontroversen Diskussion um die beste Methode der Sprachstandsmessung bezogen auf ein Hochschulstudium wird häufig unterschlagen, dass es „die beste“ Prüfung nicht gibt (vgl. Bachman/Palmer. 1996: 6). Stattdessen gilt es zu ergründen, wie eine Prüfung besser werden kann; es stellt sich die Frage nach der Sicherung und Optimierung der Qualität. Insbesondere die DSH mit ihrer langen, auf die Kriterien und Bestimmungen einer Hochschule bezogenen Prüfung wird sich hier neu orientieren müssen.

Zwar entspricht die Tradition der DSH durchaus der realen Durchführung einer Hochschulprüfung, z. B. hinsichtlich der Festlegung inhaltlicher Schwerpunkte, der Durchführung durch weitgehend erfahrene, doch nur selten geschulte Prü- 
fer ${ }^{1}$ und der Begrenzung der Menge der Prüfungsteilnehmer. Auch die mündliche Prüfung steht in dieser Tradition: Sie besteht aus einem mündlichen, durch mehr oder weniger spontan gestellte Fragen geleiteten Gespräch mit zwei Dozenten.

Diese jahrzehntelange Durchführungspraxis hat sich aufgrund der vielen inhärenten Vorteile etablieren können: Einerseits werden alle Teilnehmer sehr individuell geprüft und die DSH selbst wirkt häufig wie ein wirksames und flexibles „Zulaufventil“ ausländischer Studienbewerber.

Andererseits haben sich grundlegende Veränderungen in der Prüfungslandschaft ergeben, die diese Vorteile der DSH relativieren, ein generelles Umdenken bezogen auf die Qualität einer sprachlichen Hochschulzugangsprüfung erfordern und eben diese Qualität neu definieren. So ist die DSH nach KMK/HRK Beschluss ${ }^{2}$ einer der bundesweit anerkannten Sprachnachweise - vorausgesetzt, die DSH ist von der HRK registriert (RO-DT §3 Abs. 1, 0). Dies bedeutet offensichtlich, dass die Gebundenheit an eine Hochschule als eine der Stärken der DSH nicht mehr gegeben ist und jede registrierte DSH eine Qualität in der Erstellung, dem Inhalt und der Durchführung ausweist, die bundesweit allen Hochschulen genüge tut. Darüber hinaus hat in den letzten Jahren ein „Prüfungstourismus“ ausländischer Studienbewerber eingesetzt, die der Heterogenität der DSH-Landschaft nachreisen und an verschiedenen Orten ihr „Glück“ versuchen. Die kürzlich untersuchte Menge der Teilnehmer, Prüfungstermine, Bestehensquoten, Zulassungsbeschränkungen u. a. in der „Vorabinformation Umfrage zur DSH - Ergebnisbericht“" (Althaus, Perner, Weiß-Motz 2006) zeigt deutlich, wie weit die unterschiedlichen Institutionen von einer homogen und qualitativ gleichwertig erstellten und durchgeführten Sprachprüfung sind. Oder in Kiewegs Worten bezogen auf Gütekriterien: „eine Bestandsaufnahme der aktuellen Praxis erbringt nur die schlichte Feststellung der Divergenz" (Kieweg 1999: 4).

Das Fachsprachenzentrum der Leibniz Universität Hannover (FSZ) entwickelt seit Anfang 2005 ein Projekt, dass die dortige DSH qualitativ optimieren soll. Nach einer kurzen und simplifizierten Übersicht sowohl der existierenden als auch der praktisch umsetzbaren Qualitätskriterien sollen hier exemplarische Umsetzungsmöglichkeiten gezeigt werden. Diese Gütekriterien sollten jeder fachlichen Prüfungserstellung voran gehen, haben aber insbesondere bei der DSH-Registrierung durch die HRK und den $\mathrm{FaDaF}$ eine überragende Rolle.

Folgende Qualitätskriterien standen am Anfang des Hannoverschen Projekts:

Qualitätskriterien (nach Albers und Bolton 1995)

- Validität (Die zu überprüfenden Leistungen werden präzise erfasst.)

- Reliabilität (Die Leistungen werden zuverlässig erfasst.)

\footnotetext{
${ }^{1}$ Aus Gründen der einfacheren Lesbarkeit wird hier bei Personenbezeichnungen die kürzere maskuline Form generisch verwendet - die feminine Form ist impliziert.

${ }^{2}$ Beschluss der HRK (08.06.2004) und der KMK (25.06.2004) „,Rahmenordnung über Deutsche Sprachprüfungen für das Studium an deutschen Hochschulen (RO-DT) "sowie Beschluss der KMK (02.06.1995 i.d.F. vom 28.09.2005) „Zugang von ausländischen Studienbewerbern mit ausländischem Bildungsnachweis zum Studium an deutschen Hochschulen: Nachweis der deutschen Sprachkenntnisse“.
} 
- Objektivität (Die Bewertung ist trotz verschiedener Korrektoren einheitlich.)

Neben diesen grundlegenden Ausgangskriterien spielen weitere, inhärente Qualitätskriterien eine wichtige Rolle:

weitere Kriterien (nach Grotjahn 2000; Bachman und Palmer 1996)

- Ökonomie (Zeitaufwand bei der Prüfungserstellung, der Durchführung und der Bewertung)

- Praktikabilität (organisatorischer Aufbau)

- Fairness (bezogen auf die Teilnehmer: Wissen um den persönlichen/kulturellen Hintergrund)

- Authentizität oder Augenscheinvalidität (Nachvollziehbarkeit der Prüfung durch die Teilnehmer)

Zur Tradition der DSH gehört weiterhin, dass die Prüfungen häufig von Mitarbeitern des Deutschen Seminars, Abteilungen Deutsch als Fremdsprache oder Sprachenzentren erstellt worden sind. In den meisten Fällen müssen diese Mitarbeiter eine Prüfung neben weiteren Hauptaufgaben erstellen und durchführen. Allerdings sieht sich die DSH gegenwärtig in einer Veränderungsphase, in der die Qualität der Prüfung als primäres Ziel gesehen werden muss. Die hier angeregten Umsetzungen der Kriterien können nicht mehr als ein Gedankenanstoß sein. Das Fachsprachenzentrum der Leibniz Universität Hannover leistete sich den Luxus, einen Mitarbeiter explizit als DSH-Koordinator einzusetzen. Dennoch war ein ausgebildeter und erfahrener Prüfungsmethodiker nicht zu finanzieren, so dass das Projekt noch immer optimiert und die Kriterien noch immer nicht völlig zufrieden stellend umgesetzt werden konnten.

Für die vorläufige Umsetzung wurden am Fachsprachenzentrum die drei wichtigsten Kriterien wie folgt (um)definiert:

Die Validität sollte die Überprüfung beschreiben, mit der sich alle Teilbereiche einer Prüfung in ihrer Schwierigkeit, Fragestellung und Thematik annähern.

Die Reliabilität bezog sich auf die Vergleichbarkeit der Leistungen von Teilnehmern bei einem Vergleich von verschiedenen Prüfungen.

Die Objektivität - das zuerst umgesetzte Kriterium - bezog sich vorwiegend auf die Vergleichbarkeit und Homogenität im Korrektur- und Bewertungsverhalten der Rater ${ }^{3}$.

Als Motivation diente die Überlegung (und z.T. Erfahrung), was bei Vernachlässigung dieser Kriterien geschieht:

- Eine mangelnde (Konstrukt-)Validität testet keine einzelnen Teilbereiche, sondern undefinierte sprachliche Leistungen, wie z. B. die Fähigkeit zur Textproduktion bei bestimmten offenen Aufgaben zum Hörverstehen.

\footnotetext{
${ }^{3}$ Der nicht völlig unironische Bezug dieses Anglizismus auf das deutsche Homonym ist nicht unbedingt beabsichtigt. Testmethodisch ist lediglich die Unterscheidung von „Korrektor“", d. h. einer Person, die eine Leistung korrigiert und „Beurteiler“ oder „Bewerter“ (rater) von Bedeutung.
} 
- Die Reliabilität lässt sich gegenwärtig erschreckend am bereits erwähnten „Prüfungstourismus“ ablesen: Mit etwas Glück wird die DSH in X besser bewertet als die DSH in Y. Entsprechendes gilt beispielsweise auch für Teilnehmer einer Wiederholungsprüfung an ein und demselben Testzentrum; es stellt sich somit die Frage nach der Nachvollziehbarkeit von Unterschieden in der Bewertung beider Prüfungen.

- Die Bewertung einzelner Teilbereiche durch unterschiedliche Mitarbeiter setzt eine konsequente Anleitung und Kriterien der Bewertung, einen expliziten Erwartungshorizont und Wissen um Korrekturphänomene voraus - besonders bezogen auf die mündliche Prüfung spricht sich an einer Hochschule unter den Prüfungsteilnehmern sehr schnell herum, bei wem „bessere“ oder „schlechtere“ Leistungen zu erzielen sind - ein untrügliches Zeichen für mangelnde Objektivität. Diesen Bereich galt es in Hannover als erstes zu optimieren.

Darüber hinaus sind auch die anderen Qualitätskriterien zu bedenken:

- „Schnellschüsse“, also kurzfristig erstellte Prüfungen mit z. B. nicht ausgereiften Aufgabenstellungen sprechen für fehlende Ökonomie,

- durch einen unklaren Prüfungsablauf, durch laute Prüfungsräume oder fehlende bzw. nicht funktionierende Technik lässt sich die Praktikabilität kritisieren,

- unfaire Prüfungsbedingungen, wie z. B. kulturspezifische Themen ohne Vorentlastung

und

- die schwindende Augenscheinvalidität sowohl der Teilnehmer („Die DSH in $\mathrm{X}$ ist einfacher!") als auch von Hochschulen (wenn z. B. DSH einer Hochschule aufgrund mangelnder Qualität von anderen Hochschulen nicht mehr anerkannt werden) sind möglichst zu vermeiden.

Die Qualitätsbestrebungen sollten von der Menge der Prüfungen und Teilnehmer unabhängig sein. Am Fachsprachenzentrum der Leibniz Universität Hannover fanden 2005 acht Prüfungen mit weit über 1000 Teilnehmern statt - Grund und Möglichkeit genug, die durchgeführte Qualitätssicherung zu überprüfen. Neben dem schon erwähnten DSH-Koordinator arbeitete ein Team von vier erfahrenen und kompetenten DaF-Fachkräften an der Erstellung durch Durchführung der Prüfung mit. Zusätzlich bestand eine enge Kooperation mit dem FaDaF, in der ein Austausch stattfand und Überarbeitungen und Kritikpunkte zur DSH gesammelt wurden. Die Administration der Prüfungsanmeldung wurde durch ein An-Institut übernommen (Innovationsgesellschaft der Leibniz Universität Hannover mbH). Die Menge der Prüfungsteilnehmer machte einen externen Stamm von ca. 15 DaF-erfahrenen Bewertern (Korrektoren) unumgänglich, die eine konstante Schulung und Kalibrierung durchlaufen hatten. Neben inhaltlichen und organisatorischen Bereichen wurde stets großer Wert auf einen engen Austausch sowohl mit Fachbereichen als auch mit der zuständigen Zulassungsstelle (Hochschulbüro für Internationales) gelegt. Das Feedback sowohl dieser Stellen als auch von den 
Bewertern und dem Washback aus dem vorbereitenden Unterricht ließ sich in eine Optimierung der nächsten Prüfungserstellung umsetzen.

Die Versuche des Fachsprachenzentrums, die Validität der DSH zu optimieren, bestanden aus einer Neuorganisierung der Prüfungskonzeption. Nachdem Themenschwerpunkte einer Prüfung diskutiert und im Vorfeld definiert worden waren, wurden von den an der Erstellung beteiligten Mitarbeitern im DSH-Team Vorschläge zu den einzelnen Subtests gemacht, diskutiert und optimiert. So entwickelten sich die Mitarbeiter zu „Spezialisten“ in einzelnen Teilbereichen. Darüber hinaus konnte dann aber das Niveau bzw. die Schwierigkeit des eigenen Materials mit anderen Teilbereichen verglichen und abgestimmt werden.

Die soweit fertig gestellten Teilbereiche wurden dann vom DSH-Koordinator zusammengefasst, gelayoutet und die vorgeschlagene Bepunktung entsprechend umgesetzt. Darauf folgte eine aus Sicherheitsgründen limitierte Erprobung, zuerst durch verschiedene Muttersprachler ${ }^{4}$ und auch durch einzelne, wechselnde Fremdsprachler (z. B. ausländische Mitarbeiter). Auch hierauf folgte eine Überarbeitung der Aufgaben, der Bepunktung und/oder des präzise ausformulierten Erwartungshorizonts.

Ein Problemfall stellt erfahrungsgemäß die mündliche Prüfung dar, die traditionsgemäß weitgehend von der Erfahrung und Routine des Beurteilers abhängen. Ein sehr detaillierter Bewertungsbogen und eine ausführliche Diskussion zwischen Prüfer und Beisitzer sollten die Validität in diesem Bereich verbessern. Inzwischen sind diese Bestrebungen vom $\mathrm{FaDaF}$ übernommen worden und werden bereits erfolgreich in DSH-Prüferschulungen eingesetzt.

Bezogen auf die Reliabilität - also nach unserer Definition der gleiche Standard verschiedener Prüfungen - erstellte das FSZ eine Datenbank mit allen Teilergebnissen aller Teilnehmer. Anhand dieser umfangreichen Daten konnten zahlreiche Vergleiche und Rückschlüsse gezogen werden u. a. auf

- Leistungen von Wiederholungsteilnehmern in Bezug auf das Gesamtergebnis und/oder Teilprüfungen,

- statistische Auswertungen von Durchschnittswerten, Bestehensquoten

- die Qualität des Hörverstehens, sofern dieser Prüfungsteil in verschiedenen Hörsälen von verschiedenen Dozenten durchgeführt worden war.

Der Kern des Projektes bezog sich auf die Objektivität der Bewertung. In verschiedenen Korrektorenschulungen wurden exemplarische Testbewertungen diskutiert und die Mitarbeiter auf verschiedene Korrektureffekte vorbereitet (u. a. Serieller-, Kontaminations- und Halo-Effekt) und Strategien zu ihrer Vermeidung aufgezeigt. Außerdem wurden in den einzelnen Prüfungsdurchgängen so genannte „Dummy“-Tests (identische Prüfungsteile, Vergleichskorrekturen) ohne Wissen der Korrektoren eingefügt, deren Ergebnisse dann zu weiteren Rückschlüssen auf die Qualität und Objektivität der Korrekturen führten. Während eines Korrektur-

\footnotetext{
${ }^{4}$ Kriterium: Volle Punktzahl in der Hälfte der Zeit.
} 
durchgangs fanden weitere Sitzungen mit Austausch und Klärungsfragen innerhalb der einzelnen Teilbereiche statt. Natürlich wurden alle Kommentare der Korrektoren, die häufig sehr viel genauer die (sprachlichen) Probleme von Teilnehmern beim Lösen der Aufgaben beurteilen konnten, in die Konzeption der nächsten Prüfung aufgenommen. Und ebenso natürlich fand bei allen Teilnehmern, die sich im Randbereich eines Ergebnisses befanden, generell eine Zweitkorrektur statt. Darüber hinaus wurden zahlreiche Stichproben gemacht, deren Beobachtungen in die Schulungen einflossen. Das Angebot, Teilnehmern Einsicht in die Prüfungsunterlagen zu gewähren und ihnen die Bewertung zu erklären, wurde häufig wahrgenommen - auch hierdurch hatte der DSH-Koordinator klaren Einblick in die Objektivität der Bewertung.

Wenngleich sich dieses Projekt der Natur der DSH gemäß auch nicht mit standardisierten Sprachprüfungen vergleichen kann, ist es doch ein für die Qualitätssicherung ein notwendiges Verfahren, das auch schon konkrete Ergebnisse aufweist:

\begin{tabular}{|c|c|c|c|c|}
\hline $\begin{array}{l}\text { Teil- } \\
\text { bereich }\end{array}$ & $\begin{array}{c}\text { vorliegende } \\
\text { Exemplare }\end{array}$ & $\begin{array}{l}\text { Ergebnis } \\
\text { (P von 15) }\end{array}$ & Differenz in \% & $\begin{array}{l}\varnothing \text { Differenz } \\
\text { in } \%\end{array}$ \\
\hline HV & 2 & $\begin{array}{l}1,75 \\
2,75\end{array}$ & $6,67 \%$ & $6,67 \%$ \\
\hline HV & 3 & $\begin{array}{l}4,75 \\
6,25 \\
6,75\end{array}$ & $10 \%-3,33 \%-13,33 \%$ & $8,88 \%$ \\
\hline LV & 2 & $\begin{array}{c}8,5 \\
9,25\end{array}$ & $5 \%$ & $5,00 \%$ \\
\hline LV & 3 & $\begin{array}{l}6,75 \\
6,75 \\
7,25\end{array}$ & $3,33 \%$ & $3,33 \%$ \\
\hline Gr & 3 & $\begin{array}{c}9 \\
10 \\
10,5\end{array}$ & $6,67 \%-10 \%$ & $8,33 \%$ \\
\hline TP & 2 & $\begin{array}{l}6 \\
8\end{array}$ & $13,33 \%$ & $13,33 \%$ \\
\hline TP & 5 & $\begin{array}{c}6 \\
6,5 \\
7 \\
8,5 \\
10,5\end{array}$ & $\begin{array}{l}0,5 P=3,33 \% \\
1 P=6,67 \% \\
1,5 P=10 \% \\
2 P=13,33 \% \\
2,5 P=16,67 \\
3,5 P=23,33 \% \\
4 P=26,67 \% \\
4,5 P=30\end{array}$ & $16,25 \%$ \\
\hline & & & $\begin{array}{r}\text { Durchschnittliche } \\
\text { Korrekturdifferenz: }\end{array}$ & $\begin{array}{l}\mathbf{8 , 8 3} \% \\
(\sim 1,3 \mathrm{P})\end{array}$ \\
\hline
\end{tabular}

Tab. 1

Der erste Vergleich der ursprünglichen 20 „Dummy“-Tests zeigte eine durchschnittliche Korrekturdifferenz von 8,83\% in einzelnen Teilbereichen zwischen verschiedenen Korrekturen. So wurden z. B. von verschiedenen Korrektoren zwei bzw. drei identische Hörverstehenstests bewertet - mit einem maximalen Unterschied von 13,33\%. Offensichtlich sind die geschlossenen Aufgabenstellungen 
leichter zu bewerten; die Differenz von 30\% der zwei bzw. fünf Textproduktionen führte zu einer genaueren Untersuchung der Bewertung und schließlich zu einer Umstrukturierung der Schulung in diesem Bereich.

\begin{tabular}{|c|c|c|c|c|c|c|}
\hline $\begin{array}{c}\text { Teil- } \\
\text { bereich }\end{array}$ & $\begin{array}{c}\text { vor- } \\
\text { liegende } \\
\text { Exemplare }\end{array}$ & $\begin{array}{c}\text { Vergleichs- } \\
\text { korrektur } \\
\text { (,Dummy“) } \\
\text { vom }\end{array}$ & $\begin{array}{l}\text { Bewertung } \\
\text { in } \%\end{array}$ & $\begin{array}{l}\text { Durch- } \\
\text { schnitt- } \\
\text { liche } \\
\text { Korrektur- } \\
\text { differenz }\end{array}$ & $\begin{array}{c}\text { Maximale } \\
\text { Korrektur- } \\
\text { differenz }\end{array}$ & $\begin{array}{c}\text { Minimale } \\
\text { Korrektur- } \\
\text { differenz }\end{array}$ \\
\hline TP & 2 & 30.04 .05 & $\begin{array}{c}40 \% \\
53,33 \%\end{array}$ & $13,33 \%$ & $13,33 \%$ & $13,33 \%$ \\
\hline TP & 5 & 23.09 .05 & $\begin{array}{c}40 \% \\
43,33 \% \\
46,67 \% \\
56,67 \% \\
70 \%\end{array}$ & $16,25 \%$ & $30 \%$ & $3,33 \%$ \\
\hline TP & 3 & $10.03 .06(a)$ & $\begin{array}{c}55,5 \% \\
58 \% \\
66,5 \%\end{array}$ & $7,33 \%$ & $11 \%$ & $2,5 \%$ \\
\hline TP & 3 & $10.03 .06(b)$ & $\begin{array}{l}61,5 \% \\
63 \% \\
64 \% \\
\end{array}$ & $2 \%$ & $2,5 \%$ & $1 \%$ \\
\hline TP & 3 & $10.03 .06(c)$ & $\begin{array}{l}51,5 \% \\
73 \% \\
79 \%\end{array}$ & $18,3 \%$ & 27,5 & $6 \%$ \\
\hline \multicolumn{4}{|c|}{$\begin{array}{r}\text { Differenz (10.03.06) bezogen auf } \\
\text { Gesamtergebnis: }\end{array}$} & $2,63 \%$ & $3,9 \%$ & $0,9 \%$ \\
\hline
\end{tabular}

Tab. 2: Vergleichskorrektur - Projektentwicklung TP im Folgedurchgang 


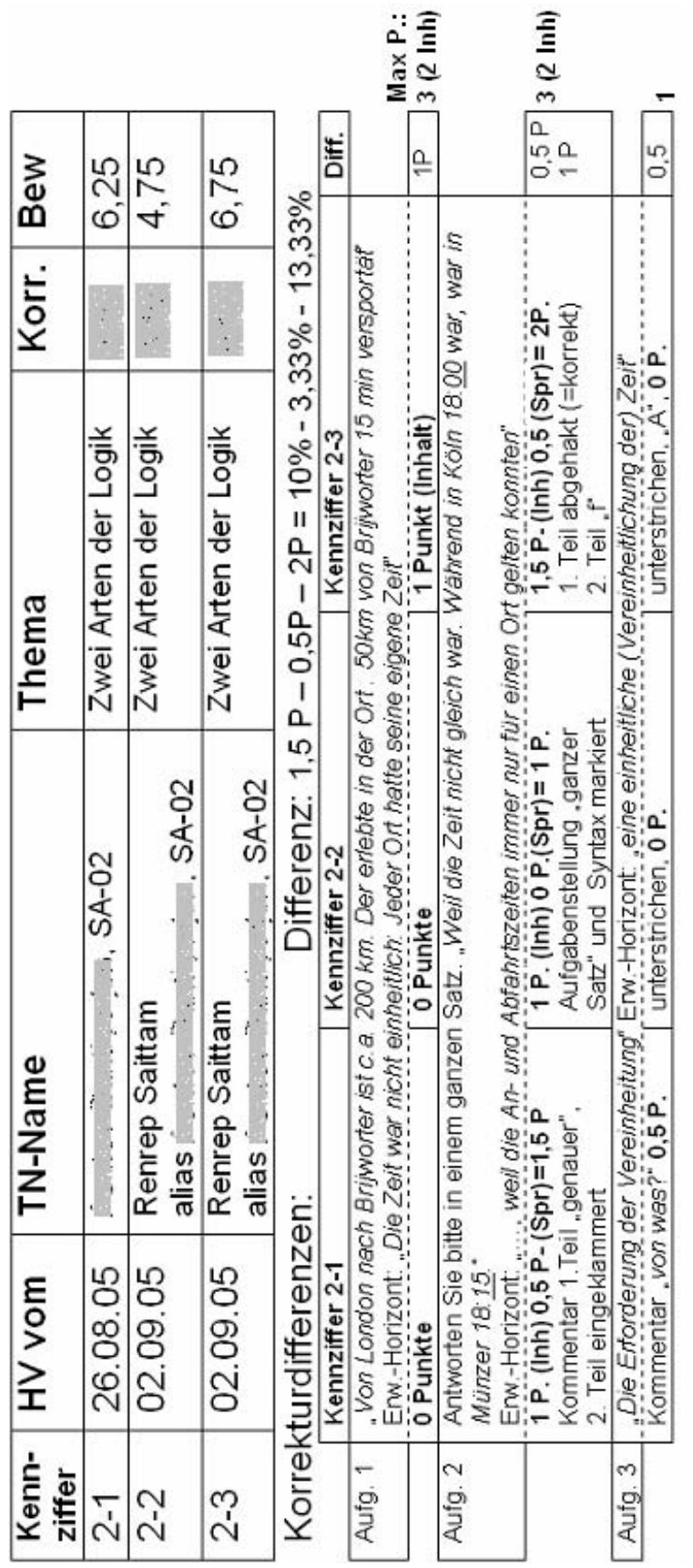

Abb. 1 

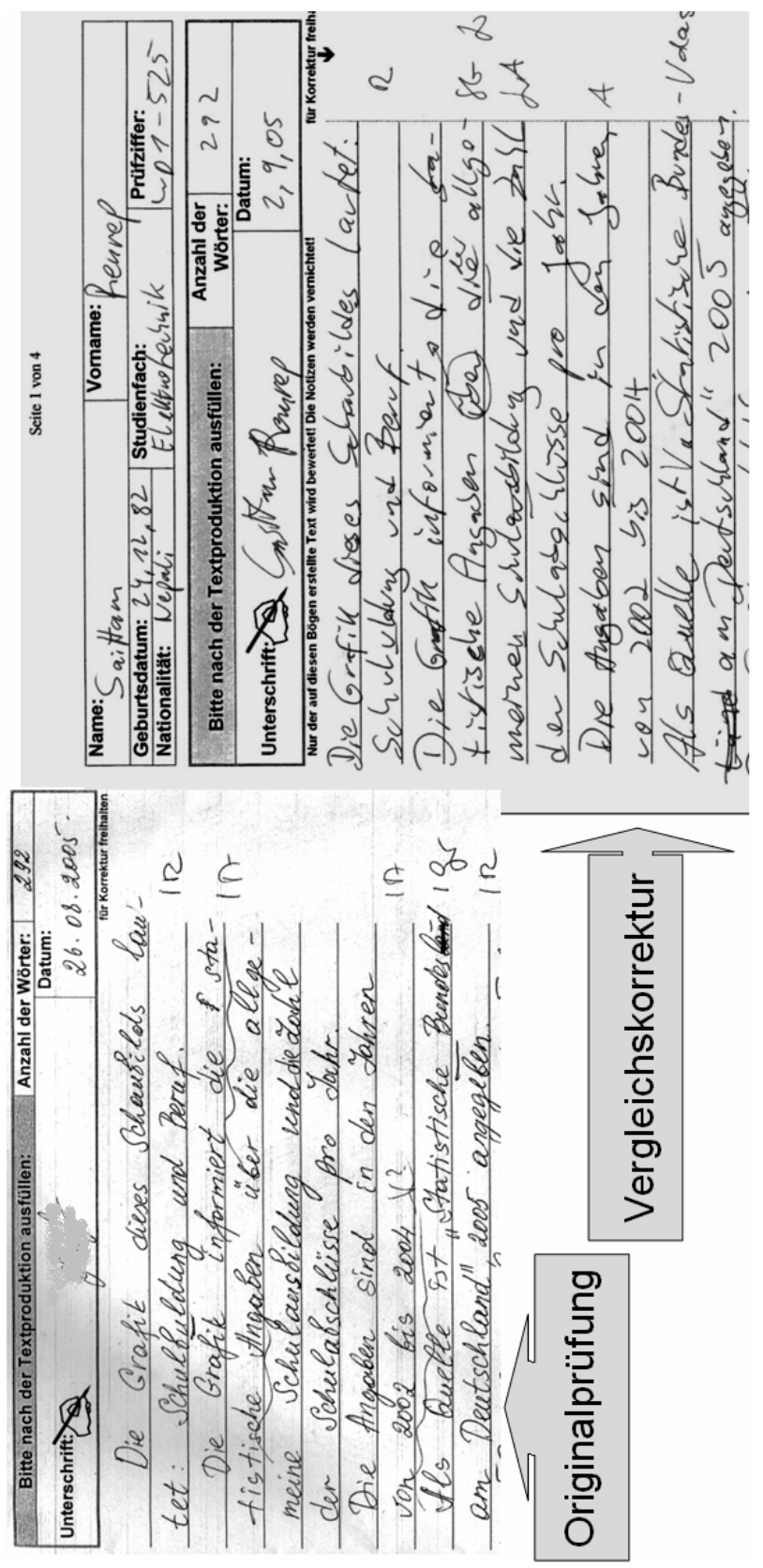

Abb. 2 
Bei einer genaueren Analyse wurde deutlich, dass sich die drei Korrektorensowohl in der Differenzierung zwischen Inhalt und Sprache, als auch in der Kenntlichmachung der Fehler nicht einig waren. Einige Mitarbeiter fügten sehr viele, zeitaufwendige Kommentare und Verbesserungsvorschläge ein, so dass die Markierung und Kategorisierung von inhaltlichen und sprachlichen Fehlern überarbeitet und in die Korrekturschulungen mit eingebunden wurden. Daneben wurde auch die unterschiedliche Umsetzung des Primats der kommunikativen Sprachkompetenz deutlich und so lange diskutiert, bis ein Konsens gefunden zu sein schien.

Der weitere Verlauf des Projektes konzentrierte sich im Bereich der RaterObjektivität vorwiegend auf die Relation von Inhalt und Sprache und deren individueller Umsetzung durch einzelne Beurteiler.

Inzwischen war unter Mitwirkung des gesamten DSH-Teams vom FaDaF ein neues DSH-Handbuch erarbeitet worden, dass u.a. die in Hannover praktizierten und bewährten Bewertungskriterien im Bereich Textproduktion abbildete.

Mit dieser sehr detaillierten Beurteilungstabelle war es möglich, sehr differenzierte Analysen in einzelnen Bereichen individueller Beurteilung durchzuführen. So war z. B. deutlich zu sehen, dass die intensiven Schulungen sowie die Vor und Nachbesprechungen Früchte trugen (Tabelle 3).

\begin{tabular}{|c|c|c|c|c|c|c|}
\hline & $\begin{array}{l}\text { Themen- } \\
\text { bezug } \\
\text { (max. } 40 \text { P.) }\end{array}$ & $\begin{array}{c}\text { Kohärenz } \\
\text { (max. } 50 \text { P.) }\end{array}$ & $\begin{array}{l}\text { Grammatik } \\
\text { (max. } 50 \text { P.) }\end{array}$ & $\begin{array}{l}\text { Wortschatz } \\
\text { (max. } 50 \text { P.) }\end{array}$ & $\begin{array}{c}\text { Ortho- } \\
\text { graphie } \\
\text { (max. 10 P.) }\end{array}$ & \\
\hline Korrektor 1 & 18 & 32 & 29 & 28 & 4 & \multirow{2}{*}{$\stackrel{-1}{x}$} \\
\hline Korrektor 2 & 30 & 35 & 32 & 30 & 6 & \\
\hline Korrektor 1 & 38 & 40 & 35 & 39 & 7 & \multirow{2}{*}{$\underset{\frac{\pi}{d}}{\frac{1}{2}}$} \\
\hline Korrektor 2 & 25 & 29 & 37 & 31 & 4 & \\
\hline Korrektor 1 & 12 & 29 & 25 & 30 & 7 & \multirow{2}{*}{$\begin{array}{l}\mathbb{D}^{-1} \\
\omega\end{array}$} \\
\hline Korrektor 2 & 25 & 27 & 37 & 31 & 4 & \\
\hline
\end{tabular}

Tab. 3

Während in den meisten Kriterien Konsens bezüglich deren Umsetzung herrschte, wies der Themenbezug einige Unstimmigkeiten zwischen den einzelnen Ratern auf. Obgleich dieser Teil nur ein Unterelement der Objektivität darstellt, wird weiterhin an einer homogenen Beurteilung gearbeitet. Weitere Ergebnisse in diesem speziellen Bereich bleiben abzuwarten. 
Insgesamt lässt sich in der Textproduktion als bezüglich der Bewertung offensten Teil der DSH mit 2,63\% Korrekturdifferenz eine sehr positive Entwicklung feststellen - im Pilotprojekt waren es noch mehr als 10\%. Diese Ergebnisse in Verbindung mit der routinemäßigen Überprüfung aller Teilnehmer, die maximal 15 Punkte (das entspricht 2,14\% des schriftlichen Ergebnisses) unter einem Schwellenwert liegen, zeigen zumindest den Erfolg der Bestrebungen zur Qualitätssicherung.

Im weiteren Verlauf des Projekts sollen verschiedene der oben erwähnten weiteren Bereiche der Qualitätssicherung in Angriff genommen und optimiert werden.

Die gegenwärtig in der Erprobung befindlichen Ansätze werden zu gegebener Zeit publiziert werden.

\section{Literatur}

Althaus, A., M. Perner und F. Weiß-Motz: „Vorabinformation Umfrage zur DSH - Ergebnisbericht”. FaDaF aktuell, 2 (2006), 15-18.

Bachman, L. F.: „Modern language testing at the turn of the century: Assuring that what we count counts".

Language Testing, Bd. 17, 1 (2000), 1-42.

Bachman, Lyle F und Palmer, Adrian S.: Language testing in practice: Designing and developing useful language tests. Oxford: Oxford University Press. 1996.

Bachman, Lyle F.: Fundamental considerations in language testing. Oxford: Oxford University Press. 1990

Bärenfänger, O. \& Kecker, G.: „Beurteilerkonsistenz und Beurteilerübereinstimmung bei der Bewertung von Kurzantwortaufgaben: Eine empirische Studie zum Bewertungsverhalten von Beurteilern im Subtest Hörverstehen des Tests", Deutsch als Fremdsprache. Zeitschrift für Fremdsprachenforschung, 15 (2004), 237-268.

Engelhard, G.: „Monitoring raters in performance assessments”. In G. Tindal \& T. M. Haladyna (Eds.), Large-scale assessment programs for all students: V alidity, technical adequacy, and implementation. Mahwah, NJ: Erlbaum, 2002. 261-287.

Grotjahn, R.: „Testtheorie: Grundzüge und Anwendungen in der Praxis“. In A. Wolff \& H. Tanzer (Hrsg.), Sprache - Kultur - Politik (Materialien Deutsch als Fremdsprache, Bd. 53, S. 304-341). Regensburg: Fachverband Deutsch als Fremdsprache.

Grotjahn, R.: „Sprachprüfungen als Instrument der Qualitätssicherung in Lehre und Studium.“. In A. Hopbach \& V. Chalvet (Hrsg.), Qualität messen - Qualität managen: Leistungsparameter in der Hochschulentwicklung. Bonn:

Hochschulrektorenkonferenz. 2005 S. 55-62 
Kieweg, W.: „Allgemeine Gütekriterien für Lernzielkontrollen“. Der Fremdspracbliche Unterricht Englisch. 33.1 (1999). 4-11.

Koreik, U. \& Mielke, F.: „DSH-Kurse auf dem Prüfstand“. In B. Voss \& E. Stahlheber (Hrsg.), Fremdsprachen auf dem Prüfstand: Innovation - QualitätEvaluation. S. 199-207. Berlin: Pädagogischer Zeitschriftenverlag. 2003

Koreik, U. (Hrsg.):: DSH und TestDaF - eine Vergleichsstudie. Hohengehren: Schneider. 2005.

McNamara, T. F.: Measuring second language performance. 1996. London: Longman.

McNamara, T. F. Language testing. Oxford, UK: Oxford University Press. 2000

Rost, J.: Lebrbuch Testtheorie, Testkonstruktion. Bern: Huber. 1996 
Themenschwerpunkt 3:

Schreiben und Sprechen in den Wissenschaften 



\section{Dimensionen wissenschaftlichen Schreibens - Problemaufriss und Vorschläge für den Unterricht in studienbegleitenden DaF-Kursen}

Henning Herrmann-Trentepobl, Bochum

Martin Lange, Kiel

1 Einführende Bemerkungen zur Themenwahl des Schwerpunktes „Schreiben und Sprechen in den Wissenschaften“

Die Mitgliederversammlung des Fachverbandes Deutsch als Fremdsprache diskutierte während der Jahrestagung 2005 in Jena sehr lebhaft die Wahl der Themenschwerpunkte für die folgende Jahrestagung, wobei unter anderem das Thema „Academic Writing"vorgeschlagen wurde. Dieser Anglizismus war allerdings einigen Mitgliedern ein Dorn im Auge, ein anderer Vorschlag lautete daher „Wissenschaftliches Schreiben". Dieser Begriff scheint von bestimmten Anforderungen an die Schreibenden im akademischen Umfeld auszugehen und wirkt aus dieser Perspektive eher normativ - oder, um mit de Saussure zu sprechen: Präskriptiv. 
Für andere Mitglieder wieder war es wichtig, auch die deskriptive Seite in die Themenstellung eines solchen Tagungsschwerpunktes einzubeziehen: Also das, was die an studienvorbereitenden und studienbegleitenden Deutschkursen teilnehmenden ausländischen Studierenden tatsächlich schriftlich produzieren. Diese Perspektive, die einerseits die Schwierigkeiten ausländischer Studierender beim Schreiben zu erfassen sucht (ob sie nun an speziellen Deutschkursen teilnehmen oder nicht) -, andererseits aber auch mögliche Lösungswege und Konzepte zur Überwindung dieser Schwierigkeiten in der Arbeit von Lehrgebieten, Studienkollegs, Schreibwerkstätten und Projekten aufzuzeigen vermag, schlug sich schließlich in der Themenwahl „Scbreiben in den Wissenschaften" nieder. Da in besagter Mitgliederversammlung aber auch ein hoher Stimmenanteil auf den Bereich der mündlichen Kommunikation entfiel, beschloss der FaDaF-Vorstand schließlich, einen Themenschwerpunkt mit dem Titel „Schreiben und Sprechen in den Wissenschaften " ins Programm aufzunehmen.

Der vorliegende Beitrag konzentriert sich vor allem aber auf verschiedene „Dimensionen wissenschaftlichen Schreibens“ und versucht, schlaglichtartig, Verweise auf die folgenden Beiträge des gesamten Themenschwerpunktes zu geben, die diese Dimensionen vorrangig behandeln.

\section{Zu den Anforderungen beim Schreiben im wissenschaft- lichen Umfeld und ihrer Bedeutung für den Studienerfolg}

Als wegweisend und zugleich als Ausgangspunkt für den Einstieg in die Thematik sind der Sammelband von Ehlich und Steets (2003) „Wissenschaftliches Schreiben - Lehren und Lernen“" sowie der Review-Article von Brian Paltridge (2004) ${ }^{1} \mathrm{zu}$ nennen, die überwiegend ähnliche Aspekte beschreiben, aber doch auch Unterschiede aufweisen, die aus der Perspektive des jeweiligen akademischen Umfeldes zu erklären sind: hier das deutsche, dort das anglo-amerikanisch geprägte.

So zeigt sich beispielsweise bei Paltridge, dass die Textsorte des wissenschaftlichen Essays im englischsprachigen Raum eine große Bedeutung hat, eine Tatsache, die einhergeht mit zum Teil völlig anderen Formen des Studierens und des Unterrichtens $^{2}$. Das betrifft Formen und Häufigkeit der Rückmeldung an die Schreibenden durch die Dozentenschaft, das Zahlenverhältnis beider an der wis-

\footnotetext{
${ }^{1}$ Paltridge, Brian, „Academic Writing” Review-Article. In: Language Teacbing 37, 87-105. Paltridge beleuchtet immer wieder die Perspektive der nicht-muttersprachlichen Studierenden. Daher sind seine Ausführungen von besonderem Interesse auch für Lehrende im Bereich deutschsprachiger Kurse in der Studienvorbereitung und -begleitung.

2 Dass es neben der Benennung des Faches auch inhaltliche Unterschiede zwischen dem "Wissenschaftlichen Schreiben" und dem "Academic Writing" gibt, zeigt sich u.a. daran, dass die Form des „academic essay“" nahezu 60\% der Schreibaufgaben der studierenden Zweitsprachlernenden im englischsprachigen akademischen Umfeld ausmachen, eine Form, die keine genaue Entsprechung im deutschsprachigen Raum hat (vgl. Moore \& Morton (1999), zit. nach Paltridge $(2004,85)$ oder den Titel von Hufeisen (2002): „Ein deutsches Referat ist kein englischsprachiges Essay “).
} 
senschaftlichen Interaktion beteiligten Seiten sowie - damit verbunden - eine oft weitaus bessere Betreuungsrate, als dies in Deutschland gegeben ist.

Diese Andersartigkeit der Formen wissenschaftlichen Arbeitens und die unterschiedlichen Rahmenbedingungen beim Studium in Deutschland bzw. im Ausland sind sicher ein weiterer guter Grund dafür, bei der Wahl dieses Themenschwerpunktes unseres Faches Deutsch als Fremdsprache im Rahmen einer national bedeutsamen Tagung nicht auf den Begriff „Academic Writing“ zurückzugreifen unabhängig davon, wie man zur Frage der Anglizismen im Wissenschaftsbetrieb stehen mag.

Wenn man nun auf die Implikationen der oben beschriebenen Unterschiede der verschiedenen Universitätssysteme näher eingeht, tun sich aber viel schwerwiegendere Probleme auf. Anlass zu großer Sorge geben in diesem Zusammenhang die Erkenntnisse einer vom DAAD in Auftrag gegebenen Studie der HIS ${ }^{3}$ zu Studienverlauf, Studienerfolg und -abbruch bei ausländischen Studierenden ${ }^{4}$ :

Der Anteil der Studienabbrecher liegt bei den ausländischen Kommiliton/inn/en ungleich höher als bei den deutschen Studierenden. Der Versuch, ein Hochschulstudium in Deutschland erfolgreich zu absolvieren, muss für den Großteil der eingereisten Studierenden als gescheitert gelten.

Bedenkt man, dass in fast allen Studiengängen dem Schreiben eine Schlüsselrolle auf dem Weg zum Examen zukommt - angefangen bei den Vorlesungsmitschriften, die vielfach Grundlage der späteren Prüfungsvorbereitung sind, über die Klausuren, Proseminararbeiten, wissenschaftlichen Hausarbeiten bis hin zur Staatsexamens-, Magister- oder Doktorarbeit -, so muss die Frage gestellt werden, ob insbesondere die ausländischen Studierenden hinreichend auf die an sie im jeweiligen Fachstudium gestellten Anforderungen vorbereitet werden.

Empirisch untermauert werden die, hier zunächst hypothetisch formulierten, offenkundigen Desiderate in der Schreibpraxis an deutschen Hochschulen durch die Studie von Ehlich und Steets (2003) ${ }^{5}$, die auf einer Befragung von Lehrenden verschiedenster Fakultäten der Ludwig-Maximilians-Universität München beruht. Aus weit über 90\% der Antworten (mit geringen Abweichungen im Bereich Medizin und Wirtschaft, wo möglicherweise mehr vorgefertigte Manuskripte ausgeteilt werden) ging hervor, dass die Studierenden des jeweiligen Fachbereichs in den Vorlesungen mitschreiben. Auf die Frage hingegen, ob die Studierenden im Grundstudium an das Mitschreiben herangeführt werden, schwanken die Angaben

\footnotetext{
${ }^{3}$ HIS $=$ Hochschul-Informations-System-GmbH. Auf der Internetseite http://www.his.de finden sich u.A. zahlreiche Publikationen zum Thema Ausländerstudium.

${ }^{4}$ Vgl. Heublein, Ulrich/Sommer, Dieter/Weitz, Brigitta, Studienverlauf im Ausländerstudium. Eine Untersucbung an vier ausgewäblten Hocbscbulen (2004), (=DAAD-Reihe Dokumente und Materialien, Mat \& Dok 55). Im Internet verfügbar unter: http://www.daad.de/de/download/zahlen/dok-undmat_55.pdf

${ }^{5}$ Die im Folgenden aufgeführten statistischen Angaben finden sich bei Ehlich, K./Steets, A., „Wissenschaftliche Schreibanforderungen in den Disziplinen. Eine Umfrage unter ProfessorInnen der LMU“. In: dies. (Hrsg.) (2003), 129-145.
} 
von Fachbereich zu Fachbereich, doch erreichen sie einzelne Spitzenwerte von lediglich 40\%, im Durchschnitt 10\%, in einigen Fakultäten gar nur ca. 4\%.

Auf die Frage, ob die Studenten zum Erwerb eines Scheins im Hauptstudium eine schriftliche Hausarbeit verfassen müssen, liegen die Antworten im Durchschnitt bei 60\% für alle Fachbereiche, in einigen davon bei 90\%, in den Sozialwissenschaften antworteten 100\% mit „Ja“. Diese Angaben machen deutlich, welche herausragende Rolle den schriftlichen Fertigkeiten auf dem Weg zum Examen zukommt. Ebenso spielen hier formale Bewertungskriterien eine wichtige Rolle, denn die Frage, ob neben dem Inhalt auch die sprachliche Form bewertet werde, bejahten durchschnittlich 70\% der antwortenden Lehrenden aller Fachbereiche (überdurchschnittliche Werte fanden Ehlich und Steets bei den Wirtschafts-, Sozial- und Geisteswissenschaften, unterdurchschnittliche in den Bereichen Mathematik und Naturwissenschaften).

Diese Zahlen verdeutlichen insgesamt, welche Hürden sich während des Studienverlaufs besonders für ausländische Kommiliton/inn/en aufbauen müssen, und wo sicher auch ein wichtiger Ansatzpunkt bei der Ursachenforschung für die oben beschriebenen hohen Abbrecherquoten besonders in dieser Gruppe der Studierenden zu suchen ist.

\section{Akademische Regeln, Erwartungshaltung der Lehrenden und Verunsicherung auf Seiten der Studierenden}

Eine allgemeine Verunsicherung in Bezug auf die Erwartungen der Lehren-den an die schriftlichen Fertigkeiten - besonders der ausländischen - Studierenden, konstatiert Dong (1997: 10), der - ähnlich wie Ehlich und Steets - zu dem Schluss kommt, dass wissenschaftliches Schreiben „das Lernen und Einüben akademischer Regeln" "bedeute" .

Eine zusätzliche Schwierigkeit liegt in der Tatsache, dass diese Regeln von Fach zu Fach variieren, sowohl nach Zielgruppe und Zweck als auch nach Form und Funktion. Zusammenfassend lassen sich diese Variationen zunächst an folgenden Punkten festmachen:

- Textmuster

- Argumentationsstruktur

- Nutzung von Fachliteratur

- Leserorientierung

- Kohäsionsmuster

- Satzkonstruktion

\footnotetext{
${ }^{6}$ Vgl. Dong, Yu Ren (1997). Supervising international students requires cultural sensitivity. In: The scientist 11, 9, 10. September 29, zit. nach Paltridge $(2004,88)$ : "[A]cademic writing [...] involves learning a new set of academic rules and learning how to play by these rules".
} 


\section{- Wortwahl.}

Diese Aspekte werden - unter besonderer Berücksichtigung des Ausländerperspektive - vor allem in den Arbeiten von Brandl, Pospiech und von Graefen behandelt (vgl. hier die besonders die Beiträge im vorliegenden Band, aber auch die weiteren Angaben in der Bibliographie).

Neben den von Fach zu Fach variierenden Textmustern und Regeln für die Abfassung wissenschaftssprachlicher Texte ist immer wieder festzustellen, dass ausländische Studierende, auch durch die Lehr- und Lerngewohnheiten, die sie aus dem Heimatland mitbringen, stark verunsichert sind, wenn sie sich im deutschsprachigen akademischen Umfeld plötzlich mit völlig anderen Erwartungen der Dozierenden bezüglich des Schreibens konfrontiert sehen. So konnte z. B. Dorothee Kaiser (2003) durch einen Vergleich von Hausarbeiten venezolanischer und deutscher Studierender gravierende Unterschiede im Umgang mit Sekundärliteratur feststellen; dies ging bis zur völligen Absenz von Literaturverzeichnissen bei den Venezolanern.

Die in diesem Fall berührte interkulturelle Dimension findet sich innerhalb dieses Bandes in den Beiträgen von Zhou und Storck. Zeilinger behandelt zudem, wie auch speziell Garcia oder Reinhardt, den Bereich des Sprechens in den Wissenschaften.

Bezüglich der an sie gestellten Anforderungen flüchten sich ausländische Studierende - sicher in weitaus größerem Umfang als deutsche - ins Internet, um dort nach Mustern und Vorbildern für die eigene Arbeit suchen. Die Existenz von Tauschbörsen für Hausarbeiten macht es leicht, sich dort zu bedienen, wo es an praktischer Anleitung fehlt. Das einmal geschriebene Wort gilt als Autorität und ist andernorts vielleicht schon positiv bewertet worden.

Die Grenze zum Plagiat ist nahe, wobei - sei es aus Bequemlichkeit oder Verzweiflung über das eigene Unvermögen oder das Fehlen geleiteter Schreibübungen - Inhalte in der Regel völlig unverdaut wiedergegeben werden. Das zugrunde liegende Verhalten für dieses neue Kopieunwesen ist oft von fehlendem Unrechtsbewusstsein gekennzeichnet, wie insbesondere aus den Ausführungen von Zhou und Storck hervorgeht. Der Plagiatsproblematik widmete sich auf der Tagung speziell der Beitrag von Weber-Wulff.

Wie Weber-Wulff fordert auch Mummert eine gezielte Schreibanleitung. Exemplarisch zeigt sie in ihrem Beitrag, wie an Studienkollegs die Form literarischer Interpretationen eingeübt werden kann. 


\section{$4 \quad$ Beobachtungen aus der Praxis in studienbegleitenden Hochschulsprachkursen}

\subsection{Schreibprozesse und Schreibstrategien}

Wie bereits im Titel des vorliegenden Beitrags mit dem Begriff „Problemaufriss“ angedeutet, ist hier ein auch nur annähernd vollständiger Überblick über die vorhandene Literatur, gar ein Forschungsbericht, nicht beabsichtigt und kann auch aus Raumgründen nicht geleistet werden 7 . Im Folgenden soll es nur darum gehen, den Themenkomplex "Schreiben in den Wissenschaften" in seinen Grundzügen und seiner Bedeutung für den DaF-Bereich knapp vorzustellen und so als generelle Einleitung für die anderen Beiträge zum Themenschwerpunkt „Sprechen und Schreiben in den Wissenschaften“ zu dienen. Da das Schreiben ein wesentlicher Bestandteil des wissenschaftlichen Arbeitens generell ist, wird auf den folgenden Seiten auch immer wieder davon die Rede sein.

Wie wichtig das Schreiben ganz allgemein für die Entwicklung des Denkens ist, ist längst unumstritten. Schreiben bedeutet nicht nur, etwas bereits FertigVorhandenes lediglich aufs Papier zu bringen, sondern im Schreiben bilden sich allererst Gedanken, die sich dann durch wiederholtes Bearbeiten in eine logischkonsequente Folge bringen lassen.

Wissenschaftliches Schreiben ist nicht allein eine Form, Erkenntnisse darzustellen, sondern auch ein Weg, Erkenntnisse zu gewinnen, zu sichten, zu vergleichen, zu ordnen, zu strukturieren, zu diskutieren und zu kommunizieren. (Kruse 2004: 11).

Nicht immer verlaufen diese Schritte linear: so können etwa spätere Arbeitsschritte Modifikationen an der Konzeption erforderlich machen, also einen kontrollierten Rückgriff auf ein früheres Stadium bei der Erstellung der Arbeit.

Schreiben ist aber auch ein hoch komplizierter psychologischer Prozess, eine kaum zu durchschauende emotionale Gemengelage von ,himmelhoch jauchzend bis ,zu Tode betrübt' ${ }^{8}$. Keineswegs nur Studierende, sondern auch Dozenten auf allen Stufen ihrer akademischen Karrieren entwickeln ausgefeilte, gelegentlich mehr irrationale als rationale Rituale, um in den Prozess des Schreibens zu kommen - oder ihn zu vermeiden Daher sind viele Probleme mit dem Schreiben auch gar nicht im engeren Sinne akademischer Natur (Bean 1996: 3). Besonders an amerikanischen Universitäten versucht man schon seit Jahren, dem Rechnung zu tragen. So gibt es an fast allen Institutionen Schreibbüros, die die Studierenden

\footnotetext{
${ }^{7}$ Verwiesen sei hier auf die Bibliographie, besonders auf den Überblick von Ehlich, Steets und Traunspurger, der die Literatur bis etwa zum Jahr 2000 sichtet.

${ }^{8}$ Nicht umsonst heißt ein von Narr und Stary herausgegebener Sammelband zum Thema „Lust und Last des wissenschaftlichen Schreibens" (vgl. Bibliographie!).
} 
betreuen (eine kleine Liste finden Sie in unserer Bibliographie). Deutschland hinkt leider (auch da) hinterher!

Seit einigen Jahren gibt es die EATAW (European Association For The Teaching Of Academic Writing), ${ }^{9}$ die sich den verschiedenen Aspekten des akademischen Schreibens widmet.

Wie auch aus unserer Bibliographie hervorgeht, gibt es inzwischen eine große Anzahl von Studien zum Thema ,Schreiben im DaF-Unterricht', die besonders auch die Schwierigkeiten behandeln, welche sich für Studierende aus verschiedenen Kulturkreisen ergeben, die in Deutschland studieren wollen. Ein Desiderat bilden allerdings konkrete Arbeitsmaterialien, die man auch als Kursgrundlage verwenden und den Studierenden zur Anschaffung empfehlen kann ${ }^{10}$.

Für Muttersprachler gibt es inzwischen eine ganze Menge an Literatur, die wir in einer Bibliographie sowohl nach studienübergreifenden, als auch nach fachspezifischen Titeln in einiger Ausführlichkeit zusammengestellt haben. Diese Titel enthalten außer Ausführungen zum akademischen Schreiben im engeren Sinne auch Kapitel zu allen anderen wichtigen akademischen Arbeitstechniken und lassen sich durchaus in Teilen für den DaF-Unterricht adaptieren.

In die Nähe eines Kurshandbuchs kommt der 2005 erschienene Band von Grit Mehlhorn et al.: „Studienbegleitung für ausländische Studierende an deutschen Hochschulen“. Hier wird versucht, ausländischen Studierenden fächerübergreifend Studierstrategien für den Bereich der mündlichen und schriftlichen Kommunikation zu vermitteln, angefangen bei Etablierung und Pflege des Kontakts zum Dozenten (ein Thema, das andere Bücher in der Regel vernachlässigen) bis zur Abgabe der fertigen Arbeit. Der zweite Teil des Buches bietet einen „Leitfaden für die Lernberatung“. Der Lernberater, der so fast in die Nähe eines Coaches rückt, soll den Studierenden bei der Klärung der Frage unterstützen, welche Ziele er in welchem Zeitraum auf welchem Weg erreichen will - und ihn auf diesem Weg auch begleiten (Mehlhorn et al. 2005, Abschnitt II: 161).

Man kann sich allerdings die Frage stellen, inwieweit Studierstrategien grundsätzlich fächerübergreifend gelehrt werden können. Zweifellos gibt es Basisstrategien wissenschaftlichen Schreibens und Arbeitens, die fächerübergreifend gebraucht und auch vermittelt werden können. Dazu würden wir zählen: Lesetechniken, Exzerpieren, Informationen ordnen und archivieren, Mitschrifttechniken, Argumentieren, Zitieren, Bibliographieren. Ein so wichtiges Thema wie das Zeitmanagement, die Frage also, wie man ein erfolgreiches Studium planen müsste, kann allerdings nur fächerspezifisch, ja sogar nur eng bezogen auf den jeweiligen

\footnotetext{
9 Der Internetauftritt der EATAW findet sich unter www.eataw.org [14.11.06].

${ }^{10} \mathrm{Zu}$ nennen wären hier u.a. die Titel von Esselborn-Krumbiegel (2004), Rost (2004), Frank / Stary (2006) oder auch Bünting, Karl-Dieter / Bitterlich, Axel / Pospiech, Ulrike (Hrsg.) (2004), Schreiben im Studium mit Erfolg. Ein Leitfaden (mit CD-ROM). Der Inhalt der CD ist auch online abrufbar unter: http:/ / www uni-essen.de/schreibwerkstatt/trainer [14.11.2006]
} 
Studienort behandelt werden ${ }^{11}$. Auch zur wichtigen Frage der Beispieltexte halten sich die Autoren zu sehr zurück. Es ist sinnvoll, dass sich die Studierenden an Texten üben, die einerseits nicht das Abstraktionsniveau von Fachtexten haben, andererseits davon aber auch nicht allzu weit entfernt sein sollten. Insgesamt liegt mit Mehlhorns Buch aber eine sehr praktikable, in besonderem Maße auch interkulturelle Gegebenheiten berücksichtigende „Handreichung“ für den Unterricht mit ausländischen Studierenden vor.

Von den speziellen Schwierigkeiten dieser Kursteilnehmer mit dem Schreiben wird in den folgenden Beiträgen noch ausführlich berichtet werden. Im Rahmen dieser Einführung möchten die Autoren zunächst in gebotener Kürze von ihren Erfahrungen an der Universitäten Kiel und Bochum berichten (von denen sie hoffen, dass sie, bis zu einem gewissen Grade jedenfalls, verallgemeinerbar sind) und zum Schluss einige Desiderate formulieren.

Wie bereits bemerkt liegen die Probleme beim Schreiben nicht immer in der Materie selbst, sondern leider auch oft in den universitären Strukturen und Mentalitäten: vom Betreuungsverhältnis war ebenfalls bereits die Rede. Deutsche Professoren scheinen auch immer noch anzunehmen, dass alle Studenten als geborene Autoren gediegenster wissenschaftlicher Prosa an die Uni kommen und einer spezifischen Schreib-Ausbildung nicht bedürften. So arbeiten die wenigen Schreibbüros an deutschen Universitäten ständig mit geringsten Mitteln, personell unterversorgt und von der Schließung bedroht. Für die Betreuung ausländischer Studierende ist da in der Regel kein Platz mehr.

Doch auch an einfachen pädagogischen Voraussetzungen hapert es auf Dozentenseite leider oft. So hat es einer der Autoren erlebt, dass einer ausländischen Studierenden bei der Wahl des Themas für eine soziologische Hausarbeit im Rahmen eines Proseminars die Lektüre eines historisch wie sachlich weit ausgreifenden Handbuchartikels zum Thema „Ausbildung und Beruf“ empfohlen wurde - in der Hoffnung, ein Thema werde ihr daraus schon von ganz allein entgegenspringen. Auch genauere Angaben des Kursleiters dazu, was genau nach welcher Methodik in welchem Zeitrahmen bearbeitet werden soll, fehlen oft.

Hier weist also bereits das „Arbeitsbündnis“ (Garbiela Ruhmann) zwischen Betreuer und Studierendem, das am Anfang eines jeden wissenschaftlichen Arbeitsprozesses steht und in dem solche elementaren Fragen geklärt werden müssten, elementare Mängel auf - womit der Erfolg des ganzen Arbeitsvorhabens schon ernsthaft in Frage gestellt ist.

\subsection{Zielgruppen}

Vorab vielleicht der Hinweis, dass ausländische Studierende prinzipiell ähnliche Probleme beim wissenschaftlichen Schreiben wie ihre inländischen Kommili-

\footnotetext{
$11 \mathrm{Vgl}$. etwa zum Jura-Studium das Buch von Lange (2005), das dem Thema Zeitmanagement immerhin mehr als die Hälfte der zur Verfügung stehenden 350 Seiten widmet.
} 
tonen haben, insbesondere beim Meistern der fachsprachlichen Voraussetzungen, aber auch bei den einzelnen, bereits genannten Arbeitsschritten wie Lesen, Exzerpieren, Recherchieren etc.. Dazu kommen allerdings die Sprachbarriere sowie vielfach interkulturelle Probleme: Unterschiedliche Vorstellungen über ,Wissenschaft' sowie der (regelmäßig mangelnde) Kontakt zum Dozenten und zu Kommilitonen sind da vielleicht die wichtigsten Punkte.

Die Abteilung Deutsch als Fremdsprache an der Ruhr-Universität etwa bietet im Wesentlichen zwei Kurse an, in denen das Schreiben eine große Rolle spielt: Das „Schreibtraining“" und die „Einführung in das wissenschaftliche Arbeiten“, beide je 3 SWS. Insgesamt kann man an den Teilnehmerzahlen (bis zu 20 pro Kurs) ein großes Interesse an diesen Veranstaltungen feststellen. Stichprobenartige Erhebungen haben ergeben, dass die Studierenden keine vergleichbaren Kurse in ihren Heimatländern besucht haben.

Nach Erfahrungen aus Bochum lassen sich die Teilnehmer an den Kursen zur „Einführung in das wissenschaftliche Arbeiten“ nach folgenden Gruppen unterscheiden:

1. Studierende mit deutscher Hochschulzugangsberechtigung, die hier für längere Zeit studieren (also mehr als nur für ein Jahr), vielleicht sogar einen Abschluss erwerben wollen; viele verfügen über gute bis sehr gute Sprachkenntnissen, meistern aber den Wissenschaftsdiskurs weder mündlich noch schriftlich in angemessener Form. Nicht wenige merken das allerdings erst kurz vor der Abschlussprüfung, so dass es erst zu einer Lernberatung kommt, wenn die Probleme als kaum noch lösbar erscheinen, wenigstens nicht in der knappen Zeit bis zu den Prüfungen. In der Regel handelt es sich bei dieser Gruppe um die sog. „Bildungsinländer“ 12 mit Migrantenhintergrund, und die beschriebenen Probleme treten in den Geistes- und Sozialwissenschaften auf, Fächern also, in denen besonders umfangreiche Leistungen gerade im schriftlichen Bereich erbracht werden müssen;

2. ERASMUS oder SOKRATES-Austauschstudierende, die sich entweder im Grund- oder Hauptstudium befinden, über sehr gute fachliche Qualifikationen, oft aber nur über sehr mäßige Deutschkenntnisse verfügen;

3. Austauschstudierende, die hier dissertieren oder sonstige größere Abschlussarbeiten auf Deutsch schreiben, in jeder Hinsicht über sehr gute Sprachkenntnisse verfügen, aber für ihre Arbeit eigentlich nur eine Oberflächenpolitur, den letzten Schliff also brauchen: Eine Arbeit, die nur ein qualifizierter Lektor leisten könnte.

12 Vgl. zum Thema etwa die Ausführungen von Ursula Neumann et al unter: www.forumbildung.de/bib/material/aydin_neumann.pdf [14.11.2006]. 
In der Regel sind in den Kursen die unterschiedlichsten Fachrichtungen vertreten: Das geht von eher wenig schreibintensiven Fächern wie Elektrotechnik/Maschinenbau bis zu den Geistes- und Sozial-, auch Rechtswissenschaften mit hohen formalen, inhaltlichen und sprachlichen Anforderungen auf dem jeweiligen Gebiet. Dazu kommen sehr unterschiedliche Vorstellungen vom Wissenschaftsbetrieb auf studentischer Seite, die sich keineswegs immer mit den westlichen Kriterien decken, wie beispielsweise eingangs schon über die vergleichende Studie von Kaiser berichtet wurde ${ }^{13}$.

Ebenfalls zu berücksichtigen ist das doch sehr unterschiedlich ausgeprägte Problembewusstsein der Studierenden, das oft dazu führt, dass die entsprechenden Kurse gar nicht erst belegt werden, sowie die mangelnde Motivation, die sich oft schon nach der Hälfte der Kursdauer erschöpft hat ${ }^{14}$. Besonders Letzteres macht es sehr schwer, den nicht nur bei Schreibkursen, sondern auch in den Veranstaltungen zur Vermittlung von Studierstrategien der Schreibforschung heute allgemein geforderten und von der psychologischen Bedeutung des Problems her auch angemessen erscheinenden ,Pakt ${ }^{`}$ zwischen Dozent und Student einzuhalten, der Rechte und Pflichte beider Seiten klar definiert ${ }^{15}$.

Ein weiteres Problem, jedenfalls in Bochum, resultiert aus der mangelnden Kooperation zwischen den einzelnen Fakultäten und der Abteilung Deutsch als Fremdsprache. Versucht man, Sprachkurse auf verschiedenen Sprachniveaus anzubieten und die Studierenden entsprechend ihrem Niveau auf die Kurse zu verteilen, kollidiert das regelmäßig mit den Stundenplänen ihrer Hauptstudienfächer. $\mathrm{Da}$ die Studierenden in der Regel ihrem Hauptstudienplan folgen, sitzen nicht immer die richtigen Leute in den richtigen Kursen ${ }^{16}$.

Vor diesem Hintergrund und der in der Regel doch mangelhaften finanziellen und personalen Mittel der Abteilungen erscheint eine allzu große Auffächerung der Teilnehmer vielleicht sogar nach Fächern, verbunden mit intensiver, coachingähnlicher Lernberatung als schwer durchführbar - zudem, wenn der Lernberater und der Kursleiter nicht dieselbe Person sein sollten ${ }^{17}$.

\footnotetext{
13 Vgl. etwa zu den Studierenden aus asiatischen Ländern die Ausführungen von Miriam Zeilinger zum Thema „Wissenschaftliches Schreiben und Diskussion für asiatische Studierende“ Mehlhorn et al. (2005), S. 191-197.

${ }_{14}$ Zur mangelnden Motivation auf Seiten der Studierenden trägt wohl auch bei, dass die Studierbzw. Schreibstrategiekurse jedenfalls in Bochum studienbegleitend und daher kostenlos sind, was zu einer gewissen Unverbindlichkeit bei der regelmäßigen Mitarbeit führen kann.

15 Vgl. zu diesem Pakt etwa das Muster eines solches Vertrages unter www.ualberta.ca/ german/ejournal/vertrag.pdf.

16 An der Christian-Albrechts-Universität Kiel hat man daher ein entsprechendes Angebot an Abendsprachkursen für ERASMUS-/SOKRATES-Studierende und ausländische Gastwissenschaftler/innen eingerichtet.

17 Vgl. dazu nur Mehlhorn et al. (2005), 214.
} 


\subsection{Erfahrungen, Vorschläge, Desiderate}

Ziel des Unterrichts sollte es zunächst sein, den Kursteilnehmern ein Grundvertrauen in die eigenen Fähigkeiten zu vermitteln und sie (auch und gerade wegen der unterschiedlichen Disziplinen, die vertreten sind) auf einer ganz basalen Ebene ,abzuholen'! Die Bereiche Schreiben, Lesen und Sprechen, in der Realität des Studiums eng miteinander verbunden, sollten auch hier im Verbund trainiert werden: wer gut und angemessen liest und darüber verbal kommuniziert, sich also gerne ausdrückt und seine Thesen zur Diskussion stellt, schreibt in der Regel auch gut bzw. hat weniger Schwierigkeiten, hier auftretenden Problemen zu begegnen.

Ebenfalls sollte den Kursteilnehmern das Wissen um die psychologischen Prozesse vermittelt werden, die beim Schreiben ablaufen, insbesondere die Fähigkeit, die einzelnen Arbeitsschritte, die von der Themenfindung bis zur Abgabe der fertigen Arbeit (oder dem Halten des Referats oder dem Schreiben der Klausur) nötig sind, zu entzerren. Das heißt etwa, nicht schon bei der Themensuche ein genaues Exposé schreiben und nicht sofort einen druckreifen Text aufs Papier bringen wollen, ohne zuerst eine Rohfassung zu erstellen.

Außerdem sollte von Dozentenseite darauf hingewiesen werden, dass es wesentlich darauf ankommt, die in den Kursen vermittelten Techniken das ganze Studium hindurch (und auch im Berufsleben) konsequent anzuwenden und sie so zu verinnerlichen: Das kann ein einzelner Kurs nicht leisten.

Was die bereits angesprochene Auswahl der Beispieltexte angeht, mit denen die Studierenden arbeiten sollen, so haben sich Texte, deren Schwierigkeitsgrad bereits dem Studium entsprechen, in den Kursen nicht bewährt. Nach Erfahrungen der Autoren ist es besser, an einfacheren Texten Schreib- und Lesestrategien zu erarbeiten, obwohl diese nicht dem akademischen Alltag der Studierenden entsprechen. Auch die ,Bearbeitung' schlechter Wissenschaftsprosa durch die Kursteilnehmer erscheint als eine mögliche Strategie. Die Gefahr, dass sich die Studierenden so mechanisch Formulierungen und Redemittel abschauen, die sie dann auf Biegen und Brechen verwenden, mag vorhanden sein, ist aber unserer Erfahrung nach im Vergleich zu den Vorteilen, die ein solches Arbeiten mit Texten bietet, vernachlässigenswert. Im Übrigen äußern die Studierenden selbst immer wieder den Wunsch nach Mustertexten ${ }^{18}$.

Sehr sinnvoll ist der Grundsatz, dass jeder Kursteilnehmer auch die individuelle Lernberatung besucht. Hier können zum einen Probleme intensiver angesprochen werden, andererseits auch Lernerfolge aus dem Kurs überprüft werden. Die Einrichtung solcher Kurse plus Lernberatungen ist allerdings sehr personalintensiv und dürfte schon bei einer Kursgröße von nur 10 Personen (die relativ leicht erreicht wird) die Möglichkeiten nur einer Lehrkraft bereits übersteigen.

\footnotetext{
${ }^{18}$ Ein guter ,Steinbruch' für Texte und Ideen bildet etwa die seit einigen Jahren bei Piper erscheinende Reihe „Denkanstöße“, in der jedes Jahr ein neuer Sammelband mit gut formulierten Texten aus allen Bereichen von Wissenschaft, Kunst und Gesellschaft erscheint.
} 
Da wissenschaftliches Schreiben und Arbeiten immer auch ein stark kreatives Element enthält, ist es sinnvoll, wissenschaftliches mit kreativem Schreiben zu verbinden. Besonders aus den Büchern Lutz von Werders lässt sich hier vieles für den Unterricht adaptieren ${ }^{19}$.

Die Kursdauer von jeweils 3 SWS bietet die Möglichkeit einer Dreiteilung: Im ersten Teil kommt nach einem spielerisch-kreativen Beginn ein Wiederholungsbzw. Übungsteil (z. B. eines Referates oder die Diskussion einer Mitschrift, die per OHP vorgelegt wird). Im zweiten Teil wird das Neue eingeführt und im dritten dann geübt.

Ebenfalls der Aktivierung kreativer Energien dient die Vermittlung von Techniken des Clusterns und des Mind-Mappings, um die eigenen Gedanken zu sortieren und ein Thema zu finden bzw. geeignete Problemstellungen innerhalb eines gegebenen Themas zu entwickeln. Das kann und sollte ruhig auf Deutsch geschehen.

Die Technik des Referats können die Teilnehmer ebenfalls an eher einfachen Themen wie etwa „Mein Lieblingsfilm“ einüben. Als Textsorte, die besonders geeignet ist, Tugenden des akademischen Schreibens wie Präzision, Klarheit und Knappheit zu vermitteln, hat sich die Inhaltsangabe (plus Stellungnahme) bewährt.

Writing summaries [...] is a superb way to develop reading and listening skills [etwa beim Mitschreiben von Vorlesungen, die Verf.] to practice decentering, and to develop the skills of precision, clarity, and succinctness [...] (Bean 1996: 128).

Eine immer wiederkehrende Empfehlung in den Ratgebern für Muttersprachler ist das Führen eines Arbeitsjournals: in der Regel eine Mischung aus privatem Tagebuch und einer kontinuierlich durchgeführten Materialsammlung. Dieses Journal kann in der Mutter-, sollte aber überwiegend in der Fremdsprache geführt werden können ${ }^{20}$. Ob diese Journale wirklich von den Dozenten - in welcher Form auch immer - zu kontrollieren sind und in welchem Umfang dies geschehen sollte, bedürfte nach Meinung der Autoren allerdings noch der Diskussion.

Die Mitarbeiter der Abteilung Deutsch als Fremdsprache in Bochum haben im vergangenen Jahr eine Ausbildung zum Schreibtutor speziell für ausländische Studierende durchlaufen, die es ihnen erlaubt, Studierende auf dem hier beschriebenen schwierigen Weg zu begleiten. Insbesondere ging es um die Entzerrung der einzelnen Schritte des Schreibprozesses von der Ideengenerierung über die Ideensortierung hin zur Formulierung und schließlich zur Überarbeitung. Ohne eine klare Unterscheidung zwischen dem ,,inneren“ und dem „äußeren Adressaten“ ist ebenfalls kein dauerhafter Arbeitserfolg denkbar. Unter ,innerem Adressaten“ ist hier die eigene Motivation zu verstehen, als ,äußeren Adressaten'bezeichnet man etwa die „scientific community“, in der sich sowohl Studierende als auch Dozen-

\footnotetext{
19 Vgl. dazu die entsprechenden Titel in der Bibliographie.

${ }^{20}$ Vgl. dazu die bemerkenswerten Ausführungen mit Beispielen bei Wagner und Magistrale (2003).
} 
ten immer bewegen ${ }^{21}$. Und schließlich das Wissen darum, dass Fehler sinnvoll sind, nicht etwas, was man möglichst vermeiden sollte - wie alle DaF-Lehrer wissen, besonders für asiatische Kulturen anathema.

Am Ende der Ausbildung zum Schreibtutor stand schließlich auch die Erkenntnis, dass, auch angesichts der bereits benannten subtilen psychologischen Prozesse beim Schreiben, E-Learning keine geeignete Plattform für die Vermittlung von Studierstrategien ist. Auf unabsehbare Zeit erscheint hier ein Unterricht ,von Mensch zu Mensch`als unabdingbar.

Zum Abschluss unserer Ausführungen noch ein paar allgemeine Empfehlungen:

- Die Schreibbüros an den Universitäten sollten materiell und personell wesentlich besser ausgestattet bzw. überhaupt erst einmal flächendeckend eingerichtet werden;

- Regelmäßige Schulung der Mitarbeiter;

- Kurse im wissenschaftlichen Arbeiten (mit Schwerpunkt Schreiben) für alle ausländischen (und inländischen) Studierenden, auch für SOKRATES- oder ERASMUS-Stipendiaten, die nur für ein Semester oder ein Studienjahr bleiben, für verbindlich erklären (ggf. die Gewährung von ECTS-Punkten als ,Belohnung)! Das würde die ,Paktsituation“ verbessern, d. h. auch auf studentischer Seite die Bereitschaft erhöhen, regelmäßig am Kurs teilzunehmen;

- für ausländische Studierende, die hier Abschlussarbeiten schreiben, wären „Korrekturbörsen' einzurichten, in der Texte lektoriert werden;

- Zwischen den Fakultäten und den Schreibbüros sollte sich ein intensiver Austausch entwickeln, damit die Studierstrategienkurse nicht schon an der mangelnden Abstimmung der Stundenpläne scheitern.

Es bleibt also noch eine Menge zu tun. Ohne einen grundlegenden Mentalitätswandel auf Seiten der Hochschulen und ohne eine bessere finanzielle Ausstattung, zu der, das sei hier im Vorübergehen bemerkt, Studiengebühren nur einen kleinen Teil werden beitragen können, wird sich die Betreuung ausländischer Studierender allerdings auf keinen Fall verbessern lassen.

${ }^{21}$ Vgl. zu den „Adressaten“ etwa die Ausführungen bei Keseling (2004). 


\section{Bibliographie zum Thema „Wissenschaftliches Schreiben“ (Stand: 14. November 2006; ohne Anspruch auf Vollständigkeit)}

\section{Wissenschaftliches Schreiben bzw. Arbeiten bzw. Lernbe- ratung allgemein / Alle Disziplinen}

Akoun, Delphine, „Schreiben an der Uni. Gespenstisch und doch so schön!“. In: Cölfen, Hermann / Januschek, Franz (Hrsg.), Linguistische Beratung im Spiegel der Praxisfelder. Oldenburg 1996, 149-167.

Bänsch, Axel, Wissenschaftliches Arbeiten. Seminar- und Diplomarbeiten. 8. Auflage.

München 2003.

Baurmann, Jürgen / Weingarten, Rüdiger, Schreiben. Prozesse, Prozeduren und Produkte. Opladen 1995.

Bean, John C., Engaging Ideas. The Professor's Guide to Integrating Writing, Critical Thinking, and Active Learning in the Classroom. San Francisco 1996.

Becker, Howard S., Die Kunst des professionellen Schreibens. Ein Leitfaden für die Geistesund Sozialwissenschaften. Frankfurt/Main 22004.

Berning, Johannes, Schreiben als Wahrnehmungs- und Denkhilfe. Elemente einer bolistischen Schreibpädagogik. Münster - New York 2002.

Blom, Herman, Der Dozent als Coach. Neuwied - Kriftel - Berlin 2000.

Boehncke, Heiner, Erfolgreich schreiben im Studium. München 2000.

Booth, Wayne C. / Colomb, Gregory J. / Williams, Joseph M., The Craft of Research. 2 ${ }^{\text {nd }}$ revised edition. Chicago - London 2003.

Bräuer, Gerd, Schreibend lernen. Grundlagen einer theoretischen und praktischen Schreibdidaktik. Innsbruck etc. 1998.

ders., Warum Schreiben? Frankfurt/Main 1996.

Bramberger, Andrea/Forster, Edgar, Wissenschaftlich schreiben: kritisch - reflexiv bandlungsorientiert. Münster 2004.

Brandt, Edmund, Rationeller Schreiben lernen: Hilfestellung zur Anfertigung wissenschaftlicher (Abschluss-)Arbeiten. Baden Baden 2002.

Brink, Alfred, Anfertigung wissenschaftlicher Arbeiten. 2. Auflage. München: 2005.

Bruce, Shanti / Rafoth, Ben, ESL Writers. Portsmouth (New Hampshire) 2004.

Brugger, Paul, Wissen schaffendes Schreiben. Herausforderungen für den (Deutsch)Unterricht. Innsbruck etc. 2004. 
Brunner, Maria, Schreibgesten. Die Entdeckung des Schreibens als Akt des Schreibens. Schreibkompetenz, durch Literaturunterricht. Neuried 1997.

Bünting, Karl-Dieter / Bitterlich, Axel / Pospiech, Ulrike (Hrsg.), Schreiben im Studium mit Erfolg. Ein Leiffaden (mit CD-ROM). Berlin 42004. Der Inhalt der $\mathrm{CD}$ ist auch online abrufbar unter:

http://www.uni-essen.de/schreibwerkstatt/trainer [14.11.2006]

Burchardt, Michael, Leichter studieren: Wegweiser für effektives wissenschaftliches Arbeiten. 4., neubearbeitete Auflage Berlin 2005.

Cioffi, Frank, Kreatives Schreiben für Studenten und Professoren. Berlin 2006.

Corsten, Hans / Deppe, Joachim, Technik des wissenschaftlichen Arbeitens. 2. Auflage. München 2002.

Eco, Umberto, Wie man eine wissenschaftliche Abschlussarbeit schreibt. Doktor-, Diplomund Magisterarbeit in den Geistes- und Sozialwissenschaften. Heidelberg ${ }^{112005 .}$

Ehlich, Konrad / Steets, Angelika (Hrsg.), Wissenschaftlich schreiben - lehren und lernen. Berlin - New York 2003.

Elbow, Peter, Everyone Can Write: Essays Toward a Hopeful Theory Of Writing and Teaching Writing. Oxford - London 2000.

Esselborn-Krumbiegel, Helga, Von der Idee zum Text. Eine Anleitung zum wissenschaftlichen Schreiben. 2., durchgesehene Auflage. Paderborn etc. 2004.

Falstauer, Renate, Wir müssen gemeinsam schreiben! Kooperatives Schreiben im fremdsprachlichen Deutschunterricht. Innsbruck 1997.

Franck, Norbert, Fit fürs Studium. Erfolgreich reden - lesen - schreiben. München 1998.

Franck, Norbert, Handbuch Wissenschaftliches Arbeiten. Frankfurt/Main 2004.

Franck, Norbert / Stary, Joachim, Die Technik wissenschaftlichen Arbeitens. 12., völlig neu bearbeitete Auflage. Stuttgart 2006.

Gibaldi, Joseph, MLA Handbook for W riters of Research Papers. New York ${ }^{6} 2003$.

Göttert, Karl-Heinz, Kleine Schreibschule für Studierende. München 2003.

Graefen, Gabriele, Der Wissenschaftliche Artikel - Textart und Textorganisation.

Frankfurt/Main et al. 1997.Im Internet abrufbar unter: http://www.daf.unimuenchen.de/DAF/PERSONEN/GRAEFEN/Wiss Artikel A4.pdf

dies., Wissenschaftliches Schreiben. Hagen 2001.

Gruber, Helmut / Rheindorf, Markus / Wetschanow, Karin / Reisigl, Martin / Muntigl, Peter / Czinglar, Christine (Hrsg.), Genre, Habitus und wissenschaftliches Schreiben. Eine empirische Untersuchung studentischer Texte. Münster - Hamburg etc. 2006. 
Grunwald, Klaus / Spitta, Johannes, Wissenschaftliches Arbeiten. Grundlagen zu Herangehensweisen, Darstellungsformen und Regeln. Eschborn 1997.

Haefner, Klaus, Gewinnung und Darstellung wissenschaftlicher Erkenntnisse. München 2000.

Heublein, Ulrich / Sommer, Dieter / Weitz, Brigitta, Studienverlauf im Ausländerstudium. Eine Untersuchung an vier ausgewählten Hoch-schulen. Bonn 2004, (=DAAD-Reihe Dokumente und Materialien, Mat \& Dok 55). Im Internet abrufbar unter: unter:

http://www.daad.de/de/download/zahlen/dok-und-mat 55.pdf

Höge, Holger, Schriftliche Arbeiten im Studium: ein Leitfaden zur Abfassung wissenschaftlicher Texte. 2., überarbeitete und erweiterte Auflage. Stuttgart-Köln 2006.

Hoffmann, Monika, Deutsch fürs Studium. Grammatik, und Rechtschreibung. Paderborn 2005.

Hornung, Antonie, Zur eigenen Sprache finden. Modell einer plurilingualen Schreibdidaktik. Tübingen 2002.

Hunziker, Alexander W., Spaß am wissenschaftlichen Arbeiten. So schreiben Sie eine gute Seminar-oder Semesterarbeit. Zürich 2002.

Jakobs, Eva-Maria, Textvernetzung in den Wissenschaften. Zitat und Verweis als Ergebnis rezeptiven, reproduktiven und produktiven Handelns. Tübingen 1999.

dies./ Knorr, Dagmar / Pogner, Karl H. (Hrsg.), Hypertext Text KonText. Frankfurt/Main 1999.

dies. / Lehnen, Katrin / Schindler, Kirsten (Hrsg.), Schreiben am Arbeitsplatz.

Wiesbaden 2005.

dies. / Knorr, Dagmar (Hrsg.), Schreiben in den Wissenschaften. Frankfurt/Main et al. 1997.

Jele, Harald, Wissenschaftliches Arbeiten in Bibliotheken. 2. Auflage. München 2003.

ders., Wissenschaftliches Arbeiten: Zitieren. München 2003.

Kaiser, Dorothee, , ,Nachprüfbarkeit' versus ,Originalität ${ }^{6}$ - Fremdes und Eigenes in studentischen Texten aus Venezuela und Deutschland“. In: Ehlich, Konrad / Steets, Angelika (Hrsg.), Wissenschaftlich schreiben - lehren und lernen. Berlin New York 2003, 305-324.

Karmasin, Matthias / Ribing, Rainer, Die inhaltliche und formale Gestaltung wissenschaftlicher Arbeiten: Ein Leitfaden für Haus, Seminar- und Diplomarbeiten sowie Dissertationen. 3., vollständig überarbeitete Auflage. Wien 2002.

Kast, Bernd (unter Mitarbeit von Eva-Maria Jenkins), Fertigkeit Schreiben. Berlin etc. 1999.

Keseling, Gisbert, Die Einsamkeit des Schreibers. Wiesbaden 2004. 
ders., Schreibprozeß und Textstruktur. Empirische Untersuchungen zur Produktion von Zusammenfassungen. Tübingen 1993.

ders., „Sprechen mit sich selbst als Strategie zur Vermeidung und Überwindung von Schreibblockaden. Erkundungen zur Funktion der inneren Sprache beim Schreiben“. In: Mauelshagen, Claudia / Seifert, Jan (Hrsg.), Sprache und Text in Theorie und Empirie. Beiträge zur Germanistischen Sprachwissenschaft. Festschrift für Wolfgang Brandt. Stuttgart 2001, 157-169.

Kissling, Walter / Perko, Gudrun (Hrsg.), Wissenschaftliches Schreiben im Studium. Reflexionen, Desiderate, Konzepte. Innsbruck 2006.

Klippert, Heinz, Kommunikations-Training. Übungsbausteine für den Unterricht.

Weinheim und Basel ${ }^{92002 .}$

Klippert; Heinz, Methoden-Training. Übungsbausteine für den Unterricht. Weinheim und Basel ${ }^{142004 .}$

Kment, Patric, Effiquientes Arbeiten mit den „Neuen Medien“. Hamburg - Münster Wien. 2006. [angekündigt!]

Kohrt, Manfred (Hg.), Schreibprozesse - Schreibprodukte. Festschrift für Gisbert Keseling. Hildesheim u.a. 1992.

Kropp, Waldemar / Huber, Alfred, Studienarbeiten interaktiv. Erfolgreich wissenschaftlich denken, schrieben, präsentieren. Köln 2006.

Kruse, Otto, Keine Angst vor dem leeren Blatt. Ohne Schreibblockaden durchs Studium. 8., durchgesehene Auflage. Frankfurt/Main 2004.

ders., / Jakobs, Eva-Maria / Ruhmann, Gabriela (Hrsg.), Schlüsselkompetenz. Schreiben. Konzepte, Methoden, Projekte für Schreibberatung und Schreibdidaktik. Neuwied 1999.

Lehnen, Katrin, Kooperative Textproduktion. Zur gemeinsamen Herstellung wissenschaftlicher Texte im Vergleich von ungeübten, fortgeschrittenen und sehr geübten SchreiberInnen. Dissertation Universität Bielefeld 2000. Im Internet abrufbar unter: http:/ / archiv.ub.uni-

bielefeld.de/disshabi/2002/0058/

Leopold-Wildburger, Ulrike / Schütze, Jörg, Verfassen und VortragenWissenschaftliche Arbeiten und Vorträge leicht gemacht. Berlin-Heidelberg 2002.

Liening, Andreas, Wissenschaftlich arbeiten - Aber wie? Studienbilfe zum Erstellen wissenschaftlicher Arbeiten. 2. Auflage. Münster 2000.

Ludwig, Otto, Geschichte des Schreibens. Band 1: Von der Antike bis zum Buchdruck. Berlin 2005. [Band 2 ist angekündigt für 2008] 
Lück, Wolfgang, Technik des wissenschaftlichen Arbeitens - Seminararbeit, Diplomarbeit, Dissertation. 9. Auflage. München 2003.

Luhmann, Niklas, „Kommunikation mit Zettelkästen. Ein Erfahrungsbericht“. In: ders.: Universität als Milieu. Kleine Schriften. Bielefeld 1992, 53-61.

Luhmann, Niklas, „Lesen lernen“. In: ders., Short Cuts. Frankfurt/Main ${ }^{42005,}$ 150-157.

Metz, Berthold / Pfeiffer, Joachim / Staiger, Michael / Wichert, Adalbert (Hrsg.), Lesen, Schreiben und Kommunizieren im Internet. Theorie und Praxis teilvirtueller Hochschullehre. Herbolzheim 2004.

Müller, C. Wolfgang, SchreibLUST. Von der Freude am wissenschaftlichen Schreiben. Münster 2001.

Narr, Wolf-Dieter / Stary, Joachim (Hrsg.), Lust und Last des wissenschaftlichen Schreibens. Hochschullehrerinnen und Hochschullehrer geben Studierenden Tipps. Frankfurt/Main 1999.

Nicol, Natascha, Wissenschaftliche Arbeiten schreiben mit Word: Formvollendete und normgerechte Examens-, Diplom- und Doktor-arbeiten. München 2004.

Niederhauser, Jürg, Die schriftliche Arbeit. Ein Leitfaden zum Schreiben von Fach-, Seminar-und Abschlussarbeiten in der Schule und beim Studium. Literatursuche, Materialsammlung und Manuskriptgestaltung mit vielen Beispielen. Mannheim Leipzig etc. 22006.

Ortner, Hanspeter, Schreiben und Denken. Tübingen 2002.

Paltridge, Brian, „Academic Writing” Review-Article. In: Language Teaching 37, 2004, 87-105.

Perrin, Daniel / Kruse, Otto / Böttcher, Ingrid / Wrobel, Arne (Hrsg.), Schreiben. Von intuitiven zu professionellen Schreibstrategien. Wiesbaden 2002.

Peterssen, Wilhelm H., Wissenschaftliches Arbeiten - eine Einführung für Schule und Studium. 6., überarbeitete und erweiterte Auflage. München 1999.

Plümper, Thomas, Effizient schreiben. München 2003.

Preißner, Andreas, Wissenschaftliches Arbeiten. 2. Auflage. München 2005.

Pyerin, Brigitte, Kreatives wissenschaftliches Schreiben. Tipps und Tricks gegen Schreibblockaden. 2., aktualisierte Auflage. Weinheim und München 2003.

Ramage, John D. / Bean, John C. / Johnson, June, Writing Arguments: A Rhetoric with Readings. Concise Edition. $4^{\text {th }}$. ed. Boston - New York 2007.

Rieder, Karl, Wissenschaftliches Arbeiten. Eine Einführung. Wien 2002. 
Rijlaarsdam, Gert / Björk, L. / Bräuer, G. et al. (Hrsg.), Teaching Academic Writing in European Higher Education. Doordrecht 2003 (= Studies in Writing, Volume 12). Zu den bisher erschienenen Bänden in dieser Reihe vgl. die Angaben unter: http://www.springeronline.com/sgw/cda/frontpage/0,11855,1-4040669-33110965-0,00.html

Rossig, Wolfram E./ Prätsch, Joachim: Wissenschaftliche Arbeiten. Ein Leitfaden für Haus-Seminar-Examens- und Diplomarbeiten sowie Präsentationen. 3., erweiterte Auflage. Bremen 2005.

Rost, Friedrich, Lern- und Arbeitstechniken für das Studium mit zablreichen Beispielen, Abbildungen, Checklisten. 4., durchgesehene Auflage. Wiesbaden 2004.

Russel-McDonald, Christina / McDonald, Robert L. (Hrsg.), Teaching Writing. Carbondale and Edwardsville 2002.

Schneider, Wolf, Deutsch fürs Leben. Reinbek 1999.

ders., Wörter machen Leute. München 1986.

Schulte-Steinicke, Barbara, Entspannung, wissenschaftliches Schreiben und NLP. Ein kreativer Übungsleitfaden für Framen an Hochschulen. Berlin 1996.

Sesink, Werner, Einführung in das wissenschaftliche Arbeiten. Mit InternetTextverarbeitung - Präsentation. München ${ }^{6} 2003$.

Sommer, Roy, Schreibkompetenzen. Erfolgreich wissenschaftlich schreiben. Stuttgart 2006.

Soven, Margot Iris, What the Writing Tutor Needs to Know. London 2005.

Standop, Ewald / Meyer, Matthias L. G., Die Form der wissenschaftlichen Arbeit. Ein unverzichtbarer Leitfaden für Studium und Beruf. 17., korrigierte und ergänzte Auflage. Wiebelsheim 2004.

Stickel-Wolf, Christine / Wolf, Joachim, Wissenschaftliches Arbeiten und Lerntechniken. Erfolgreich studieren - gewusst wie! 3. Auflage. Wiesbaden 2005.

Theisen, Manuel, Wissenschaftliches Arbeiten. München 2005.

Werder, Lutz von, Aufbaukurs der Rhetorik des wissenschaftlichen Schreibens. 2006.

ders., Brainwriting \& Co. Die 11 effektivsten Methoden des kreativen Schreibens für die Schule und das Studium. Berlin 2002

ders., Kreatives Schreiben in den Wissenschaften. Für Schule, Hochschule und Erwachsenenbildung. Berlin 1992.

ders., Kreatives Schreiben von Diplom- und Doktorarbeiten. Berlin ${ }^{3} 2000$.

ders, Kreatives Schreiben von wissenschaftlichen Hausarbeiten und Referaten. Berlin 2000.

ders., Lehrbuch des wissenschaftlichen Schreibens. Berlin 1993. 
ders. / Schulte-Steinicke, Barbara (Hrsg.), Die deutsche Schreibkrise. Empirische Umfragen von 1994 - 2002. Hohengehren 2003.

Winter, Wolfgang, Wissenschaftliche Arbeiten schreiben. Hausarbeiten/Diplom- und Magisterarbeiten/MBA-Abschlussarbeiten/Dissertationen. Frankfurt-Wien 2004.

Wrobel, Arne, Schreiben als Handlung. Überlegungen und Untersuchungen zur Theorie der Textproduktion. Tübingen 1995.

Zinsser, William, Schreiben wie ein Schriftsteller. Fach- und Sachbuch, Biographie, Reisebericht, Kritik, Business, Wissenschaft und Technik. Berlin 2002.

Einen Überblick über das einschlägige Schrifttum bis etwa 2000 bietet:

Ehlich, Konrad / Steets, Angelika / Traunspurger, Inka (Hrsg.), Schreiben für die Hochschule. Eine annotierte Bibliographie. Frankfurt/Main 2000.

Speziell zur Arbeit mit ausländischen Studierenden

Börner, Wolfgang / Vogel, Klaus (Hrsg.), Schreiben in der Fremdsprache. Prozess und Text, Lehren und Lernen. Bochum 1992.

Büker, Stella, Wissenschaftliches Arbeiten und Schreiben in der Fremdsprache Deutsch. Hohengehren 1998.

dies., "Teaching academic writing to international students: Individual Tutoring as a supplement to workshops”. In: Rijlaarsdam, Gert / Björk, L. / Bräuer, G. et al. (Hrsg.), Teaching Academic Writing in European Higher Education.

Doordrecht 2003 (= Studies in Writing, Volume 12), 41-57.

Canagarajah, A. S., Critical academic writing and multilingual students. Ann Arbor, Michigan 2002.

Esser, Ruth, „Etwas ist mir geheim geblieben am deutschen Referat“. Kulturelle Geprägtheit wissenschaftlicher Textproduktion und ihre Konsequenzen für den universitären Deutschunterricht von Deutsch als Fremdsprache.

München 1997.

Hufeisen, Britta, Ein deutsches Referat ist kein englischsprachiges Essay. Theoretische und praktische Überlegungen zu einem verbesserten textsortenbezogenen Schreibunterricht in der Fremdsprache Deutsch an der Universität. Innsbruck 2002. 
Kästner, Uwe, Freies Schreiben in der Fremdsprache Deutsch - Prozesse und ihre Didaktik. Untersuchungen zur Textproduktion im fremdsprachlichen Deutschunterricht auf dem Hintergrund neuropsychologischer Theorien. Bochum 1997.

Kaiser, Dorothee, Wege zum wissenschaftlichen Schreiben: Eine kontrastive Untersuchung zu studentischen Texten aus Venezuela und Deutschland. Tübingen 2002.

Krumm, Hans-Jürgen (Hg.), Erfahrungen beim Schreiben in der Fremdsprache Deutsch. Untersuchungen zum Schreibprozess und zur Schreibförderung im Unterricht mit Studierenden. Innsbruck-Wien-München 2000.

Lehker, Marianne, Texte im chinesischen Aufsatzunterricht. Eine kontrastive Analyse chinesischer und deutscher Aufsatzsorten. Heidelberg 1997.

Mehlhorn, Grit, Studienbegleitung für ausländische Studierende an deutschen Hochschulen. Teil I: Handreichungen für Kursleiter zum StudienstrategienKurs. Teil II: Individuelle Lernberatung - Ein Leitfaden für die Beratungspraxis. Zwei Teile in einem Band. München 2005.

Müller, Ria, Interaktives Schreiben im Unterricht Deutsch als Fremdsprache. Eine empirische Untersuchung. Baltmannsweiler 2001.

Paltridge, Brian, Approaches to Teaching Second Language Writing. http://www.englishaustralia.au/ea_conference04/proceedings/pdf/Paltridge.pdf

Portmann, Paul R., Schreiben und Lernen. Grundlagen der fremdsprachlichen Schreibdidaktik. Tübingen 1991.

Schreiter, Ina, Schreibversuche. Kreatives Schreiben bei Lernern des Deutschen als Fremdsprache. München 2002.

Stalb, Heinrich, „Die Schreibfertigkeit im Fremdsprachenunterricht“. In: Zielsprache Deutsch 4/1979, 24-34.

Steinig, Wolfgang / Frederking, Volker / Berghoff, Matthias / Jünger, Werner, „Fremde im Zug - Fremde im Netz. Ein interkulturelles Schreibprojekt“. In: Zielsprache Deutsch 1/1998, 13-24.

Wagner, Kenneth / Magistrale, Tony (Hrsg.), Writing Across Culture. An Introduction to Study Abroad and the Writing Progress. New York etc. 2003.

\section{Einzelne Fachrichtungen}

Anglistik / Amerikanistik

Purser, Emily, Studienbegleiter: Academic Writing. Berlin 2004. 
Chemie

Ebel, Hans F. / Bliefert, Claus / Russey, William E. (Hrsg.), The art of scientific writing. From student reports to professional publications in chemistry and related fields. 2., completely revised edition. Weinheim 2004.

Deutsch als Fremdsprache

Starke, Günter / Zuchewicz, Tadeusz, Wissenschaftliches Schreiben im Studium von Deutsch als Fremdsprache. Frankfurt/Main 2003.

\section{Geographie}

Baade, Jussi / Gertel, Holger / Schlottmann, Antje, Wissenschaftlich Arbeiten. Leitfaden für Studierende der Geografie. Bern 2005.

\section{Biologie}

Kremer, Bruno P., Texte schreiben im Biologiestudium. Berlin-Heidelberg 2004.

\section{Geschichte}

Kolmer, Lothar / Rob-Santer, Carmen, Geschichte schreiben. Von der Seminar- zur Doktorarbeit. Paderborn 2005.

Schmale, Wolfgang, Schreib-Guide Geschichte: Schritt für Schritt wissenschaftliches Schreiben lernen. Wien 1999.

Ingenieurwissenschaften

Landau, Kurt / Ferreira, Yvonne / Mauerhoff, Anan, Arbeitstechniken für Studierende der Ingenieurswissenschaften. Stuttgart 2002.

Jura

Kerschner, Ferdinand, Wissenschaftliche Arbeitstechnik und -methodik für Juristen (mit CD-ROM). 5. Auflage. Wien 2005.

Klaner, Andreas, Richtiges Lernen für Jurastudenten und Rechtsreferendare. Berlin: 2002.

Lange, Barbara, Jurastudium erfolgreich: Planung - Lernstrategie Zeitmanagement. 4., neu bearbeitete und erweiterte Auflage. Köln - Berlin München 2005.

Möllers, Thomas M. J., Juristische Arbeitstechnik und wissenschaftliches Arbeiten. 3. Auflage. München 2005.

\section{Literaturwissenschaft}

Bangen, Georg, Die schriftliche Form germanistischer Arbeiten. 9. Auflage. Stuttgart 1989.

Jeßing, Benedikt, Arbeitstechniken des literaturwissenschaftlichen Studiums.

Ditzingen 2001. 
Ludwig, Hans-Werner / Rommel, Thomas, Studium Literaturwissenschaften. Arbeitstechniken und Neue Medien. Bern 2003.

Mathematik

Beutelspacher, Albrecht, „Das ist o.B.d.A. trivial!“ Eine Gebrauchsanleitung zur Formulierung mathematischer Gedanken mit vielen praktischen Tips [sic !] für Studierende der Mathematik und Informatik. 7., überarbeitete Auflage. Wiesbaden 2004.

Medienwissenschaften

Dahinden, Urs / Neuroni, Alessia C. / Sturzenegger, Sabina, Wissenschaftliches Arbeiten für Medienwissenschaftler. Bern 2006.

Medizin

Deinzer, Renate, Allgemeine Grundlagen wissenschaftlichen Arbeitens in der Medizin. Stuttgart 2005.

Müllner, Marcus, Erfolgreich wissenschaftlich arbeiten in der Klinik. Wien 2004.

Pädagogik

Bohl, Thorsten, Wissenschaftliches Arbeiten im Studium der Pädagogik.

Weinheim und Basel 2005.

\section{Politikwissenschaft}

Billing, Werner / Simon, Thomas, Politikwissenschaft und die Technik des wissenschaftlichen Arbeitens. Wiesbaden 2005.

Kalina, Ondřei / Köppl, Stefan / Kranenpohl, Uwe et al., Grundkurs

Politikwissenschaft. Einführung ins wissenschaftliche Arbeiten. Wiesbaden 2003.

Technik / Naturwissenschaften allgemein

Friedrich, Christoph, Schriftliche Arbeiten im technisch-naturwissenschaft-lichen Studium. Ein Leitfaden zur effektiven Erstellung und zum Einsatz moderner Arbeitsmethoden. Mannheim 1997.

Sozialwissenschaften

Becker, Howard S., Tricks of the Trade. How to think about your research while you're doing it. Chicago - London 1998.

VWL / BWL

Burchert, Heiko / Sohr, Sven, Praxis des wissenschaftlichen Arbeitens. München 2004.

Disterer, Georg, Studienarbeiten schreiben. Diplom-, Seminar- und Hausarbeiten in den Wirtschaftswissenschaften. 3., vollständig überarbeitete und erweiterte Auflage. Berlin 2004.

Ebster, Claus / Stalzer, Lieselotte, Wissenschaftliches Arbeiten für Wirtschafts- und Sozialwissenschaftler. 2., überarbeitete Auflage. Wien 2003. 
Hansen, Katrin, Selbst- und Zeitmanagement im Wirtschaftsstudium. Effektiv arbeiten, effizient arbeiten, Stress bewältigen. Berlin 2004.

Kricsfalussy, Andreas, Format und Inhalt. Schnellkurs zur Anfertigung wirtschaftswissenschaftlicher Arbeiten. Stuttgart 32001.

Mertens, Ralf, Denk- und Lernmethodik. Gehirnjogging für Studierende. Berlin 2001.

Paetzel, Ulrich, Wissenschaftliches Arbeiten. Überblick über Arbeitstechnik und Studienmethodik. Berlin 2001.

\section{Internet-Adressen}

Schreibbüros an amerikanischen Universitäten (Auswabl)

http://www.fas.harvard.edu/ wricntr/

(Harvard)

http://www.uiowa.edu/ iww/

(Iowa)

http:/ / writing.umn.edu/

(Minnesota)

http://owl.english.purdue.edu/writinglab/

(Purdue)

http://www.scrippscol.edu/dept/writing/main.htm

(Scripps College)

http:/ / www.wisc.edu/writing/

(Wisconsin-Madison)

Schreibbüros an deutschen Universitäten

http://www.uni-bielefeld.de/slab/ bzw.

http://www.uni-bielefeld.de/Universitaet/Studium/Studienbegleitende\%20 Angebote/Punktum/

(Bielefeld)

http://www.sz.ruhr-uni-bochum.de/

(Bochum)

http://www.uni-essen.de/schreibwerkstatt/trainer/trainer/start.html

(Essen)

http://www.kstw.de/default.htm?KSTW/Seitenframe/Beratung/schreibzentrum /default3.htm

(Köln)

Schreibwerkstatt am DaF-Bereich der Universität Trier:

http://www.daf.uni-trier.de/projekte/schreibwerkstatt/Schreibwerkstatt.htm 
Internationale Vereinigungen

http://www.eataw.org/

(European Association for the Teaching of Academic Writing)

http://writingcenters.org/

(International Writing Centers Association)

Verlage, in denen das Schreiben einen oder den Programmschwerpunkt bildet:

www.autorenhaus.de

(Autorenhaus, Berlin)

www.peterlang.com

(Peter Lang Wissenschaftsverlag)

www.schibri.de

(Schibri, Berlin)

www.studienverlag.at

(Studienverlag, Innsbruck - Wien - Bozen)

(Alle Internetadressen wurden zuletzt am 14.11.2006 besucht) 



\title{
Der lästige „Kleinkram“ beim (wissenschaftlichen) Schreiben in der Fremdsprache
}

\author{
Gabriele Graefen, München
}

\section{$1 \quad$ Einleitung}

Schreiben lernen wird oft mit Alphabetisierung gleichgesetzt. Das ist doppelt falsch: Erstens gibt es nicht nur alphabetische, sondern auch logographische Schriftsysteme, in denen die Schriftzeichen Bedeutungseinheiten entsprechen; zweitens gehören zu einem alphabetischen Schriftsystem auch anders geartete, nicht alphabetische Zeichen und Ausdrucksmittel anderer Typen, die bei einer vollständigen Beherrschung der Schriftsprache mit gelernt werden müssen. Eben diese sollen im folgenden Beitrag einmal genauer betrachtet werden. Für viele deutsche Leser kann das heißen, dass hier etwas aus der Grauzone des irgendwie Vertrauten, aber kaum Reflektierten, herausgeholt wird. Für ausländische Deutschlerner und Menschen, die in dieser Fremdsprache z. B. wissenschaftliche Texte schreiben wollen oder müssen, handelt es sich oft eher um eine „terra incognita“, ein unbekanntes Gebiet. 
Das hier vorgestellte Übungsmaterial wurde entworfen und erprobt für ausländische Studierende im Magister-Studienfach „Deutsch als Fremdsprache/ Transnationale Germanistik“"12 an der LMU München³. Die Notwendigkeit einer systematischen Einführung in die wichtigsten nicht-alphabetischen Elemente der Schriftsprache ergab sich daraus, dass sowohl Seminarprotokolle als auch Hausarbeiten fast immer einen hohen Anteil an Verstößen gegen den schriftsprachlichen Usus enthalten. Diese Art von Fehlern oder Mängeln wird jedoch in den Besprechungen durch wichtigere Probleme inhaltlicher und sprachlicher Art in den Hintergrund gedrängt, da die Zeit für die Besprechung der verfassten Texte ohnehin zu knapp bemessen ist. So sehen die Dozenten sich neben vielfältigen themenund textspezifischen Korrekturen immer wieder mit denselben schriftsprachlichen Regelverstößen oder Gestaltungsmängeln konfrontiert. Sie können aber meist nur durch Markierung im studentischen Text darauf hinweisen, womit die Probleme erfahrungsgemäß nicht behoben sind. Eine selbständige Erarbeitung der einschlägigen Regeln und Normen, z. B. mit Hilfe des Duden-Rechtschreibwörterbuches (RWb), kann man von ausländischen Studierenden unter den normalen Studienbedingungen nicht erwarten. Ähnliches gilt sicher auch für den nicht-universitären DaF-Unterricht im Schreiben, soweit er über „kreatives Schreiben“ hinausgeht", außerdem für die schriftliche Kommunikation in bestimmten Berufen (Journalismus, Verlagsbranche, Übersetzungstätigkeit u. a.) sowie in der Ausbildung dazu.

Das Thema Schriftsprache hat im DaF-Unterricht einen meist geringen Stellenwert ${ }^{5}$. Auch wenn es explizit um das Schreiben geht, wird meist der „schriftliche Ausdruck" in den Vordergrund gestellt, es geht um grammatische, lexikalische und stilistische Fragen. Schon die Behandlung der Interpunktion sprengt oft den Rahmen der Curricula. Die „Vertextungsnormen“ (Jakobs 1999, 174) sind in allen Bereichen eher unklar.

Mein Ziel war es, eine für das Grundstudium brauchbare, sehr knappe Beschreibung und Erklärung mit Übungen zusammenzustellen. Abbildung 1 macht deutlich, welche Schriftzeichen und Schriftauszeichnungen darin behandelt werden. Die Studierenden, von denen viele auch im Studium noch weiterhin die Sprache lernen, sollen auf das „Produzieren“ von wissenschaftssprachlichen Texten vorbereitet werden. Kenntnisse dieser Art könnten in Zukunft zu den „Schlüssel-

1

2 Der Beiname verdankt sich dem Bemühen, die Differenz zwischen dem akademischen Fach und dem Sprachunterricht so zu verdeutlichen, dass die Studierenden und Absolventen dieses Faches nicht dem Missverständnis ausgesetzt sind, sie würden einen Sprachkurs besuchen.

${ }^{3}$ Die Zahl der ausländischen Studierenden in diesem Fach stieg im Laufe der Jahre kontinuierlich (heute über $70 \%$ ).

${ }^{4}$ Das ,kreative Schreiben“ basiert auf einer Didaktik des Mutmachens und der gelegentlichen Normüberschreitung, um die Schreiber eigene Ausdrucksmöglichkeiten finden zu lassen. Es hat also ganz anders geartete Ziele als der hier vorgestellte Schriftsprachlehrgang.

${ }^{5}$ Das in der Aufsatzdidaktik genutzte ,Zürcher Textanalyseraster“ nennt immerhin zwei schriftsprachbezogene Beurteilungskriterien („Bezugsgrößen“): 1.2.2. Äußere Gliederung (graphisch mittels Absatz, Spiegelstrich o.a.), 1.5.3. Graphische Mittel (Unterstreichung, Schriftauszeichnung u. Ä.) 
qualifikationen“ der Studiengänge in geistes-, kultur- und sprachwissenschaftlichen Bereichen gehören.

\section{Schriftsprache ist mehr als Buchstaben}

Die nichtalphabetischen Zeichen sind nach Glück (1987, S. 29) eine „eigenständige Komponente der geschriebenen Sprachform“ mit einer Art „,spezieller graphischer Syntax“. Ein gewisser Teil von ihnen wird oft summarisch zu den Interpunktionszeichen gerechnet. Es handelt sich bei vielen davon, linguistisch betrachtet, um „Syngrapheme“, deren genaue Funktion sich jeweils erst in einem bestimmten Kontext erweist. Einige gelten als Ideogramme oder Begriffszeichen ${ }^{6}$, wie die Ziffern und mathematischen Zeichen, das Paragraphenzeichen $\left\langle\int\right\rangle$ und das et-Zeichen $\langle \&\rangle$. Die „Grammatik der deutschen Sprache“ siedelt solche Zeichen im Vergleich zu den Buchstaben auf einer „höheren Ebene“ an (Zifonun et al. 1997, 254) ${ }^{7}$.

Das gilt erst recht für die semiotische Gestaltung des Geschriebenen. In semiotischer Sicht gilt „die Textgestalt als Zeichen“, so der Titel einer historischen Untersuchung von B. Frank (1994). Seit dem Mittelalter ist, wie sie zeigt, die Seitengestaltung ein Darstellungsmittel.

Die Textgestaltung ist sowohl eine Konsequenz der Herausnahme des Textes aus der Sprechsituation als auch schon ein Versuch der Problemlösung. Nach Ehlich $(1996,18)$ findet beim schriftlichen Text geradezu eine „Dissoziierung“ der Sprechsituation statt, die bei mündlicher Kommunikation homogen ist. Der Text wird damit zu einem „Verstehenspotential“ (a.a.O., 22) für einen unbekannten Leser. Es kommt sehr darauf an, wie der Autor antizipierbare Verstehensprobleme seiner Leser textintern bearbeitet. Gute und schlechte Texte unterscheiden sich demnach auch und nicht zuletzt daran, wie weit und wie gekonnt der Autor gegebene Möglichkeiten der Verstehenserleichterung und -absicherung genutzt hat. Das kann durchaus einmal bedeuten, dass eine gewisse Redundanz entsteht, z. B. bei der graphischen Hervorhebung von Wichtigkeit: Schriftartwechsel, SchriftgröBe, Abstand von umgebendem Text, besondere Positionierung auf der Textseite solche Verfahren können kombiniert und auch alle zusammen genutzt werden. Die Fixierung und „Verdauerung“ von textueller Information betrifft also keineswegs nur die äußerliche Frage, in welchem „Medium“ eine ansonsten gleichbleibende „Botschaft“ einem Adressaten mitgeteilt wird, wie es die Kommunikationsmodelle der 70-er Jahre anvisierten.

\footnotetext{
${ }^{6}$ „Begriffszeichen sind solche Elemente von alphabetischen Schriftsystemen, deren Zeichencharakter durch eine feste Verbindung zu Elementen der Bedeutungsebene konstituiert wird.“ (Glück 1987, 28)

${ }^{7}$ Peter Gallmann (1985) teilt die nichtalphabetischen Zeichen ein in Ideogramme, Klassifikatoren, Grenzsignale, Satzintentionssignale, Auslassungssignale und weiterhin in (flächige) „Supragrapheme“". Ich sehe darin keine leicht nachvollziehbare Systematik und erläutere daher die Zeichen einzeln nach ihren Funktionen.
} 
Das Gelingen der schriftlichen Kommunikation als einer Bewältigung der in sich paradoxen zerdehnten Kommunikationssituation wird zu einem wesentlichen Bereich der Autor-Tätigkeit. Sie wird zum Metier des Autors, der Autor wird zunehmend professionalisiert. (Ehlich 1996, 35)

Wissenschaftliches Schreiben zu lernen impliziert also immer, sich mit dem problematischen Autor-Leser-Verhältnis und mit den Risiken textueller Kommunikation zu befassen und sich mit Möglichkeiten der Bewältigung vertraut zu machen.

Einige der Richtlinien und Ratgeber zum wissenschaftlichen Schreiben befassen sich mit Fragen der funktionellen Seitengestaltung, und eine Fülle von Regelungen zwischen Empfehlung und Norm betreffen Absatzformate, Zitatgestaltung und Ähnliches. International und fachspezifisch gibt es diverse „publication manuals“ und „style guides“. Auch für Studierende an deutschen Universitäten existieren oft fachspezifische Anleitungen. Sie gehören nicht in einen Lehrgang zum Umgang mit Schriftsprache, schon weil sie nicht allgemein verbindlich sind. Wohl aber scheint es mir nötig, die selbständige Fähigkeit zu einer inhaltsbezogenen Textgestaltung zu verbessern. Es geht hier um die vom Autor selbst zu entscheidende Textorganisation und deren Entsprechungen im graphischen Bereich. Generell schwierig für alle Studierenden ist der Umgang mit Wort- oder Satzbeispielen im Text, die Positionierung, Gestaltung und Einbindung von Listen und Tabellen. Dabei hilft oft schon die gemeinsame genauere Betrachtung einiger gelungener Texte, z. B. aus studentischen Hausarbeiten oder Abschlussarbeiten, weiter. 


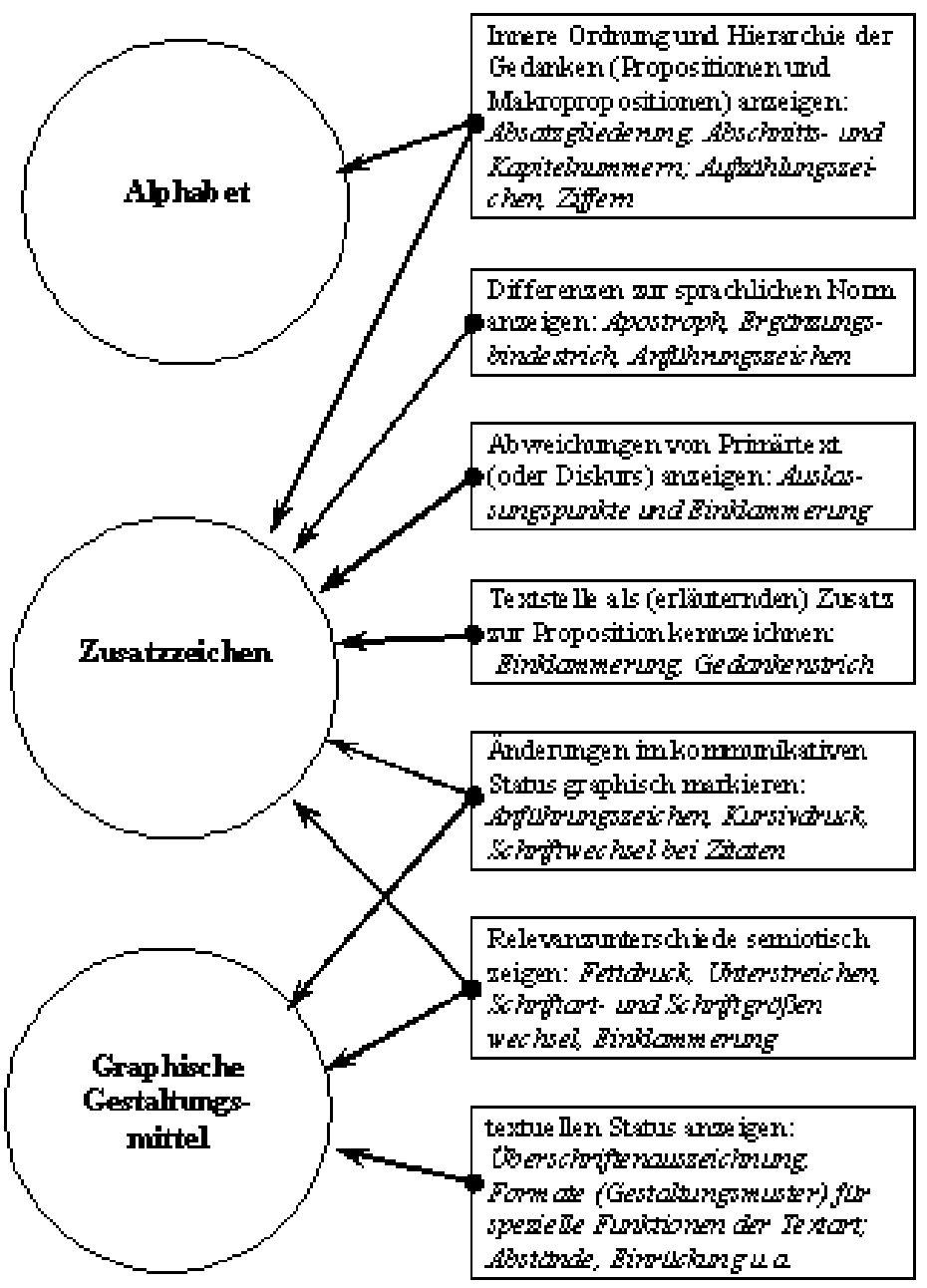

Abbildung 1: Zeichenfunktionen im Bereich der Textorganisation

Zu einem großen Teil der in Abb. 1 aufgeführten Zeichen gibt es Gebrauchsregeln im Duden-RWb, unter den Überschriften „Rechtschreibung und Zeichensetzung“ und „Textverarbeitung und E-Mails“. Die reformierte Rechtschreibung hat dem Regelwerk noch einiges hinzugefügt, z. B. zum Bindestrich. Eine wichtige Grundlage solcher Regeln sind DIN-Normen. Seit längerer Zeit existiert die Norm DIN 5008, die immer wieder aktualisiert wurde und auch weiterhin werden muss, je nach technischen Fortschritten ${ }^{8}$. Ursprünglich war das eine Vorgabe für

\footnotetext{
${ }^{8}$ Mir liegt eine Fassung von Mai 1996, also aus der Zeit vor der Rechtschreibreform, vor.
} 
,Profis` in Journalismus, Verlag und Setzerei. Heute müssen Menschen, die früher mit einer Schreibmaschine ausgekommen wären, recht viel (halb-)professionelles Wissen über Geräte, Software und nebenbei auch über die Schriftsprache, Korrekturzeichen u. a. ansammeln. Das führt auf der Seite der Fachleute, z. B. der Buchdrucker und Lektoren, zu einer Dequalifizierung. Auf der anderen Seite kommt es tendenziell zu der von Ehlich angesprochenen Professionalisierung. Die Verlage praktizieren seit längerem den doppelten Zugriff auf Autoren, die schon längst nicht mehr nur für richtiges Wissen, gute Ideen und gute Formulierungen zuständig sind, sondern daneben auch für eine große Menge an Text- und Bildgestaltungsaufwand, bis hin zum druckfertigen Aufsatz oder Buch. Diese Entwicklung auf dem wissenschaftlichen Buchmarkt schlägt sozusagen nach unten, bis in die Gestaltungsanforderungen an studentische Hausarbeiten, durch. Jakobs betont zu Recht, dass „die Einhaltung schriftsprachlicher Normen als essentielle Anforderung an professionelle Texte“ gilt $(1999,179)$; was genau als schriftsprachlich korrekt gilt, ist zu einem Teil kulturraumabhängig (vgl. a.a.O. S. 179ff).

Man könnte hoffen, dass Studenten über das Lesen von wissenschaftlicher Literatur sich intuitiv Regeln erschließen, wie Jakobs empfiehlt. Wenn aber elementares Wissen fehlt, wird diese Lücke kaum selbständig geschlossen. Solange die Aufmerksamkeit sich auf das für die Bedeutungserschließung Relevante richten muss, bleibt der „Kleinkram“ unbeachtet - was angesichts der Komplexität des lesenden und schreibenden Umgangs mit Sprache nicht erstaunlich ist (vgl. Hasert 1998). Andererseits gibt es viele Studenten, die gewandt mit Computern umgehen und viele Möglichkeiten der Textverarbeitung und des Internets gut nutzen können ${ }^{9}$. In der Schreibdidaktik gab es in den 80-er Jahren die Hoffnung, dass der Computer das fremdsprachliche Schreiben entlasten könnte (Börner 1992). Die große Fülle der Funktionen und Bedienungsmöglichkeiten heutiger Computer erlaubt es aber nicht, sie als schlichtes Schreib-Werkzeug zu gebrauchen. Stattdessen werden immer mehr und immer speziellere Kenntnisse und Fähigkeiten nötig, auch ohne die technischen Potenzen auszuschöpfen.

Im Folgenden bespreche ich exemplarisch Fragen, die im Lehrgang zu klären sind, Zeichen mit ihren Funktionen und mit typischen Schwierigkeiten des Gebrauchs. Der Lehrgang umfasst im Einzelnen: Das Leerzeichen, den Punkt, den Doppelpunkt, das Semikolon, den Apostroph, den Bindestrich, den Ergänzungsbindestrich, den Gedankenstrich und einige weitere Strichvarianten, auch die Anführungszeichen. Von den genannten Zeichen greife ich für die Darstellung hier das Leerzeichen und die wichtigsten Striche heraus. Danach gehe ich noch auf Fragen der Textgliederung ein. Im Anhang dokumentiere ich exemplarisch entsprechende Seiten des Lehrmaterials, dazu auch eine der Übungen. Interessenten,

\footnotetext{
${ }^{9}$ Soweit sie sich an Chat-Kommunikation beteiligen, lernen sie oft eine Fülle von Piktogrammen wie :-), Ideogrammen und speziellen Abkürzungszeichen kennen (inzwischen schon in Wörterbüchern festgehalten). Daran zeigt sich ein gelegentlich witziger und lockerer Umgang mit den erweiterten Möglichkeiten der Schriftsprache.
} 
die das gesamte Material oder Teile davon in einem Sprachkurs oder Tutorium einsetzen wollen, können es (20 Seiten) als PDF-Datei herunterladen von der Website des Instituts für Deutsch als Fremdsprache an der LMU-Muenchen (www.daf.uni-muenchen.de, siehe dort: „Mitglieder / Wissenschaftliche MitarbeiterInnen / Dr. Gabriele Graefen").

\section{Leerzeichen - wo und wozu braucht man Zwischenräume?}

Das Leerzeichen ist einigermaßen paradox. Handelt es sich hier überhaupt um ein Zeichen oder doch eher um ein Nicht-Zeichen? Eine Bedeutung hat es nicht, es trägt aber zur Bedeutungskonstitution bei. Das Leerzeichen (lat. Spatium) ist primär eine für den Leser erfundene Einteilung des Schriftbildes in Wörter. Der Text wird so zerlegt in seine sprachlichen Bausteine, was in der Entwicklung der Schriftkultur historisch ein großer Fortschritt war. Althochdeutsche und mittelhochdeutsche Texte wiesen nämlich noch ein ganz anderes Schriftbild auf, die „scriptio continua“, bei der ohne Wortzwischenräume und zu Beginn auch ohne Satzzeichen oder sonstige Trennzeichen geschrieben wurde. Solche Texte bestanden also tatsächlich nur aus Buchstabenreihen, ohne Unterscheidung von Groß- und Kleinbuchstaben. Hartmut Günther (1998) bietet folgendes Demonstrationsbeispiel:

\section{DIESERSATZISTINDERSCHREIBWEISEANTIKERTEXTEGESCHRIE BENDIEBISINSFRUEHMITTELALTERBEIBEHALTENWURDE}

\section{Abb. 2: Scriptio continua}

Die Wortzwischenräume sind dem Schreiber heute selbstverständlich. Besonders als Leser sind sie ihm auch ein Bedürfnis. Es ist aber auch heute noch nicht selbstverständlich, Wörter durch Spatien zu trennen. In Thailand wird z. B. ohne Wortzwischenräume geschrieben.

Solche Texte wie in Abb. 2 waren früher nur als eine Art „Partitur“ für das laute Vorlesen gedacht, was am besten der Schreiber selbst konnte. Für andere bedurfte es der Übung und Vorbereitung. Im Verlaufe des Mittelalters und danach gab es diverse Verbesserungen der Schreibweise um der Lesbarkeit willen, die in der historischen Schreibforschung ausführlich dargestellt sind (z. B. Raible 1991). Mit der Einführung weiterer Zeichen ergab es sich, dass das Spatium nicht nur zwischen Wörtern stand, sondern auch Konventionen erforderlich machte. Probleme treten dort auf, wo das Leerzeichen vor und nach einem nichtalphabetischen Zeichen auftreten soll. So dient es als Trennzeichen nach Komma, Punkt etc. (aber nicht davor!), vor und nach einer Einklammerung (aber nicht innerhalb der Einklammerung), vor einem Anführungszeichen (aber nicht danach). Damit sind schon einige falsche Verwendungen angedeutet. 
In der Vergangenheit gab es durchaus Beispiele für den Versuch, das Leerzeichen in rhetorischer Absicht anders zu gebrauchen. George B. Shaw setzte an wichtigen Textstellen ganz bewusst Leerzeichen vor einen Doppelpunkt oder ein Semikolon, um mehr Aufmerksamkeit auf diese Zeichen zu ziehen (Truss 2005, 88). Dichter können sich die Freiheit, Zeichen nach ästhetischen Gesichtspunkten zu setzen, nach wie vor nehmen. Die Texte ausländischer Studenten scheinen häufig Schreibgewohnheiten der Handschrift in das Computerschriftbild zu übertragen. Beim Schreiben von Hand gibt es - trotz gewisser Konventionen - noch eine relativ weit gehende Gestaltungsfreiheit, was die Abstände zwischen Zeilen, Wörtern, zwischen Wörtern und anderen Zeichen (z. B. Klammern) angeht. Der Schreiber kann sich selbst entscheiden, ob und wo er ästhetische Gesichtspunkte und Rücksichtnahme auf den Leser einbringt, welche Proportionen er für beides angemessen findet. Sobald er jedoch einen Computer einsetzt, ist seine Handlungsfreiheit radikal eingeschränkt, bzw. es besteht die Gefahr, mit orthographischen Normen zu kollidieren. Auch Deutsche suchen dazu öfter Rat und Hilfe ${ }^{10}$. Einschränkend sind die Normen sicher, willkürlich sind sie allerdings nicht, sie lassen sich begründen. Textverarbeitungssysteme unterwerfen den Schreiber bestimmten, ihnen einprogrammierten Abläufen. Es darf nämlich deshalb kein Leerzeichen vor zusammengehörigen Wortelementen, Ziffern und vor unselbständigen Zeichen stehen, weil sonst Fehler beim Zeilen- und Seitenumbruch passieren würden oder weil missverständliche Leseweisen entstünden. Alle Leerzeichen im Text sind potenzielle Trennstellen. Der Beginn einer neuen Zeile oder Seite kann also auf diese Stelle fallen. Dadurch würden Satzpunkte oder Fragezeichen von „ihren“ Sätzen getrennt, Worttrennungen rutschten in die nächste Zeile, Datumsangaben würden auseinandergerissen, Anführungszeichen verlieren den Kontakt zum Angeführten.

All diese Dinge passieren dann auch tatsächlich in Hausarbeiten und anderen Texten ausländischer Studierender. Daraus habe ich Material für Übungen gewonnen. Gänzlich unsystematischer Gebrauch kommt ebenso vor wie individuell zurechtgelegte, aber abweichende Systematiken. Die genaue Beobachtung von Regeln wird zwar erleichtert durch die Begründung und das Durchschauen der Systematik, aber eine ,Automatisierung" kommt nach meinen Erfahrungen nur dann zustande, wenn die Beachtung des Leerzeichens mithilfe der Übungen eine Zeitlang immer wieder explizit angestoßen wird.

\footnotetext{
${ }^{10}$ Ein häufiges Problem ist das Leerzeichen vor oder nach einem Schrägstrich. Dazu existiert keine ,dogmatische’ Regelung. Laut Duden-RWB (2006, 114) wird ,im Allgemeinen kein Leerschritt angeschlagen“.
} 


\section{Die Striche}

\subsection{Der Schrägstrich (Slash)}

Der in der Schriftsprache älteste Strich ist der Schrägstrich $</>$, zu Beginn „Virgel“ genannt. Er war in den lateinischen Texten des Mittelalters das erste Trennzeichen mit recht universeller Geltung. Es trennte sowohl Abschnitte als auch Sätze oder andere, größere Textteile. Heute erfreut sich das Virgel-Zeichen als „Slash“ einer wachsenden Beliebtheit, vermittelt über sein Vorkommen in Mailoder Web-Adressen und Pfadangaben von Computerdateien. Nicht nur im Englischen (vgl. Weber 1983), sondern auch im Deutschen wird dieser Strich zunehmend häufiger eingesetzt. Weber (a.a.O., 393) sieht in dieser Tendenz eine für die Schriftsprache typische Kondensierung:

The slash is a device in written English that contributes to its distinctive quality. Like other characteristics of written language, constructions with the slash generally serve to integrate meaning in discourse, to use Chafe's (1982) term, making the presentation of information more compressed, complex, and deeply-layered than it usually is in speech.

Unter sprachpflegerischem Aspekt erscheint der Schrägstrich häufig als hässlich, als Verunstaltung des Schriftbildes ${ }^{11}$. Das betrifft weniger die eher technischen Verwendungen, wie etwa die abkürzende Schreibung von Stundenkilometer mit $\mathrm{km} / \mathrm{h}$. Was zunimmt, sind Verwendungen des Striches im Sinne der Konjunktionen und, oder, sowohl - als auch. Nach dem Vorbild der Verkopplung von Jahreszahlen, z. B. 1974/75, bei dem eine zusammenfassende wie eine alternative Lesart möglich ist, werden auch Wörter auf diese Weise verschaltet. Es geht dabei offenbar nicht nur um eine Ökonomisierung beim Schreiben. Die logische Zweideutigkeit des Strichs, die verbal am ehesten noch mit „beziehungsweise“ ausgedrückt werden kann, hat eine offenbar erwünschte Wirkung auf die so kombinierten Begriffe oder Wörter: Ihre Verbindung wird enger, als es mit Konjunktionen der Fall wäre, die Alternative kann Teil eines Begriffsinhaltes werden. Dabei passieren aber auch Fehler. Ein Beispiel dafür stammt aus einem wissenschaftlichen Artikel des Faches Chemie: das Zink/Kupfer-Paar. Eine alternative Lesart ist hier vom Sinn her ausgeschlossen, da das Paar aus beiden Stoffen besteht. Hier wäre also der Bindestrich richtig gewesen. Weitere Belege für Vorkommen in wissenschaftlicher Kommunikation:

Soziologie: ein differenziertes Macht / Gewalt-Konzept Linguistik: der Sprecher / Schreiber

${ }^{11}$ Vgl. z. B. die Sprachtips auf der Internet-Seite www.joern.de. 
Eine weitere moderne Nutzung gibt es im Bereich der männlichen und weiblichen Wortformen, wie bei Arbeiter/in. Jenseits von Fragen der Höflichkeit und der Akzeptanz im Behördendeutsch scheinen sich solche Schreibweisen durchzusetzen. Die Duisburger Schreibwerkstatt macht dazu gleich auf einen häufigen Fehler aufmerksam: Substantive wie Kollege/Kollegin lassen sich auf diese Weise nicht abkürzend schreiben. Der Schrägstrich kann nur so gelesen werden, dass die Endung ohne weitere Wortveränderung angehängt wird.

\subsection{Der Ergänzungsbindestrich}

Eine Kondensierung tritt auch durch den Ergänzungsbindestrich ein, und zwar durch Ausdruckskürzung. Er ist ein weiteres Beispiel für eine auch mündlich mögliche Technik der Einsparung - immerhin gibt es eine Reihe von Wendungen wie bin- und herlaufen -, die schriftsprachlich viel mehr ausgebaut werden (kann). Schon bei der Ein-und Ausgang ist mündliche Realisierung unwahrscheinlich, obwohl das Beispiel ähnlich alltagssprachlich zu sein scheint wie bin- und herlaufen. Durch den Ergänzungsbindestrich wird dem drohenden Missverständnis entgegengewirkt, der unvollständige Wortteil sei schon das ganze Wort. So könnte eine ganz schlichte und plausible Begründung lauten. Die Sache ist jedoch raffinierter, da sie in Stilfragen hineinreicht. Bei der Verwendung dieses Strichs ist nämlich unterstellt, dass dem Schreiber wie dem Leser Wiederholungen zuwider sind. Ein Autor kann durch Kennzeichnung die Unschönheit der Wiederholung vermeiden, ohne missverstanden zu werden. Das Verwendungsmotiv ist also nicht die in der Fachsprachenforschung so beliebte „Ökonomisierung“ (Einsparung von Schreib- und Leseaufwand) ${ }^{12}$, sondern die Verbesserung des eigenen Stils, mit Blick auf einen ,anspruchsvollen` Leser. Dem Schreiber eröffnet sich sogar die Möglichkeit zu rhetorischen Kunstgriffen, wie ein authentisches Beispiel aus einem Magazin der Hochschulrektorenkonferenz (HRK) zeigt:

Der Bachelor, auf dessen Mehrfachbedeutung pi- und bekannterweise der ehemalige KMK-Präsident Maier ... gern hinwies, ... (HRK-Newsletter 2005)

\subsection{Der Bindestrich}

Der Bindestrich in nicht festen Komposita bietet einige Schwierigkeiten, durchaus auch für Deutsche. In funktionaler Hinsicht besteht die Schwierigkeit, genau zu verstehen, warum und wann er erforderlich ist. In formaler Hinsicht haben die

\footnotetext{
12 Coulmas $(1993,109)$ führt dazu aus, dass in Schriftsystemen zwar gelegentlich „,naturwüchsige ökonomische Entwicklungen“ festzustellen sind, aber viele Systeme im Laufe ihrer Entwicklung auch entgegengesetzte Tendenzen zeigten, dass jedenfalls „ökonomische Motive in ihrer Entwicklung nicht uneingeschränkt zum Tragen kommen“ (a.a.O., 110). Zudem neigen Schriftsysteme zur Kompensation für den Verlust von Merkmalen der Mündlichkeit.
} 
Deutschen meist kein Problem, wohl aber viele Deutschlerner: Hier gilt die strikte Regel, dass keine Leerzeichen vor oder nach dem Bindestrich stehen dürfen: $<$ abc-abc $>$. Dies ist eine der am häufigsten gebrochenen Regeln, nach meiner Erfahrung.

Was die Funktionalität des Bindestrichs betrifft, müssen ein paar linguistische Kenntnisse über Wörter eingebracht werden. Das Deutsche ist reich an Komposita, die Bildungsmöglichkeiten sind vielfältig. Warum gibt es neben „echten“ Komposita auch Kompositionen mit Bindestrich? Zwei Differenzierungen spielen hier eine Rolle. Entweder ist ein Bestandteil linguistisch von einer anderen Qualität, d.h. er ist kein deutsches Wort, sei es eine Ziffer, eine Abkürzung, ein Buchstabe oder ein Fremdwort. Die Wortbildungslehre (z. B. Fleischer/Barz 1995, 142) macht detaillierte Angaben, wann die Verständlichkeit eines Wortes durch einen Bindestrich gefördert werden muss oder kann. Der andere Fall ist die Bildung eines nicht lexikalisierten mehrteiligen Nomens, z. B. durch Nominalisierung einer Wortgruppe wie etwa „das Auf-die Spitze-Treiben“".

Im Umgang mit solchen Bildungen entsteht häufig der Fehlschluss, bei langen, mehrgliedrigen Wörtern müsse grundsätzlich ein Bindestrich stehen, weil sie schwer überschaubar seien. Das ist aber falsch. An Beispielen wie Unfallversicherungsgesetz und Krafffahrzengsteuererbebung lässt sich zeigen, dass sie wie andere Determinativkomposita zu schreiben sind.

\subsection{Der Gedankenstrich}

Der Gedankenstrich kennzeichnet, allgemein gesagt, einen Bruch oder Abbruch auf propositionaler oder syntaktischer Ebene oder einen Bruch in der Textstruktur. Wissenschaftssprachlich hat er besondere Bedeutung für die Abgrenzung von Parenthesen oder Einschüben. Es ist merkwürdig, dass im Duden-RWb die nur bedingt richtige Kennzeichnung „Zusätze und Nachträge“ gewählt wird (2005, 48). Von den genannten Gebrauchsweisen sind einige für wissenschaftliches Schreiben nicht bedeutsam; die Parenthese wird dagegen kaum verdeutlicht. Ludger Hoffmann hat (1998) die schwierige Grammatik der Parenthese untersucht. Solche Einschübe sind zwar funktional selbständig, werden aber inhaltlich in der Verarbeitung der Gesamtäußerung eng an den Trägersatz angebunden bzw. in ihn „installiert“ und sorgen für eine, Verdichtung' des Gesagten oder eine Entfaltung des Trägerausdrucks. Bei einem Nachtrag, z. B. einem angehängten weiterführenden Relativsatz, findet keine Installation statt, der Gedankenstrich deutet möglicherweise etwas Unerwartetes an. Die Erläuterung des Gedankenstrichs im Schriftsprachlehrgang für ausländische Studierende kann genutzt werden, um das operative Mittel der Parenthese an geeigneten Beispielen zu demonstrieren ${ }^{13}$.

\footnotetext{
13 Ich verwende hierzu authentische Beispiele und einen Textauszug aus einem MatDaF-Band, in dem Gedankenstriche (Parenthesen) mehrfach schlecht, verständniserschwerend, eingesetzt werden.
} 
Die Darstellung im Duden-RWb ist $\mathrm{m}$. E. auch darin kritikabel, dass mehrfach gesagt wird, der Gedankenstrich sei oft oder meistens ebenso gut durch andere Zeichen, etwa ein Komma, ersetzbar. Das mag rein orthographisch stimmen, ist aber etwas ignorant. Denn wenn es einem Autor auf den speziellen Effekt (Bruch) des Gedankenstriches ankommt, ist der gerade nicht durch das Komma ersetzbar.

\section{Die Textgliederung: Nummerierung als Beitrag zur Text- organisation}

Längere, stark informationshaltige und reflexionsbedürftige Texte bedürfen einer nachvollziehbaren, d.h. systematisch durchgeführten Gliederung. Dem liegt das Bedürfnis des Lesers nach Portionierung und Hierarchisierung, also nach leichter Überschaubarkeit des Textes, zugrunde. Aus der Lesedidaktik, der kognitiven Psychologie und der „Textwissenschaft“ (van Dijk 1980) ist bekannt, dass sowohl das Verstehen von Texten als auch die Erinnerung an Gelesenes sehr stark an der Bildung von „Makropropositionen“ hängen. Diese mentale Leistung des Lesers kann durch die Textorganisation deutlich begünstigt oder auch erschwert werden. Die Gliederung eines langen Textes in Absätze allein wäre völlig unzureichend. Einteilung in größere Abschnitte (früher gern mit römischen Ziffern überschrieben) und Zwischenüberschriften waren Problemlösungen. In wissenschaftlichen Texten wurde die systematische Ordnung dieser Überschriften zunehmend zum Standard.

Ordnungsmittel sind mehrere Zeicheninventare, Ziffern und Buchstaben. Hartmut Günther hat die Doppelnutzung des Alphabets (1996) historisch und systematisch erläutert. Dadurch, dass das Alphabet eine „geordnete Menge“ ist, deren ,kanonische“ Abfolge von jedem Schriftkundigen als „ABC“ ganz zu Anfang auswendig gelernt wird, erfährt es eine weitere Nutzung als Ordnungssystem, nicht nur in Wörterbüchern und Bibliotheken, sondern auch als Gliederungszeichen-Repertoire (1996, 1568). Verschiedene Inventare sind deshalb nötig, weil die Gliederung mehrere Text-,EEbenen“ abbilden soll. Um das Gesamtrepertoire auszubauen, sind deshalb die verwendeten Buchstaben, auch die römischen Ziffern, in großer und kleiner Variante für je zwei Ebenen in Gebrauch (siehe Tabelle 3). Die Klein-Ausgabe der römischen Ziffern sieht dann so aus:

$<\mathrm{i}$, ii, iii, iv, $\mathrm{v}$...> , parallel zu den Kleinbuchstaben des lateinischen Alphabets.

Die früher entwickelte Vielfalt wird international durch vereinheitlichte Richtlinien (DIN-Norm 5008) seit einiger Zeit eingeschränkt. Aber sie ist international noch vorhanden und nicht nur in den großen Mengen älterer Texte noch als mögliches Vorbild präsent. Tabelle 3 geht von möglichen Ebenen eines Schriftstücks aus (wobei Ebene 3 bei Bedarf weiter gestuft werden kann) und vergleicht die Inventare. Wir haben also nebeneinander: 


\begin{tabular}{|c|c|c|c|c|c|c|}
\hline Ebenen & \multicolumn{3}{|c|}{$\begin{array}{l}\text { unnormiert } \\
\text { (frühere Texte) }\end{array}$} & \multicolumn{3}{|c|}{$\begin{array}{l}\text { normiert (Großteil der } \\
\text { heutigen Texte) }\end{array}$} \\
\hline $\begin{array}{l}\text { oberste Ebene } \\
\text { (z. B. Teile } \\
\text { eines Buches) }\end{array}$ & A & $\begin{array}{l}\text { II } \\
\ldots \\
B \\
\ldots\end{array}$ & $\begin{array}{l}\text { III } \\
\text { C }\end{array}$ & 1. & & 3. \\
\hline $\begin{array}{l}\text { 2. Ebene } \\
\text { (z. B. Kapitel } \\
\text { eines Buches) }\end{array}$ & $\begin{array}{l}1 \\
1 .\end{array}$ & $\begin{array}{l}2 \\
\ldots \\
2 .\end{array}$ & $\begin{array}{l}3 \\
3 .\end{array}$ & 1.1 & $\begin{array}{l}1.2 \\
\ldots\end{array}$ & 1.3 \\
\hline $\begin{array}{l}\text { 3. Ebene } \\
\text { (z. B. Unterkapitel) }\end{array}$ & $\begin{array}{l}1.1 . \\
\text { a) } \\
\text { a. }\end{array}$ & $\begin{array}{l}1.2 \\
\ldots \\
\text { b) } \\
\ldots \\
\text { b. } \\
\ldots\end{array}$ & $\begin{array}{l}1.3 . \\
\text { c) } \\
\text { c. }\end{array}$ & 1.1.1 & $\begin{array}{l}1.1 .2 \\
\ldots\end{array}$ & 1.1 .3 \\
\hline $\begin{array}{l}\text { 4. Ebene textintern } \\
\text { (Aufzählung, Listen) }\end{array}$ & $\begin{array}{l}\text { wie } \\
\text { a. } \\
\text { i. } \\
\text { Spie } \\
\text { dive } \\
\text { zeicl }\end{array}$ & $\begin{array}{l}\text { mit: } \\
\text { a) } \\
\text { 1) } \\
\text { ii. } \\
\text { iv. } \\
\text { rich } \\
\text { ufzc }\end{array}$ & $\begin{array}{l}1 . \\
\text { iii. } \\
\text { gs- }\end{array}$ & $\begin{array}{l}\text { a) } \\
\text { 1) } \\
\text { Spieg } \\
\text { divers } \\
\text { zeich }\end{array}$ & $\begin{array}{l}\text { b) } \\
\ldots \\
2 \text { ) } \\
\ldots \\
\text { triche } \\
\text { tufzäh }\end{array}$ & c) \\
\hline
\end{tabular}

Tab. 3: Gliederungszeichen

Das Hauptargument gegen die Kombination verschiedener Zeichentypen war offenbar die Uneinheitlichkeit der Ebenenkennzeichnung, weiter die Schwierigkeit, ein übersichtliches Inhaltsverzeichnis damit zu arrangieren ${ }^{14}$. Die Kennzeichnung der unterschiedlichen Ebenen im Text selbst gelingt aber gut, u. U. besser als mit den gestaffelten arabischen Ziffern.

Den Vorteilen der Stufengliederung unter Verwendung arabischer Ziffern steht noch ein weiterer Nachteil gegenüber. Häufig stehen die letzte Gliederungszahl und die nachfolgende Überschrift so eng nebeneinander, dass die schnelle Unterscheidung von Ziffern und Buchstaben schlecht gelingt und so die Lesbarkeit gefährdet ist. Das war anders im früheren Rechtschreibwörterbuch des Leipziger Duden-Verlags, das einen abschließenden Punkt vorsah. Dieser gute Vorschlag hat sich im Westen nicht durchgesetzt. Zwar „folgen mindestens zwei Leerzeichen" nach einer Abschnittsnummer, wenn der Schreiber sich an die DIN-Norm hält. In der Praxis fehlt der Abstand meist.

${ }^{14}$ Als Grund wird die unregelmäßige Zeichenbreite genannt. 


\section{Abschließende Bemerkungen}

Die Beschäftigung mit Zeichenformen und -funktionen erfordert eine gewisse Akribie, bis hin zur Pedanterie. Man kommt in Versuchung, Normen und Konventionen mit ihren immer weitergehenden Detailfestlegungen für lästig oder überflüssig zu halten. Andererseits gilt: Eine so entwickelte und ausdifferenzierte Schriftkultur, wie sie in den europäischen und vielen anderen Ländern inzwischen existiert, braucht gerade wegen der vorhandenen Vielfalt der medialen Kommunikation und der Textarten eine gewisse Zuverlässigkeit im Gebrauch ihrer Zeichenrepertoires. Lehrende an Universitäten und Sprachlehrer/innen sollten deshalb kein schlechtes Gewissen entwickeln, wenn sie Normen als Grundlage heutiger Schreibpraxis verdeutlichen.

\section{Literatur}

Börner, Wolfgang: Das Werkzeug „Textverarbeitung“ im fremdsprachlichen Schreiben: Idealkonzeption und Realisierungen. In: Börner, W. / Vogel, Klaus (Hrg.) Schreiben in der Fremdsprache. Bochum: AKS 1992, S. 297-312

Bredel, Ursula: Die Didaktik der Gänsefüßchen. In: Bredel, U. / Siebert-Ott, Gesa / Thelen, Tobias (Hrg.) Schriftspracherwerb und Orthographie. (= Diskussionsforum Deutsch, Bd. 16) Hohengehren: Schneider 2004, 207-239

Coulmas, Florian: Ökonomie der Schrift. In: Baurmann, Jürgen / Günther, Hartmut / Knoop, Ulrich (Hgg.) Homo Scribens. Tübingen: Niemeyer 1993, 95-112

Duden: Die deutsche Rechtschreibung. (24. neubearb. Aufl.) Mannheim :

Dudenverlag 2006

Ehlich, Konrad: Funktion und Struktur schriftlicher Kommunikation. In: Baurmann, Jürgen / Coulmas, Florian / Ehlich, Konrad et al. (Hgg.) Schrift und Schriftlichkeit. Berlin, New York: de Gruyter (HSK 10.1) 1996, S. 18-40

Fleischer, Wolfgang / Barz, Irmhild (1995) Wortbildung der deutschen Gegenwartssprache. 2. Aufl. Tübingen: Niemeyer 1995

Frank, Barbara: Die Textgestalt als Zeichen. Lateinische Handschriftentradition und die Verscbriftlichung der romanischen Sprachen. Tübingen: Narr 1994

Gallmann, Peter: Graphische Elemente der geschriebenen Sprache: Grundlagen für eine Reform der Orthographie. Tübingen: Niemeyer 1985

Glück, Helmut: Schrift und Schriftlichkeit. Stuttgart: Metzler 1987

Gross, Sabine: Lesezeichen, Kognition, Medium und Materialität im Leseprozeß. Darmstadt: Wissenschaftliche Buchgesellschaft 1994 
Günther, Hartmut: Erziehung zur Schriftlichkeit. In: Eisenberg, Peter; Klotz, Peter (Hgg.) Sprache gebrauchen - Sprachwissen enverben. Stuttgart: Klett 1993, 85-96

Günther, Hartmut: Sprachwissenschaft und Sprachdidaktik. Am Beispiel kleiner und großer Buchstaben im Deutschen. (Antrittsvorlesung Köln 25.10.96). In: Didaktik Deutsch, Heft 4/1998

Günther, Hartmut: Schrift als Zahlen- und Ordnungssystem - alphabetisches Sortieren. In: Baurmann, Jürgen / Coulmas, Florian / Ehlich, Konrad et al. (Hgg.) Schrift und Schriftlichkeit. Berlin, New York: de Gruyter (HSK 10.2) 1996, S. $1568-1583$

Günther, Hartmut: „,...und hält den Verstand an“ - Eine Etüde zur Entwicklung der deutschen Interpunktion 1522-1961. In: Thieroff, Rolf / Tamrat, Matthias et al. (Hgg.) Deutsche Grammatik in Theorie und Praxis. Tübingen: Niemeyer 2000, 275-286

Hoffmann, Ludger: Parenthesen. In: Linguistische Berichte 175/1998, 299-328

Jakobs, Eva-Maria: Normen der Textgestaltung. In: Kruse, Otto / Jakobs, Eva-M. / Ruhmann, G. (Hgg.) Schlüsselkompetenz Schreiben. Luchterhand 1999, 171-190

Hasert, Jürgen W.: Fehlermaskierungen beim Schreiben. In: Osnabrücker Beiträge zur Sprachtheorie (OBST) Heft 56/1998, 28-47

Raible, Wolfgang: Zur Entwicklung von Alphabetschrift-Systemen. Heidelberg 1991

(Sitzungsberichte der Heidelb. Akad. der Wiss., Phil.-hist. Klasse, Jg. 1991, 1)

Truss, Lynne: Hier steht was alle suchen. Englische und deutsche Zeichensetzung.

Originalausg. London 2003, Übersetzung: Autorenhaus Verlag Berlin 1995

van Dijk, Teun: Textwissenschaft. Tübingen: Niemeyer 1980

Weber, Rose-Marie: Behind the Slash. In: Visible Language XVII 4/1983, 390-395

Zifonun, Gisela / Hoffmann, Ludger / Strecker, Bruno: Grammatik der deutschen Sprache. 3 Bände. Berlin: New York: de Gruyter 1997 


\section{Anhang (Beispielseiten aus dem Lehrgang)}

1 Das Leerzeichen (lat. Spatium)

Das Leerzeichen (Lz) ist notwendig zur optischen Trennung der einzelnen Wortformen in Texten. Bei nichtalphabetischen Zeichen gibt es Regeln dafür, wo das Leerzeichen stehen soll (siehe Tabelle). Allgemein gilt: Lz sollen so gesetzt werden, dass zusammengehörige Wortteile beim Zeilenwechsel nicht voneinander getrennt werden und unselbständige Zeichen (z. B. Satzpunkt) nicht in die nächste Zeile geschoben werden können.

\begin{tabular}{|c|c|}
\hline Anwendungsfall & Beispiele \\
\hline $\begin{array}{l}\text { nach Interpunktionszeichen: } \\
\text { Satzpunkt, Fragezeichen, Ausru- } \\
\text { fezeichen, Komma, Semikolon, } \\
\text { Doppelpunkt ... }\end{array}$ & $\begin{array}{l}\text { Warte, ich komme gleich. } \\
\text { Das ist nun der Erfolg: Alle sind } \\
\text { beleidigt! }\end{array}$ \\
\hline $\begin{array}{l}\text { Bei Anführungszeichen und } \\
\text { Klammern rahmen zwei Leerzei- } \\
\text { chen den ganzen Ausdruck ein, } \\
\text { wenn kein Satzzeichen folgt. }\end{array}$ & $\begin{array}{l}\text { Er nennt sich „, der Führer“. } \\
\text { Das Datum (Tag / Monat / Jahr) ist } \\
\text { oben anzugeben. }\end{array}$ \\
\hline $\begin{array}{l}\text { Allgemein: Nach einer Ordinal- } \\
\text { zahl (1. = der erste) mit Punkt } \\
\text { folgt ein Leerzeichen. In Sachtex- } \\
\text { ten als Gliederungsziffer vor } \\
\text { einer Überschrift. Dazwischen } \\
\text { steht mindestens ein Leerzeichen. } \\
\text { Ebenso bei: } \\
\text { - Aufzählungszeichen } \\
\text { - Spiegelstrich. } \\
\text { - Gliederungsbuchstaben }\end{array}$ & $\begin{array}{l}\text { 1. Planung } \\
\text { 2. Vorgehensweise } \\
\text { - Planung } \\
\text { - Vorgehensweise } \\
\text { Optionen: } \\
\text { - Verkauf } \\
\text { - Vermietung } \\
\text { a) Verkauf } \\
\text { b) Vermietung }\end{array}$ \\
\hline $\begin{array}{l}\text { zwischen Zahlangabe } \\
\text { und Maßeinheit, } \\
\text { zwischen Paragraphenzeichen } \\
\text { und Ziffer }\end{array}$ & $\begin{array}{l}120 \mathrm{~km}, 5 \mathrm{~kg}, 10 \mathrm{l} \text { (=Liter }) \\
10 \%, \\
\S 5\end{array}$ \\
\hline
\end{tabular}


nach Abkürzungen, die mit einem Punkt abgeschlossen sind.

Achtung: Einige früher mit Lz geschriebene Abkürzungen sind optisch ,zusammengewachsen“!

Lz steht nach dem Ergänzungsstrich, vor und nach dem Gedankenstrich (siehe unten),

Kein Lz in einer Bibliographie

- vor Fußnotenzeichen oder Auflagenziffern (hochgestellt)

- beim „bis-Strich“

- vor der Abkürzung f./ff. (für: folgende Seite(n) beziehungsweise: $\quad b z w$. und so weiter: usw.

z. B. z. T. d.h.

mit anderen Worten: m.a.W unter Anderem: u. $A$.

Personen- und Sachregister

Das Gesetz wird - wie geplant - im

Januar verabschiedet.

\section{Bindestrich und Ergänzungsstrich}

Der Bindestrich verbindet Wörter und andere bedeutungstragende Elemente direkt (= ohne Leerzeichen) miteinander. Dadurch entstehen keine echten Komposita, sondern eher lockere Ausdrucks-Kompositionen, Gele-

genheitszusammensetzungen.

Der Ergänzungsstrich ersetzt einen Wortteil, der sonst innerhalb derselben Wortgruppe wiederholt werden müsste (der „Rationalisierungsfachmann“ unter den Zeichen, nach Lynne Truss). Er zeigt also diese Auslassung an.

Typographie: Beide Striche entsprechen in der Form dem Trennstrich.

\begin{tabular}{l|l}
\multicolumn{1}{c|}{ Komposition mit Bindestrich } & \multicolumn{1}{|c}{ Alternativen obne Bindestrich } \\
\hline Zusammensetzung mit Zif- & \\
fern: & vierrädrig \\
4-rädrig, 1-kg-Dose & bundertprozentig \\
100-prozentig & \\
\hline Zusammensetzung mit & \\
Abkürzung: & Diplomingenieur \\
Dipl-Ing. & Fußballweltmeisterschaft \\
Fußball-WM &
\end{tabular}




\begin{tabular}{|c|c|}
\hline $\begin{array}{l}\text { Zusammensetzung mit an- } \\
\text { dersartigen Bestandteilen: } \\
\text { weil-Sat₹, dass-Satz etc. } \\
\text { Ich-Eræäblung }\end{array}$ & Nebensatz. \\
\hline $\begin{array}{l}\text { Zusammensetzung mit } \\
\text { Buchstaben: } \\
\text { der s-Laut, das a-Element etc. } \\
\text { die A-Dur-Tonleiter }\end{array}$ & Sprachlaut \\
\hline $\begin{array}{l}\text { in Wortgruppen, die als } \\
\text { Ganzes nominalisiert wer- } \\
\text { den: } \\
\text { Mund-zu-Mund-Beatmung } \\
\text { Arzt-Patient-Verbältnis } \\
\text { Das ist ja zum Aus-der-Haut- } \\
\text { Fabren! }\end{array}$ & $\begin{array}{l}\text { Beatmung von Mund zu Mund } \\
\text { das Verbältnis von Arzt und Patient } \\
\text { aus der Haut fahren }\end{array}$ \\
\hline $\begin{array}{l}\text { Zusammensetzung mit } \\
\text { Fremdwort: } \\
\text { Abend-Make-up } \\
\text { Software-Haus }\end{array}$ & \\
\hline $\begin{array}{l}\text { Zusammensetzung von und } \\
\text { mit Namen: } \\
\text { Max-Planck-Institut } \\
\text { Baden-Württemberg } \\
\text { das Deutsche-Bank-Konto }\end{array}$ & Müllerstraße \\
\hline $\begin{array}{l}\text { zwischen hierarchisch } \\
\text { gleichgestellten Adjektiven } \\
\text { (kopulativ): } \\
\text { wissenschaftlich-technisch, geistig- } \\
\text { moralisch } \\
\text { schwarz-rot-golden }\end{array}$ & \\
\hline $\begin{array}{l}\text { zur Verdeutlichung, zur Ver- } \\
\text { meidung von Missverständ- } \\
\text { nissen oder um eine Zusatz- } \\
\text { bedeutung zu erzielen. } \\
\text { Beispiele: } \\
\text { re-integrieren (Wortgliederung } \\
\text { klarer!) } \\
\text { ein guter Vor-Satz. } \\
\text { Dies war eine Hoch-Zeit des } \\
\text { deutschen Kolonialismus. }\end{array}$ & $\begin{array}{l}\text { übliche Schreibungen: } \\
\text { reintegrieren } \\
\text { Vorsatz: } \\
\text { Hochzeit }\end{array}$ \\
\hline
\end{tabular}




\begin{tabular}{l|l}
$\begin{array}{l}\text { zur Gliederung } \\
\text { von langen Zahlenreihen: } \\
\text { ISBN 3-411-00911-X }\end{array}$ & $\begin{array}{l}\text { Bei Telefon- und Kontonummern } \\
\text { kann auch ohne Gliederung geschrie- } \\
\text { ben werden }\end{array}$ \\
\hline $\begin{array}{l}\text { Ergänzungsstrich, } \\
\text { auch „Ersparungsbinde- } \\
\text { strich“: }\end{array}$ & $\begin{array}{l}\text { der Eingang und (der) Ausgang } \\
\text { bin- und herlaufen, der Ein- und } \\
\text { Ausgang, vor- und rückwärts }\end{array}$
\end{tabular}

3 Weitere Striche: Gedankenstrich - Trennstrich - Schrägstrich

Gedankenstrich: Allgemein steht er, wenn es einen Wechsel im Satz- oder Textdurchlauf gibt. (Er hieß früher auch „Pausestrich“) Er tritt auf bei einer Unterbrechung des Satzes, bei Sprecherwechsel oder Satzabbruch. Oft ist damit ein Wechsel in der Gedankenführung des Autors verbunden. Besonders charakteristisch ist die Abgrenzung eines Einschubs in den Satz (eine Parenthese) oder eine überraschende Fortsetzung des Satzes.

Typographie: Der Gedankenstrich ist (wenn technisch möglich) länger als der Trennstrich. In englischen Texten findet man sogar zwei Striche: $<-->$, um die Länge zu verdeutlichen. Vor und nach dem Gedankenstrich steht ein Leerzeichen oder ein Satzzeichen (Komma, Punkt...).

Der bis-Strich und der gegen-Strich sollen ebenfalls längere Striche sein, in der Form also dem Gedankenstrich gleich. Ein Unterschied besteht bei den Leerzeichen: in der Bedeutung „bis“ ohne Leerzeichen, bei „,gegen“ allgemein mit Leerzeichen. Trennstrich: Er dient zur Worttrennung am Zeilenrand (Funktion: Randausgleich). Die Trennstellen der Wörter sind orthographisch geregelt (einige Änderungen in der Rechtschreibreform!). Er wird stets ohne Spatium an den Wortteil angeschlossen, der am Zeilenrand steht. Form: kurzer Strich.

Der Schrägstrich war früher als „Virgel“ bekannt und entspricht am besten dem deutschen Ausdruck ,,beziehungsweise“, der die Alternative und/oder enthält. 


\begin{tabular}{|c|c|}
\hline $\begin{array}{l}\text { Bedeutung oder Funktion } \\
\text { des Strichs }\end{array}$ & Beispiele \\
\hline $\begin{array}{l}\text { Gedankenstrich } \\
\text { - markiert Einschub (Parenthese) } \\
\text { - zeigt überraschende Fortsetzung } \\
\text { der Äußerung an } \\
\text { - zeigt Sprecherwechsel } \\
\text { - zeigt Themenwechsel }\end{array}$ & $\begin{array}{l}\text { „Wir befürchten - boffentlich zu Unrecht-, } \\
\text { dass ..." } \\
\text { „Komm doch einfach mit ins Konzert- } \\
\text { oder hast du schon etwas anderes vor?" } \\
\text { Kommst du mit? - Ja, gern! } \\
\text { So weit die Regeln für die römischen Zif- } \\
\text { fern. - W as die arabischen Ziffern betrifft } \\
\text {.., }\end{array}$ \\
\hline $\begin{array}{l}\text { gegen-Strich (zu lesen als ,ge- } \\
\text { gen“) } \\
\text { mit Spatien zu schreiben }\end{array}$ & Das Fußballspiel Bochum - Frankfurt \\
\hline $\begin{array}{l}\text { bis-Strich (zu lesen als „,von - } \\
\text { bis") } \\
\text { meist ohne Leerzeichen }\end{array}$ & $\begin{array}{ll}\text { Vortrag: } & 10-12 \mathrm{Ubr} \\
\text { aber: } & 10: 00-11: 15 \mathrm{Ubr} \\
\text { und: } & \text { von } 10 \mathrm{bis} 12 \mathrm{Ubr} \\
& \text { 17.-19. Jh. } \\
& \text { (vom 17. bis zum 19. Jh.) }\end{array}$ \\
\hline $\begin{array}{l}\text { Strecken-Strich } \\
\text { (zu lesen als „,von - nach“) }\end{array}$ & der Zug Stuttgart - Berlin \\
\hline $\begin{array}{l}\text { Schrägstrich im Sinne von } \\
\text { „ pro“, je }\end{array}$ & 10 Liter $/ \mathrm{km}, 120 \mathrm{~km} / \mathrm{h}$ \\
\hline $\begin{array}{l}\text { Schrägstrich im Sinne von „bzw.“ } \\
\text { (und/oder), teils, teils, sowohl als } \\
\text { auch }\end{array}$ & $\begin{array}{l}\text { Männer und/oder Frauen } \\
\text { Er/sie trifft seinen/ibren Vater. } \\
\text { im Zeitraum 1981/1982 }\end{array}$ \\
\hline $\begin{array}{l}\text { Schrägstrich zwischen den Na- } \\
\text { men von Autoren und Koopera- } \\
\text { tionspartnern; } \\
\text { Achtung: Anders bei Firmenna- } \\
\text { men, hier steht das kaufmänni- } \\
\text { sche Zeichen }<\&>\end{array}$ & $\begin{array}{l}\text { Die Grammatik von Helbig/Buscha } \\
\text { Die Parteien CDU/CSU }\end{array}$ \\
\hline $\begin{array}{l}\text { Schrägstrich zur Trennung von } \\
\text { Bruchzahlen im Text }\end{array}$ & $\begin{array}{l}3 / 4 \\
3 / 4\end{array}$ \\
\hline $\begin{array}{l}\text { Der Trennstrich dient zur Auftei- } \\
\text { lung längerer Wörter am Zeilen- } \\
\text { rand. Er ist weitgehend auf } \\
\text { Sprechsilben bezogen, die ortho- } \\
\text { graphische Regelung steht im } \\
\text { Rechtschreibwörterbuch }\end{array}$ & $\begin{array}{l}\text { Freun- } \mid \text { des- } \mid \text { kreis } \\
\text { ein- } \mid \text { wand- } \mid \text { frei } \\
\text { ent- } \mid \text { zü- } \mid \text { ckend }\end{array}$ \\
\hline
\end{tabular}


4 Übung zum Bindestrich (auch als Diktat möglich)

Welche der folgenden zusammengeschriebenen Wörter sind echte Komposita, welche davon brauchen Bindestrich oder Ergänzungsstrich, und wo genau?

- $\quad$ einfach, 22fach, Schreibtischfach

- $\quad$ schwaryrotgolden, pechschwarz, deutschschweizerisch

- Klimaanlage, Zooordnung, Seeelefant, Bauausstellung

- Trimmdichpfad, Heldengedenktag, Gutenachtgeschichte

- Mehraweckekïchenmaschine, Straßenbahnfahrplan

- Fremdsprachenlehrundlernforschung

- $\quad$ Druckeræengnis (!?), Computertastaturreinigung

- Angestelltenunfallversicherung

- $\quad$ schaurigschön, schwerkrank

- Ubahn, Email, Deutschebahnsonderangebot

- Hardwareundsoftwareausstattung, Gepäckannabmeundausgabe

- $\quad$ KFZpapiere, Fabrzengpapiere

- das Septemberoktoberheft

- $\quad 5 \%$ Klausel, Fünfprozentklausel

- Textilgroßundeinzelhandel 



\section{Die wissenschaftliche Seminararbeit: Stolperstein für chinesische Studierende?}

Jin Zhao, Shanghai / Jena

Antje Stork, Marburg

\section{$1 \quad$ Einleitung}

Seit der Öffnungspolitik Deng Xiaopings im Jahre 1978 gehen immer mehr Chinesen zu Studienzwecken ins Ausland. Deutschland ist dabei laut einer Befragung von Madl (2002) ${ }^{1}$ in Europa (nach Großbritannien) das zweitbeliebteste Studienland. Als Begründung wird dabei am häufigsten die Qualität der Hochschulausbildung genannt, wobei wissenschaftliche Tradition, hohes Ausbildungsniveau, Hochtechnologie, akademische Forschung und ein sehr gutes Ausbildungssystem erwähnt werden (vgl. Madl 2002: 194f.). Die Zahl der Chinesen, die zum Auslandsstudium nach Deutschland kommen, ist insbesondere seit der Jahrtausend-

\footnotetext{
${ }^{1}$ Befragt wurden mittels ,face-to-face“-Interviews 175 Studierende der Universität Peking im Zeitraum vom 28. Oktober bis 22. Dezember 2000.
} 
wende so rasant gestiegen ${ }^{2}$, dass sie nunmehr den größten Anteil unter den ausländischen Studierenden in Deutschland darstellen (vgl. Blume 2002: 1, Meng 2005: 15).

Ein erfolgreicher Studienabschluss ist aber für chinesische Studierende in Deutschland besonders schwierig zu erreichen, und zwar auch im Vergleich zu den übrigen ausländischen Studierenden aus anderen Herkunftsländern. Während die Studienerfolgsquote bei deutschen Studierenden bei 70 Prozent liegt, erreichen laut DAAD (Deutscher Akademischer Austauschdienst o.J.: 6) nur 50 Prozent der ausländischen Studierenden einen Studienabschluss. Diese Zahlen veranlassten den DAAD, in seinem dritten Aktionsprogramm Maßnahmen zu ergreifen, die dafür sorgen sollen, die Erfolgsrate von ausländischen Studierenden bis zum Jahr 2010 zu verbessern. Die Erfolgsrate chinesischer Studierender ist allerdings noch wesentlich erschreckender und liegt bei nur 30 Prozent: Nach Meng (2005: 292) beenden zwei Drittel der chinesischen Studierenden in Deutschland ihr Studium ohne einen deutschen akademischen Abschluss. Studienabbrüche - vor allen Dingen in diesen immensen Dimensionen - dürfen aber nicht nur das individuelle Problem der jeweils Betroffenen sein. Sie stehen auch den deutschen Interessen der verstärkten Internationalisierung der deutschen Hochschulen entgegen.

Über die Gründe für den geringen Studienerfolg kann derzeit mangels Untersuchungen zu Studienverläufen und Studienabbrecherbiographien (vgl. Ehlich 2004: 10) sowohl für ausländische Studierende im Allgemeinen als auch für chinesische Studierende im Besonderen nur spekuliert werden. Schreiben spielt im Studium in Deutschland eine studienbestimmende Rolle (Steets 2001: 213). Während die kleineren Textarten Mitschrift und Exzerpt den Studienalltag prägen, nimmt die Hausarbeit aufgrund ihrer Rolle als Qualifikationsnachweis einen hohen Stellenwert ein: Eine Umfrage von Ehlich/Steets (2003b) unter Professorinnen und Professoren der Universität in München hat ergeben, dass 48 Prozent der Lehrenden im Grundstudium und 62 Prozent der Lehrenden im Hauptstudium eine Hausarbeit als Leistungsnachweis verlangen. Die Angaben schwanken dabei erwartungsgemäß stark in Hinblick auf die verschiedenen Disziplinen: An den philosophischen Fakultäten ${ }^{3}$ haben sie einen höheren Stellenwert (86 Prozent im Grundstudium, 95 Prozent im Hauptstudium) als in den Naturwissenschaften ${ }^{4}$ (19 Prozent im Grundstudium, 36 Prozent im Hauptstudium). In den Wirtschaftswis-

\footnotetext{
${ }^{2}$ Die Zahl der immatrikulierten chinesischen Studenten an deutschen Universitäten betrug im Wintersemester 2001/02 13.500, die um 50\% im Vergleich zum Vorjahr zugenommen hatte, wobei sich noch 7.000 chinesische Sprachschüler in Deutschland aufhielten (vgl. Blume 2002:1). Im Jahr 2004 gab es laut eines Vortrags des damaligen Bundespräsidenten Johannes Rau an der TsinghuaUniversität 14.000 chinesische Studenten in Deutschland (vgl. China-Botschaft: heruntergeladen am 23.08.2005).

${ }^{3}$ Ehlich/Steets (2003b: 131) fassen darunter folgende Fakultäten zusammen: Philosophische Fakultät für Geschichts- und Kunstwissenschaften, Fakultät für Philosophie, Wissenschaftstheorie und Statistik, Philosophische Fakultät für Altertumskunde und Kulturwissenschaften, Philosophische Fakultät für Sprachund Literaturwissenschaft I, Philosophische Fakultät für Sprach- und Literaturwissenschaft II.

${ }^{4}$ Unter Naturwissenschaften werden die Fakultät für Physik, die Fakultät für Chemie und Pharmazie, die Fakultät für Biologie und die Fakultät für Geowissenschaften gefasst.
} 
senschaften ${ }^{5}$ werden vor allem im Hauptstudium häufig Hausarbeiten verlangt (23 Prozent im Grundstudium, 79 Prozent im Hauptstudium). Die wissenschaftliche Hausarbeit hat somit - vor allem in den Geisteswissenschaften, aber auch in den anderen Disziplinen - einen hohen Anteil am Erfolg bzw. Misserfolg des Studiums.

Die Schwierigkeiten, die chinesische Studierende beim Anfertigen von schriftlichen Hausarbeiten haben, stellt ein 24-jähriger männlicher Student der Betriebswirtschaftslehre (6. Semester) in einem Interview ${ }^{6}$ im Rahmen einer von uns durchgeführten Vorstudie zur Entwicklung der interkulturellen Kompetenz chinesischer Studierender (vgl. Stork/Zhao, im Druck) folgendermaßen dar:

Was ich jedoch fürchte, sind das Schreiben von Seminararbeiten und die Präsentation, die ich noch nicht gemacht habe, und ich weiß nicht, wie ich das im nächsten Semester hinkriegen kann. Bisher habe ich nur Klausuren geschrieben. Wir Chinesen fürchten keine (schriftliche) Prüfung, aber das Verfassen von Seminararbeiten. Meine deutschen Mitstudenten schreiben doch anders als ich, finde ich wirklich gut. Ich denke, mir fehlen Fachterminologie und eigene Stellungnahmen. Meine Methode dagegen ist nun, viel zu lesen und auswendig zu lernen. Natürlich habe ich bereits eine Hausarbeit mit zwei anderen (einem Deutschen und einem aus einem anderen Land) zusammen geschrieben, dabei sollten wir diskutieren, ob eine Firma pleite wird oder nicht. An Anfang war ich auch ratlos, Gott sei Dank ist es schon vorbei. Dabei habe ich aber viel gelernt: Was uns chinesischen Studenten am Herzen liegt, ist das Ergebnis, ob die Firma wirklich in Konkurs geht oder nicht. Dagegen ist das Ziel des Lehrers, unsere Analysefähigkeit zu trainieren, er legt mehr Wert auf den Analyseprozess.

Ihre Ursache haben die von dem chinesischen Studenten geschilderten Probleme in sprachlichen Defiziten, aber vor allem auch in fremden Schreibkonventionen. Im Folgenden werden wir die kulturspezifischen Unterschiede bei wissenschaftlichen Hausarbeiten anhand ihrer Funktion (Abschnitt 2), Struktur (Abschnitt 3) und sprachlicher Gestaltung (Abschnitt 4) beschreiben. Da es bisher noch keine entsprechenden Vorarbeiten zum deutsch-chinesischen Vergleich von wissenschaftlichen Hausarbeiten gibt, können in diesem Rahmen die kulturspezifischen Unterschiede allerdings lediglich grob skizziert werden. An die Analysen schließen sich Vorschläge an, die auf eine Verbesserung der Studierfertigkeit (insbesondere in Bezug auf wissenschaftliche Hausarbeiten) der chinesischen Studierenden zielen.

\footnotetext{
5 Zu den Wirtschaftswissenschaften gehören folgende Fakultäten: Fakultät für Betriebswissenschaft, Volkswirtschaftliche Fakultät, Forstwirtschaftliche Fakultät.

${ }^{6}$ Das Interview wurde in chinesischer Sprache geführt; eine von der Interviewerin angefertigte Gesprächszusammenfassung wurde dem Interviewten mit der Bitte um Überprüfung bzw. Korrektur vorgelegt (kommunikative Validierung) und daraus Übertragungen in die deutsche Sprache angefertigt.
} 


\section{Funktion der wissenschaftlichen Hausarbeiten im Vergleich}

Nach Lutz von Werder (1993: 18) stellt das wissenschaftliche Schreiben „die höchste Stufe des Schreibprozesses“ dar, wobei Ansätze dazu zunächst in der Antike existierten und dann wieder seit der Renaissance. Die wissenschaftliche Hausarbeit ${ }^{7}$ ist innerhalb des wissenschaftlichen Schreibens eine Textsorte, in der Studenten den wissenschaftlichen Artikel einüben sollen, wobei sie allerdings im Allgemeinen - da sie nicht zur Veröffentlichung bestimmt ist - für einen simulativen Leser geschrieben wird (vgl. Ehlich 2003: 21) und zumeist eine Kommunikation von neuem Wissen unterbleibt (vgl. Guckelsberger/Stezano 2004: 419). Die Anforderungen an eine Vergleichbarkeit von wissenschaftlicher Hausarbeit und wissenschaftlichem Artikel steigen mit dem Übergang von der Proseminararbeit zur Hauptseminararbeit an und sind gradueller $\mathrm{Art}^{8}$.

Wissenschaftliche Texte unterscheiden sich in den unterschiedlichen Wissenschaftskulturen und so wird seit einigen Jahren vermehr auf ihre Kulturgebundenheit verwiesen (vgl. bspw. die Beiträge in Krumm 2000, Ehlich/Steets 2003a). Einen Einfluss auf die Herausbildung der Kulturspezifik haben die jeweilige Geistes- und Philosophiegeschichte, Aufsatzerziehung und Rhetorik (vgl. Graefen 1997: 65ff.) sowie die Organisationsform von Universitäten (z. B. wissensinnovative versus wissenskonservative Universitäten, Ehlich 2003: 13ff.) ${ }^{9}$.

In China sind - im Gegensatz zu Deutschland, wo die Einheit von Forschung und Lehre (Humboldt) betont wird - wissenskonservative Universitäten vorherrschend. Zur Tradierung des Wissens standen und stehen Prüfungen in der chinesischen Kultur im Vordergrund. Letztendlich diente das Prüfungssystem in der chinesischen Geschichte zur Rekrutierung von Beamten sowie zur Überlieferung des Wertes eines Studiums von Klassikern. Auch in der Ausbildung im heutigen China spielt dieses Prüfungssystem immer noch eine herausragende Rolle.

Anders als in Europa, wo das philosophische Interesse sowohl in der Zeit der Antike als auch seit der Renaissance hauptsächlich im Erkenntnisproblem liegt, wandte sich das altchinesische Denken eher dem Gesellschaftsproblem wie dem praktischen Handeln und dem menschlichen Gemeinschaftsleben zu (vgl. Störig 2004: 29). Dementsprechend standen im Prüfungscurriculum in der Kaiserzeit ${ }^{10}$ neben der Dichtungskunst die Auslegung der konfuzianischen Klassiker sowie die darauf basierende Stellungnahme zu zeitgeschichtlichen und tagespolitischen Themen (vgl. Chaffee 1985: 66fff.). Dies verlangte von den Prüflingen jahrelanges

\footnotetext{
${ }^{7}$ In der Literatur werden verschiedene Bezeichnungen für diese Textsorte gebraucht, z. B. studentische wissenschaftliche Arbeit, Seminararbeit, verschriftlichtes Referat.

8 Aus diesem Grund verzichten wir an dieser Stelle auf eine Unterscheidung von akademischem und wissenschaftlichem Schreiben wie sie Jakobs (1999: 173) und Dittmann/Geneuss/Nennstiel/Quast (2003: 157) vornehmen.

9 Zu den Einflussfaktoren vgl. Graefen (1997: 65ff.) sowie Esser (1997: 81ff.).

${ }^{10}$ Das Prüfungssystem zur Rekrutierung der Beamten stammte aus der Sui-Dynastie (581-618), entwickelte sich in der Song-Zeit (960-1279) und reifte in der Ming- (1368-1644) bzw. QingDynastie (1644-1911). Abgeschafft wurde es im Jahr 1905 (vgl. Wang 1988).
} 
Studieren und Auswendiglernen der konfuzianischen Klassiker in Dorfschulen bzw. an der Reichsuniversität, denn gute Prüfungsleistungen zu erzielen und schließlich Beamte zu werden war der Sinn und Zweck des jahrelangen Fleißes. Die dominierende Prüfungsform in der Geschichte war eine Art Essay namens „ba-gu wen“ (achtgliedriger Aufsatzstil), dessen Textaufbau festgelegt war (vgl. Lee 1985: 154) und weit von dem der deutschen wissenschaftlichen Arbeit entfernt ist.

Auch an den heutigen chinesischen Schulen und Universitäten wird vorwiegend Wissen über Fakten und Daten vermittelt; die Lernleistungen werden weiterhin in Form von Tests und Prüfungen festgestellt. Wissenschaftliche Arbeiten werden nicht als Textsorte in der Ausbildung thematisiert und wissenschaftliche Hausarbeiten selbst an den Hochschulen nicht als Ausbildungsform angesehen und von den Studierenden geübt. Die wissenschaftlichen Publikationen, deren Auffassung die Akademiker normalerweise durch Nachahmen autodidaktisch erlernen, unterliegen keiner strengen Form und Norm und vom Stil her sehen sie auch anders aus als deutsche wissenschaftliche Arbeiten (s. Abschnitt 3 und 4).

Im chinesischen Deutschunterricht und im Deutschkurs zur Vorbereitung der chinesischen Studierenden auf das Studium in Deutschland werden bisher die deutschen universitären wissenschaftlichen Hausarbeiten als Teil der Leistungsbewertungsform nicht ausreichend vorgestellt oder gar vernachlässigt. Oft haben die Chinesen, die sich neu an einer deutschen Hochschulen eingeschrieben haben, keine Ahnung davon, was „Seminar“", „Referat“ oder „Hausarbeit“ bedeuten (vgl. Li 2002: 22), so dass sie nicht selten wie unser Interviewpartner „ratlos" sind oder „fürchten“, wenn sie eine Hausarbeit schreiben müssen. Lange Zeit wurde und wird Schreiben im Deutschunterricht in China lediglich als Übungsmethode zur Verbesserung der zielsprachlichen Kenntnisse angewendet (vgl. Luge 1993: 140), um beispielsweise Grammatik oder Wortschatz zu üben. Auch in der neusten Zeit berichteten noch Studenten, dass sie im Deutschunterricht nicht viel schreiben, außerdem seien die Schreibaufgaben nicht komplex (vgl. Zhao 2005: 16).

Eine programmatische Ansichtsänderung gegenüber Schreiben im DaFUnterricht in China zeigt sich in dem Lehrwerk Texte verfassen, wo Schreiben nicht mehr primär zum Üben der sprachlichen Phänomen dient, sondern als Kommunikation mit einer bestimmten Funktion zwischen dem Textverfasser und seinem Leser verstanden wird (vgl. Ni 2000: 66). Jedoch wird das didaktisch vielversprechende und prozessorientierte Lehrwerk bisher nur im Germanistik-Studiengang und nicht im anderen Deutschkurs eingesetzt, wobei in den am meisten verwendeten ersten und zweiten Band Argumentation in Form von Erörterung ausführlich behandelt wird. In dem bereits erschienenen Band III wird das mündliche Referat und die schriftliche Seminararbeit thematisiert, und bei der Letzteren werden das Recherchieren im Internet, die Aufstellung eines Inhaltsverzeichnisses, das wörtliche, indirekte und sinngemäße Zitieren und das Anfertigen eines Literaturverzeichnisses im Einzelnen geübt (vgl. Ni/Kong/Stoephasius 2001: 136). Bedauerlicherweise wird dieser Band bisher für Germanistikstudenten im dritten 
Studienjahr nur an wenigen Universitäten (z. B. an der Nanjing-Universität) benutzt, oft aus studienzeitlichen Gründen. In dem bereits angekündigten Band IV wird das Verfassen einer wissenschaftlichen Abschlussarbeit eingeplant, der leider noch nicht auf den Markt gebracht worden ist.

Zusammenfassend lässt sich sagen, dass die deutsche wissenschaftliche (Haus)Arbeit für die meisten chinesischen Studierenden Neuland ist. Denn sie haben diese Textsorte weder in der schulischen Sozialisation und dem universitären Studiums in China noch im Deutschunterricht kennen gelernt und geübt.

\section{Struktur der wissenschaftlichen Hausarbeiten im Vergleich}

$\mathrm{Da}$ eine deutsche wissenschaftliche Hausarbeit argumentativ aufgebaut ist und darin eventuell auch Textsorten wie Beschreibung bzw. Bericht eingebettet werden, wird im Folgenden versucht, diesbezügliche Unterschiede der chinesischen Texte zu deutschen darzustellen, um mögliche Problemfelder der Chinesen beim Schreiben einer deutschen Hausarbeit aufzudecken.

\subsection{Textaufbau}

Während eine deutsche wissenschaftliche Arbeit deduktiv aufgebaut ist $-\mathrm{d}$. h. die Hauptinhalte oder -thesen stehen am Anfang und darauf folgen entsprechende Argumente, ist die chinesische Herangehensweise dagegen induktiv. Den chinesischen Strukturaufbau nennt Günthner (1993: 170) „das langsame Aufrollen von Hintergrundinformationen“, d. h. bevor die wichtigsten Inhalte oder die Hauptthesen präsentiert werden, wird eine lange Einführung vorgenommen, persönliche Themen ausgeführt, Beispiele genannt und Argumente vorgebracht, um einen gemeinsamen Rahmen der Hintergrundinformationen zu etablieren und das Vertrauen zueinander zu gewinnen. Diese Art und Weise des Argumentationsaufbaus sorgt im chinesischen Kontext für Höflichkeit und basiert auch auf der chinesischen sprachlichen Gewohnheit der „Weil-Deshalb-Struktur“, die in der chinesischen Alltagssprache als Konjunktion fungiert.

Eine andere Besonderheit im chinesischen Strukturaufbau eines Argumentationstextes ist die „Ja-Nein-Struktur", wenn es um eine kritische Auseinandersetzung mit einem bestimmten Gesichtspunkt oder um eine Bewertung eines wissenschaftlichen Beitrages geht. Liang (1991) hat z. B. chinesische und deutsche Rezensionen ausführlich untersucht und kommt zu dem Ergebnis, dass sich die Deutschen kritisch mit der zu rezensierenden Publikation auseinander setzen, dagegen versuchen die Chinesen, die positiven Aspekte der Publikation herauszufinden und hervorzuheben. Die häufig harmlose Kritik steht meistens erst am Schluss der Rezension und richtet sich immer nur auf einzelne Aspekte. Die negative Bewertung wird weiterhin mit anschließenden Worten im positiven Sinne relativiert. Diese oft von Deutschen als Konflikt- bzw. Kritikscheu interpretierte 
Indirektheit ist eng verbunden mit dem chinesischen Höflichkeitskonzept, das durch die Herstellung von Harmonie sowie Wahrung des Gesichts gekennzeichnet ist.

Die thematische Entfaltung im chinesischen Text verläuft nicht wie im deutschen linear, sondern sieht wie ein buddhistisches Rad aus. Das heißt, bestimmte inhaltliche Aussagen werden an verschiedenen Stellen im Text wieder aufgenommen und unter unterschiedlichen Gesichtspunkten betrachtet. In einem argumentativen Text wird dies „Schicht um Schicht vertiefende Argumentation“ genannt, wie Lehker (1997: 138-153) es an zwei chinesischen Texten ausführlich dargestellt hat. Auch bei Beschreibungen wird nicht ein Thema nach dem anderen abgehandelt, sondern man gibt z. B. zuerst mit der wichtigsten Information einen Überblick über das ganze Thema und dann geht man im Einzelnen ausführlich darauf ein, so dass dasselbe Thema wiederholt vorkommt ${ }^{11}$.

\subsection{Thesenbildung}

Es fällt den chinesischen Studierenden oft schwer, eine eigene Meinung zu bilden. Wenn sie beim Schreiben eines argumentativen Textes doch eine These aufstellen müssen, tendieren sie dazu, die so genannte ,goldene Mitte“ einzunehmen. Zhao (2005: 21) hat zum Beispiel bei der Untersuchung der Schreibaufgabe im TestDaF-Modellsatz über die Einführung der Studiengebühren herausgefunden, dass die meisten chinesischen Kursteilnehmer weder gegen noch bedingungslos für die Studiengebühren sind. Sie bilden ihre Thesen in Form von „Studiengebühren ja, aber nicht zu hoch“. Außerdem kopieren sie gern die Ansichten von öffentlichen Medien und von kollektiven Meinungen, ohne sich diese genau durch den eigenen Kopf gehen zu lassen. Dies ist vor dem chinesischen Ausbildungshintergrund nicht verwunderlich, denn in China wird im schulischen sowie universitären Lernen und Studieren nicht die kritische bzw. kreative Denkweise entwickelt, sondern das Faktenwissen vermittelt. Nach Konfuzius sollten die Lehrenden tradieren, aber nicht hinzufügen oder neu schaffen (Shu er bu zuo). Hingegen mussten die Prüflinge in der Kaiserzeit bei der Vorbereitung auf die staatliche Beamtenaufnahmeprüfung nicht nur die konfuzianischen Klassiker rezitieren, sondern nach der Südsong-Zeit (1127-1279) auch die neokonfuzianischen Auslegungen von den Klassikern auswendig lernen. Denn bestimmte Interpretationen wurden als Standardwerke festgelegt, so dass eine Abweichung davon als Fehler angesehen wurde. (vgl. Wang 1988). Diese Tradition hinterlässt Spuren in der modernen

\footnotetext{
${ }^{11}$ Zhao (2005: 20f.) gibt ein Beispiel der Grafikbeschreibung über die Studiengebühren im TestDaFModellsatz von einem chinesischen Studenten: „Unter diesen Ländern sind die Studiengebühren von Großbritannien am höchsten. Deutschland, Frankreich, Österreich und Schweden verlangen keine Gebühren. Die höchsten Gebühren erreichen in Großbritannien 7000 DM pro Jahr. Dann sind die von Niederlanden, einheitlich 2160 DM pro Jahr. In Belgien und anderen Ländern bezahlt man unterschiedliche Gebühren. Der Unterschied der Studiengebühren von Großbritannien ist am größten (1900-7000 DM).“
} 
chinesischen Didaktik, in der Frontalunterricht und Lernen von „Was“ statt Nachdenken über „Warum“ in der Ausbildung dominieren (vgl. Zhao 2002: 165-171).

\subsection{Argumentation}

In einem chinesischen argumentativen Text wird die These gern mittels narrativer Sequenzen unterstützt, wie z. B. mit Anekdoten aus dem Leben bekannter Persönlichkeiten oder persönlichen Erlebnissen in Form von Beispielen bzw. Erzählungen. Jia (2000: 193) liefert dazu ein Beispiel, in dem ein Student zum Thema „Beherrschen die Menschen die Technik oder umgekehrt?“ wie folgt geschrieben hat:

Aufgrund vieler Statistiken ist die Herzkrankheitsquote unter den Autobesitzern höher als unter den Leuten ohne Auto. Ich habe vorher eine Erzählung gelesen: Ein Mann verlor sein Auto. Deshalb mußte er jeden Tag eine halbe Stunde zu Fuß zur Arbeit gehen. Anfangs ist es ihm sehr anstrengend... Jetzt freut er sich, täglich einen schönen Morgenspaziergang machen zu können. Sein Pech ist sein Glück geworden. Das Beispiel legt dar, dass zwar die Technik uns viel Bequemlichkeit bereitet, sie aber auch eine unübersehbare Schadenseite hat.

Außerdem werden oft Zitate bekannter Persönlichkeiten oder sprichwörtliche Redensarten als Theorieargumente benutzt (vgl. Lehker 1997: 167, Yin 1999: 27f.). Um z. B. argumentativ zu begründen, dass man bescheiden bleiben soll, kann man das Zitat von Konfuzius anführen, dass „,unter drei Menschen es bestimmt einen gibt, den ich als Lehrer nehmen kann“. Oder man benutzt Worthülsen, Floskeln bzw. Behauptungen zum Bekräftigen der These, wie „es weiß alle Welt, dass ...“, „es ist allen bekannt, dass ...", „es ist ganz klar, dass ..." usw. (vgl. Jia 2000: 193).

\subsection{Beschreibung und Bericht}

Diese beiden Textsorten haben im deutschen Kontext eine deutlich informierende Funktion, welche Sachlichkeit erfordert. Dagegen haben sie aber im chinesischen Verständnis oft eine implizite argumentative Funktion, wobei der Deskriptionsbzw. der Darstellungsakt in der thematischen Entfaltung im Ganzen als Argumente für eine bestimmte Ansicht fungieren (vgl. Lehker 1997: 243, 203). Außerdem wird oft subjektiv vorgegangen, und nicht selten stehen noch Kommentare am Textende. Schließlich ist „die persönlichen Empfindungen ausdrücken, während des Erzählens (Berichtens/Beschreibens) auch kommentieren“ (You gan er fa, jia xu jia yi) das Motto des chinesischen Schreibens. Dies zeigen auch die beiden folgenden Beispiele, die von einem chinesischen Studenten verfasst wurden und in 
denen ein Klinikgebäude beschrieben und von einem Patientenbesuch berichtet wird (zitiert nach Jia 2000: 191f., Fettdruck im Original):

Die Klinik wurde allein von unserer Universität geplant und gebaut. Man darf sagen, dass sie ein erfolgreiches Werk der Fakultät für Architektur ist. Besonders beeindruckend ist die Vorhalle, die einen ausgezeichneten Eindruck durch ihre Helligkeit und Großräumigkeit macht. Der Sonnenschein flutet durch ein Glasdach. Man fühlt sich wie in einem Treibhaus. In einer so warmen Umgebung wird die Stimmung rasch leichter.

Als ich aus der Klinik trat, fühlte ich mich warm im Heržen. Ich habe nicht gedacht, dass ein Arzt die Stimmung einer Patientin so gut verstehen könnte. Einige Tage später kam ich wieder auf die Beine. Von meinen eigenen Erfahrungen weiß ich, dass es in der Klinik eine medizinische Gemeinschaft gibt, die nicht nur eine schöne Moral, sondern ach ausgezeichnete Heilkeunst besitzt. Ich bin auf unsere Klinik stol\%:

\subsection{Unterscheidung zwischen eigenen und fremden Meinungen}

In der deutschen Wissenschaftstradition sind Bezugnahme auf wissenschaftliche Autoritäten und die Nachprüfbarkeit von Quellen sehr wichtig (vgl. Kaiser 2003: 312f.). In wissenschaftlichen Texten werden fremden Daten, Ereignisse und Meinungen durch genaue Quellenangaben in Form der Einführung von Fußnoten, der verschiedenen Zitattechniken und der Auflistung von einem Literaturverzeichnis gekennzeichnet, um diese von den eigenen zu unterscheiden. Diesen Umgang mit fremdem Wissen haben die chinesischen Studenten in der Schule und an der Universität nicht kennen gelernt und er bleibt ihnen deshalb fremd. Im chinesischen Schreiben benutzt man fremde Gedanken oft als allgemeine Wahrheiten oder man signalisiert zwar, dass sie von den anderen stammen, aber ohne dass eine konkrete Quelle angegeben wird. Eingeleitet werden sie durch Ausdrücke wie „Wie alle wissen, dass ...“, „Eine bekannte Persönlichkeit hat einmal gesagt, dass ...“, „In der Zeitung steht, dass ...“" usw.. Darüber hinaus mischt man manchmal auch die fremden mit den eigenen Gedanken und formuliert sie neu. Dies wird in China nicht als Plagiat angesehen, denn man hat nicht einen fremden Text abschnittsweise abgeschrieben, sondern sich fremden Meinungen zuerst zu Eigen gemacht, sie eventuell weiter ergänzt oder überarbeitet. Diese Verwischung der Grenze zwischen fremden und eigenen Meinungen kommt nicht nur bei Studierenden, sondern auch bei Wissenschaftlern (vor allem in der Geisteswissenschaft) in China vor. In dem Sammelband Deutsch als aveite Fremdsprache in Ostasien: neue Perspektiven (Wannagat/Gerbig/Bucher 2003) sind insgesamt acht Beiträge von chinesischen DaF-Professoren aufgenommen, darunter haben jeweils 50\% der Arbeiten kein Literaturverzeichnis und keine Fußnote (Zitate in diesem Band 
werden nicht im Verlauf des Textes sondern als Fußnote eingebettet). In einigen Beiträgen dienen die spärlichen Fußnoten auch lediglich zur Begriffserklärung; Zitate kommen dort nicht einmal vor. Dies führt dazu, dass die deutschen Herausgeber im Vorwort ganz charmant formulieren (Gerbig/Wannagat 2003: 8):

Dieser erste und umfassende Einblick in die Werkstatt eines so großen und ambitionierten Projekts sowie dessen Entwicklungsstand in methodischdidaktischer Hinsicht ist sicher spannend genug, um gelegentliche Abweichungen von der Norm in der wissenschaftlichen Form der Beiträge zu tolerieren. Fußnoten, Quellenangaben und Belegtechniken sind ja westliche Standards geisteswissenschaftlichen Arbeitens, und das Fehlen derselben hier und da lässt insofern einen Blick zu auf kulturell und traditionell geprägte Unterschiede beim Verfassen wissenschaftlicher Texte. Nachhaltig bleibt bei dieser Bestandsaufnahme der Eindruck, dass der geballte Informationswert der chinesischen Beiträge insgesamt kleinere Unterschiede in der Form mehr als ausgleicht.

\section{$4 \quad$ Sprache der wissenschaftlichen Hausarbeit im Vergleich}

Wenn sich der sprachliche Stil einer deutschen wissenschaftlichen Arbeit durch Sachlichkeit, Objektivität, Komprimiertheit, Kürze und Bündigkeit usw. auszeichnet, ist der chinesische Stil im Vergleich dazu eher emotional und subjektiv, erfolgt nach der Devise „die Leser mit dem Gefühl anzurühren und ihnen mit Gründen zu erklären“ (Dong zhi yi qing, xiao zhi yi li).

In einem chinesischen Text - ob er argumentativ, deskriptiv oder darstellend ist - wird sprachlich versucht, die Rezipienten direkt oder indirekt affektiv anzusprechen (vgl. Lehker 1997: 116, 244f.). Dies zeigte sich bei der Beschreibung und dem Bericht bereits in den Beispielen zur Uni-Klinik in Abschnitt 3, wo viele emotional beladene Adjektive und bildhafte Metapher auftauchen. Außerdem weist Zhao (2005: 19) darauf hin, dass auch beim argumentativen Text die Emotion durch rhetorische Fragen oder Konjunktivsätze häufig zur Geltung kommt, wie z. B. mit den Sätzen „Aber warum muß man so viele Gebühren bezahlen?“, „Ist das die Gleichberechtigung?“", „Hätte unser Land ein noch besseres Bildungsniveau!“" usw.

Es kommt zudem im chinesischen argumentativen und darstellenden Text nicht selten vor, an die Rezipienten zu appellieren, um sie moralisch zu motivieren (vgl. Lehker 1997: 156, 203f., Yin 1999: 144). Oft wird am Ende des Textes ein Abschnitt angehängt, in dem die Leser oder eine bestimmte Gruppe aufgefordert werden, bestimmte Handlungen zu befolgen. Die entsprechenden Ausdrücke sind „Ich hoffe, dass ...“, „Wir sind uns sicher, dass ...“ usw.. Oder der Verfasser selbst bietet eine Lösung, ein Konzept bzw. einen Vorschlag an, um auf diese Art und Weise indirekt appellative Anforderungen zu äußern (vgl. Zhao 2005: 19f.). 
Im Vergleich mit deutschen wissenschaftlichen Arbeiten fehlt es bei chinesischen Texten an sprachlicher Präzision. Dies bezieht sich auf zweierlei: Zum einen gibt es in einem chinesischen Text so gut wie keine metakommunikativen Verweise, so dass dem Leser die Verantwortung für das Verstehen des Textes selbst überlassen wird (vgl. Lehker 1997: 153f.). Zhao (2005: 18) zeigt ein Beispiel von einem Argumentationsakt, in dem die These und die zwei Argumente die Leser selbst zu suchen und zu ordnen haben, ohne Verweis darauf, dass es nun darum geht ${ }^{12}$. Es fehlt nämlich an metakommunikativen Hinweisen wie „Meine Meinung ist, ...“, „Dafür gibt es folgende zwei Gründe.“ usw. Zum anderen sind viele Ausdrücke in einem chinesischen Text an sich nicht eindeutig und erst im Kontext bzw. mit Hilfe der eigenen Erfahrungen zu verstehen. Wenn man schreibt, dass „China wirtschaftlich große Schritte gemacht hat", geht man davon aus, dass es „Fortschritte“ statt „Rückschritte“ seien, denn jeder habe gesehen oder gelesen, dass die chinesische Wirtschaft seit Jahrzehnten boomt. Diese Unpräzision der sprachlichen Formulierung hat mit der chinesischen Sprache als analytischer Sprache zu tun, in der der Sprachkontext eine besondere Bedeutung besitzt, denn nach Tao (1998: 21) ist Chinesisch stark in der künstlichen Denkweise aber schwach in der Logik. Letztendlich ist beim Aufsatzschreiben im Chinesischen noch davon die Rede, dass man vieles oft nur fühlen, aber nicht mit Worten ausdrücken kann oder braucht (Zhi neng yi hui, bu neng yan chuan).

\section{Vorschläge}

Da das wissenschaftliche Schreiben eine sehr komplexe Aufgabe ist und sich die wissenschaftlichen Schreibtraditionen - wie in den vorigen Abschnitten gesehen in Deutschland sehr stark von denen in China unterscheiden, beziehen sich unsere Vorschläge zur Verbesserung der wissenschaftlichen Schreibkompetenz chinesischer Studierender auf verschiedene Ebenen, und zwar die Forschungsebene (vgl. Abschnitt 5.1 kontrastive Untersuchungen), die didaktische Ebene (vgl. Abschnitt 5.2 multiperspektivischer Ansatz) und die organisatorische Ebene (vgl. Abschnitt 5.3 studienvorbereitende und studienbegleitende Kurse).

\subsection{Kontrastive Untersuchungen}

Konkrete Vorschläge und Lehrmaterialien zum wissenschaftlichen Schreiben für chinesische Studierende können sinnvollerweise erst entwickelt werden, wenn kontrastive Untersuchungen zu relevanten Textsorten vorliegen. Kontrastive Studien sind in Bezug auf unsere Zielsetzung notwendig

\footnotetext{
12 Dieses Beispiel ist: „[Nach der Präsentation der zwei Fremdaussagen wird geschrieben]: Hochschulen sollen keine Studiengebühren verlangen. Jeder Student, ob dessen Familie reich oder arm ist, soll die gleiche Chance haben zu studieren. Bildung ist nicht nur die individuelle Aufgabe, sondern auch die der ganzen Gesellschaft" (Zhao 2005: 18).
} 
- zum wissenschaftlichen Artikel: Die wissenschaftliche Hausarbeit zielt auf die Einübung der Kompetenz, wissenschaftliche Artikel verfassen zu können. Daher dient die Analyse dazu, eigenkulturelle (chinesische wissenschaftliche Artikel) und fremdkulturelle (deutsche wissenschaftliche Artikel) Textmuster und Konventionen vergleichend gegenüberzustellen.

- zur wissenschaftlichen Hausarbeit: Da in China die wissenschaftliche Hausarbeit als Textform nicht etabliert ist und es auch keine Anleitungen zum Verfassen von Hausarbeiten gibt, bietet sich ein kontrastiver Vergleich von wissenschaftlichen Hausarbeiten deutscher und chinesischer Studierender im deutschen Wissenschaftskontext an. Dabei sollten möglichst Ergebnisse von kontrastiven Untersuchungen zum wissenschaftlichen Artikel einbezogen werden.

\subsection{Multiperspektivischer Ansatz}

In Bezug auf die didaktische Umsetzung der Ergebnisse von kontrastiven Studien plädieren wir angesichts der Komplexität der Aufgabe, chinesische Studierende zu befähigen, wissenschaftliche Hausarbeiten in einer Fremdsprache und einem fremden Wissenschaftsstil angemessen zu verfassen, für einen multiperspektivischen Ansatz wie ihn Kruse (2003) vertritt. In diesen Ansatz integriert er vier Perspektiven, aus denen Schreiben betrachtet werden kann und die wir im Folgenden auf unsere Zielgruppe fokussieren:

1. die text- und normorientierte Seite des Schreibens: Diese Perspektive ist produktorientiert und zielt auf eine sprachlich- und textsortenadäquate Erstellung von Hausarbeiten. Den chinesischen Studierenden sollten bspw. Besonderheiten und Unterschiede in der Struktur und der sprachlichen Gestaltung von wissenschaftlichen Hausarbeiten sowie die Bezugnahme auf fremdes Wissen vermittelt werden. Dabei können StandardTextmuster und Modellbeispiele guter wissenschaftlicher Hausarbeiten hilfreich sein.

2. die subjektive Steuerung des Schreibens: Hierbei handelt es sich um eine prozessorientierte Perspektive, wobei den chinesischen Studierenden bspw. wichtige Hilfsmittel für das Schreiben (Schreibtagebuch, Mind Map etc.) sowie Teilkompetenzen (Planen, Formulieren, Überarbeiten) vermittelt werden. In diesem Zusammenhang können auch in einer Schreibberatung wichtige Hilfestellungen gegeben werden.

3. die soziale Seite des Schreibens: Die Perspektive des Kontextes nimmt die sozialen und kulturellen Bezüge von wissenschaftlichen Hausarbeiten in den Blick. Für die chinesischen Studierenden ist es wichtig, die Funktion von wissenschaftlichem Schreiben für die Kommunikation von Wissen 
sowie den akademischen Diskurs und seine geistesgeschichtlichen Grundlagen zu verstehen.

4. die inhaltliche Seite des Schreibens: In der kontextbezogenen Perspektive wird das wissenschaftliche Schreiben in Verbindung mit dem Forschungsund Erkenntnisprozess betrachtet. Die chinesischen Studierenden sollen Schreiben mit kritischem Denken verbinden und als aktives Lernen verstehen. Das wissenschaftliche Schreiben wird nicht fachübergreifend, sondern innerhalb der Disziplinen gelehrt und geübt.

\subsection{Studienvorbereitende und studienbegleitende Kurse}

Fast alle chinesischen Studierenden haben bereits in China Deutschkurse besucht. Im Hinblick auf das spätere wissenschaftliche Schreiben sollten die Studierenden bereits in diesen Deutschkursen bzw. in besonderen DSH/TestDaF-Vorbereitungskursen sprachliches Handlungswissen zu relevanten Kommunikationsformen wie Argumentieren und Beschreiben im Deutschen erwerben. Didaktische Anregungen für den Schreibunterricht für chinesische Studierende liegen aus chinesischer Perspektive von Ni (2000) und Zhao (2005) vor bzw. von deutscher Seite von Schlenker (1988). Ein umfassendes Konzept sollte allerdings alle vier oben genannten Perspektiven enthalten. In der Praxis des Deutschunterrichts in China wird dies im Rahmen von Deutschkursen bzw. DSH/TestDaF-Vorbereitungskursen nur in zeitlich beschränktem Maße möglich sein (vgl. Li 2002).

Wir halten daher vor Beginn des Studiums in Deutschland Ergänzungskurse für notwendig, die in China oder in Deutschland durchgeführt werden können und unterschiedliche thematische umfassen:

- wissenschaftliche Propädeutik: Einführung in wissenschaftliche Arbeitsmethoden (selbstständiges Arbeiten, Recherchieren, Umgang mit wissenschaftlicher Literatur etc.), Wissenschaftssprache (Erarbeitung von Sprachmitteln zu zentralen Bereichen und Vermittlung der Kompetenz, selbstständig das wissenschaftssprachliche Repertoire systematisch auszubauen), wissenschaftliches Schreiben (kleinere Textsorten wie Mitschrift, Exzerpt, Protokoll) und mündliche Kommunikation (vor allem Diskussionsbeteiligung im Unterricht, aber auch Sprechstundengespräche).

- kulturelle Unterschiede im akademischen Diskurs

- interkulturelle Kommunikation: Kenntnisse über Deutschland, Selbstreflexion der eigenen Denkweisen, Sensibilisierung für Stereotypen, Sensibilisierung für die Kulturgeprägtheit von Welt- und Textmusterwissen ${ }^{13}$ (Fußnote), Entwicklung von Kommunikations- und Lernstrategien, Einführung in wichtige Hilfsmittel für das Schreiben

\footnotetext{
13 Ohne eine solche Sensibilisierung besteht die Gefahr, dass die chinesischen Studierenden auf eigenkulturelles Wissen zurückgreifen, Eßer 1997: 197.
} 
- Studienorganisation: Information über Studiensystem, Studiengänge, Veranstaltungsformen, Stundenplanerstellung, Funktion von Sprechstunden etc.

Eine Durchführung der Kurse in deutscher Sprache (auch in der chinesischen Heimat) ermöglicht eine gleichzeitige allgemeinsprachliche Weiterarbeit.

Solche Ergänzungskurse sind insbesondere für chinesische Studierende notwendig, die in China die DSH oder TestDaF erfolgreich bestanden haben und deshalb in Deutschland keine weiteren Sprachkurse besuchen, sondern direkt mit dem Studium beginnen. Zum einen entfällt bei ihnen eine dem Studium vorgelagerte Orientierungsphase, in der sie sich mit der von ihrer eigenen weit entfernten Kultur bekannt machen und in das Hochschulsystem und das studentische Leben hineinschnuppern können. Zum anderen stehen ihnen für ihr Hineinfinden in die fremden Lern-, Arbeits- und Kommunikationsformen oftmals „,nur noch“ studienbegleitende Kurse zur Verfügung. Da die Zahl der TestDaF-Absolventen in China zunehmend ist, ist in Zukunft auch mit einem steigenden Bedarf solcher studienvorbereitenden Ergänzungskurse zu rechnen.

Studienbegleitende Kurse an deutschen Hochschulen sind - nach der Vermittlung entsprechender Grundkompetenzen in studienvorbereitenden Kursen - im Hinblick auf die von Kruse (2003) als inhaltliche Seite des Schreibens bezeichnete Perspektive sehr wichtig. Das Schreiben einer wissenschaftlichen Hausarbeit kann letztendlich in seiner gesamten Komplexität erst geübt werden in Anbindung an die jeweilige Disziplin. Eine fachkundige und engagierte Betreuung ist deshalb besonders wichtig und kann - wie Koreik (2004: 111) für den studienbegleitenden DaF-Unterricht allgemein betont - auch zur Profilierung der Hochschulen im Wettbewerb um - in unserem Fall - chinesische Studierende beitragen.

\section{Literatur}

Blume, Georg: „Die zweite Wahl“, Die Zeit 52 (2002) (http://zeus.zeit.de/text/2002/52/B-China-Studenten, heruntergeladen am 08.09.2005).

Chaffee, John W.: The Gate of Learning in Sung China. A Social History of Examinations. Cambridge University Press 1985.

China-Botschaft: „Rau hält Vortrag an der Tsinghua-Universität“. In: http://www.china-botschaft. de/det/jj/t93275.htm: heruntergeladen am 23.08.2005.

Deutscher Akademischer Austauschdienst (o. J. ) (Hrsg.): Auf dem Weg zur internationalen Hochschule. Drittes Aktionsprogramm des DAAD 2004-2010, abrufbar unter http://www.daad.de/download/aktionsprogramm.pdf 
Dittmann, Jürgen/Geneuss, Katrin A./Nennstiel, Christoph/Quast, Nora A.: „Schreibprobleme im Studium - Eine empirische Untersuchung“. In: Ehlich, Konrad/Steets, Angelika (Hrsg.): Wissenschaftlich schreiben - lehren und lernen, Berlin: Walter de Gruyter 2003, 155-185.

Ehlich, Konrad: „Universitäre Textarten, universitäre Struktur“. In: Ehlich, Konrad/Steets, Angelika (Hrsg.): Wissenschaftlich schreiben - lehren und lernen, Berlin: Walter de Gruyter 2003, 13-28.

Ehlich, Konrad: „Sprachqualifizierung für die (mündliche und schriftliche) Wissenschaftskommunikation“. In: Casper-Hehne, Hiltraud/Ehlich, Konrad (Hrsg.): Kommunikation in der Wissenschaft, Regensburg: Fachverband Deutsch als Fremdsprache 2004, 1-19.

Ehlich, Konrad/Steets, Angelika (Hrsg.): Wissenschaftlich schreiben - lehren und lernen, Berlin: Walter de Gruyter 2003a.

Ehlich, Konrad/Steets, Angelika: „Wissenschaftliche Schreibanforderungen in den Disziplinen. Eine Umfrage unter ProfessorInnen der LMU“. In: Ehlich, Konrad/Steets, Angelika (Hrsg,): Wissenschaftlich schreiben - lehren und lernen, Berlin: Walter de Gruyter 2003b, 129-154.

Eßer, Ruth: „Etwas ist mir geheim geblieben am deutschen Referat“. Kulturelle Geprägtheit wissenschaftlicher Textproduktion und ibre Konsequenzen für den universitären Unterricht von Deutsch als Fremdsprache, München: iudicium 1997.

Gerbig, Jürgen/Ulrich Wannagat: „Vorwort“. In: Wannagat, Ulrich/Jürgen Gerbig/Stefan Bucher (Hrsg.): Deutsch als zweite Fremdsprache in Ostasien: neue Perspektiven. Tübingen: Stauffenburg 2003, 7-9. (= Arbeiten zur angewandten Linguistik; Bd.2)

Graefen, Gabriele: Der Wissenschaftliche Artikel - Textart und Textorganisation, Frankfurt am Main u.a.: Lang 1997.

Guckelsberger, Susanne/Stezano Cotelo, Kristin: „Vom mündlichen Referat zur Seminararbeit. Eine exemplarische Analyse und Reflexion der Erfordernisse für eine studienintegrierte Sprachqualifizierung deutscher und ausländischer Studierender“. In: Wolff, Armin/Ostermann, Torsten/Chlosta, Christoph (Hrsg.): Integration durch Sprache, Regensburg: Fachverband Deutsch als Fremdsprache [Materialien Deutsch als Fremdsprache. 73] 2004, 417-457.

Günthner, Susanne: Diskursstrategien in der interkulturellen Kommunikation: Analysen deutsch-chinesischer Gespräche. Tübingen: Niemeyer 1993. (= Linguistische Arbeiten: 286)

Jakobs, Eva-Maria: „Normen der Textgestaltung“. In: Kruse, Otto/Jakobs, EvaMaria/Ruhmann, Gabriela (Hrsg.): Schlüsselkompetenz Schreiben. Konzepte, 
Methoden, Projekte für Schreibberatung und Schreibdidaktik an der Hochschule.

Neuwied, Kriftel: Luchterhand 1999, 171-189.

Jia, Huidie (2000): „Zhongde shumian jiaoji zhong de shengcheng wenhua yinsu tanxi - yi zhongguo xuesheng yong deyu jinxing shumian biaoda zhong de wudian weili. (Analyse der tiefenstrukturellen Kulturelemente in der chinesisch-deutschen schriftlichen Kommunikation - am Beispiel von Fehlelementen im schriftlichen Ausdruck auf Deutsch von chinesischen Studierenden)“. In: Zhu, Jianhua/Gu, Shiyuan (Hrsg.): Arbeiten zur Interkulturellen Kommunikation Chinesisch-Deutsch. Shanghai: Verlag der TongjiUniversität, 188-206.

Kaiser, Dorothee (2003): „Nachprüfbarkeit' versus ,Originalität Eigenes in studentischen Texten aus Venezuela und Deutschland“. In: Ehlich, Konrad/Steets, Angelika (Hrsg.): Wissenschaftlich schreiben - lehren und lernen. Berlin: Walter de Gruyter, 305-324.

Koreik, Uwe: „Der studienvorbereitende und studienbegleitende DaF-Unterricht an deutschen Hochschulen“. In: Wolff, Armin/Ostermann, Torsten/Chlosta, Christoph (Hrsg.): Integration durch Sprache, Regensburg: Fachverband Deutsch als Fremdsprache [Materialien Deutsch als Fremdsprache. 73] 2004, 103-112.

Krumm, Hans-Jürgen (Hrsg.): Erfahrungen beim Schreiben in der Fremdsprache Deutsch. Untersuchungen zum Schreibprozess und zur Schreibförderung im Unterricht mit Studierenden, Innsbruck u.a.: StudienVerlag 2000.

Kruse, Otto: „Schreiben lehren an der Hochschule: Aufgaben, Konzepte, Perspektiven“. In: Ehlich, Konrad/Steets, Angelika (Hrsg.): Wissenschaftlich schreiben - lehren und lernen, Berlin: Walter de Gruyter 2003, 95-111.

Lee, Thomas H.C.: Government Education and Examinations in Sung China. The Chinese University of Hong Kong 1985.

Lehker, Marianne: Texte im chinesischen Aufsatzunterricht. Heidelberg: Groos 1997.

Li, Yuan: „Evaluierung der Intensivkurse am Deutschen Sprachzentrum der Zhejiang Universität. Analyse der Erfahrungen und Konsequenzen für die Weiterentwicklung“", Info DaF 29, 1 (2002), 13-36.

Liang, Yong: „Zu soziokulturellen und textstrukturellen Besonderheiten wissenschaftlicher Rezensionen. Ein kontrastive Fachtextanalyse Deutsch/Chinesisch“, Deutsche Sprache 4 (1991), 289-311.

Luge, Elisabeth: „Zum Schreibunterricht an Hochschulen in China“. In: Tütken, Gisela/Neuf-Münkel, Gabriele (Hrsg.): Schreiben im DaF-Unterricht an Hochschulen und Studienkollegs I. Forschungsergebnisse - Didaktische Konzeptionen Übungsformen. Vorträge der Fachtagung vom 4.9.-7.9.1991 in Göttingen. Regensburg: iudicium 1993. 
Madl, Benedikt: Auslandsstudium, Brain-Drain und Regierungspolitik am Beispiel der VR China, Frankfurt am Main u.a.: Lang 2002.

Meng, Hong: Das Auslandsstudium von Chinesen in Deutscbland (1861-2001). Ein Beispiel internationaler Studentenmobilität im Rahmen der chinesischen Modernisierung. Frankfurt a. M. u.a.: Lang 2005.

Ni, Jenfu: „Textlinguistik und Aufsatzschreiben“, Sprache \& Sprachen 23/24 (2000), 61-74.

$\mathrm{Ni}$, Jenfu/Kong, Deming/Stoephasius, Jutta: Texte Verfassen. Lehrerhandbuch Band III. Aufsatžbuch für Deutschlernende im Hauptstudium. Verlag der Universität Nanjing 2001.

Schlenker, Traudel: „Chinesische und westliche Lernererfahrungen. Versuch eines Brückenschlags am Beispiel eines Schreibkurses“. In: Lieber, Maria/Posset, Jürgen (Hrsg.): Texte schreiben im Germanistik-Studium, München: iudicium 1988, 113-123.

Steets, Angelika: „Überlegungen zu einem Curriculum für die Vorbereitung auf wissenschaftliches Schreiben“. In: Wolff, Armin/Winters-Ohle, Elmar (Hrsg.): Wie schwer ist die dentsche Sprache wirklich?, Regensburg: Fachverband Deutsch als Fremdsprache 2001, 211-228.

Störig, Hans Joachim: Kleine Weltgeschichte der Philosophie. Überarbeitete Nenausgabe. Frankfurt a.M.: Fischer 2004.

Stork, Antje/Zhao, Jin: „»Mit Chinesen sind wir beim ersten Mal Fremde, beim zweiten Mal schon Freunde« - Integrationsprobleme chinesischer Studierender in Deutschland“. In: Tagungsband der 24. Arbeitstagung des Arbeitskreises der Sprachenzentren, Spracheninstitute und Fremdspracheninstitute (AKS e.V.) vom 02.-04. März 2006 in Nürnberg (im Druck).

Tao, Jiawei: Xięuo yu wenhua (Schreiben und Kultur). Shanghai foreign language education press 1998.

Wannagat, Ulrich/Jürgen Gerbig/Stefan Bucher (Hrsg.): Deutsch als zweite Fremdsprache in Ostasien: neue Perspektiven. Tübingen: Stauffenburg 2003. (= Arbeiten zur angewandten Linguistik; Bd.2)

Wang, Dongcheng: Ke Ju Shi Hua (Geschichte der Beamtenaufnahmeprüfung). Zhonghua Shuju 1988.

Werder, Lutz von: Lehrbuch des wissenschaftlichen Schreibens. Ein Übungsbuch für die Praxis, Berlin, Milow: Schibri-Verlag 1993.

Yin, Lanlan: Interkulturelle Argumentationsanalyse. Frankfurt a.M.: Lang 1999. (= Werkstattreihe Deutsch als Fremdsprache; Bd. 65) 
Zhao, Jin: Wirtschaftsdeutsch als Fremdsprache: Ein didaktisches Modell dargestellt am Beispiel der chinesischen Germanistik-Studiengänge. Tübingen: Narr 2002. (=Forum für Fachsprachen-Forschung; Bd. 59)

Zhao, Jin: Probleme chinesischer Deutschlerner im Schreiben - Analyse der Schreibaufgaben im TestDaF-Modellsatz, Info DaF 32, 1 (2005), 14-27. 


\section{Sprechen in den Wissenschaften - zunächst mit Humor}

\section{Der Erwerb von Wissenschaftssprache im Daf-Unterricht: mit Abstand zum Ernstfall}

Für Muttersprachler wie für Fremdsprachler bedeutet das Lernen einer alltäglichen Wissenschaftssprache (Ehlich 1995: 340 ff) den Erwerb einer neuen sprachlichen Kompetenz, einer „Common sense-Kompetenz“ (Feilke 1994). Das außerordentlich komplexe wissenschaftssprachliche Ausdrucksspektrum ${ }^{1}$ ist im Wesentlichen durch einen dynamischen Prozess gekennzeichnet und lässt sich nicht auf Regelund Lexikonwissen reduzieren. Daher ist eine aktive Kompetenz zur Ausdrucksbildung erforderlich, die Fähigkeit, domänentypische Muster kreativ zu nutzen (vgl. Feilke/Steinhoff 2003: 117).

\footnotetext{
1 Zu Phänomenen der Wissenschaftssprache s.u.a. Kusters/ Bonset in:Ehlich / Rehbein (1983), Fluck (1992) Ehlich/Steets (2003).
} 
Feilkes und Steinhoffs Einschätzungen nach hängt die Kontextualisierung von wissenschaftlichen Texten mehr von ihrer Ausdrucktypik als vom Inhalt ab. Dies lasse sich vor allem an Wissenschaftsfälschungen und -parodien studieren.

Der Erwerb eines bestimmten Ausdrucksinventars, eines historisch gewachsenen Speichers von Verhaltensmustern, die dem „Common sense“ der Wissenschaftskommunikation entsprechen, scheint allerdings zunächst auch bei Muttersprachlern überwiegend dem Prinzip der Habitusaneignung zu folgen (vgl. Feilke/ Steinhoff: 118). Die Lernenden treten in ein fremdes Sozialisationssystem ein, dessen Werte und Normen ihnen erst im Laufe der Zeit vertrauter werden, dessen Ausdrucksweisen sie zunächst einmal imitieren. Fritz Hermanns hatte bereits 1980 auf die paradoxe Kommunikationssituation von Studierenden hingewiesen: Objektiv seien sie Novizen, müssten aber so tun, als wären sie Experten. Feilke/ Steinhoff stellen fest, dass die Studierenden entsprechend unterschiedlich mit dieser Situation umgehen. Viele reflektierten zwar die Notwendigkeit, den fremden Habitus zu übernehmen, übten sich aber in expliziter Rollendistanz (ebd. 119).

Diese offensichtliche Suche nach Abstand macht die Analogie zum Erwerb einer Fremdsprache, bei dem sich die Rollendistanz auch sehr oft als hilfreiches Mittel erweist und methodisch genutzt werden kann besonders deutlich (vgl. Reinhardt 2006).

Fremdsprachler stehen im Vergleich zu Muttersprachlern beim Erlernen der Wissenschaftssprache vor einer noch komplexeren Rollensituation. Diese schließt ihr neues „Selbst“ in der Fremdsprache und das „Experten-Selbst“ der Wissenschaftssprache mit ein.

Es bietet sich an, die Expertenrolle zunächst parodistisch zu gestalten, spielerisch wissenschaftssprachliche Formen auszuprobieren, die später im „Ernstfall“ umgesetzt werden sollen.

Im Folgenden stelle ich spielerische Sprechübungen aus dem universitären DaF-Unterricht vor, die die Studenten an eine alltägliche Wissenschaftssprache heranführen sollen. Es geht jeweils um Schritte, die ich relativ früh, mit Lernenden der Grundstufe (2. Studienjahr) durchführe, und welche teilweise auch die Arbeit zu Gespräch und Rede (Forster 1997) ${ }^{2}$ vorbereiten bzw. - je nach Sprachniveau - begleiten sollen.

Nach den Sprechübungen aus dem „normalen Unterricht“ skizziere ich kurz das Erarbeiten eines Theaterstückes als einer Art Persiflage von Wissenschaftssprache.

Im Anschluss stehen erste Ergebnisse einer Untersuchung, die ich im Rahmen der Theaterarbeit begonnen habe. Hierbei geht es hauptsächlich darum, was vom auswendig gelernten Text wie und warum behalten wird, und wie es eventuell weiter verwandt/ verarbeitet wird.

\footnotetext{
${ }^{2}$ Roland Forster plädiert 1997 für die feste Einrichtung von Gesprächs- und Redekursen im Rahmen des DaF-Unterrichts und für dieVerankerung des Bereichs der Sprechwissenschaft in das Fach $\mathrm{DaF}$. Er stellt dabei ein emanzipatorisches, an der Rhetorischen Kommunikation ausgerichtetes Konzept für mündliche Kommunikation im FSU vor, welches sich leider - ein Blick in Vorlesungsverzeichnisse deutscher Universitäten genügt - noch nicht entsprechend durchgesetzt hat.
} 
Die Verinnerlichung wissenschaftssprachlicher Strukturen erfolgt über das Sich-zu-eigen-Machen von vorgegebenen Sätzen und die schöpferische Auseinandersetzung mit diesen.

Allen vorgestellten Sprechübungen liegt das Prinzip des Abstands zugrunde. Der Abstand vom „Ernstfall“ und vom oft als defizitär empfundenen Sprachanfänger-Selbst wird über den Einsatz von Spielrahmen (vgl. Huber: 397)33, Rolle und Humor erreicht (vgl. Reinhardt 2006).

Auch das Auswendiglernen von vorgefertigtem Textmaterial ermöglicht Abstand, insofern als die Lernenden sich nicht selbst für die Textproduktion verantwortlich fühlen. Dieser Aspekt klingt sicherlich am wenigsten populär und scheint auf den ersten Blick unvereinbar mit konstruktivistischen Lerntheorien bzw. Konzepten des autonomen Lernens. Die ersten Ergebnisse der oben erwähnten Erhebung scheinen jedoch die Vorzüge des Auswendiglernens in diesem Fall zu unterstreichen.

\section{Sprechübungen im universitären DaF-Unterricht}

\subsection{Beispiel einer Unterrichtseinheit: Dekonstruktion und Rekonstruktion von Texten}

Im Rahmen des Fremdsprachenunterrichts im Ausland stellt das Sprechen in der Wissenschaftssprache eine dreifache Herausforderung dar. Denn außer der Sensibilisierung für verschiedene Sprachregister und wissenschaftssprachliche Phänomene müssen gleichzeitig der Abbau von Sprechhemmungen und eine möglichst korrekte Aussprache angestrebt werden.

Die Deutschstudenten an der Facoltà di Lettere e Filosofia in Vercelli (Italien) befinden sich im 2. Studienjahr zwischen dem Sprachniveau A2 und B1. Im Folgenden wird ein Unterrichtsbeispiel aus dem Bereich der Textarbeit mit einer Gruppe von 20-25 Teilnehmern vorgestellt:

Zur Thematik Gedächtnis und Lernstrategien wurden bereits im Unterricht und als Hausaufgaben mehrere Texte unterschiedlicher Textsorten und Schwierigkeits-

\footnotetext{
${ }^{3}$ Goffman (1980: 53/54) hatte in seiner Definition des Spiels unter anderem folgende Punkte angeführt:

a) Die spielerische Handlung wird so ausgeführt, dass ihre gewöhnliche Funktion nicht verwirklicht wird. Der stärkere und geschicktere Beteiligte hält sich soweit zurück, dass ihm der schwächere und weniger geschickte gewachsen ist.

b) Die Expansivität mancher Handlungen wird übertrieben.

c) Es kommen viele Wiederholungen vor.

d) Wenn mehrere teilnehmen sollen, müssen alle freiwillig zum Spiel bereit sein, und jeder kann eine Aufforderung zum Mitspielen ablehnen oder (wenn er schon Mitspieler ist) aus dem Spiel wieder ausscheiden.

e) Im Verlauf des Spiels kommt es häufig zu Rollenwechsel, was zu einer Auflösung der Hierarchie führt, die zwischen den Beteiligten beim nichtspielerischen Verhalten besteht.
} 
grade behandelt. In einer Doppelstunde folgen nun Sprechübungen in der Auseinandersetzung mit einem wissenschaftssprachlichen Text, einem Auszug aus Markowitsch / Welzer, Das autobiographische Gedächtnis (2005: 11). Dort heißt es unter anderem:

(...) Abstraker formuliert liefert ihm das autobiographische Gedächtnis das Vermögen, die persönliche Existenz in einem Raum-Zeit-Kontinum zu situieren und auf eine Vergangenheit zurückblicken zu können, die der Gegenwart vorausgegangen ist. Offensichtlich dient dieses Vermögen „mentale Zeitreisen“ (Endel Tulving) unternehmen zu können dem Zweck, Orientierungen für zukünftiges Handeln zu ermöglichen. Erlerntes und Erfahrenes kann auf diese Weise für die Gestaltung und Planung von Zukünftigem genutzt werden. Um diese Orientierungsleistung zu ermöglichen, muss das autobiographische Gedächtnis aber noch drei weitere Merkmale aufweisen: die Erinnerungen müssen einen Ich-Bezug haben, um sinnvoll genutzt werden zu können. (...) Damit hängt zweitens zusammen, dass autobiographische Erinnerungen einen emotionalen Index haben. (...)

Der Text enthält zahlreiche Merkmale von Wissenschaftssprache wie Partizipialformen, Passivformen, Personifizierungen, Nominalisierungen und typischen Wendungen (wie: damit bängt zusammen -...). Vom Schwierigkeitsgrad her bewegt er sich für die Zielgruppe an der oberen Grenze.

Arbeitsschritte:

1. Aufwärmphase (10 Min):

Die Teilnehmer erhalten zunächst Teile aus Zeitungen und gehen lesend durch den Raum, wobei sie den Zeitungstext vor sich hinmurmeln. Es folgen verschiedene Phasen des Sprechens, bis hin zur „Schlacht der Titel“"(vgl. Huber/Reinhardt 2005, 11)4.

2. Zergliederung des vorgegebenen Textausschnittes (s.o) in Einzelsätze:

Die Teilnehmer bekommen jeweils ein bis zwei Sätze zugeteilt, die sie leise vor sich hin lesen.

3. Einstudieren / klischeefreies Sprechen einzelner Sätze:

a) Dozent(in) spricht jeweils einzelnen Satz vor

b) Student(in) spricht/ liest ihren Satz nach

c) Gruppe spricht den gleichen Satz auswendig nach

4. Auswendiglernen der einzelnen Sätze: jeder Teilnehmer studiert den eigenen Satz möglichst gut ein, dabei Klärung letzter Intonationsprobleme

5. „Inszenieren“ der Sätze im falschen Kontext:

\footnotetext{
${ }^{4}$ Hier findet sich eine ausführliche Beschreibung dieser Aufwärmübung.
} 
Gruppe macht Vorschläge für verschiedene Kontexte. Beispiel: Studentin A soll Satz A einmal ,als Tagesschau-Sprecherin“ und einmal als „Frauchen zu ihrer Katze" sprechen.

Weitere Beispiele:

- als schlechter Schauspieler

- in einem Werbespot

- beim Zähneputzen

- schimpfend, im Straßenverkehr

- betrunken

- bei mündlicher Prüfung

- beim Schwarzfahren ertappt

$-(\ldots)$

6. Rekonstruktion des Ausgangstextes: von „Experten“ gesprochen:

Die Sätze des Textes werden wieder in ihrer richtigen Reihenfolge gesprochen. Jeder Teilnehmer spricht dabei seinen Satz möglichst auswendig, diesmal in der Rolle des stolzen „Experten“ (überzogen gespreizt/affektiert).

Erst als schriftliche Hausaufgabe erfolgt schließlich die ernsthafte Auseinandersetzung mit dem Text, indem beispielsweise Fragen zum Text beantwortet, Paraphrasierungen geübt werden o. Ä.

\subsection{Theoretische Überlegungen}

Ein möglicher Einwand zu diesem Verfahren wäre, dass bei Übungen zur gesprochenen Sprache ausgerechnet vom geschriebenen Text ausgegangen wird. Dem lassen sich leicht die Ausführungen Schwitallas (1997) entgegensetzen, welcher feststellt, dass gesprochener und geschriebener Sprache das gleiche System zugrunde liegt:

Manche scheinbaren Strukturunterschiede sind oft auf Performanzbedingungen zurückzuführen. Elisionen und Kontraktionen, die beim Sprechen häufig vorkommen, werden wieder aufgehoben, wenn man langsam oder emphatisch spricht. (Schwitalla 2003: 24)

Auf anderer Ebene stellt sich die Frage, ob man zuerst Gespräch oder zuerst Rede üben sollte. Forster plädiert mit Verweis auf Geißner dafür, zuerst das Gespräch zu üben, als Prototyp jeglicher Kommunikation. Rede entstehe häufig aus der Unmöglichkeit, ein Gespräch zu führen (Forster 1997: 51).

Für den Fremdsprachenunterricht im Ausland halte ich es persönlich für sinnvoller zunächst kleine Redeabschnitte, kurze monologische Einheiten zu proben. Denn Rede stärkt - und das bestätigt wiederum auch Forster - erwiesenermaßen das Selbstwertgefühl: 
In einer Fremdsprache sogar Reden halten zu können, als in diesem Moment vereinzelter Sprecher gibt einem Sprachenlernenden zusätzliche Sicherheit. Die Notwendigkeit, Rede in den Unterricht Deutsch als Fremdsprache zu integrieren, lässt sich demnach auch lernpsychologisch begründen. ( Forster 1997: 237)

Vom monologischen Abschnitt kann schließlich immer noch ein Bogen zum Streitgespräch geschlagen werden, wenn man als Vorarbeit das Einstudieren entsprechender Redemittel geleistet hat. Dies lässt sich ähnlich wie oben durchführen, ausgehend von fertigen Sätzen, welche zunächst auswendig gelernt und dann variiert werden, um mit humorvollem Inhalt gefüllt und in absurden Kontexten erprobt zu werden.

Ein Risiko der Habitusanpassung bleibt natürlich die angepasste Performanz ohne entsprechende Kompetenz. Feilke/Steinhoff (2003: 120) halten dagegen:

„Erste Beobachtungen an den Texten von Studierenden zeigen, dass eine imitative Übernahme von Formen in den Anfangssemestern phasenweise eine bedeutsame und positive Rolle spielen kann: Die Kompetenz wächst der Performanz nach. Studierende machen sich mit dem wissenschaftlichen Schreiben vertraut, indem sie Experten nachahmen."

Es ist anzunehmen und zu wünschen, dass es sich bei der mündlichen Sprachproduktion ähnlich verhält.

Ein letzter wichtiger Aspekt, dem die gesamte Unterrichtseinheit Rechnung tragen muss, ist das Problem der korrekten Aussprache.

Durch die Disposition zur kategorialen Sprachperzeption (List 2003) und die Ausbildung phonetischer Prototypen, die sich an den Lauten der Muttersprache orientieren, fällt es beim Erlernen einer Fremdsprache oft schwer, bestimmte Lautdifferenzierungen wahrzunehmen und zu reproduzieren. Es muss zunächst ein so genanntes reattunement stattfinden, eine perzeptive Reorganisation phonologischer Kategorien (vgl. Missaglia 2005: 66). Dies bedeutet, dass zu den Sprechübungen parallel Hörverständnis-Übungen durchgeführt werden müssen. Oder anders gesagt: dass dem Hören immer auch das Horchen, Nachahmen und Ausprobieren folgen muss. Im Fall der Übungen zur Wissenschaftssprache empfiehlt es sich, mit den Teilnehmern Aufzeichnungen von Vorlesungen zu hören und zu analysieren 5 .

Missaglia (2005: 67) unterscheidet in ihren Untersuchungen zu deutschen und italienischen phonetischen Prototypen zwischen dem akzentzählendem Rhythmus des Deutschen und dem silbenzählenden Rhythmus des Italienischen:

\footnotetext{
${ }^{5}$ Für die Arbeit mit solchen Texten bietet u.a. der Videokurs „, Präsentieren und Verhandeln“ (Goethe-Institut Internationes 2003) ein gutes Beispiel: Hier können die Lernenden Vorträge hören und in der Transkription die entsprechenden Akzente und Pausen setzen.
} 
„Die entgegengesetzeten Tendenzen zum silben- bzw. akzentzählenden Rhythmustyp wirken sich nicht nur auf die Muttersprache aus, sondern auch auf die Interlanguage der Fremdsprachenlerner. ...0 Untersuchungen zum Sprechverhalten italienischer Deutschlerner deuten auf die Tendenz zur Elaboration (z. B. Schreiben) hin, zur Lautstärkung (besonders bei Vokalen), zur Schwa-Epenthese. Dazu kommen Interferenzen auf der suprasegmentalen und segmentalenEbene, etwa in Form falscher Wort-und satzakzente, fehlerhafter Vokallaute, mangelnder Auslautverhärtung usw.“

Der Autorin nach lässt sich darüber hinaus annehmen, dass neben den Prototypen auf der segmentalen Ebene auch „prosodische Prototypen“ herausgebildet werden, etwa in Verbindung mit dem Akzentmuster, der Intonationskontur und dem Rhythmustyp, die beim Fremdspachenerwerb die Wahrnehmung der Zielsprache verzerren (Missaglia: 68).

Dass die Prosodie oft nachhaltig Schwierigkeiten bereitet, ist ein allgemeiner Erfahrungswert im Fremdsprachenunterricht. Wirksame Übungen zur Verbesserung der Prosodie lassen sich mit rhythmischen Texten erzielen (vgl. Jax 2000: 169, Huber 2003: 59, Reinhardt 2005: 491 ff.), wie unter anderem im folgenden Kapitel anklingen wird.

\title{
3 Die Inszenierung als intensive Verinnerlichung sprachlicher Muster
}

\author{
Habe nun, ach, Philosophie, Juristerei und Medizin, \\ und leider auch Theologie \\ durchaus studiert mit heißem Bemüh'n, \\ sagt Faust. \\ Wie kann Faust so viel studiert haben,... \\ Und noch so jung sein? \\ fragen sich Gabi, Susi und Hella nach einer Faust-Aufführung. \\ Ganz einfach, \\ so die Antwort von Mephisto: \\ Bachelor-Studiengang! \\ „Laurea Breve“.
}

So heißt es in der ersten Szene des Stückes „Laurea Brevis - Ein Pakt mit dem Teufel“", welches eine Gruppe meiner Studenten im Sommersemester 2005 im 
Rahmen unseres Projekts TILLIT (teatro in lingua) ${ }^{6}$ aufgeführt hat. Nachdem sie im kleinen Stadttheater eine Faust-Aufführung gesehen haben, schließen drei junge Mädchen eine Wette mit dem Teufel ab und schreiben sich für den BachelorStudiengang ein. Den Original-Faust-Text zitierend schwören sie dabei:

Susi:

Hella:

Werd ich zum Augenblicke sagen:

Gabi: Verveile doch, du bist so schön!

Susi: Dann magst du mich in Fesseln schlagen.

Dann will ich gern zugrunde gehen!

Es folgen Szenen aus dem Uni-Alltag, die die Hetze durch das Studium der so genannten Laurea Breve - dem „Kurzstudium“ (Bachelorstudiengang) ${ }^{7}$ - und das Verschwinden der Inhalte in den geisteswissenschaftlichen Fächern auf humorvolle und dramatische Weise darstellen.

Eine der drei Studentinnen muss dem Teufel schließlich auch ihre Seele überlassen, als sie bei einem Eichendorff-Gedicht ins Schwelgen gerät und Zeit verliert. Alle sind Opfer, Studenten wie Professoren. Nur die Teufel (Mephisto 1 und 2) triumphieren, und ein korrupter Hausmeister macht Geschäfte mit Kreditpunkten.

Die einzelnen Szenen der Vorlesungen folgen immer dem gleichen Grundmodell: ein Dozent/eine Dozentin spricht zu Studenten - im Hintergrund steht Mephisto mit einem Riesenwecker. Unter dieser Vorgabe haben die Studenten in Improvisationsarbeit ein Teil der weiteren Szenen zunächst auf Italienisch entwickelt. Diese wurden von mir dann auf Deutsch geschrieben, unter anderem unter Verwendung möglichst vieler wissenschaftssprachlich typischer Strukturen.

\subsection{Sprachliche Aspekte des Textes}

Das Theaterstück ist sprachlich bewusst abwechslungsreich gestaltet. Durch die Alliterationen am Anfang wird auch auf der sprachlichen Ebene schnell ein Spielrahmen erzeugt.

(Ausschnitt:)
Publikum:
Eine aus dem Publikum:
Faust als Frau.... Fragwürdig!
(...)
Chor der Zeitungsleser:
Fürchterlich!
Geniale Interpretation!

\footnotetext{
${ }^{6}$ Im Rahmen des Projekts TILLIT, vor zweieinhalb Jahren an der Universität Vercelli ins Leben gerufen (Marco Pustianaz / Michaela Reinhardt), erarbeiten Studentengruppen in den vier Fremdsprachen Englisch, Französich, Spanisch, Deutsch kurze Theaterstücke, die am Endes des Studienjahres aufgeführt werden.

${ }^{7}$ Der Bachelor-Studiengang wurde in Italien schon im Jahr 2001an den meisten Universitäten eingeführt.
} 
Fritz Feuer inszeniert in Freuden-stadt Goethes Drama.

Faust als Frau macht Furore!

Fantastisch! - Faszinierend! -

Stille - alle sehen sich verlegen an...(,funderbar?")

Phänomenal!- Funderbar!

Dem Faust-Originaltext wird etwas später im Text eine Rap-Version gegenübergestellt:

Hab Physik studiert und Mathe ausprobiert,

Hab Chemie gemacht, über Quan-ten nachgedacht.

Bin so schlau wie zuvor, doch ist mir das egal.

Mit der Uni bin ich fertig und das reicht mir allemal.

Durch die Einbindung von rhythmischen Abschnitten wird ein starker Kontrast zum restlichen Text erzeugt. Das Erlernen dieser rhythmischen Textstücke wie dem Goethe-Text im Original, dem Rap und dem Eichendorff-Gedicht bereitete den Teilnehmern am wenigsten Schwierigkeiten und oft viel Spaß.

Die wissenschaftssprachlichen Abschnitte hingegen gewinnen durch ihre parodistische Überzogenheit besonderen Reiz. In den folgenden Textausschnitten sind die typischen Strukturen von alltäglicher Wissenschaftssprache markiert:

- Ausschnitt aus Szene 2 (Philosophie - Vorlesung):

Prof: $\quad$ Der Auffassung Kants nach steht (Präsensform) hinter der Bedingtheit des Menschen seine..... Unbedingtheit ( Sprechpause vor wichtigen, neuen (Fach-)Begriffen). Die Kritik der reinen $V$ ernunft entstand...

Mephisto 2: $\quad$ Drinnng!!

Prof:

Also. Äh.....

Der kategorische Imperativ lässt sich folgendermaßen erklären...(Passiversatzform)

Mephisto 2: $\quad$ Drinnnnng!

- Ausschnitt aus Szene 3 (Vorlesung in Wirtschaftswissenschaften): 
Prof: $\quad$ Mit Blick auf die Dynamik des Weltmarktes erweist sich (Personifizierung ) Zeitökonomie als der wichtigste Faktor für Kompetitivität. (erfundener „Fachbegriff“")

- Ausschnitt aus Szene 4 (Vorlesung in Literaturwissenschaft):

Prof: Die für Goethe nötige und in seinem Werk erstrebte (Partizipialkonstruktion)_Interdependenz von Literaturund Standardsprache wird vom romantischen Subjektivismus ausgeschlossen.

Hören Sie nur:

„und meine Seele spannte

weit ihre Flügel aus,..."

- Ausschnitt aus Szene 5 (Die Synchron-Vorlesung):

Prof für Entwicklungspsychologie:

Das bei Säuglingen am häufigsten verwendete (Partizipialkonstruktion) Verfahren operanter Konditionierung ist die Konditionierung mit Hilfe eines Mobiles, die in der Regel (typische Wendung) aus einer Lernphase und einem Gedächtnistest besteht.

Theologie-Prof: Die diversen Exegeten von Lukas 4, Vers 1-13 sind sich nicht darüber einig, welche Gestalt dem Teufel als Inkarnation des Bösen in diesem Artikel zuzuschreiben wäre (Passiversatzform).

Das Stück gipfelt in der „Synchron-Vorlesung“ (s. Ausschnitt oben), in welcher aus Gründen der Zeitersparnis alle Dozenten gleichzeitig ihre Vorlesung halten. Mephisto macht sich einen Spaß daraus, ergreift einen Taktstock und dirigiert, während die Dozenten fortfahren ihre einzelnen Sätze zu wiederholen. Er gibt Tempo und Pausen an, erzeugt Crescendos und Decrescendos, so dass aus dem Sprachgewirr der Profs ein Chor in Wissenschaftssprache entsteht (s. Foto). 


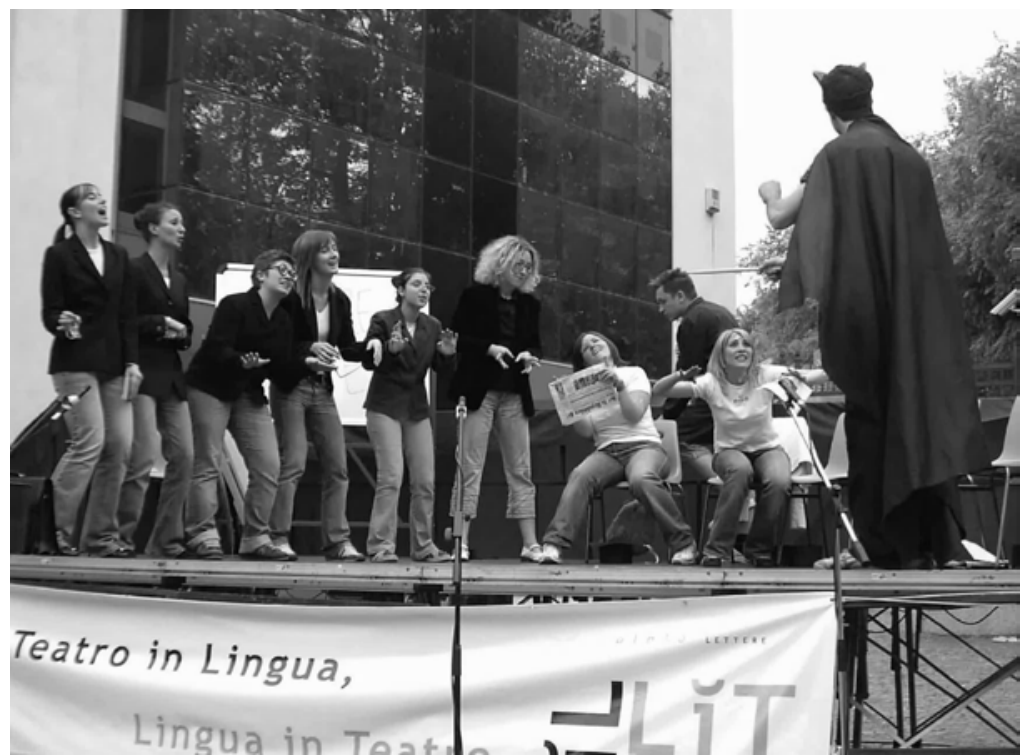

Die „Synchron-Vorlesung“: Aufführung von Laurea Brevis - Ein Pakt mit dem Teufel, Vercelli, 1.6.06

Unweigerlich endet das Stück mit der Fortsetzung des Faust-Zitats:

Faust 1/Gabi: (...)

Hier steh ich nun,

Susi: ich armer Tor,

Hella: $\quad$ und bin so klug

Alle: $\quad$ als wie zuvor!

\subsection{Didaktische Überlegungen}

Wichtig ist es bei solcher Arbeit, von Situationen auszugehen, die die Gruppe direkt betreffen, wie in diesem Fall die Hetze durch das Studium ${ }^{8}$. Von einem solchen primären Rahmen (Goffman 1980: 31) aus gerät die Gruppe leicht in den Spielrahmen. Schüler und Studenten üben sich untereinander sowieso oft in parodistischer Auseinandersetzung mit dem Schul- bzw. Uni-Alltag. Daher sind die Teilnehmer meiner Gruppe wahrscheinlich auch ohne Zögern auf das Spielange-

\footnotetext{
8 Was die geisteswissenschaftlichen Fächer betrifft, hört man oft, dass die Studenten darunter leiden, nichts mehr vertiefen zu können, keine Zeit für Arbeitsgruppen mehr zu haben. Auch Theaterarbeit schien an der Uni unter diesen Bedingungen unmöglich geworden zu sein. Mit dem Projekt TILLIT haben wir an unserer Universität durchsetzen können, dass Theaterarbeit offiziell in das Credit-

Punkt-System eingebunden wird.
} 
bot eingegangen und haben ihre Erfahrungen szenisch umgesetzt. Im Unterschied zur so genannten SAF (Simulation authentischer Fälle), wie sie als Planspiel heutzutage in vielfältigen Bereichen durchgeführt wird, gewährt hier die größere Distanz zwischen primärem Rahmen und Spielrahmen mehr Freiheit, auch, was die Wahl der Rollen betrifft (vgl. hierzu Reinhardt 2006: 484)

Durch die Authentizität der Rolle auf fiktiver Ebene erreicht man schließlich leicht den Schemakongruenz-Effekt, die Verarbeitung schemakonsistenter Informationen, welche beim Spracherwerb besonders hilfreich sind (vgl. Reinhardt 2006: 483).

\section{Schluss: Ergebnisse einer Erhebung zu Theaterarbeit}

Im Rahmen des Theaterprojekts TILLIT wurden von mir anhand eines fünfseitigen Fragebogens verschiedenste Kommentare, Erfahrungen und Bewertungen der Teilnehmer gesammelt. Das derzeitige Datenmaterial stammt aus der Befragung von ca. 50 Studenten, die in diesem und im letzten Jahr am Projekt teilgenommen haben.

Für besonders interessant halte ich in diesem Zusammenhang die Ergebnisse zum Thema Auswendiglernen. Und hier wiederum im Besonderen die Ergebnisse derjenigen Studenten, die im vergangenen Jahr teilgenommen hatten und ein Jahr später diese Arbeit beurteilen:

- Alle Befragten halten Auswendiglernen für sehr sinnvoll.

- Auf die Frage, welche Aspekte es ihnen beim Lernen des Textes geholfen hätten, waren die häufigsten Antworten: (1) die Art, in der Gruppe zu üben, im Chor zu sprechen und mit der Sprache zu experimentieren. (2) der Spaß, den diese Arbeit mit sich brachte. (3) die entspannte Atmosphäre in der Gruppe.

- Alle behaupten, auch nach einem Jahr noch fast ihren gesamten Text zu erinnern. $85 \%$ erklären, dass es ihnen oft so gehe, dass Wörtern aus dem Text begegnen von daher ihren gesamten Rollentext erneut abrufen können.

- Besonders gut eingeprägt haben sich Chunks.

- $80 \%$ der Befragten erklären, dass sie Satzstücke und Strukturen ihres Parts inzwischen anderweitig, in neuen Kontexten flexibler anwenden können. Eine Studentin schreibt: „Oft hilft mir mein Text, mich beim Sprechen ganz allein zu korrigieren“.

- Alle Befragten betonen, dass sie darüber hinaus noch große Teile der Parts der anderen auswendig können.

Solche Ergebnisse lassen hoffen, dass sich auch wissenschaftssprachliche Strukturen im Daf- Unterricht tiefer einprägen lassen, über den Weg des Auswen- 
diglernens, des Sich-zu-eigen-Machens und des Experimentierens im Spielrahmen, um dann später entsprechend flexibel angewandt werden zu können.

\section{Literatur:}

Bonset, Helge/ Kusters, Ton : Das Problem der Fachsprache im nichtfachsprachlichen Unterricht. In: Ehlich, Konrad/ Rehbein, Jochen (Hrsg): Kommunikation in Schule und Hochschule. Tübingen: Narr 1983

Feilke, Helmuth / Steinhoff, Torsten : Zur Modellierung der Entwicklung wissenschaftlicher Schreibfähigkeiten. In : Ehlich, Konrad / Steets, Angelika (Hrsg) : Wissenschaftlich schreiben- lehren und lernen. Berlin, New York : De Gruyter 2003

Fluck, Hans-Rüdiger : Didaktik der Fachsprachen. Tübingen : Narr 1993

Forster, Roland : Mündliche Kommunikation in Deutsch als Fremdsprache:

Gespräch und Rede. St.Ingbert: Röhrig Universitätsverlag 1997

Goffman, Erving : Rahmen-Analyse. Frankfurt/M.: Suhrkamp 1980

Huber, Ruth: Im Haus der Sprache wohnen. Wahrnehmung und Theater im Fremdsprachenunterricht. Tübingen: Niemeyer 2003

Huber, Ruth / Reinhardt, Michaela: „Lesen - Sprechen - Spielen. Lebendiges Zeitungstheater". In: Babylonia 4/2005

Jax, Veronika: „Was mich beim Fremdsprachenerwerb bewegt, was ich beim Fremdsprachenerwerb bewege“. In :Schlemminger,G./ Brysch,T./

Schewe,M.L ( Hrsg.): Pädagogische Konzepte für einen ganzheitlichen DaFUnterricht. Berlin: Cornelsen, 2000, 166-183

List, Gudula : „Sprachpsychologie“. In : Handbuch Fremdsprachenunterricht (4. Aufl.) Tübingen und Basel : Francke 2003, 25-31

Markowitsch, Hans J. / Welzer, Harald : Das autobiographische Gedächtnis. Stuttgart : Klett-Cotta 2005

Missaglia, Federica: „Phonetische Prototypen und Zweitspracherwerb“. In: Claudio Di Meola / Antonie Hornung / Lorenza Rega (Hgg.) (2005).

Perspektiven Eins. Akten der 1.Tagung Deutsche Sprachwissenschaft in Italien (Rom, 6.-7.2.2004). Rom: Istituto Italiano di Studi Germanici 2005

Reinhardt, Michaela: „Plädoyer für mehr Theaterarbeit im

Fremdsprachenunterricht - am Beispiel eines expressionistischen Textes (die Wandlung von Ernst Toller)“. In: Claudio Di Meola / Antonie Hornung / Lorenza Rega (Hgg.) (2005). Perspektiven Eins. Akten der 1. Tagung 
Deutsche Sprachwissenschaft in Italien (Rom, 6.-7.2.2004). Rom: Istituto Italiano di Studi Germanici 2005

Reinhardt, M.: „Was soll das Theater?" Materialien Deutsch als Fremdsprache. Heft 76: Umbrüche. Regensburg: Fachverband Deutsch als Fremdsprache 2006

Reinhardt, M. / Gruppe TILLIT-Deutsch 06 : „Laurea Brevis - Ein Pakt mit dem Teufel“". Theaterstück im Rahmen des Projekts TILLIT an der Universität Piemonte Orientale, Vercelli: 2006

Schwitalla, Johannes: Gesprochenes Deutsch. Berlin: Erich Schmidt Verlag 2003 


\section{Das mündliche Seminarreferat zwischen Theorie und Praxis}

\section{Anja Centeno García, Dresden}

Besonders in den deutschen Geisteswissenschaften bestimmt das mündliche Referat neben schriftlichen Seminararbeiten den universitären Alltag. Trotz seiner zentralen Stellung zeigt sich immer wieder, dass häufig weder deutsche noch ausländische Studierende diese „ominöse Textsorte“ (Hermanns 1980) angemessen beherrschen, obwohl scheinbar ein reichhaltiges Angebot an Ratgeberliteratur und speziellen Rhetorikkursen zur Verfügung steht.

Nach einem praxisorientierten Problemaufriss (\$1) und einer Skizze des aktuellen Forschungsstandes (\$2) werden die Ergebnisse einer 2005 durchgeführten Untersuchung ${ }^{1}$ exemplarisch für 3 zentrale Aspekte (außertextuelle Faktoren und Textfunktion; Wissenschaftlichkeit als Stilmerkmal; Visualisierung) dargestellt (\$3).

\footnotetext{
${ }^{1}$ Durchgeführt wurde eine text- und gesprächslinguistische Analyse sowie eine empirische Erhebung unter Dozenten und Studierenden der Institute für Germanistik und Romanistik an der TU Dresden zu ihren Beurteilungskriterien von Referaten. Ausgewertet wurden 11 Leitfadeninterviews mit Professoren und DozentInnen sowie eine Fragebogenerhebung $(\mathrm{N}=170)$ unter den Studierenden. Die Analyse erfolgte primär quantitativ, schloss jedoch weitgehend auch qualitative Aspekte mit ein. Eine Veröffentlichung der gesamten Untersuchung im Tectum Verlag Marburg ist Anfang 2007 geplant.
} 
Vor diesem Hintergrund werden abschließend einige didaktische Überlegungen formuliert (\$4).

\section{Problemaufriss}

Bereits ein Blick auf die curricularen Festlegungen der untersuchten Fachbereiche zeigt, dass sich die Festlegungen zu Anforderungen bei der Erbringung mündlicher Studienleistungen erheblich bzgl. ihrer Eindeutigkeit unterscheiden. Im Rahmen der Zielformulierung betonen die untersuchten Studienordnungen allgemein den Erwerb und die Vertiefung praxisorientierender Schlüsselkompetenzen, u. a. der kommunikativen Kompetenz. Lediglich eine Studienordnung fordert explizit ein Minimum von 6 Referaten als Voraussetzung für den Erwerb von Leistungsnachweisen. Alle übrigen Studienordnungen verweisen auf die Festlegungen des Kursleiters. Aus den Vorlesungsverzeichnissen wird jedoch die dominierende Stellung des Referats in den Pro- und Hauptseminaren ersichtlich. Die Umsetzung betreffend gibt es keine curricularen Festlegungen. Es ist folglich davon auszugehen, dass die Anforderungen auch innerhalb der geisteswissenschaftlichen Disziplinen variieren und Text- bzw. Handlungsmuster von den Studierenden entsprechend zu aktualisieren sind.

Praktische Ratgeber und Rhetorikkurse akzentuieren vornehmlich das reine Training oder orientieren auf allgemeingültige „Rezepturen“ zur erfolgreichen Bewältigung mündlicher Kommunikation im Studium. Im Mittelpunkt stehen dabei die Aspekte: Adressatenorientierung, klare Strukturierung, Manuskriptgestaltung, Visualisierungshilfen, Blickkontakt und Körperhaltung sowie sprachliche Umsetzung.

Lehrwerke für den (studienbegleitenden) DaF-Unterricht greifen das Thema (Kurz-) Vortrag explizit und zwangsläufig sehr begrenzt erst ab der Mittelstufe auf (z. B.: Untervegs, em, Das Mittelstufenbuch, Barthel). Schwerpunkte sind dann Gliederungshinweise, teilweise in Verbindung mit Redemitteln; Hinweise zur inhaltlichen Gestaltung sowie in Ansätzen stilistische Empfehlungen.

Da die Studierenden offensichtlich institutionelle Erwartungen der Lehrenden erkennen und sprachliche Probleme im Einzelnen lösen müssen, scheint hier das Anwenden der empfohlenen Rezepte nur kurzfristig eine Hilfe. Weiterreichend müssen sie jedoch die tiefergreifende Problem- und Lösungsstruktur durchschauen und in den systematischen Zusammenhang sprachlichen Handelns im Rahmen der Institution Universität einordnen (vgl. Redder 2002: 6f.). Die Befragung der Studierenden verdeutlichte, dass Grundkenntnisse zu formalen Kriterien der Textsorte Referat, z. B. zur Textstrukturierung, durchaus vorausgesetzt werden können. Ebenso waren den Studierenden allgemeine Empfehlungen, z. B. Adressatenorientierung, möglichst freies Sprechen, überwiegend geläufig. Großer Klärungsbedarf bestand bei Fragen, die den konkreten Zweck eines Referates und daraus resultierende Anforderungen sowie die Art der Umsetzung betrafen, da die Studierenden eine breite Varianz verschiedenster Textexemplare als Realisationen 
der Text- und Diskursart Referat beobachtet hatten. Es ist anzunehmen, dass die augenscheinliche Vielfalt der Umsetzungsmöglichkeiten und Anforderungen die Ausbildung eindeutiger Textmuster erschwert.

\section{Zum aktuellen Forschungsstand}

Die linguistische Forschung beschäftigt sich in den letzten Jahren zwar zunehmend auch mit dem akademischen Lehr- und Lerndiskurs und damit einhergehend mit studentischen Texten und Textsorten (Wiesmann 1999, Fischer / Guadatiello 2002, Ehlich/Rehbein 1986), jedoch stehen bislang entweder Aspekte der Vermittlung von Deutsch als fremder Wissenschaftsprache allgemein oder schriftliche, studentische Texte und Textsorten (z. B. Eßer 1997, Hufeisen 2002, Kaiser 2002) in ihrem Blickfeld. Das mündliche Seminarreferat hingegen bleibt daher in seiner Komplexität wenig untersucht. Erste Ansätze liefern jetzt Guckelsberger/Stenzano Cotelo (2004) oder Ribeiro-Kügler (2002). Die mangelnde empirische Untersuchung resultiert nicht zuletzt aus den Schwierigkeiten, die verschiedenen Realisationsebenen und Prozesse der Wahrnehmung des mündlichen Referats der Analyse zugänglich zu machen. Auch Guckelsberger/Stenzano Cotelo beschränken sich auf eine exemplarische Untersuchung am Einzelbeispiel mit dem Ziel Anforderungen an die Text- und Diskursart Referat festzustellen, wodurch übergeordnete funktionale, kommunikative und wahrnehmungspsychologische Aspekte keine Beachtung finden. Gezielte empirische Erhebungen zur Konkretisierung des Anforderungsprofils in der Praxis bzw. zu kollektiven Beurteilungskriterien, die der einzelnen Textrealisation übergeordnet sind, stehen noch aus.

\section{Theorie und Praxis im Vergleich}

Vor diesem Hintergrund ist es notwendig, theorie- und praxisorientierte Grundlagen für zukünftige Kursplanungen zusammenzufassen, die über die Empfehlungen gängiger Ratgeber hinausgehen.

Ausgangsbasis der Auseinandersetzung bilden die von Ruth Eßer (1997: 36) und Britta Hufeisen (2002: 46) verwendeten Modelle zu Konstituenten von Textsorten, die jedoch erst in ihrer Verbindung eine angemessene Beschreibungsgrundlage darstellen (Abb. 1). 


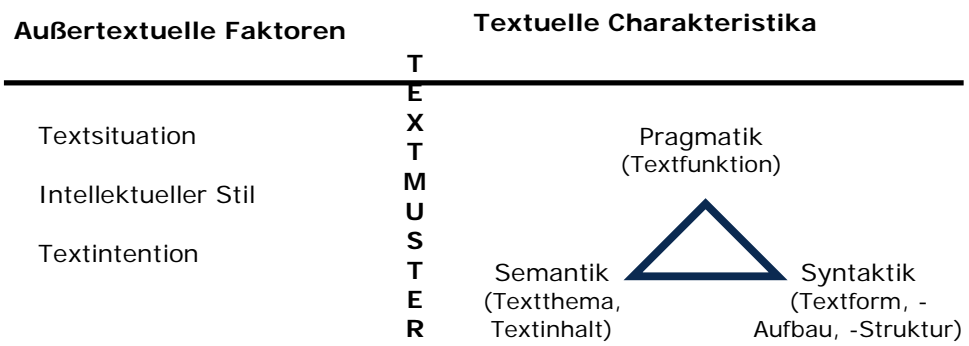

Abb. 1: Basierend auf den Modellen von Eßer 1997 und Hufeisen 2002

Beide gehen von der Feststellung aus, dass die Textmuster einer Textsorte durch spezifische Bedingungen des Kommunikationsbereiches bestimmt werden und damit einhergehend in synchroner und diachroner Dimension durch kulturund domänenbezogene Faktoren geprägt sind. Mit Hufeisen (2002: 50) gehe ich davon aus, dass (a) text- und themenexterne Faktoren funktionssteuernd wirken und (b) Textmuster hierarchisch organisierte Bedingungsgefüge textueller Charakteristika sind. Erst über die Auseinandersetzung mit der Textfunktion erschließen sich die weiteren textuellen Charakteristika².

Die theoretische Konkretisierung der Konstituenten ergab Kernthemen, die es in der Praxis zu hinterfragen galt.

1. Die Textfunktion wird durch außertextuelle Faktoren entscheidend beeinflusst. Es ist daher zu fragen, welche Ziele mit dem Einsatz von Referaten verfolgt werden und wie sich diese auf die Textfunktion auswirken.

2. Davon ausgehend, dass Informieren und Aktivieren zentrale Textfunktionen sind und sich erst über diese die semantische sowie die syntaktische Dimension erschließen, ist zu prüfen, welche Erwartungen die Rezipienten an Inhalt und Form, bzw. Wissenschaftlichkeit im Besonderen stellen.

3. Gegenseitige Wahrnehmung und Erwartungen prägen das Gesamturteil über die Qualität eines Referats. Aktuelle Entwicklungen bzgl. des Einsatzes neuer Medien lassen nach der Rolle der Visualisierung fragen.

\section{$4 \quad$ Außertextuelle Faktoren und Textfunktion}

Die außertextuellen Faktoren (nach Eßer 1997) können einerseits auf der Grundlage institutioneller Rahmenbedingungen konkretisiert werden. Andererseits wirken sie implizit im Sinne von kultureller, domänenspezifischer und individueller

2 Das deckt sich nur bedingt mit der von Eßer vertretenen Ansicht, daher werden textuelle Charakteristika (Abb. 1) mit Hilfe des Modells nach Hufeisen dargestellt. 
Prägung. Das mündliche Referat als eine besondere Form der Lehr- und Lernkommunikation ist zum einen ein Mittel der wissenschaftlichen Sozialisation für Referent und Zuhörer. Zum anderen ist es, auf der Basis curricularer Grundlagen, ein Instrument der Leistungsprüfung. Daraus ergibt sich ein spezifisches Beziehungsgefüge der Kommunikationspartner (Abb. 2), das von unterschiedlichen Erwartungen und Anforderungen geprägt ist.

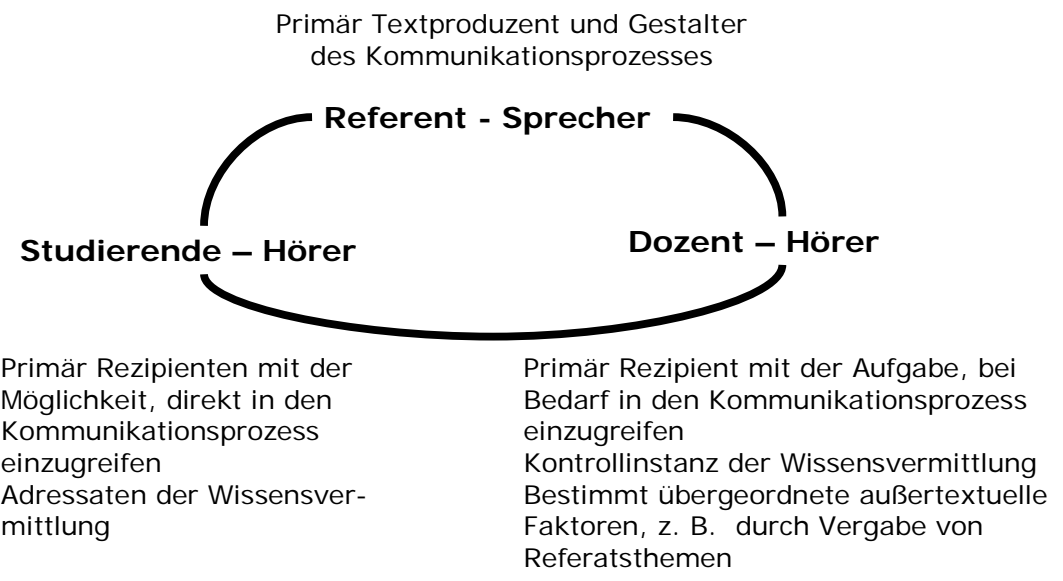

Abb. 2: Beziehung der Kommunikationspartner

Aufgrund dieser außersprachlichen Bedingungen ist das Referat multifunktional. Die zentrale Funktion ist das sachbetonte Informieren über einen bestimmten, fachspezifischen Sachverhalt unter gleichzeitiger Aktivierung der Zuhörer zum Zwecke der wissenschaftlichen Sozialisation. Aus diesem Grund dominieren bei den Zuhörern inhaltsbezogene Erwartungen, die durch individuelle Präferenzen unterschiedlich ausgeprägt sind. Allerdings ist die Bewertung durch den Dozenten für den Referenten ein zentraler Aspekt, der nicht text-, diskursimmanent, sondern institutionell bedingt ist. Die Sekundärfunktion Selbstdarstellung zum Zweck der Leistungsbewertung erhält dadurch ein verstärktes Gewicht. Hufeisen spricht von einer verschobenen Textfunktion (Hufeisen 2002: 50). Um die Akzeptanz der individuellen Umsetzung eines Textmusters zu gewährleisten, muss die Textfunktion für alle klar und gleich sein, d. h. im Zuge wechselseitiger Abhängigkeit, muss die syntaktisch-semantische Dimension für die Rezipienten wieder erkennbar und rekonstruierbar sein.

Im Vergleich mit der Praxis konnte anhand der Dozentenaussagen eine Hierarchie bezüglich der verfolgten Ziele beim Einsatz von Referaten festgestellt werden:

1. Kompetenzerwerb des Referenten

2. Wissensvermittlung durch Weitergabe von Sachinformationen 
3. Wissensvermittlung durch Aktivierung der Zuhörer zu kritischem Mitdenken (Die Diskussion und deren Moderation sind daher häufig Teil des Referats.)

Der angestrebte Kompetenzzuwachs bezieht sich auf den Referenten und soll im Referat zum Ausdruck kommen. Bzgl. der Wissensvermittlung verfolgen die Dozenten im Seminarkontext sehr unterschiedliche Ziele. So kann von einem Referat die Einführung in ein Thema genauso erwartet werden wie die Präzisierung von Teilaspekten. Es kann auch von der Wissensvermittlung im Sinne von Lernstoffvermittlung abweichen und auf eine Problematisierung oder die Positionierung des Referenten in Hinblick auf relevante Ansätze, Methoden etc. abzielen. In einigen Fällen nehmen Dozenten vom Referat in „klassischer Form“ Abstand und bevorzugen z. B. die mündliche Präsentation einer Projektarbeit.

Im Rahmen der freien Äußerungen zu Qualitätskriterien geben die Studierenden inhaltsbezogenen Aspekten ein starkes Gewicht und sehen das Referat als zentrales Mittel der Informationsvermittlung (238 von 829 Nennungen). Sie erwarten dabei, dass zum einen die Relevanz des Themas durch den Referenten herausgearbeitet wird und favorisieren systematische Überblicksdarstellungen anstelle detailreicher Einzelaspekte. Bzgl. der inhaltlichen Ausrichtung wird von ihnen die Absicherung von Lerninhalten erwartet. Hier möchten die Studierenden aktiv in die Erarbeitung der zu vermittelnde Inhalte einbezogen werden und sehen deshalb das Referat als Diskussionsvorbereitung bzw. Diskussionsgrundlage (vgl. Abb. 3). Die Daten legen den Schluss nahe, dass die Diskussion von den Studierenden als zentrales Instrument der Verständnissicherung und -prüfung betrachtet wird.

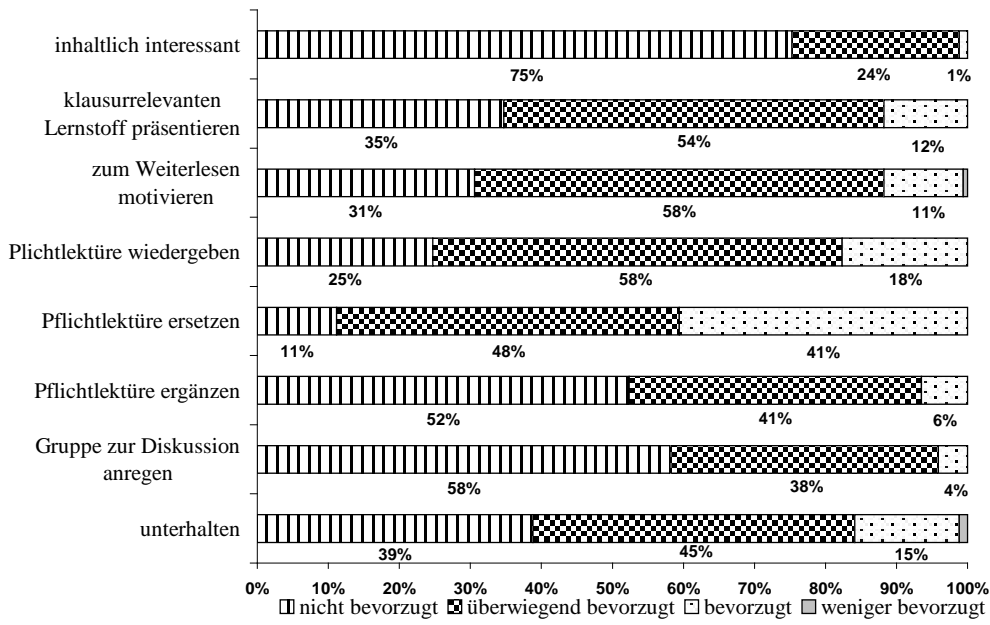

Abbildung 3: Erwartungen der Studierenden an ein Referat (Frage 3) 
Die Ausprägung der konkreten Anforderungen ist demnach von großer Varianz innerhalb der jeweiligen Zielgruppe und von Unterschieden zwischen Dozenten und Studierenden gekennzeichnet. Damit die Textfunktion als Gelingensbedingung für alle klar und gleich ist, ist sie eindeutig zu spezifizieren. Das heißt, das bloße Nennen des Referatsthemas und der Gliederung als Einleitung durch den Referenten sind nicht ausreichend. Die explizite Darstellung der Textintention in Form einer eindeutigen Zielformulierung ist zwingend erforderlich, um für alle Aktanten ein gleiches und klares Verständnis zu gewährleisten. Im Vorfeld sind Ziele und Funktionen für das erwartete Textmuster für alle allgemein, bzw. in der Planungsphase mit dem Referenten durch den Dozenten klar zu explizieren, auch wenn Informieren und Aktivieren als zentralen Funktionen festgestellt wurden.

\section{$5 \quad$ Wissenschaftlichkeit als Stilmerkmal}

Der Textinhalt ist vornehmlich unter der Prämisse zu betrachten, dass studentische Forschung weniger Beitrag zur Wissensgewinnung ist, sondern vornehmlich dem Erlernen wissenschaftlicher Arbeitstechniken dient. Im Zusammenhang mit einer, inventiven ' Wissenschaftskultur (Ehlich 1996: 84) haben studentische Textsorten einen Als-ob-Status. Daher ist Wissenschaftlichkeit bereits während der akademischen Ausbildung eine zentrale Anforderung. Laut Aussagen der Dozenten soll sich Wissenschaftlichkeit vornehmlich darin zeigen, wie der Referenten mit Thema und Literatur umgeht. Im Zentrum des Referates steht die Verarbeitung von Wissen aus Texten im Rahmen eines weitgehend vom Dozenten vorgegebenen Themas. Guckelsberger/Stenzano Cotelo (2004: 420) beschreiben die Überblicksdarstellung als zentralen Gegenstand von Referaten. Die Erhebung zeigt eindeutig, dass es sich dabei nur um eine mögliche Variante handelt. Im Referat erwarten die Dozenten eine Selektion bzw. Verdichtung der Inhalte und Informationen und das Herstellen von Beziehungen (Theorie-Praxis; TheorieBeispiel; und Theorie-allgemein fachlicher bzw. Seminarkontext). Die verwendete Literatur zum Thema wird vornehmlich als Arbeitsgrundlage gesehen. Das Erwartungsspektrum bzgl. der inhaltlichen Aufbereitung reicht von der kritischen Stellungnahme über die thesenartige Zusammenfassung bis zur Verortung der kritischen Auseinandersetzung in der Hausarbeit, also explizit nicht im Referat. Der Terminologiearbeit wird insgesamt weniger Bedeutung beigemessen, zum Teil wird sie stark auf das Grundstudium beschränkt, was sich mit den Bedürfnissen der Studierenden deckt. Dabei verändern sich die Anforderungen erwartungsgemäß mit fortschreitendem Studium (vgl. dazu Redder 2002: 17-23). Im Grundstudium dominiert die Reproduktion von angelesenem Wissen, im Hauptstudium steht die Stellungnahme des Referenten verstärkt im Vordergrund. Das Anforderungsprofil kann somit erst im Einzelfall des konkreten Seminarkontextes eindeutig bestimmt werden. 
Bei den Erwartungen der Studierenden lässt sich tendenziell feststellen, dass dem Selektieren von Informationen allgemein großes Gewicht beigemessen wird. Die spezifischen Vorstellungen sind jedoch weitgehend heterogen (Abb. 4).

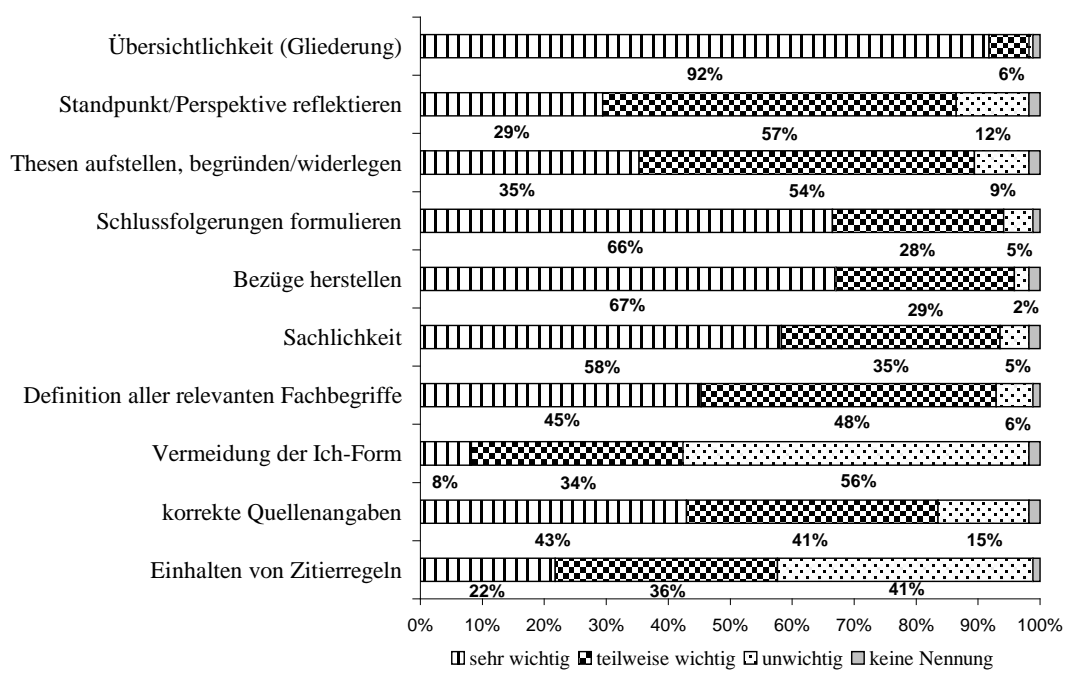

Abbildung 4: Erwartungen der Studierenden bzgl. Wissenschaftlichkeit (Frage 8)

Aufgrund der Vielfalt und Vagheit der Erwartungen beider Zielgruppen können einzelne Aspekte zu Inhalt und Form nicht präzise beschrieben werden. Im Zusammenhang mit der Textintention ist von Seiten der Dozenten dem Erwerb wissenschaftlicher Herangehensweisen besondere Bedeutung beizumessen. Daraus resultiert die Erwartung, dass wachsende Professionalität auf den Ebenen Inhalt, Struktur und Stil manifest wird.

\section{$6 \quad$ Visualisierung}

Das Referat ist ein mündlich präsentierter Fachtext, bei dessen Realisation Mündlichkeit und Schriftlichkeit kookurieren und teilweise spezifische syntaktische Muster bilden. Jedes Medium der schriftlich-optischen Dimension bringt eigene Textmuster hervor. Im Gesamtkontext des Referates handelt es sich um Teiltexte, die nicht unabhängig von der mündlichen Realisation Sinn konstituierend wirken. Dabei erfährt die visualisierte Aufbereitung von Wissen in geisteswissenschaftlichen Fächern einen Bedeutungszuwachs.

Die Auswertung der erhobenen Daten zeigt, dass der Einsatz von Hilfsmitteln zur Visualisierung allgemein akzeptiert, aus Sicht der Lehrenden jedoch häufig ambivalent gesehen wird. Es ergibt sich für die Zielgruppe der Dozenten ein Meinungsspektrum von ,prinzipiell überhaupt nicht notwendig“ über „empfehlens- 
wert immer, wenn der Inhalt sinnvoll ist“ bis „Medieneinsatz ist auf jeden Fall notwendig“. Die Wahl der Mittel ist den Studierenden vornehmlich freigestellt. Die Dozenten ordnen den Hilfsmitteln unterschiedliche Funktionen zu. Einerseits zielt ihr Einsatz auf die Unterstützung des hörerseitigen Verstehens durch Mehrfachkodierung von Informationen (visuelle Aufbereitung eines Sachverhaltes z. B. durch Schemata) bzw. durch Schaffung von Orientierungssystemen (visuelle Vermittlung von Gliederungssignalen z. B. schriftlich fixierte Gliederung) ab. Andererseits dienen sie der Illustration eines fachspezifischen Sachverhaltes (z. B. Bilder, Videos). Die Erwartungen die Funktion betreffend sind dabei sehr unterschiedlich.

Im Zentrum der Aussagen steht neben dem ambivalenten Verhältnis zu Powerpoint, das Handout, das von 3 der 11 befragten Dozenten explizit gefordert wird. Auch für das Handout werden unterschiedliche Schwerpunkte bzgl. Funktion und Inhalt gesetzt.

Anhand der Daten wird eindeutig ersichtlich, dass die Studierenden eine Visualisierung erwarten. Allerdings favorisieren sie „klassische“ Visualisierungshilfen (Abb.5).

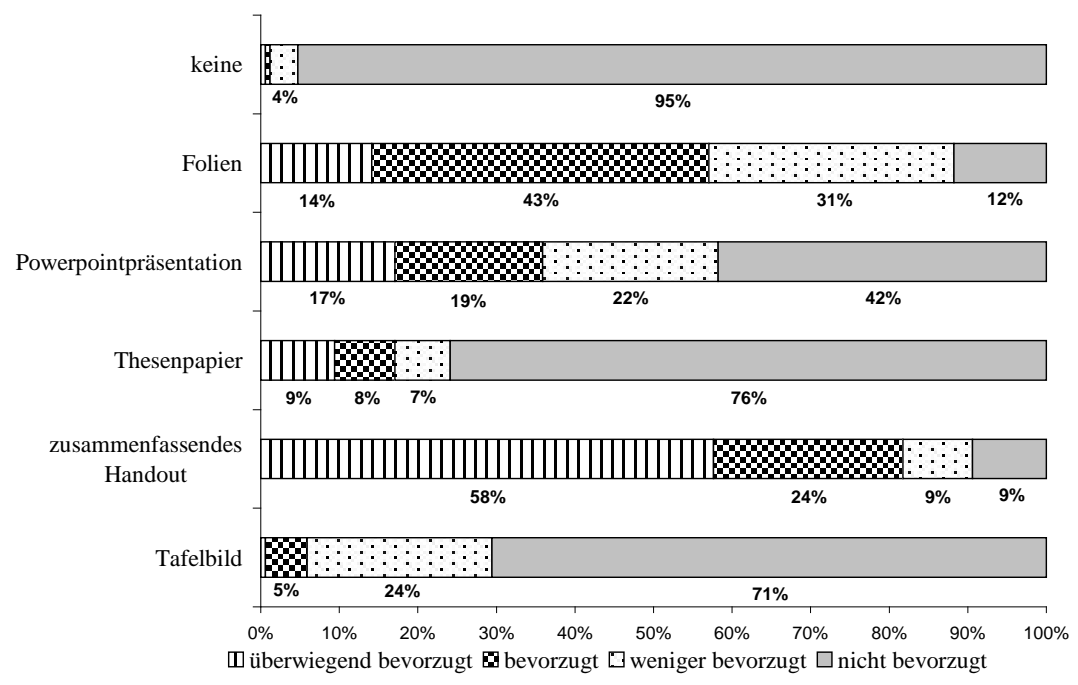

\section{Abbildung 5: Notwendigkeit von Visualisierungshilfen (Frage 10)}

Die überwiegende Mehrheit beider Zielgruppen spricht sich folglich für den Einsatz von Hilfsmitteln zur Visualisierung und Medien im Allgemeinen aus. Spezifische Anforderungen sind primär auch hier vom jeweiligen Kursleiter abhängig. Aus studentischer Perspektive wird ein übersichtliches, zusammenfassendes Handout bevorzugt, welches eine Weiterbearbeitung durch vertiefendes Selbststudium bzw. zum Zwecke einer Klausur-/Prüfungsvorbereitung ermöglicht. 


\section{$7 \quad$ Didaktische Überlegungen ${ }^{3}$}

Zusammenfassend ist festzustellen, dass im Zusammenhang mit fachspezifischer Vielfalt an Formen der Wissensgewinnung und Wissensvermittlung und im Zuge massiver Veränderungen bzgl. der institutionellen Rahmenbedingungen Unterschiede und Modifikationen auf Textsortenebene zu beobachten und weiterhin zu erwarten sind, weshalb eine Normierung der Textsorte Referat stets nur unzureichend realisiert werden kann. Daher kann es nicht Ziel didaktischer Überlegungen sein, umfassende Konzepte zur Vermittlung eineindeutiger Merkmale der Textsorte mündliches Referat zu erstellen. Vielmehr sind Grundlagen und daraus resultierende Zielformulierungen dahingehend festzuhalten, dass sie einerseits auf die Vermittlung institutions-spezifischen Handlungswissens abzielen, wobei das Sprachwissen nur einen Teil ausmacht ${ }^{4}$.

Andererseits ist der Erhalt und Ausbau einer angemessenen Redefähigkeit als ein lebenslanger Aktualisierungsprozess zu betrachten, mit der universitären Ausbildung als maßgebender Etappe im Sinne einer Professionalisierung. Seine Gewährleistung und Optimierung verlangt die systematische Strategievermittlung unter Berücksichtigung und Förderung der Lernerautonomie. Das erfordert eine grundsätzlich offene Arbeitshaltung des Lehrenden, die das Herangehen der Kursteilnehmer an ein bestimmtes Thema aus ihrer subjektiven, kulturellen und muttersprachlichen Tradition bzw. Gebrochenheit toleriert und umsetzt (dazu auch Forster 1997: 396).

Darüber hinaus ist die anteilige Integration der Vermittlung von Redefähigkeit in Fachseminare zwingend notwendig, ebenso eine Orientierung auf die zukünftige Berufspraxis. Weiterführende Kurskonzeptionen können daher nicht nur auf Textsorten, die im Studium eine besondere Rolle spielen sowie Handlungsformen zur effizienten Wissensaneignung fokussieren, sondern müssen eine Brücke zu berufspraxisrelevanten Textsorten und Handlungsformen bauen.

Zu unterstreichen ist für die Entwicklung einer umfassenden Redefähigkeit:

- Die Entwicklung einer Lese- und Hörverstehenskompetenz als basale Grundlage für die eigene produktive Tätigkeit.

- Die Fähigkeit, Hörersignale wahrzunehmen, zu deuten und entsprechend adäquat zu reagieren. Das erfordert, dass der Referenten sich von seiner Fixierung auf die eigene sprachliche Leistung lösen muss, was besonders beim Referieren in einer Fremdsprache mit erhöhten Schwierigkeiten verbunden ist (vgl. Forster 1997).

\footnotetext{
${ }^{3}$ Mit Ehlich/Redder verstehe ich Didaktik als einen „Ort der Reflexion von Unterrichtsgegenständen, Unterrichtszielen, Voraussetzungen und Zwecken der Lernenden.“ (Ehlich/Redder 1997: 1) und nicht als Verschmelzung von Didaktik-Methodik.

${ }^{4}$ Ohne die Identifikation mit den Handlungszusammenhängen, so stellt auch Wiesmann fest, gibt es keine Möglichkeit adäquaten Handelns, daher ist der ausschließliche Aufbau der sprachlichen Teilkompetenzen nicht ausreichend (Wiesmann 1999: 233).
} 
- Die Entwicklung der Fähigkeiten zur (Selbst-) Beobachtung und (Selbst-) Reflexion als elementare Bestandteile einer Fähigkeit das eigene Repertoire gezielt zu aktualisieren. Das schließt Reflexionsprozesse zur Bewusstmachung von Teilurteilen ein.

Der Erwerb fachbezogener Redefähigkeit ist stufenweise an die Phasen der Fachausbildung zu koppeln. Dabei ist zu berücksichtigen, dass die fachliche Teilkompetenz von Studienanfängern vornehmlich eine Strategieentwicklung zur Auswahl und Anordnung von Erträgen zu einem einheitlichen Ganzen erfordert. Sich in Richtung fachliche Vollkompetenz entwickelnde Wissenskonstellationen der Studierenden führen dazu, dass die Textplanung der Studierenden zunehmend auf der Anordnung von Informationselementen zu einem Textganzen basiert (vgl. dazu Buhlmann/Fearns 2000: 295). Damit einhergehend ändern sich die Anforderungen an die Textsorte Referat auf allen konstituierenden Ebenen. Die Vermittlung grundlegender kontextueller Faktoren und textueller Charakteristika ist möglichst studienvorbereitend zu gewährleisten, um unterschiedliches Textmusterwissen anhand fachübergreifender Anforderungen zu aktualisieren.

Die Erfahrungen verschiedener Programme ${ }^{5}$ zeigen, dass ein integrativer Ansatz, bei dem ausländische und deutsche Studierende gemeinsam ihre Redefähigkeit entwickeln, sinnvoll ist. Wissenschaftliches Arbeiten wird in dieser Form als gemeinsames und gleichzeitig interkulturelles Problem bewusst (Fischer/Guadatiello 2002: 29). Erfahrungen und Vorkenntnisse der Studierenden, die sie durch Referieren in Schule oder Studium, zum Teil in ihrer Muttersprache gemacht haben, bieten die Möglichkeit des kontrastiven Arbeitens.

Zusammenfassend lässt sich feststellen, dass eine fundierte Kurskonzeption zur Entwicklung fachbezogener Redefähigkeit eine interdisziplinäre Zusammenarbeit erfordert. Eine Reduktion auf Rhetorikkurse wird daher nicht als zielführend erachtet (vgl. dazu auch Redder 1999).

\section{Literatur}

Buhlmann, Rosemarie / Fearns, Anneliese: Handbuch des Fachfremdsprachenunterrichts. 6. überarb. u. erw. Aufl., Tübingen: 2000.

Ehlich, Konrad: „Wissenschaftskommunikation und Weiterbildung“, Jabrbuch Deutsch als Fremdsprache 22 (1996), 171-190.

Ehlich, Konrad / Redder, Angelika: „, Schnittstelle Didaktik‘. Empirische Untersuchungen zum DaF-Unterricht. Einleitung", Materialien Deutsch als Fremdsprache 45 (1997), 1-4.

\footnotetext{
${ }^{5}$ Exemplarisch genannt das Programm Effektiv studieren an der LMU München, dokumentiert von Redder (Hrsg.): 2002.
} 
Ehlich, Konrad / Rehbein, Jochen: Muster und Institution. Untersuchungen zur schulischen Kommunikation. Tübingen: 1986.

Eßer, Ruth: Etwas ist mir geheim geblieben am deutschen Referat. Kulturelle Geprägtheit wissenschaftlicher Textproduktion und ihre Konsequenzen für den universitären Unterricht von Deutsch als Fremdsprache. München: 1997.

Fischer, Almut / Guadatiello, Angela: „, ,Effektiv studieren“ im Überblick“. In: Redder, Angelika (Hrsg.): „Effektiv studieren“. Texte und Diskurse in der Universität. Osnabrücker Beiträge zur Sprachtheorie, Beiheft 12, Duisburg: 2002, 29-40.

Forster, Roland: Mündliche Kommunikation in Deutsch als Fremdsprache: Gespräch und Rede. Sprechen und Verstehen. Schriften zur Kommunikationstheorie und Kommunikationspädagogik, Bd. 12, St. Ingbert 1997.

Grabowski, Joachim: „Kongressvorträge und Medieneinsatz: ein Plädoyer für Professionalität", Zeitschrift für Angewandte Linguistik 39, Frankfurt a.M.: 2003, 53-73.

Geißner, Hellmut, K.: Kommunikationspädagogik. Transformation der ,Sprech'-Eræįiehung. Sprechen und Verstehen. Schriften zur Kommunikationstheorie und Kommunikationspädagogik, Bd. 17, St. Ingbert: 2000.

Guckelsberger, Susanne / Stenzano Cotelo, Kristin: „Vom mündlichen Referat zur Seminararbeit. Eine exemplarische Analyse und Reflexion der Erfordernisse für eine studienintegrierte Sprachqualifizierung deutscher und ausländischer Studierender“", Materialien Deutsch als Fremdsprache, 73 (2004), 416-456.

Hermanns, Fritz: „Das ominöse Referat. Forschungsprobleme und Lernschwierigkeiten bei einer deutschen Textsorte“. In: Wierlacher, Alois (Hrsg.): Fremdsprache Deutsch. Grundlagen und Verfahren der Germanistik als Fremdsprachenphilologie. Bd. 2., München: 1980, 593-607.

Hufeisen, Britta: Ein deutsches Referat ist kein englisch-sprachiges Essay. Theoretische und praktische Überlegungen zu einem verbesserten textsortenbezogenen Schreibunterricht in der Fremdsprache Deutsch an der Universität. Theorie und Praxis - Österreichische Beiträge zu Deutsch als Fremdsprache, Bd. 5, Innsbruck: 2002.

Kaiser, Dorothee: Wege zum wissenschaftlichen Schreiben. Eine Kontrastive Untersuchung zu studentischen Texten in Venezuela und Deutschland. Tübingen: 2002.

Pabst-Weinschenk, Marita: Reden im Studium. Ein Trainingsprogramm. Frankfurt a.M.:1995.

Redder, Angelika: „, ,Rhetorik‘ - Grundlagen einer bildungspolitischen Konjunktur und reale Basis im sprachlichen Handeln", Mitteilungen des Deutschen Germanistenverbandes, 46, 3 (1999), 316-329.

Redder Angelika: „Sprachliches Handeln in der Universität - das Einschätzen zum Beispiel“". In: Redder A. (Hrsg.): „Effektiv studieren“. Texte und Diskurse in der 
Universität. Osnabrücker Beiträge zur Sprachtheorie, Beiheft 12, Duisburg: 2002, 5-29.

Ribeiro-Kügler, Ana: „Das Referat als mündliche Wissensvermittlung von Studenten für Studenten“. In: (ebd.), 127-134.

Wiesmann, Bettina: Mündliche Kommunikation im Studium. Diskursanalysen von Lehrveranstaltungen und Konzeptualisierung der Sprachqualifizierung ausländischer Studienbewerber. Studien Deutsch, Bd. 27, München: 1999. 



\title{
Subjektivität beim Schreiben in der Fremdsprache
}

\author{
Ingrid Mummert, Hamburg
}

\section{Vorbemerkungen}

Mein Vortrag in der Sektion ,Schreiben und Sprechen in den Wissenschaften“ war der letzte von zehn Beiträgen in den drei Tagen. Ich habe fast alle, die auch in der Dokumentation nachzulesen sind, gehört. Im Rückblick darauf sehe ich meinen Beitrag auch als eine abschließende Ergänzung und Kritik, die ich an dieser Stelle vorwegnehme.

Die kritische Ergänzung betrifft die in diesen drei Tagen zu kurz gekommene Rolle der Subjektivität der ausländischen Studierenden gerade beim Schreiben wissenschaftlicher Texte, ein Mangel, der sich auch in den Schreibdidaktiken feststellen lässt, soweit ich sie kenne. Darin ist zwar theoretisch durchaus von Subjektivität, deutlich auch von der Emotionalität der Schreibenden die Rede; die Strategien, die dann aber vermittelt werden sollen, sind ganz und gar kognitiv konzipiert, losgelöst von der Subjektivität jedes Einzelnen, als wären kognitive Prozesse nicht auch schon immer sehr verschiedene, eben subjektiv verschiedene. Die Ausblendung scheint sich fortzusetzen. 
Meine Kritik richtet sich auch gegen die Praxis derjenigen DaF-Lehrer, die diesen Anteil an Subjektivität bei ihren ausländischen Studierenden schlicht ignorieren. Auf die damit verbundenen gefährlichen Folgen für die Studierenden komme ich zurück.

Mein Beitrag ist deshalb auch als Appell zu verstehen, endlich nicht nur in Theorie und Forschung, sondern auch in der Didaktik und der Praxis - auch der Universitäten - die volle Bedeutung der Subjektivität zu erkennen und zur Geltung zu bringen. Denn dass sie in jedem Schreibprozess eine wesentliche Rolle spielt, ist schon lange und hinreichend bekannt (von Werder 1993; Kruse 2002).

Sie ist nur unbequem, scheinbar; sie erweist sich aber als große Hilfe beim Vermitteln von zweifellos wichtigen Schreibtechniken.

Eine weitere kritische Ergänzung betrifft den Umgang mit dem, was ich die mitgebrachten Schätze der aus so vielen Nationen kommenden Studierenden nenne und zwar im Unterricht wie an der Universität:

Ausländische Studierende haben zu Hause in der Muttersprache alle schreiben und Texte verfassen gelernt, das muss eigentlich nicht betont werden. Sie sind mit ihren Konventionen im wissenschaftlichen Schreiben vertraut, wenn sie schon studiert haben. Untersuchungen u. a. von Eßer (1997) weisen sehr interessante Unterschiede zu deutschen Formen auf, und zwei Vorträge in dieser Sektion haben aufschlussreiche Untersuchungsergebnisse zu chinesischen Schreibkonventionen erbracht (s. Beiträge von Antje Stork und Jin Zhao sowie von Miriam Zeilinger).

Es ist wichtig, kulturspezifische Merkmale beim Schreiben zu erforschen und an die Didaktik wie die Praxis weiter zu geben. Wie aber werden diese einzelnen Ergebnisse einbezogen und umgesetzt? Bisher beziehen Schreibdidaktiken sie meines Wissens noch nicht generalisierend ein. Auch in den Beiträgen klang es eher so, als sollten diese mitgebrachten Schreibkonventionen irgendwie, durch gezielte Vermittlung deutscher Textsorten verschwinden. Zumindest erinnere ich mich nicht, dass der Umgang mit mitgebrachten Schreibkonventionen problematisiert wurde. Und in der Praxis sind die DaF- und Fachkollegen immer noch zahlreich, die es für völlig gerechtfertigt halten, bei der Vermittlung von wissenschaftlichen Textsorten „bei Null“ anzufangen, d. h. nichts vorauszusetzen, ihre Studierenden also zu unterschätzen. Sie stellen Schreibmodelle und Strategien für deutsche Textsorten vor, üben sie ein und werten Schreibweisen, die davon dennoch abweichen, als falsch oder wertlos ab. Und damit die Studierenden. Aber es kann doch nicht im Ernst einfach angenommen werden, dass z. B. chinesische Studierende in Deutschland ihre schriftlichen Höflichkeitsformen unreflektiert ablegen können und problemlos allein die hier geforderte Sachlichkeit übernehmen. Damit würden sie Gefahr laufen, später in ihrem Heimatland befremdend zu wirken und dortige Erwartungen an wissenschaftliches Schreiben zu enttäuschen.

Selbstverständlich sollen die ausländischen Studierenden in Deutschland die spezifischen Textsorten ihrer wissenschaftlichen Fächer erlernen, damit sie hier Erfolg im Studium haben. 
Aber es geht darum, dass sie nicht nur die deutschen wissenschaftlichen Schreibkonventionen kennen lernen, sondern auch sich ihrer eigenen dabei oder dadurch bewusst werden, d.h. sie in ihrem kulturellen Bezugsrahmen erkennen. Es geht darum, dass sie sie weiterhin genauso wichtig finden und gerade im Kontrast beide beherrschen und situativ anwenden können!

Dazu reicht es nicht aus, Modelle und einzelne Techniken zum Nachahmen zu vermitteln und Rezeptlisten zum Selbstkorrigieren aufzustellen. Studierende sollten sich mit den zu erlernenden Schreibtechniken auseinandersetzen können, d.h. vergleichen, Unterschiede erkennen.

Es geht darum, die Gleichwertigkeit anzuerkennen und eine dreifache Kompetenz anzustreben:

- die mitgebrachte Schreibkompetenz

- die neu zu erlernende fremde Schreibkompetenz

- $\quad$ und reflexive Distanz.

Um allen Studierenden gerecht zu werden, kann man nicht abwarten, bis die Forschung kulturspezifische Schreibweisen der etwa 50 an Studienkollegs vertretenen Länder den Didaktikern und Lehrenden zur Verfügung stellt. Und auch dann wäre zu fragen, wie daraus in multikulturellen Kursen eine Schreiblehre aussehen könnte. Inzwischen muss unterrichtet werden.

Also besser nicht hinschauen? Sicher ist es keine Lösung, „,bei Null“ anzufangen und sich einzubilden, damit seien alle gleich behandelt. Das wird den so unterschiedlichen Studierenden nicht gerecht, und unter diesen können viele nicht begreifen, warum ihnen trotz aller Bemühungen keine akzeptablen Texte gelingen. Es gibt einen anderen Weg, er ist mit den Studierenden zu gehen: Man sollte ihnen etwas zutrauen, nämlich ihr Mitgebrachtes selbst einzubringen, da wo wir überfordert und inkompetent sind. Der Weg führt über die Fähigkeit der Studierenden, selbst zu benennen oder zu erkennen, wie sie bisher geschrieben haben und wie sie jetzt schreiben sollen und warum. Und Lehrende sollten und könnten die Reflexionsprozesse leiten und dabei besser verstehen lernen, warum einzelne Studierende scheinbar so abwegig oder unverständlich schreiben.

Die jeweilige Subjektivität und Reflexionsfähigkeit der Studierenden sind dabei Wegweiser. Sie brauchen lediglich die Chance und Zeit, ihre Fähigkeiten einzubringen. Es gilt, diese eben für das Schreibenlehren in wissenschaftlichen Fächern zu nutzen.

Dass das grundsätzlich möglich ist und den Schreiblernprozess fördert, habe ich in vielen Jahren des Literaturunterrichts an Schulen und bei der Arbeit mit ausländischen Studierenden in den letzten Jahren erfahren.

Damit komme ich zu meinem in Hannover gehaltenen Vortrag. Ich befasse mich darin mit einer Schreiblehre, die ausländische Studierende aus verschiedenen Nationen und Kulturen befähigen soll, kulturspezifische deutsche Textsorten zu erfahren, zu reflektieren und zu erlernen, 
- ohne dass sie ihre mitgebrachten Schreibweisen ,abschreiben', d. h. aufgeben,

- indem sie ihre jeweilige Subjektivität in Rezeption und Produktion ausdrücken und reflektieren können.

Das Thema Schreibenlehren und -lernen in der Fremdsprache hat mich in den letzten Jahren noch einmal im Unterricht mit erwachsenen ausländischen Studierenden sehr beschäftigt, gerade weil ich als Lehrerin und Koordinatorin Einblick in die besonderen Schreibprobleme dieser Lernenden bekommen habe und sie mit denen meiner Schüler vergleichen konnte.

In der Fremdsprache schreiben zu lernen ist sicher für fast alle Ausbildungsrichtungen unerlässlich. Wenn Studierende aus dem Ausland nach Deutschland kommen, stehen sie vor dem Problem, ständig schreiben zu müssen. Sie sind aber nicht vorbereitet auf die Textsorten, die mit Selbstverständlichkeit von ihnen verlangt werden. Es geht dabei um Mitschriften von Vorlesungen, Protokollen in Seminaren, Zusammenfassungen von Ergebnissen, Interpretationen von Texten, Inhaltsangaben von Zeitungsartikeln, Analysen von wissenschaftlichen Texten, um Referate u.a.m. Ein großer Teil des Studiums besteht aus Schreiben und aus Schreiben von Texten, die bestimmten kulturbedingten Konventionen unterworfen sind, die weit oder leicht von der in der Muttersprache erlernten Form abweichen können.

Darauf sollten Studierende also vorbereitet werden und sie werden auch an manchen Orten systematisch darauf vorbereitet (Tütken 2000). Am Studienkolleg in Hamburg habe ich versucht, Studierenden mit recht guten Deutschkenntnissen aus vielen verschiedenen Ländern diese Vorbereitung für ihre Studiengänge zu ermöglichen. Darüber habe ich ausführlich, besonders unter dem Aspekt der subjektiv-kulturellen Unterschiede, in meiner 2006 erschienenen Studie - Begegnungen mit „Gertrud“ und „Elsa“ - berichtet. Ich gebe daraus einen stark gekürzten Ausschnitt zum Thema Schreiben wieder. (Seitenangaben im folgenden Text beziehen sich auf diese Veröffentlichung).

Das schreibdidaktische Konzept, das ich hier vorstelle, war und ist die Grundlage für meinen Unterricht im Schreiben sowohl für Jugendliche als auch für ausländische Erwachsene.

Es ist für Geisteswissenschaftler gedacht, die auch hermeneutische Verstehens- prozesse zu bewältigen und schriftlich zu formulieren haben. In den Kursen, die ich am Studienkolleg unterrichtet habe, saßen zukünftige Studierende der Literaturwissenschaft, Rechtswissenschaft, Geschichte, Kunstgeschichte, Erziehungswissenschaft, Psychologie etc.

Aber auch für das Schreiben in den Naturwissenschaften oder in ganz sachorientierten Fächern in der Schule kann dieses Schreibkonzept eine gute Grundlage bilden. Darauf komme ich zurück. 


\section{Das Konzept}

Dem Konzept für schriftliche Textarbeit liegt das entsprechende Konzept für mündlichen Unterricht vor, auch wenn beim Schreiben eine wesentlich andere Situation für den Schreibenden entsteht (S.72). Schreiblehre beginnt im mündlichen Unterricht: Der methodische Umgang mit Texten, die Art der Analysen, die Interpretationsschritte sind Grundlage, Vorbilder und Übungsfeld für die schriftliche Arbeit (S. 39ff.).

\section{Subjektivität}

In meinem gesamten Fremdsprachenunterricht steht die Subjektivität der ausländischen Studierenden im Zentrum, und das heißt, die jeweilige Subjektivität der Studierenden beim Lesen und Schreiben. Sie ist in mein didaktisches Konzept integriert.

Zur Definition von Subjektivität: Subjektivität beim Lesen und Interpretieren literarischer Texte umfasst, kurz gesagt, außer den kognitiven Fähigkeiten auch die beim Lesen ausgelösten Affekte, die sich entwickelnde Empathie bis zur Identifikation mit literarischen Figuren, kulturgeprägte moralische Reaktionen auf Gelesenes, persönliche Antworten, die mit der aktuellen individuellen Situation eines Lesers zu tun haben und die kulturelle Geprägtheit modifizieren. Das heißt: alle Prozesse, die das Verstehen entstehen lassen und mit bestimmen.

Grundsätzlich gilt das für das Lesen aller auch sachorientierten Texte in mehr oder weniger starkem Maße: Niemand liest und versteht ohne seine subjektiven Anteile, ohne innere unterschiedlich starke Beteiligung (s. Kruse 2002). Der Versuch, das Mitwirken nicht rein kognitiver Anteile zu eliminieren, funktioniert nicht. Sie wirken im Untergrund d. h. ungewollt, ja unbewusst fort und beeinflussen Verstehensprozesse - auch beim Schreiben - auf eine für andere schwer oder gar nicht fassbare Weise, und die schriftlichen Ergebnisse werden dann leider oft für Lehrende unverständlich, von diesen also vorschnell als falsch beurteilt. Nur wenn subjektive Lesarten sich manifestieren können/dürfen, werden sie reflektierbar. Sie werden für die Verständigung über ein literarisches Werk - wie über einen sachorientierten Text - und über den Leser also gebraucht!

Ich habe in den ersten Jahren meines Literaturunterrichts an Schulen erfahren, dass die Schüler genauso wenig mit einer rein sachlichen Textanalyse zufrieden waren wie ich und dass die Freude am Lesen und Verstehen von Literatur so nicht zu bewahren oder zu vermitteln war. Und beim Ausprobieren anderer Vorgehensweisen ist deutlich geworden, dass es unerlässlich ist, bei Muttersprachlern wie bei Fremdsprachlern, die Leser mit ihren subjektiven Leseprozessen Ernst zu nehmen, ganz im Sinne Enzensbergers, der über das Lesen von Poesie sagt:

„Soviele Köpfe, so viele Lesarten, eine richtiger als die andere. “(1985) 
Das heißt nicht Beliebigkeit, sondern Vielfalt - Vielfalt in der Einheit, die zu entdecken ist.

Ernstnehmen der Vielfalt heißt für mich darüber hinaus: die Subjektivität der Leser in die Verständigung über das Werk einzubeziehen. Das betrifft das Interpretationsgespräch wie das Schreiben über einen literarischen Text.

Nur so kommt es zu einer motivierten Arbeit, auch bei Schülern, zu einer adäquaten, auch für ausländische Leser akzeptablen Interpretation des Textes und darüber hinaus zur Selbstreflexion der Leser sowie zur interkulturellen und interpersonalen Kommunikation.

Deshalb beziehe ich subjektives Lesen konsequent nicht nur ins Interpretationsgespräch ein, sondern vermittle daraus auch methodische Schritte zum selbstständigen Schreiben über Literatur - und über sachorientierte Texte.

Auch für die Studierenden ist es unerlässlich, dass sie ihre eigene Subjektivität Ernst nehmen, d. h. ihre ganz eigenen Leseprozesse, und das bedeutet selbstverständlich auch ihre Schreibprozesse.

Sie sollen auf ihre Subjektivität achten und selbst Achtung vor ihr entwickeln, sie sollen sie in ihre Analyse und Interpretation aufnehmen und beim Schreiben selbstbewusst einsetzen, d. h. methodisch umsetzen, um den Text und sich besser zu verstehen. Das sollte im mündlichen Literaturunterricht gelehrt und gelernt werden. Auch für die schriftliche Interpretation.

Ihre ersten Eindrücke nach dem Lesen eines Textes, auch eines sachorientierten, sind also sehr wichtig, weil es ihre ganz persönlichen und kulturell geprägten sind.

\section{Methodisches}

\section{Die erste Rezeption}

Nach dem Lesen eines Textes frage ich also nicht wie üblich nach fehlendem Vokabular etc., sondern zunächst und zwar auch in dieser Reihenfolge:

1. Gefällt Ihnen der Text? Was daran gefällt Ihnen, was nicht?

2. Was fällt Ihnen dazu ein, woran erinnert er sie?

3. Was irritiert Sie?

4. Was ist Ihnen im Text nicht klar?

Die ersten drei Fragen zu beantworten sind viele ausländische Studierende nicht gewohnt - und zunächst können sie darauf verunsichert reagieren, $d$. h. schweigen.

Wie kann man das notwendige Selbstbewusstsein und die erforderliche Selbstständigkeit gerade im fremdsprachlichen Bereich entwickeln?

In meinen Kursen waren fast alle ausländischen Studierenden an zielgerichtete Textanalyse unter Ausschluss ihrer Subjektivität gewöhnt. Erst nach der sachlichen Textarbeit wurden sie - und auch das nicht alle - zu einer abschließenden 
Formulierung eigener Meinungen aufgefordert. Sie hatten in Kursen am Studienkolleg zu Beginn oft Angst, etwas Falsches zu sagen. Ich lasse sie deshalb zunächst an kleinen literarischen Texten konkrete und affektive Erfahrungen mit der Tatsache machen, dass Literatur nur durch subjektive Rezeption, durch aktive Denk- und Vorstellungsbeteiligung verstehbar wird (s. Beispiele S. 36ff.). Dabei nimmt das literarische Merkmal „Leerstelle“, ein Begriff aus der Rezeptionsästhetik, innerhalb meiner Vermittlung eine wichtige Rolle ein (s. Iser 1979).

\section{Zum Begriff „Leerstellen“:}

Leerstellen sind - kurz und unwissenschaftlich gesagt - Geheimnisse in literarischen Texten. Es sind vom Autor absichtlich gesetzte Lücken verschiedenster Art, unklare Stellen im Textverlauf. Das macht ihren Charme aus, man kann sie als solche stehen lassen. Sie machen aber auf sich aufmerksam: Sie machen Leser neugierig,

sie irritieren, sie werfen Fragen auf, sie verleiten Leser schon beim Lesen zu spontanen, intuitiven Vermutungen, zu ersten Deutungen, weil sonst beim Lesen und auch danach kein Sinn entsteht.

Die bewusste Wahrnehmung von Leerstellen führt also auf eine ganz natürliche Art zum Deuten, zum Interpretieren und damit zu methodischer Textarbeit, auch beim einsamen Schreiben über Literatur.

Studierende prägen sich übrigens den Begriff „Leerstelle“ besonders gern ein. Das liegt vielleicht daran, dass er mit der affektgeladenen Erfahrung zu tun hat, dass Verständnisprobleme (auch sprachliche) in fiktionalen Texten nicht ihre Schuld, sondern eingebaute Geheimnisse sind, die nur sie enträtseln können und zwar mit ihrer subjektiven Lesart.

Wenn die Lernenden vorher erfahren haben, dass und worin sich literarische Texte von Sachtexten unterscheiden, dass literarische Texte eben nicht chronologisch und psychologisch plausibel alles ausformulieren, dass die verbleibenden, absichtlich gesetzten Lücken, Verschiebungen, Sprünge und Andeutungen etc. zu der Subjektivität und Aktivität zwingen oder verführen, von der Enzensberger spricht, dann haben sie eher Mut, ihre affektiven Reaktionen zu äußern und auf meine Fragen spontan und unreflektiert zu antworten.

Die Studierenden, wie meine deutschen Schüler früher, üben also, ihre eigenen Einfälle wahrzunehmen, im Unterricht und nach dem Lesen - nicht erst am Schluss der gesamten Textarbeit - diese freiwillig zu äußern und evtl. aufzuschreiben. Warum schon dann?

Damit sie nicht verloren, d. h. vergessen werden oder/und im Verlauf der gemeinsamen Textarbeit nicht verändert oder danach peinlich verschwiegen werden, weil sie nicht zu passen scheinen. 
Beim Schreiben, in der Klausursituation, werden die ersten Einfälle zu kostbaren Ideenschätzen, zu einer wichtigen Vorarbeit für die anschließende methodische Textarbeit.

Diese ersten Ideen enthalten meistens:

- affektive Reaktionen

- Irritationen

- Unsicherheiten

- Fragen

- Deutungsansätze

- Verweise auf die eigene Welt

- Urteile

- Benennen von Leerstellen (nach den Übungsphasen).

Aus den ersten Einfällen entwickeln die Leser eigene Fragen, können sie dann ordnen, evtl. den gestellten Aufgaben zuordnen. Es werden ihre eigenen Aufgaben. Sie können so in die systematische Arbeit einsteigen. Und: Ihre Subjektivität bleibt erhalten und ihnen selbst bewusst.

Dieses Bewusstsein wirkt sich in der folgenden methodischen Textarbeit aus.

\section{Textarbeit: Analyse und Interpretation}

Die Deutungen der Studierenden erhalten und behalten folglich - auch das übe und unterstütze ich mit sprachlichen Mitteln (s. S. 84 ff.) - oft den Charakter des Möglichen, des Wahrscheinlichen, weil literarische Texte meist so gestaltet sind (s. Leerstellen u.a.m.), dass Eindeutigkeit nur annäherungsweise oder gar nicht hergestellt werden kann.

Diese Haltung zu entwickeln ist mir besonders wichtig, denn sie hilft den Studierenden, sich von oft voreiligen Urteilen zu lösen und sich stattdessen für andere Lösungen, Antworten, Deutungen zu öffnen.

Die sicherste Deutung entsteht nur aus der Überprüfung am Text. Auf diese Weise werden Einfälle, Deutungsansätze bestätigt. Andere werden verworfen weil sie nicht zum Text passen, nicht nur nicht zu passen scheinen. Aber zum Leser passen sie, er hat sie spontan geäußert! Sie haben also immer noch für den Leser Bedeutung.

Und so entsteht nach einer noch vorläufigen Gesamtdeutung der nächste methodische Schritt.

\section{Die Reflexionsphase}

Es geht um die Reflexion der eigenen Interpretation, aber auch um die Reflexion der ersten Reaktionen und der subjektiven Lesarten und damit um die Frage nach deren Herkunft: nach kulturbedingten oder ganz persönlichen biographischen Hintergründen. 
Hier beginnt unter den Teilnehmern oder/und beim Schreiben

- interkulturelle Kommunikation

- die Gegenüberstellung mit deutschen Verhältnissen im Text

- die Möglichkeit, den Text kritisch zu hinterfragen und

- Textaussage und Leserhaltung von einander zu unterscheiden.

Die Leser können noch besser das Besondere der Textaussage erkennen und evtl. nun begründet kritisieren, aus eigener bewusster und distanzierter Perspektive ablehnen. Bis zum Schluss spielt also die Lesersubjektivität auch beim Schreiben ihre jeweils passende Rolle.

Ich gebe hier das Schreibmodell verkürzt wieder:

\section{Das Schreibkonzept}

I. Erste Rezeptionsphase

- Lesen des Textes

- Formulieren und Aufschreiben aller spontanen Einfälle und Reaktionen auf Gelesenes

- Suche nach Unklarheiten/Leerstellen im Text

- Aus allem: Entwickeln von Fragen an den Text

- Zuordnen zu den Aufgaben, selbstständige Entwicklung eines Aufbaus für die Textarbeit

II Die Textarbeit

- Textanalyse

- Beantworten der Fragen/Füllen der Leerstellen

- Überprüfen der möglichen Antworten/Deutungen am Text und im Kontext

- Entscheidungen mit Begründungen

- Zusammenhang herstellen: Gesamtdeutung

III Reflexionsphase

- Rückbesinnung auf die ersten Einfälle

- Aufnahme der noch nicht berücksichtigten Ideen

- Einbringen der eigenen Welt

- Absetzen von der Textaussage - Gegenüberstellung

- evtl. Kritik/Selbstkritik 


\section{Praxis: Umsetzung durch die Studierenden}

Statt einer weiteren Erläuterung des obigen Modells (s. S. 70 ff.) gebe ich an dieser Stelle einen Einblick in dessen Umsetzung durch die Studierenden. Und zwar beschränke ich mich auf den Bereich, der - nach meinen Erfahrungen - immer wieder vernachlässigt wird: Die konsequente Berücksichtigung der Subjektivität der Schreibenden. Ich zeige auf, wie subjektives Schreiben sich äußert, wenn sie ihren Platz bekommt, wie ich theoretisch gefordert habe.

Dazu habe ich einen der sechs literarischen Texte ausgewählt, an denen ich in meiner Studie ausführlich Praxis dargestellt und reflektiert habe, auch mit den Beteiligten! Es geht um den oft von mir erprobten Text „Gertrud“ von K. Schwitters:

\section{K. Schwitters: GERTRUD}

Gertrud K. war ein schlankes Mädchen mit einem Ausdruck im Gesicht, dass man wußte, sie war dabei mit ibrem Herzen und ihrer Hand. Das Gesicht versprach alles, unterhaltsame Stunden, Freude und besonders Teilnahme an dem, was ihren Freund anging. Deshalb hatte ich sie lange gern, als sie noch kurze Kleider trug. In der Straßenbahn bestaunte ich sie, und wenn ich als Primaner draußen in meinen freien Zeiten Landschaft malte, war mein größter Wunsch, sie möchte vorbeikommen, stehenbleiben und meine Kunst gehörig bewundern.

Wie war ich froh, als sie zufällig an dem Tanzkursus teilnahm, bei dem ich eingeschrieben war. So konnte ich sie beim Tanzen mit dem Arm stützen und sie ganz nah betrachten. Wir lernten natürlich zuerst die leichteren Tänze. Als wir aber schon die Quadrille konnten, fragte Gertrud mich bei einer kurzen Tour, die wir zusammen tanzten; „Ist Liebe nicht ein schönes Wort?"

Natürlich errötete ich und wußte nichts darauf zu sagen. Ich mied sie sogar, da ich fürchtete, noch einmal verlegen zu werden, und sie heiratete, obne dass ich wïßte wen und wobin.

Die sechzehn Studentinnen meines multikulturellen Literaturkurses 2001 hatten dazu nach 10 Monaten Unterricht eine Abschlussklausur zu schreiben.

Die Aufgaben lauteten:

1. Interpretieren Sie bitte diese Kurzgeschichte. Berücksichtigen Sie dabei insbesondere entscheidende Leerstellen.

2. Erfinden und schreiben Sie (ab Zeile 12 (nach der Frage Gertruds)) einen anderen Schluss.

3. Zeigen Sie, wie sich rückwirkend die Deutung der Figur des Erzählers oder/und Gertruds dadurch ändert. 
Die Studentinnen waren inzwischen daran gewöhnt, zu Beginn, d. h. nach der ersten Lektüre, spontane Einfälle zu notieren. Ich zitiere einige daraus:

\section{Betül (Türkin)}

Meine Einfälle:

Warum sagt der Erzäbler nicht zu ihr, dass er sie liebt?

Warum fragt Gertrud ihn, ob die „Liebe“ "ein schönes Wort sei?

Liebt ibn Gertrud auch?

Hat sie wirklich einen Freund? (gute Frage, weil der Text unbestimmt ist)

Bemerkt sie nicht, dass er sie liebt?

Wie füblt er sich, als sie einen anderen heiratet?

Ist er enttäuscht, beleidigt, traurig?

\section{Li (Chinesin)}

\section{Einfälle:}

Die erste Liebe der beiden?

Ungewöhnliches Mädchen!

Heimliche Liebe

Geschickte Andeutung: „Ist Liebe nicht... “

Ein nicht fröbliches Ende

Leerstellen:

Wieso ging Gertrud ibren Freund an? (vielleicht sprachliche Unklarheit?)

Hat sie schon Liebe erlebt?

Liebt Gertrud den Jungen?

Wieso lebnt der Junge die Liebe ab?

Daiga (Lettin)

Einfälle, Auffälliges, Leerstellen:

- Hatte sie damals einen Freund?

- Gingen die beiden zusammen in die Schule?

- Er mag sie sebr, bewundert sie!

- Merkt sie das? Ist ibr das irgendwann aufgefallen?

- Er könnte in sie verliebt sein!

- Warum waren sie im Tan₹kurs ein Paar? Grade sie beide??

- Warum stellt sie ihm so eine Frage?

- Er stellt sich vieles über sie vor!

- Warum hat er nicht versucht, sich mit Gertrud an₹ufreunden? 
Mom (ein deutscher Schüler vom Gymnasium, 18 Jahre alt)

- Was meint G. mit diesem Satz?

- Warum sagt sie nicht: Ist Liebe nicht schön?

- Warum benutzt er diese Frage nicht als Einstieg in das Thema Liebe?

- Und warum zieht er sich mit solcher Heftigkeit zurück? Was ist passiert?

- „Natürlich errötete ich.“ Wieso natürlich? War es um 1900 natürlich zu erröten, wenn über Liebe gesprochen wurde?

- Vielleicht wollte der Erzähler gar keine richtige Beziehung zu G. sondern eine ewige Bewunderung ... Aber kann so etwas auf Dauer klappen?

Diese wenigen Beispiele mögen verdeutlichen, wie unterschiedlich gelesen und aufgenommen wird. Aber auch: Wie der literarische Text - nicht nur mit der Wortwahl, sondern auch gerade mit seinen Leerstellen - die Leser lenkt und zu gleichen Fragen bringt. Die Beispiele zeigen auch, dass die Lernenden inzwischen ein feines Gespür für die wichtigen Leerstellen bzw. offenen Fragen im Text bekommen haben. Das gilt für alle Teilnehmer des Kurses. Besonders Li unterscheidet sicher zwischen Leerstellen und persönlichen Eindrücken, Urteilen, Vermutungen und formuliert eine erste Deutung: „Ein nicht fröhliches Ende“.

Subjektive Rezeption aufzunehmen heißt also nicht, Chaos oder Beliebigkeit zuzulassen. Solche Befürchtungen erweisen sich als ungerechtfertigt.

Diese Beispiele mögen auch zeigen und - bei der Lektüre der gesamten Studie zu diesem Text - Leser davon überzeugen, dass diese Studierenden, die an das Reagieren, d. h. das Beantworten von Lehrerfragen gewöhnt waren, geradezu begeisterte Frager geworden sind: Sie sind es, die Fragen entwickeln, und sie stellen IHRE Fragen!

Und so sicher, wie sie diese Fragen aufschreiben, gehen sie auch anschließend damit an die systematische Beantwortung.

Hilfreich ist bei diesem Schreibprozess für den Aufbau der Klausur selbstverständlich, dass die Studierenden das Interpretationsmodell aus dem mündlichen Literaturunterricht kennen, an dem sie sich auch orientieren können (S.40).

\section{Zusammenfassung meiner Erfahrungen mit der Umsetzung}

Im Allgemeinen gewöhnen sich die Studierenden schnell und gern daran, ihre erste Rezeption Ernst zu nehmen, d. h. ihre spontanen, oft affektvollen Reaktionen zu notieren. Sie lassen sich dann von ihren Fragen leiten, gehen mit ihnen sicher an den Text heran, deuten zunächst vorsichtig, wägen $a b$, nehmen so eine gründliche Textanalyse vor und kommen zu schlüssigen Interpretationen - ohne sich selbst dabei verleugnen zu müssen.

Selbstsicher und selbstständig arbeiten sie so, dass ich ihren Gedankengang nachvollziehen kann. Wenn mir das nicht gelingt, suche ich das Gespräch (s. u.). 
Dieser ganze schriftliche Verständigungsprozess führt meistens zu ausführlichen und langen Klausuren. Sie hier auch nur beispielhaft wiederzugeben, würde den Rahmen dieses Beitrags sprengen. Die Klausuren zu diesem und anderen literarischen Texten habe ich in meiner Studie zum Teil vollständig wiedergegeben und analysiert, besonders dann, wenn subjektive Lesarten weit von meinen Erwartungen abwichen. Sie haben in der Gruppe oder mit den Autoren allein lehrreiche Diskussionen ausgelöst und zu einem weiteren Schritt meiner Schreiblehre beigetragen (s.u.).

\section{Zur zweiten und dritten Klausuraufgabe:}

Diese Aufgaben sind sehr anspruchsvoll, weil sie einerseits Kreativität und andererseits analytische Flexibilität und Selbstreflexion fordern - und fördern.

Erfahrungsgemäß schreiben die Studierenden ihre Wunschversion im Hinblick auf bestimmte Figuren, auf die dargestellte Beziehung und auf den Schluss einer Geschichte.

Sie stellen die eigene Version dem Original gegenüber und der Vergleich bringt den Schreibenden noch mehr Klarheit über den Text und ihre vorangegangenen Entscheidungen aus den verschiedenen Deutungs- möglichkeiten. Diese werden bestätigt oder neu aufgegriffen und modifiziert. Es tauchen neue Begründungen auf, die die eigene Welt, die Kultur der Schreibenden betreffen können.

Die zunächst kreative Phase bekommt so eine ganz wichtige Rolle im Interpretationsprozess, und so stelle ich mir die Bedeutung kreativen Schreibens mit Literatur vor.

Damit sind meine Erwartungen an eine gründliche Textarbeit und Reflexion eines literarischen Werks erfüllt. Meine Schreiblehre ist damit nicht abgeschlossen.

\section{Bearbeitung - Überarbeitung - Beurteilung}

Während ich die Klausuren eines Kurses durcharbeite, d. h. also auch korrigiere, tippe ich Teile daraus ab, die mir auffallen, die interessante Abweichungen von meinen Erwartungen an Aufbau, Deutungen, Analysen enthalten und lasse sie, vervielfältigt und selbstverständlich anonym, mit den Teilnehmern des Kurses diskutieren. Alle haben eine Klausur zu dem Thema geschrieben, alle haben ihre Denkprozesse aufgeschrieben, alle sind erfahren mit dem Text und folglich kompetent, klärend und kritisch vorzugehen.

Auf diese Weise - beim Zuhören - verstehe ich Klausurteile besser, auch weil der jeweilige Autor oft Stellung nimmt und mit der Zeit auch sich zu erkennen gibt. Es geht ums Bearbeiten und Klären VOR dem Zensieren. Letzteres wird durch die Diskussion im Plenum beeinflusst - wie ich denke, im besten Sinne.

Da ich nicht alle Klausuren, schon gar nicht vollständig, auch öffentlich in der Gruppe zur Diskussion stellen kann, nutze ich den breiten (!) Rand für meine Fragen, persönlichen Eindrücke, Vermutungen und Appelle zum klärenden Zweier-Gespräch oder zur schriftlichen Erläuterung einzelner Klausurteile. 
Erst danach sollte ,man' beurteilen, gerade bei Ausländern, deren kulturellsubjektiven Hintergrund man nicht gründlich genug kennen kann. Aber auch Schüler und Studierende der eigenen Kultur geben Lehrenden mit ihren Schreibprodukten genug Rätsel und Fragen auf, ,man' muss sie nur als spannende Rätsel aufnehmen und lösen wollen.

Auf diese Weise wird die Vermittlung des Konzepts für Textproduktion - hier Analyse und Interpretation literarischer Texte - zu einem für alle nachvollziehbaren Prozess und zu einem Lernprozess für alle Beteiligten.

Die so öffentlichen Klausurteile lassen sich von allen Teilnehmern untersuchen und am Schreibmodell messen. Die Teilnehmer können Methodisches vergleichen, ihre u. U. befremdenden Versionen einbringen, zur Diskussion stellen und sich erklärend und klärend verteidigen (S. 97ff. u. 293ff).

Die Teilnehmer entdecken so Unverständliches bei den anderen, können leichter Fragen stellen als ich und so kulturell Fremdes erfragen und die jeweilige Subjektivität der anderen berücksichtigen.

Aus einem echten Bedürfnis nach Verstehen entsteht der interkulturelle Dialog - und eine Anregung zum Weiterdenken. Auch Weiterentwicklung der bisherigen Positionen, der Einstellungen zur eigenen und deutschen Gesellschaft kann sich daraus ,mit der Zeit' ergeben. Das habe ich bei einigen Studierenden erlebt, mit vollziehen und mit den Betreffenden reflektieren können und schließlich in meinen Bericht mit aufgenommen (s.S. 166ff., S.251ff.). Subjektives Schreiben - vor allem über Begegnung mit Figuren in literarischen Texten - und die Reflexion des Geschriebenen können verändern.

Damit eine solche Selbstreflexion angeregt und unterstützt wird, genügt es eben nicht, nur Klausuren schreiben zu lassen, diese zu korrigieren und zu zensieren und Tipps zum Selbstkorrigieren zu geben.

Schreiblehre sollte weiter gehen. Sie sollte ein Kommunikationsprozess sein, in dem das eigene subjektive Schreiben thematisiert wird, so dass der Schreiber/Autor sich seiner mitgebrachten Schreibkonventionen in seinen eigenen Produkten bewusst werden kann. Ohne sie aufzugeben oder abwerten zu lassen, sondern um sie den neuen gegenüber zu stellen, damit beide reflektiert und bewusst beherrscht werden können.

\section{$7 \quad$ Abschließende Überlegungen zur Übertragung des Konzepts}

Die Studierenden in den geisteswissenschaftlichen Kursen haben diesen Umgang mit Literatur und mit ihrer Persönlichkeit schnell schätzen gelernt. Könnte ein Lehrender anderer Fachrichtungen damit etwas anfangen? Was kann ein Student mit anderen Berufszielen daraus für seine Schreibtätigkeiten lernen und für seine Interessen nutzen?

Wer als Lehrender einmal die Vielfalt erster Rezeptionen erfahren hat, wird auch beim Analysieren von Sachtexten nicht mehr mit Selbstverständlichkeit völ- 
lige Übereinstimmung unter allen Beteiligten erwarten und gar verlangen. Er wird irritierende Analysen nicht ablehnen, sondern die Studierenden befragen, nach Hintergründen forschen, zu verstehen suchen, zumindest nicht mehr am Klausurrand als „falsch“, ,abwegig“", „unsinnig“, ,unklar“" abtun, d. h. aburteilen können. Das ändert sein Verhältnis zu seiner Beurteilungsweise, er wird der Subjektivität schreibender Studenten mehr Toleranz und Neugier entgegen bringen - und viel mehr erfahren. Und das ändert sein Verhältnis zu den Studierenden, egal welcher Fakultät.

Eine Schreiblehre, die bei aller wissenschaftlichen Sachlichkeit den Studierenden auch ihre Subjektivität belässt, vermittelt diesen mehr Selbstsicherheit und erleichtert zugleich ihre Selbstkritik. Sie können „Ich“ sagen und schreiben und damit die Irrtumsmöglichkeiten mit bedenken und abwägen. D. h. ihre Reflexionsprozesse werden differenzierter.

Das Schreibenlernen von wissenschaftlichen Texten wird durch eine Schreiblehre, die Subjektivität tatsächlich einbezieht, bewusster und folglich leichter. Schreibblockaden werden seltener (s. Kruse 2002).

Warum sollte ,man' also nicht in anderen Fachbereichen, bevor man Techniken und Modelle für das Schreiben von sachorientierten Textsorten vermittelt, mit dem Interpretieren von literarischen Texten beginnen?

Warum sollte ,man' nicht ein begleitendes Programm mit einer Schreiblehre in den naturwissenschaftlichen Fachbereichen anbieten, die die Subjektivität der Studierenden integriert?

Für die Studierenden - egal welcher Fachorientierung - wäre diese zusätzliche Arbeit ein Gewinn.

Damit komme ich nochmals auf die Subjektivität beim Schreiben zurück und abschließend auf ihr Verhältnis zu den erforschten und noch zu erforschenden kulturspezifischen Merkmalen: Subjektivität schließt auch Kulturgeprägtheit ein: Kulturelle Merkmale, die das Verstehen beeinflussen, treten nicht unabhängig vom komplexen Verstehensprozess auf. Jeder verarbeitet und internalisiert kulturelle Prägung auf seine besondere Weise.

Das lässt sich in Klausuren von Studierenden aus demselben Kulturkreis sehr gut erkennen. Bei der Reflexion besonderer, d. h. subjektiver Rezeptions- und Schreibweisen treten kulturelle Hintergründe zutage und lassen sich so aus den Deutungen, Urteilen und Schreibformen erfassen und mit den jeweiligen Autoren, wenn es ihnen möglich ist und wenn sie selbst daran interessiert sind, klären.

Und: Jedes Subjekt kann sich auch aus seiner Geprägtheit, z. B. von seinen kulturbedingten Einstellungen wieder lösen, sich langsam befreien, sich distanzierter zu ihnen verhalten, andere, zunächst fremde annehmen, übernehmen und damit moralische Positionen modifizieren. Gerade solche Entwicklungen habe ich über einen längeren Zeitraum - zunächst in den Klausuren - beobachten und später in persönlicher Begleitung miterleben können, und die betroffenen Studierenden haben offen darüber gesprochen (2006). 
Zumindest in der PRAXIS des Schreibenlehrens ist es fruchtbarer, wenn Lehrende - und Studierende - ihren Blick auf die komplexe Subjektivität jedes Einzelnen richten, statt nach bestimmten Merkmalen einer Schreibkonvention innerhalb eines Kulturkreises zu suchen und sie den Betreffenden anzutragen. Studierende mögen nicht auf „typische“ Merkmale in ihrem Verhalten angesprochen werden.

Wenn sie jedoch kulturspezifische Merkmale in der Reflexion entdecken und klären, wird es - auch für sie - sinnvoll, mit ihnen zu arbeiten. Gleichzeitig wirken diese Situationen beispielhaft für Wahrnehmung und grundsätzliche Handhabung in der interkulturellen Kommunikation.

\section{Literatur:}

Enzensberger, H.M./ Thalmayr, A. (1985): Das Wasserzeichen der Poesie. Die andere Bibliothek.

Eßer, Ruth (1997): „Etwas ist mir geheim geblieben am deutschen Referat“. Kulturelle Geprägtheit wissenschaftlicher Textproduktion und ihre Konsequenzen für den universitären Unterricht von Deutsch als Fremdsprache. München.

Hermanns, F.(1989): Schreiben als Lernen. Argumente für das Schreiben im Unterricht Deutsch als Fremdsprache. In: Heid, M. (Hrsg.) (1989): Die Rolle des Schreibens im Unterricht Deutsch als Fremdsprache. Dokumentation eines Kolloquiums. München, 28-50.

Iser, W. (1979): Der Lesevorgang. Eine phänomenologische Perspektive. In: Warning, R. (Hrsg.): Rezeptionsästhetik. 2. Auflage, München, 253-276.

Kelek, N. (2002): Islam im Alltag. Islamische Religiosität und ihre Bedeutung in der Lebenswelt von Schülerinnen und Schülern türkischer Herkunft. Münster u.a.

Kreft; J. (1977): Grundprobleme der Literaturdidaktik. Eine Fachdidaktik im Konzept sozialer und individueller Entwicklung und Geschichte. Heidelberg.

Kruse, O. (2002): Keine Angst vor dem leeren Blatt. 9.Auflage, Frankfurt/M.

Mohr, I. (2000): Lernertexte untersuchen und überarbeiten: eine Übung aus dem DaF-Unterricht. In: Krumm, H.-J. (Hrsg.): Erfahrungen beim Schreiben in der Fremdsprache Deutsch. Innsbruck/Wien/München, 109-155.

Mummert, I./Kästner, U. (2000): Schreibbegegnung - Begegnungsschreiben. Ein Projekt mit polnischen und deutschen Studierenden zur interkulturellen Verständigung durch freies Schreiben. In: Krumm, H.-J. (Hrsg.): Erfahrungen beim Schreiben in der Fremdsprache Deutsch. Untersuchungen zum 
Schreibprozess und zur Schreibförderung im Unterricht mir Studierenden. Wien/München, 225-268.

Mummert, I./Pommerin, G. (2000): Über die allmähliche Verfertigung von Texten (II). In: Deutsch als Fremdsprache 37, Heft1, 3-9.

Mummert, I./Pommerin, G. (2001): Ansätze einer kreativitätsorientierten Textanalyse und Textüberarbeitung (II). In: Deutsch als Fremdsprache 38/3, Heft 3, 143-152.

Mummert, I. (2006): Begegnungen mit „Gertrud“ und „Elsa“. Mündliche undschriftliche Interpretation deutschsprachiger Literatur mit ausländischen Studierenden. Perspektiven Deutsch als Fremdsprache Band 19. Hohengehren.

Pommerin, G./Mummert, I. (1999): Über die allmähliche Verfertigung von Texten (I). In: Deutsch als Fremdsprache. 36, Heft 4, 198-204.

Pommerin, G./Mummert, I. (2001): Ansätze einer kreativitätsorientierten Textanalyse und Textüberarbeitung (I). In: Deutsch als Fremdsprache. 38, Heft 2, 67-76.

Schwitters, K.: Gertrud. In: Krusche, D. (Hrsg.) (1987): Aufschluss, Bonn, S. 170. und in: Hohler, F. (Hrsg.) (1983): 111 einseitige Geschichten. Darmstadt, Text Nr. 40. Schwitters, K. (1974): Das literarische Werk. Köln.

Tütken, G. (2000): ohne Titel. Broschüre für Studierende über das Schreiben von Sachtexten. Schriftliche Übungen - Textproduktion - Oberstufe II. Erhalten von der Universität Göttingen, Lektorat Deutsch als Fremdsprache.

Werder, L. v. (1995): Kreatives Schreiben in den Wissenschaften. Berlin/Milow.

Werder, L.v. (2002): Kreatives Schreiben von wissenschaftlichen Hausarbeiten und Referaten. 2. Auflage. Berlin/Milow. 
Themenschwerpunkt 4:

DaZ und Mehrsprachigkeit 


\section{Sprachliche Frühförderung von Kindern mit Migra- tionshintergrund $^{1}$}

They tell me every thing is gona be all right but I don't know what all right even means.

Bob Dylan

Ernst Apeltauer, Flensburg

\section{$1 \quad$ Einleitung}

In der Zeitung Die Welt vom 19. April 2006 konnte man es lesen: »Der Kontinent (Europa) wird muslimisch werden«. Das sagt der hoch angesehene Islamwissenschaftler Bernard Lewis, Emeritus aus Princeton. Werden sich die Verhältnisse also bald umkehren? Wird eine Minorität bald die Majorität in unserem Lande?

\footnotetext{
${ }^{1}$ Bei diesem Beitrag handelt es sich um die überarbeitete und erweiterte Fassung eines Plenarvortrags im Rahmen der 34. Jahrestagung des Fachverbandes Deutsch als Fremdsprache (FaDaF) am 10.Juni 2006 an der Universität Hannover zum Rahmenthema »Chance Deutsch; Schule - Studium Arbeitswelt« gehalten wurde. Der Beitrag ist auch als Heft 42/43 in den »Flensburger Papieren zur Mehrsprachigkeit und Kulturenvielfalt im Unterricht« und in Info DaF 1 (2007) Seite $3 \mathrm{ff}$. erschienen.
} 
Die neuen Daten des Mikrozensus von 2005, die Anfang Juni 2006 in einschlägigen Tageszeitungen veröffentlicht wurden, könnten als Bestätigung der oben zitierten Aussage interpretiert werden. Denn schon heute leben 15,3 Millionen Menschen mit Migrationshintergrund in Deutschland, immerhin 19\% der Bevölkerung. Man rechnet, dass in 10 Jahren jedes zweite Grundschulkind über einen Migrationshintergrund verfügen wird. Hat der Islamwissenschaftler Lewis also Recht?

Seit der Veröffentlichung der ersten Pisa-Ergebnisse wissen wir, dass 23\% der 15-jährigen Schüler in Deutschland nur die Lesekompetenzstufe I erreichen. Der OECD-Durchschnitt lag bei 18\%. In Kanada waren es sogar nur 10\%. Was bedeutet Lesekompetenzstufe I? Menschen, die nur diese Lesekompetenzstufe erreichen, können kaum lesen und sind folglich auch nicht fortbildbar. Sie werden sich einreihen in das wachsende Heer der Arbeitslosen und Sozialhilfeempfänger unserer Gesellschaft.

Und es gibt noch weitere Zahlen, die nachdenklich stimmen: 40\% der Einwanderer zwischen 25 und 35 haben keinen beruflichen Abschluss, bei türkischstämmigen Zuwanderern sind es sogar 60\% (Quelle: Hannoversche Allgemeine Zeitung vom 08.06.06, 4). Wird unsere Gesellschaft, in der heute ein großer Teil der Bevölkerung noch als gut ausgebildet gelten kann, bald eine Gesellschaft der Ungebildeten sein? Wie werden sich solche Veränderungen langfristig auf den Wirtschaftsstandort Deutschland auswirken?

Es ist bekannt, dass viele Kinder mit Migrationshintergrund (künftig: $\mathrm{KmM}$ ) wegen unzureichender Deutschkenntnisse in der Schule scheitern. Offenbar genügt das natürliche »Sprachbad« in Kindergarten und Schule nicht mehr, um ausreichende Fertigkeiten in der Zweitsprache zu entwickeln. Darum wurde in Fachkreisen und in den Medien intensiv über Fördermöglichkeiten diskutiert. Viele Bundesländer haben inzwischen Modelle zur Förderung eingerichtet. Neben den von der Stiftung Mercator geförderten Zusatzangeboten für Sekundarschüler sind das vor allem Angebote zur Frühförderung. Denn die Zweitsprache (Deutsch) soll möglichst früh und intensiv vermittelt werden.

Während weitgehend Einigkeit darüber besteht, dass Frühförderung sinnvoll ist, gehen die Meinungen darüber, ob Frühförderung ausreicht und wie die Zweitsprache vermittelt werden sollte, auseinander. Zwei Lager stehen sich dabei gegenüber.

Die einen sind der Auffassung, dass man möglichst früh und intensiv mit der Vermittlung des Deutschen beginnen sollte. Motto: Frühförderung erspart spätere Fördermaßnahmen ${ }^{2}$. Und da die Erstsprachen der Kinder (z. B. Türkisch oder Arabisch) auf dem Arbeitsmarkt nichts wert seien (so der Soziologe Esser), könnten sie vernachlässigt werden. Außerdem, so rechnet man vor, kostet die Pflege der Erstsprachen Lernzeit, Zeit, die besser für die Vermittlung der Zweitsprache

\footnotetext{
2 Man könnte hier auch von einer Frühförderungsideologie sprechen. Sie beruht auf der Annahme, dass Kinder mit Migrationshintergrund $(\mathrm{KmM})$ eine fremde Sprache im Kindergarten leichter und schneller lernen als in der Schule und dass sie in ein oder zwei Jahren das nachholen können, was deutsche Kinder in sechs Jahren entwickelt haben.
} 
verwendet werden sollte (vgl. Hopf 2005)3. ${ }^{3}$ Der Soziologe Esser geht sogar noch einen Schritt weiter. Er forderte jüngst in der Süddeutschen Zeitung: »Keine Wellness« (mit Muttersprache) sondern »Fitness einer Sprachkompetenz«, d. h. »einsprachige Assimilation«. Und weiter: »Auch in Kanada zahlt sich offenbar so gut wie nur die Assimilation an die jeweilige sprachliche »Leitkultur» aus (...)« (Süddeutsche Zeitung vom 7. April 2006)

Solche Forderungen sind freilich nicht neu. In den USA hat man schon vor 30 Jahren »English-only«-Programme eingerichtet, in denen Kinder von Zuwanderern von monolingualen Lehrkräften an fünf Tagen in der Woche unterrichtet wurden. Eine Ausklammerung der Erstsprache wurde auf der Folie der MeltingPot-Ideologie für selbstverständlich gehalten (Vgl. dazu Wiley/Lukes 1996).

Die Evaluierungsergebnisse der English-Only-Programme waren nicht ermutigend. Vor allem Berichte von Betroffenen haben gezeigt, dass die gewaltsame Unterdrückung der Erstsprache Verunsicherungen, Demotivation und Misserfolge auslösen. Rückblickend formulierte ein Betroffener:

»(...) often frightend by the sounds of los gringos, delighted by the sounds of Spanish at home« (Rodriguez 1982: 16).

was we children learned more and more English, we shared fewer and fewer words with our parents. (...) Often the parents wouldn't understand«. (Rodriguez 1982: 23).

Noch drastischer formulierte es ein anderer Betroffener:

»School was a nightmare. I dreaded going to school and facing my classmates and teacher (...) My self-image was a serious inferiority complex. I became frustrated at not being able to do anything right« (McKay 1988: 341).

Neuere Untersuchungen haben gezeigt, dass die Erstsprache immer aktiviert wird, wenn die Zweitsprache gebraucht wird. Man kann sie nicht ausschalten. Indem man die Erstsprache ausklammert, fordert man also von den Lernern eine aktive Unterdrückung von Assoziationen und Vergleichen, die sich ihnen aufdrängen. Mit anderen Worten: Lerner werden an der Nutzung ihres Lernpotentials gehindert.

Die Bayerische Staatsregierung hat am 14. September 2004 dennoch beschlossen, den muttersprachlichen Ergänzungsunterricht (d. h. den Unterricht in den

\footnotetext{
${ }^{3}$ Hopf stützt sich auf die time on task Hypothese. Sie ist in Fachkreisen umstritten. Untersuchungen in den Niederlanden haben z. B. gezeigt, dass die aufgewendete Zeit nicht maßgeblich ist für den erreichten Sprachstand (vgl. Driessen/Van der Slik/De Bot 2002). Aufgrund von Fallstudien weiß man zudem, dass die Zweitsprachentwicklung (stärker noch als die Erstsprachentwicklung) nicht kontinuierlich verläuft. Mit der time-on-task-Hypothese wird aber Kontinuität in der Entwicklung stillschweigend vorausgesetzt.
} 
Erstsprachen der Zuwandererkinder) binnen fünf Jahren abzuschaffen. Es wird dann also nur noch »Deutsch-only« unterrichtet. Auf eine Anfrage im Bayerischen Landtag hin wurde diese Entscheidung damit begründet, dass die fachlichen Argumente für den muttersprachlichen Ergänzungsunterricht zwar gesehen werden, dass aber in Abwägung der vorhandenen Mittel und der verschiedenen Interessen das frühzeitige und sichere Beherrschen der deutschen Sprache höhere Priorität habe.

Vertreter dieser Position sind zumeist Verwaltungsbeamte oder Bildungspolitiker, die mit ihren geringen finanziellen Ressourcen maximale Effekte erzielen sollen. Man kann diese Position als die ökonomisch-pragmatische Position bezeichnen.

Im anderen Lager ist man der Auffassung, dass Kinder, wenn sie in den Kindergarten kommen, bereits über Grundfertigkeiten in ihrer Erstsprache verfügen. Mit Hilfe ihrer Erstsprache haben sie begonnen, Wissen zu sammeln, welches die Grundlagen für ihr weiteres sinnstiftendes Lernen bildet. Und da die kognitive Entwicklung und der Erstspracherwerb in dieser Lebensphase intensiv aufeinander bezogen sind und über die Erstsprache zudem wichtige Wachstumsimpulse (insbesondere Impulse zur Konstruktion von Bedeutungen und Weltwissen) vermittelt werden, wäre es fatal, wenn dieser Motor der Entwicklung plötzlich abgestellt würde. Es würde dann zu einem folgenschweren Bruch in der sprachlichen und kognitiven Entwicklung kommen.

Ein anderes Argument, das von Vertretern dieses Lagers gebraucht wird, lautet: Die Erstsprache ist eine wichtige Lernvoraussetzung für den Zweitspracherwerb. Eine differenzierte Erstsprache erleichtert, eine weniger entwickelte Erstsprache erschwert die Aneignung einer Zweitsprache, eine Auffassung, die z. B. durch die Ergebnisse der DESI-Untersuchung gestützt wird. Auch Ergebnisse der Hirnforschung sprechen für diese Annahme. Man hat nämlich herausgefunden, dass beim Erwerben der Erstsprache neuronale Verarbeitungsroutinen ausgebildet werden, die sich später nicht mehr ändern lassen und auf denen alle anderen (späteren) Lernprozesse - also auch Zweitspracherwerbs- und -lernprozesse - aufbauen (vgl. Singer 2002: 5 f.). Vertreter dieser Position sind vor allem Sprachpädagogen, Sprachpsychologen und Psycholinguisten. Sie befürworten zwar ebenfalls eine frühe und intensive Vermittlung der Zweitsprache Deutsch, gehen aber davon aus, dass weitere Fördermaßnahmen auch in der Schulzeit angeboten werden müssten und die Pflege der Erstsprache (aus den oben genannten Gründen) sinnvoll ist. In der UN-Convention on the Rights of the Child heißt es in Artikel 30:

You have the right to learn and use the language and customs of your family whether or not these are shared by the majority of the people in the country where you live.

Den autochthonen Minderheiten in unserem Land (den Friesen, den Sorben und der dänischen Minderheit in Schleswig-Holstein) werden solche Rechte zugestanden. Es gibt z. B. dänische Kindergärten und dänische Schulen in Schleswig- 
Holstein. Niemand käme auf die Idee, von Dänen oder Friesen zu verlangen, ihre Sprache - immerhin einen Teil ihrer Kultur und Identität - zu vernachlässigen oder gar zu verleugnen.

Wir wollen einmal die Konsequenzen betrachten, die sich aus dieser Position ergeben. Was würde es bedeuten, wenn die Erstsprache vernachlässigt und nur noch die Zweitsprache vermittelt würde?

\section{Sprach- und Kognitionsentwicklung}

Die sprachliche Entwicklung setzt - wie wir heute wissen - im Mutterleib ein, wo bereits Sprachrhythmus und Intonation vom Fötus wahrgenommen werden. Im Alter von sechs Monaten können Kinder Satzgrenzen erkennen, indem sie auf rhythmische und prosodische Merkmale wie Pausen und Betonungen achten, was keineswegs selbstverständlich ist. Denn Sprechpausen innerhalb von Sätzen sind oft länger als zwischen Sätzen. »Ab einem Alter von ca. acht Monaten können Kinder dann auch Wortgrenzen identifizieren« (Herrmann/Fiebach 2004: 68). Kinder nutzen dazu Sprachmelodie, Akzentsetzungen und Rhythmus ihrer Erstsprache ebenso wie die Distribution von Lauten, phonotaktische Merkmale und prosodische Grenzsignale. Auf diese Weise werden typische Wortformen allmählich herausgefiltert. Am Ende ihres ersten Lebensjahres haben die Kinder eine für die von ihnen zu erlernende Sprache optimale Dekodierungsstrategie entwickelt. Damit können sie Wort- und Satzgrenzen effektiv bestimmen und so den gehörten »Lautbrei« gliedern und strukturieren. Anders formuliert:

"(....) input processing rapidly becomes attuned to the ambient language during the first year of life« (Doughty 2003: 283).

Diese Dekodierungsstrategie, die optimal auf die Erstsprache abgestimmt ist, kann später nicht mehr verändert werden. Untersuchungen haben gezeigt, dass selbst balancierte Bilinguale immer auf die Dekodierungsstrategie einer präferierten Sprache zurückgreifen. In keiner Untersuchung konnte die Beherrschung von zwei Dekodierungsstrategien nachgewiesen werden (vgl. Cutler 2001). Sollten Kinder von Zuwanderern folglich schon nach ihrer Geburt in Heime oder in deutsche Pflegefamilien abgegeben werden, damit sie die optimale Dekodierungsstrategie für Deutsch erwerben?

Gehen wir einmal davon aus, dass das niemand ernsthaft fordern wird, nicht einmal Vertreter der pragmatisch-ökonomischen Position. Gefordert wird gegenwärtig nur die Vermittlung der Zweitsprache im Vorschulalter. Aber was heißt das genau? Ab welchem Alter sollten Vorschulkinder (mit Migrationshintergrund) auf Deutsch »unterrichtet« werden? Und über welche sprachlichen Fertigkeiten in der Zweitsprache Deutsch sollen die Kinder am Ende der Kindergartenzeit (bzw. zu Schulbeginn) verfügen? 


\subsection{Sprachliche Voraussetzungen}

Kinder kommen gewöhnlich im Alter von drei oder vier Jahren in den Kindergarten. Zu diesem Zeitpunkt können sie in ihrer Erstsprache

- einfache Sätze bilden,

- die Flexion aber noch nicht sicher gebrauchen,

- 1.000 bis 2.000 Wörter produktiv verwenden und

- 3.000 bis 4.500 Wörter verstehen.

Ein Wortschatzwachstum findet zunächst überwiegend im Bereich der Nomen statt. Um das dritte Lebensjahr werden zunehmend auch Verben gebraucht und etwa ab dem vierten Lebensjahr zudem Adjektive, Adverbien und erste Präpositionen. Diese Angaben gelten natürlich auch für die Erstsprachen normal entwickelter $\mathrm{KmM}^{4}$.

Wenn Kinder in die Schule kommen, haben sie ihr sprachliches Repertoire weiterentwickelt. Ihre Sprache wird zunehmend verständlicher und abstrakter. Jetzt werden von den Kindern i. d. R.

- komplexe syntaktische und morphologische Strukturen beherrscht (z. B. Nebensätze, unterschiedliche Tempusformen),

- 5.000 bis 9.000 Wörter produktiv gebraucht,

- ca. 10.000 bis 14.000 Wörter verstanden ${ }^{5}$.

Es ist bekannt, dass Kinder aus Familien, deren Eltern über höhere Bildungsabschlüsse und ein höheres Einkommen verfügen, i. d. R. zu Schulbeginn mehr Wörter verstehen und gebrauchen können als Kinder aus sozial benachteiligten Familien. Man spricht in diesem Zusammenhang auch von high und low SES 6 . Im Falle der KmM überlagern sich häufig zwei ungünstige Faktoren: low SES und sprachliche und kulturspezifische Unterschiede.

Was bedeutet es nun, wenn Kinder mit unterschiedlichen Wortschätzen in die Schule kommen?

\footnotetext{
${ }^{4}$ Man spricht hier auch von einem altersgemäßen Entwicklungsstand in der Erstsprache. Lehrkräfte, die den sprachlichen Entwicklungsstand in einer Erstsprache nicht beurteilen können, glauben vielfach, dass Migrantenkinder über keinen altersgemäßen Sprachentwicklungsstand verfügen, dass diese Kinder vielmehr halbsprachig (oder als Steigerungsform; doppelt halbsprachig) seien. Solche "Spontanurteile" (Vorurteile) offenbaren etwas über die Person, die sie äußert, nicht aber über den Sachverhalt. Vorurteile begünstigen das Entstehen von »selbsterfüllenden Prophezeiungen«. ${ }^{5}$ Von diesen Zahlen gehen Wortschatztests für monolinguale Vorschulkinder aus, z. B. der Allgemeine Wortschatztest (AWST-R 2005). Zum Vergleich: Abiturienten müssen laut Lehrplan ca. 5.000 Wörter im Englischen beherrschen, etwa so viel, wie KmM mit sechs Jahren in ihrer Erstsprache beherrschen. ${ }^{6}$ SES bedeutet socio-economic-status. Eltern von Kindern, die über keine höhere Schulbildung verfügen und ihr Geld mit angelernten Tätigkeiten verdienen, gelten als »low SES«. In Deutschland wird das euphemistisch mit »bildungsferne Elternhäuser« umschrieben.
} 
Kindern, die zu Schulbeginn nur über einen kleinen Wortschatz verfügen, fällt das Lernen in der Klasse und das Lesenlernen schwerer, als Kindern mit einem groBen Wortschatz. Und so kommt es, dass Leistungsrückstände, die bei Schuleintritt bestehen, im Laufe der Schulzeit weiter anwachsen, statt sich zu verringern (vgl. Moser 2005: 184).

In einer niederländischen Untersuchung wurden Sprachkompetenz und Lesefertigkeiten von monolingualen (niederländischen) und zweisprachig aufwachsenden marokkanischen und türkischen Kindern vom 3. bis zum Ende des 4. Schuljahres verglichen. Es wurde festgestellt, dass während dieser Zeit die Kluft zwischen diesen Gruppen (vor allem im Wortschatzbereich) weiter wuchs (vgl. Droop/Verhoeven 2003). In einer anderen Untersuchung, in der dem Zusammenhang zwischen Sprachgebrauch in der Familie und Zweitsprachentwicklung nachgegangen wurde, kommen die Forscher zu folgendem Ergebnis:

»The result is that Turkish and Maroccan children are approximately two years behind in their (second, EA) language development at the end of primary education, compared to children of highly educated (Dutch) parents« (vgl. Driessen/Van Slik/De Bot 2002: 176; die Forscher verweisen auf weitere niederländische Untersuchungen, die zu ähnlichen Ergebnissen kommen (vgl. z. B. Driessen 1996).

Hier zeigt sich die Problematik der Frühförderungsideologie. Ihr liegen zwei Annahmen zu Grunde:

- KmM können in einem halben Jahr (so z. B. in Hessen, SchleswigHolstein) oder in einem Jahr (so seit Mai 2006 in Niedersachsen) das nachholen, was deutsche Kinder im Verlauf von fünf oder sechs Jahren an sprachlichen Fertigkeiten entwickelt haben.

- Sprachliche Frühförderung reicht aus, um erfolgreich am Regelklassenunterricht teilzunehmen?

Man vergegenwärtige sich: Sprachfördermaßnahmen werden 4-6 Stunden pro Woche in Gruppen mit sieben (in Niedersachsen) oder mit mehr als sieben Kindern durchgeführt ${ }^{8}$. Während die deutschen Kinder über mehrere Jahre eine ganz-

\footnotetext{
${ }^{7}$ In Niedersachsen wurden z. B. Sprachförderstunden (DaZ) von der Grundschule in den Kindergarten verlagert. Und der Innenminister von Niedersachsen, Schünemann, berichtete in seiner Regierungserklärung über ein Mädchen, das »vor zwei Jahren noch nicht ein einziges Wort Deutsch sprechen konnte. Dieses Mädchen, da bin ich mir sicher, (wird, EA) mit guten Aussichten auf Erfolg seine Schulkarriere starten« (vgl. http://cdl.niedersachsen.de/blob/images/C23423381_L20. pdf. [30. 07. 2006]). Um flüssig sprechen zu können genügen ca. 2000 Wörter (vgl. Nation 2005: 586). Damit kann man aber noch keinem Regelklassenunterricht folgen. Vgl. dazu auch unten Informationsaufnahme und -verarbeitung über die Zweitsprache.

${ }^{8}$ Ein Frühförderprojekt in Mannheim arbeitet mit Kleinstgruppen (»maximal drei Kinder«) 4-5 mal wöchentlich eine Stunde. www.anglistik.uni-mannheim.de/linguistik/projekte.htm [11.09.2006])
} 
tägige Einzelbetreuung haben, sollen KmM mit 1/7 von vier oder sechs Stunden pro Woche (d. h. also mit nicht einmal einer Stunde Zuwendung pro Woche) in einem halben Jahr (oder einem Jahr) dieselben sprachlichen Fertigkeiten entwickeln, wie deutsche Schulanfänger. Sind solche Vorstellungen realistisch?

Kehren wir zurück zur Wortschatzentwicklung. Da der Wortschatz eines Menschen sein Lernverhalten und seine Persönlichkeitsentwicklung beeinflusst, kann ein umfangreicher Wortschatz als eine positive Voraussetzung für seine gesamte Entwicklung betrachtet werden. Über welchen Wortschatzumfang ein Kind verfügt, hängt $u$. a. von seinen Interaktions- und Erfahrungsmöglichkeiten ab und (wie oben ausgeführt) von den Bildungsvoraussetzungen seiner Eltern. Wenn beispielsweise regelmäßig und interaktiv Gute-Nacht-Geschichten vorgelesen werden, so hat das nachweislich Auswirkungen auf den Wortschatzumfang und die begriffliche Schärfe (vgl. dazu Heath 1986).

Nun wird ein normal entwickeltes KmM in seiner Erstsprache über ähnliche sprachliche Voraussetzungen verfügen wie ein deutsches Kind aus einer Familie mit vergleichbarem SES. Es könnte also in seiner Erstsprache Informationen genauso schnell und differenziert aufnehmen und verarbeiten, wie diese deutschen Kinder.

\subsection{Informationsaufnahme und -verarbeitung über die Zweitsprache}

Was bedeutet es nun, wenn alle Informationen über die weniger entwickelte Zweitsprache aufgenommen und verarbeitet werden müssen?

In der Kognitionspsychologie wird heute davon ausgegangen, dass es so etwas wie ein konzeptuelles System gibt, in dem wir Informationen speichern und verarbeiten. Mit diesem System ist das mentale Lexikon verbunden, das aus unterschiedlichen Teilsystemen besteht, im rezeptiven Bereich aus einem akustischen, visuellen und einem abstrakten Wortmarkensystem, im produktiven Bereich aus Sprech- und Schreibprogrammen. Ein Mensch, der zwei Sprachen beherrscht, verfügt über zwei solche Lexika, die auch untereinander vernetzt sind. Ein Teil der Wortbedeutungen (von vertrauten, häufig gebrauchten oder auf konkrete Objekte verweisenden Wörtern) scheint dabei überlappend im semantischen Gedächtnis gespeichert zu sein, ein anderer Teil (Bedeutungen von nichtverwandten, abstrakten, selten gebrauchten oder kulturspezifischen Wörtern und insbesondere von Wörtern, die Gefühle bezeichnen) scheinen dagegen in gesonderten Teilsystemen gespeichert zu werden (ausführlicher dazu Apeltauer 2006c).

Wenn Wörter in einer Zweitsprache gelernt werden, so werden diese anfangs an Wörter der Erstsprache angekoppelt und zwar sowohl in phonologischer als auch in konzeptueller Hinsicht. Aufgrund ihrer in der Erstsprache entwickelten Hörgewohnheiten (bzw. Dekodierungsstrategien, s. o.) nehmen Lerner die fremde Sprache anfangs gefiltert durch ihre Erstsprache wahr und passen neue Wörter in der Aussprache den ihnen bekannten Aussprachemustern an. Je besser sie neue Wörter nachsprechen können (was korrektes Hören voraussetzt!), desto leichter 
können sie neue Wörter auch lernen (vgl. dazu auch Service 1992). Sie müssen Äußerungen dazu viele Male gehört haben. Dann entwickeln sie allmählich ein Gefühl für die mögliche Abfolge von Lauten und für mögliche Wortformen. Erst danach werden sie sich auf das Lernen neuer Wortinhalte konzentrieren. Anders formuliert: Erst wenn sich Kinder in die neue Sprache eingehört haben, wenn ihre Aufmerksamkeit und Energie nicht mehr von den Dekodierungsprozessen absorbiert wird, wird sich ihr Wortschatzwachstum beschleunigen, wird sprachliche Förderung im Wortschatzbereich sinnvoll (vgl. dazu Apeltauer 2004b, Kapitel 3).

Wir haben oben darauf hingewiesen, dass beim Sprechen der Zweitsprache die Erstsprache automatisch aktiviert wird. Entsprechend wird die Bedeutung eines neuen Wortes der Zweitsprache mit Hilfe der Erstsprache erschlossen. Der Zugriff auf das konzeptuelle System erfolgt also über die Erstsprache. Während einer Übergangsphase werden Wörter der Zweitsprache vorübergehend mit situationsspezifischen Teilbedeutungen oder mit entsprechenden Bedeutungen der Erstsprache assoziiert. Es dauert einige Zeit, bis für Wörter der Zweitsprache ergänzend zu den erstsprachlichen Bedeutungen eigenständige Bedeutungen entwickelt werden. Erst viel später, wenn die Zweitsprache auf hohem Niveau beherrscht wird, kann auch von der Zweitsprache direkt (d. h. ohne Umweg über die Erstsprache) auf das konzeptuelle System zugegriffen werden. Doch selbst Bilinguale, die ihre beiden Sprachen gut beherrschen, verfügen meist über eine präferierte Sprache, in der sie leichter verstehen und sich ausdrücken können. Man hat nachgewiesen, dass Verbindungen von der präferierten Sprache (i. d. R. die Erstsprache) zum jeweiligen Konzept schneller hergestellt werden können als von der Zweitsprache, ein Effekt, der allerdings mit zunehmendem Sprachstand in der Zweitsprache geringer wird (vgl. Kroll/Sunder-mann 2003). Längere Reaktionszeiten wurden auch beim Übersetzen von der Erst- in die Zweitsprache nachgewiesen (vgl. de Groot/Dannenburg/van Hell 1994).

Im semantischen Gedächtnis werden aber nicht nur Wortbedeutungen gespeichert, sondern auch Wissen über Gegenstände, mit denen Erfahrungen gesammelt werden konnten, d. h. also auch Weltwissen. Lernforscher betonen, dass Individuen vor allem dann klug handeln können, wenn sie über ausreichendes Wissen für eine Problemlösung verfügen. Es geht dabei nich um auswendig gelerntes Wissen, sondern um vintelligentes Wissen«, d. h. Wissen, das von Lernenden auf der Grundlage vorausgehender Erfahrungen selbst konstruiert wurde.

»(...) Wissen und nicht Intelligenz (ist) der Schlüssel zum Können (...) Während fehlendes Wissen nicht kompensierbar ist, können Defizite bei Intelligenz und speziellen Begabungen durch besonders intensives Üben in gewissem $\mathrm{Maß}$ ausgeglichen werden. (...) Kinder, die unabhängig von ihrer Intelligenz schon zu Beginn des Schuljahres Wissen mitbringen, haben die besten Chancen, etwas dazuzulernen« (Stern 2003: 11). 
Hinzu kommt, dass der Umgang mit einer differenzierten Sprache auch Rückwirkungen auf das Vorstellungs- und Denkvermögen von Menschen hat.

»Offenbar kann man also durch den übenden Umgang mit einer differenzierten Sprache, die abstrakte Konstrukte auszudrücken erlaubt, erlernen, solche Konstrukte auch zu denken und sich vorzustellen« (Singer 2001: 7).

Zusammenfassend kann man also sagen, dass man mit einer differenzierten Sprache (i. d. R. die Erstsprache) Informationen schneller aufnehmen und präziser verarbeiten kann als mit einer weniger entwickelten Zweitsprache. Kinder können mit Hilfe ihrer Erstsprache schneller und genauer verstehen und sich auch differenzierter ausdrücken. Sie können damit sowohl Erfahrungen als auch Informationen besser verarbeiten und so ihr Weltwissen rascher erweitern. Da der Gebrauch einer differenzierenden Sprache zugleich auch das Denk- und Vorstellungsvermögen anregt, stellt der Gebrauch einer differenzierenden Sprache also auch ein Anregungspotential für die kognitive Entwicklung dar.

Im Gegensatz dazu benötigt man in einer Zweitsprache für Informationsaufnahme und -verarbeitung nachweislich mehr Zeit und Energie (was u. a. daran erkennbar wird, dass man beim Gebrauch einer Zweitsprache i. d. R. schneller ermüdet). Außerdem kann man - z. B. aufgrund des geringeren Wortschatzumfangs - oft nur Grob- oder Teilinformationen aufnehmen. Daraus folgt, dass für Kinder, die nicht über gute oder sehr gute Zweitsprachkenntnisse verfügen, die Erstsprache als Motor für die Entwicklung und als Medium zum Sammeln von (auch schulrelevantem) Weltwissen unentbehrlich ist, zumindest so lange, bis die Zweitsprache so weit entwickelt wurde, dass sie die oben erwähnten Funktionen der Erstsprache übernehmen kann.

Wir wollen nun einmal die sprachlichen Fertigkeiten genauer betrachten, über die KmM bei der Einschulung in ihrer Zweitsprache Deutsch verfügen und im Anschluss daran überlegen, welche zweitsprachlichen Fertigkeiten erforderlich wären, um einem Regelklassenunterricht problemlos folgen zu können.

\subsection{Zweitsprachkenntnisse von KmM im Schuleingangsbereich}

Es gibt verschiedene Versuche, die sprachlichen Voraussetzungen von Schulanfängern, insbesondere von $\mathrm{KmM}$, zu ermitteln. In einer breit angelegten Untersuchung (Grimm 2003b) wurden 1395 Kinder erfasst. Etwa 60\% der untersuchten KmM zeigten sprachliche Auffälligkeiten. Der Anteil an Risikokindern war bei den $\mathrm{KmM}$ mit 34,5\%, mehr als dreimal so hoch wie bei den deutschsprachigen Kindern. Sprachliche Probleme zeigten sich bei KmM besonders im Bereich von Wortschatz und Syntax.

In zwei kleineren Untersuchungen (Hamburger Erhebung von 1999/2000, 130 Schüler, vgl. Reich 2000; Essener Erhebung 2000, 90 Schüler, vgl. Bericht zur 
Sprachstandserhebung 2000), die aufgrund der Probandenzahlen vielleicht nicht gerade als repräsentativ bezeichnet werden können, wurde weiter differenziert als bei der Bielefelder Untersuchung. Demnach scheint ein kleinerer Teil der Kinder (in absteigender Reihenfolge) über gute $(16 \%)$ oder über stabile und ausgebaute $(18 \%)$ Deutschkenntnisse zu verfügen. Die Mehrzahl der untersuchten Kinder (ca. 66\%) verfügt nur über eingeschränkte Deutschkenntnisse, d. h. über grundlegende (21\%), einfache (23\%), elementare (13\%) oder fast keine (8\%) Deutschkenntnisse. Diesen Aussagen kommt Gewicht zu, da beide Untersuchungen zu ähnlichen Ergebnissen kommen. Sie relativieren die Ergebnisse der Bielefelder Untersuchung (vgl. Grimm 2003b).

Man könnte nun festlegen, dass Kinder mit guten und solche mit stabilen und ausgebauten Deutschkenntnissen keinen Förderbedarf mehr haben. Und man könnte weiter annehmen, dass $\mathrm{KmM}$, die über solche Zweitsprachkenntnissen verfügen, am Regelklassenunterricht erfolgreich teilnehmen werden. Das klingt zwar plausibel, ist aber in Anbetracht unserer gegenwärtigen Kenntnisse über solche Zusammenhänge spekulativ.

Neuere Untersuchungen zur Bedeutungsentwicklung legen die Vermutung nahe, dass auch KmM, die Deutsch flüssig sprechen und nach einer kurzen Sprachprobe als gut eingestuft werden, im Regelklassenunterricht Probleme haben werden. Viele Erhebungsverfahren (z. B. das hessische MSS oder das hamburgische HAVAS 5) kommen mit einer Durchführungszeit von 10 bis 15 Minuten aus. In der Praxis wird diese Zeit durch Anweisungen, Fragen und Nachfragen der Person, die das Verfahren durchführt, weiter reduziert. Kann man auf der Grundlage von 5 oder 10 Minuten Sprechproben ein Urteil über die Sprachkompetenz eines Kindes fällen? (vgl. dazu auch Apeltauer 2004a).

Es wurde nachgewiesen, dass fortgeschrittene Lerner einer fremden Sprache nur halb so viele Assoziationen zu einzelnen Wörtern produzierten wie Muttersprachler. Weit fortgeschrittene Lerner, d. h. Lerner, die über einen etwa gleich großen Wortschatz verfügen wie Muttersprachler, produzieren zwar genauso viele Assoziationen wie die Muttersprachler. Aber diese Assoziationen unterscheiden sich deutlich von denen der Muttersprachler. So reagierten Muttersprachler z. B. auf ruler mit school, mathematics, inche(s), während weit fortgeschrittene Nicht-Muttersprachler darauf mit authority, king, monarch antworten (vgl. Zareva/Schwanenflugel/Nikolova 2005). Ein anderes Beispiel: Im Mathematikunterricht reagiert ein türkisches Mädchen auf die Abbildung von Gewichten, die verglichen werden sollen, mit der Äußerung: Gift - wegen so schwar: Es hatte die Abbildungen offenbar mit Flaschen assoziiert und die Farbe schwarz mit Gift (vgl. Wiedemann 2005: 95). Was das für das Lese- (aber auch für das Hörverstehen) und das Lösen von Aufgaben bedeutet, ist unschwer vorstellbar: Kinder, denen wegen flüssiger Aussprache gute Deutschkenntnissen attestiert wurden, werden nicht immer über die benötigten sprachlichen Voraussetzungen verfügen, um dem Regelklassenunterricht zu folgen, weshalb Lehrkräfte im Unterricht vermehrt verständnissichernde 
Fragen stellen und im Rahmen von Wortschatzarbeit immer wieder Bedeutungen klären und Begriffsgrenzen herausarbeiten sollten?

\section{Förderbedarf ermitteln}

Um Fördermaßnahmen durchführen zu können, muss man zunächst einmal wissen, welche Kinder einen Förderbedarf haben. Und es muss geklärt werden, in welchen Bereichen ein Förderbedarf besteht, damit Förderangebote entsprechend abgestimmt werden können. Zu fragen ist daher, über welche Zweitsprachkenntnisse Kinder verfügen, die nicht über gute oder stabile und ausgebaute Zweitsprachkenntnisse verfügen?

Eine Beantwortung dieser Fragen ist schwierig, da die Forschungslage im Bereich Zweitspracherwerb von $\mathrm{KmM}$ noch sehr viele Wünsche offen lässt. Es gibt nur wenige Fallstudien von Schulkindern und kaum Untersuchungen zum Zweitspracherwerb von KmM im Vorschulbereich. Weil Forschungsergebnisse fehlen, sollten Aussagen über einen erreichten Entwicklungsstand bei KmM vorsichtig und mit Vorbehalten formuliert werden.

Man hat versucht, sich an den Kenntnissen monolingualer deutscher Kinder $\mathrm{zu}$ orientieren. Letztlich ist das aber ein untauglicher Versuch, weil zweisprachig aufwachsende Kinder in ihrer Zweitsprache erst nach zwei bis drei Lernjahren einen Sprachstand erreichen, den man als flüssig und (vielleicht auch) als gut bezeichnen kann, sprachliche Fertigkeiten, über die monolinguale Kinder bereits im Alter von vier bis fünf Jahren verfügen ${ }^{10}$. Mit anderen Worten: Auch wenn KmM ab dem vierten Lebensjahr einen Kindergarten besuchen, können sie bei der Einschulung noch nicht über die gleichen sprachlichen Voraussetzungen verfügen, wie ihre monolingualen Klassenkameraden. Man sollte also der Euphorie, mit der gegenwärtig aller Orten über sprachliche Frühförderung geredet wird, mit einer gesunden Portion Skepsis begegnen, zumal auch die vielfältigen Maßnahmen zur Frühförderung sehr heterogen sind und kaum wissenschaftliche Evaluationen vorliegen. Es gibt inzwischen eine Vielzahl von Sprachstandserhebungsverfahren. Viele Bundesländer haben eigene Verfahren ${ }^{11}$ entwickeln lassen, wobei (offenbar nach

\footnotetext{
${ }^{9}$ Im Regelklassenunterricht wird meist noch immer so unterrichtet, als ob nur Kinder mit der Erstsprache Deutsch zu unterrichten wären. Systematische Wortschatzarbeit, die gerade für KmM für den weiteren Ausbau ihres Wortschatzes und die Schärfung ihrer Begriffe wichtig wäre, wird im Unterricht vielfach vernachlässigt.

${ }^{10}$ Hier ist zu berücksichtigen, dass die sprachliche und kulturelle Differenz zwischen Ausgangssprache (z. B. Italienisch oder Türkisch) und -kultur und Zielsprache (Deutsch) und -kultur sowie familiale und lernerspezifische Voraussetzungen (z. B. Interesse und Motivation) die Aneignung des Deutschen erleichtern oder erschweren, weshalb mehr oder weniger Zeit für den Erwerb zu veranschlagen ist. Hinzu kommt, dass Aneignungsprozesse phasenweise immer auch diskontinuierlich verlaufen, weshalb Vorhersagen schwierig sind.

${ }^{11}$ Die Verfahren, die auf dem Markt sind, sind entweder nicht normierte Tests oder Beobachtungsverfahren. In Bremen der SFD (Hobusch/Lutz/Wiest 2002), in Hamburg HAVAS 5 (Reich/Roth 2004), in Hessen das »Marburger Screening [MSS]« (Holler-Zittlau u. a. 2003), in Bayern das »Bayerische Screening« (Hölscher u. a. 2002), in Niedersachsen »Fit in Deutsch«, ebenfalls ein
} 
dem Zufallsprinzip) in einem Falle Sprachheilpädagoginnen und -pädagogen bei der Konzeption federführend waren (so z. B. beim Marburger Screening MSS), in anderen Fällen Pädagoginnen und Sonderpädagoginnen (z. B. beim niedersächsischen Fit in Deutsch) oder Sprachdidaktiker und Pädagogen (z. B. bei HAVAS 5 und beim Bayerischen Screening). Ja es gibt sogar einen Fall, wo sich ein Schulpsychologe, eine Rektorin und eine Sonderschullehrerin zusammenfanden und ein Verfahren (das SFD) entwickelt haben.

Unter diesen Bedingungen wird es kaum überraschen, wenn jedes dieser Verfahren sich auf andere Kriterien stützt und unterschiedliche sprachliche Bereiche fokussiert werden. Das hessische Verfahren orientiert sich z. B. an Erkenntnissen über den Erstspracherwerb und an Entwicklungsskalen für deutsche Kinder sowie an allgemeinen Sprachstörungen deutscher Kinder (vgl. MSS, S. 19), was im Hinblick auf zweisprachig aufwachsende Kinder höchst problematisch ist.

Es gibt ein Verfahren (HAVAS 5), das sich an Ergebnissen der Zweitspracherwerbsforschung orientiert und sprachliche Indikatoren ${ }^{12}$ verwendet. Eine Transkription der aufgezeichneten Daten wird aber freigestellt. Ohne eine Transkription ist eine differenzierte Auswertung von Daten aber nicht möglich. Neben einer für Kinder sicher anregenden Bildfolge wird ein problematisches »Küchenbild« verwendet, das die Sprachdomäne Familie thematisiert, eine Domäne, in der die Zweitsprache funktionslos ist. Den durchführenden Personen wird empfohlen, sich bei Erzählungen der Kinder neutral zu verhalten. Aus testtheoretischen Gründen mag das berechtigt sein. Ein natürlicher Erzählkontext kann dadurch aber nicht entstehen. Schüchterne Kinder oder Kinder mit eingeschränkten Erzählfähigkeiten werden ihr sprachliches Wissen kaum entfalten werden.

Andere Verfahren orientieren sich an der Schulpraxis und versuchen von dieser ausgehend erforderliche sprachliche Fertigkeiten von KmM zu bestimmen (z. B. SFD oder Fit in Deutsch) ${ }^{13}$. Jedes Verfahren verspricht relevante Informationen zu liefern. Doch keinem gelingt es, mehr als Globalauskünfte zu erteilen: Ein Kind hat (oder es hat keinen) Förderbedarf. Hilfen zur Förderplanung werden nicht (oder nur in sehr allgemeiner und unverbindlicher Form) bereitgestellt (Vgl. dazu auch die Kritik von Lütje-Klose 2006 an SFD und HAVAS 5).

Der Einsatz der meisten Verfahren erfolgt punktuell, d. h. ein Jahr oder ein halbes Jahr vor Schulbeginn. Zu diesen Zeitpunkten werden die Kinder (i. d. R. von ihnen unbekannten Lehrkräften in für sie fremden Räumen (kritisch dazu Apeltauer 2004a; vgl. auch Schnieders/Komor 2005) überprüft und im Bedarfsfalle Fördermaßnahmen zugewiesen. Es gibt auch Verfahren, die über einen längeren

Screeningverfahren (Lütje-Klose 2003). Es gibt weitere Verfahren (z. B. die Profilanalyse von Grießhaber oder das »Penner-Screening«), die hier vernachlässigt werden, weil sie kaum zum Einsatz kommen.

12 Als Sprachstandsindikatoren werden sprachliche Phänomene bezeichnet, von denen wir (aufgrund von Untersuchungsergebnissen) wissen, in welchen Entwicklungsphasen sie in welcher Form auftreten. Das sind z. B. Satzmuster oder der verbale Wortschatz oder Formen und Stellungen des Verbs im Satz.

${ }^{13}$ Kinder müssen demnach Anweisungen verstehen können und wissen, was Präpositionen bedeuten. 
Zeitraum (d. h. mehrfach) genutzt werden können, die aber sprachlich wenig differenziert sind (dazu gehört z. B. das von Uhlich/Mayr entwickelte Sismik [Sprachverhalten und Interesse an Sprache bei Migrantenkindern in Kindertageseinrichtungen] oder die in Wuppertal entwickelten Sprachentwicklungsbögen (vgl. Maiworm-Jäckel 2005). In Österreich werden Erzieherinnen und Lehrkräfte dazu angehalten, das Sprachverhalten vor Förderbeginn und nach Förderende zu dokumentieren. In Deutschland muß sich das erst noch einbürgern.

Viele Verfahren benötigen erstaunlich wenig Zeit zur Datenerhebung ${ }^{14}$. Wenn man bedenkt, dass die Durchführungszeiten immer Fragen und Instruktionen der durchführenden Person mit einschließen, so kann man die angegebenen Zeiten getrost halbieren. Es bleiben also 20 bzw. 10 Minuten für die eigentliche Datenaufnahme. Sind Datensätze, die in einem solchen Zeitrahmen gewonnen werden, für eine Beurteilung ausreichend? Muss nicht davon ausgegangen werden, dass es eine kritische Datenmenge gibt, die nicht unterschritten werden darf, damit relevante Urteile gefällt oder Empfehlungen für Fördermaßnahmen gegeben werden können ${ }^{15}$ ? Müsste nicht auch gefragt werden, ab welcher Datenmenge relevante Einblicke in die sprachlichen Fertigkeiten eines Kindes gewonnen werden können ${ }^{16}$ ?

Es gibt zu einigen Verfahren Übersetzungen in die Erstsprachen der Kinder, so dass auch erstsprachliche Fertigkeiten erfasst werden könnten. SFD bietet z. B. zwei CDs, mit denen Wortschatzkenntnisse in Sprachen wie Türkisch, Russisch, Polnisch, Persisch, Kroatisch/Serbisch, Kurdisch, Tamil und Spanisch (Teil 1) und Englisch, Italienisch, Arabisch, Portugiesisch, Griechisch, Französisch und Albanisch (Teil 2) überprüft werden können. Die CDs werden abgespielt und die Kinder haben dann zu einzelnen Wörtern passende Bilder zu zeigen. Die Auswahl des Wortmaterials wird nicht begründet, die Durchführung soll auch ohne Sprachkenntnisse in den Migrantensprachen möglich sein. Das Verhältnis zwischen Erst- und Zweitsprache wird nicht thematisiert.

Auch ,Fit in Deutsch' wurde in die großen Migrantensprachen (Russisch, Arabisch, Türkisch, Kurdisch, Italienisch und Spanisch) übersetzt und ebenso HAVAS 5, so dass Sprachstandseinschätzungen nun auf der Grundlage beider Sprachen möglich wären, wenn entsprechende Normen vorliegen würden und Spezialisten zur Auswertung zur Verfügung stünden. Lütje-Klose schreibt über Fit in Deutsch:

\footnotetext{
${ }^{14}$ SFD und Fit in Deutsch benötigen (nach eigenen Angaben) für die Datenerhebung ca. 30 bis 45 Minuten, HAVAS 5 ca. 15-20 Minuten (vgl. dazu auch Fußnote 16).

${ }^{15}$ Diese Frage müsste grundsätzlich geklärt werden, damit angemessene Sprachstandserhebungsverfahren entwickelt werden können.

${ }_{16}$ Eine meiner Studentinnen hat das Verfahren HAVAS 5 mit zwei Kindern im Rahmen eines mehrwöchigen Fachpraktikums durchgeführt. Zur Kommentierung der Bildfolge Katze und Vogel benötigte das eine Kind ca. 35 Sekunden, das andere etwa eine Minute. Die Datenauswertung ergab, dass die Kinder nach HAVAS 5 keinen Förderbedarf hatten. Betreuerin und Praktikantin hatten dagegen den Eindruck, dass in beiden Fällen Förderbedarf besteht. Die Kinder waren von ihnen allerdings auch mehrere Wochen beobachtet worden.
} 
»Eine Berücksichtigung der Herkunftssprachen durch eine eigene Sprachüberprüfung ist theoretisch möglich, wird aber nicht gefordert" (vgl. Lütje-Klose 2006: 11).

Abschließend bleibt festzuhalten, dass die vorliegenden Sprachstandserhebungsverfahren keineswegs das bereitstellen, was benötigt wird. Sie vermitteln $-z$. T. auf der Grundlage fragwürdiger Kriterien aber auch aufgrund des noch unzureichenden Forschungsstandes - Globaleinschätzungen, ermitteln aber keine differenzierten Sprachstandsprofile (vgl. dazu auch die kritischen Anmerkungen bei Jeuk 2006). Um herauszufinden, wo ein Kind in seiner sprachlichen Entwicklung (und das sollte auch heißen; in seiner Erstsprache) steht und welche nächsten Entwicklungsstufen es voraussichtlich durchlaufen wird, so dass sinnvolle Fördermaßnahmen geplant und durchgeführt werden können, bedarf es geeigneter Beobachtungsverfahren, die nicht nur punktuell eingesetzt werden, die ausreichende Datenmengen generieren und Ergebnisse liefern, die einen differenzierten Einblick in den jeweiligen Entwicklungstand ermöglichen. Auch zur Bildung unterschiedlicher Fördergruppen (z. B. KmM mit keinen/geringen oder mit elementaren Deutschkenntnissen) wären solche differenzierteren Informationen wichtig. Sie lassen sich nur auf einer soliden Grundlage (d. h. anhand von längeren und mehrfachen Beobachtungen) ermitteln ${ }^{17}$.

\section{$4 \quad$ Förderschwerpunkte}

\subsection{Phonologische Analysefähigkeiten}

Wir haben oben darauf hingewiesen, dass Kinder im Verlaufe ihres ersten Lebensjahres eine für das Verstehen der Erstsprache optimale Dekodierungsstrategie entwickeln. So wird es möglich, Wörter und Phrasen zu isolieren. Der Ausgangspunkt für Wortschatz- und Syntaxerwerb ist die phonologische Analyse der Sprache.

Doch nicht nur für das Hörverstehen ist ein solches Vorgehen von Bedeutung. Auch das Lesen- und Schreibenlernen basiert zu einem großen Teil auf diesen Fertigkeiten. Gerade der Zusammenhang zwischen phonologischer Analyse und der Entwicklung von Fertigkeiten in den Bereichen Lesen und Schreiben wurde in den letzten Jahren intensiv erforscht (vgl. dazu z. B. Christophe./Guasti/Nespor/ Dupoux/Van Ooyen 1997; Cutler 2001). Fähigkeiten wie das Erkennen von sprachlichen Rhythmen oder Betonungsstrukturen, das Isolieren von Lauten (z. B. am Wortanfang oder Wortende) werden zu den Vorläuferfertigkeiten für den

\footnotetext{
${ }^{17}$ Um SISMIK Beobachtungsbögen auszufüllen, benötigen Erzieherinnen, die darin trainiert wurden, 1-2 Wochen Beobachtungszeit. Es ist davon auszugehen, dass sie während dieser Zeit differenziertere Einsichten gewinnen können als Lehrkräfte, die einzelne Kinder in 10 oder 15 Minuten begutachten sollen.
} 
Schriftspracherwerb gerechnet. Man unterscheidet dabei zwischen phonemanalytischen Kompetenzen in einem weiteren und einem engeren Sinne. Im weiteren Sinne gehört dazu das Analysieren von Wörtern in größere Einheiten (z. B. in Silben) oder das Erkennen von Reimen.

In einem engeren Sinne gehört dazu das Segmentieren (Zerlegen) eines Wortes in Laute, das Identifizieren (z. B. Feststellen, ob ein bestimmter Laut in einem Wort vorkommt), Synthetisieren (z. B. die Laute $r-o-t$ zu / rot/ zusammenfügen) und Manipulieren (z. B. aus Zebra und Löwe Zewe bilden). Denn solche Fertigkeiten werden beim Lesen und Schreiben benötigt. Beim Lesen müssen aus kleinen Einheiten größere gebildet werden, d. h. Buchstaben müssen zu Phonemen in Beziehung gesetzt werden und diese zu Silben und schließlich zu Wörtern. Beim Schreiben müssen in umgekehrter Reihenfolge Wörter in Silben, Laute und Buchstaben zerlegt werden.

Man betrachtet fehlende Kompetenzen im Bereich phonologischer Analysefähigkeiten als mögliche Ursache für Lese- und Rechtschreibschwierigkeiten (in der psychologischen Fachliteratur wird von phonologischer Bewusstheit gesprochen; vgl. Küspert/Schneider 1999). Und weil man herausgefunden hat, dass spätere Schulleistungen durch Vorläuferkompetenzen im Lesen und Schreiben besser vorhergesagt werden können als mittels nonverbaler Intelligenztests, wurden entsprechende Präventionsmaßnahmen (insbesondere für Kinder mit Dyslexie-Risiko) entwickelt (zu den dafür entwickelten Trainingsprogrammen gehören z. B. Küspert/ Schneider 1999; Martschinke/Kirschhock/Frank, 2001; Christiansen 2005 ) 18. In einigen Bundesländern werden diese Trainingsprogramme inzwischen für alle Kinder (d. h. auch für $\mathrm{KmM}$ ) angeboten.

Nun trägt der Erwerb einer fremden Sprache dazu bei, dass Kinder sprachliche Formen früher fokussieren und auch früher ein Bewußtsein für die Konventionalität von Sprache entwickeln. Demnach würden $\mathrm{KmM}$ also über bessere Voraussetzungen für ein phonlogisches Training verfügen, als monolinguale deutsche Kinder. Und insofern wäre die Einbeziehung von KmM in solche Trainingsprogramme (die nur für monolinguale deutsche Kinder entwickelt wurden) zu rechtfertigen, wenn das Training in der jeweiligen Erstsprache erfolgen würde. Wenn es aber in der Zielsprache Deutsch erfolgt (wie das gegenwärtig der Fall ist), sind die damit verbundenen Aufgaben für 66\% (bzw. 60\%) der KmM schwieriger zu lösen als für deutsche Kinder, weil diese KmM i. d. R. nicht über den gleichen Wortschatz verfügen, wie monolinguale deutsche Kinder. Wenn sie z. B. noch keine komplexen Wörter in ihrer Zweitsprache beherrschen (vgl. Moser/Berweger/Stamm 2005) ${ }^{19}$, werden sie mit dem Wortmaterial, das in den Trainingsprogrammen angebo-

\footnotetext{
${ }^{18}$ Unter Dyslexie versteht man Leserechtschreibschwächen, d. h. Störungen bei der Aneignung der Schriftsprache.

${ }_{19}$ Moser/Berweger/Stamm unterscheiden drei Kompetenzniveaus der Wort-schatzentwicklung. Auf Stufe I können einfache Basisobjekte benannt oder umschrieben werden, auf Stufe II werden morphologisch komplexere Wörter verwendet und Unterbegriffe benannt, auf Stufe III verfügen die Lerner über ein flexible Auswahl an Wörtern und gebrauchen auch mehrsilbige Wörter.
} 
ten wird, überfordert (bei Küspert/Schneider 1999: 46 finden sich z. B. Wortungetüme wie Schreibmaschine [veraltet?], Blumenwiese und Streichbolssschachtel). Was deutschen Kindern geläufig ist, kann von $\mathrm{KmM}$ oft noch nicht verstanden werden. Wenn KmM aber zum Nachsprechen und Hantieren mit Lautsequenzen genötigt werden, denen sie noch keine Bedeutung zuordnen können, kann damit auch kaum "phonologische Bewusstheit« trainiert werden. Gräsel/Gutenberg/Pietzsch/ Schmidt 2004 glauben bei ihrer Evaluation des Würzburger Trainingsprogramms (vgl. Küspert/Schneider 1999) auch eine positive Wirkung für KmM nachweisen zu können. Eine solche Wirkung wird (aus den oben genannten Gründen) nur für $\mathrm{KmM}$ mit guten Deutschkenntnissen nachweisbar sein.

Für die Entwicklung von Literalität ist ein metasprachliches Bewußtsein hilfreich, das sich gewöhnlich in der starken Erstsprache früher entwickelt als in der (schwachen) Zweitsprache (vgl. Verhoeven 2003). Ein Training phonologischer Analysefähigkeiten sollte darum in der starken Sprache beginnen. Man hat empfohlen, Minoritätenkinder zunächst in ihrer starken Sprache zu alphabetisieren. Und Minoritäteneltern wurde geraten, ihre Kinder vor Schuleintritt in der Erstsprache zu alphabetisieren, weil ein Transfer in die Zweitsprache danach leichter fällt (vgl. dazu Saville/Troike 1971; ähnlich Boyd/Arvidson 1998). Solche Empfehlungen sind freilich ohne entsprechende Fortbildungsangebote für Eltern kaum umsetzbar.

In der jüdischen Tradition werden Kinder übrigens bereits ab dem 3. Lebensjahr unterrichtet, können mit 6 Jahren lesen und schreiben (vgl. Rosten 2002, 134 f.). Die bekannte schwedische Kindersprachforscherin Söderbergh betont, dass Kinder schon lange vor Schuleintritt lesen und schreiben lernen könnten, wenn unsere pädagogischen Traditionen sie nicht daran hindern würden. Denn lesen und schreiben lernen - so Söderbergh - kann man auch ohne Grammatikkenntnisse.

Würde man Wortmaterial aus der starken Sprache (der Erstsprache) für ein solches Training verwenden, so würde KmM das Segmentieren, Identifizieren, Synthetisieren und Manipulieren leichter fallen als gleich alten monolingualen deutschen Kindern, weil sie im Verlauf ihres Zweitspracherwerbs ja schon gelernt haben, sprachliche Formen zu fokussieren und weil sie auf vertrautes (und hochgradig automatisiertes) Wortmaterial zurückgreifen könnten. In der Zweitsprache aber können sie z. B. ähnlich klingende Wörter verwechseln und Analyseaufgaben u. U. darum nicht lösen ${ }^{20}$. Damit ein phonologisches Training für $\mathrm{KmM}$ wirksam werden kann, müßte man also entweder erstsprachliches Wortmaterial einbeziehen (was für Betreuungspersonen ohne Grundkenntnisse in einer solchen Sprache nicht möglich ist) bzw. ein solches Training in der Erstsprache beginnen und das

\footnotetext{
20 Wenn ein Lerner in der fremden Sprache Deutsch ein Wort wie Reise hört und leise dekodiert, so wird er sich aufgrund des Kontextes meist selbst korrigieren können. Wenn ihm dagegen isoliertes Wortmaterial vorgesetzt wird (wie in den Trainingsprogrammen) und z. B. der Wortanlaut bestimmt werden soll, kann eine Aufgabenlösung an einer solchen Verwechslung scheitern. Bleibt diese Verwechslung verdeckt (d. h. merkt die Betreuungsperson nicht, dass hier eine Verwechslung vorliegt) entsteht der Eindruck, dass das Kind über keine guten phonologischen Analysefähigkeiten verfügt. In der Erstsprache wäre dem gleichen Kind eine solche Verwechslung kaum passiert.
} 
Wortmaterial allmählich durch die Hereinnahme von zweitsprachlichem Material anreichern. Parallel dazu müsste eine intensive Wortschatzarbeit geleistet werden, damit die Kinder Wörter lernen, die später im phonologischen Training gebraucht werden.

Wortschatzarbeit ist aber erst sinnvoll, wenn die Einhörphase vorbei ist, $d . h$. wenn die Lerner schon über sprachliche Grundkenntnisse verfügen, weil sie sonst viele Wörter rasch wieder vergessen (bei den von uns untersuchten Kindern dauerte die Einhörphase drei bis vier Monate, bei einigen Kindern sogar sechs bis neun Monate; vgl. Apeltauer 2004 b). Es ist daher sinnvoll, KmM, die ohne oder mit geringen Deutschkenntnissen in den Kindergarten kommen, beim Einhören in die fremde Sprache zu helfen, insbesondere dann, wenn keine Mehrheit deutschsprachiger Kinder in den Gruppen vorhanden ist. Dazu können Kinderlieder, Abzählreime, Rätsel und Kindergedichte verwendet werden. Durch begleitendes Klatschen, Pantomime oder Gesten hilft man, Höreindrücke zu strukturieren, so dass die neuen Laute und Lautfolgen allmählich genauer gehört und schließlich auch korrekt artikuliert werden können. Natürlich könnte man auch Eltern anregen, ihren Kindern zu Hause Kinderreime, Abzählverse und Lieder in ihrer Erstsprache beizubringen. Auch dadurch würde die Entwicklung phonologischer Analysefähigkeiten unterstützt (es kann sinnvoll sein, wenn Eltern von KmM dazu fortgebildet werden; vgl. Apeltauer 2006a). Vor allem aber sollte man $\mathrm{KmM}$, die über keine guten Deutschkenntnisse verfügen, Überforderungserlebnisse ersparen, die im Rahmen der für deutsche Kinder entwickelten Trainingsprogramme zur phonologischen Bewusstheit fast unvermeidlich sind.

Zusammenfassend kann man sagen, dass das Training der phonologischen Bewußtheit heute in vielen Kindergärten zu einem festen Bestandteil der Förderangebote geworden ist. Man kann sogar von einer Euphorie in diesem Bereich sprechen, weil viele Erzieherinnen und Lehrkräfte glauben, dass damit auch KmM geholfen werden kann (kritisch zu den Trainingsverfahren Brüggelmann 2003; vgl. dazu auch Einsiedler u. a. 2000). Tatsächlich kann mit den Trainingsprogrammen Risikokindern (KmM gehören i. d. R. nicht dazu) kurzfristig (nicht langfristig!) geholfen werden (vgl. dazu Ehri u. a. 2001).

In niederländischen Untersuchungen zum Lesen und zum Leseverstehen hat man nachgewiesen, dass viele $\mathrm{KmM}$ das Dekodieren von Buchstaben schneller bewältigen als niederländische Kinder aus low SES Familien, dass KmM also schneller lesen als diese Kinder. Diese Fertigkeiten scheinen also (auch ohne phonologisches Training) gut entwickelt zu werden. Dennoch haben diese KmM Schwierigkeiten mit dem Textverstehen. Dekodierungsfähigkeiten und das Leseverstehen scheinen sich also relativ unabhängig voneinander zu entwickeln. Was aber sind dann zuverlässige Indikatoren zur Vorhersage für späteres Leseverstehen?

Vor allem zwei Faktoren scheinen dabei eine Rolle zu spielen:

- der jeweilige Wortschatzumfang und

- vorausgehende Leseerfahrungen. 
Droop/Verhoeven stellen dazu fest:

"(...) the strongest predictor of reading comprehension is (...) prior reading comprehension« (Droop/Verhoeven 2003: 16).

Ist das regelmäßige und interaktive Vorlesen von Gute-Nacht-Geschichten vielleicht wichtiger, als ein Training phonologischer Bewusstheit? Im Hinblick auf die Wortschatzentwicklung zweifellos. Und da ein umfangreicher Wortschatz in der Zweitsprache (das haben wir oben zu zeigen versucht) auch für ein phonologisches Training (im engeren Sinne) mit KmM Voraussetzung ist, sollten solche Trainingsprogramme erst dann eingesetzt werden, wenn mit den KmM schon ein umfangreicherer Wortschatz erarbeitet wurde.

\subsection{Wortschatzarbeit}

Kinder lernen nicht alle Wörter gleichzeitig. Sie bevorzugen am Anfang Inhaltswörter, d. h. also Nomen und Verben, später auch Adjektive und Adverbien. Nomen lassen sich z. B. mit Hilfe semantischer Merkmale und mit lexikalischen Hierarchien (Ober- und Unterbegriffen) beschreiben, Verben mit Handlungssequenzen, mit motorischen Programmen und syntaktischen Rahmenvorgaben (Valenzen). Funktionswörter werden zunächst nur vereinzelt aufgegriffen. Erst wenn ca. 400 Wörter erworben wurden, beginnt der Ausbau der Funktionswörter.

Bei der Aneignung eines neuen Wortes werden mehrere Stufen durchlaufen. Zuerst muss das neue Wort aus dem Lautstrom herausgefiltert werden. Erst dann kann ihm eine (erste, vorläufige) Bedeutung zugeordnet werden, die meist an die Lernsituation gekoppelt ist. Von diesem situativen Kontext muss abstrahiert werden und es muss auch die Bedeutung generalisiert werden. Bedeutungen werden also zunächst mit dem jeweiligen Kontext gespeichert, später allmählich vom Kontext abgelöst, abstrahiert und verdichtet. Parallel dazu muss auch die Artikulation geübt werden ${ }^{21}$. Im weiteren Verlauf der Aneignung muss das neue Wort mit anderen Wörtern vernetzt werden. Zusätzlich müssen auch grammatische Kenntnisse (z. B. Wortbildungsmöglichkeiten oder die Wortartzugehörigkeit) erschlossen werden. Das alles kostet Zeit (man lernt z. B. dass man eine Mütze abnehmen oder aufsetzen kann, dass es Zipfelmützen und Schirmmützen gibt usw. Solche Entwicklungsprozesse brauchen viel Zeit. Aitchison 1994: 180 spricht von der Geschwindigkeit einer Schildkröte, mit der diese Entwicklung voranschreitet). Erst wenn alle Stufen durchlaufen sind und auch automatisiert wurden, können wir sagen, dass ein Wort wirklich beherrscht wird (vgl. ausführlicher dazu Apeltauer 2005: 64 f.).

Im Rahmen der Erstspracherwerbsforschung hat man festgestellt, dass es nicht immer genügt, wenn ein neues Wort im Zusammenhang mit einem zu be-

\footnotetext{
${ }^{21}$ Wenn ein Kind z. B. Dornbröschen sagt, so zeigt das, dass es das Wort noch nicht in sinnvolle Bestandteile zerlegen kann und dass es wahrscheinlich noch mit der Artikulation beschäftigt ist.
} 
nennenden Objekt erwähnt wird, z. B. indem auf das Objekt gedeutet wird. Kindern hilft es offenbar mehr, wenn das Objekt, über das gesprochen wird, mit Blicken markiert wird. Dies scheint vor allem in der Anfangsphase der Bedeutungserschließung wichtig, weshalb gemeinsame Interaktionen in der frühen Kindheit einen hohen Stellenwert haben (vgl. Tomasello 2003). Doch auch dann unterscheidet sich das, was Erwachsene mit solchen Situationen verbinden von dem was Kinder denken, weil Kinder sich Bedeutungen auf der Grundlage anderer kognitiver Operationen erarbeiten als Erwachsene. Mit anderen Worten:

»Wenn Kinder Wörter aus dem Erwachsenenangebot erwerben, so geben sie diesen Wörtern Bedeutungen, die ihren Erkenntnisstrukturen entsprechen. Man kann daher nicht davon ausgehen, dass das gleiche Wort bei Kind und Erwachsenem den gleichen Begriff bedeutet« (Szagun 1983: 215).

Selbst wenn ein Kind ein Wort korrekt artikulieren kann, verfügt es also i. d. R. nicht über die Bedeutungen, die Erwachsene mit dem Wort verbinden und schon gar nicht über das »Bedeutungspotential« 22 eines Wortes, weil Inhalte des semantischen Gedächtnisses nur schrittweise ausgebaut, differenziert und vernetzt und so den jeweiligen Bedürfnissen und Interessen angepaßt werden. Und wie ist das beim Zweitspracherwerb?

Kinder im Alter von drei oder vier Jahren haben in ihrer Erstsprache schon einen umfangreichen Wortschatz entwickelt und gelernt, wie man die Bedeutung von Wörtern durch Beobachten und Zuhören erschließen kann. Doch wenn sie in den Kindergarten kommen und nur über geringe (oder keine) Deutschkenntnisse verfügen, sind sie vorübergehend wieder zurückgeworfen auf ihre Anfänge beim Spracherwerb. Wenn sie nur wenige Wörter der fremden Sprache verstehen, sind sie bei der Bedeutungserschließung wieder auf einen »sprachlichen Laufstall« angewiesen, d. h. auf Segmentierungshilfen, auf Wiederholungen und dialogische Einbettungen $^{23}$. Derartige Hilfestellungen können in größeren Gruppen nicht gegeben werden. Ähnliches gilt für Objektmarkierungen durch Blicke. Darum sollte in der Anfangsphase möglichst nur mit zwei (maximal drei) Kindern gearbeitet werden ${ }^{24}$.

\footnotetext{
22 Unter Bedeutungspotential versteht man die möglichen Bedeutungen eines Wortes in unterschiedlichen Kontexten. Je gebildeter ein Sprecher einer Sprache ist, desto mehr vom Bedeutungspotential eines Wortes wird er kennen und zum Verstehen nutzen können. Um komplexer (insbesondere schriftliche) Texte verstehen zu können, sollte man möglichst viel vom Bedeutungspotential eines Wortes kennen.

${ }^{23}$ In der angelsächsischen Fachliteratur spricht man vom „scaffolding“. Damit ist gemeint, dass Lernern geholfen wird (z. B. durch Gestik und Mimik, durch Fragen, dialogische Vorgaben, Einsagen oder begleitendes Mitsprechen), so dass Äußerungen verstanden oder unvollständige Äußerungen ergänzt werden.

${ }^{24}$ Im Kieler Modell wurde beim interaktiven Erzählen und Vorlesen mit zwei bis maximal drei Kindern gearbeitet; ähnlich in den Fördermaßnahmen der Universität Mannheim.
} 
Wie lernt man ein neues Wort in einer fremden Sprache? Dazu sind i. d. R. ähnliche Schritte erforderlich wie beim Erstspracherwerb. Es müssen Wortgrenzen und eine erste Bedeutung bestimmt werden. In der Anfangsphase werden dazu bestehende Wortbedeutungen der Erstsprache genutzt. Das neue Wort der Zweitsprache wird an ein bereits existierendes Wort der Erstsprache angekoppelt (vgl. Kroll/Tokowicz 2005: 543 ff.). Es kommt also zu einer ersten Vokabelgleichung, die jedoch aufgrund weiterer Interaktionserfahrungen überprüft und gegebenenfalls modifiziert werden sollte ${ }^{25}$. Wenn eine Bedeutung im Verlaufe des Erstspracherwerbs noch nicht erworben wurde, dann erfordert die Konstruktion dieser Bedeutung (z. B. höflich) zusätzliche Zeit ${ }^{26}$. Denn eine erste Bedeutungszuordnung erfolgt immer bezogen auf die Ausgangssituation. Für den weiteren Bedeutungsausbau sind Kommunikations- und Interaktionserfahrungen und/oder Beobachtungen erforderlich sowie Abstraktionen von der Ausgangssituation.

Es kann helfen, wenn beim Auftreten eines neuen Wortes gesondert auf die Wortbetonung hingewiesen wird, weil in vielen Sprachen der Wortakzent festgelegt ist (z. B. im Polnischen immer auf der vorletzten Silbe, im Französischen auf der letzten). Der im Deutschen veränderliche (dynamische) Wortakzent bereitet daher vielen Lernern (sowohl bei der Rezeption als auch bei der Produktion) Schwierigkeiten. Ähnliches gilt für Langvokale, weil es in vielen Sprachen keine Langvokale gibt (In den Materialien von Tophinke 2003 findet man dazu brauchbare Übungsvorschläge). Solche Verweise auf Besonderheiten oder auch Kontrastierungen mit der Erstsprache können ein differenziertes Wahrnehmen erleichtern. Vor allem aber gilt: Ein Wort muss korrekt gehört werden, damit es auch korrekt artikuliert werden kann. Erst dann werden sich Lerner auch mit dem Bedeutungsausbau und der Vernetzung des Wortes beschäftigen.

Von einem Wort müssen also zunächst charakteristische perzeptuelle und phonologische Merkmale gespeichert werden. Parallel dazu werden auch erste semantische Kategorien und Merkmale erfasst (z. B. Vögel fliegen, haben Federn ...). Mit Hilfe vorhandenen (Welt-) Wissens und aufgrund laufender Erfahrungen werden diese anfänglichen Bedeutungen beständig erweitert und angereichert.

Nicht alle Wörter werden gleich korrekt verstanden. Häufig sind Lerner zum Raten gezwungen oder zur Aufstellung von Vermutungen (Arbeitshypothesen). Denn nur ein Bruchteil der Wörter, die Lerner hören, wird z. B. im Unterricht erklärt (Nagy/Hermann 1987: 23) ${ }^{27}$. Es kann zu abweichenden Bedeutungszu-

\footnotetext{
${ }^{25} \mathrm{Vgl}$. z. B. das türkische dost und das deutsche Freund. Sprachanfänger (und kleinere Wörterbücher) speichern beide als Äquivalente. Für Fortgeschrittene sind das Wörter, die im Bereich der Bedeutung über eine größere Schnittmenge verfügen, die aber auch Bedeutungsunterschiede aufweisen. ${ }^{26}$ Bedeutungserwerb ist immer mit Begriffsarbeit verbunden, die ein Lerner leisten muss. Neue Erfahrungen und Einsichten müssen in Bedeutungen umgewandelt und in das Netzwerk von Bedeutungen eingepasst werden. Und das erfordert Zeit. Lernpsychologen sprechen vom »intelligentem Wissen«, das so entsteht und das flexibel nutzbar ist (im Gegensatz zum toten oder »nachgebeteten « Wissen). ${ }^{27}$ Sie gehen davon aus, dass im Verlauf eines Unterrichtsjahres nur wenige hundert Wörter explizit vermittelt werden, dass aber etwa 3.000 neue Wörter pro Jahr präsentiert werden. Muttersprachler
} 
schreibungen kommen, die sich - wenn es keinen Anstoß zur Korrektur gibt negativ auf weiteres Verstehen auswirken können. So wurde z. B. grob von KmM in einem fünften Schuljahr (Realschule) mit schlecht drauf sein, ärgerlich sein, böse sein, gemein sein paraphrasiert, Deutungen, die im Kontext des Vorkommens (einem Kinderbuchtext) möglich schienen, womit die intendierte Bedeutung (nicht rücksichtsvoll sein) jedoch nicht adäquat erfasst wurde. Effekte eines solchen ungenauen Verstehens können kumulieren.

Beim Bedeutungsausbau eignen sich Lerner Merkmale und Zusammenhänge nicht nur zielgerichtet (bzw. intentional) an, sondern auch beiläufig (inzidentell). Inzidentelles Lernen spielt z. B. beim »aufschnappen« von Wörtern und Wendungen eine Rolle. Inzidentell wird meist langsamer gelernt. Dafür wird Gelerntes aber vergessensresistenter gespeichert. Was man also zielgerichtet lernt, kann man sich schneller aneignen. Es wird aber auch rascher wieder vergessen. Beiläufig Gelerntes wird i. d. R. dauerhafter gespeichert. Vorschulkinder lernen eher inzidentell. Beim intentionalen Lernen ist ihre Konzentrationsspanne i. d. R. begrenzt, sofern sie nicht interessengeleitet Informationen suchen und sammeln.

Viele Erzieherinnen merken, wenn Kinder sich für etwas ernsthaft interessieren und ermutigen sie dann. Daraus können Interessengruppen entstehen und Kleinprojekte. Im Rahmen solcher Projekte entwickeln interessierte Kinder große Ausdauer und Konzentrationsfähigkeit. Und sie haben im Anschluss an die Projekte ein großes Mitteilungsbedürfnis, das ihnen hilft, ihrer Zweitsprache zu gebrauchen und auszubauen (vgl. dazu auch Swain 2005: 471; die Beschreibung eines praktischen Beispiels findet man in Apeltauer 2006a: 35).

Dort, wo Zusammenhänge einfach und visualisierbar sind (z. B. bei Konkreta oder Antonymen wie dick und dünn), lassen sich Bedeutungen durch Erläuterungen i. d. R. rascher ausbauen. Es wird vermutet, dass für häufig gebrauchte, konkrete und verwandte Wörter zweier Sprachen nur ein (gemeinsames) konzeptuelles System existiert. Bedeutungsübertragungen werden in diesen Bereichen also unproblematisch sein. Bei nicht verwandten Sprachen (oder entfernteren Kulturen) kann das aber anders sein. Hier können selbst scheinbar harmlose Bedeutungsübertragungen das Entstehen von Missverständnissen begünstigen ${ }^{28}$.

Noch schwieriger ist das bei abstrakten, seltener gebrauchten oder kulturspezifischen Wörtern. Dort, wo Zusammenhänge komplex sind (vgl. z. B. höflich), werden Lerner mehr Zeit und mehr sprachlichen Input benötigen, um Regelhaftigkeiten (bzw. Wahrscheinlichkeit des Auftretens) entdecken zu können. Instruktionen sind in solchen Fällen von begrenztem Wert. Lerner erschließen sich diese Bedeutungen aufgrund von Interaktions- und Kommunikationserfahrungen, wobei inzidentelle Prozesse eine wichtige Rolle spielen, weil Zusammenhänge dabei ganz-

\footnotetext{
können das mit Hilfe ihres potentiellen Wortschatzes bewältigen. Zweitsprachler verlieren dadurch zunehmend den Anschluss an die Klasse (vgl. oben Seite 6 f.).

${ }^{28}$ Tisch oder Haus kann z. B. in arabischen oder afrikanischen Ländern auf andere prototypische

Formen bezogen werden als bei uns.
} 
heitlich erfasst werden können, während intentionales Lernen immer mit einem Fokussieren verbunden ist, d. h. mit dem Ausblenden von (möglicherweise relevanten) Aspekten.

Zur Beherrschung eines Wortes und zum Ausbau seiner Bedeutung(en) gehören natürlich auch sprachliche Kontexte, in denen ein Wort besonders häufig vorkommt. Kompetente Sprecher einer Sprache wissen z. B., mit welchen vorausgehenden oder nachfolgenden Wörtern ein Wort häufig gebraucht wird ${ }^{29}$. Nur wenn man das weiß, kann man auch entscheiden, ob ein Wort in einem bestimmten Kontext angewandt werden kann oder nicht. Verbindungen zu anderen, verwandten Wörtern (z. B. der Wortfamilie oder zu Synonymen oder Antonymen, zu Unter- und Oberbegriffen) werden erst später erworben ${ }^{30}$, ebenso grammatisches Wissen (z. B. die Wortartzugehörigkeit oder Wortbildungsmöglichkeiten).

Wie wird nun in der Praxis verfahren? Lehrkräfte, die im Rahmen von Frühfördermaßnahmen eingesetzt sind, berichten, dass sie Wortschatzarbeit zumeist mit Bildmaterial durchführen sowie mit Memories und/oder Kartenspielen. Wortschatzarbeit wird auch mit Grammatikarbeit verbunden. Es werden z. B. Verben vermittelt und »die sollen dann auch gebeugt werden« (Lehrkraft). Oder man nimmt Material wie »erzähl mir was« (vgl. Rucys 2004) und »arbeitet dann an der Pluralbildung oder an Artikeln« (Lehrkraft). Manche Lehrkräfte berichten auch von Reimwörtern als Merkhilfen und von Silbenklatschen, Elementen aus dem Training zur phonologischen Bewusstheit, die sie im Rahmen der Fördermaßnahmen einsetzen.

Erzieherinnen bevorzugen dagegen eher einen ganzheitlichen Zugang. Für sie sind z. B. Bewegungs- und Kreisspiele wichtig, um die Kinder zum Sprechen zu bringen und ihnen Sprechfreude zu vermitteln. Auch das Erzählen oder Vorlesen von Geschichten oder Gedichten gehört für sie zur Wortschatzarbeit, wenn z. B. zu einzelnen Wörtern agiert werden kann. Solche Interaktionsformen müssen allerdings ritualisiert werden, weil Kinder, die das von zu Hause nicht kennen, anfangs rasch abschweifen. Vereinzelt wird auch über die Durchführung von Kleinprojekten berichtet. An denen teilweise auch Mütter teilgenommen und in der jeweiligen Erstsprache Verstehenshilfen gegeben zu haben scheinen. So etwas könnte ausgebaut werden.

In den Handreichungen DaZ (Senatsverwaltung Berlin) wird unter Wortschatzarbeit auf Inhalts- und Strukturwortschatz verwiesen und es wird ein Mitteilungswortschatz von einem Verstehenswortschatz und einem potentiellen Wortschatz abgegrenzt.

Normalerweise werden zunächst Formeln und Inhaltswörter (Nomen, Verben) erworben und nur vereinzelt Funktionswörter. Beim Erstspracherwerb werden Funktionswörter erst dann vermehrt aufgegriffen, wenn eine Schwelle (bei ca.

\footnotetext{
${ }^{29}$ Man nennt dieses erwartbare Miteinandervorkommen von Lexemen auch Kollokation bzw. Kollokationen.

${ }^{30}$ Vorausgehende und nachfolgende Wörter stehen in einer syntagmatischen Relation zu einem Wort. Wörter, die mit einem bestimmten Wort ausgetauscht werden können (z. B. Synonyme), stehen zu ihm in einer paradigmatischen Relation. Gewöhnlich werden syntagmatische Bezüge vor paradigmatischen erlernt (bzw. erworben).
} 
400 Wörtern) erreicht wurde (vgl. Kauschke 1999: 135). Ergebnisse zum Zweitspracherwerb liegen in diesem Bereich nicht vor.

Ein potentieller Wortschatz ist das, was uns hilft, die Bedeutung eines neuen Wortes zu erschließen aufgrund von Regeln der Wortbildung oder des Ko-Textes (d. h. der umgebenden Wörter). Muttersprachler verfügen i. d. R. über einen groBen potentiellen Wortschatz. Wird eine verwandte Zweitsprache erlernt, erleichtert dieser potentielle Wortschatz vielfach das Erraten von Wortbedeutungen. Ist die Zweitsprache mit der Erstsprache nicht verwandt (wie z. B. Türkisch oder Arabisch), ist ein potentieller Wortschatz praktisch nicht vorhanden. Mit der Zunahme an Kenntnissen und Fertigkeiten in der Zweitsprache entwickelt sich auch ein potentieller Wortschatz.

Semantisierungen sollen »sicht- und erlebbar« gemacht werden und auch die Wortbildung wird »zur Erklärung von Wörtern« für sinnvoll erachtet (vgl. Rösch u. a. 2001: 47 f.).

Zusammenfassend kann man sagen, dass eine erste Bedeutung eines neuen (zweitsprachlichen) Wortes im Falle von Konkreta mit einem Objekt oder Bild vermittelt werden kann. Zunächst muss aber auch bei Konkreta (z. B. Schatzkä̈stlein) eine Form erfasst und gespeichert und die Artikulation beherrscht werden, ehe auf der Inhaltsebene weiter gearbeitet werden kann. Denn auch bei Konkreta werden am Anfang meist nur situationsbezogene Teilbedeutungen gespeichert. Objekte müssen kategorisiert werden, es muss von der Lernsituation abstrahiert werden und es müssen die mit dem Wort verbundenen Konnotationen (Schätrkästlein, ein kleiner Kasten, der wertvolle Gegenstände enthält) und Kollokationen erlernt werden, damit das Wort flexibel einsetzbar wird.

Ein längeres oder komplexes Wort (z. B. Streichholzschachte) oder ein Wort, das schwierig auszusprechen ist oder das eine abstraktere Bedeutung hat oder eine Bedeutung, die es in der Erstsprache nicht gibt, wird i. d. R. schwerer erworben als ein kurzes, einfach auszusprechendes Wort, das auf konkrete Dinge verweist. Erst wenn zu einem Wort eine Grundbedeutung gespeichert wurde, wenn syntagmatische und paradigmatische Bezüge hergestellt werden können, wenn grammatische Kenntnisse (Wortart, Wortbildung) vorhanden sind und ein Lerner auch weiß, in welchem stilistischen Kontext (z. B. formell oder informell) das Wort gebraucht und mit welchen Konnotationen das Wort verbunden wird und dies alles automatisiert wurde, können wir sagen, dass das Wort beherrscht wird (Maier 1999: 51 empfiehlt darum, »systematisch zu überprüfen, ob die scheinbar flüssig deutsch sprechenden Kinder die jeweiligen Wörter und Ausdrücke tatsächlich aktiv beherrschen, d. h., ob sie sie von sich aus richtig anwenden können«). Bevor ein Wort automatisch (d. h. schnell) abgerufen werden kann, besteht die Gefahr des Vergessens. Deshalb sollten neue Wörter nach einer ersten Semantisierung möglichst in unterschiedlichen Kontexten wiederholt und vor allem auch gebraucht werden. 


\section{Fördermaterialien}

Über die Bedeutung des Wortschatzumfangs, des Bedeutungsausbaus und die Vernetzung und Automatisierung von Wörtern für das Hör- und Leseverstehen (und damit auch für die Schullaufbahn) sind sich Fachleute weitgehend einig. Dagegen gehen die Meinungen darüber, wie neue Wörter vermittelt werden sollten, auseinander. In der Praxis findet man eine Fülle von Materialien, die teilweise auch von Verlagen vertrieben werden (vgl. z. B. Schlösser 2001; Tophinke 2003; Piepho 2003; Loos 2004; Boehrer 2004; Ott/Stanscheva 2005). Die Materialien sind sehr unterschiedlich, basieren z. T. auf wissenschaftlichen Erkenntnissen ${ }^{31}$, z. T. auf didaktischen Ideen und Vorstellungen.

Lernszenarien wurden in der Englischdidaktik in den 80er Jahren eingeführt (vgl. Di Pietro 1987). Ursprünglich sollten Szenarien eine „dramatische“ Komponente enthalten, so dass Lerner sich stärker engagieren (minvolvment«). Die Lernszenarien von Hölscher u. a. sind dagegen Rahmenthemen, die z. T. in traditioneller Manier gefüllt werden. Ein Beispiel: Im Lernszenario Zablenfresser steht unter dem Stichwort: Schatそ̌kästlein »Ein individuelles Schatzkästlein für ausgeschnittene Ziffern wird angelegt«. Für KmM mit geringen Deutschkenntnissen ist Schatzkästlein ein langes und schweres Wort. Positive Konnotationen (Mitbedeutungen) werden damit nicht verbunden. Fortgeschrittene, die diese Konnotationen schon kennen, werden sich fragen, was an ausgeschnittenen Ziffern wertvoll sein soll.

Die meisten Materialien konzentrieren sich auf die Vermittlung von Nomen (bzw. Konkreta), die überwiegend mit Hilfe von Bildern, z. T. auch mit Gegenständen semantisiert werden.

Boehrer empfiehlt z. B.: »Ist der Wortschatz nicht bekannt, wird er durch die einbezogenen Realgegenstände automatisch eingeführt« (Boehrer 2004: 65). Ähnlich Hölscher u. a.: »In den Lernszenarien erfolgt die Erweiterung des Wortschatzes (...) über die Präsenz eines Objekts, das benannt wird« (Hölscher u. a. 2006: 12). Genügt das zur Einführung von Konkreta? Und müssen im Deutschen nur Konkreta vermittelt werden? Sind in der Anfangsphase nicht auch Formeln, Verben und Verweiswörter wichtig?

Bei Schlösser 2001 finden wir z. B. auf Seite 26 »Ein Koffer voller Kleider«, dazu Kopiervorlagen mit 12 Bildern zu 31 Wörtern, die als »Sprachziel« deklariert werden. Das methodische Vorgehen erinnert an den Fremdsprachenunterricht der 70er Jahre. Die Kinder sollen mit ganzen Sätzen (vgl. »Das ist die Hose«.) antworten. Es sollen also nicht nur neue Bezeichnungen gelernt werden, sondern zugleich auch Genusformen und Satzstrukturen, eine kaum bewältigbare Lernaufga-

\footnotetext{
31 Tophinke stützt sich bei ihren Wortbetonungsmustern und bei den Wortabwandlung auf neuere wissenschaftliche Erkenntnisse (phonologische Analysefähigkeiten). Ihre Bausteine zur Vermittlung von Satzmustern erinnern dagegen an die Muttersprachendidaktik der 70er Jahre. Sie lassen sich mit neueren Ergebnissen der Instruktionsforschung (SLA) nicht vereinbaren. Gleichwohl scheinen damit Erwartungen von Erzieherinnen und Lehrkräften befriedigt zu werden.
} 
be. Als Variante wird auf derselben Seite angegeben: Kinder »kommentieren ihr Tun: Ich ziehe die Hose an. Ich ziehe den Rock an. Ich ziehe den Hut auf ... «32.

Loos 2004 bezieht sich explizit auf einen Grundwortschatz, gibt aber nicht an, auf welchen ${ }^{33}$. Positiv zu vermerken ist bei seinen Materialien, dass nicht nur Konkreta (z. B. beim Thema Kleidung) behandelt werden, sondern auch Formeln (Ritualia wie Guten Tag, Auf Wiedersehen) einbezogen werden und Verben, Fragewörter und Adverbien, die in diesem thematischen Rahmen (Kleidung) sinnvoll sind (anziehen, ausziehen, aufsetzen, zumachen). Vermittelt werden sollen die Wörter u. a. über Reime und Lieder, die durch begleitendes Tun veranschaulicht werden. Lieder und Reime sind gute Hilfen zum Einhören, zum Üben der Artikulation und zum Wiederholen. Zur Bedeutungsvermittlung sind sie dagegen weniger geeignet.

Zusammenfassend kann man sagen, dass Wortschatzarbeit in allen Fördermaßnahmen geleistet werden soll. Die Auswahl der Wörter, die vermittelt werden sollen, ist unklar, folgt häufig einem Zufallsprinzip. Unklar sind auch die Prozesse, die bei der Aneignung eines neuen Wortes bewältigt werden müssen. Es scheint die Vorstellung zu dominieren, dass einem Gegenstand nur ein Name (»label«) angehängt werden muss. Über Verarbeitungsprozesse und die Stufen der Bedeutungsentwicklung scheint es vielfach unklare Vorstellungen zu geben. Es gibt Material, das Hilfen zum Einhören anbietet oder zum Einhören und zur Aussprache (z. B. Tophinke 2003 zum Einhören, Loos 2004 mit Liedern und Reimen zum Einhören, Artikulieren und Üben). Manche Konzeption orientiert sich am Muttersprachunterricht (z. B. Tophinke 2003), andere wiederum am traditionellen Fremdsprachenunterricht (z. B. Schlösser 2001). Alle methodischen Empfehlungen beginnen mit Konkreta, die überwiegend mit Hilfe von Bildern semantisiert werden sollen. Dass Vorschüler besser mit Objekten lernen, dass sie kategorisieren und von situativen Kontexten abstrahieren lernen müssen, dass sie Wörter funktional gebrauchen lernen sollten, dass sie Beziehungen zu verwandten Wörtern (Stichwort: Wortfamilie, Wortfeld, Wortbildungsmuster) herstellen und ein Gespür dafür entwickeln müssen, welche vorausgehenden oder nachfolgenden Wörter häufig mit dem neuen Wort vorkommen sowie über stilistische (z. B. informell oder formell) und emotionale Mitbedeutungen (Konnotationen) informiert sein sollten, wird nirgends thematisiert. Auch dass Interferenzen aus der Erstsprache auftreten oder dass Wortbedeutungen unklar sein können, wird nicht in den Blick genommen, ebenso wenig wie die Möglichkeit, begriffliche Grenzen zu schärfen, z. B. durch eine Kontrastierungen mit Objekten, die nicht zu einer Kategorie gehören oder durch eine Kontrastierung mit erstsprachlichen Bedeutungen (vgl. dagegen Apeltauer 2005: 64 ff.). Meist wird davon ausgegangen, dass es genügt, wenn bestimmte Themen (z. B. Verkehrserziehung) behandelt werden,

\footnotetext{
32 Zieht man tatsächlich einen Hut auf? Und was ist mit der Brille, die hier unter Kleidungsstücke subsumiert wird? Zieht man die auch an oder auf? Schließlich: Wer kommentiert sein „Tun“ in dieser Weise? Wird Sprache hier funktional gebraucht?

${ }_{33}$ Unklar ist bei den meisten Materialien, nach welchen Kriterien der jeweilige Wortschatz ausgewählt wurde.
} 
zu denen Lehrkräfte Wörter spontan gesammelt haben ${ }^{34}$. Dass es Untersuchungen zum Wortschatz monolingualer Schulanfänger gibt, scheint unbekannt (vgl. z. B. Augst 1984 oder Pregel / Rickheit 1987; vergleichbare Nachschlagewerke für $\mathrm{KmM}$ sind ein Desiderat der Forschung). Sprachförderunterricht sollte solche Orientierungsmöglichkeiten nutzen, auch wenn das nur vorläufige Hilfen sein können und entsprechende Nachschlagewerke für KmM erst noch entwickelt werden müssen. Vor allem aber sollten Erzieherinnen und Lehrkräfte Material modifizieren, so dass aktuelle Erlebnisse und Erfahrungen von Kindern einbezogen werden können, dass nicht (nur) über irgend ein vorgegebenes Bild (vgl. »erzähl mir was«) »erzählt« wird, sondern über Geschehnisse, die ein Kind bewegt haben, über die Informationen z. B. von Eltern oder von anderen Erzieherinnen bereits vorliegen, so dass darüber mit dem Kind auch eine „authentische Konversation" stattfinden kann.

\section{Anbahnen von Literalität $\mathrm{t}^{35}$}

Wir wissen heute, dass Kinder schon in einem sehr frühen Alter Interesse an Literalität entwickeln. Sie scheinen sogar in der Lage, sich Fertigkeiten spontan anzueignen, wenn sie entsprechende Vorbilder haben (vgl. dazu Scollon/Scollon 1981). Kann dieses Interesse auch für den Zweitspracherwerb genutzt werden?

Zunehmend mehr Kindertageseinrichtungen bieten im Rahmen von Fördermaßnahmen neben einem Training phonologischer Bewusstheit, neben Wortschatz- und Grammatikarbeit auch Aktivitäten an, die als Vorbereitung für den Schriftspracherwerb gedacht sind. Zwei Ansätze lassen sich dabei unterscheiden: Ein fertigkeitsorientierter Ansatz und ein Ansatz, der ein ganzheitliches Vorgehen bevorzugt.

Vertreter des fertigkeitsorientierten Ansatzes gehen davon aus, dass zuerst Dekodierungsfähigkeiten fokussiert werden müssen. Dazu sollen die graphophonetischen Grundlagen von Literalität erarbeitet werden. Es wird also die Zuordnung von Lauten zu Buchstaben vermittelt und diese werden dann zu Morphemen, Lexemen und deren Bedeutung in Beziehung gesetzt. Kurz: Literalitätsentwicklung wird als deduktiver Prozess organisiert von kleinsten zu immer größer werdenden Einheiten. Ein solches Vorgehen ist möglich, wenn alle Wörter, die zerlegt oder aus Einzellauten (bzw. Buchstaben) zusammengesetzt werden sollen, bereits vertraut und automatisiert sind. Mit anderen Worten: Sie eignen sich für monolinguale Kinder mit einem großen Wortschatz, sind für KmM mit einem kleinen Wortschatz ungeeignet.

\footnotetext{
34 So finden wir z. B. im Bericht über die Pilotphase der "Sprachintensivförde-rung« in SchleswigHolstein (Schuljahr 2002/2003) unter »Verkehrserziehung« Wörter wie Fußgängerweg, obwohl Fußweg kürzer und daher zweifellos leichter lernbar und für einen aktiven Gebrauch auch ausreichend wäre.

${ }^{35}$ Unter Literalität versteht man mehr als schriftsprachliche Fertigkeiten. Dazu gehört die Fähigkeit zum Umgang mit unterschiedlichen Textsorten, mit Tabelle oder Fahrplänen ebenso wie z. B. mit statistischen Grafiken oder mit animierten Texten im Internet. Kurz: Literalität ist die Fähigkeit, sich die für eine Zielsetzung relevanten Informationen durch die Erschließung unterschiedlichster Texte zu beschaffen.
} 
Im Unterschied dazu wird beim ganzheitlichen Ansatz (den i. d. R. Erzieherinnen bevorzugen) davon ausgegangen, dass Sprachenlernen ein kollaborativer Prozess der Bedeutungskonstruktion und des Bedeutungsaushandelns ist, in dessen Verlauf von einem Ganzen (z. B. einer Erzählung) auf induktivem Wege zu immer kleineren Teilen übergegangen wird. Durch interaktives Erzählen oder Vorlesen werden Bedeutungen mit Hilfe von Gestik und Mimik verständlich gemacht, werden Wörter erforderlichen Falles auch wiederholt oder paraphrasiert, werden zusätzlich Fragen zur Erzählung gestellt und so zum Mitdenken und zum Mitmachen angeregt (ausführlicher dazu Apeltauer 2003 sowie 2004 b). Eine Erzählung soll so mit möglichst vielen Sinnen erfassbar werden, durch

- langsames, betontes Erzählen oder Vorlesen unter Einsatz von Mimik und Gestik;

- Fragen zum Mitdenken und Mitmachen (z. B. Wie könnte es weiter gehen? Was könnte er/sie jetzt tun?);

- das Visualisieren von Inhalten mit Hilfe von Gegenständen oder Bildern

- ein gestalterisches Reproduzieren (z. B. Malen von „Helden“ oder Szenen, verkleiden und spontanes nachspielen von Szenen);

- kollektive Diktate (Kinder erzählen gemeinsam eine Geschichte weiter oder berichten über Erlebnisse z. B. auf einem Spaziergang; eine Betreuungsperson schreibt auf, was gesagt wird und liest es anschließend vor. Das »Diktat« wird in Form eines Plakats im Raum aufgehängt, so dass es immer wieder in den Morgenkreis einbezogen werden kann);

- das Inszenieren einer Geschichte, die oft gehört oder vorgelesen wurde z. B. für Eltern (evtl. dokumentiert durch Fotos oder Videoaufnahmen);

Dieser Ansatz wird inzwischen erfolgreich zur Vermittlung fremdsprachlicher Kenntnisse genutzt (vgl. dazu z. B. das Förderprogramm Hocus \& Lotus von Taeschner u. a., dessen Effektivität wissenschaftlich evaluiert wurde oder das Kieler Modell, das ebenfalls wissenschaftlich evaluiert wurde (vgl. Apeltauer 2004b).

Nun kann man fragen, ob ein solcher Ansatz nicht besser in der Erstsprache Verwendung finden sollte, wenn die Erstsprache die starke Sprache ist. (In einer niederländischen Untersuchung wurde festgestellt, dass selbst nach zwei Schuljahren bei $\mathrm{KmM}$ die Erstsprache immer noch die dominante Sprache war (vgl. Narain/Verhoeven 1993). Sowohl das Hörverstehen als auch die Erzählkompetenz sind ja gewöhnlich in einer starken Erstsprache weiter entwickelt als in einer schwachen Zweitsprache. Es empfiehlt sich daher, vor oder parallel zu Fördermaßnahmen Angebote zur Elternfortbildungen zu machen, um damit eine Aktivierung von Eltern von $\mathrm{KmM}$ zu erreichen (vgl. dazu Apeltauer 2006 a und die dort angeführten Beispiele). Denn wenn Geschichten vorlaufend zu Hause erzählt werden, kann Erzähltes in der Zweitsprache leichter erschlossen werden, was Rehbein in einem eindrucksvollen Versuch nachgewiesen hat (vgl. Rehbein 1987). 
Es kann für KmM durchaus motivierend sein, wenn ihnen zu Hause zuerst z. B. eine türkische oder polnische oder russische usw. Version von Aschenputtel erzählt wird und anschließend im Kindergarten die deutsche. Solche Varianten lassen sich auch kombinieren oder collagieren, so dass daraus neue und (auch für deutsche Kinder und Erzieherinnen/Lehrkräfte) interessante und anregende Erzählungen entstehen können (ein anregendes Buch zu dieser Thematik ist Rodari 1973, ein guter Videofilm von der Stadt Frankfurt am Main ist »Erzähl mir 'ne Geschichte«).

Wenn wir davon ausgehen, dass das Übermitteln von Gedanken und Gefühlen Kindern ein Bedürfnis ist, dann muss auch beim Anbahnen von Literalität für die Kinder durchschaubares und sinnvolles Handeln im Vordergrund stehen. Eine besondere Herausforderung besteht dabei darin zu lernen, wie man eigene Gedanken und Gefühle in der fremden Sprache differenziert ausdrücken kann und wie man sich mit Hilfe von Wörtern und Texten die Welt erschließen kann. In diesem Sinne ist Anbahnen von Literalität immer auch eine Vorbereitung auf die Schule und auf das Lesen lernen, weil im Rahmen solcher Maßnahmen auch Weltwissen gesammelt, geordnet und verfügbar gemacht wird, verfügbar insbesondere über die Zweitsprache.

Es gibt weitere Gründe, weshalb das Anbahnen von Literalität (in diesem Sinne) zur Aneignung der Zweitsprache Deutsch genutzt werden sollte. Wir gebrauchen gesprochene und geschriebene Sprache in ganz unterschiedlichen Situationen. Wenn wir beispielsweise eine Geschichte erzählen, werden wir andere sprachliche Mittel verwenden als wenn wir sie vorlesen. Während beim mündlichen Erzählen im Deutschen z. B. Endungen häufig verschluckt werden, werden dieselben Endungen beim Vorlesen i. d. R. artikuliert. Und wenn Rollen getauscht werden, d. h. also Kinder eine Geschichte (die sie schon auswendig gelernt haben) erzählen, so bemühen sie sich um eine korrektere Aussprache ${ }^{36}$. Insbesondere dann, wenn Geschichten oder Erlebnisse von Kindern in Form von kollektiven Diktaten verschriftlicht werden, ergeben sich ganz natürliche Möglichkeiten zum Fokussieren sprachlicher Formen: zum Erläutern von Endungen, zum Abwägen von Wörtern, zur Bewusstmachung syntaktischer Formen. Wenn solche formalen Aspekte in funktionalen Zusammenhängen thematisiert werden, z. B. wenn ein Kind einen Brief an seine Eltern diktieren will, oder wenn es eine Beobachtung, die es gemacht hat, schriftlich festgehalten haben möchte (vgl. das Schneckenbuch in Apeltauer 2006a: 35), verfügt es i. d. R. über eine große Aufnahmebereitschaft und eine höhere Konzentrationsspanne als wenn Betreuungspersonen Rahmenthemen vorgeben ${ }^{37}$. Vor allem aber sollte man wissen, dass beiläufige (implizite)

\footnotetext{
${ }^{36} \mathrm{Im}$ Rahmen des Kieler Modells haben wir beobachtet, dass Kinder, wenn sie so tun als ob sie vorlesen würden, langsamer, betonter und korrekter sprechen als in sonstigen Interaktionen.

${ }^{37}$ Kleinprojekte können durch Betreuungspersonen initiiert werden (z. B. wir machen Eis/einen Obstsalat). Sie können aber auch aus Ideen oder Interessen der Kinder entstehen. Wenn Betreuungspersonen Ideen der Kinder aufgreifen und mit den Kindern zusammen weiterentwickeln (was man freilich nicht im Rahmen von einer Förderstunde pro Tag machen kann), entsteht etwas
} 
Erläuterungen oder Korrekturen meist wirksamer sind als explizite Korrekturen oder Erklärungen und dass durch selbst initiiertes und selbst gesteuertes Lernen langfristiges Behalten und dauerhafte sprachliche Entwicklungen begünstigt werden (Doughty 2003: 270 f. verweist darauf, dass viele Untersuchungsergebnisse zeigen: Mit Hilfe grammatischer Instruktionen erzielt man meist nur kurzzeitige Effekte). Zudem wird später in der Schule eine zunehmend formellere Sprache gebraucht, weshalb Kinder auf diesen anderen Sprachgebrauch vorbereitet werden sollten (Cummins 1984 hat die Unterscheidung BICS (basic interpersonal communicative skills) und CALP (cognitive academic language proficiency) geprägt. Mit BICS wird auf die informelle Umgangssprache verwiesen, mit CALP auf eine eher formelle Sprache, insbesondere auf Schriftsprache).

Zusammenfassend lassen sich die Unterschiede zwischen gesprochener und geschriebener Sprache folgendermaßen darstellen:

Merkmale gesprochener Sprache

\section{Merkmale geschriebener Sprache}

\begin{tabular}{|c|c|}
\hline $\begin{array}{l}\text { In Interaktion eingebettet, d. h. flüchtig und daher } \\
\rightarrow \text { schwerer wahrnehmbar, z. B. Endungen }\end{array}$ & $\begin{array}{l}\text { nicht unter Zeitdruck } \rightarrow \text { leichter wahrnehm- } \\
\text { bar }\end{array}$ \\
\hline $\begin{array}{l}\text { wegen Zeitdruck weniger strukturiert und geplant } \\
\rightarrow \text { Wiederholungen, Floskeln (du weißt schon } \\
\text { wie ich das meine ne ...) }\end{array}$ & $\begin{array}{l}\text { stärker durchdacht und sprachlich struktu- } \\
\text { riert, Wörter werden sorgfältig gewählt und } \\
\text { „gewogen“, Verzicht auf Floskeln }\end{array}$ \\
\hline $\begin{array}{l}\text { Situation/Kontext wird als Verstehens-hilfe ge- } \\
\text { nutzt (durch Mimik und Gestik, Verweiswörter wie } \\
\text { da, genau hier ...) eher implizites Formulieren }\end{array}$ & $\begin{array}{l}\text { Verweiswörter und Kontexthilfen entfallen } \rightarrow \\
\text { expliziteres Formulieren, u. U. auch abstrak- } \\
\text { tere Sprache }\end{array}$ \\
\hline $\begin{array}{l}\text { nutzt Elemente wie Tonhöhenverlauf, Lautstärke, } \\
\text { Sprechgeschwindigkeit, Rhythmus, } \\
\text { Akzentgebung }\end{array}$ & $\begin{array}{l}\text { nutzt grafische Gestaltungsmöglichkeiten } \\
\text { wie Normal- und Fettdruck, Überschriften, } \\
\text { Absätze, Fußnoten etc. }\end{array}$ \\
\hline $\begin{array}{l}\text { eher emotional aufgeladen durch Sprechweise } \\
\text { (bzw. Prosodie) }\end{array}$ & $\begin{array}{l}\text { eher sachlich, auf Fakten bezogen (bzw. } \\
\text { Emotionen werden anders evoziert) }\end{array}$ \\
\hline $\begin{array}{l}\text { Korrekturmöglichkeiten während des Formulie- } \\
\text { rens sind wahrnehmbar }\end{array}$ & $\begin{array}{l}\text { scheinbar perfekte Sprache, weil Korrektu- } \\
\text { ren in Endfassung nicht sichtbar sind }\end{array}$ \\
\hline
\end{tabular}

Die Übergänge zwischen diesen beiden Formen sind bekanntlich fließend. Es gibt z. B. ein formelles Sprechen (z. B. Nachrichtensprecher) und ein informelles Schreiben (z. B. SMS, Liebesbriefe etc.). Für den Zweitspracherwerb bedeutet das, dass Themen in unterschiedlicher Weise behandelt werden können. Geschichten können z. B. (mit oder ohne Requisiten) erzählt werden und sie können (mit oder ohne Requisiten) vorgelesen werden. Anhand von ein paar Requisiten könnte eine Geschichte aber auch (gemeinsam mit den Kindern) entwickelt oder weiterentwi-

qualitativ Neues. Kinder wollen dann auch korrekt formulieren, sind also an formalen Hilfen interessiert und werden auf diese Weise mehr lernen, als wenn ihnen - wie im traditionellen Sprachunterricht - Themen und Formulierungen vorgegeben werden bzw. sie »zum Nachsprechen abgerichtet werden«. 
ckelt werden. Und am Ende können eigene Versionen vorgetragen werden (Die Kinder der Kieler Modellgruppe haben ihre Bilderbücher übrigens mit "gucken«, »lesen« aber auch mit "gemütlich machen« und »lernen« assoziiert (vgl. Apeltauer 2006 b). Und sie hatten CDs (Hörbücher), die sie nach Bedarf nutzen konnten, d. h. also »klingende Versionen« dieser Texte). Genau darin besteht der qualitative Unterschied zur oben beschriebenen Wortschatz- und Grammatikarbeit, die nicht dadurch attraktiver wird, dass nebenbei auch Lieder gesungen werden. Dort wird Sprache überwiegend in ihrer beschreibenden Funktion (z. B. Was ist das? Das ist Bus. Und was ist das? Das ist rote Rose) verwendet. Eine solche Sprache macht kaum Appetit auf mehr. Lehrkräfte in Sprachfördermaßnahmen sagen selbst: »Eine halbe Stunde Sprachförderung reicht komplett«. Dass Sprache auch klingen kann, dass Inhalte anregen können zum Nachdenken, zum Nachahmen und Weiterlernen, dass Sprache uns bei der Strukturierung unserer Wahrnehmung und Erfahrung helfen kann, dass wir Beziehungen mit ihrer Hilfe vertiefen können oder dass Geschichten geliebt werden, das alles wissen wir. Traditionelle und auch neuere Kinderliteratur eröffnet dabei viele Möglichkeiten ${ }^{38}$. Dennoch wird in vielen Fördermaßnahmen offenbar zuerst einmal trockenes Brot (Beschreibungssprache) verteilt, werden Lerner zum intentionalen Lernen gezwungen, werden Möglichkeiten des inzidentellen Lernens beim Zuhören oder beim Tun (d. h. instrumenteller, interaktiver oder imaginativer Gebrauch von Sprache) viel zu wenig genutzt. Dadurch kann sich bei KmM der Eindruck verstärken, dass die deutsche Sprache eine schwere Sprache ist.

Betreuungspersonen sollten lernen, wie solche antisuggestiven Eindrücke ${ }^{39}$ vermieden werden können. Und sie sollten lernen, dass explizites Korrigieren und Disziplinieren bei Vorschulkindern häufig kontraproduktiv ist. Vorschulkinder sollten zum Sprechen ermutigt werden. Sie sollten gute Sprachvorbilder haben und es sollte ihnen interessantes und reiches Sprachmaterial (u. U. auch über CDs und (Lehr-)Filme) zugänglich gemacht werden, damit sie sich anregen lassen können. Vor allem aber sollten sie sich die fremde Sprache nicht nur nach Vorgaben aneignen dürfen, sondern sie sich durch eigenen Gebrauch erschließen, in der Kommunikation mit Betreuungspersonen und anderen Kindern sowie im Rahmen von selbst initiierten Kleinprojekten.

\footnotetext{
38 Über Wörter im Kontext und über funktionalen Zusammenhängen lassen sich Bedeutungen von Wörtern und Beziehungen zwischen Wörtern leichter erschließen als über isoliertes Wortmaterial. ${ }^{39}$ Der rumänische Arzt Lozanov hat in seiner Fremdsprachenlehrmethode Suggestopädie auf Lernhemmungen aufmerksam gemacht, die durch antisuggestive Maßnahmen entstehen. Um solche Hemmungen abzubauen, setzt er Barockmusik als Hintergrundsmusik ein, damit sich (erwachsene) Lerner entspannen und beiläufig mehr Wörter aufnehmen und speichern können. Dazu werden Dialoge (nicht Einzelwörter!), die zuvor praktisch (mit Gestik und Mimik) geübt wurden, in einem freundlichen, halblauten Ton (suggestiv) vorgetragen.
} 


\section{$7 \quad$ Zusammenfassung und Ausblick}

Ausgangspunkt unserer Darstellung war der Zusammenhang zwischen Sprachund Kognitionsentwicklung und die Feststellung, dass im Verlaufe des Erstspracherwerbs neuronale Verarbeitungsroutinen entwickelt werden, die zur Grundlage spätere Lernprozesse - also auch von Aneignungsprozessen im Bereich der Zweitsprache - werden. Weil die Erstsprache beim Gebrauch der Zweitsprache immer aktiviert wird, muss sie auch als »Denkwerkzeug« zugelassen und weiter gepflegt werden, zumindest so lange, bis die Zweitsprache diese Funktion der Erstsprache übernehmen kann.

Es wurde auf die während des ersten Lebensjahres entwickelten sprachlichen Dekodierungsstrategie hingewiesen und auf den Wortschatzumfang, mit dem deut-sche (monolinguale) Kinder in die Schule kommen. Untersuchungsergebnisse zeigen, dass die Kluft im Wortschatzbereich zwischen einsprachig und zweisprachig aufwachsenden Kindern während der ersten Schuljahre wächst, weshalb die Vorstellung, dass man mit sprachlicher Frühförderung die Lösung aller Probleme in der Hand habe, als unrealistisch verworfen wurde.

Wir haben zu zeigen versucht, wie die Bedeutung von Wörtern gelernt wird und was alles zum Wissen über ein Wort gehört. Weil Prozesse, die dabei durchlaufen werden, eng mit der Erstsprache verbunden sind, gehören der Entwicklungsstand der Erstsprache und bereits erarbeitetes Weltwissen zu den Sprachlernvoraussetzungen für den Zweitspracherwerb. Und darum können sich Anregungen im Bereich der Erstsprache positiv auf den Zweitspracherwerb und auf eine Sensibilisierung für Sprachlernprozesse auswirken. Auch die Aufnahme und Verarbeitung von neuem Wissen und von Erfahrungen erfolgt in der dominanten Sprache (i. d. R. die Erstsprache) bzw. - wenn die Zweitsprache dafür gebraucht wird - indirekt über die dominante Sprache, weshalb eine Pflege der Erstsprache weiterhin wichtig ist. Darum sollten Eltern von $\mathrm{KmM}$ - vorlaufend oder parallel zu Förderangeboten für ihre Kinder - Fortbildungsangebote gemacht werden, damit sie lernen, wie sie die sprachliche Entwicklung ihre Kinder unterstützen können.

Grundlage für Frühförderangebote sollten Sprachstandsbeurteilungen sein. Aufgrund der unzureichenden Forschungslage und wegen der unterschiedlichen Kompetenzen, mit denen „Diagnoseverfahren“ konzipiert wurden, sind gegenwärtig nur vorläufige Globalaussagen (bzw. im Falle von HAVAS oder dem Verfahren von Grießhaber) nur differenziertere Aussagen über wissenschaftlich abgesicherte Teilbereiche möglich. Die Zweitsprachentwicklung von KmM, insbesondere von Vorschulkindern, ist zweifellos ein Desiderat der Forschung. Ohne eine entsprechende Grundlagenforschung werden Verfahren zur Sprachstandsbeurteilung weder sinnvoll weiterentwickelt werden können, noch Fördermaßnahmen sich optimieren lassen.

Drei Schwerpunkte werden gegenwärtig im Rahmen von Fördermaßnahmen gesetzt: 1. Das Training der phonologischen Bewusstheit, 2. Wortschatz und Grammatikarbeit und 3. das Anbahnen von Literalität. Phonologische Analysefä- 
higkeiten bilden sich zunächst in der starken Sprache (bei KmM i. d. R. die Erstsprache) aus. KmM sollten also ein solches Training in ihrer Erstsprache durchlaufen können oder über entsprechende Verfahren (z. B. das Lernen von Abzählreime oder Kinderlieder) Anregungen in ihrer Erstsprache erhalten. Ein Transfer dieser Fertigkeiten in die Zweitsprache ist möglich. Da das Training der "phonologischen Bewusstheit« für monolinguale (deutsche) Kindern entwickelt und erprobt wurde, wird ein Wortschatz vorausgesetzt, der dem Wortschatz deutscher Kinder im fünften Lebensjahr entspricht, ein Wortschatz, über den $60 \%$ der $\mathrm{KmM}$ in diesem Alter i. d. R. nicht verfügen. Aus diesem Grunde halten wir ein Training phonologischer Bewusstheit (im engeren Sinne) für dies KmM für problematisch. Nur KmM, die über gute bzw. sehr gute Deutschkenntnisse verfügen, werden von einem solchen Training profitieren.

Bei der Wortschatzarbeit wurde darauf verwiesen, dass Erzieherinnen einen ganzheitlichen Zugang bevorzugen, d. h. es wird viel erzählt, auch vorgelesen, um Wörter zu semantisieren. Zudem werden Kreis- und Bewegungsspiele gemacht und Lieder gesungen, um neue Wörter zu wiederholen und $\mathrm{zu}$ verankern und Sprechfreude $\mathrm{zu}$ entwickeln. Eine solche lebendige, funktionale und kontextualisierende Vermittlung von neuen Wörtern ist in jedem Fall dem isolierten vermitteln einzelner Wörter mit Hilfe von Bildkarten (wie das z. T. durch Fördermaterialien angeregt wird) vorzuziehen. Realobjekte sind immer besser zu »begreifen«.

Problematisch ist die Vorstellung von Lehrkräften, dass parallel zur Einführung eines neuen Wortes auch gleich grammatisches Wissen über das Wort (z. B. Flexionsformen beim Verb) vermittelt werden können. Hier besteht Fortbildungsbedarf. Es sollte Wissen über Wortschatzentwicklung und Bedeutungsdifferenzierungen, aber auch Wissen über Stufen der Lernersprachentwicklung (in den Bereichen von Syntax und Morphologie) vermittelt werden, so dass Fördermaßnahmen angemessener konzipiert werden können. Im Hinblick auf Schule und die dort verwendete formalere Sprache (vgl. CALP) wird empfohlen, bereits im Vorschulbereich mit dem Anbahnen von Literalität zu beginnen, um so ein Bewußtsein für Sprache und insbesondere formale Aspekte von Sprache (Endungen, Wortbildungsmöglichkeiten, Satzmuster, Kohäsion etc.) zu schaffen, so dass auch schlussfolgerndes Denken und abstraktere Vorstellungen entwickelt werden können. Dabei sollten sowohl rezeptive als auch produktive Aspekte in funktionalen Zusammenhängen fokussiert werden und - wo immer möglich - auch Kontrastierungen mit der/den Erstsprache/n stattfinden, um Literalität (wenigstens in Ansätzen) in eine Biliteralität zu überführen, so dass $\mathrm{KmM}$ ihr kognitives Potential besser nutzen können. Gelernt werden sollte von $\mathrm{KmM}$ auch, wie man Informationen (z. B. im Rahmen von Kleinprojekten) sammelt und daraus Texte (bzw. Bücher) produziert, weil sie sich im Rahmen solcher Tätigkeiten Strategien zur Informationsbeschaffung und zur Strukturierung von Informationen aneignen und so gleichzeitig ein erfolgreiches Durchlaufen der Schule vorbereitet wird. 


\section{Literatur}

Adybasova, Anastasia; Altmüller, Sonja; Kuyumcu, Reyhan: Sprachentwicklung beobachten, erfassen, beschreiben, Eine Analyse des Beobachtungsverfahrens SISMIK. In: Apeltauer, Ernst (Hrsg.): Flensburger Papiere zur Mehrsprachigkeit und Kulturenvielfalt im Unterricht. Flensburg, Heft 44/45 (2006).

Aitchison, Jean: Words in the Mind, an introduction to the mental lexicon. Oxford: Blackwell, 1989. [zitiert nach der 2. Auflage 1994].

Apeltauer, Ernst: Literalität und Spracherwerb. Flensburg, 2003, (= Flensburger Papiere zur Mehrsprachigkeit und Kulturenvielfalt im Unterricht, Heft 32.

Apeltauer, Ernst: Sprachlerndispositionen - eine Alternative zu Sprachtests im Vorschulalter? In: Frühes Deutsch Heft 2 (2004a), 48-55.

Apeltauer, Ernst: Sprachliche Frühförderung von zweisprachig aufwachenden türkischen Kindern im Vorschulbereich. Flensburg, 2004b (= Sonderheft 1 der Flensburger Papiere zur Mehrsprachigkeit und Kulturenvielfalt im Unterricht).

Apeltauer Ernst: Wortbedeutungsentwicklung bei zweisprachig aufwachsenden türkischen Vorschulkindern. In: Gogolin, Ingrid; Krüger-Patrak, Marianne; Kuhs, Katharina, Neumann, Ursula;; Wittek, Fritz (Hrsg.): Migration und sprachliche Bildung. Münster: Waxmann, 2005, 63-81.

Apeltauer, Ernst: Kooperation mit zugewanderten Eltern. Flensburg, 2006a (= Flensburger Papiere zur Mehrsprachigkeit und Kulturenvielfalt im Unterricht, Heft 40/41).

Apeltauer Ernst: Bedeutungsentwicklung bei zweisprachig aufwachsenden türkischen Vorschulkindern. In: Ahrenholz, Bernt; Apeltauer, Ernst (Hrsg.):

Zweitspracherwerb und curriculare Dimensionen, Empirische Untersuchungen zum Deutschlernen in Kindergarten und Grundschule. Tübingen: Stauffenburg, 2006b, 31-54.

Apeltauer Ernst 2006c: Förderprogramme, Modellvorstellungen und empirische

Befunde. Zur Wortschatz- und Bedeutungsentwicklung bei türkischen Vorschulkindern. In: Ahrenholz, Bernt (Hrsg.): Kinder mit

Migrationshintergrund - Spracherwerb und Fördermöglichkeiten. Freiburg: Fillibach, 11-33.

Augst, Gerhard 1984: Kinderwort. Der aktive Kinderwortschatz (kurz vor der Einschulung) nach Sachgebieten geordnet mit einem alphabetischen Register. (= Augst, Gerhard; Beier, Rudolf (Hrsg.): Theorie und Vermittlung der Sprache, Band 1), Frankfurt am Main: Lang, 1984. 
Benholz, Claudia; Lipkowski, Eva; Schroeder, Christoph: Bericht zur Sprachstandserhebung bei Grundschülerinnen und Grundschülern mit Migrationshintergrund zum Zeitpunkt der Einschulung 1999. Universität Essen, 2000 (Ms).

Bericht zur Sprachstandserhebung bei Grundschülerinnen und Grundschülern mit Migrationshintergrund zum Zeitpunkt der Einschulung. Essen 2000 [Projekt der RAA Essen].

Bertschi-Kaufmann, Andrea: Lesen und Schreiben in einer Medienumgebung, Die literalen Aktivitäten von Primarschulkindern. Aargau: Zentrum Lesen FHA, 2003.

Boehrer, Helga: Deutsch mit Spaß und Spiel, Basiswissen und Praxismaterialien DaZ. Stuttgart: Klett, 2004

Boyd, Stephen; Arvidson, G.: The acquisition of literacy by immigrant children in Sweden. In: Durgunoglu, Aydin.Y.; Verhoeven, Ludo (eds.) 1998: Literacy development in a multilingual context. Mahawh, N.J.: Erlbaum, 1998, 203-224.

Brockmeier, Jens: Literales Bewußtsein, Schriftlichkeit und das Verhältnis von Sprache und Kultur. München: Fink, 1998.

Brüggelmann, Hans: Fördern: Nur das Reparieren einer unzulänglichen Technik? In: Grundschule, 35 Jahrgang, Heft 10 (2003), 54-57.

Christiansen, Christiane: WUPPIs Abenteuer-Reise durch die phonologische Bewusstheit. Oberursel: Finken, 2005.

Christophe, Anne; Guasti, Teresa; Nespor, Marina; Dupoux, Emmanuel; Van Ooyen, Brit 1997: Reflections on Phonological Bootstrapping: Its Role for lexical and Syntactic Acqusition. In: Language and cognitive Processes, (1997), 12, 5/6, 1997, 58-612.

Cutler, Anne: Listening to a second language through the ears of a first. In: Interpreting 5 (2001), 1-18.

de Groot, Annette M. B.; Danneburg, A.; Van Hell, J. G.: Forward and backward translation. In: Journal of Memory and Language 33, (1994), 600-629.

Deutsches Jungendinstitut (Hrsg.): Sprachförderung im Vor- und Grundschulalter, Konzepte und Methoden für den außerschulischen Bereich; München: Verlag DJI, 2002.

Di Pietro, Robert J.: Strategic Interaction, Learning Languages through Scenarios. Cambridge: Cambridge University Press, 1987.

Doughty, Catherine J.: Instructed SLA: Constraints, Compensations, and Enhancement. In: Doughty, Catherine J.; Long, Michael H. (eds.): The Handbook of Second Language Acquisition; Oxford: Blackwell, 2003, 256-310. 
Driessen, Gert: De taalvaardigkeid Nederlands van allochtone en autochtone leerlingen. De ontwikkeling in het basisononderwijs in kaart gebracht. Gramma/T'T'T Tijdschrift voor Taalwetenschap 5 (1996), 31-40.

Drissen, Geert; Van der Slik, Frans; De Bot, Kees: Home Language and Language Proficiency: A Large-scale Longitudinal Study in Dutch Primary Schools. In: Journal of Multilingual and Multicultural Development Vol. 23, 3 (2002), 175-194.

Droop, Mienke; Verhoeven, Ludo: Language Proficiency and Reading Ability in Firstand Second-Language Learners. In: Readign Research Quarterly Vol. 38 (2003).

Ehri, Linnea; Nunes, Simone R.; Willows, Dale M.; Valeska-Schuster, Barbara; Yaghoub-Zadeh, Zohreh; Shanahan, Timothy: Phonemic awareness instruction helps children learn to read: Evidence from the National Reading Panel's meta analysis. In: Reading Research Quarterly 36/3 (2001), 250-287.

Einsiedler, Wolfgang: Der Einfluß verschiedener Unterrichtsmethoden auf die phonologische Bewußtheit sowie auf Lese- und Rechtschreibleistungen im 1. Schuljahr. Bericht Nr. 93 IfG (Universität Erlangen-Nürnberg 2000).

Erzähl' mir 'ne Geschichte, Praktische Beispiele für die Sprachförderung im Kindergarten hrg. von der Stadt Frankfurt am Main (Dezernat für Schule, Bildung und Frauen - Stadtschulamt o. J.

Extra, Guus.; Verhoeven, Ludo (eds.): Community languages in the Netherland. European Studies on Multilingualism; Amsterdam: Swets\&Zeitlinger Volume 3, 1993.

Gräsel, Cornelia; Gutenberg, Norbert; Pietzsch, Thomas; Schmidt, Elke: Zwischenbericht zum Forschungsprojekt »Hören - Lauschen - Lernen: Umsetzung und Evaluation des Würzburger Trainingsprogramms zur Vorbereitung auf den Erwerb der Schriftsprache«. Saarbrücken, Universität des Saarlandes, 2004.

Grießhaber, Wilhelm: Testen nichtdeutschsprachiger Kinder bei der Einschulung mit dem Verfahren der Profilanalyse - Konzeption und praktische Erfahrung. In: Ahrenholz, Bernt; Apeltauer, Ernst (Hrsg.): Zweitspracherwerb und curriculare Dimensionen. Empirische Untersuchungen zum Deutschlernen in Kindergarten und Grundschule; Tübingen: Stauffenburg, 2006, 73-90 (Forum Sprachlehrforschung Band 6).

Grimm, Hannelore (unter Mitarbeit von Mareen Aktaş und S. Frevert): SETK 3-5. Sprachentwicklungstest für drei- bis fünfjährige Kinder. Diagnose von Sprachverarbeitungsfähigkeiten und auditiven Gedächtnisleistungen. Göttingen: Hogrefe, 2001. 
Grimm, Hannelore. (unter Mitarbeit von Mareen Aktaş und U. Kießig): SSV. Sprachscreening für das Vorschulalter. Kurzform des SETK 3-5. Göttingen: Hogrefe. 2003a..

Grimm, Hannelore: Wie viele Vorschulkinder brauchen tatsächlich eine Sprachförderung? - Bedarfsanalyse am Beispiel der Stadt Bielefeld (Pressemitteilung der Universität Bielefeld Nr. 163/2003 vom 30.09.2003), 2003b.

Hamburger Erhebung zum Sprachstand türkisch-deutscher Schulanfänger des Schuljahres 1999/2000. Bericht über die Erhebung mündlicher Sprachkenntnisse im Sommer 1999; Universität Landau, Institut für Interkulturelle Bildung 2000 (Ms).

Heath, Shirley B. 1986: What no bedtime story means. Narrative skills at home and school. In: Schiefelin, Bamla B.; Ochs, Elena (eds.): Language socialization across cultures. Cambridge: Cambridge University Press, 1986, 97-124.

Herrmann, Christoph; Fiebach, Christian: Gehirn und Sprache. Frankfurt am Main: Fischer, 2004.

Hobusch, Anna; Lutz, Nevin; Wiest, Uwe: Sprachstandsüberprüfung und Förderdiagnostik für Ausländer- und Aussiedlerkinder. Horneburg: Persen, 2002.

Hölscher, Petra; Angelsperger, Ulrike: Kenntnisse in Deutsch als Zweitsprache erfassen, Screening-Modell für Schulanfänger; Stuttgart: Klett, 2002 (= Bayerisches Screening.

Hölscher, Petra: Lernszenarien. In: Frühes Deutsch, 2. Jahrgang, Heft 5, (2005) 4-5.

Holler-Zittlau, Inge; Dux, Winfried; Berger, Roswitha: Marburger Sprachscreening für 4 bis 6-jährige Kinder (MSS). Horneburg: Persen, 2003.

Hopf, Diether: Zweisprachigkeit und Schulleistung bei Migrantenkindern. In: Zeitschrift für Pädagogik, 51, 2 (2005), 236-249.

Jeuk, Stefan: Sprachstandsmessung bei Kindern nichtdeutscher Herkunftssprache zum Zeitpunkt der Einschulung. In: Didaktik Deutsch 20, (2006), 52 - 69.

Kauschke, Christina: Früher Wortschatzerwerb im Deutschen: Eine empirische Studie zum Entwicklungsverlauf und zur Komposition des kindlichen Lexikons. In: Meibauer, Jörg; Rothweiler, Monika (Hrsg.): Das Lexikon im Spracherwerb. Tübingen: Francke, 1999, 128-157.

Kies-Himmel, Christiane: Aktiver Wortschatztest für 3 - 6 jährige Kinder (AWST). Weinheim: Beltz, 1979 [revidierte und aktualisierte Fassung AWST-R 2005].

Kim, K. H. S.: Distinct cortical areas associated with native and second languages. In: Nature 388 (1997), 171-174. 
Kroll, Judith; Sunderman, Gretchen: Cognitive Processes in Second Language Learners and Biliguals: The Development of Lexical and Conceptual

Representations. In: Doughty, Catherine J.; Long, Michael H. (eds.): The Handbook of Second Language Acquisition. Oxford: Blackwell, 2003, 104-129.

Kroll, Judith; Tokowicz, Natasha: Models of Bilingual Representation and Processing. In: Kroll, Judith F.; De Groot, Annette M. B. (eds.): Handbook of Bilingualism; Oxford: Uni Press, 2005, 531-553.

Kucer, Stephen B. 2005: Dimensions of Literacy, a Conceptual Base for Teaching Reading and Writing in School Settings. Mahwah /NJ: Erlbaum, 2005.

Küspert, Petra; Schneider, Wolfgang: Hören, lauschen, lernen, Sprachspiele für Kinder im Vorschulalter, Würzburger Trainingsprogramm zur Vorbereitung auf den Erwerb der Schriftsprache. Göttingen: Vandenhoek\&Ruprecht, 1999 [5. Auflage 2006].

Loos, Roger 2004: Praxisbuch Spracherwerb, Sprachförderung im Kindergarten. München: Don Bosco, 2004 [2 Bände]

Lütje-Klose, Birgit 2004: Zwischenbericht zum Forschungsprojekt »Prävention von Sprach- und Lernstörungen bei Kinder nichtdeutscher Herkunftssprache«. Niedersächsisches Kultusministerium, 2004.

Lütje-Klose, Birgit 2006: Sprachstandserhebungen und Sprachförderung im Vorschulalter. In: Fleischer, Th./Grewe, N./Jötten, B./Seifried, K./Sieland, B. (Hrsg.): Psychologie für die Schule. Stuttgart: Kohlhammer, 2006 [im Druck, zitiert nach dem Text im Internet]

Maier, Wolfgang: Deutsch lernen in Kindergarten und Grundschule, Grundlagen, Methoden und Spielideen zur Sprachförderung und Integration. München (Don Bosco, 1999 [zitiert nach der 4. Auflage 2003].

Maiworm-Jäckel, Monika: Sprachförderung in Tageseinrichtungen für Kinder, Das Beispiel der Stadt Wuppertal. In: Röhner, Charlotte (Hrsg.): Erziehungsziel Mehrsprachigkeit, Diagnose von Sprachentwicklung und Förderung von Deutsch als Zweitsprache. Weinheim: Juventa, 139-160.

Martschinke, Sabine; Kirschhock, Eva-Maria; Frank, Angela: Diagnose und Förderung im Schriftspracherwerb. Der Rundgang durch Hörhausen, Erhebungsverfahren zur phonologischen Bewußtheit. Donauwörth: Auer, 2001 [3. Auflage 2004].

Martschinke, Sabine; Kirschhock, Eva-Maria; Frank, Angela: Leichter lesen und schreiben lernen mit der Hexe Susi - Diagnose und Förderung im Schriftspracherwerb. Donauwörth: Auer, 2000 [3. Auflage 2003]. 
McKay, Sandra Lee: Weighning Educational Alternatives. In: McKay, Sandra Lee; Wong, Sau-ling Cynthia (eds.): Language Diversity: Problem or Resource? New York: Newbury, 1988 [zitiert nach Baker, Colin; Prys-Jones, Sylvia: Encyclopedia of Bilingualism and Bilingual Education. Clevedon: Multilingual Matters, 1998.

Moser, Urs: Lernvoraussetzungen in Schulklassen zu Beginn der 1.Klasse. In: Moser, Urs; Stamm, Margit; Hollenweger, Judith (Hrsg.): Für die Schule bereit? Lesen, Wortschatz und soziale Kompetenz beim Schuleintritt. Oberentfelden: Sauerländer, 2005, 167-187.

Moser, Urs; Berweger, Simone; Stamm, Margit: Lesekompetenzen bei Schuleintritt. In: Moser, Urs; Stamm, Margit; Hollenweger, Judith (Hrsg.): Für die Schule bereit? Lesen, Wortschatz, Mathematik und soziale Kompetenzen bei Schuleintritt. Oberentfelden: Sauerländer, 2005, 37-58.

Moser, Urs; Berweger, Simone; Stamm, Margit: Wortschatz bei Schuleintritt. In: Moser, Urs; Stamm, Margit; Hollenweger, Judith (Hrsg.): Für die Schule bereit? Lesen, Wortschatz, Mathematik und soziale Kompetenzen bei Schuleintritt. Oberentfelden: Sauerländer, 2005, 59-76.

Narain, G.; Verhoeven, Ludo: Ontwikkeling van tweetaligheid bij allochtone kleuters; Tilburg: uni press, 1994

Nation, I.S.P.: Teaching and Learning Vocabulary. In: Hinkel, Eli (ed.): Handbook of Research in Second Language Teaching and Learning; Mahwah /NJ: Erlbaum, 2005, 581-595.

Nagy, W. E.; Herman, P. A.: Breadth and Depth of Vocabulary Knowledge. In: McKeown, M.; Curtis, M. E. (eds.): The Nature of Vocabulary Acquisition. Hillsdale/New York: Lawrence Erlbaum, 9-35.

Ott, Irene; Stanscheva, Nadja: Hippo. Für Kinder mit Deutsch als Zweitsprache (1. Teil: Mündlicher Vorkurs für die Vorschule). Donauwörth:Auer, 2005.

Perani, Daniela; Paulesu, Eraldo; Sebastian-Galles, Nuria; Dupoux, Emmanuel; Dehaene, Stanislas; Bettinardi, Valentino; Cappa, Stefano F.; Fazio, Ferrucco; Mehler, Jacques: The bilingual brain: proficiency and age of acquisition of the second language. In: Brain 121 (1998), 1841-1852.

Piepho, Hans-Eberhard: Lerneraktivierung im Fremdsprachenunterricht. "Szenarien« in Theorie und Praxis. Hannover: Schroedel, 2003.

Pregel, Dietrich; Rickheit, Gert: Der Wortschatz im Grundschulalter. Häufigkeitswörterbuch zum verbalen, substantivischen und adjektivischen Wortgebrauch. Hildesheim: Olms, 1987. 
Rehbein, Jürgen: Diskurs und Verstehen. Zur Rolle der Muttersprache bei der Textverarbeitung in der Zweitsprache. In: Apeltauer, Ernst Hrsg.: Gesteuerter Zweitspracherwerb, Voraussetzungen und Konsequenzen für den Unterricht. Ismaning: Hueber, 1987, 113-172.

Reich, Hans H.: Hamburger Erhebung zum Sprachstand türkisch-deutscher Schulanfänger des Schuljahres 1999/2000. Bericht über die Erhebung mündlicher Sprachkenntnisse im Sommer 1999. Lande (MS).

Reich, Hans H.; Roth, Hans Joachim: Spracherwerb zweisprachig aufwachsender Kinder und Jugendlicher - ein Überblick über den Stand der nationalen und internationalen Forschung. Hamburg: Behörde für Bildung und Sport,2002.

Reich, Hans H.; Roth, Hans Joachim: HAVAS 5 - Hamburger Verfahren zur Analyse des Sprachstandes bei 5-jährigen. Hamburg: Behörde für Bildung und Sport, 2004.

Reich, Hans H: Forschungsstand und Desideratenaufweis zu Migrations-linguistik und Migrationspädagogik für die Zwecke des »Anforderungsrahmens«. In: Ehlich: Anforderungen an Verfahren der regelmäßigen Sprachstandsfeststellung als Grundlage für die frühe und individuelle Förderung von Kindern mit und ohne Migrationshintergrund. Bonn: BMBF, 2005 (= Bildungsreform Band 11), 121-169.

Rickheit, Gert; Müller, Horst M.: Neurokognition der Sprache; Tübingen: Stauffenburg, 2002 (= Neurokognition Band 1).

Rodari, Gianni: Grammatik der Phantasie, Die Kunst Geschichten zu erfinden. Leipzig: Reclam, 1973.

Rodriguez, Richard: Hunger of memory, the education of Richard Rodriguez, an autobiography; New York, Toronto: Bantam, 1982.

Rösch, Heidi: Handreichungen für Lehrerinnen und Lehrer an Berliner Schulen herausgegeben von der Senatsverwaltung für Schule, Jugend und Sport; Berlin, 2001.

Rosten, Leo: Jiddisch, Eine kleine Enzyklopädie; München: dtv, 2002.

Rucys, Dagmar: Erzähl mir was! Lernspiele zur Sprachförderung; Oberursel: Finken, 2004.

Ruoff, Arno: Häufigkeitswörterbuch gesprochener Sprache. Idiomatica Band 8. Tübingen: Niemeyer, 1981 [2. Auflage 1990].

Saville, Muriel; Troike, Rudolph C.: A Handbook of Bilingual Education; Washington D.C.: TESOL, 1971.

Schlösser, Elke: Wir verstehen uns gut; Münster: Ökotopia, 2001. 
Schneider, Wolfgang; Stefanek, Jan Dotzler, Hans 1997: Erwerb des Lesens und Rechtschreibens. Ergebnisse aus dem Scholastikprojekt. In: Helmke, Andreas; Weinert, F. (Hrsg.): Entwicklung im Grundschulalter; Weinheim: Beltz, 1997.

Schneider, Wolfgang; Roth, E.; Ennemoser, Marco: Training phonological Skills and Letter Knowledge in Children at Risk for Dyslexia: A Comparison of Three Kindergarten Intervention Programs. In: Journal of Educational Psychology Volume 92, 2 (2000), 284-295.

Schnieders, G.; Komor, A.: Eine Synopse aktueller Verfahren der Sprachstandsfeststellung. In: Ehlich, Konrad: Anforderungen an Verfahren der regelmäßigen Sprachstandsfeststellung als Grundlage für die frühe und individuelle Förderung von Kindern mit und ohne Migrationshintergrund. Eine Expertise des Bundesministeriums für Bildung und Forschung. Bonn, Berlin: BMBF, 2005, 261-342 (= Bildungsreform Band 11).

Scolon, Ron; Scolon, Suzanne B. K.: Narrative, literacy and face in interethnic communication; Norwood/NJ: Ablex, 1981.

Service, Elisabet: Phonology, working memory, and foreign language learning. In: Quarterly Journal of Experimental Psychology, Heft 45 (1992), 21-50.

Singer, Wolf 2001: Was kann ein Mensch wann lernen? http://www.mpih-frankfurt.mpg.de/global/Np/Pubs/mckinsey.pdf

Stern, Elsbeth: Wissen ist der Schlüssel zum Können. In: Psychologie Heute 7 (2003), zitiert nach dem Text im Internet (siehe unten).

www.ipn.uni-kiel.de/projekte/quiss-prosa/pdf/Tagung_Soltau.pdf [10. 08. 2006].

Stern, Elsbeth 2004: Sprache als wichtigstes Denkwerkzeug. (2004).

http://www.mckinseybildet.de/download/04_bildungswerkstatt/bw_1_stern.pdf [30.08.2006].

Stern, Elsbeth: Je früher desto besser? Über Lernstrategien von Vorschulkindern. Sendung im SWR 2 am 28. August 2005

http://db.swr.de/upload/manuskriptdienst/aula/au20050401_3076.rtf [28. 07. 2006].

Swain, Merrill. 2005: The Output-Hypothesis: Theory and Research. In: Hinkel, Eli (ed.): Handbook of research in Second Language Teaching and Learning; Mahwah/NJ: Erlbaum, 2005.

Tesser, P.; Mewrens, J.; Van Praag, C.; Iedema, J.: Rapportage Minderheden. Positie in het Onderwijs op de Arbeidsmarkt. Den Haag: SCP, 1999. 
Tophinke, Doris: Sprachförderung im Kindergarten - Julia, Elena und Fatih entdecken gemeinsam die deutsche Sprache; Weinheim: Beltz, 2003.

Tomasello, Michael: Constructing A Language, A Usage-Based Theory of Language Acquisition. Cambridge/Mass.: Harvard University Press, 2003.

Uhlich, Michaela; Mayr, Toni.: Sismik (= Sprachverhalten und Interesse an Sprache bei Migrantenkindern in Kindergarteneinrichtungen). Freiburg: Herder, 2003.

Verhoeven, Ludo: Literacy Development in Immigrant Groups. In: IMIS-Beiträge 2003, Heft 21, (2003), 162-179.

Wiedemann, Marianne: Kompetenzorientierte Ansätze zu einer pädagogischen Diagnostik der Sprachleistungen von Kindern mit Migrationshintergrund. In: Röhner, Charlotte (Hrsg.): Erziehungsziel Mehrsprachigkeit, Diagnose von Sprachentwicklung und Förderung von Deutsch als Zweitsprache; Weinheim: Juventa, 2005, 77-103.

Wiley, Terrence; Lukes, M.: English-only and standard English ideologies in the United States. In: TESOL Quarterly 30, 3 (1996), 511-535.

Wygotski, Lew S.: Denken und Sprechen; Frankfurt am Main: Fischer, 1964.

Wygotski, Lew S.: Ausgewählte Schriften; Köln: Pahl-Rugenstein, 1987 (= Band 2: Arbeiten zur psychischen Entwicklung der Persönlichkeit).

Zareva, Alla; Schwanenflugel, Paula J.; Nikolova, Yolanka: Relationsship between lexical competence and language proficiency. In: Studies in Second Language Acquisition, 27, (2005); 567-595. 


\title{
Förderunterricht Deutsch als Sprache
}

\author{
Gunde Kurtr, Kaiserslautern
}

\section{Situierung und Zusammenfassung}

In dem 2005 gestarteten Versuch an der BBS II Wirtschaft und Verwaltung in Kaiserslautern wird ein neuer Ansatz des Förderunterrichts für Deutsch als Mutter-/Zweitsprache entwickelt und erprobt ${ }^{1}$. Einbezogen sind rund 150 Schüler aus fünf Parallelklassen der Berufsfachschule 1 (BF1), Jugendliche, die nach dem Hauptschulabschluss keinen Ausbildungsplatz finden konnten, aber noch schulpflichtig sind, und solche, die auf diesem Weg einen weiterführenden Schulabschluss anstreben. Ihre Sprachprofile, besonders der Schriftlichkeit, weisen viele Gemeinsamkeiten auf, was Textkompetenz, semantische Konzepte und das vielleicht geringste, aber auffälligste Problem, Rechtschreibung und Morphologie betrifft.

Grundkonzept ist individuelle Förderung ohne Aufteilung der Klassen nach Gesichtspunkten von Leistung oder sprachlichen Voraussetzungen. Dies geht

${ }^{1}$ Team: Daniela Cetto, Bernadette Collins, Andreas Kuntz, Gunde Kurtz, Mona Richtscheid, Claudia Wilhelm. 
zum einen auf vorgängige Negativerfahrung mit solchen Teilungen zurück, zum anderen auf die Tatsache, dass die Sprachprofile muttersprachlicher Schüler nur wenig von denen der „DaZ“-Schüler abweichen².

Im Folgenden gehe ich zuerst kurz auf Mehrsprachigkeit als Normalität in den meisten Schulklassen und Überlegungen zur chancengleichen Förderung aller Schüler ein (1). Im zweiten Teil zeige ich eine Möglichkeit zur Erstellung und Nutzung von Sprachprofilen sowohl ganzer Klassen als auch einzelner Schüler als Förderinstrument (2). Schließlich wird das Zusammenwirken von DaF/Z mit DaM und die Zusammenarbeit der Lehrertandems aus DaM und DaF/Z-Lehrern (also Lehrern, deren Ausbildung auf muttersprachlichen Unterricht gerichtet war, und Lehrer für fremd- oder zweitsprachlichen Unterricht) vorgestellt sowie die daraus entstehenden Arbeits- und Lösungsansätze (3).

Förderunterricht sollte langfristig keine Ressourcenfrage, sondern eine der veränderten Lehrerausbildung und Bereitstellung von Angeboten (Konzepten, Materialien) sein. Er kann im Zuge der allgemeinen Erneuerung(sbedarfe!) und Umdenkprozesse in der Organisation und Realisation schulischen Alltags aufgehen, also als solcher wieder verschwinden. Für die Schule ist das Konzept „Förderung“ eine Chance zum Einstieg in weniger notenorientiertes Unterrichten, für den Moment ein Name für eine neue Anstrengung und Sichtweise beim Beschulen.

\section{Deutsch im mehrsprachigen Schulraum}

\section{1 $\mathrm{DaM}-\mathrm{DaF} / \mathrm{Z}$}

Die Abtrennung von DaM gegenüber DaF/DaZ ist im mehrsprachigen Schulraum längst nicht mehr sinnvoll. Reich (2002) weist darauf hin, dass die traditionelle Aufteilung der sprachdidaktischen Forschung weit hinter der sprachlichen Wirklichkeit von Schule und Gesellschaft zurückbleibt. Diese ist bestimmt von vielfältig mehrsprachigen Situationen. In den o.g. Klassen, wo ca. 50\% der Schüler einen z. T. jüngeren Migrationshintergrund aufweisen, wurde die Frage „Welches ist deine beste Sprache? ${ }^{\text {“ }}$ von den weitaus meisten mit Deutsch beantwortet (128 Antworten, davon 102 Deutsch, 16 Deutsch + X gleich gut, 10 X (andere als Deutsch), wobei von den 16 Nennungen beide 4 Schüler angeben, dass Deutsch ihre einzige Schriftsprache ist. Diese Selbsteinschätzung verdeutlicht, dass die meisten der von außen als Zweitsprachler eingestuften Betroffenen sich selbst eher als deutschsprachig bzw. Zweisprachler betrachten.

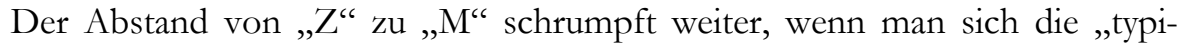
schen Fehler" ansieht: Hätten wir nur die Schüler mit Migrationshintergrund im Förderunterricht (FöU), würden wir bei denjenigen, die ihre gesamte Schulzeit in

\footnotetext{
2 Das gilt nicht für Schüler mit Aufenthaltsdauer unter 2 Jahren, die aber in keiner der Klassen vertreten waren.
} 
Deutschland verbracht haben, deutliche Spuren der Familiensprache anhand der „typischen Fehler“ feststellen. Da wir aber die ganze Klasse in den FöU einbeziehen, können wir fast alle diese Febler auch bei Deutsch-Einsprachigen beobachten und bearbeiten. Das betrifft nicht nur Ungenauigkeiten bei Endungen und Vermeidungsstrategien in den Bereichen Syntax und Lexik. Es geht auch von Artikelfehlern über fehlende Kollokationen und Idiomatik bis hin zu Problemen mit Wortgrenzen, ganz zu schweigen von Textualität. Zur Verdeutlichung exemplarische Beispiele häufiger Fehlerformen, die von Einsprachigen stammen:

der Briefformigeumschlag; ich wäble die bekomme Nummer; die Auskunft berichtet mir die Nummer; warten Sie, bis das Freizeichen erscheint; man muss die Teile in das Handy anbringen; Gebrauchseinleitung; erviedernd (meint: erneut); die Karikatur will uns damit sagen; etwa (anstelle von entweder); wenn (anstelle von bis, indem und während); mündliche Syntax; geschriebener Dialekt; strukturlose Assoziationsketten usw.

Hier soll nun aber nicht der Eindruck entstehen, dass wir fehlerbasiert fördern (dazu mehr unter 2.1). Vielmehr sollen die Beispiele gerade darauf hinweisen, dass eine DaZ-orientierte Febleranalyse und -behebung für Scbüler mit Migrationshintergrund zu kure greift:

- sie benachteiligt den Rest der Klasse, der ebenfalls Sprachförderung in diesen Punkten benötigt,

- Fehlerursachen sind offensichtlich auch in anderen als den familiensprachlichen Gegebenheiten zu suchen, also auch zu bearbeiten,

- man verstärkt durch die Abtrennung der „Migranten“ Segregation und verliert damit Potenziale des voneinander Lernens.

So wenig die Teilung der Schüler nach DaM/DaZ hilfreich ist, so wichtig sind Kenntnisse zu DaF/Z/M dagegen für Lehrer und zwar als Bezugspunkte

- für ihr eigenes sprachliches Handeln,

- für richtige Beurteilung und entsprechende Rückmeldung an Schüler

- für den Umgang mit Aufgaben und Texten.

Dabei könnte in Zukunft Sprachlehrern aufgrund detaillierterer linguistischer und systematischer Kenntnisse Beraterfunktion für Sachfachlehrer zukommen, die in vielen Punkten auch Fertigkeiten zur Sprachförderung brauchen.

Aufgabe des FöU ist Förderung von Sprache im Allgemeinen und von hauptsprachlichen ${ }^{3}$ Kenntnissen im Speziellen. Methoden und Werkzeuge des Fremd- bzw. Zweit-

\footnotetext{
${ }^{3}$ Hauptsprache steht hier (im Unterschied zu Zielsprache, die andere Sprachen völlig ausblendet) für die im öffentlichen Alltag dominante Sprache.
} 
sprachunterrichts sind in vielerlei Hinsicht bei der Vermittlung von Wissen nützlich: Sowohl die notwendige Sprachbewusstheit (Fragen von Bedeutung, Ausdrucksweise, Textkonventionen u. Ä. rücken häufig ins Zentrum der Aufmerksamkeit) als auch die gezielte Arbeit an Sprachaufbau und Sprachausbau sind wirksame Instrumente im Lernprozess (Näheres unter 2.2 und 3.).

\subsection{Suchen \& Versuchen: Welche Sprachförderung fördert Chancengleichheit?}

Sind heterogene Gruppen erfolgreich förderbar? Diese Frage wird meist intuitiv mit nein beantwortet, weshalb bei versagendem FöU stets die Heterogenität Schuld hat: Ganz gleich, wie man sortiert, die Gruppe erweist sich bei näherem Hinsehen stets als sehr heterogen. Für unsere Schüler haben wir festgestellt, dass wir je nach Arbeitsbereich Stunde für Stunde neu sortieren müssten und auch dann keine im engeren Sinne homogenen Gruppen erhalten könnten. Denn Gründe für und Hintergründe von bestimmten Sprachleistungen (also Erscheinungen, nach denen sortiert werden kann) und somit auch entsprechende Bearbeitungsformen sind sehr verschieden. Die homogene Gruppe erweist sich, je mehr Möglichkeiten zu differenzieren man hat, desto mehr als Phantom.

Schwerwiegender ist allerdings, dass die Schüler selbst Sortierungen fast panisch zu fürchten scheinen. Wenn wir aufgrund bestimmter Aufgaben die Klasse völlig beliebig in zwei Hälften teilen mussten, löste das stets große Misstrauen aus, ob die Gruppierungen etwas ,zu bedeuten“ hätten (im Sinne von „,die Guten/die Schlechten“).

Eine der erstaunlichsten Erfahrungen in unserem FöU ist, wie stark die individuellen Leistungen bei verschiedenen Aufgaben(typen) schwanken können. Nicht stabil gute/mittlere/schwache Schüler sind die Hauptgruppe, sondern solche mit starken, teilweise sehr starken Leistungsschwankungen. Dies war nicht nur stimmungsund ggf. gruppenabhängig, sondern vor allem aufgabenabhängig, sowohl bezüglich der Aufgabeninhalte (manche Schüler gaben sich je nach Thema viel/kaum Mühe), als auch bezüglich der Aufgabentypen (z. B. Sorgfalts- vs. Masseaufgaben).

Aufgrund der faktischen Heterogenität muss Sprachförderung immer eine individuelle sein, auf den Einzelnen eingehen und dessen Möglichkeiten nutzen. Das bedeutet nicht, dass man nur noch mit allerkleinsten Gruppen arbeiten kann. Vielmehr geht es darum, die Parameter in Aufgabenstellung und Feedback an die Scbüler zu individualisieren, sie vom Vorhandenen ausgehend zu fordern (s. 2.2 und 3). Die ganze Gruppe mit einer zunächst gleichen Aufgabe konfrontieren und dann individuell weitere Aufgaben bzw. Überarbeitungsschritte folgen zu lassen ermöglicht in einem bisher wenig bedachten Punkt Chancengleichheit, nämlich in der persönlichen Entwicklungsfähigkeit: Zu oft werden Schüler v.a. aufgrund von in einem bestimmten Moment gemessener Leistung sortiert. Dabei wird aber ihr dynamisches Potenzial weitgehend außer Acht gelassen und somit verkümmern sie nicht selten unterfordert in gut gemeinten Maßnahmen. Wendet sich FöU aber an ganze Klassen, ist die Durchlässigkeit zu elementaren wie anspruchsvollen Aufgaben(niveaus) jede 
Stunde gegeben. Dagegen ist Durchlässigkeit zwischen einmal separierten Gruppen wesentlich geringer, zum einen, da die Leistungen deutlich vom Erwarteten abweichen müssen, bis ein Wechsel befürwortet wird; meist geschieht er auch dann aus organisatorischen Gründen nicht mehr. Zum anderen haben nur wenige abgestufte Schüler die Energie zu beweisen, dass sie unterschätzt wurden. Am schlimmsten: Sehr entwicklungsfähige Schüler sind der Gelegenheit beraubt, mit anspruchsvolleren Aufgaben in Berührung zu kommen, gute Schüler mit Lücken im Basisbereich treffen nicht mehr auf entsprechende Aufgaben.

Soll FöU individuell fördern, treten vergleichende Maßstäbe (Musterlösungen, Richtzeiten u. Ä.) in den Hintergrund. Wichtig dagegen sind individualisierte Anforderungen im Rahmen der gleichen Aufgabenkomplexe und häufige persönliche Rückmeldung durch Lehrer während der schülerseitigen Bearbeitung der Aufgabe oder Überarbeitung aufgrund schriftlichen Feedbacks (Förderkorrekturen, s. 3.2). Bei vielen Aufgabenformen haben sich ansteigende Arbeitsaufträge bewährt: Ausgehend von einem Basisauftrag gibt es weitere aufbauende Übungen und Aufgaben, die aber nur von einem Teil der Schüler bearbeitet werden. Diese sind klar als Zusatzanfgaben deklariert, so dass langsamere Lerner nicht das Gefühl bekommen etwas zu versäumen. Dazu kommt, dass das Angebot besteht (und gelegentlich genutzt wird), diese Aufgaben mit nach Hause zu nehmen, also nicht zu verlieren ${ }^{4}$. FöU enthält durch Rückmeldungen, Korrekturen und differenz̨ierte Aufgabenstellung viele Züge von Lernberatung, was gerade bei der Altersgruppe 15+ auch den Übergang vom kindlichen zum erwachsenen Lernen fördert.

Ein Wort noch zur Situation in den BF1-Klassen: Diese Schüler sind bereits durch etliche Sortierraster gefallen. Es liegt daher nahe, sie als benachteiligt, sei es vom sozialen Umfeld, sei es von ihrer persönlichen Leistungsfähigkeit o.Ä., zu betrachten. Die Gefahr dabei ist, dass man als Lehrender nicht mehr an das Potenzial der Klassen glaubt, also die Scbüler nicht ernsthaft fordert (Rosenthal-Effekt). Nach meinem Eindruck ist die Mischung in diesen Klassen daher so fatal, dass ein geringer Teil der Schüler tatsächlich sehr früh an die Grenzen des ihnen Möglichen stößt, ein großer Teil aber durchaus belastbar und leistungsfähig ist, was allerdings durch verschiedene Verhaltensmechanismen überdeckt wird. Eine weitere interessante Erfahrung im FöU ist nämlich, dass unabbängig vom Scbwierigkeitsgrad der Aufgabe die Klasse als Ganze ibre Standard-Leistung erreicht. Ein Viertel kommt kaum zurecht, die Hälfte schlägt sich durch und ein Viertel macht die Sache gut bis sehr gut. Das legt die Vermutung nahe, dass man mit leichteren Aufgaben (Niveausenkung) nicht fördert, sondern Potenzial verschenkt. Es weist auch darauf hin, dass sich bei den Schülern über Jahre eine Einstellung verfestigt hat, wie weit man eine Aufgabe bearbeiten muss, um sie loszuwerden. Die Wieder- und Wiederaufnahme der

\footnotetext{
${ }^{4}$ M.E. kann man hier auch „eilige“ Lerner mit mehr Aufgaben eindecken, die nicht zu sorgfältigem Arbeiten zu zwingen sind, sondern eher über Masse lernen. Bei einigen Aufgaben kann man sie sogar erst durch den schwierigeren Arbeitsauftrag motivieren sich mit dem Basisauftrag auseinanderzusetzen.
} 
Aufgaben (begleitete Überarbeitung) bis zu einem für den jeweiligen Schüler akzeptablen Ergebnis soll dieser fatalistischen Haltung entgegensteuern.

Sprachförderung, die ja immer im Zusammenhang mit kulturellem Hintergrund gedacht werden muss, kann mehr als anderer FöÜ Diversity nutzen. Primär betrifft das die Vielfalt der Sprachen im Raum, z. B. für Sprachvergleiche, Spiele, Arbeit an Wortbedeutungen, Sprichwörter usw., aber auch für die Zusammensetzung von Gruppen und die vielperspektivische Bearbeitung bestimmter Themen. Ein (zumindest im süddeutschen Raum) wichtiger Nebeneffekt ist in diesem Zusammenhang die Aufwertung der Dialekte, die wie andere Sprachen einbezogen werden können.

Auch interkulturelle Arbeit wird durch die sehr verschiedenen familiären Hintergründe erst wirklich lebendig - man lernt nicht über andere Kulturen, sondern von und mit ihnen. Dabei ist es aber wichtig, die Freiwilligkeit der Beiträge zu gewährleisten und nicht Schüler auf die Rolle der Vertreter der Kultur X festzulegen.

Interessant, gerade für Teenager, sind Aufgaben, in denen man etwas über sich erfährt, z. B. Assoziationsspiele (die ersten vier Wörter, die einem zu einem Wort/ Bild/Sprichwort/Text ... einfallen), und deren Auswertung dann Facetten von persönlichen, kultur- oder geschlechtsspezifischen Varianten ans Licht bringt. Auch hier geht es aber nicht darum, typische Muster zu entdecken, sondern letztlich Gemeinsamkeiten zu fokussieren. Denn der gesellschaftliche Aspekt dieser Arbeit ist es, übergreifende Systeme zu finden, die Diverses einbinden können.

\section{Sprachprofile 5 /„Haben“: Wie ist die Ausgangssituation hier \& jetzt?}

\subsection{Warum es ineffektiv ist, von Problembereichen/,Soll““ auszugehen}

Vielen Berufsschülern fehlt neben der Sprachbewußtheit (Bewußtsein der eigenen Febler, Defizite und Probleme) und Reflexion des eigenen sprachlichen Könnens und Handelns auch die Einsicht in die Notwendigkeit des (selbständigen Bemühens um) sprachlichen Kompetenzausbau(s) für eine berufliche (und soziale) Integration. Die Motivation, sich mit Sprache und Texten auseinanderzusetzen, fehlt aufgrund der mangelnden Erkenntnis, dass Sprachkompetenz die notwendige Grundlage für lebenslanges Lernen und beruflichen Erfolg ist. Wie bereits angedeutet, fehlt bier oft auch den Lehrern das Problembewußtsein. Die Schule muß aber... (Efing, o.J., Abs. II; Hervorhebungen G.K.)

Warum sollten diese Schüler sich reflektierend um eine Bilanz bemühen, deren Ergebnis ein vernichtendes Urteil über sie sein wird? Ihre Herabwürdigung ist ihnen

\footnotetext{
${ }^{5}$ Dass wir ein eigenes Instrument zur Erstellung sprachförderorientierter Profile versuchen, liegt daran, dass derzeit kein auch nur ansatzweise sinnvolles vorliegt, wobei insbesondere unsere Altersgruppe überhaupt kaum beachtet wird (vgl. Ehlich u.a., 2005). Dies ist auch Konsens sämtlicher Beiträge in Gogolin u.a. (2005).
} 
längst hinreichend klar. In dieser Situation richten sie ihre Energien im Sinne eines positiven Selbstbildes besser auf Ersatzstrategien. Denn das Geforderte scheint kaum mehr aufzuholen und es ist allemal ehrenhafter, schulisch an Frechbeit und Faulheit gescheitert zu sein, also aus eigenem Willen, als trotz Anstrengung, was als Grund Versagen nahelegen würde.

Förderung auf Basis einer Defiziterhebung mit anschließendem Einsatz von Mitteln gegen die festgestellten Fehler und Lücken scheint uns doppelt ineffektiv. Erstens, weil Sprache mehr ist als das, was durch Febler ins Auge springt, weil mehr entwickelt werden muss als die sich zunächst aufdrängenden Bereiche (s. Kategorien und Kriterien unter 2.2). Zweitens: Wessen Kenntnisse und Fähigkeiten nur als Fehlerhäufung wahrgenommen und behandelt werden, der wird verkannt, unterfordert und wobl kaum motiviert.

Geht man von den erhobenen Fehlerdaten aus, müsste man quasi bei Null anfangen. Das hat unattraktive Aufgaben(niveaus) zur Folge, schadet also wieder der Motivation. Der Neuanfang ist aber gar nicht nötig, denn vieles ist zumindest teilweise bekannt, wird aber nicht angewandt/umgesetzt (inert-knowledge-Problem, s. 3.1). Ein einfaches Beispiel sind (der Normalfall in unseren Klassen) grobe Fehler bei der Registerwahl (Umgangssprache/Dialekt in schriftlichen Textsorten), die geringe Nutzung konzeptioneller Schriftlichkeit 6 . Daraus leitet sich nun aber nicht ab, dass der Unterricht diesbezüglich bei Null ansetzen muss mit der Hinführung zur Erkenntnis, dass es verschiedene Register gibt: Hochdeutsch und Dialekt, Umgangssprache, Slang, Schriftsprache usw. Von solchen Einführungen fühlen sich die Schüler unterfordert (auch wenn sie es nicht sein sollten), bauen dadurch Abwehrhaltung auf, was mögliche Lerneffekte blockiert. Zielführender, gerade bei Bereichen mit drastischen Fehlern, ist es, darauf zu achten, was schon da ist und wie man darauf aufbauen bzw. mit dem Vorhandenen ansetzen kann. Auf solche Aufgaben reagieren Schüler postiv(er), da sie sich (a) nicht unterschätzt fühlen, (b) mit einem Guthaben an den Start gehen. Sogar wenn Registerwissen nur sehr rudimentär bzw. vorbewusst vorhanden ist, kann man es nutzen. Im Falle der Registerarbeit sind mehrfache Textbeobachtungsaufgaben ideal: Die Schüler sind in der attraktiven Rolle des Urteilenden, wobei sie schrittweise ihre Urteile verteidigen und verfeinern können. Aufgaben wie „Was gehört zusammen? Was klingt falsch/besser? Was passt nicht in den Text? Wie könnte man es besser sagen? ... "bilden einen Übergang von Textbeobachtung (dadurch Kriterienbildung und Erweiterung von Kenntnissen) zu eigenen kleineren Produktionen (Korrekturen). Erst dann folgen umfassendere Produktionsaufgaben wie das Überführen mündlicher Erzählung in Berichts- oder Protokollform.

Anstelle der Fehler steht so stets umfassende und integrierte Spracharbeit im Mittelpunkt. Das wird im Beispiel konzeptioneller Schriftlichkeit besonders deutlich, da

\footnotetext{
${ }^{6}$ Begriff nach Koch/Oesterreicher $(1985,1994)$. Schülertexte sind zwar medial schriftlich, weisen aber viele Züge mündlicher Texte auf, was nicht nur eine Stilfrage ist, sondern mangels Situationskontext zu erschwerter Verständlichkeit führt.
} 
hier mit den großen Fragen Register und Textsorte auch Grammatik und Lexik bis hin zur korrekten Rechtschreibung eine Rolle spielen.

\subsection{Mit der „Haben-Seite“ arbeiten: Sprachprofile}

Unsere Sprachprofile (schülerspezifische Daten als Grundlage für Aufgaben und Feedback, s. u.) suchen v. a. Anknüpfungspunkte: Was ist da, womit können wir beginnen, worauf aufbauen? In welchen Punkten zeigt der Schüler selbst Interesse und Bereitschaft zur Spracharbeit? Die Aussage: „Nichts ist da, das ist ja das Problem. “ ist zynisch und verkennt, dass ein großer Teil dieser Schüler außerordentlich lernund denkfähig ist. Nur: Sie richten diese Fähigkeiten auf anderes, auf ausgeklügelte Vermeidungsstrategien, auf Gesichtswahrung, Selbstschutz bzw. -verteidigung, auf effizienten Umgang mit Systemen und Inhalten, die sie nicht treffen. (Beispiele zur Förderung des Vorhanden s. 3.2).

Wir beginnen mit einer weiten Bestandsaufnahme als Ausgangspunkt, wobei die Selbsteinschätzung der Schüler ebenso wichtig ist wie ihre Sprachbiografie und ihre Lösungen spezieller Aufgaben, also Schülertexte. Auf Basis der Sprachprofile kann dann Sprachstand erfasst und Schüler gefördert werden.

$\mathrm{Zu}$ Beginn des Schuljahres bitten wir (mit Begründung warum und wozu!) die Schüler um die schriftliche Beantwortung folgender Fragen:

Delche Sprachen verstehst und sprichst du? Nenne auch Dialekte und Sprachen, die du in der Schule gelernt hast oder die du nur en bisschen kannst.

$>$ Wo und wie hast du diese Sprachen gelernt?

$>$ Welche Bereiche jeder Sprache (Verstehen, Sprechen, Lesen, Schreiben) kannst du besser oder weniger gut? Wie würdest du dich selbst einschätzen?

$>$ In welchen Situationen verwendest du welche Sprachen?

$>$ Mit welchen Personen verwendest du diese Sprachen?

$>$ Wie verteilt sich deine Benutzung der Sprachen (Anteil pro Tag/wie oft pro Woche)?

$>$ Welche Sprache(n) beherrscht du am besten?

$>$ Welche Sprache(n) benutzt du besonders gerne oder ungerne? Warum?

$>$ Welche Sprachen würdest du gerne neu oder besser lernen? Warum?

$>$ Was möchstest du zum Thema "Sprachen“ noch sagen?

Daraus entstehen nicht nur individuelle Sprachbiografien für die einzelnen Schülerprofile, sondern auch ein Klassenprofil (Übersichts-Tabelle), das allen Lehrern dieser Klasse wertvolle Hinweise zur Gesamtsituation gibt, also auch die Möglichkeit vorhandene Potenziale zu nutzen.

Mit der Abgabe der ersten Schreibaufgabe bitten wir die Schüler um eine stichwortartige Selbsteinschätrung bęüglich des Schulfaches Deutsch: 
Wo liegen deine Stärken und Schwächen im Schulfach Deutsch?

Freies Sprechen, lautes Lesen, stilles Lesen (Schnelligkeit, Sinn erfassen) Rechtschreibung, Aufsätze schreiben (versch. Typen), Sachtexte schreiben, Phantasie, Merkfähigkeit (Gedächtnis); Selbstkorrektur (z. B. vor dem Abgeben einer Klassenarbeit), Lexika benutzen, Bücher lesen?

Was würdest du gerne in diesem Schuljahr in Deutsch lernen? Wo würdest du dich gerne verbessern?

Diese Selbsteinschätzung ist in vielerlei Hinsicht wichtig:

- Selbstüber- bzw. Selbstunterschätzer kann man bei Feedbacks entsprechend berücksichtigen

- auf zu hoch gesteckte Ziele oder starke Abwehrhaltungen kann man früh reagieren

- Interessen, also mögliche Ansatzpunkte, werden sicht- also nutzbar

- Schüler werden auf Breite und Ziele des Faches Deutsch aufmerksam, einschließlich seiner übergreifenden Bedeutung für andere Bereiche.

Dritter und aufwändigster Bestandteil der Sprachprofile ist die Auswertung der Abgaben schriftlicher Texte und Lösungen ${ }^{7}$, die sich über das gesamte Schuljabr erstreckt und neben dem Ausgangsprofil (Aufgaben 1.-3.) auch die Entwicklung jedes einzelnen erfassen kann.

Das Ausgangsprofil entsteht aufgrund verschiedener Aufgaben, wobei wir im ersten Versuch folgende Kombination erprobt haben, die sich bewährt hat und im Folgejahr leicht überarbeitet zum Einsatz kommen wird:

(1) Freie Schreibanfgabe:

Stelle deine Lieblingsmusik oder deinen Lieblingsfilm vor. Gehe dabei in drei Schritten vor und unterteile den Text streng in drei Teile:

> Beschreibe die Musik/den Film sehr genau, so dass auch eine Person, die sie nie gehörtlihn nie gesehen hat, eine genaue Vorstellung davon bekommt.

$>$ Erkläre, was du daran besonders wichtig und besonders gut findest. Erkläre auch, warum du gerade diese Eigenschaften gut bzw. wichtig findest.

> Nenne Argumente von Personen, die diese Musik/diesen Film nicht mögen, Was könnte man diesen Personen sagen, um sie davon zu überzeugen, dass die Musik/der Film doch gut ist?

\footnotetext{
${ }^{7}$ Unser FöU konzentriert sich auf schriftliche Texte.
} 
Dass die Aufgabe eine Struktur vorgibt, führt beim ersten Anlauf durchaus nicht zu strukturierten Ergebnissen. Viele Schüler schreiben in einem Textblock, meist Aspekte aus dem ersten und/oder zweiten Teil der Aufgabe. Der dritte Teil fehlt zunächst bei fast allen gänzlich, was weniger ein Zeitproblem ist, er wird häufig sogar explizit abgelehnt.

(2) Vertextungsaufgabe, d. h. Textanfang und einige Teile sind vorgegeben, einige Textpassagen liegen nur stichwortartig vor und sollen ausformuliert werden; Ziel ist ein Einblick, wie weit die Schüler einen vorgegebenen Rahmen (Register; Explizitheit, v. a. Konnektoren und Überleitungen) erkennen und aufnehmen; hierbei spielen Lesefertigkeiten eine wichtige Rolle.

(3) Lückentext mit wenigen Lücken (nur Verben, Nomen, Adjektive: Teile von idiomatischen Ausdrücken/Kollokationen u. Ä.), um einen Eindruck von Umfang und Abrufbarkeit bestimmter Wortschatzbereiche zu bekommen; bei dieser Aufgabe spielt Lesekompetenz die Hauptrolle ${ }^{8}$.

Im Sprachprofil werden aufgrund der Abgaben in ff. Kategorien (zu den jeweiligen Kriterien) für jeden Schüler Daten notiert:

zu (1) Freie Schreibaufgabe:

- Rechtschreibung $\rightarrow$ Wortgrenzen, Konsistenz, Groß-klein, DehnungSchärfung, Auffälligkeiten, Fremdwörter, Interpunktion

- Ausdruck $\rightarrow$ Flüssigkeit, Sicherheit, Norm/Umgangssprache/Mündlichkeit; Explizitheit, Sätze, Satzmuster, Kohärenz, Wortschatzreichtum und -präzision; DaZ-Effekte (Kollokationen, Pronominalisierung, Artikel u. Ä.); Risiko / Vermeidung

- Text $\rightarrow$ Texthaftigkeit; Lesbarkeit, Leserorientierung (nachvollziehbar); Strukturiertheit; Kohärenz, sprachlich / inhaltlich stimmig; Detailliertheit, Vollständigkeit; verwendete Mittel (erzählen, erklären, kommentieren, argumentieren ...); Risiko / Vermeidung

- Bemerkung $\rightarrow$ Qualität der Überarbeitungen; Sonstiges (Umfang, Vollständigkeit, Metaebene z. B. Ablehnung o. ̈.,, Memos usw.).

zu (2) Für die Vertextungsaufgabe kommen hinzu:

- Sinn erfasst $\rightarrow$ Sinn nachvollzogen (genauer: mit den Gedanken des Autors mitvollzogen); eigene Variante erstellt; Grauzone (vage bis chaotisch)

- Verbindung/Vertextung $\rightarrow$ minimal bis maximal; Dichte vs. Sprünge; Logik - Stimmigkeit - Klarheit; Vielfalt der Verbindungen; Risiko/Vermeidung

- Konzentration $\rightarrow$ gleichbleibend, abfallend, ansteigend; Tempo.

\footnotetext{
${ }^{8}$ Aufgaben und Details zur Durchführung unter http://www.daz-daf.de
} 
zu (3) Für die Wortschatz/Leseroutine-Aufgabe kommen hinzu:

- Lesegeschwindigkeit $\rightarrow$ während der Bearbeitung (Einzelarbeit) v.a. zu Beginn der Stunde Tempo und Anteil der sinnvoll ausgefüllten Lücken beobachten - nach der Stunde auffällig langsame / schnelle Schüler notieren

- Automatisierungsgrad $\rightarrow$ Wörter im Kontext, erwartete vs. ungewöhnliche Lösungen

- Erfasste Sinnspanne $\rightarrow$ Menge der sinnvollen Einsetzungen: Menge der Einsetzungen, die nur in ihrer Mikro-Umgebung sinnvoll sind, im Gesamtzusammenhang aber völlig unpassend

- Lottostrategie $\rightarrow$ Menge der wahllos eingesetzten Wörter, die auch aus der Mikroumgebung nicht abzuleiten sind, sondern z. B. aus falschem Abschreiben/Aufschnappen resultieren.

Nach der Schreibaufgabe (1) schließen sich mehrere Überarbeitungseinheiten an, u. a. zu Mündlichkeit/Schriftlichkeit, bevor es mit (2) weitergeht. Das Sprachprofil muss nicht in Eile erstellt werden, sondern alles Unterrichtsgeschehen dient vorrangig dem intensiven, vertiefenden Lernen, nicht dem „Abhaken“.

Die Vielzahl der Kategorien und Kriterien lässt einen kaum noch handhabbaren Datenwust befürchten. Da aber pro Schüler nur jeweils einige Punkte relevant sind, bleibt das Ganze schon in Tabellenform übersichtlich'. Im Laufe des Schuljahres kommen mit jeder neuen Abgabe Ergänzungen zu den Profilen hinzu, wobei man sich bald daran gewöhnt, beim Lesen und Kommentieren der Abgaben jeweils eine Notiz mitabzulegen; der zeitliche Mehraufwand ist nur in der Lernphase erheblich, rechnet sich insgesamt aber bald.

Die Schülerprofile dienen weniger der Dokumentation von Leistung als der von Entwicklung. Quasi als Nebeneffekt bekommt man so auch einen Eindruck von der persönlichen Entwicklungsfähigkeit (Tempo, stagnierende und wachsende Sprachbereiche). Diese Notizen sind Grundlage für die durch Feedback und Aufgabenstellung fokussierten Bereiche, an denen ein Schüler gerade besonders arbeiten soll. Dabei behält man Lücken im Auge, aber nicht im Fokus.

Leitziele wie Textkompetenz, Wortschatzausbau usw. dienen als Grundlage von Aufgabenstellung und Sequenzierung. Davor und darüber stehen aber die Möglichkeiten und der Bedarf des einzelnen: Wer noch an Wortgrenzen arbeitet, wird darin bestärkt und die Angemessenheit seiner Abgaben wird nur zweitrangig mitbesprochen. Wichtig sind auch die von den Schülern selbst aufgestellten Ziele. Diese sind oft eng mit Aufstiegswünschen oder individuellen Bedeutsamkeiten verbunden, was als Motivationsfaktor eine enorme Rolle spielen kann und daher unbedingt genutzt werden sollte.

\footnotetext{
${ }^{9}$ Ein einfaches, datenbankbasiertes Werkzeug ist in Arbeit.
} 


\section{Sprachförderung: DaM + DaZ $\rightarrow$ Deutsch}

\subsection{Wo lernt DaM von DaF? Wo bleibt DaZ?}

Elemente aus DaF, die im Deutschunterricht in der Schule weiterhelfen, sind:

\section{(1) Kenntnis des ,inert knowledge problem"10}

Die Diskrepanz zwischen Wissen (bzw. schon gehört haben) und Können dürfte einen Teil der Schulmüdigkeit der Schüler ausmachen, die im 10. Jahr ihres Schulbesuchs noch immer an teilweise einfachsten Anforderungen scheitern. Sie haben jedoch deutlich mehr Wissen, als ihre Leistungen vermuten lassen. Das betrifft alle Bereiche von der Rechtschreibung über Wortschatzkenntnisse bis hin zu Wissen über normgerechte Textstrukturen: Z. B. kann Regelwissen zur Großschreibung korrekt erklärt werden, wird beim freien Schreiben aber kaum umgesetzt; machen wird problemlos durch speziellere Verben ersetzt, Kriterien zur Textbeurteilung sind vorhanden.

So, wie es bei den Schülern vorliegt, trägt dieses Wissen aber offensichtlich kaum zum Können bei. Neuerlicher Erklärungsinput nutzt hier wenig, es mangelt vielmehr an integrierten Übungsformen oberhalb der Konditionierungsebene:

\section{(2) Gezielte Fertigkeitenorientierung u. -verbindung zu Sprachtraining}

Schwerpunktmäßig an einer bestimmten Fertigkeit im Umgang mit Sprache arbeiten; an einem Teilziel solange arbeiten, bis ein gewisser Stand erreicht ist; attraktive und relevante Themen und Aufgabenstellungen finden, mit denen vielfach wiederholte Übung an einer Sache lohnend wird; für Lerner mit geringem Kenntnisstand Aufgabenformen finden, die ihnen trotzdem attraktive Rollen anbieten, das ist Alltag im DaF-Unterricht. In der Schule kann das ähnlich genutzt werden: Textbeobachtungs- oder Korrekturaufgaben weisen Schülern Experten- bzw. Prüferrollen zu, Texte in Grafiken umarbeiten ist attraktiver als Schlüsselwörter markieren, da der Schüler hier selbst etwas Neues schaffen kann, eine lückenoder fehlerhafte Mitschrift zu einem Kurzvortrag oder Film bei laufendem Text zu ergänzen bzw. verbessern bietet mehr Aussicht auf Erfolg, als aus dem Stand eine eigene Mitschrift anzufertigen. Mit solchen Vorübungen wird die später notwendige volle Fertigkeit (z. B. Textmerkmale erkennen, Normen verinnerlichen, exzerpieren, mitschreiben) erreichbarer.

\footnotetext{
10 Begriff nach Larsen-Freeman (2003), die damit explizites Grammatikwissen von Fremdsprachenlernern bezeichnet, das diese kaum in ihrem sprachlichen Handeln anwenden können.
} 


\section{(3) Integrierte Wortschatz- und Grammatikarbeit}

Was in $\mathrm{DaF}$ als ein Hauptproblem bekannt ist, sind die schwer zugänglichen Bereiche in der Wortbedeutung, die Auswirkungen auf Kombinierbarkeit mit anderen Wörtern haben, auf Möglichkeiten der Verwendung im Satz oder auch auf (nicht) anzuwendende grammatische Regeln. Schwer zugänglich sind diese Bereiche z. B., weil Wörter normalerweise unbewusst kategorisiert sind, etwa als Gefäße oder Objekte mit definierter Standfläche (was zur Folge hat, dass man stehen anstelle von liegen anwenden muss, auch wenn ein Objekt flach und nicht hochformatig ist). Viele Unsicherheiten (,unglückliche Formulierungen“ z. B.) der Schüler gehen auf Schwierigkeiten mit dem Erfassen der vollen Wortbedeutung und damit Verwendungsumfang und -beschränkungen für Wörter und Ausdrücke zurück. Rein formale Erklärungen (das muss Akkusativ sein/den heißen) oder Korrekturen (es heißt nicht erscheint, sondern ertönt) helfen daher wenig. Wenn man korrigiert, dann

bitte mehr: Z. B. nachfragen, was alles erscheinen kann, feststellen, dass es sich auf Sichtbares beschränkt, während Geräusche ein eigenes Wort nutzen. Diese Erklärungen kommen stets aus der Klasse, meist sogar von den Betroffenen selbst, wenn man einen kleinen Anstoß gibt, und sie bleiben im Gedächtnis, weil sie häufig zu Wortspielen und Neckereien führen.

\section{(4) Unerschütterlichkeit angesichts Blockaden, Lücken, Bebelfslösungen}

In DaF-Kursen steht man als Lehrer aufgrund der „fremden“ Herkunft der Lerner täglich in Situationen, die man nicht beurteilen kann, zu denen man sich jedoch verhalten muss. Dabei entwickelt man einen kreativen, positiven Umgang mit Unbekanntem, lernt Kompetenzen an die Lerner abzugeben und dabei selbst viel Neues zu erfahren. Diese Einstellung ist nicht nur wichtig im Umgang mit multikulturellen Klassen, sondern auch bei Schülern mit verschiedenen sozialen und familiären Hintergründen, also mit allen. Im Zusammenhang mit der relativen Fremdheit wird man im DaF-Unterricht gelegentlich auch mit Blockaden oder Abwehrhaltungen an Stellen überrascht, wo man keinerlei Konfliktpotenzial erwartet hätte. Eventuell vorgebrachte Argumente erscheinen einem vielleicht absurd, schweigende Ablehnung bereitet fast noch mehr Probleme. Auch dazu muss man sich verhalten, einen gemeinsamen Weg zum Lernen herausfinden, nicht aufgeben. Dies lässt sich leicht auch im Umgang mit schwierigen Lernergruppen anwenden.

Ein weiterer wichtiger Punkt: Man hat in DaF gelernt, Sprachentwicklung von Null an zu begleiten, auch rudimentärste Äußerungen und Texte als Schritt auf dem Weg zu mehr zu beachten und zu nutzen. Vergleichsmaßstab für Freude über die Qualität von Lernerleistungen ist dabei nicht die Standardsprache, sondern die vorhergehende Lernerleistung, die relative Steigerung. Das ist auch im Förderunterricht grundlegend. 
Die unter (1) bis (4) genannten, m. E. leicht aus DaF auf DaZ übertragbaren Punkte bleiben aber weitgehend unberücksichtigt. Dagegen existieren an anderen Stellen fragwürdige Übertragungen aus $\mathrm{DaF}$ in DaZ:

Wo bleibt DaZ überhaupt in diesem Zusammenhang? Vielleicht am ehesten im Bewusstsein lehrerseits für mögliche tieferliegende Ursachen von Fehlleistungen (s. 3.2). Interessanterweise orientiert sich DaZ in seiner Argumentation/Selbstlegitimation zwar sehr stark an der Spezifik der Zielgruppe (längerfristig in Deutschland lebend), nicht aber in seinem Sprachlehransatz. Hier wird ohne Umschweife auf Lebrwerke und Grammatiken (!) aus DaF zurückgegriffen. DaZ sollte m.E. aber gerade auf diesem Gebiet völlig eigene Ansätze vertreten und entsprechende Lehrkonzepte und Materialien haben. Hier spielen Ergebnisse der Forschung zur „Fossilisierung“ (Han 2004) eine Rolle, z. B. die Trennlinie zwischen eher auf kognitivem Weg erlernbaren Sprachbereichen (z. B. Wortschatz, Textsorten) und eher auf intuitivem Weg erlernbaren Sprachbereichen (z. B. Genus, Morphologie). Erstere werden eher durch Erklärungen und Übungen, Letztere eher durch Masse und negative Evidenz (Korrektur) gefördert.

Insgesamt können Unterrichtskonzepte, Aufgaben \& Materialien aus dem $\mathrm{DaF} /$ Z-Bereich über den Förderunterricht in den Deutschunterricht gelangen. Das stützt wiederum den fächerübergreifenden Ansatz, Sprache als Werkezeng bei der Bearbeitung von Themen und der Erschließung und Darstellung von Inhalten benutzen zu lernen.

\subsection{Arbeit im schulischen Alltag $\rightarrow$ Praxissituation kennen $u$. mitgestalten}

Als neben dem „,normalen“ Unterricht eingesetzte DaZ- oder Förderlehrerin hätte ich viel Wichtiges nicht wahrgenommen, hätte einige nur mit Hilfe der DaM-Lehrer erreichte Ziele verpasst und damit wäre die eingesetzte Unterrichtszeit wesentlich ineffektiver geblieben. Durch die Arbeit im Tandem bekommt man nicht nur Einblicke in die alltäglichen schulischen Abläufe, den „normalen“ Deutschunterricht und in das gesamte Umfeld, sondern man lernt auch mögliche Aufgabenstellungen mit den Augen der Routiniers auf Schwierigkeit, Umfang, Verständlichkeit und auch Bedarf bin zu betrachten. Dadurch konnten häufig ohne zeitraubende Absprachen kurzfristig Unterrichtsinhalte und Aufgabenstellungen justiert werden und so den Scbülern unnötige Experimente erspart bleiben.

Förderlehrer, die neben dem regulären Unterricht arbeiten, klagen meist über die weitgehende Abtrennung vom sonstigen schulischen Alltag. In speziellen Projekten wird das z. T. gezielt durch Teamsitzungen und umfangreichen Informationsaustausch einschließlich Absprachen aufgefangen, was allerdings mit im Schulalltag kaum zu erbringendem Zeiteinsatz verbunden ist. Insofern scheint das Tandem letżtlich eine žeitökonomische Variante zu sein: Die Gruppengröße pro Lehrer (eine halbe normale Klasse) ist immer noch größer als in Förderunterrichten sonst üb- 
lich, die gegenseitige Information und Fortbildung (also Austausch DaM $\leftarrow \rightarrow$ $\mathrm{DaF} / \mathrm{Z})$ läuft dabei automatisch mit.

Wichtig war auch, dass durch die enge Anbindung an den Regelunterricht die Normalität dieser Stunde für die Schüler gegeben war und so die Akzeptanz des Konzepts FöU einschließlich der zusätzlichen Lehrperson in den Klassen maßgeblich getragen wurde.

Die wöchentliche Arbeit im FöU fokussiert auf Sprachaufbau und -training: Es gibt knappe Arbeitsanweisungen zu Beginn, im Wesentlichen sind alle Schüler während der gesamten Unterrichtszeit aktiv, meist in Einzelarbeit oder Paaren, gelegentlich in Kleingruppen. Die Aufgabenstellungen versuchen dabei vor allem tieferliegende Sprachbereiche anzugehen: Lexikogrammatische, semantische und idiomatisch-kulturelle Ebene ${ }^{11}$ werden schon durch die Konzeption der Aufgaben herausgefordert, aber v.a. durch Rückmeldungen (Einzelfeedback und Besprechungen in Gruppen oder Plenum) und Korrekturen (angeleitete Überarbeitungssequenzen) auch nachhaltig bearbeitet. Gerade in diesen Bereichen besteht für Mehrsprachige häufig erhöhter Bedarf an Klärung und Beispielen - von der Unbekanntheit des „Rumpelstilzchens“ bis zur Frage der Passivfähigkeit von Verben. Daneben zielen die Aufgaben natürlich auf den Ausbau übergreifender Bereiche wie Registerbewusstsein und Textkompetenz ab, bauen dazu aber sehr auf die genannten Teilbereiche.

Der Tandem-Unterricht ermöglicht auch ein weiteres, v. a. im FSU für Erwachsene übliches Instrument zu übernehmen: Die Lernberatung, wenn auch in kleiner Form. Diese besteht nicht nur aus den fast wöchentlichen persönlichen Rückmeldungen (schriftliches Feedback zur Abgabe, Korrekturhinweise usw.), sondern v.a. auch in der Hilfe beim Tun: Die Schüler starten ihre Aktivität, während beide Lebrer im Raum Ansprechpartner für Fragen sind. So kann man durchaus auch für längere Zeit an einem Tisch verweilen und Fragen klären oder Anregungen geben und zwar genau in dem Moment, in dem die Schüler beim Arbeiten auf Schwierigkeiten stoßen.

Die Erfahrung, eine Lösung auch gegen Widerstände zu erarbeiten, ist für viele unserer Scbüler neu: Bisherige Strategien waren (1) aufgeben und abbrechen („das kann ich nicht"), (2) abschreiben/fertige Lösung suchen (,,wer hat das, wie geht das?") oder (3) irgendetwas hinschreiben, um guten Willen zu zeigen, wohl wissend, dass es nicht das Verlangte trifft.

$\mathrm{Zu}$ Beginn des Schuljahres sind 13-15 Schüler pro Lehrer noch deutlich zu viel, man wird permanent gerufen, da das Hilfsangebot v.a. Strategie (2) auslöst, man muss die Strategie (1) wählenden Schüler möglichst parallel zu Stundenbeginn ,anschieben“. Über die Zeit machen sich die Schüler dann z. T. Antworten der Lehrer zu eigen, was die Arbeitsatmosphäre im Raum langsam intensiviert („Du kommst nicht bier her, um das zu können, sondern um es zu lernen. "bei (1), bei (2) „Mach wenigstens einen Versuch, bevor du Hilfe rufst / Stell eine Frage, die etwas mit der Aufgabe zu tun hat. / Was genau verstehst du denn nicht an der Aufgabe?").

\footnotetext{
11 Modalität, Kausalität, ,,vollständige“ Sätze, präzise Wortbedeutungen, routinisierte Formulierungen, anschauliche Darstellung / Metaphorik usw.
} 
Letztlich ist es also gerade die wohldosierte Ressourcenknappheit (Hilfe vom Lehrer), die dazu führt, dass nach einigen Monaten die Qualität der Fragen steigt und die eigene Initiative bei Schwierigkeiten zum Normalfall wird. (Zu Beginn des Schuljahres muss die Initiative häufig noch vom Lehrer ausgehen, Schüler, die keinen Anfang finden oder nicht weiterwissen, verhalten sich zum Teil einfach abwartend bzw. völlig passiv: „Wieso schreibst du nicht? Weil ich nicht weiß, was ich da überhaupt machen soll. Warum fragst du dann nicht, du kannst dich doch melden, wenn du nicht weiterweißt! oder: Hol doch bitte mal ein Blatt und einen Schreibstift raus! ..." - solche und ähnliche Aufforderungen sind v. a. in den ersten Wochen ständig nötig.)

Dadurch, dass der FöU keinen für alle gleichen Plan abarbeiten und keine Noten liefern muss, werden Förderkorrekturen in doppelter Hinsicht möglich: Rückmeldungen zu den Schülerleistungen können fokussiert und personalisiert geschehen.

Beim Korrigieren kann man bestimmte Ausschnitte der Scbülerleistung fokussieren. Es geht nicht darum, Fehler auf allen Ebenen (z. B. von Textstruktur über Ausdruck bis Rechtschreibung) anzustreichen, sondern man kann sich auf einen Aspekt konzentrieren oder - je nach Ziel und Schülertyp - aus allen Gebieten zunächst die „Spit$z e n$ " bearbeiten. Es geht weiterhin bei fokussierten Korrekturen nicht darum, jeden einzelnen Fehler im fokussierten Gebiet aufzuzeigen, sondern auch hier kann man Prioritäten setzen. Dazu kommt, dass man ja nicht nur Vorhandenes, sondern auch Feblendes kommentieren muss, um Vermeidungsstrategien zu lösen. Dabei fällt es allerdings meist leichter, sich auf Ausschnitte, einzelne Anregungen zu beschränken.

Für die fokussierte Förderkorrektur hat sich auch das Zwei-Farben-System bewährt, wobei „rot“ Angestrichenes unbedingt und gründlich vom Schüler überarbeitet werden muss, während „grüne“ Anmerkungen im Moment nicht drängen (Motto: Mach erst mal das Rote klar, wenn du dann noch Zeit und Lust hast, geh an den Rest.). Diese Teilung hilft auch dabei, dem vorläufig noch dominanten Bedürfnis (bei Lehrern, Schülern und Eltern) zu entsprechen, dass Fehler nicht „unbemerkt“ stehen bleiben dürfen.

Ebenso wichtig wie die gerade geschilderte fokussierte Rückmeldung ist die personalisierte Rückmeldung: Hierbei kann erstens sprachbiografisches, interkulturelles und aus dem Sprachprofil stammendes Hintergrundwissen zum Einsatz kommen (etwa gezielte Hinweise aufgrund der Familiensprachen bei „typischen“ Fehlern; es können Anregungen, Aufforderungen oder Ermutigungen konkret und persönlich formliert werden). Zweitens ist hier der Platz die o. g. Stärken der Scbüler als Ausgangspunkt für Sprachaufbau zu nehmen. Dies möchte ich am Beispiel einer Schreibaufgabe veranschaulichen. Verlangt war eine Gebrauchsanleitung für das eigene Handy zu schreiben, Adressat sollte ein „Opa“ sein, der den gleichen Typ Handy neu bekommen hat. Die Textsorte Gebrauchsanleitung war vorher nicht thematisiert worden. Bei der Lektüre der Abgaben fanden sich nun (in der Reihenfolge der Häufigkeit) verschiedenste Textmuster: 
- Texte wie Transkription einer mündlichen Instruktion (jetzt nimmst du ... und dann schauen wir mal ... / teilw. auch Sie-Form)

- Erzählende Texte (bei vielen Handys ...; wenn man ..., dann ...)

- Texte wie typische Gebrauchsanleitungen (stark gegliedert, teilweise stichwortartig, mit Skizzen; Sie/man/Infinitiv-Form), oft mit einem Maximum an Präzision und Informationsdichte

- Lektionsform (Gliederung in Schritt / Übung / Lektion 1, 2 ...; oft wir-Form)

- Briefform (Lieber Opa, ... du ..., persönliche Bezüge), + sehr fürsorgliche Anleitung.

Die Schüler wählen ibnen leicht fallende und angemessen erscheinende Textsorten. Als Förderlehrer kann man hier zunächst beobachten, welche Werkzeuge ein Schüler wie gut einsetzt und ihm dabei helfen, diese zu optimieren. Das Feedback richtet sich nicht auf eine bestimmte formale Erwartung, sondern versucht den Text als solchen mit Fokus auf Sprache und Schriftlichkeit zu verbessern. Nachdem die Schüler ihre Texte überarbeitet haben, können sie sich darüber austauschen, welche Form ihnen am angemessensten (a) für den „Opa“ und (b) als Gebrauchsanleitung im Allgemeinen erscheint. Hieran kann sich im Bedarfsfall Textsortenarbeit anschließen, sie muss aber nicht das Ziel der Übung sein. Wir haben den „Handy-Text“ z. B. hauptsächlich für allgemeine Texthaftigkeit (Aufbau, Stimmigkeit, Nachvollziehbarkeit) und zur Übung des Ausdrucks von komplexeren Bedingungsgefügen eingesetzt.

Der Ansatz, gemeinsam (DaM + DaF/Z) nach Bedarf Aufgaben zu wählen und inhaltlich sowie in Details der Aufgabenstellung genau auf die Klasse bzw. einzelne nachzujustieren, entspricht einem Fördergedanken, der Sprachförderung als Aufbau enveiterter Kompetenz ausgehend von den Möglichkeiten der Schüler versteht. Was mehr in die Sprachförderung einbezogen werden muss, sind fächerübergreifende Bausteine, also Sprachentwicklung anhand von Inhalten, die „sowieso“ gelernt werden müssen.

FöU im Tandem DaM $+\mathrm{DaF} / \mathrm{Z}$ ist im Schulalltag praktikabel und erweist sich (quasi nebenbei) als gutes Instrument gegenseitiger Fortbildung. Dabei werden zur Zeit noch - nicht nur bei uns - Konzepte und Materialien erprobt, die sich am Maßstab ihres langfristigen Gesamtnutzens messen lassen müssen.

\section{$5 \quad$ Zum Schluss}

Der Weg von der Theorie (linguistisches, psycholinguistisches und sprachdidaktisches Werkzeug, Wissen um soziale Faktoren bei Sprachlernen und -verwendung, Sichtweise des Problems als Aufgabe) in die Praxis, gemeint ist die erfolgreiche Praxis, kann kein direkter sein. Dazwischen steht eine mühsame Phase des Konzipierens und Erprobens. Dieses Erproben muss zwei Dinge berücksichtigen, nämlich dass die Zeit und Anstren- 
gungen von Schülern und Lehrern keine für „Forscher“ frei verfügbaren Güter sind ${ }^{12}$ und dass die Suchrichtung weniger auf Ausnahmeprojekte zugeschnitten sein sollte als auf spätere Verwendbarkeit im schulischen Alltag.

Die Erprobung durch oder zusammen mit Lehrkräfte(n), die Einbeziehung der Lehrer in die Ideenfindung und Konkretisierung von theoretischen Erkenntnissen $\mathrm{zu}$ Unterrichtskonzepten ist die derzeit vielerorts stattfindende Phase. In unserem Fall hat sich dabei das Teamteaching, die Arbeit mit Sprach(förder)profilen und das nichtsegregierende Fördern mit gestuften Aufgaben als wirkungsvoll und praktikabel gezeigt.

Allerdings fehlen bisher nicht nur in unserem Projekt großangelegte Evaluierungen ${ }^{13}$, also mehrfach durchgeführte, ernsthafte und ergebnisoffene Praxistests mit vergleichbaren Ergebnissen, um festzustellen, welche der Ansätze besonders hilfreich für eine umfangreiche und verhältnismäßig rasche/einfach durchzuführende Sprachförderung sind.

Fragen, die im Zusammenhang mit „Problemklassen“ \& Sprachförderung mitschwingen, betreffen Schulmüdigkeit, durch frühere Schulerlebnisse hervorgerufene Lernblockaden, Verhaltensauffälligkeiten als Abwehrmechanismen und die fragwürdige Mischung von tatsächlich lernschwachen Schülern mit eigentlich leistungsfähigen Kindern aus sozial schwachen Bevölkerungsgruppen. Mehr Freiarbeit mit klaren Zielvorgaben und ein systematischer Einsatz von Lernberatung könnten, vor allem für Jugendliche, hier erfolgversprechend sein.

\section{Literatur}

Efing, Christian (o.J.): „Berufsbezogene Schreib- und Lesekompetenz - ein

Sprachenportfolio als Mittel der Wissensdokumentation in der Berufsschule." http://s1.teamlearn.de/QuickPlace/b-1mv_voli/Main.nsf/h_BD2CA3C4FD98030FC1256E2100598C49/8C74EA0 C567F4F26C1256F2C002C419E/?OpenDocument (zuletzt aufgerufen 10.09.2006).

Ehlich, Konrad u.a. (2005): „Anforderungen an Verfahren der regelmäßigen Sprachstandsfeststellung als Grundlage für die frühe und individuelle Förderung von Kindern mit und ohne Migrationshintergrund.“ Bonn, Berlin : BMBF Bildungsreform Band 11.

Gogolin, Ingrid u.a. (Hrsg.) (2005): „Sprachdiagnostik bei Kindern und Jugendlichen mit Migrationshintergrund.“ Münster u.a.: Waxmann.

\footnotetext{
12 Es werden tatsächlich noch erschreckende Datenerhebungsmethoden verwendet, z. B. Schüler um Aufsätze oder sonstige aufwändige schriftliche Arbeiten bitten, diese im Unterricht anfertigen lassen und damit ohne jede weitere Rückmeldung an die Probanden zu verschwinden.

${ }^{13}$ Gemeint sind Evaluierungen der Effektivität von (Förder)unterricht in der schulischen Praxis.
} 
Gogolin, Ingrid (1994): „Der monolinguale Habitus der multilingualen Schule.“ Münster u.a.: Waxmann.

Han, ZhaoHong (2004): „Fossilization in Adult Second Language Acquisition.“ Clevedon u.a.: Multilingual Matters.

Kniffka, Gabriele (im Druck): „Sprachförderung zwischen Theorie und Praxis: Neue Wege in der Lehrerausbildung. “ erscheint in: Hug, M. / Siebert-Ott, G. (Hrsg.): „Sprachbewusstheit und Mehrsprachigkeit.“ Hohengehren: Schneider.

Koch, Peter \& W. Oesterreicher (1994): „Schriftlichkeit und Sprache.“ In:

Günther, Hartmut u.a. (Hrsg.): „Schrift und Schriftlichkeit“, 587-604. Berlin: de Gruyter.

dies. 1985: "Sprache der Nähe - Sprache der Distanz. Mündlichkeit und Schriftlichkeit im Spannungsfeld von Sprachtheorie und Sprachgeschichte", Romanistisches Jahrbuch 36: 15-43.

Larsen-Freeman, Diane (2003): „Teaching Language. From Grammar to Grammaring." Boston: Thompson/Heinle.

Reich, Hans H. u.a. (2002): „Spracherwerb zweisprachig aufwachsender Kinder und Jugendlicher." Hamburg: Behörde für Bildung und Sport. 



\section{DaF/DaZ, Mehrsprachigkeit und gelebte Interna- tionalität - Beispiele aus dem Bildungsangebot und Alltag an einer International School in Deutschland}

Andrea Wagner, Düsseldorf

\section{Allgemeine Informationen über das Schulsystem der In- ternational Schools}

Um den Anforderungen der Mobilität in der internationalen Berufswelt einerseits und den Bildungsbedürfnissen der betroffenen Familien andererseits nachkommen zu können, sind weltweit etwa 800 Internationale Schulen gegründet worden, die in der Unterrichtssprache Englisch ein weltweit kompatibles Unterrichtsprogramm anbieten, das Schülern einen häufigen Schulwechsel ohne erkennbare Nachteile in Bezug auf fehlende Sprachkenntnisse des Ziellandes ermöglicht und gleichzeitig ihre Mehrsprachigkeit fördert.

Die International Baccalaureate Organization (IBO), die 1968 gegründet wurde, arbeitet derzeit mit über 1.700 Schulen in 122 Ländern zusammen und bietet Lernprogramme für über 200.000 Schüler im Alter von 3 bis 19 Jahren. Das In- 
ternational Baccalaureate Diplom wird weltweit für die Zulassung zum Studium an einer Universität anerkannt. Im Jahr 1951 wurde auf einer Konferenz im UNESCO Gebäude in Paris beschlossen, ein zweijähriges akademisches Programm zu entwickeln, das eine neue Qualifizierung zur Universitätszulassung darstellt. Das führte zur Gründung des International Baccalaureate Büros in Genf. In den letzen Jahren wurde dieses für die letzten beiden Schuljahre entwickelte Programm auf die Primar- und Sekundarstufe ausgeweitet, um den internationalen Schulen ein einheitliches Lemprogramm vom Kindergarten bis zur Klasse 12 anzubieten. Der European Council of International Schools (ECIS) mit Sitz in Großbritannien sowie Partnerorganisationen in den Vereinigten Staaten wie die New England Association of Schools and Colleges überprüfen und bewerten regelmäBig als externe Instanz die internationalen Schulen und deren Lehrpläne. Diese Qualitätskontrolle wird mit dem Begriff ,,accredited“ als erfolgreich bestanden bezeichnet.

Allein in Deutschland gibt es zurzeit 18 Internationale Schulen mit circa 10.000 Schülern. Zu den Ausbildungsgrundlagen der Internationalen Schulen gehört, dass das Erlernen der jeweiligen Landessprache für alle Altersstufen angeboten wird. Das bedeutet, dass in Deutschland in jeder der Internationalen Schulen Deutsch als Fremdsprache in verschiedenen Niveaus angeboten und von insgesamt mehreren tausend Schülern aller Altersstufen erlernt wird. Schüler, die sich lange genug im Land aufhalten, erhalten die Gelegenheit Deutsch nicht nur als Fremdsprache zu erlernen, sondern auch als Zweitsprache zu nutzen.

Die überwiegende Mehrheit der Schüler an International Schools ist mehrsprachig, d. h. lernt zusätzlich zu den in der Schule geforderten Sprachen (Englisch und Landessprache) weitere Fremdsprachen und spricht häufig darüber hinaus mit den Eltern noch andere Sprachen. Mehrsprachigkeit, insbesondere das Angebot zusätzlichen muttersprachlichen Unterrichts, wird von Seiten der Schulen durch Bereitstellen von Unterrichtsräumen und Unterstützung bei der Suche nach Lehrkräften gefördert, weil sich gezeigt hat, dass es Schülern, die über eine gute Basis in ihrer ersten Sprache verfügen, weniger Schwierigkeiten bereitet, ihre allgemeine Sprachkompetenz ständig zu erweitern oder eine neue Sprache zu erlernen. Eines der zentralen Erziehungsziele der International Schools ist somit die Erziehung zum „life-long-learner“, der seine Ausbildung, sein Lernen und seine Erfahrung nicht mit dem letzten Jahr der schulischen oder universitären Ausbildung beendet, sondern die ständige Erweiterung seines Erfahrungshorizontes und Wissensstandes als Teil seines Lebens versteht.

Am Beispiel der International School of Düsseldorf soll gezeigt werden, was Mehrsprachigkeit und gelebte Internationalität im Bereich Bildung bedeuten kann, welcher Stellenwert dem Fach DaF bzw. DaZ eingeräumt wird und wie Kontakte zu International Schools von Universitäten sinnvoll genutzt werden können. 


\section{$2 \mathrm{DaF} / \mathrm{DaZ} / \mathrm{Mehrsprachigkeit} \mathrm{an} \mathrm{der} \mathrm{International} \mathrm{School}$ of Düsseldorf}

\subsection{Deutsch als Fremdsprache}

Da das Erlernen der jeweiligen Landessprache für alle Altersstufen zu der Ausbildungsgrundlage der International Schools gehört, bedeutet dies, dass jede International School in Deutschland das Unterrichtsfach Deutsch als Fremdsprache in verschiedenen Lernstufen anbietet. Für die Schüler, die neu in Deutschland sind, werden Anfängerkurse angeboten. Dies gilt für Kinder im Grundschulbereich ebenso wie für Schüler in der Oberstufe. Das bedeutet, dass das Fach Deutsch als Fremdsprache in jedem einzelnen Schuljahr für Anfänger angeboten wird und dem Fach eine weitaus größere Bedeutung zukommt als einer Fremdsprache, die beispielsweise an einem Gymnasium angeboten wird, wo die Klassenstufe, in der man mit einer bestimmten Sprache beginnen kann, festgelegt ist.

In der International School of Düsseldorf werden in jeder Klassenstufe - abhängig von der Schülerzahl - die folgenden Lernstufen angeboten: Basic (Anfänger ohne Vorkenntnisse), Advanced Basic (fortgeschrittene Anfänger), Intermediate (3. Lernjahr) und Advanced (ab 4. Lernjahr). Wenn Schüler im ersten Schuljahr beginnen Deutsch zu lernen, können sie im 4. Schuljahr die Stufe Advanced erreicht haben. In dieser Stufe bleiben sie in den folgenden Jahren. Das Programm ist entsprechend darauf abgestimmt, dass einerseits die Schüler, die bereits seit Jahren am DaF-Unterricht teilnehmen, fortlaufend ihre Kenntnisse erweitern und dass andererseits Schüler, die erst seit drei Jahren Deutsch gelernt haben, in der gleichen Klasse unterrichtet werden und fachlich so unterstützt werden wie es ihren Kenntnissen entspricht. Für den Lehrer bedeutet das, dass keine Lerngruppe homogen ist. Es wird vorausgesetzt, dass die Lehrkraft das Unterrichtsprogramm den Bedürfnissen der Schüler anpasst, was bedeutet, dass Zusatzmaterial für kleine Teilgruppen der Klasse erstellt wird, um den Schülern zu helfen, Lücken zu füllen oder schwierige Aspekte der deutschen Sprache in kleinen Gruppen zu vertiefen oder einfach nur, um ihren Wortschatz gezielt zu erweitern, wenn er im Wesentlichen von den durchschnittlichen Kenntnissen der Klassenkameraden abweicht.

Der Unterricht im Fach Deutsch als Fremdsprache beschränkt sich zudem nicht nur auf die reine Sprachvermittlung, sondern schließt Informationen zur Landeskunde ebenso ein wie konkrete Möglichkeiten, die neu erlernten Aspekte im praktischen Leben anzuwenden. Die durchschnittliche Aufenthaltsdauer eines Schülers in einer International School beträgt zwischen zwei und drei Jahren. Somit haben einige Schüler, deren Eltern aus beruflichen Gründen sehr mobil sein und häufig das Aufenthaltsland wechseln müssen, bis zu ihrem siebten oder achten Schuljahr bereits in mehr als drei Ländern gelebt. Das Erlernen der jeweiligen Landessprache wird deshalb von diesen Kindern oft nicht als Chance verstanden, sich besser in die neue Umgebung integrieren zu können, sondern als zusätzliche 
Belastung oder sogar als deutliches Zeichen für die fremde Umgebung, die für die Schüler mit einem weiteren Schulwechsel, dem Verlust von engen Kontakten zu alten Freunden und Klassenkameraden und anderen Schwierigkeiten in engem Zusammenhang steht.

Schwierig ist es außerdem, wenn die Eltern die neue Sprache lediglich als notwendiges Übel sehen, das in Kauf genommen werden muss, weil die Landessprache Pflichtfach ist.

Für den Unterricht des Faches Deutsch als Fremdsprache an einer International School in Deutschland bedeutet das konkret, dass der Lehrer nicht mit einer Selbstverständlichkeit davon ausgehen kann, dass die Schüler grundsätzlich motiviert sind, die neue Sprache zu erlernen oder ihre neue Umgebung zu erkunden. Da die International Schools Ganztagsschulen sind, ist es für diese Schüler sehr oft schwierig - wenn nicht sogar unmöglich - Kontakte zu gleichaltrigen Muttersprachlern aufzubauen, weil der Tagesablauf eines Schülers einer deutschen Schule sich wesentlich von dem Tagesrhythmus eines Schülers einer International School unterscheidet. Während die Schüler eines Gymnasiums nachmittags zum Training eines Sportvereins gehen können, kommen die anderen Schüler erst am späten Nachmittag nach Hause und haben kaum mehr die Möglichkeit, nachbarschaftliche Kontakte zu knüpfen. Diese besondere Situation erschwert nicht nur das Erlernen der Landessprache, sondern die Integration und das Kennenlernen des neuen Landes, was insgesamt zu einem angenehmen Aufenthalt in diesem Land führen kann. So lässt sich auch erklären, weshalb einige Schüler, die bereits seit Jahren in Deutschland leben, kaum bessere Sprachkenntnisse aufweisen, als Schüler, die DaF als Schulfach lernen, aber in Ländern leben, die weit von deutschsprachigen Gebieten entfernt sind, so dass diese Schüler kaum jemals die Gelegenheit erhalten, ihre erlernten Sprachkenntnisse mit Muttersprachlern auszutauschen oder durch ständige Präsens der Zielsprache ihre Sprachkompetenz zu erweitern.

\subsection{Deutsch als Zweitsprache}

Eine Sprache in einem Land zu erlernen, in dem diese Sprache gesprochen wird, sollte den Lernfortschritt normalerweise im positiven Sinne erhöhen können. Dieses hängt jedoch wesentlich von verschiedenen Faktoren ab. Einerseits sind Schüler, die zum ersten Mal im Ausland leben und Deutschland als erstes neues Land kennen lernen, manchmal sehr motiviert die Sprache, die im neuen Land gesprochen wird - in diesem Fall Deutsch - zu lernen und anzuwenden, da sie häufig die Erfahrung machen, dass sie in diesem Aspekt ihren Eltern überlegen sein können, weil sie die Sprache schneller lernen als die Erwachsenen. Dabei können sie von der deutschsprachigen Umgebung profitieren. Andererseits ermöglichen ihnen die Sprachkenntnisse eine direkte Kontaktaufnahme mit gleichaltrigen Jugendlichen und somit die Möglichkeit von Integration. 
Wichtig ist auch die grundsätzliche Einstellung der Eltern. Wenn die Eltern ihren Kindern vermitteln, dass das Erlernen einer neuen Sprache nicht nur Bestandteil des neuen Stundenplans ist, sondern auch die Möglichkeiten bietet, neue Freundschaften zu knüpfen und später auf den neu erworbenen Sprachkenntnissen aufzubauen und diese Kenntnisse beruflich anzuwenden, fällt es den Schülern wesentlich leichter, die neue Sprache nicht nur anzunehmen, sondern auch als Möglichkeit für neue Erfahrungen zu verstehen. Hilfreich ist es, wenn die Eltern ihre Kinder zusätzlich dabei unterstützen, indem sie ihnen Möglichkeiten bieten, ihre erworbenen Sprachkenntnisse anzuwenden. Gerne nehmen Familien, die neu in Deutschland sind, die Gelegenheit wahr, sich in Sportvereinen anzumelden. Gemeinsames Radiohören oder Fernsehen deutscher Programme und nicht nur „übersetzter" Fernsehserien, die sie aus ihren Ländern kennen, hilft bei der Kompetenzerweiterung im Bereich Hörverstehen sowie bei der Erweiterung der mündlichen Kompetenz durch die Wortschatzerweiterung, die außerhalb des schulischen Umfeldes stattfindet. Die Schüler, die Kontakte zu Muttersprachlern haben, erweitern und festigen ihre Sprachkenntnisse wesentlich schneller als ihre Klassenkameraden. Da das Deutschprogramm parallel stattfindende Deutschklassen in verschiedenen Niveaustufen anbietet, stellt es kein Problem dar, von einer Niveaustufe zu einer anderen zu wechseln, wenn sich die Sprachkenntnisse eines Schülers schneller entwickeln als die des Klassendurchschnitts. Dies ist häufig bei Schülern, die aus anderen europäischen Ländern kommen und mit fremdsprachlichem Spracherwerb aus ihren Heimatländern vertraut sind, der Fall.

Einige Schüler, die aus skandinavischen oder aus Benelux-Ländern stammen, erreichen eine sehr hohe Sprachkompetenz, die weit über der Kompetenz eines Schülers einer DaF-Klasse liegt, aber noch nicht ganz das Sprachniveau der Muttersprachler erreicht hat, die in Native-Klassen unterrichtet werden. Für diese Schüler, die den Sprung von Deutsch als Fremdsprache zu Deutsch als Zweitsprache erreicht haben, gibt es in Klassenstufen, in denen die Zahl der Schüler mit gleichem Sprachniveau gering ist, die Möglichkeit, am muttersprachlichen Unterricht teilzunehmen um ihre guten Sprachkenntnisse zu erweitern. Wenn die Schülerzahl es zulässt, werden separate Lernstufen gebildet. Ab der 9. Klasse werden in Vorbereitung auf das International Baccalaureate Lerngruppen eingerichtet, die es den Schülern ermöglichen, sich gezielt auf die Abschlussprüfungen vorzubereiten, die für Muttersprachler A-level (A1) und für Schüler mit nahezu muttersprachlicher Kompetenz A-level (A2) genannt werden. Zusätzlich werden die Prüfungen im fremdsprachlichen Bereich (Deutsch als Fremdsprache, Spanisch, Französisch etc.) (B-level) angeboten.

\subsection{Mehrsprachigkeit}

An der International School of Düsseldorf werden zurzeit etwa 1.000 Schüler aus 40 Ländern unterrichtet. Die Unterrichtssprache für alle Fächer ist Englisch. Da 
nur etwa $35 \%$ der Schüler Englisch als Muttersprache haben, ist das Fremdsprachenprogramm in Bereich ESL (English as a Second Language) sehr umfassend und wird in allen Klassenstufen von Grundschule bis Oberstufe angeboten. Als Pflichtfach müssen die Schüler Deutsch als Landessprache lernen. Ab der sechsten Klasse ist das Erlernen einer weiteren Fremdsprache obligatorisch: Entweder wählen die Schüler Spanisch oder Französisch. Da sich gezeigt hat, dass das Erlernen und Beherrschen der Muttersprache eine wesentliche Voraussetzung für das erfolgreiche Erlernen weiterer Fremdsprachen ist, unterstützt die International School of Düsseldorf die Bemühungen der Eltern, die jeweilige Muttersprache während der Unterrichtszeit in den Stundenplan zu integrieren - wie es in Düsseldorf aufgrund der hohen Schülerzahl koreanischer und japanische Schüler möglich ist. Während des Spanisch- oder Französischunterrichts wird somit zeitgleich Koreanisch und Japanisch für Muttersprachler angeboten. Alternativ kann die Schule am Nachmittag nach dem regulären Unterricht die Unterrichtsräume anbieten, so dass auf private Initiative hin Schwedisch, Finnisch, Dänisch, Italienisch und weitere Sprachen für Muttersprachler angeboten werden können.

Das Beibehalten oder Vertiefen der Muttersprache ist auch für die schulische Laufbahn der Schüler wichtig, denn in der Oberstufe können sie ihre Muttersprache (A-level: A1) als beste Sprache für ihre International Baccalaureate Prüfung wählen, auch wenn diese Sprache nicht an der Schule unterrichtet wird. Da die Prüfungen zentral erstellt werden, ist die Wahl der Sprache unabhängig vom Angebot der jeweiligen Schule, was den Schülern zugute kommt, die unter Umständen erst in der Oberstufe das Land wechseln und dann an eine Schule kommen, die ihre Muttersprache nicht als Unterrichtsfach anbietet.

Ein englischsprachiger Schüler lernt an der International School Deutsch als Fremdsprache sowie Spanisch oder Französisch ab Klasse 6 und kann dann ab Klasse 9 und 11 noch einmal zusätzlich weitere Fremdsprachen wählen. Dieser Schüler spricht also mindestens zwei Fremdsprachen zusätzlich zu seiner Muttersprache. Ein Schüler, der erst an der International School Englisch lernt, beherrscht nach Ende seiner Schulzeit mindestens drei Sprachen als Fremdsprachen - es sei denn, dass seine Muttersprache Deutsch ist. In diesem Fall beherrscht er mindestens zwei Fremdsprachen.

Viele Schüler sind allerdings schon in ihren Familien mehrsprachig, d. h. sie sprechen mit einem Elternteil eine Sprache, mit einem anderen Elternteil eine andere Sprache, lernen unter Umständen Englisch als Fremdsprache, dann Deutsch als Landessprache und zusätzlich Spanisch, Französisch usw. Das bedeutet nicht automatisch, dass die Schüler Sprachtalente sind, die problemlos zwischen den einzelnen Sprachen hin- und herwechseln können. Manchmal sprechen sie viele Sprachen, beherrschen aber nur eine Sprache oder in wenigen Fällen auch keine Sprache mit muttersprachlicher Kompetenz. Häufig ist das der Fall, wenn die Eltern zwar zwei verschiedene Sprachen sprechen, untereinander aber in einer dritten gemeinsamen Sprache kommunizieren, die beide Elternteile nicht in mut- 
tersprachlicher Kompetenz beherrschen. Ein Beispiel: Der Vater spricht Schwedisch, die Mutter Spanisch, untereinander kommunizieren die Eltern auf Englisch, das beide unterschiedlich gut beherrschen; in der Schule lernt der Schüler deshalb Englisch auch noch als Fremdsprache, da er weder Englisch noch Spanisch oder Schwedisch gut beherrscht. Zusätzlich zum Deutschunterricht wählt er Französisch, da er im Spanischen den reinen Anfängern sprachlich überlegen ist, obwohl er die spanische Sprache nicht kompetent beherrscht. Dieser Schüler spricht im Alter von 11 Jahren 5 Sprachen, keine jedoch in vergleichbarer muttersprachlicher Kompetenz. Die asiatischen Schüler, die entweder Japanisch oder Koreanisch sprechen, erhalten zusätzlich zu ihrem Englisch- und Deutsch-als-FremdspracheUnterricht muttersprachlichen Unterricht, der ihnen helfen soll, ihre allgemeine Sprachkompetenz zu erweitern.

Im Alltag bedeutet diese besondere Situation für die Schüler, dass ihnen Mehrsprachigkeit nicht nur aus ihrer familiären Situation, sondern auch aus dem Schulalltag vertraut ist. Im Unterricht - außer in den Fremdsprachenklassen - sprechen sie Englisch, in den Pausen unterhalten sie sich häufig in ihrer Muttersprache, aber sobald Schüler dazukommen, die diese Sprache nicht sprechen, wechseln sie mit Selbstverständlichkeit zur gemeinsamen Sprache Englisch. Oft bieten die Pausen die einzige Gelegenheit, ihre Muttersprache außerhalb ihrer Familie zu praktizieren. Vielen Schülern vermittelt diese Gelegenheit ein Gefühl von Sicherheit, das für sie gerade zu Beginn eines neuen Schuljahres in fremder Umgebung und in einem Land mit unbekannter Sprache wichtig ist.

Gefördert wird die Mehrsprachigkeit von Seiten der Schulorganisation durch das Einrichten zahlreicher muttersprachlicher Zusatzklassen, so dass Schüler ihre Muttersprache - häufig auch „beste Sprache“ genannt - vertiefen können. Im täglichen Schulalltag gibt es von den Fremdsprachenlehrern geförderte Projekte und Ausstellungen, in denen die Schüler ihren Mitschülern zeigen können, was sie in einer anderen Sprache erstellen können. Wichtig ist dabei nicht, ob ihre Mitschüler die Texte verstehen können, sondern es geht um die Wahrnehmung, die Kenntnis und damit auch die Akzeptanz anderer Sprachen und Kulturen. Die Schüler geben gerne Präsentationen in verschiedenen Sprachen, die bei diversen Versammlungen vor der gesamten Schüler- und Lehrerschaft vorgetragen werden. Bei zahlreichen Schulfesten und -veranstaltungen repräsentieren auch die Eltern der Schüler nicht nur ihr Land, sondern auch ihre Sprache und Kultur. Somit finden alle Sprachen ihren Stellenwert und keine Sprache ist bedeutender als die andere. Hilfreich ist es auch - nicht nur bei Elternsprechtagen - wenn die Lehrkräfte mehrere Sprachen beherrschen, weil dies den Schülern einerseits zeigt, dass es durchaus möglich ist, mehr als eine Sprache zu beherrschen, und andererseits, dass die Lehrer, die neu im Land sind, auch Fortschritte beim Erlernen der neuen Sprache machen, was ihnen die Integration und somit das Wohlfühlen in der neuen Umgebung erleichtert. 
Das alles trägt zu einer größeren Akzeptanz fremder Sprachen und Kulturen bei, die für Schüler an International Schools zum selbstverständlichen Bestandteil ihres Alltags wird.

\section{Voraussetzungen für Mehrsprachigkeit und Internationalität}

Eine Grundvoraussetzung für ein erfolgreiches Unterrichtsprogramm, in dem Mehrsprachigkeit eine Rolle spielt, ist die grundsätzliche Akzeptanz gegenüber anderen Sprachen und Kulturen. Viele Schüler lernen erst in ihrer schulischen Umgebung eine Vielzahl von bisher unbekannten Sprachen kennen, wenn sich ihre Mitschüler untereinander in ihrer Muttersprache unterhalten. Für einige der Schüler kann diese Situation eine besondere Motivation bedeuten, die sie ermuntert, diese Sprache auch zu erlernen. Die grundsätzliche Einstellung der Eltern und anderer Familienmitglieder spielt dabei eine nicht unbedeutende Rolle. Wenn die eigene Familie Neugier gegenüber fremden Kulturen besitzt und das Kennenlernen des „Fremden“ als etwas Positives vermittelt, fällt es den Kindern wesentlich leichter, neue Sprachen, fremde Umgebungen und Kulturen nicht nur zu akzeptieren, sondern interessant zu finden, was helfen kann bei dem Interesse, mehr über die jeweilige Sprache und Kultur zu erfahren. Fehlt diese Unterstützung durch die Familien oder etwa die Akzeptanz anderen Kulturen und Sprachen gegenüber, so kommt der Motivation des Schülers eine größere Bedeutung zu.

Die Bedeutung von Fremdsprachenkenntnissen und die Motivation mehr darüber zu erfahren oder sich sogar Kenntnisse dieser Sprache anzueignen, kann durch die Anwendbarkeit des Gelernten im täglichen Leben zusätzlich erhöht werden. Im konkreten Fall bedeutet dies beispielsweise, dass ein Schüler, der die mitgebrachten Süßigkeiten eines anderen Schülers probieren möchte, sehr schnell die Begriffe für „danke“ und „bitte“ unabhängig davon lernt, ob diese Sprache an seiner Schule unterrichtet wird oder nicht. Diese positiven Lernerfahrungen, die außerhalb des Unterrichts stattfinden, helfen den Schülern nicht nur schnelle Lernerfolge zu erzielen, die ohne zusätzlichen Druck in Form von Notengebung stattfinden. Sie unterstützen gleichzeitig die Möglichkeiten eigene Mehrsprachigkeit zu entwickeln.

Eine Gelegenheit fremde Sprachen und Kulturen kennenzulernen, bietet sich schnell an: Sehr oft sind Eltern von Schülern gerne bereit, den Klassenkameraden ihrer Kinder etwas über ihre Heimat zu erzählen, ihnen Spezialitäten mitzubringen und ihnen in ihrer Sprache vorzusingen oder auch Ausdrücke wie Begrüßungen beizubringen. Andere Kulturen auf diese Weise kennenzulernen ist mit geringem finanziellem Aufwand zu realisieren. Die Akzepetanz fremder Kulturen kann gerade über den Weg des Kennenlernens ausländischer Spezialitäten gelingen. In Schulen und Universitäten bietet es sich an, Vertreter verschiedener Kulturen einzuladen und um Information über ihr Land zu bitten. 
Das Bereitstellen von Unterrichtsräumen für von Eltern organisierten muttersprachlichen Unterricht ist eine weitere Möglichkeit Mehrsprachigkeit ohne den Einsatz finanzieller Mittel zu fördern. Anstelle von ausgebildeten Lehrkräften, die für den Unterricht bezahlt werden müssen, ist es manchmal - insbesondere für junge Lerner - angebracht, Spielgruppen zu organisieren, in denen die gewünschte Sprache von Muttersprachlern gesprochen wird. Dabei sollte nicht die didaktische Schwerpunktsetzung im Vordergrund stehen, wenn Lehrkräfte entweder nicht zur Verfügung stehen oder nicht bezahlt werden können. Oft ist es insbesondere für kleinere Kinder wichtig, überhaupt die Muttersprache außerhalb ihrer Familie hören und sprechen zu können und somit ihre Sprachkenntnisse vertiefen zu können.

Eine weitere Möglichkeit Mehrsprachigkeit ohne den Einsatz aufwändiger finanzieller Mittel zu organisieren, ist die Organisation eines Treffens von Interessierten, die entweder die angebotene Sprache als Muttersprache sprechen, Interesse an dieser Sprache bekunden oder bereits vorhandene Sprachkenntnisse vertiefen möchten. An der International School of Düsseldorf hat es zahlreiche Treffen für verschiedene Sprachen gegeben, bei denen beispielsweise Lesematerial, d. $h$. Zeitschriften für Kinder, Jugendliche oder Erwachsene, Bücher etc. ausgetauscht werden. Für kleinere Besucher werden Spiele angeboten, die von älteren Jugendlichen in ihrer Muttersprache erklärt und gezeigt werden. Für die Erwachsenen bietet sich die Gelegenheit sich untereinander kennenzulernen und vorhandene Sprachkenntnisse anzuwenden. Das bedeutet für einige Teilnehmer, dass sie ihre Muttersprache sprechen und für andere Besucher, dass sie eine Fremdsprache in zwangloser Umgebung sprechen oder lernen können.

\section{Konsequenzen für das Fach Deutsch als Fremdsprache/ für den FaDaF / für Universitäten}

Da der Unterricht im Fach Deutsch als Fremdsprache an allen 18 Internationalen Schulen in Deutschland unterrichtet wird und circa 10.000 Schüler diese Sprache erlernen, ist eine entsprechend hohe Zahl von Lehrern mit einer Ausbildung und mit Unterrichtserfahrung im Fach Deutsch als Fremdsprache nötig. Diese große Zielgruppe ist bisher auf den FaDaF-Tagungen kaum berücksichtigt worden. Dies mag einerseits daran liegen, dass die Tagungen und jeweiligen Schwerpunktthemen sich auf den universitären und weniger auf den schulischen Bereich beziehen, andererseits sicherlich auch, weil vielen Lehrkräften, die an International Schools unterrichten, der Fachbereich Deutsch als Fremdsprache nicht vertraut ist. Einige Lehrkräfte sind für den DaF-Unterricht eingestellt worden, weil sie muttersprachliche Kompetenz besitzen, nicht aber, weil sie Unterrichtsmethoden oder Fachdidaktik im Bereich Fremdsprachenvermittlung beherrschen. Die mittlerweile zahlreichen Möglichkeiten, sich im Bereich DaF aus- und fortzubilden, sind nicht überall bekannt. Sinnvoll wäre deshalb eine bessere Zusammenarbeit, die es dieser Zielgruppe ermöglichen würde, die universitäre Ausbildung und die angebotenen 
Fachtagungen zu nutzen und die eigene fachliche Kompetenz zu erweitern. Für den $\mathrm{FaDaF}$ und seine Mitglieder, aber auch für die Universitäten, die $\mathrm{DaF}$ als Studienfach anbieten, können sich Möglichkeiten für enge Zusammenarbeit ergeben, die für beide Seiten fruchtbare Ergebnisse erzielen können.

Die Heinrich-Heine-Universität Düsseldorf vermittelt seit einigen Jahren sehr erfolgreich Praktikanten, die im Rahmen ihres DaF-Studiums ihr Praktikum an der International School of Düsseldorf absolvieren. Die International Schools suchen immer wieder qualifizierte Lehrkräfte. Da die Stellensituation im Bereich des Fremdsprachenunterrichts insgesamt schwierig ist, bietet sich eine engere $\mathrm{Zu}$ sammenarbeit mit den International Schools in Deutschland und dem deutschsprachigen Ausland an, da die Schulen auch häufig Stellenangebote mit unbefristeten Verträgen anbieten können. Eine Unterstützung in der Qualitätssicherung im Fachbereich Deutsch als Fremdsprache wäre sicherlich auch ein interessanter Aspekt für eine sinnvolle Zusammenarbeit.

Dieser Beitrag soll einerseits ein Beispiel für ein erfolgreiches Miteinander zahlreicher Sprachen und Kulturen aufzeigen, andererseits den DaF-Unterricht und die Mehrsprachigkeit an der International School of Düsseldorf darstellen und nicht zuletzt als Anregung dienen, einen engeren Kontakt zwischen Schule und Universität zu knüpfen um beiderseits davon profitieren zu können.

\section{Nützliche Websites:}

www.ecis.org (European Council of International Schools)

www.ibo.org (International Baccalaureate Organization)

www.agis-schools.org (Association of German International Schools) 


\section{Korrekturen und Ausdruckshilfen im Deutsch-als Zweitsprache-Unterricht. Fallstudien und Verglei- che mit anderen Kontexten der Sprachförderung}

Martina Rost-Roth, Berlin

\section{Einführung}

Im Rahmen des DFG-Projektes „Förderunterricht und Deutsch-als-Zweitsprache-Erwerb" steht die Untersuchung von mündlichen Sprachkompetenzen im Vordergrund. Im Zentrum des vorliegenden Beitrags steht die Frage, wie Ausdrucksmöglichkeiten im Förderunterricht ,Deutsch als Zweitsprache' so gestaltet werden können, dass sie möglichst günstige Bedingungen für eine Erweiterung der Sprachkompetenzen darstellen. Ausgangspunkt sind Beobachtungen und Analysen zu Situationen im Förderunterricht der 3. und 4. Grundschulklasse. Auf der Basis von gesprächsanalytischen Kriterien werden Ausdrucksmöglichkeiten und Korrekturen sowie der Umgang mit Ausdrucksschwierigkeiten analysiert und Schlussfolgerungen für eine Effektivierung der Unterrichtinteraktion abgeleitet. 


\section{Forschungshintergrund}

Kommunikation im Fremdsprachenunterricht ist bereits ein traditionsreicher Untersuchungsbereich. Das Spektrum der Untersuchungen reicht von quantifizierenden Analysen des gesamten sprachlichen Handelns (z. B. Flanders 1970, Fanselow 1977) bis hin zu detaillierten Analysen von einzelnen sprachlichen Handlungen (z. B. Gumperz/Herasimhuk 1975). Auch zu einzelnen Aspekten der Interaktion wie insbesondere Korrekturen im Fremdsprachenunterricht gibt es zahlreiche Veröffentlichungen; von empirischen Analysen (Chaudron 1977, Kleppin/Königs 1991, Rost-Roth 1989, Lochtman 2002 oder Blex 2003) bis hin zu Korrekturempfehlungen wie sie in der Fernstudieneinheit ,Fehler und Fehlerkorrektur' ausgesprochen werden (vgl. Kleppin 1998).

Ausdruckshilfen sind hingegen noch weniger untersucht (vgl. hierzu auch Rost 1989, Rost-Roth 1995 und 1999). Auch speziell in Hinblick auf Interaktionen im Förderunterricht Deutsch als Zweitsprache gibt es noch kaum empirische Studien. Publikationen gibt es hier vor allem in Form von Erfahrungsberichten und Projektdarstellungen. Auch Korrekturen und Feedback sind für den DaZ-Bereich noch vergleichsweise wenig thematisiert, (dies gilt $u$. a. auch für didaktische Literatur in der Deutschlehrerausbildung, in der im Allgemeinen eher pauschale Empfehlungen gegeben werden, vgl. (Rösch 2004: 37f.).

Nichtsdestotrotz scheinen aufgrund zweitspracherwerbstheoretischer Annahmen und aktueller Diskussionen der Sprachlehr- und -lernforschung Interaktionen und Reaktionen der Unterrichtenden auf lernersprachliche Äußerungen bezogen auf den DaZ-Unterricht von Interesse. Dem freien Sprechen und mündlichen Ausdruck wird nach der sog. ,kommunikativen Wende wie in anderen Bereichen der Sprachförderung große Bedeutung beigemessen.

\section{Methodisch-theoretischer Rahmen}

Die hier interessierenden sprachlichen Aktivitäten der Unterrichtenden werden als Reparaturen im Sinne der ethnomethodologischen Konversationsanalyse analysiert. Hierüber hat man nicht nur ein methodisch konsistentes und weitreichenes Analyseinstrumentarium (vgl. ausführlich Rost 1989 und Rost-Roth 1995), auf dieser Beschreibungsebene lässt sich sprachliche Interaktion im Unterricht auch in Bezug auf Erwerbsprozesse und deren Förderung im zweitsprachlichen Unterricht in Verbindung mit Erwerbstheorien diskutieren.

Zur Erörterung von Erwerbspotentialen und Möglichkeiten der Förderung von Erwerbsprozessen sind weiter Theorien des Zweitspracherwerbs von Interesse. Hierbei wird an lernersprachliche Ansätze in der Tradition des InterlanguageKonzeptes angeknüpft (vgl. hierzu Kasper 1989 und Klein 2001), in der Lernersprachen als eigenständige Systeme angesehen werden, die sich in Richtung auf Zielsprachen entwickeln können (Selinker 1972). Als wesentlich für Erwerbs- 
prozesse wird dabei angesehen, dass Lerner Hypothesen bilden und testen und dass auch sprachliche Interaktion und Feedback Auswirkungen auf die Entwicklung von lernersprachlichen Kompetenzen haben. Vor diesem Hintergrund interessiert vor allem auch die Frage, inwiefern die Unterrichtsinteraktion und LehrerFeedback geeignet sind, die Entwicklung des lernersprachlichen Systems und mündliche Kompetenzen zu fördern.

\section{Datengrundlage}

Datengrundlage ist das Projekt „Förderunterricht und Deutsch-als-ZweitspracheErwerb. Eine longitudinale Untersuchung zur mündlichen Sprachkompetenz bei Schülerinnen und Schülern nicht-deutscher Herkunftssprache (ndH) in Berlin“ (im Folgenden ,FöDaZ) an der Technischen Universität Berlin¹. Im Rahmen des Projektes werden mündliche Kompetenzen und der Spracherwerb bei insgesamt 37 Kindern an zwei Berliner Grundschulen untersucht, wobei es sich bei den NichtmuttersprachlerInnen um SchülerInnen handelt, die Förderunterricht erhalten $^{2}$. Als Vergleichsgruppe wurden auch muttersprachliche Kinder in die Untersuchung einbezogen. Die Datenerhebung umfasst Einzelaufnahmen mit unterschiedlichen Aufgabenstellungen sowie Unterrichtsaufzeichnungen und erstreckt sich über 2 Jahre vom Beginn der 3. Klasse bis zum Ende der 4. Klasse.

Grundlage für die hier dargelegten Fallstudien sind Unterrichtsphasen aus dem DaZ-Förderunterricht, in denen der mündliche Ausdruck der LernerInnen im Vordergrund steht. Die Teilnehmerzahl schwankt zwischen 3 und 7 SchülerInnen. In den dokumentierten Unterrichtssituationen konnten im Wesentlichen drei Arten von Sprechanlässen beobachtet werden:

- Erlebnisbezogenes oder thematisch vorgegebenes Erzählen (ERZ),

- Texte oder Bilder als Sprechanlässe (T/B),

- Sprechanlässe, mit Impulsen, die die Produktion bestimmter Sprachstrukturen anregen sollen (z. B. Gebrauch von Präpositionen) (SPR).

Dokumentiert sind Unterrichtssituationen von 3 (weiblichen) Unterrichtenden (U1, U2, U3) an zwei Berliner Grundschulen Übersicht 1).

\footnotetext{
${ }^{1}$ Laufzeit dieses von der Deutschen Forschungsgemeinschaft geförderten Projektes ist 2003-2006, die Leitung hat Ulrich Steinmüller, Co-Leitung Bernt Ahrenholz, Mitarbeiterinnen sind Beate Lütke und Martina Rost-Roth. Genauere Angaben zur Konzeption und Datenerhebung des Projektes finden sich in Ahrenholz (2003) sowie Ahrenholz (2006).

2 Die Zahl der Informanten ermöglichte es, trotz ,Ausfällen` wie z. B. Wegzug eine ausreichende Zahl von Schülerinnen und Schülern über einen Zeitraum von zwei Jahren untersuchen zu können. Ausgangssprachen sind u. a. Türkisch, Russisch, Arabisch und Polnisch.
} 


\begin{tabular}{|l|l|l|l|l|l|}
\hline & Thema & Min. & $\begin{array}{l}\text { Sprach- } \\
\text { orientiert }\end{array}$ & Erzählen & Texte/Bilder \\
\hline U1 & Moschee & 15 & & $\mathrm{X}$ & \\
\hline U1 & Nikolaus I & 20 & & & $\mathrm{X}$ \\
\hline U1 & Nikolaus II & 9 & & $\mathrm{X}$ & $\mathrm{X}$ \\
\hline U1 & Morgenkreis & 1 & & $\mathrm{X}$ & \\
\hline U1 & Adjektiv-Paare & 8 & $\mathrm{X}$ & & \\
\hline U1 & Zwei Wiewörter & 7 & $\mathrm{X}$ & & \\
\hline U2 & Zimmer & 7 & $\mathrm{X}$ & $\mathrm{X}$ & \\
\hline U2 & Bild I & 8 & & & $\mathrm{X}$ \\
\hline U2 & Bild II & 3 & & & $\mathrm{X}$ \\
\hline U3 & Morgenkreis & 4 & & $\mathrm{X}$ & \\
\hline U3 & Richtungswörter & 8 & $\mathrm{X}$ & & \\
\hline U3 & Präpositionen & 5 & $\mathrm{X}$ & & \\
\hline
\end{tabular}

Übersicht 1: Fallstudien zu freiem Sprechen im DaZ-Förderunterricht

Im Folgenden soll nun genauer betrachtet werden, welcher Art die hier zu beobachtenden Reaktionen von Unterrichtenden in Phasen freien mündlichen Ausdrucks sind.

\section{$5 \quad$ Korrekturen und Ausdruckshilfen - Reparaturen}

In der vorliegenden Untersuchung wird auf einen Analyserahmen zurückgegriffen, der bereits mit Bezug auf andere Unterrichtskontexte zur Anwendung kam und hierüber Vergleichsmöglichkeiten mit anderen Kontexten der Sprachförderung eröffnet. Zum einen sind dies Beobachtungen zum Sprachunterricht mit Erwachsenen in Kleingruppen, wie er z. B. von Volkshochschulen oder Sprachinstituten angeboten wird (vgl. Rost 1989), zum anderen sind dies Beobachtungen zum Sprachenlernen im Tandem (vgl. Rost-Roth 1995).

Korrekturen im engeren Sinne, d. h. Reaktionen auf Äußerungen, die etwas als fehlerhaft behandeln, können im Prinzip auf zwei Arten durchgeführt werden:

a) indem die Person, die auf einen Fehler reagiert, diesen selbst berichtigt,

b) indem die Person, die auf den Fehler hinweist, die Person, die die als fehlerhaft angesehene Äußerung produziert hat, selbst korrigieren lässt. Man spricht hier von Selbst- und Fremdkorrektur und kann diesen Unterschied auch im übergeordneten Konzept der ,Reparatur' der Konversationsanalyse fassen ${ }^{3}$.

\footnotetext{
3 Zu den Grundlagen vgl. Schegloff /Jefferson/ Sacks 1977, die Reparatur ('repair') als interaktiven Mechanismus der Störungsbehandlung beschreiben. Als Störung ('trouble') können unterschiedliche Arten von Problemen wie Fehler, Irrtümer unterschiedlichster Art, Verständnisschwierigkeiten oder Ausdrucksschwierigkeiten u.v.m. in Erscheinung treten.
} 
In der Konversationsanalyse werden vier Arten von Reparaturen, je nach Art der Initiierung und der Durchführung des Reparaturschritts unterschieden (ausführlicher hierzu vgl. Rost 1989 und Rost-Roth 1995).

Zunächst ein Beispiel für ,Fremdinitiierte Fremdkorrektur ${ }^{4}$ :

*S1: $\quad<$ ein junge $>[/ /]$ ich beschreibe ein junge

*U: jungen

*S1: ja, jungen, der trägt ein käppi,

*U: $\quad$ woran erkennen wir die Barbara?

$<$ name S4>

*S4: $\quad$ bat einen rock an

*U: ja, sie trägt einen rock

Die Unterrichtende initiiert hier die Korrektur und berichtigt den Fehler im Sinne einer Fremdreparatur.

In den folgenden Beispielen für ,fremdinitiierte Selbstkorrekturen' werden hingegen die Lerner aufgefordert, den Fehler selbst zu korrigieren, d. h. es handelt sich um Fremdinitiierung und Selbstkorrektur.

*S1: ja, jungen, der trägt ein käppi,

der hat roller [/] rollerskater,

er geht von links nach rechts

*U: geht er von links nach rechts?

*S1: nein, fährt

*U: $\quad$ er fährtT von links nach rechts,

*S: $\quad$ \# und \# ähm \# \#̈ber mein Bett' \# ist ein großer Scbrank -', $\%$ COM:Kind pfeifft

*U2: \# \# über deinem Bett -?

*S: nein .

*S: $\quad$ ähm rechts neben meinem + /

Entsprechende Korrekturaufforderungen können sich auch an einzelne andere Teilnehmer oder die gesamte Lerngruppe richten (Delegation der Korrektur).

\footnotetext{
${ }^{4}$ Mit U bzw. U1 etc. wird hier auf die Unterrichtenden verwiesen, mit S bzw. S1 auf SchülerInnen; Kommentarzeilen sind mit \%COM gekennzeichnet. Die Tonband- und Videoaufzeichnungen wurden in Anlehnung an CA-CHAT transkribiert. Vgl. hierzu die Transkriptkonventionen im Anhang.
} 


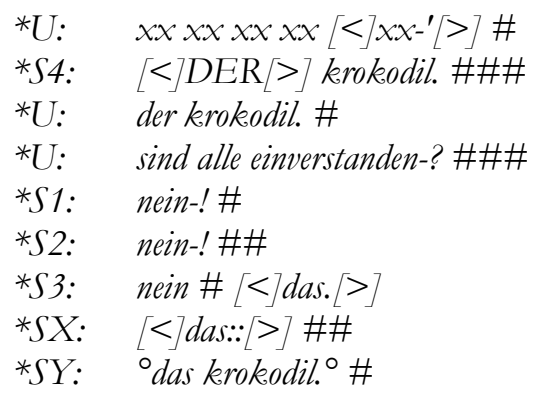

Während es sich bei Fremdkorrektur vs. Aufforderung zur Selbstkorrektur um Unterschiede handelt, die in der fremdsprachendidaktischen Diskussion schon recht weitgehend diskutiert wurden, hat die Komponente der Reparaturinitiierung noch weniger Beachtung gefunden. Als Initiierung einer Reparatur im Sinne der Konversationsanalyse gelten alle Anzeichen und Signale, aus denen hervorgeht, dass der Produzent der Äußerung selbst diese in irgendeiner Weise als problematisch ansieht. Anzeichen dieser Art können Zögern, Abbrüche, Wiederholungen und Paraphrasierungen oder andere Bekundungen der Unsicherheit sowie des Nicht-Wissens sein. Dabei sind in Hinblick auf die Durchführung des Reparaturschritts wiederum zwei Möglichkeiten zu unterscheiden.

Im Falle einer selbstinitiierten Fremdreparatur wird der Reparaturschritt vom Gesprächspartner - hier U - übernommen.

*S6: $\quad$ da ham wir uns so in den bus gesessen [//] gese

*U: $\quad{ }^{\text {gesettrt }} t^{\circ}$

mehrere: gesetzt

Bei Selbstinitiierungen ist es auch möglich, dass die Lerner sich selbst berichtigen; in diesen Fällen handelt es sich um sog. selbstinitiierte Selbstreparaturen, auch bezeichnet als Selbstkorrekturen, die jedoch hier weniger interessieren, da die Befassung mit der Interaktion zwischen Lernern und Unterrichtenden im Zentrum des Interesses steht. Von Bedeutung ist jedoch für Unterrichtskontexte eine weitere Unterscheidung, auf die vor allem in Rost (1989) hingewiesen wurde, nämlich ob die Selbstiniitierungen der Lerner gewissermaßen interaktive Obligationen für die Unterrichtenden implizieren. Dies ist z. B. dann der Fall, wenn eine Frage gestellt wird. Grenzfälle stellen Äußerungen wie die folgende dar, denn hier wird nur Nicht-Wissen bekundet, entsprechende Äußerungen werden jedoch oft - wie im folgenden Beispiel - als Hilfsappelle behandelt:

*S: $\quad$ ich hab vergessen wie das heißt -

*U3: das Regal -; 
In Fällen wie diesen liegt eine Ausdruckshilfe im Sinne einer selbstinitiierten Fremdreparatur vor.

In Hinblick auf grundsätzlichere Erwägungen der Fremd- und Zweitsprachendidaktik und Analysen von Unterrichtskommunikation scheint es sinnvoll, gerade auch den letztgenannten Reparatur-Möglichkeiten, d. h. Selbstinitiierungen und Ausdruckshilfen, mehr Aufmerksamkeit zu widmen. Zum einen birgt dies die Chance, Lernersprachen und Sensibilitäten der Lerner für sprachliche Problembereiche stärkere Beachtung zu schenken. Damit lässt sich auch eine Sichtweise überwinden, die Feedback oft verengt als Reaktionen auf Fehler behandelt. Von Bedeutung ist diese Perspektive auch in Hinblick auf die an Zweitspracherwerbstheorien anknüpfende Annahme, lernersprachlichen Ausdruck als Potential zur Sprachentwicklung zu nutzen.

Ein Vergleich der Unterrichtssituationen zeigt, dass die verschiedenen Arten von Reparaturen mit unterschiedlicher Häufigkeit zum Einsatz kommen (vgl. Übersicht 2).

\begin{tabular}{|l|l|l|l|l|l|}
\hline & $\begin{array}{l}\text { Selbst- } \\
\text { korrektur }\end{array}$ & $\begin{array}{l}\text { Fremd- } \\
\text { korrektur }\end{array}$ & $\begin{array}{l}\text { Korrektur- } \\
\text { Delegation }\end{array}$ & sonstige & Summe \\
\hline U1 T/B & 14 & 3 & 0 & 4 & 21 \\
\hline U1 SPR & 7 & 2 & 0 & 1 & 10 \\
\hline U2 T/B & 0 & 3 & 0 & 0 & 3 \\
\hline U2 SPR & 3 & 0 & 1 & 0 & 4 \\
\hline U3 ERZ & 4 & 5 & 0 & 0 & 9 \\
\hline U3 SPR & 17 & 8 & 8 & 3 & 36 \\
\hline Summe & 45 & 21 & 9 & 8 & 83 \\
\hline
\end{tabular}

Übersicht 2: Häufigkeiten von Selbst- und Fremdkorrekturen sowie Korrektur-Delegationen

Aufforderung zur Selbstkorrektur wird bei zwei Unterrichtenden deutlich häufiger eingesetzt als Fremdkorrektur, bei einer Unterrichtenden gleich häufig. Korrekturdelegationen sind hier nur in einigen Situationen zu beobachten.

\section{Sprachbereiche}

Zur Analyse des Erwerbspotentials ist des Weiteren von Interesse, auf welche Sprachbereiche sich die Interventionen der Unterrichtenden, d. h. Reparaturinitiierungen im Sinne von Fremdkorrekturen und Aufforderungen zu Selbstkorrekturen richten. Hier können in Anlehnung an Chaudron (1977) folgende Sprachbereiche unterschieden werden: 
- morphologische Korrekturen:

*S: $\quad$ da hab ich mich weh getan -

*U: \#\# habe ich mir weh getan -

(S2 DaZ-Ur 040604 MK)

- lexikalische Korrekturen:

*S: $\quad$ das ist von dieser frau-' \# und diesen mann-' \#\#

*S: das haus-' \#\#

*U: ja-? \#\#\#

*S: $\quad$ es ist ein [/]ein prinz̧aus-? +... \#\#

*U: $\quad$ mh hmm. \#\#\#

*U: $\quad$ ein schloss-? \#\#

*S: ja:- \#\#\#

(S2 DaZ-Ur 170105 BB)

- phonetische Korrekturen:
S: $\quad$ Barbara
U: $\quad$ wie heißt der name?
S: $\quad$ Barbara
S: $\quad$ Barbara
U: $\quad$ die' Barbara, ganz genau.

(S2 Daz-Ur 040604)

- inhaltliche Korrekturen:

*S: $\quad$ der könig haltet die post von den junge-' \#

*U: ja- \#\#

*S: $\quad$ und die frau haltet da: \#\# eintausend euro. \#2\#

*U: $\quad$ ob das euro sind-' \#\#

*U: $\quad$ könnte sein. \#\#

*U: könntn auch TALER sein. \#\#

*U: $\quad$ oder \# ich weiß nicht $[<]$ was. $[>] \#$

*S: $\quad[<$ Joder gold. $[>] \# \#$

*U: $\quad$ aber aufjeden fall \#\#\#

*U: $\quad$ man kann sie gut erkennen. \#\#

*U: $\quad$ sie sind geld. \# na-? \#

*U: richtig. \#

(S2 DaZ-Ur 170105) 
- Diskursfehler:

*U: aha, wie viel personen kommen von links, <Name *S1>

*S1: $\quad \underline{\text { sechs }}$

*U: $\quad$ sprich bitte den sat;.

*S1: $\quad$ sechs kommen von links.

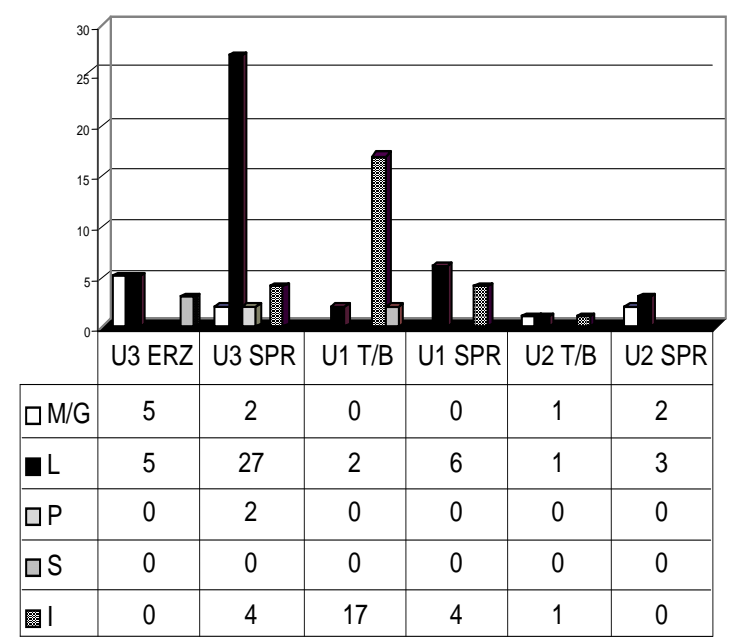

Diagramm 1: Korrigierte Fehlerarten (in absoluten Zahlen)

Die Übersicht zu den einzelnen Situationen in Diagramm 1 zeigt, dass es recht unterschiedliche Gewichtungen geben kann. Auffallend ist, dass lexikalische (L) und inhaltliche (I) Fehler in zwei Unterrichtssituationen im Vordergrund stehen.

Für morphologische $(\mathrm{M} / \mathrm{G})$ Fehler zeigt sich, dass diese relativ selten korrigiert werden, syntaktische (S) Fehler überhaupt nicht und auch phonologische/ phonetische (P) Fehler nur in Ausnahmen ${ }^{5}$.

\section{$7 \quad$ Vergleich mit anderen Unterrichtskontexten}

Im Folgenden werden die Befunde zu DaZ-Förderunterricht mit Befunden zu anderen Unterrichtskontexten verglichen. Zum einen werden Ergebnisse freier

${ }^{5}$ Dass dies nicht auf den lernersprachlichen Ausdruck zurückzuführen ist, zeigen andere Analysen des Projektes sowie auch die vorausgehenden Beispiele. Die Lernersprachen sind hier durch vielfältige Abweichungen auch in den nicht korrigierten Bereichen gekennzeichnet. Vgl. hierzu auch RostRoth (2006) und Lütke (2006). 
Konversationen im Gruppenunterricht mit Erwachsenen herangezogen (FKErw, vgl. Rost 1989), zum anderen Tandem-Interaktionen (Tandem, vgl. Rost-Roth 1995).

Der Vergleich der Unterrichtskommunikation mit anderen Kontexten der Sprachförderung in Hinblick auf Reparaturen zeigt in verschiedenen Punkten Unterschiede. In Bezug auf die korrigierten Fehlerarten ergibt sich, dass die Korrektur morphologischer und anderer grammatischer Fehler in anderen Kontexten durchschnittlich häufiger ist (vgl. Diagramm 2).

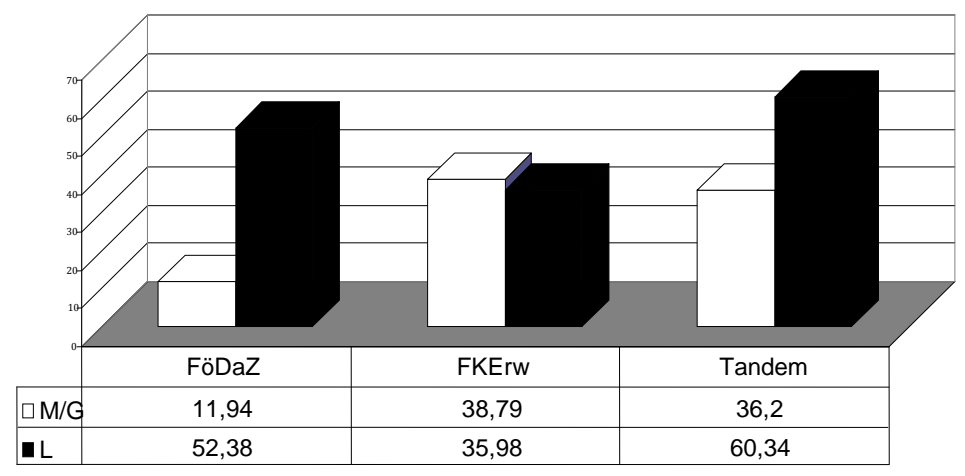

Diagramm 2: Korrigierte Fehlerarten - Sprachförderkontekxte im Vergleich (M/G= Morphologie/Grammatik, L= Lexik, prozentuale Anteile) ${ }^{7}$

\footnotetext{
${ }^{6}$ In Rost 1989 wurden 12 Unterrichtsituationen mit verschiedenen Unterrichtenden an Volkshochschulen und anderen Sprachschulen und Einrichtungen im Bereich 'Deutsch für Ausländer' analysiert. In Rost-Roth 199512 Situationen aus Tandem-Interaktionen in deutsch-italienischen TandemPaaren, so dass sich eine gewisse Vergleichbarkeit mit den hier untersuchten 12 Situationen (die allerdings insgesamt kürzer sind) ergibt.

${ }^{7}$ Es handelt sich hier um pauschale Vergleiche, in denen Durchschnittswerte für verschiedene Unterrichtssituationen verglichen werden. Auf Variationsspektren in den Vergleichskontexten kann hier aus Platzgründen nicht eingegangen werden.Vgl. hierzu jedoch die Analysen in Rost (1989) und Rost-Roth (1995) sowie (1999).
} 
Auch in Bezug auf die Häufigkeiten von Aufforderungen zu Selbstkorrekturen zeigen sich Unterschiede (vgl. Diagramm 3).

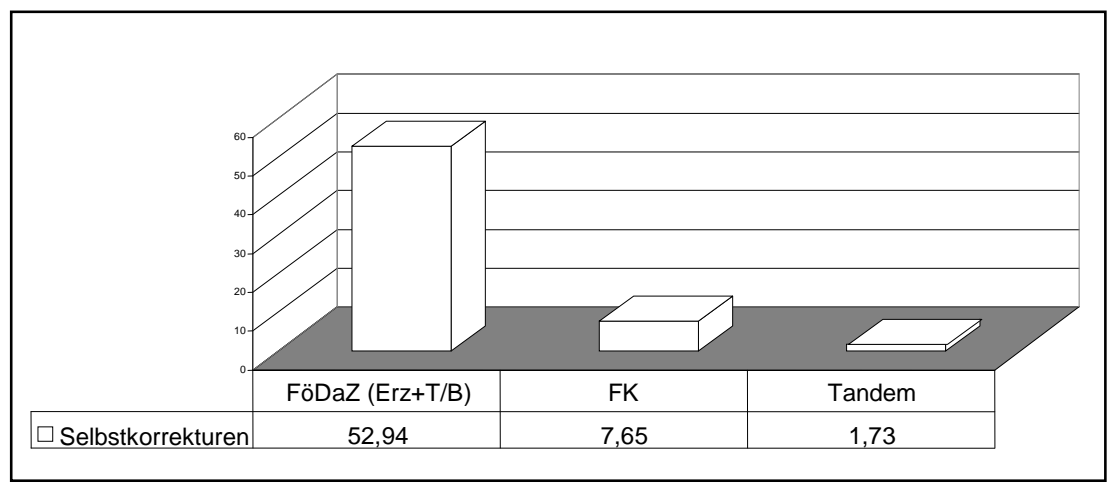

Diagramm 3: Anteile von Aufforderungen zu Selbstkorrekturen und KorrekturDelegationen an Korrekturen insgesamt (in Prozent) - Sprachförderkontexte im Vergleich

Aufforderungen zu Selbstkorrekturen sind im DaZ-Förderunterricht wesentlich häufiger als in den anderen Sprachförderkontexten. Dies mag unter anderem darauf zurückgeführt werden, dass dieser Art von Korrektur in der diaktischen Diskussion - vor allem auch bezogen auf den schulischen Bereich - relativ hohe Wirksamkeit zugeschrieben wird, da die kognitive Aktivierung und Beteiligung der Lerner hier am größten ist. Der Einsatz der Selbstkorrektur passt nicht zuletzt auch in das gängige Schema von dreischrittiger Unterrichtskommunikation und Aufgabe-Lösungssequenzen mit abschliessender Lehrerbewertung ${ }^{8}$. In anderen Situationen scheint hingegen eine Unterbrechung wie sie mit dieser Art der Reparatur einhergeht, eher gemieden zu werden.

8 Vgl. Rost (1989: 179ff). 
Auch Ausdruckshilfen sind mit unterschiedlicher Häufigkeit repräsentiert Diagramm 4).

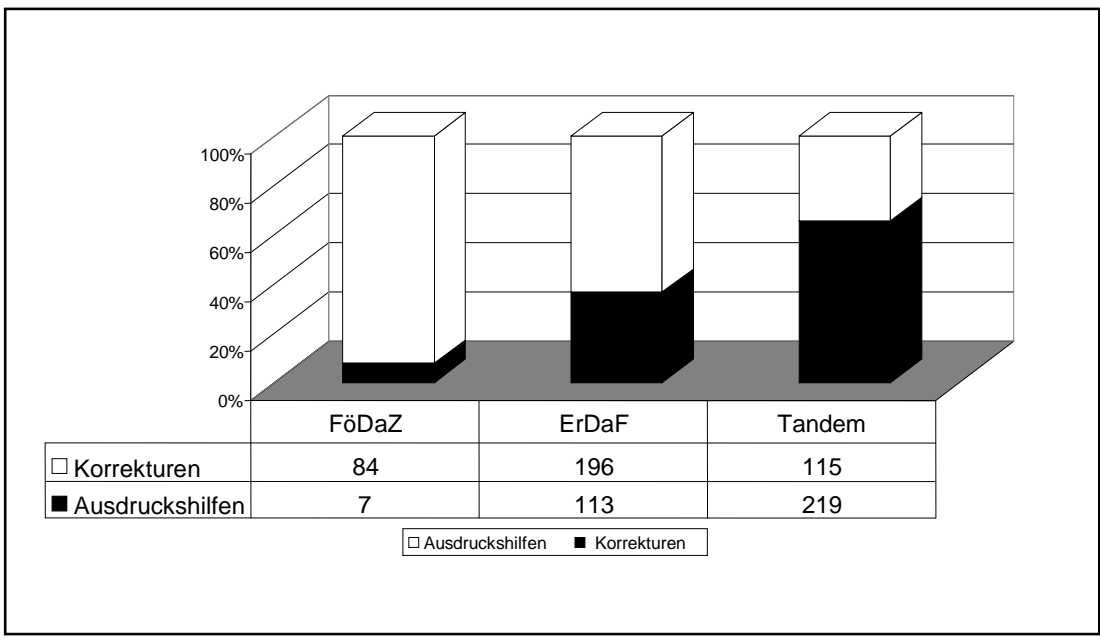

Diagramm 4: Ausdrucksschwierigkeiten und Korrekturen - Sprachförderkontexte im Vergleich

Ausdruckshilfen sind in den Tandem-Interaktionen am häufigsten, in Freien Konversationen des Gruppenunterrichts seltener und im DaZ-Förderunterricht am seltensten.

\section{Resümee}

Mit Fallstudien zu Situationen des DaZ-Förderunterrichts wurde untersucht, wie sich Unterrichtsinteraktionen in konkreten Einzelfällen gestalten. Dabei zeigen sich in einigen Punkten Unterschiede für die untersuchten Situationen, die insbesondere die Korrekturdurchführung betrafen. Es zeigten sich aber auch in verschiedenen Punkten Gemeinsamkeiten, wenn man die hier untersuchten Situationen mit anderen Unterrichtskontexten wie Freien Konversationen im Erwachsenen DaF-Unterricht oder Tandem-Interaktionen vergleicht. Es werden im DaZUnterricht anteilmäßig häufiger Selbstkorrekturen durchgeführt, Fehlerkorrekturen zielen häufiger auf Diskursfehler und inhaltliche Fehler, seltener auf grammatische/morphologische Fehler. Zudem gibt es weniger Ausdruckshilfen.

Freies Sprechen im zweit- oder fremdsprachlichen Unterricht ist für die Unterrichtspraxis ein diffiziler Bereich. Zum einen ist der freie Ausdruck zu fördern, zum anderen soll Feedback und Korrektur geleistet werden, was wiederum den freien Ausdruck hemmen kann. Es kommt hier zu Maximen-Konflikten im Sinne von Ehlich/Rehbein (1982: 179ff). Theoretisch und empirisch gesicherte Befunde 
für Empfehlungen sind noch ein Desiderat und Empfehlungen sind sicherlich auch nicht pauschal für alle Kontexte der Sprachförderung und Lernertypen oder Zielgruppen zu geben. Aber auch wenn Vergleiche von DaZ-Förderunterricht mit anderen Sprachfördermaßnahmen nicht unproblematisch sind, da sich Erwerbsprozesse und Fördermöglichkeiten unterscheiden, können Vergleiche zur Perspektiverweiterung beitragen und zur Diskussion anregen.

Auch bezogen auf Förderunterricht im Bereich Deutsch als Zweitsprache scheint es plausibel anzunehmen, dass eine stärkere Orientierung an Selbstinitiierungen und Ausdrucksschwierigkeiten der Lerner der Sprachförderung dienlich sein kann. Desgleichen scheint es vor dem Hintergrund fachdidaktischer ebenso wie zweitspracherwerbstheoretischer Diskussionen sinnvoll, Interaktion und Feedback in Gesprächssituationen des zweitsprachlichen Unterrichts weniger ausschließlich unter dem Aspekt der Fehlerbehandlung sondern unter stärkerer Berücksichtigung der lernersprachlichen Andeutung von Ausdrucksschwierigkeiten zu betrachten.

\section{Literatur}

Ahrenholz, Bernt: „Förderunterricht und Deutsch-als-Zweitsprache-Erwerb. Eine longitudinale Untersuchung zur mündlichen Sprachkompetenz bei Schülerinnen und Schülern nicht-deutscher Herkunftssprache (ndH) in Berlin“, Zeitschrift für Fremdsprachenforschung, 14, 2 (2003), 291-300.

Ahrenholz, Bernt: „Zur Entwicklung mündlicher Sprachkompetenzen bei Schülerinnen und Schülern mit Migrationshintergrund“. In: Ahrenholz, Bernt/Apeltauer, Ernst (Hrsg.): Zweitspracherwerb und curriculare Dimensionen. Empirische Untersuchungen zum Deutschlernen in Kindergarten und Grundschule. Tübingen: Stauffenburg 2006, 91-109.

Blex, Klaus: „Mündliche Fehlerkorrekturen und kurzzeitiger Fremdsprachenerwerb - einige Daten zur Komplexität dieser Relation“. In: Eckerth, J. (ed.): Empirische Arbeiten aus der Fremdsprachenerwerbsforschung. Beiträge des Hamburger Promovierendenkolloquiums Sprachlehrforschung. (Fremdsprachen in Lehre und Forschung, 33) Bochum: AKS 2003, 23-52.

Chaudron, Craig: "A Descriptive Model of Discourse in the Corrective Treatment of Learners’ Errors”, Language Learning, 27, 1 (1977), 29-46.

Chaudron, Craig: Second Language Classrooms. Research on Teaching and Learning. Cambridge: University Press 1988.

Deutsches PISA-Konsortium (Hrsg.): PISA 2000. Basiskompetenzen von Schülerinnen und Schülern im internationalen Vergleich. Opladen: Leske + Budrich 2001. 
Ehlich, Konrad/Rehbein, Jochen: Augenkommunikation. Methodenreflexion und Beispielanalyse. (Linguistik Aktuell) Amsterdam: John Benjamins B.V 1982.

Fanselow, John F.: "Beyond Rashomon - conceptualising and describing the teacher act”, TESOL Quarterly, 11, 1 (1977), 17-39.

Flanders, Ned A.: Analysing Teaching Behavior. Reading, Mass.: Addison-Wesley 1970.

Gumperz, John J./Heramsimchuk, E.: "The conversational analysis of social meaning. A study in classroom interaction". In: Jäger, Siegfried (ed.): Probleme der Soziolinguistik. Zeitschrift für Literaturwissenschaft und Linguistik, Beiheft 1975, 79-110.

Henrici, Gert: Spracherwerb durch Interaktion? Eine Einführung in die fremdsprachenerwerbsspezifische Diskursanalyse. Baltmannsweiler: Schneider 1995.

Jefferson, Gail: "On Exposed and Embedded Correction in Conversation", Studium Linguistik, 14 (1983), S. 58-68.

Kasper, Gabriele: "Repair in Foreign Language Teaching”. In: Kasper, Gabriele (ed.): Learning, Teaching and Communication in the Foreign Language Classroom. Aarhus 1986, 23-41.

Kasper, Gabriele: "Funktionen und Formen der Lernersprachenanalyse". In: Bausch, K.-R./Christ, H./Hüllen, W./Krumm, H.-J. (Hrsg.): Handbuch Fremdsprachenunterricht. Tübingen: Francke 1989, S. 218-222.

Klein, Wolfgang: „Typen und Konzepte des Spracherwerbs“. In: Helbig, Gerd et al. (Hrsg.): Deutsch als Fremdsprache. Ein internationales Handbuch, 2 Hbde. (Handbücher zur Sprach- und Kommunikationswissenschaft; Bd. 19). Berlin/New York: de Gruyter 2001, 604-617.

Kleppin, Karin: Fehler und Fehlerkorrektur. Fernstudieneinheit 19. Berlin usw.: Langenscheidt 1998.

Kleppin, Karin/Königs, Frank G.: Der Korrektur auf der Spur - Untersuchungen zum mündlichen Korrekturverhalten von Fremdsprachenlehrern. (Manuskripte zur Sprachlehrforschung 34) Bochum: Brockmeyer 1991.

Lochtman, Katja: Korrekturhandlungen im Fremdsprachenunterricht. Bochum: AKS-Verlag 2002.

Lochtman, Katja: „Die mündliche Fehlerkorrektur im DaF-Unterricht“, German as a foreign Language, 3, (2003), 1-19 [http://www.gfl-journal.de/3-2003/ lochtman.html] 2003.

Lütke, Beate: „Vorfeldbesetzungen in Erzählungen von Kindern mit Migrationshintergrund“. In: Ahrenholz, B. (ed.): Kinder mit Migrationshintergrund. Spracherwerb und Fördermöglichkeiten. Freiburg i. Br.: Fillibach 2006, 266-286. 
Rösch, Heidi (ed.): Sprachförderung, Grundlagen, Übungsideen, Kopiervorlagen. Deutsch als Zweitsprache. Braunschweig: Schroedel 2004.

Rost, Martina: Sprechstrategien in ,freien Konversationen'.Eine linguistische Untersuchung zu Interaktionen im zweitsprachlichen Unterricht. Tübingen: Narr 1989.

Rost-Roth, Martina: „Formulierungshilfen und Fehlerkorrekturen als Erwerbspotentiale. Freie Konversationen im Gruppenunterricht und TandemInteraktionen", Deutsch als Fremdsprache, 36, 3 1999, 160-165.

Rost-Roth, Martina: „'Mütterkurse': Förderung pragmatischer Kompetenzen in Deutschkursen für Frauen mit Migrationshintergrund. Lernvoraussetzungen und Kommunikationsbedürfnisse". In: Wolff, Armin/Riemer, Claudia/Neubauer, Fritz (Hrsg.): Sprache lehren - Sprache lernen. Regensburg: Fachverband Deutsch als Fremdsprache 2005, 129-154.

Rost-Roth, Martina (unter Mitarbeit von Oliver Lechlmair): Sprachenlernen im direkten Kontakt. Autonomes Tandem in Südtirol. Eine Fallstudie. Bozen, Meran: Alpha\&Beta 1995.

Rost-Roth, Martina: „Komplexe Fragerealisierungen. SchülerInnen der 3. und 4. Grundschulklasse mit Deutsch als Zweit- und Deutsch als Muttersprache“. In: Ahrenholz, Bernt (ed.): Kinder mit Migrationshintergrund, Zweitspracherwerb und Sprachförderung. Freiburg i. Br.: Fillibach 2006, 241-265.

Rost-Roth, Martina: Nachfragen. Formen und Funktionen äußerungsbezogener Interrogationen. Berlin, New York: De Gruyter (im Druck).

Schegloff, Emanuel A./Jefferson, Gail/Sacks, Harvey: "The Preference for SelfCorrection in the Organization of Repair in Conversation", Language, 53, 2 (1977), 361-382.

Selinker, Larry: "Interlanguage", International Review of Applied Linguistics in Language Teaching, 10, 3 1972, 209-231. 


\section{Anhang:}

Mit U bzw. U1 etc. wird hier auf die Unterrichtenden verwiesen, mit S bzw. S1 auf SchülerInnen.

Die Tonband- und Videoaufzeichnungen wurden in Anlehnung an CA-CHAT transkribiert. Hier kommen die folgenden Zeichen zur Anwendung:

Frage, Aussage, Ausruf

komplexe Satzgefüge

Intonierung innerhalb der Äußerung als Ausruf Stimmhöhe steigend,

Stimmhöhe fallend, fallende Intonationskontur (Ende der Äußerung) (innerhalb der Äußerung)

Pausen

Wiederholungen von Äußerungsteilen ohne Veränderung

Wiederholung/Wiederaufnahmen mit Veränderung

Abbruch/Selbstkorrektur

Unterbrechung

'trailing off' Abklingen (leiser werdend)

Dehnung

Betonung

leiser gesprochen

Lachen

lachend gesprochen

nicht genau verständlich, vermuteter Text bei mehreren Wörtern unverständlicher Text

Kommentare

- kurze Kommentare innerhalb der Zeile

- Kommentarzeile für längere Kommentare ((unter Sprecherzeile))

Beginn und Ende von Überlappung

Namen im Text

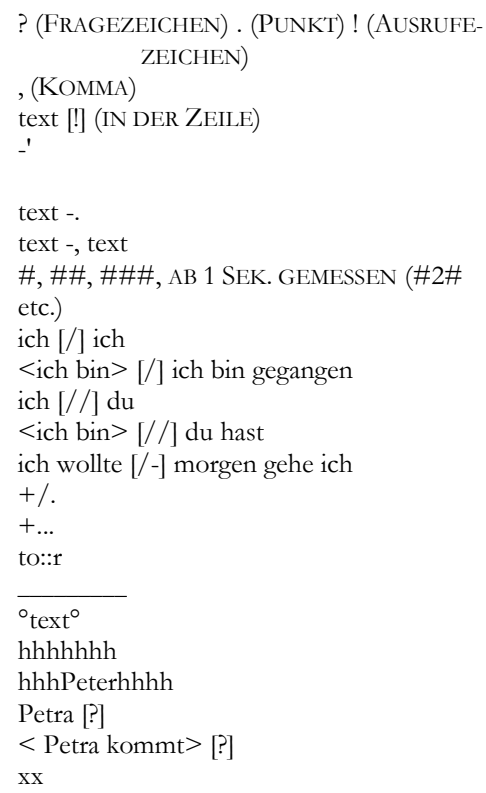




\section{Sprachmischungen im simultanen Erwerb zweier Muttersprachen: Ein Fall von Sprachdominanz?}

Katja Francesca Cantone, Bremen

\section{$1 \quad$ Einleitung}

Diese Arbeit befasst sich mit bilingualen Kindern, die ihre beiden Sprachen von Geburt an erworben haben. Es wird gezeigt, dass in der frühen Phase des Spracherwerbs gemischt wird, jedoch nicht in einem großen Umfang. Die Daten lassen auch erkennen, dass es keinen Zusammenhang zwischen Sprachmischungen und Sprachdominanz geben muss. Darüber hinaus werde ich zeigen, dass die kindlichen Sprachmischungen keine Verletzung von soziolinguistischen oder grammatischen Regeln darstellen, sondern ein Zeichen für den kreativen Umgang mit den beiden 
Muttersprachen sind. Somit sind Mischungen kein Beleg für die fehlerhafte Trennung oder der fehlerhaften Nutzung der Muttersprachen (siehe auch Cantone). Diese Ergebnisse bieten eine wichtige Grundlage zur Diskussion über die Verwendung der beiden Muttersprachen seitens bilingualer Kinder im Alltag, d. h., mit Eltern und Familie, sowie im Kindergarten und nicht zuletzt in der Schule.

\section{Definitionen und Theorie}

Das Thema Mehrsprachigkeit ist hochaktuell und wird auf vielen Gebieten diskutiert, z. B. in der Schule, im Beruf, auf kultureller Ebene. Oft wird der Begriff bilingual in der Alltagssprache benutzt, um jemand zu beschreiben, der mehr als eine Sprache kann. Dabei spielt es keine Rolle, wann die auf die Muttersprache folgende(n) Sprache(n) gelernt worden ist/sind. Auch die Umstände, z. B. gesteuert oder ungesteuert, werden außer Acht gelassen. Ich werde mich hier nicht mit den unzähligen Beschreibungen von bilingual beschäftigen, sondern nur mit einem bestimmten, kleinen Bereich der Mehrsprachigkeit, nämlich mit dem gleichzeitigen Erwerb von zwei Sprachen von Geburt an.

\subsection{Der simultane Erwerb von zwei (oder mehr) Sprachen}

Der Begriff doppelter Erstspracherwerb geht auf den englischen Terminus Bilingual First Language Acquisition (BFLA) zurück, der bei Meisel (1989: 20) zuerst erwähnt wird. Mit diesem Begriff wird deutlich, dass beide Sprachen als Muttersprachen, also Erstsprachen, gesehen werden sollten. Es stellt sich die Frage, wie gleichzeitig der Erwerb stattfinden muss: Muss das Kind vom Tag der Geburt an (oder bereits im Mutterleib?) mit beiden Sprachen in Kontakt kommen? Oder reicht es aus, wenn die zweite Sprache innerhalb des ersten Lebensjahres hinzukommt? Die Diskussion, ab wann der Spracherwerb als simultan gesehen werden kann, ist bis heute unter Spracherwerbsforschern offen. Manche Studien nennen tatsächlich den Zeitpunkt der Geburt (plus ca. eine Woche) als Erwerbsbeginn (De Houwer 1990: 3), andere akzeptieren das dritte Lebensjahr als Grenze (McLaughlin 1984). Das heißt, vor dieser Grenze wird der Erwerb zweier Sprachen als simultan angesehen, danach als sukzessiv. Man kann sagen, dass keine Einigkeit darüber herrscht, zu welchem Zeitpunkt eine Sprache nicht mehr als Muttersprache bezeichnet werden kann. Grob gesagt befindet er sich zwischen dem 4. und dem 7. Lebensjahr. Dass die Muttersprache sich von der Zweitsprache unterscheidet, kann der heutige Forschungsstand in der Neurolinguistik beweisen, z. B. anhand der Stelle, in der sie im Gehirn gespeichert wird, oder anhand der Bereiche, die zur Verarbeitung der Sprachen verwendet werden. 


\subsection{Sprachentrennung von Anfang an?}

Der Nachweis, dass das bilinguale Kind seine beiden Sprachen auf dieselbe Weise erwirbt wie monolinguale Kinder, kann als eines der wichtigsten Ziele der Bilinguismusforschung gesehen werden.

Die Hypothese, dass die zwei Sprachen zunächst in einem Sprachsystem vereint sind und dass es erst später zur Sprachentrennung kommt, ist vielfach widerlegt worden. Heutzutage wird davon ausgegangen, dass Kinder von Anfang an über zwei getrennte Systeme verfügen, eins für jede Sprache, die sie erwerben (Genesee 1989, Meisel 1989). Die bekanntesten Anhänger der so genannten Fusionshypothese sind Volterra \& Taeschner (1978, vgl. auch Taeschner 1983). Nach ihrem Drei-Phasen-Modell werden Kinder graduell zweisprachig. Zunächst sind Wörter aus beiden Sprachen in nur einem Lexikon gespeichert. Später entstehen zwar zwei Lexika (eins pro Sprache), es wird jedoch nur ein syntaktisches System für beide Sprachen verwendet. In der dritten Phase schließlich, wenn auch die Syntax sprachspezifisch gespeichert ist, sind die Kinder in der Lage, ihre Sprachen zu trennen. Abgesehen davon, dass unklar bleibt, wodurch diese Entwicklungsphase eingeleitet wird, muss Volterra \& Taeschner vorgehalten werden, dass ihre Methodik nicht einwandfrei ist. Nur ein genaues methodisches Vorgehen jedoch kann garantieren, dass die Ergebnisse einer Studie glaubwürdig sind. Insbesondere bei der Untersuchung zweisprachiger Kinder muss sichergestellt sein, dass die Aufnahmebedingungen eindeutig einsprachig sind und keine Sprachmischungen oder Sprachwechsel seitens der Forscher stimuliert werden. Mit anderen Worten, wenn man analysieren will, ob zweisprachige Kinder ihre Sprachwahl sowohl soziolinguistisch als auch grammatisch beherrschen, dürfen die Erwachsenen keinen Kontext schaffen, in dem beide Sprachen gewählt werden können. Sonst kann die Adäquatheit der Sprachwahl nicht getestet werden!

Volterra \& Taeschner (1978) gehen zum Beispiel davon aus, dass die von ihnen untersuchten Kinder von klein auf den Elternteil in seiner jeweiligen Muttersprache ansprechen sollten, obwohl die deutsche Mutter den Tonbandaufnahmen und Tagebuchaufzeichnungen nach sowohl Italienisch als auch Deutsch mit ihnen gesprochen hat und die Kinder immer in Anwesenheit beider Elternteile getestet wurden. Bei Deuchar \& Quay $(1998,2000)$ machen die Forscherinnen einen ähnlichen Folgefehler: Da sie nicht immer über eine spanischsprechende Person für die Aufnahmen verfügte, hat die englischsprachige Mutter, die für den englischen Teil der Aufnahmen zuständig war, teilweise die spanische Aufnahme übernommen. Da erscheint es nur logisch, dass das Kind gemischt hat und abwechselnd beide Sprache verwendet hat.

Müller, Cantone, Kupisch \& Schmitz (2002) haben in ihrer Studie gezeigt, dass es trotz Sprachentrennung von früh auf Einfluss zwischen den beiden Sprachen geben kann. Dieser kann drei unterschiedliche Auslegungen haben (vgl. Paradis \& Genesee 1996: 3): Er kann Beschleunigung bedeuten, in dem Sinne, dass ein Phänomen, das in einer Sprache bereits erworben worden ist, auf die andere übertra- 
gen wird. Hiernach können bilinguale Kinder im Vergleich zu einsprachigen Kindern sogar einen Zeitvorteil davon haben, dass sie bilingual sind. Es kann aber auch zu einer Verlangsamung kommen: In diesem Fall treten bestimmte Phänomene im bilingualen Kind später auf, als es der Fall sein sollte. Die dritte Erscheinung von Spracheinfluss ist Transfer, wenn also bestimmte Eigenschaften der Sprache A auch in Sprache B benutzt werden, obwohl dies nicht zielsprachlich ist. Transfer kommt z. B. in der Wortstellung zum Vorschein. Ein Beispiel ist der italienische Satz „Ho mangiato una mela", in dem, anders als im deutschen Äquivalent "Ich habe einen Apfel gegessen", das Objekt nach dem Partizip steht. Es kann temporär dazu kommen, dass ein zweisprachig italienisch/ deutsches Kind das Objekt auch im Deutschen nach dem Verb stellt, und "Ich babe gegessen den Apfel" sagt. Müller et al. haben jedoch gezeigt, dass diese Konstruktionen im simultanen Erwerb von zwei Sprachen nur kurzfristig auftreten, und dass das Kind schnell begreift, dass viele Regeln sprachspezifisch sind und nicht verallgemeinert werden können.

Man muss aber erwähnen, dass Spracheneinfluss auch positiver Natur sein kann, wenn nämlich von zweisprachigen Kindern ein Phänomen, das sie bereits in einer Sprache erworben haben, auf die zweite Sprache übertragen wird. Zum Beispiel ist es im Italienischen einfacher, Genus zu markieren, weil es nur zwei Formen gibt (Maskulinum und Femininum) und nicht drei wie im Deutschen (plus Neutrum) und weil die Artikel nicht mit Kasus markiert werden. So könnte man sich vorstellen, dass ein bilingual italienisch/deutsches Kind, das bereits im Italienischen Artikel benutzt und diese mit Genus markiert, dies auch fürs Deutsche anwendet und damit früher beginnt als ein monolinguales deutsches Kind ${ }^{1}$. Aus Platzgründen kann hier nicht näher auf die grammatischen Bereiche eingegangen werden, bei denen Einfluss nachgewiesen worden ist. Für eine detaillierte Diskussion verweise ich auf Kapitel 6 in Müller, Kupisch, Schmitz \& Cantone (2006).

Zusammenfassend lässt sich feststellen, dass bilinguale Kinder ihre beiden Sprachsysteme von Beginn an getrennt speichern. Dies soll aber nicht bedeuten, dass sie keinen Vorteil, und manchmal sogar auch einen Nachteil, daraus ziehen, zwei Sprachen gleichzeitig zu erwerben.

\subsection{Sprachdominanz}

Der folgende Abschnitt umreißt den Begriff Sprachdominanz (für eine genauere Darstellung siehe Kupisch 2006 sowie Cantone, Kupisch, Müller \& Schmitz 2006). Einfach dargestellt meint man mit Dominanz, dass eine Sprache beim bilingualen Individuum stärker ist oder besser beherrscht wird als die andere. Leider gibt es unterschiedliche Definitionen, insbesondere in der Literatur zum Spracherwerb, und die Kriterien, die zum Messen von Dominanz benutzt werden, variieren ebenfalls von Studie zu Studie. Im Spracherwerb wird von Dominanz gespro-

\footnotetext{
1 Für den Erwerb von Genus bei zweisprachigen Kindern vgl. Cantone (1999), Müller (1999), Kupisch, Müller \& Cantone (2002).
} 
chen, wenn die Entwicklung der beiden Sprachen zeitlich versetzt verläuft, also eine Sprache schneller erworben wird als die andere. Cantone et al. (2006) stellen in Frage, weshalb der Begriff Dominanz verwendet wird, da es nicht darum geht, dass die schnellere Sprache eine Einwirkung auf die schwächere Sprache hat, sondern lediglich darum, dass sie in unterschiedlicher Geschwindigkeit erworben werden.

Die Kriterien, die in der Forschung zu diesem Thema verwendet werden, sind z. B. die durchschnittliche Äußerungslänge (MLU, vgl. Brown 1973), Hesitationen (De Houwer 1990), die Lexikongröße (Genesee et al. 1995) und der Lexikonanstieg (Müller \& Kupisch 2003) in der jeweiligen Sprache, und der Erwerb grammatischer Kategorien (Bernardini \& Schlyter 2004).

Petersen (1988) geht in der Dominance Language Hypothesis davon aus, dass funktionale Elemente (wie z. B. Determinanten oder Flektionsmorpheme) immer aus der dominanten Sprache in die schwache Sprache gemischt werden, jedoch nie umgekehrt. So kann z. B. bei einem deutsch/englischen Kind, dessen dominante Sprache das Deutsche ist, die gemischte Äußerung ,der $c a r^{\text {" }}$ auftreten, jedoch nicht „the Auto“. Das bedeutet, Petersen greift Sprachmischungen als direktes Kriterium auf, um Dominanz bei bilingualen Kindern festzustellen. Ich werde jedoch zeigen, dass ein gewisser Vorteil in einer Sprache keineswegs bedeutet, dass man die Mischrichtung vorhersagen kann.

Cantone et al. (2006) schlagen vor, die unterschiedliche Entwicklung der beiden Sprachen im bilingualen Kind anders zu analysieren. Sie plädieren dafür, die Sprachentwicklung bei bilingualen Kindern nicht anhand eines direkten Vergleichs der beiden Sprachen untereinander zu überprüfen, sondern beim Vergleichen der Sprachproduktion mehrerer bilingualer Kinder in der jeweiligen Sprache. Um zu wissen, ob eine Sprache schneller als die andere erworben wird, soll sie nicht mit der zweiten Muttersprache verglichen werden, sondern es soll untersucht werden, ob das Kind im Vergleich zu anderen Kindern in der Muttersprache im Durchschnitt liegt oder schneller oder langsamer ist. Die monolingualen Daten eines zweisprachigen Kindes sollten nur mit Daten anderer zweisprachiger Kinder verglichen werden und nicht mit Daten eines monolingualen Kindes, um die Ganzheitlichkeit des bilingualen Individuums zu wahren.

\subsection{Code-switching}

Während man bei erwachsenen Sprechern davon ausgeht, dass dieser Sprachstil ein Zeichen von guter Kompetenz in beiden Sprachen ist und aus psycho- und soziolinguistischen Gründen eingesetzt wird, nimmt man bei kindersprachlichen Mischungen an, dass es sich um nichtregelgeleitetes und unbeabsichtigtes Mischen der Sprachen handelt. Bei vielen Forschern herrscht die Vorstellung, dass Kinder erst erwerben müssen, ihre beiden Sprachen grammatisch und im Sprachgebrauch auseinander zu halten. 


\subsubsection{Sprachmischungen bei Kindern}

Viele Studien zum Spracherwerb bilingualer Kinder haben festgestellt, dass es eine Phase zu Beginn gibt, in der alle Kinder in beiden Sprachen viel mischen (vgl. z. B. Lanza 1992, Köppe \& Meisel 1995, Köppe 1996, Deuchar \& Quay 1998, 2000). Es herrscht jedoch keine Einigung darüber, weshalb es diese Phase gibt und warum sie abrupt endet.

Wie bereits erwähnt, geht der heutige Forschungsstand davon aus, dass die beiden Sprachen von Anfang an zwei getrennte Systeme darstellen. Demnach kann die hohe Mischungsrate nicht damit zusammenhängen, dass Kinder nur ein Lexikon besitzen, in dem Wörter aus beiden Sprachen gespeichert werden. Fehlende Sprachentrennung im Gehirn kann also ausgeschlossen werden. Möglich ist allerdings, dass es eine fehlende Sprachentrennung im Sprachgebrauch bzw. in den Aufnahmemodi gibt, die zu verstärkten Mischungen führen könnten. Es ist z. B. ungeklärt, ob ein starkes Mischverhalten seitens der Eltern dazu führt, dass die Kinder auch viel Mischen (Goodz 1989 vs. Genesee, Nicoladis \& Paradis 1995).

Eine Möglichkeit, warum Kinder zu einem höheren Maße mischen, kann dadurch gegeben sein, dass sie das äquivalente Wort in der anderen Sprache noch nicht können. Da ihnen das entsprechende Wort noch fehlt, greifen sie zu der Vokabel, die sie schon können. Daraus kann eine Mischung oder ein Sprachwechsel entstehen. Eine andere Erklärung für die hohe Rate an Sprachmischungen am Anfang des Spracherwerbs ist, dass das grammatische Verständnis bei Kindern nicht von Geburt an vorhanden ist. Erst wenn die Grammatik maturiert, soll das Kind lernen, die Sprachen auseinander zu halten und mischt daher weniger (Meisel 1994 sowie Köppe \& Meisel 1995).

Die hier vorliegende Arbeit stützt sich auf die Annahme von Cantone (erscheint), die davon ausgeht, dass Sprachmischungen bei bilingualen Kindern von Anfang an als Code-switching bezeichnet werden können, weil sie sowohl soziolinguistische als auch grammatische Regularitäten befolgen.

\subsubsection{Sprachmischungen bei Erwachsenen}

Verschiedene Studien mit unterschiedlichen Ansätzen haben das Auftreten von zwei Sprachen bei bilingualen Sprechern innerhalb eines Diskurses oder eines Satzes untersucht. Zunächst beschäftigte sich die Soziolinguistik mit diesem als Code-switching bezeichneten Sprachstil (vgl. u. a. Gumperz 1976, 1982). Dabei wurde analysiert, in welchen Kontexten Bilinguale von einer Sprache zu anderen wechselten bzw. welche Wörter zu denen gehörten, die gemischt wurden. Nach vielen soziolinguistischen Untersuchungen, die auch die Akzeptanz dieses Sprachstils im Fokus hatten, begann eine systematische Analyse der Grammatikalität von Sprachmischungen. Eine chronologische Darstellung der vorgeschlagenen Restriktionen würde den hier vorgegebenen Rahmen sprengen, es sei jedoch erwähnt, dass die unterschiedlichen Regeln, die fürs Code-switching postuliert worden sind, nicht studienübergreifend bestätigt werden konnten: Während z. B. eine Untersu- 
chung Sprachmischungen zwischen dem Artikel und dem Nomen als ungrammatisch bezeichnet (Belazi, Rubin \& Toribio 1994), gehen andere Studien davon aus, dass diese Mischungen grammatisch sind und geben an, dass sie oft unter Zweisprachigen auftreten (Poplack 1980, DiSciullo, Muysken \& Singh 1986).

Vor einigen Jahren hat die Diskussion darüber, welche Mischungen als gesichert grammatisch oder ungrammatisch gesehen werden können, und zwar sprachübergreifend, eine Wendung genommen. Der Sprach- und Erziehungswissenschaftler Jeff MacSwan hat vorgeschlagen, dass die Architektur der Sprachfähigkeit bei Bilingualen genauso sein sollte wie bei einsprachigen Individuen (1999, 2000). Vereinfacht dargestellt schlägt MacSwan vor, dass die im Minimalismus (Chomsky 1995) angenommenen Vorgänge, die zur Entwicklung einer Äußerung führen, dieselben bei ein- und zweisprachigen Menschen sind. Der einzige Unterschied liegt darin, dass Bilinguale über zwei Lexika verfügen, die jeweils aus den Wörtern der beiden Muttersprachen bestehen, und über zwei phonologische Ebenen, um diese Wörter jeweils sprachspezifisch auszusprechen.

Diese Hypothese hat einschneidende Konsequenzen für das Untersuchen von Sprachmischungen: Demnach benötigt man keine so genannte dritte Grammatik und keine Sonderregeln mehr, um das Code-switching zu begrenzen, denn nach MacSwan sind alle Sprachmischungen grammatisch, solange sie keine Regeln der beiden betroffenen Sprachen verletzen (1999: 146). Es muss also beispielsweise nicht mehr infragegestellt werden, ob zwischen Artikel und Nomen gemischt werden darf, sondern es stellt sich die Frage, ob an dieser Stelle gemischt werden kann, wenn die zwei betroffenen Sprachen unterschiedliche Wortstellungen haben: Kann eine Mischung stattfinden, wenn ein Artikel vor dem Nomen steht, aber die Sprache aus der das Nomen kommt eigentlich nur postnominale Artikel erlaubt? Es geht nicht mehr um das Erfinden von neuen Regeln, die das Code-switching beschränken, sondern um das Einhalten der beiden involvierten Grammatiken.

Cantone nimmt an, dass die Hypothese von MacSwan $(1999,2000)$ auch auf Kinderdaten angewendet werden kann. Wie wir im Folgenden sehen werden, können diese theoretischen Annahmen dabei helfen, eine gründliche Analyse von Code-switching-Daten durchzuführen, ohne mit erhobenen Zeigefinger auf Fehlersuche zu gehen. 


\section{Analyse der Daten}

Die hier diskutierten Daten stammen aus dem Projekt: ,Frühkindliche Zweisprachigkeit: Italienisch/Deutsch und Französisch/Deutsch im Vergleich`2. Die fünf untersuchten Kinder sind bilingual mit den Sprachen Italienisch und Deutsch in Hamburg aufgewachsen, wobei alle Mütter Italienerin sind. Die Eltern sprachen ihre jeweilige Muttersprache mit dem Kind. Die Familiensprache variierte: Bei drei Familien wurde Italienisch als Familiensprache gewählt, um die Minderheitensprache zu unterstützen, bei zwei Kindern sprachen die Eltern zu Hause untereinander Deutsch. In nur einem Fall konnte der Vater nur ausreichend Italienisch. Drei der untersuchten Kinder haben ältere Geschwister, die bereits bilingual aufwuchsen. Bei vier der fünf Familien bestand regelmäßiger Kontakt zur italienischen Verwandtschaft; außerdem reisten die Familien mindestens einmal im Jahr nach Italien. Man kann sagen, die Familien hatten sehr großes Interesse daran, dass die Kinder genügend Kontakt zur italienischen Sprache hatten.

Die Zeitspanne der Analyse streckt sich vom Alter 1,7 (Jahr; Monat) bis ca. 5 Jahren. Die Sprachentwicklung der Kinder wurde anhand von Videoaufnahmen dokumentiert, die alle zwei Wochen bei den Kindern zu Hause stattgefunden haben. Dabei haben zwei Muttersprachlerinnen jeweils eine halbe bis dreiviertel Stunde mit dem Kind gespielt. Das heißt, die Sprachen sind getrennt aufgenommen worden. In allen Fällen war die italienischsprachige Interaktionspartnerin bilingual; dies hat jedoch keine Auswirkung auf die Ergebnisse gehabt, wie wir später sehen werden. Die Aufnahmen wurden transkribiert, kontrollgehört und anschließend auf unterschiedliche sprachliche Phänomene untersucht.

Die Mischungen der Kinder sind in zwei Phasen eingeteilt worden (vgl. Cantone erscheint), um die Daten besser mit früheren Studien zu vergleichen, die zum einen frühe Mischungen nicht als Code-switching, sondern als Code-mixing bezeichnen, und zum anderen davon ausgehen, dass viel in der frühen Phase gemischt wird und dass das Mischen abrupt aufhört.

Im Folgenden werde ich mich aus Platzgründen auf zwei Fragen konzentrieren: Mischen die Kinder in der frühen Phase des Spracherwerbs tatsächlich mehr als später (siehe 2.1.1)? Gibt es einen Zusammenhang zwischen Sprachmischungen und Sprachdominanz (2.1.2)?

\footnotetext{
${ }^{2}$ Das Forschungsprojekt, das am Sonderforschungsbereich Mehrsprachigkeit an der Universität Hamburg von 1999-2005 angesiedelt war, wurde durch Drittmittel finanziert, die die Deutsche Forschungsgemeinschaft an Frau Natascha Müller vergeben hat. Die wissenschaftlichen Mitarbeiterinnen waren Katja F. Cantone, Tanja Kupisch und Katrin Schmitz (siehe Müller, Cantone, Kupisch \& Schmitz 2002, Müller, Kupisch, Schmitz \& Cantone 2006). Heutige Postanschrift: Prof. Dr. Natascha Müller, Bergische Universität Wuppertal, Fachbereich A Geistes- und Kulturwissenschaften Romanistik, Gaußstr. 20, D-42119 Wuppertal, nmueller@uni.wuppertal.de
} 


\subsection{Die frühe Phase des Spracherwerbs}

In der frühen Phase des Spracherwerbs, die von ca. 1 Jahr und 7 Monate bis zu 2 Jahre und 7 Monate geht, von Kind zu Kind variiert, und jeweils zwischen 3 und 6 Monaten dauert, mischen die Kinder überraschend wenig.

Das Kind Carlotta mischt in beiden Sprachen selten, im Deutschen aber etwas mehr als im Italienischen. Das ist insofern interessant, als dass die Interaktionspartnerin in der italienischen Aufnahme zweisprachig ist und Carlotta einen Grund zum Wechseln bzw. Mischen der Sprachen hätte geben können. Beim Kind Lukas finden wir eine hohe Rate an Mischungen im Italienischen, jedoch fast keine gemischten Äußerungen im Deutschen. Lukas hat auch eine bilinguale Interaktionspartnerin in der italienischen Aufnahme, jedoch nicht dieselbe Person wie bei Carlotta. Ob das der Grund fürs Mischen ist, kann an dieser Stelle nicht beantwortet werden. Das Kind Jan mischt selten im Deutschen. In der italienischen Aufnahme überschreitet er die 10\%-Mischrate nicht und verhält sich ähnlich wie Carlotta. Die beiden haben dasselbe Aufnahmeteam gehabt. Das Kind Aurelio mischt in beiden Sprachen viel. Im Italienischen reduziert sich die Prozentzahl der Mischungen, wenn wir die Fälle ausschließen, in denen er Italienisch spricht und das deutsche ja oder nein hineinmischt. Das Kind Marta schließlich sagt nur ein deutsches Wort in der italienischen Aufnahme, nämlich ja. Im Deutschen mischt sie etwas mehr als Carlotta.

Keines der Kinder mischt in beiden Aufnahmeteilen, sodass die Mischungen auf keinen Fall ein Zeichen dafür sein können, dass die Kinder ihre Sprachen nicht trennen können. Hier ein paar Beispiele für Mischungen in dieser frühen Phase ${ }^{3}$ :

(1) $\underline{\mathrm{da}}$, quello

(2) $\underline{\text { luna }}$

da, dieses (Carlotta,1;11,12,IK)

(3) bullo noch $=$ burro

Mond

(Carlotta, 1;10,8, DK)

(4) musica

Butter noch

(Lukas, $1 ; 11,1, \mathrm{IK}$ )

(5) noch

Musik.

(Lukas, 1;9,13, DK)

(6) ja , io

noch

(Jan, 2;1,3, IK)

(7)

ja, ich

(Aurelio, 1;11,28,IK)

$$
\text { bautele qua }=\text { Baustelle }
$$

casa is hier $=$ ist

Haus ist hier

Baustelle hier

(Aurelio, 2;0,14, DK)

Alle Kinder mischen in dieser frühen Phase überwiegend einzelne Elemente. In den Beispiel (1), (3), (5), (6) und (7) werden so genannte Funktionswörter gemischt, bei (2), (4) und (8) handelt es sich um Nomina.

\footnotetext{
${ }^{3}$ Die Angaben sind wie folgt zu lesen: (Name des Kindes/Alter in Jahr;Monat,Tag/DK= Deutscher Kontext, IK= Italienischer Kontext). Das gemischte Wort ist jeweils unterstrichen.
} 


\subsubsection{Miscbraten}

Man kann allgemein sagen, dass Mischungsraten bis zu 10\% einer niedrigen Mischrate entsprechen und verdeutlichen, dass Sprachmischungen keine Strategie im Spracherwerb sein können. Bei allen hier untersuchten Kindern ist die Mutter fast die einzige Person, die zum Kind auf Italienisch spricht. Mit anderen Worten, Italienisch muss als Minderheitensprache bewertet werden und ist ein guter Kandidat dafür, von den Kindern wenig verwendet zu werden.

Bei Carlotta haben wir jedoch gesehen, dass sie in beiden Sprachen wenig mischt. Das könnte z. B. daran liegen, dass Italienisch die Familiensprache ist. Bei Lukas spricht Vieles dafür, dass er nicht genug mit Italienisch in Kontakt kommt, weil zu Hause Deutsch gesprochen wird. Das gilt auch für Jan, der außerdem von einem nichtitalienischsprachigen Au-pair-Mädchen betreut wird. Bei Aurelio sprechen die Mutter und der ältere Bruder viel Italienisch, jedoch hat die Mutter viel gemischt. Dieses scheint einen Einfluss auf ihn gehabt zu haben, da er in beiden Sprachen viel mischt. In Martas Umgebung ist Italienisch die meistgesprochene Sprache, weil sie die Familiensprache ist und von der Mutter, dem älteren Bruder und dem Kindermädchen gesprochen wird.

Nachdem über die Mischraten gesprochen wurde, soll kurz auf die Zahlen verwiesen werden, die zeigen, wie viel die Kinder die in den Aufnahmen nicht erwünschte Sprache gesprochen haben.

Carlotta hat in den italienischen Aufnahmen nicht einen deutschen Satz zu der italienischen Interviewerin gesagt, während sie in der italienischen Aufnahme einige wenige Äußerungen auf Deutsch gemacht hat. Lukas hat sehr selten Sätze auf Deutsch im italienischen Kontext geäußert, jedoch kein Italienisch in den deutschen Aufnahmen gesprochen. Die Kinder Jan und Marta haben nie die im Kontext nicht erwünschte Sprache gesprochen. Auch Aurelio hat sich fast 100\% an den von den Erwachsenen vorgegebenen Sprachkontext gehalten.

Die Zahlen verdeutlichen, dass Kinder schon früh in der Lage sind zu erkennen, welche Sprache sie zu wem und in welchem Kontext sprechen können. Das zeigt, dass sie sehr sensibel für psycho- und soziolinguistische Aspekte des Sprachgebrauches sind und die Sprachwahl korrekt beherrschen. Tabelle 1 zeigt die Sprachproduktion der Kinder während der Sprachaufnahmen. 


\begin{tabular}{|c|c|c|c|c|c|}
\hline Kind & Alter & Kontext & Italienisch & Deutsch & Mischungen \\
\hline \multirow{2}{*}{ Carlotta } & \multirow{2}{*}{$1 ; 10,8-2 ; 2,19$} & Italienisch & $1.131(94,6 \%)$ & 0 & $65(5,4 \%)$ \\
\hline & & Deutsch & $47(5,2 \%)$ & $670(75,5 \%)$ & $171(19,3 \%)$ \\
\hline \multirow{2}{*}{ Lukas } & \multirow{2}{*}{$1 ; 8,14-2 ; 1,23$} & Italienisch & $790(69,7 \%)$ & $21(2 \%)$ & $321(28,3 \%)$ \\
\hline & & Deutsch & 0 & $2.312(97,3 \%)$ & $65(2,7 \%)$ \\
\hline \multirow{2}{*}{ Jan } & \multirow{2}{*}{$2 ; 1,3-2 ; 7,28$} & Italienisch & $905(91,8 \%)$ & 0 & $80(8,2 \%)$ \\
\hline & & Deutsch & 0 & $1.632(99,1 \%)$ & $15(0,9 \%)$ \\
\hline \multirow{2}{*}{ Aurelio } & \multirow{2}{*}{$1 ; 10,23-2 ; 1,23$} & Italienisch & $538(47,4 \%)$ & 0 & $564(52,6 \%)$ \\
\hline & & Deutsch & $4(0,8 \%)$ & $384(80,3 \%)$ & $91(18,9 \%)$ \\
\hline \multirow{2}{*}{ Marta } & \multirow{2}{*}{$1 ; 7,10-2 ; 0,16$} & Italienisch & $946(99,9 \%)$ & 0 & $1(0,1 \%)$ \\
\hline & & Deutsch & 0 & $524(85,4 \%)$ & $90(14,6 \%)$ \\
\hline
\end{tabular}

Tabelle 1: Sprachgebrauch in den Aufnahmen (aus Cantone erscheint)

\subsubsection{Sprachdominanz}

Vier der hier untersuchten Kinder sind in der Studie von Cantone et al. (2006) getestet worden, das Kind Aurelio wurde bei Kupisch (2006) daraufhin geprüft, ob es einen unbalancierten Spracherwerbsverlauf hat.

Nachdem mehrere Kriterien (z. B. MLU und Anzahl der Äußerungen) ausgezählt wurden, haben Cantone et al. (2006) zunächst festgestellt, in welcher der beiden Sprachen die einzelnen Kinder (die in Phasen aufgeteilt wurden) die höchsten Werte verzeichnen. Damit ist gemeint, in welcher Sprache zum Beispiel die höchsten MLU-Werte verzeichnet wurden. Bei Carlotta und Marta sind die höchsten Werte in der für diese Studie relevanten Phase immer im Italienischen. Bei Jan sind die höchsten Werte im Deutschen. Lukas hat abwechselnd hohe Werte im Italienischen und im Deutschen, wobei eine Tendenz zu höheren Werten im Deutschen vorhanden ist. Vergleicht man diese Kategorisierung mit der Entwicklung des Lexikons, so zeigt sich, dass die Kinder in der oben genannten Sprache mehr Wörter erworben haben als in der anderen Sprache. Die Autorinnen haben anschließend die Werte aller Kinder summiert und für jede Sprache den Durchschnittswert (Norm) errechnet. Vergleicht man die Normwerte mit den Einzelwerten der Kinder, so sieht man, dass bei denen, die immer höhere Werte im Italienischen haben, nämlich Carlotta und Marta, die persönlichen Werte den Normwerten im Italienischen entsprechen bzw. darüber liegen. Im Deutschen liegen beide unter der Norm. Bei Jan verhält es sich genau umgekehrt: im Deutschen liegen seine Werte in der Norm und darüber, im Italienischen unter der Norm. Bei Lukas finden wir wieder abwechselnde Werte in beiden Sprachen, d. h., er liegt in keiner Sprache außergewöhnlich über oder unter den Normwerten. Bei Aurelio ist der Kontrast zwischen den beiden Sprachen sehr groß, d. h., sein Italienisch entwi- 
ckelt sich schneller als das Deutsche. Tabelle 2 zeigt einen Überblick dieser Ergebnisse. Hinzu kommen die Informationen zu den Sprachmischungen.

\begin{tabular}{|l|l|l|l|l|}
\hline Kind & Höchste Werte & Größeres Lexikon & $\begin{array}{l}\text { Werte über } \\
\text { Norm }\end{array}$ & $\begin{array}{l}\text { Mischungen im } \\
\text { Sprachkontext }\end{array}$ \\
\hline Carlotta & ttalienisch & Italienisch & Italienisch & Deutschen \\
\hline Lukas & Italienisch/Deutsch & Italienisch/Deutsch & $\begin{array}{l}\text { Italienisch/ } \\
\text { Deutsch }\end{array}$ & Italienischen \\
\hline Jan & Deutsch & Deutsch & Deutsch & Italienischen \\
\hline Aurelio & Italienisch & Italienisch & Italienisch & $\begin{array}{l}\text { Italienischen/ } \\
\text { Deutschen }\end{array}$ \\
\hline Marta & Italienisch & Italienisch & Italienisch & Deutschen \\
\hline
\end{tabular}

Tabelle 2: Zusammenfassung der Ergebnisse zur Sprachdominanz und zu den Mischungen

Die Tatsache, dass Carlotta und Marta im Deutschen und Lukas im Italienischen mischen, stimmt tendenziell mit den Ergebnissen der Normwerte überein. Auf der anderen Seite muss man betonen, dass die Kinder Mischraten von 8-19\% haben: Können diese Prozentzahlen wirklich untermauern, dass die drei dominant in der anderen Sprache sind? Denken wir an den Sprachgebrauch zurück: Warum haben die drei nicht in der Sprache, in der sie niedrigere Werte haben, die stärkere Sprache benutzt? Bei keinem dieser Kinder ist eine Äußerung in der starken Sprache im Kontext der so genannten schwachen Sprache zu finden. Lukas hat abwechselnd hohe Werte im Italienischen und im Deutschen. Wie kommt es, dass er nur im italienischen Kontext mischt? Kann das daran liegen, dass die italienische Interviewerin bilingual ist? Wenn das der Fall ist, muss Sprachdominanz als Erklärung ausgeschlossen werden. Aurelio mischt in der Sprache, in der er die höchsten Werte hat. Wenn man aber bedenkt, dass er überwiegend die Partikel ja/nein mischt, und man nicht annehmen kann, dass er die entsprechenden Äquivalente im Italienischen nicht kann, muss man davon ausgehen, dass er nicht aus einer Notwendigkeit heraus mischt.

Dass ein Kind regelmäßig höhere Werte in der einen Sprache hat, muss nicht als Folge haben, dass von der stärkeren Sprache in die schwächere gemischt wird bzw. dass die schwächere Sprache gar nicht gesprochen wird. Bei Kindern, die von Geburt an zweisprachig aufwachsen und beide Sprachen relativ gleichmäßig entwickeln, hat weder die Tatsache, dass eine Sprache höhere Werte hat, Auswirkungen auf die andere Sprache, noch muss es zwingend bedeuten, dass gemischt werden muss. Wie bereits erwähnt, scheint es vielmehr einen Zusammenhang zwischen dem Sprachgebrauch in der Familie und dem der Kinder zu geben: Bei Carlotta und Marta ist Italienisch die Familiensprache, bei Aurelio auch, aber es wird auch viel gemischt, und bei Lukas und Jan wird zu Hause Deutsch gesprochen. 


\subsubsection{Flüssiges Sprechen}

Cantone \& Müller (2005) haben eine andere Erklärung für Sprachmischungen bei jungen bilingualen Kindern vorgeschlagen. Die Autorinnen haben vier Kinder untersucht (Carlotta, Lukas, Jan und Aurelio) und die Hypothese formuliert, dass frühe Mischungen damit zusammenhängen, dass Kinder noch keinen routinierten Umgang damit haben, Wörter aus der richtigen Sprache zu wählen. Wie im Abschnitt 1.4.2 erwähnt, soll die Architektur der Sprachfähigkeit bei bilingualen Sprechern genauso aussehen wie bei Einsprachigen. Im Minimalismus (Chomsky 1995) nimmt man an, dass die Operation Select dafür verantwortlich ist, Wörter aus dem Lexikon zu entnehmen, um daraus Äußerungen zu bilden. Die Tatsache, dass das bilinguale Kleinkind in der ersten Phase des Spracherwerbs ist und noch nicht flüssig spricht, könnte als Erklärung für die falschen Zugriffe von Select sein. In der Tat haben Cantone \& Müller (2005) herausgefunden, dass es einen Zusammenhang zwischen der Anzahl von Mischungen und der Anzahl von Äußerungen gibt. Je mehr und flüssiger die Kinder sprechen, desto weniger wird gemischt. Man kann also behaupten, dass Sprachmischungen bei Kindern zwischen 1;7 und 2;7 Jahre eine Performanzerscheinung sind, also auf Anwendungsfehler basieren, und nicht auf Kompetenzfehler, also auf Fehler des Sprachwissens, zurückzuführen sind. Das heißt, die Mischungen in der frühen Phase haben weder mit dem Erwerb der Grammatiken zu tun oder ausschließlich damit, dass die Vokabeln erst erworben werden müssen, noch damit, dass Kinder dominant in einer Sprache sind.

\subsection{Die spätere Phase des Mischens}

Da ich annehme, dass das kindliche Mischen mit dem erwachsensprachlichen Code-switching gleichgesetzt werden kann, kann die Grammatikalität der kindlichen Sprachmischungen auf dieselbe Weise untersucht werden. Cantone (erscheint) hat die Restriktionen, die vorgeschlagen wurden, um diesen Sprachstil zu regulieren, auf die Kinderdaten angewendet und festgestellt, dass die Kinder mehrere Restriktionen verletzen. Geht man allerdings von MacSwans (1999, 2000) Annahme aus, dass nur die Regeln der involvierten Sprachen im Code-switching gelten und nicht extra formulierte Regeln, so stellt man fest, dass die hier vorgestellten Kinder keine ungrammatischen Mischungen äußern. Hier einige Sprachmischungen (aus Cantone erscheint) in der späteren Phase (ab ca. 2;7):

(9) das ist ein pannolino klein

(10) ho fatto zimtsterne

(11) la mosca geht

(12) pecché ich war kleiner= perché
Das ist eine kleine Windel

(Carlotta, 2;9,25, DK)

Ich babe Zimtsterne gemacht

Die Fliege geht

(Carlotta, 4;6,8, IK)

(Lukas, 3;8,17, IK)

Weil ich kleiner war 
(13) perché hanno fatto un kampf

(14) noi abbiamo gewonnen

(15) nein das ist die motore
(Lukas, 4;0,5, IK)

Weil sie einen Kampf gemacht haben

Wir haben gewonnen

$$
\text { (Jan, 4;4,6, IK) }
$$

(Aurelio, 3;8,13,IK)

Nein das ist der Motor

Bestimmte Mischpunkte sind beliebter als andere, z. B. mischen alle Kinder zwischen einem Artikel und einem Nomen, wie in (9), (13) und (15). Viel wird auch zwischen Verb und Objekt gemischt, wie in (10), oder zwischen dem Hilfsverb und dem Partizip (14). Andere Punkte kommen seltener vor, wie z. B. zwischen einer Konjunktion und dem Nebensatz (12), oder zwischen einem Nomen und einem Adjektiv, wie in (9). Selten tauchen Mischungen zwischen Subjekt und Verb auf (11) ${ }^{4}$. In Beispiel (9) sieht man, dass der Artikel mit dem Genus des gemischten Wortes markiert ist und nicht mit dem Genus der Sprache des Kontextes. Das italienische Wort für Windel ist Maskulin, während Windel im Deutschen Feminin ist. Der begleitende deutsche Artikel hier ist der maskuline. In Beispiel (13) ist das Wort Kampf im Deutschen und im Italienischen ein Maskulinum; Beispiel (15) ist ein Zeichen, dass die Schwa-Endung in motore vermutlich das Kind veranlasst hat, das Femininum zu wählen, da Motor im Deutschen und im Italienischen ein Maskulinum ist. Wir konnten in einer anderen Studie zeigen, dass bilinguale Kinder in 84\% der Fälle den Artikel mit dem Genus des gemischten Wortes markieren (Cantone \& Müller, im Druck).

Insgesamt kann man sagen, dass Code-Switching kein Zeichen für Chaos oder für einen unkontrollierten Gebrauch der beiden Sprachen ist, da die gemischten Äußerungen zielsprachlich sind und keine der beiden Grammatiken verletzen.

Auch in dieser Phase zeigen die Kinder einen korrekten Sprachgebrauch an, d. h., sie sprechen während der Aufnahme die von den Erwachsenen vorgegebene Sprache. Die Menge der Sprachmischungen variiert von Kind zu Kind. Während Lukas zunächst fast aufhört im Italienischen zu mischen, um dann wieder verstärkt gemischte Äußerungen zu produzieren, hört Aurelio nie auf, viel im Italienischen zu mischen. Carlotta, Jan und Marta mischen sehr wenig im Italienischen. Die Mischrate variiert zwischen 1-3\%. Im Deutschen mischen Jan und Lukas weiterhin fast gar nicht, während die anderen zwar etwas mischen, aber keine hohen Raten aufweisen.

\footnotetext{
${ }^{4}$ Eine detaillierte quantitative und qualitative Analyse aller Sprachmischungen findet sich in Cantone (erscheint).
} 


\section{Abschluss}

Meine Untersuchung hat gezeigt, dass bilinguale Kinder von früh an ihre Sprachen trennen und diese auch zielsprachlich im angegebenen Sprachkontext benutzen. Die Anzahl der Sprachmischungen variiert von Kind zu Kind, wodurch ausgeschlossen werden kann, dass es eine allgemeingültige Phase geben muss, in der alle Kinder viel mischen. Vielmehr kann man das Sprachverhalten bzw. die Sprachpräferenz der Kinder gut mit der sprachlichen Situation in der Familie erklären.

Sprachdominanz kann nicht der einzige Grund fürs Mischen bzw. für die Mischrichtung sein, da einerseits der Begriff selbst missverständlich ist und andererseits Kinder auch dann mischen, wenn sie die Sprache gut beherrschen.

Ein wichtiges Ergebnis ist, dass die Kinder beim Mischen nicht die grammatischen Regeln ihrer Sprachen verletzen. Die Daten zeigen eher, wie spielerisch bilinguale Kinder mit ihren beiden Sprachen umgehen, wie die folgenden Beispiele (aus Cantone erscheint) verdeutlichen:

(16) die habn-die habn-die habn fruchtgust

(17) himbeerodore

(18) e poi è finiert

(19) león

(20) volp

$$
\text { = gusto }
$$

Himbeergeruch

Fruchtgeschmack

(Carlotta, 4;3,23,DK)

Und dann ist es zu Ende gegangen

$$
\text { (Carlotta, 4;9,1, IK) }
$$

(Aurelio, 4;0,28, IK)

=leone, Löwe

$=$ volpe, Fuchs

(Marta, 2;6,10, DK)

(Marta, 2;6,10, DK)

In (16) lässt Carlotta, um das italienische gusto deutsch klingen zu lassen, den Endvokal weg. In (17) erfinden sie erneut ein neues Kompositum: es riecht nach Himbeere, hat also Himbeergeruch. In (18) benutzt Aurelio ein italienisches Partizip, finito, das jedoch eine deutsche Partizipendung bekommt, (i)ert. In (19) und (20) sieht man, wie Marta systematisch die italienischen Wörter, die sie in der deutschen Aufnahme äußert, verdentscht, indem sie den Endvokal auslässt. Diese Äußerungen sind ein gutes Beispiel dafür, wie kreativ und ausgesprochen schlau bilinguale Kinder sein können und wie falsch es ist, sie aufgrund ihrer Sprachmischungen negativ zu beurteilen. 


\section{Literatur}

Belazi, Hedi M./ Rubin, Edward J. / Toribio, A. Jacqueline: "Code switching and x-bar theory: The functional head constraint”, Linguistic Inquiry, 25, 2 (1994), 221-237.

Bernardini, Petra / Schlyter, Suzanne: "Growing syntactic structure and code-mixing in the weaker language: The ivy hypothesis”, Bilingualism 7,1, (2004), 49-69.

Brown, Roger: A first language: The early stages, Cambridge, MA.: Harvard University Press 1973.

Cantone, Katja F.: Das Genus im Italienischen und Deutschen: Empirische Untersuchung zum bilingualen Spracherwerb, Unveröffentlichte Magisterarbeit, Universität Hamburg 1999.

Cantone, Katja F.: "Evidence against a third grammar: Code-switching in ItalianGerman bilingual children". In Cohen, James / McAlister, Kara / Rolstad, Kellie / MacSwan, Jeff (Hgg.), ISB4: Proceedings of the 4th International Symposium on Bilingualism. Somerville, MA: Cascadilla Press 2005, 477-496.

Cantone, Katja F.: Code-switching in bilingual children, Dordrecht: Springer, erscheint.

Cantone, Katja / Kupisch, Tanja / Müller, Natascha / Schmitz, Katrin: „Rethinking language dominance in bilingual children”, Manuskript, Universität Bremen 2006.

Cantone, Katja F. / Müller, Natascha: „Code-switching at the interface of language-specific lexicons and the computational system", International Journal of Bilingualism, Vol. 8,2 (2005), 205-225.

Cantone, Katja F. / Müller, Natascha: „Un Nase or una Nase? What gender marking within switched DPs reveals about the architecture of the bilingual language faculty", Lingua, im Druck.

Cohen, James / McAlister, Kara / Rolstad, Kellie / MacSwan, Jeff (Hgg.), ISB4: Proceedings of the 4th International Symposium on Bilingualism, Somerville, MA: Cascadilla Press 2005.

Chomsky, Noam: The Minimalist Program, Cambridge, MA: MIT Press 1995.

De Houwer, Annick: The acquisition of two languages from birth: A case study, Cambridge: Cambridge University Press 1990.

Deuchar, Margaret / Quay, Suzanne: “One vs. two systems in early bilingual syntax: Two versions of this question”, Bilingualism 1 (1998), 231-243.

Deuchar, Margaret / Quay, Suzanne: Bilingual acquisition: Theoretical implications of a case study, Oxford University Press 2000. 
DiSciullo, Anne-Marie / Muysken, Pieter / Singh, Rajendra: "Government and code-Switching”, Journal of Linguistics 22 (1986), 1-24.

Genesee, Fred: “Early bilingual development: One language or two?”, Journal of Child Language 16 (1989), 161-179.

Genesee, Fred / Nicoladis, Elena / Paradis, Johanne: "Language differentiation in early bilingual development", Journal of Child Language 22 (1995), 611-631.

Goodz, Naomi: "Parental language mixing in bilingual families", Infant Mental Health Journal 10 (1989), 25-43.

Gumperz, John J.: "The sociolinguistic significance of conversational codeswitching", Papers on Language and Context: Working papers 46, Berkeley: University of California, Language Behavior Research Laboratory (1976), 1-46.

Gumperz, John J. Discourse strategies, Cambridge: Cambridge University Press 1982.

Hyltenstam, Kenneth / Obler, Loraine K. (Hgg.), Bilingualism across the lifespan: Aspects of acquisition, maturity and loss, Cambridge: Cambridge University Press 1989.

Köppe, Regina: "Language differentiation in bilingual children: The development of grammatical and pragmatic competence", Linguistics 34 (1996), 927-954.

Köppe, Regina / Meisel, Jürgen M.: „Code-Switching in bilingual first language acquisition". In Milroy, Leslie / Muysken, Pieter (Hgg.), One speaker-two languages: Cross-disciplinary perspectives on code-switching, Cambridge: Cambridge University Press 1995, 276-301.

Kupisch, Tanja: The acquisition of determiners in bilingual German-Italian and German-French children, München: Lingcom Europa 2006.

Kupisch, Tanja / Müller, Natascha / Cantone, Katja: „Gender in monolingual and bilingual first language acquisition: Comparing Italian and French", Lingue e Linguaggio 1,1 (2002), 107-149.

Lanza, Elizabeth: "Can bilingual two-year-olds code-switch?”, Journal of Child Language 19 (1992), 633-658.

MacSwan, Jeff: A minimalist approach to intrasentential code switching, New York, Garland 1999.

MacSwan, Jeff. "The architecture of the bilingual language faculty: Evidence from intrasentential codeswitching", Bilingualism 3,1 (2000), 37-54.

McLaughlin, Barry. Second language acquisition in childhood, Vol 1: Preschool Children, Hillsdale, N.J.: Erlbaum 1984. 
Meisel, Jürgen M.: „Early differentiation of languages in bilingual children”. In Hyltenstam, Kenneth / Obler, Loraine K. (Hgg.), Bilingualism across the lifespan: Aspects of acquisition, maturity and loss, Cambridge: Cambridge University Press 1989, 13-40.

Meisel, Jürgen M.: "Code-switching in young bilingual children. The acquisition of grammatical constraints", Studies in Second Language Acquisition 16 (1994), 413-439.

Milroy, Leslie / Muysken, Pieter (Hgg.): One speaker-two languages: Crossdisciplinary perspectives on code-switching, Cambridge: Cambridge University Press 1995.

Müller, Natascha: "Gender and number in acquisition". In Unterbeck, Barbara / Rissanen, Matti (Hgg), Gender in grammar and cognition, I. Approaches to gender, II, The Hague: Mouton de Gruyter 1999, 351-399.

Müller, Natascha / Cantone, Katja / Kupisch, Tanja / Schmitz, Katrin: „Zum Spracheneinfluss im bilingualen Erstsprachenerwerb: Italienisch-Deutsch“, Linguistische Berichte 190 (2002), 157-206.

Müller, Natascha / Kupisch, Tanja: „Zum simultanen Erwerb des deutschen und des Französischen bei (un)ausgeglichenen bilingualen Kindern“, Vox Romanica 62 (2003), 1-25.

Müller, Natascha / Kupisch, Tanja / Schmitz, Katrin / Cantone, Katja: Einführung in die Mehrsprachigkeitsforschung, Tübingen: Narr 2006.

Paradis, Johanne / Genesee, Fred: "Syntactic acquisition in bilingual children: Autonomous or interdependent?", Studies on Second Language Acquisition 18 (1996), 1-15.

Petersen, Jennifer: "Word-internal code-switching constraints in a bilingual child's grammar”, Linguistics 26 (1988), 479-493.

Poplack, Shana: "Sometimes I'll start a sentence in Spanish y termino en Español: Toward a typology of code-switching”, Linguistics, 18 (1980), 581-618.

Taeschner, Traute: The sun is feminine. A study of language acquisition in bilingual children, Berlin: Springer 1983.

Unterbeck, Barbara / Rissanen, Matti (Hgg), Gender in grammar and cognition, I. Approaches to gender, II, The Hague: Mouton de Gruyter 1999.

Volterra, Virginia / Taeschner, Traute: "The acquisition and development of language by bilingual children", Journal of Child Language, 5 (1978), 311- 326. 


\title{
Deutsch als Zweitsprache und Mehrsprachigkeit - Widerspruch oder Möglichkeit?
}

\author{
Sigrid Luchtenberg, Essen
}

Deutsch als Zweitsprache ist zur Zeit hochaktuell im Rahmen der Integrationsdebatten, aber auch auf Grund der jüngsten Ergebnisse der OECD-Vergleichsuntersuchungen (vgl. OECD 2006 für vergleichende Ergebnisse bei Schüler/innen mit Migrationshintergrund)). Demgegenüber nimmt Mehrsprachigkeitserziehung im derzeitigen öffentlichen Diskurs eine sehr viel weniger beachtete Position ein.

Im Folgenden soll zunächst kurz auf die Entwicklung von Deutsch als Zweitsprache einerseits und Mehrsprachigkeitserziehung andererseits eingegangen werden. Danach wird nach den Möglichkeiten ihrer Verbindung gefragt. Dazu wird auf folgende Punkte eingegangen: Die Verbindung von Deutsch als Zweitsprache und Mehrsprachigkeitserziehung im Deutschunterricht, in Sachfächern sowie in den Fremd- und Erstsprachen, ferner auf Multiliteralitätsaspekte in Deutsch als Zweitsprache. Nach dieser Diskussion der sich aus dem Zusammenführen beider Konzepte ergebenden Möglichkeiten stellt sich die Frage nach der Lehrerausbildung, denn ohne entsprechend ausgebildete Lehrkräfte bleiben solche Vorstellungen leer. 


\section{Die Entwicklung von Deutsch als Zweitsprache und Mehrsprachigkeitserziehung}

Sowohl Deutsch als Zweitsprache wie auch Mehrsprachigkeitserziehung sind in ihrer Entwicklung eng verbunden mit der (Bildungs)politik als Reaktion auf Einwanderung nach Deutschland seit den $60 \mathrm{er}$ Jahren, die zunächst keine adäquaten Antworten fand wie die wechselnden Empfehlungen der Kultusministerkonferenz deutlich zeigen. Auch die Erziehungswissenschaft musste sich auf eine bis dahin weitgehend unbekannte Situation in Deutschland (d. h. hier der alten BRD) einstellen und Konzepte entwickeln. Dabei wurde zunächst ein ausländerpädagogischer Ansatz gefunden, der jedoch zunehmend durch einen interkulturellen ersetzt wurde. Deutsch als Zweitsprache war von Anfang an ein wichtiges Thema, während Mehrsprachigkeitserziehung erst mit der Interkulturellen Erziehung an Relevanz gewann.

Zweifellos lassen sich beide Konzepte didaktisch sinnvoll separat für verschiedene Fächer diskutieren. Es ist jedoch ein Verdienst der DaF-Tagung 2006, beide zusammenzuführen, denn zwar ist eine Einbindung der beiden Bereiche - unabhängig voneinander - in die Schule möglich, aber nur ihre Verbindung erlaubt eine bildungspolitische Integration von Schülern und Schülerinnen mit Migrationshintergrund. Eine ausschließliche Förderung in Deutsch als Zweitsprache wird oft mit den dadurch erreichbaren Partizipationschancen von jungen Einwanderern begründet (vgl. Esser 2006), hat aber diskriminierenden Charakter, wenn gleichzeitig eine europäisch postulierte und geförderte Mehrsprachigkeitserziehung in den gängigen Schulfremdsprachen stattfindet, da dies einer Abwertung der Erstsprachen von Einwanderern entspricht.

\subsection{Deutsch als Zweitsprache}

Zwar spielte die Vermittlung der deutschen Sprache in der Bildungspolitik wie auch in der Erziehungswissenschaft von Anfang an eine wichtige Rolle, jedoch musste ein „Fach“ Deutsch als Zweitsprache erst konzipiert werden, was im Wesentlichen im Rahmen der sich parallel entwickelnden Ausländerpädagogik geschah. Die vorhandene Disziplin des Deutschen als Fremdsprache - in Schulen im Ausland wie in der Erwachsenenbildung - erwies sich als unzureichend für die Anforderungen des Zweitsprachenerwerbs im Zielsprachenland. Pädagogische Untersuchungen machten zudem deutlich, dass auch die marginalisierte Rolle ausländischer Arbeitnehmer/innen und ihrer Kinder Bedeutung für ihren Zweitsprachenerwerb hatte und noch hat (Kuhs 1989; Neumann 2002).

Die Vermittlung der deutschen Sprache an die Kinder der Arbeitsmigranten sollte es ihnen ermöglichen, sich im deutschen Schulsystem zurechtzufinden, wobei der Besuch einer Regelschule und -klasse vorgesehen war. 
Deutsch als Zweitsprache hat starke Wurzeln in der Ausländerpädagogik, da sich die gesamte Konzeption ausschließlich mit Kindern mit Migrationshintergrund befasst. Zugleich entwickelte sich das neue Fach weitgehend unabhängig vom muttersprachlichen Deutschunterricht, so dass interkulturelle Deutschdidaktik im Kontext des regulären Deutschunterrichts parallel zu Deutsch als Zweitsprache entstand, ohne dass es zunächst Berührungen gegeben hätte (vgl. Luchtenberg 2004 zum Verhältnis von Deutsch als Zweitsprache und Interkultureller Pädagogik). Wie stark Deutsch als Zweitsprache aus der Entwicklung interkultureller Pädagogik ausgeklammert blieb, zeigt die KMK-Empfehlung von 1996 (Sekretariat 1996) zu Interkultureller Pädagogik, da hier nicht auf Deutsch als Zweitsprache eingegangen wird.

Unabhängig von dieser Entwicklung gibt es in der Disziplin Deutsch als Zweitsprache durchaus Bestrebungen, zu einem interkulturellen Selbstverständnis zu finden, indem vom Ziel der Defizitreduzierung abgegangen wird zu Gunsten des Ziels einer interkulturellen Kommunikationskompetenz in deutschsprachigen Ländern (vgl. Barkowski 2001).

\subsection{Mehrsprachigkeitserziehung}

Der Begriff der Mehrsprachigkeitserziehung ist weniger eindeutig definiert. Wir finden seine Verwendung in unterschiedlichen Kontexten wie vor allem:

- Aufwachsen mit 2 (und mehr) Sprachen in bilingualen Familien

- Aufwachsen in mehrsprachigen Regionen bzw. Ländern

- Fremdsprachenunterricht

- Bilingualer Unterricht/Bilinguale Klassen

- Deutsch als Zweitsprache unter Berücksichtigung der Erstsprachen

- Entwicklung eines Gesamtsprachenkonzepts

- EU/Europarat-Sprachenpolitik

- Rezeptive Mehrsprachigkeit

- Deutschunterricht unter Berücksichtigung vorhandener Erstsprachen (Ansatz des Handbuchs für Deutschdidaktik (Bredel u.a. 2003))

- Integration der Herkunftssprachen in den Regelunterricht

- Mehrsprachigkeitserziehung und Languages Awareness.

Die meisten der hier aufgezählten Ansätze sind spracherwerbsorientiert, lediglich die letzten vier sind eher nicht spracherwerbsorientiert. Deutsch als Zweitsprache lässt sich in die spracherwerbsorientierten Kontexte gut einbinden, wenn, ,Zweitsprache ${ }^{6}$ ernst genommen wird, denn dann ergibt sich durch den Erwerb einer zweiten Sprache Mehrsprachigkeit, da diese zweite Sprache zu einer ersten Sprache hinzukommt. Bei ausreichender Förderung beider Sprachen kommt es zu einer ausgebauten Mehrsprachigkeit. In diesem Sinne gehört die Verbindung von 
Deutsch als Zweitsprache und Mehrsprachigkeitserziehung in den weiteren Rahmen der Fremdsprachenpolitik.

Im Folgenden soll jedoch nicht spracherwerbsorientierte Mehrsprachigkeit (in Verbindung mit Deutsch als Zweitsprache) im Mittelpunkt stehen, d. h. die Berücksichtigung von anderen Sprachen im Unterricht, unabhängig davon, ob es sich um Sprachunterricht oder andere Fächer handelt, und auch unabhängig davon, ob es sich um Herkunftssprachen oder Fremdsprachen handelt. Allerdings gehen wir davon aus, dass in mehrsprachigen Klassen auf jeden Fall die jeweiligen Herkunftssprachen Relevanz haben. Mehrsprachigkeitserziehung in diesem Sinn steht den Konzepten von Language Awareness sehr nahe (vgl. Luchtenberg 1998).

\section{Welche Möglichkeiten ergeben sich aus ihrer Verbindung?}

Die folgenden Überlegungen beruhen teilweise auf Praxisberichten, teilweise sind sie als Anregungen zu verstehen. Aus erziehungswissenschaftlicher Sicht können sie als ein Versuch angesehen werden, die beiden Stränge interkultureller Erziehung zusammenzuführen, ohne die unabhängige Bedeutung beider in Frage zu stellen (vgl. auch Luchtenberg 2004):

Interkulturelle Pädagogik

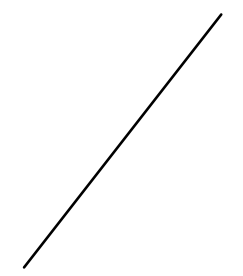

Herstellung von Chancengleichheit

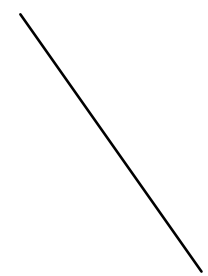

Vorbereitung aller auf ein Leben in einer mehrsprachig-mehrkulturellen Gesellschaft

Mehrsprachigkeitserziehung unter Berücksichtigung der migrationsbedingten Mehrsprachigkeit des rechten Strangs soll hier mit Deutsch als Zweitsprache als einer der wesentlichen Aufgaben des linken Strangs zusammengeführt werden unter der Prämisse, dass beide gleichberechtigte Aufgaben interkultureller Erziehung sind, zu der allerdings noch weitere wie antirassistische Erziehung oder Citizenship Eduation gehören. Beide Stränge verstehen sich auch als Teil einer integrationsorientierten Erziehung, die sich allerdings deutlich von Assimilation abgrenzt. 


\subsection{Deutschunterricht}

Es scheint nahe zu liegen, mit dem Deutschunterricht zu beginnen als dem Fach, das Deutsch als Zweitsprache am nächsten liegt. Viele Diskussionen um Deutsch als Zweitsprache haben allerdings auch deutlich gemacht, dass diese Verbindung nicht so eindeutig ist, wie es auf den ersten Blick scheint, so dass eine Verortung von Deutsch als Zweitsprache immer noch schwierig ist. Die Verortung von Deutsch als Zweitsprache in der Deutschdidaktik ist aus verschiedenen Gründen problematisch:

- Bei der Entwicklung von Deutsch als Zweitsprache spielte die Deutschdidaktik nur eine geringe Rolle

- Die herkömmliche Deutschdidaktik integriert den Bereich Deutsch als Zweitsprache nur zögerlich, was auch für die Lehrerausbildung gilt

- Es gibt viele Bedenken, ob der Deutschunterricht es leisten kann, gleichzeitig die Belange von Deutsch als Zweitsprache zu bedienen (vgl. Kuhs 2002).

Andererseits ist die Einbindung von Deutsch als Zweitsprache in die Deutschdidaktik dringend notwendig, weil

- der Deutschunterricht von den übrigen Fächern als zuständig erachtet wird

- der Deutschunterricht sich zur Koordination der Zusatzprogramme in Deutsch als Zweitsprache anbietet

- die Anforderungen des Deutschunterrichts als Richtschnur für die Erfordernisse in Deutsch als Zweitsprache gelten können

- im Deutschunterricht die Verbindung zur Mehrsprachigkeitserziehung bereits konzeptionell erarbeitet ist.

Im Rahmen der interkulturellen Erziehung und ihrer Didaktik wurde auch eine interkulturelle Deutschdidaktik entwickelt. Ihr wesentliches Merkmal ist die bewusste Berücksichtigung von Mehrsprachigkeit, was der Vorbereitung aller auf ein Leben in der mehrsprachigen und mehrkulturellen Gesellschaft entspricht, also dem rechten Strang in der obigen Definition.

Ausgehend von der „faktischen Mehrsprachigkeit und Plurikulturalität fast aller Klassen“ entwickelte Oomen-Welke $(1998,287)$ einen Ansatz, der „die vorhandene Zweisprachigkeit der anwesenden Schüler/innen berücksichtigt, für Lehrpersonen praktikabel ist und den Deutschunterricht inhaltlich und methodisch bereichert". Ausgangspunkt einer solchen nicht spracherwerbsorientierten Mehrsprachigkeitsdidaktik im Rahmen des Deutschunterrichts sind einerseits das Vorhandensein mehrerer Sprachen in einer Klasse sowie andererseits die Existenz von Sprachaufmerksamkeit, die allerdings auch entwickelt werden kann (OomenWelke 1999, 17). In einem mehrschrittigen Verfahren kann nun die Sprachenviel- 
falt für Lerninhalte des Deutschunterrichts auf sehr verschiedenen Gebieten des Lernbereichs Reflexion über Sprache, aber auch Textunterricht genutzt werden. Methodisch steht der - spontane oder angeleitete - Sprachenvergleich im Mittelpunkt, der im Allgemeinen zu neuen Einsichten über die deutsche Sprache, aber auch über Vielfalt von Sprachen führt.

Mehrsprachigkeit im Deutschunterricht zu berücksichtigen verlangt Sprachaufmerksamkeit. Dies ergibt sich in allen Ansätzen und vielen weiteren Beiträgen zum Unterricht in mehrsprachigen Klassen (vgl. auch Reich 2000). Dem deutschen ,Sprachaufmerksamkeit" entspricht im Englischen ,Language Awareness', wobei es sich zugleich um eine in den 70er Jahren in Großbritannien entwickelte Konzeption handelt, die zunächst den schlechten Ergebnissen englischer Schüler/innen im Englisch- und Fremdsprachenlernen entgegenwirken sollte. Da auch in Großbritannien Mehrsprachigkeit eher der Normalfall in den Schulen ist, wurde die Konzeption erweitert zu ,Languages Awareness' und ,Languages and Cultural Awareness'. Mit ,Critical Language(s) Awareness` gelang schließlich die Einbeziehung von Sprachmissbrauch und sprachlicher Diskriminierung (vgl. Luchtenberg 1998). ,Language(s) Awareness' kann als in mehrfacher Hinsicht holistisch bezeichnet werden, da alle Sprachen, die im Umfeld eine Rolle spielen, berücksichtigt werden; Sprache ganzheitlich einbezogen wird, d. h. also alle ihre Phänomene von Lauten bis zu Texten und zudem kognitive, soziale und emotionale Zugänge zu Sprache(n) eine Rolle spielen. ,Language(s) Awareness' kann - ebenso wie das Mehrsprachigkeitskonzept von Oomen-Welke - den gesamten Deutschunterricht betreffen.

Im Wesentlichen findet die Entwicklung einer Einbindung von Deutsch als Zweitsprache in den Deutschunterricht und dessen Öffnung hin zu einer interkulturellen Mehrsprachigkeitskonzeption im Sinne der gerade skizzierten Vorstellungen unabhängig voneinander statt, obwohl die Akteure die gleichen sind:

- Die Lernenden des Deutschen als Zweitsprache sind zugleich diejenigen, die die Experten für die im Mehrsprachigkeitsansatz verwandten Sprachen sind.

Hier liegt also eine erste Möglichkeit der Verbindung beider Konzepte. Die zweite liegt in den Inhalten der Mehrsprachigkeitsansätze, in denen es in aller Regel um Phänomene des Wortschatzes, der Texte oder auch grammatischer Strukturen geht, wobei der Wortschatz der deutschen Sprache mit denen der anderen Sprachen ebenso verglichen wird wie Textsorten oder Strukturen. Es sollte zu denken geben, dass deutsche Schüler/innen einen enormen Lernzuwachs verzeichnen, da sie durch den Vergleich beginnen, über Phänomene in ihrer Sprache nachzudenken.

Wie lässt sich ein Lernzuwachs für alle Kinder erreichen?

- Die Ergebnisse des Sprachvergleichs können in den Förderstunden Deutsch als Zweitsprache systematisiert werden 
- Die Ergebnisse können in unterschiedlichen Formen der Gruppen- und Partnerarbeit wie beispielsweise Lerntandems vertieft werden.

- Die zweisprachigen Schüler/innen geben nicht nur Impulse des Vergleichs, sondern bringen auch ihre Fragen zum Deutschen ein, was vielfach wiederum zum Vergleich führt (z. B. die Frage nach Geschlecht und Diminutiva)

Für den Deutschunterricht bieten sich also vielfältige Möglichkeiten, die Belange von Deutsch als Zweitsprache mit denen einer nicht spracherwerbsorientierten Mehrsprachigkeitserziehung zu verbinden. Neben den Herkunftssprachen und Deutsch lassen sich auch die jeweiligen Dialekte und Fremdsprachen berücksichtigen, wie es etwa im Kontext Internationalismen, Werbesprache oder Entlehnungen ohnehin üblich ist (vgl. Schader 2000 für eine Fülle anregender Unterrichtsvorschläge).

Im Deutschunterricht Mehrsprachigkeitskonzepte mit denen von Deutsch als Zweitsprache zu verbinden gibt dem Deutschunterricht also wesentlich neue Möglichkeiten im Bereich der Sprachreflexion wie auch der Textproduktion. Was aber ist mit dem Teil des Deutschunterrichts, der sich mit Literatur befasst? Hier scheinen auf den ersten Blick weder Deutsch als Zweitsprache noch Mehrsprachigkeitserziehung allzu relevant zu sein. Untersuchungen zur Kinder- und Jugendliteratur haben allerdings ergeben, dass Sprache und insbesondere Sprachen wie auch Mehrsprachigkeit selbst in einer Reihe von Büchern eine wesentliche Rolle spielen, was sich in interkultureller Kinder- und Jugendliteratur noch erhöht, wenn es um Themen im Migrationskontext geht (vgl. Luchtenberg 2001). Vergleichbare Aspekte finden sich auch in literarischen Werken wie etwa die Sprachenverteilung in Thomas Mann Buddenbrooks oder die autobiographische Auseinandersetzung mit den Sprachen seiner Kindheit bei Elias Canetti. Die im Sprachunterricht Deutsch geweckte Neugierde auf Sprache und die damit verbundene Sprachaufmerksamkeit wird auch im Literaturunterricht Früchte tragen. Welche Rolle kommt Deutsch als Zweitsprache hier zu? Es ergeben sich verschiedene Aspekte:

- Sprachtandems etc. zur Aufarbeitung sprachlicher Besonderheiten

- Formen der Textentlastung finden Anwendung

- Zweitsprachenlerner/innen bringen (sprach)kulturelle Fragen ein, z. B. bei Texten, in denen Religion, Geschichte oder Kultur eine Rolle spielen.

Medienerziehung ist ein weiterer Bereich des Deutschunterrichts, in dem Mehrsprachigkeitserziehung und Deutsch als Zweitsprache gemeinsam zu neuen Perspektiven führen können, etwa bei der Arbeit im Internet, bei E-Mail-Kontakten oder auch in den Bereichen kritischer Medienanalyse.

Der Deutschunterricht enthält also Möglichkeiten der Erweiterung auf Mehrsprachigkeitserziehung und Einbeziehung von Deutsch als Zweitsprache, 
aber es sollte betont werden, dass dies keine Hilfsfunktion des Deutschunterrichts ist, sondern dass er durch diese Erweiterung für seine eigenen Fragestellungen gewinnt.

\subsection{Sachfächer}

Eine Empfehlung aus NRW (Ministerium 1999) fordert, Förderung in der deutschen Sprache als Aufgabe des Unterrichts in allen Fächern anzusehen, so dass vor allem (aber nicht ausschließlich), Fachsprachenvermittlung in den Fächern selbst stattfinden kann. Außerdem wird empfohlen, berufsrelevante mündliche und schriftliche Sprachkompetenz ebenfalls in die Sachfächer einzubinden. Es liegt nahe zu überlegen, in diese Empfehlung die Zweitsprachenlernenden explizit einzubeziehen. Bereits Deppner (1989, 216ff.) hat die Forderung erhoben, dass der Charakter aller Fächer als Zweitsprachenunterricht in der Lehrerausbildung ebenso wie in den Lehrplänen berücksichtigt werden müsse, so dass etwa Konzepte der Textentlastung, der Wortschatzvermittlung, des Gebrauchs von Wörterbüchern oder des Sprachvergleichs auch Teil der jeweiligen Fächer werden würden. Eine solche Einbeziehung von Deutsch als Zweitsprache in alle Fächer würde die Sprachlernsituation der mehrsprachigen Schüler/innen, für die jeder Unterricht in deutscher Sprache immer auch Unterricht in Deutsch als Zweitsprache ist, wesentlich verbessern. Die erhöhte Sprachaufmerksamkeit in den Sachfächern dürfte aber allen Schüler/innen nützen, da insbesondere die Vermittlung von Fachsprache stärker sprachbezogen erfolgt und nicht - wie bislang häufig - implizit in der Vermittlung von Fachinhalten.

Welche Rolle aber kann Mehrsprachigkeitserziehung im Fachunterricht einnehmen und wo liegt die Verbindung zu Deutsch als Zweitsprache?

In den meisten Fächern ergeben sich Bezüge zu anderen Sprachen, oft auch den Herkunftssprachen der Schüler/innen mit Migrationshintergrund. Dies wird im Geographie- und Geschichtsunterricht besonders deutlich, wenn es um die Aussprache von Namen geht. Namen spielen auch in Migrationen eine vielfältige Rolle: Viele Auswanderer haben die Namen ihrer Städte mitgenommen, andererseits haben viele ihre Namen der jeweiligen Landessprache angepasst. Darüber hinaus kann es auch eine Rolle spielen, welche Sprachen in welchen Regionen gesprochen werden, auch auf Grund von Migrationen. Auch Sprachen haben eine Geschichte: Wie wurde früher gesprochen? Wie sind die Sprachen gewandert? Woher beziehen Sprachen neue Wörter? Hier sind die Sprachen der Kinder mit Migrationshintergrund besonders interessant. Dies betrifft unter anderem auch Kolonialprozesse und Sprachen wie etwa die Entstehung von Pidgin- und Creolesprachen, aber auch die Unterdrückung vorhandener Sprachen.

Fächerübergreifend können die Sprachbiographien der Schüler/innen aufgearbeitet werden, auch in Anlehnung an entsprechende literarische Vorbilder. Mehrsprachige Schülerzeitungen, die Behandlung mehrsprachiger Witze und Rätsel bzw. Unterschiede zwischen ihnen in verschiedenen Sprachen, Gastarbeiterdeutsch 
und Foreigner Talk - auch im Kontext gegenwärtiger Integrationsthemen - , aber auch rassistischer Umgang mit Mehrsprachigkeit können weitere Themen sein.

Deutsch als Zweitsprache kann als Impulsgeber für die Einbeziehung sprachlicher Aspekte fungieren, so dass die nicht spracherwerbsorientierte Mehrsprachigkeitserziehung auch in Sachfächern Normalität wird.

\subsection{Fremdsprachen}

Fremdsprachenunterricht stellt in der Liste der hier angesprochenen Fächer eine Besonderheit dar, da es sich bereits um spracherwerbsorientierte Mehrsprachigkeitserziehung handelt. Eine Integration von Deutsch als Zweitsprache ist dennoch möglich, und zwar auf unterschiedlichen Ebenen:

- die Sprachlernerfahrungen in Deutsch als Zweitsprache werden explizit einbezogen

- kontrastive Elemente fließen in den Unterricht ein (DeutschFremdsprache)

- die Sprachreflexion wird erhöht.

Voraussetzung ist allerdings, dass der Fremdsprachenunterricht insbesondere in sprachreflexiven Teilbereichen das Prinzip der Einsprachigkeit zu Gunsten des Sprachvergleichs aufgibt.

Dies ist auch notwendig, um im Fremdsprachenunterricht der Mehrsprachigkeitserziehung Raum zu geben. In allen sprachlichen Bereichen kann auch der Fremdsprachenunterricht durch Vergleich gewinnen wie es oben für den Deutschunterricht ausführlicher diskutiert wurde. Der Vergleich kann sich auf die den Schüler/innen bereits bekannten Fremdsprachen beziehen, also etwa Gemeinsamkeiten zwischen romanischen Sprachen herausstellen, oder aber durch Einbeziehung der Erstsprachen der Schüler/innen erfolgen. Die Basis für den Sprachvergleich wird hier durch Deutsch als Zweitsprache gelegt. Ein weiterer Aspekt ist auch die Chance, Spracherwerbsstrategien selbst anzusprechen, denn die am Fremdsprachenunterricht teilnehmenden Schüler/innen mit einer nichtdeutschen Erstsprache können hierzu Beiträge leisten. Sprachlernphänomene wie Interferenzen oder der Umgang mit den ,falschen Freunden' werden durch die Berücksichtigung von Deutsch als Zweitsprache wie auch weiterer Sprachlernerfahrungen auf eine breitere Basis gestellt und können entsprechend aufgearbeitet werden. Auch Interkulturelle Kommunikation kann in diesem erweiterten Rahmen gut thematisiert und ihre Strategien erlernt werden. Anregungen und Hilfen könnten möglicherweise auch aus den Konzepten des Bilingualen Fachunterrichts gewonnen werden.

\subsection{Erstsprachen}

Gegenüber dem Fremdsprachenunterricht ist der Unterricht in den Erstsprachen dadurch gekennzeichnet, dass er zwar auch spracherwerbsorientiert ist, sich aber 
in den meisten Fällen an eine homogene Gruppe wendet, die die zu erwerbende Sprache bereits als Erstsprache spricht, wenn auch sehr oft in stark unterschiedlichem Maße. In der Regel ist Deutsch für alle teilnehmenden Schüler/innen Zweitsprache. In einigen Klassen können auch Schüler/innen anderer Erstsprachen wie z. B. Deutsch an diesem Sprachunterricht teilnehmen, wodurch die sprachliche Heterogenität deutlich erhöht wird.

Die Verbindung des Erstsprachenunterrichts zum Unterricht in Deutsch als Zweitsprache würde eine Koordination beider Sprachen erlauben, wie sie sonst nur in bilingualen Unterrichtsformen möglich ist. Allerdings sind hier auch einige - überwiegend organisatorisch-strukturelle - Hindernisse zu benennen wie etwa die Tatsache, dass der Erstsprachenunterricht nur selten ein reguläres Unterrichtsfach ist, sondern dass er stattdessen vielfach noch außerhalb des Regelunterrichts und zum Teil schulübergreifend stattfindet. Dies behindert eine Kooperation zwischen den beteiligten Lehrkräften. In den höheren Klassen, in denen eher Unterricht in den Erstsprachen als Schulfach anzutreffen ist, fehlt dagegen meist ein Zusatzunterricht in Deutsch als Zweitsprache, da die Schüler/innen dem deutschsprachigen Unterricht zumindest folgen können.

Wie kann das Sprachenfach, Erstsprachenunterricht ${ }^{6}$ in Verbindung mit Deutsch als Zweitsprache zur Mehrsprachigkeitserziehung beitragen? Hierzu könnte zum einen die europäische Sprachen-Portfolio-Entwicklung zur Berücksichtigung der individuellen Sprachenvielfalt beitragen und andererseits eine kontrastive Form des Sprachunterrichts, der nicht nur Deutsch berücksichtigt, sondern auch Schulsprachen oder mit der Erstsprache verwandte Sprachen bzw. Sprachen, die den Schüler/innen bekannt sind.

\subsection{Multiliteralität}

Die bisherigen Überlegungen zur sinnvollen gegenseitigen Ergänzung von Deutsch als Zweitsprache und Mehrsprachigkeitserziehung waren an Schulfächern orientiert. Multiliteralität ließe sich im Kontext des Deutsch- oder Sprachenunterrichts behandeln, ist aber Aufgabe aller Fächer und soll daher hier kurz als eigenes Thema angesprochen werden. In diesem Begriff werden mehrere Aspekte zusammengefasst, die den gesamten Bereich der Schriftlichkeit in den letzten Jahren in der internationalen Diskussion erneuert haben. Zum einen ist die Vorstellung des Schriftspracherwerbs stark erweitert worden von einer ursprünglichen Alphabetisierung mit dem Ziel des Erwerbs von Lesekompetenz hin zu einer umfassenden Literalität, die auch Textsortenkompetenz und Semiotik umfasst, zum anderen wird Sprachenerwerb auch mit dem Erwerb einer Literalität in den neuen Sprachen verbunden, so dass Schüler/innen mit Migrationshintergrund durch das Erlernen von Deutsch als Zweitsprache die Chance zu Biliteralität erhalten, sofern sie in ihrer Erstsprache auch im Schriftsprachbereich ausreichend gefördert werden. Im Multiliteracies-Ansatz wird zeitgemäße Lesekompetenz mit kritischer Aus- 
einandersetzung und zudem ,lokale Diversität mit gleichzeitiger ,globaler Verbundenheit' verknüpft (vgl. Cope/Kalantzis 2000). Es werden zudem unterschiedliche Anforderungen an Lesen berücksichtigt: Bilder und Schrift müssen entschlüsselt, gegeneinander geprüft und verbunden werden, aber auch graphische Anordnungen und Punktuation müssen verstanden werden, versteckte Botschaften sowie kulturelle Prägungen müssen gefunden und mit den eigenen Erfahrungen verbunden werden. Oft muss ein Bezug zwischen Text und Realität hergestellt werden. Es kommt hinzu, dass hier auch die mediale Welt erfasst wird.

Der Ansatz von Multiliteracies kann gerade auch dazu dienen, die unterschiedlichen literalen Kenntnisse und Bedürfnisse von Schüler/innen mit Deutsch als Erst- und Zweitsprache in mehrsprachigen Klassen zu erkennen und sprach- und literaturdidaktisch aufzuarbeiten. Auch die sprachliche Vielfalt lässt sich in diesem Ansatz gut vermitteln. Ein kritischer Aspekt kommt darin zum Tragen, dass beim Lesen zugleich eine 'kompetente Kontrolle' ausgeübt werden soll (Cope/Kalantzis 2000, 9). Kritisches Lesen (Critical Literacy) wird zu Critical Multiliteracies, wenn kritische Lesekompetenz zugleich die Vielfalt der Lesekompetenzen und der Mehrsprachigkeit berücksichtigt. Kritische Lesekompetenzen werden vor allem für Medienkonsum entwickelt.

Literacy in mehr als einer Sprache ist ein in der deutschen Schule angestrebtes Ziel, das sich allerdings im Wesentlichen fremdsprachlich versteht. Ein solches Ziel ist im Hinblick auf europäische und globale Anforderungen im späteren Berufsleben der heutigen Schüler/innen durchaus begrüßenswert, sollte jedoch eine Erweiterung in Bezug auf die ,natürliche' Biliteralität von Schüler/innen mit Migrationshintergrund erfahren, die allerdings durchaus nicht ,natürlich' ist und auch nur selten ohne schulische Unterstützung erworben werden kann - im Gegensatz zu einer kommunikativen Zweisprachigkeit.

Der Multiliteralitäts-Ansatz ist darüber hinaus dazu geeignet, mit Literalität verbundene Fragen in Deutsch als Zweitsprache mit Mehrsprachigkeitserziehung zu verbinden, da Literalität nicht auf eine Sprache begrenzt verstanden wird, sondern sprachenübergreifend. Zudem sind die Sachfächer einbezogen, in denen beispielsweise die Kompetenzen der Entschlüsselung von Bildern und graphischen Darstellungen ebenso eine Rolle spielen können wie auch kulturell-sprachliche Aspekte anderer Sprachen oder Sprachzeiten wie etwa bei historischen Texten.

\section{Lehrerausbildung}

In der bisherigen Lehrerausbildung bleiben die Fächer ähnlich voneinander getrennt wie es auch in der Schule der Fall ist. Studierende des Fachs Germanistik werden demzufolge wenige Chancen haben, sich mit Mehrsprachigkeitserziehung auseinanderzusetzen. Möglicherweise werden sie nicht einmal mit den Belangen von Deutsch als Zweitsprache vertraut gemacht werden. Wer im Hinblick auf ein Lehramt eine oder mehrere Fremdsprachen studiert, wird sich nur in seltenen 
Fällen mit Deutsch als Zweitsprache befassen können, aber möglicherweise etwas über Mehrsprachigkeitserziehung im Sinne des Europarats erfahren. Der einzige Bereich, in dem alle Lehramtsstudierende in etwa vergleichbare Erfahrungen und Kenntnisse sammeln können, ist das erziehungswissenschaftliche Studium, in dem die hier vorgestellte Thematik allerdings bislang auch eher am Rande eine Rolle spielt im Kontext der interkulturellen Erziehung.

Angesichts der Tatsache, dass das ,Integrations'fach Deutsch als Zweitsprache in der bundesdeutschen Bildungsdiskussion zur Zeit hohe Bedeutung genießt und Mehrsprachigkeitserziehung ein europäisch verankertes Erziehungsziel ist, bestehen aber wiederum gute Chancen, eine solche Verbindung herzustellen. Hierzu könnte die ,Didaktische Landkarte، (Schader 2000, 51) erste Anregungen geben. $\mathrm{Zu}$ den dort aufgelisteten Lehrkompetenzen zählen Wissen um den Zweitspracherwerb und seine Didaktik, sprachfördernde Unterrichtsgestaltung und die Öffnung des (jeden!) Unterrichts für das sprachliche Potenzial einer Klasse, was durchaus auch Schulsprachen, Dialekte und außerschulische Sprachkenntnisse neben den Erstsprachen umfassen könnte. Hinzu kommen Basiskompetenzen wie Linguistik, methodisch-didaktisches Wissen, interkulturelles Wissen und eine Grundhaltung der Offenheit gegenüber kultureller und sprachlicher Vielfalt. Zu ergänzen ist diese Landharte um die Möglichkeiten einzelner Fächer wie auch um einen erweiterten Begriff der Mehrsprachigkeitserziehung.

Lehrkräfte müssen ebenso wie Lehramtsstudierende auch befähigt werden, sich - kritisch - mit den Integrationsdiskursen und ihren Implikationen auseinanderzusetzen und eine eigene auf Erfahrung basierende Einstellung zu Mehrsprachigkeit gewinnen.

\section{Abschlussbemerkungen}

Deutsch als Zweitsprache ist ein Sprachfach, ohne allerdings den Status eines regulären Schulfachs für sich in Anspruch nehmen zu können. Seine Herkunft in der Ausländerpädagogik hat lange Zeit verhindert, es im Kontext einer eher interkulturell ausgerichteten Mehrsprachigkeitserziehung wahrzunehmen. Inzwischen wird dieser Zusammenhang aus Sicht der bildungsbezogenen Integrationspolitik in Frage gestellt, denn die natürliche Mehrsprachigkeit, in der Deutsch als Zweitsprache zunächst anzusiedeln ist - der der Migrationsmehrsprachigkeit - wird inzwischen bildungspolitisch wie integrationssoziologisch als nicht wünschenswert angesehen. Dies ist in mehrfacher Hinsicht unverständlich:

- Mehrsprachigkeitserziehung gilt in Europa als anzustrebendes Ziel

- Mehrsprachigkeitserziehung ohne Migrationsmehrsprachigkeit ist diskriminierend 
Deutsch als Zweitsprache ohne Mehrsprachigkeitserziehung vergibt Möglichkeiten für andere Fächer.

Die Verbindung von Deutsch als Zweitsprache mit Mehrsprachigkeitserziehung lässt sich leicht in das Konzept der Europäischen Sprachenportfolios einbinden und gewinnt damit eine international anerkannte Basis. Dies wird deutlich, wenn man sich wesentliche Ziele des Portfolio-Konzepts vergegenwärtigt: Förderung der Mehrsprachigkeit, Förderung des kulturellen Dialogs, Erleichterung der Mobilität in Europa, Stärkung und Bewahrung der kulturellen Vielfalt, Förderung des autonomen Lernens, Ermutigung zum lebenslangen Sprachenlernen.

Es bleibt jedoch das Problem bestehen, dass in den universitären Lehramtsstudiengängen wenig Möglichkeiten zu erkennen sind, Studierende mit Deutsch als Zweitsprache in Verbindung mit Mehrsprachigkeitserziehung vertraut zu machen.

Zukünftige Überlegungen müssen auch das Phänomen der Transmigration einbeziehen, das auch für Deutsch als Zweitsprache in Verbindung mit Mehrsprachigkeitserziehung neue Aufgaben beinhaltet (vgl. Gogolin/Pries 2004 zu Erziehung und Transmigration).

\section{Literatur}

Barkowski,. Hans: Curriculumentwicklung und Lehrziele Deutsch als Zweitsprache. In: Helbig, Gerhard / Götze, Lutz / Henrici, Gert \& HansJürgen-Krumm (Hrsg.): Deutsch als Fremdsprache. Ein internationales Handbuch. Berlin \& New York: De Gruyter, 2001, 810 - 827.

Bredel, Ursula / Hartmut Günther / Peter Klotz / Jakob Ossner \& Gesa SiebertOtt (Hrsg.): Didaktik der deutschen Sprache -- ein Handbuch. Band 1+2 Paderborn: Schöningh (UTB), 2003.

Cope, Bill \& Mary Kalantzis: Multiliteracies. Literacy Learning and the Design of Social Futures. London and New York: Routledge, 2000.

Deppner, Jutta: Fachsprache der Chemie in der Schule. Heidelberg: Groos 1989.

Esser, Hartmut: Migration, Sprache und Integration. AKI-Forschungsbilanz 4. Berlin: WZB, 2006.

Gogolin, Ingrid \& Ludger Pries: Stichwort: Transmigration und Bildung. Zeitschrift für Bildungswissenschaft 7:1 (2004), 5-19.

Kuhs, Katharina: Soæialpsychologische Faktoren im Zweitspracherwerb. Tübingen: Narr, 1989.

Kuhs, Katharina: Unerfüllte Hoffnungen: Deutsch als Zweitsprache in der Regelklasse. In: Barkowski, Hans und Renate Faistauer (Hrsg.): ... in Sachen Deutsch als Fremdsprache. Sprachenpolitik und Mehrsprachigkeit, Unterricht, Interkulturelle Begegnung. Festschrift für Hans-Jürgen Krumm zum 60. Geburtstag. Hohengehren: Schneider, 2002, 263-279 
Luchtenberg, Sigrid: Möglichkeiten und Grenzen von Language Awareness zur Berücksichtigung von Mehrsprachigkeit im (Deutsch-)Unterricht. In: Katharina Kuhs, Wolfgang Steinig (Hrsg.): Pfade durch Babylon. Konzepte und Beispiele für den Umgang mit sprachlicher Vielfalt in Schule und Gesellschaft. Freiburg: Fillibach Verlag,1998, 137-156.

Luchtenberg, Sigrid: Interkulturelle Kinder- und Jugendliteratur: Welche Rolle spielt Mehrsprachigkeit? In: Jörg Steitz-Kallenbach (Hrsg.): Kinder- und Jugendliteraturforschung interdisziplinär. Oldenburg: bis, 2001, 59-83.

Luchtenberg, Sigrid: Interkulturelle Erziehung und Deutsch als Zweitsprache. In: Armin Wolff; Torsten Ostermann \& Christoph Chlosta (Hgg.): Integration durch Sprache (= Band 73 der Materialien DaF). Regensburg: Fachverband Deutsch als Fremdsprache, 2004, 235-259.

Ministerium für Schule, Jugend und Kinder des Landes Nordrhein-Westfalen (Hrsg.): Förderung in der deutschen Sprache als Aufgabe des Unterrichts in allen Fächern. Empfehlungen. Frechen: Ritterbach, 1999.

Neumann, Ursula: Zwischen Scheitern und Karriere - Kinder und Jugendliche aus Migrantenfamilien im deutschen Bildungssystem. In: Barkowski, Hans und Renate Faistauer (Hrsg.): ... in Sachen Deutsch als Fremdsprache. Sprachenpolitik und Mehrsprachigkeit, Unterricht, Interkulturelle Begegnung. Festschrift für Hans-Jürgen Krumm zum 60. Geburtstag. Hohengehren: Schneider, 2002, 132-144.

OECD: Where Immigrant Students Succeed. A comparative review of performance and engagement in PIS A 2003. Paris, 2006.

Oomen-Welke, Ingelore: Kultur der Mehrsprachigkeit im Deutschunterricht. In:

Köhnen, Ralph (Hrsg.): Wege zur Kultur. Perspektiven für einen integrativen Deutschunterricht. Germanistentag der Deutschlehrerinnen und Deutschlehrer in Bochum vom 29. September bis 2. Oktober 1996. Frankfurt/Main u.a.: Peter Lang, 1998, 287-305.

Oomen-Welke, Ingelore: Sprachen in der Klasse, Praxis Deutsch 157 (1999), 14-23.

Reich, Hans H.: Deutsch: Sprache. In: Hans H. Reich / Alfred Holzbrecher \& Hans Joachim Roth (Hrsg.): Fachdidaktik interkulturell. Ein Handbuch. Leverkusen: Leske + Budrich, 2000, 235-256.

Schader, Basil: Sprachenvielfalt als Chance. Handbuch für den Unterricht in mehrsprachigen Klassen. Hintergründe und 95 Unterrichtsvorschläge für Kindergarten bis Sekundarstufe I. Zürich: Orell Füssli, 2000.

Sekretariat: Empfehlung „Interkulturelle Bildung und Erziehung in der Schule“. Bonn: Sekretariat der Ständigen Konferenz der Kultusminister der Länder in der Bundesrepublik Deutschland. Bonn, 1996. 


\section{Deutsch als Zweitsprache im Rahmen der Lehrer- ausbildung an der Universität Duisburg-Essen}

Christoph Chlosta, Essen

Im Rahmen des Themenschwerpunkts „Deutsch als Zweitsprache und Mehrsprachigkeit" wurden verschiedenste Aspekte des Themenfeldes in eigenen Vorträgen behandelt - so wie es auch in den hier abgedruckten Tagungsakten zum Ausdruck kommt. Ein immer wieder auftauchender Diskussionspunkt, war die Frage der Ausbildung, speziell die der Lehrerausbildung, die in den heute vorliegenden Formen vor allem in den Diskussionen als Sackgasse dargestellt wurde. Unterstrichen wurde dieser Eindruck auch durch den Verweis auf und die Darstellung von Fortbildungsnotwendigkeiten und -defiziten im Bereich DaZ/Mehrsprachigkeit, was bereits im Eingangsreferat durch das niedersächsische Bildungsministerium deutlich wurde, 
denn die Fort- und Weiterbildung ist eben notwendig, weil es keine grundlegende Ausbildung gibt - nicht weil grundgelegtes Wissen weiter ausgebaut werden soll.

Gerade im Rahmen von Lehrerausbildung ist man schnell bei einem scheinbar unauflöslichen Dilemma: In den meisten Fällen orientiert sich die Lehrerausbildung an den zu unterrichtenden Schulfächern, die wiederum in unmittelbarer Beziehung zu universitären Studienfächern stehen. Eine Ausnahme stellt etwa der Lernbereich Sachunterricht in der Primarstufe dar, da hier mehrere akademische Fächer eine gemeinsame Ausbildung betreiben (sollen). In diesem Bedingungsgeflecht von Schulfach und Studienfach ist die Frage, wie etwa DaZ in die Schule integriert werden sollte, Endpunkt der Diskussion. Als eigenes Unterrichtsfach, was sowohl die Einsatzfähigkeit der Lehrenden einschränken als auch zur Segregation der Schülerinnen und Schüler führen würde, ist DaZ wohl kaum denkbar.

Im Folgenden soll es nun weder um die Frage gehen, ob DaZ oder DaZ/Mehrsprachigkeit ein eigenständiges Schulfach sein sollten und wie sich dieses Schulfach in den Kanon bestehender Schulfächer einreihen könnte, noch um die Diskussion der Verortung des Fachs DaZ im universitären Fächerspektrum etwa am Beispiel der Bezugswissenschaften. Diese Diskussionen sind ausgiebig geführt und belegt. Ziel ist es vielmehr, am Beispiel der Lehrerausbildung der Universität Duisburg-Essen (UDE) und hier besonders am Beispiel der Ausbildung von Deutschlehrern aller Schulstufen eine Möglichkeit aufzuzeigen, wie zumindest Grundlagenwissen DaZ in die Lehrerausbildung aufgenommen werden kann. Anschließend wird ein neuer Studienabschnitt in der nordrheinwestfälischen Lehrerausbildung vorgestellt, der die Reichweite von DaZ-Ausbildungsabschnitten nochmals deutlich verbessert und durchaus modellbildend sein könnte.

\section{DaZ/DaF an der Universität Duisburg-Essen (UDE)}

Wie viele andere Universitäten wurde an der UDE Mitte der achtziger Jahre des vergangenen Jahrhunderts eine Lehreinheit ${ }^{1}$ Deutsch als Zweit- und Fremdsprache gegründet, deren Aufgabe die Durchführung des „Zusatzstudiums Deutsch als Zweitsprache/Interkulturelle Pädagogik“ ist. Dieser Zusatzstudiengang richtet sich an alle Lehramtsstudierende und kann von diesen belegt werden. Folgt man der Lehramtsprüfungsordnung (LPO), so kann man die Zusatzqualifikation erst nach der grundständigen Ausbildung ablegen. In der Praxis werden die Veranstaltungen aus diesem Studiengang jedoch oft schon während des grundständigen Studiums belegt, das Examen kann gleichwohl erst (in der logischen Sekunde) nach dem Ersten Staatsexamen abgelegt werden.

\footnotetext{
1 Unter einer Lehreinheit versteht man eine organisatorisch und inhaltlich abgeschlossene Einheit, die einen eigenen Studiengang betreut und für diesen vorrangig verantwortlich ist. Inhaltlich könnte man von einem Fach sprechen.
} 
Das Studium erfolgt in vier thematischen Bereichen, die gleichrangig mit jeweils acht SWS zu studieren sind, wobei ein Bereich als individueller Schwerpunkt mit zusätzlichen acht SWS verstärkt studiert werden kann:

- Deutsch als Zweitsprache/Mehrsprachigkeit,

- Interkulturelle Pädagogik,

- Migration und gesellschaftliche Partizipation,

- Sprache der Migrantinnen und Migranten.

Inhaltlich ist es sicher nicht unbedeutend, dass das Studium des Zusatzstudiums Deutsch als Zweitsprache/Interkulturelle Pädagogik an keine weiteren Voraussetzungen geknüpft ist als die oben beschriebenen. Die Studierenden sind sowohl Studierende der Fächer Deutsch oder moderner Fremdsprachen als auch Studierende der übrigen Unterrichtsfächer also etwa Sport und Musik. Dies hat natürlich Auswirkungen auf die anzusetzenden Vorkenntnisse.

Die Reichweite der Zusatzausbildung ist aus verschiedenen Gründen beschränkt. Obwohl solides Wissen im Bereich Deutsch als Zweitsprache und Mehrsprachigkeit eine gesellschaftliche Notwendigkeit ist, wie die aktuelle politische Diskussion deutlich belegt, bleibt es doch dem Engagement des einzelnen Studierenden überlassen, sich ein zusätzliches Studium zu leisten. In der Realität führte dies oft zu unterschiedlich großen Studiengruppen aber vor allem zu wenigen Absolventen, denn es gibt eigentlich kaum einen Grund, die Zusatzausbildung zu beenden. Versuche, die Attraktivität zu steigern, hängen vor allem von außeruniversitären Faktoren ab.

Es sind vor allem die beiden folgenden Parameter, die Einfluss nehmen. Zunächst ist es der Markt, der wesentlich auf die Zusatzausbildungen wirkt. Interessantweise ist es aber nicht wie in anderen Fällen ökonomischer Steuerung der Bedarf bzw. die Nachfrage, die die Attraktivität des Studiengangs bestimmt, denn am Bedarf von Wissen und Fähigkeiten im Bereich Deutsch als Zweitsprache in der Schule gibt es keinen Zweifel. Es ist vielmehr allein ein Belohnungsprinzip, welches steuert, ob Studierende die Zusatzausbildung studieren oder nicht. Gerade auf der Belohnungsseite ist aber eine klare Fehlanzeige zu vermelden: Weder werden Lehrer mit dieser Qualifikation besser bezahlt, noch haben sie bessere Einstellungschancen, noch Gratifikationen etwa über Stundenreduktion. Oft ist gerade das Gegenteil zu beobachten. Wer auch nur im Geringsten zu erkennen gibt, sich mit DaZ beschäftigt zu haben, wird als Wunderwaffe durch die Schule gereicht, erhält Fördergruppen - soweit diese eingerichtet sind - und „Problemklassen“ und dieses vornehmlich am Nachmittag ...

Dies ist natürlich nur soweit möglich, wie es wahrscheinlich und glaubhaft ist, dass die Kolleginnen und Kollegen im Kollegium eine Fehlanzeige in DaZKenntnisse melden. Anders gesagt: Wenn alle nichts wissen, wächst der soziale Druck auf jene, die sich Wissen aneigneten, zur Übernahme von Fördergruppen. 
Hiermit wird aber gleichzeitig verhindert, dass sie sich etwa auf ebenso wichtige Funktionen wie die Unterstützung des Kollegiums bei der Schulprogrammentwicklung konzentrieren. Um also die Zusatzausbildung wirkungsvoller gestalten zu können, wäre vor allem das flächendeckende Grundwissen bei allen Lehrerinnen und Lehren zu erhöhen.

\section{DaZ und Deutschlehrerausbildung}

Will man Deutsch als Zweitsprache/Mehrsprachigkeit im Rahmen einer Lehramtsausbildung verankern, so wird die Deutschlehrerausbildung zunächst im Vordergrund stehen. Natürlich muss man sehen, dass die Deutschlehrerausbildung ebenso wie der Deutschunterricht eine Fülle von Aufgaben übernehmen muss, als Beispiele seien literar-ästhetische Bildung, Umgang mit Medien und kommunikative Kompetenz (Schrader/Trampe 2002) genannt - und dass diese sicher auch für die einzelnen Schulformen einerseits spezifiziert werden müssen, andererseits originäre schulformbezogene Aufgaben hinzukommen (für das Berufskolleg vgl. Grundmann 2002). Im Zentrum stellt der Deutschunterricht wie die Deutschlehrerausbildung aber das wesentliche Gerüst zur sprachlichen Bildung aller Schülerinnen und Schüler zur Verfügung, somit auch jener die Deutsch als Zweit(e)sprache sprechen. Insofern ist zu fragen, ob nicht neben der klassischen Dreiteilung Linguistik, Literaturwissenschaft und Mediävistik ein vierter Ausbildungsstrang, nämlich Deutsch als Zweitsprache einbezogen werden könnte. Genau diesen Weg hat die Germanistik der UDE zusammen mit der Lehreinheit Deutsch als Zweitsprache beschritten.

Mit dem Lehrerausbildungsgesetz von 2002 hat das Land NRW die Stufenlehrerausbildung durch eine schulformbezogene Lehrerausbildung ersetzt. Als Schulformen und entsprechende Lehrämter wurde das Lehramt

- Grundschule,

- Haupt- und Realschule sowie die entsprechenden Jahrgangsstufen der Gesamtschule,

- Gymnasium sowie die entsprechenden Jahrgangsstufen der Gesamtschule und das

- Berufskolleg eingeführt.

Die „Lehrämter“ Grundschule und Haupt- und Realschule sowie die entsprechenden Jahrgangsstufen der Gesamtschule wurden wiederum zum Lehramt Grund-, Haupt- und Realschule zusammengefasst, wobei die Studierenden bei Studienantritt sich für einen Schwerpunkt entsprechend des Lehramts entscheiden müssen.

In der Germanistik der UDE wird für alle vier Lehrämter ausgebildet. In nachfolgender Übersicht sind die auf den Bereich DaZ entfallenden Einheiten der Lehramtsausbildung Deutsch abzulesen. 


\begin{tabular}{|l|l|l|l|l|}
\hline & Grundschule & $\begin{array}{l}\text { Haupt-, Real-, } \\
\text { Gesamtschule }\end{array}$ & $\begin{array}{l}\text { Gymnasium und } \\
\text { Gesamtschule }\end{array}$ & Berufskolleg \\
\hline $\begin{array}{l}\text { Einführung } \\
\text { DaZ/DaF 2 SWS }\end{array}$ & Pflicht & Pflicht & Pflicht & Pflicht \\
\hline $\begin{array}{l}\text { Proseminare zu } \\
\text { DaZ/DaF 2 SWS }\end{array}$ & & & Pflicht & Pflicht \\
\hline $\begin{array}{l}\text { Sprachkurse } \\
\text { 4 SWS }\end{array}$ & Wahlpflicht & Wahlpflicht & Wahlpflicht & Wahlpflicht \\
\hline $\begin{array}{l}\text { Hauptsseminare } \\
\text { Mehrsprachigkeit } \\
\text { 2 SWS }\end{array}$ & Pflicht & Pflicht & Pflicht & Pflicht \\
\hline $\begin{array}{l}\text { Schulforschung } \\
\text { und Praktikum } \\
\text { 2 SWS }\end{array}$ & $\begin{array}{l}\text { DaZ-Angebot ist } \\
\text { fakultativ }\end{array}$ & $\begin{array}{l}\text { DaZ-Angebot ist } \\
\text { fakultativ }\end{array}$ & $\begin{array}{l}\text { DaZ-Angebot ist } \\
\text { fakultativ }\end{array}$ & $\begin{array}{l}\text { DaZ-Angebot } \\
\text { ist fakultativ }\end{array}$ \\
\hline
\end{tabular}

- Bei den Sprachkursen handelt es sich zu einem Großteil um „Problemorientierte Sprachkurse“, so wie sie auch im Zusatzstudiengang angeboten werden. Problemorientiert sind sie deshalb, weil neben dem Erlernen der Fremdsprache eine Reflexion von Sprachlernen, eine Kontrastierung zum Deutschen und eine Diskussion von Lernschwierigkeiten für Lerner des Deutschen als Zweitsprache aus der vom Studierenden belegten Sprache heraus stattfindet.

- Die Angebote im Bereich „Schulforschung und Praktikum“ sind insofern fakultativ, als die Lehrkapazität der Lehreinheit DaZ ein regelmäßiges Angebot im Moment nicht möglich macht.

- Die verminderte Stundenanzahl in den Studiengängen Grund-, Haupt, Realschule gegenüber Gymnasium und Gesamtschule überrascht sicher, würde man doch die Notwendigkeit gerade in diesen Schulformen höher einschätzen. Sie ist allerdings der LPO geschuldet, die ja generell weniger Ausbildungszeiten für das Lehramt Grund-, Haupt, Realschule zur Verfügung stellt, ein durch die Bildungspolitik oft beklagter Missstand, der allein dem Beamtenrecht geschuldet ist.

Die Deutschlehrerausbildung an der UDE ist modularisiert, d. h. zwischen drei bis fünf Lehrveranstaltungen bilden gemeinsam ein Modul, welches einen thematischen Rahmen darstellt. Eine solche Struktur legt es zunächst nahe, neben die bestehenden mediävistischen, literaturwissenschaftlichen und linguistischen Module ein eigenständiges Modul DaZ zu setzen. Bei einem solchen Vorgehen werden jedoch Einheiten getrennt, Bezüge zwischen Inhalten aus DaZ und anderen Lehrveranstaltungen sind nicht mehr unmittelbar vorhanden, DaZ erhält erneut eine zwar eigene jedoch in Konkurrenz bzw. Kontrast zu den anderen Mo- 
dulen stehende Position. Aus diesen Überlegungen heraus wurde in DuisburgEssen der Weg beschritten, auf ein eigenständiges Modul zu verzichten und an entscheidenden Stellen des Studienverlaufs Lehrveranstaltungen in die Module zu integrieren. Hierdurch erhalten die Studierenden schon im Grundstudium Bezüge zu DaZ und müssen sich auch im Hauptstudium mit der Fragestellung beschäftigen.

Mit dem oben beschriebenen Einheiten kann man zumindest davon ausgehen, dass jeder Deutschlehrer eine gewisse Grundkompetenz haben sollte. Es gibt aber darüber hinaus in der Lehrerausbildung NRW und auch an der UDE Möglichkeiten, DaZ zu integrieren.

\section{DaZ und „Didaktisches Grundlagenstudium Deutsch“ (DGD)}

\section{$3.1 \quad$ Hintergrund}

Mit Einführung des Lehrerausbildungsgesetzes (LABG) NRW von (2002) wurden einige Neuerungen in die nordrheinwestfälische Lehrerausbildung aufgenommen. Im Mittelpunkt der Diskussion standen und stehen besonders jene Ausführungen, die als Öffnungsklausel für die Einführung konsekutiver Studienstrukturen in Form von BA- und MA-Studien dienen.

Etwas weniger Beachtung genießt eine weitere Änderung: Im LABG wurde ein Studienabschnitt implementiert, der als Didaktisches Grundlagenstudium Deutsch oder Mathematik genannt wird. In $\ 13$ heißt es zum Studium für das Lehramt an Grund-, Haupt- und Realschulen und den entsprechenden Jahrgangsstufen der Gesamtschulen:

(1) „Das Studium für das Lehramt an Grund-, Haupt- und Realschulen und den entsprechenden Jahrgangsstufen der Gesamtschulen umfasst

1. das erziehungswissenschaftliche Studium,

2. das Studium von zwei Unterrichtsfächern einschließlich schulformbezogener Schwerpunktbildung und

3. das didaktische Grundlagenstudium in den Unterrichtsfächern Deutsch oder Mathematik.

(2) Bei der Wahl des Studienschwerpunktes Grundschule ist eines der beiden Unterrichtsfächer nach Absatz 1 Nr. 2 Deutsch oder Mathematik. Das didaktische Grundlagenstudium nach Absatz $1 \mathrm{Nr} .3$ erfolgt in dem nicht gewählten Fach.“

Nicht näher erläutert findet man zunächst, was dieser Studienabschnitt beinhalten soll.

Auch die Lehramtsprüfungsordnung NRW (2003) gibt zunächst keine weiteren Hinweise, wie und für wen dieser Studienabschnitt ausgestaltet werden soll. Allerdings wird dem Didaktischen Grundlagenstudium mit immerhin 20 SWS ein nicht geringes Gewicht beigemessen. 


\subsection{Fächerspezifische Vorgaben Didaktisches Grundlagestudium Deutsch}

Nur zu wenigen Fächern und Studienabschnitten hat das Ministerium Ausführungsbestimmungen erlassen. Zum Didaktischen Grundlagestudium wurden allerdings zwei Expertenkommissionen eingesetzt, die Vorschläge erarbeitet haben, die anschließend - wohl mit einigen ,ministeriellen Korrekturen' - auch als fächerspezifische Vorgaben erlassen wurden.

„Das Konzept des didaktischen Grundlagenstudiums Deutsch orientiert sich an der Zielsetzung, Sprache und Sprachkompetenz in allen Fächern zu fördern. Lehrerinnen und Lehrer sollen die jeweilige Fachsprache und unterrichtliche Kommunikation in ihren Fächern reflektieren und darauf bezogene Lernprozesse ihrer Schülerinnen und Schüler anleiten." (DGS 2004: 2)

Ziel ist es also, dass Lehrerinnen und Lehrer die sprachliche Seite des Unterrichts reflektieren und gestalten können. Insofern macht es keinen Sinn, dass das DGD ein kleines Fach darstellt, denn es gehört bislang wohl nicht zu den zentralen germanistischen Inhalten des Deutschstudiums, Unterrichtskommunikation zu analysieren und Alternativen zu erörtern.

Eher schon stellt die Betrachtung von Sprachentwicklungsprozessen der Schülerinnen und Schüler einen solchen Inhalt dar. Betrachtet man jedoch die exemplarischen Modulbeschreibungen, so wird die Diskrepanz zum „Kanon“ einer grundständigen Ausbildung im Fach Deutsch nochmals deutlich. Einerseits fehlen die sonst so prägenden literaturwissenschaftlichen Inhalte, wie die nachfolgende thematische Liste zeigt:

„Modul A

Sprach- und Medientheorien

Mündlichkeit und Schriftlichkeit

Fachsprachliche Kommunikation

Virtuelle Kommunikation und Medien

Mehrsprachigkeif"

(DGS 2004: 8; Herv. Ch)

„Modul B

Sprachliche Entwicklungsprozesse

Spracherwerb zwei- und mebrsprachiger Kinder"

(DGS 2004: 10; Herv. Ch)

„Modul C

Unterrichtliche Kommunikation

Schulische Kommunikation

Professionsspezifische Vermittlungsfähigkeit

Interkulturelle Fäbigkeit"

(DGS 2004: 11; Herv. Ch) 
Andererseits ist mit den drei kursivierten Veranstaltungen eine inhaltlichthematische Perspektive eröffnet, die heute noch nicht Bestandteil aller Lehrerausbildungen, nicht mal aller Deutschlehrerausbildungen ist.

Mehrsprachigkeit, Spracherwerb zwei- und mehrsprachiger Kinder, Interkulturelle Fähigkeit sind Themenbereiche, die „traditionell“ eher im Bereich Deutsch als Zweitsprache angesprochen werden als in der Germanistik.

Es ist hier nicht der Ort, die Diskussion um eine fachsystematische Positionierung von Deutsch als Zweit- und Fremdsprache zwischen Germanistik, Fremdsprachenphilologie und Sprachlehrforschung erneut aufzurollen. Es reicht für die Überlegungen an dieser Stelle vollständig aus, dass bislang nicht in jedem Hochschulcurriculum des Lehramtsfachs Deutsch Themen aus dem Bereich Deutsch als Zweitsprache integriert sind und viele angehende Lehrerinnen und -lehrer immer noch vor dem Hintergrund einer vermeintlichen monolingualen Schülerschaft ausgebildet werden.

Mit der Einführung des Didaktischen Grundlagenstudiums Deutsch ist mit dieser Perspektive zwar nicht aufgeräumt - so können Studierende des Unterrichtsfachs Deutsch auch Mathematik als didaktisches Grundlagenstudium wählen, ja müssen es wie im Falle des Grundschullehramts sogar - allerdings hat Deutsch als Zweitsprache im Rahmen der nordrheinwestfälischen Lehrerausbildung erstmals einen festen und verbindlichen Platz.

Die Hoffnung, dass über den Umweg Didaktisches Grundlagenstudium Deutsch das Thema Deutsch als Zweitsprache auch in die Ausbildung des Unterrichtsfachs Deutsch an anderen Universitäten Einzug findet, resultiert aus zwei Überlegungen:

- Das Didaktische Grundlagestudium, so wie es hier dargestellt wurde, erfordert auf Grund der fachspezifischen Vorgaben zwingend die Perspektive Deutsch als Zweitsprache, was zur Einrichtung von entsprechenden Stellen in den Universitäten führen muss. Bislang jedenfalls verfügen nicht alle Germanistiken (bzw. Universitäten mit Deutschlehrerausbildungen) über entsprechendes Personal.

- Wenn man dieses Personal erst einmal hat, dürften die Chancen groß sein, es ebenfalls in der Deutschlehrerausbildung einzusetzen und damit alle Lehramtsstudierenden der Lehrämter für Grund-, Haupt- und Realschulen sowie den entsprechenden Jahrgangsstufen der Gesamtschule zu erreichen.

Natürlich sind die im Rahmen des Didaktischen Grundlagenstudiums zur Verfügung gestellten Stunden (6 SWS) nur eine Basis, die es auszubauen gilt. Vor allem müsste auch diskutiert werden, warum Studierende des Lehramts Gymnasium und Berufskolleg von dieser Basis im Moment ausgeschlossen bleiben. 


\section{Zusammenfassung}

Wenn man die obigen Studienabschnitte und Studienangebote betrachtet, so wird deutlich, dass es nur einen kleinen Teil von Studierenden gibt, die ein Lehramt an der UDE studieren ohne auch DaZ zu studieren. Ergänzend zu vier Semesterwochenstunden Interkultureller Pädagogik, die tatsächlich für alle Lehramtsstudierenden verpflichtend, studiert ein Großteil der Studierenden die oben dargestellten Einheiten im Bereich DaZ.

Zusammengenommen müssen diese Studienanteile die Grundlage an Förderung Deutsch als Zweitsprache und der Mehrsprachigkeit übernehmen. Auf der Grundlage einer solchen integrativen Ausbildung erhält dann freilich die Zusatzausbildung DaZ einen neuen Sinn, da sie tatsächliche Spezialisten ausbildet, die an den Schulen auf der allgemeinen Förderung in DaZ aufbauend wirken können.

\section{Literatur}

Grundmann, Hilmar (2002): „Der gegenwärtige Umgang mit dem

Deutschunterricht an berufsbildenden Schulen - Eine der größten

Herausforderungen der berufsschulischen Fachdidaktik." in: Josting, Petra;

Peyer, Ann (Hrsg.) (2002): Deutschdidaktik, und berufliche Bildung.

Baltmannsweiler. S. 8-27.

Schrader, Heinrich; Trampe, Wilhelm (2002): Kommunikative Kompetenz als Schlüsselqualifikation im Deutschunterricht an berufsbildenden Schulen. in: Josting, Petra; Peyer, Ann (Hrsg.) (2002): Deutschdidaktik und berufliche Bildung. Baltmannsweiler. 28-41.

LPO (2003) Ordnung der Ersten Staatsprüfungen für Lehrämter an Schulen (Lehramtsprüfungsordnung - LPO) vom 27. März 2003. NRW.

DGS (2004): Ministerium für Schule, Jugend und Kinder des Landes NordrheinWestfalen: Fächerspezifische Vorgaben Didaktisches Grundlagenstudium Deutsch für das Lehramt an Grund-, Haupt- und Realschulen sowie den entsprechenden Jahrgangsstufen der Gesamtschulen.

LABG (2002) Gesetz über die Ausbildung für Lehrämter an öffentlichen Schulen (Lehrerausbildungsgesetz - LABG) vom 2. Juli 2002 geändert durch Gesetz vom 8. Juli 2003 NRW. 



\title{
Förderung zweisprachiger Kinder
}

\author{
Angela Esgen, Düsseldorf
}

Das Problem der mangelnden Deutschkenntnisse von Kindern mit Migrationshintergrund bleibt ein kontrovers diskutiertes Thema. Wichtige Fragen sind dabei:

\section{Wie früh soll Deutsch als Zweitsprache gefördert werden?}

Das Problem ist bekannt: Kinder, die erst mit zwei oder drei Jahren nach Deutschland gekommen sind, oder die zwar hier geboren wurden, deren Eltern aber kein Deutsch sprechen, schneiden in der Schule im Durchschnitt schlechter ab. Das heißt: Sie kommen durchaus gut in alltäglichen Situationen zurecht. Nicht das informelle Gespräch wird zur Herausforderung, sondern die Lese- und Schreibfähigkeit, das selbständige Formulieren abstrahierender Gedanken, kurz: Die so genannte „literacy“. 
Das sind nach Ansicht der Autorin Folgen eines ungesteuerten Zweitsprachenerwerbs. In diesem Beitrag wird deshalb die Meinung vertreten, dass eine systematische Förderung von Deutsch als Zweitsprache bereits früh, und zwar im Kindergarten, beginnen muss.

\section{Wie kann eine parallele Förderung der Erstsprache ge- schehen?}

Nach der auf Cummins beruhenden Schwellentheorie bestimmen die Fähigkeiten in der Erstsprache die Fähigkeiten in der Zweitsprache. Erzieher/-innen und Lehrer/-innen haben aber nur begrenzt Einfluss auf eine systematische Entwicklung der Erstsprache: entweder im Rahmen von bilingualen Einrichtungen (Kindertagesstätten und Grundschulen), oder durch intensive Elternarbeit. Angesichts der Vielzahl von Herkunftssprachen, die in deutschen Kindergärten und Schulen gesprochen werden, wie auch in Anbetracht der knappen finanziellen Mittel, ist es illusorisch anzunehmen, in nächster Zukunft könne ein ausreichendes institutionelles Förderangebot für die diversen Herkunftssprachen geschaffen werden. Umso wichtiger ist die Kooperation mit den Eltern ausländischer Kinder. Wie die von der Autorin dieses Beitrags begonnene Umfrage unter Kindertageseinrichtungen in verschiedenen Bundesländern zeigt, könnte eine solche Zusammenarbeit noch beträchtlich verbessert werden ${ }^{1}$.

Betrachtet man bilinguale Schulen, die bereits Grundschulkindern Sprach- und Fachunterricht in einer anderen als der deutschen Sprache anbieten, lässt sich ein besonders positiver Effekt für die leistungsstarken deutschen Schüler feststellen (vgl. Sandfuchs 1999: 159-170). Der wünschenswerte Ausbau bilingualer Kindertageseinrichtungen und Schulen sollte deshalb nicht anstelle einer frühen, systematischen Förderung von Deutsch als Zweitsprache geschehen, sondern parallel dazu.

\section{Wie sieht eine optimale Förderung von Deutsch als Zweit- sprache in Kindergärten aus?}

Ein gutes Beispiel bietet die Stadt Recklinghausen, die im Rahmen eines Gesamtkonzepts des Fachbereiches Kinder, Jugend und Familie Bausteine entwickelt hat, nach denen eine Sprachförderung unter Einbeziehung der Familien stattfindet. Dort gibt es Spielgruppen und Angebote der Familienbildung für die Unter-3Jährigen und deren Mütter. In Tageseinrichtungen werden Kinder in ihrer Sprachentwicklung unterstützt: im Rahmen einer Sprachförderung für alle Kinder, durch Beobachtungsbögen, mit Hilfe derer besonderer Bedarf ermittelt wird, in regelmäßigen Spracheinheiten, über deren Inhalte auch die Eltern informiert werden,

\footnotetext{
${ }^{1}$ Siehe Tabelle am Ende dieses Beitrags
} 
und bei ständigen Spielen und Übungen während des Alltags. Eltern sind zur Zusammenarbeit verpflichtet und können zu „Stadtteileltern“ ausgebildet werden².

Dieses nur kurz skizzierte Beispiel enthält viele Elemente, die besonders wichtig erscheinen: Niedrigschwelligkeit, eine systematisierte Sprachstandsfeststellung und -beobachtung, ganzheitliches, lebensbedeutsames Lernen, kontinuierliches Angebot von der Vorkindergarten- bis zur Schulzeit, Integration der Eltern, interkulturelle Ausbildung der Erzieher, Schaffung eines Netzwerks im Stadtteil.

Niedrigschwelligkeit und interkulturelle Ausbildung der Erzieher sind von großer Bedeutung, um Eltern zur Teilnahme bewegen zu können und um diese Teilnahme dann auch von der Seite der Einrichtung erfolgreich gestalten zu können. Warum ist heute ein interkulturelles Training für Manager und Projektleiter in großen Wirtschaftsunternehmen selbstverständlich, während von Erziehern noch häufig angenommen wird, sie könnten ohne entsprechende Schulung mit Angehörigen anderer Kulturen kooperieren?

Eine einheitliche Sprachstandsfeststellung und ein Verfahren zur Beobachtung von Sprachentwicklung sind, obwohl von allen Seiten als wichtig erachtet, doch noch lange nicht überall etabliert.

Die Vermittlung von Techniken und Methoden im Fach Deutsch als Zweitsprache sollte unbedingt zur allgemeinen Ausbildung von Erzieher/-innen in Kindertageseinrichtungen gehören, denn die Wirklichkeit besteht vielerorts nicht aus homogenen, deutschsprachigen, sondern eben aus gemischten Gruppen mit oft weitaus mehr als nur zwei verschiedenen Sprachen.

Und schließlich: Nur eine kontinuierliche Förderung vom Elementar- über den Primar- bis zum Sekundärbereich ist wirklich effizient. Eine - wenigstens landesweite - Vereinheitlichung der Förderkonzepte an Kindgärten und Schulen ist notwendig. Was spricht dagegen, das Fach Deutsch als Zweitsprache durchgängig als Pflichtfach für alle Schüler, für die Deutsch eben Zweitsprache ist, einzuführen, und zwar in einem Umfang von mindestens 4 Stunden pro Woche? Dies könnte - zumindest in der Grundschule, aber je nach Bedarf auch an weiterführenden Schulen - anstelle der Teilnahme am regulären Deutschunterricht stattfinden, wenn Inhalte des Fachunterrichts Deutsch mit integriert würden. Alle Einwände dagegen - wie Unbezahlbarkeit, Nichtvereinbarkeit mit Plänen und Vorgaben, Stofflücken - sind entweder kurzsichtig oder bürokratisch. Es ist sowohl teurer als auch ineffektiver, Schüler am Fach Deutsch zu beteiligen, wenn sie sprachlich und daher auch inhaltlich überfordert sind. Einen bestimmten Stoff vorgesetzt zu bekommen ist schließlich noch lange nicht gleichbedeutend mit einer Bewältigung dieses Stoffes.

Noch ein Wort zur Ausbildung der Lehrer: Könnte nicht ein Modul über die Techniken der Vermittlung von Fremdsprachen zum festen Bestandteil des Studiums werden? Natürlich kann und soll nicht in jedem Fach der Schwerpunkt auf

\footnotetext{
${ }^{2}$ Für eine detaillierte Beschreibung: Kita aktuell NRW, Nr. 1/2004, S. 13-17; Internet: Ulrich Braun, Diplom-Pädagoge, Münster: www.u.-braun.de/veroeff42.htm
} 
der Spracharbeit liegen, doch würde zumindest das Verständnis der Lehrenden für die Situation der Schüler erhöht werden, deren Herkunftssprache nicht Deutsch ist. Jeder Fremdsprachenlehrer weiß, wie wichtig es ist, seine Worte auf die Goldwaage zu legen und das zu behandelnde Thema klar zu präsentieren. Und welchem deutschen Schüler würde es letztendlich schaden, wenn der Lehrer knapp, einfach und präzise formuliert und seine Schüler zu ebensolchen Antworten anleitet?

Die vorläufige Auswertung der Befragung von Kindertageseinrichtungen zur Feststellung von Weiterbildungsbedarf wird in der folgenden Tabelle präsentiert. Hintergrund ist die Planung eines Weiterbildungsangebotes für Kindertageseinrichtungen in den Bereich Sprachförderung und Interkulturelle Erziehung.

Die Themen des Fragebogens sind im Einzelnen:

- Art und Zeitpunkt der Sprachstandserfassung

- System der Sprachförderung

- Integration, Förderung und Teilnahme der Eltern

- Interkulturelle Zielsetzung

- Zusatzqualifizierung der Erzieher/-innen.

Angeschrieben wurden 200 Einrichtungen in Nordrhein-Westfalen und Bayern mit höherem und niedrigerem Ausländeranteil. 80 Bögen konnten bisher ausgewertet werden, mehr als 60\% davon wurden allerdings von Einrichtungen zurückgeschickt, die einen Anteil von weniger als 10\% Kindern mit Migrationshintergrund haben.

In der Tabelle wird der Anteil der Einrichtungen in Prozent angegeben, die auf die entsprechende (markierte) Frage mit „Ja“ antworteten. 
Fragebogen zur Feststellung von Weiterbildungsbedarf in Kindertageseinrichtungen

Familienanamnese

$90 \%$

Sprachstandserfassung

vor Eintritt in den Kindergarten

$5 \%$

vor Eintritt in die Grundschule

$50 \%$

gezielte Sprachförderung

für alle Kinder (auch deutsche)

$20 \%$

für Kinder mit Migrationshintergrund

$10 \%$

bilinguale Erzieher

$10 \%$

davon französisch oder englisch

$50 \%$

Qualifizierung und Freistellung der Erzieher

für sprachliche Arbeit mit den Kindern

Interkulturelle Ausgestaltung und Angebote

fremdsprachliche Bilderbücher

$50 \%$

vereinzelte Projekte und Spiele

$20 \%$

Elternintegration durch fremdsprachliche Prospekte,

$8 \%$

Elternbesuche und Projekte

Aus den Ergebnissen, soweit sie bereits vorliegen, sollen drei vorläufige Schlussfolgerungen formuliert werden:

1. Es gibt keine flächendeckende, systematisierte Sprachförderung im Bereich Deutsch als Zweitsprache in Kindergärten.

2. Die interkulturelle Arbeit in Kindergärten ist aufgrund beschränkter Kapazitäten und Qualifikation des Fachpersonals stark entwicklungsbedürftig.

3. Die Tatsache, dass deutlich mehr Kindergärten mit einem geringen Anteil an nicht deutschsprachigen Kindern Zeit finden, sich an der Umfrage zu beteiligen, deutet auf eine Überbelastung der Einrichtungen mit sprachlich heterogenen Gruppen hin.

Zum Abschluss daher die - bewusst provozierende - Frage: Wäre es nicht einen Versuch wert, eine standardisierte Sprachförderung in Kindertageseinrichtungen zu organisieren? Abgesehen von allen theoretischen Vorüberlegungen kann eine Veränderung einer übereinstimmend als nicht zufriedenstellend empfundenen Situationen doch nur zu wünschenswerten neuen Erkenntnissen führen. 


\section{Weiterführende Literatur}

Bach, Gerhard und Niemeier, Susanne: Bilingualer Unterricht. Grundlagen, Methoden, Praxis, Perspektiven. Lang: 2005

Bartnitzky, Horst und Speck-Hamdan, Angelika (Hg.): Deutsch als Zweitsprache lernen. Grundschulverband - Arbeitskreis Grundschule e.V.: 2005

Butzkamm, Wolfgang: Unterrichtssprache Deutsch. Hueber: 1996

De Florio-Hansen, Inez (Hg.) : Plurilingualität und Identität. Zur Selbst- und Fremdwahrnehmung mehrsprachiger Menschen. Stauffenburg: 2003

Gemende, Marion: Zwischen den Kulturen. Juventa: 1999

Gereke, Iris, Meinhardt, Rolf, Renneberg, Wilm: Sprachförderung in Kindertagesstätten und Grundschulen - ein integrierendes Fortbildungskonzept. Abschlussbericht des Pilotprojekts. Bibliotheks- und Informatinossystem der Universität Oldenburg: 2005

Gogolin, Ingrid, Hohmann, Manfred: Vom bikulturellen zum interkulturellen Unterricht. Die Limburger Modellversuche zum Aufnahmeunterricht von Einwandererkindern in Kindergarten und Primarschule. Waxmann: 1993

Günther, Britta und Günther, Herbert: Erstsprache und Zweitsprache, Beltz: 2004

Guggenberger, Eva: Einflussfaktoren auf Migrantensprachen. Bausteine für ein migrationslinguistisches Modell, in: Jürgen Erfurt, Gabriele Budach, Sabine Hofmann: Mehrsprachigkeit und Migration, Europäischer Verlag der Wissenschaften: 2003, S. 37-62

Ministerium für Frauen, Jugend, Familie und Gesundheit des Landes NRW $(\mathrm{Hg})$ : Wie Kinder sprechen lernen. Entwicklung und Förderung der Sprache im Elementarbereich auf der Grundlage des situationsbezogenen Ansatzes. 2001

Nagel, Helga: Integration gestalten, in: Erfurt, Jürgen (Hg.): Mehrsprachigkeit und Migration. Ressourcen sozialer Identifikation. Lang: 2003, S. 211-216

Sandfuchs, U.: Migrantenkinder in Schule und Unterricht, S. 159-170, in: Marion Gemende et al: Zwischen den Kulturen, Juventa Verlag: 1999

Siebert-Ott, Gesa: Zweisprachigkeit und Schulerfolg: die Wirksamkeit von schulischen Modellen zur Förderung von Kindern aus zugewanderten Sprachminderheiten. Verlag für Schule und Weiterbildung Kettler: 2001

Tophinke, Doris: Sprachförderung im Kindergarten. Julia, Elena und Fatih entdecken gemeinsam die deutsche Sprache. Beltz: 2003 
Ulich, Michaela, Oberhuemer, Pamela, Soltendieck, Monika: Die Welt trifft sich im Kindergarten. Interkulturelle Arbeit und Sprachförderung in Kindertageseinrichtungen. Beltz: 2005

Johann, Ellen, Michely, Hildegard, Springer, Monika: Interkulturelle Pädagogik. Methodenhandbuch für sozialpädagogische Berufe. Cornelsen: 1998 

Forum DaF 



\section{Anfängerintensivkurse als Vorbereitung auf das Fachstudium}

Dagmar Schimmel/Linda Meier/Florian tom Wörden, Hannover

Ein Verzahnungsmodell zur Integration eines Allgemein- und Fachsprachenkurses in das 1. Fachsemester eines technischen Studiengangs 1

An der Fakultät für Bauingenieurwesen und Geodäsie der Leibniz Universität Hannover bietet die „Arbeitsgruppe für technologische Zusammenarbeit mit

\footnotetext{
${ }^{1}$ Der Vortrag wurde am 8. Juni 2006 auf der Jahrestagung an der Leibniz Universität Hannover von Dagmar Schimmel als Projektleiterin an der IGEL, Florian tom Wörden als Projektkoordinator an der Fakultät für Geodäsie und Bauingenieurwesen der Leibniz Universität Hannover sowie Linda Meier als Fachsprachenlehrkraft de IGEL im Verzahnungskurs gehalten. Die Umsetzung dieses Modells hatte zwei Tage zuvor, also am 6. Juni mit der allgemeinsprachlichen Phase des Deutschkurses begonnen. Diese schriftliche Fassung wurde erstellt, nachdem die Vorbereitungsphase bereits abgeschlossen war und die Verzahnung von Deutschkurs und Fachsprachenkurs bereits angefangen hatte, sodass erste Ergebnisse in die Schriftfassung einfließen konnten.
} 
Entwicklungsländern“ (AG TZE) seit 1987 einen Ergänzungsstudiengang „M.Sc. in Geotechnik und Infrastruktur" an. Für diesen deutschsprachigen Masterstudiengang bewerben sich jährlich ca. 90-130 Studierende vorrangig aus Entwicklungs- und Schwellenländern, welche bereits mindestens einen B.Sc.-Abschluss in Bauingenieurwesen sowie 2-jährige Berufserfahrung nachweisen müssen. Ausgewählt werden 10-20 Studierende pro Jahrgang, von denen etwa die Hälfte ein Stipendium des Deutschen Akademischen Austauschdienstes (DAAD) erhält. Diese Förderung umfasste bislang für die Studierenden auch einen 6-monatigen Sprachkurs, welcher die TeilnehmerInnen von der Grundstufe bis zu den Sprachprüfungen für das Studium in Deutschland, TestDaF und DSH, führen sollte.

Dieser Sprachkurs wurde seit 2004 bei der Innovationsgesellschaft Universität Hannover mbH (kurz: IGEL) durchgeführt, im direkten Anschluss besuchten die TeilnehmerInnen das 4-semestrige Fachstudium, welches vollständig in deutscher Sprache abgehalten wird.

In Reflexionsgesprächen zwischen den Fachdozenten des Studiengangs und den Sprachkursorganisatoren der IGEL betonten die Fachdozenten zwar die grundsätzliche Zufriedenheit mit den allgemeinsprachlichen Kompetenzen und den guten Prüfungsergebnissen der Studierenden in DSH und TestDaF. Jedoch wurde sich auch darüber beklagt, dass den TeilnehmerInnen die für das Studium absolut relevanten, fachsprachlichen Fertigkeiten fehlen würden, sodass im 1. Fachsemester beispielsweise große Teile der Veranstaltungen zur Vermittlung des Fachvokabulars aufgewendet werden mussten.

Diese Klage der Fachdozenten wird sicherlich die meisten im studienvorbereitenden bzw. universitären DaF-Bereich Tätigen nicht allzu sehr überraschen und wird zudem auch in der Fachliteratur bestätigt:

[Es] wird deutlich, dass es vom allgemeinsprachlichen Unterricht nicht ohne weiteres einen direkten Weg zum Überleben im Fachunterricht gibt: Es werden nicht nur Fertigkeiten entwickelt, die im Fachunterricht nicht benötigt werden, sondern auch solche, die unvereinbar mit den tatsächlich benötigten sind bzw. ihre Entwicklung verhindern können. Der allgemeinsprachliche Unterricht entwickelt jedenfalls nicht automatisch die Fertigkeiten, die im Fachunterricht erforderlich sind und die dort vorausgesetzt werden.

Verfechter der These, dass Ausländer mit guten allgemeinsprachlichen Kenntnissen vor dem Fachunterricht keinen Fachsprachenunterricht mehr benötigen, verschlechtern damit die Startbedingungen für die ausländische Studenten, sowohl absolut betrachtet, als auch im Vergleich zu ihren deutschen Kommilitonen [...]. (Buhlmann / Fearns 2000: 82)

Zusätzlich zu dem allgemeinsprachlichen 6-monatigen Intensivkurs, dessen oberstes Ziel zwangsläufig das Bestehen von TestDaF (hier 4 x TDN 3) oder der 
DSH (hier auf Stufe I) war, wurde den TeilnehmerInnen bei der IGEL zwar ab der Mittelstufe Fachsprachenunterricht für Bauingenieure angeboten. Da das Sprachkursprogramm jedoch eine sehr hohe und schnelle Progression haben musste - man bedenke, dass die Studierenden vorab in der Regel keine Deutschkenntnisse hatten und der Kurs deshalb für Nullanfänger konzipiert war, welche in 6 Monaten bis zu den Prüfungen gebracht werden mussten - konnte solch ein Fachsprachenunterricht nur mit 4 SWS (insgesamt 64 Unterrichtsstunden) angeboten werden.

Beobachtet wurde zudem auch seitens der Programmkoordinatorin der IGEL bei beiden Jahrgängen des bisherigen Intensivkursprogramms die absolut hohe Motivation und vor allem das außerordentlich große Interesse der Studierenden an diesem Fachsprachenunterricht, die hierin - so gaben sie bei Umfragen an - einen ganz konkreten Bezug und die Notwendigkeit für ihr zukünftiges Studium sahen.

So trat die AG TZE schließlich im Juli 2005 mit der Frage an die IGEL heran, ob es nicht möglich sei, den studienvorbereitenden Sprachkurs inhaltlich enger mit dem Studium zu verknüpfen und somit eine effektivere Vorbereitung auf das Fachstudium zu erzielen.

In weiteren Gesprächen wurden gemeinsam Ideen entwickelt, bei denen schnell deutlich wurde, dass durch eine engere Verknüpfung und gezieltere Ausrichtung des Sprachkurses auf die Fachveranstaltungen durchaus Synergien zwischen Deutschkurs und Fachstudium erzeugt werden können, was nicht nur zur Verbesserung der fachsprachlichen Kompetenzen - und damit für die Dozenten zu einer Verbesserung des 1. Fachsemesters - führt, sondern auch weitere positive Effekte mit sich bringt. Für den Sprachkurs bedeutet eine solche Verzahnung natürlich eine deutliche Erhöhung des fachsprachlichen Unterrichtsanteils, inhaltlich bezogen auf die Veranstaltungen des 1. Fachsemesters. Eine solche inhaltliche und - damit auch verbundene - organisatorische Verknüpfung erhöht zudem die gesamte Attraktivität des Studiengangs erheblich, da der Sprachkurs zu einem festen Bestandteil des fachlichen Angebots wird und den Studierenden somit von Beginn ein gut überschaubares Programm geboten werden kann. So entsteht ein komplettes, den Fremd- und Fachsprachenerwerb integrierendes, deutschsprachiges Studienangebot, welches auch für die Studierenden zeitlich gut planbar ist und schließlich sogar eine Verkürzung der Aufenthaltsdauer der ausländischen Studierenden für Spracherwerb und Studium um bis zu 10\% zur Folge hat - ein durchaus positiver Effekt für das kostenintensive Auslandsstudium insbesondere für InteressentInnen aus Entwicklungs- und Schwellenländern. Durch eine stärkere Verzahnung, welche auch eine engere Zusammenarbeit in der Betreuung der Studierenden mit sich bringt, kann somit ein sehr attraktives deutschsprachiges Studienangebot entstehen, welches im Rahmen der zunehmenden Internationalisierung der Universitäten und des internationalen Wettbewerbs um ausländische Studierende entscheidende Vorteile bietet. 
Diese erste Projektidee lässt sich im Vergleich zum bisherigen Sprachkurs- und Studienprogramm folgendermaßen graphisch darstellen:

Bislang:

Sprachkurs vor 1. Fachsemester

\begin{tabular}{|c|c|}
\hline 6 Monate & 1. Fachsemester \\
Sprachkurs & \\
\hline
\end{tabular}

Neu:

Verzahnung von Sprachkurs und Fachstudium

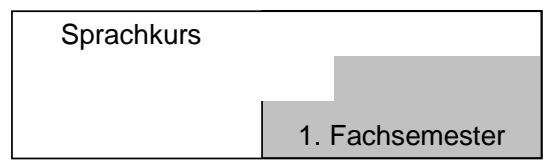

Eine Verzahnung von Deutschkurs und dem 1. Fachsemester integriert somit einerseits das 1. Fachsemester in den Sprachkurs, andererseits aber gleichzeitig auch den Sprachkurs in das 1. Fachsemester, sodass es sich um einen wechselseitigen Prozess handelt, der Synergien mit sich bringt, die beiderseitig genutzt und entwickelt werden sollten. Die Integration des 1. Fachsemesters erfordert eine inhaltliche Anpassung des Deutschunterrichts durch gezielten Fachsprachenunterricht sowie eine sprachliche Vor- und Nachbereitung der Fachveranstaltungen. Andererseits erfordert die Integration des Sprachkurses in das 1. Fachsemester auch in gewissem Maße eine sprachliche Anpassung der Fachveranstaltungen an das aktuelle Sprachniveau der TeilnehmerInnen. Es ist nicht unbedingt davon auszugehen, dass die Studierenden problemlos beispielsweise die syntaktischen Beziehungen in einem Satz wie dem folgenden verstehen, welcher einem Skript der Bauingenieure zum Thema „Hydraulischer Grundbruch“ entnommen ist:

Dabei wird in der Bruchfuge die an der jeweils betrachteten Stelle gegenüber dem Unterwasserspiegel noch nicht abgebaute Stahlrohrdifferenz n * $\Delta \mathrm{h}$ multipliziert mit $\Upsilon_{\mathrm{w}}$ als ideelle Druckfläche aufgetragen.

So gilt es bei einer solchen Verzahnung und damit verbundenen Verkürzung der allgemeinsprachlichen Vorbereitungszeit auch, sprachliche Sensibilität bei den FachdozentInnen zu wecken, ihnen beispielsweise Hinweise zu Satzverkürzungen zu geben, sodass inhaltlich nicht notwendige, sprachlich hochkomplexe Konstruktionen reduziert werden können. Gleichfalls können solche Konstruktionen, gerade da sie authentischem Material entnommen sind, ab einem bestimmten Sprachniveau im Fachsprachenunterricht als Übungsmöglichkeit aufgegriffen werden. 
Für dieses Verzahnungsmodell wurden drei Pflichtkurse als Fachveranstaltungen des 1. Semesters festgelegt, welche eine geeignete fachsprachliche und fachliche Progression mit sich bringen, die für eine Verknüpfung mit fachsprachlichem Unterricht günstig ist. In Abstimmung hiermit mussten die Inhalte des fachsprachenbezogenen Deutschkurses festgelegt werden. Da die Studierenden des Aufbaustudiengangs bereits ein B.Sc.-Studium abgeschlossen haben, konnte von einem entsprechenden fachlichen Wissen ausgegangen werden - auch wenn dieses erfahrungsgemäß im Hinblick auf die verschieden Herkunftsländer und die unterschiedlichen bisher absolvierten Studiengänge nicht wirklich einheitlich ist ${ }^{2}$. Es bietet aber den entscheidenden Vorteil, dass die grundlegenden Inhalte der Fachveranstaltungen, und damit auch die Inhalte des fachsprachlichen Unterrichts, grundsätzlich vorausgesetzt werden können, den Studierenden jedoch die Möglichkeiten zur Versprachlichung fehlen.

So wurde nach diesen ersten Vorgesprächen und gemeinsam weiterentwickelten Ideen zunächst versucht, einen geeigneten Zeitpunkt zur Verzahnung von Sprachkurs und Fachstudium auszumachen. Hierbei war es von wesentlicher Bedeutung herauszufinden, ab wann ein auf das Studium bezogener Fachsprachenunterricht einsetzen kann und wie viel Vorlaufzeit in einem allgemeinsprachlichen Deutschkurs notwendig bzw. wünschenswert ist. Deshalb wurden die sprachlichen Fertigkeiten in Grammatik und Syntax der einzelnen Kursstufen analysiert und ihre Relevanz für einen fachsprachlichen Unterricht festgelegt. Die grammatischen Phänomene, welche in Grund-, Mittel- und Oberstufe erworben werden, wurden zudem zwischen ihrer rezeptiven und produktiven Bedeutung für die Fachveranstaltungen und somit für den studienvorbereitenden Fachsprachenunterricht unterschieden.

Die grammatische Analyse ergab zunächst, dass bereits zum Ende der Grundstufe alle wesentlichen sprachlichen Grundlagen vorhanden sind, wenn auch in recht einfacher Form. Beispielsweise werden in der Grundstufe bereits Partizipialsowie Aktiv- und Passivkonstruktionen eingeführt, Zusammenhänge und Beziehungen sollten bereits durch unterschiedlichste Haupt- und Nebensatzkonstruktionen ausgedrückt bzw. verstanden werden können - alles Konstruktionen, welche in der Fachsprache für technische Disziplinen von wesentlicher Bedeutung sind.

Die weitere Analyse ergab, dass all die grammatischen Konstruktionen, welche in der Grundstufe in relativ einfacher Form eingeführt wurden, in der Mittelstufe anhand komplexerer Beispiele vertieft werden. Hier werden Fähigkeiten für Verstehen und Produktion erworben, die sich prinzipiell auch an fachsprachlichen Unterrichtsbeispielen erarbeiten ließen, da die Grundlagen aus der Grundstufe vorauszusetzen sind. Fast alle grammatischen Phänomene, welche in der allgemeinsprachlichen Mittelstufe vertieft werden, z. B. Adjektive, Konnektoren, Partizipialkonstruktionen, Aktiv/Passiv, aber auch Syntax und Wortbildungsmöglichkeiten, sind für die Fachsprache absolut relevant und treten hier mit gewissen, sich

2 Vgl. hierzu z. B. Fluck 1992, S. 32 f. 
von der Allgemeinsprache unterscheidenden, Präferenzen auf. Eigentlich, so unsere erste Hypothese, könnte somit bereits ab der Mittelstufe, bzw. bei einem sehr intensivem Sprachkursangebot sogar bereits nach ca. zwei Monaten Unterrichtszeit, eine Konzentration auf Fachsprachenunterricht erfolgen, in welchem die grammatischen Phänomene, welche in einfacher Form aus der Grundstufe bekannt sind, in einer fachsprachlich ausgerichteten Mittelstufe anhand entsprechender Unterrichtsbeispiele vertieft werden.

Die Möglichkeit eines frühzeitigen fachsprachlichen Unterrichts wird auch in der Literatur belegt:

Kann man die entsprechenden lexikalischen Grundlagen voraussetzen, so kann man mit jeder Fachsprache direkt beim Zertifikatsniveau beginnen. Haben die Lerner dieses Niveau erst einmal erreicht, gibt es keine morphologischen und kaum mehr syntaktische Schwierigkeiten.

Die Lexikvermittlung und Einführung in die Lektüre von Texten kann immer unter Zertifikatsniveau einsetzen, während man den für das Fach und den Spezialisierungsgrad des Adressaten angemessenen schriftlichen oder mündlichen Ausdruck kaum unter Zertifikatsniveau ansetzen bzw. vermitteln kann. (Buhlmann / Fearns 2000: 86)

Nachdem es also möglich schien, die Projektidee grundsätzlich umzusetzen, mussten weitere Institutionen, ohne deren Kooperation eine Realisierung unmöglich gewesen wäre, einbezogen werden. So musste bei der Fakultät für Geodäsie und Bauingenieurwesen eine Änderung der Studienordnung für den Masterstudiengang befürwortet werden, da eine Verzahnung von Sprachkurs und 1. Fachsemester zu einer Neukonzeption des 1. und 2. Fachsemesters führt. Weiterhin wurde die Idee dem DAAD vorgestellt, der die Projektentwicklung für die StipendiatInnen des Studiengangs daraufhin auch finanziell unterstützte. Zudem mussten mit dem Fachsprachenzentrum der Leibniz Universität Hannover (FSZ) die Möglichkeiten einer fachsprachenbezogenen DSH geklärt werden. Auch hier wurde Kooperationsbereitschaft signalisiert, zumal die DSH-Rahmenordnung entsprechende Möglichkeiten einer fachsprachlich ausgerichteten Prüfung bietet ${ }^{3}$. Mittlerweile unterstützt auch die Leitung der Universität Hannover das Projekt, welches von der Grundidee auch auf andere Studiengänge übertragbar ist, obwohl an dieser Stelle auch explizit erwähnt werden muss, dass jede Umsetzung einen enormen Aufwand insbesondere in der Erarbeitung entsprechender Unterrichtsmaterialien bedeutet und auf eine intensive Zusammen- und Mitarbeit des ent-

\footnotetext{
3 In diesem Zusammenhang sollte erwähnt werden, dass der damalige Leiter des Fachsprachenzentrums, Dr. Uwe Koreik, diesem Projekt nicht nur wohlwollend gegenüber stand, sondern auch als Fachaufsicht tatkräftig mitgewirkt und wesentliche Ideen zum Konzept beigesteuert hat. Es ist zudem geplant, das Projekt in Zusammenarbeit mit Dr. Sigrun Schroth-Wiechert und Dr. Klaus Schwienhorst als neuem Leiter des FSZ auf den studienbegleitenden Bereich auszuweiten.
} 
sprechenden Studiengangs angewiesen ist, welche keinesfalls als selbstverständlich vorausgesetzt werden kann.

Die konkrete Umsetzung des „Verzahnungskurse“, welcher am 5. Juni 2006 erstmals begonnen hat, sieht nun folgendermaßen aus:

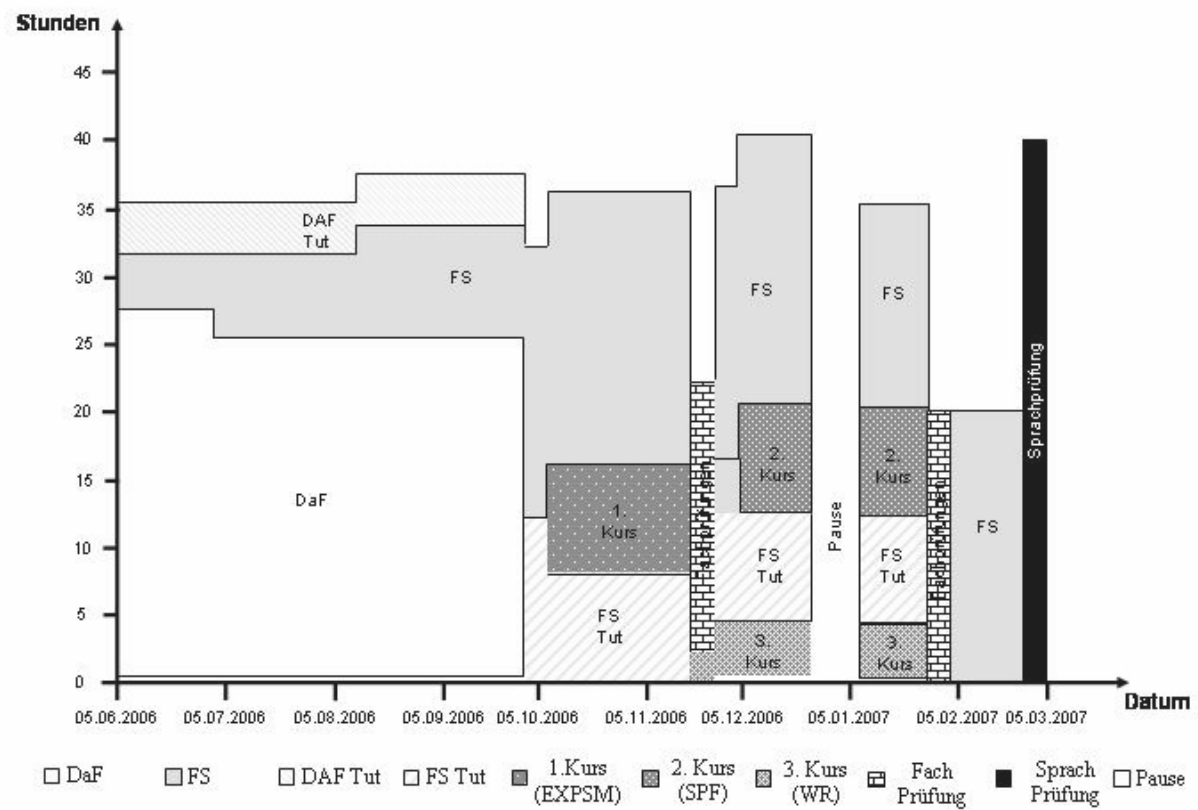

Die Studierenden besuchen demnach zunächst einen 4-monatigen (genau: 17wöchigen) allgemeinsprachlichen Deutschkurs (DaF) mit 25-27 SWS, in welchem sie ein mittleres Mittelstufenniveau (B1 / B2, GERR) erreichen sollen. Wir halten diese allgemeinsprachliche Vorbereitungszeit für angemessen, um auch die allgemeinsprachlichen Fertigkeiten der Studierenden ausreichend zu fördern. Dieser Deutschkurs wird sowohl durch ein DaF-Tutorium (DaF-Tut) mit 4 SWS als auch von Beginn an durch Fachsprachenunterricht (FS), dessen Stundenanteil im Verlauf von 4 SWS auf 8 SWS ansteigt, ergänzt. Vor Beginn des 1. Fachsemesters findet in der 18. Kurswoche eine intensive Studienvorbereitung mit 20 SWS fachsprachlichem Unterricht statt, welcher zudem durch ein Fach(sprachen)tutorium (FS-Tut) mit 12 SWS ergänzt wird, in welchem die Lerner mit einem Studierenden aus einem höheren Semester nach Bedarf sowohl fachinhaltliche, die Studiengrundlagen betreffende, als auch fachsprachliche Schwierigkeiten bearbeiten können. Bereits vor Studienbeginn wird mit sprachlich didaktisierten Materialien gearbeitet, welche sich auf die folgenden drei Veranstaltungen der Verzahnungsphase beziehen. 
In der anschließenden, insgesamt 5-monatigen Verzahnungsphase von Fachveranstaltungen und Fachsprachenunterricht, findet zunächst als 6-wöchige Blockveranstaltung der Kurs „Bodenmechanik“ (EXPSM) statt, welcher 8 SWS umfasst und durch 20 SWS Fachsprachenunterricht sprachlich vor- und nachbereitet werden kann. Diese Fachveranstaltung wurde deshalb als erste ausgewählt, weil sie durch ihren fachlich grundlegenden und einführenden Charakter einerseits in großem Maße unmittelbar Inhalte aufgreift und ausführt, welche den Studierenden durch ihr vorheriges B.Sc.-Studium gut bekannt sind. Andererseits ist sie somit Grundlage für folgende, darauf aufbauende Kurse. Weiterhin werden in den Vorlesungen und Skripten besonders viele sprachlich relativ reduzierte Elemente wie Formeln, Tabellen, Grafiken usw. eingebracht, deren Inhalte für die Studierende durch ihre Kenntnisse relativ leicht zu erfassen sind.

Da mit großer Sicherheit davon auszugehen ist, dass sich die fachsprachlichen Kompetenzen parallel zu dieser ersten Blockveranstaltung weiter verbessern werden, finden nach deren Abschluss und einer weiteren intensiven fachsprachlichen Vorbereitungswoche ab der 26. Kurswoche zwei Fachveranstaltungen als Blockveranstaltungen statt, wobei die Veranstaltung „Gründungen“ (SPF) 8 SWS umfasst, der Kurs „Wasserwirtschaft“ (WR) jedoch nur 4 SWS, da er im 2. Fachsemester fortgesetzt wird. Diese beiden Kurse sollen zunächst von 20 SWS, später von 15 SWS Fachsprachenunterricht begleitet werden. Während der gesamten Verzahnungsphase findet unterrichts- und vorlesungsbegleitend weiterhin ein Fach(sprachen)tutorium mit 6-12 SWS statt.

Nachdem die Studierenden ihre Fachprüfungen abgelegt haben, soll eine 3wöchige Prüfungsvorbereitung auf die zeitlich genau abgestimmte DSH stattfinden, in welcher noch einmal die spezifischen sprachlichen Erfordernisse für diese Sprachprüfung trainiert werden sollen. Wir planen, die Studierenden auch an einer TestDaF-Prüfung teilnehmen zu lassen, um anhand dieses standardisierten Tests zu kontrollieren, inwieweit sich auch die allgemeinsprachlichen Kompetenzen entwickelt haben. Allerdings bietet die Rahmenordnung der DSH einen gewissen, für unsere Belange vorteilhaften, Spielraum für eine fachsprachlich ausgerichtete Sprachprüfung, was keineswegs bedeutet, dass die Studierenden dadurch einen einfachern Zugang zum Hochschulstudium in Deutschland erhalten, sondern lediglich, dass sie ihre Sprachkenntnisse etwas fachspezifischer nachweisen müssen, wie es bisher auch an einigen Studienkollegs üblich war ${ }^{4}$.

Da sich das Konzept mit dem ersten Durchgang von Studierenden in einer Erprobungsphase befindet, ist es für uns wesentlich, durch eine entsprechende Flexibilität auf Rückmeldungen von Sprach- und FachdozentInnen, aber auch auf Rückmeldungen von den Studierenden einzugehen und die Stundenverteilung des Sprach- und Fachsprachenunterrichts entsprechend den aktuellen Erfordernissen auch kurzfristig anzupassen und zu verändern. Wir schließen hierbei auch nicht

\footnotetext{
${ }^{4}$ Vgl. hierzu Krekeler 2005, S.160.
} 
aus, dass selbst in der Verzahnungsphase der Fachsprachenunterricht ergänzt werden kann durch allgemeinen DaF-Unterricht. Eine gründliche Evaluation erfolgt insbesondere während des ersten Kursjahrgangs, so dass diese ebenfalls Änderungen in einigen Details mit sich bringen und zu einem leicht veränderten Kursplan führen könnte.

Bei der weiteren Lektüre entsprechender Fachliteratur stellte sich heraus, dass Modelle mit einem großen Anteil von studienvorbereitendem fachsprachlichem Unterricht bereits in früheren Jahren beispielsweise in Leipzig vom Herder-Institut oder auch in einigen osteuropäischen Ländern umgesetzt wurden.

Die folgende Tabelle vergleicht im Jahr 1984 die Verhältnisse von studienvorbereitenden allgemeinsprachlichem Unterricht (SVASU) und studienvorbereitenden Fachsprachenunterricht (SVFSU) in Kursen von der Grundstufe bis zur Oberstufe am Beispiel verschiedener Institutionen nach Unterrichtsstunden sowie im Verhältnis zueinander:

\begin{tabular}{|l|l|l|l|}
\hline & SVASU & SVFSU & SVASU/ SVFSU \\
\hline Leipzig & $452 \mathrm{~h}$ & $484 \mathrm{~h}$ & $0,9: 1$ \\
\hline Budapest & $526 \mathrm{~h}$ & $434 \mathrm{~h}$ & $1,2: 1$ \\
\hline Sofia & $712 \mathrm{~h}$ & $532 \mathrm{~h}$ & $1,4: 1$ \\
\hline Bratislava & $764 \mathrm{~h}$ & $432 \mathrm{~h}$ & $1,8: 1$ \\
\hline Moskau & $764 \mathrm{~h}$ & $360 \mathrm{~h}$ & $2,0: 1$ \\
\hline
\end{tabular}

(Pudzuhn 1994: 31)

Wir können das geplante Unterrichtsmodell unseres Verzahnungsprojekts in einer ähnlichen Tabelle veranschaulichen, in welcher einerseits zwischen Studienvorbereitung und Verzahnung unterschieden wird, andererseits zwischen allgemeinsprachlichem Unterricht $(\mathrm{DaF})$ und Tutorium (DaF-Tut) sowie Fachsprachenunterricht (FS) und fach(-sprachlichem) Tutorium (FS-Tut / FachTut) unterschieden wird. Aufgeführt werden zusätzlich die Fachveranstaltungen EXPSM, SPF und WR.

\begin{tabular}{|l|l|l|l|l|l|l|l|}
\hline Verzahnungsmodell Leibniz Universität Hannover \\
\hline & DaF & $\begin{array}{l}\text { DaF } \\
\text { Tut }\end{array}$ & FS & $\begin{array}{l}\text { FS-Tut / } \\
\text { FachTut }\end{array}$ & $\begin{array}{l}\text { Kurs1 } \\
\text { EXPSM }\end{array}$ & $\begin{array}{l}\text { Kurs2 } \\
\text { SPF }\end{array}$ & $\begin{array}{l}\text { Kurs3 } \\
\text { WR }\end{array}$ \\
\hline Vorbereitung & 433 & 68 & 128 & 12 & & & \\
\hline Verzahnung & & & 305 & 108 & 48 & 48 & 30 \\
\hline Total & & & 433 & 120 & & & \\
\hline Gesamt & 501 & 553 & 126 & & \\
\hline & 0,94 & 1 & & & & \\
\hline
\end{tabular}

Hiernach würde das Modell von IGEL und AGTZE mit 501 h: $553 \mathrm{~h}$ und somit in einem Verhältnis von 0,94: 1 in der ersten Tabelle direkt hinter Leipzig 
stehen, wobei in Hannover allerdings bereits der Sprachkurs mit dem Studium verzahnt wird.

Für die umfangreiche Materialerstellung zum Fachsprachenunterricht wurden von der AG TZE zahlreiches Material zu den neu konzipierten Blockveranstaltungen in Form von Vorlesungsskripten, Übungsaufgaben, Literatur, Beispiele für Prüfungsaufgaben, Hausarbeiten usw. erarbeitet und zur Verfügung gestellt, welche möglichst alle Unterrichts- und Übungsformen der Veranstaltungen abdecken sollten. Für die Erstellung von Hörverstehensübungen wurden Videoaufzeichnungen von Vorlesungen und Repetitorien bei den Bauingenieuren angefertigt, welche authentisches Arbeitsmaterial für entsprechende Übungen bieten und in didaktisierter und aufbereiteter Form im Fachsprachenunterricht eingesetzt werden. Durch die finanzielle Unterstützung des DAAD konnte vorübergehend eine Mitarbeiterin im Sprachbereich eingestellt werden, die anhand der zahlreichen Materialien zu den Fachveranstaltungen entsprechendes Unterrichtsmaterial für den Fachsprachenunterricht auf unterschiedlichen Niveaustufen entwickelte. Hierbei war eine Durchsicht und Kontrolle der Unterrichtsmaterialien durch die FachdozentInnen unbedingt notwendig, um auszuschließen, dass sich bei sprachlicher Korrektheit inhaltliche Fehler in das Fachsprachenmaterial eingeschlichen haben. Deutlich wird hier, dass sich Fach- und FachsprachendozentInnen keinesfalls gegenseitig ersetzen sollen und können, das Projekt aber auf eine Kooperation miteinander unbedingt angewiesen ist.

Für eine erfolgreiche Umsetzung des Projekts war zudem eine umfangreiche Analyse des Materials aus den Fachveranstaltungen notwendig, für welche ebenfalls eine Mitarbeit der Fachdozenten unabdingbar ist. So mussten die drei Fachkurse zunächst in ihrer sprachlichen Progression, aber auch in ihrer detaillierten thematischen Abfolge, in ihrer allgemeinen Struktur und ihrem Gesamtaufbau sowie auch in Gliederung und Struktur der Einzelveranstaltungen mit ihren unterschiedlichen Unterrichtsformen wie z. B. Vorlesung, Übung, Hausübung, Prüfung, Laborpraktikum usw. systematisiert und analysiert werden. Hier gilt es also, Unterschiede in den Textsorten sowie in den Mikro- und Makrostrukturen der Texte herauszuarbeiten ${ }^{5}$. An diesen Abfolgen orientiert sich natürlich auch der Aufbau des Fachsprachenunterrichts insbesondere in der Verzahnungsphase, weiterhin werden auf dieser Grundlage auch Übungsformen für den Fachsprachenunterricht entwickelt, welche den Aufbau und die Struktur der Veranstaltungen thematisieren. So mussten schließlich auch die unterschiedlichen Methoden und Kommunikationsverfahren sprachlich analysiert und systematisiert werden, sodass im Fachsprachenunterricht entsprechende Redemittel für unterschiedliche Arbeitsweisen im Fach, wie z. B. die Beschreibung von Grafiken oder Tabellen, Versuchsbeschreibungen, Anwendung von Normen usw. zur Verfügung gestellt werden können.

\footnotetext{
${ }^{5}$ Vgl. z. B. Roelcke 1999, S. 42 ff sowie S. 88 ff.
} 
Weiterhin können die beteiligten Disziplinen und einzelnen Fachsprachen wie bspw. Mathematik, Physik, Chemie unterschieden und systematisiert werden, welche in die Fachsprache für Bauingenieurwesen einfließen, da sie meist wesentliche Grundlagen für die inhaltliche Arbeit darstellen. Eine Differenzierung erleichtert und systematisiert die Arbeit im Fachsprachenunterricht sowohl für die LehrerInnen als auch für die Studierende.

Wie bereits erwähnt, muss anhand des Materials aus den Fachveranstaltungen auch die fachsprachliche Lexik, Morphologie und Syntax in ihrer jeweiligen Progression und Relevanz für die Fachveranstaltungen und den Fachsprachenunterricht untersucht werden ${ }^{6}$. Dies ist wesentlich für den konkreten Aufbau des Fachsprachenkurses und kann so auch den FachdozentInnen als Orientierungsmöglichkeit für den aktuellen Sprachstand der Studierenden dienen. In diesem $\mathrm{Zu}$ sammenhang muss auch festgehalten und bei der Materialerstellung berücksichtigt werden, welche lexikalischen, morphologischen und syntaktischen Mittel die Studierenden mit welcher Progression und Relevanz vorrangig rezeptiv bzw. zu welchem Zeitpunkt und in welcher Relevanz auch produktiv beherrschen sollten, um eine entsprechende Progression im Fachsprachenunterricht und den notwendigen Fähigkeiten entsprechendes Unterrichtsmaterial zu erarbeiten.

Schließlich ist eine Unterscheidung zwischen der Fachsprache und einer allgemeinen Wissenschaftssprache hilfreich, um den Studierenden auch allgemeine Mittel zum wissenschaftlichen Arbeiten an die Hand zu geben. Sie sollen innerhalb des (Fach-) Sprachkurses eine „studiengangsbezogene Studierfähigkeit“ und entsprechende Fertigkeiten erlangen, welche auch auf das praktische, studienrelevante Arbeiten bezogen sind, wie z. B. das Erstellen von Hausarbeiten, das Halten von Kurzvorträgen und Referaten sowie zudem das Arbeiten und Recherchieren in der Bibliothek usw. ${ }^{7}$.

Durch eine Analyse des Materials lassen sich zudem fachspezifische Unterschiede zwischen Fachsprache und Allgemeinsprache herausarbeiten. Hierbei ist es vor allem notwendig, Überschneidungen im Wortschatz, welche mit einer Bedeutungsveränderung einhergehen, herauszuarbeiten und den Studierenden im Fachsprachenunterricht aufzuzeigen.

Wir streben an, nach Ende des ersten Kursdurchlaufs eine entsprechende Materialsammlung mit den im Fachsprachenunterricht eingesetzten Materialien als Projektprodukt zu erstellen. Die im Unterricht eingesetzten Materialien sollen hierfür nach ihrem erstmaligen Einsatz im Unterricht erprobt werden und nach

\footnotetext{
${ }^{6}$ Hier gibt es Untersuchungen zu fachsprachlichen Besonderheiten in Verteilung und Relevanz von grammatischen Funktionen, Lexik, Syntax usw., an welchen sich grob orientiert werden kann. Vgl. hierzu z. B. Buhlmann / Fearns 2000, Kapitel „Fachsprachliche Grundlagen“ oder auch allgemeinerer Art in Beneš 1981. Unter Berücksichtigung verschiedener Sprachen siehe Hoffmann 1985, Kaptitel 2.

${ }^{7}$ Dieses „wissenschaftliche Arbeiten“, welches Elemente der allgemeinen Wissenschaftssprache beinhaltet, wird in der Literatur teilweise auch grundlegend der Fachsprache zugeordnet (vgl. z. B. Hoffmann 1978, S. 8f). Da es sich bei unserem Konzept aber um eine Fachsprache mit einem sehr hohen Spezialisierungsgrad handelt und auch neuere Autoren diese Unterscheidung wieder einführen (z. B. Graefen 1994 sowie Ehlich 1999), halten wir es für sinnvoll, diese Unterscheidung hier aufzugreifen.
} 
einer evtl. Überarbeitung oder Ergänzung in einer Sammlung zusammengefasst werden. Zusätzlich hierzu werden Fachglossare erstellt, welche den Fachsprachenunterricht ergänzen und den Studierenden insbesondere Hilfestellung beim selbstständigen Lexikerwerb leisten sollen.

Wesentlich, nicht nur für die Erstellung einer Materialsammlung, ist insbesondere für den ersten Durchlauf des Kurses eine umfangreiche Evaluation bei den Studierenden, aber auch bei den FachsprachenlehrerInnen, TutorInnen und FachdozentInnen, um die Stärken und Schwächen des bisherigen Konzeptes herauszufinden und dieses entsprechend für die nächsten Durchgänge, aber auch in seiner Übertragbarkeit für andere Studiengänge, zu optimieren. Während der Verzahnungsphase sind regelmäßige Treffen und Rücksprachen zwischen Sprach- und FachdozentInnen notwendig, um sich über aktuelle Erfordernisse und bisherige Beobachtungen und Einschätzungen zu informieren und die Sprach- oder Fachveranstaltungen ggf. daraufhin anzupassen. Allgemeine Hinweise und Ratschläge sowohl für Fach- als auch FachsprachlehrerInnen werden, sofern notwendig, schließlich in einem Dozenten-Handbuch festgehalten, welches den Fach- und Sprachlehrkräften entsprechende sprachliche und fachliche Anweisungen und Hilfestellungen beim Lehren im Verzahnungskurs geben soll.

Abschließend sollen auszugsweise und für diesen Vortrag reduzierte, bereits erstellte und teilweise auch schon im Fachsprachenunterricht eingesetzte Materialien für unterschiedliche Niveaustufen vorgestellt werden, welche in knapper Form für ihren Unterrichtseinsatz kommentiert sind. Die auf der Jahrestagung in Hannover vorgestellten Übungen zum Hörverstehen lassen sich in diesem Beitrag verständlicherweise nicht darstellen, da diese nur in Verbindung mit den bearbeiteten Videoausschnitten präsentiert werden können. 
1. Materialbeispiel für die Grundstufe:

Bodenstrukturen

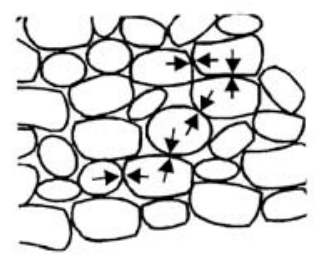

Sand / Kies:

Große Körner; Verhalten wird durch

Korn-zu-Korn-Spannung (Reibung)

bestimmt

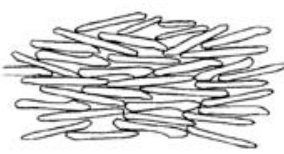

Ton:

Kleine „Körner" (Plättchen); aus Oberflăchenladungen der Tonminerale resultiert

Anziehungskraft

$\rightarrow$ Festigkeit auch ohne Normalspannung

(Kohäsion)

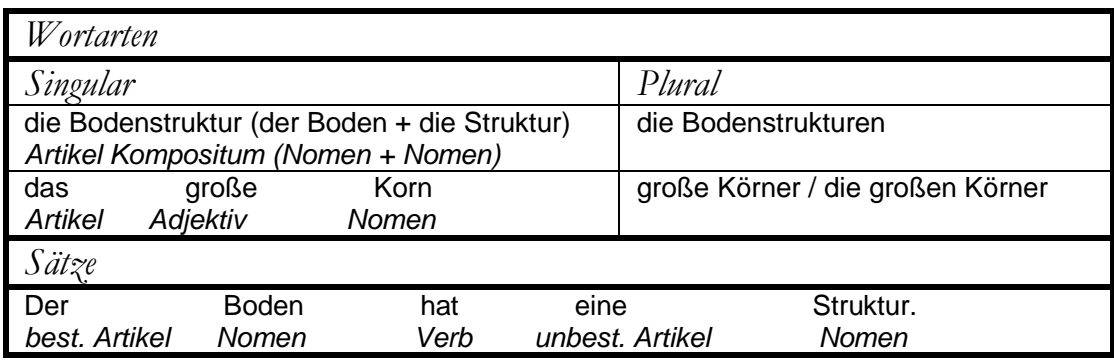

Die Abbildung und der kurze Text sind authentisches Material aus der Vorlesung Bodenmechanik und zeigen Bodenstrukturen für Sand, Kies und Ton. Wegen des hohen Spezialisierungsgrads sind die Texte kurz. Die Bilder werden von den TN erkannt, die dadurch eine inhaltliche Vorstellung haben, die die Motivation weckt. Der fachliche Inhalt gehört zu den Grundlagen, die vorausgesetzt werden, als Begriffe in Deutsch aber nicht vorhanden sind. Jede deutsche Bezeichnung muss im Einzelnen erklärt werden.

Zu Beginn werden einige Wortarten vorgestellt und Hinweise auf das Lernen von Artikeln und Pluralformen gegeben. Im nächsten Schritt werden Satzbeispiele gegeben um ein Wiedererkennen der gelernten Begriffe zu erreichen und als phonetische Übung, damit TN das Gelernte auch sprechen können. Die damit verbundene Aufgabe ist, Wörter nach Artikeln zu ordnen. Die TN erhalten einen 
Hinweis auf den Artikel im Femininum bei den Nomen im Text mit den Endungen -ung und -keit.

\section{Materialbeispiel für die Mittelstufe:}

\section{Räumlicher Erddruck}

\subsection{Allgemeines}

Für viele Aufgabenstellungen im Grundbau ist es ausreichend, ebene (2-dimensionale)

Erddruckprobleme zu betrachten (z. B. Erddruckbelastung von Baugrubenwänden oder Stützmauern, Erdwiderstand vor Spundwänden und Ortbetonwänden etc.). Es gibt aber zahlreiche Fälle, in denen die Berücksichtigung der räumlichen Verhältnisse notwendig ist, wie z. B.:

a) Aktiver Erddruck: Erddruck auf flüssigkeitsgestützte Einzellamellen einer Schlitzwand, Erddruck auf schmale Wände, Erddruck auf Schächte

b) Passiver Erddruck: Erdwiderstand vor Bohlträgern und vor Ankerplatten, Erdwiderstand vor horizontal belasteten Pfählen

1. Fragen zum Text

a) Wie heißt der Erddruck, der 3-dimensional wirkt?

b) Wie wird der Erddruck auf Schächte bezeichnet?

2. Erklären Sie:

a) Die Ortbetonwand:

3. Welche Wörter im Text haben die gleiche Bedeutung wie die kursiv gedruckten Wörter?
a. flächig
Lösung: 2-dimensional
b. ist erforderlich
Lösung: ist notwendig

Dieser Text entspricht einer Seite aus dem Skript „Erddruck“. Auf diesem Niveau werden etwas längere Texte bearbeitet. Im Vorfeld untersucht die Lehrkraft fachliche und sprachliche Aspekte des Textes, auf die sie im Kurs eingehen wird, wenn erforderlich auch mit Hilfe und Korrektur der Fachleute aus dem FB Bauingenieurwesen.

Der Text wird auf den OHP gelegt, damit alle die gleiche Blickrichtung und keine Schwierigkeiten beim Auffinden von Textstellen haben. Dabei erklärt die Lehrkraft als erstes alle neuen Fachbegriffe und regt ein Gespräch mit den TN und Fragen an. Wichtig ist, dass die TN sich auch gegenseitig zuhören und zum Fachgespräch bereit sind. Das ist nicht in allen Kulturen gegeben, einige sind nur auf Lehrer fixiert.

Nach der Klärung der Fachbezeichnungen weist die Lehrkraft auf die Redemittel des Textes hin, die in Übungen wieder aufgegriffen werden, damit die TN sprachlich flexibler werden. 
Im Anschluss bearbeiten die TN Leseverständnisfragen, die sie in ganzen Sätzen beantworten müssen, um Formulieren zu üben. Die Antwortmöglichkeiten werden miteinander verglichen.

Eine weitere Übung ist, die eben gelernten Begriffe mit eigenen Worten zu erklären oder eine Art Definition zu finden. Diese Übung lässt sich auch als Spiel auf Kärtchen durchführen und entwickelt sich meistens zu einem Anlass für Fachgespräche. Geübt werden auch sprachliche Alternativen der im Text vorkommenden Redemittel und Ausdrücke zur Erweiterung des Wortschatzes.

\section{Materialbeispiel für die Oberstufe:}

Wasseraufnahmefähigkeit

Definition: $\quad$ Die Wasseraufnahmefähigkeit $w_{A}$ ist das Verhältnis der von der getrockneten Bodenprobe im Versuchsgerät aufgesaugten Wassermasse $m_{w g}$ zur Trockenmasse $m_{d}$ der Bodenprobe. Dabei ist $\mathrm{m}_{\mathrm{wg}}$ der Grenzwert der aufge-

Vorschrift: saugten Wassermasse.

Bodenprobe: DIN 18132(1995)

Probenmenge: $\quad$ ca. $20 \mathrm{~g}$ trockener Boden ohne Körner mit $\mathrm{d}>0,4 \mathrm{~mm}$

Versuchsgerät: Versuchsanordnung nach Enslin/Neff (s. Abb. 4.32), Trocknungsofen, Waage, destilliertes Wasser, Exsikkator, Mörser etc. (vgl. DIN 18132, 1995) 
Die benötigten Geräte zur Bestimmung der Wasseraufnabmefähigkeit:

Wasseraufnahmefähigkeit

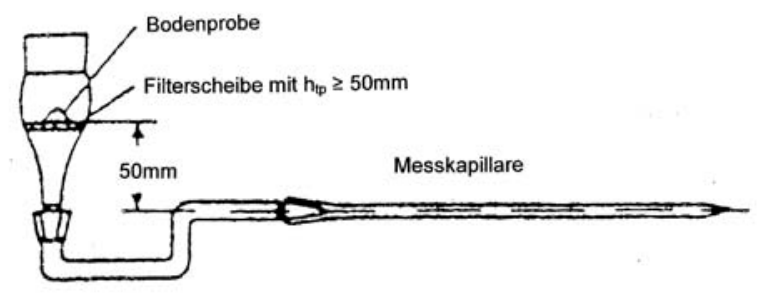

Abb. 4.32 Gerăte zur Bestimmung der Wasseraufnahmefähigkeit (Schultze et al., 1967)

\section{Versuchsdurchführung:}

Die getrocknete Bodenprobe wird zerkleinert $(<0,2 \mathrm{~mm})$, gewogen und eine Probenmenge von $1 \mathrm{~g}$ für $\mathrm{w}_{\mathrm{A}}<100 \%$ bzw. $0,2 \mathrm{~g}$ für $W_{A} \geq 100 \%$ in das Gerät eingebracht. Ober eine Messkapillare wird der Probe Wasser zugeführt. Nachdem die Ablesungen der Menge des zugeführten Wassers $\left(V_{\text {wg }}\right)$ keine Änderungen mehr anzeigen, wird der Wassergehalt mit $m_{w g}=V_{w g}, / p_{w}$ wie folgt bestimmt:

$$
w_{A}=\left(m_{w_{0}} / m_{e}\right) \cdot 100 \quad[\%]
$$


1. Geben Sie Anweisungen für die einzelnen Schritte der Versuchsdurchführung für wA $<100 \%$. Verwenden Sie dabei die Imperativform.

Lösungsbeispiel:

Verwenden Sie eine Bodenprobe von ca. $20 \mathrm{~g}$ ohne Körner mit $d>0,4 \mathrm{~mm}$.

Trocknen Sie die Bodenprobe im Trocknungsofen.

$\ldots$

2. Schreiben Sie eine Beschreibung der Versuchsdurchführung für $w A \geq 100 \%$. Verwenden Sie dabei die Passivform Präsens.

Lösungsbeispiel:

Eine Bodenprobe von ca. $20 \mathrm{~g}$ ohne Körner mit d >0,4 $\mathrm{mm}$ wird verwendet.

Die Bodenprobe wird im Trocknungsofen getrocknet.

$\cdots$

3. Schreiben Sie einen Bericht über die Versuchsdurchführung für $w A<100 \%$. Verwenden Sie dabei die Passivform Präteritum.

Lösungsbeispiel:

- Eine Bodenprobe von ca. $20 \mathrm{~g}$ ohne Körner mit d >0,4 mm wurde verwendet.

- Die Bodenprobe wurde im Trocknungsofen getrocknet.

- $\quad \ldots$

Dieses Beispiel ist eine Versuchsbeschreibung aus dem Skript „Experimentelle Bodenmechanik“. Die Wasseraufnahmefähigkeit des Bodens wird mit Hilfe eines Versuchs im Labor festgestellt. Dabei ist es wichtig auf Normen hinzuweisen, die Durchführung des Versuchs ist genormt. Solche Normierungen sind wesentlich, werden international aber nicht unbedingt einheitlich gehandhabt ${ }^{8}$. Die Begriffe werden in ihrer Anwendung präsentiert und mit den fachlichen Inhalten in Verbindung gebracht.

Im Text sind die einzelnen Schritte der Versuchsdurchführung genannt, die Ausgangspunkte für die folgenden Übungen sind.

Mit den Vorgaben der Versuchsdurchführung werden einfache Anweisungen im Imperativ geschrieben. Danach müssen diese Schritte sprachlich umgestellt werden für eine Beschreibung der Versuchsdurchführung im Passiv Präsens. Das Schreiben eines Berichtes über die Versuchsdurchführung verlangt eine nochmalige Umstellung, diesmal im Passiv Präteritum. Im Verlauf ihres Studiums müssen die TN eine Reihe Texte dieser Art schreiben, z. B. Laborberichte. Formeln im Text werden Schritt für Schritt auf Deutsch mit den TN gelesen.

In den Höraufgaben wird konzentriertes Zuhören in der Vorlesung geübt. Das Beantworten der Fragen kann sowohl fachliches als auch sprachliches Verständnis erfordern. Die Videoaufnahmen machen außerdem mit der Struktur und dem Ablauf der Vorlesungen vertraut, welche die TN später besuchen.

8 Vgl. Roelcke 1999, S. $111 \mathrm{ff}$ 
Erste Ergebnisse der noch bis April 2007 laufenden Evaluation dieses Verzahnungsprojektes geben Anlass zu der Hoffnung, dass die Studierenden in sprachlicher Hinsicht ausreichend vorbereitet sind, dem Fachunterricht unseren Erwartungen entsprechend zu folgen. Inwieweit ihre allgemeinsprachlichen Fähigkeiten oder auch die Kompetenz in einzelnen Fertigkeiten (z. B. Schreiben) durch die Verkürzung der allgemeinsprachlichen Lernzeit gelitten haben, wird sich bei den Sprachprüfungen oder auch dem Anfertigung von Hausarbeiten zum Kursende zeigen.

\section{Literatur:}

Beneš; Eduard: „Die formale Struktur der wissenschaftlichen Fachsprachen in syntaktischer Hinsicht.“ In: Bungarten, Theo (Hrsg.): Wissenschaftssprache. Beiträge zur Methodologie, theoretischen Fundierung und Deskription. München: Fink 1981, 185-212.

Buhlmann, Rosemarie / Fearns, Anneliese: Handbuch des Fachsprachenunterrichts. Unter besonderer Berücksichtigung naturwissenschaftlich-technischer Fächer. Tübingen: Narr 2000.

Ehlich, Konrad: „Alltägliche Wissenschaftssprache.“ In: Info DaF, 26, 1 (1999), 3-24.

Fluck, Hans-Rüdiger. Didaktik der Fachsprachen. Aufgaben und Arbeitsfelder, Konzepte und Perspektiven im Sprachbereich Deutsch. Tübingen: Narr 1992.

Graefen, Gabriele: „Wissenschaftssprache - ein Thema für den Deutsch-alsFremdspracheunterricht?" In: Wolff, Armin / Schleyer, Walter (Hrsg.): Fachund Sprachunterricht. Gemeinsamkeiten und Unterschiede. - Studiengänge Deutsch als Fremdsprache: Von der Theorie zur Praxis. Materialien Deutsch als Fremdsprache. Heft 43. Regensburg: 1997.

Hoffmann, Lothar: „Sprache in Wissenschaft und Technik“. In: Hoffmann, Lothar (Hrsg.): Sprache in Wissenschaft und Technik. Ein Sammelband. Leipzig: Enzyklopädie 1978, 5-11.

Hoffmann, Lothar: Kommunikationsmittel Fachsprache. Eine Einführung. Berlin: Akademie - Verlag, 1985.

Krekeler, Christian: „Die DSH-TestDaF-Vergleichsstudie an der Fachhochschule Konstanz." In: Koreik, Uwe (Hrsg.): DSH und TestDaF - eine Vergleichsstudie. Hohengehren: Schneider 2005, 153-185.

Pudzuhn, Manfred: Fachunterricht versus Sprachunterricht. Untersuchungen zum studienvorbereitenden Fachsprachenunterricht (DaF für Ausländer). Frankfurt am Main: Lang 1994.

Roelcke, Thorsten: Fachsprachen. Berlin: Schmidt 1999. 


\section{Interkulturelle Kommunikation und Geschlecht?}

Roya Moghaddam, Bremen

Der Titel dieses Beitrags ist mit einem Fragezeichen versehen, um deutlich zu machen, dass es sich hierbei um einen offenen Diskurs handelt, der erneut und intensiviert werden soll.

Als Soziolinguistin habe ich mich bisher vorrangig mit kontrastiv angewandter Linguistik und der Rolle von Geschlecht in Interaktionen beschäftigt, wobei ich Geschlecbt im Sinne von Gender benutze. In meiner Forschung arbeite ich vorrangig qualitativ-empirisch. Dabei untersuche ich Gesprächsinteraktionen beispielsweise mit Blick auf den non- und paraverbalen gleichrangig zum verbalen Bereich der gesprochenen Sprache. Interkulturell konzentriert sich der Forschungsblick auf die Relevanz von Geschlecht und deren Funktion als Gesprächskategorie im Gesprächssetting und hinterfragt spezifische Zuschreibungen, Handlungsinteraktionen und Stereotypisierungen in Bezug auf nationale und kulturelle Zugehörigkeitszuschreibungen.

Folgende Fragestellungen stehen in diesem Kontext im Vordergrund: 
- Was haben Interkulturelle Kommunikation und Geschlecht miteinander zu tun?

- Welche soziokulturellen Aspekte von Geschlecht sind relevant?

- Wie können Tabuisierungen von Geschlecht im DaF-Unterricht transparent gemacht und verringert werden?

- Welche Rolle spielt Geschlecht und dessen Benennung für Deutsch als Fremdsprache als Disziplin, als Unterrichtsfach und in der Interkulturellen Kommunikation?

In einer immer stärker globalisierten Welt werden die Entfernungen immer kleiner und die Differenzen zwischen Menschen, ihren Lebensweisen und Sprechgewohnheiten verschieben sich, wodurch nicht mehr nur die Unterschiede, sondern vielmehr auch kulturelle Gemeinsamkeiten anhaltend stärker in den Vordergrund treten.

Zunächst stelle ich die Begriffe Kultur und Interkulturelle Kommunikation und Geschlecht kurz dar.

\section{$1 \quad$ Was bedeutet Kultur?}

Zum Begriff Kultur definiert Böhm diesen im Wörterbuch der Pädagogik wie folgt: Kultur.

Das lat. cultura (Ackerbau) wurde schon von Cicero metaphorisch auf den Menschen übertragen [...] stellte dem rohen Naturzustand des Menschen seinen höheren Kulturzustand entgegen (Naturalismus, päd.) Herder fasste Kultur dann auch als histor. Erscheinungsform von Völkern, Nationen und Gemeinschaften auf. Kultur stellt die zweite Natur des Menschen dar, ohne die er nicht menschenwürdig, ja wohl überhaupt nicht zu leben vermöchte. Der Mensch als "Mängelwesen" (so Herder, Gehlen) mit reduzierten Instinkten, uneingefasst [...] Tradition bringt die gesellschaftliche Zusammenarbeit (Solidarität) hervor, erhebt sich zu objektiver Erkenntnis und Weltoffenheit (Scheler), denkt in die Zukunft und macht Entwürfe, gestaltet die Welt und fragt nach einem transzendenten Sinn. Erziehung und Bildung stehen in diesem menschl. - geschichtl. Kultur Zusammenhang, führen den Menschen in die (objektive) Kultur ein und verhelfen ihm so zu einer eigenen (subjektiven) Kultur Anthropologie ${ }^{1}$.

Somit zeigt sich in den Begriffsdefinitionen von Kultur: Strukturen werden nach immer wieder neu interaktiv hergestellten Querschnitten vorgenommen, die wichtige Bereiche für Menschen darstellen, um die Welt erfassen zu können und die wiederum Vorstellungen und Maßstäbe erschaffen.

\footnotetext{
${ }^{1}$ Böhm (1994:411), s. hierzu auch Bussmann (1990:429)
} 
1.1 Was bedeutet demnach interkulturell und Interkulturelle Kommunikation?

Interkulturell bedeutet zwischen den Kulturen, genauer gesagt, es handelt sich um zwischenmenschliche Konstellationen, bei denen davon ausgegangen werden kann, dass die beteiligten Menschen in diesen unterschiedlich aufgewachsen und dementsprechend anders sozialisiert sind. Interkulturelle Kommunikation beinhaltet Gesprächshandlungen zwischen Personen, die diesen konstruierten Grenzen zuzuordnen sind und über dessen Demarkationslinien hinweg miteinander interagieren, wobei diese Grenzlinien ebenso wie die Kulturen beweglich sind. Nestvogel (2000: 23f.)

Zur Analyse der Interkulturellen Kommunikation und der Kommunikationskontexte zwischenmenschlicher Interaktionen betrachten wir das verbale, nonund paraverbale und auch das soziale Setting, also den gesamten Gesprächskontext Moghaddam (2006). Dazu gehören im Allgemeinen und auch in interkulturellen Kontexten, Bereiche wie: Alter, Nationalität, Beruf, Familienstand, Geschlecht, Bildungsstand, Hautfarbe, Interessen etc. Diese haben ebenso Gültigkeit in Interaktionsbeziehungen im Deutsch als Fremd- oder auch Zweitsprachenunterricht.

Insbesondere in den heutigen globalisierten und individualisierten Gesellschaften können Interaktionen nicht mehr nur aus traditioneller Perspektive Interkultureller Kommunikationssituationen betrachtet werden. Eine adäquate Perspektive nach anderen Quer- und Längsschnitten in Fremd- und Eigengruppen beziehungsweise In-/Out- und Non-groups wäre wünschenswert. Eine derartige Perspektive sollte nicht nur nach Ländergrenzen, sondern wesentlich detaillierter betrachtet werden, da die Vorstellung homogener Nationalkulturen und entsprechender Gesprächsstile mehr als fragwürdig ist. Das bedeutet, dass die Kommunikation in Institutionen, in Berufszusammenhängen, durch Interessensgebiete, durch Familienstrukturen und Denkstrukturen der jeweiligen GesprächspartnerInnen geprägt und beeinflusst sein kann. Dabei sind die unterschiedlichen Lebensweisen und ggf. auch regional vielfältigen geographischen Kontexte mitzudenken, die interkulturell eingestuft werden können und flexibel gehandhabt werden müssen, wodurch sich die Grenzen zwischen Intrakulturell und Interkulturell verschieben. Hier formieren sich Gruppen, zu denen sich die Beteiligten zuordnen oder sich von diesen distanzieren. Mit dieser Definition einer eher flexibel wandelbaren Interkulturalität schließe ich mich interdisziplinär ForscherInnen wie Nestvogel (2000: 223f.), Apeltauer (2002) und Kotthoff (2002: 7f.) im Diskurs um den Begriff Interkulturell an.

Dementsprechend gehören die zwischenmenschlichen Interaktionen nach ethnischen, nationalen, sprachlichen und räumlichen Querschnitten, aber auch nach individuellen Längsschnitten wie Interessen, Berufsgruppen, Sprachvariationen, Geschlecht, Familienstand, Bildungsstand in den Bereich der Interkulturellen Kommunikation. Ebenso ist zu berücksichtigen, wie die Individuen diese Einflüsse handhaben, (re-)produzieren, verändern oder ihnen widersprechen. 
1.2 Welche Rolle spielt Geschlecht in der Kommunikation und in der interkultu rellen Kommunikation speziell?

Menschen, die miteinander kommunizieren, also sprachlich handeln, befinden sich immer auch in bestimmten Rahmenbedingungen von Gesprächssituationen. $\mathrm{Zu}$ diesen Rahmenbedingungen gehören soziale Settings wie äußere Erscheinungsformen, Befinden, Geschlecht und andere ebenfalls bereits genannte Faktoren, die in der face-to-face Interaktion sichtbar sind und die Wahrnehmung beeinflussen können. Auch klassisch interkulturelle Kommunikationssituationen sollten für den $\mathrm{DaF}-$ Unterricht genutzt und berücksichtigt werden. In den Unterrichtskontexten zeigen sich ebenfalls immer wieder Situationen, die einen adäquaten didaktischen und linguistischen Sprachgebrauch fordern. Ebenso ist zu berücksichtigen, dass Geschlecht in unterschiedlichen Ebenen auf das sprachliche Handeln wie folgt Einfluss nehmen kann:

a) Geschlecht als beeinflussende Gesprächskategorie, die in ein Konglomerat von Kategorien einzuordnen ist

b) Geschlecht in der Gesprächsthematik

c) Geschlecht kann durch sprachliche Benennung, aber auch durch Tabuisierung relevant werden

d) Geschlecht kann als das assoziierte Bild der jeweiligen GesprächspartnerInnen gesehen werden.

Geschlecht ist der assoziierte Bereich im Gespräch, der weniger in den Sprechhandlungen der SprecherInnen sichtbar sein muss, sondern viel stärker durch die Erwartungen der RezipientInnen gesteuert werden kann. Geschlecht kann in Interaktionen als Gesprächskategorie in den Vordergrund treten, kann die Rahmenbedingungen beeinflussen, kann die Thematik bestimmen und kann den Gesprächsverlauf leiten, kann aber auch völlig zurücktreten.

Um dieses näher erläutern zu können möchte ich an dieser Stelle kurz auf meine Forschungsarbeit eingehen, in der ich unter anderem auch Geschlecht als Gesprächskategorie untersucht und das Gesprächsverhalten der TeilnehmerInnen im Hinblick auf mögliche Stereotypisierungen nach Geschlecht beispielsweise auf Dominanz und Kooperation überprüft habe.

In meiner Forschungsarbeit WELCHE BEDEUTUNG HAT GESCHLECHT IN GESPRÄCHSINTERAKTIONEN? Eine empirische Studie zum universitären Sprachgebrauchsverhalten im Iran - kontrastiv zu ausgewählten Untersuchungen des deutschsprachigen Raumes (Moghaddam: 2006) habe ich eine Gesprächsanalyse durchgeführt. Dabei handelt es sich um eine empirische, soziolinguistische, geschlechtsspezifische und interdisziplinär ausgerichtete Untersuchung, deren Fokus auf der verbalen, nonverbalen und paraverbalen Kommunikation liegt.

Obwohl die Differenz- und Dichotomie-Hypothese der feministischen Linguistik längst vom doing-gender-Ansatz (Gottburgsen: 2000) der linguistischen Gen- 
derforschung abgelöst wurden, existieren weiterhin Aussagen empirischer Untersuchungen, die davon ausgingen und teilweise noch davon ausgehen, dass sich generalisierende sprachliche Stereotypisierungen nach Geschlecht vornehmen lassen, wie kooperatives und kompetetives Gesprächsverhalten².

\section{Die Bedeutung von Gender / Geschlecht in Interaktionen?}

Im Rahmen meiner qualitativen Forschungsarbeit untersuchte ich, inwiefern Geschlechter im Gespräch different agieren, ob Geschlecht an sich Einfluss auf Gesprächshandlungen nimmt, inwiefern das Geschlecht Einfluss auf Handlungsmuster der Agierenden nehmen kann und ob geschlechtsspezifische Gesprächsstile festschreibbar für ein Geschlecht zu finden sind oder der Forschungsblick an sich überdacht werden muss.

Methodisch habe ich mit der Gesprächsanalyse, der doing-gender und nach der Grounded Theory Strauss/Corbin (1996) gearbeitet.

Parallel dazu interessierte hier die Einbringung einer nichteuropäischen Sprache, das Farsi (Standardsprache im Iran) in die Kontrastive Linguistik: Obwohl das Farsi sich im Bereich der grammatischen Strukturen wie etwa bzgl. Artikel und Genera sehr vom Deutschen unterscheidet, zeigen sich im Sprachgebrauch dieser Sprachen wichtige Gemeinsamkeiten wie bei den Verbalphrasen, Kürzungen und Vereinfachung umgangssprachlicher Wendungen und bspw. bezüglich der Benennung von Geschlecht. Als weiterer Aspekt wurde in dieser Untersuchung die interdisziplinäre Verknüpfung des Gesprochenen mit der Art, wie gesprochen wurde (z. B. Intonation, Mimik, Gestik), also des Verbalen mit dem Non- und Paraverbalen und dem sozialen Gefüge des Gesprächs erforscht.

Für die einjährige Feldforschungsphase gründete ich im Iran Gesprächsgruppen an freien und staatlichen Universitäten in Teheran. Diese Gruppen trafen sich zu Diskussionen über selbst gewählte Themen in ein- und gemischt-geschlechtlichen Konstellationen. Diese Arbeitssitzungen habe ich audiovisuell aufgezeichnet, transkribiert und der Veröffentlichung beigelegt.

Bei der Durchsicht des Datenmaterials traten Gesprächsbereiche wie Höflichkeit, Konflikte und Gruppenbildungsprozesse in den Vordergrund, anhand derer sich Bereiche wie Dominanz, Unterdrückung, Lachen und Fragen qualitativ mit erweiterter Forschungsperspektive analysieren ließen.

Einerseits arbeitete ich dabei heraus, dass diese Gesprächselemente wesentlich detaillierter zu untersuchen sind, indem z. B. Dominanz sehr unterschiedlich eingesetzt wird und folglich diverse Formen annimmt. Es kann festgehalten werden, dass Stereotypisierungen nach der Kategorie Geschlecht in den Gesprächen nicht haltbar sind. Sie sind nicht nur durch Abzählungen etwa nach quantitativen Auffälligkeiten wie Anzahl der Unterbrechungen, der Gesprächsschrittübernahme

\footnotetext{
2 Vgl. Günthner (1992; 1997); C. Schmidt (1992); A. Schmidt (1998) und Schoenthal (1998) u. a.
} 
oder von lautem Sprechen alleine abhängig, sondern auch immer im Kontext der Gesamtsituation zu erfassen und zu analysieren. Das bedeutet, dass nicht nur die Sprechakte bzw. -handlungen der SprecherInnen für die Relevanz von Geschlecht in Gesprächsinteraktionen wichtig sind, sondern auch deren Realisierung sowie die Erwartungen, die von den HörerInnen an diese gestellt werden. Dadurch wird deutlich, welches Gewicht der non- und paraverbale Gesprächsbereich neben dem Verbalen einnimmt. Aufgrund des Datenmaterials und der Analyse kann das Geschlechterbild interkulturell und bezogen auf das Farsi zu Forschungsaussagen des Gesprächsverhaltens im deutschsprachigen Raum als einseitig festlegbares nicht bestätigt werden.

Dabei hat sich im Iran aber auch gezeigt, dass die Thematisierung von Geschlecht ein wichtiger sprachlicher Aspekt ist, weswegen ich diesen auch auf den $\mathrm{DaF} / \mathrm{DaZ}$-Unterricht übertragen und näher untersuchen möchte.

\subsection{Interaktionsbeispiele}

Ich komme nun zu zwei Beispielen, die für den Bereich Geschlecht als soziokulturellem Aspekt sprachlichen Handelns, aber auch des Lernens und Lehrens äußerst wichtig sind.

Einleitend möchte ich darauf hinweisen, dass in dem ersten Beispiel im Iran Geschlecht mehrfach eine Rolle im Gespräch spielt. Besonders interessant ist die interaktionsbedingte Veränderung des Sprachgebrauchs, da das standardsprachliche Farsi über kein Genus und auch nicht über Artikel verfügt Moghaddam (2006). In dem zweiten Beispiel in Deutschland stehen diese sprachlichen Optionen zur Verfügung, die nicht genutzt werden. Hier zeigen sich eher Rollenzuschreibungen per Geschlechtszugehörigkeit.

\subsubsection{Beispiel aus dem Iran}

Bei dem Beispiel aus dem Iran, das ich kurz beschreibe, handelt es sich um eine Gruppe von sechs Personen, zwei Studentinnen und vier Studenten. Sie sprechen zu dem Thema Frauenrechte im Iran, das von einem der Studenten provokativ in den Raum gestellt wurde. Eine der Studentinnen, ist eine aktive und engagierte Sprecherin, die sich mit der Thematik gut auskennt und die andere ist eine eher ruhige und schüchterne Studentin. Diese beiden treffen auf zwei Studenten. Von denen zwei aktive, engagierte und zwei eher schüchterne Studenten sind. Die aktive Studentin und die beiden aktiven Studenten handeln die Gesprächssituation und die Gesprächsrichtung aus, wobei sich in den ersten 20 Minuten eine Konfliktsituation zwischen diesen drei GesprächsteilnehmerInnen anbahnt. Einer der aktiveren Studenten stellt eine Frage, die die Studentin ungefragt beantwortet, woraufhin der fragende Student sie direkt und wenig begeistert anspricht. Sein Ton ist dabei deutlich sanktionierend lauter und hastig gesprochen; er weist daraufhin, dass er nur noch auf ihren Rat gewartet habe. Die Studentin lässt sich 
davon nicht beeindrucken, fährt fort und setzt ihre Gesprächsrichtung trotz der Gegenwehr der Studenten nach ca. 50 Minuten durch, wodurch sie die Dominanzposition übernimmt.

Im Iran ist durch die derzeitige Kleiderordnung eine Assoziation nach Geschlecht wesentlich stärker gegeben, die das Gespräch beeinflussen könnte. Dieses geschieht hier dadurch, dass die Kategorie Geschlecht im Gespräch mitschwingt und die Gesprächsthematik und Gesprächshandlung beeinflusst sein kann. Somit können Konflikte von der Kategorie Geschlecht beeinflusst werden, sie erweist sich jedoch selten als dominante Kategorie. Vielmehr ist von einem Einfluss vielfacher Kategorien, die sich gegenseitig beeinflussen, zu sprechen.

\subsubsection{Beispiel aus dem Integrationskurs in Deutschland}

In einer Unterrichtsstunde arbeiteten die TeilnehmerInnen (13 Frauen und 2 Männer) zum Thema Berufswahl. In der Stunde sollten die TeilnehmerInnen Zeitungsanzeigen/Stellenanzeigen lesen und Anzeigen auswählen, auf die sie sich bewerben würden und dieses erläutern und begründen. In Kleingruppen arbeiteten sie sich in das Thema ein und tauschten sich aus. Bei den Gruppengesprächen wurde schnell deutlich, dass die TeilnehmerInnen in der Regel eher das generische Maskulin benutzen. Als Kursleiterin spreche ich nicht mit dem generischen Maskulin, so dass sich den TeilnehmerInnen die unterschiedlichen Sprechvarianten bieten $^{3}$. Interessant sind hierbei insbesondere die Differenzierungen der TeilnehmerInnen im Sprachgebrauch und die abweichenden sprachlichen Lernstrukturen, die sich dabei herausbilden können. Trotzdem sind im Kurs noch Äußerungen zu vernehmen, die einseitig festgeschriebene Geschlechterbilder aufzeigen und die Notwendigkeit eines Umdenkens bestätigen, wie beispielsweise, dass einer der Männer in der Gruppe zu gesprächig sei, was sich für Männer nicht gehöre und ein Privileg der Frauen sei. Auch in dieser face-to-face Interaktionssituation sind äußere und soziale Geschlechterzuschreibungen für diese Annahme zuständig. Die Assoziation und gesellschaftliche Zuschreibung ist die Grundlage dieser Sichtweise, die realitätsnah und neu gedacht werden sollte.

\section{$3 \quad$ Fazit}

Resümierend lässt sich daraus ablesen, dass in den Kleingruppengesprächen beispielsweise durch Hinweise oder im Plenum durch Wiederholung mit Beidbenennung begegnet werden kann und sollten. Dadurch können Lerngruppen die Geschlechtermarkierung sehr gut kennen lernen und lernen. Die Berufswahl entspricht anteilig Stereotypisierungen nach Geschlecht, die im Kontext der Thematik nicht explizit besprochen werden, sondern mehr unterschwellig mitschwin-

\footnotetext{
${ }^{3}$ Dieser Bereich ist in Deutschland noch zu wenig untersucht und beschrieben worden, was mit diesem Forschungsprojekt nachgeholt werden soll.
} 
gen. Dieses lässt sich durch die Benennung der Geschlechter im Deutschen verringern.

Gute kommunikative und lernspezifische Möglichkeiten boten sich der Gruppe beispielsweise in Kontexten wie folgt:

- $\quad$ Gespräche über Berufsgruppen

- Zeitungsanzeigen analysieren

- Wahlen und Wahlprozesse besprechen

- Benennung der Geschlechtermarkierung

- Gespräche über gesellschaftliche Konstruktionen geschlechts-spezifischer Berufsfelder

- $\quad$ Vorstellungen der TN bezüglich geschlechtsspezifischen Verhaltens

Eine Fortschreibung und Festschreibung von Geschlecht kann zumindest durch eine Benennung und Enttabuisierung verringert werden und spiegelt ein reales Bild in interkulturellen Kommunikationssituationen, in Unterrichtskontexten im Allgemeinen und in DaF / DaZ-Kontexten im Speziellen wieder.

Die kulturelle, lebensweltliche Vielfalt der Lernenden und Lehrenden bietet eine gute Plattform für den adäquaten Erfahrungsaustausch interkulturell und geschlechtsspezifisch. Hier könnte zeitweise gut auf diese Bereiche eingegangen werden, da eine reale Darstellung deutschsprachiger Lebenswelt sowohl das Lehrmaterial als auch der Referenzrahmen zulassen würden. Hierfür sind im Folgenden einige Rahmendaten, die die Kurse, TeilnehmerInnen und Lehrenden betreffen können, aufgeführt:

Lernende / lernen

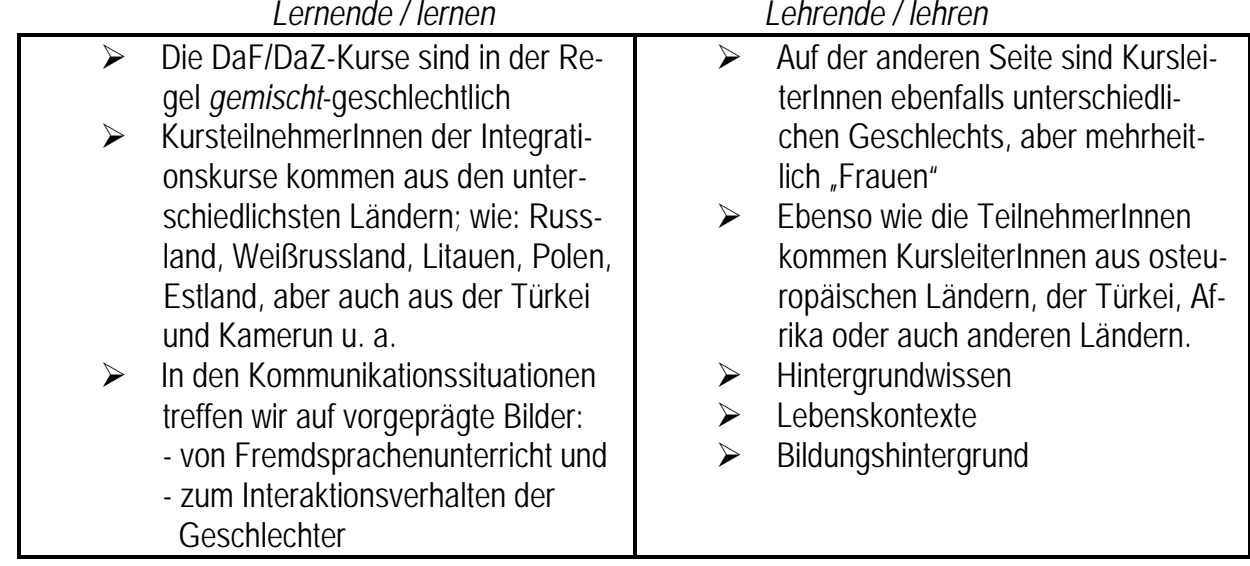

Die angeführten Aspekte, die den Aneignungsprozess des Deutschen beeinflussen, werden häufig noch reduziert oder auch völlig ignoriert. Zusätzlich finden Tabuisierungen nach Gescblecht sowohl sprachlich, inhaltlich als auch strukturell Eingang in den Lernprozess. Die Berücksichtigung, Enttabuisierung und letztend- 
lich auch die Benennung von Geschlecht kann in wichtigen kleinen Schritten ein realisierbarer Prozess sein.

Themen wie die aufgeführten, werden im DaF / DaZ-Unterricht behandelt:

Städte, Orte und Politike (Landeskunde)

Deutsch / typisch Deutsch - Frauen und Männer

Berufswabl

Wie bewerbe ich mich? / Zeitungsanzeigen

Lebensläufe / Berufsbilder

Reisen und Freizeit

Filme - Der Himmel über Berlin

Einkaufen u. a.

Diese Themen bieten optional gute sprachliche, pragmatische und soziale Strukturen an. Sie sollten und können als Raum zur Enttabuisierung genutzt werden und genügend Raum bieten Deutsch so zu vermitteln, dass die Lernenden ein realistisches Werkzeug der deutschen Sprache erwerben und sich dadurch Tabuisierungen verringern, indem bspw. auf differenzierte Formen der Genusmarkierung hingewiesen wird, bei Gesprächen zusätzlich die Femininmarkierung mit genannt wird oder bei Gesprächen Gemeinsamkeiten und Unterschiede von Gescblecht thematisiert werden.

Abschließend möchte ich betonen, dass ich die Thematisierung von Geschlecht als Bestandteil interkultureller Kommunikation für sehr wichtig für die Zuordnung von sozialer Identität und das Erfassen der deutschen Kultur, die sich hier über Sprache präsentiert, halte.

Hier bieten sich interkulturelle Möglichkeiten der Sprache und des Sprachgebrauchs an, mittels derer Sprache ohne Tabuisierung gelehrt und gelernt werden kann, indem beispielsweise nicht mit dem generischen Maskulin agiert wird, Stereotypisierungen aufgedeckt und ein übergreifendes Bewusstsein für die Bedeutsamkeit der Geschlechter geschaffen wird, das zu deren Gleichberechtigung beitragen und wie folgt realisiert werden kann und eingehender untersucht werden soll:

- Einsatz sprachlicher Geschlechtermarkierung im Gespräch/Unterricht

- Einbinden von Geschlecht in Unterrichtsthematiken, um Tabuisierungen zu vermeiden

- Benennung von Rollenstereotypisierungen nach Geschlecht, um Gemeinsamkeiten hervorzuheben und von der Differenzorientiertheit wegzuführen

- Sprachliche Vermeidung von Benachteiligungen 


\section{Literatur:}

Apeltauer, Ernst: „Interkulturelle Kommunikation“, Guter Narr Verlag 2002.

Böhm, Winfried: „Wörterbuch der Pädagogik“, 14. überarb. Auflage. Stuttgart: Kröner Verlag 1994.

Bußmann, Hadumod: „Lexikon der Sprachwissenschaft“, 2. überarb. Auflage. Stuttgart: Kröner 1990.

Gottburgsen, Anja: „Stereotype Muster des sprachlichen Doing Gender“. Eine empirische Untersuchung. Wiesbaden: Westdeutscher Verlag 2000.

Günthner, Susanne: „Zur kommunikativen Konstruktion von Geschlechterdifferenzen im Gespräch", Friederike Braun \& Ursula Pasero (Hrsg.). Kommunikation von Geschlecht. Pfaffenweiler: Centaurus-Verl.-Ges. (1997), 122-146.

Güntner, Susanne: „Die interaktive Konstruktion von Geschlechterrollen, kulturellen Identitäten und institutioneller Dominanz.

Sprechstundengespräche zwischen Deutschen und Chinesen/innen“, Susanne Günthner \& Helga Kotthoff (Hrsg.). Die Geschlechter im Gespräch:

Kommunikation in Institutionen. Stuttgart: Metzler (1992), 91-125.

Moghaddam, Roya: „Welche Bedeutung hat Geschlecht in Gesprächsinteraktionen? Eine empirische Studie zum universitären Sprachgebrauchsverhalten im Iran kontrastiv zu ausgewählten Untersuchungen des deutschsprachigen Raumes“, Frankfurt: Peter Lang 2006.

Nestvogel, Renate: „Konstrukte zum Geschlechterverhältnis und Konsequenzen für eine interkulturelle Pädagogik", Doris Lemmermöble, Dietlind Fischer, Dorle Klika und Anne Scblüter (Hrsg.). Lesarten des Geschlechts. Zur

Konstruktionsdebatte in der erziehungswissenschaftlichen

Geschlechterforschung. Opladen: Leske + Budrich (2000), 223-244.

Schoenthal, Gisela: „Geschlechtstypisches Kommunikationsverhalten: Ergebnisse, Konsequenzen, Perspektiven“, Gisela Schoenthal (Hrsg.). Feministische Linguistik - Linguistische Geschlechterforschung. Hildesheim u.a.: Olms (1998), 155-174.

Strauss, Anselm L. Corbin, Juliet M.: „Grounded Theory. Grundlagen Qualitativer Sozialforschung“", Berlin: Beltz Verlag 1995. 


\section{Besser sprechen durch „Loreley“ und „Mondnacht“.}

Anke Stöver-Blahak, Hannover

\section{Ein Projekt zur Verbesserung der mündlichen Kommunikation am Fachsprachenzentrum der Leibniz Universität Hannover}

Dem hier vorgestellten Projekt liegt die Erkenntnis zugrunde, dass die Einbeziehung von Emotion und Literatur ${ }^{1}$ den Sprachlernprozess entscheidend verbessern kann. Dies ist in verschiedenen Zusammenhängen vielfach thematisiert worden wenn man z. B. die Begriffe „Literatur, Emotion und Fremdsprachenlernen“ bei Google eingibt, findet man 793 Einträge ${ }^{2}$.

Wie man diese Erkenntnis in der Praxis erfolgreich umsetzen kann, ist Thema dieses Beitrags.

${ }^{1}$ Siehe Fußnote 5

2 Abfrage vom 05.06.2006 


\section{Rahmenbedingungen}

Durchgeführt wurde der Kurs bisher dreimal am Fachsprachenzentrum der Leibniz Universität Hannover als Angebot zur Verbesserung der mündlichen Kommunikation ${ }^{3}$.

Er findet mit zwei Semesterwochenstunden in der Vorlesungszeit statt, die TeilnehmerInnen erhalten einen qualifizierten Schein, wenn sie regelmäßig teilgenommen und am Ende „öffentlich“4 ein Gedicht vorgetragen haben.

Der Kurs wird auf C1 Niveau (nach dem Gemeinsamen Europäischen Referensrahmen für Sprachen) ausgeschrieben. Die KursteilnehmerInnen können vier ECTS Punkte erhalten.

Die Kurse waren international besetzt mit TeilnehmerInnenzahlen zwischen 8 und 16, in allen Kursen gab es mehr Frauen als Männer. Das vertretene Fächerspektrum reichte von Maschinenbau über Jura, Sportwissenschaften, Wirtschaftswissenschaften bis zu Germanistik.

\section{Ausgangspunkt}

Ausgangspunkt für diesen Kurs war die Beobachtung, dass viele Studierende Probleme hatten, ganze Sätze in angemessener Intonation, Phonetik und richtigem Rhythmus auf Deutsch zu sprechen. Am Fachsprachenzentrum gab (und gibt) es Angebote für Phonetik und für das Präsentieren von wissenschaftlichen Ergebnissen, diese Kurse haben aber andere Ziele als die von mir beobachteten Defizite zu bearbeiten. So entstand der Gedanke, mit Literatur als schon vorliegendem Text zu arbeiten und gleichzeitig den Bereich von Affektion und Emotion lernunterstützend zu nutzen in dem Sinne, dass nicht über Gefühle gesprochen wird wie im herkömmlichen Literaturunterricht, sondern dass die in den Gedichten thematisierten Emotionen wie Liebe, Trauer, Sehnsucht, Einsamkeit etc. im Sprechen/durch das Sprechen nachvollzogen und dargestellt werden (Affektion) und dass die SprecherInnen in ihrem Bemühen, die gefühlten Inhalte adäquat zu artikulieren auch Verbesserungen in den o. g. Bereichen des Sprechens erreichen ${ }^{5}$.

Das Projekt „Besser sprechen mit Gedichten“ umfasst also zwei Komponenten:

„Besser sprechen..."

bedeutet, dass in den Bereichen Phonetik, Intonation, Ausdruck aber auch Mimik und Gestik Verbesserungen erreicht werden sollen.

\footnotetext{
${ }^{3}$ SoSe 2005; WiSe 2005/6; SoSe 2006 (war zum Zeitpunkt des mündlichen Vortrags noch nicht abgeschlossen).

${ }^{4}$ Wird im Folgenden spezifiziert.

${ }^{5}$ Es ist mir bewusst, dass es zu den Begriffen Emotion, Affektion, Kognition und Motivation im Zusammenhang mit der Behandlung von Literatur im Fremdsprachenunterricht eine umfassende wissenschaftliche Diskussion gibt. Ich möchte sie in diesem Praxisbericht nicht aufgreifen, sondern verwende die genannten Begriffe in der kurz umrissenen eher alltagssprachlichen Bedeutung. Eine wissenschaftliche Einordnung ist geplant.
} 
„,...mit Gedicbten“

Gedichte als fertige, abgeschlossene relativ kurze Texteinheiten entlasten den Vortragenden/die Vortragende vom Produzieren von Inhalt, d. h. die Aufmerksamkeit kann beim „richtigen“ Sprechen bleiben und wird nicht abgelenkt durch Fragen wie „Habe ich die richtigen Wörter, die richtige Grammatik benutzt?“

Gedichte - zumindest die hier zugrunde gelegten - sind in der Regel rhythmische, durchkomponierte Sprache. Durch diese Sprache und die Inhalte ermöglichen sie den SprecherInnen im o. g. Sinne einen affektiven Zugang zur Sprache und erleichtern dadurch den Sprachlernprozess.

\section{Kursverlauf}

\section{Übersicht}

Der Kurs von 13 bis 14 Doppelstunden im Semester gliedert sich in drei Phasen:

I. Phase

Vertrauen aufbauen, sensibilisieren, Kriterien schaffen

II. Phase

Auswählen und Einüben der Gedichte/Dialoge

III. Phase

Vorbereitung einer „öffentlichen Aufführung“

Diese Phasen sind nicht statisch voneinander getrennt zu betrachten, sondern markieren Schwerpunkte im Unterrichtsgeschehen.

\subsection{Phase: Vertrauen aufbauen, sensibilisieren, Kriterien schaffen}

Sprechen ist immer mehr als das bloße Produzieren von Lauten oder Wörtern. Wenn in einer Gruppe gemeinsam Sprechen geübt werden soll, bedeutet das auch, dass sich die einzelnen öffnen müssen, dass sie Fehler direkt offenbaren und sich verletzbar und angreifbar machen. Deshalb ist es sehr wichtig, von Anfang an für eine Atmosphäre des gegenseitigen Vertrauens und Respekts zu sorgen. Die KursteilnehmerInnen in einer sehr heterogenen Gruppe müssen unter anderem erkennen, dass

- es sehr verschiedene Sprechprobleme gibt,

- diese Sprechprobleme ihre Ursache in der Ausgangssprache aber auch in der Person haben können,

- sie sich gegenseitig bei verschiedenen Problemen helfen und unterstützen können,

- unter keinen Umständen jemand ausgelacht werden darf. 
Das gemeinsame Ziel ist „richtiges Sprechen“, das heißt in diesem Zusammenhang Sprechen nach standardsprachlichen Vorgaben in Rhythmus, Intonation, etc. Drei Methoden haben sich auf dem Weg dorthin als besonders sinnvoll erwiesen:

a) Das Erstellen von Sprechfassungen.

Sinnvoll sind hier Textanalysen nach Anregungen der Sprecherziehung ${ }^{6}$, wie z. B. das Erarbeiten und Kennzeichnen von Pausen, Senkungen, Hebungen und Betonungen.

Als Basistexte eignen sich Märchen oder auch kurze Wilhelm Busch Gedichte.

b) Sprechübungen nach Anregungen aus dem Bereich der Theaterpädagogik und der Sprecherziehung

Dazu gehören Übungen wie z. B.

laut-leise Sprechen, rhythmisches Sprechen,

Vokale/Konsonanten verschieden artikulieren, z. B. durch Dehnungen/Kürzungen, Verbindungen von Vokalen mit Plosivlauten etc.,

Ketten- oder Partnerübungen für die Reaktion und Interaktion

Gefühle ausdrücken

c) Körperübungen nach Anregungen aus dem Bereich der Theaterpädagogik Wichtig ist hier, dass die KursteilnehmerInnen ihre Körper und deren Möglichkeiten spüren. Übungen sind z. B.

Gehen im Raum nach Vorgaben

Das allmähliche Finden einer guten „Sprechhaltung“ bzw. das Vermeiden von Kommunikationshindernissen

Das Darstellen von Gefühlen durch Mimik und Gestik

Das Spielen von kleinen Pantomimen

Das Erstellen von Sprechfassungen kann noch an den Arbeitstischen durchgeführt werden, die anderen Übungen erfordern einen Sitzkreis, bzw. einen freien Raum. Das bedeutet, dass ab der zweiten oder dritten Stunde immer am Anfang die Tische zur Seite geräumt werden müssen.

Sprechübungen und Körperübungen sollten am Anfang einer jeden Stunde durchgeführt werden.

Von den ersten geübten Sprechfassungen werden Videoaufnahmen gemacht und anschließend gemeinsam analysiert. Videoaufnahmen eignen sich für eine kritische Analyse besser als eine Kritik direkt am Anschluss an einen Vortrag, weil zum einen der/die Vortragende selber mit kritisieren und Einwände auch gleich überprüfen kann, zum anderen geben sie die Möglichkeit zu einer ausgewogeneren

\footnotetext{
${ }^{6}$ Vgl. R. Forster Ästhetische Kommunikation: „Von der Leselehre zum Textsprechen“. Ein workshop der FaDaF-Tagung Kiel, 25.05.01. Veröffentlicht in: Materialien DaF, Heft 65 „Europäisches Jahr der Sprachen: Mehrsprachigkeit nmin Europa“, Hrsg. Armin Wolff und Martin Lange, Regensburg 2002
} 
Stellungnahme, weil durch das Medium Distanz geschaffen wird und man Teile mehrfach ansehen und auch mit anderen vergleichen kann. Durch dieses in gewisser Weise objektivierbare Reflektieren wird ein gößeres Sprachbewußtsein geschaffen - sowohl für das eigene Sprechen als auch für das Sprechen der anderen.

Bei einer solchen Analyse wird schnell klar, wo Verbesserungsbedarf besteht. Die Kritikpunkte werden gesammelt und in einem Kriterienkatalog zusammengefasst. So ein Katalog, in den unbedingt die Formulierungen der KursteilnehmerInnen aufzunehmen sind, kann wie folgt aussehen:

Fachsprachenzentrum der Universität Hannover

Kurs: Mündliche Kommunikation

SoSe 2006

Bewertungskriterien für einen mündlichen Vortrag

Name:

Gedicht:

\begin{tabular}{|l|l|l|l|l|}
\hline & sehr gut & mittel & $\begin{array}{l}\text { verbesserungs } \\
\text { bedürttig }\end{array}$ & Bemerkungen \\
\hline $\begin{array}{l}\text { Aussprache } \\
\text { z. B. Artikulation } \\
\text { Lippenbewegung } \\
\text { Besonderheiten }\end{array}$ & & & & \\
\hline Lautstärke & & & & \\
\hline Rhythmus & & & & \\
\hline Tempo & & & & \\
\hline Pausen & & & & \\
\hline $\begin{array}{l}\text { Betonung } \\
\text { Akzente gesetzt } \\
\text { inhaltliche Überein- } \\
\text { stimmung }\end{array}$ & & & & \\
\hline $\begin{array}{l}\text { Melodie } \\
\text { Führung } \\
\text { inhaltliche Überein- } \\
\text { stimmung }\end{array}$ & & & & \\
\hline
\end{tabular}

\begin{tabular}{|l|l|l|l|l|}
\hline Augenkontakt & & & & \\
\hline Mimik & & & & \\
Einsatz & & & \\
inhaltliche Überein- & & & & \\
stimmung & & & & \\
\hline
\end{tabular}




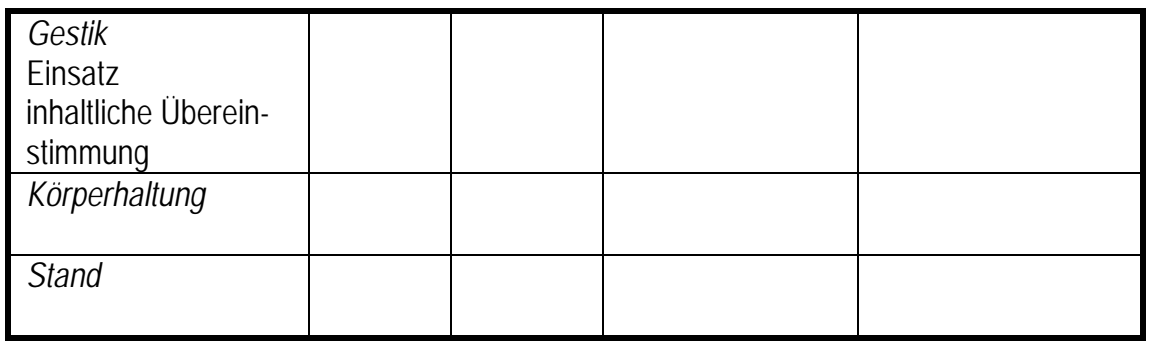

Datum: von:

\subsection{Phase: Auswählen und Einüben der Gedichte/Dialoge}

Nun bekommen die KursteilnehmerInnen einen Reader mit ca. 20 klassischen deutschen Gedichten ausgehändigt. Die Auswahl orientiert sich an den Kriterien Länge (sie sollten nicht zu lang sein), Themen (sie sollten möglichst allgemein, ohne viel Vorwissen zugänglich sein) und ,gute Sprechbarkeit“ (ich habe mich hier mit einer erfahrenen Rezitatorin beraten und mich auch an ihrer CD mit gesprochenen Gedichten orientiert 7 ).

Die KursteilnehmerInnen sollen zu Hause die Gedichte lesen, sich eines aussuchen, das sie besonders anspricht, und dieses üben.

Wichtig ist hier darauf hinzuweisen, dass kein Literaturunterricht im herkömmlichen Sinn stattfindet. Die Gedichte bekommen keine weitere Einführung, sie werden nicht interpretiert, es gibt keine weiteren Informationen zu den AutorInnen oder zu der Entstehungszeit. Im Zentrum stehen das gesprochene Gedicht, der Sprecher/die Sprecherin mit seiner/ihrer Interpretation und der Hörer/die Hörerin. Es hat sich herausgestellt, dass die Studierenden sehr wenig Nachfragen zu unbekannten und im Wörterbuch nicht auffindbaren Wörtern und so gut wie nie Fragen zu den DichterInnen oder der Historie haben. Die Gedichte sprechen offensichtlich für sich, auch die ungewohnte, ältere Sprache war nie ein Kritikpunkt oder eine Barriere für das Verstehen.

Die gewählten und geübten Gedichte werden dann zunächst vor der Gruppe vorgetragen, die ein erstes Feedback nach den Kriterien des Bewertungsbogens gibt. Wichtig ist hier, immer den Vortragenden/die Vortragende zuerst nach seinem/ihrem Eindruck zu fragen. Häufig wird dadurch schon viel Kritik vorweggenommen, die Situation eines „Angeklagten“ wird vermieden.

Danach kann ein zweiter Vortragsdurchgang folgen, der wiederum aufgezeichnet wird.

\footnotetext{
${ }^{7}$ C. Kühn-Leitz „Jahreszeit des Lebens“, Deutsche Gedichte vom Barock bis zur Gegenwart, CD, Bestellnr. 222923-215.
} 
Zur Beurteilung dieses Durchgangs kann jeder Teilnehmer/jeder Teilnehmerin einen Bewertungsbogen für jeden anderen/jede andere bekommen, diese nach dem Vortrag oder der Analyse ausfüllen und an den Sprecher/die Sprecherin zurückgeben. Am Ende hat dann jeder/jede viele Bewertungsbögen zum eigenen Vortrag. Diese sind ausschließlich zur Fehleranalyse und Unterstützung gedacht, nicht zur Bewertung nach Noten. Der Kursleiter/die Kursleiterin bekommt die Auswertungen gar nicht zu Gesicht.

Bei einem weiteren Vortragsdurchgang kann dann jeder Kursteilnehmer/jede Kursteilnehmerin vor seinem/ihren Vortrag sagen, was er/sie bei dem neuen Versuch besser machen will. Wichtig ist hierbei, dass die TeilnehmerInnen angeleitet werden, positiv zu formulieren, also z. B. „Ich möchte laut sprechen!“ und nicht „Ich darf nicht so leise sprechen!“

Auch hier kann man, wenn es die Zeit und die Kursgröße zulassen, wieder Videoaufnahmen machen und diese dann (evtl. im Vergleich zu den vorherigen) auswerten.

Entscheidend ist, dass die Studierenden lernen, sich selbst und andere konstruktiv zu kritisieren und dadurch zu unterstützen. Dem Hörer/der Hörerin kommt in einer Kommunikationssituation eine wichtige Rolle zu: Wenn der Hörer/die Hörerin den Sprecher/die Sprecherin nicht versteht, ist die Kommunikation missglückt, wenn die ZuhörerInnen das Gedicht nicht verstehen, ist der Vortrag missglückt.

Im Sommersemester 2005 hatte ich die Rezitatorin Cornelia Kühn-Leitz eingeladen, mit den Studierenden gemeinsam an ihren Gedichtinterpretationen zu arbeiten. Sie stellte ganz besonders den emotionalen Zugang zu den Gedichten in den Mittelpunkt, ohne den ihrer Meinung nach eine Interpretation nicht gelingen kann. Zitat: „Man muss sich zu einem Gedicht bekennen, sonst kann man es nicht sprechen!“ Tatsächlich war in den Aufzeichnungen eine Verbesserung des Sprechens zu erkennen, obwohl an der Aussprache nicht explizit gearbeitet wurde.

Auch Frau Kühn-Leitz wies auf die Funktion des Zuhörers/der Zuhörerin hin, für die ein Gedichtvortrag letztlich gemacht wird. (Zitat: „Du nimmst den Zuhörer an die Hand wie einen Freund und sagst zu ihm ,Hör zu, ich sag Dir jetzt etwas ganz Schönes' - und dann sprichst Du das Gedicht.")

\subsection{Phase: Vorbereitung einer öffentlichen Aufführung}

Um die Dimension des Zuhörens realitätsnah in dem Kurs zu verankern, ist es sehr sinnvoll, ihn mit einer öffentlichen Aufführung zu beenden. Folgende Varianten sind bisher erprobt: eine Radio-Livesendung „Ausländische Studierende sprechen deutsche Gedichte“, eine Aufführung für die Institutsöffentlichkeit „Kulturreise“ und ein Videofilm, der öffentlich gezeigt wurde „Kulturelle Leckerbissen“.

Da die Vorbereitung einer solchen Unternehmung sehr zeitintensiv sein kann, empfiehlt es sich, rechtzeitig etwa nach der Hälfte des Kurses zu beginnen. 
Nach der endgültigen Auswahl und Festlegung der deutschen Gedichte kann und sollte an dieser Stelle auch das kreative Potential und die kulturelle Vielfalt der Kurse ausgeschöpft werden.

Die Heimatkulturen können in verschiedener Form eingebracht werden, z. B. durch das Vortragen von Gedichten aus den Muttersprachen, dazu können entweder deutsche Übersetzungen oder kurze Inhaltsangaben erstellt werden, oder man kann eine Videoaufnahme mit deutschen Untertiteln versehen. In der öffentlichen Aufführung gab es polnische und irische Tänze, in der Radiosendung klassische und moderne Musik aus den Heimatländern, bei der Vorführung des Videofilms mit Vorträgen von deutschen Gedichten und Gedichten aus den Heimatländern gab es auch internationale Speisen ... Die inhaltliche Gestaltung liegt bei den KursteilnehmerInnen.

Zwischenziel der Vorbereitung sollte die Erstellung eines Planes sein, nach der eine Radiosendung oder eine Aufführung ablaufen kann. Zwei Beispiele für solche Pläne, die auch noch einmal die Vielfältigkeit des Programms deutlich machen, finden Sie in Auszügen in der Anlage ${ }^{8}$.

Auch die Auswahl der Veranstaltungstitel und die Werbung9 waren Aufgabe der Studierenden. Sie wurden mit großem Engagement angenommen und mit viel kreativem Potential gelöst.

Ein solcher Abschluss des Kurses ist eine kaum zu überschätzende Motivation für die TeilnehmerInnen: Sie möchten sich selbst und ihr Heimatland/ihre Muttersprache vor einem „unbekannten“ Publikum so positiv wie möglich präsentieren. Eine DVD oder CD mit einer Aufzeichnung nehmen sie meist mit großem Stolz in ihre Heimatländer zurück.

\section{$4 \quad$ Fazit und Ausblick}

Die Kurse haben die gesteckten Ziele erreicht: Im Bereich des „Besser Sprechens ..." waren deutliche Verbesserungen in der Artikulation, der Intonation, der Melodieführung und des Rhythmus' zu hören, nachzuprüfen in den Aufzeichnungen, die zu Beginn und am Ende der Kurse gemacht wurden ${ }^{10}$.

Ebenso wichtig für den Bereich der mündlichen Kommunikation ist aber auch, dass die KursteilnehmerInnen Mimik und Gestik kontrollierter und zielgerichteter einsetzen konnten, ihre Haltung und ihr Bezug zu den ZuhörerInnen sicherer geworden war. Auch diese Aspekte sind für ein Gelingen von mündlicher Kommunikation wesentlich.

Das Sprechen von Gedichten hat m. E. diesen Erfolg ganz wesentlich mit bestimmt. Das häufige Wiederholen von fertigen, abgeschlossenen Texteinheiten in

\footnotetext{
8 Anlage 1: Sendeprotokoll von Radio Flora, SoSe 2005Anlage 2: Ablaufplan der „Kulturreise“, WiSe $2005 / 6$

${ }^{9}$ Anlage 3: Werbung für die „Kulturreise“.

${ }^{10}$ Bisher wurden die Ergebnisse nicht systematisch evaluiert.
} 
Gedichtform lässt eine Konzentration auf die o. g. Bereiche der gesprochenen Sprache zu, die mit selbstproduzierten Texten nur schwer zu erreichen wäre. Ein gelungener Gedichtvortrag basiert auf einer intellektuellen Verstehensleistung und auf einem affektiven Zugang des Sprechers/der Sprecherin. Beides manifestiert sich in einem dem Inhalt des Gedichtes angemessenen und der persönlichen Interpretation des Sprechers/der Sprecherin entsprechenden Vortrag. Wenn man den Vortrag eines Gedichtes als kommunikativen Akt begreift, dann ist dieser erst dann erfolgreich, wenn alle Aspekte gelingen. In den Audio- und Videobeispielen während des mündlichen Vortrags in Hannover wurde deutlich, dass dieses in den Kursen der Fall war.

Der für die Studierenden ungewohnte Zugang zur Literatur führte wiederholt zu mündlich geäußerten Kommentaren wie „Früher habe ich überhaupt keine Gedichte gelesen, auch nicht in meiner Heimatsprache - aber jetzt interessiert mich das!“ Die Aufforderung, für die Aufführung Gedichte in den Muttersprachen zu suchen, war für einige eine schwierige Herausforderung. Wenn man Gedichte darüber hinaus als wichtige Manifestationen von Kultur begreift, dann finden in solchen Kursen tatsächlich intensive Kulturbegegnungen statt, die spürbar sind, auch wenn sie nicht explizit thematisiert werden... und die wiederum nach drauBen getragen werden, wenn die KursteilnehmerInnen ihre Begegnung mit der deutschen Sprache (und anderen) in Form einer CD mit nach Hause nehmen.

Ein weiteres wichtiges Merkmal dieser Kurse ist die intensive Gruppenerfahrung, die entsteht, wenn gemeinsam Sprechen geübt wird. Wie oben schon gesagt, setzt dies die Bereitschaft voraus, Fehler zu offenbaren, sich zu öffnen und verletzbar zu machen - andererseits wird auch Toleranz, Respekt und die Fähigkeit zu konstruktiver Kritik verlangt. Daraus entsteht nach wenigen Kurssitzungen eine ausgesprochen freundliche und offene Kursatmosphäre und führt zu Kommentaren wie: „In diesem Kurs haben wir kritisieren gelernt.“ (Im Sinne von „Hier fühlte ich mich sicher und konnte Kritik annehmen aber auch angemessen äußern.")

Das Ziel dieses Berichtes aus der Praxis ist es, zu interessieren und zum Nachahmen aufzufordern. Ich möchte ein Fenster aufstoßen für eine andere Möglichkeit, Literatur im Fremdsprachenunterricht einzusetzen, die Aspekte Emotion und Affektion in den Sprachlernprozess mit einzubeziehen, einen Raum für Kulturbegegnungen zu schaffen und dabei die Fertigkeit des Sprechens zu verbessern ... dieses Beispiel soll mehr sein als ein „Guck-mal-das-da-an“, wie Willis Edmonson es in seinem provozierenden Artikel „Literatur im Fremdsprachenunterricht Wozu?" "11 nennt. Es könnte in seiner neuen Kombination ein neuer Ansatz im Bereich der mündlichen Kommunikation sein oder werden, der vielleicht nicht für jeden Lernenden/jede Lernende - und auch nicht für jeden Lehrer/jeder Leh-

\footnotetext{
${ }^{11}$ Edmondson, Willis: Literatur im Fremdsprachenunterricht - Wozu? In: Johannes-Peter Timm/Helmut Johannes Vollmer (Hg); Kontroversen in der Fremdsprachenforschung, Bochum, 1993, S. 275 bis 284.
} 
rerin geeignet ist, aber das bestehende Spektrum der Möglichkeiten durchaus erweitern kann.

\section{Literatur}

Edmondson, Willis: „Literatur im Fremdsprachenunterricht - Wozu?“ In:

Johannes-Peter Timm/Helmut Johannes Vollmer (Hg); Kontroversen in der Fremdsprachenforschung, Bochum, 1993, S. 275 bis 284

Forster, Roland: „Ästhetische Kommunikation: „Von der Leselehre zum Textsprechen“"Ein workshop der FaDaF-Tagung Kiel“", 25.05.01.

Veröffentlicht in: Materialien DaF, Heft 65 „Europäisches Jahr der Sprachen: Mehrsprachigkeit in Europa“, Hrsg. Armin Wolff und Martin Lange, Regensburg 2002

Kühn-Leitz, Cornelia: http:/ /www.cornelia-kuehn-leitz.de. ,JJahreszeit des Lebens“, Deutsche Gedichte vom Barock bis zur Gegenwart, CD, Bestellnr. 222923-215

Marita Pabst-Weinschenk: „Ästhetische Kommunikation“, 180. 3.1. Grundlagen des interpretierenden Textsprechens (Ortwin Lämke); 3.3. Sprecherische Erarbeitung künstlerischer Texte (Baldur Neuber); Beispiel für eine sprechkünstlerische Erarbeitung (Martina Haase) ... www.bsz-bw.de/rekla/ show.php?mode=source\&eid=UNI_0_11215917inh(Stand: 25.05.2006)

Marita Pabst-Weinschenk (Hg): ,Grundlagen der Sprechwissenschaft und Sprecherziehung.“ München: Reinhardt 2004.

Reinhardt, Michaela: „Theaterformen im DaF-Unterricht - Spaß am Sprechen Ein workshop der FaDaF-Tagung Jena.“ 05.05.05 


\section{Anlage 1: Sendeprotokoll von Radio Flora}

Seite 2 Sendung: Sendetag : Sonntag,03. Juli 2005, 14.00 Uhr

\begin{tabular}{|c|c|c|c|c|c|c|}
\hline$\overline{N r}$ & was läuft? Thema/Titel & $\begin{array}{l}\text { AutorIn } \\
/ \\
\text { Interpre- } \\
\text { tIn }\end{array}$ & $\begin{array}{l}\text { Tonträ- } \\
\text { ger/ } \\
\text { Träck }\end{array}$ & $\begin{array}{l}\text { Uhr } \\
\text { zeit }\end{array}$ & Länge & $\begin{array}{l}\text { Bemer } \\
\text { kungen }\end{array}$ \\
\hline & $\begin{array}{l}\text { Tschechische Musik } \\
\text { Nr. } 2 \\
\text { Überleitung von der } \\
\text { Musik }\end{array}$ & & $\mathrm{CD}$ & & $2 \mathrm{~min}$ & \\
\hline & $\begin{array}{l}\text { Einleitung/Erklärung } \\
\text { Tschechisches Gedicht }\end{array}$ & Sarka & & & $2 \mathrm{~min}$ & \\
\hline & $\begin{array}{l}\text { Mondnacht } \\
\text { von J. von Eichendorff }\end{array}$ & Radka & & & $1 \mathrm{~min}$ & \\
\hline & Moderation & & & & & \\
\hline & $\begin{array}{l}\text { Geile Zeit Juli } \\
\text { Ausgesucht v. Rosalia }\end{array}$ & & $\mathrm{CD}$ & & $2 \mathrm{~min}$ & \\
\hline & $\begin{array}{l}\text { Überleitung / Einleitung } \\
\text { Lied von Lynceus } \\
\text { von J. W. von Goethe }\end{array}$ & Rosalia & & & $1 \mathrm{~min}$ & \\
\hline & $\begin{array}{l}\text { Einleitung/Erklärung } \\
\text { Italienisches Gedicht }\end{array}$ & Carmen & & & $2 \mathrm{~min}$ & \\
\hline & $\begin{array}{l}\text { Einleitung: Rosalia } \\
\text { Italienische Musik }\end{array}$ & & $\mathrm{CD}$ & & $2 \min$ & \\
\hline & $\begin{array}{l}\text { Persische Musik } \\
\text { Persisches Gedicht }\end{array}$ & $\begin{array}{l}\text { Be- } \\
\text { hrouz }\end{array}$ & $\mathrm{CD}$ & & $4 \mathrm{~min}$ & $\begin{array}{l}\text { Die Mu- } \\
\text { sik läuft } \\
\text { während } \\
\text { des Ge- } \\
\text { dichtvor- } \\
\text { trags leise } \\
\text { im Hin- } \\
\text { tergrund } \\
\text { und wird } \\
\text { danach } \\
\text { lauter }\end{array}$ \\
\hline
\end{tabular}




\section{Anlage 2: Auszug aus dem Ablaufplan zur „Kulturreise“}

Auffuibrung: 24.01.2006, 20.00 Ubr

\begin{tabular}{|c|c|c|c|c|}
\hline $\begin{array}{l}2 \\
\min \end{array}$ & $\begin{array}{l}\text { Michal, Veroni- } \\
\text { ka }\end{array}$ & „Weibsbilder“ Dialog & & \\
\hline $\begin{array}{l}20 \\
\sec \end{array}$ & Sinéad & Ansage & & \\
\hline $\begin{array}{l}4 \\
\min \end{array}$ & Jin Meng & $\begin{array}{l}\text { chinesisches Lied + Ge- } \\
\text { dicht - erklären und spre- } \\
\text { chen }\end{array}$ & $\begin{array}{l}\text { CD } \\
\text { Die Musik } \\
\text { wird laut } \\
\text { eingespielt } \\
\text { und läuft } \\
\text { während des } \\
\text { Vortrags lese } \\
\text { mit }\end{array}$ & \\
\hline & Jin Meng & $\begin{array}{l}\text { „Mondnacht" von } \\
\text { Joseph Freiherr von Ei- } \\
\text { chendorff }\end{array}$ & & \\
\hline $\begin{array}{l}20 \\
\sec \end{array}$ & Sinéad & Ansage & & \\
\hline $\begin{array}{l}2.30 \\
\min \end{array}$ & $\begin{array}{l}\text { Tjasa, Sylvia, } \\
\text { Beata } \\
(+, \text {,Kamera- } \\
\text { mann“) }\end{array}$ & $\begin{array}{l}\text { „Ansprache des Ministers“ } \\
\text { Dialog }\end{array}$ & & $\begin{array}{l}\text { Jackett, } \\
\text { Kravatte, } \\
\text { Kamera, } \\
\text { Tisch? }\end{array}$ \\
\hline $\begin{array}{l}20 \\
\sec \end{array}$ & Sinéad & Ansage & & \\
\hline 2 & Sita & $\begin{array}{l}\text { „Lorelei“ von } \\
\text { Heinrich Heine }\end{array}$ & & \\
\hline $\begin{array}{l}20 \\
\sec \end{array}$ & Sinéad & Ansage & & \\
\hline $\begin{array}{l}2.30 \\
\min \end{array}$ & Tjasa & $\begin{array}{l}\text { slowenisches Gedicht mit } \\
\text { Übersetzung }\end{array}$ & & \\
\hline $\begin{array}{l}20 \\
\sec \end{array}$ & Tjasa & Ansage von Sinéad & & \\
\hline $\begin{array}{l}2-3 \\
\min \end{array}$ & Sinéad & $\begin{array}{l}\text { Einleitung/Erklärung } \\
\text { Tanz aus Irland }\end{array}$ & & Platz \\
\hline
\end{tabular}




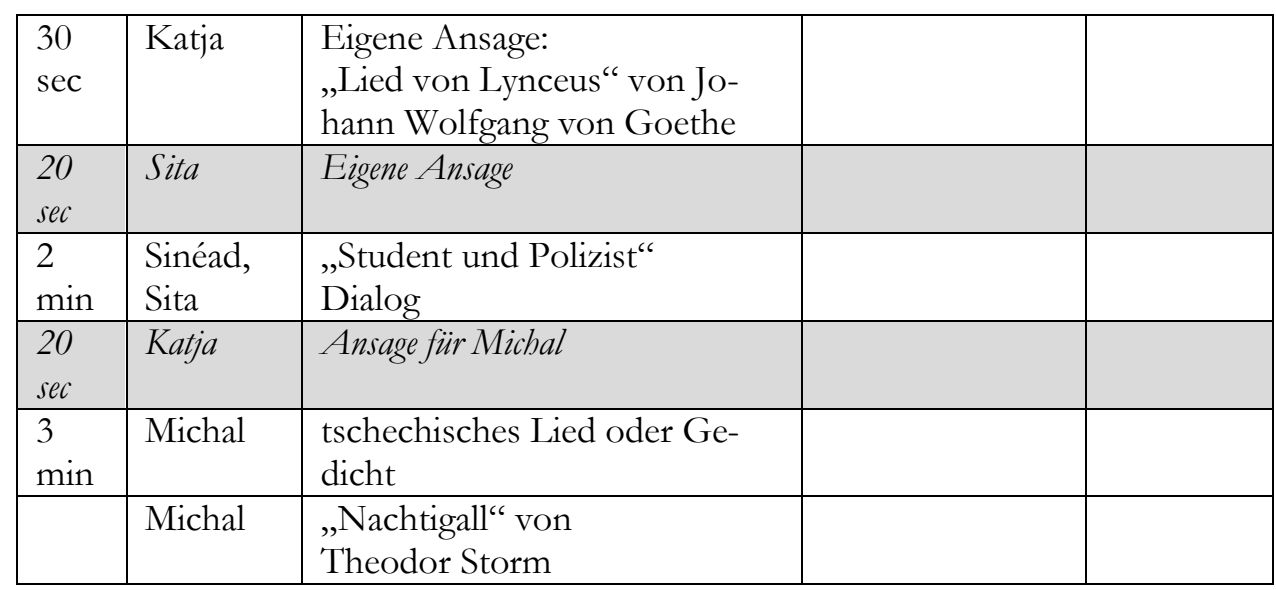


Anlage 3

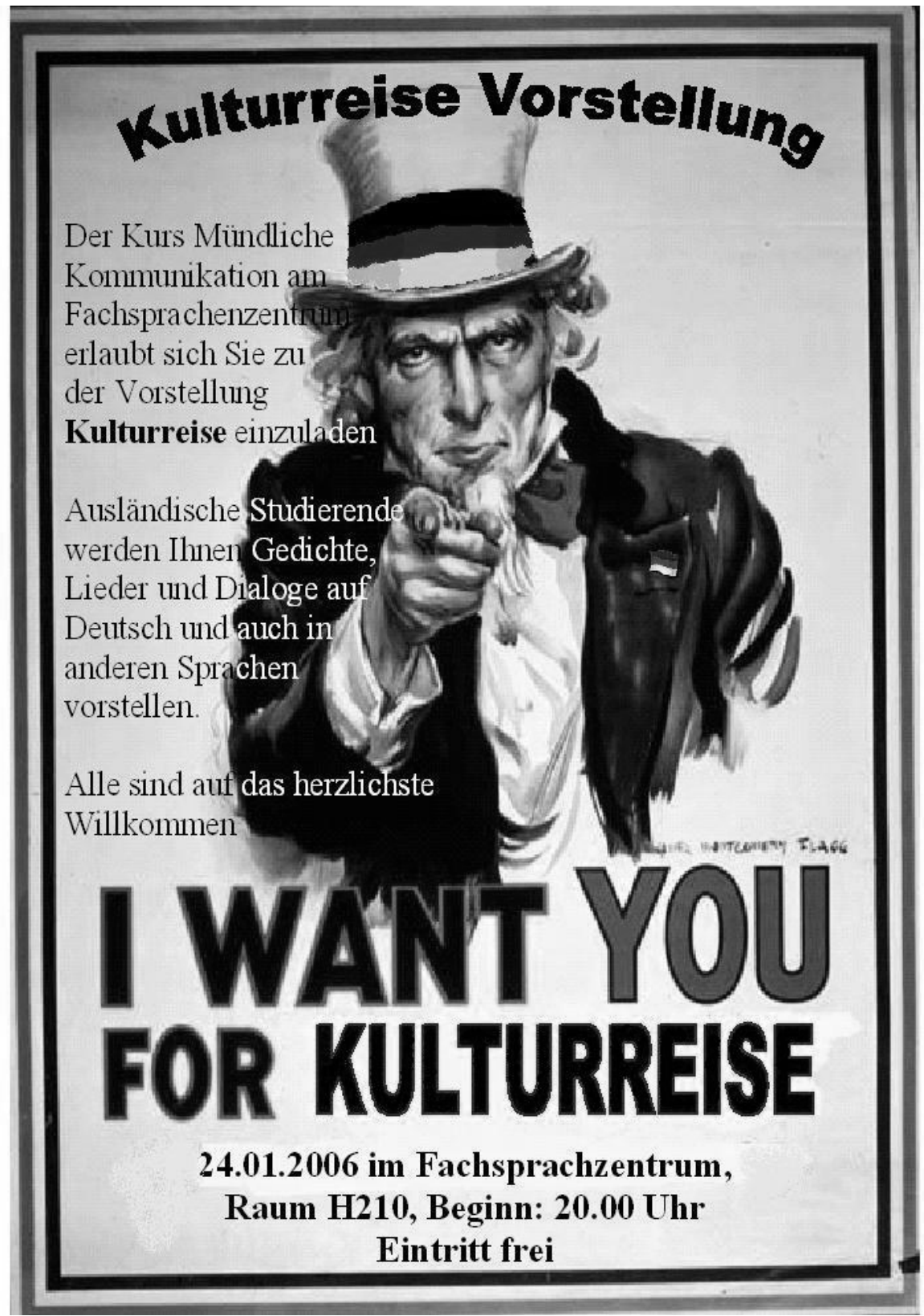




\section{Aktive Mitarbeit deutschsprachiger Hospi- tant/innen im DaF-Unterricht, eine Methode der sprachlichen Förderung und Integration internationa- ler Studierender - ein Praxisbericht aus Tübingen}

Wolfgang Rug, Tübingen/Dornburg b. Jena

\section{Vorbemerkung}

Ich berichte über die mehr als 20jährige erfolgreiche Praxis an der „Abteilung Internationale Sprachprogramme“ (vormals „Lektorat für Deutsche Sprache“) an der Universität Tübingen, deutschsprachige Studierende verschiedener Studienfächer (in der Mehrzahl Studierende der neuphilologischen Fächer) einzuladen, sich für die Dauer eines Semesters und auf freiwilliger Grundlage als Hospitant/innen an den Sprach- und Landeskundekursen für internationale Studierende zu beteiligen.

An dieser Praxis beteiligen sich die in Tübingen lehrenden Dozent/innen in unterschiedlicher Weise, die Skala reicht von „gar nicht" über „gelegentlich" und „partiell“ und bis hin zu „sehr engagiert und umfangreich“. Kurse, deren didaktische Konzeption die Mitwirkung deutscher Hospitant/innen fest eingeplant, gelegentlich geradezu Voraussetzung ist, sind z. B. Grammatik- und Wortschatzkurse für Fortgeschrittene, Aufbaukurse der Grund- und Mittelstufe, Projektkurse zum Inter- 
nationalen Wortschatz, Kurse zur gesprochenen Umgangssprache, spezielle Fehlerkurse, Rhetorikkurse, Schreibkurse und projektorientierte Landeskundekurse.

Dieser Bericht ist nicht einfach ein „Ist“-Bericht, und das in zweierlei Hinsicht: Zum einen ist er ein „War“-Bericht insofern, als ich selbst meine Berufstätigkeit als Sprachdozent an der Tübinger DaF-Abteilung Ende 2005 beendet habe und die beschriebene Praxis selbst nicht mehr weiterführen, beeinflussen oder weiterentwickeln kann. Es liegt also in der Verantwortung und Entscheidung der in der Abteilung weiterarbeitenden Kolleginnen und Kollegen, in welcher Form und in welchem Umfang dieses Praxis-Element in Zukunft fortgeführt wird.

Zum anderen enthält der Bericht auch „idealtypische“ Aspekte insofern, als das Hospitanten-Modell nicht in jedem einzelnen unserer Kurse in gleichmäßig aktiver Weise gehandhabt und didaktisch kreativ gemacht und ausgenutzt worden ist. Wir haben also durchaus nicht zu jeder Zeit und nicht in allen Kursen, an den sich Hospitant/innen beteiligt haben, alles als Praxis verwirklicht, was in dem Hospitanten-Modell selbst als didaktische Möglichkeit angelegt und hier skizziert ist. Es handelt sich also auch um einen „Hätte-sein-können/Könnte-sein“Bericht. Mir kommt es darauf an, das Modell und seine Möglichkeiten aufzuzeigen, in der Überzeugung, dass didaktisch kreative und engagierte DaF-Kolleginnen und Kollegen daraus für ihre Praxis Nutzen ziehen können.

\section{Der Hintergrund}

2.1 Wie DaF an der Universität Tübingen (eher unbefriedigend) vertreten ist

Die DaF-Abteilung an der Universität Tübingen war seit den 70er Jahren dadurch gekennzeichnet,

- dass es trotz mehrerer Versuche nicht gelungen ist, Deutsch als Fremdsprache als wissenschaftliches Fach bei der Fakultät Neuphilologie oder im Rahmen eines Zentralen Sprachlehrinstituts zu etablieren ${ }^{1}$,

- dass sie anfangs als einfacher Funktionsbereich des Akademischen Auslandsamts, ab Ende der 80er Jahre dann als eigenständige Abteilung im reorganisierten „Dezernat für internationale Beziehungen“ eingerichtet war,

- dass sie in dieser Zeit mit lediglich zwei etatisierten BAT IIa/Ib-Stellen auszukommen hatte (Lektoren- bzw. Wiss. Angest.-Stellen) und den ständig wachsenden Unterrichtsbedarf durch eine wachsende Zahl von Lehrbeauftragten bewältigen musste ${ }^{2}$.

\footnotetext{
${ }^{1}$ Ein früher Bericht dazu findet sich in der Tübinger Universitätszeitung 4/1974.

2 Immerhin konnten inzwischen für eine Reihe von Lehrauftragsverhältnissen unabhängig vom Landesetat auf der wirtschftlichen Grundlage von Kurs- und Programmgebühren einige ganze und
} 


\subsection{Aber es gibt auch Vorteile}

Diese besondere, aus dem Blickwinkel des Faches Deutsch als Fremdsprache eher negativ zu beurteilende Tübinger Situation hatte jedoch die positiven Auswirkungen,

- dass alle fachdidaktischen Überlegungen und Bemühungen in der Abteilung von vornherein an der eigenen Praxis orientiert und auf die Verbesserung der eigenen Praxis hinzielend angelegt waren ${ }^{3}$

- und dass die DaF-Abteilung zunehmend zu einem aktiven Mitgestalter der Internationalen Beziehungen der Universität Tübingen werden konnte, zunächst im Rahmen des Uni-Dezernats ${ }^{4}$, dann aber auch in Kooperation mit dem anfangs der 90er Jahre von Tübingen aus ins Leben gerufenen „Internationalen Zentrum“ 5 .

So konnte eine dezidiert praxisorientiert arbeitende DaF-Abteilung entstehen, die sich durch eine große Zahl von sprachlichen oder landeskundlichen Sonderprogrammen für internationale Partnerhochschulen und sonstige Programmpartner auch wirtschaftlich eine gewisse Selbstständigkeit erarbeiten konnte.

\subsection{DaF mit großen Gruppen? Aber bitte nicht so!}

Auch wenn das fremdsprachendidaktische Argument, dass Sprachunterricht in kleinen Klassen für den sprachlichen Erfolg sinnvoll und daher erstrebenswert ist, nicht bestritten wird, haben wir in Tübingen darüber nachgedacht, wie eine sinnvolle und effektive DaF-Unterrichtspraxis auch mit großen Gruppen, mit 25, 30 oder 40 Teilnehmern, zu bewerkstelligen wäre. Das didaktisch sicher oft gut begründete Rufen nach mehr Lehrerstellen, das hartnäckige Streiten mit den übergeordneten universitären und staatlichen Stellen für eine besserere Personalausstat-

partielle BAT-Stellen geschaffen werden; mit also nach wie vor nur zwei vollen Etatstellen kann inzwischen ein Programm von jährlich weit mehr als 6000 Unterrichtsstunden in Semesterkursen und Sonderprogrammen angeboten werden.

3 Auf der Basis der Tübinger DaF-Praxis sind auch die eigenen Beiträge zum Fach DaF entstanden, etwa „Arbeit mit Texten“ (1976), ,,50 praktische Tips zum Deutschlernen“ (1991), ,,Grammatik mit Sinn und Verstand“ (1993 und überarbeitet 2001), „Meine 199 liebsten Fehler“ (1996): Alle bei Klett.

${ }^{4}$ Die Universität Tübingen hat sich bei Umfang und Intensität internationaler Partnerschaften und beim internationalen Studentenaustausch in Deutschland eine Spitzenstellung erworben, mit der Folge, dass der Bedarf an Sprach- und Landeskundekursen für Austauschstudenten sowie an speziellen Sonderprogrammen stark angestiegen ist; dieses Sprachangebot ist zugleich ein aktives Element, mit dem Studentenaustausch und Partnerschaften vorteilhaft verhandelt und ausgestaltet werden können.

${ }^{5}$ Das durch den damaligen Tübinger Universitätspräsidenten Adolf Theis zu Anfang der 90er Jahre initiierte "Internationale Zentrum" war eine Kooperation von Hochschulen aus Ost- und Mittelosteuropa, Westeuropa und den USA, dessen Programm von internationalen Sommerschulen in einem breiten Fächerspektrum mehr als ein Jahrzehnt lang von Tübingen aus organisiert wurde, mit dem Ziel, im Zuge des gesellschaftlichen Wandels in Osteuropas den wissenschaftlichen Austausch zwischen West und Ost in Gang zu bringen und zu fördern; in diesem Wissenschaftsdialog spielte neben Englisch auch Deutsch eine wichtige Rolle, sei es als zu fördernde Kommunikati Kommunikationssprache oder auch direkt als Thema der Fortbildung von Deutsch- und Deutschlandkundelehrern an osteuropäischen Hochschulen. 
tung ist ja, so zeigt es die Erfahrung, häufig und zunehmend erfolglos und deshalb demotivierend. Ebenso abgeschreckend erschienen uns Beispiele von Mammut-Kursen des Typs „Spanisch (oder Italienisch) für Nicht-Romanisten“ mit 60 und mehr Teilnehmer/innen, wo der bedauernswerte Lektoren-Kollege im groBen, hörsaalartigen Seminarraum einsam und verloren die Aufgabe zu bewältigen versuchte, eine lebendige Fremdsprache zu vermitteln.

\section{Umfang der DaF-Hospitanten-Praxis}

Wir haben daher in der Tübinger DaF-Abteilung eine Praxis entwickelt, pro Semester zwischen 40 und 60 Studierende mit Deutsch als Muttersprache einzuladen, an den Sprach- und Landeskundekursen für internationale Studierende als Hospitanten und auf freiwilliger Grundlage teilzunehmen ${ }^{6}$. Besondere Fachrichtungen, linguistische, DaF- oder Fremdsprachenkenntnisse werden dabei begrüßt, aber bis auf Ausnahmen ${ }^{7}$ ausdrücklich nicht vorausgesetzt; hingegen sind interkulturelle Offenheit und Neugier besonders erwünscht.

\section{Elemente der Tübinger Hospitations-Praxis: Ein Katalog}

a) Wichtig ist, dass die Institution ein starkes Interesse an der Hospitationspraxis hat und damit eine sorgfältige Betreunng garantiert; das geschieht am besten dadurch, dass ein/e Mitarbeiter/in für den gesamten Rahmen des Hospitationsprogramms verantwortlich zeichnet.

b) Die Hospitationen erfolgen grundsätzlich auf freiwilliger und kostenloser Basis; das setzt voraus, dass sich die Institution und jeder einzelne beteiligte Sprachdozent darüber im Klaren sein müssen, nicht nur Mitarbeit anwerben und entgegennehmen zu können, sondern Kenntnisse, Erfahrungen und Chancen vermitteln zu müssen.

c) Man braucht ein Instrumentarium für intensive Anwerbung, vor jedem Semester aufs Neue (und bei zunehmendem Zeit- und Leistungsdruck der Studierenden um so mehr); die Werbung richtet sich vor allem an Studierende der Germanistik und der anderen neusprachlichen philologischen Fächer, geht aber auch fächerunabhängig darüber hinaus. Das geht durch

\footnotetext{
${ }^{6}$ Schätzungsweise haben bis 2006 weit mehr als 2000 deutschsprachige Studierende als Hospitant/innen mitgearbeitet.

7 So werden z. B. bei spezifischen „Fehlerkursen“ je nach Sprache deutsche Studierende mit guten Englisch-, Spanisch- oder Italienisch-Kenntnissen besonders angesprochen, bei Landeskundekursen mit zeitgeschichtlicher oder politischer Thematik Studierende der Fächer Geschichte oder Politikwissenschaft.
} 
- Zettelwerbung im Vorsemester (s. Anlage 1)

- Plakate auf den Institutsstellwänden und auf einer abteilungseigenen Litfaßsäule

- Massenrundmail an die Studierenden mit Uni-Account zwei Wochen vor Semesterbeginn (s. Anlage 2)

- eine Serie von vier bis, fünf oder sechs Anwerbeterminen in den ersten beiden Semesterwochen

- Handzettelwerbung in der Neuphilologischen Fakultät zum Semesterstart

- gelegentlich persönlichen Werbeauftritt bei großen Anfangsvorlesungen der neuphilologischen Fächer, bes. der Germanistik, Anglistik, Romanistik.

d) Erwartet (aber nicht als Forderung) wird die regelmäßige Hospitanz über ein Semester für zwei, maximal drei Semesterwochenstunden; die Wabrung der Freiwilligkeit ist dabei wichtig, d. h., dass man „abspringende“ Hospitanten nicht mit Vorwürfen konfrontiert oder partout zu halten versucht; wobei die Praxis-Erfahrung zeigt, dass es nur eine geringe Zahl von „Abspringern“ gibt.

e) Es muss darauf geachtet werden, dass Hospitant/innen nicht in Bereichen und für Aufgaben eingesetzt werden, die als Arbeit Studentischer Hilfskräfte gewertet werden müssen (Korrekturen, Bewältigung von Infrastrukturen bestimmter Kurse, Kursvorbereitung etc.); wobei es immer wieder dazu kommt, dass motivierte Hospitant/innen darum bitten, intensiver in die Kursgestaltung einbezogen zu werden; als erfahrener DaF-Dozent wird man in solchen Fällen das richtige $\mathrm{Maß}$ und die richtige Form finden.

f) Es ist sehr sinnvoll, den Hospitanten während des Kurses didaktisch-methodische Anleitung und Hilfen anzubieten; die Lehrkraft fungiert als jederzeit zugänglicher didaktischer und methodischer Ansprechpartner. Das ist umso leichter möglich, als die Lehrkraft durch die Anwesenheit und Mitarbeit von Hospitanten aus ihrer zentralen Beanspruchung deutlich entlastet wird. Zusätzlich wird mindestens eine gemeinsame Hospitantenbesprechung veranstaltet, spätestens in der Semestermitte, um Erfahrungen auszutauschen, Schwierigkeiten zu besprechen, Vorschläge der Hospitanten zu diskutieren, persönliche Motivationen kennenzulemen etc.

g) Die Abteilung stellt den Hospitanten zum Semesterende eine qualifizierte, nicht qualifizierende Bescheinigung aus (s. die Beispiele in den Anlagen 3.1-3.3); die Bescheinigung beschreibt also nicht die individuelle Leistung der Hospitantin oder des Hospitanten (,qualifizierend“), sondern alles das, was man DaFdidaktisch in diesem Kurs als Mitarbeit hat leisten und an Erfahrung hat sammeln können (,qualifiziert"). 
h) Die im Kurs verwendeten Eigenprint-Lehrmaterialien werden kostenlos zur Verfügung gestellt; für Lehrwerke aus den DaF-Buchverlagen besteht die Möglichkeit, ein Exemplar aus der "Hospitanten-Bibliothek" zu entleihen.

i) Für die Hospitant/innen kostenlos oder preislich stark reduziert ist auch die Beteiligung an Exkursionen in Landeskundekursen, bei Blockseminaren oder Sonderveranstaltungen.

\section{$5 \quad$ Hospitation als erster DaF-Schritt, dem weitere folgen können}

Bei dem Kreis von 40-60 Hospitant/innen pro Semester handelt es sich bei einer DaF-Abteilung wie in Tübingen, wo ein lehrerausbildendes Fach Deutsch als Fremdsprache nicht etabliert ist, um den einzigen organisierten Kontakt zu deutschsprachigen Studierenden ${ }^{8}$. Aus diesem Kreis erfolgt deshalb in der Regel auch die Personenauswahl für die nicht unerhebliche ${ }^{9}$ Zahl vertraglich gestalteter und vergüteter Tutoren- oder Hiwi-Stellen der Abteilung, sowohl für die Sprachund Landeskundekurse des Semesterprogramms als auch für die verschiedenen zusätzlichen Sonderprogramme: für eine Anzahl der zunächst als Hospitanten angeworbenen Studierenden ein zweiter Schritt ins Fach Deutsch als Fremdsprache.

In besonderen, qualitativ gut begründeten Fällen können Hospitant/innen - in der Regel erst nach einem zweiten oder dritten Semester der Mitarbeit - individuelle Befürwortungen und Begutachtungen für Bewerbungen erwarten, z. B. für Assistant-Teaching-Stellen, für Stellenangebote im Rahmen von Programmen der Robert Bosch Stiftung, für Bewerbungen auf DAAD-Lektorat im Ausland etc. ${ }^{10}$.

So können sich also - auch ohne einen etablierten DaF-Studiengang an der Universität Tübingen - bei besonders motivierten und qualifizierten Hospitant/innen erste Elemente von DaF-Karrieren ergeben (und haben sich in den zurückliegenden zweieinhalb Jahrzehnten schon häufig ergeben).

\section{$6 \quad$ Beobachtungen aus der Tübinger Praxis: Ein Katalog}

a) Die Tübinger Praxis führt weg von frontalen und stark lehrerbezogenen Unterrichtsformen und hin zu Arbeitsformen in kleinen Gruppen von 4 bis 5

\footnotetext{
${ }^{8}$ Nur ausnahmsweise richten sich Kurse wie z. B. „Amerikanisch-Deutsche Kulturkontak-

te/Germany and Amerika - Cultures in Contact“ oder die „Bausteinseminaren Deutsch als Fremdsprache“ (s. Anlage 4) auch an deutschsprachige Studierende.

${ }^{9}$ Für alle Sprachkurse an der Tübinger DaF-Abteilung stehen Studentische Hilfskräfte mit begrenztem Stundendeputat zur Verfügung, was durch die Einwerbung von Gebühren für Kurse und Programme, die nicht studienbegleitend sind, erleichtert wird.

${ }^{10}$ Seit Anfang der 80er Jahre waren es allein bei mir weit über 100 solcher qualifizierender Begutachtungen.
} 
internationalen Teilnehmern mit einem Hospitanten/einer Hospitantin um einen Tisch herum; nicht zu enge Übungsräume mit gut beweglichem Mobiliar und guter Akustik sind also Voraussetzung.

b) Die Lehrkraft kann den eigenen Sprechanteil erheblich reduzieren, kann eine ambulierende und moderierende Position einnehmen, kann aber trotzdem den Kursverlauf maßgeblich mitgestalten (Lernziele vorgeben, Zeitphasen strukturieren, Schwierigkeiten hervorheben, Lernerfahrungen und Ergebnisse zusammenfassen, den Stil und die Atmosphäre des Unterrichts prägen und beeinflussen, sich einzelnen Tischgruppen und Lernern zuwenden etc.)

c) Die Lernsprache Deutsch wird automatisch und ohne besondere didaktische Appelle oder Zwang zur Kommunikationssprache während des Unterrichts; gleichzeitig wird die Sprechgelegenheit für die internationalen Studenten stark erweitert, Sprechbarrieren werden, weil gleichaltrige deutsche Studierende die Gruppenarbeit moderieren, spürbar gesenkt ${ }^{11}$.

d) Es entsteht eine besondere "Arbeitsatmosphäre" (gedämpftes bis halblautes Gemurmel gleichzeitig an vier bis acht Arbeitstischen, Kontakte zwischen den Tischgruppen) statt der traditionell dominanten Lehrerzentriertheit oder lähmender Kursatmosphäre.

e) Je nach Kursinhalt können sich - freiwillig zwischen den Teilnehmern vereinbart - feste Arbeitsgruppen etablieren, sei es als Element der Binnendifferenzierung (stärkere/schnellere und schwächere/langsamere Tischgruppen), sei es zur gleichzeitigen Erledigung verschiedener Arbeitsaufgaben oder auch nur auf der Grundlage persönlicher Sympathie oder Bekanntheit. Andererseits kann, wenn es sinnvoll erscheint, durch Beratung und Vereinbarung erreicht werden, sprachlich homogene bzw. nationale Grüppchen auseinander zu ziehen (oder genau umgekehrt), um die Kommunikationsbereitschaft und den Lernerfolg zu fördern.

f) Es können Aspekte der Feblerdidaktik einbezogen werden (Korrigieren, Hilfen bei Vorbereitungen, Nacharbeit von Tests etc.); Grammatik- und Wortschatzkurse, sprachkontastive Projektkurse, Schreib- und Tagebuchkurse sind dafür besonders geeignet.

${ }^{11}$ Dabei müssen oft didaktisch noch wenig geübte deutsche Hospitant/innen von der Lehrkraft dazu angeleitet werden, die eigene Sprechfrequenz zu drosseln und sich in zurückhaltender Moderationstechnik zu üben. 
g) Bei Kursen mit landeskundlicher Thematik ist die Mitarbeit einer Gruppe deutscher Studierender über den sprachlichen Aspekt hinaus von besonderer Wirkung, als zeitgeschichtliche oder politische Themen aus einem breiteren „deutschen“ Spektrum von Argumenten und Erfahrungen diskutiert werden können, statt nur aus der Perspektive der deutschen Lehrkraft.

h) Natürlich profitiert die soziale und interkulturelle Rabmengestaltung von Sprachkursen in besonderer Weise von der Beteiligung der Hospitanten, z. B. bei Kompaktkursen, Kursfreizeiten, Kursfesten, Projektarbeit, Kursdokumentation in Seminarausstellungen, Text- oder Projektreadern etc.

i) Damit weitet sich der Blick in das hochschuldidaktische und soziale Umfeld der internationalen Kursteilnehmer, in „,normalen“ Sprachkursen sind persönliche Kontakte zwischen den Kursteilnehmern und den deutschsprachigen Hospitanten noch eher ein Randaspekt, wenn auch oft genug mit der Perspektive Tandem-Partnerschaft oder engerem persönlichen Kontakt im Hochschulalltag; stärker im Blick ist dieser Aspekt bei spezifischen „Sprachberatungskursen“, indem Fragen des Studiums, der Lernmethoden, des alltäglichen Lebens am Hochschulort, Fragen der interkulturellen Durchdringung der Lebensbereiche als Themen ins Zentrum der Kursdidaktik und Kursgestaltung rücken.

\section{Exkurs: Eine Frage an die Kollegen in den anderen neu- sprachlichen Fächern}

Aus meiner Tübinger Praxis heraus habe ich nie verstanden, wieso die Kolleg/innen der anderen „lebendigen“ Sprachfächer die internationale Studentenschaft an ihrer Universität nicht in ähnlicher Weise als „,native speaker“-Hospitanten einladen, in ihren Kursen zu hospitieren und mitzuarbeiten. So könnte sich quasi von unten her die Atmosphäre einer ,internationaler Sprachlernpraxis“ quer zu den Fächern und Instituten entwickeln, im Interesse der deutschen Anglistikoder Romanistik-Studierenden, sicher auch zum Vorteil der Berufspraxis der beteiligten Kollegenschaft selbst und ganz bestimmt auch zum Nutzen und Lob der betreffenden Hochschule. Vor allem aber wäre es zum Vorteil der internationalen Studierenden, die in ihrer muttersprachlichen und kulturellen Kompetenz ernst genommen und zu akademischer Mitarbeit und zu einem eigenen Beitrag zur Internationalität der Universität eingeladen würden. 


\section{Fünf persönliche Erfahrungen}

a) Die Praxis mitarbeitender Hospitant/innen hat meine eigene DaF-Lehrtätigkeit erheblich angeregt, erleichtert und methodisch vertieft.

b) Sie hat wenn das Urteil in eigener Sache erlaubt ist - zu einer ,jungen ", studenten- bzw. lernerbezogenen Arbeitsatmosphäre spürbar beigetragen.

c) Auch wenn das für die beschrieben Praxis erforderliche Engagement (das heißt auch: Gebotene Aufmerksamkeit, Toleranz und Flexibilität sowie investierte Arbeitszeit) nicht unerheblich war und als wichtige Voraussetzung für den Erfolg zu werten ist, bestand für mich immer ein absolut positives Verhältnis von Aufwand und Nutren.

d) Die Hospitationspraxis hat - neben den auf der Hand liegenden großen Vorteilen für die internationalen Studierenden - vielen der deutschsprachigen Hospitant/innen eine erhebliche internationale/interkulturelle Erweiterung des persönlichen Horizonts und der Gestaltung des Studiums ermöglicht.

e) Die Tübinger Praxis hat zudem - auch ohne DaF-Studiengang - manche Tübinger Hospitantin und manchen Hospitanten auf berufliche Wege in Richtung DaF oder Internationalität im In- und Ausland, zu Buchverlagen, zur Erwachsenenbildung, Kulturarbeit oder zu Internationalen Organisationen gebracht.

\section{Mögliche Erweiterungen der Tübinger Praxis - ein Kata- $\log$ nicht nur für Tübingen}

a) Es wäre notwendig und nützlich, einen kleinen „Didaktischen Bausteinkasten“ mit praxisorientierten Handreichungen für die Arbeit der Hospitanten bereitzustellen, um so mehr an einer Hochschule, die wie Tübingen nicht einmal über einen eigenen DaF-Aufbaustudiengang verfügt. Auch wenn das Prinzip des Hospitanten-Modells, keine besonderen fremdsprachendidaktischen Vorkenntnisse für die Hospitation vorauszusetzen, richtig ist, könnte eine solche Handreichung den Zugang zur Praxis und die Selbstbewertung der eigenen Erfahrungen erleichtern.

b) Die Lücke zwischen einer DaF-theoretisch unreflektierten Unterrichtspraxis und den Möglichkeiten, die ein etablierter Studiengang Deutsch als Fremdsprache bieten könnte, haben wir in Tübingen wiederholt mit „Fortbildungsbaustein-Seminaren Deutsch als Fremdsprache" zu schließen versucht (s. Anlage 4). Die- 
ses Instrument hat sich sehr bewährt, sowohl für die teilnehmenden, überwiegend deutschsprachigen Studierenden, vor allem aber auch für die eigene Kollegenschaft als DaF-Dozenten, die diese Gelegenheit zur Systematisierung und theoretischen Reflektierung ihrer Lehrtätigkeit nutzen konnten. Es wäre anzustreben, derartige Seminare regelmäßig und besonders den Hospitantinnen und Hospitanten anzubieten. Das unter a) beschriebene Baukastensystem mit praktischen DaF-Handreichungen könnte sich dabei als ein nützliches Nebenprodukt ergeben.

c) Die oben im Abschnitt 7 aufgezeigte Perspektive würde verbindliche Querkontakte zu den anderen Kursleitern für moderne Fremdsprachen (in Sprachzentren oder in den Neusprachen-Philologien) bewirken, und zwar

- in didaktisch-methodischer Hinsicht und

- für die Einwerbung internationaler Studierender als Hospitant/innen in diesen Kursen.

d) Die Tübinger DaF-Abteilung betreibt ein kleines, sprachendidaktisch eher ansprucbsloses „Tandem"-Vermittlungssystem in einem breiten Spektrum von Sprachen, im Umfang von etwa 60 bis 100 Vermittlungen pro Semester. Schon aus eigener Initiative erweitern manche deutsche Studierende ihre Hospitationspraxis mit einem festen Tandem-Kontakt. Dieser Ansatz wäre aber weiterzuentwickeln in Richtung auf eine fremdsprachendidaktische und interkulturelle Tandem-Beratung und Begleitung, vor allem auch in Richtung auf die Vermittlung und Organisation von fachspezifischen, auf den Studiengang und Studienerfolg bezogenen Tandem-Kontakten. Ein derartiges Modell würde auf eine weiter gefasste Dimension von Internationalität und Interlingualität der Hochschulausbildung zielen und könnte die Hochschule bei ihrer internationalen Präsentation unterstützen und auszeichnen.

e) Auch die aktuelle Entwicklung des Themas „Spracbberatung "12 gehört in diesen Kontext. Die Mitarbeit deutschsprachiger Hospitanten kann sinnvoll genutzt werden, Sprachkurse für internationale Studierende generell in Richtung auf „Sprachberatungskurse“ und „Autonomes Lernen“ weiter zu entwickeln.

f) Man kann sich eine intensivere Verbindung zum hochschuleigenen AlumniProgramm vorstellen mit dem Ziel, über den Abschluss des Studienaufenthalts hinaus internationale und interlinguale Kontakte zu fördern und zu festigen.

\footnotetext{
12 Dazu die von karin Kleppin und Grit Mehlhorn herausgebenen Berichte der FaDaF-Tagung in Bochum vom Juni 2005, zu finden im Internet unter www.ualberta.ca/ german/ejournal.html, und der Beitrag von Kleppin und Mehlhorn über Sprachlernberatung in „Materialien Deutsch als Fremdsprache“ Bd. 74, 2005, 81-98.
} 
g) Es ist nützlich, die Instrumente der Öffentlichkeitsarbeit an der Hochschule (Hochschulzeitung, Uniradio, Lokalpresse, Landes-Lehrpreis-Verleihung etc.) immer wieder zu beanspruchen und zu nutzen, um die vorhandenen Elemente von „Internationalität" innerhalb der Hochschule und darüber hinaus bekanntzumachen und weiterzuentwickeln.

\section{Gibt es ein DaF-politisches Gegenargument?}

Wir haben uns in der Vergangenheit in Tübingen dazu entschlossen, ein solches Praxiselement bewusst unterhalb „wissenschaftlicher“ Organisationsformen zu etablieren, quasi aus fachlicher Notlage heraus. Darin kann, wer das entsprechende Interesse hat, ein Argument zu „Billiglösungen“ an einer doch generell auf die sprachliche Förderung und fachliche wie interkulturelle Integration ihrer Studierenden verpflichteten Universität erkennen. Das mag in einem Land wie BadenWürttemberg mit seiner - sieht man von den DaF/DaZ-Bereichen an den Pädagogischen Hochschulen einmal ab - nur schwachen und unbefriedigenden Institutionalisierung des Faches Deutsch als Fremdsprache besonders ins Gewicht fallen.

Mindert also eine solche ausgesprochene (und dazu noch billige) „PraxisLösung“ die Wirkung und den Druck der Argumente, die an die einschlägigen Personen und Strukturen zu richten wären

- in der Germanistik bzw. in anderen einschlägigen Wissenschaften

- in der Hochschulleitung

- auf der staatlichen Ebene?

Das Ziel, trotz fehlender wissenschaftlicher DaF-Einbindung auf eine Verbesserung der DaF-Unterrichtspraxis nicht zu verzichten, hat uns das beschriebene Modell erfinden und entwickeln lassen, sogar ein Erfolgsmodell, wie die Erfahrungen und die vielfältig positiven Rückmeldungen der Beteiligten zeigen. Zunächst sei deshalb die Empfehlung ausgesprochen, auch an anderen Hochschulen, an denen DaF institutionell schwach etabliert ist, mit einem solchen Praxis-Modell zu beginnen, zumal es außer dem beschriebenen Engagement und der notwendigen DaF-didaktischen Sorgfalt mit keinerlei Kosten verbunden ist.

Auch wenn es Widerspruch erregen mag: Ich bin fest davon überzeugt, dass das Tübinger Hospitanten-Modell nicht per se eine kontraproduktive Praxis im Blick auf eine weitergehende und notwendige Fachentwicklung Deutsch als Fremdsprache darstellt.

Zudem wird der Leser schon vermutet und erkannt haben, dass das Tübinger Modell auch an Hochschulen mit wissenschaftlich eingerichteten DaF-Schwerpunkten ohne Einschränkung übernommen werden kann, als Verbindungsstück zwischen dem eher langfristig wirkenden Zielen und Nutzen der DaF-Institution selbst und dem generell an jeder Hochschule in der Breite vorhandenen Bedarf an qualifiziertem und effektiven Deutschunterricht auf allen Stufen für die internationalen 
Studierenden, seien Sie nun Austauschstudenten im Rahmen der internationalen Partnerschaftsbeziehungen der Hochschule oder Vollstudenten in den Studiengängen.

Der Ausbau der Internationalität der deutschen Hochschulen ist nicht nur im Kontext der internationalen Hochschulpartnerschaften, der im Gange befindlichen Internationalisierung der Studiengänge, der internationalen Kompatibilität der Studienabschlüsse und der wichtiger werdenden Rolle des Englischen im deutschen Hochschulwesen zu diskutieren. Es wird auch wesentlich darauf ankommen, inwieweit es den Hochschulen gelingt, durch eine internationale, interkulturelle und interlinguale Atmosphäre im Innern den Studien- und Lebensalltag in Deutschland attraktiv zu gestalten, gleichermaßen zugunsten der nicht deutschsprachigen und der deutschen Studierenden. Dazu scheint uns das HospitantenModell ein kleiner und mit einfachen Mitteln zu praktizierender, vor allem ein wirkungsvoller Beitrag zu sein. 


\title{
Anlagen:
}

\author{
Anlage 1: Werbeflyer
}

Anlage 1: Worbeflyer Ss 2005 Können Sie Ihre
Muttersprache Deutsch erklären?
Hier können Sie es lernen!

Auch im Sommersemester 2005 können wieder 40 bis 50 deutschsprachige Studentinnen und Studenten (aller Fächer, aber mit Deutsch als Muttersprache) bei der ABTEILUNG INTERNATIONALE SPRACHPROGRAMME als

\section{Hospitant/innen für Deutsch als Fremdsprache}

mitarbeiten, für 2-3 Stunden pro Woche, auf freiwilliger Grundlage, und zwar in den folgenden Kursen:

GRAMMATIK FÜR FORTGESCHRITTENE - DEUTSCHE UMGANGSSPRACHE AUFBAUKURSE - KONVERSATIONSKURSE - FEHLERKURS ITALIENISCH- und SPANISCH-DEUTSCH - INTERKULTURELLE KOMMUNIKATION - DISKUTIEREN, ARGUMENTIEREN, REFERIEREN und andere Kurse

Für die internationalen Kursteilnehmerinnen und -teilnehmer liegen die Vorteile auf der Hand, wenn sich deutschsprachige Studierende an den Kursen beteiligen:

- mehr Sprechpraxis und Fehlerkorrektur auf Deutsch

Deutschlernen in kleinen Gruppen

mehr Kontakt und Kommunikation mit Deutschen, mehr soziale und kulturelle Integration.

Für die deutschsprachigen Studentinnen und Studenten bieten sich:

- viel Gelegenheit zum praktischen und theoretischen Umgang mit der eigenen Sprache

- Vermittlung praktischer Erfahrungen für eigene Unterrichtstätigkeit im In- und Ausland

- Bescheinigung der Hospitation am Semesterende

- in begründeten Făllen auch Begutachtung (z.B. für Bewerbungen)

- Informationen über das Fach Deutsch als Fremdsprache und über weitere Aus- und

Fortbildungsmöglichkeiten.

Spezifische Vorkenntnisse (Linguistik, Fremdsprachen, DaF-Didaktik) werden nicht vorausgesetzt; man kann einfach mitmachen. Eine Vergütung kann nicht angeboten werden.

Interessent/innen mit der Muttersprache Deutsch bitten wir, zu einer der folgenden VORBESPRECHUNGEN zu kommen:

\begin{tabular}{|lll|}
\hline Wann/Wo? & Donnerstag, & 7. April 2005, 12 Uhr c.t., Neuphilologie, Raum 031 \\
oder & Dienstag, & 12. April 2005, 12 Uhr c.t., Neuphilologie, Raum 031 \\
oder & Mittwoch, & 13. April 2005, 12 Uhr c.t., Neuphilologie, Raum 031 \\
oder & Donnerstag. & 14. April 2005, 13! Uhr c.t., Neuphilologie, Raum 031 \\
oder & Dienstag, & 19. April 2005, 12 Uhr c.t., Neuphilologie, Raum 031 \\
oder & Donnerstag, 21. April 2005, 13! Uhr c.t., Neuphilologie, Raum 031 \\
oder & einfach nachfragen, anrufen oder mailen \\
Ansprechpartner: & Dr. Wolfgang Rug, Dr. Volker Schmidt oder Michaela Rödel \\
\hline
\end{tabular}

Sie können auch gern interessierte Freund/innen auf dieses Angebot hinweisen und sie zum Mitmachen einladen!

\begin{tabular}{|c|}
\hline EBERHARD-KARLS-UNIVERSITÄT TŨBINGEN - Abteilung Internationale Sprachprogramme \\
Wilthelmstr. 9 (Alte Archäologie) 72074 Tübingen \\
Tel.: 07071/29-74401 oder $29-75409$ oder $29-74392$ \\
Bürozeiten: montags bis freitags $10-12.30$ Uhr \\
Mailadressen: lektorat@uni-tuebingen.de oder wolfgang.rug@uni-tuebingen.de \\
\hline
\end{tabular}


Anlage 2: Massenmail der Universität an alle Studierende mit Uni-Account vom WS 2004/05

Wie in früheren Semestern können im Wintersemester 2004/05 wieder bis zu 50 deutschsprachige studentinnen und studenten aller Fächer, aber mit Deutsch als Muttersprache) bei der ABTEILUNG INTERNATIONALE SPRACHPROGRAMME als Hospitant/innen in verschiedenen Sprachkursen für Deutsch als Fremdsprache mitarbeiten: für 2-3 Stunden pro woche, auf freiwilliger Grundlage, und zwar in den folgenden Kursen:

1) GRAMMATIK FÜR FORTGESCHRITTENE

2) AUFBAUKURSE verschiedener Sprachstufen

3) SPRACHBERATUNGSKURS für Teilnehmer mit Englisch als Mut tersprache

3) QUASSELKURS (Konversationstraining)

4) DISKUTIEREN, ARGUMENTIEREN, REFERIEREN

5) Landeskundekurs PANORAMA DEUTSCHLAND

6) BESSERE AUSSSPRACHE UND INTONATION

- für Teilnehmer mit slawischen Muttersprachen, Ungarisch und Rumänisch

- für Teilnehmer mit ostasiatischen Muttersprachen

- für Teilnehmer mit Spanisch als Muttersprache

Alle Kursbeschreibungen finden Sie unter:

http://wWw. uni-tuebingen.de/isp

Die internationalen Kursteilnehmer haben davon den Vorteil, Sprechtraining und Fehlerkorrektur in kleinen Arbeitsgruppen mit viel deutscher Hilfe organisieren zu können.- Für die deutschsprachigen Hospitanten bietet sich viel Gelegenheit zum praktischen und theoretischen Umgang mit der eigenen Sprache und zur Einübung von Unterrichtspraxis für Deutsch als Fremdsprache. Die Hospitation wird am Semesterende bescheinigt. Eine Vergütung kann nicht angeboten werden. Spezifische Vorkenntnisse (Linguistik, Fremdsprachen, DaF-Didaktik) nicht vorausgesetzt. Sie können einfach bei uns mitmachen.

Interessent/innen mit der Muttersprache Deutsch bitten wir, zu einer der folgenden VORBESPRECHUNGEN zu kommen:

Donnerstag, 14. Oktober 2004, $12 \mathrm{Uhr}$ c.t. oder

Dienstag, 19. Oktober 2004, 12 Uhr c.t. oder

Mittwoch, 20. Oktober 2004, $13 \mathrm{Uhr}$ c.t. oder

Donnerstag, 21. Oktober 2004, $13 \mathrm{Uhr}$ c.t. oder

Dienstag, 26. Oktober 2004, $12 \mathrm{Uhr}$ c.t. oder

Donnerstag, 28. Oktober 2004, $12 \mathrm{Uhr}$ c.t.

- jeweils Neuphilologie, Raum 031

Sie können auch einfach bei uns nachfragen, anrufen oder mailen - Adressen untenstehend; Ansprechpartner: Dr.Wolfgang Rug, Michaela Rödel, Andrea Werner, Dr. Volker Schmidt. Bitte weisen Sie auch interessierte Freunde auf dieses Angebot hin.

EBERHARD-KARLS-UNIVERSITÄT TÜBINGEN - Abteilung Internationale Sprachprogramme - Wilhelmstr. 9 (Alte Archäologie) 72074 Tübingen - Tel.: $07071 / 29-74401$ oder 29-75409 oder 29-74392 - Bürozeiten: montags bis freitags 10 - 12.30 Uhr Mailadresse: lektorat@uni-tuebingen.de oder: wolfgang.rug@uni-tuebingen.de 


\section{Anlage 3: Bescheinigungen}

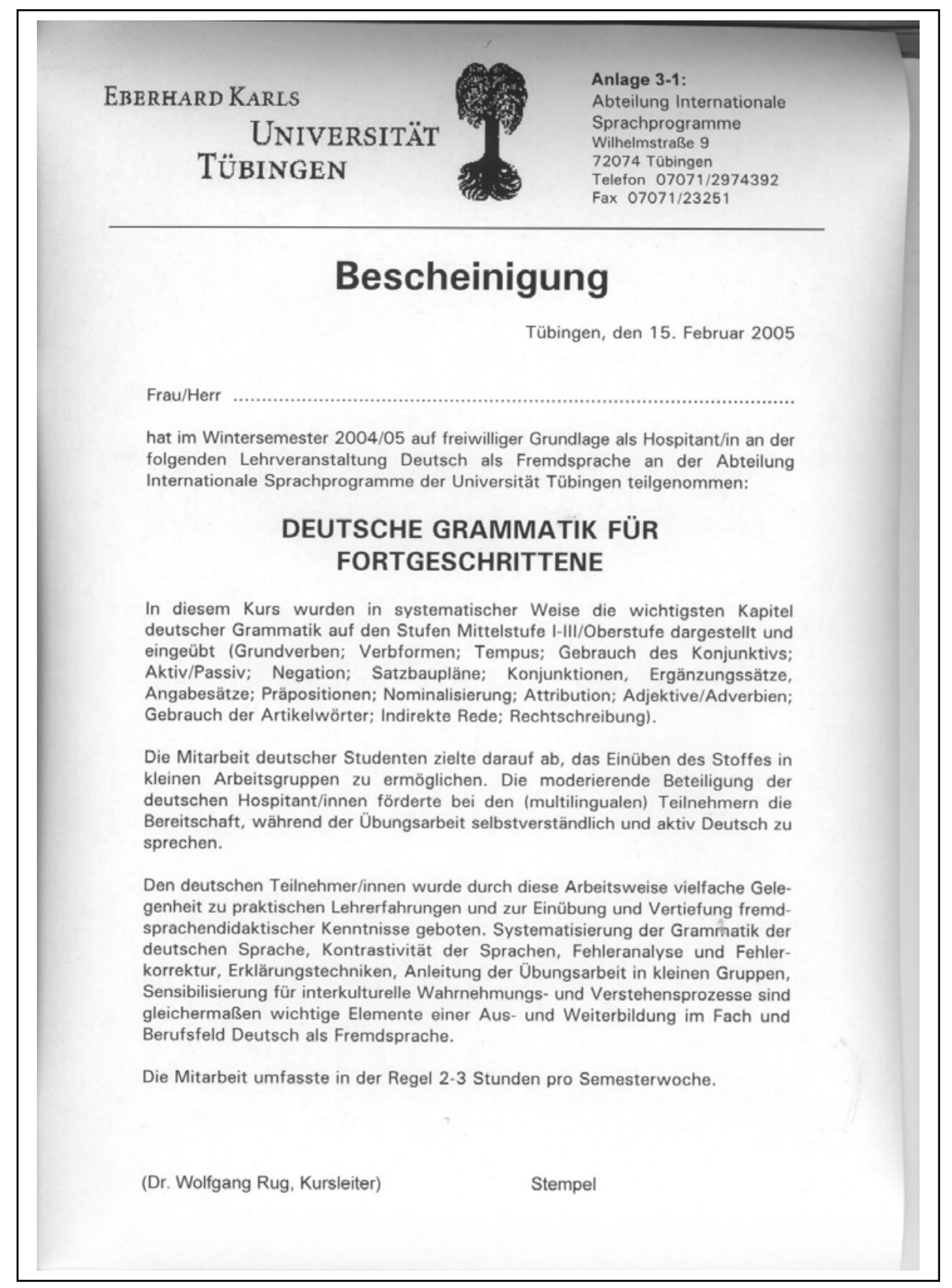


EBERHARD KARLS TÜBINGEN
Anlage 3-2:

Abteilung Internationale Sprachprogramme

Wilhelmstraße 9, D-72074 Tübingen,

Tel. (0) $7071 / 2974392$, Fax (0) $7071 / 23251$

\section{Bescheinigung}

Frau/Herr

hat im Winter-Semester 2004/05 auf freiwilliger Grundlage als Hospitant/in an der folgenden Lehrveranstaltung Deutsch als Fremdsprache an der Abteilung Internationale Sprachprogramme der Universităt Tübingen teilgenommen:

PRAKTISCHE SPRACHBERATUNG

FÜR AUSTAUSCH-STUDIERENDE MIT DER MUTTERSPRACHE ENGLISCH

(USA, Kanada, Großbritannien, Australien, Neuseeland u.a.)

Leitung: Dr. Wolfgang Rug unter Mitarbeit von Katrin Saur

Kurstermine: $28.10 .04 ; 04.11 .04 ; 11.11 .04 ; 09.12 .04 ; 10.02 .05$ und 17.02 .05

(12 Kontaktstunden und individuelle Arbeitstreffen)

Themen: $\quad$ Erarbeitung eines Programms mit typischen Aussprache- und Intonationsfehlern von Lernern mit Englisch als Muttersprache; Anleitung und Tipps zum selbststandigen Oben der Aussprache; Erarbeitung eines Programms mit typischen Grammatikfehlern und Anleitung zum selbstandigen Öben; Empfehlungen zum systematischen Worterlernen und zur lernorientierten Textarbeit; Empfehlungen zur kontinuierlichen Deutsch-Lekture: Projekt "Sprachtagebuch" zur Begleitung und Förderung des eigenen Deutschlernens: Erarbeitung eines Reportage-Essays: Beschreibung der eigenen Praxis und Erfahrungen beim Deutschlernen und zur Begegnung mit der deutschen Sprache und Alltagskultur.

Die Mitarbeit deutscher Studenten zielte neben der Einarbeitung in kontrastiv gestaltete Lehrmatieralien für anglophone Deutschlerner (Materialien zur Phonetik und Intonation, Fehlerkartenbuch für die Bereiche Grammatik und Wortschatz) darauf, die Lernarbeit der internationalen Studenten in kleinen Lerngruppen anzuleiten und sie bei eigenen, selbstbestimmten Lernperpsektiven helfend $z u$ begleiten. Den deutschen Teilnehmern wurde dabe Gelegenheit zu praktischen Lehrerfahrungen und zur Vertiefung sprach- und fremdsprachendidaktischer Kenntnisse geboten: die Kontrastivităt Englisch-Deutsch, Fehleranalyse und Fehlerkorrektur, Praktizieren geeigneter Erklärungstechniken bei Aussprache, Grammatik und Wortschatz, Redaktionshilfe bei schriftlichen Arbeiten sowie die Erörterung interkultureller Aspekte (Kulturkontraste anglophone Lănder vs. Deutschland) - all dies sind wichtige Elemente einer Aus- und Weiterbildung im Fach und Berufsfeld Deutsch als Fremdsprache.

Die Mitarbeit umfasste insgesamt 12 Stunden und mehr im Verlauf des Semesters

Tübingen, den 17. Februar 2005

(Dr. Wolfgang Rug, Kursleiter)

(Stempel) 


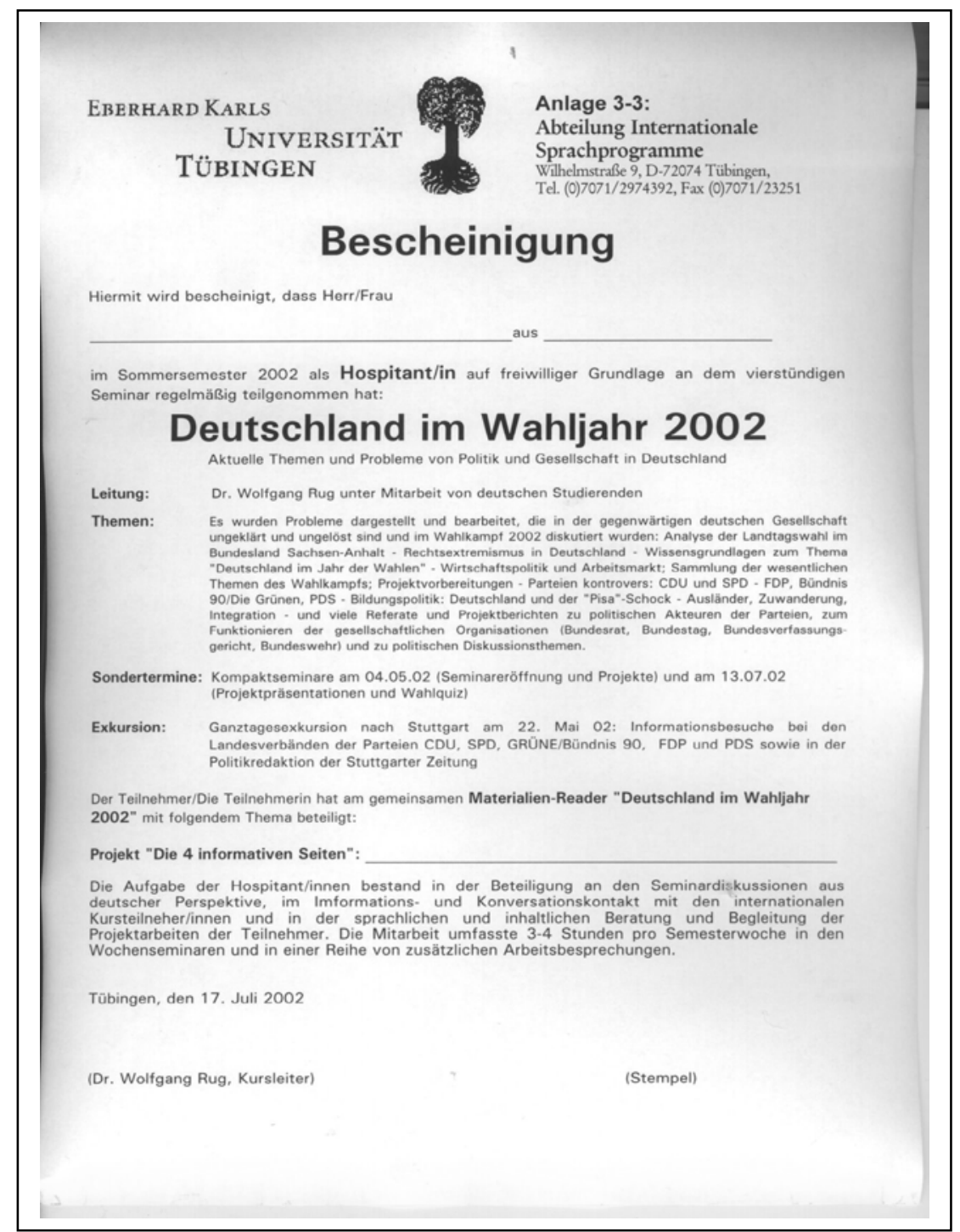


Anlage 4: Universität Tübingen - Abt. Internationale Sprachprogramme - SS 2005 Fortbildungsbausteine Deutsch als Fremdsprache, Leitung: Dr. Wolfgang Rug

\begin{tabular}{|c|c|c|c|}
\hline \multicolumn{4}{|c|}{$\begin{array}{c}\text { Programm: 14 Termine: Freitag, } 10-13 \text { Uhr } \\
\text { zwei Terminverschiebungen (4 u. 7) am Freitag Nachmittag, } \\
\text { ein abschließendes Blockseminar }\end{array}$} \\
\hline 15.04 .05 & 1 & $\begin{array}{l}\text { Einführungsseminar: Seminarorganisation; Erwartungen an die } \\
\text { Mitarbeit der Teilnehmer/innen; Qualifikationen: Hospitationen, DaF- } \\
\text { Recherchen - Themenpuzzle: "Deutsch als Fremdsprache" - ein } \\
\text { Überblick }\end{array}$ & Dr. Wolfgang Rug \\
\hline 22.04 .05 & 2 & Methoden des fremdsprachlichen Deutschunterrichts & Dr. Peter Kelbel \\
\hline 29.04 .05 & 3 & $\begin{array}{l}\text { Tipps und Tricks zum kreativen Gestalten kleinerer Unterrichtseinhei- } \\
\text { ten - Lebendiges Grammatiklernen }\end{array}$ & Michaela Rödel \\
\hline $\begin{array}{l}29.04 .05 \\
14-17 \mathrm{~h}\end{array}$ & 4 & $\begin{array}{l}\text { Linguistische Grundlagen für das Unterrichten - Umrisse einer didak- } \\
\text { tischen Grammatik Deutsch als Fremdsprache }\end{array}$ & Dr. Wolfgang Rug \\
\hline 13.05 .05 & 5 & Interkulturelle Kommunikation & Donato Tangredi \\
\hline 20.05 .05 & 6 & Grundlagen und Praxis der Phonetik des Deutschen & Dr. Wolfgang Rug \\
\hline $\begin{array}{l}20.05 .05 \\
14-17 \mathrm{~h}\end{array}$ & 7 & Lernen aus/mit Fehlern & Dr. Wolfgang Rug \\
\hline 03.06 .05 & 8 & $\begin{array}{l}\text { Sprechen - Kommunikation - } \\
\text { Motiviertes Deutschlernen }\end{array}$ & Dr. Wolfgang Rug \\
\hline 10.06 .05 & 9 & Sozialformen im Unterricht Deutsch als Fremdsprache & Dr. Peter Kelbel \\
\hline 17.06 .05 & 10 & $\begin{array}{l}\text { Wie Lehrwerke entstehen und wie konzeptionelle und unterrichts- } \\
\text { praktische Überlegungen zu handhabbaren Unterrichtsentwürfen in } \\
\text { Buchform werden }\end{array}$ & $\begin{array}{l}\text { Andreas } \\
\text { Tomaszewski, } \\
\text { Hueber Verlag }\end{array}$ \\
\hline 24.06 .05 & 11 & Filme im DaF-Unterricht & Dr. Peter Kelbel \\
\hline 01.07 .05 & 12 & $\begin{array}{l}\text { Vom Lesen zum Schreiben und Deutsch in den USA - ein Gespräch } \\
\text { mit Dr. Burkhard Henke, Davidson College, North Carolina, USA }\end{array}$ & Ursula Kimpel \\
\hline $\begin{array}{l}08.07 .05 \\
14-17 \mathrm{~h}\end{array}$ & 13 & $\begin{array}{l}\text { Deutsch als Fremdsprache im Internet } \\
\text { im Computerraum } 04 \text { - Neuphilologie }\end{array}$ & Ursula Kimpel \\
\hline $\begin{array}{l}15.07 .05 \\
10-14 \mathrm{~h}\end{array}$ & 14 & $\begin{array}{l}\text { Abschluss-Blockseminar: Themenblock: Vom Umgang mit den neu- } \\
\text { en Rechtschreibregelungen im DaF-Unterricht - Gespräch über die } \\
\text { Hospitationserfahrungen - Kurzreferate der Seminar- } \\
\text { teilnehmer/innen über die DaF-Recherchen - Seminarkritik - Ge- } \\
\text { spräch über Arbeits- und Berufsperspektiven im Bereich DaF; Gäste: } \\
\text { Christina Hahn, Robert Bosch Stiftung und Jochen Krakowski, Uh- } \\
\text { land-Gymnasium Tübingen }\end{array}$ & $\begin{array}{l}\text { Leitung: } \\
\text { Dr. Wolfgang Rug }\end{array}$ \\
\hline \multicolumn{4}{|c|}{$\begin{array}{l}\text { Themen der Recherchen, die im Rahmen des Seminars von den Teilnehmern angefertigt wurden: } \\
\text { DaF-Didaktik, DaF-Lehrwerke: Wer-Wie-Was (Gilde) - Wörterbücher für den Einsatz im Bereich DaF - Die Zeitschrift } \\
\text { "Fremdsprache Deutsch" - Wirtschaftskommunikation Deutsch - Deutsch im Beruf - Deutsch für Kinder - Die Zeitschrift } \\
\text { "Primar" - Das Computerprogramm LingoFox - Kreative DaF-Links im Internet - Interkulturelle Kommunikation } \\
\text { Deutsch als Fremdsprache in der Welt: Polen -Tschechien - Türkei - China - USA } \\
\text { Institutionelle Bedingungen des Bereichs DaF: Der gemeinsame europäische Referenzrahmen für Sprachen - Prüfungen } \\
\text { im DaF-Bereich - Studiengänge DaF an Hochschulen - Der Fernstudiengang DaF - Das deutsche Auslandsschulwesen - } \\
\text { Internationale Sommerkurse an deutschen Hochschulen - Möglichkeiten für Praktika im In- und Ausland - DaF-Verbände } \\
\text { (FaDaF, IDV) - Rezension "Deutsch als Fremdsprache, ein internationales Handbuch" (Helbig/Götze/Henrici/Krumm) } \\
\text { Andere Themen: "Deutsch ist eine schwere Sprache", eine Umfrage unter internationalen Studenten - Grammatik, Stil und } \\
\text { Ton (Rezensionen zu B. Sick, "Der Dativ ist dem Genitiv sein Tod" u. A. Thalmayr, "Heraus mit der Sprache") }\end{array}$} \\
\hline
\end{tabular}




\title{
„Das klingt irgendwie komisch“. Ausspracheschu- lung für DaF-Studierende
}

\author{
Julia Richter, Bielefeld
}

\section{$1 \quad$ Einleitung}

Dieser Beitrag stellt ein Konzept der Ausspracheschulung vor, das im BachelorStudiengang $\mathrm{DaF}$ an der Universität Bielefeld seit vier Semestern läuft. Das Besondere des Modells besteht darin, dass nicht nur Nichtmuttersprachler (als DaFLernende), sondern auch Muttersprachler (als Tutoren) an dem Kurs teilnehmen. Es wird erläutert, inwiefern alle am Kurs Beteiligten voneinander profitieren können und inwiefern sich hier ein Weg aufzeigt, angehenden DaF-Lehrenden praktische didaktische Kompetenzen im Bereich der Ausspracheschulung zu vermitteln.

Im ersten Teil werden einige Bemerkungen zur Rolle der Ausspracheschulung im DaF-Unterricht gemacht. Anschließend wird das Bielefelder Konzept der Ausspracheschulung vorgestellt. Dabei wird insbesondere darauf eingegangen, welche Kompetenzen angehende $\mathrm{DaF}-L$ hehrer brauchen, um Ausspracheschulung geben zu können, welche Elemente Ausspracheschulung beinhalten sollte, wie die Bielefelder Aussprache- 
schulung im Semesterverlauf aufgebaut ist und welche Vor- und Nachteile das Konzept im Vergleich zu konventionellen Aussprachekursen bislang aufweist.

\section{Zur Rolle der Ausspracheschulung im DaF-Unterricht}

Dass Phonetik eine wichtige Rolle im DaF-Unterricht spielen sollte, ist wohl unumstritten. Als Argumente für den Erwerb einer guten Aussprache werden z. B. die Relevanz der Aussprache für die Verständlichkeit und Verarbeitbarkeit von Sprache durch den Hörer und damit den Erfolg der intendierten Sprechhandlung sowie für die soziale Akzeptanz des Sprechers und sogar Auswirkungen auf den Sprachlernerfolg allgemein genannt (Stichwort: Sprechangst und ihre Konsequenzen) (vgl. Kelz 1992: 25; Grotjahn 1998: 40-42; Hirschfeld 2001: 872).

Da jedoch nicht jede Art von Aussprachetraining zum Erfolg führt, ist die generelle Effizienz von Ausspracheschulung lange angezweifelt worden (vgl. z. B. die immer wieder angeführten Studien von Suter 1976 und Purcell und Suter 1980; vgl. Pennington 1998: 324-327 zu einer Kritik an der Aussagekraft dieser Studien). Mittlerweile haben jedoch zahlreiche Untersuchungen gezeigt, dass Instruktion grundsätzlich zur Verbesserung der Aussprache beitragen kann (vgl. z. B. de Bot und Mailfert 1982, Champagne-Muzar, Schneiderman und Bourdages 1993, Derwing, Munro und Wiebe 1998). Als Bedingungen für den Erfolg von Ausspracheschulung werden häufig genannt: Schwerpunkt der Arbeit auf Suprasegmentalia, Arbeit mit bedeutungsvollen sprachlichen Einheiten, ein kognitiver Zugang und Berücksichtigung von voice setting features und koartikulatorischen Phänomenen (vgl. z. B. Pennington und Richards 1986; Scarcella und Oxford 1994; Celce-Murcia, Brinton und Goodwin 1996: 25-29). Allerdings scheint es im Bereich des Ausspracheerwerbs große individuelle Unterschiede zu geben, was die Wirksamkeit verschiedener Instruktionsformen betrifft (Macdonald, Yule, Powers 1994; Yule und Macdonald 1995), so dass Pennington und Richards (1986: 219) zugestimmt werden kann, dass es wohl keine 1:1-Beziehung zwischen Ausspracheschulung und -erwerb geben kann.

Trotz der Relevanz einer guten Aussprache und dem Konsens, dass Instruktion unter bestimmten Voraussetzungen zur Verbesserung der Aussprache beitragen kann, kann Aussprachevermittlung nach wie vor als das oder zumindest ein Stiefkind des DaF-Unterrichts gelten. Zurückgeführt wird dieses Manko gemeinhin auf Versäumnisse in der DaF-Lehrer-Ausbildung (vgl. z. B. Kelz 1992: 30-31; Grotjahn 1998: 36-37; Hirschfeld 2001: 872-873; Hirschfeld 2003: 193-194).

Während lange Zeit zusätzlich die Vernachlässigung der Phonetik in DaFLehrwerken ins Feld geführt wurde (vgl. z. B. die Studie von Dieling 1994), so hat sich auf diesem Gebiet in den letzten Jahren viel getan. Alle gängigen DaFLehrwerke haben Ausspracheaspekte integriert, einige sogar in relativ großem Umfang. Auch das Angebot an Zusatzmaterialien zur Ausspracheschulung ist seit Mitte der 90er Jahre stark angestiegen. Wie ist es also zu erklären, dass Lehrer 
immer noch häufig, wenn auch etwas zerknirscht, von sich sagen, den PhonetikTeil der Lektion würden sie immer überspringen?

Meiner Einschätzung nach liegt hier sowohl in der Lehrerausbildung als auch in der Gestaltung der Lehrwerke noch Potential. Während Vorschläge zur Lehrwerkgestaltung nur am Rande erwähnt werden sollen, wird im Folgenden ein Modell der Lehrerausbildung im Bereich der Ausspracheschulung vorgestellt.

\section{Ein alternatives Konzept zur Ausspracheschulung im BA- Studiengang DaF in Bielefeld}

Die Kursteilnehmer an der Ausspracheschulung sind in der Regel im zweiten bis vierten Fachsemester. Die Nichtmuttersprachler besuchen den Kurs, um sich auf die Prüfung Dentsche Wissenschaftssprache (PDW) vorzubereiten. Diese Prüfung, auf die mit 6 SWS vorbereitet wird, ist der einzige sprachpraktische Teil im Bielefelder BA DaF, aber auch über die PDW hinaus trägt eine Aussprache deutlich oberhalb des DSH-Niveaus zum Studienerfolg bei, da sie in die Bewertung mündlicher Kursbeiträge, Referate und Prüfungen miteinfließt. Die Muttersprachler absolvieren anstelle der PDW einen Kurs zur Sprecherziehung und erlernen eine Kontrastsprache.

Da die Aussprachekurse mit durchschnittlich 20 bis 25 Teilnehmern, die darüber hinaus im Normalfall bis zu 15 unterschiedliche Muttersprachen sprechen und dementsprechend ganz eigene Ausspracheschwierigkeiten zeigen, viel zu groß für ein sinnvolles Arbeiten waren, entstand die Idee, einige Muttersprachler als Tutoren statt an der Sprecherziehung an der Ausspracheschulung teilnehmen zu lassen. Ziel war dabei zunächst, eine intensivere Betreuung der Nichtmuttersprachler zu gewährleisten. So wurde pro fünf Nichtmuttersprachler je ein Muttersprachler in die Ausspracheschulung aufgenommen.

\section{$4 \quad$ Zu erwerbende Kompetenzen angehender DaF-Lehrer}

Um den Lehrenden sinnvoll unterstützen zu können, mussten die Muttersprachler zunächst in Hinblick auf bestimmte Aspekte der Ausspracheschulung ausgebildet werden. Denn in der Tat sehen sich die derart ,in die Praxis gestoßenen“ muttersprachlichen Studierenden anfangs nicht geringen Schwierigkeiten gegenüber. Natürlich bemerken sie im Unterschied zu Nichtmuttersprachlern (zumindest die meisten) Abweichungen von der deutschen Standardaussprache, können diese jedoch zunächst weder analysieren noch korrigieren.

Und genau vor dieser Schwierigkeit stehen meiner Einschätzung nach auch die meisten DaF-Lehrer, die ihr Studium bereits abgeschlossen haben. Die theoretischen Kenntnisse über Phonetik und Phonologie sind wieder verblasst und reichen darüber hinaus auch nicht aus, um Abweichungen von der Norm tatsächlich zu korrigieren. Die Lehrer befänden sich also in der misslichen Lage, Aussprache- 
fehler weder zutreffend erklären noch effektiv korrigieren zu können, was einem Gefühl der Ohnmacht im Bereich der Phonetik gleichkäme. So erschiene es verständlich, dass viele Lehrer es vorziehen, dieses für alle Beteiligten frustrierende Kapitel schnell zu überspringen ${ }^{1}$.

Im hier beschriebenen Aussprache-Kurs entsteht jedoch aus einem ersten, wenig präzisen „Das ist so nicht richtig.“ oder „Das klingt irgendwie komisch.“, dem versucht wird mit „Sprich mal deutlicher... oder vielleicht härter?“ entgegenzuwirken, im Allgemeinen schnell die Motivation, Wissen zu erwerben, mit dem den festgestellten Ausspracheabweichungen tatsächlich entgegengewirkt werden kann.

Und zwar brauchen die angehenden DaF-Lehrer dazu meines Erachtens Informationen zu den folgenden fünf Fragen:

- Welche artikulatorischen Kategorien gibt es im Deutschen?

- Wie klingen typische Fehler in diesen Kategorien, und wie kann ich sie artikulatorisch beschreiben?

- Welche Wege gibt es, diese Fehler zu korrigieren?

- Wie sollte eine Ausspracheschulung didaktisch aufgebaut sein?

- Welche Aussprachelehrmaterialien sind zu empfehlen?

Während die ersten vier Fragen im Rahmen des Kurses explizit geklärt werden, wird bei der letzten, die Aussprachelehrmaterialien betreffenden, darauf gesetzt, den Studierenden durch regelmäßige Unterrichtshospitation, Literaturhinweise, Semesterapparat und eine eigenständig geplante und durchgeführte Unterrichtseinheit einen Einblick zu vermitteln.

\section{$5 \quad$ Elemente der Ausspracheschulung}

Um die Integration der Muttersprachler in den Ausspracheunterricht zu erläutern, ist es sinnvoll, zunächst ein paar grundlegende Überlegungen zum didaktischen Vorgehen in der Ausspracheschulung vorzustellen.

Als Elemente jeder Ausspracheschulung können die folgenden angesehen werden:

\footnotetext{
${ }^{1}$ Meines Wissens fehlen allerdings empirische Untersuchungen, die der Frage nachgehen, wie Lehrer im DaF-Unterricht tatsächlich mit dem Bereich Aussprache umgehen und was die Beweggründe ihres Handelns sind. Hier würden Befragungen sicherlich wichtige Hinweise darauf liefern, wie Ausbildung und Unterrichtsmaterialien optimiert werden könnten. Erste Untersuchungen aus dem anglophonen Bereich liegen vor (vgl. Macdonald 2003).
} 


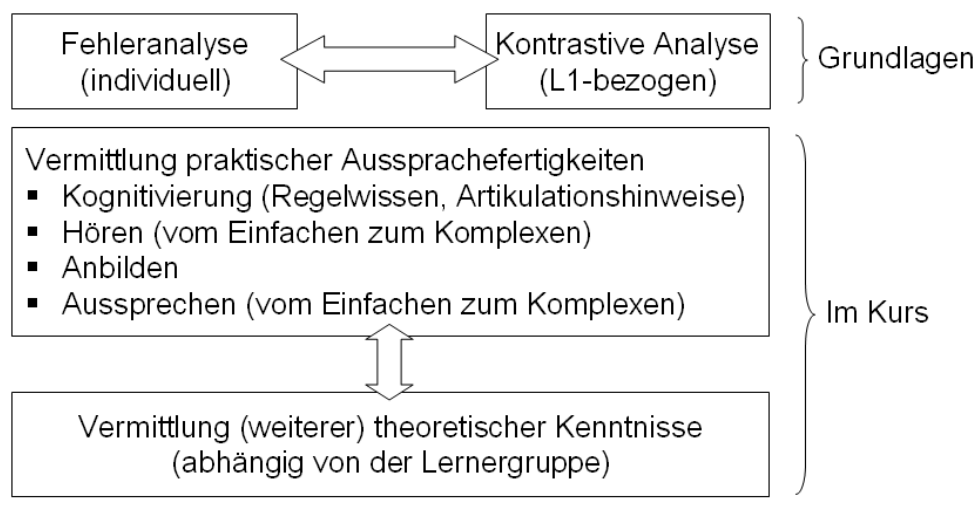

\section{Abbildung 1: Elemente von Ausspracheschulung}

Fehleranalyse und Kontrastive Analyse stellen dabei quasi die Vorarbeiten dar, die einen effektiven Unterricht überhaupt erst ermöglichen. Idealerweise sollten beide durchgeführt werden, da sie sich in gewisser Hinsicht ergänzen. So stellt die Kontrastive Analyse zunächst Gemeinsamkeiten und Unterschiede zwischen den sprachlichen Systemen der L1 und der L2 heraus. Dass die Kontrastive Analyse keine prognostische, sondern nur explikative Funktion haben kann, ist schon lange klar, aber sie kann die Aufmerksamkeit beim Hören auf bestimmte phonetische Kategorien lenken. Wenn der Lehrer z. B. weiß, dass es im Englischen zwei unterschiedliche L-Laute (nämlich / / und / 1 / gibt) und dass diese koartikulatorisch auftreten (nämlich das apiko-alveolare prävokalisch, wie z. B. in letter, und das palatalisierte postvokalisch, wie z. B. in milk), dann kann er bei der Fehleranalyse besonders darauf achten, ob das im Deutschen nicht vorkommende $/ \mathfrak{l} /$ in den einschlägigen phonetischen Kontexten zu hören ist. Solange er von der Existenz zweier so ähnlicher Laute jedoch nichts weiß, ist die Gefahr, dass das / $1 /$ durch den phonologischen Filter als /1/ eingeht, sehr groß. Außerdem kann die Kontrastive Analyse dabei helfen, eruierte Fehler artikulatorisch genauer zu beschreiben, was für die Korrektur hilfreich ist. Um erneut das L-Beispiel zu bemühen: Wenn der Lehrer weiß, dass das /1/ mit der Zungenspitze an den Alveolen gebildet wird und dass sich beim / $/$ zusätzlich der Zungenrücken Richtung Palatum wölbt, dann kann er seine Lerner instruieren darauf zu achten, nur leicht, wie beim /d/, mit der Zungenspitze die hinteren Vorderzähne zu berühren. Da in den DaF-Kursen allerdings, wie oben erwähnt, bis zu 15 verschiedene L1 vertreten sind, kann der hohe Aufwand einer Kontrastiven Analyse für all diese Sprachen leider nicht geleistet werden. Er ist wohl grundsätzlich nur in Gruppen mit heterogener L1 realistisch. In besonders schwierigen Fällen kann jedoch auf Kontras- 
tive Analysen zurückgegriffen werden. Außerdem entstehen im Rahmen der Besprechung der Fehleranalyse oft Gespräche, in denen die Nichtmuttersprachler hilfreiche Informationen über ihre L1 geben.

Diese eben angesprochene Fehleranalyse eruiert dann, welche Fehler tatsächlich gemacht werden. Zum einen zeigt sie jedem Lerner auf, wo seine individuellen Stärken und Schwächen liegen und in welchen Bereichen er noch an sich arbeiten sollte. Zum anderen können auf Grundlage der Diagnose-Bögen Arbeitsschwerpunkte für den jeweiligen Kurs festgelegt werden. Die Kategorien, in denen die meisten Lerner eines Kurses Schwierigkeiten haben, haben Prioriät.

Der eigentliche Unterricht läuft dann nach einem relativ festen Grundschema ab. Zunächst werden Ausspracheregeln erarbeitet und eventuell Erläuterungen zur Artikulationsweise einzelner Phänomene gegeben. Insbesondere im Unterricht mit erwachsenen Lernern sollte ein stark kognitiv ausgerichteter Zugang gewählt werden. Studien zum Faktor Alter (vgl. z. B. den Forschungsüberblick bei Grotjahn 1998: 46-53; Molnár und Schlak 2005) zeigen, dass auch die Aussprache erwachsener Lerner noch sehr gut werden kann. Zwar geht die Imitationsfähigkeit mit steigendem Alter zurück, dafür bieten aber im Vergleich zu Kindern größere kognitive Fähigkeiten einen Ausgleich. Möglicherweise kann in einigen Fällen sogar ein rein kognitiver Zugang zur Aussprache Verbesserungen bewirken, wie z. B. die Studie von Rebuschat (2004) zeigt.

Im Anschluss werden Hörübungen gemacht. Wenn die Teilnehmer in der Lage sind, eine phonetische Kategorie sicher zu hören, wird die richtige Aussprache angebildet. Techniken des „Anbildens“ (Slembek 1995: 57) bzw. der „Ableitung“ (Rausch; Rausch 1994: 95) zu beherrschen, bietet auf Grundlage artikulatorischer Kenntnisse einen Ausweg aus der oben skizzierten „Ohnmacht“ des Aussprachelehrers. Denn Aussprache umfasst im Gegensatz zu anderen Sprachebenen auch physische Aspekte, weswegen die häufig verwendete Sportmetapher durchaus heranzuziehen ist; bestimmte Muskeln müssen umlernen. Und auch im Sport würde man neue Bewegungen mit Hilfestellungen einführen, da davon auszugehen ist, dass sie sich nicht sofort von allein einstellen werden. Genauso wenig Sinn macht es in der Ausspracheschulung, darauf zu bauen, dass der Lerner eine für ihn neue artikulatorische Bewegung automatisch richtig machen wird - insbesondere dann nicht, wie oben erläutert, wenn er bereits erwachsen ist. Das Anbilden stellt außerdem sicher, dass die Zielform zumindest einmal von den Lernern richtig produziert wurde, bevor das Aussprechen trainiert wird, um nicht etwa eine falsche Form zu automatisieren. Ist das Anbilden gelungen, wird mit dem Aussprechen begonnen. Sowohl beim Hören als auch beim Aussprechen wird von einfachen Übungen über komplexe zum freien Sprechen übergegangen.

Pro Kurs werden in dieser Weise ein bis zwei phonetische Kategorien schwerpunktmäßig trainiert. Am Ende des Kurses dient außerdem eine komplexe Ausspracheübung dazu, den Fokus auch auf andere, nicht in diesem Kurs angesprochene Ausspracheschwierigkeiten zu legen, um so den Teilnehmern möglichst in 
jeder Sitzung die Gelegenheit zu geben, auch individuelle Übungsschwerpunkte zu setzen. Weiter wird versucht, durch unterschiedliche Textsorten und spielerische Übungsformen Abwechslung in den Kurs zu bringen, um der Gefahr, durch die notwendige große Anzahl an Wiederholungen Langeweile zu erzeugen, zu begegnen.

An dieser Stelle soll noch ein kurzer Blick auf Verbesserungsmöglichkeiten von Lehrwerken geworfen werden. Es gibt mittlerweile in Hinblick auf die Phonetik sehr gute Lehrwerke, wie Berliner Platz oder Stufen International, um Beispiele zu nennen. Viele andere Lehrwerke, auch reine Aussprachelehrwerke, berücksichtigen aber nach wie vor nicht alle Bausteine, die für eine sinnvolle Ausspracheschulung erforderlich sind, beschränken sich z. T. auf reines Hören und Nachsprechen. Insbesondere Tipps zum Anbilden fehlen häufig. Wie oben erläutert, stellen diese Techniken jedoch ein notwendiges Werkzeug des Lehrers in der Ausspracheschulung dar und sollten daher vom Lehrwerk mitgeliefert werden. Auch Erläuterungen zu phonetischen Kategorien sind nicht immer vollständig. Z. B. wird zwar stets auf die Quantitäts-, aber sehr selten auf die Qualitätsunterschiede zwischen Vokalen hingewiesen, obwohl diese perzeptiv stark zum fremden Akzent beitragen. Und schließlich wären mehr kreative Übungsformen wünschenswert. Ein bis zwei Hör- und Imitationsübungen sind zwar ein guter Anfang, reichen aber nicht aus, um Aussprache nachhaltig zu verbessern. Außerdem könnten komplexere Übungen und Texte oder spielerische Formen die Motivation der Lerner für die Ausspracheschulung steigern.

\section{Ausspracheschulung im Semesterverlauf}

$\mathrm{Zu}$ Beginn des Semesters machen die Nichtmuttersprachler ihre DiagnoseAufnahmen. Jeder Nichtmuttersprachler nimmt dazu einen zweiseitigen Text auf. Wir arbeiten im Moment mit dem Anfang von Dürrenmatts Die Physiker. Weniger komplexe Diagnose-Texte schlägt z. B. Phonetik aktuell (Bunk 2005: 75) vor. Wichtig ist bei der Auswahl der Texte vor allem, dass alle phonetischen Kategorien enthalten sind, also auch seltener vorkommende Phänomene, wie z. B. Knacklaut oder Auslautverhärtung innerhalb eines Wortes.

Im Kurs, an dem sowohl die Nichtmuttersprachler als auch die Muttersprachler teilnehmen, werden phonetische Kategorien besprochen. Außerdem werden typische Beispiele für Fehler in den einzelnen Kategorien vorgespielt und erörtert. Die Beispiele stammen aus den Diagnose-Aufnahmen und stellen somit real existierende Ausspracheschwierigkeiten dar. Drittens werden Möglichkeiten des Anbildens für die verschiedenen Fehlertypen vorgestellt. Diese Informationen wurden dieses Semester in Form eines Vortrags vermittelt, sind aber mittlerweile auch online verfügbar und können so auch von externen Interessenten genutzt werden (Richter 2006).

Die Muttersprachler treffen sich zusätzlich mit dem Lehrenden, um gemeinsam exemplarisch eine Diagnose-Aufnahme auszuwerten. Die Auswertung der Diagnose-Aufnahmen erfolgt auf Grundlage eines Diagnose-Bogens, der eng an 
den Vorschlag aus der Fernstudieneinheit Phonetik lebren und lernen (Dieling; Hirschfeld 2000: 198) angelehnt ist. Diese Übung dient zum einen dazu sicherzustellen, dass die einzelnen phonetischen Kategorien beherrscht werden, zum anderen dazu zu testen, welche Kategorien man jeweils leicht hört und welche man des Öfteren überhört. Meiner Erfahrung nach gibt es hier große individuelle Unterschiede. So lernen die Muttersprachler ihre eigenen Stärken und Schwächen beim Hören kennen. Dies ermöglicht ihnen, gezielt auf die Kategorien zu achten, von denen sie wissen, dass sie sie leicht überhören.

An diese gemeinsame Diagnose-Aufnahme schließt sich ein Auswertungsgespräch an, in dem der Lehrende gemeinsam mit einem Nichtmuttersprachler dessen Diagnose-Aufnahme analysiert und in dem die Muttersprachler hospitieren. Den Rahmen des Gesprächs bilden eine Selbsteinschätzung des Nichtmuttersprachlers zu Beginn und eine kooperative Festlegung von zwei bis vier phonetischen Kategorien, an denen während des Semesters besonders gearbeitet werden soll, zum Ende des Gesprächs. Im Hauptteil werden die Fehler in den einzelnen Kategorien anhand von konkreten Beispielen aus der Diagnose-Aufnahme besprochen. Außerdem kann im Rahmen dieser Besprechung ausprobiert werden, ob bestimmte Fehler auf Hör- oder Aussprechschwierigkeiten oder gar einfach auf mangelndes Regelwissen zurückgeführt werden können. Zusätzlich ist es motivierend, einige Kategorien anzubilden, um so aufzuzeigen, dass eine Verbesserung in diesem Bereich durchaus möglich ist.

Nachdem die Muttersprachler in dieser Weise ausgebildet worden sind, werten sie in Eigenregie ca. fünf Diagnose-Aufnahmen aus und besprechen sie mit dem jeweiligen Nichtmuttersprachler. Eine selbständige Auswertung wird noch vom Lehrenden gegengehört, und der Lehrende hospitiert auch in der (wenn möglich) ersten Besprechung und gibt Feedback auf die Vorgehensweise und das Beraterverhalten.

Im weiteren Verlauf des Semesters helfen die Muttersprachler wie Praktikanten oder Tutoren bei der Ausspracheschulung, indem sie mit Teilgruppen arbeiten oder auch einzelnen Lernern mit besonderen Schwierigkeiten zur Seite stehen. Darüber hinaus können sich die Teilnehmer, sobald eine Frage auftritt, an einen in ihrer Umgebung sitzenden Muttersprachler wenden und ihn um seine Meinung bitten².

Gegen Ende des Semesters führen die Muttersprachler außerdem eine Einheit eigenen Ausspracheunterrichts durch, um so auch Aussprachelehrerfahrung zu sammeln. Eine Evaluation durch die Kursteilnehmer und auf Grundlage einer Aufzeichnung des Unterrichts auch mit dem Lehrenden ermöglicht ein umfassendes Feedback auf den Unterricht.

Auch die Nichtmuttersprachler gestalten ihren Unterricht mit. In zweieinhalb Sitzungen werden in Gruppen Spiele und Texte didaktisiert, die dann unmittelbar

\footnotetext{
2 Auch wenn es sicherlich auch Ziel von Ausspracheschulung sein muss, die nichtmuttersprachlichen Teilnehmer selbst zu befähigen, zwischen falscher und richtiger Aussprache zu unterscheiden, bleibt meiner Erfahrung nach immer eine Grauzone bestehen, in der Uneinigkeit zwischen den nichtmuttersprachlichen Hörern besteht und gern ein muttersprachliches Ohr zur Klärung herangezogen wird.
} 
im Anschluss ausprobiert und evaluiert werden. So haben die Teilnehmer zum einen die Möglichkeit, gegen Ende des Kurses und kurz vor der Prüfung noch einmal Übungs- bzw. Wiederholungsschwerpunkte zu setzen, zum anderen werden sie motiviert, selbst einen Blick in Aussprachelernmaterialien zu werfen und eine Form von Ausspracheschulung zu planen, durchzuführen und zu evaluieren. Dies ist insofern wichtig, als dass natürlich auch viele von den Nichtmuttersprachlern angehende DaF-Lehrer sind.

\section{$7 \quad$ Erste Evaluation des Konzepts}

Eine umfassende Evaluation des vorgestellten Kurskonzeptes steht zwar noch aus, aber es gibt bereits erste positive Signale, wenn auch einschränkend einige Schwierigkeiten zu nennen sind.

Der Hauptkritikpunkt besteht sicherlich in dem relativ hohen Arbeitsaufwand, der sowohl für die Muttersprachler als auch für den Lehrenden mit diesem Konzept verbunden ist. Dem möchte ich allerdings grundsätzlich zwei Argumente entgegenhalten: Zum einen sind sprachpraktische Kurse immer mit einem relativ hohen Aufwand verbunden, wenn man nur an die Korrekturen von eingereichten Übungen und Tests denkt. Für einen geübten Hörer dauert die Auswertung einer Diagnose-Aufnahme ca. 10 Minuten, die anschließende Besprechung im Durchschnitt 20 Minuten. Wenn man die investierte Zeit mit der Zeit, die gewöhnlich für die Korrektur von Vokabel- und Grammatik-Tests notwendig ist, vergleicht, ist der Aufwand kaum höher. Zum anderen müssen die muttersprachlichen Studierenden auch in anderen Kursen vor- und nachbereiten, so dass der Unterschied eher nicht im Arbeitsumfang besteht, sondern darin, dass statt theoretischem Lesen praktisches Arbeiten gefordert ist, was vor allem ein Mehr an Organisation bedeutet. Auch für den Lehrenden ist festzustellen, dass das Verfahren durch die Schulungselemente zwar insgesamt relativ arbeitsintensiv ist, er jedoch im Gegenzug ja auch Hilfe bei der Auswertung der Diagnose-Aufnahmen bekommt und darüber hinaus im Kurs unterstützt wird ${ }^{3}$.

Weiter kann der Wert einer Diagnose-Aufnahme, die in diesem Konzept den Hauptarbeitsaufwand ausmacht, für die Ausspracheschulung meiner Erfahrung nach gar nicht hoch genug eingeschätzt werden. Sie liefert den Lernern eine individuelle Grundlage zur Arbeit an ihrer Aussprache. Einige wissen zwar schon sehr genau, wo ihre Schwierigkeiten liegen, andere jedoch kaum, und allen gemeinsam ist eine gewisse Unsicherheit bei der Selbsteinschätzung. Diese rührt nicht zuletzt daher, dass die Diagnose-Aufnahme für die meisten die erste strukturierte Rückmeldung ist, die sie auf ihre Aussprache bekommen. Der große Vorteil des Systems besteht außerdem darin, dass die Nichtmuttersprachler über die Diagnose-

\footnotetext{
${ }^{3}$ Eine weitere Möglichkeit, den Lehrenden zu entlasten, ist die Einstellung einer studentischen Hilfskraft. Ab dem kommenden Semester soll die Schulungsarbeit von einer Tutorin übernommen werden, die das Modell bereits selbst durchlaufen hat.
} 
Aufnahme hinaus im Rahmen des Kurses an Feedback und individueller Förderung ein Vielfaches von dem erhalten, was ein einziger Lehrender im Kurs zu leisten imstande wäre ${ }^{4}$. Dies ist auch insofern wichtig, als dass im Bereich der Aussprache nicht mit für alle Teilnehmer gültigen „Lösungsschlüsseln“ für durchgeführte Übungen gearbeitet werden kann, wie dies im Bereich der Lexik und Grammatik gang und gäbe ist. Vielmehr benötigt jeder Lerner ein individuelles Feedback, ob seine „Lösung“, d. h. seine Aussprache, richtig ist oder nicht. Die Muttersprachler erleben so gleichzeitig unmittelbar, welche Schwierigkeiten bei den unterschiedlichen phonetischen Kategorien auftreten und erproben Möglichkeiten, diese zu beheben.

Ein weiterer potentiell kritischer Punkt ist, dass natürlich nicht alle Muttersprachler gleich hoch motiviert, verantwortungsbewusst und kompetent sind. Insbesondere in Bezug auf die Auswertung und Besprechung der DiagnoseAufnahmen, aber auch in Bezug auf die Hilfe im Unterricht ist diese Schwierigkeit nicht von der Hand zu weisen, denn die Muttersprachler übernehmen in diesem Konzept viel Verantwortung für das Fortkommen der Nichtmuttersprachler. Ein gangbarer Weg scheint mir hier zu sein, die oben erwähnte Sprecherziehung (s. 3.) weiterhin als Standardkurs für die muttersprachlichen DaF-Studierenden beizubehalten und nur besonders interessierte und engagierte Muttersprachler in die Ausspracheschulung aufzunehmen. Jemand, der diesen Kurs nur „absitzt“, würde die Effizienz des vorgestellten Konzepts in erheblichem Maße stören. Genau in dieser Verantwortlichkeit der Muttersprachler liegt aber auch wiederum ein Vorteil des Konzeptes. Soft skills, wie die Übernahme von Verantwortung für andere, Selbstorganisation und Beratungskompetenz, werden geschult.

Ein weiterer positiver Nebeneffekt des vorgestellten Konzepts besteht darin, dass es Muttersprachler und Nichtmuttersprachler miteinander in einen relativ persönlichen Arbeitskontakt bringt. Dieses Kennenlernen und Zusammenarbeiten entsteht auch im Rahmen eines DaF-Studiengangs nicht immer automatisch, da doch zunächst einige Barrieren zu überwinden sind. Das gemeinsame Ziel kann hier integrierend wirken.

Positive Effekte des Konzepts lassen sich außerdem an der Semesterevaluation der Studierenden ablesen. Wie eine erste kleine Pilotstudie aus dem Sommersemester 2005 zeigt, wurden insbesondere die Diagnose-Aufnahmen und die Teilnahme der Muttersprachler von den Kursteilnehmern sehr positiv bewertet. Die Studierenden wurden am Ende des Kurses gebeten, alle im Kurs durchgeführten Übungen mit Schulnoten von eins bis sechs zu bewerten. Im Anschluss an diesen Fragebogen wurde noch eine offene Diskussion geführt. Die Diagnose-Aufnahme erhielt bei der Befragung die Bestnote, wurde $17 \mathrm{Mal}$ mit eins und $3 \mathrm{Mal}$ mit zwei

\footnotetext{
${ }^{4}$ Dass die Arbeit damit immer noch nicht komplett im Kurs erledigt werden kann, sondern dass die Lerner auch für sich unter der Woche regelmäßig trainieren müssen, ist sicherlich richtig. Aber die Grundlagen ihrer Arbeit sind viel besser. Die Weiterentwicklung von Selbstlernmaterialien zur Ausspracheschulung ist in diesem Zusammenhang als Desiderat zu nennen.
} 
bewertet. In der Abschlussdiskussion wurde außerdem das Engagement der Muttersprachler besonders gelobt ${ }^{5}$.

Zusammenfassend lässt sich sagen, dass die Integration muttersprachlicher DaF-Studierender in die Ausspracheschulung bzw. in sprachpraktische Kurse für nichtmuttersprachliche Studierende im Allgemeinen in zweifacher Hinsicht gewinnbringend sein kann: Während die Nichtmuttersprachler von der erheblich größeren Menge an Feedback profitieren, werden die Muttersprachler durch praxisbezogene Schulung und learning by doing in die Lage versetzt, Aussprache wirklich selbst vermitteln zu können ${ }^{6}$.

\section{Literatur}

Bunk, Gerhard J. S.: Phonetik aktuell, Ismaning: Hueber 2005.

Celce-Murcia, Marianne / Brinton, Donna M. / Goodwin, Janet M.: Teacbing pronunciation. A reference for teachers of English to speakers of other languages, Cambridge: Cambridge University Press 1996.

Cenoz, Jasone / Garcia Lecumberri, M. Luisa: „Learners’ views on the acquisition of an L2 phonetic system", PTLC 1999. Online: http:// wnw.phon.ucl.ac.uk/ home/johnm/vitoria.htm (3.7.2006)

Champagne-Muzar, C. / Schneiderman, E. I. / Bourdages, J. S.: „Second language accent. The role of the pedagogical environment", IRAL 16 (1993), 143-160.

de Bot, Kees / Mailfert, Kate: „The teaching of intonation. Fundamental research and classroom applications", TESOL Quaterly 16 (1982), 71-77.

Derwing, Tracey M. / Rossiter, Marian J.: „ESL learners' perceptions of their pronunciation needs and strategies", System 30 (2002), 155-166.

Derwing, Tracey / Munro, Murray J. / Wiebe, Grace: „Evidence in favour of a broad framework for pronunciation instruction", Language Learning 48 (1998), 393-410.

\footnotetext{
5 Auch Informationen aus Lernerbefragungen zur Ausspracheschulung könnten sehr zur weiteren Optimierung von Ausspracheschulung beitragen. Vgl. z. B. die Studie von Frey (1993), die interessanterweise auch den Wunsch nach individueller Betreuung der Lerner sehr deutlich zeigt. Vgl. ferner Studien aus dem anglophonen Bereich, z. B. de Bot und Mailfert (1982), Cenoz und Garcia Lecumberri (1999), Vitanova und Miller (2002), ferner Derwing und Rossiter (2002).

Neben Lerner- und Lehrerbefragungen fehlen im Bereich der Ausspracheschulung insbesondere empirische Untersuchungen, die die Effizienz vorgeschlagener Verfahrensweisen vergleichend prüfen.

${ }^{6}$ Herzlichen Dank an Claudia Riemer und Markus Schumacher für ihre hilfreichen Kommentare zu früheren Versionen dieses Aufsatzes.
} 
Dieling, Helga: „Phonetik in Lehrwerken Deutsch als Fremdsprache von 1980 bis 1992. Eine Analyse“. In: Breitung, Horst (Hrsg.): Phonetik. Intonation. Kommunikation, München: Rother Druck 1994, 13-20.

Dieling, Helga / Hirschfeld, Ursula: Phonetik lehren und lernen, Berlin u.a.: Langenscheidt 2000.

Dürrenmatt, Friedrich: Die Physiker. Komödie, Zürich: Diogenes 1998 (1962).

Frey, Evelyn: „Angewandte Phonetik im Unterricht Deutsch als Fremdsprache. Methoden und Erfahrungen“, Zielsprache Deutsch 24 (1993), 195-202.

Grotjahn, Rüdiger: „Ausspracheunterricht. Ausgewählte Befunde aus der Grundlagenforschung und didaktisch-methodische Implikationen“, Zeitschrift für Fremdsprachenforschung 9 (1998), 35-83.

Grotjahn, Rüdiger: „Der Faktor ,Alter‘ beim Fremdsprachenlernen. Mythen, Fakten, didaktisch-methodische Implikationen“, Deutsch als Fremdsprache 40 (2003), 32-41.

Hirschfeld, Ursula: „Vermittlung der Phonetik“. In: Helbig, Gerhard / Götze, Lutz / Henrici, Gert / Krumm, Hans-Jürgen (Hrsg.): Deutsch als Fremdsprache. Ein internationales Handbuch. 2. Halbband, Berlin; New York: de Gruyter 2001, 872-879.

Hirschfeld, Ursula: „Phonologie und Phonetik in Deutsch als Fremdsprache“. In: Altmayer, Claus / Forster, Roland (Hrsg.): Deutsch als Fremdsprache.

Wissenschaftsanspruch - Teilbereiche - Bezugsdisziplinen, Frankfurt am Main u.a.: Lang 2003, 189-223.

Kelz, Heinrich P.: „Lernziel deutsche Aussprache“. In: Vorderwülbecke, Klaus (Hrsg.): Phonetik, Ausspracheschulung und Sprecherziehung im Bereich Deutsch als Fremdsprache, Aachen: Becker-Kuns 1992, 23-38.

Lemcke, Christiane / Rohrmann, Lutz / Scherling, Theo: Berliner Platz 1. Deutsch im Alltag für Erwachsene. Lehr- und Arbeitsbuch 1. In Zusammenarbeit mit Anne Köker, Berlin u.a.: Langenscheidt 2002.

Macdonald, Doris / Yule, George / Powers, Maggie: „Attempts to improve English pronunciation. The variable effects of different types of instruction", Language Learning 44 (1994), 75-100.

Macdonald, Shem: „Pronunciation. Views and practices of reluctant teachers”, Prospect 17 (2003). Online: http://www.nceltr.mq.edu.au/prospect/17/pros17_3smac.asp (22.6.2006)

Pennington, Martha C.: „,The teachability of phonology in adulthood. A reexamination", IRAL 36 (1998), 323-341. 
Pennington, Martha C. / Richards, Jack C.: „Pronunciation revisited”, TESOL Quaterly 20 (1986), 207-225.

Purcell, Edward T. / Suter, Richard W.: „Predictors of pronunciation accuracy. A re-examination", Language Learning 30 (1980), 271-287.

Rausch, Rudolf / Rausch, Ilka: Deutsche Phonetik für Ausländer. 4. Auflage, Leipzig u.a.: Langenscheidt 1994.

Rebuschat, Patrick: „Aufmerksamkeit und Formfokussierung im Erwerb fremdsprachlicher Lautsysteme“. In: Börner, Wolfgang / Vogel, Klaus (Hrsg.): Emotion und Kognition im Fremdsprachenunterricht. Tübingen: Narr 2004, 217-241.

Richter, Julia: „Kursmaterialien“, Online 2006:

http:/ / www.uni-bielefeld.de/lili/studiengaenge/daf/personen/

richter_julia/lehre/ausspracheschulung/kursmaterialien/

Scarcella, Robin C. / Oxford, Rebecca L.: „Second language pronunciation. State of the art in instruction", System 22 (1994), 221-230.

Slembek, Edith: Lebrbuch der Febleranalyse und Feblertherapie. Deutsch bören, sprechen und schreiben. Für Lernende mit griechischer, italienischer, polnischer, russischer oder türkischer Muttersprache. 2., erweiterte Auflage, Heinsberg: Agentur Dieck 1995.

Suter, Richard W.: „Predictors of pronunciation accuracy in second language learning", Language Learning 26 (1976), 233-253.

Vitanova, Gergana / Miller, Ann: „Reflective practice in pronunciation learning”, The Internet TESL Journal 1 (2002). Online:

http:/ / iteslj.org/Articles/Vitanova-Pronunciation.html (22.6.2006)

Vorderwülbecke, Anne / Vorderwülbecke, Klaus: Stufen International. Deutsch als Fremdsprache für Jugendliche und Erwachsene. Lehr- und Arbeitsbuch 1, Stuttgart: Klett 1995.

Yule, George / Macdonald, Doris: „The different effects of pronunciation teaching", IRAL 33 (1995), 345-350. 



\section{Präsentationstraining auf der Mittelstufe}

Das menschliche Gehirn ist eine großartige Sache. Es funktioniert bis zu dem Zeitpunk.t, wo du aufstehst, um eine Rede zu halten.

Mark Twain

Susanne Duxa, Marburg

Im folgenden Beitrag werden das Trainingskonzept und Unterrichtsmaterialien vorgestellt, mit denen die Referentin seit zwei Semestern in studienbegleitenden Deutschkursen am Sprachenzentrum der Philipps-Universität Marburg arbeitet. Die Teilnehmer ${ }^{1}$ an den Lehrveranstaltungen Wie balte ich ein Referat? Präsentations-

\footnotetext{
${ }^{1}$ Im Folgenden wird zwecks besserer Lesbarkeit auf Doppelformen verzichtet. Mit der generisch verwendeten maskulinen Form sind immer beide Geschlechter gemeint.
} 
und Diskussionstraining ${ }^{2}$ sind Austauschstudierende aus verschiedenen Ländern, die Deutschkenntnisse unterhalb des für ein Vollstudium geforderten DSH 2 bzw. TDN 4 Niveaus haben; dennoch haben sie in den Lehrveranstaltungen ihrer jeweiligen Fachbereiche u.a. durch die Übernahme von Referaten Leistungspunkte zu erbringen. Während die Alltagskommunikation im universitären Betrieb diesen Studierenden wenig Schwierigkeiten bereitet, stellt das monologische Sprechen über abstraktere Themen für sie eine doppelte Herausforderung dar: Zum einen sind sie oft nicht damit vertraut, Lehrveranstaltungen durch eigene Präsentationen mit zu gestalten, zum anderen verfügen sie nur unzureichend über die dafür notwendigen spezifischen Deutschkenntnisse. Auf diese Diskrepanz reagieren die Studierenden nicht selten mit Panik und/oder mit Inanspruchnahme von Hilfeleistungen wie dem Übersetzen ihrer Vorlagen, durch die sie die benötigten Fähigkeiten nicht erwerben.

Das Präsentationstraining von 3 SWS soll den Studierenden für die Bewältigung dieser Herausforderung Planungs- und Handlungsstrategien verfügbar machen und ihnen so zu mehr Sicherheit und Erfolg verhelfen. Sie gliedert sich in einen einführenden Teil mit der Bewusstmachung von Erfolgskriterien für Präsentationen und mit der Erarbeitung der notwendigen Vorbereitungsschritte sowie in eine Übungsphase, in der die Umsetzung der zuvor erarbeiteten Grundsätze in eigenen MiniPräsentationen von 10-15 Minuten Dauer erprobt und reflektiert wird.

\section{$1 \quad$ Lernziele und Bewertung}

Als wichtigstes Lernziel gilt es zunächst, den Kursteilnehmern die Prinzipien für das Gelingen von Präsentationen bewusst zu machen und diese durch praktische Übungen für ihr Verhaltensrepertoire verfügbar zu machen. Dazu gehört die Einsicht, dass der Erfolg einer Präsentation von mehr Aspekten als der sprachlichen Gestalt des gesprochenen Textes abhängig ist und dass diese wiederum mehr umfasst als grammatisch und lexikalisch korrekt formulierte Sätze. Nur wenn hier Überzeugungsarbeit geleistet wird, sehen Studierende davon ab, in ihren Referaten größere ,Anleihen' bei schriftsprachlichen Vorlagen zu machen und/oder ihre von muttersprachlichen Kommilitonen korrigierten ausformulierten Vorlagen abzulesen oder auswendig gelernt ,abzuspulen'.

Ein weiteres wichtiges Ziel ist die Befähigung zur Selbst- und Fremdevaluation anhand von Kriterien, die auf den o. g. Prinzipien beruhen und auf die man sich für die praktischen Phasen des Kurses geeinigt hat.

\footnotetext{
2 Im Veranstaltungstitel werden sowohl der an der Universität übliche Terminus Referat als auch der weitere Begriff Präsentation verwendet; da die Teilnehmer aus unterschiedlichen Fachrichtungen kommen, tragen sie in der Veranstaltung allerdings nicht zu fachspezifischen akademischen Themen vor, so dass als gemeinsamer Nenner beider Begriffe die Vermittlungsfähigkeit als grundlegende transferierbare Kompetenz im Vordergrund steht.
} 
Bedingung für das Erlangen eines Leistungsnachweises ist neben der regelmäBigen Mitarbeit die Vorbereitung und Durchführung einer eigenen Präsentation inklusive der Anfertigung von Hand-outs o. ä. sowie einer nachträglichen schriftlichen Reflexion des eigenen Lernprozesses, in die die Rückmeldung der Zuhörer einfließt.

\section{$2 \quad$ Einstieg}

Um die Teilnehmer von Anfang an für die Kursziele zu sensibilisieren, sind bereits die Kennenlern-Aktivitäten in der ersten Sitzung auf die Selbsterfahrung des Kontrasts zwischen dem seminarüblichen dialogischen Sprechen im Sitzen und einem monologischen Sprechen vor der gesamten Gruppe im Stehen ausgerichtet, indem die Teilnehmer sich zunächst bei einem Speed Dating am Tisch nacheinander mit jeweils nur einer anderen Person bekannt machen und anschließend in einem Kreis stehend anhand eines gewählten Objekts oder einer gewählten Bildpostkarte über ihre Einstellung zum Kursthema und ihre Motivation zum Besuch des Kurses sprechen. Schon hier kann durch gelenkte Fragen oder Spiegeln lassen von Körperhaltungen das Bewusstsein für die bedeutsame Wirkung von Blickkontakt und Körpersprache geschärft werden.

Als eine Art Ausgangsbasis für die weitere Arbeit wird das hier bewusst Gemachte und das dadurch aktivierte Vorwissen in einer ersten Kriterienliste für gelungene Präsentationen fixiert und für alle sichtbar im Kursraum ausgehängt, damit die Kriterien immer präsent sind und im weiteren Verlauf des Kurses ergänzt bzw. verfeinert werden können ${ }^{3}$. Dabei ist vor allem der Konkretheitsgrad der Kriterien wichtig, da sie in der Praxisphase des Kurses als Maßstab für die Evaluationen der Präsentationen dienen sollen ${ }^{4}$. Sollte die erste Ausbeute eher dürftig ausfallen, können Texte wie Tucholskys Ratschläge für einen schlecbten Redner ${ }^{5}$ weitere Gesichtspunkte liefern.

Um für die nächsten Schritte der Grundlagenerarbeitung den Kenntnisstand der Gruppenmitglieder besser einschätzen zu können, bieten sich zum Abschluss des Seminareinstiegs Aktivitäten wie eine Erfahrungsschlange an. Es zeigt sich nämlich immer wieder, dass große Unterschiede in dem Grad an Vorab-Training in Schule oder heimischer Universität bestehen.

\footnotetext{
${ }^{3}$ Eine Verfeinerung ist wichtig, da die genannten Kriterien häufig wenig operationalisierbar sind, z. B. Der Inhalt muss gut organisiert sein.

${ }_{4}^{4}$ Als Beispiel für eine im Kursverlauf (üb)erarbeitete Zusammenstellung von Kriterien ist im Anhang eine Mindmap beigefügt (= Anlage 1).

${ }_{5}$ Eine bebilderte Version des Texts mitsamt Audiodatei ist im Internet $\mathrm{zu}$ finden unter:

http://www.mediaculture-online.de/Ratschlaege.381.0.html (15.10.2006)
} 


\section{Drei Schritte für die Vorbereitung einer Präsentation}

Die allgemeine, gemeinsame Vorbereitung der studentischen Präsentationen gliedert sich in die drei Schritte Planungsfaktoren Zubörer, Rahmenbedingungen, Situation sowie Strukturierung des Inhalts und Medieneinsatz. Sie umfasst mindestens 3 Seminarsitzungen und wird von der Lehrperson angeleitet, wobei je nach Vorwissen der Studierenden einzelne Teilnehmer einbezogen werden können.

\subsection{Planungsfaktoren Zuhörer, Rahmenbedingungen und Situation}

Erfahrungsgemäß neigen Studierende mit wenig Präsentationserfahrung dazu, bei der Planung eines Referates ausschließlich dessen Thema und vielleicht noch die Zeitvorgabe vor Augen zu haben, so dass sie die Vermittlungssituation und insbesondere ihre Zuhörer mit deren Erwartungen und Bedürfnissen ausblenden. Häufig zu erlebende Konsequenzen sind dann beispielsweise das Fixieren des Blickkontakts auf die Seminarleiterin oder das Jonglieren mit selbst soeben erst erworbenem Fachvokabular ohne Rücksicht auf Verständlichkeit. Einem solchen Fehlverhalten kann mit einem grundlegenden Kommunikationsmodell auf der Basis von Bühlers Organon-Modell' ${ }^{6}$ entgegen gewirkt werden, indem die Studierenden sich der Wichtigkeit von Hörer-Sprecher-Beziehungen und der Einbeziehung der situativen Rahmenbedingungen ihrer Vortragssituation für eine gelungene Kommunikation bewusst werden: Wer spricht wo zu wem warum wie?

Verdeutlicht wird dies durch eine Übung, in der die Studierenden in Partneroder Gruppenarbeit für die Gestaltung von verschiedenen Präsentationen zu einem identischen Grundthema Vorüberlegungen hinsichtlich der Zielsetzungen, Inhalte und rhetorischen Mittel treffen sollen. Indem die Faktoren Rabmensituation und Zubörer bei den verschiedenen Gruppen jeweils unterschiedlich sind, wird bei der Auswertung der Gruppenarbeit die Tragweite dieser Planungsfaktoren auf wirkungsvolle Weise evident ${ }^{7}$.

Die Umsetzung dieser Erfahrungen in das eigene Tun erfolgt anschließend in einer Hausaufgabe, bei der die Studierenden aus einer Reihe vorgeschlagener Themenstellungen für ein Kurzreferat zwei Themen auswählen, zu denen sie in Gedanken einen kurzen Beitrag für die Gruppe der Kursteilnehmer entwerfen. Die Aufgabe besteht darin, zu jedem Thema einen Eingangssatz zu formulieren und aufzuschreiben, aus dem das Ziel dieses gedachten Beitrags hervorgeht. Nacheinander werden dann in der nächsten Sitzung diese Eingangssätze vor dem Plenum präsentiert und ein Abgleich zwischen der eigenen Planung und den durch den Satz geweckten Zuhörererwartungen vorgenommen. Falls hierbei gröBere Diskrepanzen festgestellt werden, wird der Eingangssatz gemeinsam revidiert

\footnotetext{
${ }^{6} \mathrm{Vgl}$. Anlage $2 \mathrm{im}$ Anhang.

7 Ein Beispiel für diese Ubung ist als Anlage 3 beigefügt. Die dort zur Verfügung gestellten Auswahlantworten können bei fortgeschrittenen Gruppen weggelassen werden.
} 
und noch einmal vorgetragen. Die Beschränkung auf ein oder zwei Äußerungen macht einsichtig, welche zentrale Rolle die Zuhörerorientierung am Anfang einer Präsentation spielt, d. h. wie die Zuhörer hier bereits ,gewonnen' oder ,verloren werden können. Zudem wird schüchternen Teilnehmern durch die Kürze des Auftritts vor der Gruppe eine Brücke zu längeren Präsentationen gebaut ${ }^{8}$. Mit den korrigierten Versionen der Eingangssätze aus der Gruppe und den ergänzenden Beispielen für Zielsatzformulierungen von der Dozentin starten die Studierenden ihre eigene Sammlung von Redemitteln für die verschiedenen Phasen einer Präsentation, die im weiteren Seminarverlauf ergänzt wird.

\subsection{Planung des Inhalts und der Struktur}

Der Auftrag für die nächste Sitzung lautet, für die weitere Planungsarbeit eine Stoffsammlung zu einem der gewählten Themen aus dieser letzten Hausaufgabe zu machen. Im Unterricht gleichen Studierende mit identischer Themenwahl in Partnerarbeit ihre Stoffsammlungen ab und einigen sich auf eine Anordnung als ersten groben Plan für die Struktur ihres Referats, den sie dem Plenum auf Folie oder als Wandplakat vorstellen. Der Vergleich dieser Gliederungsentwürfe lässt verschiedene Strategien bei diesem Planungsschritt erkennen, z. B. die Vorteile einer graphisch strukturierten Inhaltsplanung vor einer linearen, in die spätere Änderungen schwerer einzufügen sind. Sollten sich solche Vergleichsmöglichkeiten nicht, von selbst ${ }^{\star}$ ergeben, ist hier ein Vorstellen und Erläutern alternativer Möglichkeiten wie Mindmaps, Flussdiagramme o. ä. angebracht.

Da bei den Präsentationen der eigenen Gliederungsmuster einfache Strukturen wie additives Nebeneinander oder chronologisches Hintereinander weit häufiger vorkommen als komplexere logische Strukturen wie etwa einerseits-andererseits, schließen sich weitere Übungen an, bei der visualisierte Strukturen fremder Textentwürfe gemeinsam nachvollzogen werden bzw. ,leere' Strukturvorgaben zu einem Thema mit Inhalten gefüllt werden ${ }^{9}$. Auf diese Weise soll der ,Röntgenblick ${ }^{6}$ für die Gliederungsstruktur eines Textes trainiert und das Spektrum eigener Handlungsmöglichkeiten erweitert werden. Erfahrungsgemäß ist für das Unterrichtsgespräch über verschiedene textgrammatische Funktionen intensive Vokabelarbeit notwendig (z. B. Ausgangsfrage: $\rightarrow$ sich mit einer Frage beschäftigen, einer Frage nachgehen etc.; Übergänge $\rightarrow$ die Kernpunkte zusammenfassen, zum nächsten Punkt kommen etc.).

\footnotetext{
${ }^{8}$ Je nachdem, wie gelassen oder nervös die Kursteilnehmer mit dieser Aufgabe umgehen, können diese ersten Mini-Präsentationen auf Video aufgezeichnet werden. In dem Fall hat man für den Seminarabschluss erfahrungsgemäß ebenso eindrucksvolle wie erheiternde Bilder für einen VorherNachher Vergleich. Mehr dazu in Kapitel 5.3.

${ }^{9}$ Ein Beispiel dafür findet sich im Anhang als Anlage 4. Als weiteres Training bietet sich hier natürlich auch die Analyse von Musterreden an, ähnlich wie es beim Training von Leseverstehen praktiziert wird.
} 


\subsection{Einsatz von Medien}

Die Sitzung zum Erstellen visueller Hilfsmittel und zum Medieneinsatz verläuft vornehmlich als Anschauungsunterricht; (möglichst authentische) positive und negative Beispiele hinsichtlich der Faktoren Aufmerksamkeitslenkung, Informationsfülle, Schriftgröße, Prägnanz von Folien sprechen eine deutlichere Sprache als langatmige Belehrungen.

Auch werden Studierende animiert, auf eigene Erfahrungen zurück zu greifen und daraus Empfehlungen und Warnungen für den Medieneinsatz abzuleiten, die sich im Sinne von Dos and Don'ts gut als Zickzack-Sprechübung präsentieren lassen, um den Sprechanteil der Teilnehmer nicht übermäßig hinter dem Informationsinput seitens der Dozentin zurück stehen zu lassen.

Spätestens an dieser Stelle werden durch spielerische Aufgabenstellungen auch Berührungsängste mit Overheadprojektoren, Beamern etc. abgebaut, und es wird den Studierenden klar gemacht, wie gut sie sich bereits mit den Gegebenheiten des Raumes, in dem sie in nächster Zeit eine Präsentation halten werden, vertraut gemacht haben.

Eine Erfahrung, die ich in der Arbeit mit Studierenden häufig gemacht habe, ist die, dass Studierende zwar anschauliche Folien erstellen, sie nach dem Auflegen aber nicht wirklich in ihren Vortrag einbinden, so dass ihre unterstützende Funktion nicht zum Tragen kommt. Das bei den Studierenden immer populärer werdende Power Point lässt dieses Manko nicht aufkommen und bietet zugleich eine Alternative für das Erstellen von traditionellen Formen der Gedächtnisstütze wie beispielsweise Karteikarten. Das Medium erfordert freilich eigene Grundsätze für eine sinnvolle Unterstützung von Präsentationen, die in den meisten Handbüchern zum Präsentationstraining noch nicht ausreichend thematisiert sind und deshalb umso mehr im Kurs reflektiert werden müssen.

Damit die Kriterien für eine effektive Gestaltung und Verwendung visueller Hilfsmittel nicht in Vergessenheit geraten, fertigen die Studierenden Checklists darüber an. Als Muster für eine solche Liste erhalten sie eine Checklist für das Anfertigen eines Hand-outs, die sie bei der Ausarbeitung ihrer Referate zu Hilfe ziehen können.

Zum Abschluss der allgemeinen Planungsphase werden die zwischenzeitlich ergänzten Kriterien für eine gelungene Präsentation aus der ersten Sitzung noch einmal gesichtet und revidiert. Sie sind Grundlage für das gemeinsame Erstellen des Feedbackbogens, mit dem im zweiten Kursteil individuelle Rückmeldungen auf jede Präsentation gegeben werden ${ }^{10}$.

\section{Die Übungsphase}

Die praktische Phase des Seminars beginnt in der Regel mit der fünften Sitzung. Zuvor haben die Studierenden sich allein oder zu zweit mit einem Referatthema in

\footnotetext{
${ }^{10}$ Ein Beispiel für einen solchen Bogen ist im Anhang als Anlage 5 beigefügt.
} 
einen Sitzungsplan eingetragen ${ }^{11}$. Jede Präsentation wird spätestens drei Tage zuvor mit der Dozentin durchgesprochen und ggf. modifiziert. Entsprechend den im Seminar durchlaufenen Planungsschritten stellen die Studierenden bei diesem Vorgespräch ihre Zielsetzung und die Struktur ihrer Präsentation vor und zeigen die Medien, die sie einsetzen wollen. Diese Vorgespräche können zwischen 15 und 90 Minuten dauern; eine Zeitinvestition, die angesichts der positiven Bilanz lohnenswert erscheint, dass bis dato alle Studierenden eine zufriedenstellende Präsentation haben geben können und niemand ein Misserfolgserlebnis hat erleben müssen. Auch wird in den Seminarauswertungen - insbesondere von den Teilnehmern mit wenig Präsentationserfahrung - immer wieder betont, wie entlastend diese ,Vorrunde auf die Studierenden wirkt und als wie hilfreich sie die individuelle Beratung empfinden ${ }^{12}$.

Auf die Durchführung der Präsentation im Kurs und die sich anschließende Frage- bzw. Diskussionsrunde erfolgt als nächstes eine kurze Feedbackrunde im Plenum mit der Reihenfolge Referent/en, Zuhörer, Kursleiterin. Sie dient in erster Linie dazu, den/die Referenten zu entlasten und ihm/ihnen die Möglichkeit zu geben, auf spezielle Fragen eine Rückmeldung zu bekommen. Auch wenn Regeln für ein konstruktives Feedback zuvor abgesprochen wurden, erfordert es in der Regel einiges Einüben, bis sie konsequent angewendet werden: Positives zuerst! Direkt den/die Referenten ansprechen! Ich-Äußerungen! Das umfassendere Feedback wird mittels des zuvor erarbeiteten Feedbackbogens schriftlich fixiert und direkt den Referenten übergeben, damit diese es in Ruhe aufnehmen können. Wie nachhaltig diese Rückmeldungen von Kommilitonen sein können, zeigt folgender Auszug aus einer Selbstreflexion, die eine Referentin am Kursende einreichte:

„Bei Inhalt und Struktur waren meine Zubörer fast alle einverstanden, dass alles nachvollziehbar strukturiert wurde und dass die Beispiele gut veranschaulichten. (...) Viele haben mir geschrieben, dass sie mich nicht immer so gut verstanden baben, wegen meiner zu schnell Sprechgeschwindigkeit, der undeutlichen Aussprache und meiner eintönigen Stimme. Ich brauche mehr üben und ich werde nächstes Mal alles mehr auswendig lernen, oder mindestens die längere Sätze."

Ein weiterer Inhalt in der Übungsphase richtet sich auf die Interaktion nach dem Referat, auf die ich hier aus Platzgründen nur ganz kurz eingehen werde. In Einklang mit der Zuhörerorientierung bei der Planung und Durchführung einer Präsentation ist es die Aufgabe der Referenten, stimulierende Fragen oder Appelle für die Diskussionsrunde zu überlegen. Bei den Auswertungsgesprächen stellt sich allerdings häufig heraus, dass dieser Teil einer Präsentation aufgrund seiner Unvorhersehbarkeit am meisten angstbesetzt ist. Um den Studierenden hier Strate-

\footnotetext{
${ }^{11}$ Das paarweise Referieren hat sich doppelt bewährt: zum einen kommt so auch in größeren Gruppen jeder Studierende zum Zuge und zum anderen fördert das gemeinsame Planen ein umsichtiges Durchdenken der Gliederung und des Vortrags der Präsentation.

12 Vgl. hierzu aber einschränkend Kapitel 5.2
} 
gien an die Hand zu geben, werden Übungen zum Thema Verständlich Fragen stellen/Souverän auf Fragen reagieren gemacht, bei denen zunächst die Prinzipien verdeutlicht und dann sprachliche Realisierungsmöglichkeiten zusammengestellt werden. Auf diese Bewusstmachung von Möglichkeiten erfolgt die Übung z. T. in Rollenspielen mit verschriftlichten Szenarios und Rollenkarten, um Verhaltensoptionen in einem möglichst breiten Situationsspektrum erproben zu können.

Hier zeigt sich auch der fließende Übergang von Übungen, die auf die Förderung von Interaktivität ausgerichtet sind und solchen, die der Korrektur und Erweiterung der sprachlichen Äußerungen dienen. Für letzteres ist am Ende jeder Sitzung eine Phase vorgesehen; hier kommen revisionsbedürftige Passagen aus den Präsentationen oder den anschließenden Diskussionen zur Sprache, wobei in erster Linie auf die Beachtung der vereinbarten Grundsätze sowie auf Verständlichkeit geachtet wird.

\section{$5 \quad$ Entscheidungsfragen für Lehrende}

\subsection{Themen vorgeben oder nicht?}

Eine erste Frage bei der Planung der studentischen Präsentationen ist die, ob Themen dafür vorgegeben werden sollten oder nicht. Dass in akademischen Veranstaltungen in der Regel Referate zu bestimmten Themen vergeben werden, kann einerseits als Plädoyer dafür gesehen werden, im Trainingsseminar ebenso zu verfahren. Auf diese Weise ist es auch möglich, unterschiedliche Textstrategien präsent zu haben (Darstellung eines Sachverhalts; Vergleiche von verschiedenen Systemen oder von Vor- und Nachteilen; Entwicklung einer logischen Argumentation etc.). Die besten Referate in meinen Veranstaltungen waren allerdings solche, wo Studierende z. T. zu recht ungewöhnlichen Themen referiert und dabei großes persönliches Engagement gezeigt haben, das in einem fremdbestimmten Thema so vielleicht nicht entstanden wäre. Am besten erscheint es mir daher, eine Themenpalette für Unentschlossene bereit zu halten, sie aber nicht zur Pflicht zu machen.

\subsection{Was und wie viel kommentieren?}

Ähnlich wie bei der Fehlerkorrektur im Sprachunterricht stellt sich beim Präsentationstraining für die Lehrenden die Frage, was und wie viel sie kommentieren sollen. So muss die Dozentin bei der Vorbesprechung abwägen, wie weit sie Studierende aus eigenen Erfahrungen lernen lassen will, oder ob sie ihnen durch Einflussnahme vorab bestimmte Erfahrungen ersparen will. Maßgeblich ist hier letzten Endes die Verantwortung für den Erfolg des Gesamtseminars; es wäre es sicherlich sehr motivationshemmend, wenn mehrere Präsentationen hintereinander nicht als gelungen bezeichnet werden könnten, weil grundlegende Prinzipien 
nicht ausreichend berücksichtigt wurden. Gleichwohl kann es frustrierend für die Dozentin sein, bei den Vorgesprächen durch zeitaufwendige Verbesserungsvorschläge wiederholt die gleichen Fehler auszumerzen, die so nie Gesprächsgegenstand im Plenum werden. Hier muss ein jeder für sich entscheiden, wie er im Einzelnen verfahren will. Die Grenze ist meiner Meinung nach auf jeden Fall da zu ziehen, wo Studierende jegliche Appelle an ein vernünftiges Zeitmanagement außer Acht schlagen und schlicht und ergreifend schlecht vorbereitet sind. Solche Versäumnisse kann ein Präsentationstraining nicht vermeiden helfen, da ist ein Seminar zu Zeitmanagement anzuraten.

Wie die Reflexion einer Studentin oben zeigte, sind die Rückmeldungen der anderen Kursteilnehmer häufig effektiver als die der Kursleiterin, so dass so manche Lernerfahrung getrost dem Feedback durch die Zuhörer überlassen werden kann. Dies kann aber nur funktionieren, wenn die Zuhörer die Präsentation auch kritisch genug verfolgen und sich nicht einfach ,berieseln` lassen. Zur Erhöhung der Zuhörer-Aufmerksamkeit hat sich eine Übung bewährt, bei der die Studierenden nach beendeter Präsentation die Aufgabe bekommen, gemeinsam mit einem Partner den Verlauf der Präsentation zu rekonstruieren. Für den Fall, dass sie an einer Stelle Unstimmigkeiten entdecken oder ihnen der weitere Verlauf unklar ist, sollen sie klären, wo die Ursache dafür liegt und zur Klärung Fragen an den Referenten formulieren. Stellt sich in der anschließenden Fragerunde heraus, dass es häufige Übereinstimmung bei der Identifikation solch kritischer Stellen in der Präsentation gibt, ist dies ein wichtiges Zeichen zur Revisionserfordernis für den Referenten, die andernfalls vielleicht nicht zutage getreten wäre.

\subsection{Videoaufzeichnungen auf jeden Fall?}

Eine letzte Frage ist die nach der Bedeutung von Videoaufzeichnungen der studentischen Präsentationen für die Trainingsarbeit. Hier kann es kein deutliches Votum geben, sondern es ist zwischen Vor- und Nachteilen abzuwägen. Der von mir sehr geschätzten Möglichkeit des „Erkenne Dich selbst“ steht der Kommentar nicht weniger Seminarteilnehmer gegenüber, dass das Arbeiten damit ein Grund sei, sich nicht für das Training anzumelden (bei Vorab-Ankündigung) oder ihm fernzubleiben (bei konsequentem Einsatz), weil das Thema schon so angstbesetzt genug sei.

Einerseits ermöglicht es das Medium, Verhaltensalternativen direkt am Beispiel auszuprobieren, doch birgt das zuvor als zu revidieren identifizierte Verhalten andererseits immer auch die Gefahr der Bloßstellung. Spielt dagegen die Seminarleiterin ein bestimmtes Verhalten nach und überzieht es dabei ausdrücklich, macht sie sich selbst zum Objekt der Lacher und lenkt von der eigentlichen Person ab. Doch auch diese Strategie ist selbstverständlich kein Patentrezept, sondern muss mit Vorsicht und Blick auf die jeweiligen Teilnehmer abgewogen werden.

Videomitschnitte ermöglichen des Weiteren stellenweise Texttranskriptionen, um den Studierenden sowohl ihre Stärken deutlich zu machen (Diese Formulierungen 
können Sie sich merken!), als auch an immer wiederkehrenden Schwachstellen zu arbeiten (Wie kann man dies besser formulieren?). Hier dient der Mitschnitt aber eher der Unterrichtsvorbereitung der Lehrenden als dem Einsatz im Unterricht, da das Spulen von einer signifikanten Stelle zur anderen schnell lästig wird.

Ein großer Erfolg kann schließlich ein Zusammenschnitt von lustigen und/ oder beeindruckenden Momenten aus dem Kursverlauf sein, der - mit Zwischentiteln versehen - in der letzten Seminarsitzung gemeinsam angeschaut wird und häufig sehr eindrucksvoll das Ergebnis des Trainingsprozesses zeigt. Kopien davon werden von den Studierenden gerne als Dokumentation ihrer erworbenen Fähigkeiten mit nach Hause genommen.

\section{Schluss}

Auf der Mittelstufe kämpfen Lernende wie Lehrende häufig mit dem Phänomen, dass der Lernerfolg nicht mehr so deutlich spürbar ist und die Motivation zur weiteren Anstrengung darunter leidet. Daher gehört es zu den schönsten Erfolgen eines Präsentationstrainings, wenn ein sprachlich eher schwacher Teilnehmer aufgrund einer konsequenten Einbeziehung seiner Zuhörer, einer nachvollziehbaren Struktur seiner Ausführungen, prägnanter Beispiele mit angemessen eingesetzten Visualisierungen etc. trotz vieler kleinerer sprachlicher Fehler eine von allen gut verstandene und gut bewertete Präsentation gibt. Für den Betreffenden ist das ein ungeheures Erfolgserlebnis und für die leistungsstärkeren Studierenden eine große Motivation, sich nicht auf ihre guten Sprachkenntnisse zu verlassen, die für sich allein genommen eben noch keine Garantie für eine gut gelungene Präsentation darstellen.

\section{Literatur:}

Bartsch, Tim Christian u.a.: Trainingsbuch Rhetorik. Paderborn: Schöningh 2005

Franck, Norbert: Fit für's Studium. Erfolgreich reden, lesen, schreiben. München: dtv 1999

Franck, Norbert: Rhetorik für Wissenschaftler. Selbstbewusst auftreten, selbstsicher reden. München: Vahlen 2001

Mehlhorn, Grit (unter Mitarbeit von Bausch, Karl-Richard u. a.): Studienbegleitung für ausländische Studierende an deutschen Hochschulen. München: iudicium 2005

Papst-Weinschenk, Marita: Reden im Studium. Ein Trainingsprogramm. Berlin: Cornelsen / Scriptor 1995 
Anhang:

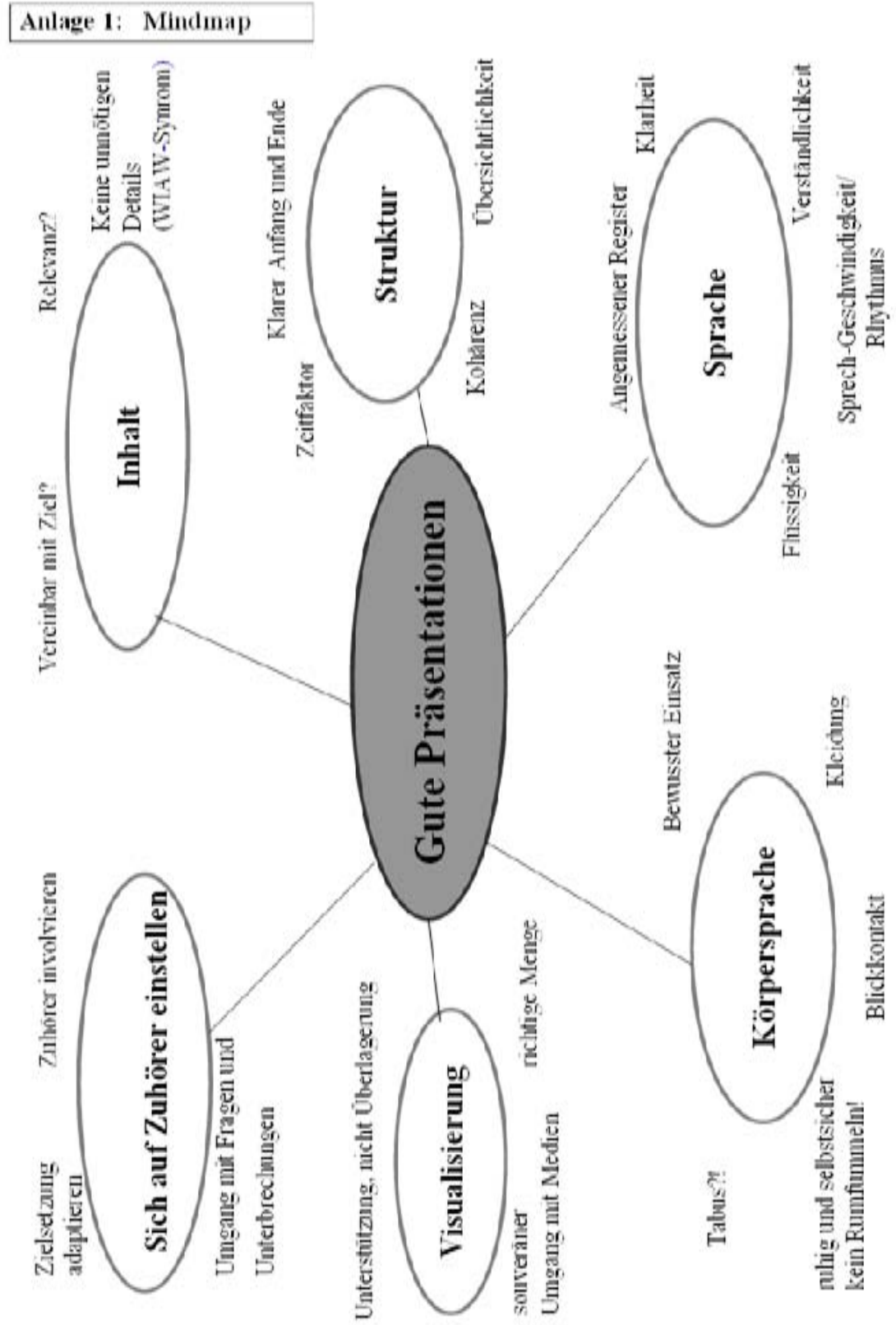




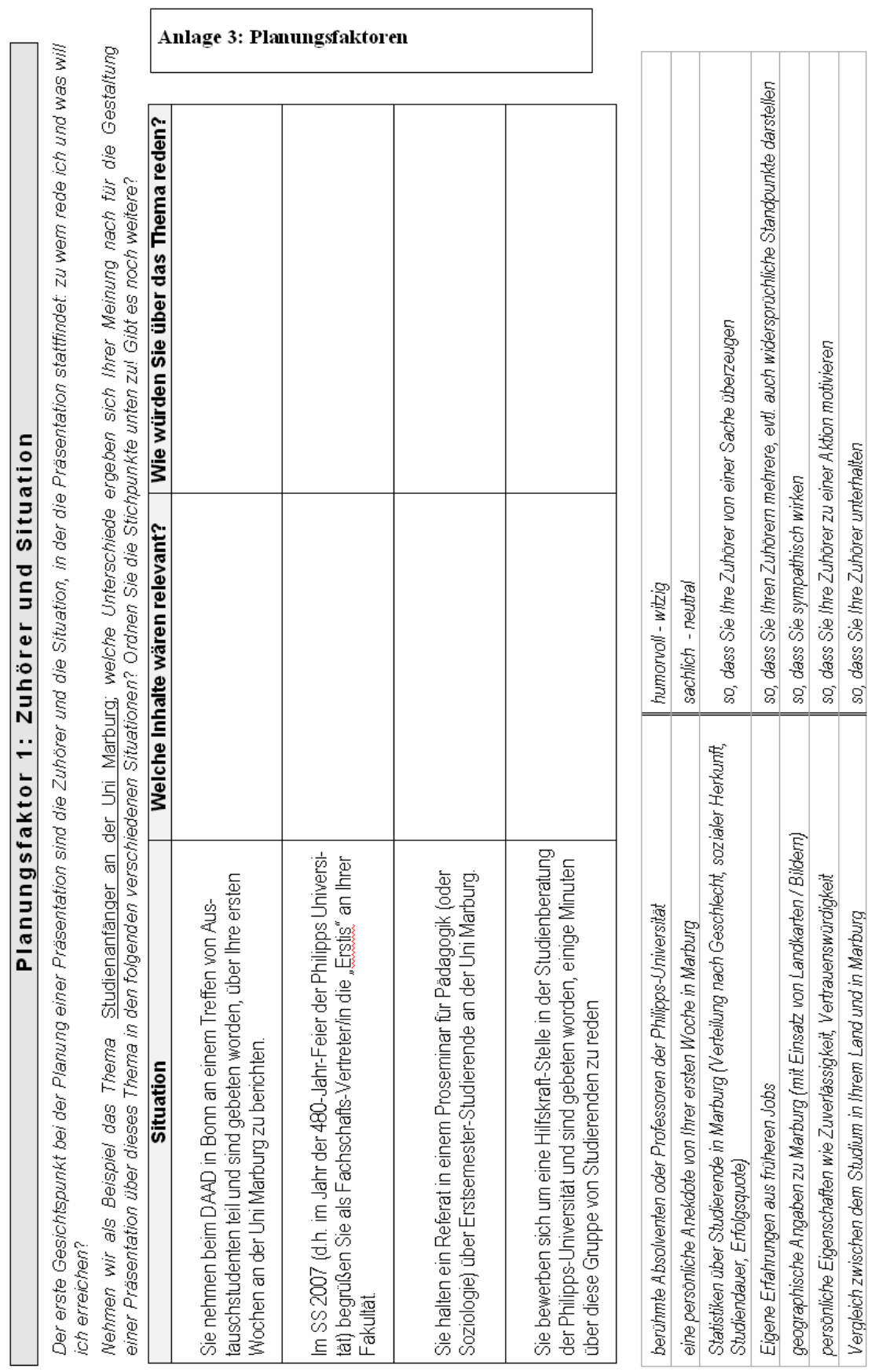




\section{Akademisches Schreiben für ausländische Studieren- de: Ein Lehr- und Übungsbuch für Fortgeschrittene}

Christiane Beinke/Stefan Thürmer, Osnabrück

Melanie Brinkschulte/Lothar Bunn, Münster

Lehrenden in universitären DaF-Schreibkursen dürften Texte wie der folgende vertraut $\operatorname{sein}^{1}$ :

o Ethik im Internet.

Heute zu Tage fast alles oder alles ist möglich im Internet. Wie weit kann man kommen? Heute es ist möglich sogar Kinder im Internet einkaufen und verkaufen. Und was noch? Ich meine, dass wenn Personen sind als Ware benutzt alles das übrige ist auch möglich. Wer kann sagen wie viel kostet eine Person? Es ist möglich Preis zu einer Person stellen? Ich würde nicht sagen, aber es gibt Leute die importieren sich nicht was Sie machen um Geld zu verdienen. Das ist das wichtigste und größste Problem auf dem Welt und deswegen muss man bestimmte Grenzen einfübren, weil Ethik existiert nicht.

1 Text einer Studentin aus einem UNIcert®-II-Schreibkurs der Universität Münster (WS 2004/2005). 
Die Verfasserin bemüht sich, wie bereits in ihrer - hier nicht abgedruckten Einleitung angekündigt, eine Fragestellung zu entwickeln, diese zu beantworten und auch den Konventionen, wie sie für akademische Texte gelten, gerecht zu werden. Ins Auge fallen jedoch neben den deutlichen sprachlichen Mängeln auch die fehlende Leserorientierung, die durchgängig fehlenden Belege sowie der subjektive Schreibstil.

Wesentliche Probleme für nichtdeutsche (und deutsche) Studierende bei der Studienbewältigung bestehen erstens in der fehlenden Kenntnis darüber, welche Konventionen eine Haus- oder auch Abschlussarbeit erfüllen muss und zweitens in der erfolgreichen Bewältigung des Schreibprozesses. Die auf dem Markt vorhandenen Einfübrungen in das wissenschaftliche Schreiben können bei diesen Problemen nicht helfen: Einerseits richten sie sich nicht speziell an Deutschlernende und berücksichtigen demnach nicht deren spezifische Schwierigkeiten. Andererseits lassen sie den Prozess des Schreibens größtenteils unberücksichtigt.

Diese beiden Lücken soll unser Lehr- und Übungsbuch füllen. Durch methodische Anleitungen sowie systematische Schreibübungen sollen die Studierenden Schritt für Schritt in die Lage versetzt werden, die formale und inhaltliche Gestaltung einer Hausarbeit nachzuvollziehen, dieses Wissen zunächst in einzelnen Phasen praktisch umzusetzen, um dann auf dieser Basis eine eigenständige Hausarbeit selber zu verfassen.

\section{$1 \quad$ Hintergründe der Lehrbuchentwicklung}

Seit Ende der 90er Jahre werden an der Westfälischen Wilhelms-Universität Münster mit zunehmendem Erfolg - die Teilnehmerzahlen hatten sich zwischen dem Sommersemester 1998 und dem Sommersemester 2000 verdoppelt Schreibkurse auf Mittel- und Oberstufenniveau (B1/B2-UNIcert ${ }^{\circledR}$ II; C1UNIcert ${ }^{\circledR}$ III) durchgeführt. Um die Unterrichtskonzeption und die Fortentwicklung der Schreibkurse empirisch abzusichern, haben wir im Wintersemester 2000/01 eine umfangreiche Befragung von Lehrenden der Universität und internationalen Studierenden durchgeführt.

Ermittelt werden sollten auf der einen Seite die Anforderungen der Lehrenden an die Qualität der geschriebenen Texte der internationalen Studierenden. Auf der anderen Seite wollten wir in Erfahrung bringen, welche Erwartungen internationale Studierende an die Schreibkurse haben und über welche Schreib-Erfahrungen sie bereits verfügen.

Die Befragung der Lehrenden² ergab, dass ihre Ansprüche an die Qualität der Arbeiten internationaler Studierender nur unwesentlich von denjenigen abwichen,

\footnotetext{
${ }^{2}$ Der Fragebogen wurde von 75 Lehrenden der geistes-, sozial-, rechts- und wirtschaftswissenschaftlichen Fakultäten der WWU beantwortet.
} 
die sie an deutsche Studierende stellten. Ihre konkreten Anforderungen betrafen vor allem

die Form:

„denselben Anforderungen wie deutsche Studierende genügen"

"dass es eine Einleitung, dass es einen Schluss geben muss"

„,korrektes Erscheinungsbild"

"Zitierregeln beachten"

den Inhalt:

"Logisch einwandfreie Argumentationen"

„eine eigenständige Behandlung von Literatur mit einer kritischen Auseinandersetzung anhand einer Fragestellung"

die Sprache:

„Dass der Text nicht perfekt ist, ist kein Problem, wenn inhaltlich die Sache erkannt ist." „da Germanistik - gut!"

Generell hat die Befragung der Studierenden ${ }^{3}$ ergeben, dass diese das Schreiben in der Zielsprache - unabhängig vom Stand der Deutschkenntnisse - als wesentlich schwieriger ansehen als die anderen Fertigkeiten. Wie zu erwarten, ergaben sich jedoch auch Unterschiede entsprechend dem Stand der Deutschkenntnisse: In den Mittelstufenkursen überwiegen sprachpraktische Probleme, die Teilnehmenden verfügen kaum über Textartenwissen und die alltägliche Wissenschaftssprache ist nur bedingt bekannt. Ihr vorrangiges Ziel besteht in einer grundsätzlichen Verbesserung ihrer Deutschkenntnisse. In den Oberstufenkursen dagegen verfügen die Teilnehmenden bereits über umfangreiche Sprachkenntnisse und Schreib-Erfahrungen. Es geht ihnen insbesondere um das Erlernen schriftsprachlicher Konventionen für deutschsprachige akademische Texte, wobei ein spezifisches Training in komplexem Wortschatz, Syntax, Grammatik und Wissenschaftssprache als ,Mittel' angesehen wird, um dieses Ziel zu erreichen.

Hieraus wurde folgendes Kurskonzept entwickelt:

Für die Niveaustufen B1 und B2 eine sprachpraktische, fächerübergreifende Ausrichtung:

- Kürzung der erforderlichen Texte

- Populärwissenschaftliche Themenwahl

- Lernmodule zur Grammatik

- Charakteristika von geistes- und sozialwissenschaftlichen Texten

\footnotetext{
${ }^{3}$ Befragt wurden 88 Studierende.
} 
Für die Niveaustufe C1 eine die Anforderungen der einzelnen Fächer berücksichtigende Ausrichtung:

- spezifisches Textartenwissen (begleiteter Schreibprozess)

- akademisches Schreiben in der Zielsprache, darin integriert:

- Charakteristika von Wissenschaftssprache

- Schriftsprachliche Grammatik für Fortgeschrittene

Dieses Konzept hat sich bis heute bewährt. Dies zeigen die Nachfrage nach den Kursen und die regelmäßig durchgeführten Evaluationen.

\section{Konzept des Lehr- und Übungsbuchs}

Vor dem beschriebenen Hintergrund entstand die Idee der Entwicklung eines Lehr- und Übungsbuchs zum akademischen Schreiben für Geistes- und Sozialwissenschaften. Zielgruppe sind internationale Studierende mit Deutschkenntnissen auf den Stufen B2 und C1, unabhängig vom Studienstand (Grundstudium/ Hauptstudium/Prüfungsvorbereitung). Das Lehrbuch kann, wie die Erprobungsphasen gezeigt haben, auf diesen Stufen Kurs tragend und im Selbststudium eingesetzt werden. (Auf der Stufe B1 dagegen eignet es sich für einen punktuellen, auf ausgewählte, leichtere Kapitel/Arbeitsphasen beschränkten Einsatz im Unterricht.)

Im Lehrteil wird das Erstellen einer Hausarbeit dargestellt. An den Ergebnissen der Schreibprozessforschung orientiert ${ }^{4}$ wurde ein Konzept entwickelt, bei dem das Schreiben in Einzeltätigkeiten untergliedert wird, wie z. B. Einleiten, Gliedern, Definieren, Argumentieren etc. Die Konzeption des Lehrwerks trägt der Tatsache Rechnung, dass diese Tätigkeiten nicht in einem geradlinigen Prozess ablaufen. Die einzelnen Kapitel können deshalb unabhängig voneinander und in beliebiger Reihenfolge bearbeitet werden.

Alle Kapitel enthalten:

- motivationsfördernde Einführungen in die jeweiligen Themen,

- theoretische Erläuterungen,

- authentische Beispiele von Hausarbeiten,

- exemplarische Analysen der jeweils behandelten Bestandteile einer Hausarbeit (Inhaltsverzeichnis, Einleitung, Schluss etc.),

- tabellarische Übersichten wichtiger Merkmale des Textaufbaus,

- leicht nachzuvollziehende Darstellungen zentraler Elemente von Hausarbeiten,

- Leitfragen und Regeln für die Bearbeitung der einzelnen Kapitel.

\footnotetext{
4 Auf eine Aufbereitung der wissenschaftlichen Grundlagen der Schreibprozess-Forschung wird in diesem Beitrag verzichtet, da die unterrichtspraktische Seite im Vordergrund steht.
} 
Im Anhang finden sich:

- Kommunikationsmittellisten,

- Wissenschaftssprache für ausländische Studierende: Übersichten zu grammatischen Aspekten, die aus den einzelnen Lehrbuchkapiteln resultieren,

- Schreibtipps.

Diese sind so konzipiert, dass die Studierenden sie beim Schreiben eigener Texte nutzen können.

Auf die jeweiligen Arbeitsphasen ausgerichtete Übungen beziehen sich auf stilistische und grammatische Erscheinungen und auf Merkmale des Textaufbaus. Sie erscheinen als zweiter Band des Lehr- und Übungsbuches.

\section{1 Überblick über das Lehrbuch}

Entsprechend der bereits erwähnten Prozessorientierung stehen in den einzelnen Kapiteln die jeweiligen Arbeitsschritte im Fokus. Die Kapitel bauen, wie bereits erwähnt, nicht aufeinander auf, so dass sie von Lernenden und Lehrenden jeweils nach Bedarf bearbeitet werden können:

1. Finden eines Themas und Konzipieren der Arbeit

2. Erstellen eines Inhaltsverzeichnisses

3. Verfassen einer Einleitung

4. Argumentieren

5. Einbinden fremder Textteile

6. Verfassen eines Schlusskapitels

7. Der rote Faden

8. Überarbeiten

Hinzu kommen neben dem bereits erwähnten Anhang zwei weitere Kapitel:

- Der Prüfer als korrigierender und bewertender Leser

- Beachten von Formalia

Zur Veranschaulichung dieser recht allgemein formulierten Ausführungen sollen exemplarisch Auszüge aus zwei Kapiteln des Lehrbuchs näher vorgestellt werden.

\subsection{Auszüge aus dem Lehr- und Übungsbuch}

Zur Illustration eines motivationsfördernden Einstiegs in ein Thema mag der folgende Beginn des Kapitels „Erstellen einer Inhaltsverzeichnisses“ dienen. An- 
hand eines alltäglichen Beispiels wird die Problematik des behandelten Themas verdeutlicht - hier die Notwendigkeit einer klar gegliederten Übersicht:

\section{Lehrbuch-Auszug 1:}

\section{Labyrinthisches}

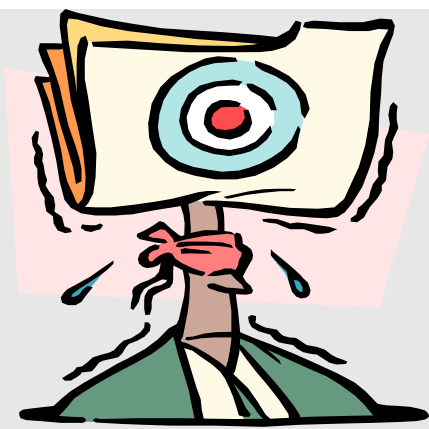

Tourist: $\quad$ Entschuldigung, können Sie mir bitte helfen? Ich möchte zum Picasso-Museum.

Passant: Ja, das ist nicht so schwer. Also: Sie gehen erst mal geradeaus bis zur nächsten Kreuzung. Dann gehen Sie nach rechts und dann weiter geradeaus bis zur dritten Ampel. Da sehen Sie ein großes weißes Gebäude, und schräg dahinter liegt das Picasso-Museum.... Das heißt, jetzt hab' ich mich vertan - an der ersten Kreuzung gehen Sie besser einfach geradeaus, bis Sie zu einer Kirche kommen. Kurz vorher kommen Sie an einem Schnellimbiss vorbei, aber das ist eigentlich nicht so wichtig. Den können Sie auch nicht richtig erkennen, weil das Gebäude gerade renoviert wird. Und um diese Uhrzeit ist der auch geschlossen.

Obwohl: vielleicht wäre es doch besser gewesen, Sie wären da schon nach links abgebogen. Ach ja, und was ich noch vergessen habe: an der ersten Kreuzung können Sie gar nicht abbiegen, weil die Straße seit gestern gesperrt ist.

Konnten Sie diese konfuse Wegebeschreibung nachvollziehen? Es wäre nicht erstaunlich, wenn der Tourist nach einer solchen Wegbeschreibung das Picasso-Museum gar nicht mehr besichtigen wollte. Bei dieser Wegbeschreibung ist Einiges durcheinander: So haben z. B. für diese Wegbeschreibung wichtige Informationen denselben Stellenwert wie unwichtige, es lässt sich keine sinnvolle Abfolge der Richtungsangaben erkennen, und es fehlen Angaben zu markanten Punkten, die eine bessere Orientierung ermöglichen. Anders im folgenden Beispiel:

$\begin{array}{ll}\text { Tourist: } & \text { Entschuldigung, können Sie mir bitte helfen. Ich möchte zum Picasso-Museum. } \\ \text { Passant: } & \text { Klar, das ist ganz einfach. Zu Fuß brauchen Sie ungefähr } 10 \text { Minuten. Zunächst einmal gehen Sie } \\ & \text { von hier aus auf der Windthorststraße ca. } 500 \text { Meter einfach geradeaus. Dann kommen Sie an einen } \\ & \text { Platz: Den erkennen Sie sofort an einer großen Skulptur mit zwei großen roten Kirschen. Den Platz } \\ & \text { überqueren Sie und biegen dann nach links ab. Dann kommen Sie auf die Fußgängerzone. Die über- } \\ & \text { queren Sie ebenfalls. Am Ende gelangen Sie zu einem Eis-Café. Jetzt stoßen Sie auf die Königspas- } \\ & \text { sage und müssen sich nach rechts wenden. Von da aus können Sie das Picasso-Museum schon se- } \\ & \text { hen. Es sind nur noch } 100 \text { Meter. } \\ \text { Tourist: } & \text { Herzlichen Dank. Das finde ich. }\end{array}$

Der Tourist kann nach einer solch klaren Beschreibung den Weg ohne Schwierigkeiten finden. Das gleiche Prinzip gilt für Hausarbeiten. Eine klare Gliederung hilft dem Leser, sich über den Inhalt einer Hausarbeit zu orientieren. 
Darüber hinaus enthält das Kapitel Erläuterungen zu den Kennzeichen eines Inhaltsverzeichnisses sowie eine Analyse eines authentischen Beispiels.

Ziel des Kapitels „Einbinden fremder Textteile“ ist, die Lernenden dazu zu befähigen, Texte zusammenzufassen und damit Textbausteine zu erstellen, die wie beispielsweise auch Zitate, Paraphrasen und Definitionen in die eigene Hausarbeit integriert werden müssen.

Das Kapitel „Der rote Faden“, aus dem jetzt ein längerer Auszug folgt, zeigt anhand zahlreicher Beispiele, wie diese Integration funktioniert und die einzelnen Teile einer Hausarbeit so miteinander verbunden werden, dass ein zusammenhängender Text entsteht und nicht eine Aneinanderreihung einzelner Teile.

\section{Lebrbuch-Auszug 2:}

$[\ldots]$

1. Funktion des Roten Fadens

Beispieltext:

\section{STEP 21-Medienbox: Die Materialanalyse}

[...]

Ziel dieses Kapitels ist es unter anderem, zu analysieren, wie diese von Bastian formulierten Potenziale der Medienbox sich in den Materialien wiederfinden. Untersucht wird dazu, welche medialen Angebote die Medienbox enthält und [...].

Zuvor wird die Jugendinitiative STEP 21 vorgestellt, um die Darstellung der Medienbox und die Herkunft der Materialien zu systematisieren.

\subsection{Die J ugendinitiative}

[...]

(Breer, Martina: Jugend - Medien - Bildung : die STEP 21-Medienbox als Beitrag zur Modernisierung von schulischen und außerschulischen Entwicklungsprozessen. http://nbn-resolving.de/urn:nbn: de:hbz:6-23689465034; online am 19.07. 2006.) 
Die Schreiberin gibt dem Leser zu Beginn dieses 4. Kapitels ihrer Dissertation eine sprachliche Hilfe, den Text zu verstehen. Sie orientiert den Leser darüber

- was das Ziel des Kapitels ist,

- $\quad$ wie das Kapitel an die Ausführungen des vorherigen Kapitels anknüpft,

- $\quad$ welche Beziehung der Textabschnitt zum gesamten Text hat.

Um den Leser darüber zu orientieren, hat die Verfasserin eine Vielzahl von sprachlichen und textbaulichen Möglichkeiten, die sie benutzen kann. Mit innen macht sie ihren Textbauplan durchsichtig und trägt dazu bei, dass sich der Leser einen Leseplan aufbaut.

\section{Sprachlich explizite Leserführung}

\subsection{Satzebene}

Der Aufbau eines Textverständnisses erfolgt $u$. a. durch die Verknüpfung von Sätzen. Beim Leser wird das Verständnis erleichtert durch

- $\quad$ eine geordnete Gedankenführung (vgl. sprachlich implizite Leserführung),

- Konjunktionen, Pronomen usw.,

- Kommunikationsmittel wie z. B. „Daraus kann gefolgert werden, ...“

Diese sprachlichen Mittel benutzt der Schreiber, um den Leser zu führen und inm zu zeigen, welche logische Verknüpfung von Aussagen er herstellt.

\subsection{Kapitelebene}

Unter Kapitel-Ebene werden Textelemente verstanden, die einen Absatz oder ein Kapitel umfassen. Auf dieser Ebene versucht der Schreiber dem Leser zu helfen, einen Zusammenhang zwischen Textabschnitten mit dem Thema des Kapitels oder des gesamten Textes herzustellen.

\section{Beispieltext:}

\subsection{Die J ugendinitiative}

„STEP 21 - die jugendinitiative für toleranz und verantwortung“ ist die vollständige Bezeichnung der gemeinnützigen Gesellschaft, die sich 1994 aus einem von dem Unternehmen Bertelsmann ausgeschriebenen Projektwettbewerb entwickelt hat. Der nachfolgende chronologische Abriss gibt einen kurzen Überblick über Entstehung und Entwicklung der Initiative. ${ }^{270}$

1994: Projektwettbewerb „Bertelsmann für Toleranz" - Aufgrund der zu Beginn der 1990er Jahre stark ansteigenden fremdenfeindlichen Übergriffe [...].

1994-1998: Konkretisierung des Projekts „Aktion: Jugend fordert!“ - In dieser Zeit wird das Grobkonzept der Projektidee überarbeitet und [...].

Diese sehr verkürzte Chronologie verdeutlicht anschaulich, wie aus der ersten Projektidee innerhalb von 10 Jahren eine etablierte Jugendinitiative wird.

(Quelle: s. o.)

Der letzte Satz liefert hier keine Informationen über die Initiative STEP 21, sondern weist den Leser an, wie er die vorher dargelegten Informationen verstehen soll: als eine chronologische Darstellung von der Gründung bis zur etablierten Initiative. Diese Absicht wird durch Betitelungen der Zeitphasen unterstützt, die kursiv hervorgehoben sind.

Um den Leser zum Aufbau eines Leseplans zu führen, bedient sich die Schreiberin folgender Schreibhandlungen:

\section{Betiteln}

Wiederholen (eines Zwischenergebnisses/ eines Problems/ ...)

Wiederaufgreifen

Ziele vorgeben

Ankündigen (des weiteren Verlaufs) 
Bezug herstellen (zu vorherigem/ zukünftigem Kapitel)

Rückverweisen

Anknüpfen

Zusammenfassen

Das Kapitel 4.1 aus dem obigen Beispiel endet folgendermaßen:

\subsection{Die J ugendinitiative}

[...]

STEP 21 versucht die Vielfalt sowohl der Jugend als auch die jeweils subjektive Vielfalt des einzelnen Jugendlichen zu berücksichtigen. Ob und wie dies durch das Angebot der Medienbox gelingt, wird im weiteren Verlauf dieser Arbeit dargestellt.

Wie sich die Auseinandersetzung mit demokratischen Grundwerten und die Förderung der Entwicklung eines Wertebewusstseins in dem Angebot "Medienbox [Die Clique]" wiederfindet, welche medienpädagogischen Ansätze [...] dazu beitragen sollen, ist unter anderem Gegenstand der folgenden Kapitelabschnitte.

(Quelle: s. o.)

Während der erste Satz ein Fazit/die Zusammenfassung eines Zwischenergebnisses der vorhergehenden Aussagen darstellt, schließt sich daran eine Lesehilfe für die kommenden Kapitel an. Ein Beispiel dafür, wie der Schreiber das Wissen des Lesers durch Rückverweisen strukturiert, findet sich im Kapitel 4.2.

[...]

Dies mag als Beispiel genügen, das Ende dieses Kapitels ist hier ausgelassen. Es dürfte deutlich geworden sein, wie wir in diesem Kapitel durch kürzere und jeweils ausführlich kommentierte Textbeispiele den Einsatz sprachlich expliziter Leserführung auf Satz- und Kapitelebene zeigen.

\subsection{Einsatżöglichkeiten des Lehr- und Übungsbuches}

Folgende Gruppen werden besonders angesprochen:

- Teilnehmende an studienvorbereitenden Sprachkursen auf dem Niveau B2-C1 (Lehrgebiete u. ä. Institutionen)

- Teilnehmende an studienbegleitenden Sprachkursen auf dem Niveau B2-

C1 für ERASMUS-Stipendiaten

- Regelstudierende

- Teilnehmende an Kursen zur Vermittlung von Studierstrategien

- Studierende in deutschsprachigen Studiengängen im Ausland mit den Schwerpunkten Geistes- und Sozialwissenschaften

- Gastwissenschaftler/innen

- Teilnehmende an Sprachkursen, die auf das Studium in Deutschland vorbereiten

- Studierende in Schreibberatungen 
- Teilnehmende an studienvorbereitenden Kursen mit dem Studienziel Abitur (Studienkollegs)

- am Einsatz im Selbststudium Interessierte

\section{Ausblick}

Auf der Grundlage des Lehr- und Übungsbuches ist geplant, über das Internet weitere Übungsformen und ergänzendes Unterrichtsmaterial zur Verfügung zu stellen. Damit können die individuellen Bedürfnisse der Lernenden

- fächerspezifisch

- niveauspezifisch

- kulturspezifisch

berücksichtigt werden. Diese Erweiterung des Lehrbuches würde auch einen Fernstudienkurs mit Teilnehmern im Ausland möglich machen. 


\section{Erinnerungsorte im Unterricht Deutsch als Fremd- sprache}

Manfred Kaluza/Karin Schmidt/Sabine Schmidt, Berlin

\section{Einleitung}

Im Rahmen des DAAD-Lektorennetzwerkes Berlin hat sich eine Arbeitsgruppe zum Thema „Geschichte und Deutsch als Fremdsprache“ konstituiert, die Unterrichtsmaterialien zu ausgewählten deutschen Erinnerungsorten erstellt. In der Arbeitsgruppe konnte durch die Erinnerungsthematik ein Ansatz gefunden werden, der, so die Hoffnung, wegen seiner Universalität bei Deutschlernern im Inund Ausland auf Interesse stoßen wird. Der Landeskundeunterricht wird hier durch eine historische Dimension erweitert, ohne die Lerner auf herkömmliches „Geschichte lernen“ zu verpflichten - ein Desiderat im Fach Landeskunde. AuBerdem wurde es möglich, die vielfältigen und verschiedenen Interessen und Arbeitsfelder der einzelnen Mitglieder zusammenzuführen. 
Die Relevanz der Erinnerungsthematik ist sowohl dem Paradigmenwechsel in der Geschichtswissenschaft - von der Sozial- und Gesellschaftsgeschichte zur Kulturgeschichte - als auch der wachsenden Bedeutung der Kulturwissenschaften geschuldet. Über die Grenzen der Wissenschaft hinaus zeigt sich ihre Bedeutung in einer quantitativen Dimension, immer mehr Gedenktage und -stätten für immer mehr gesellschaftliche Gruppen, und einer qualitativen Dimension, z. B. woran erinnern, und wie erinnert man „,richtig“".

Innerhalb dieser weit gefächerten und komplexen Thematik einigte sich die Arbeitsgruppe darauf, dem von Pierre Nora $(1984,1986,1992)$ in Frankreich entwickelten Konzept der Erinnerungsorte („Lieux de mémoire“) zu folgen, allerdings mit der wesentlichen, auf Maurice Halbwachs $(1925,1941)$ zurückgehenden Einschränkung, nur topografisch fixierbare Orte zu bearbeiten. Diesen Erinnerungsorten, wie auch den abstrakten, nur symbolisch verortbaren, „ist gemeinsam, dass sie einen Überschuss an symbolischer Bedeutung besitzen, der sich allerdings ändern, auch gänzlich verlieren kann" (François/Schulze, Bd.1, 2003: 16).

Eine wichtige Inspirationsquelle für die Arbeitsgruppe stellte das dreibändige Werk „Deutsche Erinnerungsorte“, herausgegeben von Etienne François und Hagen Schulze (François/Schulze 2003) dar. Hilfreich war darüber hinaus, dass die Erinnerungsthematik in den Lehrplänen für die Gymnasien mittlerweile verankert ist, und somit didaktisierte Lehrwerke (Brütting/Gümbel 2005) und Materialien erhältlich sind. Einem größeren Publikum bekannt gemacht und durch viele Materialien veranschaulicht wurde diese Thematik durch zwei Ausstellungen des Deutschen Historischen Museums in Berlin. Zunächst wurde den mythologisch überhöhten „Urszenen“ europäischer Nationen nachgegangen (Flacke 1988), 16 Jahre später wurde das europäische Schlüsseljahr 1945 in der Erinnerung verschiedener Nationen nachgezeichnet (Flacke 2004).

Um die Bearbeitung der einzelnen Erinnerungsorte zu strukturieren, und für die Nutzer - Lernende und Lehrende - übersichtlich zu machen, wurden didaktischmethodische Leitlinien festgelegt, die im Folgenden kurz dargestellt werden sollen.

Im zweiten Teil sollen am Beispiel der Wartburg verschiedene Dimensionen des Erinnerns beschrieben und, wo erforderlich, durch konkrete Hinweise auf verwendete Materialien verdeutlicht werden.

\section{Didaktisch-methodische Leitlinien}

Die Entscheidung für topografisch fixierbare Orte wurde auch von dem didaktischen Prinzip der Anschaulichkeit geleitet.

Die von der Arbeitsgruppe ausgewählten Erinnerungsorte stellen lediglich ein paar Bäume im riesigen Wald der Erinnerungen dar, sie beanspruchen daher nur einschränkend Repräsentativität für die Geschichte und Erinnerungskultur Deutschlands.

Mit der Ausweitung der Zielgruppe auf Deutschlerner aus der ganzen Welt, in der Regel aus dem universitären Kontext, und sprachlich den Niveaustufen des 
europäischen Referenzrahmens von B2 bis C1 entsprechend, ergibt sich automatisch auch eine Perspektivverschiebung. Egal, ob es sich um heterogene Lerngruppen in Deutschland oder weitgehend homogene Lerngruppen im Ausland handelt, Erinnerung mit historisch bedeutsamen Orten in Deutschland zu verbinden, bedeutet für die Zielgruppe sich mit einer fremden Erinnerungskultur auseinanderzusetzen, die, als Folge der Universalität des Erinnerns, ständig vergleichend auf die eigene bezogen werden kann. Dementsprechend fokussieren Aufgabenstellungen zu einzelnen Erinnerungsaspekten immer wieder auf diese Verschränkung von fremder und eigener Erinnerungskultur. (Es soll hier jedoch nicht vergessen werden, dass auch innerhalb der gewählten deutschen Erinnerungsorte Möglichkeiten des Vergleichs gegeben sind.)

Die Vermittlung historischer Kenntnisse an ausländische Deutschlerner führt zu einem wechselseitigen Bezug von geschichts- und fremdsprachendidaktischen Kategorien. In Verbindung mit den historischen Inhalten, und nicht als Nebenprodukt, sollen die sprachlichen Fertigkeiten Sprechen, Lesen, Hören und Schreiben verbessert werden. Bei der Auswahl der Materialien, den Vermittlungsformen und Arbeitsaufgaben waren Vielfältigkeit und Variabilität die leitenden Kriterien. Den Hauptanteil an den Materialien nehmen Texte ein, die sprachlich entlastet und didaktisiert werden, wobei texterschließende Methoden durch quellenkritische ergänzt und erweitert werden. Immer wurde darauf geachtet, dass eine Balance zwischen eher kognitiv/analytischen Arbeitsaufträgen und eher kommunikativ orientierten Handlungs- bzw. Produktionsaufträgen besteht.

Sprachübungen zu Redemitteln und Wortschatz sollen immer aus den Materialien hervorgehen bzw. an die Materialien angebunden werden. Im besten Fall haben sie nicht nur übende und wiederholende Funktion für die sprachlichen Fertigkeiten, sondern dienen der Vertiefung des historischen Stoffes.

Bei einzelnen Erinnerungsorten, z. B. der Wartburg, wird die Sprache selbst zur Gewinnung von historischem Wissen benutzt oder sie dient der Illustration von Sprachgeschichte und Sprachwandel. Es soll somit zur Auseinandersetzung mit und Reflexion über Sprache anregt werden. Bezugsdisziplinen sind Teilbereiche der Linguistik wie Sprachgeschichte, Etymologie, historische Semantik und Dialektologie.

Was die historischen Inhalte betrifft, so legen die Unterrichtsmaterialien zu den Erinnerungsorten den Schwerpunkt nicht auf die Vermittlung von Geschichtswissen in Form von Daten und Fakten, diese haben dienende, orientierende Funktion. Die Lerner sollen in die Lage versetzt werden zu reflektieren, wie durch die Rekonstruktion von Vergangenheit (=Geschichte) ein bestimmtes Verständnis der Gegenwart erreicht wird, das gleichzeitig legitimatorischen Charakter für die Zukunft hat. Diese Zusammenführung von Vergangenheit, Gegenwart und Zukunft bildet das Geschichtsbewusstsein, das wiederum der Identitätsstiftung von Kollektiven, hier der Nation, dient (Jeismann 1997).

Wenn möglich, soll in den Materialien ein Gegenwartsbezug vorhanden sein. Zum einen ist der Gegenwartsbezug durch die Anknüpfung an Weltwissen und 
Erfahrungswissen der Lernenden ein motivierender Faktor für den Unterricht. Zum anderen wird das Geschichtswissen selbst („Wissenschaftswissen“) mit der Gegenwart verbunden, eingeschränkt durch den Primat der Auswahl bzw. der Relevanz. Vermittelt werden sollen solche Inhalte, die über Kontinuität, auch im Sinne einer Kausalbeziehung, bis in die Gegenwart verfügen und bei der Lösung gegenwärtiger und zukünftiger Probleme Orientierung geben (Bergmann 1997).

Der Gegenwartsbezug stößt dort an seine Grenzen, insbesondere im Hinblick auf die Zielgruppe, wo eine Kontinuität zur Gegenwart nur schwer herzustellen ist, statt dessen eine substantielle Andersartigkeit (Alterität) vorherrscht, die die Gegenwart als einen Bruch empfinden lässt, wie Teilaspekte einzelner Erinnerungsorte, die bis ins Mittelalter zurückreichen (siehe unten die Module 3 und 4 der Wartburg).

\section{Dimensionen des Erinnerns am Beispiel der Wartburg}

Aufbau und Inhalt der Unterrichtseinheit zur Wartburg haben keinen exemplarischen Charakter für das Gesamtprojekt. Dazu sind die gewählten Erinnerungsorte: Die Berliner Mauer, Dresden, Weimar/Buchenwald, die Paulskirche, die Hanse, der Kölner Dom, der Führerbunker, das Mahnmal zur Bücherverbrennung, Schloss Neuschwanstein und Preußen in ihrer Topografie, ihrer Geschichte und Bedeutung, sowie in ihrem Erinnerungswert zu heterogen.

Die Unterrichtseinheit ist in Module unterteilt, die auch einzeln unterrichtet werden und/oder auch mit einzelnen Modulen anderer Erinnerungsorte kombiniert werden können.

Es wird Wert darauf gelegt, dass ein Erinnerungsort nicht alle Dimensionen des Erinnerns ausschöpfen kann. Beispielsweise kann das eminent wichtige ,kontroverse Erinnern“ an der Wartburg nicht gut gezeigt werden. Zwar gibt es eine Kontroverse um ein Wartburgtreffen 1990, anlässlich dessen noch einmal an die nunmehr negativ besetzten Aspekte des Wartburgfestes von 1817, Antisemitismus einiger Teilnehmer, Bücherverbrennung, erinnert wurde (Zwiener 1990), aber die Reaktion in der Öffentlichkeit war gleich Null. Anders bei anderen Erinnerungsorten: so enthält der Erinnerungsort Dresden mit dem Wiederaufbau der Frauenkirche geradezu exemplarisch kontroverses Erinnern. Dasselbe gilt für „,mahnendes Erinnern“", das viel besser am Buchenwalddenkmal aufgezeigt werden kann.

\subsection{Aufbau der Unterrichtseinheit:}

\section{Einführungsmodul}

Ausgehend von einigen Schlüsseldaten, aus deren Zeitabstand geschlossen werden kann, dass die Bedeutung der Wartburg, vielleicht bis auf die Gründungsphase im Mittelalter und die Phase nach der Restauration in der zweiten Hälfte des 19. Jahrhunderts, immer eher punktueller Natur war und Höhepunkte nur lange Pha- 
sen des Niedergangs unterbrachen, sollen die unten in den einzelnen Modulen angeführten Ereignisse kurz eingeführt werden. Dies geschieht mit kurzen Texten und Bildern, die zunächst einander, später einer Zeitleiste mit den entsprechenden Daten zugeordnet werden sollen.

Im zweiten Teil sollen am Beispiel der Wartburg die Topografie einer Burg sowie deren Funktionen gezeigt werden.

\section{Modul 2: 1999: Die Wartburg wird Weltkulturerbe}

\section{Dimension: Globalisiertes Erinnern}

Hintergrund: Die internationale Staatengemeinschaft verpflichtet sich im Jahre 1972 durch die Welterbe-Konvention, als wertvoll deklariertes Kultur- und Naturerbe zu erhalten und zu pflegen. Damit erhielt die Erinnerung an kulturgeschichtliche Leistungen und natürliche Gewordenheiten ihren globalen Maßstab. Es werden drei Arten von Welterbe unterschieden: Kulturerbe, Naturerbe, Kulturlandschaften. Unterstellt wurde den Stätten ein universeller Wert, dessen Zerstörung ein Schaden für die ganze Menschheit bedeutet (UNESCO-Kommission 2002).

Das Weltkulturerbe repräsentiert einen globalen Konsens hinsichtlich bedeutender, schützens- und erinnernswerter Orte. Demgemäß kann diese Form der Erinnerung und die damit verbundene Problematik - exemplarisch dargestellt anhand der Wartburg - überall im DaF-Unterricht im Hinblick auf die eigene Kultur thematisiert werden.

In den nächsten, konkreteren Schritten sollen die Kriterien für ein Weltkulturgut sprachlich und inhaltlich erarbeitet werden und dann, auf der Basis der bereits gewonnenen Kenntnisse über die Wartburg, Hypothesen gebildet werden, welche der Kriterien auf die Wartburg zutreffen. Da die Wartburg den Status „Weltkulturerbe“ aufgrund des Kriteriums „ein einzigartiges oder zumindest außergewöhnliches Zeugnis einer untergegangenen Kultur oder kulturellen Tradition" verliehen bekam, kann von diesem Punkt aus direkt ins Mittelalter ,zurückgesprungen“ werden, um zu verdeutlichen, welche „Kultur oder kulturelle Tradition“ untergegangen ist.

Am Ende der gesamten Unterrichtseinheit soll die Bedeutung des Status „Weltkulturerbe“" wieder aufgenommen werden. Dabei sollen aktuelle Probleme, die mit der Verleihung verbunden sind, angesprochen werden:

Welterbe und Umwelt:

Die UNESCO verfügt über Sanktionspotential, sie kann den Status nicht nur verleihen, sondern, nach einem abgestuften System („Rote Liste“), auch wieder entziehen. Spektakuläre Fälle sind der Kölner Dom (ebenfalls ein Erinnerungsort 
in unserem Projekt) und das Dresdner Elbtal. Aber auch die Wartburg hat durch den geplanten Bau eines sog. „Windparks“ Ärger bekommen.

Welterbe und Tourismus:

Ein zweiter Aspekt ist der Kulturtourismus, dessen eher erfreuliche ökonomische Seite mit den belastenden Faktoren, die die Pflege und den Erhalt des Weltkulturerbes betreffen, konfrontiert werden soll. Methodisch interessant wäre hier, ob empirisch ein Nachweis über den Zusammenhang vom Weltkulturerbestatus mit steigenden Touristenzahlen erbracht werden kann.

\section{Modul 3: Das Mittelalter (Erster Teil): Der Sängerkrieg auf der W artburg}

Dimension: Imaginiertes Erinnern

Hier liegt der Fokus auf einem Ereignis, das so nicht stattgefunden hat, aber in der Erinnerung als literarisches Dokument mit seinen verschiedenen Überlieferungen untrennbar mit der Wartburg verbunden ist. Dabei sollen, neben der eigentlichen Geschichte, Einblicke in das kulturelle Schaffen des Mittelalters und in die Bedingungen, denen es unterlag, gegeben werden. Der Fremdheitsaspekt überwiegt, da ein Anschluss an die Lebenswirklichkeit der Studenten nur schwer oder sehr gekünstelt möglich ist. Mögliche Referenzpunkte bieten Ursprungsmythen verschiedener Nationen, wobei die Wahrscheinlichkeit, mit der ein solches Ereignis stattgefunden haben könnte, problematisiert werden muss.

\section{Modul 4: Das Mittelalter (Zweiter Teil): Die Heilige Elisabeth}

Dimension: Selektives Erinnern

Historische Persönlichkeiten, noch dazu wenn ihr Leben aufgrund des Zeitabstands von 700 Jahren und aufgrund der Überlieferungsprobleme nur fragmentarisch rekonstruiert werden kann, sind aufgrund ihrer Komplexität nur in Ausschnitten zu erfassen und fordern per se ein selektives Erinnern. In diesem Modul soll nicht in einem diachronen Längsschnitt gezeigt werden, wie unterschiedlich $\mathrm{zu}$ welchen Zeiten an Elisabeth erinnert wurde, sondern in einem synchronen Verfahren sollen drei aktuelle Webseiten, die die Erinnerung an Elisabeth in unterschiedlichen institutionellen Kontexten und mit unterschiedlicher Handlungslegitimation wach halten, erschlossen werden.

Modul 5: Luther auf der Wartburg:

Dimension: Gespaltenes Erinnern 
Schwerpunkt dieses Moduls ist die Übersetzung der Bibel und deren sprachgeschichtliche Bedeutung. Luther wurde auf die Wartburg gebracht, gerade weil sie zu dieser Zeit unbedeutend und beinahe verfallen war. Sein Aufenthalt dort steht für das komplizierte Verhältnis von Spaltung und Einheit: Religionsspaltung und gleichzeitig sprachliche Einheit.

\section{Modul 6: Das 19. Jahrbundert (Erster Teil): Romantische Restauration}

Dimension: Verklärendes Erinnern

Hier ist zunächst der Epochenwechsel von der Klassik zur Romantik wichtig, gekennzeichnet auch durch den Wechsel von der griechisch-römischen Antike hin zum christlichen deutschen Mittelalter als der verehrten Referenzepoche. In verklärender Weise schufen sich Künstler ein Idealbild einer ständisch organisierten harmonischen Gemeinschaft von Herrscher und Beherrschten, versehen mit den Segnungen einer einheitlichen Kirche. An der Wartburg kann dies an der Rezeption der mittelalterlichen, bedeutsamen Topoi Sängerkrieg und Heilige Elisabeth in Literatur und Musik gezeigt werden. Was das konkrete Bauwerk anbetrifft, ist hier die Restauration der Wartburg in der zweiten Hälfte des 19. Jahrhunderts gemeint, mit ihren zahlreichen Bezügen zum Mittelalter, etwa die Wandfresken Moritz von Schwinds.

Modul 7: Das 19. Jabrbundert (Zweiter Teil): Das Wartburgfest von 1817

Dimension: Individuelles und historisches Erinnern

Neben Informationen zu Organisation, Ablauf und Bedeutung dieser ersten deutschen politischen „Demo“ soll die Festrede des Theologiestudenten Riemann analysiert werden. Diese Rede zeigt nicht nur die für ein Geschichtsbewusstsein konstitutive Verbindung von Vergangenheit, Gegenwart und Zukunft auf. Sie ist geradezu paradigmatisch für die Verschränkung von individuellem Erinnern: Teilnahme an den Napoleonischen Befreiungskriegen und die Enttäuschung nach dem Sieg über die europäische Neuordnung auf dem Wiener Kongress, die wegen der gescheiterten deutschen Einheit als Niederlage interpretiert wird, und historischem Erinnern an die Reformation. Hier kann aufgezeigt werden, wie diese Erinnerungen für die Formung eines kollektiven, hier nationalen Gedächtnisses konstitutiv sind. 


\section{Literatur}

Bergmann, Klaus: Gegenwarts- und Zukunftsbezogenheit. In: Bergmann, Klaus / Fröhlich, Klaus / Kuhn, Annette / Rüsen, Jörn / Schneider, Gerhard (Hrsg.): Handbuch der Geschichtsdidaktik, 5., überarb. Aufl., Seelze-Velber: Kallmeyer 1997, S.266-268.

Brütting, Rolf / Grümbel, Annette: Erinnerung und Geschichte. Geschichte und Geschehen exempla. Themenheft für die Sekundarstufe II, Leipzig: Ernst Klett Schulbuchverlag 2005.

Flacke, Monika: Mythen der Nationen. Ein europäisches Panorama. Berlin: Deutsches Historisches Museum 1988.

Flacke, Monika: Mythen der Nationen. 1945 - Arena der Erinnerungen, 2 Bde., Berlin: Deutsches Historisches Museum 2004.

François, Etienne / Schulze, Hagen (Hrsg.): Deutsche Erinnerungsorte, 3 Bde., München: Beck (Broschierte Sonderausgabe) 2003.

Halbwachs, Maurice: Les cadres sociaux de la mémoire, Paris: 1925.

Halbwachs, Maurice: La topographie légendaire des évangiles en Terre Sainte, Paris: 1941.

Jeismann, Karl-Ernst: Geschichtsbewusstsein - Theorie. In: Bergmann, Klaus / Fröhlich, Klaus / Kuhn, Annette / Rüsen, Jörn / Schneider, Gerhard (Hrsg.): Handbuch der Geschichtsdidaktik, 5., überarb. Aufl., Seelze-Velber: Kallmeyer 1997, S.42-44.

Nora, Pierre (Hrsg.): Les lieux de mémoire I: La république, Paris: Gallimard 1984.

Nora, Pierre (Hrsg.): Les lieux de mémoire II: La Nation, Paris: Gallimard 1986.

Nora, Pierre (Hrsg.): Les lieux de mémoire III: Les France. Paris: Gallimard 1992.

Österreichische UNESCO-Kommission / Deutsche UNESCO-Kommission / Deutsche Stiftung Denkmalschutz (Hrsg.): Welterbe für junge Menschen. Entdecken - Erforschen - Erhalten. Eine Unterrichtsmappe für Lehrerinnen und Lehrer, Bonn: 2003.

Zwiener, Ulrich (Hrsg.): Wartburgtreffen 1990. Ein demokratisches Deutschland für Europa, Jena: Universitätsverlag 1990, S.33-37. 


\section{Spiele zur Vermittlung und Übung von Fachwort- schatz}

Sabine Jentges, Marburg

\section{Ausgangsposition}

Sprachlernspiele gehören heute zum festen Bestandteil der von Lehrpersonen, Lehrmaterialautoren und Sprachlehrforschern vorgeschlagenen Übungsformen für den Fremdsprachenunterricht. Gleichzeitig aber ist der Einsatz von Sprachlernspielen im Unterricht immer noch umstritten (vgl. u. a. Klippel 1980: 90, Stellfeld 1994: 49ff). Der Grund hierfür scheint in dem immer wieder in den Mittelpunkt gestellten Gegensatz von Spiel und Arbeit zu sehen zu sein, basierend auf der Annahme, dass das unterhaltende Spiel einen Gegensatz zur anstrengenden Arbeit darstelle und darüber hinaus Lernen nicht mit Spaß, sondern mit Anstrengung und somit mit Arbeit gleichgesetzt wird (vgl. Jentges 2006: 94f). Daraus folgt bis heute in der Unterrichtspraxis ein Gegensatz zwischen einer positiven Einschätzung von Spielen einerseits und dem tatsächlich erfolgenden Spieleinsatz andererseits. Noch heute werden Spiele auf die Funktion einer Belohnung, eines Lückenoder Pausenfüllers reduziert oder nur eingesetzt, um die Aufmerksamkeit der 
Lernenden wieder zu wecken (vgl. Stellfeld 1994: 49ff). Als Gründe hierfür wird von DaF-Lehrenden vor allem angegeben, dass nicht alle Teilnehmer Spiele als Übungsform und somit Lernmittel annehmen und Spiele eine zu zeitintensive Übungsform darstellen würden (vgl. Mensmann 2006: 130).

Aus der vielfach geäußerten Befürchtung, mit Spielen lerne man ja nichts, resultiert die Notwendigkeit zu überprüfen, ob Lernen mit Spielen im Fremdsprachenunterricht möglich ist, also ob und falls wie erfolgreich mit Spielen gelernt werden kann. Denn nur wenn positive Lernerfolge mit dem Einsatz von Spielen erreicht werden, können diese ohne Bedenken für den Einsatz im Unterricht empfohlen werden - andernfalls hätten Bedenkenträger Recht und von einem Spieleinsatz als ernst zu nehmendes Lernmittel müsste abgeraten werden.

\section{Untersuchung zur Wortschatzvermittlung mit Spielen}

Um der Frage nach der Lerneffizienz von Spielen nachzugehen, habe ich im Rahmen von universitären Lehrveranstaltungen für ausländische Studierende an der Philipps-Universität Marburg in den Jahren 2000 bis 2002 eine Untersuchung mit über 100 Teilnehmern durchgeführt, bei der verschiedene Spiele, Spielformen und spielerische Übungen eingesetzt und in der für Wortschatzvermittlung qualitative Ergebnisse erfasst wurden ${ }^{1}$.

Für die Untersuchung wurden zwei methodische Vorgehensweisen entwickelt - die basierend auf gleiche Materialien und identische Unterrichtssituation sowie Zeitaufwand - den gleichen Lernstoff einmal ausschließlich mit Spielen vermittelten und einmal ohne Spieleinsatz ${ }^{2}$. Zu lernen war jeweils ein Set von 20 Vokabeln innerhalb einer Unterrichtszeit von 45 Minuten. Als zu vermittelnde Vokabeln wurde zum einen ein Set aus dem Bereich Blumen-, und zum anderen eines aus dem Bereich Tierbezeichnungen ausgewählt ${ }^{3}$.

Die Untersuchungsergebnisse zeigten, dass kurzfristig mit Spielen besser gelernt wurde; in einer Gruppe sogar signifikant. Mittel- und langfristig blieben die Lernerfolge ohne Signifikantsniveau. Die zunächst deutlich besseren Lernerfolge mit Spielen bestätigten sich also nicht dauerhaft, denn der Vorsprung des mit Spielen mehr Gelernten reduziert sich langfristig 4 .

\footnotetext{
${ }^{1}$ Wortschatzvermittlung wurde für die Lernerfolgsmessungen ausgewählt, da hier am ehesten fassbare, konkret gelernte Inhalte für eindeutige Messungen des Lernerfolgs möglich sind.

${ }^{2}$ Gearbeitet wurde darüber hinaus mit einem Lateinischen Quadrat und einem „unabhängige Gruppen-Design“".

${ }^{3} \mathrm{Da}$ es sich sowohl von der Muttersprache als auch vom Sprachkenntnisstand her um eine sehr heterogene Lerngruppe im Zielsprachenland handelte, musste Wortschatz gefunden werden, bei dem jeder Teilnehmer, unabhängig vom Sprachniveau und der vorherigen Sprachlern-Erfahrung (noch) etwas lernen konnte. Außerdem sollten weniger alltagsrelevante Themenbereiche ausgewählt werden, um idealere Rahmenbedingungen für das Experiment zu erhalten.

${ }_{4}^{4}$ Für genaue Angaben zu den Lernerfolgen (Rohzahlenmaterial, statistische Berechnung, Überprüfung von Listeneffekten usw.) und zur Diskussion der Ergebnisse sowie den eingesetzten Materialien, dem Unterrichtsverlauf usw. vgl. Jentges 2007.
} 
Generell sind aber für beide Vermittlungswege die Behaltensleistungen, auch wenn diese nur rezeptiv überprüft wurden, als sehr gut zu bezeichnen. So wurden kurzfristig (also direkt nach dem Unterricht) in der o. g. Gruppe, von der langfristige Behaltensleistungen gemessen werden konnten, mit Spielen 89,2\% der zu lernenden Vokabeln behalten, nach vier Wochen waren es immerhin noch 73,6 \% (=82,5\% der vorab gelernten) und nach drei Monaten 65,9\% (=89,5\% der vorab gelernten). Ohne Spiele wurden direkt nach dem Unterricht $71 \%$ der zu lernenden Vokabeln memorisiert, nach vier Wochen waren es $69,2 \%(=97,4 \%$ der vorab gelernten) und nach drei Monaten noch immer 68,2\% (=98,6\% der vorab gelernten $)^{5}$.

Die durchgeführte Untersuchung konnte somit zeigen, dass der Unterricht ausschließlich auf Basis von Sprachlernspielen in einer vergleichbaren Unterrichtssituation ebenso effizient wie der Unterricht ohne Einbezug von Sprachlernspielen war $^{6}$. Die Untersuchungsergebnisse weisen also daraufhin, dass Sprachlernspiele eine empfehlenswerte, da effiziente Lernform im Fremdsprachenunterricht sind. Da beide Vermittlungswege (basierend auf Spiele und ohne Spiele) mit identischem Zeitaufwand durchgeführt wurden, kann darüber hinaus dem Gedanken, Spiele seien eine zeitintensivere Übungsform als andere, widersprochen werden. Aus didaktisch-methodischer Sicht kann der Unterricht mit Sprachlernspielen, wenn sie lernziel- und zielgruppenorientiert ausgewählt werden, im Vergleich mit anderen Unterrichtsverfahren ein ebenso guter, wenn nicht gar besserer Vermittlungsweg sein. Dies mag die eingangs benannten Bedenken von Lernenden gegenüber dem Spieleinsatz nicht zwangsläufig ausräumen, gibt aber Lehrpersonen ein Argument für den Spieleinsatz an die Hand und dürfte auch von Lemenden positiv aufgenommen werden, zumindest sobald diese selbst die Erfahrung machen, dass sie tatsächlich effizient mit Spielen und spielerischen Übungen lernen können?7.

Insbesondere aufgrund der - wenn auch nur rezeptiv überprüften - hohen Behaltensleistungen (bis zu 89,2\% kurzfristige Behaltensleistung sowie nach drei

\footnotetext{
${ }^{5}$ Hierbei sei angemerkt, dass die Teilnehmer innerhalb der Unterrichtsstunden, in denen die Untersuchung durchgeführt wurde, sehr viel mehr gelernt haben, als nur den reinen, die Untersuchung betreffenden Wortschatz. Z. B. erforderte die Durchführung der Spiele und Übungsformen natürlich auch andere sprachliche Leistungen. Da es sich um heterogene Gruppen handelte, verlief jegliche Kommunikation im Unterricht auf Deutsch, also auch die Verständigung über Spielhandlungen bzw. Arbeitsanweisungen usw. Auch weitere konkrete sprachliche Inhalte kamen zwangsläufig zur Vermittlung bzw. wurden in allen Unterrichtseinheiten thematisiert, so z. B. die Artikel und Pluralendungen der zu lernenden Vokabeln und in diesem Zusammenhang auch weitere Regeln zu deren Bildung, wie z. B. zum Diminutiv.

${ }^{6}$ Die hier präsentierten Ergebnisse sind natürlich nicht pauschal auf den Einsatz von Spielen in jeglicher gesteuerten fremdsprachlichen Lernsituation übertragbar. Sie sind ohne Zweifel abhängig von der untersuchten Lernergruppe, den hier ausgewählten Spieltypen usw. zu sehen. Dennoch zeigen sie deutlich, dass es möglich ist, mit Spielen zu lernen und zu behalten und dies auch durchaus effizient.

${ }^{7}$ Bei der durchgeführten Untersuchung belegen Rückmeldungen der Teilnehmer, dass einige erst durch den Einsatz der zur Messung des Lernerfolgs eingesetzten Prüfbogen ihren Lernerfolg bemerkten; beispielsweise schreibt ein Teilnehmer: „Ich habe etwas gelernt, ohne es zu bemerken. Erst am Ende dieses Unterrichts (Liste) habe ich bemerkt, dass ich viele Wörter gelernt hatte“.; eine weitere Person bemerkt z. B.: „Die Tabellen waren nur wie eine Prüfung (nötig auch für Ihre Forschung), aber gut: Man bemerkt, wie der Lernprozess sich entwickelt.“ (Anonyme Teilnehmerzitate, vgl. Jentges 2007).
} 
Monaten noch bis zu 68,2\%) halte ich die für die Untersuchung erstellten Materialien und eingesetzten Vermittlungswege für eine im Unterricht stattfindende Wortschatzvermittlung, insbesondere für spezifischen Wortschatz, für sehr empfehlenswert. So konnte selbst für den doch recht ungewöhnlichen Begriff Eichelhäher, der das prozentual gesehen am schlechtesten behaltene Wort darstellt, immerhin kurzfristig noch $63,6 \%$ Lernerfolg nachgewiesen werden, übrigens gefolgt von der Meise mit 64,6 \%, dem Veilchen mit $65 \%$, der Elster mit 67,1\% und dem Enzian mit 69,2\%. Für alle anderen zu lernenden Begriffe konnten kurzfristig, prozentual gesehen, Behaltensleistungen zwischen $70 \%$ und $100 \%$ ermittelt werden.

\section{Vermittlung von Fachwortschatz}

Wortschatzvermittlung und vor allem das Lernen von Wortschatz wird in der Unterrichtsrealität häufig auf das selbstständige Lernen zu Hause verlagert. Im „,normalen“ Fremdsprachenunterricht ist es relativ selten, dass tatsächlich ausschließlich die Wortschatzvermittlung und -übung zum Inhalt einer ganzen Unterrichtseinheit oder -stunde erklärt wird. Dies dürfte daran liegen, dass das Memorieren von Vokabular ein sehr individueller Vorgang ist und man daher den Lernenden eher verschiedene Verfahren zur Memorisierung von Wortschatz vorstellt, die diese dann selbst erproben, je nach Vorliebe anwenden und dann eigenständig so lernen (können), wie es für sie am angenehmsten, leichtesten bzw. effizientesten ist, als diese Arbeit im Unterricht zu integrieren.

Insbesondere im Bereich der Fachsprachen stellt Wortschatzvermittlung aber in aller Regel einen zentralen Aspekt dar. Denn hier wird - nicht nur - aber zu einem gewissen Teil und zwar meist einem recht großen, die Allgemeinsprache um ein entsprechendes Vokabular ergänzt. Fachsprache verfügt über einen (im GroBen und Ganzen zusätzlichen) Spezialwortschatz; darüber hinaus natürlich noch über weitere Komponenten, z. B. dass im beruflichen Umgang spezielle Formen existieren können, die Außenseiter nicht kennen. Ziel im Fachsprachenunterricht ist es jedenfalls, die Lernenden in die Lage zu versetzen, in der Zielsprache entsprechende Gespräche zu führen und auch Fachtexte zu verstehen. Dabei braucht man neben Entschlüsselungsstrategien auch die Kenntnis der entsprechenden Terminologie. Und meistens ist es zudem bzw. daher so, dass Fachsprachenunterricht nur in Ausnahmefällen im Anfängerunterricht stattfindet, meist werden bereits 300 oder mehr Stunden Fremdsprachenunterricht vorausgesetzt. D. h., ein Großteil des Fachsprachenunterrichts besteht aus der Vermittlung bestimmter Floskeln und Termini, die ergänzend zum allgemeinen Wortschatz der Sprache, die gelernt wird, vermittelt werden müssen. Wie diese Fachwortschatzvermittlung im Unterricht integriert und mittels Spielen und spielerischer Übungen mit Lerneffizienz und Freude am Lernen gleichzeitig vermittelt werden kann, möchte ich im Folgenden beschreiben, wozu ich das genauere Vorgehen der ursprünglich von 
mir für die Untersuchung konzipierten und hier für fachspezifisches Vokabular weiterentwickelten Wortschatzvermittlungswege vorstellen möchte.

\section{$4 \quad$ Vorschlag zur Vermittlung fachspezifischen Wortschatzes}

Gesteuerte Wortschatzvermittlung im Fremdsprachenunterricht besteht klassischerweise - wie auch die Vermittlung anderer Phänomene - aus einer Einstiegsphase, aus der Erwerbsphase, also der Bedeutungsvermittlung, einer Übungsphase und einer Vertiefungsphase, in der das Gelernte angewendet werden soll, sowie späteren Wiederholungsphasen ${ }^{8}$. Dieser Phasenstruktur entsprechen auch die in der Untersuchung eingesetzten, im Folgenden vorgestellten Vermittlungswege.

Darüber hinaus ist es üblich und sinnvoll, im Unterricht nicht zusammenhangslos beliebigen Wortschatz einzuführen, sondern die Auswahl des zu vermittelnden Wortschatzes erfolgt nach Wortfeldern oder thematischen Bereichen, z. B. dem Thema einer bestimmten Lehrbuchlektion. Wichtig bei der Auswahl des zu vermittelnden Vokabulars ist darüber hinaus, ein vom Schwierigkeitsgrad her angemessenes Lernset zusammen zu stellen, wobei natürlich eine Prognose darüber, welches Wort für jeden einzelnen Lerner als eher leicht oder eher schwer zu erwerben einzustufen wäre, nahezu unmöglich ist. Bekanntlich ist Lernen ein individueller Vorgang, nicht nur abhängig von Lernertyp und Lerntraditionen, sondern auch von individuellen Interessen und Vorlieben sowie stark von Gefühlen und Erfahrungen geprägt. Dennoch lassen sich generelle Tendenzen festhalten, die bei der konkreten Auswahl eines vom Schwierigkeitsgrad der Lernergruppe angemessenen Sets zu lernender Vokabeln berücksichtigt werden sollten?.

Im Folgenden werden nun die einzelnen Schritte des hier vorgeschlagenen Vermittlungsweges am Beispiel von Vokabular aus dem Hotel- und Gaststättengewerbe bzw. aus dem Bereich „Deutsch für Tourismus“ vorgestellt. Dieser Bereich dürfte im Übrigen nach „Wirtschaftsdeutsch“ - zumindest nach hierfür auf

\footnotetext{
${ }^{8}$ Natürlich sieht die heutige Fremdsprachenlehr- und -lernforschung Wiederholungsphasen für die Vermittlung neuer Inhalte vor. Dies gilt selbstverständlich auch für eine ideale Wortschatzvermittlung (vgl. z. B. Kleinschroth 1992: 84ff). Im Rahmen des zuvor beschriebenen Experiments musste auf entsprechende Wiederholungsphasen des zu Lernenden jedoch aus untersuchungstechnischen Gründen verzichtet werden.

${ }^{9}$ Zunächst einmal geht man davon aus, dass Konkreta besser behalten werden als Abstrakta und Nomen besser als Verben (vgl. u. a. de Groot/Nas 1991: 114 sowie de Groot 1993: 37ff). Neuner spricht in diesem Zusammenhang von visualisierbaren Wörtern. Dies müssen nicht zwangsweise konkrete Nomina sein, diese sind aber auf jeden Fall visualisierbar (vgl. Neuner 1991: 79). Auch einoder zweisilbige Begriffe können als leichter zu lernen eingestuft werden als mehrsilbige. Ebenso kann die Aussprechbarkeit eines Wortes den Schwierigkeitsgrad des Memorierens beeinflussen. Inhaltliche und/oder formale Ähnlichkeit mit der Muttersprache, also insbesondere Cognates und Internationalismen, dürften gegenüber Begriffen ohne Übereinstimmung mit der Muttersprache, insbesondere Falsche Freunde und Regionalismen, als leichter zu lernen gesehen werden können. Gleiches gilt für Komposita, bestehend aus bekannten Wortteilen, im Gegensatz zu solchen Komposita, die aus unbekannten Wortteilen bestehen.
} 
dem Markt erhältlichen Lehrbüchern ${ }^{10}$ zu urteilen - der größte im Bereich Fachsprachenunterricht sein. Im besagten fachspezifischen Unterricht muss u. a. ein sehr viel größeres Spektrum an Lebensmitteln, Nahrungsprodukten und Gerichten erworben werden, als dies im allgemeinsprachlichen Unterricht der Fall ist. Hier ist es beispielsweise von Nöten bei dem Begriff „Pilz“ nicht nur zwischen Speise- und Giftpilz differenzieren zu können, sondern auch verschiedene Speisepilzarten wie z. B. Pfifferling, Steinpilz und Trüffel oder Champignon, Shiitake und Austernpilz namentlich zu kennen. In den im Folgenden vorgestellten konkreten Beispielen geht es nicht um die deutschsprachigen Bezeichnungen von bestimmten Gerichten und Lebens- bzw. Nahrungsmitteln ${ }^{11}$, sondern im Speziellen um die deutschsprachigen Bezeichnungen verschiedener Kräuter- und Gewürzsorten. Dies wird lediglich in einem der Lehrwerke für diesen Bereich gemacht (vgl. Lévy-Hillerich 2005: 51f) und zwar auch nur in sehr kurzer Form und zu einem großen Teil unter Einbezug von Internationalismen (z. B. Curry, Safran). Übrigens ist dies auch in allgemeinsprachlichen Lehrbüchern ein stark vernachlässigter Bereich; in einem normalen DaF-Lehrwerk lernt man außer Zucker, Pfeffer und Salz kaum Würzmöglichkeiten auf Deutsch kennen.

Hier werden daher ausschließlich solche Gewürzbezeichnungen und Kräuter thematisiert, die zum einen auf einem Mittelstufen-Niveau (ab B1) nicht als bekannt vorausgesetzt werden können (Pfeffer, Salz z. B.). Außerdem bleiben Begriffe unberücksichtigt, die als Internationalismen oder als solche mit starker inhaltlicher und formaler Ähnlichkeit ${ }^{12}$ mit mehreren Sprachen eingestuft werden können ${ }^{13}$. Außerdem wurden nur solche berücksichtigt, die traditionell im deutschsprachigen Raum Verwendung finden. Eine auf dieser Basis mögliche, zu vermittelnde Wortliste mit einem Set von 20 zu lernenden Vokabeln könnte wie folgt aussehen:

\footnotetext{
${ }^{10}$ Man denke beispielsweise an „Kommunikation im Beruf: Kommunikation im Tourismus“ (LévyHillerich 2005), „Zimmer frei“ "(Cohen 2000), „Deutsch im Hotel, Teil 1 und 2“ (Barberis / Bruno 2000 sowie 2001), ,Herzlich willkommen“ (Cohen 2001) sowie auch „Culinary Guidebook Germany. A Culinary Journey through Germany“ (Lennert 2003), „Fachwörterbuch für Hotellerie und Gastronomie. Deutsch - Englisch/English - German“" (Schaetzing 2006).

${ }_{11}$ Dies geschieht in den meisten Lehrwerken für diesen Bereich (vgl. z. B. Barberis/Bruno 2000: $17-$ 32, Lévy-Hillerich 2005: 49-64: insb. 51-52, Cohen 2001: 18-42 sowie Cohen 2000: 22f, 44, 58).

${ }^{12}$ Gemeint sind gleicher etymologischen Ursprung als auch Wortbildungsmuster, die von einer auf die andere Sprache übertragen wurden, also Lehnwörter.

${ }^{13}$ Vgl. z. B. Anis (engl. anise, franz. anis, span. anís), Chili (engl. chili, franz. chili, span. Chile), Curry (engl. curry, franz. curry, span. curry), aber auch: Kardamom (engl. cardamom, franz.: cardamome, span. cardamomo), Koriander (engl. coriander, franz. coriandre, span. culantro), Safran (engl. saffron, franz. safran, span. azafrán), Vanille (engl. vanilla, franz. vanille, span. vainilla), vgl. Small 1997. Natürlich sollten auch solche Begriffe im Unterricht thematisiert werden. Hierbei steht aber weniger das Einprägen der Bedeutung im Mittelpunkt als das Einprägen der korrekten Schreibweise und Aussprache im Deutschen sowie ggf. eine von der Muttersprache abweichende

Genuszuweisung. Darüber hinaus wurden auch solche Begriffe nicht berücksichtigt, bei denen die ursprüngliche deutsche Bezeichnung inzwischen von der internationalen verdrängt wurde, wie z. B. bei Dost als deutsche Bezeichnung für Oregano bzw. wilden Thymian, die inzw. weitestgehend von der internationalen (auf der lateinischen Fachterminologie basierende Bezeichnung) gewichen ist. Gleiches gilt z. B. auch für Gelbwurz(el) (= Kurkuma), Kreuzkümmel (= Cumin), Süßholz (= Lakritze) usw.
} 
Bärlauch, Beifuß, Bohnenkraut, Bor(r)etsch, Brunnenkresse, Dill, Ingwer, Kapuzinerkresse, Kerbel, Kümmel, Liebstöckel, Lorbeer, Petersilie, Salbei, Sauerampfer, Schnittlauch, Wacholder, Waldmeister, Zimt, Zitronenmelisse.

Erfahrungsgemäß würde ich für eine Unterrichtseinheit von 45-60 Minuten raten, nicht mehr als bis zu 30 neue Vokabeln zu vermitteln.

\subsection{Einstieg}

Ein gelungener Einstieg in die Unterrichtsstunde bzw. in die Thematik ist von großer Bedeutung, Einstiege beeinflussen nicht unwesentlich den weiteren Verlauf der Stunde. Mit einem gelungenen Einstieg sollte einerseits Interesse und Aufmerksamkeit bei den Lernenden geweckt, aber auch ein affektiv-emotionaler Kontakt zum Thema hergestellt werden.

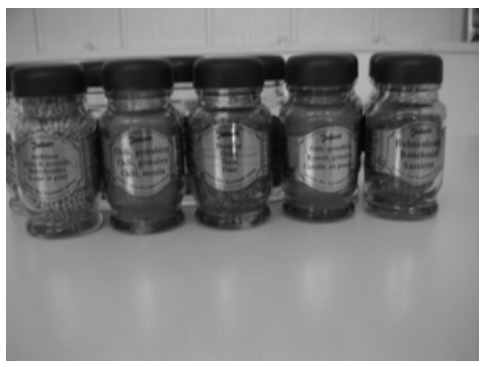

Hierzu können z. B. Illustrationen zum Thema eingebracht werden. Aus drucktechnischen Gründen können Beispiele hierfür nur in Graustufen abgebildet werden, im Unterricht ist es aber mehr als sinnvoll mit farbigen Illustrationen zu arbeiten, um zu gewährleisten, dass die Illustrationen eindeutig und nicht verallgemeinernd sind.
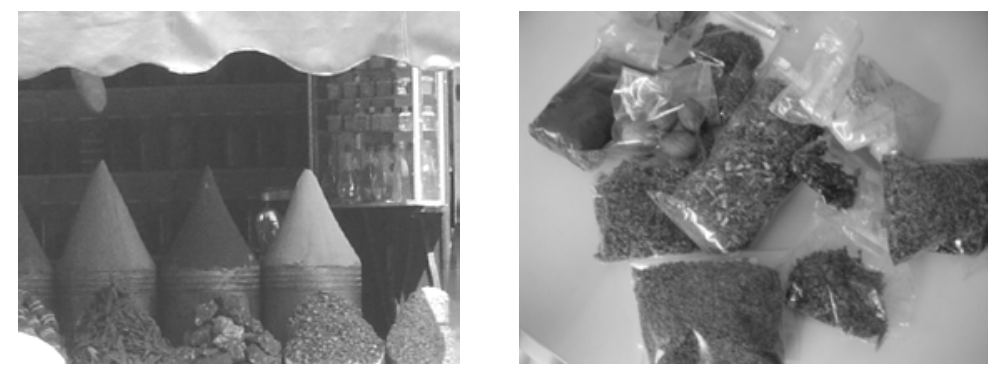

Die Lernenden erhalten z. B. jeweils aus einem Bild hergestellte Puzzle, die zusammengesetzt werden sollen, um sich so selbst das Thema der Stunde zu erschließen.

Eine weitere, durchaus auch ergänzend sinnvoll einzusetzende Variante wäre das Einbringen echter Produkte, also Kräuter und Gewürzsorten. Man bringt die entsprechenden Produkte z. B. in kleinen Behältern mit, stellt diese auf einem Tisch und lässt sie von den Lernen „beschnuppern“ sowie ggf. auch schmecken. Ein solcher Überraschungseffekt sichert die Aufmerksamkeit der Lerner und wird diese auf das, was dann folgt, neugierig machen. Die mitgebrachten Originalpro- 
dukte können übrigens leicht für folgende Gruppenarbeiten zur Partnerfindung eingesetzt werden, ebenso können sie als Preise für die folgenden Spielsequenzen genutzt werden.

Hilfreich im Falle des Erriechens und Erschmeckens als Einstieg dürfte es auch sein, entsprechende Adjektive zur Geruchs- bzw. Geschmacksbezeichnung wiederholend sammeln zu lassen; dies sollte auch in schriftlicher Form, z. B. an der Tafel, festgehalten werden.

\subsection{Bedeutungsvermittlung}

Besonders wesentlich ist eine gelungene Bedeutungsvermittlung, da diese Voraussetzung für ein späteres erfolgreiches Wiedererkennen, Behalten und Anwenden ist. Daher sollte versucht werden, möglichst vielfältige Verfahren der Bedeutungsvermittlung einzusetzen, also z. B. auch morphologische Komponenten bei Komposita. Bedacht werden muss auch, dass Wortschatzvermittlung, insbesondere die Bedeutungsvermittlung, immer abhängig ist vom zu vermittelnden Wortschatz (Substantiv oder Verb, Abstrakta oder Konkreta usw.), von der Lernergruppe sowie der Individualität eines jeden einzelnen Lernenden (Lernertypen, Lerntraditionen etc.). Bei der Wortschatzvermittlung, zunächst bestehend aus der Formund Bedeutungsvermittlung, erscheint für das hier vorliegende Wortmaterial eine Kombination verschiedener erklärender Ansätze sinnvoll. Geht man von einer heterogenen Lernergruppe aus, kann nur mit einsprachigen Erklärungen gearbeitet werden. Inhaltliche oder formale Ähnlichkeit von Wörtern mit anderen Sprachen und Internationalismen können in diesem Fall nicht erklärend genutzt werden ${ }^{14}$. Bedacht werden muss auch, dass eventuell (einzelne) Lerner einzelne der zu lernenden Begriffe als Konzept nicht kennen und ihre Muttersprache über keine Bezeichnung dafür verfügt, da diese in der Herkunftskultur nicht existieren.

Nur mit Visualisierungen zu arbeiten, wäre schwierig, da zunächst einmal überlegt werden muss, ob das Gewürz in seiner natürlichen Form als Pflanze gezeigt werden sollte oder als getrocknetes Gewürzprodukt, Pulver oder Blätter oder in einer Kombination von beiden. Hier dürfte das Einbringen echter Produkte zum „(Er)riechen“ wesentlich sein, diese lassen sich nämlich viel einfacher „erriechen“ als dies durch ihre visuelle Erscheinung ${ }^{15}$ oder durch das Er- bzw. Abtasten möglich wäre. Hier bietet sich eine olfaktorische Präsentation, ggf. ergänzt durch den Einsatz von Visualisierungen ${ }^{16}$, zur Erschließung der zu lernenden Konkreta an, die das Verstehen erleichtern kann, da das Dargestellte in seiner Bedeutung veran-

\footnotetext{
${ }^{14}$ Für die Arbeit mit Gruppen einer gemeinsamen Muttersprache bietet es sich natürlich an, auf die Muttersprache als erklärende Übersetzung zurückzugreifen.

${ }^{15}$ Kreuzkümmel, Kurkuma, Safran, Curry usw. lassen sich z. B. farblich kaum von der rein visuellen Erscheinung unterscheiden, insbesondere, wenn diese als Abbildung präsentiert werden.

${ }^{16}$ In gängigen Gewürzlexika und Enzyklopädien, z. B. im Internet, findet sich eine Vielzahl an entsprechenden Illustrationen. Für besonders gelungen halte ich solche Zeichnungen, die die Abbildung der jeweiligen Gewürzpflanze mit dem daraus gewonnen Küchengewürz kombinieren.
} 
schaulicht wird. Auch zu bedenken ist, dass für die hier angenommene Lerngruppe die Nutzung der Gewürze und Kräuter im Küchenkontext interessant ist und von daher auch diese Berücksichtigung finden sollte. Eine Kombination verschiedener Erklärungsansätze zur Erschließung der Bedeutung ist in diesem Fall also unerlässlich.

Neben dem Duft echter Gewürze und minimalen Informationen zum Gebrauch im Küchenkontext, die zur Erklärung des Begriffs dienen, sollten jedoch auch die zu lernenden Vokabeln selbst ermittelt werden. Da es sich um neuen, also den Lernenden unbekannten Wortschatz handelt, muss in diesem ersten Vermittlungsschritt auf reine Zuordnungsübungen z. B. verzichtet werden. Dennoch sollten Lernende die Chance haben, sich den neuen Begriff auf sprachlicher, also auch orthografischer Ebene selbst zu entschlüsseln, also zu selbstentdeckendem Lernen angeregt zu werden. Hierzu schlage ich vor, zu jeder Vokabel auch kurze Rätsel zu erstellen, die die Möglichkeit eröffnen, die Bezeichnung des jeweiligen Gewürzes selbst herauszufinden. Vergleichbar der von Mnemotechniken vorgeschlagenen Lernstrategien können hierzu Bilderrätsel, Sprüche und Verse, Buchstaben- und Worträtsel eingesetzt werden, wie in den folgenden Beispielen dargestellt ${ }^{17}$.

\begin{tabular}{|c|c|}
\hline $\begin{array}{l}\text { Lösen Sie das Rätsel, dann finden Sie den } \\
\text { Namen von diesem Gewürz heraus: } \\
\text { (Plural) } \\
\text { Tipp: Der zweite Wortteil (unteres Bild) ist } \\
\text { übrigens der griechische Begriff für Biene! }\end{array}$ & $\begin{array}{l}\text { Ein Gedicht von Ringelnatz heißt: „Das } \\
\text { arme Kräutlein“, darin heißt es: } \\
\text { „Ein Sauerampfer auf dem Damm stand zwi- } \\
\text { schen Bahngleisen, [...] Der arme Sauerampfer } \\
\text { sah Eisenbahn um Eisenbahn, sah niemals einen } \\
\text { Dampfer.“ } \\
\text { Wie heißt das gesuchte Kraut? } \\
\text { Tipp: Das gesuchte Kraut ist natürlich ein } \\
\text { Substantiv und zwar das mit den meisten } \\
\text { Vokalen! }\end{array}$ \\
\hline $\begin{array}{l}\text { Wenn Sie die Endbuchstaben der folgen- } \\
\text { den Gewürze zusammensetzen, erhalten } \\
\text { Sie den Namen dieses Gewürzes: } \\
\text { Salz, Chili, Basilikum, Piment }\end{array}$ & $\begin{array}{l}\text { Lösen Sie das Rätsel, um den Namen dieses } \\
\text { Krautes zu erhalten: } \\
\text { Tipp: Der zweite Wortteil ist ein anderer } \\
\text { Begriff für „Porree“! }\end{array}$ \\
\hline
\end{tabular}

${ }_{17}$ Die hier und im nächsten Abschnitt verwendeten Zeichnungen stammen aus der sehr empfehlenswerten Sammlung „Sprachspiele - Tipps und Ideen zum Sprachenlernen“ von Manuela Macedonia 2000. Der genau Bildquellennachweis ist: Zitrone: 66, Melone: 66, Bär: 98, Kerze: 40, Nebel: 42, Wald: 94, Füße: 85, Hund: 93 und Salbe: 69. 


\begin{tabular}{|l|l|}
\hline Stellen Sie sich eine Kerze & $\begin{array}{l}\text { Mehrere Bäume sind ein }- \\
---? \\
\text { Und wie bezeichnet man einen sehr, sehr } \\
\text { guten, hervorragenden Koch? Das ist ein } \\
-1\end{array}$ im Nebelvor. \\
$\begin{array}{l}\text { Setzen Sie die erste Silbe des ersten fettge- } \\
\text { druckten Wortes und die zweite Silbe des } \\
\text { zweiten fettgedruckten Wortes zusammen, } \\
\text { so erhalten Sie den Namen dieser Pflanze. }\end{array}$ & $\begin{array}{l}\text { Ergänzen Sie jetzt die beiden Begriffe und } \\
\text { Sie haben den Namen dieser Pflanze. }\end{array}$ \\
\hline
\end{tabular}

In einigen Fällen bieten sich auch Erklärungsansätze, die mit der Schlüsselwort-Methode ${ }^{18}$ vergleichbar sind, an. Hier können als Merkhilfe zum Teil Visualisierungen von ähnlich klingenden Wörtern benutzt werden, z. B. für „Beifuß“ (= bei Fuß) und „Salbei“ (= Salbe + i oder: $=$ Salbe + Ei). Allerdings kann - vorausgesetzt es handelt sich um eine heterogene Lernergruppe mit unterschiedlichen Muttersprachen - hier kein ähnlich klingendes Wort in der Muttersprache der Lerner angebracht werden, sondern nur ein als bereits bekannt anzunehmendes in der Fremdsprache, also Deutsch, wie in den folgenden Beispielen vorgestellt.

\begin{tabular}{l} 
Wenn ein Besitzer seinen \\
Hund zu sich ruft, sagt er: \\
$\begin{array}{l}\text { So heißt auch diese Pflanze, es ist aber nur } \\
\text { ein Wort. }\end{array}$ \\
$\begin{array}{l}\text { Tipp: Es ist keine Creme, sondern eine } \\
\text { gen).-- (benutzt man z. B. bei Verletzun- }\end{array}$ \\
\hline Sie den Namen dieser Pflanze:
\end{tabular}

Diese Lernhilfen können Üben nicht ersetzen, aber eventuell Behaltensleistungen steigern. Wie erfolgreich die hier angebotenen Lernhilfen aus dem Bereich der Mnemotechniken (natürlich zunächst um Bezeichnungen überhaupt herauszufinden, in Kombination mit dem echten Gewürz) von den Lernenden aufgenommen werden, ist schwer zu beurteilen, da das Erstellen solcher Merkhilfen ja ein sehr individueller Vorgang ist. Sie können aber durchaus als zusätzliches Angebot der Merkhilfe angesehen werden, die wie von einigen Untersuchungsteilnehmern betont, im Rahmen des Experiments auch genutzt wurden: „Ich habe auch gesehen, dass die Wortbildungsübungen (z. B. Rätsel, Bildung der Tierna-

\footnotetext{
18 Der Lerner assoziiert zu jedem zu lernenden Wort ein Bild, das in der Muttersprache des Lerners ähnlich klingt wie das zu lernende fremdsprachige Wort und baut als Gedächtnisstütze ein interaktives Bild auf, in dem die Bedeutung dieses muttersprachlichen Wortes und des zu lernenden Wortes in Zusammenhang gebracht werden. Für einen Überblick zu dieser Methode siehe z. B.

Pressley/Levin/McDaniel 1987: 107ff sowie Stork 2002: 119ff.
} 
men) eine gute Hilfe sein können“, „Die Rätsel sind gut, weil man Bilder oder Wörterspiele (Fuchs+ie, Stiefmütter+chen) mit den Vokabeln verbindet und damit ist es schwerer zu vergessen." (Anonyme Teilnehmerzitate, vgl. Jentges 2007).

Daher halte ich für eine gesteuerte Wortschatzvermittlung das hier vorgestellte Verfahren zur Bedeutungsvermittlung, bestehend aus einer Kombination von nichtsprachlichen (nonverbalen) und sprachlichen (verbalen) Erklärungs- und Entschlüsselungsverfahren, besonders für fachspezifischen Wortschatz für äußerst empfehlenswert.

In der Untersuchung wurden die so erstellten Materialien $^{19}$ zum einen als Partnerarbeitsblätter eingesetzt, zum anderen in einer Spielversion in Anlehnung an die Fernsehsendung „Der Große Preis“ (vergleichbar auch mit Jeopardy, engl.). Für die dort eingesetzte Spielvariante suchen sich die Lerner Felder auf der Spielwand aus und versuchen, die dahinter „versteckten“ Fragen zu beantworten. Gelingt ihnen

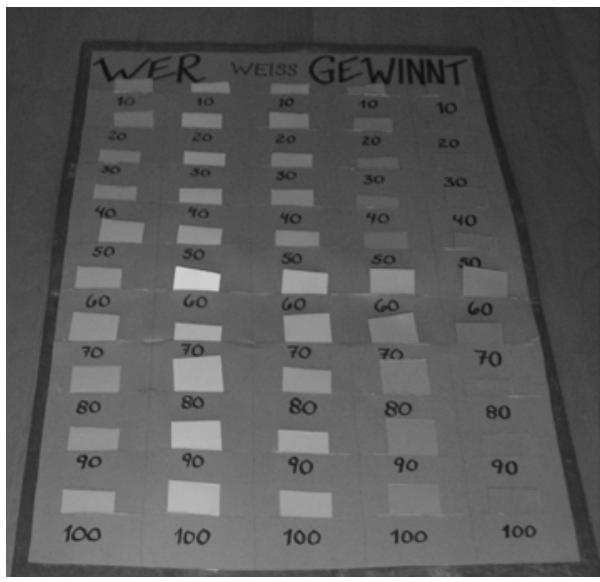
dies, erhalten sie die auf dem Feld genannten Punkte.

Gewinner ist selbstverständlich die Person oder Gruppe, die am Ende die meisten Punkte hat ${ }^{20}$.

Im Falle der Gewürze wäre für jede Variante empfehlenswert, die mitgebrachten Gewürz-Behältnisse mit Nummern zu versehen, mit welchen wiederum die entsprechenden Rätsel und Kurzbeschreibungen auf allen eingesetzten Materialien versehen sind.

Zu empfehlen wäre für diese Phase auch ein domino-ähnliches Spiel, bei dem ein Spielstein nicht wie üblich aus zwei, sondern aus drei Feldern besteht, wie im Folgenden präsentiert.

\footnotetext{
${ }^{19}$ Hier kamen für die Bedeutungsvermittlung nur Rätsel und Abbildungen der zu lernenden Blumenund Tierbezeichnungen zum Einsatz.

${ }^{20}$ In der eingangs beschriebenen Untersuchung zur Überprüfung der Lerneffizienz mit Spielen wurden für den Unterricht mit Spielen nur solche Aktivitäten ausgewählt, die als klassische Spiele gelten und von jedem deutlich als Spiel erkannt werden, also alle Kriterien des Spiels erfüllen inkl. Gewinner-Verlierer-Aspekt sowie Glücksfaktor.
} 


\begin{tabular}{|c|c|c|}
\hline START & $\begin{array}{l}\text { Dieses Kraut schmeckt } \\
\text { kräftig, würzig und erinnert } \\
\text { an Thymian, ist aber } \\
\text { etwas bitterer. }\end{array}$ & $\begin{array}{l}\text { Das ist ein typisches } \\
\text { Gewürz für Hülsenfrüchte. } \\
\text { Daher kommt auch sein } \\
\text { Name, es heißt nicht } \\
\text { Erbsen, nicht Linsen, } \\
\text { sondern _______kraut. }\end{array}$ \\
\hline das Bohnenkraut & $\begin{array}{l}\text { Diese Pflanze wird wegen } \\
\text { ihres Gurkengeruchs auch } \\
\text { Gurkenkraut genannt. Die } \\
\text { frischen Blätter werden z. } \\
\text { B. für Salate verwendet. } \\
\text { Sie ist auch Bestandteil } \\
\text { der Frankfurter Grünen } \\
\text { Soße. }\end{array}$ & $\begin{array}{l}\text { Wenn Sie die Anfangs- } \\
\text { buchstaben der folgenden } \\
\text { Küchenkräuter und Ge- } \\
\text { würzmittel zusammenset- } \\
\text { zen, erhalten Sie den } \\
\text { Namen dieses Krautes: } \\
\text { Basilikum, Oregano, } \\
\text { Rosmarin, Rosenblätter, } \\
\text { Essig, Tomate, Salz, Chili, } \\
\text { Honig }\end{array}$ \\
\hline der Borretsch & $\begin{array}{l}\text { Diese Pflanze riecht an- } \\
\text { genehm, ihr Geschmack } \\
\text { ist aber scharf und würzig. } \\
\text { Man benutzt sie bei Ge- } \\
\text { flügel, aber auch Lebku- } \\
\text { chen und Printen werden } \\
\text { damit verfeinert. }\end{array}$ & $\begin{array}{l}\text { Wer ist ein Ing.? Ing. ist } \\
\text { die Abkürzung für einen } \\
\text { Ingenieur. Beim Zusam- } \\
\text { mensetzen der beiden fett } \\
\text { gedruckten Wörter in der } \\
\text { Frage ergibt sich die } \\
\text { gesuchte Pflanzenbe- } \\
\text { zeichnung; der Anfangs- } \\
\text { buchstabe ist „„“. }\end{array}$ \\
\hline
\end{tabular}

\section{3 Üben und Anwenden}

Für den zweiten und dritten Schritt der Wortschatzvermittlung, also dem Üben und Anwenden des Wortschatzes, schlage ich vor, sowohl intentionales (also bewusstes) als auch inzidenzielles (also unbewusstes) Lernen zu integrieren, da so verschiedene Lerntraditionen angesprochen werden können ${ }^{21}$. Hierzu können klassische Bild-Text- bzw. Produkt-Text-Zuordnungsaufgaben erstellt und in einen anderen Kontext gebracht werden. Ein typisches Spiel für diesen Schritt wäre ein Memory-Spiel oder eine Aufgabe mit spielerischem Charakter bei der die Bild-

${ }^{21}$ Die Teilnehmerrückmeldungen aus der Untersuchung legen nahe, dass Spiele oft, gerade weil das in ihnen stattfindende „unbewusste“ Lernen ungewohnt ist, zwar als besonders positiv empfunden werden, aber nicht als „lästige“ Lernform wahrgenommen werden. Dies könnte zur Folge haben, dass Lerner insofern ,überfordert" werden, als dass sie (noch) nicht auf einen solchen Vermittlungsweg eingestellt sind und somit ihre üblichen, persönlichen, individuellen Lernstrategien für einen ungewohnten Unterrichtstil nicht aktivieren können. Wenn sie nur unbewusst lernen, tun sie vielleicht weniger, um bewusst zu lernen. 
Text-Zuordnung durch eine übertragene Aufgabenstellung in einen anderen Kontext (,maskierte Aufgabe") und somit attraktiver gestaltet wird.

Für die hier betrachteten Gewürzbezeichnungen würde ich z. B. anstelle eines Bildmemories ein „Riech“memory - bestehend aus Filmhüllen, in denen zum einen das Gewürz ist, zum anderen die auf Papier geschriebenen Gewürzbezeichnungen - vorschlagen.

Ergänzend könnte hier z. B. ein Wortfinder (die neuen Begriffe sollen in einem Buchstabengewirr entdeckt werden) oder Kreuzworträtsel eingesetzt werden. Auch ein Bingo z. B. wäre denkbar. In diesem Fall werden die Lernenden aufgefordert, die Namen der neu gelernten Gewürze in eine 9-zellige Tabelle einzutragen. Die Lehrperson ruft Gewürzbezeichnungen aus. Wer den entsprechenden Eintrag auf seinem Blatt hat, darf das Wort in seiner Tabelle ausstreichen. Gewonnen hat der Spieler, der alle Wörter als Erster durchgestrichen hat! Bewegungsaktiver lässt sich dies gestalten, indem die Lerner ein vorgefertigtes Blatt mit kurzen Beschreibungstexten, z. B. den bereits zur Bedeutungsvermittlung eingesetzten, erhalten und aufgefordert sind, durch den Unterrichtsraum zu gehen und die anderen Teilnehmer zu fragen, auf welches Gewürz die jeweilige Beschreibung zutrifft. In diesem Fall wäre derjenige Sieger, der zuerst Antworten auf alle auf seinem Arbeitsblatt enthaltene Beschreibungen erhalten hat. Die gerade genannten Spielideen sollten nur zusätzlich eingesetzt werden, da bei ihnen erfahrungsgemäß nur ein Teil der neu zu lernenden Vokabeln geübt werden kann.

Möglich ist auch, dass die Lehrperson zu den einzelnen Gewürzen treffende Charakteristika formuliert, z. B. für Zitronenmelisse: a) schmeckt frisch und zitronig, b) wird besonders im Sommer verwendet, c) der zweite Wortteil bedeutet auf Griechisch Biene. Wer als erstes weiß, um welches Gewürz es sich handelt, gewinnt das Gewürz.

Auch zwei Arbeitsblätter als Zuordnungsaufgabe von Text zu Illustrationen der Gewürze mit z. B. folgender Aufgabenstellung sind denkbar:

Arbeitsblatt 1: Der Gewürzhändler „Gewürze der Welt“ möchte ein Werbeblatt mit seinen Sonderangeboten machen. Leider fehlen einige Gewürzabbildungen und einige Gewürzbezeichnungen.

Helfen Sie dem Gewürzhändler wieder Ordnung in sein Werbeblatt zu bekommen! Bitte ergänzen Sie auch Artikel und Plural.

Arbeitsblatt 2: Was auf dem Werbeblatt vom Gewürzhändler fehlt, finden Sie hier. Nehmen Sie Schere und Klebstoff und bringen Sie die Werbung in Ordnung.

Einen Wettbewerbscharakter kann diese Aufgabe erhalten, indem man entweder ein zeitliches Limit einbaut oder die erste Gruppe, die alles korrekt gelöst hat, als Gewinner kürt.

Für die zweite Übungsphase der Wortschatzvermittlung, der Anwendung, kann z. B. eine Variante des bekannten Kinderspiels „Die Reise nach Jerusalem“ 
(=engl. musical chairs) eingesetzt werden. Dieses Spiel eignet sich besonders für Gruppen, die auch Bewegungsspielen gegenüber offen sind. Es werden pro Gruppe der Teilnehmerzahl -1 (minus eins) entsprechend viele Stühle abwechselnd nach vorne und nach hinten offen in einer Reihe aufgestellt. Die Lehrperson zieht eine Karte mit der Abbildung eines Gewürzes, die allen gezeigt wird, ohne den Namen des abgebildeten Gewürzes zu nennen. Die Lehrperson nennt nun beliebig viele Gewürzbezeichnungen. Wird der Name des Gewürzes von der zuvor gezogenen Karte ausgesprochen, müssen sich alle Teilnehmer setzen. Derjenige, der keinen Platz mehr bekommt, scheidet aus. Es wird ein Stuhl weggenommen, der Ausgeschiedene zieht eine neue Gewürzkarte, zeigt diese wieder allen Lernern und beginnt mit der Nennung von Gewürzbezeichnungen.

Eine bewegungsärmere Variante wäre das Verfassen von eigenen kleinen Rätseln zu dem zu lernenden Vokabular. Jeder Teilnehmer erhält ein Bildkärtchen²2, in Gruppen werden dann kleine Rätsel erstellt, wie sie den Teilnehmern bereits aus der Phase der Bedeutungsvermittlung bekannt sind. Die neuen Rätsel werden abschlieBend im Plenum vorgetragen. Alle gemeinsam versuchen, die Rätsel zu lösen.

Einige der in dieser Phase im Rahmen der Untersuchung entstandenen Rätsel zu Tier- und Blumenbezeichnungen sind in den folgenden Illustrationen zu sehen.

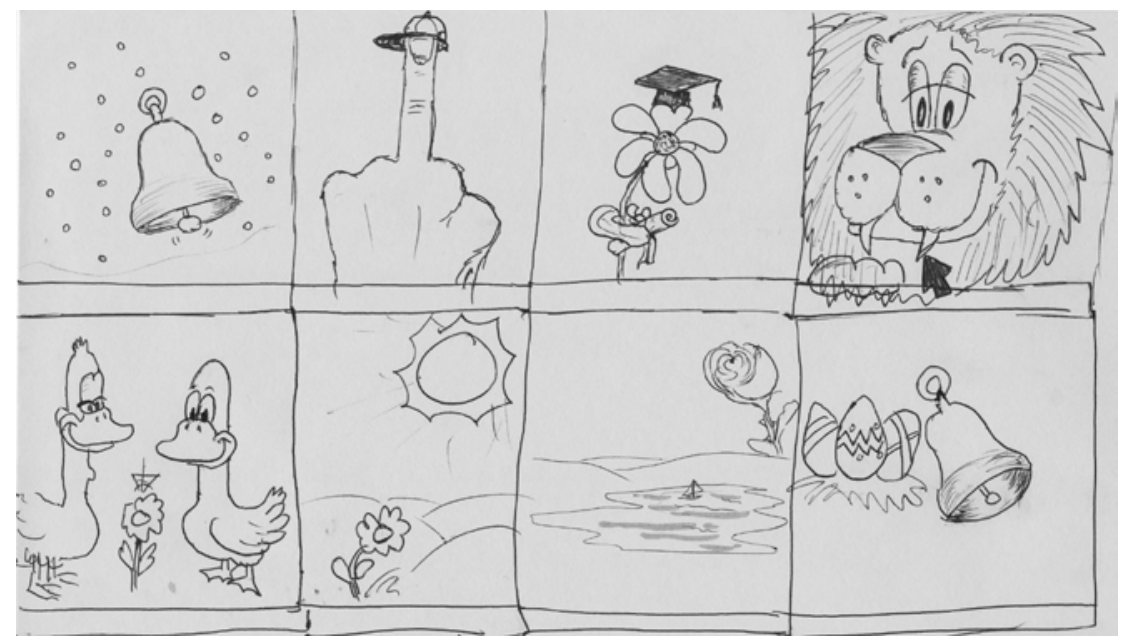

22 Um diese Übung tatsächlich lerneffizient zu gestalten, ist es wichtig, die Teilnehmer anzuhalten, nur zu solchen Vokabeln Rätsel zu erstellen, die ihnen tatsächlich vorab, also vor dem Unterricht, noch unbekannt waren. 


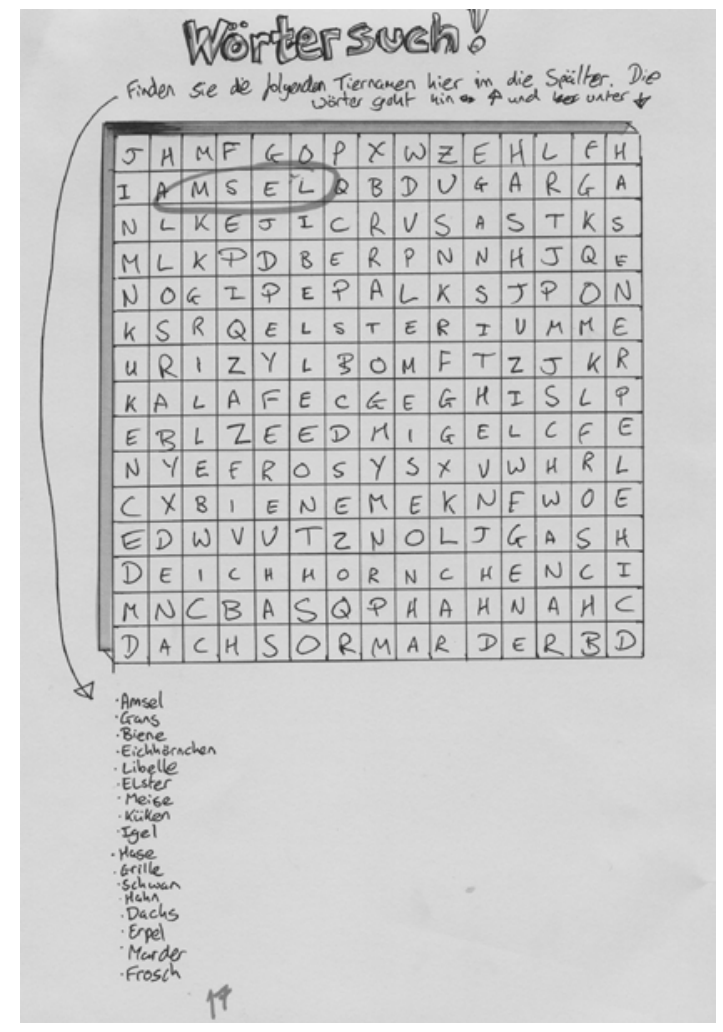

Weitere hier ergänzend einzusetzende Aktivitäten könnten z. B. Wortketten sein, die sich in zahlreichen Varianten einsetzen lassen, z. B. in alphabetischer. Hierbei soll möglichst schnell zu möglichst vielen Buchstaben des Alphabets eine Gewürzsorte mit dem entsprechenden Anfangsbuchstaben gefunden werden ${ }^{23}$, also z. B.: A = Anis, $\mathrm{B}=$ Bärlauch, $\mathrm{C}=$ Chili, $\mathrm{D}=$ Dill usw. Eine Variante hiervon mit höherem Schwierigkeitsgrad wäre das Bilden einer Wortkette, bei der Gewürze gefunden werden müssen, die den letzten Buchstaben des vorherigen Wortes als Anfangsbuchstaben haben, z. B.: Salz - Zimt - Thymian - Nelke usw. Eine ähnliche Möglichkeit wäre der Einsatz eines Wörter-Spiels, bei dem ein Begriff vorgegeben wird und mit jedem Buchstaben dieses Begriffes ein Wort aus dem Themenbereich gefunden werden muss.

\footnotetext{
${ }^{23}$ Selbstverständlich müssen bzw. können hierbei mehr Gewürze als die gerade neu gelernten und jetzt zu wiederholenden zum Einsatz kommen.
} 


$\begin{array}{llllllllllll}1 & \text { S } & \text { A } & \text { U } & \text { E } & \text { R } & \text { A } & \text { M } & \text { P } & \text { E } & \text { R } & \\ 2 & \text { A } & \text { N } & \text { I } & \text { S } & & & & & & & \\ 3 & \text { L } & \text { O } & \text { R } & \text { B } & \text { E } & \text { E } & \text { R } & & & & \\ 4 & \text { B } & \text { O } & \text { H } & \text { N } & \text { E } & \text { N } & \text { K } & \text { R } & \text { A } & \text { U } & \text { T } \\ 5 & \text { E } & \text { S } & \text { T } & \text { R } & \text { A } & \text { G } & \text { O } & \text { N } & & & \\ 6 & \text { I } & \text { N } & \text { G } & \text { W } & \text { E } & \text { R } & & & & & \end{array}$

Darüber hinaus würden sich hier natürlich auch für die Zielgruppe „realistischere" Aufgabenstellungen anbieten. Ein Beispiel für eine übertragene Aufgabenstellung wäre die Ermittlung von Preisen der gelernten Produktbezeichnungen. Diese Spielvariante würde sich besonders als Wiederholungsphase nach der Vermittlung mehrerer Themenbereiche anbieten, könnte also in unserem Fall neben Gewürzbezeichnungen auch weitere Nahrungsmittel mit einbeziehen. Jeder Spieler enthält eine Einkaufsliste, auf der aus insgesamt 50 Produkten fünf notiert sind. Außerdem wird ein Arbeitsblatt mit Abbildungen (aber nicht Bezeichnungen) aller Produkte zusammengestellt, das beschriftet werden soll, sowie ein Lösungsblatt auf dem die genauen und somit zu erratenden Preise und die Produktbezeichnungen sind. Gewonnen hat derjenige, der am genauesten die Preise auf seiner Einkaufsliste schätzen konnte.

Daran anschließen könnte sich „Das Überraschungs-Menü“. Mit Hilfe eines Einkaufszettels stellen Kleingruppen oder Paare im Plenum ein Menü vor. Gewonnen hat die Gruppe, für deren Menü sich die meisten Teilnehmer begeistern können. Die Arbeitsanweisung hierzu könnte lauten: Als Grundzutaten für ein Abendessen haben Sie alle neu gelernten Gewürzbezeichnungen zur Verfügung, außerdem Salz, Pfeffer, Butter oder Öl zum Brutzeln. Denken Sie sich jetzt ein Menü aus, für das Sie einkaufen gehen! Bedingung ist nur, dass Sie nicht mehr als zehn Produkte kaufen und mindestens fünf der neu gelernten Gewürze benutzen! Für die Übungs- und Wiederholungsphase gäbe es unzählige Spielvarianten. Hier können neben den bereits genannten auch komplexere Brettspiele, Quartetts, Quizspiele wie Varianten von „Wer wird Millionär?“, Buchstabenspiele wie Scrabble, Varianten zu bestimmten Themenbereichen von Stadt, Land, Fluss, Tabu-ähnliche Spiele usw. entwickelt werden.

\section{4 Übertragbarkeit des Verfahrens auf andere Wortschatzbereiche}

Prinzipiell lassen sich die vorgeschlagenen Arbeitsformen und insbesondere die vorgestellte Form der Bedeutungsvermittlung auf jegliche Wortschatzfelder übertragen.

Eine vergleichbare Materialienerstellung für Wortschatz, der sich bildlich darstellen lässt, dürfte transparent und klar sein. Aber natürlich kann man vergleichbare Verfahren auch für die Vermittlung von Verben, die sich bei Fachwortschatz z. B. oft über entsprechende Objekte (also Substantive) darstellen lassen, einfüh- 
ren. Beispielsweise hat Henryk Dominczak (1997) Materialien für den praktischen Fachsprachenunterricht für die Bereiche Handwerk, Technik, Biologie und Medizin zusammengestellt. Auch er arbeitet zur Erklärung, also zur Bedeutungsvermittlung, mit Bildern. Allerdings werden bei ihm die Bilder direkt mit dem deutschen Begriff präsentiert. Im Beispiel Handwerk verbindet er aber die visuelle Darstellung von Substantiven (z. B. Schraube, Stiftschraube) mit den entsprechenden Tätigkeiten, also Verben, z. B.: „die Schraube anziehen/lösen“.

Bei Abstrakta bzw. abstrakten Begriffen sind Visualisierungen natürlich nur über Symbole möglich, wenn diese (halbwegs) eindeutig verstanden werden können. Hier bieten sich aber andere Präsentationsverfahren an, wieder versehen mit kleinen Rätseln bzw. Merkhilfen zur Entschlüsselung. Ein Beispiel hierfür wäre die Erklärung aus dem Kontext. Der zu lernende Begriff wird im Kontext in mehreren Sätzen präsentiert, allerdings in ,ge-x-ter“ Form. Die Beispielsätze müssen dabei so gewählt sein, dass sie eindeutig sind, also die Bedeutung des ,ge-x-ten“ Wortes zu entschlüsseln ist, ohne das Wort an sich zu kennen oder zu benennen. Zur Entschlüsselung des gesuchten Wortes können wieder die präsentierten Rätselformen genutzt werden.

Auch wenn dieses Verfahren insbesondere im Bereich der Materialerstellung als recht zeitaufwändig erscheinen mag, dürfte sich dieser „Aufwand“ wirklich lohnen, wenn es auf diese Weise gelingt, dass „wichtiger" Wortschatz, der unbedingt gelernt werden soll, nicht nur gut gelernt, sondern auch tatsächlich behalten wird.

\section{$5 \quad$ Tipps für die Unterrichtspraxis}

Abschließend möchte ich noch einige Tipps für die Unterrichtspraxis geben:

Spiele am besten einmal kurz vormachen (Spielregeln zu erklären ist nicht einfach und braucht viel Zeit).

$>$ Wer öfter im Unterricht spielt bzw. spielen lässt: Kleine Sammlung an Spielmaterialien (Spielfiguren, Würfel, Eieruhren usw.) anlegen. Praktisch zum Aufbewahren dafür sind kleine Werkzeugkästchen aus dem Baumarkt!

$>$ Sinn und Zweck von Spielen (zumindest nach dem Spiel) transparent machen! Dies ist besonders wichtig für Teilnehmer, die Bedenken gegen das Lernen mittels Spiel haben, dieses nicht ernst nehmen bzw. nicht als seriös empfinden! Auch Überprüfungen des Wissensstandes bzw. Lernzuwachses vor und nach dem Spieleinsatz können zum Abbau von Bedenken gegen den Spieleinsatz beitragen.

$>$ Bei der Erstellung von Spielen die Teilnehmer einbeziehen bzw. diese Spiele erstellen lassen!

$>$ Ein Charakteristikum von Spielen ist der Aspekt des Gewinns (und Verlierens). Spiele werden lebendiger, und ggf. auch eher als echte Spiele akzeptiert, wenn es auch im Unterrichtsspiel etwas zu gewinnen gibt. Dabei 
muss es nicht um materielle Dinge gehen, auch ein besonders schönes Wort, ein Gedicht oder ähnliche symbolische Dinge können als Preise zu Verfügung stehen.

\section{Literatur}

Barberis, Paola /Bruno Elena: Deutsch im Hotel - Neubearbeitung, Teil 1:

Gespräche führen, Ismaning: Hueber 2000.

Barberis, Paola / Bruno, Elena: Deutsch im Hotel - Neubearbeitung, Teil 2: Korrespondenz, Ismaning: Hueber 2001.

Cohen, Ulrike: Zimmer frei. Deutsch im Hotel, Berlin: Langenscheidt 2000.

Cohen, Ulrike: Herzlich willkommen. Deutsch in Restaurant und Tourismus, Berlin: Langenscheidt 2001.

Jentges, Sabine: „Sprachlernspiele: Mit Spielen Sprachen lernen“. In: Jung, Udo O. H. (Hrgs.): Praktische Handreichungen für Fremdsprachenlehrer. 4., vollständig neu bearbeitete Auflage, Frankfurt: Lang 2006, 94-99.

Jentges, Sabine: Effektivität von Sprachlernspielen, Baltmannsweiler: Schneider Hohengehren 2007.

Kleinschroth, Robert: Sprachen lernen: der Schlüssel zur richtigen Technik, Reinbek bei Hamburg: Rowohlt 1992.

Klippel, Friederike: Spieltheoretische und pädagogische Grundlagen des Lernspieleinsatzes im Fremdsprachenunterricht, Frankfurt: Lang 1980.

Lennert, Joachim: Culinary Guidebook Germany. A Culinary Journey through Germany, Berlin: Langenscheidt 2003.

Lévy-Hillerich, Dorothea: Kommunikation im Beruf: Kommunikation im Tourismus, Kursbuch, Berlin: Cornelsen 2005.

Macedonia, Manuela: Sprachspiele. Tipps \& Ideen zum Sprachenlernen, Frankfurt: Scriptor/Cornelsen 2000.

Mensmann, Tanja: Sprachlernspiele im DaF-Unterricht (Diplomarbeit), Marburg: Philipps-Universität 2006.

Schaetzing, Edgar E.: Fachwörterbuch für Hotellerie und Gastronomie. Deutsch Englisch / English - German, Frankfurt: Deutscher Fachverlag 2006.

Small, Ernest: Culinary Herbs, Ottawa: Research Press 1997.

Stellfeld, Elke: Zu Schreibspielen als Sprachlernspiele im

Fremdsprachenunterricht des mittleren Schulalters (Russischunterricht), 
Magdeburg: Fakultät für Geistes-, Sozial- und Erziehungswissenschaften der Otto-von-Guericke-Universität 1994.

\section{Weiterführende Hinweise auf neuere Spielesammlungen}

Dreke, Michael / Salgueiro, S. Prestes: Wechselspiel junior - Bilder und mehr. Interaktive Arbeitsblätter für junge Deutschlernende, Berlin: Langenscheidt 2002.

Dreke, Michael: Wortschatz \& Grammatik. Üben mit Bildern, Berlin: Langenscheidt 2004.

Koliander-Bayer, Claudia \& Faistauer, Renate: Spiele für den

Fremdsprachenunterricht. Arbeitsunterlagen für den

Fremdsprachenunterricht, Wien: Pädagogische Arbeits- und Forschungsstelle (PAF) des Verbandes österreichischer Volkshochschulen 1999.

Macedonia, Manuela: Sprachspiele 2. Tipps \& Ideen zum Sprachenlernen, Linz: Veritas 2004.

Pfau, Anita / Schmidt, Ann (Hrgs.): 22 Brettspiele - Deutsch als Fremdsprache, Stuttgart: Klett 2001.

Piel, Alexandra: Sprache(n) lernen mit Methode. 170 Sprachspiele für den Deutsch- und Fremdsprachenunterricht, Mülheim a.d.R.: Verlag an der Ruhr 2002.

Prange, Lisa: 44 Sprechspiele für Deutsch als Fremdsprache, Ismaning: Hueber 2000.

Rinvolucri, Mario / Davis, Paul: 66 Grammatik-Spiele Deutsch als Fremdsprache, Stuttgart: Klett 2002.

Sánchez, Juana / Sanz, Carlos / Dreke, Michael: Spielend Deutsch lernen. Interaktive Arbeitsblätter für Anfänger und Fortgeschrittene, Berlin: Langenscheidt 2004.

Scheller, Ingo: Szenisches Spiel. Handbuch für die pädagogische Praxis, Berlin: Cornelsen 1998.

Spier, Anne: Mit Spielen Deutsch lernen. Spiele und spielerische Übungsformen für den Unterricht mit ausländischen Kindern, Jugendlichen und Erwachsenen, Frankfurt: Scriptor/Cornelsen 2002.

Tselikas, Elektra: Dramenpädagogik im Sprachunterricht, Zürich: Orell Füssli 1999.

Westenfelder, Frank: Sprech- und Grammatikspiele DaF/DaZ, Hamburg: AOL 2005.

Wicke, Rainer: Handeln und Sprechen im Deutschunterricht. Spielerische Sprachaktivitäten, Ismaning: Hueber 1995. 
Chance Deutsch - solide Sprachkenntnisse erhöhen die Chancen in der Schule, im Studium und in der Arbeitswelt. Ausgehend von dieser Erkenntnis haben sich zahlreiche Fachwissenschaftler/innen und Vertreter/innen aus der Praxis auf der 34. Jahrestagung des Fachverbands Deutsch als Fremdsprache an der Leibniz Universität Hannover mit spezifischen Fragen und Überlegungen zu Wegen der Optimierung beschäftigt. In diesem Band sind die Beiträge nach den Themenschwerpunkten der Tagung wiedergegeben:

- Integrationskurse DaZ; · Testen und Prüfen in DaZ / DaF; · Schreiben und Sprechen in den Wissenschaften; · DaZ und Mehrsprachigkeit; · Forum DaF

Der Band bildet damit eine Basis für eine weiter führende konstruktive Diskussion zwischen Wissenschaftlern aus den verschiedenen für das Fach relevanten Forschungsrichtungen und Lehrenden an verschiedenen Institutionen, die auf unterschiedlichen Niveaustufen unterrichten und verschiedene Adressatengruppen im Blick haben müssen.

\section{fadaf $f$}

fadaf.de 